\title{
Assessment of a composite CC2/DFT procedure for calculating 0-0 excitation energies of organic molecules
}

\author{
Baswanth Oruganti, Changfeng Fang and Bo Durbeej \\ Journal Article
}

Tweet

N.B.: When citing this work, cite the original article.

This is an electronic version of an article published in:

Baswanth Oruganti, Changfeng Fang and Bo Durbeej, Assessment of a composite CC2/DFT procedure for calculating $0-\& \# 128 ; \& \# 147 ; 0$ excitation energies of organic molecules, Molecular Physics, 2016.

Molecular Physics is available online at informaworldTM:

http://dx.doi.org/10.1080/00268976.2016.1235736

Copyright: Taylor \& Francis: STM, Behavioural Science and Public Health Titles http://www.tandf.co.uk/journals/default.asp

Postprint available at: Linköping University Electronic Press

http://urn.kb.se/resolve?urn=urn:nbn:se:liu:diva-132610

I.U UNNGENGS 


\section{Assessment of a composite CC2/DFT procedure for calculating 0-0 excitation energies of organic molecules}

Baswanth Oruganti ${ }^{\dagger}$, Changfeng Fang ${ }^{\dagger}$ and Bo Durbeej*

Division of Theoretical Chemistry, IFM, Linköping University, Linköping, Sweden

${ }^{\dagger}$ These authors contributed equally to the work.

*Corresponding author. E-mail: bodur@ifm.liu.se. Tel: +46-(0)13-282497.

Supplemental data for this article can be accessed at http://dx.doi.org/10.1080/x. 


\begin{abstract}
The task to assess the performance of quantum chemical methods in describing electronically excited states has in recent years started to shift from calculation of vertical $\left(\Delta E_{\mathrm{ve}}\right)$ to calculation of 0-0 excitation energies $\left(\Delta E_{00}\right)$. Here, based on a set of 66 excited states of organic molecules for which high-resolution experimental $\Delta E_{00}$ energies are available and for which the approximate coupled-cluster singles and doubles (CC2) method performs particularly well, we explore the possibility to simplify the calculation of CC2-quality $\Delta E_{00}$ energies using composite procedures that partly replace CC2 with more economical methods. Specifically, we consider procedures that employ CC2 only for the $\Delta E_{\mathrm{ve}}$ part and density functional theory methods for the cumbersome excited-state geometry optimizations and frequency calculations required to obtain $\Delta E_{00}$ energies from $\Delta E_{\mathrm{ve}}$ ones. The results demonstrate that it is indeed possible to both closely (to within $0.06-0.08 \mathrm{eV}$ ) and consistently approximate 'true' $\mathrm{CC} 2 \Delta E_{00}$ energies in this way, especially when CC2 is combined with hybrid density functionals. Overall, the study highlights the unexploited potential of composite procedures, which hitherto have found widespread use mostly in ground-state chemistry, to also play an important role in facilitating accurate studies of excited states.
\end{abstract}

\title{
Keywords
}

excited states $\bullet$ quantum chemistry $\bullet 0-0$ excitation energies $\bullet$ composite procedures 


\section{Introduction}

A common approach for benchmarking the performance of quantum chemical methods in calculating electronically excited states of molecular systems is to focus on vertical excitation energies $\left(\Delta E_{\mathrm{ve}}\right)$ and use as reference data either experimental absorption maxima or $\Delta E_{\mathrm{ve}}$ energies obtained with high-level ab initio methods. Although this approach has been invaluable for establishing how well different methods in the field describe different types of excited states [1-7], it rests on two key assumptions. First, it is assumed that the nuclei remain fixed in their ground-state geometric configuration during the electronic transition (the Franck-Condon principle). Second, it is assumed that vibrational effects do not influence the positions of experimental absorption maxima. Also, when the reference data are taken from high-level calculations, it has to be presupposed that these are indeed accurate [2]. In a similar vein, the use of reference data from experimental spectra measured in solution calls for a treatment of solvent effects, which makes it less straightforward to directly assess the intrinsic quality of the quantum chemical description of an excited state. In this regard, gas-phase data are preferable for benchmarking purposes, despite the many methodological possibilities [8-10] to model solvatochromic shifts [11-14].

Instead of calculating $\Delta E_{\mathrm{ve}}$ energies and comparing these with experimental absorption maxima, a few excited-state benchmarks have rather focused on adiabatic or $0-0$ excitation energies [15-29], herein denoted $\Delta E_{\text {ad }}$ and $\Delta E_{00}$, respectively. As illustrated in Figure 1, these are energy differences between ground and excited states at their respective equilibrium geometries, without $\left(\Delta E_{\text {ad }}\right)$ or with $\left(\Delta E_{00}\right)$ inclusion of the lowest vibrational level. Although increasing the computational cost by requiring excited-state geometry optimizations and frequency calculations to obtain zero-point vibrational energy (ZPVE) corrections, such studies are nowadays feasible for a wide variety of excited-state methods thanks to efficient implementations of analytic gradients [15,30-48]. In one of the first extensive $\Delta E_{00}$-based benchmarks, Furche and coworkers [21] first surveyed high-resolution gas-phase experiments to compile a large set (91 molecules, 109 states) of $\Delta E_{00}$ energies, including both organic molecules and inorganic maingroup and transition-metal compounds. Then, they tested how well these energies are reproduced by time-dependent density functional theory (TD-DFT) [49-56], using six different functionals, and two correlated ab initio methods: the approximate coupled-cluster singles and doubles (CC2) 
[57] and second-order algebraic diagrammatic construction approximation $(\operatorname{ADC}(2))[58,59]$ methods.

In light of the emphasis on relatively small molecules in Furche's study [21], another extensive $\Delta E_{00}$-based benchmark by Hättig and co-workers [25] rather dealt with medium-sized and large organic molecules. For such systems, a similarly large set (66 states) of high-resolution gas-phase $\Delta E_{00}$ energies was assembled and used to assess the performance of TD-DFT with the B3LYP hybrid functional [60-62], CC2, ADC(2) and the spin-scaled SCS-CC2 and SOS-CC2 variants of $\mathrm{CC} 2[18,46,63]$. Also recognizing the value of an investigation focused on larger molecules, Jacquemin and co-workers [23] considered a 40-member set of excited states of conjugated dyes, which they subjected to TD-DFT calculations exclusively. In contrast to the studies by Furche [21] and Hättig [25], however, these authors' interest lay in the reproducibility of experimental $\Delta E_{00}$ energies in solution, as determined from the intersection of measured absorption and fluorescence bands. This work was later followed by a second extensive $\Delta E_{00^{-}}$ based study of medium-sized and large conjugated dyes in solution [29], but this time investigating the performance of ab initio methods like $\mathrm{CC} 2$ and $\mathrm{ADC}(2)$.

As shown by some research groups [64-73] but not easily afforded in broad benchmark studies, it is also possible to go beyond the $\Delta E_{00}$ description of excited states and successfully simulate - in both the gas phase and in solution - the full vibronic structure of an electronic absorption spectrum. Such simulations are very helpful for determining which type of transition (vertical or $0-0$ ) best represents the experimental absorption maximum [68].

In a recent study, we contributed to the benchmarking of excited-state methods from a different angle. Namely, instead of primarily focusing our interest on the accuracy with which excited-state methods reproduce experimental absorption maxima or experimental $\Delta E_{00}$ energies, we investigated to what extent calculated differences between $\Delta E_{\mathrm{ve}}, \Delta E_{\mathrm{ad}}$ and $\Delta E_{00}$ energies vary with the choice of method [26]. To the best of our knowledge, this was the first investigation of its kind to be reported for a large set of excited states and methods. Specifically, for a slightly reduced version of Furche's 109-member set of excited states [21], $\Delta E_{\mathrm{ve}}, \Delta E_{\mathrm{ad}}$ and $\Delta E_{00}$ energies were calculated using TD-DFT with seven different functionals, CC2 and the configuration interaction singles (CIS) method [31]. Thereby, it was found that while the average standard deviations for the $\Delta E_{\mathrm{ve}}, \Delta E_{\mathrm{ad}}$ and $\Delta E_{00}$ energies obtained with the nine methods amount to $\sim 0.4$ $\mathrm{eV}$, the corresponding values for the $\Delta \Delta E_{\mathrm{ad}}=\Delta E_{\mathrm{ad}}-\Delta E_{\mathrm{ve}}$ and $\Delta \Delta E_{00}=\Delta E_{00}-\Delta E_{\mathrm{ad}}$ energy 
differences are only 0.10 and $0.02 \mathrm{eV}$, respectively [26]. These results are a clear, quantitative indication that while calculating excitation energies is a notoriously challenging and methoddependent task in quantum chemistry, determining the photochemically relevant $\Delta \Delta E_{\text {ad }}$ (excitedstate relaxation energy) and $\Delta \Delta E_{00}$ (difference in ZPVE corrections between the excited state and the ground state) quantities is much less sensitive to the choice of method.

A frequent observation regarding single-reference organic excited states in $\Delta E_{00}$-based benchmarks is that TD-DFT, which is currently the most popular tool for modeling excited states, cannot fully match the performance of CC2 [25,26,29]. Indeed, for such states, CC2 typically shows excellent agreement $(\sim 0.1 \mathrm{eV})$ with experimental $\Delta E_{00}$ energies $[25,26,29]$. However, although CC2 is one of the most cost-effective approaches to account for dynamic electron correlation effects in excited states, it is still an expensive method compared to TDDFT, especially when it comes to performing excited-state geometry optimizations and frequency calculations. Therefore, it is desirable to develop and assess computational procedures capable of yielding $\Delta E_{00}$ energies of $\mathrm{CC} 2$ quality at a lower cost, particularly for large molecules.

In this work, we investigate to what extent the aforementioned method-insensitivity of $\Delta \Delta E_{\mathrm{ad}}$ and $\Delta \Delta E_{00}$ documented [26] for Furche's benchmark set [21] of relatively small organic and inorganic molecules can be exploited to obtain CC2-quality $\Delta E_{00}$ energies for the mediumsized and large (up to 78 atoms) organic chromophores contained in Hättig's benchmark set [25], without having to use $\mathrm{CC} 2$ for excited-state geometry optimizations and frequency calculations. Specifically, we explore how well 'true' $\mathrm{CC} 2 \Delta E_{00}$ energies, denoted $\Delta E_{00}(\mathrm{CC} 2)$ and obtained using CC2 for all parts of the required calculations as (see Figure 1)

$\Delta E_{00}(\mathrm{CC} 2)=\Delta E_{\mathrm{ve}}(\mathrm{CC} 2)+\Delta \Delta E_{\mathrm{ad}}(\mathrm{CC} 2)+\Delta \Delta E_{00}(\mathrm{CC} 2)$

are approximated when CC2 is used for the $\Delta E_{\mathrm{ve}}$ and $\Delta \Delta E_{\mathrm{ad}}$ terms or the $\Delta E_{\mathrm{ve}}$ term only, and a cheaper method ' $\mathrm{X}$ ' is used to calculate the remaining term(s):

$\Delta E_{00}\left(\mathrm{CC} 2 / \mathrm{X}^{\prime}\right)=\Delta E_{\mathrm{ve}}(\mathrm{CC} 2)+\Delta \Delta E_{\mathrm{ad}}(\mathrm{CC} 2)+\Delta \Delta E_{00}(\mathrm{X})$

and 
$\Delta E_{00}\left(\mathrm{CC} 2 / \mathrm{X}^{\prime \prime}\right)=\Delta E_{\mathrm{ve}}(\mathrm{CC} 2)+\Delta \Delta E_{\mathrm{ad}}(\mathrm{X})+\Delta \Delta E_{00}(\mathrm{X})$,

respectively. To this end, we first calculate $\Delta E_{\mathrm{ve}}, \Delta E_{\mathrm{ad}}$ and $\Delta E_{00}$ energies and the corresponding $\Delta \Delta E_{\text {ad }}=\Delta E_{\text {ad }}-\Delta E_{\mathrm{ve}}$ and $\Delta \Delta E_{00}=\Delta E_{00}-\Delta E_{\text {ad }}$ energy differences for each of the 66 states in Hättig's benchmark set [25] using CC2 as well as TD-DFT with the BP86 [74,75], PBE0 [76], M06-2X [77], M06-HF [78], CAM-B3LYP [79] and $\omega B$ 97X-D [80,81] functionals and CIS. Then, we evaluate how well $\Delta E_{00}\left(\mathrm{CC} 2 / \mathrm{X}^{\prime}\right)$ and $\Delta E_{00}\left(\mathrm{CC} 2 / \mathrm{X}^{\prime \prime}\right)$ approximate $\Delta E_{00}(\mathrm{CC} 2)$ when the latter eight methods are combined with $\mathrm{CC} 2$ according to equations (2) and (3). Furthermore, besides identifying ways to simplify the calculation of accurate $\Delta E_{00}$ energies, we also present valuable new data on the performance of six other density functionals than B3LYP - the only functional hitherto applied to Hättig's benchmark set [25] - in calculating experimentally available gas-phase $\Delta E_{00}$ energies of organic chromophores.

Finally, we note that although the use of composite procedures that combine different quantum chemical methods to obtain reliable $\Delta E_{00}$ energies at a lower computational cost is not a new endeavor, with earlier contributions from Grimme [16,19,20], Hättig [25] and Jacquemin $[29,82-84]$ and their co-workers, the merits of such procedures for studies of excited states are nonetheless much less explored than their usefulness for studies of electronic ground states, which is well-established, especially in the calculation of accurate thermochemistry [85-88]. Thus, a study like the present one is timely, particularly since it involves a set of excited states with quite uniform character that (as we will see) are similarly described by the methods considered and for which composite procedures therefore have the greatest potential. In this light, the study should be viewed as an extensive initial assessment of the merits of composite

procedures under favorable circumstances, rather than as a broad test of the general applicability of these approaches to different kinds of excited states.

\section{Computational details}

\subsection{Composition of the benchmark set}


The 66-member set of excited states of organic molecules considered is presented in Table 1 and in Figures S1-S4 of the Supplemental data. For ease of presentation, the molecules were divided into two groups: group I (comprising aromatic heterocyclic compounds, shown in Figure S1) and group II (comprising aromatic [or aliphatic] hydrocarbons and substituted aromatic hydrocarbons, shown in Figure S3). For example, of biological relevance, group I includes three porphyrins, of which Zn-tetraphenylporphine (77 atoms) and tetraphenylporphine (78 atoms) are the largest systems in the study. Some of the 66 states pertain to different conformational isomers of the same chromophore, such as the $2^{1} \mathrm{~A}$ state of seven isomers of tryptamine (see Figure S2) and the $2^{1} \mathrm{~A}$ state of the gauche isomer and the $1^{1} \mathrm{~A}^{\prime \prime}$ state of the anti isomer of $3 \mathrm{P}$ propionic acid (see Figure S4). In each of these cases, the assignment of the experimental $\Delta E_{00}$ energies to the different isomers was done already in Ref. [25] and the original experimental studies cited therein. Importantly, all of the 66 states are the lowest excited singlet state $\left(\mathrm{S}_{1}\right)$ of the chromophore/isomer in question, and can be described as originating from a one-electron excitation out of a closed-shell single-reference ground state $\left(\mathrm{S}_{0}\right)$. As such, the states are qualitatively well-described by the TD-DFT, CIS and CC2 methods employed in this work, and do not require a generally applicable excited-state method like complete active space secondorder perturbation theory (CASPT2) [89,90] or multi-reference CI (MRCI) [91,92].

\subsection{Electronic structure methods}

To evaluate how accurately $\Delta E_{00}\left(\mathrm{CC} 2 / \mathrm{X}^{\prime}\right)$ and $\Delta E_{00}\left(\mathrm{CC} 2 / \mathrm{X}^{\prime \prime}\right)$ computed through equations (2) and (3) reproduce $\Delta E_{00}(\mathrm{CC} 2)$ obtained through equation (1), $\Delta E_{\mathrm{ve}}, \Delta E_{\mathrm{ad}}$ and $\Delta E_{00}$ energies and the corresponding $\Delta \Delta E_{\mathrm{ad}}=\Delta E_{\mathrm{ad}}-\Delta E_{\mathrm{ve}}$ and $\Delta \Delta E_{00}=\Delta E_{00}-\Delta E_{\mathrm{ad}}$ energy differences were calculated using nine different methods: BP86, B3LYP, PBE0, M06-2X, M06-HF, CAMB3LYP, $\omega$ B97X-D, CIS and CC2. Throughout all calculations, the TZVP valence triple- $\zeta$ plus polarization basis set [93] was employed (as deemed appropriate from complementary calculations with the larger aug-cc-pVTZ basis set [94,95]). Thereby, the geometries, electronic energies and ZPVE corrections needed to obtain the 'true' $\Delta E_{\mathrm{ve}}, \Delta E_{\mathrm{ad}}$ and $\Delta E_{00}$ energies at a particular level of theory were computed at that very level, with a few exceptions specified below. All CC2 calculations were carried out within the resolution-of-the-identity approximation, and with an auxiliary TZVP basis set [96]. The omission of diffuse functions 
through calculations with the TZVP basis set is motivated by the observation in Ref. [25] that, for the same set of excited states but using different methods (see Introduction), the effect of such basis functions on electronic energies is consistently smaller than $0.06 \mathrm{eV}$ at the triple- $\zeta$ level. This result reflects the compact valence character that these excited states exhibit. It should also be re-iterated that the main focus of this work is not to achieve the best possible agreement with experimental data, which would certainly require diffuse functions, but rather to compare $\Delta E_{00}\left(\mathrm{CC} 2 / \mathrm{X}^{\prime}\right)$ and $\Delta E_{00}\left(\mathrm{CC} 2 / \mathrm{X}^{\prime \prime}\right)$ with $\Delta E_{00}(\mathrm{CC} 2)$ at a reasonable basis-set level.

All seven density functionals used are based on the generalized gradient approximation (GGA), and have shown good performance in many TD-DFT benchmarks [1,3,5-7]. BP86 is a pure GGA without any exact Hartree-Fock (HF) exchange in the exchange-correlation potential; B3LYP and PBE0 are global hybrid GGAs that include a fixed fraction of HF exchange (20\% and 25\%, respectively); M06-2X (54\%) and M06-HF (100\%) are global hybrid meta-GGAs from the Minnesota family of functionals [97] that additionally include a dependence on the kinetic energy density; and CAM-B3LYP and $\omega$ B97X-D are range-separated hybrid GGAs whose potentials have the correct long-range asymptotic behavior, owing to the inclusion of a variable amount of HF exchange (larger at long interelectronic distances) by means of a suitable $[98,99]$ partitioning of the Coulomb operator into short-range and long-range parts.

$\Delta E_{\mathrm{ve}}, \Delta E_{\mathrm{ad}}$ and $\Delta E_{00}$ energies were calculated in the following way. First, ground- and excited-state geometries were optimized with each of the nine methods using analytic gradients not only for the DFT, HF and CC2 [100] ground-state optimizations, but also for the TD-DFT [42], CIS [31] and CC2 [39] excited-state optimizations. Based on the resulting geometries, ground- and excited-state frequency calculations were then carried out to obtain ZPVE corrections for both states, and to confirm that the geometries exhibit real vibrational frequencies only and hence correspond to potential-energy minima. While the DFT, HF and CIS frequencies were calculated with analytic second-derivative methods, the TD-DFT and CC2 frequencies were determined through numerical differentiation of analytic gradients using finite differences. Finally, $\Delta E_{\mathrm{ve}}$ energies were obtained by performing TD-DFT, CIS and CC2 excited-state singlepoint calculations on the DFT, HF and $\mathrm{CC} 2$ ground-state geometries, respectively. $\Delta E_{\text {ad }}$ energies, in turn, were obtained as (pure electronic) energy differences between ground and excited states at their respective equilibrium geometries, and $\Delta E_{00}$ energies were obtained by adding ZPVE corrections to the $\Delta E_{\text {ad }}$ energies. 
As for the goal to derive the 'true' $\Delta E_{\mathrm{ve}}, \Delta E_{\text {ad }}$ and $\Delta E_{00}$ energies at a specific level of theory by executing all calculations of geometries, electronic energies and ZPVE corrections at that very level, this was achievable in all cases but for the CC2 $\Delta E_{00}$ energies of the five largest molecules in the benchmark set: porphycene, porphine, chlorin, Zn-tetraphenylporphine and tetraphenylporphine. For these molecules, it is simply too expensive to determine ZPVE corrections numerically at the CC2/TZVP level. Therefore, for porphycene, porphine and chlorin, the $\mathrm{CC} 2$ ground- and excited-state geometries and ZPVE corrections were calculated with a smaller auxiliary SVP basis set [96]. For Zn-tetraphenylporphine and tetraphenylporphine, however, numerical frequency calculations are not feasible with this basis set either. As a workaround, the CC2/TZVP ZPVE corrections for these two molecules were instead taken from B3LYP/TZVP calculations.

All calculations were carried out with the GAUSSIAN 09 [101] and TURBOMOLE 6.3 $[102,103]$ (for CC2 calculations with the RICC2 module [104]) suites of programs.

\section{Results and discussion}

\section{1. $\Delta E_{00}$ energies with different methods}

Before analyzing how the $\Delta E_{00}\left(\mathrm{CC} 2 / \mathrm{X}^{\prime}\right)$ and $\Delta E_{00}\left(\mathrm{CC} 2 / \mathrm{X}^{\prime \prime}\right)$ energies of the 66 states compare to the $\Delta E_{00}(\mathrm{CC} 2)$ energies obtained from $\mathrm{CC} 2$ calculations exclusively, it is worthwhile to first evaluate how accurately the nine methods considered reproduce the corresponding experimental $\Delta E_{00}$ energies by themselves. This is done in Table 2, which compares the full set of calculated 'true' $\Delta E_{00}$ energies for each method with the experimental data assembled by Hättig and coworkers [25]. Provided is also a statistical analysis of the results in terms of the methods' mean absolute errors (MAEs), maximum absolute errors (MaxAEs) and mean signed errors (MSEs) relative to the experimental values.

Starting with the results over the full benchmark set, it is clear that none of the density functionals can rival the average accuracy and robustness achieved by $\mathrm{CC} 2$, whose MAE and MaxAE values amount to 0.11 and $0.22 \mathrm{eV}$, respectively. In terms of MAEs, the best-performing functionals are the B3LYP $(0.20 \mathrm{eV})$ and PBE0 $(0.24 \mathrm{eV})$ global hybrids, followed by the 
$\omega B$ 97X-D $(0.32 \mathrm{eV})$ and CAM-B3LYP $(0.33 \mathrm{eV})$ range-separated hybrids. The M06-2X and M06-HF meta-GGA global hybrids, in turn, have MAEs of 0.36 and $0.57 \mathrm{eV}$, respectively, that fall on either side of the $0.40 \mathrm{eV}$ MAE shown by the pure BP86 functional. Thus, even though M06-HF through the inclusion of full HF exchange is more broadly applicable than B3LYP and PBE0 to different types of excited states - including Rydberg and charge-transfer states [77,78] that pose a considerable challenge to TD-DFT [52,55,105-108] - this functional is by some margin the least accurate functional for the current benchmark set of low-lying organic valence states. Since the MAE for M06-HF is $0.21 \mathrm{eV}$ larger than the MAE for M06-2X (with 54\% HF exchange), which in turn is $0.12-0.16 \mathrm{eV}$ larger than the MAEs for PBE0 (25\%) and B3LYP $(20 \%)$, the inclusion of successively larger fractions of HF exchange beyond the B3LYP and PBE0 values appears to have a negative effect on the accuracy of calculated $\Delta E_{00}$ energies of such states.

Notably, it is well-documented that a $20-25 \%$ fraction of HF exchange in a hybrid functional is also what yields the best performance for the calculation of $\Delta E_{\mathrm{ve}}$ energies of singlet organic valence states using TD-DFT [5], with increasing amounts of HF exchange typically having a blue-shifting effect. As can be inferred from the MSE values in Table 2, a similar effect is present in the calculation of $\Delta E_{00}$ energies. Specifically, the MSE is negative for BP86 ( -0.39 $\mathrm{eV}$ ), which thus tends to underestimate these $\Delta E_{00}$ energies, but increasingly positive for B3LYP (0.05 eV), PBE0 (0.16 eV), M06-2X (0.33 eV) and M06-HF (0.55 eV). For CIS, the MSE is a substantial $1.08 \mathrm{eV}$. On a positive note, however, CIS is the only method whose errors are 'perfectly' systematic, because the $\Delta E_{00}$ estimates are without exception larger than their experimental counterparts (i.e., MSE $=\mathrm{MAE}$ ).

Given that the excited states are of valence type, it is perhaps not surprising that CAMB3LYP and $\omega$ B97X-D show larger MAEs than B3LYP and PBE0, as the range separation of the Coulomb operator used in the former methods is primarily aimed at improving the description of long-range charge-transfer excitations [79-81]. It is possible that the CAM-B3LYP and wB97XD MAEs would have been smaller if, in a simplified computational procedure, the nine sets of $\Delta E_{00}$ energies had been obtained from nine sets of singlepoint calculations performed using a common set of geometries and ZPVE corrections (obtained with, e.g., B3LYP). In this regard, Rohrdanz and Herbert have shown that it is indeed difficult to assign a value to the rangeseparation parameter employed by range-separated functionals that is simultaneously suitable for 
both excitation energies and ground-state properties [109]. Nonetheless, we believe that the most pertinent assessment of the 'true' performance of a method in calculating $\Delta E_{00}$ energies is to use the method not only for singlepoint calculations, but also for geometry optimizations and frequency calculations. Notably, although CAM-B3LYP and $\omega$ B97X-D have larger MAEs than B3LYP and PBE0, a comparison of the MaxAEs - 0.56 eV (CAM-B3LYP and wB97X-D), 0.65 $\mathrm{eV}$ (PBE0) and $0.72 \mathrm{eV}$ (B3LYP) - indicates that the former methods are more robust, albeit by no means as robust as CC2 (whose MaxAE is only $0.22 \mathrm{eV}$ ).

By comparing the overall MAEs for the full benchmark set with (also given in Table 2) the MAEs within groups I and II of the benchmark set, it is found that the overall CC2 > B3LYP/PBE0 > $>$ B97X-D/CAM-B3LYP > M06-2X/M06-HF > CIS performance ordering applies separately to these two groups as well. Also, with one notable exception, each method shows a smaller (by $0.03-0.16 \mathrm{eV}$ ) MAE for the heterocyclic compounds of group I, than for the hydrocarbons and substituted hydrocarbons of group II. The outlier in this pattern is BP86, whose MAE of $0.60 \mathrm{eV}$ within group I is markedly larger than its MAE of $0.27 \mathrm{eV}$ within group II. One strongly contributing factor to the large MAE of BP86 within group I, and thus to the comparatively poor performance of this method for the full benchmark set (MAE of $0.40 \mathrm{eV}$ ), is that it severely underestimates the $\Delta E_{00}$ energies of the $2^{1} \mathrm{~A}$ state of the seven different tryptamine isomers included in group I (see Figure S2). Specifically, for each of these isomers, BP86 underestimates the experimental $\Delta E_{00}$ energy by at least $1.15 \mathrm{eV}$. As a consequence, if the tryptamine isomers are excluded from the benchmark set, the group I and overall BP86 MAEs are reduced by 0.24 (from 0.60 to 0.36 ) and 0.10 (from 0.40 to 0.30 ) eV, respectively, whereas the corresponding MAEs of all other methods are affected by at most 0.03 and $0.02 \mathrm{eV}$, respectively. In fact, without tryptamine, the overall BP86 MAE of $0.30 \mathrm{eV}$ is surpassed only by CC2 (0.11 eV), B3LYP (0.20 eV) and PBE0 (0.26 eV).

It is also worthwhile to compare the overall statistics for how well the nine methods employed in this work perform in calculating $\Delta E_{00}$ energies with the corresponding statistics documented in previous benchmarks investigating other methods and/or chemical systems. Comparing with the study by Hättig and co-workers [25], who considered the same exact 66member set of organic excited states (but kept a clear focus on ab initio methods and carried out TD-DFT calculations with B3LYP alone), the present results reaffirm their finding that CC2 is favored over TD-DFT by at least $\sim 0.1 \mathrm{eV}$ for such systems. Comparing also with our own recent 
results [26] for 96 excited states of smaller organic and inorganic compounds obtained with the same nine methods used herein, it is noteworthy that the present MAEs offer a ranking of the methods that is identical to the ranking offered by the MAEs found in that study: CC2 $(0.11 / 0.19$ eV), B3LYP (0.20/0.24), PBE0 (0.24/0.26), wB97X-D (0.32/0.27), CAM-B3LYP (0.33/0.27), M06-2X (0.36/0.30), BP86 (0.40/0.42), M06-HF (0.57/0.50) and CIS (1.08/0.88) [26]. Accordingly, B3LYP and PBE0 appear especially suitable density functionals for calculating $\Delta E_{00}$ energies.

Finally, comparing with the TD-DFT benchmark by Jacquemin and co-workers [23], who considered 40 excited states of medium-sized and large conjugated dyes in solution, the $0.24 \mathrm{eV}$ MAE of PBE0 reported in the present study is close to the value of $0.22 \mathrm{eV}$ found in their study. For other functionals tested in both studies, the differences in MAEs are slightly larger: 0.20 vs. $0.27 \mathrm{eV}$ for B3LYP; 0.33 vs. $0.25 \mathrm{eV}$ for CAM-B3LYP; and $0.36 \mathrm{vs}$. $0.26 \mathrm{eV}$ for M06-2X.

\subsection{Performance of the composite procedures}

We now turn to analyzing how well the $\Delta E_{00}\left(\mathrm{CC} 2 / \mathrm{X}^{\prime}\right)$ and $\Delta E_{00}\left(\mathrm{CC} 2 / \mathrm{X}^{\prime \prime}\right)$ energies, obtained by combining CC2 calculations with calculations using BP86, B3LYP, PBE0, M06-2X, M06-HF, CAM-B3LYP, $\omega$ B97X-D and CIS according to equations (2) and (3), approximate the 'true' $\Delta E_{00}(\mathrm{CC} 2)$ energies obtained when $\mathrm{CC} 2$ is used for all parts of the required calculations. This is done in Figures 2 and 3, which respectively show the absolute errors (AEs) of $\Delta E_{00}\left(\mathrm{CC} 2 / \mathrm{X}^{\prime}\right)$ and $\Delta E_{00}\left(\mathrm{CC} 2 / \mathrm{X}^{\prime \prime}\right)$ relative to $\Delta E_{00}(\mathrm{CC} 2)$ for each of the 66 states in the benchmark set.

Starting with the results for the $\Delta E_{00}\left(\mathrm{CC} 2 / \mathrm{X}^{\prime}\right)$ scheme in which TD-DFT or CIS replaces CC2 for the calculation of the $\Delta \Delta E_{00}$ term of $\Delta E_{00}=\Delta E_{\mathrm{ve}}+\Delta \Delta E_{\mathrm{ad}}+\Delta \Delta E_{00}$, Figure 2 shows that the AEs introduced by this procedure consistently average to very small values (MAEs of 0.03$0.04 \mathrm{eV}$ ) over the 66 states. Thus, for states of the current type it is generally possible to compute the expensive $\Delta \Delta E_{00}$ term with a cheap method, without it affecting the quality of the final $\Delta E_{00}$ energy.

Continuing with the results for the $\Delta E_{00}\left(\mathrm{CC} 2 / \mathrm{X}^{\prime \prime}\right)$ scheme in which also the $\Delta \Delta E_{\mathrm{ad}}$ term is obtained with TD-DFT or CIS, Figure 3 reveals that the AEs remain small even after this step is taken. This holds true especially for the hybrid functionals (MAEs of 0.06-0.08 eV). 
Accordingly, for a clear majority of the 66 states it is perfectly feasible to compute $\Delta E_{00}$ energies of $\mathrm{CC} 2$ quality by employing $\mathrm{CC} 2$ only for the vertical $\Delta E_{\mathrm{ve}}$ term, and resorting to hybrid functionals for both the $\Delta \Delta E_{00}$ and $\Delta \Delta E_{\text {ad }}$ terms. This, we believe, is the first time that such a trend is extensively validated for a variety of different density functionals in one single study, and helps reinforce previous positive results on whether one specific functional can partly replace a correlated ab initio method in accurate $\Delta E_{00}$ calculations $[25,29,82-84]$. This also means that photochemical problems whose modeling requires reliable calculation of the $\Delta \Delta E_{00}$ and $\Delta \Delta E_{\text {ad }}$ terms, such as understanding isotope effects in excited states [110,111], are more well-suited for cheap methods than one may at first envision.

At this point, it may be re-emphasized that all three terms of $\Delta E_{00}(\mathrm{CC} 2), \Delta E_{00}\left(\mathrm{CC} 2 / \mathrm{X}^{\prime}\right)$ and $\Delta E_{00}\left(\mathrm{CC} 2 / \mathrm{X}^{\prime \prime}\right)$ have consistently been calculated with a single basis set lacking diffuse functions (TZVP), without adopting the common practice to perform singlepoint calculations with a larger basis set. This procedure allows for a balanced comparison of $\Delta E_{00}\left(\mathrm{CC} 2 / \mathrm{X}^{\prime}\right)$ and $\Delta E_{00}\left(\mathrm{CC} 2 / \mathrm{X}^{\prime \prime}\right)$ to $\Delta E_{00}(\mathrm{CC} 2)$ at a reasonable basis-set level, and has been prioritized over comparing $\Delta E_{00}\left(\mathrm{CC} 2 / \mathrm{X}^{\prime}\right)$ and $\Delta E_{00}\left(\mathrm{CC} 2 / \mathrm{X}^{\prime \prime}\right)$ to as accurate $\Delta E_{00}(\mathrm{CC} 2)$ energies as possible. However, it is also of interest to use $\Delta E_{00}(\mathrm{CC} 2)$ energies obtained with diffuse functions as reference. For the present benchmark set, such energies are available from Hättig's original study [25], which used the aug-cc-pVTZ basis set [94,95] for CC2 singlepoint calculations on CC2/TZVPP ground- and excited-state geometries. Since the $\Delta E_{\mathrm{ve}}(\mathrm{CC} 2)$ term needed to compute $\Delta E_{00}\left(\mathrm{CC} 2 / \mathrm{X}^{\prime}\right)$ and $\Delta E_{00}\left(\mathrm{CC} 2 / \mathrm{X}^{\prime \prime}\right)$ is not included in the data reported by Hättig, this term was for each of the 66 states re-calculated using the aug-cc-pVTZ basis set, so as to enable an appropriate comparison with Hättig's $\Delta E_{00}(\mathrm{CC} 2)$ energies. The results of this comparison are given in Table $\mathrm{S} 1$ of the Supplemental data. As can be seen, the MAEs of $\Delta E_{00}\left(\mathrm{CC} 2 / \mathrm{X}^{\prime}\right)$ and $\Delta E_{00}\left(\mathrm{CC} 2 / \mathrm{X}^{\prime \prime}\right)$ relative to Hättig's $\Delta E_{00}(\mathrm{CC} 2)$ energies are consistently very similar to the original MAEs obtained when all calculations are performed with the TZVP basis set, with a maximum discrepancy of $0.02 \mathrm{eV}$ only. This is a clear indication that the conclusions drawn regarding the usefulness of the $\Delta E_{00}\left(\mathrm{CC} 2 / \mathrm{X}^{\prime}\right)$ and $\Delta E_{00}\left(\mathrm{CC} 2 / \mathrm{X}^{\prime \prime}\right)$ schemes are not biased by the choice of basis set.

Returning to the results in Figures 2 and 3, there are also a few outlier states with quite large MaxAEs where especially the $\Delta E_{00}\left(\mathrm{CC} 2 / \mathrm{X}^{\prime \prime}\right)$ scheme faces problems. Given the number of states considered, such outliers are expected and will be further discussed below. First, however, it is 
pertinent to ask why $\Delta E_{00}\left(\mathrm{CC} 2 / \mathrm{X}^{\prime}\right)$ and $\Delta E_{00}\left(\mathrm{CC} 2 / \mathrm{X}^{\prime \prime}\right)$ are overall so surprisingly good approximations to $\Delta E_{00}(\mathrm{CC} 2)$ for the current set of excited states. Of course, it should be clearly pointed out that we have deliberately focused our investigation on states with single-reference character whose descriptions by the different methods are (as we will see) qualitatively similar and for which composite procedures therefore have the greatest potential. Notwithstanding, an extensive quantitative assessment of the performance of composite procedures under these favorable circumstances is much desirable, before exploring their general applicability to more variable sets of excited states in future work.

From their very definitions, it follows directly that the reason why the $\Delta E_{00}\left(\mathrm{CC} 2 / \mathrm{X}^{\prime}\right)$ and $\Delta E_{00}\left(\mathrm{CC} 2 / \mathrm{X}^{\prime \prime}\right)$ schemes perform well is a lesser method-dependence in calculating $\Delta \Delta E_{\text {ad }}$ and $\Delta \Delta E_{00}$ values than in calculating the $\Delta E_{\mathrm{ve}}, \Delta E_{\text {ad }}$ and $\Delta E_{00}$ excitation energies themselves, which in turn stems from a systematic cancellation of errors. Thus, it is illuminating to establish these relationships quantitatively, which we have done in Figure 4 and Table 3 using the methoddependence of $\Delta E_{\mathrm{ve}}$ as reference (we could just as well have used $\Delta E_{\text {ad }}$ or $\Delta E_{00}$ for this purpose; this choice does not affect the analysis). For each of the 66 excited states in the benchmark set, Figure 4 compares standard deviations $\sigma(X)$ (SDs), evaluated as

$\sigma(X)=\sqrt{\frac{\sum(x-\bar{x})^{2}}{n}}$,

for the calculated $\Delta \Delta E_{\text {ad }}$ and $\Delta \Delta E_{00}$ energy differences with the corresponding SD for the calculated $\Delta E_{\mathrm{ve}}$ energies. In each case, the SDs pertain to the variation between all nine methods employed for the calculations. The raw data underlying the analysis are given in Tables S2 (group I of the benchmark set) and S3 (group II) of the Supplemental data. Table 3, in turn, lists the average SDs (denoted ASDs) for the $\Delta E_{\mathrm{ve}}, \Delta \Delta E_{\text {ad }}$ and $\Delta \Delta E_{00}$ calculations within groups I and II separately, and within the full benchmark set (i.e., groups I and II merged together). Thus, the ASDs in group I are obtained by averaging the SDs for all states in group I, and so on.

From Table 3, the ASD for the calculation of $\Delta E_{\mathrm{ve}}$ energies is $0.39 \mathrm{eV}$ for the full benchmark set, and is only slightly different in the two sub-groups: $0.41 \mathrm{eV}$ for the heterocyclic compounds of group I and $0.38 \mathrm{eV}$ for the hydrocarbons of group II. These values illustrate the 
notorious method-dependency plaguing the calculation of molecular excitation energies in quantum chemistry. Notably, however, the ASDs for $\Delta \Delta E_{\text {ad }}$ and $\Delta \Delta E_{00}$ over the full benchmark set are markedly smaller, amounting to a mere 0.06 and $0.02 \mathrm{eV}$, respectively. The same goes for the corresponding ASDs over the individual sub-groups, which fall within 0.05-0.08 $\left(\Delta \Delta E_{\mathrm{ad}}\right)$ and $0.02-0.03 \mathrm{eV}\left(\Delta \Delta E_{00}\right)$. These results solidify that accurate estimates of $\Delta \Delta E_{\mathrm{ad}}$ and $\Delta \Delta E_{00}$ energy differences are well-amenable to cheap methods, which is why $\Delta E_{00}\left(\mathrm{CC} 2 / \mathrm{X}^{\prime}\right)$ and $\Delta E_{00}\left(\mathrm{CC} 2 / \mathrm{X}^{\prime \prime}\right)$ on the whole approximate $\Delta E_{00}(\mathrm{CC} 2)$ so well.

It could be argued that it is more noteworthy that $\Delta \Delta E_{\mathrm{ad}}$ varies little with the choice of computational method, than that the same holds true for $\Delta \Delta E_{00}$, which is simply the difference in ZPVE corrections between the excited state and the ground state. However, as shown on geometric grounds in Table S4 of the Supplemental data, there is a large degree of similarity between the methods in their descriptions of the excited states, enabled by the fact that all states have single-reference character, which helps explain why also the predicted $\Delta \Delta E_{\text {ad }}$ excited-state relaxation energies are similar.

Finally, it is also of interest to explore if certain criteria can be established that help anticipate beforehand whether the $\Delta E_{00}\left(\mathrm{CC} 2 / \mathrm{X}^{\prime}\right)$ and $\Delta E_{00}\left(\mathrm{CC} 2 / \mathrm{X}^{\prime \prime}\right)$ schemes can be successfully applied to a particular excited state, and that also help explain the origin of the aforementioned outlier states in Figures 2 and 3. An attempt to achieve this is reported in Figure S5 of the Supplemental data, focusing on the $\Delta E_{00}\left(\mathrm{CC} 2 / \mathrm{X}^{\prime \prime}\right)$ scheme (whose errors are larger). Specifically, first a subset of states was selected for which each state is such that the MAE relative to $\Delta E_{00}(\mathrm{CC} 2)$ for the eight methods complementing $\mathrm{CC} 2$ in the $\Delta E_{00}\left(\mathrm{CC} 2 / \mathrm{X}^{\prime \prime}\right)$ scheme exceeds a certain threshold, as defined in the Supplemental data. Second, for each of these states, this MAE value, denoted MAE-X", was associated with the corresponding geometric 'similarity measures' from Table S4 that quantify the variation in calculated excited-state bond lengths between the methods. Then, the MAE-X" values were plotted as functions of these measures in Figure S5. Notably, with quite reasonable $R^{2}$ values of $0.72-0.77$, the resulting plots indicate that $\Delta E_{00}\left(\mathrm{CC} 2 / \mathrm{X}^{\prime \prime}\right)$ will come close to $\Delta E_{00}(\mathrm{CC} 2)$ provided that the methods yield similar geometries of the excited state in question. Thus, it appears useful to perform precisely this test before employing a composite procedure to estimate the $\Delta E_{00}$ energy of a single-reference organic excited state. 


\section{3. $\Delta \Delta E_{a d}$ and $\Delta \Delta E_{00}$ energy differences for different chemical systems}

In this section we briefly assess how calculated $\Delta \Delta E_{\text {ad }}$ and $\Delta \Delta E_{00}$ energy differences vary from one chemical system to another, with some notable findings. Figure 5 shows histograms of the $\Delta \Delta E_{\text {ad }}$ and $\Delta \Delta E_{00}$ values obtained for the 66 states in the benchmark set. For each state, the histograms consider the average $\Delta \Delta E_{\text {ad }}$ and $\Delta \Delta E_{00}$ values for that state as obtained from nine separate sets of calculations using BP86, B3LYP, PBE0, M06-2X, M06-HF, CAM-B3LYP, $\omega \mathrm{B} 97 \mathrm{X}-\mathrm{D}, \mathrm{CIS}$ and CC2. Histograms for the individual methods are presented in Figures S6 and S7 of the Supplemental data. Since the statistics of these histograms are almost identical to what Figure 5 shows, they will not be discussed in further detail.

From Figure 5 (a), the variation in $\Delta \Delta E_{00}$ between the states is seen to be extraordinary small, with a SD of only $0.04 \mathrm{eV}$ despite that a broad range of molecular sizes is covered (from 10 atoms in pyrazine to 78 atoms in tetraphenylporphine). The mean $\Delta \Delta E_{00}$ value for the 66 states is $-0.14 \mathrm{eV}$, which, given the small $\mathrm{SD}$, appears a very reasonable a priori estimate of this quantity for excited states of similar organic molecules that preclude quantum chemical investigation. Qualitatively, the clear tendency of $\Delta \Delta E_{00}$ to be negative (i.e, the tendency of $\Delta E_{00}$ excitation energies to be smaller than $\Delta E_{\text {ad }}$ ones) can be rationalized in terms of weakened bonding in the excited state, whereby the associated potential energy surface is flattened and the ZPVE correction reduced with respect to the situation in the ground state.

As for the $\Delta \Delta E_{\text {ad }}$ histogram in Figure 5 (b), finally, this negative quantity also shows surprisingly little variation between the states. Specifically, the $\mathrm{SD}$ amounts to $0.09 \mathrm{eV}$, which is larger than the variation in $\Delta \Delta E_{00}$, yet sufficiently small that the mean value of $-0.24 \mathrm{eV}$ holds some predictive power.

\section{Conclusions}

Benchmark studies are indispensable in computational chemistry for assessing the applicability of different methods in the field to different chemical problems. Calculating electronically excited states is one example where such studies are especially important, because of the many 
challenges associated with describing excited states from first principles. In this work, we have contributed to the benchmarking of excited-state methods from a different angle than the usual one. Specifically, rather than having as main goal to assess how well different methods reproduce reference $\Delta E_{\mathrm{ve}}$ or $\Delta E_{00}$ energies, we have explored the possibility to simplify the calculation of accurate $\Delta E_{00}$ energies by means of composite procedures. In these, a cheap method replaces a more expensive one for the cumbersome excited-state geometry optimizations and frequency calculations that are needed to estimate the $\Delta \Delta E_{\mathrm{ad}}$ (excited-state relaxation energy) and $\Delta \Delta E_{00}$ (difference in ZPVE corrections between the excited state and the ground state) terms of $\Delta E_{00}=\Delta E_{\mathrm{ve}}+\Delta \Delta E_{\mathrm{ad}}+\Delta \Delta E_{00}$.

To this end and with the goal to reduce the cost to obtain $\Delta E_{00}$ energies of CC2 quality, we consider a benchmark set of 66 single-reference excited states of medium-sized and large organic molecules [25] and calculate the three different terms in $\Delta E_{00}$ using on the one hand CC2 and on the other less expensive TD-DFT (with the BP86, B3LYP, PBE0, M06-2X, M06-HF, CAMB3LYP and $\omega$ B97X-D functionals) and CIS methods. Although composite procedures that combine different levels of theory are not a new idea in excited-state quantum chemistry $[16,19,20,25,29,82-84]$, their applicability to excited states has not been as thoroughly explored as is the case for problems in ground-state chemistry, where such approaches are integral tools for, e.g., the calculation of accurate thermochemistry [85-88].

The resulting data show that it is perfectly possible to replace CC2 with TD-DFT or CIS for the $\Delta \Delta E_{00}$ part of the calculations, without deteriorating the quality of the final $\Delta E_{00}$ energy. Indeed, irrespective of which of the alternative eight methods is invoked for this part, the MAE of the corresponding $\Delta E_{00}$ energies for the 66 states relative to the $\Delta E_{00}$ energies obtained when CC2 is used for all parts of the calculations, is consistently a mere 0.03-0.04 eV. Furthermore, the MAEs remain small even when also the $\Delta \Delta E_{\mathrm{ad}}$ term is computed with TD-DFT or CIS instead of CC2, particularly so for the hybrid functionals (MAEs of 0.06-0.08 eV). In fact, for a clear majority of the 66 states CC2-quality $\Delta E_{00}$ energies can be calculated by employing CC2 only for the vertical $\Delta E_{\mathrm{ve}}$ term, and using any of the hybrid functionals for both of the $\Delta \Delta E_{\mathrm{ad}}$ and $\Delta \Delta E_{00}$ terms. To the best of our knowledge, this is the first time that such a trend is supported by computational evidence derived for both a statistically meaningful number of chemical systems and a variety of different density functionals. 
Having thus demonstrated the potential of composite procedures for studies of singlereference organic excited states, it is a natural goal of future research in the field to also test the performance of composite procedures that combine more versatile methods than CC2 and TDDFT for applications to sets of more variable and challenging types of excited states. Relevant systems that come to mind are, for example, excited states of transition-metal compounds [112] and polar intramolecular charge-transfer excited states of dual-fluorescent aromatic compounds [113].

Through the calculations, we have also been able to present valuable new benchmark data on how closely TD-DFT, CIS and CC2 reproduce experimentally available $\Delta E_{00}$ energies of organic chromophores, when the $\Delta E_{00}$ energies produced by each method are exclusively based on geometries, electronic energies and ZPVE corrections computed with that method. Thereby, CC2 is found to yield impressively accurate and robust results, with MAE and MaxAE values of only 0.11 and $0.22 \mathrm{eV}$, respectively. With a MAE of $0.20 \mathrm{eV}$, the best-performing functional is B3LYP, followed by PBE0 (0.24 eV), $\omega$ B97X-D (0.32 eV) and CAM-B3LYP $(0.33 \mathrm{eV})$.

Finally, it is also found that the $\Delta \Delta E_{\mathrm{ad}}$ and $\Delta \Delta E_{00}$ quantities vary remarkably little from one system in the benchmark set to another. Actually, the SD for the $\Delta \Delta E_{00}$ values of the 66 states is only $0.04 \mathrm{eV}$, which means that this quantity falls within a very narrow range around the mean value of $-0.14 \mathrm{eV}$. This mean value can therefore be regarded as a 'typical' $\Delta \Delta E_{00}$ value for these and similar systems. Equaling $0.09 \mathrm{eV}$, the SD for the $\Delta \Delta E_{\mathrm{ad}}$ values is also small. 


\section{Acknowledgments}

We acknowledge the Swedish National Infrastructure for Computing (SNIC) for providing resources at the National Supercomputer Centre (NSC) at Linköping University.

\section{Disclosure statement}

No potential conflict of interest was reported by the authors.

\section{Funding}

This work was supported by the Swedish Research Council [grant number 621-2011-4353]; the Olle Engkvist Foundation [grant number 2014/734]; the Carl Trygger Foundation [grant number CTS 15:134]; the Wenner-Gren Foundations; and Linköping University. 


\section{References}

[1] D. Jacquemin, E.A. Perpète, G.E. Scuseria, I. Ciofini, and C. Adamo, J. Chem. Theory Comput. 4, 123 (2008).

[2] M. Schreiber, M.R. Silva-Junior, S.P.A. Sauer, and W. Thiel, J. Chem. Phys. 128, 134110 (2008).

[3] M.R. Silva-Junior, M. Schreiber, S.P.A. Sauer, and W. Thiel, J. Chem. Phys. 129, 104103 (2008).

[4] S.P.A. Sauer, M. Schreiber, M.R. Silva-Junior, and W. Thiel, J. Chem. Theory Comput. 5, 555 (2009).

[5] D. Jacquemin, V. Wathelet, E.A. Perpète, and C. Adamo, J. Chem. Theory Comput. 5, 2420 (2009).

[6] M. Caricato, G.W. Trucks, M.J. Frisch, and K.B. Wiberg, J. Chem. Theory Comput. 6, 370 (2010).

[7] S.S. Leang, F. Zahariev, and M.S. Gordon, J. Chem. Phys. 136, 104101 (2012).

[8] C.J. Cramer and D.G. Truhlar, Chem. Rev. 99, 2161 (1999).

[9] J. Tomasi, B. Mennucci, and R. Cammi, Chem. Rev. 105, 2999 (2005).

[10] I. Carnimeo, C. Cappelli, and V. Barone, J. Comput. Chem. 36, 2271 (2015).

[11] R. Rajamani and J. Gao, J. Comput. Chem. 23, 96 (2002).

[12] M. Parac, M. Doerr, C.M. Marian, and W. Thiel, J. Comput. Chem. 31, 90 (2010).

[13] A. Marini, A. Muñoz-Losa, A. Biancardi, and B. Mennucci, J. Phys. Chem. B 114, 17128 (2010).

[14] A.V. Marenich, C.J. Cramer, D.G. Truhlar, C.A. Guido, B. Mennucci, G. Scalmani, and M.J. Frisch, Chem. Sci. 2, 2143 (2011).

[15] F. Furche and R. Ahlrichs, J. Chem. Phys. 117, 7433 (2002).

[16] S. Grimme and E.I. Izgorodina, Chem. Phys. 305, 223 (2004).

[17] D. Rappoport and F. Furche, J. Chem. Phys. 122, 064105 (2005).

[18] A. Hellweg, S.A. Grün, and C. Hättig, Phys. Chem. Chem. Phys. 10, 4119 (2008).

[19] L. Goerigk, J. Moellmann, and S. Grimme, Phys. Chem. Chem. Phys. 11, 4611 (2009).

[20] L. Goerigk and S. Grimme, J. Chem. Phys. 132, 184103 (2010).

[21] R. Send, M. Kühn, and F. Furche, J. Chem. Theory Comput. 7, 2376 (2011). 
[22] M. Uppsten and B. Durbeej, J. Comput. Chem. 33, 1892 (2012).

[23] D. Jacquemin, A. Planchat, C. Adamo, and B. Mennucci, J. Chem. Theory Comput. 8, 2359 (2012).

[24] A. Chantzis, A.D. Laurent, C. Adamo, and D. Jacquemin, J. Chem. Theory Comput. 9, 4517 (2013).

[25] N.O.C. Winter, N.K. Graf, S. Leutwyler, and C. Hättig, Phys. Chem. Chem. Phys. 15, 6623 (2013).

[26] C. Fang, B. Oruganti, and B. Durbeej, J. Phys. Chem. A 118, 4157 (2014).

[27] D. Jacquemin, B. Moore, II, A. Planchat, C. Adamo, and J. Autschbach, J. Chem. Theory Comput. 10, 1677 (2014).

[28] L. Barnes, S. Abdul-Al, and A.-R. Allouche, J. Phys. Chem. A 118, 11033 (2014).

[29] D. Jacquemin, I. Duchemin, and X. Blase, J. Chem. Theory Comput. 11, 5340 (2015).

[30] Y. Osamura, Y. Yamaguchi, and H.F. Schaefer, III, J. Chem. Phys. 77, 383 (1982).

[31] J.B. Foresman, M. Head-Gordon, J.A. Pople, and M.J. Frisch, J. Phys. Chem. 96, 135 (1992).

[32] J.F. Stanton and J. Gauss, J. Chem. Phys. 100, 4695 (1994).

[33] N. Yamamoto, T. Vreven, M.A. Robb, M.J. Frisch, and H.B. Schlegel, Chem. Phys. Lett. 250, 373 (1996).

[34] T. Nakajima and H. Nakatsuji, Chem. Phys. Lett. 280, 79 (1997).

[35] S.R. Gwaltney and R.J. Bartlett, J. Chem. Phys. 110, 62 (1999).

[36] C. Van Caillie and R.D. Amos, Chem. Phys. Lett. 308, 249 (1999).

[37] C. Van Caillie and R.D. Amos, Chem. Phys. Lett. 317, 159 (2000).

[38] H. Lischka, M. Dallos, and R. Shepard, Mol. Phys. 100, 1647 (2002).

[39] A. Köhn and C. Hättig, J. Chem. Phys. 119, 5021 (2003).

[40] P. Celani and H.-J. Werner, J. Chem. Phys. 119, 5044 (2003).

[41] S.V. Levchenko, T. Wang, and A.I. Krylov, J. Chem. Phys. 122, 224106 (2005).

[42] G. Scalmani, M.J. Frisch, B. Mennucci, J. Tomasi, R. Cammi, and V. Barone, J. Chem. Phys. 124, 094107 (2006).

[43] M. Caricato, B. Mennucci, J. Tomasi, F. Ingrosso, R. Cammi, S. Corni, and G. Scalmani, J. Chem. Phys. 124, 124520 (2006).

[44] M. Chiba, T. Tsuneda, and K. Hirao, J. Chem. Phys. 124, 144106 (2006). 
[45] Y.M. Rhee, D. Casanova, and M. Head-Gordon, J. Phys. Chem. A 113, 10564 (2009).

[46] N.O.C. Winter and C. Hättig, Chem. Phys. 401, 217 (2012).

[47] W. Györffy, T. Shiozaki, G. Knizia, and H.-J. Werner, J. Chem. Phys. 138, 104104 (2013).

[48] M.G. Delcey, L. Freitag, T.B. Pedersen, F. Aquilante, R. Lindh, and L. González, J. Chem. Phys. 140, 174103 (2014).

[49] E. Runge and E.K.U. Gross, Phys. Rev. Lett. 52, 997 (1984).

[50] R. Bauernschmitt and R. Ahlrichs, Chem. Phys. Lett. 256, 454 (1996).

[51] M. Petersilka, U.J. Gossmann, and E.K.U. Gross, Phys. Rev. Lett. 76, 1212 (1996).

[52] M.E. Casida, C. Jamorski, K.C. Casida, and D.R. Salahub, J. Chem. Phys. 108, 4439 (1998).

[53] R.E. Stratmann, G.E. Scuseria, and M.J. Frisch, J. Chem. Phys. 109, 8218 (1998).

[54] M.A.L. Marques and E.K.U. Gross, Annu. Rev. Phys. Chem. 55, 427 (2004).

[55] A. Dreuw and M. Head-Gordon, Chem. Rev. 105, 4009 (2005).

[56] M.E. Casida and M. Huix-Rotllant, Annu. Rev. Phys. Chem. 63, 287 (2012).

[57] O. Christiansen, H. Koch, and P. Jørgensen, Chem. Phys. Lett. 243, 409 (1995).

[58] J. Schirmer, Phys. Rev. A 26, 2395 (1982).

[59] J. Schirmer and A.B. Trofimov, J. Chem. Phys. 120, 11449 (2004).

[60] C. Lee, W. Yang, and R.G. Parr, Phys. Rev. B 37, 785 (1988).

[61] A.D. Becke, J. Chem. Phys. 98, 5648 (1993).

[62] P.J. Stephens, F.J. Devlin, C.F. Chabalowski, and M.J. Frisch, J. Phys. Chem. 98, 11623 (1994).

[63] N.O.C. Winter and C. Hättig, J. Chem. Phys. 134, 184101 (2011).

[64] M. Dierksen and S. Grimme, J. Chem. Phys. 120, 3544 (2004).

[65] T. Petrenko and F. Neese, J. Chem. Phys. 127, 164319 (2007).

[66] V. Barone, J. Bloino, M. Biczysko, and F. Santoro, J. Chem. Theory Comput. 5, 540 (2009).

[67] E. Kamarchik and A.I. Krylov, J. Phys. Chem. Lett. 2, 488 (2011).

[68] F.J. Avila Ferrer, J. Cerezo, E. Stendardo, R. Improta, and F. Santoro, J. Chem. Theory Comput. 9, 2072 (2013).

[69] A. Charaf-Eddin, A. Planchat, B. Mennucci, C. Adamo, and D. Jacquemin, J. Chem. Theory Comput. 9, 2749 (2013).

[70] V. Barone, M. Biczysko, M. Borkowska-Panek, and J. Bloino, ChemPhysChem 15, 3355 (2014). 
[71] B. Moore, II, A. Charaf-Eddin, A. Planchat, C. Adamo, J. Autschbach, and D. Jacquemin, J. Chem. Theory Comput. 10, 4599 (2014).

[72] D. Barton, C. König, and J. Neugebauer, J. Chem. Phys. 141, 164115 (2014).

[73] F. Santoro and D. Jacquemin, WIREs Comput. Mol. Sci. DOI: 10.1002/wcms. 1260 (2016).

[74] J.P. Perdew, Phys. Rev. B 33, 8822 (1986).

[75] A.D. Becke, Phys. Rev. A 38, 3098 (1988).

[76] C. Adamo and V. Barone, J. Chem. Phys. 110, 6158 (1999).

[77] Y. Zhao and D.G. Truhlar, Theor. Chem. Acc. 120, 215 (2008).

[78] Y. Zhao and D.G. Truhlar, J. Phys. Chem. A 110, 13126 (2006).

[79] T. Yanai, D.P. Tew, and N.C. Handy, Chem. Phys. Lett. 393, 51 (2004).

[80] J.-D. Chai and M. Head-Gordon, Phys. Chem. Chem. Phys. 10, 6615 (2008).

[81] J.-D. Chai and M. Head-Gordon, J. Chem. Phys. 128, 084106 (2008).

[82] A. Charaf-Eddin, B. Le Guennic, and D. Jacquemin, RSC Adv. 4, 49449 (2014).

[83] P. Boulanger, S. Chibani, B. Le Guennic, I. Duchemin, X. Blase, and D. Jacquemin, J. Chem. Theory Comput. 10, 4548 (2014).

[84] B. Le Guennic and D. Jacquemin, Acc. Chem. Res. 48, 530 (2015).

[85] L.A. Curtiss, K. Raghavachari, and J.A. Pople, J. Chem. Phys. 98, 1293 (1983).

[86] J.A. Montgomery, Jr., M.J. Frisch, J.W. Ochterski, and G.A. Petersson, J. Chem. Phys. 110, 2822 (1999).

[87] J.M.L. Martin and G. de Oliveira, J. Chem. Phys. 111, 1843 (1999).

[88] A. Karton, WIREs Comput. Mol. Sci. 6, 292 (2016).

[89] K. Andersson, P.-Å. Malmqvist, B.O. Roos, A.J. Sadlej, and K. Wolinski, J. Phys. Chem. 94, 5483 (1990).

[90] K. Andersson, P.-Å. Malmqvist, and B.O. Roos, J. Chem. Phys. 96, 1218 (1992).

[91] R.J. Buenker and S.D. Peyerimhoff, Theoret. Chim. Acta 35, 33 (1974).

[92] P.G. Szalay, T. Müller, G. Gidofalvi, H. Lischka, and R. Shepard, Chem. Rev. 112, 108 (2012).

[93] A. Schäfer, C. Huber, and R. Ahlrichs, J. Chem. Phys. 100, 5829 (1994).

[94] T.H. Dunning, Jr., J. Chem. Phys. 90, 1007 (1989).

[95] R.A. Kendall, T.H. Dunning, Jr., and R.J. Harrison, J. Chem. Phys. 96, 6796 (1992).

[96] F. Weigend, M. Häser, H. Patzelt, and R. Ahlrichs, Chem. Phys. Lett. 294, 143 (1998). 
[97] Y. Zhao and D.G. Truhlar, Acc. Chem. Res. 41, 157 (2008).

[98] T. Leininger, H. Stoll, H.-J. Werner, and A. Savin, Chem. Phys. Lett. 275, 151 (1997).

[99] Y. Tawada, T. Tsuneda, S. Yanagisawa, T. Yanai, and K. Hirao, J. Chem. Phys. 120, 8425 (2004).

[100] C. Hättig, J. Chem. Phys. 118, 7751 (2003).

[101] M.J. Frisch, G.W. Trucks, H.B. Schlegel, G.E. Scuseria, M.A. Robb, J.R. Cheeseman, G. Scalmani, V. Barone, B. Mennucci, G.A. Petersson, H. Nakatsuji, M. Caricato, X. Li, H.P. Hratchian, A.F. Izmaylov, J. Bloino, G. Zheng, J.L. Sonnenberg, M. Hada, M. Ehara, K. Toyota, R. Fukuda, J. Hasegawa, M. Ishida, T. Nakajima, Y. Honda, O. Kitao, H. Nakai, T. Vreven, J.A. Montgomery, Jr., J.E. Peralta, F. Ogliaro, M. Bearpark, J.J. Heyd, E. Brothers, K.N. Kudin, V.N. Staroverov, R. Kobayashi, J. Normand, K. Raghavachari, A. Rendell, J.C. Burant, S.S. Iyengar, J. Tomasi, M. Cossi, N. Rega, J.M. Millam, M. Klene, J.E. Knox, J.B. Cross, V. Bakken, C. Adamo, J. Jaramillo, R. Gomperts, R.E. Stratmann, O. Yazyev, A.J. Austin, R. Cammi, C. Pomelli, J.W. Ochterski, R.L. Martin, K. Morokuma, V.G. Zakrzewski, G.A. Voth, P. Salvador, J.J. Dannenberg, S. Dapprich, A.D. Daniels, Ö. Farkas, J.B. Foresman, J.V. Ortiz, J. Cioslowski, and D.J. Fox, Gaussian 09 Revision D.01 (Gaussian Inc., Wallingford CT, 2009).

[102] R. Ahlrichs, M. Bär, M. Häser, H. Horn, and C. Kölmel, Chem. Phys. Lett. 162, 165 (1989).

[103] TURBOMOLE V6.3 2011, a development of University of Karlsruhe and Forschungszentrum Karlsruhe GmbH, 1989-2007, TURBOMOLE GmbH, since 2007; available at: http://www.turbomole.com (accessed January 22, 2016).

[104] C. Hättig and F. Weigend, J. Chem. Phys. 113, 5154 (2000).

[105] D.J. Tozer and N.C. Handy, J. Chem. Phys. 109, 10180 (1998).

[106] D.J. Tozer, R.D. Amos, N.C. Handy, B.O. Roos, and L. Serrano-Andrés, Mol. Phys. 97, 859 (1999).

[107] S. Hirata, C.-G. Zhan, E. Aprà, T.L. Windus, and D.A. Dixon, J. Phys. Chem. A 107, 10154 (2003).

[108] A.J.A. Aquino, D. Nachtigallova, P. Hobza, D.G. Truhlar, C. Hättig, and H. Lischka, J. Comput. Chem. 32, 1217 (2011).

[109] M.A. Rohrdanz and J.M. Herbert, J. Chem. Phys. 129, 034107 (2008). 
[110] K.C. Toh, E.A. Stojković, I.H.M. van Stokkum, K. Moffat, and J.T.M. Kennis, Proc. Natl. Acad. Sci. USA 107, 9170 (2010).

[111] A. Walter, M. Andresen, S. Jakobs, J. Schroeder, and D. Schwarzer, J. Phys. Chem. B 119, 5136 (2015).

[112] F. Neese, T. Petrenko, D. Ganyushin, and G. Olbrich, Coord. Chem. Rev. 251, 288 (2007).

[113] Z.R. Grabowski and K. Rotkiewicz, Chem. Rev. 103, 3899 (2003). 
Table 1. Groups (I and II) of molecules and excited states in the benchmark set. ${ }^{a}$

\begin{tabular}{|c|c|c|c|c|c|}
\hline Group I ${ }^{b}$ & Label $^{\mathrm{c}}$ & State & Group II $^{\mathrm{d}}$ & Label $^{\mathrm{c}}$ & State \\
\hline pyrazine & 1 & $\mathrm{~S}_{1} 1^{1} \mathrm{~A}_{\mathrm{u}}$ & tetrafluorobenzene & 1 & $\mathrm{~S}_{1} 1^{1} \mathrm{~B}_{1}$ \\
\hline 2,6-difluoropyridine & 2 & $\mathrm{~S}_{1} 1^{1} \mathrm{~B}_{2}$ & benzonitrile & 2 & $\mathrm{~S}_{1} 1^{1} \mathrm{~B}_{2}$ \\
\hline 2-fluoropyridine & 3 & $\mathrm{~S}_{1} 2^{1} \mathrm{~A}^{\prime}$ & $o$-fluorophenol, cis & 3 & $\mathrm{~S}_{1} 2^{1} \mathrm{~A}$ \\
\hline 2-hydroxypyridine & 4 & $\mathrm{~S}_{1} 2^{1} \mathrm{~A}^{\prime}$ & $o$-fluorophenol, trans & 4 & $\mathrm{~S}_{1} 2^{1} \mathrm{~A}$ \\
\hline 2-pyridone & 5 & $\mathrm{~S}_{1} 2^{1} \mathrm{~A}^{\prime}$ & $m$-fluorophenol, cis & 5 & $\mathrm{~S}_{1} 2^{1} \mathrm{~A}$ \\
\hline 2-methylpyrimidine & 6 & $\mathrm{~S}_{1} 1^{1} \mathrm{~A}^{\prime \prime}$ & $m$-fluorophenol, trans & 6 & $\mathrm{~S}_{1} 2^{1} \mathrm{~A}^{\prime}$ \\
\hline 5-methylpyrimidine & 7 & $\mathrm{~S}_{1} 1^{1} \mathrm{~A}^{\prime \prime}$ & $p$-fluorophenol & 7 & $\mathrm{~S}_{1} 2^{1} \mathrm{~A}^{\prime}$ \\
\hline 7-azaindole & 8 & $\mathrm{~S}_{1} 2^{1} \mathrm{~A}^{\prime}$ & phenylacetylene & 8 & $\mathrm{~S}_{1} 1^{1} \mathrm{~B}_{2}$ \\
\hline 7-hydroxyquinoline, cis & 9 & $\mathrm{~S}_{1} 2^{1} \mathrm{~A}^{\prime}$ & aniline & 9 & $\mathrm{~S}_{1} 1^{1} \mathrm{~A}^{\prime \prime}$ \\
\hline 7-hydroxyquinoline, trans & 10 & $\mathrm{~S}_{1} 2^{1} \mathrm{~A}$ & catechol & 10 & $\mathrm{~S}_{1} 2^{1} \mathrm{~A}$ \\
\hline 2-hydroxyquinoline, enol & 11 & $\mathrm{~S}_{1} 2^{1} \mathrm{~A}^{\prime}$ & resorcinol, isomer 1 & 11 & $\mathrm{~S}_{1} 2^{1} \mathrm{~A}$ \\
\hline 2-hydroxyquinoline, keto & 12 & $\mathrm{~S}_{1} 2^{1} \mathrm{~A}^{\prime}$ & resorcinol, isomer 2 & 12 & $\mathrm{~S}_{1} 1^{1} \mathrm{~B}_{2}$ \\
\hline dibenzofuran & 13 & $\mathrm{~S}_{1} 2^{1} \mathrm{~A}_{1}$ & resorcinol, isomer 3 & 13 & $\mathrm{~S}_{1} 1^{1} \mathrm{~A}^{\prime \prime}$ \\
\hline pyrrolo[3,2- $h]$ quinoline & 14 & $\mathrm{~S}_{1} 2^{1} \mathrm{~A}^{\prime}$ & hydroquinone, cis & 14 & $\mathrm{~S}_{1} 2^{1} \mathrm{~A}_{1}$ \\
\hline carbazole & 15 & $\mathrm{~S}_{1} 2^{1} \mathrm{~A}_{1}$ & hydroquinone, trans & 15 & $\mathrm{~S}_{1} 1^{1} \mathrm{~B}_{\mathrm{u}}$ \\
\hline tryptamine, A-ph ${ }^{\mathrm{e}}$ & 16 & $\mathrm{~S}_{1} 2^{1} \mathrm{~A}$ & salicylic acid & 16 & $\mathrm{~S}_{1} 2^{1} \mathrm{~A}^{\prime}$ \\
\hline tryptamine, A-py ${ }^{\mathrm{f}}$ & 17 & $\mathrm{~S}_{1} 2^{1} \mathrm{~A}$ & $o$-cresol, cis & 17 & $\mathrm{~S}_{1} 2^{1} \mathrm{~A}$ \\
\hline tryptamine, A-up ${ }^{\mathrm{g}}$ & 18 & $\mathrm{~S}_{1} 2^{1} \mathrm{~A}$ & $o$-cresol, trans & 18 & $\mathrm{~S}_{1} 2^{1} \mathrm{~A}$ \\
\hline tryptamine, $\mathrm{Ph}$-out ${ }^{\mathrm{h}}$ & 19 & $\mathrm{~S}_{1} 2^{1} \mathrm{~A}$ & $m$-cresol, cis & 19 & $\mathrm{~S}_{1} 2^{1} \mathrm{~A}$ \\
\hline tryptamine, $\mathrm{Ph}-\mathrm{up}^{\mathrm{i}}$ & 20 & $\mathrm{~S}_{1} 2^{1} \mathrm{~A}$ & $m$-cresol, trans & 20 & $\mathrm{~S}_{1} 2^{1} \mathrm{~A}^{\prime}$ \\
\hline tryptamine, $P y$-out ${ }^{\mathrm{j}}$ & 21 & $\mathrm{~S}_{1} 2^{1} \mathrm{~A}$ & $p$-cresol & 21 & $\mathrm{~S}_{1} 2^{1} \mathrm{~A}$ \\
\hline tryptamine, Py-up ${ }^{\mathrm{k}}$ & 22 & $\mathrm{~S}_{1} 2^{1} \mathrm{~A}$ & $o$-methoxyphenol & 22 & $\mathrm{~S}_{1} 2^{1} \mathrm{~A}$ \\
\hline porphycene & 23 & $\mathrm{~S}_{1} 2^{1} \mathrm{~A}^{\prime}$ & $m$-methoxyphenol, isomer 1 & 23 & $\mathrm{~S}_{1} 2^{1} \mathrm{~A}$ \\
\hline porphine & 24 & $\mathrm{~S}_{1} 1{ }^{1} \mathrm{~B}_{1 \mathrm{u}}$ & $m$-methoxyphenol, isomer 2 & 24 & $\mathrm{~S}_{1} 2^{1} \mathrm{~A}$ \\
\hline chlorin & 25 & $\mathrm{~S}_{1} 1^{1} \mathrm{~B}_{2}$ & $p$-methoxyphenol, cis & 25 & $\mathrm{~S}_{1} 2^{1} \mathrm{~A}$ \\
\hline Zn-tetraphenylporphine & 26 & $\mathrm{~S}_{1} 1^{1} \mathrm{~B}_{2}$ & p-methoxyphenol, trans & 26 & $\mathrm{~S}_{1} 2^{1} \mathrm{~A}$ \\
\hline \multirow[t]{13}{*}{ tetraphenylporphine } & 27 & $\mathrm{~S}_{1} 1^{1} \mathrm{~B}$ & 1-naphthol, cis & 27 & $\mathrm{~S}_{1} 2^{1} \mathrm{~A}$ \\
\hline & & & 1-naphthol, trans & 28 & $\mathrm{~S}_{1} 2^{1} \mathrm{~A}^{\prime}$ \\
\hline & & & 2-naphthol, cis & 29 & $\mathrm{~S}_{1} 2^{1} \mathrm{~A}^{\prime}$ \\
\hline & & & 2-naphthol, trans & 30 & $\mathrm{~S}_{1} 2^{1} \mathrm{~A}^{\prime}$ \\
\hline & & & 5-methoxysalicylic acid & 31 & $\mathrm{~S}_{1} 2^{1} \mathrm{~A}^{\prime}$ \\
\hline & & & $m$-dimethoxybenzene, trans & 32 & $\mathrm{~S}_{1} 2^{1} \mathrm{~A}^{\prime}$ \\
\hline & & & 3P-propionic acid, ${ }^{1}$ gauche & 33 & $\mathrm{~S}_{1} 2^{1} \mathrm{~A}$ \\
\hline & & & 3P-propionic acid, ${ }^{1}$ anti & 34 & $\mathrm{~S}_{1} 1^{1} \mathrm{~A}^{\prime \prime}$ \\
\hline & & & fluorene & 35 & $\mathrm{~S}_{1} 1^{1} \mathrm{~B}_{2}$ \\
\hline & & & phenanthrene & 36 & $\mathrm{~S}_{1} 2^{1} \mathrm{~A}_{1}$ \\
\hline & & & 2,4,6,8-decatetraene & 37 & $\mathrm{~S}_{1} 1^{1} \mathrm{~B}_{\mathrm{u}}$ \\
\hline & & & tetracene & 38 & $\mathrm{~S}_{1} 1^{1} \mathrm{~B}_{1 \mathrm{u}}$ \\
\hline & & & perylene & 39 & $\mathrm{~S}_{1} 1^{1} \mathrm{~B}$ \\
\hline
\end{tabular}

${ }^{\mathrm{a}}$ The molecules in groups I and II are depicted in Figures S1 and S3 of the Supplemental data. Symmetry labels reflect molecular geometries after excited-state relaxation. ${ }^{\mathrm{b}}$ Group I: aromatic heterocyclic compounds. ${ }^{\mathrm{c}}$ Label used in subsequent figures. ${ }^{\mathrm{d}}$ Group II: aromatic (or aliphatic) hydrocarbons and substituted aromatic hydrocarbons. ${ }^{\mathrm{e}}$ Anti-ph. ${ }^{\mathrm{f}}$ Anti-py. ${ }^{\mathrm{g}}$ Anti-up. ${ }^{\mathrm{h}}$ gauche-phenyl-out. ${ }^{\mathrm{i}}$ gauche-phenyl-up. ${ }^{j}$ gauche-pyrrole-out. ${ }^{\mathrm{k}}$ gauche-pyrrole-up. ${ }^{1}$ 3-phenyl-1-propionic acid. 
Table 2. Calculated $\Delta E_{00}$ energies for all states in the benchmark set $(\mathrm{eV})$.

Method

\begin{tabular}{|c|c|c|c|c|c|c|c|c|c|c|c|}
\hline Group/Label/Molecule & State & Exp. $^{\mathrm{a}}$ & $\begin{array}{l}\infty \\
\infty \\
\stackrel{\infty}{n}\end{array}$ & 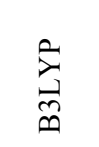 & $\begin{array}{l}0 \\
\text { 핌 } \\
\text { 口. }\end{array}$ & 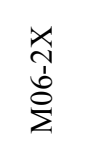 & 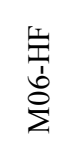 & 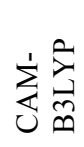 & 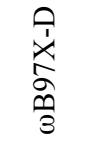 & $\tilde{u}$ & U \\
\hline I/1/pyrazine & $1^{1} A_{u}$ & 3.83 & 3.13 & 3.61 & 3.68 & 3.68 & 3.47 & 3.86 & 3.83 & 4.82 & 3.91 \\
\hline I/2/2,6-difluoropyridine & $1^{1} \mathrm{~B}_{2}$ & 4.69 & 4.72 & 5.03 & 5.13 & 5.24 & 5.43 & 5.18 & 5.17 & 6.02 & 4.79 \\
\hline I/3/2-fluoropyridine & $2^{1} \mathrm{~A}^{\prime}$ & 4.71 & 4.83 & 5.05 & 5.16 & 5.27 & 5.58 & 5.21 & 5.19 & 5.98 & 4.75 \\
\hline I/4/2-hydroxypyridine & $2^{1} \mathrm{~A}^{\prime}$ & 4.48 & 4.35 & 4.73 & 4.84 & 4.98 & 5.17 & 4.92 & 4.90 & 5.74 & 4.57 \\
\hline I/5/2-pyridone & $2^{1} \mathrm{~A}^{\prime}$ & 3.70 & 3.40 & 3.90 & 3.94 & 4.14 & 4.28 & 4.09 & 4.09 & 4.95 & 3.56 \\
\hline I/6/2-methylpyrimidine & $1^{1} \mathrm{~A}^{\prime \prime}$ & 3.78 & 3.16 & 3.61 & 3.70 & 3.76 & 3.72 & 3.88 & 3.85 & 5.16 & 3.84 \\
\hline I/7/5-methylpyrimidine & $1^{1} \mathrm{~A}^{\prime \prime}$ & 3.82 & 3.19 & 3.65 & 3.74 & 3.79 & 3.75 & 3.93 & 3.90 & 5.18 & 3.84 \\
\hline I/8/7-azaindole & $2^{1} \mathrm{~A}^{\prime}$ & 4.29 & 3.67 & 4.11 & 4.25 & 4.54 & 4.85 & 4.50 & 4.50 & 5.28 & 4.39 \\
\hline I/9/7-hydroxyquinoline & $2^{1} \mathrm{~A}^{\prime}$ & 3.82 & 3.36 & 3.78 & 3.89 & 4.15 & 4.48 & 4.12 & 4.12 & 4.94 & 3.92 \\
\hline I/10/7-hydroxyquinoline & $2^{1} \mathrm{~A}$ & 3.78 & 2.92 & 3.51 & 3.61 & 4.09 & 4.59 & 4.06 & 4.06 & 4.90 & 3.86 \\
\hline I/11/2-hydroxyquinoline & $2^{1} \mathrm{~A}^{\prime}$ & 3.89 & 3.48 & 3.84 & 3.95 & 4.20 & 4.50 & 4.17 & 4.16 & 4.94 & 4.00 \\
\hline I/12/2-hydroxyquinoline & $2^{1} \mathrm{~A}^{\prime}$ & 3.61 & 3.25 & 3.63 & 3.74 & 3.97 & 4.21 & 3.96 & 3.96 & 4.84 & 3.74 \\
\hline I/13/dibenzofuran & $2^{1} \mathrm{~A}_{1}$ & 4.17 & 3.72 & 4.15 & 4.28 & 4.55 & 4.86 & 4.52 & 4.53 & 5.51 & 4.25 \\
\hline I/14/pyrrolo[3,2-h]quinolone & $2^{1} \mathrm{~A}^{\prime}$ & 3.66 & 3.10 & 3.50 & 3.62 & 3.95 & 4.36 & 3.92 & 3.95 & 4.67 & 3.78 \\
\hline I/15/carbazole & $2^{1} \mathrm{~A}_{1}$ & 3.82 & 3.38 & 3.81 & 3.94 & 4.20 & 4.48 & 4.19 & 4.21 & 4.91 & 3.92 \\
\hline I/16/tryptamine & $2^{1} \mathrm{~A}$ & 4.32 & 3.16 & 4.14 & 4.27 & 4.56 & 4.86 & 4.54 & 4.55 & 5.26 & 4.43 \\
\hline I/17/tryptamine & $2^{1} \mathrm{~A}$ & 4.32 & 3.15 & 4.11 & 4.25 & 4.54 & 4.84 & 4.52 & 4.53 & 5.26 & 4.41 \\
\hline I/18/tryptamine & $2^{1} \mathrm{~A}$ & 4.32 & 3.17 & 4.06 & 4.21 & 4.53 & 4.85 & 4.52 & 4.52 & 5.26 & 4.41 \\
\hline I/19/tryptamine & $2^{1} \mathrm{~A}$ & 4.32 & 2.94 & 4.14 & 4.27 & 4.56 & 4.84 & 4.53 & 4.53 & 5.26 & 4.44 \\
\hline I/20/tryptamine & $2^{1} \mathrm{~A}$ & 4.32 & 2.95 & 4.01 & 4.15 & 4.48 & 4.80 & 4.48 & 4.48 & 5.23 & 4.36 \\
\hline I/21/tryptamine & $2^{1} \mathrm{~A}$ & 4.33 & 2.96 & 4.12 & 4.26 & 4.55 & 4.85 & 4.53 & 4.55 & 5.27 & 4.40 \\
\hline I/22/tryptamine & $2^{1} \mathrm{~A}$ & 4.33 & 3.04 & 4.10 & 4.25 & 4.56 & 4.86 & 4.54 & 4.57 & 5.27 & 4.44 \\
\hline I/23/porphycene & $2^{1} \mathrm{~A}^{\prime}$ & 2.00 & 1.89 & 2.10 & 2.16 & 2.13 & 2.05 & 2.08 & 2.01 & 2.51 & 2.07 \\
\hline I/24/porphine & $1^{1} \mathrm{~B}_{1 \mathrm{u}}$ & 2.02 & 2.05 & 2.20 & 2.26 & 2.25 & 2.52 & 2.19 & 2.14 & 2.73 & 2.14 \\
\hline I/25/chlorin & $1^{1} \mathrm{~B}_{2}$ & 1.97 & 2.09 & 2.19 & 2.24 & 2.20 & 2.15 & 2.15 & 2.11 & 2.57 & 2.15 \\
\hline I/26/Zn-tetraphenylporphine & $1^{1} \mathrm{~B}_{2}$ & 2.17 & 1.98 & 2.20 & 2.23 & 2.23 & 2.21 & 2.21 & 2.18 & 2.55 & 2.24 \\
\hline I/27/tetraphenylporphine & $1^{1} \mathrm{~B}$ & 1.94 & 1.87 & 2.08 & 2.12 & 2.10 & 2.32 & 2.09 & 2.02 & 2.71 & 2.00 \\
\hline $\operatorname{MAE}(\mathrm{I})^{\mathrm{b}}$ & & & 0.60 & 0.17 & 0.15 & 0.27 & 0.50 & 0.24 & 0.23 & 1.01 & 0.09 \\
\hline $\operatorname{MaxAE}(\mathrm{I})^{\mathrm{b}}$ & & & 1.38 & 0.34 & 0.45 & 0.56 & 0.87 & 0.50 & 0.48 & 1.39 & 0.17 \\
\hline MSE (I) ${ }^{\mathrm{b}}$ & & & -0.57 & -0.04 & 0.06 & 0.25 & 0.46 & 0.24 & 0.23 & 1.01 & 0.08 \\
\hline II/1/tetrafluorobenzene & $1^{1} \mathrm{~B}_{1}$ & 4.53 & 4.34 & 4.73 & 4.82 & 4.98 & 5.12 & 4.94 & 4.93 & 5.92 & 4.58 \\
\hline II/2/benzonitrile & $1^{1} \mathrm{~B}_{2}$ & 4.53 & 4.49 & 4.82 & 4.94 & 5.10 & 5.42 & 5.06 & 5.05 & 5.78 & 4.74 \\
\hline II/3/o-fluorophenol & $2^{1} \mathrm{~A}$ & 4.56 & 4.50 & 4.73 & 4.89 & 5.06 & 5.29 & 5.00 & 5.02 & 5.94 & 4.68 \\
\hline $\mathrm{II} / 4 / o$-fluorophenol & $2^{1} \mathrm{~A}$ & 4.58 & 4.44 & 4.77 & 4.89 & 5.03 & 5.17 & 4.98 & 4.98 & 5.82 & 4.63 \\
\hline II $/ 5 / m$-fluorophenol & $2^{1} \mathrm{~A}$ & 4.54 & 4.51 & 4.85 & 4.96 & 5.09 & 5.25 & 5.04 & 5.04 & 5.88 & 4.69 \\
\hline II/6/m-fluorophenol & $2^{1} \mathrm{~A}^{\prime}$ & 4.57 & 4.49 & 4.83 & 4.95 & 5.09 & 5.30 & 5.03 & 5.02 & 5.86 & 4.72 \\
\hline II/7/p-fluorophenol & $2^{1} \mathrm{~A}^{\prime}$ & 4.35 & 4.21 & 4.55 & 4.65 & 4.82 & 5.05 & 4.74 & 4.73 & 5.59 & 4.47 \\
\hline II/8/phenylacetylene & $1^{1} \mathrm{~B}_{2}$ & 4.45 & 4.31 & 4.69 & 4.83 & 5.01 & 5.29 & 4.99 & 4.98 & 5.31 & 4.67 \\
\hline II/9/aniline & $1^{1} \mathrm{~A}^{\prime \prime}$ & 4.22 & 3.95 & 4.32 & 4.44 & 4.61 & 4.78 & 4.57 & 4.58 & 5.42 & 4.40 \\
\hline II/10/catechol & $2^{1} \mathrm{~A}$ & 4.42 & 4.33 & 4.65 & 4.76 & 4.90 & 5.11 & 4.84 & 4.84 & 5.68 & 4.49 \\
\hline II/11/resorcinol & $2^{1} \mathrm{~A}$ & 4.49 & 4.41 & 4.76 & 4.88 & 4.99 & 5.15 & 4.96 & 4.95 & 5.79 & 4.57 \\
\hline II/12/resorcinol & $1^{1} \mathrm{~B}_{2}$ & 4.48 & 4.40 & 4.74 & 4.87 & 4.99 & 5.20 & 4.94 & 4.94 & 5.79 & 4.61 \\
\hline II/13/resorcinol & $1^{1} \mathrm{~A}^{\prime \prime}$ & 4.46 & 4.32 & 4.67 & 4.79 & 4.92 & 4.99 & 4.88 & 4.89 & 5.75 & 4.51 \\
\hline II/14/hydroquinone & $2^{1} \mathrm{~A}_{1}$ & 4.16 & 3.94 & 4.29 & 4.39 & 4.56 & 4.79 & 4.49 & 4.49 & 5.36 & 4.25 \\
\hline II/15/hydroquinone & $1^{1} B_{u}$ & 4.15 & 3.93 & 4.28 & 4.38 & 4.56 & 4.79 & 4.48 & 4.48 & 5.36 & 4.25 \\
\hline II/16/salicylic acid & $2^{1} \mathrm{~A}^{\prime}$ & 3.70 & 3.22 & 3.73 & 3.83 & 4.12 & 4.32 & 4.07 & 4.10 & 5.17 & 3.68 \\
\hline $\mathrm{II} / 17 / o$-cresol & $2^{1} \mathrm{~A}$ & 4.51 & 4.44 & 4.76 & 4.88 & 5.04 & 5.24 & 4.97 & 4.97 & 5.77 & 4.65 \\
\hline $\mathrm{II} / 18 / o$-cresol & $2^{1} \mathrm{~A}$ & 4.49 & 4.49 & 4.75 & 4.86 & 5.01 & 5.20 & 4.95 & 4.94 & 5.72 & 4.63 \\
\hline II $/ 19 / m$-cresol & $2^{1} \mathrm{~A}$ & 4.46 & 4.41 & 4.73 & 4.84 & 4.97 & 5.16 & 4.92 & 4.91 & 5.71 & 4.61 \\
\hline $\mathrm{II} / 20 / m$-cresol & $2^{1} \mathrm{~A}^{\prime}$ & 4.47 & 4.39 & 4.72 & 4.83 & 4.96 & 5.17 & 4.93 & 4.92 & 5.72 & 4.62 \\
\hline
\end{tabular}


Table 2. Continued.

\begin{tabular}{|c|c|c|c|c|c|c|c|c|c|c|c|}
\hline \multirow[b]{2}{*}{ Group/Label/Molecule } & \multirow[b]{2}{*}{ State } & \multirow[b]{2}{*}{ Exp. $^{\text {a }}$} & \multicolumn{9}{|c|}{ Method } \\
\hline & & & $\begin{array}{l}\infty \\
\infty \\
\stackrel{\infty}{\infty}\end{array}$ & 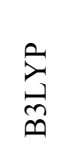 & $\begin{array}{l}\text { 워 } \\
\text { 童 }\end{array}$ & $\begin{array}{l}\underset{x}{c} \\
\text { bे } \\
\stackrel{0}{\Sigma}\end{array}$ & 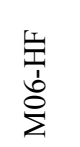 & 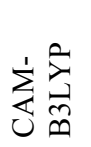 & $\begin{array}{l}\stackrel{0}{\dot{x}} \\
\hat{\alpha} \\
\frac{\hat{\sigma}}{3}\end{array}$ & $\stackrel{\Omega}{0}$ & $\underset{U}{U}$ \\
\hline $\mathrm{II} / 21 / p$-cresol & $2^{1} \mathrm{~A}$ & 4.38 & 4.38 & 4.62 & 4.72 & 4.87 & 5.06 & 4.80 & 4.80 & 5.59 & 4.53 \\
\hline II/22/o-methoxyphenol & $2^{1} \mathrm{~A}$ & 4.45 & 4.32 & 4.64 & 4.76 & 4.91 & 5.07 & 4.86 & 4.86 & 5.71 & 4.49 \\
\hline II/23/m-methoxyphenol & $2^{1} \mathrm{~A}$ & 4.46 & 4.34 & 4.68 & 4.80 & 4.93 & 4.98 & 4.89 & 4.90 & 5.74 & 4.50 \\
\hline II/24/m-methoxyphenol & $2^{1} \mathrm{~A}$ & 4.47 & 4.41 & 4.74 & 4.86 & 4.99 & 5.16 & 4.94 & 4.93 & 5.79 & 4.55 \\
\hline II/25/p-methoxyphenol & $2^{1} \mathrm{~A}$ & 4.17 & 3.96 & 4.32 & 4.42 & 4.58 & 4.83 & 4.50 & 4.49 & 5.42 & 4.23 \\
\hline II/26/p-methoxyphenol & $2^{1} \mathrm{~A}$ & 4.16 & 3.96 & 4.30 & 4.40 & 4.57 & 4.86 & 4.49 & 4.50 & 5.41 & 4.22 \\
\hline II/27/1-naphthol & $2^{1} \mathrm{~A}$ & 3.87 & 3.27 & 3.66 & 3.78 & 4.06 & 4.32 & 4.02 & 4.04 & 4.72 & 3.98 \\
\hline II/28/1-naphthol & $2^{1} \mathrm{~A}^{\prime}$ & 3.90 & 3.38 & 3.74 & 3.85 & 4.11 & 4.40 & 4.07 & 4.07 & 4.71 & 4.08 \\
\hline II/29/2-naphthol & $2^{1} \mathrm{~A}^{\prime}$ & 3.83 & 3.48 & 3.81 & 3.90 & 4.12 & 4.43 & 4.08 & 4.09 & 4.78 & 3.98 \\
\hline II/30/2-naphthol & $2^{1} \mathrm{~A}^{\prime}$ & 3.79 & 3.43 & 3.75 & 3.84 & 4.07 & 4.38 & 4.03 & 4.03 & 4.71 & 3.92 \\
\hline II/31/5-methoxysalicylic acid & $2^{1} \mathrm{~A}^{\prime}$ & 3.49 & 2.89 & 3.36 & 3.47 & 3.76 & 3.97 & 3.68 & 3.70 & 4.81 & 3.41 \\
\hline II/32/m-dimethoxybenzene & $2^{1} \mathrm{~A}^{\prime}$ & 4.43 & 4.21 & 4.59 & 4.71 & 4.87 & 5.02 & 4.81 & 4.79 & 5.68 & 4.42 \\
\hline II/33/3P-propionic acid & $2^{1} \mathrm{~A}$ & 4.67 & 3.71 & 5.04 & 5.17 & 5.25 & 5.43 & 5.22 & 5.23 & 5.92 & 4.86 \\
\hline II/34/3P-propionic acid & $1^{1} \mathrm{~A}^{\prime \prime}$ & 4.66 & 4.22 & 5.04 & 5.16 & 5.24 & 5.44 & 5.22 & 5.21 & 5.91 & 4.86 \\
\hline II/35/fluorene & $1^{1} \mathrm{~B}_{2}$ & 4.19 & 3.86 & 4.22 & 4.34 & 4.52 & 4.75 & 4.47 & 4.50 & 4.87 & 4.37 \\
\hline II/36/phenanthrene & $2^{1} \mathrm{~A}_{1}$ & 3.64 & 3.36 & 3.73 & 3.85 & 4.06 & 4.35 & 4.06 & 4.07 & 4.65 & 3.81 \\
\hline II/37/2,4,6,8-decatetraene & $1^{1} \mathrm{~B}_{\mathrm{u}}$ & 4.31 & 3.36 & 3.59 & 3.66 & 3.88 & 4.18 & 3.86 & 3.87 & 4.56 & 4.18 \\
\hline II $/ 38 /$ tetracene & $1{ }^{1} \mathrm{~B}_{1 \mathrm{u}}$ & 2.78 & 1.91 & 2.17 & 2.22 & 2.47 & 2.81 & 2.47 & 2.51 & 3.17 & 2.57 \\
\hline II/39/perylene & $1^{1} \mathrm{~B}$ & 2.98 & 2.35 & 2.62 & 2.68 & 2.94 & 3.28 & 2.93 & 2.96 & 3.51 & 2.91 \\
\hline MAE (II) ${ }^{\mathrm{b}}$ & & & 0.27 & 0.22 & 0.30 & 0.43 & 0.62 & 0.39 & 0.39 & 1.14 & 0.12 \\
\hline $\operatorname{MaxAE}(\mathrm{II})^{\mathrm{b}}$ & & & 0.96 & 0.72 & 0.65 & 0.59 & 0.90 & 0.56 & 0.56 & 1.48 & 0.22 \\
\hline $\operatorname{MSE}(\mathrm{II})^{\mathrm{b}}$ & & & -0.27 & 0.11 & 0.22 & 0.39 & 0.61 & 0.35 & 0.35 & 1.14 & 0.09 \\
\hline $\operatorname{MAE}(\mathrm{I}-\mathrm{II})^{\mathrm{b}}$ & & & 0.40 & 0.20 & 0.24 & 0.36 & 0.57 & 0.33 & 0.32 & 1.08 & 0.11 \\
\hline $\operatorname{MaxAE}(\mathrm{I}-\mathrm{II})^{\mathrm{b}}$ & & & 1.38 & 0.72 & 0.65 & 0.59 & 0.90 & 0.56 & 0.56 & 1.48 & 0.22 \\
\hline $\operatorname{MSE}(\mathrm{I}-\mathrm{II})^{\mathrm{b}}$ & & & -0.39 & 0.05 & 0.16 & 0.33 & 0.55 & 0.30 & 0.30 & 1.08 & 0.09 \\
\hline
\end{tabular}

${ }^{a}$ Experimental values compiled in Ref. [25], see also references therein. ${ }^{b}$ Mean absolute error (MAE), maximum absolute error (MaxAE) and mean signed error (MSE) relative to the experimental values for all states in the indicated group(s) of molecules. 
Table 3. ASD values for calculated $\Delta E_{\mathrm{ve}}$ energies and $\Delta \Delta E_{\mathrm{ad}}$ and $\Delta \Delta E_{00}$ energy differences for all states in different groups of the benchmark set $(\mathrm{eV}){ }^{\mathrm{a}}$

\begin{tabular}{lccc}
\hline Group(s) & $\Delta E_{\mathrm{ve}}$ & $\Delta \Delta E_{\mathrm{ad}}$ & $\Delta \Delta E_{00}$ \\
\hline I & 0.41 & 0.08 & 0.03 \\
II & 0.38 & 0.05 & 0.02 \\
I-II & 0.39 & 0.06 & 0.02 \\
\hline
\end{tabular}

${ }^{a}$ Average standard deviation (ASD) for calculations with the BP86, B3LYP, PBE0, M06-2X, M06-HF, CAMB3LYP, $\omega$ B97X-D, CIS and CC2 methods. 


\section{Figure captions}

Figure 1. Different types of excitation energies.

Figure 2. Absolute errors in calculated $\Delta E_{00}\left(\mathrm{CC} 2 / \mathrm{X}^{\prime}\right)$ energies relative to $\Delta E_{00}(\mathrm{CC} 2)$.

Figure 3. Absolute errors in calculated $\Delta E_{00}\left(\mathrm{CC} 2 / \mathrm{X}^{\prime \prime}\right)$ energies relative to $\Delta E_{00}(\mathrm{CC} 2)$.

Figure 4. Standard deviations for calculated $\Delta E_{\mathrm{ve}}$ energies and $\Delta \Delta E_{\mathrm{ad}}$ and $\Delta \Delta E_{00}$ energy differences of individual states in groups I and II of the benchmark set using the BP86, B3LYP, PBE0, M06-2X, M06-HF, CAM-B3LYP, wB97X-D, CIS and CC2 methods. For both groups, states are labeled following Table 1.

Figure 5. (a) Histogram of calculated $\Delta \Delta E_{00}$ energy differences. (b) Histogram of calculated $\Delta \Delta E_{\mathrm{ad}}$ energy differences. 


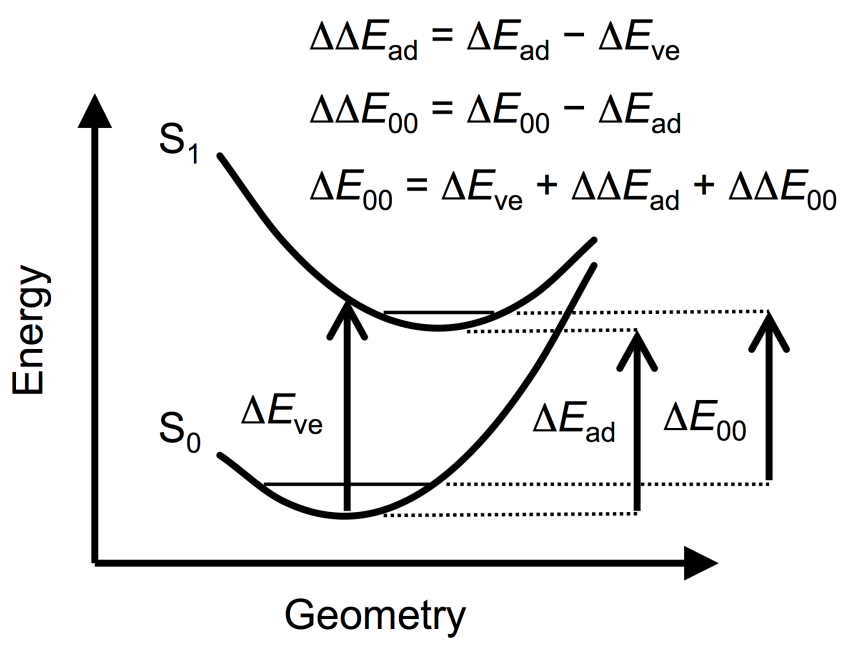

Figure 1 

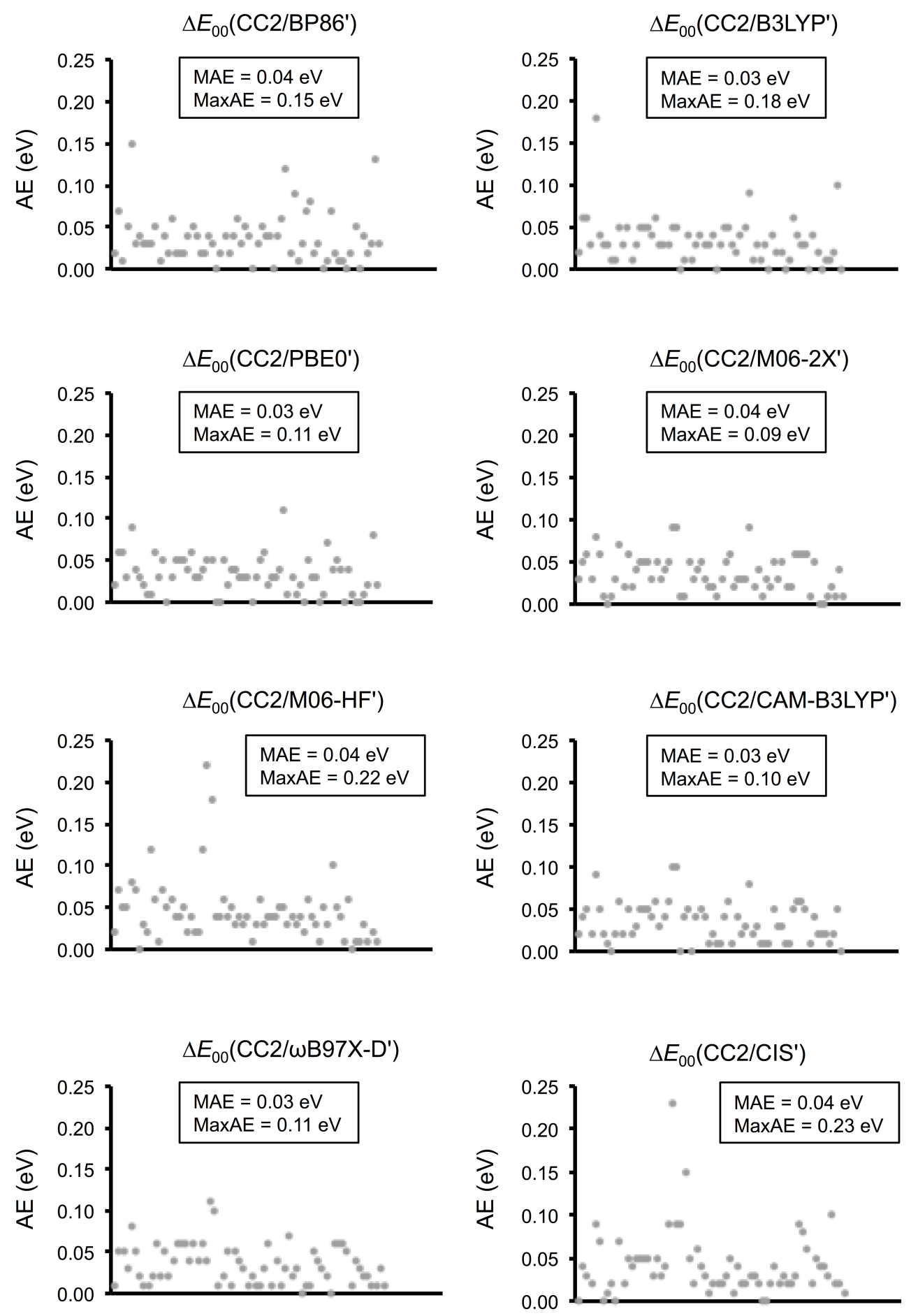

Figure 2 

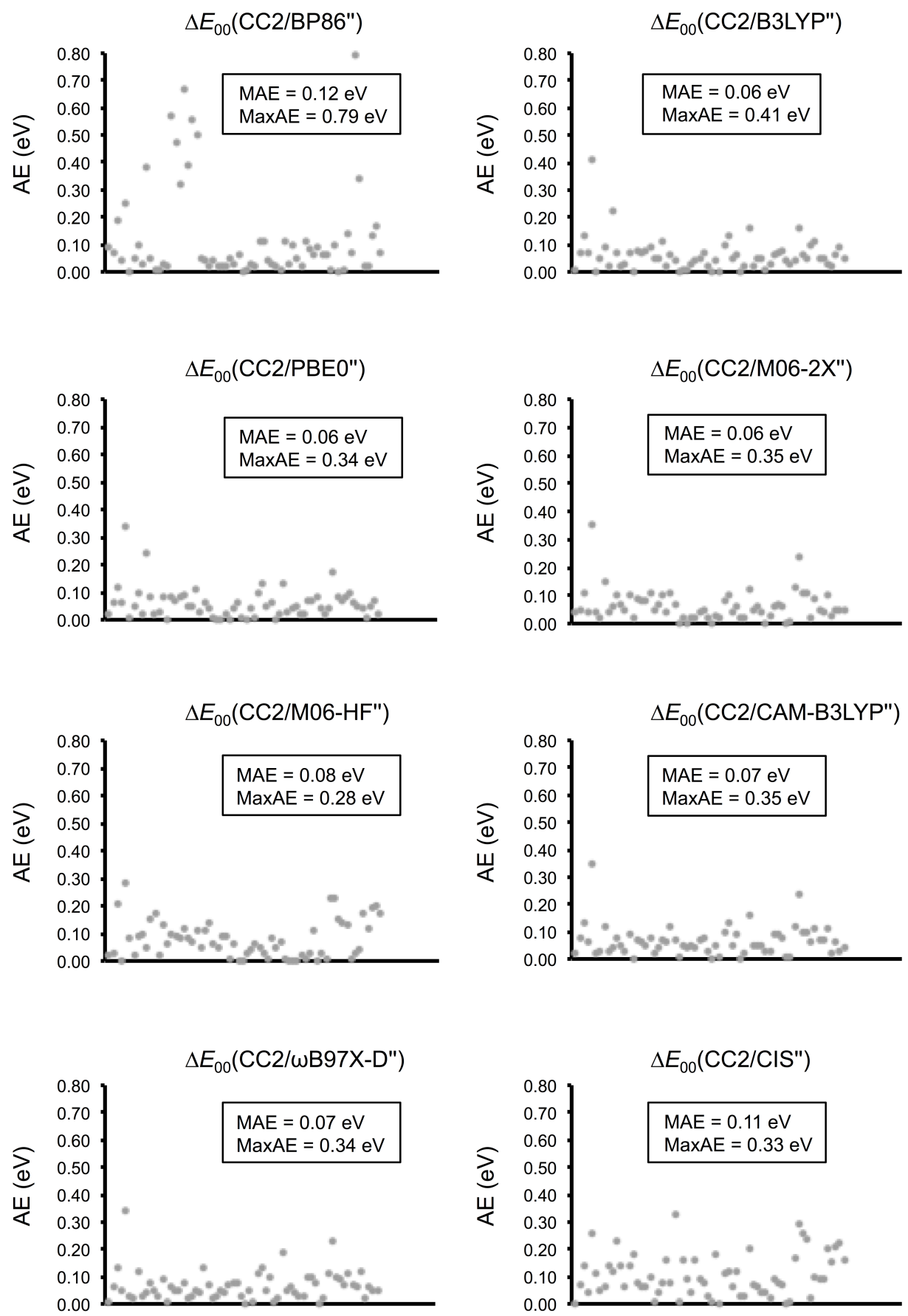

Figure 3 

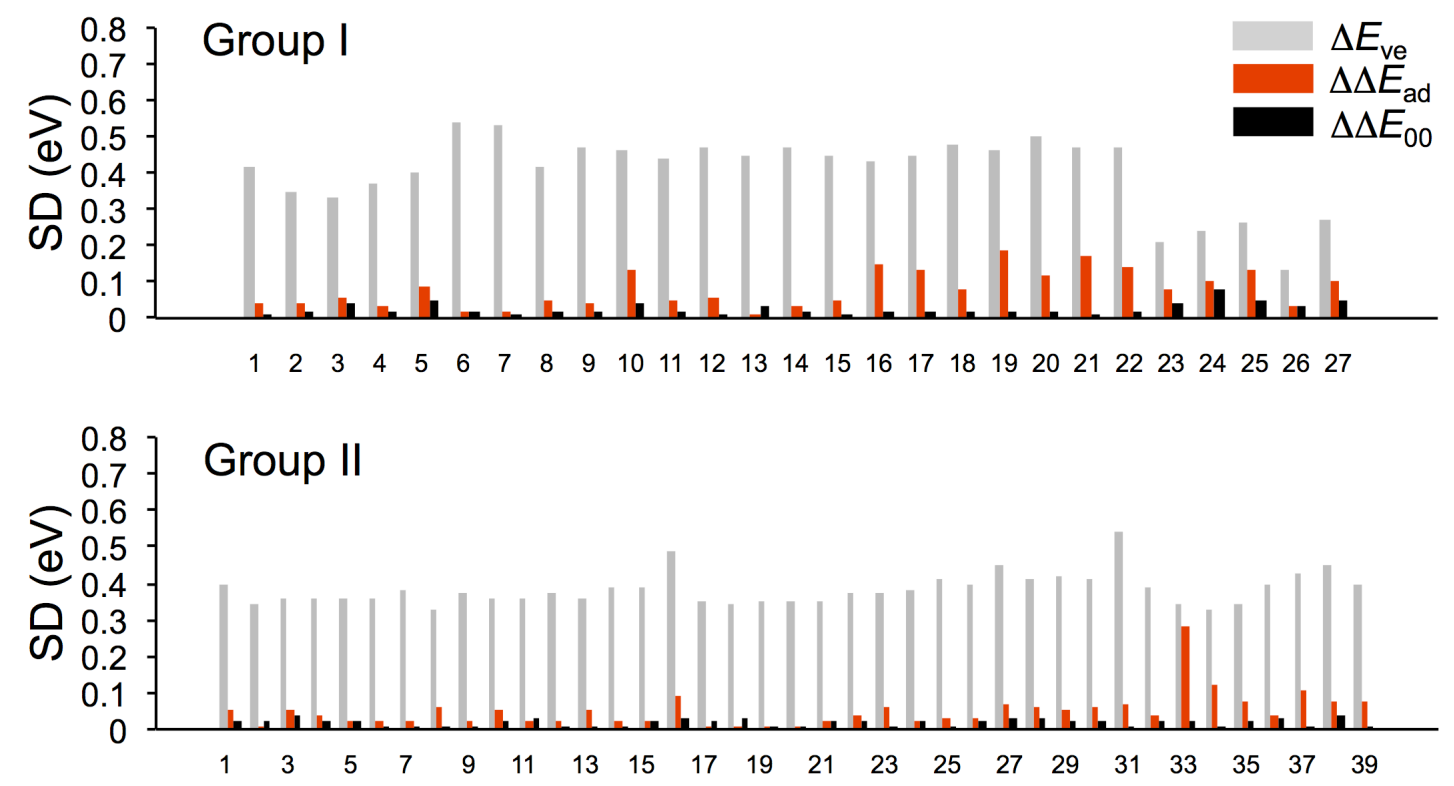

Figure 4 

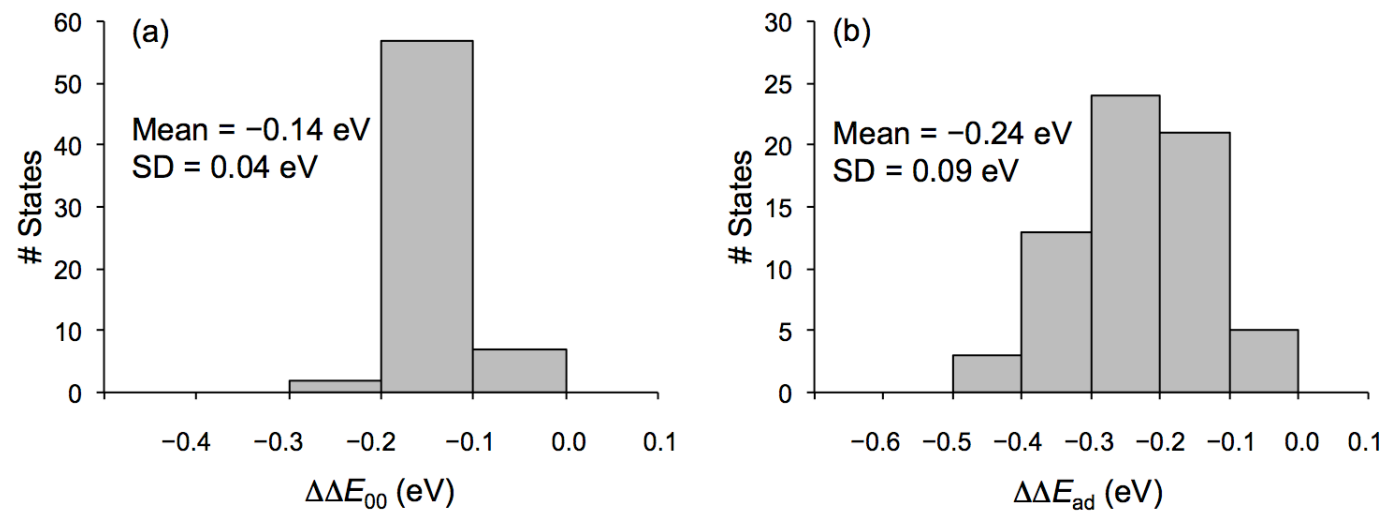

Figure 5 


\section{Graphical abstract}

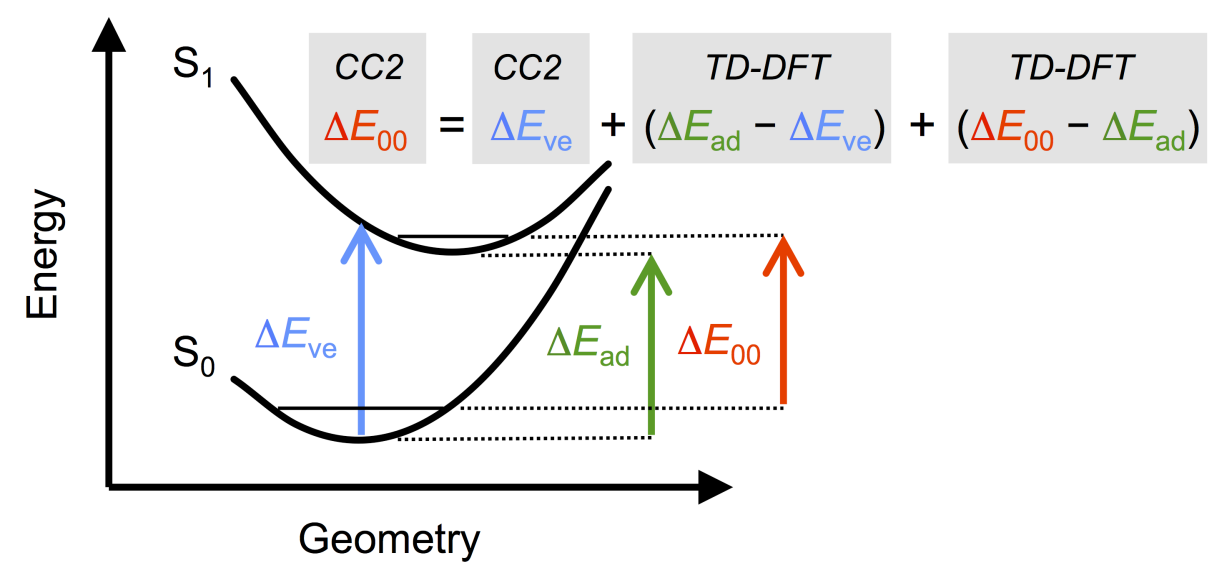


Supplemental data for

\section{Assessment of a composite CC2/DFT procedure for calculating $0-0$ excitation energies of organic molecules}

Baswanth Oruganti ${ }^{\dagger}$, Changfeng Fang ${ }^{\dagger}$ and Bo Durbeej*

Division of Theoretical Chemistry, IFM, Linköping University, Linköping, Sweden

\footnotetext{
${ }^{\dagger}$ These authors contributed equally to the work.

*Corresponding author. E-mail: bodur@ifm.liu.se. Tel: +46-(0)13-282497.
} 


\section{Table of Contents}

Figure S1

page $\mathrm{S} 3$

Figure S2

page S5

Figure S3

page $\mathrm{S} 6$

Figure S4

page $\mathrm{S} 8$

Figure S5

page S9

Figure S6

page $\mathrm{S} 10$

Figure S7

page $\mathrm{S} 11$

Table S1

page S12

Table S2

page $\mathrm{S} 13$

Table S3

page S16

Table S4

page $\mathrm{S} 20$

Atomic coordinates of optimized ground- and excited-state geometries

page $\mathrm{S} 21$

BP86 geometries

page S21

B3LYP geometries

page $\mathrm{S} 88$

PBE0 geometries

page $\mathrm{S} 155$

M06-2X geometries

page $\mathrm{S} 222$

M06-HF geometries

page S290

CAM-B3LYP geometries

page $\mathrm{S} 357$

$\omega B 97 X-D$ geometries

page $\mathrm{S} 424$

CIS geometries

page $\mathrm{S} 491$

CC2 geometries

page S559 


\section{Group I}
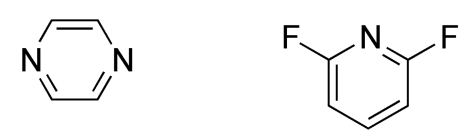

pyrazine<smiles>O=c1cccc[nH]1</smiles>

2,6-difluoropyridine<smiles>Cc1ncccn1</smiles><smiles>Fc1ccccn1</smiles>

2-fluoropyridine<smiles>Cc1cncnc1</smiles><smiles>Oc1ccccn1</smiles>

2-hydroxypyridine<smiles>c1cnc2[nH]ccc2c1</smiles>

7-azaindole

2-pyridone 2-methylpyrimidine

5-methylpyrimidine<smiles>Oc1ccc2ccccc2n1</smiles>

7-hydroxyquinoline, cis<smiles>O=c1ccc2ccccc2[nH]1</smiles>

2-hydroxyquinoline, keto

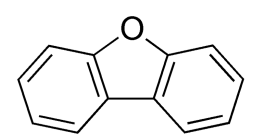

dibenzofuran

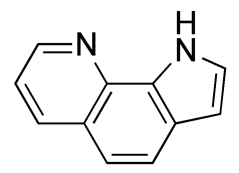

pyrrolo[3,2-h]quinoline<smiles>c1ccc2c(c1)[nH]c1ccccc12</smiles>

carbazole<smiles>NCc1c[nH]c2ccccc12</smiles>

tryptamine*

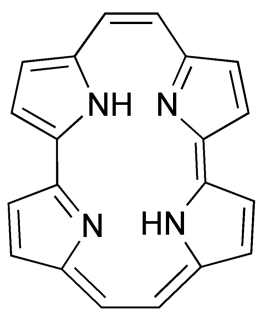

porphycene

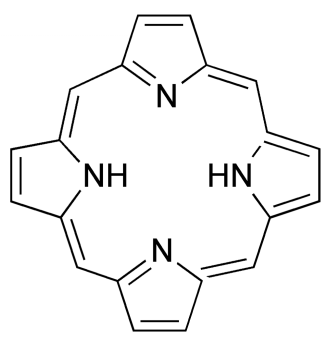

porphine

${ }^{*}$ Different tryptamine isomers are shown in Figure S2.

Figure S1. Chemical structures of the molecules in group I of the benchmark set (continued on page S4). 


\section{Group I continued}

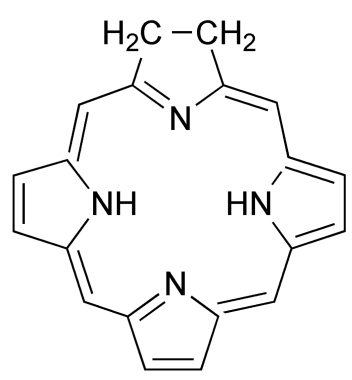

chlorin

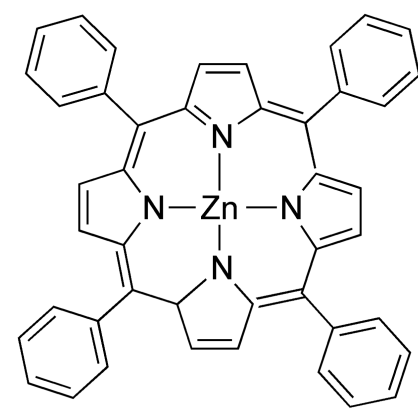

Zn-tetraphenylporphine

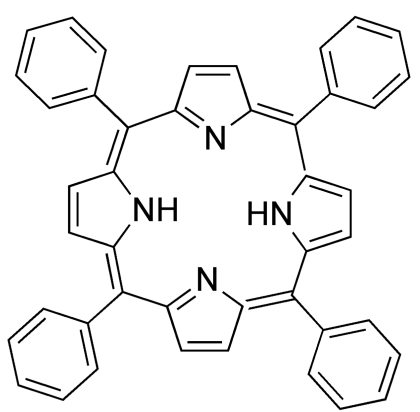

tetraphenylporphine

Figure S1. Continued. 


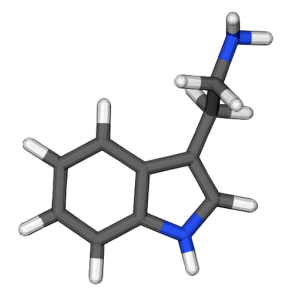

tryptamine, A-ph

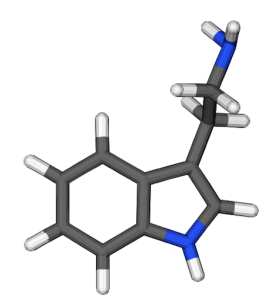

tryptamine, A-py

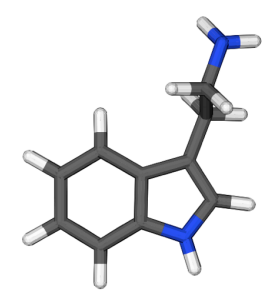

tryptamine, A-up

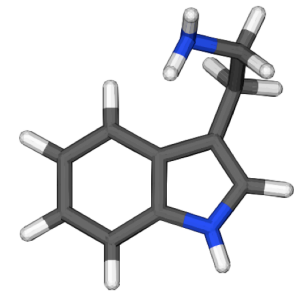

tryptamine, $\mathrm{Ph}$-out

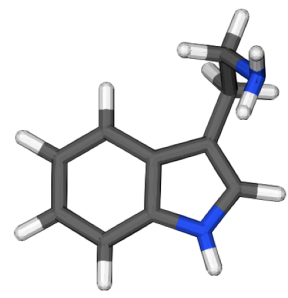

tryptamine, Py-out

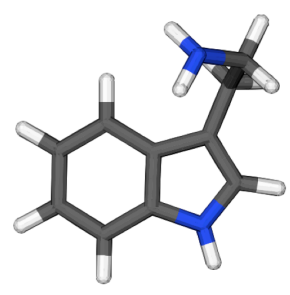

tryptamine, $\mathrm{Ph}$-up

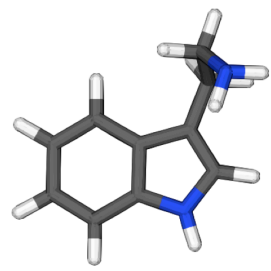

tryptamine, Py-up

Figure S2. Different tryptamine isomers. 


\section{Group II}<smiles>Fc1cc(F)c(F)cc1F</smiles>

tetrafluorobenzene

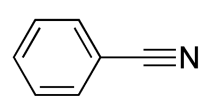

benzonitrile

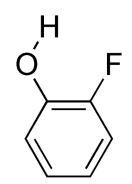

o-fluorophenol, cis<smiles>Oc1ccccc1F</smiles>

o-fluorophenol, trans<smiles>Oc1cccc(F)c1</smiles>

$m$-fluorophenol, cis<smiles>Nc1ccccc1</smiles>

aniline<smiles>Oc1cccc(O)c1</smiles>

resorcinol, isomer 3

hydroquinone, cis<smiles>Oc1ccc(O)cc1</smiles><smiles>Oc1ccc(O)cc1</smiles><smiles>Oc1cccc(O)c1</smiles>

resorcinol, isomer 1

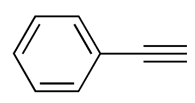

phenylacetylene<smiles>Cc1ccccc1O</smiles>

o-cresol, cis<smiles>Cc1ccccc1O</smiles>

o-cresol, trans<smiles>Cc1cccc(O)c1</smiles>

$m$-cresol, cis<smiles>Cc1cccc(O)c1</smiles><smiles>Cc1ccc(O)cc1</smiles>

$m$-cresol, trans $\quad p$-cresol<smiles>COc1ccccc1O</smiles>

o-methoxyphenol

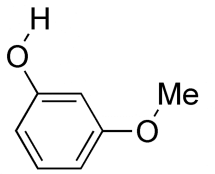

$m$-methoxyphenol, isomer 1<smiles>O=C(O)c1ccccc1O</smiles>

salicylic acid<smiles>Oc1cccc(O)c1</smiles>

resorcinol, isomer 2 


\section{Group II continued}<smiles>COc1ccc(O)cc1</smiles>

p-methoxyphenol, cis<smiles>COc1ccc(O)cc1</smiles>

p-methoxyphenol, trans<smiles>Oc1cccc2ccccc12</smiles><smiles>Oc1cccc2ccccc12</smiles><smiles>Oc1ccc2ccccc2c1</smiles><smiles>Oc1ccc2ccccc2c1</smiles>

2-naphthol, trans<smiles>COc1ccc(O)c(C(=O)O)c1</smiles>

5-methoxysalicylic acid<smiles>c1ccc2c(c1)Cc1ccccc1-2</smiles>

fluorene<smiles>c1ccc2cc3cc4ccccc4cc3cc2c1</smiles>

tetracene<smiles>COc1ccccc1OC</smiles>

m-dimethoxybenzene, trans

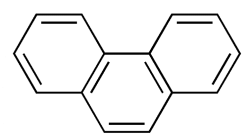

phenanthrene

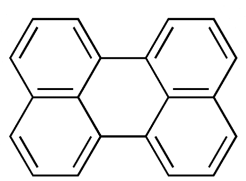

perylene

*Different 3P-propionic acid isomers are shown in Figure S4.

Figure S3. Continued. 


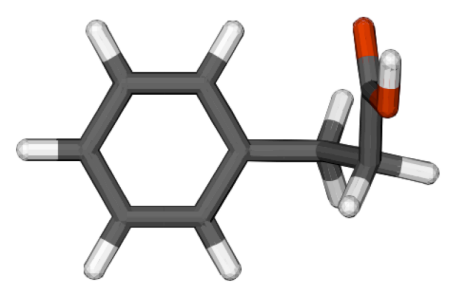

3P-propionic acid, gauche

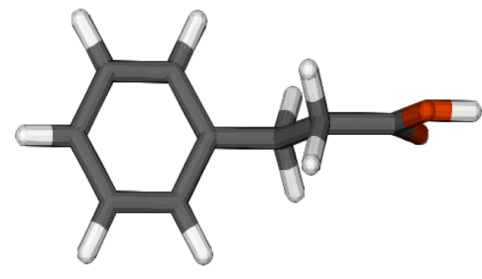

3P-propionic acid, anti

Figure S4. Different 3P-propionic acid isomers. 

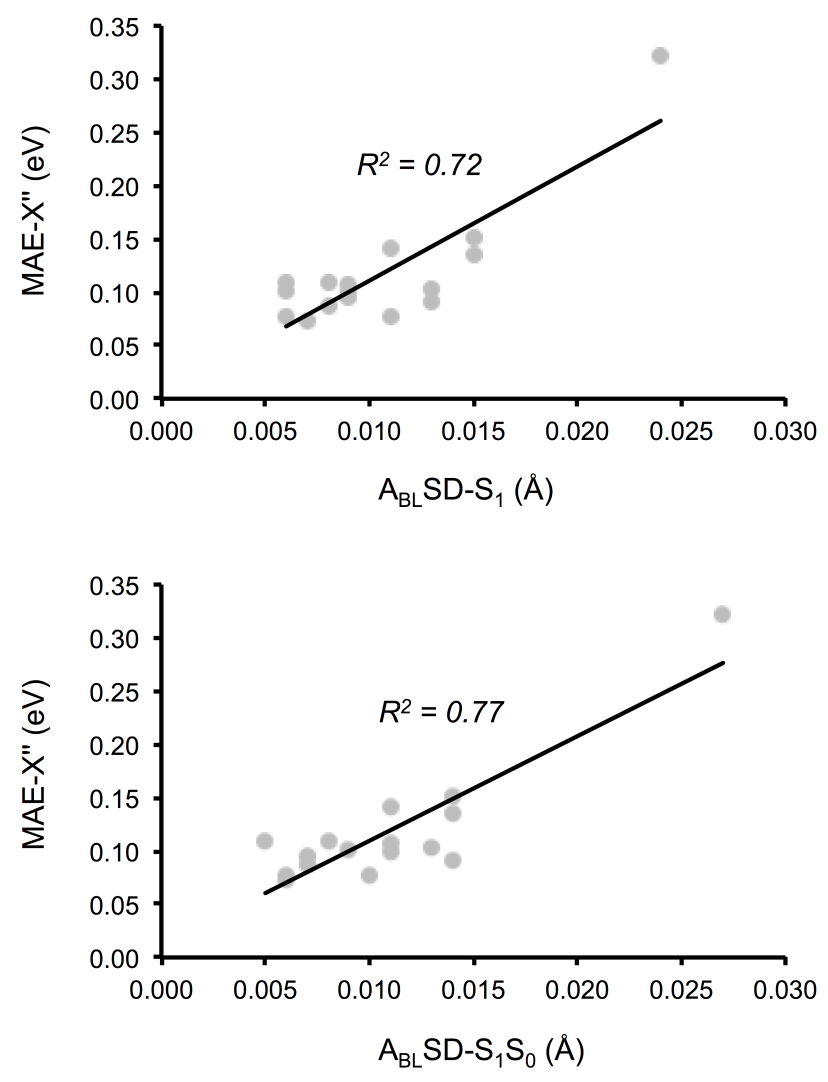

Figure S5. MAE- $X "$ values as functions of $A_{B L} S D-S_{1}$ and $A_{B L} S D-S_{1} S_{0}$ values (see Table $S 4$ ) for selected states in the benchmark set.

\section{Comments on Figure S5}

In order to make possible trends more easily discernible, the analysis in Figure S5 only includes states in the benchmark set whose MAE-X" values are larger than a specific threshold value. This threshold value was defined as the average MAE for the eight $\Delta E_{00}(\mathrm{CC} 2 / \mathrm{X} ")$ schemes in Figure 3 , and amounts to $0.078 \mathrm{eV}$. 

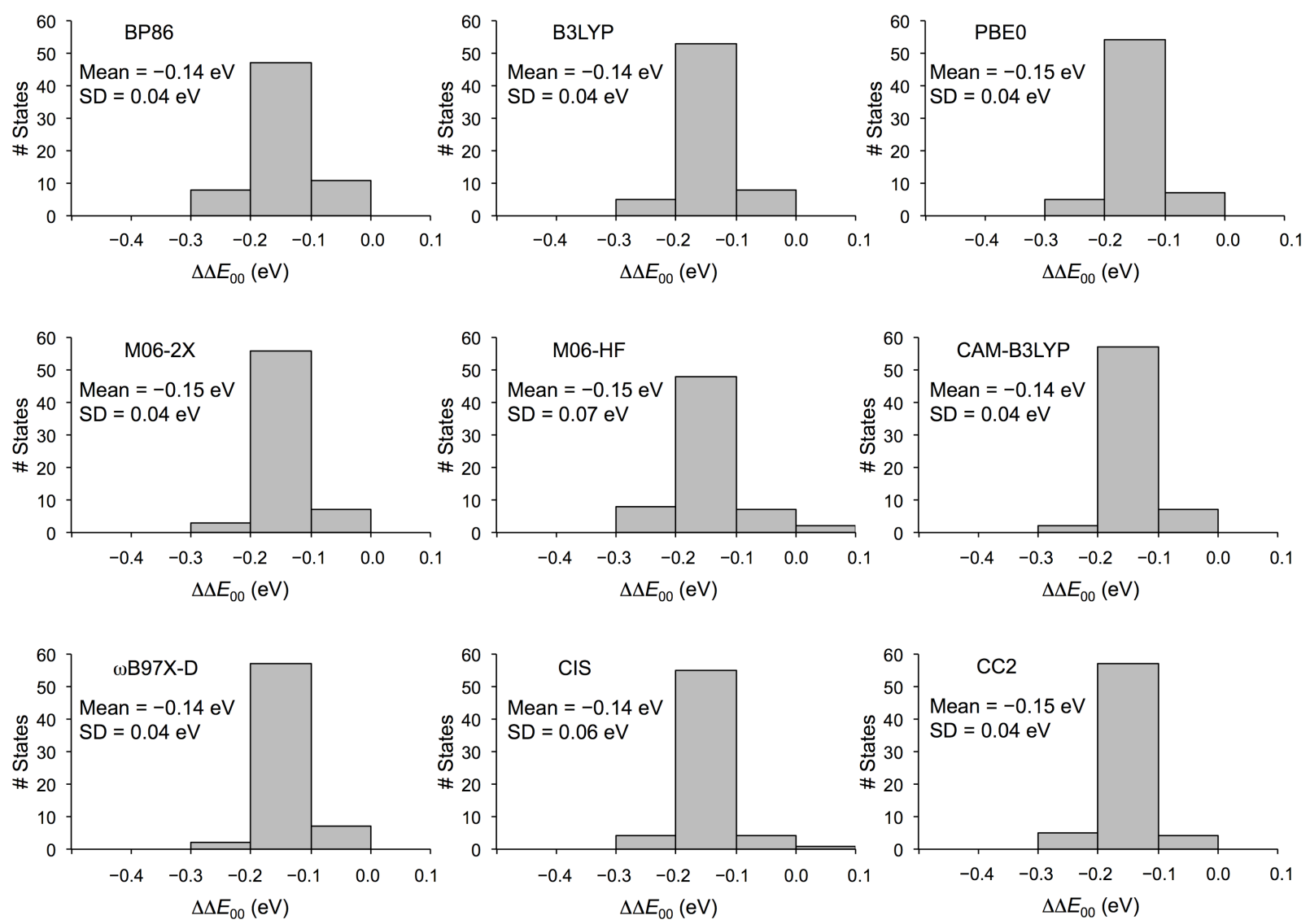

Figure S6. Histograms of calculated $\Delta \Delta E_{00}$ energy differences at different levels of theory. 

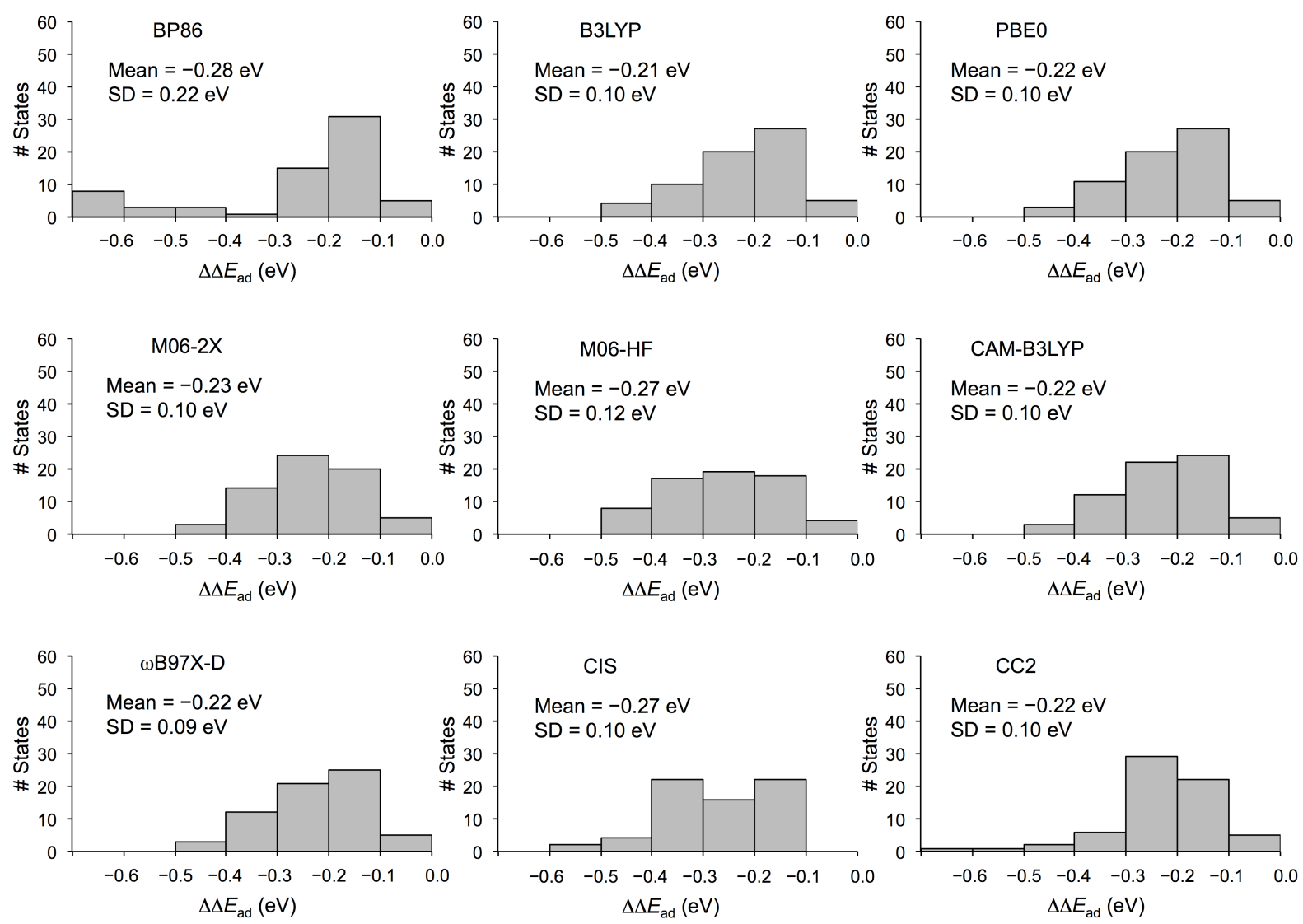

Figure S7. Histograms of calculated $\Delta \Delta E_{\text {ad }}$ energy differences at different levels of theory. 
Table S1. Comparison of MAE and MaxAE values for the $\Delta E_{00}\left(\mathrm{CC} 2 / \mathrm{X}^{\prime}\right)$ and $\Delta E_{00}\left(\mathrm{CC} 2 / \mathrm{X}^{\prime \prime}\right)$ schemes relative to the $\Delta E_{00}(\mathrm{CC} 2)$ energies reported in this work and by Hättig $(\mathrm{eV})$.

\begin{tabular}{|c|c|c|c|c|}
\hline \multirow[b]{2}{*}{ Scheme } & \multicolumn{2}{|c|}{$\begin{array}{l}\text { Relative to } \Delta E_{00}(\mathrm{CC} 2) \\
\text { energies of this work }\end{array}$} & \multicolumn{2}{|c|}{$\begin{array}{c}\text { Relative to } \Delta E_{00}(\mathrm{CC} 2) \\
\text { energies of Hättig }\end{array}$} \\
\hline & MAE & MaxAE & MAE & MaxAE \\
\hline \multicolumn{5}{|l|}{$\Delta E_{00}\left(\mathrm{CC} 2 / \mathrm{X}^{\prime}\right)$} \\
\hline$\Delta E_{00}\left(\mathrm{CC} 2 / \mathrm{BP}^{2} 6^{\prime}\right)$ & 0.04 & 0.15 & 0.03 & 0.08 \\
\hline$\Delta E_{00}\left(\mathrm{CC} 2 / \mathrm{B} 3 \mathrm{LY} \mathrm{P}^{\prime}\right)$ & 0.03 & 0.18 & 0.02 & 0.08 \\
\hline$\Delta E_{00}\left(\mathrm{CC} 2 / \mathrm{PBE} 0^{\prime}\right)$ & 0.03 & 0.11 & 0.02 & 0.09 \\
\hline$\Delta E_{00}\left(\mathrm{CC} 2 / \mathrm{M} 06-2 \mathrm{X}^{\prime}\right)$ & 0.04 & 0.09 & 0.02 & 0.13 \\
\hline$\Delta E_{00}\left(\mathrm{CC} 2 / \mathrm{M} 06-\mathrm{HF}^{\prime}\right)$ & 0.04 & 0.22 & 0.04 & 0.20 \\
\hline$\Delta E_{00}(\mathrm{CC} 2 / \mathrm{CAM}-\mathrm{B} 3 \mathrm{LYP})$ & 0.03 & 0.10 & 0.02 & 0.12 \\
\hline$\Delta E_{00}\left(\mathrm{CC} 2 / \omega \mathrm{B} 97 \mathrm{X}-\mathrm{D}^{\prime}\right)$ & 0.03 & 0.11 & 0.02 & 0.14 \\
\hline$\Delta E_{00}\left(\mathrm{CC} 2 / \mathrm{CIS}^{\prime}\right)$ & 0.04 & 0.23 & 0.03 & 0.19 \\
\hline \multicolumn{5}{|l|}{$\Delta E_{00}\left(\mathrm{CC} 2 / \mathrm{X}^{\prime \prime}\right)$} \\
\hline$\Delta E_{00}(\mathrm{CC} 2 / \mathrm{BP} 86 ")$ & 0.12 & 0.79 & 0.14 & 0.79 \\
\hline$\Delta E_{00}(\mathrm{CC} 2 / \mathrm{B} 3 \mathrm{LYP} ")$ & 0.06 & 0.41 & 0.08 & 0.54 \\
\hline$\Delta E_{00}(\mathrm{CC} 2 / \mathrm{PBE} 0 ")$ & 0.06 & 0.34 & 0.07 & 0.54 \\
\hline$\Delta E_{00}\left(\mathrm{CC} 2 / \mathrm{M} 06-2 \mathrm{X}^{\prime \prime}\right)$ & 0.06 & 0.35 & 0.08 & 0.50 \\
\hline$\Delta E_{00}\left(\mathrm{CC} 2 / \mathrm{M} 06-\mathrm{HF}^{\prime \prime}\right)$ & 0.08 & 0.28 & 0.10 & 0.47 \\
\hline$\Delta E_{00}(\mathrm{CC} 2 / \mathrm{CAM}-\mathrm{B} 3 \mathrm{LYP} ")$ & 0.07 & 0.35 & 0.08 & 0.51 \\
\hline$\Delta E_{00}(\mathrm{CC} 2 / \omega \mathrm{B} 97 \mathrm{X}-\mathrm{D} ")$ & 0.07 & 0.34 & 0.08 & 0.50 \\
\hline$\Delta E_{00}(\mathrm{CC} 2 / \mathrm{CIS} ")$ & 0.11 & 0.33 & 0.11 & 0.50 \\
\hline
\end{tabular}

${ }^{\mathrm{a}}$ Taken from Ref. [25]. 
Table S2. Calculated $\Delta E_{\mathrm{ve}}$ energies and $\Delta \Delta E_{\mathrm{ad}}$ and $\Delta \Delta E_{00}$ energy differences for all states in group I of the benchmark set $(\mathrm{eV}){ }^{\mathrm{a}}$

\begin{tabular}{|c|c|c|c|c|c|c|c|c|c|c|c|c|c|}
\hline \multirow[b]{2}{*}{ Label/Molecule } & \multirow[b]{2}{*}{ State } & \multirow[b]{2}{*}{$\Delta E$} & \multicolumn{11}{|c|}{ Method and statistics $^{\mathrm{b}}$} \\
\hline & & & BP86 & B3LYP & PBE0 & M06-2X & M06-HF & CB3LYP & $\omega B 97 X-D$ & CIS & $\mathrm{CC} 2$ & SD & MaxAD \\
\hline \multirow[t]{3}{*}{ 1/pyrazine } & $1^{1} \mathrm{~A}_{\mathrm{u}}$ & $\Delta E_{\mathrm{ve}}$ & 3.58 & 3.96 & 4.02 & 4.00 & 3.82 & 4.20 & 4.17 & 5.18 & 4.27 & 0.42 & 1.60 \\
\hline & & $\Delta \Delta E_{\mathrm{ad}}$ & -0.24 & -0.14 & -0.13 & -0.12 & -0.13 & -0.13 & -0.13 & -0.13 & -0.13 & 0.04 & 0.12 \\
\hline & & $\Delta \Delta E_{00}$ & -0.21 & -0.21 & -0.21 & -0.20 & -0.21 & -0.21 & -0.22 & -0.23 & -0.23 & 0.01 & 0.03 \\
\hline \multirow[t]{3}{*}{ 2/2,6-difluoropyridine } & $1^{1} \mathrm{~B}_{2}$ & $\Delta E_{\mathrm{ve}}$ & 5.10 & 5.41 & 5.52 & 5.64 & 5.86 & 5.56 & 5.55 & 6.40 & 5.24 & 0.35 & 1.30 \\
\hline & & $\Delta \Delta E_{\mathrm{ad}}$ & -0.20 & -0.21 & -0.22 & -0.24 & -0.24 & -0.22 & -0.23 & -0.23 & -0.34 & 0.04 & 0.13 \\
\hline & & $\Delta \Delta E_{00}$ & -0.18 & -0.17 & -0.17 & -0.16 & -0.18 & -0.15 & -0.16 & -0.15 & -0.11 & 0.02 & 0.07 \\
\hline \multirow[t]{3}{*}{ 3/2-fluoropyridine } & $2^{1} \mathrm{~A}^{\prime}$ & $\Delta E_{\mathrm{ve}}$ & 5.16 & 5.45 & 5.56 & 5.67 & 5.89 & 5.60 & 5.59 & 6.35 & 5.27 & 0.33 & 1.19 \\
\hline & & $\Delta \Delta E_{\mathrm{ad}}$ & -0.18 & -0.19 & -0.20 & -0.21 & -0.22 & -0.20 & -0.20 & -0.21 & -0.38 & 0.06 & 0.20 \\
\hline & & $\Delta \Delta E_{00}$ & -0.15 & -0.20 & -0.20 & -0.20 & -0.09 & -0.19 & -0.19 & -0.17 & -0.13 & 0.04 & 0.11 \\
\hline \multirow[t]{3}{*}{ 4/2-hydroxypyridine } & $2^{1} \mathrm{~A}^{\prime}$ & $\Delta E_{\mathrm{ve}}$ & 4.74 & 5.09 & 5.21 & 5.37 & 5.61 & 5.29 & 5.28 & 6.13 & 5.00 & 0.37 & 1.39 \\
\hline & & $\Delta \Delta E_{\mathrm{ad}}$ & -0.21 & -0.20 & -0.21 & -0.23 & -0.25 & -0.22 & -0.22 & -0.24 & -0.30 & 0.03 & 0.10 \\
\hline & & $\Delta \Delta E_{00}$ & -0.18 & -0.16 & -0.16 & -0.16 & -0.18 & -0.15 & -0.16 & -0.15 & -0.13 & 0.02 & 0.06 \\
\hline \multirow[t]{3}{*}{ 5/2-pyridone } & $2^{1} \mathrm{~A}^{\prime}$ & $\Delta E_{\mathrm{ve}}$ & 4.03 & 4.36 & 4.47 & 4.66 & 4.87 & 4.62 & 4.61 & 5.57 & 4.43 & 0.40 & 1.54 \\
\hline & & $\Delta \Delta E_{\mathrm{ad}}$ & -0.53 & -0.40 & -0.38 & -0.36 & -0.43 & -0.37 & -0.37 & -0.46 & -0.63 & 0.09 & 0.27 \\
\hline & & $\Delta \Delta E_{00}$ & -0.09 & -0.06 & -0.15 & -0.16 & -0.16 & -0.15 & -0.16 & -0.15 & -0.24 & 0.05 & 0.18 \\
\hline \multirow[t]{3}{*}{ 6/2-methylpyrimidine } & $1^{1} \mathrm{~A}^{\prime \prime}$ & $\Delta E_{\mathrm{ve}}$ & 3.73 & 4.19 & 4.29 & 4.38 & 4.38 & 4.48 & 4.46 & 5.85 & 4.42 & 0.54 & 2.12 \\
\hline & & $\Delta \Delta E_{\mathrm{ad}}$ & -0.45 & -0.44 & -0.45 & -0.46 & -0.49 & -0.45 & -0.46 & -0.52 & -0.48 & 0.02 & 0.08 \\
\hline & & $\Delta \Delta E_{00}$ & -0.13 & -0.14 & -0.14 & -0.16 & -0.17 & -0.15 & -0.15 & -0.17 & -0.10 & 0.02 & 0.07 \\
\hline \multirow[t]{3}{*}{ 7/5-methylpyrimidine } & $1^{1} \mathrm{~A}^{\prime \prime}$ & $\Delta E_{\mathrm{ve}}$ & 3.76 & 4.23 & 4.33 & 4.41 & 4.40 & 4.52 & 4.50 & 5.86 & 4.47 & 0.53 & 2.10 \\
\hline & & $\Delta \Delta E_{\mathrm{ad}}$ & -0.45 & -0.44 & -0.44 & -0.45 & -0.48 & -0.45 & -0.46 & -0.51 & -0.46 & 0.02 & 0.08 \\
\hline & & $\Delta \Delta E_{00}$ & -0.13 & -0.14 & -0.14 & -0.16 & -0.17 & -0.15 & -0.15 & -0.17 & -0.17 & 0.01 & 0.05 \\
\hline \multirow[t]{3}{*}{ 8/7-azaindole } & $2^{1} \mathrm{~A}^{\prime}$ & $\Delta E_{\mathrm{ve}}$ & 4.20 & 4.63 & 4.77 & 5.12 & 5.36 & 5.06 & 5.05 & 5.77 & 4.82 & 0.42 & 1.57 \\
\hline & & $\Delta \Delta E_{\mathrm{ad}}$ & -0.41 & -0.40 & -0.40 & -0.43 & -0.34 & -0.41 & -0.41 & -0.33 & -0.28 & 0.05 & 0.15 \\
\hline & & $\Delta \Delta E_{00}$ & -0.12 & -0.12 & -0.13 & -0.15 & -0.18 & -0.14 & -0.14 & -0.16 & -0.14 & 0.02 & 0.06 \\
\hline \multirow[t]{3}{*}{ 9/7-hydroxyquinoline } & $2^{1} \mathrm{~A}^{\prime}$ & $\Delta E_{\mathrm{ve}}$ & 3.68 & 4.13 & 4.25 & 4.54 & 4.92 & 4.49 & 4.49 & 5.42 & 4.26 & 0.47 & 1.75 \\
\hline & & $\Delta \Delta E_{\mathrm{ad}}$ & -0.19 & -0.22 & -0.22 & -0.22 & -0.27 & -0.22 & -0.21 & -0.31 & -0.19 & 0.04 & 0.13 \\
\hline & & $\Delta \Delta E_{00}$ & -0.12 & -0.14 & -0.14 & -0.16 & -0.17 & -0.15 & -0.16 & -0.17 & -0.14 & 0.02 & 0.05 \\
\hline \multirow[t]{3}{*}{ 10/7-hydroxyquinoline } & $2^{1} \mathrm{~A}$ & $\Delta E_{\mathrm{ve}}$ & 3.63 & 4.06 & 4.18 & 4.48 & 4.87 & 4.43 & 4.43 & 5.35 & 4.19 & 0.46 & 1.72 \\
\hline & & $\Delta \Delta E_{\mathrm{ad}}$ & -0.61 & -0.43 & -0.45 & -0.23 & -0.27 & -0.22 & -0.22 & -0.32 & -0.20 & 0.13 & 0.42 \\
\hline & & $\Delta \Delta E_{00}$ & -0.10 & -0.12 & -0.12 & -0.16 & -0.01 & -0.15 & -0.15 & -0.13 & -0.13 & 0.04 & 0.15 \\
\hline \multirow[t]{3}{*}{ 11/2-hydroxyquinoline } & $2^{1} \mathrm{~A}^{\prime}$ & $\Delta E_{\mathrm{ve}}$ & 3.82 & 4.22 & 4.34 & 4.59 & 4.95 & 4.54 & 4.54 & 5.47 & 4.30 & 0.44 & 1.65 \\
\hline & & $\Delta \Delta E_{\mathrm{ad}}$ & -0.20 & -0.22 & -0.22 & -0.23 & -0.29 & -0.22 & -0.22 & -0.36 & -0.20 & 0.05 & 0.17 \\
\hline & & $\Delta \Delta E_{00}$ & -0.15 & -0.15 & -0.16 & -0.17 & -0.16 & -0.16 & -0.16 & -0.17 & -0.11 & 0.02 & 0.06 \\
\hline
\end{tabular}


Table S2. Continued.

\begin{tabular}{|c|c|c|c|c|c|c|c|c|c|c|c|c|c|}
\hline \multirow[b]{2}{*}{ Label/Molecule } & \multirow[b]{2}{*}{ State } & \multirow[b]{2}{*}{$\Delta E$} & \multicolumn{11}{|c|}{ Method and statistics ${ }^{b}$} \\
\hline & & & BP86 & B3LYP & PBE0 & M06-2X & M06-HF & CB3LYP & $\omega B 97 X-D$ & CIS & $\mathrm{CC} 2$ & SD & MaxAD \\
\hline \multirow[t]{3}{*}{ 12/2-hydroxyquinoline } & $2^{1} \mathrm{~A}^{\prime}$ & $\Delta E_{\mathrm{ve}}$ & 3.61 & 4.01 & 4.13 & 4.41 & 4.76 & 4.38 & 4.38 & 5.35 & 4.11 & 0.47 & 1.75 \\
\hline & & $\Delta \Delta E_{\mathrm{ad}}$ & -0.23 & -0.24 & -0.24 & -0.30 & -0.41 & -0.28 & -0.28 & -0.37 & -0.25 & 0.06 & 0.19 \\
\hline & & $\Delta \Delta E_{00}$ & -0.13 & -0.15 & -0.15 & -0.14 & -0.13 & -0.14 & -0.14 & -0.14 & -0.11 & 0.01 & 0.04 \\
\hline \multirow[t]{3}{*}{ 13/dibenzofuran } & $2^{1} \mathrm{~A}_{1}$ & $\Delta E_{\mathrm{ve}}$ & 4.02 & 4.47 & 4.61 & 4.89 & 5.19 & 4.85 & 4.86 & 5.75 & 4.55 & 0.45 & 1.72 \\
\hline & & $\Delta \Delta E_{\mathrm{ad}}$ & -0.11 & -0.12 & -0.12 & -0.13 & -0.09 & -0.12 & -0.12 & -0.13 & -0.14 & 0.01 & 0.05 \\
\hline & & $\Delta \Delta E_{00}$ & -0.20 & -0.21 & -0.21 & -0.22 & -0.23 & -0.21 & -0.21 & -0.11 & -0.16 & 0.03 & 0.12 \\
\hline \multirow[t]{3}{*}{$14 /$ pyrrolo $[3,2-h]$ quinoline } & $2^{1} \mathrm{~A}^{\prime}$ & $\Delta E_{\mathrm{ve}}$ & 3.46 & 3.90 & 4.04 & 4.38 & 4.82 & 4.34 & 4.37 & 5.14 & 4.11 & 0.47 & 1.69 \\
\hline & & $\Delta \Delta E_{\mathrm{ad}}$ & -0.24 & -0.27 & -0.27 & -0.27 & -0.27 & -0.26 & -0.26 & -0.29 & -0.19 & 0.03 & 0.11 \\
\hline & & $\Delta \Delta E_{00}$ & -0.12 & -0.13 & -0.14 & -0.16 & -0.19 & -0.16 & -0.16 & -0.18 & -0.14 & 0.02 & 0.07 \\
\hline \multirow[t]{3}{*}{ 15/carbazole } & $2^{1} \mathrm{~A}_{1}$ & $\Delta E_{\mathrm{ve}}$ & 3.66 & 4.07 & 4.20 & 4.48 & 4.80 & 4.46 & 4.48 & 5.35 & 4.18 & 0.45 & 1.69 \\
\hline & & $\Delta \Delta E_{\text {ad }}$ & -0.11 & -0.12 & -0.12 & -0.13 & -0.15 & -0.12 & -0.12 & -0.28 & -0.15 & 0.05 & 0.17 \\
\hline & & $\Delta \Delta E_{00}$ & -0.17 & -0.14 & -0.14 & -0.15 & -0.17 & -0.14 & -0.15 & -0.16 & -0.12 & 0.01 & 0.05 \\
\hline \multirow[t]{3}{*}{ 16/tryptamine } & $2^{1} \mathrm{~A}$ & $\Delta E_{\mathrm{ve}}$ & 4.11 & 4.62 & 4.74 & 5.04 & 5.34 & 4.99 & 5.00 & 5.73 & 4.82 & 0.43 & 1.62 \\
\hline & & $\Delta \Delta E_{\mathrm{ad}}$ & -0.78 & -0.32 & -0.32 & -0.33 & -0.33 & -0.31 & -0.31 & -0.32 & -0.19 & 0.15 & 0.59 \\
\hline & & $\Delta \Delta E_{00}$ & -0.18 & -0.15 & -0.15 & -0.15 & -0.16 & -0.15 & -0.14 & -0.15 & -0.21 & 0.02 & 0.06 \\
\hline \multirow[t]{3}{*}{ 17/tryptamine } & $2^{1} \mathrm{~A}$ & $\Delta E_{\mathrm{ve}}$ & 4.04 & 4.59 & 4.72 & 5.03 & 5.34 & 4.99 & 4.99 & 5.72 & 4.82 & 0.45 & 1.69 \\
\hline & & $\Delta \Delta E_{\mathrm{ad}}$ & -0.70 & -0.33 & -0.33 & -0.34 & -0.34 & -0.32 & -0.32 & -0.32 & -0.21 & 0.13 & 0.49 \\
\hline & & $\Delta \Delta E_{00}$ & -0.18 & -0.15 & -0.15 & -0.15 & -0.16 & -0.15 & -0.14 & -0.15 & -0.20 & 0.02 & 0.06 \\
\hline \multirow[t]{3}{*}{ 18/tryptamine } & $2^{1} \mathrm{~A}$ & $\Delta E_{\mathrm{ve}}$ & 3.90 & 4.55 & 4.69 & 5.02 & 5.34 & 4.98 & 4.98 & 5.73 & 4.82 & 0.48 & 1.83 \\
\hline & & $\Delta \Delta E_{\mathrm{ad}}$ & -0.55 & -0.34 & -0.34 & -0.34 & -0.34 & -0.31 & -0.32 & -0.32 & -0.21 & 0.08 & 0.34 \\
\hline & & $\Delta \Delta E_{00}$ & -0.18 & -0.15 & -0.15 & -0.15 & -0.15 & -0.15 & -0.14 & -0.15 & -0.20 & 0.02 & 0.06 \\
\hline \multirow[t]{3}{*}{ 19/tryptamine } & $2^{1} \mathrm{~A}$ & $\Delta E_{\mathrm{ve}}$ & 3.99 & 4.61 & 4.74 & 5.04 & 5.35 & 5.00 & 5.00 & 5.73 & 4.82 & 0.46 & 1.74 \\
\hline & & $\Delta \Delta E_{\mathrm{ad}}$ & -0.90 & -0.32 & -0.32 & -0.33 & -0.33 & -0.31 & -0.31 & -0.32 & -0.19 & 0.19 & 0.71 \\
\hline & & $\Delta \Delta E_{00}$ & -0.15 & -0.15 & -0.15 & -0.16 & -0.17 & -0.15 & -0.15 & -0.16 & -0.20 & 0.02 & 0.05 \\
\hline \multirow[t]{3}{*}{ 20/tryptamine } & $2^{1} \mathrm{~A}$ & $\Delta E_{\mathrm{ve}}$ & 3.79 & 4.52 & 4.65 & 5.00 & 5.34 & 4.96 & 4.96 & 5.71 & 4.82 & 0.50 & 1.92 \\
\hline & & $\Delta \Delta E_{\mathrm{ad}}$ & -0.70 & -0.37 & -0.37 & -0.36 & -0.38 & -0.34 & -0.34 & -0.32 & -0.26 & 0.12 & 0.43 \\
\hline & & $\Delta \Delta E_{00}$ & -0.15 & -0.14 & -0.14 & -0.15 & -0.16 & -0.14 & -0.14 & -0.15 & -0.20 & 0.02 & 0.06 \\
\hline \multirow[t]{3}{*}{$21 /$ tryptamine } & $2^{1} \mathrm{~A}$ & $\Delta E_{\mathrm{ve}}$ & 3.94 & 4.60 & 4.73 & 5.04 & 5.35 & 4.99 & 5.00 & 5.73 & 4.82 & 0.47 & 1.79 \\
\hline & & $\Delta \Delta E_{\mathrm{ad}}$ & -0.84 & -0.32 & -0.32 & -0.34 & -0.33 & -0.31 & -0.31 & -0.31 & -0.24 & 0.17 & 0.60 \\
\hline & & $\Delta \Delta E_{00}$ & -0.14 & -0.15 & -0.15 & -0.15 & -0.16 & -0.15 & -0.14 & -0.15 & -0.18 & 0.01 & 0.05 \\
\hline \multirow[t]{3}{*}{$22 /$ tryptamine } & $2^{1} \mathrm{~A}$ & $\Delta E_{\mathrm{ve}}$ & 3.92 & 4.60 & 4.73 & 5.04 & 5.34 & 5.00 & 5.00 & 5.74 & 4.82 & 0.47 & 1.82 \\
\hline & & $\Delta \Delta E_{\mathrm{ad}}$ & -0.71 & -0.33 & -0.33 & -0.33 & -0.32 & -0.30 & -0.30 & -0.31 & -0.19 & 0.14 & 0.53 \\
\hline & & $\Delta \Delta E_{00}$ & -0.17 & -0.16 & -0.16 & -0.15 & -0.17 & -0.15 & -0.13 & -0.15 & -0.20 & 0.02 & 0.07 \\
\hline 23/porphycene & $2^{1} \mathrm{~A}^{\prime}$ & $\Delta E_{\mathrm{ve}}$ & 2.11 & 2.24 & 2.29 & 2.26 & 2.16 & 2.19 & 2.14 & 2.83 & 2.24 & 0.21 & 0.72 \\
\hline
\end{tabular}


Table S2. Continued.

\begin{tabular}{|c|c|c|c|c|c|c|c|c|c|c|c|c|c|}
\hline \multirow[b]{2}{*}{ Label/Molecule } & \multirow[b]{2}{*}{ State } & \multirow[b]{2}{*}{$\Delta E$} & \multicolumn{11}{|c|}{ Method and statistics ${ }^{b}$} \\
\hline & & & BP86 & B3LYP & PBE0 & M06-2X & M06-HF & CB3LYP & $\omega B 97 X-D$ & CIS & $\mathrm{CC} 2$ & SD & MaxAD \\
\hline \multirow{5}{*}{ 24/porphine } & \multirow{5}{*}{$1^{1} \mathrm{~B}_{1 \mathrm{u}}$} & $\Delta \Delta E_{\mathrm{ad}}$ & -0.07 & -0.05 & -0.05 & -0.05 & -0.11 & -0.04 & -0.04 & -0.29 & -0.04 & 0.08 & 0.25 \\
\hline & & $\Delta \Delta E_{00}$ & -0.15 & -0.10 & -0.09 & -0.08 & -0.01 & -0.07 & -0.09 & -0.04 & -0.13 & 0.04 & 0.14 \\
\hline & & $\Delta E_{\mathrm{ve}}$ & 2.15 & 2.28 & 2.34 & 2.29 & 2.59 & 2.22 & 2.16 & 2.96 & 2.29 & 0.24 & 0.81 \\
\hline & & $\Delta \Delta E_{\mathrm{ad}}$ & -0.03 & -0.02 & -0.02 & -0.01 & -0.14 & -0.01 & -0.01 & -0.34 & -0.03 & 0.10 & 0.33 \\
\hline & & $\Delta \Delta E_{00}$ & -0.08 & -0.07 & -0.07 & -0.03 & 0.10 & -0.02 & -0.01 & 0.11 & -0.12 & 0.08 & 0.23 \\
\hline \multirow[t]{3}{*}{ 25/chlorin } & \multirow[t]{3}{*}{$1^{1} \mathrm{~B}_{2}$} & $\Delta E_{\mathrm{ve}}$ & 2.19 & 2.28 & 2.32 & 2.25 & 2.10 & 2.20 & 2.16 & 3.03 & 2.27 & 0.26 & 0.93 \\
\hline & & $\Delta \Delta E_{\mathrm{ad}}$ & -0.01 & -0.01 & -0.01 & -0.02 & -0.04 & -0.03 & -0.03 & -0.42 & 0.00 & 0.13 & 0.42 \\
\hline & & $\Delta \Delta E_{00}$ & -0.09 & -0.07 & -0.07 & -0.03 & 0.06 & -0.02 & -0.02 & -0.03 & -0.12 & 0.05 & 0.18 \\
\hline \multirow[t]{3}{*}{ 26/Zn-tetraphenylporphine } & \multirow[t]{2}{*}{$1^{1} \mathrm{~B}_{2}$} & $\Delta E_{\mathrm{ve}}$ & 2.12 & 2.30 & 2.34 & 2.33 & 2.24 & 2.30 & 2.25 & 2.64 & 2.34 & 0.13 & 0.52 \\
\hline & & $\Delta \Delta E_{\mathrm{ad}}$ & -0.08 & -0.04 & -0.05 & -0.03 & -0.02 & -0.03 & -0.03 & -0.12 & -0.04 & 0.03 & 0.10 \\
\hline & \multirow{4}{*}{$1^{1} \mathrm{~B}$} & $\Delta \Delta E_{00}$ & -0.06 & -0.06 & -0.06 & -0.07 & -0.02 & -0.06 & -0.05 & 0.03 & -0.06 & 0.03 & 0.10 \\
\hline \multirow[t]{3}{*}{ 27/tetraphenylporphine } & & $\Delta E_{\mathrm{ve}}$ & 1.97 & 2.16 & 2.20 & 2.16 & 2.35 & 2.12 & 2.07 & 2.95 & 2.08 & 0.27 & 0.98 \\
\hline & & $\Delta \Delta E_{\mathrm{ad}}$ & -0.04 & -0.04 & -0.04 & -0.03 & -0.03 & -0.03 & -0.03 & -0.35 & -0.04 & 0.10 & 0.32 \\
\hline & & $\Delta \Delta E_{00}$ & -0.06 & -0.05 & -0.04 & -0.03 & 0.00 & 0.00 & -0.02 & 0.11 & -0.05 & 0.05 & 0.17 \\
\hline
\end{tabular}

${ }^{\mathrm{a}} \Delta \Delta E_{\mathrm{ad}}=\Delta E_{\mathrm{ad}}-\Delta E_{\mathrm{ve}}$ and $\Delta \Delta E_{00}=\Delta E_{00}-\Delta E_{\mathrm{ad}}$. ${ }^{\mathrm{b}}$ Standard deviation (SD) and maximum absolute deviation (MaxAD) for calculations with the nine methods. 
Table S3. Calculated $\Delta E_{\mathrm{ve}}$ energies and $\Delta \Delta E_{\mathrm{ad}}$ and $\Delta \Delta E_{00}$ energy differences for all states in group II of the benchmark set (eV). ${ }^{\mathrm{a}}$

\begin{tabular}{|c|c|c|c|c|c|c|c|c|c|c|c|c|c|}
\hline \multirow[b]{2}{*}{ Label/Molecule } & \multirow[b]{2}{*}{ State } & \multirow[b]{2}{*}{$\Delta E$} & \multicolumn{11}{|c|}{ Method and statistics ${ }^{\mathrm{b}}$} \\
\hline & & & BP86 & B3LYP & PBE0 & M06-2X & M06-HF & CB3LYP & $\omega B 97 X-D$ & CIS & $\mathrm{CC} 2$ & SD & MaxAD \\
\hline \multirow[t]{3}{*}{ 1/tetrafluorobenzene } & $1^{1} \mathrm{~B}_{1}$ & $\Delta E_{\mathrm{ve}}$ & 4.84 & 5.19 & 5.31 & 5.46 & 5.70 & 5.37 & 5.36 & 6.31 & 5.06 & 0.40 & 1.47 \\
\hline & & $\Delta \Delta E_{\mathrm{ad}}$ & -0.37 & -0.34 & -0.36 & -0.36 & -0.46 & -0.32 & -0.31 & -0.27 & -0.31 & 0.05 & 0.19 \\
\hline & & $\Delta \Delta E_{00}$ & -0.13 & -0.13 & -0.12 & -0.12 & -0.11 & -0.12 & -0.12 & -0.12 & -0.18 & 0.02 & 0.07 \\
\hline \multirow[t]{3}{*}{ 2/benzonitrile } & $1^{1} \mathrm{~B}_{2}$ & $\Delta E_{\mathrm{ve}}$ & 4.80 & 5.12 & 5.25 & 5.41 & 5.66 & 5.34 & 5.34 & 6.07 & 5.07 & 0.34 & 1.26 \\
\hline & & $\Delta \Delta E_{\mathrm{ad}}$ & -0.15 & -0.15 & -0.15 & -0.14 & -0.14 & -0.14 & -0.14 & -0.13 & -0.19 & 0.01 & 0.05 \\
\hline & & $\Delta \Delta E_{00}$ & -0.16 & -0.15 & -0.16 & -0.17 & -0.10 & -0.14 & -0.15 & -0.16 & -0.15 & 0.02 & 0.07 \\
\hline \multirow[t]{3}{*}{ 3/o-fluorophenol } & $2^{1} \mathrm{~A}$ & $\Delta E_{\mathrm{ve}}$ & 4.84 & 5.16 & 5.28 & 5.43 & 5.66 & 5.35 & 5.34 & 6.17 & 5.07 & 0.36 & 1.33 \\
\hline & & $\Delta \Delta E_{\mathrm{ad}}$ & -0.15 & -0.31 & -0.28 & -0.26 & -0.18 & -0.24 & -0.22 & -0.14 & -0.24 & 0.05 & 0.18 \\
\hline & & $\Delta \Delta E_{00}$ & -0.19 & -0.12 & -0.11 & -0.11 & -0.20 & -0.11 & -0.10 & -0.09 & -0.15 & 0.04 & 0.10 \\
\hline \multirow[t]{3}{*}{ 4/o-fluorophenol } & $2^{1} \mathrm{~A}$ & $\Delta E_{\mathrm{ve}}$ & 4.81 & 5.13 & 5.26 & 5.40 & 5.63 & 5.32 & 5.31 & 6.15 & 5.04 & 0.36 & 1.33 \\
\hline & & $\Delta \Delta E_{\mathrm{ad}}$ & -0.28 & -0.24 & -0.25 & -0.26 & -0.34 & -0.23 & -0.21 & -0.20 & -0.25 & 0.04 & 0.14 \\
\hline & & $\Delta \Delta E_{00}$ & -0.10 & -0.12 & -0.12 & -0.11 & -0.13 & -0.11 & -0.12 & -0.12 & -0.16 & 0.02 & 0.07 \\
\hline \multirow[t]{3}{*}{ 5/m-fluorophenol } & $2^{1} \mathrm{~A}$ & $\Delta E_{\mathrm{ve}}$ & 4.84 & 5.16 & 5.28 & 5.42 & 5.64 & 5.34 & 5.34 & 6.18 & 5.07 & 0.36 & 1.34 \\
\hline & & $\Delta \Delta E_{\mathrm{ad}}$ & -0.19 & -0.18 & -0.19 & -0.20 & -0.18 & -0.18 & -0.17 & -0.17 & -0.22 & 0.02 & 0.05 \\
\hline & & $\Delta \Delta E_{00}$ & -0.13 & -0.13 & -0.13 & -0.13 & -0.20 & -0.12 & -0.13 & -0.13 & -0.16 & 0.02 & 0.08 \\
\hline \multirow[t]{3}{*}{ 6/m-fluorophenol } & $2^{1} \mathrm{~A}^{\prime}$ & $\Delta E_{\mathrm{ve}}$ & 4.85 & 5.17 & 5.30 & 5.43 & 5.66 & 5.36 & 5.35 & 6.19 & 5.08 & 0.36 & 1.34 \\
\hline & & $\Delta \Delta E_{\mathrm{ad}}$ & -0.15 & -0.15 & -0.16 & -0.16 & -0.17 & -0.16 & -0.16 & -0.16 & -0.20 & 0.02 & 0.05 \\
\hline & & $\Delta \Delta E_{00}$ & -0.21 & -0.19 & -0.19 & -0.18 & -0.19 & -0.17 & -0.17 & -0.17 & -0.16 & 0.01 & 0.05 \\
\hline \multirow[t]{3}{*}{ 7/p-fluorophenol } & $2^{1} \mathrm{~A}^{\prime}$ & $\Delta E_{\mathrm{ve}}$ & 4.56 & 4.91 & 5.01 & 5.18 & 5.44 & 5.10 & 5.09 & 5.96 & 4.83 & 0.38 & 1.40 \\
\hline & & $\Delta \Delta E_{\mathrm{ad}}$ & -0.16 & -0.17 & -0.18 & -0.19 & -0.20 & -0.19 & -0.19 & -0.20 & -0.21 & 0.02 & 0.05 \\
\hline & & $\Delta \Delta E_{00}$ & -0.19 & -0.19 & -0.18 & -0.17 & -0.19 & -0.17 & -0.17 & -0.17 & -0.14 & 0.01 & 0.05 \\
\hline \multirow[t]{3}{*}{ 8/phenylacetylene } & $1^{1} \mathrm{~B}_{2}$ & $\Delta E_{\mathrm{ve}}$ & 4.62 & 4.99 & 5.13 & 5.32 & 5.59 & 5.27 & 5.27 & 5.83 & 5.01 & 0.33 & 1.21 \\
\hline & & $\Delta \Delta E_{\mathrm{ad}}$ & -0.15 & -0.14 & -0.14 & -0.14 & -0.13 & -0.14 & -0.14 & -0.34 & -0.18 & 0.06 & 0.21 \\
\hline & & $\Delta \Delta E_{00}$ & -0.16 & -0.16 & -0.16 & -0.17 & -0.17 & -0.15 & -0.15 & -0.18 & -0.17 & 0.01 & 0.03 \\
\hline \multirow[t]{3}{*}{ 9/aniline } & $1^{1} \mathrm{~A}^{\prime \prime}$ & $\Delta E_{\mathrm{ve}}$ & 4.39 & 4.75 & 4.87 & 5.05 & 5.25 & 4.98 & 4.99 & 5.84 & 4.82 & 0.37 & 1.44 \\
\hline & & $\Delta \Delta E_{\mathrm{ad}}$ & -0.23 & -0.21 & -0.22 & -0.23 & -0.27 & -0.22 & -0.22 & -0.22 & -0.24 & 0.02 & 0.05 \\
\hline & & $\Delta \Delta E_{00}$ & -0.21 & -0.21 & -0.21 & -0.21 & -0.21 & -0.19 & -0.19 & -0.20 & -0.17 & 0.01 & 0.04 \\
\hline \multirow[t]{3}{*}{ 10/catechol } & $2^{1} \mathrm{~A}$ & $\Delta E_{\mathrm{ve}}$ & 4.69 & 5.01 & 5.13 & 5.29 & 5.53 & 5.21 & 5.20 & 6.04 & 4.96 & 0.36 & 1.35 \\
\hline & & $\Delta \Delta E_{\mathrm{ad}}$ & -0.16 & -0.17 & -0.17 & -0.19 & -0.21 & -0.18 & -0.18 & -0.18 & -0.32 & 0.05 & 0.16 \\
\hline & & $\Delta \Delta E_{00}$ & -0.20 & -0.20 & -0.20 & -0.20 & -0.21 & -0.19 & -0.18 & -0.18 & -0.15 & 0.02 & 0.06 \\
\hline \multirow[t]{3}{*}{ 11/resorcinol } & $2^{1} \mathrm{~A}$ & $\Delta E_{\mathrm{ve}}$ & 4.72 & 5.05 & 5.17 & 5.31 & 5.53 & 5.24 & 5.24 & 6.09 & 4.99 & 0.36 & 1.37 \\
\hline & & $\Delta \Delta E_{\mathrm{ad}}$ & -0.17 & -0.16 & -0.17 & -0.20 & -0.18 & -0.17 & -0.17 & -0.17 & -0.24 & 0.02 & 0.08 \\
\hline & & $\Delta \Delta E_{00}$ & -0.14 & -0.13 & -0.12 & -0.12 & -0.21 & -0.12 & -0.12 & -0.13 & -0.17 & 0.03 & 0.09 \\
\hline
\end{tabular}


Table S3. Continued.

\begin{tabular}{|c|c|c|c|c|c|c|c|c|c|c|c|c|c|}
\hline \multirow[b]{2}{*}{ Label/Molecule } & \multirow[b]{2}{*}{ State } & \multirow[b]{2}{*}{$\Delta E$} & \multicolumn{11}{|c|}{ Method and statistics ${ }^{b}$} \\
\hline & & & BP86 & B3LYP & PBE0 & M06-2X & M06-HF & CB3LYP & $\omega B 97 X-D$ & CIS & $\mathrm{CC} 2$ & SD & $\operatorname{Max} A D$ \\
\hline \multirow[t]{3}{*}{ 12/resorcinol } & $1^{1} \mathrm{~B}_{2}$ & $\Delta E_{\mathrm{ve}}$ & 4.73 & 5.06 & 5.19 & 5.33 & 5.56 & 5.26 & 5.26 & 6.10 & 4.98 & 0.37 & 1.37 \\
\hline & & $\Delta \Delta E_{\mathrm{ad}}$ & -0.13 & -0.13 & -0.14 & -0.15 & -0.16 & -0.15 & -0.15 & -0.14 & -0.21 & 0.02 & 0.08 \\
\hline & & $\Delta \Delta E_{00}$ & -0.20 & -0.19 & -0.18 & -0.18 & -0.20 & -0.17 & -0.17 & -0.17 & -0.17 & 0.01 & 0.03 \\
\hline \multirow[t]{3}{*}{ 13/resorcinol } & $1^{1} \mathrm{~A}^{\prime \prime}$ & $\Delta E_{\mathrm{ve}}$ & 4.71 & 5.03 & 5.15 & 5.28 & 5.49 & 5.21 & 5.21 & 6.05 & 4.93 & 0.36 & 1.35 \\
\hline & & $\Delta \Delta E_{\mathrm{ad}}$ & -0.24 & -0.23 & -0.24 & -0.24 & -0.39 & -0.22 & -0.21 & -0.19 & -0.27 & 0.05 & 0.20 \\
\hline & & $\Delta \Delta E_{00}$ & -0.15 & -0.13 & -0.12 & -0.12 & -0.11 & -0.11 & -0.11 & -0.11 & -0.15 & 0.01 & 0.04 \\
\hline \multirow[t]{3}{*}{ 14/hydroquinone } & $2^{1} \mathrm{~A}_{1}$ & $\Delta E_{\mathrm{ve}}$ & 4.29 & 4.66 & 4.76 & 4.95 & 5.22 & 4.86 & 4.85 & 5.76 & 4.62 & 0.39 & 1.46 \\
\hline & & $\Delta \Delta E_{\mathrm{ad}}$ & -0.16 & -0.18 & -0.19 & -0.21 & -0.23 & -0.20 & -0.20 & -0.23 & -0.22 & 0.02 & 0.07 \\
\hline & & $\Delta \Delta E_{00}$ & -0.19 & -0.19 & -0.18 & -0.18 & -0.19 & -0.17 & -0.16 & -0.17 & -0.15 & 0.01 & 0.05 \\
\hline \multirow[t]{3}{*}{ 15/hydroquinone } & $1^{1} \mathrm{~B}_{\mathrm{u}}$ & $\Delta E_{\mathrm{ve}}$ & 4.29 & 4.65 & 4.75 & 4.94 & 5.21 & 4.85 & 4.84 & 5.74 & 4.60 & 0.39 & 1.45 \\
\hline & & $\Delta \Delta E_{\mathrm{ad}}$ & -0.16 & -0.18 & -0.19 & -0.20 & -0.23 & -0.20 & -0.20 & -0.22 & -0.21 & 0.02 & 0.06 \\
\hline & & $\Delta \Delta E_{00}$ & -0.20 & -0.19 & -0.18 & -0.17 & -0.19 & -0.17 & -0.17 & -0.16 & -0.14 & 0.02 & 0.06 \\
\hline \multirow[t]{3}{*}{ 16/salicylic acid } & $2^{1} \mathrm{~A}^{\prime}$ & $\Delta E_{\mathrm{ve}}$ & 3.74 & 4.21 & 4.34 & 4.64 & 4.94 & 4.54 & 4.54 & 5.61 & 4.32 & 0.49 & 1.87 \\
\hline & & $\Delta \Delta E_{\mathrm{ad}}$ & -0.30 & -0.28 & -0.29 & -0.32 & -0.47 & -0.29 & -0.27 & -0.30 & -0.53 & 0.09 & 0.26 \\
\hline & & $\Delta \Delta E_{00}$ & -0.23 & -0.20 & -0.22 & -0.20 & -0.16 & -0.19 & -0.18 & -0.14 & -0.12 & 0.03 & 0.11 \\
\hline \multirow[t]{3}{*}{ 17/o-cresol } & $2^{1} \mathrm{~A}$ & $\Delta E_{\mathrm{ve}}$ & 4.78 & 5.10 & 5.21 & 5.35 & 5.59 & 5.29 & 5.28 & 6.06 & 5.01 & 0.35 & 1.28 \\
\hline & & $\Delta \Delta E_{\mathrm{ad}}$ & -0.19 & -0.19 & -0.18 & -0.17 & -0.17 & -0.17 & -0.17 & -0.16 & -0.20 & 0.01 & 0.04 \\
\hline & & $\Delta \Delta E_{00}$ & -0.14 & -0.15 & -0.15 & -0.14 & -0.19 & -0.14 & -0.14 & -0.13 & -0.16 & 0.02 & 0.06 \\
\hline \multirow[t]{3}{*}{ 18/o-cresol } & $2^{1} \mathrm{~A}$ & $\Delta E_{\mathrm{ve}}$ & 4.78 & 5.09 & 5.21 & 5.35 & 5.58 & 5.28 & 5.27 & 6.06 & 5.02 & 0.34 & 1.28 \\
\hline & & $\Delta \Delta E_{\mathrm{ad}}$ & -0.21 & -0.20 & -0.21 & -0.20 & -0.18 & -0.20 & -0.19 & -0.18 & -0.22 & 0.01 & 0.04 \\
\hline & & $\Delta \Delta E_{00}$ & -0.08 & -0.14 & -0.14 & -0.13 & -0.21 & -0.14 & -0.14 & -0.15 & -0.16 & 0.03 & 0.12 \\
\hline \multirow[t]{3}{*}{ 19/m-cresol } & $2^{1} \mathrm{~A}$ & $\Delta E_{\mathrm{ve}}$ & 4.71 & 5.04 & 5.15 & 5.29 & 5.52 & 5.23 & 5.23 & 6.02 & 4.97 & 0.35 & 1.31 \\
\hline & & $\Delta \Delta E_{\mathrm{ad}}$ & -0.16 & -0.16 & -0.16 & -0.17 & -0.17 & -0.16 & -0.16 & -0.16 & -0.20 & 0.01 & 0.04 \\
\hline & & $\Delta \Delta E_{00}$ & -0.15 & -0.15 & -0.15 & -0.15 & -0.19 & -0.15 & -0.16 & -0.16 & -0.16 & 0.01 & 0.05 \\
\hline \multirow[t]{3}{*}{ 20/m-cresol } & $2^{1} \mathrm{~A}^{\prime}$ & $\Delta E_{\mathrm{ve}}$ & 4.71 & 5.04 & 5.15 & 5.30 & 5.53 & 5.23 & 5.23 & 6.03 & 4.96 & 0.35 & 1.31 \\
\hline & & $\Delta \Delta E_{\mathrm{ad}}$ & -0.14 & -0.15 & -0.15 & -0.16 & -0.17 & -0.15 & -0.15 & -0.15 & -0.19 & 0.01 & 0.04 \\
\hline & & $\Delta \Delta E_{00}$ & -0.18 & -0.18 & -0.17 & -0.18 & -0.19 & -0.16 & -0.16 & -0.15 & -0.16 & 0.01 & 0.04 \\
\hline \multirow[t]{3}{*}{$21 / p$-cresol } & $2^{1} \mathrm{~A}$ & $\Delta E_{\mathrm{ve}}$ & 4.63 & 4.95 & 5.05 & 5.19 & 5.43 & 5.13 & 5.13 & 5.93 & 4.89 & 0.35 & 1.30 \\
\hline & & $\Delta \Delta E_{\mathrm{ad}}$ & -0.16 & -0.17 & -0.18 & -0.19 & -0.23 & -0.18 & -0.18 & -0.20 & -0.20 & 0.02 & 0.07 \\
\hline & & $\Delta \Delta E_{00}$ & -0.09 & -0.16 & -0.16 & -0.14 & -0.14 & -0.15 & -0.15 & -0.14 & -0.16 & 0.02 & 0.07 \\
\hline \multirow[t]{3}{*}{ 22/o-methoxyphenol } & $2^{1} \mathrm{~A}$ & $\Delta E_{\mathrm{ve}}$ & 4.69 & 5.02 & 5.14 & 5.29 & 5.53 & 5.21 & 5.20 & 6.06 & 4.93 & 0.37 & 1.37 \\
\hline & & $\Delta \Delta E_{\mathrm{ad}}$ & -0.25 & -0.23 & -0.23 & -0.24 & -0.34 & -0.21 & -0.20 & -0.20 & -0.25 & 0.04 & 0.14 \\
\hline & & $\Delta \Delta E_{00}$ & -0.11 & -0.15 & -0.14 & -0.14 & -0.13 & -0.14 & -0.14 & -0.15 & -0.18 & 0.02 & 0.07 \\
\hline 23/m-methoxyphenol & $2^{1} \mathrm{~A}$ & $\Delta E_{\mathrm{ve}}$ & 4.69 & 5.02 & 5.14 & 5.27 & 5.49 & 5.21 & 5.21 & 6.06 & 4.91 & 0.37 & 1.38 \\
\hline
\end{tabular}


Table S3. Continued.

\begin{tabular}{|c|c|c|c|c|c|c|c|c|c|c|c|c|c|}
\hline \multirow[b]{2}{*}{ Label/Molecule } & \multirow[b]{2}{*}{ State } & \multirow[b]{2}{*}{$\Delta E$} & \multicolumn{11}{|c|}{ Method and statistics ${ }^{b}$} \\
\hline & & & BP86 & B3LYP & PBE0 & M06-2X & M06-HF & CB3LYP & $\omega B 97 X-D$ & CIS & $\mathrm{CC} 2$ & SD & MaxAD \\
\hline \multirow{5}{*}{ 24/m-methoxyphenol } & \multirow{5}{*}{$2^{1} \mathrm{~A}$} & $\Delta \Delta E_{\mathrm{ad}}$ & -0.21 & -0.20 & -0.21 & -0.21 & -0.40 & -0.19 & -0.19 & -0.19 & -0.25 & 0.06 & 0.21 \\
\hline & & $\Delta \Delta E_{00}$ & -0.14 & -0.14 & -0.13 & -0.13 & -0.12 & -0.13 & -0.12 & -0.14 & -0.16 & 0.01 & 0.04 \\
\hline & & $\Delta E_{\mathrm{ve}}$ & 4.70 & 5.04 & 5.16 & 5.30 & 5.54 & 5.24 & 5.23 & 6.10 & 4.93 & 0.38 & 1.40 \\
\hline & & $\Delta \Delta E_{\mathrm{ad}}$ & -0.15 & -0.15 & -0.16 & -0.20 & -0.18 & -0.16 & -0.16 & -0.17 & -0.21 & 0.02 & 0.06 \\
\hline & & $\Delta \Delta E_{00}$ & -0.14 & -0.15 & -0.14 & -0.12 & -0.20 & -0.14 & -0.14 & -0.14 & -0.17 & 0.02 & 0.08 \\
\hline \multirow[t]{2}{*}{ 25/p-methoxyphenol } & \multirow[t]{2}{*}{$2^{1} \mathrm{~A}$} & $\Delta E_{\mathrm{ve}}$ & 4.26 & 4.65 & 4.76 & 4.95 & 5.23 & 4.86 & 4.86 & 5.79 & 4.60 & 0.41 & 1.53 \\
\hline & & $\Delta \Delta E_{\mathrm{ad}}$ & -0.16 & -0.18 & -0.18 & -0.20 & -0.26 & -0.20 & -0.20 & -0.24 & -0.22 & 0.03 & 0.10 \\
\hline \multirow{3}{*}{ 26/p-methoxyphenol } & \multirow{3}{*}{$2^{1} \mathrm{~A}$} & $\Delta \Delta E_{00}$ & -0.15 & -0.15 & -0.15 & -0.17 & -0.14 & -0.16 & -0.17 & -0.13 & -0.15 & 0.01 & 0.04 \\
\hline & & $\Delta E_{\mathrm{ve}}$ & 4.25 & 4.63 & 4.74 & 4.93 & 5.21 & 4.85 & 4.84 & 5.76 & 4.58 & 0.40 & 1.50 \\
\hline & & $\Delta \Delta E_{\mathrm{ad}}$ & -0.16 & -0.17 & -0.18 & -0.20 & -0.25 & -0.19 & -0.19 & -0.22 & -0.21 & 0.03 & 0.10 \\
\hline \multirow{3}{*}{ 27/1-naphthol } & \multirow{3}{*}{$2^{1} \mathrm{~A}$} & $\Delta \Delta E_{00}$ & -0.14 & -0.16 & -0.16 & -0.17 & -0.10 & -0.16 & -0.15 & -0.13 & -0.15 & 0.02 & 0.07 \\
\hline & & $\Delta E_{\mathrm{ve}}$ & 3.60 & 4.04 & 4.16 & 4.54 & 4.89 & 4.48 & 4.48 & 5.23 & 4.32 & 0.45 & 1.63 \\
\hline & & $\Delta \Delta E_{\mathrm{ad}}$ & -0.26 & -0.30 & -0.31 & -0.39 & -0.40 & -0.37 & -0.37 & -0.40 & -0.20 & 0.07 & 0.20 \\
\hline \multirow{3}{*}{ 28/1-naphthol } & \multirow{3}{*}{$2^{1} \mathrm{~A}^{\prime}$} & $\Delta \Delta E_{00}$ & -0.07 & -0.08 & -0.07 & -0.08 & -0.17 & -0.09 & -0.08 & -0.11 & -0.14 & 0.03 & 0.10 \\
\hline & & $\Delta E_{\mathrm{ve}}$ & 3.77 & 4.19 & 4.31 & 4.64 & 4.92 & 4.59 & 4.59 & 5.30 & 4.37 & 0.41 & 1.52 \\
\hline & & $\Delta \Delta E_{\mathrm{ad}}$ & -0.24 & -0.28 & -0.29 & -0.34 & -0.29 & -0.34 & -0.33 & -0.36 & -0.16 & 0.06 & 0.21 \\
\hline \multirow{3}{*}{ 29/2-naphthol } & \multirow{3}{*}{$2^{1} \mathrm{~A}^{\prime}$} & $\Delta \Delta E_{00}$ & -0.15 & -0.17 & -0.17 & -0.19 & -0.23 & -0.19 & -0.19 & -0.22 & -0.13 & 0.03 & 0.10 \\
\hline & & $\Delta E_{\mathrm{ve}}$ & 3.78 & 4.16 & 4.27 & 4.52 & 4.87 & 4.48 & 4.48 & 5.33 & 4.27 & 0.42 & 1.56 \\
\hline & & $\Delta \Delta E_{\mathrm{ad}}$ & -0.17 & -0.21 & -0.21 & -0.23 & -0.28 & -0.22 & -0.22 & -0.36 & -0.18 & 0.05 & 0.19 \\
\hline \multirow{4}{*}{ 30/2-naphthol } & \multirow{3}{*}{$2^{1} \mathrm{~A}^{\prime}$} & $\Delta \Delta E_{00}$ & -0.12 & -0.14 & -0.16 & -0.17 & -0.16 & -0.17 & -0.17 & -0.19 & -0.12 & 0.02 & 0.07 \\
\hline & & $\Delta E_{\mathrm{ve}}$ & 3.71 & 4.09 & 4.20 & 4.46 & 4.81 & 4.41 & 4.42 & 5.25 & 4.21 & 0.41 & 1.54 \\
\hline & & $\Delta \Delta E_{\mathrm{ad}}$ & -0.16 & -0.20 & -0.21 & -0.23 & -0.28 & -0.23 & -0.22 & -0.36 & -0.18 & 0.06 & 0.20 \\
\hline & \multirow{3}{*}{$2^{1} \mathrm{~A}^{\prime}$} & $\Delta \Delta E_{00}$ & -0.12 & -0.14 & -0.15 & -0.17 & -0.15 & -0.16 & -0.16 & -0.17 & -0.11 & 0.02 & 0.07 \\
\hline \multirow[t]{2}{*}{ 31/5-methoxysalicylic acid } & & $\Delta E_{\mathrm{ve}}$ & 3.24 & 3.74 & 3.87 & 4.22 & 4.59 & 4.10 & 4.10 & 5.30 & 3.89 & 0.54 & 2.07 \\
\hline & & $\Delta \Delta E_{\mathrm{ad}}$ & -0.19 & -0.23 & -0.25 & -0.30 & -0.45 & -0.28 & -0.27 & -0.37 & -0.33 & 0.07 & 0.26 \\
\hline \multirow{3}{*}{ 32/m-dimethoxybenzene } & \multirow{3}{*}{$2^{1} \mathrm{~A}^{\prime}$} & $\Delta \Delta E_{00}$ & -0.15 & -0.15 & -0.15 & -0.16 & -0.16 & -0.14 & -0.14 & -0.13 & -0.15 & 0.01 & 0.04 \\
\hline & & $\Delta E_{\mathrm{ve}}$ & 4.63 & 4.97 & 5.10 & 5.26 & 5.51 & 5.18 & 5.17 & 6.07 & 4.91 & 0.39 & 1.44 \\
\hline & & $\Delta \Delta E_{\mathrm{ad}}$ & -0.24 & -0.22 & -0.23 & -0.25 & -0.36 & -0.22 & -0.22 & -0.24 & -0.29 & 0.04 & 0.14 \\
\hline \multirow{3}{*}{ 33/3P-propionic acid } & \multirow{3}{*}{$2^{1} \mathrm{~A}$} & $\Delta \Delta E_{00}$ & -0.18 & -0.16 & -0.16 & -0.15 & -0.14 & -0.16 & -0.16 & -0.15 & -0.19 & 0.02 & 0.05 \\
\hline & & $\Delta E_{\mathrm{ve}}$ & 4.83 & 5.33 & 5.45 & 5.54 & 5.74 & 5.50 & 5.50 & 6.16 & 5.20 & 0.34 & 1.33 \\
\hline & & $\Delta \Delta E_{\mathrm{ad}}$ & -1.02 & -0.11 & -0.11 & -0.13 & -0.15 & -0.13 & -0.14 & -0.13 & -0.18 & 0.28 & 0.90 \\
\hline & & $\Delta \Delta E_{00}$ & -0.11 & -0.18 & -0.17 & -0.16 & -0.16 & -0.14 & -0.13 & -0.12 & -0.15 & 0.02 & 0.07 \\
\hline 34/3P-propionic acid & $1^{1} \mathrm{~A}^{\prime \prime}$ & $\Delta E_{\mathrm{ve}}$ & 4.91 & 5.33 & 5.45 & 5.54 & 5.74 & 5.50 & 5.49 & 6.16 & 5.20 & 0.33 & 1.25 \\
\hline & & $\Delta \Delta E_{\mathrm{ad}}$ & -0.52 & -0.13 & -0.13 & -0.14 & -0.15 & -0.13 & -0.14 & -0.13 & -0.18 & 0.12 & 0.40 \\
\hline
\end{tabular}


Table S3. Continued.

\begin{tabular}{|c|c|c|c|c|c|c|c|c|c|c|c|c|c|}
\hline \multirow[b]{2}{*}{ Label/Molecule } & \multirow[b]{2}{*}{ State } & \multirow[b]{2}{*}{$\Delta E$} & \multicolumn{11}{|c|}{ Method and statistics ${ }^{\mathrm{b}}$} \\
\hline & & & BP86 & B3LYP & PBE0 & M06-2X & M06-HF & CB3LYP & $\omega B 97 X-D$ & CIS & $\mathrm{CC} 2$ & SD & MaxAD \\
\hline \multirow{4}{*}{ 35/fluorene } & \multirow{3}{*}{$1{ }^{1} \mathrm{~B}_{2}$} & $\Delta \Delta E_{00}$ & -0.16 & -0.16 & -0.16 & -0.16 & -0.15 & -0.14 & -0.14 & -0.12 & -0.16 & 0.01 & 0.04 \\
\hline & & $\Delta E_{\mathrm{ve}}$ & 4.19 & 4.55 & 4.68 & 4.92 & 5.23 & 4.88 & 4.92 & 5.37 & 4.67 & 0.34 & 1.18 \\
\hline & & $\Delta \Delta E_{\mathrm{ad}}$ & -0.12 & -0.16 & -0.18 & -0.25 & -0.32 & -0.27 & -0.28 & -0.37 & -0.14 & 0.08 & 0.25 \\
\hline & \multirow{3}{*}{$2^{1} \mathrm{~A}_{1}$} & $\Delta \Delta E_{00}$ & -0.20 & -0.17 & -0.16 & -0.15 & -0.15 & -0.14 & -0.14 & -0.13 & -0.16 & 0.02 & 0.08 \\
\hline \multirow[t]{3}{*}{ 36/phenanthrene } & & $\Delta E_{\mathrm{ve}}$ & 3.60 & 3.98 & 4.11 & 4.37 & 4.74 & 4.35 & 4.36 & 5.07 & 4.08 & 0.40 & 1.47 \\
\hline & & $\Delta \Delta E_{\mathrm{ad}}$ & -0.12 & -0.13 & -0.14 & -0.17 & -0.25 & -0.17 & -0.17 & -0.21 & -0.16 & 0.04 & 0.13 \\
\hline & \multirow{4}{*}{$1^{1} B_{u}$} & $\Delta \Delta E_{00}$ & -0.13 & -0.12 & -0.12 & -0.13 & -0.14 & -0.12 & -0.12 & -0.21 & -0.11 & 0.03 & 0.09 \\
\hline \multirow[t]{3}{*}{$37 / 2,4,6,8$-decatetraene } & & $\Delta E_{\mathrm{ve}}$ & 3.64 & 3.94 & 4.03 & 4.34 & 4.78 & 4.33 & 4.34 & 5.18 & 4.59 & 0.43 & 1.53 \\
\hline & & $\Delta \Delta E_{\mathrm{ad}}$ & -0.15 & -0.23 & -0.24 & -0.35 & -0.49 & -0.35 & -0.36 & -0.50 & -0.31 & 0.11 & 0.35 \\
\hline & & $\Delta \Delta E_{00}$ & -0.13 & -0.12 & -0.12 & -0.11 & -0.11 & -0.12 & -0.11 & -0.12 & -0.11 & 0.01 & 0.02 \\
\hline \multirow[t]{3}{*}{ 38/tetracene } & \multirow[t]{3}{*}{$1^{1} \mathrm{~B}_{1 \mathrm{u}}$} & $\Delta E_{\mathrm{ve}}$ & 2.15 & 2.47 & 2.56 & 2.92 & 3.41 & 2.91 & 2.96 & 3.73 & 2.97 & 0.45 & 1.59 \\
\hline & & $\Delta \Delta E_{\mathrm{ad}}$ & -0.13 & -0.18 & -0.18 & -0.26 & -0.39 & -0.25 & -0.25 & -0.37 & -0.17 & 0.08 & 0.26 \\
\hline & & $\Delta \Delta E_{00}$ & -0.10 & -0.13 & -0.15 & -0.19 & -0.21 & -0.18 & -0.20 & -0.25 & -0.23 & 0.04 & 0.14 \\
\hline \multirow[t]{3}{*}{$39 /$ perylene } & \multirow[t]{3}{*}{$1{ }^{1} \mathrm{~B}$} & $\Delta E_{\mathrm{ve}}$ & 2.53 & 2.82 & 2.91 & 3.24 & 3.70 & 3.22 & 3.26 & 3.92 & 3.16 & 0.40 & 1.39 \\
\hline & & $\Delta \Delta E_{\mathrm{ad}}$ & -0.12 & -0.17 & -0.18 & -0.26 & -0.38 & -0.26 & -0.26 & -0.37 & -0.22 & 0.08 & 0.26 \\
\hline & & $\Delta \Delta E_{00}$ & -0.06 & -0.03 & -0.05 & -0.04 & -0.04 & -0.03 & -0.04 & -0.04 & -0.04 & 0.01 & 0.03 \\
\hline
\end{tabular}

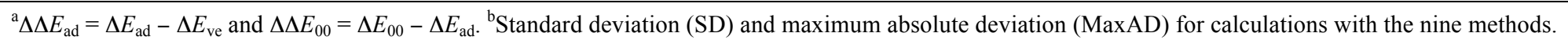


Table S4. $<\mathrm{A}_{\mathrm{BL}} \mathrm{SD}-\mathrm{S}_{1}>,<\mathrm{A}_{\mathrm{BL}} \mathrm{SD}-\mathrm{S}_{1} \mathrm{~S}_{0}>$ and $<\mathrm{A}_{\mathrm{BL}} \mathrm{SD}-\mathrm{S}_{0}>$ values for bonds between heavy atoms for selected states in groups I and II of the benchmark set $(\AA))^{a}$

\begin{tabular}{lccc}
\hline Group $(\mathrm{s})^{\mathrm{b}}$ & $\left\langle\mathrm{A}_{\mathrm{BL}} \mathrm{SD}_{-} \mathrm{S}_{1}>\right.$ & $\left\langle\mathrm{A}_{\mathrm{BL}} \mathrm{SD}-\mathrm{S}_{1} \mathrm{~S}_{0}>\right.$ & $\left\langle\mathrm{A}_{\mathrm{BL}} \mathrm{SD}-\mathrm{S}_{0}>\right.$ \\
\hline I & 0.013 & 0.013 & 0.007 \\
II & 0.009 & 0.009 & 0.007 \\
I-II & 0.011 & 0.010 & 0.007 \\
\hline
\end{tabular}

\begin{abstract}
${ }^{\mathrm{a}}$ Average ASDs for excited-state bond lengths $\left(<\mathrm{A}_{\mathrm{BL}} \mathrm{SD}-\mathrm{S}_{1}>\right)$, for excited-state bond lengths relative to ground-state bond lengths $\left(<\mathrm{A}_{\mathrm{BL}} \mathrm{SD}-\mathrm{S}_{1} \mathrm{~S}_{0}>\right)$, and for ground-state bond lengths $\left(<\mathrm{A}_{\mathrm{BL}} \mathrm{SD}_{-} \mathrm{S}_{0}>\right)$ with calculations performed using the BP86, B3LYP, PBE0, M06-2X, M06-HF, CAM-B3LYP, $\omega$ B97X-D, CIS and CC2 methods. ${ }^{b}$ From group I, the states considered in the analysis are the $\mathrm{S}_{1}$ states of pyrazine, 2,6-difluoropyridine, 2-pyridone, 2-methylpyrimidine, 7azaindole, 7-hydroxyquinoline ( $c i s)$, dibenzofuran, pyrrolo[3,2- $h]$ quinoline, carbazole, tryptamine (A-ph) and porphycene. From group II, the states considered in the analysis are the $\mathrm{S}_{1}$ states of tetrafluorobenzene, benzonitrile, o-fluorophenol (cis), phenylacetylene, aniline, catechol, resorcinol (isomer 1), hydroquinone (cis), salicylic acid, o-cresol (cis), $o$ methoxyphenol, 1-naphthol (cis), 5-methoxysalicylic acid, m-dimethoxybenzene (trans), 3Ppropionic acid (gauche), fluorene, phenanthrene, 2,4,6,8-decatetraene, tetracene and perylene.
\end{abstract}

\title{
Comments on Table S4
}

The similarity between the methods (BP86, B3LYP, PBE0, M06-2X, M06-HF, CAM-B3LYP, $\omega \mathrm{B} 97 \mathrm{X}-\mathrm{D}, \mathrm{CIS}$ and $\mathrm{CC} 2)$ in their descriptions of the excited states in the benchmark set was quantified geometrically in the following way. First, for a representative subset of excited states (see footnote [b]), SDs were computed for the estimates of each excited-state bond length, as well as for the estimates of each excited-state bond length relative to the corresponding groundstate bond length. Throughout, only bonds between heavy atoms were considered. Then, by averaging the resulting SDs over all bonds in the excited state in question, average bond-length SDs denoted $\mathrm{A}_{B L} \mathrm{SD}-\mathrm{S}_{1}$ and $\mathrm{A}_{\mathrm{BL}} \mathrm{SD}-\mathrm{S}_{1} \mathrm{~S}_{0}$, respectively, were obtained for that excited state. Finally, by averaging the $\mathrm{A}_{\mathrm{BL}} \mathrm{SD}-\mathrm{S}_{1}$ and $\mathrm{A}_{\mathrm{BL}} \mathrm{SD}-\mathrm{S}_{1} \mathrm{~S}_{0}$ values over all excited states considered, 'similarity measures' denoted $<\mathrm{A}_{\mathrm{BL}} \mathrm{SD}-\mathrm{S}_{1}>$ and $<\mathrm{A}_{\mathrm{BL}} \mathrm{SD}-\mathrm{S}_{1} \mathrm{~S}_{0}>$, respectively, were obtained. These are the values given in Table S4. Notably, their smallness $(0.011$ and $0.010 \AA$ for the full subset of excited states) are indicative of a large degree of similarity between the descriptions of the excited states by the methods, which helps explain why also the estimates of $\Delta \Delta E_{\text {ad }}$ are similar. 
Atomic coordinates of optimized ground- and excited-state geometries

\section{BP86/TZVP geometries - Group I}

pyrazine $\mathrm{S}_{0}$

$\begin{array}{lrrr}\mathrm{C} & 1.136741 & -0.700091 & 0.000000 \\ \mathrm{C} & 1.136741 & 0.700091 & 0.000000 \\ \mathrm{~N} & 0.000000 & 1.415836 & 0.000000 \\ \mathrm{C} & -1.136741 & 0.700091 & 0.000000 \\ \mathrm{C} & -1.136741 & -0.700091 & 0.000000 \\ \mathrm{~N} & 0.000000 & -1.415836 & 0.000000 \\ \mathrm{H} & -2.075256 & -1.263288 & 0.000000 \\ \mathrm{H} & 2.075256 & -1.263288 & 0.000000 \\ \mathrm{H} & 2.075256 & 1.263288 & 0.000000 \\ \mathrm{H} & -2.075256 & 1.263288 & 0.000000\end{array}$

pyrazine $S_{1}$

$\begin{array}{lrrr}\mathrm{C} & -1.194537 & 0.711534 & 0.000000 \\ \mathrm{C} & -1.143270 & -0.791758 & 0.000000 \\ \mathrm{~N} & 0.045994 & -1.339174 & 0.000000 \\ \mathrm{C} & 1.194537 & -0.711534 & 0.000000 \\ \mathrm{C} & 1.143270 & 0.791758 & 0.000000 \\ \mathrm{~N} & -0.045994 & 1.339174 & 0.000000 \\ \mathrm{H} & 2.054296 & 1.386842 & 0.000000 \\ \mathrm{H} & -2.144173 & 1.242961 & 0.000000 \\ \mathrm{H} & -2.054296 & -1.386842 & 0.000000 \\ \mathrm{H} & 2.144173 & -1.242961 & 0.000000\end{array}$

2,6-difluoropyridine $\mathrm{S}_{0}$

$\begin{array}{lrrr}\mathrm{C} & 1.122223 & 0.000000 & -0.881003 \\ \mathrm{~N} & 0.000000 & 0.000000 & -1.580445 \\ \mathrm{C} & -1.122223 & 0.000000 & -0.881003 \\ \mathrm{C} & -1.210941 & 0.000000 & 0.511558 \\ \mathrm{C} & 0.000000 & 0.000000 & 1.210052 \\ \mathrm{C} & 1.210941 & 0.000000 & 0.511558 \\ \mathrm{~F} & -2.262310 & 0.000000 & -1.606181 \\ \mathrm{H} & -2.179095 & 0.000000 & 1.010283 \\ \mathrm{H} & 0.000000 & 0.000000 & 2.301497 \\ \mathrm{H} & 2.179095 & 0.000000 & 1.010283 \\ \mathrm{~F} & 2.262310 & 0.000000 & -1.606181\end{array}$

2,6-difluoropyridine $\mathrm{S}_{1}$ 


$\begin{array}{crcr}\mathrm{C} & -1.108049 & 0.000000 & -0.338787 \\ \mathrm{~N} & 0.000000 & 0.000000 & -1.116400 \\ \mathrm{C} & 1.108049 & 0.000000 & -0.338787 \\ \mathrm{C} & 1.204198 & 0.000000 & 1.075616 \\ \mathrm{C} & 0.000000 & 0.000000 & 1.854217 \\ \mathrm{C} & -1.204198 & 0.000000 & 1.075616 \\ \mathrm{~F} & 2.264104 & 0.000000 & -1.030409 \\ \mathrm{H} & 2.195547 & 0.000000 & 1.529601 \\ \mathrm{H} & 0.000000 & 0.000000 & 2.939434 \\ \mathrm{H} & -2.195547 & 0.000000 & 1.529601 \\ \mathrm{~F} & -2.264104 & 0.000000 & -1.030409\end{array}$

2-fluoropyridine $\mathrm{S}_{0}$

$\begin{array}{lrrr}\mathrm{C} & -1.322697 & 0.506378 & 0.000000 \\ \mathrm{~N} & -1.378282 & -0.809808 & 0.000000 \\ \mathrm{C} & -0.198302 & -1.462325 & 0.000000 \\ \mathrm{C} & 1.037487 & -0.813803 & 0.000000 \\ \mathrm{C} & 1.051633 & 0.587518 & 0.000000 \\ \mathrm{C} & -0.158188 & 1.281127 & 0.000000 \\ \mathrm{H} & -0.261117 & -2.554215 & 0.000000 \\ \mathrm{H} & 1.963751 & -1.389807 & 0.000000 \\ \mathrm{H} & 1.995960 & 1.135691 & 0.000000 \\ \mathrm{H} & -0.212925 & 2.369854 & 0.000000 \\ \mathrm{~F} & -2.517331 & 1.149381 & 0.000000\end{array}$

2-fluoropyridine $\mathrm{S}_{1}$

$\begin{array}{rrrr}\mathrm{C} & -1.326387 & 0.498671 & 0.000000 \\ \mathrm{~N} & -1.439048 & -0.845692 & 0.000000 \\ \mathrm{C} & -0.219221 & -1.482539 & 0.000000 \\ \mathrm{C} & 1.040544 & -0.807114 & 0.000000 \\ \mathrm{C} & 1.107080 & 0.618887 & 0.000000 \\ \mathrm{C} & -0.156124 & 1.303235 & 0.000000 \\ \mathrm{H} & -0.256008 & -2.573252 & 0.000000 \\ \mathrm{H} & 1.952830 & -1.405795 & 0.000000 \\ \mathrm{H} & 2.052744 & 1.155366 & 0.000000 \\ \mathrm{H} & -0.244051 & 2.388791 & 0.000000 \\ \mathrm{~F} & -2.512370 & 1.149433 & 0.000000\end{array}$

2-hydroxypyridine $\mathrm{S}_{0}$
$\begin{array}{llll}\text { C } & -0.987526 & 0.759336 & 0.000000\end{array}$
$\begin{array}{llll}\text { C } & -0.980023 & -0.647264 & 0.000000\end{array}$
$\begin{array}{llll}\mathrm{N} & 0.129751 & -1.390420 & 0.000000\end{array}$ 


$\begin{array}{rrrc}\mathrm{C} & 1.311177 & -0.742977 & 0.000000 \\ \mathrm{C} & 1.425935 & 0.646491 & 0.000000 \\ \mathrm{C} & 0.244763 & 1.404775 & 0.000000 \\ \mathrm{O} & -2.169982 & -1.309237 & 0.000000 \\ \mathrm{H} & 2.199908 & -1.381029 & 0.000000 \\ \mathrm{H} & 2.407328 & 1.122038 & 0.000000 \\ \mathrm{H} & 0.287381 & 2.496152 & 0.000000 \\ \mathrm{H} & -1.932498 & 1.303031 & 0.000000 \\ \mathrm{H} & -1.936215 & -2.260895 & 0.000000\end{array}$

2-hydroxypyridine $\mathrm{S}_{1}$

$\begin{array}{rrrl}\mathrm{C} & -1.021693 & 0.778762 & 0.000000 \\ \mathrm{C} & -0.969554 & -0.616073 & 0.000000 \\ \mathrm{~N} & 0.097208 & -1.449809 & 0.000000 \\ \mathrm{C} & 1.303659 & -0.777410 & 0.000000 \\ \mathrm{C} & 1.396848 & 0.640959 & 0.000000 \\ \mathrm{C} & 0.258656 & 1.465932 & 0.000000 \\ \mathrm{O} & -2.161910 & -1.320034 & 0.000000 \\ \mathrm{H} & 2.201421 & -1.396173 & 0.000000 \\ \mathrm{H} & 2.395256 & 1.085665 & 0.000000 \\ \mathrm{H} & 0.326107 & 2.550868 & 0.000000 \\ \mathrm{H} & -1.978760 & 1.298088 & 0.000000 \\ \mathrm{H} & -1.847238 & -2.260775 & 0.000000\end{array}$

2-pyridone $\mathrm{S}_{0}$

$\begin{array}{crrc}\mathrm{C} & -1.255560 & 0.727076 & 0.000000 \\ \mathrm{~N} & -1.139695 & -0.693862 & 0.000000 \\ \mathrm{C} & 0.032294 & -1.395488 & 0.000000 \\ \mathrm{C} & 1.232287 & -0.733907 & 0.000000 \\ \mathrm{C} & 1.213893 & 0.691964 & 0.000000 \\ \mathrm{C} & 0.034842 & 1.394353 & 0.000000 \\ \mathrm{H} & -2.033284 & -1.185810 & 0.000000 \\ \mathrm{H} & -0.055318 & -2.482848 & 0.000000 \\ \mathrm{H} & 2.166357 & -1.292818 & 0.000000 \\ \mathrm{H} & 2.163020 & 1.234449 & 0.000000 \\ \mathrm{H} & 0.012725 & 2.484345 & 0.000000 \\ \mathrm{O} & -2.371562 & 1.252558 & 0.000000\end{array}$

2-pyridone $\mathrm{S}_{1}$
$\begin{array}{llll}\text { C } & -1.356335 & 0.100156 & 0.000000\end{array}$
$\begin{array}{llll}\mathrm{N} & -0.735684 & -1.103967 & 0.000000\end{array}$
$\begin{array}{llll}\text { C } & 0.649837 & -1.265576 & 0.000000\end{array}$
$\begin{array}{llll}\text { C } & 1.433611 & -0.076395 & 0.000000\end{array}$ 


$\begin{array}{rrrr}\mathrm{C} & 0.836795 & 1.159264 & 0.000000 \\ \mathrm{C} & -0.621783 & 1.273714 & 0.000000 \\ \mathrm{H} & -1.365197 & -1.907608 & 0.000000 \\ \mathrm{H} & 1.034962 & -2.279803 & 0.000000 \\ \mathrm{H} & 2.520070 & -0.176679 & 0.000000 \\ \mathrm{H} & 1.445526 & 2.064217 & 0.000000 \\ \mathrm{H} & -1.140285 & 2.227827 & 0.000000 \\ \mathrm{O} & -2.701516 & -0.015161 & 0.000000\end{array}$

2-methylpyrimidine $\mathrm{S}_{0}$

$\begin{array}{rrrr}\mathrm{C} & 0.000122 & 1.975040 & 0.000000 \\ \mathrm{C} & -1.183179 & 1.231325 & 0.000000 \\ \mathrm{~N} & -1.194446 & -0.108706 & 0.000000 \\ \mathrm{C} & 0.010225 & -0.722325 & 0.000000 \\ \mathrm{~N} & 1.208566 & -0.101620 & 0.000000 \\ \mathrm{C} & 1.186866 & 1.241463 & 0.000000 \\ \mathrm{H} & -2.162031 & 1.723264 & 0.000000 \\ \mathrm{H} & 2.162610 & 1.739118 & 0.000000 \\ \mathrm{H} & -0.004416 & 3.066081 & 0.000000 \\ \mathrm{C} & 0.005379 & -2.225763 & 0.000000 \\ \mathrm{H} & 1.031864 & -2.610222 & 0.000000 \\ \mathrm{H} & -0.530785 & -2.603827 & 0.883900 \\ \mathrm{H} & -0.530785 & -2.603827 & -0.883900\end{array}$

2-methylpyrimidine $\mathrm{S}_{1}$

$\begin{array}{crcc}\mathrm{C} & -0.018858 & 2.029709 & 0.000000 \\ \mathrm{C} & -1.169082 & 1.270719 & 0.000000 \\ \mathrm{~N} & -1.189313 & -0.089862 & 0.000000 \\ \mathrm{C} & 0.017607 & -0.789122 & 0.000000 \\ \mathrm{~N} & 1.089680 & -0.059136 & 0.000000 \\ \mathrm{C} & 1.224301 & 1.299739 & 0.000000 \\ \mathrm{H} & -2.154939 & 1.747376 & 0.000000 \\ \mathrm{H} & 2.222721 & 1.724372 & 0.000000 \\ \mathrm{H} & -0.036952 & 3.119174 & 0.000000 \\ \mathrm{C} & 0.016291 & -2.277144 & 0.000000 \\ \mathrm{H} & 1.039967 & -2.671670 & 0.000000 \\ \mathrm{H} & -0.520711 & -2.652083 & 0.886434 \\ \mathrm{H} & -0.520711 & -2.652083 & -0.886434\end{array}$

5-methylpyrimidine $\mathrm{S}_{0}$
$\begin{array}{llll}\text { C } & 0.879602 & 0.857605 & 0.000000\end{array}$
$\begin{array}{llll}\text { C } & -0.364866 & 0.212339 & 0.000000\end{array}$
$\begin{array}{llll}\text { C } & -0.302884 & -1.190745 & 0.000000\end{array}$ 


$\begin{array}{rrrr}\mathrm{N} & 0.849372 & -1.877217 & 0.000000 \\ \mathrm{C} & 1.970446 & -1.136444 & 0.000000 \\ \mathrm{~N} & 2.052048 & 0.202174 & 0.000000 \\ \mathrm{H} & 2.917407 & -1.684694 & 0.000000 \\ \mathrm{H} & -1.222210 & -1.788937 & 0.000000 \\ \mathrm{H} & 0.936253 & 1.952854 & 0.000000 \\ \mathrm{C} & -1.669157 & 0.965077 & 0.000000 \\ \mathrm{H} & -1.498635 & 2.051164 & 0.000000 \\ \mathrm{H} & -2.273688 & 0.718401 & -0.886916 \\ \mathrm{H} & -2.273688 & 0.718401 & 0.886916\end{array}$

5-methylpyrimidine $\mathrm{S}_{1}$

$\begin{array}{rrrr}\mathrm{C} & 0.861160 & 0.852296 & 0.000000 \\ \mathrm{C} & -0.383415 & 0.252388 & 0.000000 \\ \mathrm{C} & -0.352328 & -1.206926 & 0.000000 \\ \mathrm{~N} & 0.881128 & -1.763930 & 0.000000 \\ \mathrm{C} & 2.045812 & -1.189799 & 0.000000 \\ \mathrm{~N} & 2.051015 & 0.197330 & 0.000000 \\ \mathrm{H} & 2.974080 & -1.754994 & 0.000000 \\ \mathrm{H} & -1.222214 & -1.857196 & 0.000000 \\ \mathrm{H} & 0.940503 & 1.945626 & 0.000000 \\ \mathrm{C} & -1.685431 & 0.987362 & 0.000000 \\ \mathrm{H} & -1.526048 & 2.074947 & 0.000000 \\ \mathrm{H} & -2.292137 & 0.731453 & -0.887061 \\ \mathrm{H} & -2.292137 & 0.731453 & 0.887061\end{array}$

7-azaindole $\mathrm{S}_{0}$

$\begin{array}{lrrr}\mathrm{N} & -0.637571 & 1.854213 & 0.000000 \\ \mathrm{C} & -0.679511 & 0.473966 & 0.000000 \\ \mathrm{C} & 0.675768 & 0.007402 & 0.000000 \\ \mathrm{C} & 1.518202 & 1.169427 & 0.000000 \\ \mathrm{C} & 0.685344 & 2.268532 & 0.000000 \\ \mathrm{C} & 0.851846 & -1.386156 & 0.000000 \\ \mathrm{C} & -0.293259 & -2.183689 & 0.000000 \\ \mathrm{C} & -1.573117 & -1.589901 & 0.000000 \\ \mathrm{~N} & -1.791715 & -0.264286 & 0.000000 \\ \mathrm{H} & -2.467982 & -2.219282 & 0.000000 \\ \mathrm{H} & 1.849073 & -1.832587 & 0.000000 \\ \mathrm{H} & -0.212143 & -3.272119 & 0.000000 \\ \mathrm{H} & -1.458869 & 2.449906 & 0.000000 \\ \mathrm{H} & 2.604262 & 1.197604 & 0.000000 \\ \mathrm{H} & 0.929692 & 3.326970 & 0.000000\end{array}$

7-azaindole $\mathrm{S}_{1}$ 


$\begin{array}{rrrr}\mathrm{N} & -0.599324 & 1.887010 & 0.000000 \\ \mathrm{C} & -0.653417 & 0.435413 & 0.000000 \\ \mathrm{C} & 0.645217 & -0.018078 & 0.000000 \\ \mathrm{C} & 1.505701 & 1.175265 & 0.000000 \\ \mathrm{C} & 0.663380 & 2.329672 & 0.000000 \\ \mathrm{C} & 0.895504 & -1.426687 & 0.000000 \\ \mathrm{C} & -0.308488 & -2.197892 & 0.000000 \\ \mathrm{C} & -1.566653 & -1.604025 & 0.000000 \\ \mathrm{~N} & -1.834543 & -0.239381 & 0.000000 \\ \mathrm{H} & -2.457209 & -2.239496 & 0.000000 \\ \mathrm{H} & 1.890965 & -1.863250 & 0.000000 \\ \mathrm{H} & -0.255094 & -3.289060 & 0.000000 \\ \mathrm{H} & -1.440955 & 2.462362 & 0.000000 \\ \mathrm{H} & 2.593310 & 1.204377 & 0.000000 \\ \mathrm{H} & 0.921617 & 3.383791 & 0.000000\end{array}$

7-hydroxyquinoline, cis $\mathrm{S}_{0}$

$\begin{array}{rrrr}\mathrm{N} & 0.473337 & 1.984315 & 0.000000 \\ \mathrm{C} & 0.440268 & 0.614229 & 0.000000 \\ \mathrm{C} & -0.793479 & -0.128534 & 0.000000 \\ \mathrm{C} & -2.003173 & 0.608711 & 0.000000 \\ \mathrm{C} & -1.955890 & 1.989373 & 0.000000 \\ \mathrm{C} & -0.688076 & 2.623904 & 0.000000 \\ \mathrm{C} & 1.672429 & -0.088711 & 0.000000 \\ \mathrm{C} & 1.685676 & -1.473717 & 0.000000 \\ \mathrm{C} & 0.470596 & -2.212648 & 0.000000 \\ \mathrm{C} & -0.736999 & -1.550379 & 0.000000 \\ \mathrm{H} & -0.631037 & 3.718356 & 0.000000 \\ \mathrm{H} & -1.671173 & -2.118359 & 0.000000 \\ \mathrm{H} & 0.524371 & -3.302566 & 0.000000 \\ \mathrm{O} & 2.839694 & -2.217607 & 0.000000 \\ \mathrm{H} & 2.594777 & 0.498826 & 0.000000 \\ \mathrm{H} & -2.958032 & 0.075399 & 0.000000 \\ \mathrm{H} & -2.866518 & 2.591054 & 0.000000 \\ \mathrm{H} & 3.603230 & -1.611636 & 0.000000\end{array}$

7-hydroxyquinoline, cis $\mathrm{S}_{1}$
$\begin{array}{llll}\mathrm{N} & 0.508803 & 1.987365 & 0.000000\end{array}$
$\begin{array}{llll}\text { C } & 0.421489 & 0.636200 & 0.000000\end{array}$
$\begin{array}{llll}\text { C } & -0.784317 & -0.130827 & 0.000000\end{array}$
$\begin{array}{llll}\text { C } & -2.027462 & 0.605165 & 0.000000\end{array}$
$\begin{array}{llll}\text { C } & -1.939475 & 2.010291 & 0.000000\end{array}$
$\begin{array}{llll}\text { C } & -0.700495 & 2.652588 & 0.000000\end{array}$ 


$\begin{array}{rrrr}\mathrm{C} & 1.650216 & -0.105402 & 0.000000 \\ \mathrm{C} & 1.669321 & -1.530941 & 0.000000 \\ \mathrm{C} & 0.485455 & -2.285183 & 0.000000 \\ \mathrm{C} & -0.709522 & -1.556392 & 0.000000 \\ \mathrm{H} & -0.637934 & 3.743122 & 0.000000 \\ \mathrm{H} & -1.653624 & -2.110103 & 0.000000 \\ \mathrm{H} & 0.514941 & -3.372798 & 0.000000 \\ \mathrm{O} & 2.863854 & -2.182389 & 0.000000 \\ \mathrm{H} & 2.586100 & 0.464333 & 0.000000 \\ \mathrm{H} & -2.985730 & 0.085798 & 0.000000 \\ \mathrm{H} & -2.850947 & 2.614085 & 0.000000 \\ \mathrm{H} & 3.589326 & -1.524903 & 0.000000\end{array}$

7-hydroxyquinoline, trans $\mathrm{S}_{0}$

$\begin{array}{rrrc}\mathrm{N} & 0.523191 & 2.057576 & 0.000000 \\ \mathrm{C} & 0.488120 & 0.685727 & 0.000000 \\ \mathrm{C} & -0.750764 & -0.051395 & 0.000000 \\ \mathrm{C} & -1.959310 & 0.689799 & 0.000000 \\ \mathrm{C} & -1.907032 & 2.069089 & 0.000000 \\ \mathrm{C} & -0.635293 & 2.699385 & 0.000000 \\ \mathrm{C} & 1.718098 & -0.016025 & 0.000000 \\ \mathrm{C} & 1.728144 & -1.400899 & 0.000000 \\ \mathrm{C} & 0.508913 & -2.133892 & 0.000000 \\ \mathrm{C} & -0.700929 & -1.470723 & 0.000000 \\ \mathrm{H} & -0.575595 & 3.793838 & 0.000000 \\ \mathrm{H} & -1.635594 & -2.037829 & 0.000000 \\ \mathrm{H} & 0.537483 & -3.228252 & 0.000000 \\ \mathrm{O} & 2.944934 & -2.039226 & 0.000000 \\ \mathrm{H} & 2.649659 & 0.550723 & 0.000000 \\ \mathrm{H} & -2.915894 & 0.159515 & 0.000000 \\ \mathrm{H} & -2.815176 & 2.674581 & 0.000000 \\ \mathrm{H} & 2.797027 & -3.001971 & 0.000000\end{array}$

7-hydroxyquinoline, trans $\mathrm{S}_{1}$
$\begin{array}{llll}\mathrm{N} & 0.372242 & 1.980247 & -0.000001\end{array}$
$\begin{array}{llll}\text { C } & 0.523576 & 0.657087 & -0.000001\end{array}$
$\begin{array}{llll}\text { C } & -0.761534 & -0.061573 & -0.000002\end{array}$
$\begin{array}{llll}\text { C } & -1.951866 & 0.714301 & 0.000000\end{array}$
$\begin{array}{llll}\text { C } & -1.935829 & 2.123749 & 0.000001\end{array}$
$\begin{array}{llll}\text { C } & -0.703902 & 2.770227 & -0.000001\end{array}$
$\begin{array}{llll}\text { C } & 1.755112 & -0.030073 & -0.000001\end{array}$
$\begin{array}{llll}\text { C } & 1.736858 & -1.437379 & 0.000000\end{array}$
$\begin{array}{llll}\text { C } & 0.542420 & -2.161936 & -0.000001\end{array}$
$\begin{array}{llll}\text { C } & -0.695178 & -1.472058 & -0.000001\end{array}$ 


$\begin{array}{rrrr}\mathrm{H} & -0.549992 & 3.848524 & 0.000000 \\ \mathrm{H} & -1.632762 & -2.029422 & -0.000001 \\ \mathrm{H} & 0.561597 & -3.254698 & 0.000000 \\ \mathrm{O} & 2.967225 & -2.050467 & 0.000001 \\ \mathrm{H} & 2.699823 & 0.508617 & 0.000000 \\ \mathrm{H} & -2.908920 & 0.188908 & 0.000001 \\ \mathrm{H} & -2.846296 & 2.720646 & 0.000003 \\ \mathrm{H} & 2.827408 & -3.014677 & 0.000002\end{array}$

2-hydroxyquinoline, enol $\mathrm{S}_{0}$

$\begin{array}{rrrr}\mathrm{C} & 0.436651 & 1.912871 & 0.000000 \\ \mathrm{C} & 0.491862 & 0.494504 & 0.000000 \\ \mathrm{C} & -0.730653 & -0.263113 & 0.000000 \\ \mathrm{C} & -1.966269 & 0.437406 & 0.000000 \\ \mathrm{C} & -1.987543 & 1.820359 & 0.000000 \\ \mathrm{C} & -0.782209 & 2.565966 & 0.000000 \\ \mathrm{C} & 1.707527 & -0.242774 & 0.000000 \\ \mathrm{C} & 1.674956 & -1.617476 & 0.000000 \\ \mathrm{C} & 0.399240 & -2.263754 & 0.000000 \\ \mathrm{~N} & -0.751076 & -1.631402 & 0.000000 \\ \mathrm{O} & 0.311532 & -3.631254 & 0.000000 \\ \mathrm{H} & 1.371843 & 2.479882 & 0.000000 \\ \mathrm{H} & -0.820345 & 3.657229 & 0.000000 \\ \mathrm{H} & -2.944305 & 2.347636 & 0.000000 \\ \mathrm{H} & -2.884849 & -0.152050 & 0.000000 \\ \mathrm{H} & 2.662110 & 0.290235 & 0.000000 \\ \mathrm{H} & 2.598636 & -2.203043 & 0.000000 \\ \mathrm{H} & 1.212894 & -4.001224 & 0.000000\end{array}$

2-hydroxyquinoline, enol $\mathrm{S}_{1}$

$\begin{array}{rrrc}\mathrm{C} & 0.422191 & 1.889184 & 0.000000 \\ \mathrm{C} & 0.520478 & 0.483228 & 0.000000 \\ \mathrm{C} & -0.709274 & -0.260517 & 0.000000 \\ \mathrm{C} & -1.955471 & 0.449215 & 0.000000 \\ \mathrm{C} & -2.021773 & 1.861989 & 0.000000 \\ \mathrm{C} & -0.833972 & 2.579551 & 0.000000 \\ \mathrm{C} & 1.757040 & -0.233384 & 0.000000 \\ \mathrm{C} & 1.705600 & -1.657608 & 0.000000 \\ \mathrm{C} & 0.433599 & -2.257228 & 0.000000 \\ \mathrm{~N} & -0.760317 & -1.627183 & 0.000000 \\ \mathrm{O} & 0.287962 & -3.614183 & 0.000000 \\ \mathrm{H} & 1.342651 & 2.480590 & 0.000000 \\ \mathrm{H} & -0.837578 & 3.671204 & 0.000000 \\ \mathrm{H} & -2.987918 & 2.367744 & 0.000000\end{array}$




$\begin{array}{lrrr}\mathrm{H} & -2.864192 & -0.156863 & 0.000000 \\ \mathrm{H} & 2.706644 & 0.301116 & 0.000000 \\ \mathrm{H} & 2.614773 & -2.261713 & 0.000000 \\ \mathrm{H} & 1.179548 & -4.015122 & 0.000000\end{array}$

2-hydroxyquinoline, keto $\mathrm{S}_{0}$

$\begin{array}{lrrr}\mathrm{N} & 0.561446 & 1.713477 & 0.000000 \\ \mathrm{C} & 0.608135 & 0.331682 & 0.000000 \\ \mathrm{C} & -0.622025 & -0.385187 & 0.000000 \\ \mathrm{C} & -1.844111 & 0.376019 & 0.000000 \\ \mathrm{C} & -1.842704 & 1.737590 & 0.000000 \\ \mathrm{C} & -0.600353 & 2.509746 & 0.000000 \\ \mathrm{C} & 1.831982 & -0.365596 & 0.000000 \\ \mathrm{C} & 1.833526 & -1.756792 & 0.000000 \\ \mathrm{C} & 0.625645 & -2.479665 & 0.000000 \\ \mathrm{C} & -0.584594 & -1.796142 & 0.000000 \\ \mathrm{H} & -1.530025 & -2.344564 & 0.000000 \\ \mathrm{H} & 0.641716 & -3.570720 & 0.000000 \\ \mathrm{H} & 2.786073 & -2.290712 & 0.000000 \\ \mathrm{H} & 2.772624 & 0.190974 & 0.000000 \\ \mathrm{H} & -2.791351 & -0.171666 & 0.000000 \\ \mathrm{H} & -2.764364 & 2.319980 & 0.000000 \\ \mathrm{O} & -0.516402 & 3.740285 & 0.000000 \\ \mathrm{H} & 1.434799 & 2.241310 & 0.000000\end{array}$

2-hydroxyquinoline, keto $\mathrm{S}_{1}$

$\begin{array}{rrrr}\mathrm{N} & 0.538592 & 1.720435 & 0.000000 \\ \mathrm{C} & 0.593069 & 0.312068 & 0.000000 \\ \mathrm{C} & -0.664701 & -0.385980 & 0.000000 \\ \mathrm{C} & -1.843833 & 0.358475 & 0.000000 \\ \mathrm{C} & -1.850630 & 1.789420 & 0.000000 \\ \mathrm{C} & -0.614963 & 2.426720 & 0.000000 \\ \mathrm{C} & 1.816512 & -0.344827 & 0.000000 \\ \mathrm{C} & 1.848682 & -1.754708 & 0.000000 \\ \mathrm{C} & 0.635009 & -2.472531 & 0.000000 \\ \mathrm{C} & -0.588365 & -1.826560 & 0.000000 \\ \mathrm{H} & -1.520080 & -2.395812 & 0.000000 \\ \mathrm{H} & 0.661939 & -3.565087 & 0.000000 \\ \mathrm{H} & 2.804859 & -2.277579 & 0.000000 \\ \mathrm{H} & 2.747219 & 0.229498 & 0.000000 \\ \mathrm{H} & -2.802413 & -0.163679 & 0.000000 \\ \mathrm{H} & -2.765524 & 2.372576 & 0.000000 \\ \mathrm{O} & -0.416068 & 3.748636 & 0.000000 \\ \mathrm{H} & 1.420695 & 2.228946 & 0.000000\end{array}$




\begin{tabular}{lrrr}
\multicolumn{4}{l}{ dibenzofuran $\mathrm{S}_{0}$} \\
C & 1.729100 & 0.000000 & -1.367760 \\
$\mathrm{C}$ & 0.726408 & 0.000000 & -0.385839 \\
$\mathrm{C}$ & 1.104014 & 0.000000 & 0.977017 \\
$\mathrm{C}$ & 2.427642 & 0.000000 & 1.404042 \\
$\mathrm{C}$ & 3.408991 & 0.000000 & 0.405810 \\
$\mathrm{C}$ & 3.065556 & 0.000000 & -0.959978 \\
$\mathrm{C}$ & -0.726408 & 0.000000 & -0.385839 \\
$\mathrm{C}$ & -1.104014 & 0.000000 & 0.977017 \\
$\mathrm{O}$ & 0.000000 & 0.000000 & 1.813402 \\
$\mathrm{C}$ & -1.729100 & 0.000000 & -1.367760 \\
$\mathrm{C}$ & -3.065556 & 0.000000 & -0.959978 \\
$\mathrm{C}$ & -3.408991 & 0.000000 & 0.405810 \\
$\mathrm{C}$ & -2.427642 & 0.000000 & 1.404042 \\
$\mathrm{H}$ & -2.681217 & 0.000000 & 2.464910 \\
$\mathrm{H}$ & -4.461938 & 0.000000 & 0.694695 \\
$\mathrm{H}$ & -3.858559 & 0.000000 & -1.710351 \\
$\mathrm{H}$ & -1.472103 & 0.000000 & -2.429082 \\
$\mathrm{H}$ & 1.472103 & 0.000000 & -2.429082 \\
$\mathrm{H}$ & 3.858559 & 0.000000 & -1.710351 \\
$\mathrm{H}$ & 4.461938 & 0.000000 & 0.694695 \\
$\mathrm{H}$ & 2.681217 & 0.000000 & 2.464910
\end{tabular}

dibenzofuran $\mathrm{S}_{1}$

$\begin{array}{lrrr}\mathrm{C} & 0.000000 & 1.746522 & -1.539553 \\ \mathrm{C} & 0.000000 & 0.717569 & -0.567768 \\ \mathrm{C} & 0.000000 & 1.115446 & 0.801066 \\ \mathrm{C} & 0.000000 & 2.426595 & 1.265606 \\ \mathrm{C} & 0.000000 & 3.448073 & 0.264882 \\ \mathrm{C} & 0.000000 & 3.098957 & -1.089951 \\ \mathrm{C} & 0.000000 & -0.717569 & -0.567768 \\ \mathrm{C} & 0.000000 & -1.115446 & 0.801066 \\ \mathrm{O} & 0.000000 & 0.000000 & 1.621991 \\ \mathrm{C} & 0.000000 & -1.746522 & -1.539553 \\ \mathrm{C} & 0.000000 & -3.098957 & -1.089951 \\ \mathrm{C} & 0.000000 & -3.448073 & 0.264882 \\ \mathrm{C} & 0.000000 & -2.426595 & 1.265606 \\ \mathrm{H} & 0.000000 & -2.651891 & 2.331764 \\ \mathrm{H} & 0.000000 & -4.496308 & 0.562695 \\ \mathrm{H} & 0.000000 & -3.893630 & -1.839706 \\ \mathrm{H} & 0.000000 & -1.521443 & -2.606319 \\ \mathrm{H} & 0.000000 & 1.521443 & -2.606319 \\ \mathrm{H} & 0.000000 & 3.893630 & -1.839706\end{array}$




$$
\begin{array}{llll}
\mathrm{H} & 0.000000 & 4.496308 & 0.562695 \\
\mathrm{H} & 0.000000 & 2.651891 & 2.331764
\end{array}
$$

pyrrolo[3,2-h]quinoline $\mathrm{S}_{0}$

$\begin{array}{lrrr}\mathrm{C} & 0.130960 & -0.692703 & 0.000000 \\ \mathrm{~N} & 0.055343 & -2.053518 & 0.000000 \\ \mathrm{C} & 1.203035 & -2.726128 & 0.000000 \\ \mathrm{C} & 2.477026 & -2.113304 & 0.000000 \\ \mathrm{C} & 2.555135 & -0.729839 & 0.000000 \\ \mathrm{C} & 1.369084 & 0.042001 & 0.000000 \\ \mathrm{H} & 1.121214 & -3.818410 & 0.000000 \\ \mathrm{H} & 3.377101 & -2.730888 & 0.000000 \\ \mathrm{H} & 3.524741 & -0.223874 & 0.000000 \\ \mathrm{C} & -1.058132 & 0.080527 & 0.000000 \\ \mathrm{C} & -1.064495 & 1.497380 & 0.000000 \\ \mathrm{C} & 0.179715 & 2.190832 & 0.000000 \\ \mathrm{C} & 1.358428 & 1.476182 & 0.000000 \\ \mathrm{H} & 0.196630 & 3.283134 & 0.000000 \\ \mathrm{H} & 2.318325 & 1.999117 & 0.000000 \\ \mathrm{C} & -2.439953 & 1.903130 & 0.000000 \\ \mathrm{C} & -3.203318 & 0.750162 & 0.000000 \\ \mathrm{~N} & -2.362992 & -0.348124 & 0.000000 \\ \mathrm{H} & -2.631402 & -1.327124 & 0.000000 \\ \mathrm{H} & -2.824571 & 2.919141 & 0.000000 \\ \mathrm{H} & -4.281866 & 0.622308 & 0.000000\end{array}$

pyrrolo[3,2-h]quinoline $\mathrm{S}_{1}$

$\begin{array}{rrrr}\mathrm{C} & 0.159611 & -0.701294 & 0.000000 \\ \mathrm{~N} & -0.002491 & -2.058953 & 0.000000 \\ \mathrm{C} & 1.201315 & -2.746471 & 0.000000 \\ \mathrm{C} & 2.453326 & -2.143902 & 0.000000 \\ \mathrm{C} & 2.593078 & -0.734852 & 0.000000 \\ \mathrm{C} & 1.359489 & 0.018354 & 0.000000 \\ \mathrm{H} & 1.115336 & -3.836331 & 0.000000 \\ \mathrm{H} & 3.341845 & -2.780743 & 0.000000 \\ \mathrm{H} & 3.563942 & -0.241991 & 0.000000 \\ \mathrm{C} & -1.032117 & 0.109063 & 0.000000 \\ \mathrm{C} & -1.075847 & 1.562283 & 0.000000 \\ \mathrm{C} & 0.147521 & 2.247099 & 0.000000 \\ \mathrm{C} & 1.316229 & 1.471931 & 0.000000 \\ \mathrm{H} & 0.199272 & 3.336084 & 0.000000 \\ \mathrm{H} & 2.279398 & 1.991591 & 0.000000 \\ \mathrm{C} & -2.472871 & 1.922163 & 0.000000 \\ \mathrm{C} & -3.193464 & 0.756652 & 0.000000\end{array}$




$\begin{array}{lrrr}\mathrm{N} & -2.294891 & -0.341560 & 0.000000 \\ \mathrm{H} & -2.505982 & -1.339586 & 0.000000 \\ \mathrm{H} & -2.887226 & 2.925806 & 0.000000 \\ \mathrm{H} & -4.265485 & 0.584656 & 0.000000\end{array}$

carbazole $\mathrm{S}_{0}$

$\begin{array}{lrrr}\mathrm{C} & 1.136766 & 0.000000 & -0.904103 \\ \mathrm{~N} & 0.000000 & 0.000000 & -1.704322 \\ \mathrm{C} & -1.136766 & 0.000000 & -0.904103 \\ \mathrm{C} & -0.725723 & 0.000000 & 0.462792 \\ \mathrm{C} & 0.725723 & 0.000000 & 0.462792 \\ \mathrm{C} & -1.703175 & 0.000000 & 1.470120 \\ \mathrm{C} & -3.052064 & 0.000000 & 1.110249 \\ \mathrm{C} & -3.436972 & 0.000000 & -0.245648 \\ \mathrm{C} & -2.487832 & 0.000000 & -1.270441 \\ \mathrm{C} & 2.487832 & 0.000000 & -1.270441 \\ \mathrm{C} & 3.436972 & 0.000000 & -0.245648 \\ \mathrm{C} & 3.052064 & 0.000000 & 1.110249 \\ \mathrm{C} & 1.703175 & 0.000000 & 1.470120 \\ \mathrm{H} & -2.793752 & 0.000000 & -2.319194 \\ \mathrm{H} & -4.498461 & 0.000000 & -0.502402 \\ \mathrm{H} & -3.820302 & 0.000000 & 1.885918 \\ \mathrm{H} & -1.412841 & 0.000000 & 2.523231 \\ \mathrm{H} & 1.412841 & 0.000000 & 2.523231 \\ \mathrm{H} & 3.820302 & 0.000000 & 1.885918 \\ \mathrm{H} & 4.498461 & 0.000000 & -0.502402 \\ \mathrm{H} & 2.793752 & 0.000000 & -2.319194 \\ \mathrm{H} & 0.000000 & 0.000000 & -2.717681\end{array}$

carbazole $\mathrm{S}_{1}$

$\begin{array}{lrrr}\mathrm{C} & 1.152087 & 0.000000 & -0.837701 \\ \mathrm{~N} & 0.000000 & 0.000000 & -1.623315 \\ \mathrm{C} & -1.152087 & 0.000000 & -0.837701 \\ \mathrm{C} & -0.721521 & 0.000000 & 0.528155 \\ \mathrm{C} & 0.721521 & 0.000000 & 0.528155 \\ \mathrm{C} & -1.718987 & 0.000000 & 1.529529 \\ \mathrm{C} & -3.082373 & 0.000000 & 1.125711 \\ \mathrm{C} & -3.474349 & 0.000000 & -0.216292 \\ \mathrm{C} & -2.484598 & 0.000000 & -1.247902 \\ \mathrm{C} & 2.484598 & 0.000000 & -1.247902 \\ \mathrm{C} & 3.474349 & 0.000000 & -0.216292 \\ \mathrm{C} & 3.082373 & 0.000000 & 1.125711 \\ \mathrm{C} & 1.718987 & 0.000000 & 1.529529 \\ \mathrm{H} & -2.761418 & 0.000000 & -2.303633\end{array}$




$\begin{array}{rrrr}\mathrm{H} & -4.531959 & 0.000000 & -0.479996 \\ \mathrm{H} & -3.853612 & 0.000000 & 1.900041 \\ \mathrm{H} & -1.458384 & 0.000000 & 2.588274 \\ \mathrm{H} & 1.458384 & 0.000000 & 2.588274 \\ \mathrm{H} & 3.853612 & 0.000000 & 1.900041 \\ \mathrm{H} & 4.531959 & 0.000000 & -0.479996 \\ \mathrm{H} & 2.761418 & 0.000000 & -2.303633 \\ \mathrm{H} & 0.000000 & 0.000000 & -2.641687\end{array}$

tryptamine, A-ph $\mathrm{S}_{0}$

$\begin{array}{lrrr}\mathrm{C} & -2.137641 & 0.128895 & 0.890611 \\ \mathrm{~N} & -1.256383 & 0.117171 & 1.963965 \\ \mathrm{C} & 0.040530 & 0.043549 & 1.488431 \\ \mathrm{C} & -0.039271 & 0.002469 & 0.061111 \\ \mathrm{C} & -1.438474 & 0.059402 & -0.297232 \\ \mathrm{C} & 1.262466 & 0.014463 & 2.173050 \\ \mathrm{C} & 2.427109 & -0.056214 & 1.408943 \\ \mathrm{C} & 2.375076 & -0.093250 & -0.002554 \\ \mathrm{C} & 1.157847 & -0.064180 & -0.679987 \\ \mathrm{C} & -2.015935 & 0.009871 & -1.682426 \\ \mathrm{C} & -2.020620 & -1.404153 & -2.294212 \\ \mathrm{~N} & -2.504215 & -1.358798 & -3.684882 \\ \mathrm{H} & 1.306079 & 0.046778 & 3.264436 \\ \mathrm{H} & 3.396476 & -0.080603 & 1.911184 \\ \mathrm{H} & 3.307488 & -0.144255 & -0.568879 \\ \mathrm{H} & 1.133260 & -0.089075 & -1.772023 \\ \mathrm{H} & -1.522132 & 0.180956 & 2.939445 \\ \mathrm{H} & -3.209625 & 0.192865 & 1.060852 \\ \mathrm{H} & -1.451216 & 0.671442 & -2.360702 \\ \mathrm{H} & -3.050971 & 0.394102 & -1.664815 \\ \mathrm{H} & -2.604179 & -2.074053 & -1.628570 \\ \mathrm{H} & -0.990227 & -1.793124 & -2.307730 \\ \mathrm{H} & -3.494196 & -1.097814 & -3.705118 \\ \mathrm{H} & -2.438656 & -2.284065 & -4.115528\end{array}$

tryptamine, A-ph $\mathrm{S}_{1}$

$\begin{array}{rrrr}\mathrm{C} & -2.216695 & 0.086047 & 0.956564 \\ \mathrm{~N} & -1.264778 & 0.122930 & 1.979584 \\ \mathrm{C} & 0.024036 & 0.059296 & 1.468574 \\ \mathrm{C} & -0.091887 & 0.032540 & 0.046386 \\ \mathrm{C} & -1.485835 & 0.066680 & -0.248819 \\ \mathrm{C} & 1.255870 & -0.005090 & 2.151954 \\ \mathrm{C} & 2.435796 & -0.080647 & 1.334567 \\ \mathrm{C} & 2.350551 & -0.094858 & -0.056516\end{array}$




$\begin{array}{rrrr}\mathrm{C} & 1.114246 & -0.051010 & -0.746421 \\ \mathrm{C} & -2.094245 & 0.012675 & -1.623362 \\ \mathrm{C} & -2.024583 & -1.410300 & -2.187841 \\ \mathrm{~N} & -2.509602 & -1.556141 & -3.555296 \\ \mathrm{H} & 1.312677 & 0.029559 & 3.240806 \\ \mathrm{H} & 3.415456 & -0.131300 & 1.815214 \\ \mathrm{H} & 3.276609 & -0.142588 & -0.638649 \\ \mathrm{H} & 1.095216 & 0.020056 & -1.836395 \\ \mathrm{H} & -1.481319 & 0.132237 & 2.968501 \\ \mathrm{H} & -3.256209 & 0.338862 & 1.149501 \\ \mathrm{H} & -1.561083 & 0.697133 & -2.308536 \\ \mathrm{H} & -3.147953 & 0.337922 & -1.587591 \\ \mathrm{H} & -2.600324 & -2.134803 & -1.589144 \\ \mathrm{H} & -0.994626 & -1.801034 & -2.200394 \\ \mathrm{H} & -2.803517 & -0.746118 & -4.104025 \\ \mathrm{H} & -2.515232 & -2.459668 & -4.031335\end{array}$

tryptamine, A-py $\mathrm{S}_{0}$

$\begin{array}{lrrr}\mathrm{C} & -2.131185 & 0.108799 & 0.867612 \\ \mathrm{~N} & -1.260687 & 0.112513 & 1.949328 \\ \mathrm{C} & 0.041326 & 0.046177 & 1.487459 \\ \mathrm{C} & -0.023946 & -0.006852 & 0.059395 \\ \mathrm{C} & -1.420031 & 0.034852 & -0.312897 \\ \mathrm{C} & 1.256166 & 0.032643 & 2.185004 \\ \mathrm{C} & 2.429619 & -0.033490 & 1.434216 \\ \mathrm{C} & 2.392709 & -0.081892 & 0.022630 \\ \mathrm{C} & 1.182243 & -0.069164 & -0.667275 \\ \mathrm{C} & -1.996087 & -0.020440 & -1.698770 \\ \mathrm{C} & -1.970402 & -1.428304 & -2.322934 \\ \mathrm{~N} & -2.577085 & -1.399252 & -3.665631 \\ \mathrm{H} & 1.287650 & 0.073232 & 3.276524 \\ \mathrm{H} & 3.393705 & -0.045896 & 1.946891 \\ \mathrm{H} & 3.331401 & -0.130067 & -0.533504 \\ \mathrm{H} & 1.170688 & -0.106004 & -1.759259 \\ \mathrm{H} & -1.536939 & 0.179790 & 2.921681 \\ \mathrm{H} & -3.205392 & 0.165394 & 1.025513 \\ \mathrm{H} & -1.438971 & 0.669095 & -2.359873 \\ \mathrm{H} & -3.039408 & 0.333050 & -1.693851 \\ \mathrm{H} & -2.571154 & -2.104033 & -1.693031 \\ \mathrm{H} & -0.930895 & -1.815055 & -2.302132 \\ \mathrm{H} & -1.992547 & -0.849179 & -4.301689 \\ \mathrm{H} & -2.620867 & -2.343527 & -4.056296\end{array}$

tryptamine, A-py $\mathrm{S}_{1}$ 


$\begin{array}{lrrr}\mathrm{C} & -2.198011 & 0.097370 & 0.871474 \\ \mathrm{~N} & -1.283151 & 0.131660 & 1.927795 \\ \mathrm{C} & 0.022777 & 0.060513 & 1.463314 \\ \mathrm{C} & -0.042322 & 0.031304 & 0.038021 \\ \mathrm{C} & -1.424652 & 0.071711 & -0.306945 \\ \mathrm{C} & 1.229048 & -0.008614 & 2.190532 \\ \mathrm{C} & 2.437010 & -0.091771 & 1.416009 \\ \mathrm{C} & 2.401536 & -0.108503 & 0.022790 \\ \mathrm{C} & 1.190945 & -0.060091 & -0.711043 \\ \mathrm{C} & -1.983775 & 0.017489 & -1.702280 \\ \mathrm{C} & -1.900896 & -1.407108 & -2.260713 \\ \mathrm{~N} & -2.336700 & -1.553935 & -3.644587 \\ \mathrm{H} & 1.246954 & 0.028035 & 3.280645 \\ \mathrm{H} & 3.398625 & -0.146298 & 1.931422 \\ \mathrm{H} & 3.347616 & -0.162050 & -0.525663 \\ \mathrm{H} & 1.211245 & 0.009419 & -1.801091 \\ \mathrm{H} & -1.534811 & 0.144436 & 2.908321 \\ \mathrm{H} & -3.242484 & 0.355722 & 1.026551 \\ \mathrm{H} & -1.423087 & 0.697784 & -2.369388 \\ \mathrm{H} & -3.036461 & 0.347919 & -1.704922 \\ \mathrm{H} & -2.501414 & -2.127419 & -1.681661 \\ \mathrm{H} & -0.873045 & -1.802804 & -2.235239 \\ \mathrm{H} & -2.605268 & -0.743734 & -4.205902 \\ \mathrm{H} & -2.329769 & -2.458658 & -4.118338\end{array}$

tryptamine, A-up $\mathrm{S}_{0}$

$\begin{array}{lrrr}\mathrm{C} & -2.130030 & 0.122014 & 0.878796 \\ \mathrm{~N} & -1.256912 & 0.095595 & 1.958170 \\ \mathrm{C} & 0.043763 & 0.034999 & 1.491834 \\ \mathrm{C} & -0.024593 & 0.017451 & 0.063268 \\ \mathrm{C} & -1.421253 & 0.076813 & -0.305080 \\ \mathrm{C} & 1.260041 & -0.001781 & 2.185986 \\ \mathrm{C} & 2.431235 & -0.056282 & 1.430825 \\ \mathrm{C} & 2.391072 & -0.070893 & 0.018636 \\ \mathrm{C} & 1.179259 & -0.034215 & -0.667955 \\ \mathrm{C} & -1.992296 & 0.036248 & -1.693123 \\ \mathrm{C} & -1.991811 & -1.379430 & -2.328521 \\ \mathrm{~N} & -2.507972 & -1.469706 & -3.699897 \\ \mathrm{H} & 1.294301 & 0.011826 & 3.278061 \\ \mathrm{H} & 3.396417 & -0.086769 & 1.940656 \\ \mathrm{H} & 3.328189 & -0.111967 & -0.540628 \\ \mathrm{H} & 1.165342 & -0.045603 & -1.760483 \\ \mathrm{H} & -1.530702 & 0.136583 & 2.932711 \\ \mathrm{H} & -3.203477 & 0.178593 & 1.042083 \\ \mathrm{H} & -1.423904 & 0.715978 & -2.354090\end{array}$




$\begin{array}{llll}\mathrm{H} & -3.029286 & 0.414882 & -1.680650 \\ \mathrm{H} & -2.586563 & -2.055129 & -1.692352 \\ \mathrm{H} & -0.966298 & -1.781791 & -2.323097 \\ \mathrm{H} & -1.954550 & -0.880895 & -4.328432 \\ \mathrm{H} & -3.468732 & -1.118610 & -3.743481\end{array}$

tryptamine, A-up $\mathrm{S}_{1}$

$\begin{array}{lrrr}\mathrm{C} & -2.208150 & 0.034654 & 0.947945 \\ \mathrm{~N} & -1.268762 & 0.026559 & 1.983129 \\ \mathrm{C} & 0.026735 & 0.016420 & 1.485387 \\ \mathrm{C} & -0.072426 & 0.073162 & 0.062811 \\ \mathrm{C} & -1.463300 & 0.100626 & -0.247215 \\ \mathrm{C} & 1.251681 & -0.067605 & 2.178962 \\ \mathrm{C} & 2.441987 & -0.073380 & 1.373299 \\ \mathrm{C} & 2.372919 & -0.005421 & -0.017095 \\ \mathrm{C} & 1.143946 & 0.058134 & -0.718524 \\ \mathrm{C} & -2.054621 & 0.118151 & -1.630131 \\ \mathrm{C} & -1.952615 & -1.267234 & -2.277167 \\ \mathrm{~N} & -2.418792 & -1.339765 & -3.656971 \\ \mathrm{H} & 1.295416 & -0.097849 & 3.268550 \\ \mathrm{H} & 3.416866 & -0.135891 & 1.862210 \\ \mathrm{H} & 3.306283 & -0.001935 & -0.589416 \\ \mathrm{H} & 1.136049 & 0.195274 & -1.802318 \\ \mathrm{H} & -1.496726 & -0.026259 & 2.968106 \\ \mathrm{H} & -3.254077 & 0.257950 & 1.142716 \\ \mathrm{H} & -1.525879 & 0.851350 & -2.266557 \\ \mathrm{H} & -3.114371 & 0.422412 & -1.588568 \\ \mathrm{H} & -2.522190 & -2.036000 & -1.729972 \\ \mathrm{H} & -0.915660 & -1.638549 & -2.299645 \\ \mathrm{H} & -2.402308 & -2.213209 & -4.185962 \\ \mathrm{H} & -2.720785 & -0.503675 & -4.160354\end{array}$

tryptamine, $\mathrm{Ph}$-out $\mathrm{S}_{0}$

$\begin{array}{lrrr}\mathrm{C} & 0.011451 & 0.007133 & 0.075691 \\ \mathrm{C} & 0.051646 & 0.063444 & 1.504867 \\ \mathrm{C} & 1.254347 & 0.077452 & 2.223368 \\ \mathrm{C} & 2.441033 & 0.038771 & 1.491881 \\ \mathrm{C} & 2.428230 & -0.014157 & 0.079744 \\ \mathrm{C} & 1.231089 & -0.029240 & -0.632892 \\ \mathrm{~N} & -1.258619 & 0.100707 & 1.945735 \\ \mathrm{H} & 1.266337 & 0.119171 & 3.315252 \\ \mathrm{H} & 3.396662 & 0.049374 & 2.020330 \\ \mathrm{H} & 3.377095 & -0.042371 & -0.460363 \\ \mathrm{H} & 1.231223 & -0.085445 & -1.723485\end{array}$




$\begin{array}{lrrr}\mathrm{C} & -1.380432 & 0.018452 & -0.319642 \\ \mathrm{C} & -2.111531 & 0.080434 & 0.850739 \\ \mathrm{H} & -1.550850 & 0.174202 & 2.913043 \\ \mathrm{H} & -3.188644 & 0.123208 & 0.993086 \\ \mathrm{C} & -1.940945 & -0.038701 & -1.714923 \\ \mathrm{H} & -1.292968 & 0.531324 & -2.401476 \\ \mathrm{C} & -2.103353 & -1.464348 & -2.277591 \\ \mathrm{H} & -2.929640 & 0.449797 & -1.729171 \\ \mathrm{H} & -2.573739 & -1.395054 & -3.272870 \\ \mathrm{~N} & -0.797444 & -2.120163 & -2.443863 \\ \mathrm{H} & -2.807583 & -2.020596 & -1.621963 \\ \mathrm{H} & -0.352503 & -2.246452 & -1.529967 \\ \mathrm{H} & -0.915891 & -3.052833 & -2.846318\end{array}$

tryptamine, $\mathrm{Ph}$-out $\mathrm{S}_{1}$

$\begin{array}{rrrr}\mathrm{C} & -0.082018 & 0.007914 & 0.055796 \\ \mathrm{C} & -0.001433 & 0.085892 & 1.469545 \\ \mathrm{C} & 1.213125 & -0.012609 & 2.192473 \\ \mathrm{C} & 2.399584 & -0.166373 & 1.404589 \\ \mathrm{C} & 2.359626 & -0.226784 & 0.018560 \\ \mathrm{C} & 1.132500 & -0.202963 & -0.729327 \\ \mathrm{~N} & -1.298425 & 0.229058 & 1.943893 \\ \mathrm{H} & 1.249739 & 0.065705 & 3.279224 \\ \mathrm{H} & 3.364380 & -0.237506 & 1.913623 \\ \mathrm{H} & 3.304256 & -0.296633 & -0.530149 \\ \mathrm{H} & 1.181244 & 0.190099 & -1.757389 \\ \mathrm{C} & -1.471634 & 0.077528 & -0.286070 \\ \mathrm{C} & -2.207171 & 0.224336 & 0.894486 \\ \mathrm{H} & -1.540059 & 0.352189 & 2.919805 \\ \mathrm{H} & -3.269088 & 0.374099 & 1.063459 \\ \mathrm{C} & -2.041764 & -0.056773 & -1.673696 \\ \mathrm{H} & -1.514685 & 0.596801 & -2.390885 \\ \mathrm{C} & -2.013276 & -1.495927 & -2.216292 \\ \mathrm{H} & -3.095417 & 0.264108 & -1.696454 \\ \mathrm{H} & -2.449742 & -1.601381 & -3.228201 \\ \mathrm{~N} & -0.688201 & -2.062879 & -2.261300 \\ \mathrm{H} & -2.602266 & -2.181028 & -1.574516 \\ \mathrm{H} & 0.113534 & -1.596023 & -1.743464 \\ \mathrm{H} & -0.557818 & -3.006733 & -2.628497\end{array}$

tryptamine, $\mathrm{Ph}$-up $\mathrm{S}_{0}$
$\begin{array}{llll}\text { C } & -2.085183 & 0.014536 & 0.818790\end{array}$
$\begin{array}{llll}\mathrm{N} & -1.245584 & 0.005275 & 1.923847\end{array}$
$\begin{array}{llll}\text { C } & 0.069848 & 0.032539 & 1.499515\end{array}$ 


$\begin{array}{rrrc}\mathrm{C} & 0.046425 & 0.052332 & 0.068880 \\ \mathrm{C} & -1.338892 & 0.039957 & -0.343983 \\ \mathrm{C} & 1.263649 & 0.047022 & 2.232733 \\ \mathrm{C} & 2.459042 & 0.087141 & 1.516041 \\ \mathrm{C} & 2.463950 & 0.113690 & 0.103063 \\ \mathrm{C} & 1.274812 & 0.097034 & -0.622346 \\ \mathrm{C} & -1.874337 & 0.015274 & -1.748486 \\ \mathrm{C} & -2.144086 & -1.411757 & -2.298199 \\ \mathrm{~N} & -0.994657 & -2.316552 & -2.394683 \\ \mathrm{H} & 1.262161 & 0.030054 & 3.325275 \\ \mathrm{H} & 3.407807 & 0.099950 & 2.056400 \\ \mathrm{H} & 3.418944 & 0.150102 & -0.425441 \\ \mathrm{H} & 1.295031 & 0.121678 & -1.714486 \\ \mathrm{H} & -1.551650 & 0.009321 & 2.889739 \\ \mathrm{H} & -3.164460 & 0.010552 & 0.950421 \\ \mathrm{H} & -1.166861 & 0.528479 & -2.423130 \\ \mathrm{H} & -2.816877 & 0.588926 & -1.798430 \\ \mathrm{H} & -2.592758 & -1.325438 & -3.302331 \\ \mathrm{H} & -2.900285 & -1.898117 & -1.659214 \\ \mathrm{H} & -0.543140 & -2.411196 & -1.480482 \\ \mathrm{H} & -0.289249 & -1.931171 & -3.028435\end{array}$

tryptamine, $\mathrm{Ph}$-up $\mathrm{S}_{1}$

$\begin{array}{lrrr}\mathrm{C} & -2.201460 & 0.162441 & 0.903261 \\ \mathrm{~N} & -1.300475 & 0.132577 & 1.958984 \\ \mathrm{C} & 0.003323 & 0.054565 & 1.488418 \\ \mathrm{C} & -0.065030 & 0.057683 & 0.071728 \\ \mathrm{C} & -1.453633 & 0.107291 & -0.277459 \\ \mathrm{C} & 1.215037 & -0.052089 & 2.214805 \\ \mathrm{C} & 2.411076 & -0.125484 & 1.429673 \\ \mathrm{C} & 2.382634 & -0.104969 & 0.042218 \\ \mathrm{C} & 1.160696 & -0.071522 & -0.713541 \\ \mathrm{C} & -2.010551 & 0.038545 & -1.675223 \\ \mathrm{C} & -1.936354 & -1.364819 & -2.300953 \\ \mathrm{~N} & -0.595020 & -1.889926 & -2.368004 \\ \mathrm{H} & 1.241883 & -0.036724 & 3.304555 \\ \mathrm{H} & 3.373820 & -0.199423 & 1.942181 \\ \mathrm{H} & 3.332687 & -0.115551 & -0.501471 \\ \mathrm{H} & 1.205321 & 0.381786 & -1.716634 \\ \mathrm{H} & -1.552568 & 0.188226 & 2.938424 \\ \mathrm{H} & -3.268211 & 0.274109 & 1.071363 \\ \mathrm{H} & -1.498818 & 0.748362 & -2.348757 \\ \mathrm{H} & -3.073128 & 0.329225 & -1.687374 \\ \mathrm{H} & -2.362575 & -1.423052 & -3.321015 \\ \mathrm{H} & -2.509419 & -2.103054 & -1.705408\end{array}$




$$
\begin{array}{cccc}
\mathrm{H} & 0.189212 & -1.431752 & -1.816799 \\
\mathrm{H} & -0.434786 & -2.806836 & -2.787882
\end{array}
$$

tryptamine, Py-out $\mathrm{S}_{0}$

$\begin{array}{rrrr}\mathrm{C} & 0.525253 & 0.457812 & 0.215756 \\ \mathrm{C} & 0.601121 & 0.528236 & 1.642554 \\ \mathrm{C} & 1.819395 & 0.489954 & 2.333091 \\ \mathrm{C} & 2.985605 & 0.380239 & 1.576042 \\ \mathrm{C} & 2.938346 & 0.314137 & 0.165601 \\ \mathrm{C} & 1.724472 & 0.352504 & -0.517664 \\ \mathrm{~N} & -0.695701 & 0.635715 & 2.112159 \\ \mathrm{H} & 1.858985 & 0.545397 & 3.423685 \\ \mathrm{H} & 3.952243 & 0.347453 & 2.082992 \\ \mathrm{H} & 3.871825 & 0.233047 & -0.395435 \\ \mathrm{H} & 1.704625 & 0.303248 & -1.608929 \\ \mathrm{C} & -0.872404 & 0.533922 & -0.148236 \\ \mathrm{C} & -1.573909 & 0.639764 & 1.036590 \\ \mathrm{H} & -0.963204 & 0.721052 & 3.085609 \\ \mathrm{H} & -2.645622 & 0.723704 & 1.197947 \\ \mathrm{C} & -1.457773 & 0.472449 & -1.531178 \\ \mathrm{H} & -0.891051 & 1.135717 & -2.207317 \\ \mathrm{C} & -1.469611 & -0.946727 & -2.133235 \\ \mathrm{H} & -2.494913 & 0.843967 & -1.508051 \\ \mathrm{H} & -0.422951 & -1.304142 & -2.230803 \\ \mathrm{~N} & -2.328707 & -1.831733 & -1.331338 \\ \mathrm{H} & -1.889887 & -0.893493 & -3.151143 \\ \mathrm{H} & -2.332611 & -2.776198 & -1.723815 \\ \mathrm{H} & -1.943506 & -1.906025 & -0.384862\end{array}$

tryptamine, Py-out $\mathrm{S}_{1}$

$\begin{array}{lrrr}\mathrm{C} & 0.572597 & 0.510209 & 0.158535 \\ \mathrm{C} & 0.598476 & 0.432777 & 1.597129 \\ \mathrm{C} & 1.789664 & 0.394470 & 2.333219 \\ \mathrm{C} & 3.014170 & 0.449255 & 1.604074 \\ \mathrm{C} & 3.014258 & 0.532007 & 0.206047 \\ \mathrm{C} & 1.825395 & 0.557462 & -0.542464 \\ \mathrm{~N} & -0.710437 & 0.367379 & 2.028631 \\ \mathrm{H} & 1.781940 & 0.335085 & 3.423545 \\ \mathrm{H} & 3.961839 & 0.419192 & 2.145353 \\ \mathrm{H} & 3.972174 & 0.573061 & -0.320628 \\ \mathrm{H} & 1.861151 & 0.633011 & -1.631645 \\ \mathrm{C} & -0.783711 & 0.486223 & -0.229210 \\ \mathrm{C} & -1.622417 & 0.279911 & 0.935040 \\ \mathrm{H} & -0.967747 & 0.185994 & 2.991669\end{array}$




$\begin{array}{lrrr}\mathrm{H} & -2.571769 & 0.829745 & 1.056920 \\ \mathrm{C} & -1.319690 & 0.449979 & -1.634555 \\ \mathrm{H} & -0.613035 & 0.907515 & -2.342566 \\ \mathrm{C} & -1.597170 & -0.988083 & -2.126571 \\ \mathrm{H} & -2.256459 & 1.029459 & -1.721813 \\ \mathrm{H} & -0.656209 & -1.553375 & -2.276719 \\ \mathrm{~N} & -2.320312 & -1.737273 & -1.130976 \\ \mathrm{H} & -2.141915 & -1.059873 & -3.083691 \\ \mathrm{H} & -2.560821 & -2.717176 & -1.286166 \\ \mathrm{H} & -2.269984 & -1.316965 & -0.153167\end{array}$

tryptamine, Py-up $\mathrm{S}_{0}$

$\begin{array}{rrrr}\mathrm{C} & -2.124936 & 0.225857 & 0.890332 \\ \mathrm{~N} & -1.246453 & 0.202484 & 1.965782 \\ \mathrm{C} & 0.049095 & 0.090654 & 1.493926 \\ \mathrm{C} & -0.027888 & 0.038290 & 0.066780 \\ \mathrm{C} & -1.424766 & 0.129296 & -0.296485 \\ \mathrm{C} & 1.267253 & 0.034716 & 2.183353 \\ \mathrm{C} & 2.431821 & -0.076063 & 1.424148 \\ \mathrm{C} & 2.383321 & -0.126142 & 0.013174 \\ \mathrm{C} & 1.169625 & -0.069897 & -0.668832 \\ \mathrm{C} & -2.003041 & 0.071831 & -1.679579 \\ \mathrm{C} & -2.039638 & -1.360803 & -2.281835 \\ \mathrm{~N} & -2.859144 & -2.344831 & -1.568908 \\ \mathrm{H} & 1.307888 & 0.076551 & 3.274473 \\ \mathrm{H} & 3.398473 & -0.123016 & 1.929912 \\ \mathrm{H} & 3.315672 & -0.209876 & -0.549243 \\ \mathrm{H} & 1.149454 & -0.106935 & -1.760590 \\ \mathrm{H} & -1.512173 & 0.280498 & 2.940315 \\ \mathrm{H} & -3.194542 & 0.327290 & 1.056735 \\ \mathrm{H} & -1.422354 & 0.723707 & -2.357452 \\ \mathrm{H} & -3.031282 & 0.473832 & -1.667886 \\ \mathrm{H} & -1.012478 & -1.756589 & -2.336610 \\ \mathrm{H} & -2.406516 & -1.299232 & -3.319913 \\ \mathrm{H} & -3.835016 & -2.035576 & -1.535814 \\ \mathrm{H} & -2.543855 & -2.416424 & -0.596893\end{array}$

tryptamine, Py-up $\mathrm{S}_{1}$

$\begin{array}{lrrr}\mathrm{C} & -2.260694 & 0.459295 & 0.957006 \\ \mathrm{~N} & -1.311625 & 0.439170 & 1.979658 \\ \mathrm{C} & -0.049381 & 0.134841 & 1.491357 \\ \mathrm{C} & -0.168982 & 0.010440 & 0.073930 \\ \mathrm{C} & -1.547943 & 0.227009 & -0.244675 \\ \mathrm{C} & 1.158672 & -0.062296 & 2.193008\end{array}$




$\begin{array}{lrrr}\mathrm{C} & 2.312963 & -0.372340 & 1.396974 \\ \mathrm{C} & 2.227482 & -0.482135 & 0.010517 \\ \mathrm{C} & 1.010283 & -0.313764 & -0.695273 \\ \mathrm{C} & -2.164291 & 0.250885 & -1.617745 \\ \mathrm{C} & -2.171081 & -1.124977 & -2.344357 \\ \mathrm{~N} & -2.084305 & -2.222413 & -1.428162 \\ \mathrm{H} & 1.221719 & 0.056795 & 3.275306 \\ \mathrm{H} & 3.274566 & -0.527807 & 1.891140 \\ \mathrm{H} & 3.136989 & -0.705878 & -0.555755 \\ \mathrm{H} & 1.002632 & -0.318895 & -1.787111 \\ \mathrm{H} & -1.522193 & 0.564411 & 2.962589 \\ \mathrm{H} & -3.248300 & 0.885547 & 1.114629 \\ \mathrm{H} & -1.643559 & 0.956564 & -2.286059 \\ \mathrm{H} & -3.205257 & 0.602903 & -1.551545 \\ \mathrm{H} & -1.291860 & -1.261324 & -3.003429 \\ \mathrm{H} & -3.041744 & -1.289525 & -3.000876 \\ \mathrm{H} & -2.083325 & -3.194322 & -1.735081 \\ \mathrm{H} & -1.762246 & -1.962555 & -0.477131\end{array}$

porphycene $\mathrm{S}_{0}$

$\begin{array}{lrrr}\mathrm{C} & -3.447292 & 1.920092 & 0.000000 \\ \mathrm{C} & -2.421788 & 0.915125 & 0.000000 \\ \mathrm{~N} & -1.216520 & 1.563133 & 0.000000 \\ \mathrm{C} & -1.401995 & 2.924102 & 0.000000 \\ \mathrm{C} & -2.822253 & 3.152616 & 0.000000 \\ \mathrm{C} & -0.370377 & 3.886216 & 0.000000 \\ \mathrm{C} & 1.018408 & 3.735940 & 0.000000 \\ \mathrm{C} & 1.841182 & 2.584641 & 0.000000 \\ \mathrm{H} & -0.221601 & 1.162087 & 0.000000 \\ \mathrm{~N} & 1.408428 & 1.284814 & 0.000000 \\ \mathrm{C} & 3.297121 & 2.614936 & 0.000000 \\ \mathrm{C} & 3.720801 & 1.310356 & 0.000000 \\ \mathrm{C} & 2.518127 & 0.498362 & 0.000000 \\ \mathrm{C} & 2.421788 & -0.915125 & 0.000000 \\ \mathrm{C} & 3.447292 & -1.920092 & 0.000000 \\ \mathrm{C} & 2.822253 & -3.152616 & 0.000000 \\ \mathrm{C} & 1.401995 & -2.924102 & 0.000000 \\ \mathrm{~N} & 1.216520 & -1.563133 & 0.000000 \\ \mathrm{C} & 0.370377 & -3.886216 & 0.000000 \\ \mathrm{C} & -1.018408 & -3.735940 & 0.000000 \\ \mathrm{C} & -1.841182 & -2.584641 & 0.000000 \\ \mathrm{C} & -3.297121 & -2.614936 & 0.000000 \\ \mathrm{C} & -3.720801 & -1.310356 & 0.000000 \\ \mathrm{C} & -2.518127 & -0.498362 & 0.000000 \\ \mathrm{~N} & -1.408428 & -1.284814 & 0.000000\end{array}$




$\begin{array}{lrrr}\mathrm{H} & 0.221601 & -1.162087 & 0.000000 \\ \mathrm{H} & 3.294523 & -4.132071 & 0.000000 \\ \mathrm{H} & 4.516438 & -1.725409 & 0.000000 \\ \mathrm{H} & 0.731988 & -4.918244 & 0.000000 \\ \mathrm{H} & -1.579890 & -4.675090 & 0.000000 \\ \mathrm{H} & -3.911918 & -3.513212 & 0.000000 \\ \mathrm{H} & -4.745038 & -0.944241 & 0.000000 \\ \mathrm{H} & -4.516438 & 1.725409 & 0.000000 \\ \mathrm{H} & -3.294523 & 4.132070 & 0.000000 \\ \mathrm{H} & -0.731988 & 4.918245 & 0.000000 \\ \mathrm{H} & 1.579890 & 4.675090 & 0.000000 \\ \mathrm{H} & 3.911918 & 3.513212 & 0.000000 \\ \mathrm{H} & 4.745038 & 0.944241 & 0.000000\end{array}$

porphycene $\mathrm{S}_{1}$

$\begin{array}{crrr}\mathrm{C} & -3.471614 & 1.912772 & 0.000000 \\ \mathrm{C} & -2.456791 & 0.909704 & 0.000000 \\ \mathrm{~N} & -1.240205 & 1.557904 & 0.000000 \\ \mathrm{C} & -1.422649 & 2.917867 & 0.000000 \\ \mathrm{C} & -2.837814 & 3.148529 & 0.000000 \\ \mathrm{C} & -0.384720 & 3.887583 & 0.000000 \\ \mathrm{C} & 0.997947 & 3.750931 & 0.000000 \\ \mathrm{C} & 1.852315 & 2.608220 & 0.000000 \\ \mathrm{H} & -0.261406 & 1.150965 & 0.000000 \\ \mathrm{~N} & 1.425882 & 1.297647 & 0.000000 \\ \mathrm{C} & 3.288462 & 2.643497 & 0.000000 \\ \mathrm{C} & 3.731607 & 1.323646 & 0.000000 \\ \mathrm{C} & 2.562116 & 0.508217 & 0.000000 \\ \mathrm{C} & 2.456791 & -0.909704 & 0.000000 \\ \mathrm{C} & 3.471614 & -1.912772 & 0.000000 \\ \mathrm{C} & 2.837814 & -3.148529 & 0.000000 \\ \mathrm{C} & 1.422649 & -2.917867 & 0.000000 \\ \mathrm{~N} & 1.240205 & -1.557904 & 0.000000 \\ \mathrm{C} & 0.384720 & -3.887583 & 0.000000 \\ \mathrm{C} & -0.997947 & -3.750931 & 0.000000 \\ \mathrm{C} & -1.852315 & -2.608220 & 0.000000 \\ \mathrm{C} & -3.288462 & -2.643497 & 0.000000 \\ \mathrm{C} & -3.731607 & -1.323646 & 0.000000 \\ \mathrm{C} & -2.562116 & -0.508217 & 0.000000 \\ \mathrm{~N} & -1.425882 & -1.297647 & 0.000000 \\ \mathrm{H} & 0.261406 & -1.150965 & 0.000000 \\ \mathrm{H} & 3.307085 & -4.129362 & 0.000000 \\ \mathrm{H} & 4.541387 & -1.723306 & 0.000000 \\ \mathrm{H} & 0.753653 & -4.916778 & 0.000000 \\ \mathrm{H} & -1.548395 & -4.696973 & 0.000000\end{array}$




$\begin{array}{rrrr}\mathrm{H} & -3.902700 & -3.542194 & 0.000000 \\ \mathrm{H} & -4.762070 & -0.976243 & 0.000000 \\ \mathrm{H} & -4.541387 & 1.723306 & 0.000000 \\ \mathrm{H} & -3.307085 & 4.129362 & 0.000000 \\ \mathrm{H} & -0.753653 & 4.916778 & 0.000000 \\ \mathrm{H} & 1.548395 & 4.696973 & 0.000000 \\ \mathrm{H} & 3.902699 & 3.542194 & 0.000000 \\ \mathrm{H} & 4.762070 & 0.976243 & 0.000000\end{array}$

porphine $\mathrm{S}_{0}$

$\begin{array}{lrrr}\mathrm{N} & 0.000000 & -2.030802 & 0.000000 \\ \mathrm{~N} & 2.123919 & 0.000000 & 0.000000 \\ \mathrm{C} & 1.089600 & -2.862419 & 0.000000 \\ \mathrm{C} & 0.681164 & -4.266630 & 0.000000 \\ \mathrm{C} & 2.429949 & -2.446695 & 0.000000 \\ \mathrm{C} & 2.906854 & -1.133327 & 0.000000 \\ \mathrm{C} & 4.274586 & -0.688651 & 0.000000 \\ \mathrm{H} & 1.355839 & -5.121063 & 0.000000 \\ \mathrm{H} & 3.190924 & -3.230154 & 0.000000 \\ \mathrm{H} & 5.135567 & -1.352518 & 0.000000 \\ \mathrm{H} & 1.099723 & 0.000000 & 0.000000 \\ \mathrm{~N} & 0.000000 & 2.030802 & 0.000000 \\ \mathrm{~N} & -2.123919 & 0.000000 & 0.000000 \\ \mathrm{C} & 1.089600 & 2.862419 & 0.000000 \\ \mathrm{C} & -1.089600 & 2.862419 & 0.000000 \\ \mathrm{C} & -1.089600 & -2.862419 & 0.000000 \\ \mathrm{C} & 0.681164 & 4.266630 & 0.000000 \\ \mathrm{C} & -0.681164 & 4.266630 & 0.000000 \\ \mathrm{C} & -0.681164 & -4.266630 & 0.000000 \\ \mathrm{C} & 2.429949 & 2.446695 & 0.000000 \\ \mathrm{C} & -2.429949 & 2.446695 & 0.000000 \\ \mathrm{C} & -2.429949 & -2.446695 & 0.000000 \\ \mathrm{C} & 2.906854 & 1.133327 & 0.000000 \\ \mathrm{C} & -2.906854 & 1.133327 & 0.000000 \\ \mathrm{C} & -2.906854 & -1.133327 & 0.000000 \\ \mathrm{C} & 4.274586 & 0.688651 & 0.000000 \\ \mathrm{C} & -4.274586 & 0.688651 & 0.000000 \\ \mathrm{C} & -4.274586 & -0.688651 & 0.000000 \\ \mathrm{H} & 1.355839 & 5.121063 & 0.000000 \\ \mathrm{H} & -1.355839 & 5.121063 & 0.000000 \\ \mathrm{H} & -1.355839 & -5.121063 & 0.000000 \\ \mathrm{H} & 3.190924 & 3.230154 & 0.000000 \\ \mathrm{H} & -3.190924 & 3.230154 & 0.000000 \\ \mathrm{H} & -3.190924 & -3.230154 & 0.000000 \\ \mathrm{H} & 5.135567 & 1.352518 & 0.000000\end{array}$




$$
\begin{array}{rrrr}
H & -5.135567 & 1.352518 & 0.000000 \\
H & -5.135567 & -1.352518 & 0.000000 \\
H & -1.099723 & 0.000000 & 0.000000
\end{array}
$$

porphine $\mathrm{S}_{1}$

$\begin{array}{lrrr}\mathrm{N} & 0.000000 & -2.055470 & 0.000000 \\ \mathrm{~N} & 2.120716 & 0.000000 & 0.000000 \\ \mathrm{C} & 1.095084 & -2.892891 & 0.000000 \\ \mathrm{C} & 0.685657 & -4.287710 & 0.000000 \\ \mathrm{C} & 2.436608 & -2.454601 & 0.000000 \\ \mathrm{C} & 2.906466 & -1.136040 & 0.000000 \\ \mathrm{C} & 4.274376 & -0.690076 & 0.000000 \\ \mathrm{H} & 1.357874 & -5.143810 & 0.000000 \\ \mathrm{H} & 3.205544 & -3.230517 & 0.000000 \\ \mathrm{H} & 5.135854 & -1.353319 & 0.000000 \\ \mathrm{H} & 1.098278 & 0.000000 & 0.000000 \\ \mathrm{~N} & 0.000000 & 2.055470 & 0.000000 \\ \mathrm{~N} & -2.120716 & 0.000000 & 0.000000 \\ \mathrm{C} & 1.095084 & 2.892891 & 0.000000 \\ \mathrm{C} & -1.095084 & 2.892891 & 0.000000 \\ \mathrm{C} & -1.095084 & -2.892891 & 0.000000 \\ \mathrm{C} & 0.685657 & 4.287710 & 0.000000 \\ \mathrm{C} & -0.685657 & 4.287710 & 0.000000 \\ \mathrm{C} & -0.685657 & -4.287710 & 0.000000 \\ \mathrm{C} & 2.436608 & 2.454601 & 0.000000 \\ \mathrm{C} & -2.436608 & 2.454601 & 0.000000 \\ \mathrm{C} & -2.436608 & -2.454601 & 0.000000 \\ \mathrm{C} & 2.906466 & 1.136040 & 0.000000 \\ \mathrm{C} & -2.906466 & 1.136040 & 0.000000 \\ \mathrm{C} & -2.906466 & -1.136040 & 0.000000 \\ \mathrm{C} & 4.274376 & 0.690076 & 0.000000 \\ \mathrm{C} & -4.274376 & 0.690076 & 0.000000 \\ \mathrm{C} & -4.274376 & -0.690076 & 0.000000 \\ \mathrm{H} & 1.357874 & 5.143810 & 0.000000 \\ \mathrm{H} & -1.357874 & 5.143810 & 0.000000 \\ \mathrm{H} & -1.357874 & -5.143810 & 0.000000 \\ \mathrm{H} & 3.205544 & 3.230517 & 0.000000 \\ \mathrm{H} & -3.205544 & 3.230517 & 0.000000 \\ \mathrm{H} & -3.205544 & -3.230517 & 0.000000 \\ \mathrm{H} & 5.135854 & 1.353319 & 0.000000 \\ \mathrm{H} & -5.135854 & 1.353319 & 0.000000 \\ \mathrm{H} & -5.135854 & -1.353319 & 0.000000 \\ \mathrm{H} & -1.098278 & 0.000000 & 0.000000\end{array}$

chlorin $\mathrm{S}_{0}$ 


\begin{tabular}{rrrr}
$\mathrm{C}$ & 0.000000 & -4.268309 & 0.929314 \\
$\mathrm{C}$ & 0.000000 & -2.907547 & 1.384049 \\
$\mathrm{~N}$ & 0.000000 & -2.114507 & 0.248658 \\
$\mathrm{C}$ & 0.000000 & -2.887333 & -0.886102 \\
$\mathrm{C}$ & 0.000000 & -4.257158 & -0.449585 \\
$\mathrm{C}$ & 0.000000 & -2.432628 & 2.693763 \\
$\mathrm{C}$ & 0.000000 & -1.090461 & 3.114148 \\
$\mathrm{~N}$ & 0.000000 & 0.000000 & 2.281450 \\
$\mathrm{C}$ & 0.000000 & 1.090461 & 3.114148 \\
$\mathrm{C}$ & 0.000000 & 0.682947 & 4.513052 \\
$\mathrm{C}$ & 0.000000 & -0.682947 & 4.513052 \\
$\mathrm{C}$ & 0.000000 & 2.432628 & 2.693763 \\
$\mathrm{C}$ & 0.000000 & 2.907547 & 1.384049 \\
$\mathrm{~N}$ & 0.000000 & 2.114507 & 0.248658 \\
$\mathrm{C}$ & 0.000000 & 2.887333 & -0.886102 \\
$\mathrm{C}$ & 0.000000 & 4.257158 & -0.449585 \\
$\mathrm{C}$ & 0.000000 & 4.268309 & 0.929314 \\
$\mathrm{C}$ & 0.000000 & 2.423006 & -2.212239 \\
$\mathrm{C}$ & 0.000000 & 1.107241 & -2.669059 \\
$\mathrm{C}$ & 0.000000 & 0.769964 & -4.160752 \\
$\mathrm{C}$ & 0.000000 & -0.769964 & -4.160752 \\
$\mathrm{C}$ & 0.000000 & -1.107241 & -2.669059 \\
$\mathrm{~N}$ & 0.000000 & 0.000000 & -1.883996 \\
$\mathrm{C}$ & 0.000000 & -2.423006 & -2.212239 \\
$\mathrm{H}$ & -0.884872 & 1.196079 & -4.658474 \\
$\mathrm{H}$ & 0.000000 & 3.205499 & -2.974765 \\
$\mathrm{H}$ & 0.000000 & 5.112938 & -1.120118 \\
$\mathrm{H}$ & 0.000000 & 1.356526 & 5.368397 \\
$\mathrm{H}$ & 0.000000 & -1.356526 & 5.368397 \\
$\mathrm{H}$ & -0.884872 & -1.196079 & -4.658474 \\
$\mathrm{H}$ & 0.000000 & 3.194243 & 3.476950 \\
$\mathrm{H}$ & 0.000000 & -3.194243 & 3.476950 \\
$\mathrm{H}$ & 0.000000 & -3.205499 & -2.974765 \\
$\mathrm{H}$ & 0.000000 & 5.135026 & 1.585780 \\
$\mathrm{H}$ & 0.000000 & -5.135026 & 1.585780 \\
$\mathrm{H}$ & 0.000000 & -5.112938 & -1.120118 \\
$\mathrm{H}$ & 0.884872 & -1.196079 & -4.658474 \\
$\mathrm{H}$ & 0.884872 & 1.196079 & -4.658474 \\
$\mathrm{H}$ & 0.000000 & -1.092797 & 0.276726 \\
$\mathrm{H}$ & 0.000000 & 1.092797 & 0.276726 \\
& & & \\
\hline
\end{tabular}

chlorin $\mathrm{S}_{1}$

$\begin{array}{llll}\text { C } & 0.000000 & -4.278537 & 0.929959 \\ \text { C } & 0.000000 & -2.915209 & 1.382410\end{array}$ 


$\begin{array}{lrrr}\mathrm{N} & 0.000000 & -2.122743 & 0.246517 \\ \mathrm{C} & 0.000000 & -2.902570 & -0.890697 \\ \mathrm{C} & 0.000000 & -4.269444 & -0.453570 \\ \mathrm{C} & 0.000000 & -2.438802 & 2.698589 \\ \mathrm{C} & 0.000000 & -1.092916 & 3.119258 \\ \mathrm{~N} & 0.000000 & 0.000000 & 2.283378 \\ \mathrm{C} & 0.000000 & 1.092916 & 3.119258 \\ \mathrm{C} & 0.000000 & 0.682681 & 4.520981 \\ \mathrm{C} & 0.000000 & -0.682681 & 4.520981 \\ \mathrm{C} & 0.000000 & 2.438802 & 2.698589 \\ \mathrm{C} & 0.000000 & 2.915209 & 1.382410 \\ \mathrm{~N} & 0.000000 & 2.122743 & 0.246517 \\ \mathrm{C} & 0.000000 & 2.902570 & -0.890697 \\ \mathrm{C} & 0.000000 & 4.269444 & -0.453570 \\ \mathrm{C} & 0.000000 & 4.278537 & 0.929959 \\ \mathrm{C} & 0.000000 & 2.432572 & -2.219318 \\ \mathrm{C} & 0.000000 & 1.108812 & -2.670828 \\ \mathrm{C} & 0.000000 & 0.768859 & -4.156761 \\ \mathrm{C} & 0.000000 & -0.768859 & -4.156761 \\ \mathrm{C} & 0.000000 & -1.108812 & -2.670828 \\ \mathrm{~N} & 0.000000 & 0.000000 & -1.877846 \\ \mathrm{C} & 0.000000 & -2.432572 & -2.219318 \\ \mathrm{H} & -0.883866 & 1.196009 & -4.657772 \\ \mathrm{H} & 0.000000 & 3.210512 & -2.986117 \\ \mathrm{H} & 0.000000 & 5.126600 & -1.122203 \\ \mathrm{H} & 0.000000 & 1.357267 & 5.375250 \\ \mathrm{H} & 0.000000 & -1.357267 & 5.375250 \\ \mathrm{H} & -0.883866 & -1.196009 & -4.657772 \\ \mathrm{H} & 0.000000 & 3.200022 & 3.481427 \\ \mathrm{H} & 0.000000 & -3.200022 & 3.481427 \\ \mathrm{H} & 0.000000 & -3.210512 & -2.986117 \\ \mathrm{H} & 0.000000 & 5.143871 & 1.587875 \\ \mathrm{H} & 0.000000 & -5.143871 & 1.587875 \\ \mathrm{H} & 0.000000 & -5.126600 & -1.122203 \\ \mathrm{H} & 0.883866 & -1.196009 & -4.657772 \\ \mathrm{H} & 0.883866 & 1.196009 & -4.657772 \\ \mathrm{H} & 0.000000 & -1.101182 & 0.270019 \\ \mathrm{H} & 0.000000 & 1.101182 & 0.270019 \\ & & & \end{array}$

Zn-tetraphenylporphine $\mathrm{S}_{0}$

$\begin{array}{lrrr}\mathrm{C} & -3.709343 & 4.333997 & 1.125859 \\ \mathrm{C} & -3.513648 & 3.513648 & 0.000000 \\ \mathrm{C} & -4.333997 & 3.709340 & -1.125860 \\ \mathrm{C} & -5.321407 & 4.699931 & -1.126751 \\ \mathrm{C} & -5.508376 & 5.508373 & -0.000002\end{array}$




$\begin{array}{rrrr}\mathrm{C} & -4.699935 & 5.321406 & 1.126748 \\ \mathrm{C} & -2.454158 & 2.454158 & 0.000001 \\ \mathrm{C} & -1.108851 & 2.880059 & -0.012094 \\ \mathrm{~N} & 0.000000 & 2.056199 & 0.033806 \\ \mathrm{C} & 1.108851 & 2.880059 & -0.012094 \\ \mathrm{C} & 0.683602 & 4.260857 & -0.103504 \\ \mathrm{C} & -0.683602 & 4.260857 & -0.103504 \\ \mathrm{C} & 2.454158 & 2.454158 & 0.000001 \\ \mathrm{C} & 3.513648 & 3.513648 & 0.000000 \\ \mathrm{C} & 4.333997 & 3.709340 & -1.125860 \\ \mathrm{C} & 5.321407 & 4.699931 & -1.126751 \\ \mathrm{C} & 5.508376 & 5.508373 & -0.000002 \\ \mathrm{C} & 4.699935 & 5.321406 & 1.126748 \\ \mathrm{C} & 3.709343 & 4.333997 & 1.125859 \\ \mathrm{C} & -2.880059 & 1.108851 & 0.012097 \\ \mathrm{~N} & -2.056199 & 0.000000 & -0.033805 \\ \mathrm{C} & -2.880059 & -1.108851 & 0.012097 \\ \mathrm{C} & -4.260857 & -0.683602 & 0.103506 \\ \mathrm{C} & -4.260857 & 0.683602 & 0.103506 \\ \mathrm{C} & -2.454158 & -2.454158 & 0.000001 \\ \mathrm{C} & -3.513648 & -3.513648 & 0.000000 \\ \mathrm{C} & -4.333997 & -3.709340 & -1.125860 \\ \mathrm{C} & -5.321407 & -4.699931 & -1.126751 \\ \mathrm{C} & -5.508376 & -5.508373 & -0.000002 \\ \mathrm{C} & -4.699935 & -5.321406 & 1.126748 \\ \mathrm{C} & -3.709343 & -4.333997 & 1.125859 \\ \mathrm{C} & 2.880059 & 1.108851 & 0.012097 \\ \mathrm{~N} & 2.056199 & 0.000000 & -0.033805 \\ \mathrm{C} & 2.880059 & -1.108851 & 0.012097 \\ \mathrm{C} & 4.260857 & -0.683602 & 0.103506 \\ \mathrm{C} & 4.260857 & 0.683602 & 0.103506 \\ \mathrm{C} & 2.454158 & -2.454158 & 0.000001 \\ \mathrm{C} & 3.513648 & -3.513648 & 0.000000 \\ \mathrm{C} & 4.333997 & -3.709340 & -1.125860 \\ \mathrm{C} & 5.321407 & -4.699931 & -1.126751 \\ \mathrm{C} & 5.508376 & -5.508373 & -0.000002 \\ \mathrm{C} & 4.699935 & -5.321406 & 1.126748 \\ \mathrm{C} & 3.709343 & -4.333997 & 1.125859 \\ \mathrm{C} & 1.108851 & -2.880059 & -0.012094 \\ \mathrm{~N} & 0.000000 & -2.056199 & 0.033806 \\ \mathrm{C} & -1.108851 & -2.880059 & -0.012094 \\ \mathrm{C} & -0.683602 & -4.260857 & -0.103504 \\ \mathrm{C} & 0.683602 & -4.260857 & -0.103504 \\ \mathrm{H} & 1.348047 & -5.116906 & -0.177248 \\ \mathrm{H} & 5.116906 & -1.348047 & 0.177251 \\ \mathrm{H} & 1.348047 & 5.116906 & -0.177248\end{array}$




$\begin{array}{crrr}\mathrm{H} & -1.348047 & 5.116906 & -0.177248 \\ \mathrm{H} & -1.348047 & -5.116906 & -0.177248 \\ \mathrm{H} & 5.116906 & 1.348047 & 0.177251 \\ \mathrm{H} & -5.116906 & 1.348047 & 0.177251 \\ \mathrm{H} & -5.116906 & -1.348047 & 0.177251 \\ \mathrm{H} & 4.185472 & 3.082819 & -2.008303 \\ \mathrm{H} & 5.944428 & 4.842351 & -2.012785 \\ \mathrm{H} & 6.280700 & 6.280697 & -0.000003 \\ \mathrm{H} & 4.842356 & 5.944428 & 2.012781 \\ \mathrm{H} & 3.082823 & 4.185474 & 2.008303 \\ \mathrm{H} & 4.185472 & -3.082819 & -2.008303 \\ \mathrm{H} & 5.944428 & -4.842351 & -2.012785 \\ \mathrm{H} & 6.280700 & -6.280697 & -0.000003 \\ \mathrm{H} & 4.842356 & -5.944428 & 2.012781 \\ \mathrm{H} & 3.082823 & -4.185474 & 2.008303 \\ \mathrm{H} & -4.185472 & -3.082819 & -2.008303 \\ \mathrm{H} & -5.944428 & -4.842351 & -2.012785 \\ \mathrm{H} & -6.280700 & -6.280697 & -0.000003 \\ \mathrm{H} & -4.842356 & -5.944428 & 2.012781 \\ \mathrm{H} & -3.082823 & -4.185474 & 2.008303 \\ \mathrm{H} & -4.185472 & 3.082819 & -2.008303 \\ \mathrm{H} & -5.944428 & 4.842351 & -2.012785 \\ \mathrm{H} & -6.280700 & 6.280697 & -0.000003 \\ \mathrm{H} & -4.842356 & 5.944428 & 2.012781 \\ \mathrm{H} & -3.082823 & 4.185474 & 2.008303 \\ \mathrm{Zn} & 0.000000 & 0.000000 & 0.000000\end{array}$

Zn-tetraphenylporphine $\mathrm{S}_{1}$

$\begin{array}{lrrr}\mathrm{C} & -3.550612 & 4.555748 & 0.941506 \\ \mathrm{C} & -3.515152 & 3.510252 & -0.004376 \\ \mathrm{C} & -4.510981 & 3.478908 & -1.003032 \\ \mathrm{C} & -5.498473 & 4.464534 & -1.061442 \\ \mathrm{C} & -5.522476 & 5.495836 & -0.113875 \\ \mathrm{C} & -4.546744 & 5.535059 & 0.888724 \\ \mathrm{C} & -2.459364 & 2.463751 & 0.040576 \\ \mathrm{C} & -1.111489 & 2.875676 & -0.020619 \\ \mathrm{~N} & 0.000000 & 2.060197 & 0.146253 \\ \mathrm{C} & 1.111489 & 2.875676 & -0.020619 \\ \mathrm{C} & 0.683837 & 4.226144 & -0.307362 \\ \mathrm{C} & -0.683837 & 4.226144 & -0.307362 \\ \mathrm{C} & 2.459364 & 2.463751 & 0.040576 \\ \mathrm{C} & 3.515152 & 3.510252 & -0.004376 \\ \mathrm{C} & 4.510981 & 3.478908 & -1.003032 \\ \mathrm{C} & 5.498473 & 4.464534 & -1.061442 \\ \mathrm{C} & 5.522476 & 5.495836 & -0.113875\end{array}$




$\begin{array}{rrrr}\mathrm{C} & 4.546744 & 5.535059 & 0.888724 \\ \mathrm{C} & 3.550612 & 4.555748 & 0.941506 \\ \mathrm{C} & -2.885365 & 1.109247 & 0.134211 \\ \mathrm{~N} & -2.068373 & 0.000000 & -0.025504 \\ \mathrm{C} & -2.885365 & -1.109247 & 0.134211 \\ \mathrm{C} & -4.230609 & -0.688393 & 0.413416 \\ \mathrm{C} & -4.230609 & 0.688393 & 0.413416 \\ \mathrm{C} & -2.459364 & -2.463751 & 0.040576 \\ \mathrm{C} & -3.515152 & -3.510252 & -0.004376 \\ \mathrm{C} & -4.510981 & -3.478908 & -1.003032 \\ \mathrm{C} & -5.498473 & -4.464534 & -1.061442 \\ \mathrm{C} & -5.522476 & -5.495836 & -0.113875 \\ \mathrm{C} & -4.546744 & -5.535059 & 0.888724 \\ \mathrm{C} & -3.550612 & -4.555748 & 0.941506 \\ \mathrm{C} & 2.885365 & 1.109247 & 0.134211 \\ \mathrm{~N} & 2.068373 & 0.000000 & -0.025504 \\ \mathrm{C} & 2.885365 & -1.109247 & 0.134211 \\ \mathrm{C} & 4.230609 & -0.688393 & 0.413416 \\ \mathrm{C} & 4.230609 & 0.688393 & 0.413416 \\ \mathrm{C} & 2.459364 & -2.463751 & 0.040576 \\ \mathrm{C} & 3.515152 & -3.510252 & -0.004376 \\ \mathrm{C} & 4.510981 & -3.478908 & -1.003032 \\ \mathrm{C} & 5.498473 & -4.464534 & -1.061442 \\ \mathrm{C} & 5.522476 & -5.495836 & -0.113875 \\ \mathrm{C} & 4.546744 & -5.535059 & 0.888724 \\ \mathrm{C} & 3.550612 & -4.555748 & 0.941506 \\ \mathrm{C} & 1.111489 & -2.875676 & -0.020619 \\ \mathrm{~N} & 0.000000 & -2.060197 & 0.146253 \\ \mathrm{C} & -1.111489 & -2.875676 & -0.020619 \\ \mathrm{C} & -0.683837 & -4.226144 & -0.307362 \\ \mathrm{C} & 0.683837 & -4.226144 & -0.307362 \\ \mathrm{H} & 1.347637 & -5.058568 & -0.520838 \\ \mathrm{H} & 5.065531 & -1.352603 & 0.614674 \\ \mathrm{H} & 1.347637 & 5.058568 & -0.520838 \\ \mathrm{H} & -1.347637 & 5.058568 & -0.520838 \\ \mathrm{H} & -1.347637 & -5.058568 & -0.520838 \\ \mathrm{H} & 5.065531 & 1.352603 & 0.614674 \\ \mathrm{H} & -5.065531 & 1.352603 & 0.614674 \\ \mathrm{H} & -5.065531 & -1.352603 & 0.614674 \\ \mathrm{H} & 4.489180 & 2.681052 & -1.748287 \\ \mathrm{H} & 6.250164 & 4.431109 & -1.853486 \\ \mathrm{H} & 6.298191 & 6.263523 & -0.156249 \\ \mathrm{H} & 4.564124 & 6.329153 & 1.638815 \\ \mathrm{H} & 2.798339 & 4.580635 & 1.732682 \\ \mathrm{H} & 4.489180 & -2.681052 & -1.748287 \\ \mathrm{H} & 6.250164 & -4.431109 & -1.853486 \\ & & & \end{array}$




$\begin{array}{crrr}\mathrm{H} & 6.298191 & -6.263523 & -0.156249 \\ \mathrm{H} & 4.564124 & -6.329153 & 1.638815 \\ \mathrm{H} & 2.798339 & -4.580635 & 1.732682 \\ \mathrm{H} & -4.489180 & -2.681052 & -1.748287 \\ \mathrm{H} & -6.250164 & -4.431109 & -1.853486 \\ \mathrm{H} & -6.298191 & -6.263523 & -0.156249 \\ \mathrm{H} & -4.564124 & -6.329153 & 1.638815 \\ \mathrm{H} & -2.798339 & -4.580635 & 1.732682 \\ \mathrm{H} & -4.489180 & 2.681052 & -1.748287 \\ \mathrm{H} & -6.250164 & 4.431109 & -1.853486 \\ \mathrm{H} & -6.298191 & 6.263523 & -0.156249 \\ \mathrm{H} & -4.564124 & 6.329153 & 1.638815 \\ \mathrm{H} & -2.798339 & 4.580635 & 1.732682 \\ \mathrm{Zn} & 0.000000 & 0.000000 & 0.056494\end{array}$

tetraphenylporphine $\mathrm{S}_{0}$

$\begin{array}{lrrr}\mathrm{C} & -0.691018 & 4.280797 & 0.142043 \\ \mathrm{C} & -1.135974 & 2.914295 & 0.014677 \\ \mathrm{~N} & 0.000000 & 2.130387 & -0.049416 \\ \mathrm{C} & 1.135974 & 2.914295 & 0.014676 \\ \mathrm{C} & 0.691019 & 4.280797 & 0.142043 \\ \mathrm{C} & 2.476387 & 2.457809 & -0.024448 \\ \mathrm{C} & 2.876525 & 1.092437 & -0.074537 \\ \mathrm{~N} & 2.045874 & 0.000000 & -0.010549 \\ \mathrm{C} & 2.876525 & -1.092437 & -0.074536 \\ \mathrm{C} & 4.278102 & -0.683720 & -0.217519 \\ \mathrm{C} & 4.278102 & 0.683720 & -0.217519 \\ \mathrm{C} & 2.476387 & -2.457809 & -0.024447 \\ \mathrm{C} & 1.135974 & -2.914295 & 0.014677 \\ \mathrm{~N} & 0.000000 & -2.130387 & -0.049416 \\ \mathrm{C} & -1.135974 & -2.914295 & 0.014676 \\ \mathrm{C} & -0.691019 & -4.280797 & 0.142043 \\ \mathrm{C} & 0.691018 & -4.280797 & 0.142043 \\ \mathrm{C} & -2.476387 & -2.457809 & -0.024448 \\ \mathrm{C} & -2.876525 & -1.092437 & -0.074537 \\ \mathrm{~N} & -2.045874 & 0.000000 & -0.010549 \\ \mathrm{C} & -2.876525 & 1.092437 & -0.074536 \\ \mathrm{C} & -4.278102 & 0.683720 & -0.217519 \\ \mathrm{C} & -4.278102 & -0.683720 & -0.217519 \\ \mathrm{C} & -2.476387 & 2.457809 & -0.024447 \\ \mathrm{H} & -5.136818 & -1.355700 & -0.327257 \\ \mathrm{C} & -3.544947 & -3.510406 & 0.000637 \\ \mathrm{H} & -1.356611 & -5.144933 & 0.238508 \\ \mathrm{H} & 0.000000 & -1.101120 & -0.084406 \\ \mathrm{H} & 5.136819 & -1.355700 & -0.327256\end{array}$




\begin{tabular}{|c|c|c|c|}
\hline & 5.136818 & & \\
\hline & & & \\
\hline & 3.544947 & -3.510406 & \\
\hline & & & \\
\hline & -3.544947 & & \\
\hline & & & \\
\hline & 1.356611 & & \\
\hline & -1.356610 & & \\
\hline & 0.000000 & & \\
\hline & 3.689769 & -4.4 & \\
\hline & & & \\
\hline & 5.56 & -5.5 & \\
\hline & & & \\
\hline & 4.4 & -3.6 & \\
\hline & & & \\
\hline & 4.7 & -6 . & \\
\hline & & & \\
\hline & 6.1 & -4.6 & \\
\hline & 4.3 & -2.9 & \\
\hline & & -4.4 & \\
\hline & -4.6 & -5.4 & \\
\hline & & & \\
\hline & -5.4 & -4.5 & \\
\hline & -4.4 & -3.6 & \\
\hline & -3.0 & & \\
\hline F & & & \\
\hline & -6.3 & & \\
\hline & -6.1 & -4.6 & \\
\hline & -4.3 & -2.5 & \\
\hline & -3.6 & & \\
\hline C & -4.6 & 5.4 & -1 . \\
\hline & -5.5 & & \\
\hline & -5.4 & 4.5 & \\
\hline & -4.4 & 3.61 & \\
\hline $\mathrm{H}$ & & & \\
\hline $\mathrm{H}$ & -4.7 & & 580 \\
\hline $\mathrm{H}$ & -6.3 & & \\
\hline $\mathrm{H}$ & -6.107711 & 4.66 & 5815 \\
\hline & -4.3 & & \\
\hline$C$ & 3.689768 & 4.427467 & -1.067068 \\
\hline & 4.691721 & 5.4 & \\
\hline & & & \\
\hline & 5.431125 & 4.597193 & 1.128638 \\
\hline & 4.431689 & & \\
\hline & 3.014276 & 4.352900 & -1.934062 \\
\hline & 4.793795 & 6.111621 & -1.883585 \\
\hline
\end{tabular}




$\begin{array}{llll}\mathrm{H} & 6.349776 & 6.274320 & 0.082935 \\ \mathrm{H} & 6.107711 & 4.662452 & 1.995810 \\ \mathrm{H} & 4.323720 & 2.907476 & 1.940041\end{array}$

tetraphenylporphine $\mathrm{S}_{1}$

$\begin{array}{rrrr}\text { C } & -4.263962 & -0.691863 & 0.299069 \\ \mathrm{C} & -2.913960 & -1.138779 & 0.054113 \\ \mathrm{~N} & -2.135718 & 0.000000 & -0.070800 \\ \mathrm{C} & -2.913960 & 1.138778 & 0.054106 \\ \mathrm{C} & -4.263963 & 0.691864 & 0.299062 \\ \mathrm{C} & -2.465510 & 2.482554 & -0.036975 \\ \mathrm{C} & -1.096033 & 2.894134 & -0.155954 \\ \mathrm{~N} & 0.000000 & 2.065925 & -0.029641 \\ \mathrm{C} & 1.096033 & 2.894135 & -0.155949 \\ \mathrm{C} & 0.688346 & 4.267966 & -0.420197 \\ \mathrm{C} & -0.688346 & 4.267965 & -0.420198 \\ \mathrm{C} & 2.465510 & 2.482555 & -0.036963 \\ \mathrm{C} & 2.913960 & 1.138779 & 0.054113 \\ \mathrm{~N} & 2.135718 & 0.000000 & -0.070800 \\ \mathrm{C} & 2.913960 & -1.138778 & 0.054106 \\ \mathrm{C} & 4.263963 & -0.691864 & 0.299062 \\ \mathrm{C} & 4.263962 & 0.691863 & 0.299069 \\ \mathrm{C} & 2.465510 & -2.482554 & -0.036975 \\ \mathrm{C} & 1.096033 & -2.894134 & -0.155954 \\ \mathrm{~N} & 0.000000 & -2.065925 & -0.029641 \\ \mathrm{C} & -1.096033 & -2.894135 & -0.155949 \\ \mathrm{C} & -0.688346 & -4.267966 & -0.420197 \\ \mathrm{C} & 0.688346 & -4.267965 & -0.420198 \\ \mathrm{C} & -2.465510 & -2.482555 & -0.036963 \\ \mathrm{H} & 1.357606 & -5.114843 & -0.608520 \\ \mathrm{C} & 3.511413 & -3.546437 & 0.002380 \\ \mathrm{H} & 5.116391 & -1.355036 & 0.478488 \\ \mathrm{H} & 1.110698 & 0.000000 & -0.154571 \\ \mathrm{H} & 1.357607 & 5.114843 & -0.608517 \\ \mathrm{H} & -1.357606 & 5.114843 & -0.608520 \\ \mathrm{H} & -1.357607 & -5.114843 & -0.608517 \\ \mathrm{C} & 3.511414 & 3.546437 & 0.002398 \\ \mathrm{C} & -3.511413 & 3.546437 & 0.002380 \\ \mathrm{C} & -3.511414 & -3.546437 & 0.002398 \\ \mathrm{H} & 5.116390 & 1.355033 & 0.478500 \\ \mathrm{H} & -5.116391 & 1.355036 & 0.478488 \\ \mathrm{H} & -5.116390 & -1.355033 & 0.478500 \\ \mathrm{H} & -1.110698 & 0.000000 & -0.154571 \\ \mathrm{C} & 4.562711 & 3.580444 & -0.948009 \\ \mathrm{C} & 5.543348 & 4.585095 & -0.901981\end{array}$




$\begin{array}{lrrr}\mathrm{C} & 5.499389 & 5.572877 & 0.097833 \\ \mathrm{C} & 4.462803 & 5.551440 & 1.049405 \\ \mathrm{C} & 3.476692 & 4.554104 & 0.999218 \\ \mathrm{H} & 4.590385 & 2.818819 & -1.743054 \\ \mathrm{H} & 6.344146 & 4.599982 & -1.658661 \\ \mathrm{H} & 6.270124 & 6.359108 & 0.134802 \\ \mathrm{H} & 4.424850 & 6.316143 & 1.841758 \\ \mathrm{H} & 2.672336 & 4.534957 & 1.751013 \\ \mathrm{C} & 4.562707 & -3.580441 & -0.948031 \\ \mathrm{C} & 5.543343 & -4.585094 & -0.902011 \\ \mathrm{C} & 5.499386 & -5.572880 & 0.097800 \\ \mathrm{C} & 4.462804 & -5.551444 & 1.049376 \\ \mathrm{C} & 3.476694 & -4.554107 & 0.999196 \\ \mathrm{H} & 4.590379 & -2.818814 & -1.743074 \\ \mathrm{H} & 6.344139 & -4.599979 & -1.658694 \\ \mathrm{H} & 6.270120 & -6.359111 & 0.134762 \\ \mathrm{H} & 4.424853 & -6.316151 & 1.841726 \\ \mathrm{H} & 2.672341 & -4.534962 & 1.750994 \\ \mathrm{C} & -4.562711 & -3.580444 & -0.948009 \\ \mathrm{C} & -5.543348 & -4.585095 & -0.901981 \\ \mathrm{C} & -5.499389 & -5.572877 & 0.097833 \\ \mathrm{C} & -4.462803 & -5.551440 & 1.049405 \\ \mathrm{C} & -3.476692 & -4.554104 & 0.999218 \\ \mathrm{H} & -4.590385 & -2.818819 & -1.743054 \\ \mathrm{H} & -6.344146 & -4.599982 & -1.658661 \\ \mathrm{H} & -6.270124 & -6.359108 & 0.134802 \\ \mathrm{H} & -4.424850 & -6.316143 & 1.841758 \\ \mathrm{H} & -2.672336 & -4.534957 & 1.751013 \\ \mathrm{C} & -4.562707 & 3.580441 & -0.948031 \\ \mathrm{C} & -5.543343 & 4.585094 & -0.902011 \\ \mathrm{C} & -5.499386 & 5.572880 & 0.097800 \\ \mathrm{C} & -4.462804 & 5.551444 & 1.049376 \\ \mathrm{C} & -3.476694 & 4.554107 & 0.999196 \\ \mathrm{H} & -4.590379 & 2.818814 & -1.743074 \\ \mathrm{H} & -6.344139 & 4.599979 & -1.658694 \\ \mathrm{H} & -6.270120 & 6.359111 & 0.134762 \\ \mathrm{H} & -4.424853 & 6.316151 & 1.841726 \\ \mathrm{H} & -2.672341 & 4.534962 & 1.750994\end{array}$

BP86/TZVP geometries - Group II

tetrafluorobenzene $\mathrm{S}_{0}$
$\begin{array}{llll}\text { C } & -1.200749 & 0.699908 & 0.000000\end{array}$
$\begin{array}{lrrr}\text { C } & -1.200749 & -0.699908 & 0.000000\end{array}$
$\begin{array}{llll}\text { C } & 0.000000 & -1.406578 & 0.000000\end{array}$ 


$\begin{array}{cccc}\text { C } & 1.200749 & -0.699908 & 0.000000 \\ \text { C } & 1.200749 & 0.699908 & 0.000000 \\ \text { C } & 0.000000 & 1.406578 & 0.000000 \\ \text { F } & -2.375441 & -1.366172 & 0.000000 \\ \text { H } & 0.000000 & -2.496729 & 0.000000 \\ \text { F } & 2.375441 & -1.366172 & 0.000000 \\ \text { F } & 2.375441 & 1.366172 & 0.000000 \\ \text { H } & 0.000000 & 2.496729 & 0.000000 \\ \text { F } & -2.375441 & 1.366172 & 0.000000\end{array}$

tetrafluorobenzene $\mathrm{S}_{1}$

$\begin{array}{lrrr}\text { C } & 1.172990 & -0.712093 & 0.110382 \\ \text { C } & 1.172990 & 0.712093 & 0.110382 \\ \text { C } & 0.000000 & 1.473170 & 0.300645 \\ \text { C } & -1.172990 & 0.712093 & 0.110382 \\ \text { C } & -1.172990 & -0.712093 & 0.110382 \\ \text { C } & 0.000000 & -1.473170 & 0.300645 \\ \text { F } & 2.333101 & 1.334912 & -0.221015 \\ \text { H } & 0.000000 & 2.519151 & 0.618745 \\ \text { F } & -2.333101 & 1.334912 & -0.221015 \\ \text { F } & -2.333101 & -1.334912 & -0.221015 \\ \text { H } & 0.000000 & -2.519151 & 0.618745 \\ \text { F } & 2.333101 & -1.334912 & -0.221015\end{array}$

benzonitrile $\mathrm{S}_{0}$

$\begin{array}{rrrr}\mathrm{C} & 0.000000 & 0.000000 & -1.066347 \\ \mathrm{C} & -1.220721 & 0.000000 & -0.361575 \\ \mathrm{C} & -1.213243 & 0.000000 & 1.033840 \\ \mathrm{C} & 0.000000 & 0.000000 & 1.732710 \\ \mathrm{C} & 1.213243 & 0.000000 & 1.033840 \\ \mathrm{C} & 1.220721 & 0.000000 & -0.361575 \\ \mathrm{H} & -2.161362 & 0.000000 & -0.914349 \\ \mathrm{H} & -2.159392 & 0.000000 & 1.578562 \\ \mathrm{H} & 0.000000 & 0.000000 & 2.824687 \\ \mathrm{H} & 2.159392 & 0.000000 & 1.578562 \\ \mathrm{H} & 2.161362 & 0.000000 & -0.914349 \\ \mathrm{C} & 0.000000 & 0.000000 & -2.498254 \\ \mathrm{~N} & 0.000000 & 0.000000 & -3.665743\end{array}$

benzonitrile $S_{1}$
C $\quad 0.000000$
$0.000000-1.120467$
C $\quad-1.235788 \quad 0.000000 \quad-0.376924$
C $\quad-1.220250 \quad 0.000000 \quad 1.055562$ 


$\begin{array}{lrrr}\mathrm{C} & 0.000000 & 0.000000 & 1.772932 \\ \mathrm{C} & 1.220250 & 0.000000 & 1.055562 \\ \mathrm{C} & 1.235788 & 0.000000 & -0.376924 \\ \mathrm{H} & -2.182937 & 0.000000 & -0.915769 \\ \mathrm{H} & -2.171096 & 0.000000 & 1.589976 \\ \mathrm{H} & 0.000000 & 0.000000 & 2.862614 \\ \mathrm{H} & 2.171096 & 0.000000 & 1.589976 \\ \mathrm{H} & 2.182937 & 0.000000 & -0.915769 \\ \mathrm{C} & 0.000000 & 0.000000 & -2.518310 \\ \mathrm{~N} & 0.000000 & 0.000000 & -3.702458\end{array}$

$o$-fluorophenol, cis $\mathrm{S}_{0}$

$\begin{array}{rrrr}\mathrm{C} & 0.327737 & 1.462676 & 0.000000 \\ \mathrm{C} & -0.879085 & 0.779392 & 0.000000 \\ \mathrm{C} & -0.956585 & -0.621240 & 0.000000 \\ \mathrm{C} & 0.239871 & -1.347723 & 0.000000 \\ \mathrm{C} & 1.466494 & -0.676138 & 0.000000 \\ \mathrm{C} & 1.517649 & 0.722926 & 0.000000 \\ \mathrm{~F} & -2.070405 & 1.466106 & 0.000000 \\ \mathrm{O} & -2.157485 & -1.279392 & 0.000000 \\ \mathrm{H} & 0.186518 & -2.437779 & 0.000000 \\ \mathrm{H} & 2.391615 & -1.255457 & 0.000000 \\ \mathrm{H} & 2.476886 & 1.242460 & 0.000000 \\ \mathrm{H} & 0.325083 & 2.553821 & 0.000000 \\ \mathrm{H} & -2.868282 & -0.609673 & 0.000000\end{array}$

$o$-fluorophenol, cis $\mathrm{S}_{1}$

$\begin{array}{cccc}\text { C } & 0.327542 & 1.523969 & 0.000000 \\ \text { C } & -0.899997 & 0.793763 & 0.000000 \\ \text { C } & -0.946891 & -0.622470 & 0.000000 \\ \text { C } & 0.242864 & -1.414869 & 0.000000 \\ \text { C } & 1.476357 & -0.696394 & 0.000000 \\ \text { C } & 1.502323 & 0.725616 & 0.000000 \\ \text { F } & -2.101472 & 1.457172 & 0.000000 \\ \text { O } & -2.149892 & -1.270584 & 0.000000 \\ \text { H } & 0.175838 & -2.500124 & 0.000000 \\ \text { H } & 2.420212 & -1.241629 & 0.000000 \\ \text { H } & 2.472276 & 1.227571 & 0.000000 \\ \text { H } & 0.341306 & 2.610680 & 0.000000 \\ \text { H } & -2.860455 & -0.592724 & 0.000000\end{array}$

$o$-fluorophenol, trans $\mathrm{S}_{0}$

$\begin{array}{llll}\text { C } & 0.293369 & 1.272884 & 0.000000\end{array}$ 


$\begin{array}{cccc}\mathrm{C} & -0.954164 & 0.623235 & 0.000000 \\ \mathrm{C} & -0.968349 & -0.778431 & 0.000000 \\ \mathrm{C} & 0.227641 & -1.505777 & 0.000000 \\ \mathrm{C} & 1.456653 & -0.840558 & 0.000000 \\ \mathrm{C} & 1.486067 & 0.560256 & 0.000000 \\ \mathrm{O} & -2.080357 & 1.403423 & 0.000000 \\ \mathrm{H} & -1.930340 & -1.300577 & 0.000000 \\ \mathrm{H} & 0.191359 & -2.596674 & 0.000000 \\ \mathrm{H} & 2.391833 & -1.402415 & 0.000000 \\ \mathrm{H} & 2.426232 & 1.114466 & 0.000000 \\ \mathrm{~F} & 0.320123 & 2.630245 & 0.000000 \\ \mathrm{H} & -2.860077 & 0.819923 & 0.000000\end{array}$

$o$-fluorophenol, trans $\mathrm{S}_{1}$

$\begin{array}{lrrr}\mathrm{C} & 0.829251 & 1.060149 & 0.137700 \\ \mathrm{C} & -0.588126 & 0.976929 & 0.087976 \\ \mathrm{C} & -1.265299 & -0.282880 & 0.066631 \\ \mathrm{C} & -0.463601 & -1.436480 & -0.099158 \\ \mathrm{C} & 0.945166 & -1.344372 & -0.053419 \\ \mathrm{C} & 1.614597 & -0.128902 & 0.236839 \\ \mathrm{O} & -1.274851 & 2.149249 & -0.030217 \\ \mathrm{H} & -2.305551 & -0.363556 & 0.407494 \\ \mathrm{H} & -0.932982 & -2.413783 & -0.228545 \\ \mathrm{H} & 1.537323 & -2.247002 & -0.240714 \\ \mathrm{H} & 2.689647 & -0.080901 & 0.407705 \\ \mathrm{~F} & 1.414614 & 2.191452 & -0.411713 \\ \mathrm{H} & -2.200218 & 1.920097 & -0.280579\end{array}$

$m$-fluorophenol, cis $\mathrm{S}_{0}$

$\begin{array}{cccc}\text { C } & 0.310209 & 1.418041 & 0.000000 \\ \mathrm{C} & -0.934874 & 0.792199 & 0.000000 \\ \mathrm{C} & -0.974052 & -0.610417 & 0.000000 \\ \mathrm{C} & 0.216528 & -1.353330 & 0.000000 \\ \mathrm{C} & 1.441766 & -0.685328 & 0.000000 \\ \mathrm{C} & 1.510532 & 0.714190 & 0.000000 \\ \mathrm{O} & -2.155420 & -1.311772 & 0.000000 \\ \mathrm{~F} & 0.341216 & 2.781001 & 0.000000 \\ \mathrm{H} & 0.161780 & -2.442396 & 0.000000 \\ \mathrm{H} & 2.366756 & -1.265277 & 0.000000 \\ \mathrm{H} & 2.459876 & 1.249389 & 0.000000 \\ \mathrm{H} & -1.845038 & 1.396616 & 0.000000 \\ \mathrm{H} & -2.899278 & -0.682916 & 0.000000\end{array}$

$m$-fluorophenol, cis $\mathrm{S}_{1}$ 


$\begin{array}{lrrr}\mathrm{C} & 1.221006 & -0.067249 & -0.692560 \\ \mathrm{C} & -0.067546 & 0.038406 & -0.105612 \\ \mathrm{C} & -0.024381 & 0.042279 & 1.328710 \\ \mathrm{C} & 1.162206 & 0.033008 & 2.099195 \\ \mathrm{C} & 2.433427 & 0.102647 & 1.439945 \\ \mathrm{C} & 2.435518 & -0.034957 & 0.032412 \\ \mathrm{O} & -1.200145 & -0.051340 & 2.027965 \\ \mathrm{~F} & 1.296662 & -0.238641 & -2.044728 \\ \mathrm{H} & 1.081217 & -0.131675 & 3.175325 \\ \mathrm{H} & 3.361666 & 0.179405 & 2.001771 \\ \mathrm{H} & 3.370148 & -0.103568 & -0.529834 \\ \mathrm{H} & -0.903626 & 0.486351 & -0.661770 \\ \mathrm{H} & -1.910792 & -0.254666 & 1.379130\end{array}$

$m$-fluorophenol, trans $\mathrm{S}_{0}$

$\begin{array}{lrrc}\mathrm{C} & 0.270424 & 1.313194 & 0.000000 \\ \mathrm{C} & -0.956250 & 0.633905 & 0.000000 \\ \mathrm{C} & -0.991036 & -0.769758 & 0.000000 \\ \mathrm{C} & 0.207718 & -1.489769 & 0.000000 \\ \mathrm{C} & 1.443844 & -0.836340 & 0.000000 \\ \mathrm{C} & 1.437847 & 0.558394 & 0.000000 \\ \mathrm{O} & -2.092370 & 1.406534 & 0.000000 \\ \mathrm{H} & -1.950073 & -1.295347 & 0.000000 \\ \mathrm{H} & 0.177123 & -2.581071 & 0.000000 \\ \mathrm{H} & 2.388063 & -1.380502 & 0.000000 \\ \mathrm{~F} & 2.629810 & 1.218414 & 0.000000 \\ \mathrm{H} & 0.304931 & 2.402509 & 0.000000 \\ \mathrm{H} & -2.870033 & 0.819846 & 0.000000\end{array}$

$m$-fluorophenol, trans $\mathrm{S}_{1}$

$\begin{array}{lccc}\mathrm{C} & 1.095027 & -0.017330 & 0.000000 \\ \mathrm{C} & 0.328400 & 1.182257 & 0.000000 \\ \mathrm{C} & -1.093381 & 1.224460 & 0.000000 \\ \mathrm{C} & -1.832028 & -0.009727 & 0.000000 \\ \mathrm{C} & -1.089643 & -1.226044 & 0.000000 \\ \mathrm{C} & 0.320265 & -1.211420 & 0.000000 \\ \mathrm{O} & 1.056264 & 2.345737 & 0.000000 \\ \mathrm{H} & -1.607009 & 2.189355 & 0.000000 \\ \mathrm{H} & -2.919176 & -0.018146 & 0.000000 \\ \mathrm{H} & -1.596677 & -2.192503 & 0.000000 \\ \mathrm{~F} & 0.981361 & -2.402096 & 0.000000 \\ \mathrm{H} & 2.181447 & -0.011910 & 0.000000 \\ \mathrm{H} & 0.434001 & 3.101516 & 0.000000\end{array}$


$p$-fluorophenol $\mathrm{S}_{0}$

$\begin{array}{cccc}\mathrm{C} & 0.273901 & 1.325969 & 0.000000 \\ \mathrm{C} & -0.957087 & 0.653338 & 0.000000 \\ \mathrm{C} & -0.987487 & -0.748986 & 0.000000 \\ \mathrm{C} & 0.206098 & -1.479675 & 0.000000 \\ \mathrm{C} & 1.415408 & -0.791666 & 0.000000 \\ \mathrm{C} & 1.467226 & 0.601339 & 0.000000 \\ \mathrm{O} & -2.097887 & 1.425743 & 0.000000 \\ \mathrm{H} & -1.944759 & -1.278679 & 0.000000 \\ \mathrm{H} & 0.199982 & -2.570436 & 0.000000 \\ \mathrm{~F} & 2.583074 & -1.500143 & 0.000000 \\ \mathrm{H} & 2.433577 & 1.107460 & 0.000000 \\ \mathrm{H} & 0.282852 & 2.416857 & 0.000000 \\ \mathrm{H} & -2.874918 & 0.838869 & 0.000000\end{array}$

p-fluorophenol $\mathrm{S}_{1}$

$\begin{array}{lrrr}\mathrm{C} & 1.276428 & 0.686366 & 0.000000 \\ \mathrm{C} & 0.030312 & 1.360117 & 0.000000 \\ \mathrm{C} & -1.239007 & 0.717377 & 0.000000 \\ \mathrm{C} & -1.257867 & -0.713204 & 0.000000 \\ \mathrm{C} & -0.006877 & -1.371265 & 0.000000 \\ \mathrm{C} & 1.258411 & -0.745324 & 0.000000 \\ \mathrm{O} & 0.095692 & 2.731125 & 0.000000 \\ \mathrm{H} & -2.162098 & 1.300796 & 0.000000 \\ \mathrm{H} & -2.179141 & -1.291810 & 0.000000 \\ \mathrm{~F} & -0.027604 & -2.726267 & 0.000000 \\ \mathrm{H} & 2.165598 & -1.345540 & 0.000000 \\ \mathrm{H} & 2.201284 & 1.260071 & 0.000000 \\ \mathrm{H} & -0.818434 & 3.083151 & 0.000000\end{array}$

phenylacetylene $S_{0}$

$\begin{array}{rrrr}\mathrm{C} & 1.216684 & 0.000000 & -0.014491 \\ \mathrm{C} & 0.000000 & 0.000000 & -0.731782 \\ \mathrm{C} & -1.216684 & 0.000000 & -0.014491 \\ \mathrm{C} & -1.211212 & 0.000000 & 1.381094 \\ \mathrm{C} & 0.000000 & 0.000000 & 2.083602 \\ \mathrm{C} & 1.211212 & 0.000000 & 1.381094 \\ \mathrm{C} & 0.000000 & 0.000000 & -2.158837 \\ \mathrm{C} & 0.000000 & 0.000000 & -3.374760 \\ \mathrm{H} & -2.158918 & 0.000000 & -0.565143 \\ \mathrm{H} & -2.158775 & 0.000000 & 1.924222 \\ \mathrm{H} & 0.000000 & 0.000000 & 3.175653\end{array}$




$\begin{array}{lrrr}\mathrm{H} & 2.158775 & 0.000000 & 1.924222 \\ \mathrm{H} & 2.158918 & 0.000000 & -0.565143 \\ \mathrm{H} & 0.000000 & 0.000000 & -4.445257\end{array}$

phenylacetylene $\mathrm{S}_{1}$

$\begin{array}{rrrr}\mathrm{C} & 1.247661 & 0.000000 & -0.036145 \\ \mathrm{C} & 0.000000 & 0.000000 & -0.750713 \\ \mathrm{C} & -1.247661 & 0.000000 & -0.036145 \\ \mathrm{C} & -1.232432 & 0.000000 & 1.401107 \\ \mathrm{C} & 0.000000 & 0.000000 & 2.089744 \\ \mathrm{C} & 1.232432 & 0.000000 & 1.401107 \\ \mathrm{C} & 0.000000 & 0.000000 & -2.143479 \\ \mathrm{C} & 0.000000 & 0.000000 & -3.379952 \\ \mathrm{H} & -2.184741 & 0.000000 & -0.591169 \\ \mathrm{H} & -2.172532 & 0.000000 & 1.952725 \\ \mathrm{H} & 0.000000 & 0.000000 & 3.182199 \\ \mathrm{H} & 2.172532 & 0.000000 & 1.952725 \\ \mathrm{H} & 2.184741 & 0.000000 & -0.591169 \\ \mathrm{H} & 0.000000 & 0.000000 & -4.450836\end{array}$

aniline $\mathrm{S}_{0}$

$\begin{array}{rrrr}\mathrm{C} & 0.928070 & 0.025211 & 0.000000 \\ \mathrm{C} & 0.206799 & 0.033620 & 1.211415 \\ \mathrm{C} & -1.189124 & 0.043569 & 1.205438 \\ \mathrm{C} & -1.901204 & 0.049527 & 0.000000 \\ \mathrm{C} & -1.189124 & 0.043569 & -1.205438 \\ \mathrm{C} & 0.206799 & 0.033620 & -1.211415 \\ \mathrm{H} & 0.749662 & 0.033301 & 2.161019 \\ \mathrm{H} & -2.992438 & 0.056486 & 0.000000 \\ \mathrm{H} & -1.725845 & 0.046876 & 2.157210 \\ \mathrm{H} & -1.725845 & 0.046876 & -2.157210 \\ \mathrm{H} & 0.749662 & 0.033301 & -2.161019 \\ \mathrm{~N} & 2.325992 & 0.079001 & 0.000000 \\ \mathrm{H} & 2.778304 & -0.262474 & -0.844037 \\ \mathrm{H} & 2.778304 & -0.262474 & 0.844037\end{array}$

aniline $\mathrm{S}_{1}$
$\begin{array}{llll}\text { C } & 0.878818 & -0.033600 & 0.000000\end{array}$
$\begin{array}{llll}\text { C } & 0.229327 & -0.008781 & 1.248675\end{array}$
$\begin{array}{llll}\text { C } & -1.214493 & 0.046412 & 1.240085\end{array}$
$\begin{array}{llll}\text { C } & -1.882284 & 0.071950 & 0.000000\end{array}$
$\begin{array}{llll}\text { C } & -1.214493 & 0.046412 & -1.240085\end{array}$
$\begin{array}{llll}\text { C } & 0.229327 & -0.008781 & -1.248675\end{array}$ 


$\begin{array}{rrrr}\mathrm{H} & 0.801662 & -0.030720 & 2.178027 \\ \mathrm{H} & -2.976002 & 0.113760 & 0.000000 \\ \mathrm{H} & -1.775135 & 0.067822 & 2.173794 \\ \mathrm{H} & -1.775135 & 0.067822 & -2.173794 \\ \mathrm{H} & 0.801662 & -0.030720 & -2.178027 \\ \mathrm{~N} & 2.285115 & -0.087302 & 0.000000 \\ \mathrm{H} & 2.805810 & -0.107147 & -0.873379 \\ \mathrm{H} & 2.805810 & -0.107147 & 0.873379\end{array}$

catechol $\mathrm{S}_{0}$

$\begin{array}{rrrr}\mathrm{C} & 0.472952 & 1.286986 & 0.000000 \\ \mathrm{C} & -0.753381 & 0.623139 & 0.000000 \\ \mathrm{C} & -0.804125 & -0.785965 & 0.000000 \\ \mathrm{C} & 0.386742 & -1.514733 & 0.000000 \\ \mathrm{C} & 1.618180 & -0.848052 & 0.000000 \\ \mathrm{C} & 1.665149 & 0.549185 & 0.000000 \\ \mathrm{O} & -1.990964 & 1.249117 & 0.000000 \\ \mathrm{O} & -2.010148 & -1.438882 & 0.000000 \\ \mathrm{H} & 0.329997 & -2.604718 & 0.000000 \\ \mathrm{H} & 2.542956 & -1.427770 & 0.000000 \\ \mathrm{H} & 2.622982 & 1.071922 & 0.000000 \\ \mathrm{H} & 0.497496 & 2.381240 & 0.000000 \\ \mathrm{H} & -1.868460 & 2.214081 & 0.000000 \\ \mathrm{H} & -2.709376 & -0.755549 & 0.000000\end{array}$

catechol $\mathrm{S}_{1}$

$\begin{array}{rrrr}\mathrm{C} & 0.477478 & 1.354865 & 0.000000 \\ \mathrm{C} & -0.767106 & 0.626268 & 0.000000 \\ \mathrm{C} & -0.807272 & -0.787276 & 0.000000 \\ \mathrm{C} & 0.390878 & -1.581922 & 0.000000 \\ \mathrm{C} & 1.610607 & -0.861179 & 0.000000 \\ \mathrm{C} & 1.650704 & 0.559774 & 0.000000 \\ \mathrm{O} & -1.993046 & 1.263667 & 0.000000 \\ \mathrm{O} & -2.007031 & -1.448750 & 0.000000 \\ \mathrm{H} & 0.329644 & -2.667107 & 0.000000 \\ \mathrm{H} & 2.556115 & -1.406337 & 0.000000 \\ \mathrm{H} & 2.625408 & 1.052143 & 0.000000 \\ \mathrm{H} & 0.502433 & 2.444264 & 0.000000 \\ \mathrm{H} & -1.847954 & 2.227342 & 0.000000 \\ \mathrm{H} & -2.720858 & -0.775752 & 0.000000\end{array}$

resorcinol, isomer $1 \mathrm{~S}_{0}$

$\begin{array}{llll}\text { C } & 0.076388 & 1.155736 & 0.000000\end{array}$ 


$\begin{array}{rrrr}\mathrm{C} & -1.145853 & 0.470836 & 0.000000 \\ \mathrm{C} & -1.176887 & -0.930850 & 0.000000 \\ \mathrm{C} & 0.032265 & -1.636445 & 0.000000 \\ \mathrm{C} & 1.259915 & -0.974591 & 0.000000 \\ \mathrm{C} & 1.272984 & 0.429184 & 0.000000 \\ \mathrm{O} & -2.287213 & 1.240800 & 0.000000 \\ \mathrm{H} & -2.132088 & -1.463126 & 0.000000 \\ \mathrm{H} & 0.012882 & -2.728360 & 0.000000 \\ \mathrm{H} & 2.204613 & -1.518768 & 0.000000 \\ \mathrm{O} & 2.502055 & 1.047039 & 0.000000 \\ \mathrm{H} & 0.072712 & 2.248969 & 0.000000 \\ \mathrm{H} & -3.059777 & 0.647684 & 0.000000 \\ \mathrm{H} & 2.368005 & 2.011882 & 0.000000\end{array}$

resorcinol, isomer $1 \mathrm{~S}_{1}$

$\begin{array}{rrrc}\mathrm{C} & 0.003164 & 1.081988 & 0.041215 \\ \mathrm{C} & -1.194667 & 0.310641 & -0.014303 \\ \mathrm{C} & -1.208776 & -1.109594 & -0.037623 \\ \mathrm{C} & 0.001273 & -1.855450 & 0.073625 \\ \mathrm{C} & 1.217710 & -1.114928 & 0.023946 \\ \mathrm{C} & 1.201886 & 0.303445 & 0.042839 \\ \mathrm{O} & -2.366808 & 1.027631 & -0.062339 \\ \mathrm{H} & -2.165887 & -1.627652 & -0.159857 \\ \mathrm{H} & -0.002390 & -2.942586 & 0.083478 \\ \mathrm{H} & 2.183399 & -1.615872 & -0.067635 \\ \mathrm{O} & 2.423316 & 0.929196 & -0.021390 \\ \mathrm{H} & -0.025678 & 2.105644 & 0.430531 \\ \mathrm{H} & -3.116598 & 0.401966 & -0.030009 \\ \mathrm{H} & 2.261050 & 1.861691 & -0.282826\end{array}$

resorcinol, isomer $2 \mathrm{~S}_{0}$

$\begin{array}{crcc}\mathrm{C} & 1.206694 & 0.000000 & -0.551383 \\ \mathrm{C} & 0.000000 & 0.000000 & -1.258141 \\ \mathrm{C} & -1.206694 & 0.000000 & -0.551383 \\ \mathrm{C} & -1.216761 & 0.000000 & 0.853683 \\ \mathrm{C} & 0.000000 & 0.000000 & 1.540460 \\ \mathrm{C} & 1.216761 & 0.000000 & 0.853683 \\ \mathrm{O} & -2.363168 & 0.000000 & -1.297186 \\ \mathrm{O} & 2.363168 & 0.000000 & -1.297186 \\ \mathrm{H} & -2.163656 & 0.000000 & 1.400580 \\ \mathrm{H} & 0.000000 & 0.000000 & 2.632513 \\ \mathrm{H} & 2.163656 & 0.000000 & 1.400580 \\ \mathrm{H} & 0.000000 & 0.000000 & -2.348317 \\ \mathrm{H} & -3.124012 & 0.000000 & -0.689191\end{array}$




\section{H $\quad 3.124012 \quad 0.000000 \quad-0.689191$}

resorcinol, isomer $2 \mathrm{~S}_{1}$

$\begin{array}{rrrr}\mathrm{C} & 1.203985 & 0.000000 & -0.331008 \\ \mathrm{C} & 0.000000 & 0.000000 & -1.092165 \\ \mathrm{C} & -1.203985 & 0.000000 & -0.331008 \\ \mathrm{C} & -1.221365 & 0.000000 & 1.089832 \\ \mathrm{C} & 0.000000 & 0.000000 & 1.834686 \\ \mathrm{C} & 1.221365 & 0.000000 & 1.089832 \\ \mathrm{O} & -2.374490 & 0.000000 & -1.053833 \\ \mathrm{O} & 2.374490 & 0.000000 & -1.053833 \\ \mathrm{H} & -2.183288 & 0.000000 & 1.611562 \\ \mathrm{H} & 0.000000 & 0.000000 & 2.921840 \\ \mathrm{H} & 2.183288 & 0.000000 & 1.611562 \\ \mathrm{H} & 0.000000 & 0.000000 & -2.178541 \\ \mathrm{H} & -3.125032 & 0.000000 & -0.429005 \\ \mathrm{H} & 3.125032 & 0.000000 & -0.429005\end{array}$

resorcinol, isomer $3 \mathrm{~S}_{0}$

$\begin{array}{rrrr}\mathrm{C} & 1.212768 & 0.000000 & -0.291046 \\ \mathrm{C} & 0.000000 & 0.000000 & -0.996783 \\ \mathrm{C} & -1.212768 & 0.000000 & -0.291046 \\ \mathrm{C} & -1.220016 & 0.000000 & 1.109784 \\ \mathrm{C} & 0.000000 & 0.000000 & 1.790771 \\ \mathrm{C} & 1.220016 & 0.000000 & 1.109784 \\ \mathrm{O} & -2.431779 & 0.000000 & -0.930590 \\ \mathrm{O} & 2.431779 & 0.000000 & -0.930590 \\ \mathrm{H} & -2.172978 & 0.000000 & 1.639424 \\ \mathrm{H} & 0.000000 & 0.000000 & 2.882889 \\ \mathrm{H} & 2.172978 & 0.000000 & 1.639424 \\ \mathrm{H} & 0.000000 & 0.000000 & -2.092995 \\ \mathrm{H} & 2.284851 & 0.000000 & -1.893275 \\ \mathrm{H} & -2.284851 & 0.000000 & -1.893275\end{array}$

resorcinol, isomer $3 \mathrm{~S}_{1}$
$\begin{array}{llll}\text { C } & -0.035949 & -0.302229 & 1.191467\end{array}$
$\begin{array}{llll}\text { C } & -0.145130 & -1.085589 & 0.000000\end{array}$
$\begin{array}{llll}\text { C } & -0.035949 & -0.302229 & -1.191467\end{array}$
$\begin{array}{llll}\text { C } & 0.013837 & 1.117548 & -1.208182\end{array}$
$\begin{array}{llll}\text { C } & -0.053878 & 1.857697 & 0.000000\end{array}$
$\begin{array}{llll}\text { C } & 0.013837 & 1.117548 & 1.208182\end{array}$
$\begin{array}{llll}\mathrm{O} & 0.090115 & -0.926566 & -2.408007\end{array}$
$\begin{array}{llll}\mathrm{O} & 0.090115 & -0.926566 & 2.408007\end{array}$ 


$\begin{array}{rrrr}\mathrm{H} & 0.141429 & 1.612652 & -2.173456 \\ \mathrm{H} & -0.079779 & 2.945363 & 0.000000 \\ \mathrm{H} & 0.141429 & 1.612652 & 2.173456 \\ \mathrm{H} & -0.745853 & -2.015938 & 0.000000 \\ \mathrm{H} & 0.280162 & -1.873074 & 2.228345 \\ \mathrm{H} & 0.280162 & -1.873074 & -2.228345\end{array}$

hydroquinone, cis $\mathrm{S}_{0}$

$\begin{array}{lrrr}\mathrm{C} & -0.700473 & 0.000000 & 1.098584 \\ \mathrm{C} & 0.700473 & 0.000000 & 1.098584 \\ \mathrm{C} & 1.405113 & 0.000000 & -0.110980 \\ \mathrm{C} & 0.696785 & 0.000000 & -1.321985 \\ \mathrm{C} & -0.696785 & 0.000000 & -1.321985 \\ \mathrm{C} & -1.405113 & 0.000000 & -0.110980 \\ \mathrm{O} & 2.784137 & 0.000000 & -0.180499 \\ \mathrm{O} & -2.784137 & 0.000000 & -0.180499 \\ \mathrm{H} & 1.253258 & 0.000000 & -2.260754 \\ \mathrm{H} & -1.253258 & 0.000000 & -2.260754 \\ \mathrm{H} & -1.239283 & 0.000000 & 2.051095 \\ \mathrm{H} & 1.239283 & 0.000000 & 2.051095 \\ \mathrm{H} & -3.141561 & 0.000000 & 0.724828 \\ \mathrm{H} & 3.141561 & 0.000000 & 0.724828\end{array}$

hydroquinone, cis $\mathrm{S}_{1}$

$\begin{array}{lrrr}\mathrm{C} & 0.714308 & 0.000000 & -1.134790 \\ \mathrm{C} & -0.714308 & 0.000000 & -1.134790 \\ \mathrm{C} & -1.376979 & 0.000000 & 0.122181 \\ \mathrm{C} & -0.714438 & 0.000000 & 1.371619 \\ \mathrm{C} & 0.714438 & 0.000000 & 1.371619 \\ \mathrm{C} & 1.376979 & 0.000000 & 0.122181 \\ \mathrm{O} & -2.747440 & 0.000000 & 0.167851 \\ \mathrm{O} & 2.747440 & 0.000000 & 0.167851 \\ \mathrm{H} & -1.297964 & 0.000000 & 2.290345 \\ \mathrm{H} & 1.297964 & 0.000000 & 2.290345 \\ \mathrm{H} & 1.285138 & 0.000000 & -2.065791 \\ \mathrm{H} & -1.285138 & 0.000000 & -2.065791 \\ \mathrm{H} & 3.083959 & 0.000000 & -0.750644 \\ \mathrm{H} & -3.083959 & 0.000000 & -0.750644\end{array}$

hydroquinone, trans $\mathrm{S}_{0}$
C 0.022536
$\begin{array}{ll}1.399812 & 0.000000\end{array}$
$\begin{array}{llll}\text { C } & -1.203917 & 0.724564 & 0.000000\end{array}$
$\begin{array}{llll}\text { C } & -1.218799 & -0.678010 & 0.000000\end{array}$ 


$\begin{array}{crrr}\mathrm{C} & -0.022536 & -1.399812 & 0.000000 \\ \mathrm{C} & 1.203917 & -0.724564 & 0.000000 \\ \mathrm{C} & 1.218799 & 0.678010 & 0.000000 \\ \mathrm{O} & -2.353677 & 1.489219 & 0.000000 \\ \mathrm{H} & -2.171758 & -1.216326 & 0.000000 \\ \mathrm{H} & -0.029968 & -2.491016 & 0.000000 \\ \mathrm{O} & 2.353677 & -1.489219 & 0.000000 \\ \mathrm{H} & 2.171758 & 1.216326 & 0.000000 \\ \mathrm{H} & 0.029968 & 2.491016 & 0.000000 \\ \mathrm{H} & -3.123006 & 0.892997 & 0.000000 \\ \mathrm{H} & 3.123006 & -0.892997 & 0.000000\end{array}$

hydroquinone, trans $\mathrm{S}_{1}$

$\begin{array}{lrrr}\mathrm{C} & 1.271591 & 0.683445 & 0.000000 \\ \mathrm{C} & 0.037850 & 1.376373 & 0.000000 \\ \mathrm{C} & -1.234094 & 0.744713 & 0.000000 \\ \mathrm{C} & -1.271591 & -0.683445 & 0.000000 \\ \mathrm{C} & -0.037850 & -1.376373 & 0.000000 \\ \mathrm{C} & 1.234094 & -0.744713 & 0.000000 \\ \mathrm{O} & 0.122646 & 2.744789 & 0.000000 \\ \mathrm{H} & -2.150696 & 1.338357 & 0.000000 \\ \mathrm{H} & -2.204212 & -1.244732 & 0.000000 \\ \mathrm{O} & -0.122646 & -2.744789 & 0.000000 \\ \mathrm{H} & 2.150696 & -1.338357 & 0.000000 \\ \mathrm{H} & 2.204212 & 1.244732 & 0.000000 \\ \mathrm{H} & -0.786419 & 3.106532 & 0.000000 \\ \mathrm{H} & 0.786419 & -3.106532 & 0.000000\end{array}$

salicylic acid $\mathrm{S}_{0}$

$\begin{array}{rrrr}\mathrm{C} & 1.110815 & 1.607987 & 0.000000 \\ \mathrm{C} & 1.139976 & 0.200295 & 0.000000 \\ \mathrm{C} & -0.091951 & -0.520069 & 0.000000 \\ \mathrm{C} & -1.312503 & 0.191433 & 0.000000 \\ \mathrm{C} & -1.325545 & 1.579430 & 0.000000 \\ \mathrm{C} & -0.104995 & 2.281456 & 0.000000 \\ \mathrm{O} & 2.339350 & -0.413767 & 0.000000 \\ \mathrm{C} & -0.040690 & -1.981872 & 0.000000 \\ \mathrm{O} & -1.250606 & -2.602696 & 0.000000 \\ \mathrm{O} & 1.009351 & -2.643953 & 0.000000 \\ \mathrm{H} & -2.245144 & -0.373926 & 0.000000 \\ \mathrm{H} & 2.061086 & 2.144001 & 0.000000 \\ \mathrm{H} & -2.272378 & 2.121176 & 0.000000 \\ \mathrm{H} & -0.108845 & 3.373968 & 0.000000 \\ \mathrm{H} & 2.149897 & -1.399836 & 0.000000\end{array}$




\begin{tabular}{lrrr}
$\mathrm{H}$ & -1.057816 & -3.563628 & 0.000000 \\
\multicolumn{4}{c}{ salicylic acid $\mathrm{S}_{1}$} \\
& & \\
$\mathrm{C}$ & 0.996462 & 1.918583 & 0.000000 \\
$\mathrm{C}$ & 0.962728 & 0.506598 & 0.000000 \\
$\mathrm{C}$ & -0.286253 & -0.205877 & 0.000000 \\
$\mathrm{C}$ & -1.444381 & 0.551644 & 0.000000 \\
$\mathrm{C}$ & -1.413418 & 2.004888 & 0.000000 \\
$\mathrm{C}$ & -0.212707 & 2.678118 & 0.000000 \\
$\mathrm{O}$ & 2.098171 & -0.190389 & 0.000000 \\
$\mathrm{C}$ & -0.227959 & -1.674748 & 0.000000 \\
$\mathrm{O}$ & -1.427748 & -2.337899 & 0.000000 \\
$\mathrm{O}$ & 0.888465 & -2.328070 & 0.000000 \\
$\mathrm{H}$ & -2.411583 & 0.045365 & 0.000000 \\
$\mathrm{H}$ & 1.973770 & 2.404161 & 0.000000 \\
$\mathrm{H}$ & -2.361149 & 2.545619 & 0.000000 \\
$\mathrm{H}$ & -0.172570 & 3.767671 & 0.000000 \\
$\mathrm{H}$ & 1.751458 & -1.259112 & 0.000000 \\
$\mathrm{H}$ & -1.202707 & -3.289604 & 0.000000
\end{tabular}

$o$-cresol, cis $\mathrm{S}_{0}$

$\begin{array}{rrrr}\mathrm{C} & 1.975343 & 0.515940 & 0.000000 \\ \mathrm{C} & 0.733206 & 1.157665 & 0.000000 \\ \mathrm{C} & -0.469713 & 0.435828 & 0.000000 \\ \mathrm{C} & -0.392889 & -0.973500 & 0.000000 \\ \mathrm{C} & 0.845267 & -1.628252 & 0.000000 \\ \mathrm{C} & 2.025454 & -0.882801 & 0.000000 \\ \mathrm{C} & -1.811133 & 1.129704 & 0.000000 \\ \mathrm{O} & -1.520076 & -1.767439 & 0.000000 \\ \mathrm{H} & 0.861487 & -2.719647 & 0.000000 \\ \mathrm{H} & 2.987676 & -1.399462 & 0.000000 \\ \mathrm{H} & 2.895451 & 1.103082 & 0.000000 \\ \mathrm{H} & 0.686991 & 2.250381 & 0.000000 \\ \mathrm{H} & -2.307989 & -1.194395 & 0.000000 \\ \mathrm{H} & -2.412530 & 0.876132 & 0.891632 \\ \mathrm{H} & -1.684014 & 2.220641 & 0.000000 \\ \mathrm{H} & -2.412530 & 0.876132 & -0.891632\end{array}$

$o$-cresol, cis $\mathrm{S}_{1}$
$\begin{array}{llll}\text { C } & 1.862687 & -0.024492 & -0.784016\end{array}$
$\begin{array}{llll}\text { C } & 0.627567 & 0.154466 & -1.457803\end{array}$
$\begin{array}{llll}\text { C } & -0.605671 & 0.120107 & -0.710837\end{array}$
$\begin{array}{llll}\text { C } & -0.468708 & 0.076565 & 0.716150\end{array}$ 


$\begin{array}{rrrc}\mathrm{C} & 0.772288 & -0.014391 & 1.405974 \\ \mathrm{C} & 1.956278 & -0.115440 & 0.624096 \\ \mathrm{C} & -1.896980 & -0.099905 & -1.430928 \\ \mathrm{O} & -1.575445 & 0.104681 & 1.521541 \\ \mathrm{H} & 0.787303 & 0.098013 & 2.489871 \\ \mathrm{H} & 2.928264 & -0.235857 & 1.102469 \\ \mathrm{H} & 2.777756 & -0.099241 & -1.378078 \\ \mathrm{H} & 0.599736 & 0.237489 & -2.544681 \\ \mathrm{H} & -2.360265 & 0.276184 & 0.951974 \\ \mathrm{H} & -2.780822 & -0.202048 & -0.775687 \\ \mathrm{H} & -2.118054 & 0.726616 & -2.132402 \\ \mathrm{H} & -1.856514 & -1.022238 & -2.055234\end{array}$

o-cresol, trans $\mathrm{S}_{0}$

$\begin{array}{rrrr}\mathrm{C} & 2.425523 & 0.000022 & 0.029247 \\ \mathrm{C} & 1.244809 & -0.000031 & -0.724862 \\ \mathrm{C} & 0.021988 & -0.000002 & -0.021561 \\ \mathrm{C} & -0.007166 & 0.000061 & 1.378894 \\ \mathrm{C} & 1.188590 & 0.000113 & 2.104556 \\ \mathrm{C} & 2.411814 & 0.000088 & 1.429769 \\ \mathrm{C} & 1.253404 & -0.000114 & -2.231584 \\ \mathrm{O} & -1.130378 & -0.000052 & -0.781061 \\ \mathrm{H} & -0.969324 & 0.000086 & 1.901786 \\ \mathrm{H} & 1.157215 & 0.000158 & 3.196146 \\ \mathrm{H} & 3.350418 & 0.000136 & 1.986979 \\ \mathrm{H} & 3.381424 & 0.000003 & -0.502025 \\ \mathrm{H} & -1.897272 & -0.000048 & -0.180581 \\ \mathrm{H} & 2.283267 & -0.000072 & -2.614672 \\ \mathrm{H} & 0.732255 & -0.882631 & -2.635478 \\ \mathrm{H} & 0.732139 & 0.882282 & -2.635583\end{array}$

$o$-cresol, trans $\mathrm{S}_{1}$

$\begin{array}{lrrr}\mathrm{C} & 2.427985 & 0.281573 & -0.002517 \\ \mathrm{C} & 1.206011 & 0.139252 & -0.763407 \\ \mathrm{C} & 0.005188 & -0.000124 & -0.015160 \\ \mathrm{C} & -0.058852 & -0.105939 & 1.415168 \\ \mathrm{C} & 1.181125 & -0.093200 & 2.111301 \\ \mathrm{C} & 2.391971 & 0.091191 & 1.395386 \\ \mathrm{C} & 1.255960 & -0.026309 & -2.244764 \\ \mathrm{O} & -1.163568 & -0.102310 & -0.725951 \\ \mathrm{H} & -1.005375 & 0.076933 & 1.934831 \\ \mathrm{H} & 1.203911 & -0.183076 & 3.198095 \\ \mathrm{H} & 3.330905 & 0.099945 & 1.956097 \\ \mathrm{H} & 3.372240 & 0.447446 & -0.521106\end{array}$




$\begin{array}{rrrr}\mathrm{H} & -1.853916 & -0.418602 & -0.100366 \\ \mathrm{H} & 1.838398 & -0.928442 & -2.529872 \\ \mathrm{H} & 0.259377 & -0.103650 & -2.696382 \\ \mathrm{H} & 1.787331 & 0.825312 & -2.711373\end{array}$

$m$-cresol, cis $\mathrm{S}_{0}$

$\begin{array}{lrrr}\mathrm{C} & 1.159709 & -0.005281 & 2.099258 \\ \mathrm{C} & 2.378039 & 0.008493 & 1.420544 \\ \mathrm{C} & 2.422807 & 0.019291 & 0.020407 \\ \mathrm{C} & 1.235711 & 0.014417 & -0.726935 \\ \mathrm{C} & 0.008981 & 0.002515 & -0.041973 \\ \mathrm{C} & -0.030912 & -0.007876 & 1.358350 \\ \mathrm{C} & 1.263137 & 0.002010 & -2.237924 \\ \mathrm{O} & -1.213939 & -0.018132 & 2.064373 \\ \mathrm{H} & 1.110476 & -0.011093 & 3.189146 \\ \mathrm{H} & 3.308757 & 0.013946 & 1.992509 \\ \mathrm{H} & 3.385736 & 0.032730 & -0.495435 \\ \mathrm{H} & -0.928456 & 0.002710 & -0.610032 \\ \mathrm{H} & -1.954276 & -0.018335 & 1.431365 \\ \mathrm{H} & 2.266718 & 0.237237 & -2.619281 \\ \mathrm{H} & 0.981076 & -0.987949 & -2.633167 \\ \mathrm{H} & 0.558737 & 0.734687 & -2.661256\end{array}$

$m$-cresol, cis $\mathrm{S}_{1}$

$\begin{array}{rrrr}\mathrm{C} & 1.162168 & 0.013115 & 2.141002 \\ \mathrm{C} & 2.412701 & 0.087388 & 1.451483 \\ \mathrm{C} & 2.412231 & 0.043524 & 0.037249 \\ \mathrm{C} & 1.223309 & -0.010646 & -0.751226 \\ \mathrm{C} & -0.040941 & 0.013525 & -0.067807 \\ \mathrm{C} & -0.017019 & 0.009399 & 1.360071 \\ \mathrm{C} & 1.266313 & -0.047885 & -2.242973 \\ \mathrm{O} & -1.201833 & -0.042066 & 2.050118 \\ \mathrm{H} & 1.092675 & -0.143679 & 3.217762 \\ \mathrm{H} & 3.351169 & 0.125946 & 2.002725 \\ \mathrm{H} & 3.371418 & 0.067135 & -0.489188 \\ \mathrm{H} & -0.956737 & 0.297196 & -0.600787 \\ \mathrm{H} & -1.915277 & -0.217661 & 1.398167 \\ \mathrm{H} & 2.293353 & 0.038936 & -2.625263 \\ \mathrm{H} & 0.833727 & -0.986925 & -2.644328 \\ \mathrm{H} & 0.665023 & 0.772069 & -2.687064\end{array}$

$m$-cresol, trans $\mathrm{S}_{0}$

$\begin{array}{llll}\text { C } & -1.196757 & -1.102431 & 0.000000\end{array}$ 


$\begin{array}{rrrr}\mathrm{C} & 0.203770 & -1.215989 & 0.000000 \\ \mathrm{C} & 0.978794 & -0.046946 & 0.000000 \\ \mathrm{C} & 0.368496 & 1.212763 & 0.000000 \\ \mathrm{C} & -1.030623 & 1.321015 & 0.000000 \\ \mathrm{C} & -1.803914 & 0.157477 & 0.000000 \\ \mathrm{C} & 0.875773 & -2.570029 & 0.000000 \\ \mathrm{O} & 1.196931 & 2.314087 & 0.000000 \\ \mathrm{H} & -1.508134 & 2.305735 & 0.000000 \\ \mathrm{H} & -2.893359 & 0.237535 & 0.000000 \\ \mathrm{H} & -1.812636 & -2.004692 & 0.000000 \\ \mathrm{H} & 2.070184 & -0.099053 & 0.000000 \\ \mathrm{H} & 0.645793 & 3.117042 & 0.000000 \\ \mathrm{H} & 0.135757 & -3.382433 & 0.000000 \\ \mathrm{H} & 1.517506 & -2.697779 & -0.886316 \\ \mathrm{H} & 1.517506 & -2.697779 & 0.886316\end{array}$

$m$-cresol, trans $\mathrm{S}_{1}$

$\begin{array}{rrrr}\mathrm{C} & -1.207069 & -1.059183 & 0.000000 \\ \mathrm{C} & 0.205306 & -1.245611 & 0.000000 \\ \mathrm{C} & 1.035124 & -0.068629 & 0.000000 \\ \mathrm{C} & 0.395963 & 1.198264 & 0.000000 \\ \mathrm{C} & -1.013965 & 1.386954 & 0.000000 \\ \mathrm{C} & -1.833440 & 0.215339 & 0.000000 \\ \mathrm{C} & 0.827644 & -2.602530 & 0.000000 \\ \mathrm{O} & 1.232758 & 2.287297 & 0.000000 \\ \mathrm{H} & -1.441076 & 2.392721 & 0.000000 \\ \mathrm{H} & -2.919690 & 0.294592 & 0.000000 \\ \mathrm{H} & -1.842105 & -1.950052 & 0.000000 \\ \mathrm{H} & 2.123473 & -0.122140 & 0.000000 \\ \mathrm{H} & 0.682498 & 3.097475 & 0.000000 \\ \mathrm{H} & 0.068803 & -3.398208 & 0.000000 \\ \mathrm{H} & 1.481128 & -2.755636 & -0.883431 \\ \mathrm{H} & 1.481128 & -2.755636 & 0.883431\end{array}$

$p$-cresol $\mathrm{S}_{0}$
$\begin{array}{llll}\text { C } & 0.712530 & 0.008458 & -1.197831\end{array}$
$\begin{array}{llll}\text { C } & -0.682364 & 0.010982 & -1.207207\end{array}$
$\begin{array}{llll}\text { C } & -1.385613 & 0.000723 & 0.005496\end{array}$
$\begin{array}{llll}\text { C } & -0.678518 & -0.014765 & 1.214514\end{array}$
$\begin{array}{llll}\text { C } & 0.720881 & -0.017126 & 1.203734\end{array}$
$\begin{array}{llll}\text { C } & 1.445277 & -0.003676 & 0.003072\end{array}$
$\begin{array}{llll}\mathrm{O} & -2.762808 & 0.001193 & -0.057291\end{array}$
$\begin{array}{llll}\text { C } & 2.956387 & 0.021973 & -0.004336\end{array}$
H $\quad-1.218516 \quad-0.028629 \quad 2.166757$ 


$\begin{array}{rrrr}\mathrm{H} & 1.257666 & -0.032079 & 2.156318 \\ \mathrm{H} & 1.246936 & 0.013828 & -2.152063 \\ \mathrm{H} & -1.241474 & 0.017366 & -2.144585 \\ \mathrm{H} & -3.114866 & -0.008452 & 0.850655 \\ \mathrm{H} & 3.368406 & -0.657140 & -0.766560 \\ \mathrm{H} & 3.341552 & 1.031514 & -0.226210 \\ \mathrm{H} & 3.366083 & -0.278794 & 0.970838\end{array}$

$p$-cresol $\mathrm{S}_{1}$

$\begin{array}{cccc}\mathrm{C} & 0.735912 & -0.095589 & -1.241099 \\ \mathrm{C} & -0.693564 & -0.040969 & -1.252064 \\ \mathrm{C} & -1.358902 & -0.017108 & -0.003740 \\ \mathrm{C} & -0.695530 & -0.002134 & 1.256124 \\ \mathrm{C} & 0.732813 & -0.004752 & 1.242780 \\ \mathrm{C} & 1.434511 & -0.032916 & 0.001413 \\ \mathrm{O} & -2.729791 & 0.013177 & -0.049574 \\ \mathrm{C} & 2.926815 & 0.042856 & -0.000237 \\ \mathrm{H} & -1.260248 & -0.138962 & 2.181982 \\ \mathrm{H} & 1.295096 & 0.008083 & 2.177153 \\ \mathrm{H} & 1.296936 & -0.125755 & -2.175313 \\ \mathrm{H} & -1.270862 & 0.069256 & -2.169744 \\ \mathrm{H} & -3.058880 & 0.147408 & 0.864137 \\ \mathrm{H} & 3.356509 & -0.507125 & -0.853040 \\ \mathrm{H} & 3.263570 & 1.094080 & -0.099754 \\ \mathrm{H} & 3.357165 & -0.344170 & 0.936267\end{array}$

o-methoxyphenol $\mathrm{S}_{0}$

$\begin{array}{lrrr}\mathrm{C} & 2.043074 & -0.089256 & 0.000000 \\ \mathrm{C} & 0.881933 & 0.701181 & 0.000000 \\ \mathrm{C} & -0.370325 & 0.084892 & 0.000000 \\ \mathrm{C} & -0.471717 & -1.326321 & 0.000000 \\ \mathrm{C} & 0.686731 & -2.101612 & 0.000000 \\ \mathrm{C} & 1.943955 & -1.481703 & 0.000000 \\ \mathrm{O} & -1.596141 & 0.724625 & 0.000000 \\ \mathrm{O} & -1.704445 & -1.925565 & 0.000000 \\ \mathrm{H} & 0.587431 & -3.188571 & 0.000000 \\ \mathrm{H} & 2.845933 & -2.096494 & 0.000000 \\ \mathrm{H} & 3.020782 & 0.395705 & 0.000000 \\ \mathrm{H} & 0.964042 & 1.788873 & 0.000000 \\ \mathrm{C} & -1.600320 & 2.154667 & 0.000000 \\ \mathrm{H} & -2.365428 & -1.203533 & 0.000000 \\ \mathrm{H} & -2.655381 & 2.452805 & 0.000000 \\ \mathrm{H} & -1.105062 & 2.555152 & 0.900847 \\ \mathrm{H} & -1.105062 & 2.555152 & -0.900847\end{array}$




\begin{tabular}{lrrr}
\multicolumn{5}{c}{$o$-methoxyphenol $\mathrm{S}_{1}$} \\
$\mathrm{C}$ & 2.486573 & 0.008773 & 0.043460 \\
$\mathrm{C}$ & 1.385618 & -0.396760 & -0.745577 \\
$\mathrm{C}$ & 0.108827 & -0.388116 & -0.084668 \\
$\mathrm{C}$ & 0.015096 & -0.165226 & 1.318233 \\
$\mathrm{C}$ & 1.176184 & 0.026249 & 2.132635 \\
$\mathrm{C}$ & 2.387156 & 0.219904 & 1.440790 \\
$\mathrm{O}$ & -1.101448 & -0.276832 & -0.749564 \\
$\mathrm{O}$ & -1.204987 & 0.088519 & 1.878620 \\
$\mathrm{H}$ & 1.116361 & -0.056670 & 3.219236 \\
$\mathrm{H}$ & 3.285413 & 0.514960 & 1.990873 \\
$\mathrm{H}$ & 3.463679 & 0.140028 & -0.433891 \\
$\mathrm{H}$ & 1.535105 & -0.860005 & -1.725401 \\
$\mathrm{C}$ & -1.092923 & 0.203129 & -2.130170 \\
$\mathrm{H}$ & -1.871305 & -0.002923 & 1.153016 \\
$\mathrm{H}$ & -2.148845 & 0.235695 & -2.421013 \\
$\mathrm{H}$ & -0.639718 & 1.203289 & -2.191671 \\
$\mathrm{H}$ & -0.542226 & -0.494021 & -2.776829
\end{tabular}

$m$-methoxyphenol, isomer $1 \mathrm{~S}_{0}$

$\begin{array}{lrrc}\mathrm{C} & 0.935618 & 1.457561 & 0.000000 \\ \mathrm{C} & -0.262351 & 0.723164 & 0.000000 \\ \mathrm{C} & -0.232040 & -0.680477 & 0.000000 \\ \mathrm{C} & 1.008068 & -1.342475 & 0.000000 \\ \mathrm{C} & 2.206725 & -0.622422 & 0.000000 \\ \mathrm{C} & 2.151603 & 0.775643 & 0.000000 \\ \mathrm{H} & -1.154794 & -1.264864 & 0.000000 \\ \mathrm{O} & 1.093836 & -2.717612 & 0.000000 \\ \mathrm{H} & 3.155438 & -1.159702 & 0.000000 \\ \mathrm{H} & 3.083147 & 1.345842 & 0.000000 \\ \mathrm{H} & 0.887638 & 2.546928 & 0.000000 \\ \mathrm{O} & -1.418341 & 1.464457 & 0.000000 \\ \mathrm{H} & 0.193293 & -3.088452 & 0.000000 \\ \mathrm{C} & -2.661682 & 0.761561 & 0.000000 \\ \mathrm{H} & -3.439853 & 1.534070 & 0.000000 \\ \mathrm{H} & -2.773153 & 0.133388 & -0.901266 \\ \mathrm{H} & -2.773153 & 0.133388 & 0.901266\end{array}$

m-methoxyphenol, isomer $1 \mathrm{~S}_{1}$

$\begin{array}{lrrr}\text { C } & 1.172234 & -0.023551 & -0.628328 \\ \text { C } & -0.025441 & -0.048161 & 0.130176 \\ \text { C } & -0.054213 & -0.089513 & 1.560044\end{array}$ 


$\begin{array}{lrrc}\mathrm{C} & 1.245264 & 0.000377 & 2.155945 \\ \mathrm{C} & 2.458574 & 0.008869 & 1.420472 \\ \mathrm{C} & 2.444583 & -0.089334 & 0.008248 \\ \mathrm{H} & -0.866890 & -0.602305 & 2.098053 \\ \mathrm{O} & 1.352736 & 0.181988 & 3.514698 \\ \mathrm{H} & 3.392171 & 0.129793 & 1.974958 \\ \mathrm{H} & 3.365239 & -0.142327 & -0.569031 \\ \mathrm{H} & 1.083210 & 0.069315 & -1.712799 \\ \mathrm{O} & -1.167954 & 0.050071 & -0.632018 \\ \mathrm{H} & 0.464382 & 0.455819 & 3.837926 \\ \mathrm{C} & -2.443284 & 0.028396 & 0.025686 \\ \mathrm{H} & -3.161162 & 0.437753 & -0.694988 \\ \mathrm{H} & -2.727100 & -1.008353 & 0.274168 \\ \mathrm{H} & -2.424041 & 0.641164 & 0.939531\end{array}$

$m$-methoxyphenol, isomer $2 \mathrm{~S}_{0}$

$\begin{array}{lrrc}\mathrm{C} & 0.350334 & 2.173580 & 0.000000 \\ \mathrm{C} & -0.842867 & 1.457304 & 0.000000 \\ \mathrm{C} & -0.800017 & 0.049706 & 0.000000 \\ \mathrm{C} & 0.428308 & -0.623639 & 0.000000 \\ \mathrm{C} & 1.617761 & 0.121687 & 0.000000 \\ \mathrm{C} & 1.591032 & 1.520923 & 0.000000 \\ \mathrm{O} & -2.018307 & -0.580947 & 0.000000 \\ \mathrm{C} & -2.027468 & -2.011036 & 0.000000 \\ \mathrm{O} & 2.791782 & -0.600282 & 0.000000 \\ \mathrm{H} & 0.498630 & -1.710545 & 0.000000 \\ \mathrm{H} & 2.522334 & 2.094107 & 0.000000 \\ \mathrm{H} & 0.320695 & 3.265342 & 0.000000 \\ \mathrm{H} & -1.811955 & 1.957139 & 0.000000 \\ \mathrm{H} & -3.085103 & -2.300323 & 0.000000 \\ \mathrm{H} & -1.536657 & -2.419282 & -0.900355 \\ \mathrm{H} & -1.536657 & -2.419282 & 0.900355 \\ \mathrm{H} & 3.538145 & 0.025556 & 0.000000\end{array}$

$m$-methoxyphenol, isomer $2 \mathrm{~S}_{1}$

$\begin{array}{lrrr}\mathrm{C} & 1.244598 & -0.078145 & -0.720224 \\ \mathrm{C} & 0.047721 & -0.040239 & 0.066272 \\ \mathrm{C} & 0.111435 & -0.054314 & 1.481084 \\ \mathrm{C} & 1.338637 & -0.022433 & 2.211043 \\ \mathrm{C} & 2.512712 & 0.026202 & 1.399539 \\ \mathrm{C} & 2.478829 & 0.038540 & -0.016782 \\ \mathrm{O} & -1.119848 & -0.031258 & 2.099653 \\ \mathrm{C} & -1.172272 & 0.033576 & 3.530653 \\ \mathrm{O} & 3.705992 & 0.100695 & 2.084751\end{array}$




$\begin{array}{rrrr}\mathrm{H} & 1.422919 & -0.290464 & 3.263875 \\ \mathrm{H} & 3.418945 & 0.148666 & -0.568013 \\ \mathrm{H} & 1.209221 & -0.072991 & -1.806694 \\ \mathrm{H} & -0.936446 & 0.024819 & -0.400609 \\ \mathrm{H} & -2.223918 & 0.214311 & 3.781309 \\ \mathrm{H} & -0.847370 & -0.921728 & 3.975779 \\ \mathrm{H} & -0.542070 & 0.851173 & 3.913346 \\ \mathrm{H} & 4.436047 & 0.073591 & 1.436918\end{array}$

$p$-methoxyphenol, cis $\mathrm{S}_{0}$

$\begin{array}{lrrr}\mathrm{C} & -0.320710 & 0.736713 & 0.000000 \\ \mathrm{C} & -1.560547 & 0.072247 & 0.000000 \\ \mathrm{C} & -1.619873 & -1.317105 & 0.000000 \\ \mathrm{C} & -0.436788 & -2.073816 & 0.000000 \\ \mathrm{C} & 0.797194 & -1.418101 & 0.000000 \\ \mathrm{C} & 0.861048 & -0.015954 & 0.000000 \\ \mathrm{O} & -0.562918 & -3.449465 & 0.000000 \\ \mathrm{O} & -0.377773 & 2.113402 & 0.000000 \\ \mathrm{C} & 0.862157 & 2.819626 & 0.000000 \\ \mathrm{H} & 1.838101 & 0.467313 & 0.000000 \\ \mathrm{H} & -2.474276 & 0.669180 & 0.000000 \\ \mathrm{H} & -2.580598 & -1.834745 & 0.000000 \\ \mathrm{H} & 1.726816 & -1.995892 & 0.000000 \\ \mathrm{H} & 0.599610 & 3.884422 & 0.000000 \\ \mathrm{H} & 1.460411 & 2.592518 & 0.900474 \\ \mathrm{H} & 1.460411 & 2.592518 & -0.900474 \\ \mathrm{H} & 0.327734 & -3.842841 & 0.000000\end{array}$

p-methoxyphenol, cis $\mathrm{S}_{1}$

$\begin{array}{rrrr}\mathrm{C} & 1.325430 & -0.031152 & -0.609225 \\ \mathrm{C} & 0.041116 & -0.047853 & -0.000334 \\ \mathrm{C} & -0.027165 & -0.005861 & 1.426431 \\ \mathrm{C} & 1.184183 & -0.006863 & 2.148210 \\ \mathrm{C} & 2.470533 & -0.012276 & 1.542927 \\ \mathrm{C} & 2.540771 & -0.020876 & 0.114180 \\ \mathrm{O} & 1.080813 & 0.009665 & 3.515767 \\ \mathrm{O} & 1.281219 & -0.042438 & -1.982554 \\ \mathrm{C} & 2.500486 & 0.033093 & -2.734457 \\ \mathrm{H} & 3.506079 & -0.028925 & -0.387558 \\ \mathrm{H} & -0.847747 & -0.051434 & -0.627845 \\ \mathrm{H} & -0.971325 & 0.094531 & 1.959739 \\ \mathrm{H} & 3.365608 & -0.173107 & 2.148898 \\ \mathrm{H} & 2.199362 & 0.003576 & -3.787387 \\ \mathrm{H} & 3.032915 & 0.972066 & -2.517714\end{array}$




$$
\begin{array}{cccc}
\mathrm{H} & 3.151713 & -0.824907 & -2.505439 \\
\mathrm{H} & 1.979539 & 0.132744 & 3.883362
\end{array}
$$

$p$-methoxyphenol, trans $\mathrm{S}_{0}$

$\begin{array}{rrrc}\mathrm{C} & -0.662984 & 1.314440 & 0.000000 \\ \mathrm{C} & -1.901717 & 0.675185 & 0.000000 \\ \mathrm{C} & -1.975928 & -0.726978 & 0.000000 \\ \mathrm{C} & -0.796299 & -1.476293 & 0.000000 \\ \mathrm{C} & 0.449790 & -0.837913 & 0.000000 \\ \mathrm{C} & 0.523530 & 0.563899 & 0.000000 \\ \mathrm{O} & -3.174320 & -1.414640 & 0.000000 \\ \mathrm{H} & 1.353459 & -1.447760 & 0.000000 \\ \mathrm{O} & 1.694476 & 1.290542 & 0.000000 \\ \mathrm{H} & -0.596758 & 2.403777 & 0.000000 \\ \mathrm{H} & -2.818060 & 1.273916 & 0.000000 \\ \mathrm{H} & -3.902546 & -0.768496 & 0.000000 \\ \mathrm{H} & -0.859205 & -2.565799 & 0.000000 \\ \mathrm{C} & 2.919095 & 0.557258 & 0.000000 \\ \mathrm{H} & 3.717251 & 1.309382 & 0.000000 \\ \mathrm{H} & 3.015103 & -0.075269 & 0.900356 \\ \mathrm{H} & 3.015103 & -0.075269 & -0.900356\end{array}$

$p$-methoxyphenol, trans $\mathrm{S}_{1}$

$\begin{array}{rrrr}\mathrm{C} & 0.123524 & -0.019430 & -1.401273 \\ \mathrm{C} & -1.295399 & -0.018280 & -1.222791 \\ \mathrm{C} & -1.793320 & 0.014796 & 0.105194 \\ \mathrm{C} & -0.974858 & 0.058908 & 1.255619 \\ \mathrm{C} & 0.444439 & 0.038228 & 1.079718 \\ \mathrm{C} & 0.945772 & 0.010349 & -0.242084 \\ \mathrm{O} & -3.145866 & 0.017938 & 0.333295 \\ \mathrm{H} & 1.103511 & 0.064562 & 1.944410 \\ \mathrm{O} & 2.289783 & 0.001517 & -0.529104 \\ \mathrm{H} & 0.589022 & -0.046560 & -2.384594 \\ \mathrm{H} & -1.972135 & -0.184635 & -2.064260 \\ \mathrm{H} & -3.597170 & 0.082093 & -0.532280 \\ \mathrm{H} & -1.432735 & 0.168949 & 2.237421 \\ \mathrm{C} & 3.243720 & -0.030546 & 0.541779 \\ \mathrm{H} & 4.227626 & -0.025178 & 0.060014 \\ \mathrm{H} & 3.134448 & 0.855772 & 1.186037 \\ \mathrm{H} & 3.120427 & -0.944173 & 1.143600\end{array}$

1-naphthol, cis $\mathrm{S}_{0}$

$\begin{array}{llll}\text { C } & 0.836377 & 1.526706 & 0.017846\end{array}$ 


$\begin{array}{rrrc}\mathrm{C} & 0.533412 & 0.125746 & 0.006488 \\ \mathrm{C} & -0.853546 & -0.269358 & -0.010806 \\ \mathrm{C} & -1.869081 & 0.724769 & -0.016167 \\ \mathrm{C} & -1.529634 & 2.064344 & -0.004852 \\ \mathrm{C} & -0.177585 & 2.470860 & 0.012188 \\ \mathrm{C} & 1.531331 & -0.887676 & 0.011439 \\ \mathrm{C} & 1.193311 & -2.227885 & 0.000126 \\ \mathrm{C} & -0.168168 & -2.617168 & -0.016794 \\ \mathrm{C} & -1.162307 & -1.658843 & -0.022078 \\ \mathrm{O} & 2.128900 & 1.993000 & 0.034587 \\ \mathrm{H} & -2.214167 & -1.956453 & -0.035167 \\ \mathrm{H} & -0.427768 & -3.677899 & -0.025653 \\ \mathrm{H} & 1.976919 & -2.988144 & 0.004208 \\ \mathrm{H} & 2.595529 & -0.627937 & 0.024320 \\ \mathrm{H} & -2.915769 & 0.413050 & -0.029306 \\ \mathrm{H} & -2.310742 & 2.827624 & -0.008994 \\ \mathrm{H} & 0.090630 & 3.528534 & 0.021177 \\ \mathrm{H} & 2.742369 & 1.236740 & 0.037434\end{array}$

1-naphthol, cis $\mathrm{S}_{1}$

$\begin{array}{lrrr}\mathrm{C} & 0.859861 & 1.481730 & 0.015066 \\ \mathrm{C} & 0.529611 & 0.086792 & -0.073572 \\ \mathrm{C} & -0.842836 & -0.245448 & -0.006985 \\ \mathrm{C} & -1.799905 & 0.825712 & -0.023722 \\ \mathrm{C} & -1.443544 & 2.189371 & -0.029884 \\ \mathrm{C} & -0.099162 & 2.533957 & -0.018973 \\ \mathrm{C} & 1.542596 & -0.930647 & -0.120938 \\ \mathrm{C} & 1.117539 & -2.295755 & -0.029565 \\ \mathrm{C} & -0.228088 & -2.621893 & 0.058427 \\ \mathrm{C} & -1.240817 & -1.623856 & 0.047290 \\ \mathrm{O} & 2.144604 & 1.850289 & 0.171406 \\ \mathrm{H} & -2.297060 & -1.885487 & 0.102656 \\ \mathrm{H} & -0.518338 & -3.672827 & 0.133004 \\ \mathrm{H} & 1.867312 & -3.089840 & -0.037313 \\ \mathrm{H} & 2.570589 & -0.708574 & -0.421763 \\ \mathrm{H} & -2.860064 & 0.558222 & -0.038013 \\ \mathrm{H} & -2.215607 & 2.958923 & -0.056382 \\ \mathrm{H} & 0.249915 & 3.566445 & 0.012446 \\ \mathrm{H} & 2.663395 & 1.022878 & 0.316795\end{array}$

1-naphthol, trans $\mathrm{S}_{0}$
$\begin{array}{llll}\text { C } & -0.721390 & -0.544613 & 0.000000\end{array}$
$\begin{array}{llll}\mathrm{C} & -0.677830 & -1.967010 & 0.000000\end{array}$
$\begin{array}{llll}\text { C } & 0.526200 & -2.644609 & 0.000000\end{array}$ 


$\begin{array}{crrc}\mathrm{C} & 1.748778 & -1.928940 & 0.000000 \\ \mathrm{C} & 1.748879 & -0.546390 & 0.000000 \\ \mathrm{C} & 0.524630 & 0.174605 & 0.000000 \\ \mathrm{H} & -1.622109 & -2.517894 & 0.000000 \\ \mathrm{H} & 0.538869 & -3.736919 & 0.000000 \\ \mathrm{H} & 2.695251 & -2.473965 & 0.000000 \\ \mathrm{H} & 2.686767 & 0.010350 & 0.000000 \\ \mathrm{C} & -1.950004 & 0.174544 & 0.000000 \\ \mathrm{C} & -1.947421 & 1.554834 & 0.000000 \\ \mathrm{C} & -0.730203 & 2.277506 & 0.000000 \\ \mathrm{C} & 0.480801 & 1.604404 & 0.000000 \\ \mathrm{H} & -2.890427 & -0.381195 & 0.000000 \\ \mathrm{H} & -2.889841 & 2.106747 & 0.000000 \\ \mathrm{H} & -0.746778 & 3.371896 & 0.000000 \\ \mathrm{O} & 1.694183 & 2.253127 & 0.000000 \\ \mathrm{H} & 1.531656 & 3.213512 & 0.000000\end{array}$

1-naphthol, trans $\mathrm{S}_{1}$

$\begin{array}{rrrr}\mathrm{C} & -0.930155 & -0.393383 & 0.000000 \\ \mathrm{C} & -1.324727 & -1.766630 & 0.000000 \\ \mathrm{C} & -0.318967 & -2.781227 & 0.000000 \\ \mathrm{C} & 1.025754 & -2.445765 & 0.000000 \\ \mathrm{C} & 1.451522 & -1.082461 & 0.000000 \\ \mathrm{C} & 0.455973 & -0.056270 & 0.000000 \\ \mathrm{H} & -2.384100 & -2.024594 & 0.000000 \\ \mathrm{H} & -0.617867 & -3.831776 & 0.000000 \\ \mathrm{H} & 1.781597 & -3.234744 & 0.000000 \\ \mathrm{H} & 2.508142 & -0.824087 & 0.000000 \\ \mathrm{C} & -1.892064 & 0.665361 & 0.000000 \\ \mathrm{C} & -1.542411 & 2.036506 & 0.000000 \\ \mathrm{C} & -0.201195 & 2.381965 & 0.000000 \\ \mathrm{C} & 0.776436 & 1.338169 & 0.000000 \\ \mathrm{H} & -2.951232 & 0.393453 & 0.000000 \\ \mathrm{H} & -2.318657 & 2.802371 & 0.000000 \\ \mathrm{H} & 0.124351 & 3.426467 & 0.000000 \\ \mathrm{O} & 2.094433 & 1.661227 & 0.000000 \\ \mathrm{H} & 2.187489 & 2.634490 & 0.000000\end{array}$

2-naphthol, cis $\mathrm{S}_{0}$
$\begin{array}{llll}\text { C } & 0.602132 & 2.019601 & 0.000000\end{array}$
$\begin{array}{llll}\text { C } & 0.627600 & 0.598914 & 0.000000\end{array}$
$\begin{array}{llll}\text { C } & -0.617493 & -0.123897 & 0.000000\end{array}$
$\begin{array}{llll}\text { C } & -1.833355 & 0.616100 & 0.000000\end{array}$
$\begin{array}{llll}\text { C } & -1.822458 & 1.998243 & 0.000000\end{array}$ 


$\begin{array}{rrrr}\mathrm{C} & -0.596158 & 2.708916 & 0.000000 \\ \mathrm{C} & 1.842898 & -0.143283 & 0.000000 \\ \mathrm{C} & 1.842947 & -1.520800 & 0.000000 \\ \mathrm{C} & 0.611263 & -2.228690 & 0.000000 \\ \mathrm{C} & -0.592959 & -1.545320 & 0.000000 \\ \mathrm{H} & -1.541190 & -2.093479 & 0.000000 \\ \mathrm{H} & 1.552092 & 2.561172 & 0.000000 \\ \mathrm{H} & -0.602344 & 3.800890 & 0.000000 \\ \mathrm{H} & -2.765404 & 2.549783 & 0.000000 \\ \mathrm{H} & -2.782039 & 0.072405 & 0.000000 \\ \mathrm{H} & 2.791637 & 0.399820 & 0.000000 \\ \mathrm{H} & 2.772622 & -2.092380 & 0.000000 \\ \mathrm{O} & 0.704749 & -3.601373 & 0.000000 \\ \mathrm{H} & -0.194550 & -3.976612 & 0.000000\end{array}$

2-naphthol, cis $\mathrm{S}_{1}$

$\begin{array}{lrrl}\mathrm{C} & 0.602628 & 2.008413 & 0.000000 \\ \mathrm{C} & 0.640231 & 0.585782 & 0.000000 \\ \mathrm{C} & -0.625023 & -0.114368 & 0.000000 \\ \mathrm{C} & -1.843128 & 0.609429 & 0.000000 \\ \mathrm{C} & -1.848481 & 2.033622 & 0.000000 \\ \mathrm{C} & -0.632602 & 2.711517 & 0.000000 \\ \mathrm{C} & 1.855712 & -0.155447 & 0.000000 \\ \mathrm{C} & 1.899188 & -1.568081 & 0.000000 \\ \mathrm{C} & 0.663622 & -2.233078 & 0.000000 \\ \mathrm{C} & -0.578573 & -1.536124 & 0.000000 \\ \mathrm{H} & -1.512317 & -2.110055 & 0.000000 \\ \mathrm{H} & 1.541908 & 2.565879 & 0.000000 \\ \mathrm{H} & -0.616607 & 3.804135 & 0.000000 \\ \mathrm{H} & -2.792634 & 2.579312 & 0.000000 \\ \mathrm{H} & -2.786769 & 0.058218 & 0.000000 \\ \mathrm{H} & 2.795414 & 0.402708 & 0.000000 \\ \mathrm{H} & 2.830998 & -2.129583 & 0.000000 \\ \mathrm{O} & 0.665621 & -3.597270 & 0.000000 \\ \mathrm{H} & -0.259178 & -3.915000 & 0.000000\end{array}$

2-naphthol, trans $\mathrm{S}_{0}$
$\begin{array}{llll}\text { C } & 0.514694 & 2.021392 & 0.000000\end{array}$
$\begin{array}{llll}\text { C } & 0.545244 & 0.599838 & 0.000000\end{array}$
$\begin{array}{llll}\text { C } & -0.697333 & -0.129908 & 0.000000\end{array}$
$\begin{array}{llll}\text { C } & -1.916660 & 0.607112 & 0.000000\end{array}$
$\begin{array}{llll}\text { C } & -1.910409 & 1.988324 & 0.000000\end{array}$
$\begin{array}{llll}\text { C } & -0.686022 & 2.704706 & 0.000000\end{array}$
$\begin{array}{llll}\text { C } & 1.761431 & -0.136101 & 0.000000\end{array}$ 


$\begin{array}{rrrr}\mathrm{C} & 1.760509 & -1.516066 & 0.000000 \\ \mathrm{C} & 0.531481 & -2.229634 & 0.000000 \\ \mathrm{C} & -0.673403 & -1.548535 & 0.000000 \\ \mathrm{H} & -1.608191 & -2.113053 & 0.000000 \\ \mathrm{H} & 1.462381 & 2.567043 & 0.000000 \\ \mathrm{H} & -0.697231 & 3.796671 & 0.000000 \\ \mathrm{H} & -2.855187 & 2.536766 & 0.000000 \\ \mathrm{H} & -2.862394 & 0.058813 & 0.000000 \\ \mathrm{H} & 2.709750 & 0.407643 & 0.000000 \\ \mathrm{H} & 2.705727 & -2.068655 & 0.000000 \\ \mathrm{O} & 0.500407 & -3.606234 & 0.000000 \\ \mathrm{H} & 1.415216 & -3.940122 & 0.000000\end{array}$

2-naphthol, trans $\mathrm{S}_{1}$

$\begin{array}{lrrl}\mathrm{C} & 0.514569 & 2.013151 & 0.000000 \\ \mathrm{C} & 0.553178 & 0.590361 & 0.000000 \\ \mathrm{C} & -0.708647 & -0.114882 & 0.000000 \\ \mathrm{C} & -1.927698 & 0.606779 & 0.000000 \\ \mathrm{C} & -1.935019 & 2.031514 & 0.000000 \\ \mathrm{C} & -0.722276 & 2.713391 & 0.000000 \\ \mathrm{C} & 1.771269 & -0.146468 & 0.000000 \\ \mathrm{C} & 1.814420 & -1.559338 & 0.000000 \\ \mathrm{C} & 0.575661 & -2.226723 & 0.000000 \\ \mathrm{C} & -0.665030 & -1.536941 & 0.000000 \\ \mathrm{H} & -1.583652 & -2.127212 & 0.000000 \\ \mathrm{H} & 1.452993 & 2.572145 & 0.000000 \\ \mathrm{H} & -0.709613 & 3.806049 & 0.000000 \\ \mathrm{H} & -2.880751 & 2.574761 & 0.000000 \\ \mathrm{H} & -2.869701 & 0.053385 & 0.000000 \\ \mathrm{H} & 2.710038 & 0.413355 & 0.000000 \\ \mathrm{H} & 2.757843 & -2.107733 & 0.000000 \\ \mathrm{O} & 0.475635 & -3.587933 & 0.000000 \\ \mathrm{H} & 1.376780 & -3.967651 & 0.000000\end{array}$

5-methoxysalicylic acid $\mathrm{S}_{0}$

$\begin{array}{lrrc}\mathrm{C} & 2.293537 & -0.165828 & 0.000000 \\ \mathrm{C} & 1.577297 & -1.350334 & 0.000000 \\ \mathrm{C} & 0.163186 & -1.346481 & 0.000000 \\ \mathrm{C} & -0.517289 & -0.133767 & 0.000000 \\ \mathrm{C} & 0.204787 & 1.086945 & 0.000000 \\ \mathrm{C} & 1.625975 & 1.076207 & 0.000000 \\ \mathrm{O} & -0.428880 & -2.587227 & 0.000000 \\ \mathrm{C} & -1.857679 & -2.627561 & 0.000000 \\ \mathrm{C} & -0.474879 & 2.380442 & 0.000000\end{array}$




$\begin{array}{rrrr}\mathrm{O} & -1.836029 & 2.319524 & 0.000000 \\ \mathrm{O} & 2.371628 & 2.202656 & 0.000000 \\ \mathrm{O} & 0.105917 & 3.477826 & 0.000000 \\ \mathrm{H} & -2.125640 & -3.690641 & 0.000000 \\ \mathrm{H} & -2.276136 & -2.144413 & -0.900416 \\ \mathrm{H} & -2.276136 & -2.144413 & 0.900416 \\ \mathrm{H} & -1.604901 & -0.088026 & 0.000000 \\ \mathrm{H} & 3.384514 & -0.167845 & 0.000000 \\ \mathrm{H} & 2.092496 & -2.312942 & 0.000000 \\ \mathrm{H} & 1.720152 & 2.965184 & 0.000000 \\ \mathrm{H} & -2.141902 & 3.250696 & 0.000000\end{array}$

5-methoxysalicylic acid $\mathrm{S}_{1}$

$\begin{array}{crcc}\mathrm{C} & 2.184434 & -0.137167 & 0.000000 \\ \mathrm{C} & 1.747690 & -1.468296 & 0.000000 \\ \mathrm{C} & 0.360052 & -1.694849 & 0.000000 \\ \mathrm{C} & -0.579672 & -0.617535 & 0.000000 \\ \mathrm{C} & -0.146087 & 0.711962 & 0.000000 \\ \mathrm{C} & 1.273234 & 0.939722 & 0.000000 \\ \mathrm{O} & -0.032130 & -3.003194 & 0.000000 \\ \mathrm{C} & -1.430646 & -3.346815 & 0.000000 \\ \mathrm{C} & -1.046698 & 1.861210 & 0.000000 \\ \mathrm{O} & -2.397761 & 1.563894 & 0.000000 \\ \mathrm{O} & 1.733827 & 2.199347 & 0.000000 \\ \mathrm{O} & -0.645758 & 3.074811 & 0.000000 \\ \mathrm{H} & -1.456661 & -4.441491 & 0.000000 \\ \mathrm{H} & -1.928775 & -2.960356 & -0.901648 \\ \mathrm{H} & -1.928775 & -2.960356 & 0.901648 \\ \mathrm{H} & -1.651620 & -0.814410 & 0.000000 \\ \mathrm{H} & 3.248996 & 0.102602 & 0.000000 \\ \mathrm{H} & 2.439406 & -2.308966 & 0.000000 \\ \mathrm{H} & 0.873930 & 2.797287 & 0.000000 \\ \mathrm{H} & -2.844932 & 2.433379 & 0.000000\end{array}$

m-dimethoxybenzene, trans $\mathrm{S}_{0}$
$\begin{array}{llll}\text { C } & 0.000000 & -0.135184 & 0.711983\end{array}$
$\begin{array}{llll}\text { C } & 0.000000 & -0.135184 & -0.711983\end{array}$
$\begin{array}{llll}\text { C } & 0.000000 & 1.082545 & -1.398050\end{array}$
$\begin{array}{llll}\text { C } & 0.000000 & 2.299441 & -0.695600\end{array}$
$\begin{array}{llll}\text { C } & 0.000000 & 2.299441 & 0.695600\end{array}$
$\begin{array}{llll}\text { C } & 0.000000 & 1.082545 & 1.398050\end{array}$
$\begin{array}{llll}\mathrm{O} & 0.000000 & -1.371152 & -1.302558\end{array}$
$\begin{array}{llll}\mathrm{H} & 0.000000 & 1.092312 & -2.488169\end{array}$
H $\quad 0.000000 \quad 3.239239 \quad-1.250886$ 


$\begin{array}{crrr}\mathrm{H} & 0.000000 & 3.239239 & 1.250886 \\ \mathrm{H} & 0.000000 & 1.092312 & 2.488169 \\ \mathrm{O} & 0.000000 & -1.371152 & 1.302558 \\ \mathrm{C} & 0.000000 & -1.415431 & 2.730111 \\ \mathrm{H} & 0.000000 & -2.480230 & 2.991977 \\ \mathrm{H} & 0.900239 & -0.935165 & 3.152134 \\ \mathrm{H} & -0.900239 & -0.935165 & 3.152134 \\ \mathrm{C} & 0.000000 & -1.415431 & -2.730111 \\ \mathrm{H} & 0.000000 & -2.480230 & -2.991977 \\ \mathrm{H} & -0.900239 & -0.935165 & -3.152134 \\ \mathrm{H} & 0.900239 & -0.935165 & -3.152134\end{array}$

$m$-dimethoxybenzene, trans $\mathrm{S}_{1}$

$\begin{array}{lrrr}\mathrm{C} & -0.142696 & -1.075503 & -1.451846 \\ \mathrm{C} & -0.375533 & 0.138575 & -0.710118 \\ \mathrm{C} & -0.375533 & 0.138575 & 0.710118 \\ \mathrm{C} & -0.142696 & -1.075503 & 1.451846 \\ \mathrm{C} & 0.284088 & -2.193693 & 0.706303 \\ \mathrm{C} & 0.284088 & -2.193693 & -0.706303 \\ \mathrm{O} & -0.397135 & 1.374908 & -1.311289 \\ \mathrm{C} & 0.049974 & 1.479080 & -2.680343 \\ \mathrm{O} & -0.397135 & 1.374908 & 1.311289 \\ \mathrm{C} & 0.049974 & 1.479080 & 2.680343 \\ \mathrm{H} & -0.464329 & -1.188220 & 2.491967 \\ \mathrm{H} & 0.573228 & -3.110266 & 1.230892 \\ \mathrm{H} & 0.573228 & -3.110266 & -1.230892 \\ \mathrm{H} & -0.464329 & -1.188220 & -2.491967 \\ \mathrm{H} & 0.011230 & 2.550094 & -2.910401 \\ \mathrm{H} & 1.075640 & 1.097387 & -2.793486 \\ \mathrm{H} & -0.621377 & 0.930077 & -3.358767 \\ \mathrm{H} & 0.011230 & 2.550094 & 2.910401 \\ \mathrm{H} & -0.621377 & 0.930077 & 3.358767 \\ \mathrm{H} & 1.075640 & 1.097387 & 2.793486\end{array}$

3P-propionic acid, gauche $\mathrm{S}_{0}$

$\begin{array}{lrrr}\mathrm{C} & -1.040556 & 0.911483 & -1.176587 \\ \mathrm{C} & -0.388392 & 0.669179 & 0.044961 \\ \mathrm{C} & -1.054543 & -0.094354 & 1.018498 \\ \mathrm{C} & -2.334918 & -0.603833 & 0.775404 \\ \mathrm{C} & -2.971955 & -0.357155 & -0.445420 \\ \mathrm{C} & -2.320232 & 0.403857 & -1.422521 \\ \mathrm{C} & 1.007418 & 1.207867 & 0.291516 \\ \mathrm{C} & 2.124358 & 0.281955 & -0.229822 \\ \mathrm{C} & 2.339323 & -0.947807 & 0.630593\end{array}$




$\begin{array}{rrrr}\mathrm{O} & 2.079663 & -1.047832 & 1.815311 \\ \mathrm{O} & 2.919712 & -1.962255 & -0.081536 \\ \mathrm{H} & 1.939710 & -0.032074 & -1.267757 \\ \mathrm{H} & 3.088847 & 0.821110 & -0.235008 \\ \mathrm{H} & 1.161966 & 1.373087 & 1.368417 \\ \mathrm{H} & 1.113832 & 2.184053 & -0.207503 \\ \mathrm{H} & -0.557601 & -0.291899 & 1.971070 \\ \mathrm{H} & -2.838128 & -1.192896 & 1.545794 \\ \mathrm{H} & -3.973178 & -0.751260 & -0.633297 \\ \mathrm{H} & -2.812452 & 0.608576 & -2.376207 \\ \mathrm{H} & -0.542750 & 1.514748 & -1.941961 \\ \mathrm{H} & 3.059887 & -2.694570 & 0.556064\end{array}$

3P-propionic acid, gauche $\mathrm{S}_{1}$

$\begin{array}{lrrr}\mathrm{C} & -1.275543 & 1.198949 & -1.160431 \\ \mathrm{C} & -0.400956 & 0.581292 & -0.205526 \\ \mathrm{C} & -0.924573 & -0.445729 & 0.666178 \\ \mathrm{C} & -2.252683 & -0.794442 & 0.595752 \\ \mathrm{C} & -3.102527 & -0.173203 & -0.358983 \\ \mathrm{C} & -2.600021 & 0.830971 & -1.238518 \\ \mathrm{C} & 1.011084 & 1.033806 & -0.050270 \\ \mathrm{C} & 2.152922 & -0.000320 & -0.270308 \\ \mathrm{C} & 2.213706 & -1.133298 & 0.770431 \\ \mathrm{O} & 1.738559 & -0.931264 & 1.965179 \\ \mathrm{O} & 3.540461 & -1.710048 & 0.632159 \\ \mathrm{H} & 2.113011 & -0.408904 & -1.294188 \\ \mathrm{H} & 3.071037 & 0.612147 & -0.226825 \\ \mathrm{H} & 1.092589 & 1.343583 & 1.009936 \\ \mathrm{H} & 1.179501 & 1.921987 & -0.676052 \\ \mathrm{H} & -0.201162 & -0.882738 & 1.371911 \\ \mathrm{H} & -2.661465 & -1.553663 & 1.263764 \\ \mathrm{H} & -4.151581 & -0.467769 & -0.426402 \\ \mathrm{H} & -3.269487 & 1.293389 & -1.965062 \\ \mathrm{H} & -0.870792 & 1.965858 & -1.822491 \\ \mathrm{H} & 3.597929 & -2.280625 & 1.419758\end{array}$

3P-propionic acid, anti $\mathrm{S}_{0}$
$\begin{array}{llll}\text { C } & 1.471008 & -0.449292 & 0.000000\end{array}$
$\begin{array}{llll}\text { C } & 0.547020 & 0.780187 & 0.000000\end{array}$
$\begin{array}{llll}\text { C } & 2.941487 & -0.087266 & 0.000000\end{array}$
$\begin{array}{llll}\mathrm{H} & 1.277305 & -1.090371 & 0.876346\end{array}$
$\begin{array}{llll}\mathrm{H} & 1.277305 & -1.090371 & -0.876346\end{array}$
$\begin{array}{llll}\text { C } & -0.916689 & 0.390321 & 0.000000\end{array}$
$\begin{array}{llll}\mathrm{H} & 0.775185 & 1.400213 & 0.880484\end{array}$ 


$\begin{array}{lrrr}\mathrm{H} & 0.775185 & 1.400213 & -0.880484 \\ \mathrm{C} & -1.609082 & 0.191046 & 1.206148 \\ \mathrm{C} & -2.951972 & -0.200616 & 1.208958 \\ \mathrm{C} & -3.627861 & -0.399568 & 0.000000 \\ \mathrm{C} & -2.951972 & -0.200616 & -1.208958 \\ \mathrm{C} & -1.609082 & 0.191046 & -1.206148 \\ \mathrm{H} & -1.090826 & 0.354289 & 2.155685 \\ \mathrm{H} & -3.473769 & -0.344805 & 2.157907 \\ \mathrm{H} & -4.677458 & -0.701410 & 0.000000 \\ \mathrm{H} & -3.473769 & -0.344805 & -2.157907 \\ \mathrm{H} & -1.090826 & 0.354289 & -2.155685 \\ \mathrm{O} & 3.728979 & -1.209464 & 0.000000 \\ \mathrm{O} & 3.407053 & 1.036460 & 0.000000 \\ \mathrm{H} & 4.655617 & -0.888159 & 0.000000\end{array}$

3P-propionic acid, anti $\mathrm{S}_{1}$

$\begin{array}{lrrr}\mathrm{C} & 1.478108 & -0.561142 & 0.000000 \\ \mathrm{C} & 0.549475 & 0.689780 & 0.000000 \\ \mathrm{C} & 2.898422 & -0.047965 & 0.000000 \\ \mathrm{H} & 1.237479 & -1.190831 & 0.885145 \\ \mathrm{H} & 1.237479 & -1.190831 & -0.885145 \\ \mathrm{C} & -0.892084 & 0.352300 & 0.000000 \\ \mathrm{H} & 0.806535 & 1.289177 & 0.884935 \\ \mathrm{H} & 0.806535 & 1.289177 & -0.884935 \\ \mathrm{C} & -1.610791 & 0.173314 & 1.236435 \\ \mathrm{C} & -2.945231 & -0.158419 & 1.237189 \\ \mathrm{C} & -3.628885 & -0.327718 & 0.000000 \\ \mathrm{C} & -2.945231 & -0.158419 & -1.237189 \\ \mathrm{C} & -1.610791 & 0.173314 & -1.236435 \\ \mathrm{H} & -1.063841 & 0.304750 & 2.169863 \\ \mathrm{H} & -3.487471 & -0.295789 & 2.173327 \\ \mathrm{H} & -4.686844 & -0.594745 & 0.000000 \\ \mathrm{H} & -3.487471 & -0.295789 & -2.173327 \\ \mathrm{H} & -1.063841 & 0.304750 & -2.169863 \\ \mathrm{O} & 3.824859 & -1.159603 & 0.000000 \\ \mathrm{O} & 3.284902 & 1.191969 & 0.000000 \\ \mathrm{H} & 4.681525 & -0.695961 & 0.000000\end{array}$

fluorine $\mathrm{S}_{0}$
$\begin{array}{llll}\text { C } & 0.000000 & 0.000000 & -1.799774\end{array}$
$\begin{array}{llll}\text { C } & 0.000000 & 1.185985 & -0.855157\end{array}$
$\begin{array}{llll}\text { C } & 0.000000 & 0.735034 & 0.487206\end{array}$
$\begin{array}{llll}\text { C } & 0.000000 & -0.735034 & 0.487206\end{array}$
$\begin{array}{llll}\text { C } & 0.000000 & 1.656632 & 1.542545\end{array}$ 


$\begin{array}{rrrr}\mathrm{C} & 0.000000 & 3.024524 & 1.246352 \\ \mathrm{C} & 0.000000 & 3.469297 & -0.084207 \\ \mathrm{C} & 0.000000 & 2.549684 & -1.143617 \\ \mathrm{H} & 0.000000 & 2.903408 & -2.178124 \\ \mathrm{H} & 0.000000 & 4.540763 & -0.296392 \\ \mathrm{H} & 0.000000 & 3.754318 & 2.059176 \\ \mathrm{H} & 0.000000 & 1.318860 & 2.581746 \\ \mathrm{C} & 0.000000 & -1.656632 & 1.542545 \\ \mathrm{C} & 0.000000 & -3.024524 & 1.246352 \\ \mathrm{C} & 0.000000 & -3.469297 & -0.084207 \\ \mathrm{C} & 0.000000 & -2.549684 & -1.143617 \\ \mathrm{C} & 0.000000 & -1.185985 & -0.855157 \\ \mathrm{H} & 0.000000 & -1.318860 & 2.581746 \\ \mathrm{H} & 0.000000 & -3.754318 & 2.059176 \\ \mathrm{H} & 0.000000 & -4.540763 & -0.296392 \\ \mathrm{H} & 0.000000 & -2.903408 & -2.178124 \\ \mathrm{H} & -0.885019 & 0.000000 & -2.459631 \\ \mathrm{H} & 0.885019 & 0.000000 & -2.459631\end{array}$

fluorine $\mathrm{S}_{1}$

$\begin{array}{lrrr}\mathrm{C} & 0.000000 & 0.000000 & -1.867837 \\ \mathrm{C} & 0.000000 & 1.169744 & -0.918856 \\ \mathrm{C} & 0.000000 & 0.713845 & 0.444094 \\ \mathrm{C} & 0.000000 & -0.713845 & 0.444094 \\ \mathrm{C} & 0.000000 & 1.618911 & 1.537773 \\ \mathrm{C} & 0.000000 & 2.997499 & 1.242876 \\ \mathrm{C} & 0.000000 & 3.443785 & -0.099773 \\ \mathrm{C} & 0.000000 & 2.550420 & -1.195288 \\ \mathrm{H} & 0.000000 & 2.927638 & -2.218996 \\ \mathrm{H} & 0.000000 & 4.519575 & -0.293074 \\ \mathrm{H} & 0.000000 & 3.732638 & 2.049262 \\ \mathrm{H} & 0.000000 & 1.255193 & 2.565755 \\ \mathrm{C} & 0.000000 & -1.618911 & 1.537773 \\ \mathrm{C} & 0.000000 & -2.997499 & 1.242876 \\ \mathrm{C} & 0.000000 & -3.443785 & -0.099773 \\ \mathrm{C} & 0.000000 & -2.550420 & -1.195288 \\ \mathrm{C} & 0.000000 & -1.169744 & -0.918856 \\ \mathrm{H} & 0.000000 & -1.255193 & 2.565755 \\ \mathrm{H} & 0.000000 & -3.732638 & 2.049262 \\ \mathrm{H} & 0.000000 & -4.519575 & -0.293074 \\ \mathrm{H} & 0.000000 & -2.927638 & -2.218996 \\ \mathrm{H} & -0.883100 & 0.000000 & -2.547694 \\ \mathrm{H} & 0.883100 & 0.000000 & -2.547694\end{array}$

phenanthrene $\mathrm{S}_{0}$ 


$\begin{array}{rrrr}\mathrm{C} & 0.729369 & 0.000000 & -0.353670 \\ \mathrm{C} & -0.729369 & 0.000000 & -0.353670 \\ \mathrm{C} & -1.426405 & 0.000000 & 0.897974 \\ \mathrm{C} & -0.682667 & 0.000000 & 2.126108 \\ \mathrm{C} & 0.682667 & 0.000000 & 2.126108 \\ \mathrm{C} & 1.426405 & 0.000000 & 0.897974 \\ \mathrm{C} & -1.501116 & 0.000000 & -1.542365 \\ \mathrm{C} & -2.844260 & 0.000000 & 0.908866 \\ \mathrm{H} & -1.238085 & 0.000000 & 3.067854 \\ \mathrm{H} & 1.238085 & 0.000000 & 3.067854 \\ \mathrm{C} & 1.501116 & 0.000000 & -1.542365 \\ \mathrm{C} & 2.887874 & 0.000000 & -1.506018 \\ \mathrm{C} & 3.568855 & 0.000000 & -0.271171 \\ \mathrm{C} & 2.844260 & 0.000000 & 0.908866 \\ \mathrm{H} & 1.002857 & 0.000000 & -2.512896 \\ \mathrm{H} & 3.454232 & 0.000000 & -2.439976 \\ \mathrm{H} & 4.660711 & 0.000000 & -0.247913 \\ \mathrm{H} & 3.359448 & 0.000000 & 1.873297 \\ \mathrm{C} & -3.568855 & 0.000000 & -0.271171 \\ \mathrm{C} & -2.887874 & 0.000000 & -1.506018 \\ \mathrm{H} & -1.002857 & 0.000000 & -2.512896 \\ \mathrm{H} & -3.454232 & 0.000000 & -2.439976 \\ \mathrm{H} & -4.660711 & 0.000000 & -0.247913 \\ \mathrm{H} & -3.359448 & 0.000000 & 1.873297\end{array}$

phenanthrene $S_{1}$

$\begin{array}{rrrr}\mathrm{C} & 0.716817 & 0.000000 & -0.376275 \\ \mathrm{C} & -0.716817 & 0.000000 & -0.376275 \\ \mathrm{C} & -1.441516 & 0.000000 & 0.888488 \\ \mathrm{C} & -0.702332 & 0.000000 & 2.094681 \\ \mathrm{C} & 0.702332 & 0.000000 & 2.094681 \\ \mathrm{C} & 1.441516 & 0.000000 & 0.888488 \\ \mathrm{C} & -1.499512 & 0.000000 & -1.574802 \\ \mathrm{C} & -2.857430 & 0.000000 & 0.891211 \\ \mathrm{H} & -1.242833 & 0.000000 & 3.044739 \\ \mathrm{H} & 1.242833 & 0.000000 & 3.044739 \\ \mathrm{C} & 1.499512 & 0.000000 & -1.574802 \\ \mathrm{C} & 2.902542 & 0.000000 & -1.535197 \\ \mathrm{C} & 3.592248 & 0.000000 & -0.313329 \\ \mathrm{C} & 2.857430 & 0.000000 & 0.891211 \\ \mathrm{H} & 1.004956 & 0.000000 & -2.545973 \\ \mathrm{H} & 3.461193 & 0.000000 & -2.473862 \\ \mathrm{H} & 4.682950 & 0.000000 & -0.292581 \\ \mathrm{H} & 3.378297 & 0.000000 & 1.851939\end{array}$



$\begin{array}{llll}\text { C } & -3.592248 & 0.000000 & -0.313329\end{array}$
$\begin{array}{llll}\text { C } & -2.902542 & 0.000000 & -1.535197\end{array}$
$\mathrm{H} \quad-1.004956 \quad 0.000000 \quad-2.545973$
$\mathrm{H} \quad-3.461193 \quad 0.000000 \quad-2.473862$
$\begin{array}{llll}\mathrm{H} & -4.682950 & 0.000000 & -0.292581\end{array}$
$\begin{array}{llll}\mathrm{H} & -3.378297 & 0.000000 & 1.851939\end{array}$

\section{2,4,6,8-decatetraene $\mathrm{S}_{0}$}

$\begin{array}{crcc}\mathrm{C} & -2.601171 & -5.034927 & 0.000000 \\ \mathrm{C} & -2.488572 & -3.542433 & 0.000000 \\ \mathrm{C} & -1.325475 & -2.848691 & 0.000000 \\ \mathrm{C} & -1.225999 & -1.410938 & 0.000000 \\ \mathrm{C} & -0.050800 & -0.715576 & 0.000000 \\ \mathrm{C} & 0.050800 & 0.715576 & 0.000000 \\ \mathrm{C} & 1.225999 & 1.410938 & 0.000000 \\ \mathrm{C} & 1.325475 & 2.848691 & 0.000000 \\ \mathrm{C} & 2.488572 & 3.542433 & 0.000000 \\ \mathrm{C} & 2.601171 & 5.034927 & 0.000000 \\ \mathrm{H} & -0.380326 & -3.407830 & 0.000000 \\ \mathrm{H} & -2.168103 & -0.847893 & 0.000000 \\ \mathrm{H} & 0.890251 & -1.280647 & 0.000000 \\ \mathrm{H} & -0.890251 & 1.280647 & 0.000000 \\ \mathrm{H} & 2.168103 & 0.847893 & 0.000000 \\ \mathrm{H} & 0.380326 & 3.407830 & 0.000000 \\ \mathrm{H} & 3.430549 & 2.979420 & 0.000000 \\ \mathrm{H} & -3.430549 & -2.979420 & 0.000000 \\ \mathrm{H} & 1.612224 & 5.516875 & 0.000000 \\ \mathrm{H} & 3.157514 & 5.395836 & 0.882858 \\ \mathrm{H} & 3.157514 & 5.395836 & -0.882858 \\ \mathrm{H} & -1.612224 & -5.516875 & 0.000000 \\ \mathrm{H} & -3.157514 & -5.395836 & 0.882858 \\ \mathrm{H} & -3.157514 & -5.395836 & -0.882858\end{array}$

2,4,6,8-decatetraene $\mathrm{S}_{1}$

$\begin{array}{lrrr}\mathrm{C} & -2.597285 & -5.053147 & 0.000000 \\ \mathrm{C} & -2.535838 & -3.566384 & 0.000000 \\ \mathrm{C} & -1.362268 & -2.832307 & 0.000000 \\ \mathrm{C} & -1.270063 & -1.417725 & 0.000000 \\ \mathrm{C} & -0.054632 & -0.702091 & 0.000000 \\ \mathrm{C} & 0.054632 & 0.702091 & 0.000000 \\ \mathrm{C} & 1.270063 & 1.417725 & 0.000000 \\ \mathrm{C} & 1.362268 & 2.832307 & 0.000000 \\ \mathrm{C} & 2.535838 & 3.566384 & 0.000000 \\ \mathrm{C} & 2.597285 & 5.053147 & 0.000000\end{array}$




$\begin{array}{crrr}\mathrm{H} & -0.414760 & -3.389237 & 0.000000 \\ \mathrm{H} & -2.204039 & -0.841669 & 0.000000 \\ \mathrm{H} & 0.875442 & -1.285252 & 0.000000 \\ \mathrm{H} & -0.875442 & 1.285252 & 0.000000 \\ \mathrm{H} & 2.204039 & 0.841669 & 0.000000 \\ \mathrm{H} & 0.414760 & 3.389237 & 0.000000 \\ \mathrm{H} & 3.490023 & 3.024549 & 0.000000 \\ \mathrm{H} & -3.490023 & -3.024549 & 0.000000 \\ \mathrm{H} & 1.592856 & 5.503178 & 0.000000 \\ \mathrm{H} & 3.146284 & 5.444935 & 0.880083 \\ \mathrm{H} & 3.146284 & 5.444935 & -0.880083 \\ \mathrm{H} & -1.592856 & -5.503178 & 0.000000 \\ \mathrm{H} & -3.146284 & -5.444935 & 0.880083 \\ \mathrm{H} & -3.146284 & -5.444935 & -0.880083\end{array}$

tetracene $\mathrm{S}_{0}$

$\begin{array}{lrrr}\mathrm{C} & 3.717924 & 1.412143 & 0.000000 \\ \mathrm{C} & 2.456948 & 0.728329 & 0.000000 \\ \mathrm{C} & 2.456948 & -0.728329 & 0.000000 \\ \mathrm{C} & 3.717924 & -1.412143 & 0.000000 \\ \mathrm{C} & 4.900754 & -0.715477 & 0.000000 \\ \mathrm{C} & 4.900754 & 0.715477 & 0.000000 \\ \mathrm{C} & 1.237451 & 1.410929 & 0.000000 \\ \mathrm{C} & 0.000000 & 0.729086 & 0.000000 \\ \mathrm{C} & 0.000000 & -0.729086 & 0.000000 \\ \mathrm{C} & 1.237451 & -1.410929 & 0.000000 \\ \mathrm{C} & -1.237451 & 1.410929 & 0.000000 \\ \mathrm{C} & -2.456948 & 0.728329 & 0.000000 \\ \mathrm{C} & -2.456948 & -0.728329 & 0.000000 \\ \mathrm{C} & -1.237451 & -1.410929 & 0.000000 \\ \mathrm{C} & -3.717924 & 1.412143 & 0.000000 \\ \mathrm{C} & -4.900754 & 0.715477 & 0.000000 \\ \mathrm{C} & -4.900754 & -0.715477 & 0.000000 \\ \mathrm{C} & -3.717924 & -1.412143 & 0.000000 \\ \mathrm{H} & -3.716259 & -2.505536 & 0.000000 \\ \mathrm{H} & -5.853071 & -1.250374 & 0.000000 \\ \mathrm{H} & -5.853071 & 1.250374 & 0.000000 \\ \mathrm{H} & -3.716259 & 2.505536 & 0.000000 \\ \mathrm{H} & -1.237471 & -2.505220 & 0.000000 \\ \mathrm{H} & -1.237471 & 2.505220 & 0.000000 \\ \mathrm{H} & 1.237471 & -2.505220 & 0.000000 \\ \mathrm{H} & 1.237471 & 2.505220 & 0.000000 \\ \mathrm{H} & 3.716259 & -2.505536 & 0.000000 \\ \mathrm{H} & 5.853071 & -1.250374 & 0.000000 \\ \mathrm{H} & 5.853071 & 1.250374 & 0.000000\end{array}$




\section{$\begin{array}{llll}\mathrm{H} & 3.716259 & 2.505536 & 0.000000\end{array}$}

tetracene $\mathrm{S}_{1}$

$\begin{array}{lrrr}\mathrm{C} & 3.723567 & 1.404860 & 0.000000 \\ \mathrm{C} & 2.481694 & 0.724335 & 0.000000 \\ \mathrm{C} & 2.481694 & -0.724335 & 0.000000 \\ \mathrm{C} & 3.723567 & -1.404860 & 0.000000 \\ \mathrm{C} & 4.936185 & -0.701765 & 0.000000 \\ \mathrm{C} & 4.936185 & 0.701765 & 0.000000 \\ \mathrm{C} & 1.237928 & 1.403860 & 0.000000 \\ \mathrm{C} & 0.000000 & 0.726429 & 0.000000 \\ \mathrm{C} & 0.000000 & -0.726429 & 0.000000 \\ \mathrm{C} & 1.237928 & -1.403860 & 0.000000 \\ \mathrm{C} & -1.237928 & 1.403860 & 0.000000 \\ \mathrm{C} & -2.481694 & 0.724335 & 0.000000 \\ \mathrm{C} & -2.481694 & -0.724335 & 0.000000 \\ \mathrm{C} & -1.237928 & -1.403860 & 0.000000 \\ \mathrm{C} & -3.723567 & 1.404860 & 0.000000 \\ \mathrm{C} & -4.936185 & 0.701765 & 0.000000 \\ \mathrm{C} & -4.936185 & -0.701765 & 0.000000 \\ \mathrm{C} & -3.723567 & -1.404860 & 0.000000 \\ \mathrm{H} & -3.725670 & -2.498055 & 0.000000 \\ \mathrm{H} & -5.881080 & -1.248832 & 0.000000 \\ \mathrm{H} & -5.881080 & 1.248832 & 0.000000 \\ \mathrm{H} & -3.725670 & 2.498055 & 0.000000 \\ \mathrm{H} & -1.239150 & -2.498341 & 0.000000 \\ \mathrm{H} & -1.239150 & 2.498341 & 0.000000 \\ \mathrm{H} & 1.239150 & -2.498341 & 0.000000 \\ \mathrm{H} & 1.239150 & 2.498341 & 0.000000 \\ \mathrm{H} & 3.725670 & -2.498055 & 0.000000 \\ \mathrm{H} & 5.881080 & -1.248832 & 0.000000 \\ \mathrm{H} & 5.881080 & 1.248832 & 0.000000 \\ \mathrm{H} & 3.725670 & 2.498055 & 0.000000\end{array}$

perylene $\mathrm{S}_{0}$

$\begin{array}{lrrr}\mathrm{C} & 0.021956 & 1.481761 & -2.434445 \\ \mathrm{C} & 0.011903 & 0.737528 & -1.251351 \\ \mathrm{C} & 0.012741 & 1.440221 & 0.000000 \\ \mathrm{C} & 0.034039 & 2.881388 & 0.000000 \\ \mathrm{C} & 0.043358 & 3.582016 & -1.235351 \\ \mathrm{C} & 0.037644 & 2.888932 & -2.429853 \\ \mathrm{C} & 0.043633 & 3.582010 & 1.235354 \\ \mathrm{C} & 0.038180 & 2.888923 & 2.429854 \\ \mathrm{C} & 0.022415 & 1.481753 & 2.434445\end{array}$




$\begin{array}{lrrr}\mathrm{C} & 0.012019 & 0.737525 & 1.251350 \\ \mathrm{C} & -0.011903 & -0.737528 & -1.251351 \\ \mathrm{C} & -0.012741 & -1.440221 & 0.000000 \\ \mathrm{C} & -0.012019 & -0.737525 & 1.251350 \\ \mathrm{C} & -0.034039 & -2.881388 & 0.000000 \\ \mathrm{C} & -0.043633 & -3.582010 & 1.235354 \\ \mathrm{C} & -0.038180 & -2.888923 & 2.429854 \\ \mathrm{C} & -0.022415 & -1.481753 & 2.434445 \\ \mathrm{C} & -0.021956 & -1.481761 & -2.434445 \\ \mathrm{C} & -0.037644 & -2.888932 & -2.429853 \\ \mathrm{C} & -0.043358 & -3.582016 & -1.235351 \\ \mathrm{H} & 0.056964 & 4.674670 & 1.221679 \\ \mathrm{H} & 0.046501 & 3.427793 & 3.379874 \\ \mathrm{H} & 0.020100 & 0.973374 & 3.399025 \\ \mathrm{H} & -0.056964 & -4.674670 & 1.221679 \\ \mathrm{H} & -0.046501 & -3.427793 & 3.379874 \\ \mathrm{H} & -0.020100 & -0.973374 & 3.399025 \\ \mathrm{H} & -0.056673 & -4.674676 & -1.221675 \\ \mathrm{H} & -0.045696 & -3.427805 & -3.379873 \\ \mathrm{H} & -0.019441 & -0.973390 & -3.399028 \\ \mathrm{H} & 0.056673 & 4.674676 & -1.221675 \\ \mathrm{H} & 0.045696 & 3.427805 & -3.379873 \\ \mathrm{H} & 0.019441 & 0.973390 & -3.399028\end{array}$

perylene $\mathrm{S}_{1}$

$\begin{array}{lrrr}\text { C } & -0.006797 & 1.475809 & -2.461927 \\ \text { C } & -0.006547 & 0.723014 & -1.248933 \\ \text { C } & 0.010499 & 1.430046 & 0.000002 \\ \text { C } & 0.008759 & 2.868081 & -0.000002 \\ \text { C } & 0.009395 & 3.565038 & -1.237625 \\ \text { C } & -0.001623 & 2.868449 & -2.447970 \\ \text { C } & 0.009655 & 3.565045 & 1.237613 \\ \text { C } & -0.001079 & 2.868461 & 2.447967 \\ \text { C } & -0.006323 & 1.475824 & 2.461932 \\ \text { C } & -0.006419 & 0.723017 & 1.248942 \\ \text { C } & 0.006547 & -0.723014 & -1.248933 \\ \text { C } & -0.010499 & -1.430046 & 0.000002 \\ \text { C } & 0.006419 & -0.723017 & 1.248942 \\ \text { C } & -0.008759 & -2.868081 & -0.000002 \\ \text { C } & -0.009655 & -3.565045 & 1.237613 \\ \text { C } & 0.001079 & -2.868461 & 2.447967 \\ \text { C } & 0.006323 & -1.475824 & 2.461932 \\ \text { C } & 0.006797 & -1.475809 & -2.461927 \\ \text { C } & 0.001623 & -2.868449 & -2.447970 \\ \text { C } & -0.009395 & -3.565038 & -1.237625\end{array}$




$\begin{array}{rrrr}\mathrm{H} & 0.014010 & 4.657751 & 1.228120 \\ \mathrm{H} & -0.004749 & 3.418325 & 3.391812 \\ \mathrm{H} & -0.028624 & 0.960490 & 3.421482 \\ \mathrm{H} & -0.014010 & -4.657751 & 1.228120 \\ \mathrm{H} & 0.004749 & -3.418325 & 3.391812 \\ \mathrm{H} & 0.028624 & -0.960490 & 3.421482 \\ \mathrm{H} & -0.013732 & -4.657744 & -1.228137 \\ \mathrm{H} & 0.005578 & -3.418305 & -3.391819 \\ \mathrm{H} & 0.029441 & -0.960464 & -3.421464 \\ \mathrm{H} & 0.013732 & 4.657744 & -1.228137 \\ \mathrm{H} & -0.005578 & 3.418305 & -3.391819 \\ \mathrm{H} & -0.029441 & 0.960464 & -3.421464\end{array}$

\section{B3LYP/TZVP geometries - Group I}

pyrazine $\mathrm{S}_{0}$

$\begin{array}{lrrr}\mathrm{C} & 1.131190 & -0.696330 & 0.000000 \\ \mathrm{C} & 1.131190 & 0.696330 & 0.000000 \\ \mathrm{~N} & 0.000000 & 1.402180 & 0.000000 \\ \mathrm{C} & -1.131190 & 0.696330 & 0.000000 \\ \mathrm{C} & -1.131190 & -0.696330 & 0.000000 \\ \mathrm{~N} & 0.000000 & -1.402180 & 0.000000 \\ \mathrm{H} & -2.062100 & -1.253940 & 0.000000 \\ \mathrm{H} & 2.062100 & -1.253940 & 0.000000 \\ \mathrm{H} & 2.062100 & 1.253940 & 0.000000 \\ \mathrm{H} & -2.062100 & 1.253940 & 0.000000\end{array}$

pyrazine $\mathrm{S}_{1}$

$\begin{array}{lrrl}\mathrm{C} & -1.210743 & 0.677974 & 0.000000 \\ \mathrm{C} & -1.115368 & -0.746154 & 0.000000 \\ \mathrm{~N} & 0.020130 & -1.350897 & 0.000000 \\ \mathrm{C} & 1.210743 & -0.677974 & 0.000000 \\ \mathrm{C} & 1.115368 & 0.746154 & 0.000000 \\ \mathrm{~N} & -0.020130 & 1.350897 & 0.000000 \\ \mathrm{H} & 2.040304 & 1.318591 & 0.000000 \\ \mathrm{H} & -2.157658 & 1.189742 & 0.000000 \\ \mathrm{H} & -2.040304 & -1.318591 & 0.000000 \\ \mathrm{H} & 2.157658 & -1.189742 & 0.000000\end{array}$

2,6-difluoropyridine $\mathrm{S}_{0}$
$\begin{array}{llll}\text { C } & 1.116540 & 0.000000 & -0.876120\end{array}$
$\begin{array}{llll}\mathrm{N} & 0.000000 & 0.000000 & -1.567360\end{array}$ 


$\begin{array}{lrrr}\mathrm{C} & -1.116540 & 0.000000 & -0.876120 \\ \mathrm{C} & -1.203790 & 0.000000 & 0.508170 \\ \mathrm{C} & 0.000000 & 0.000000 & 1.203840 \\ \mathrm{C} & 1.203790 & 0.000000 & 0.508170 \\ \mathrm{~F} & -2.248090 & 0.000000 & -1.594690 \\ \mathrm{H} & -2.164990 & 0.000000 & 1.001360 \\ \mathrm{H} & 0.000000 & 0.000000 & 2.286500 \\ \mathrm{H} & 2.164990 & 0.000000 & 1.001360 \\ \mathrm{~F} & 2.248090 & 0.000000 & -1.594690\end{array}$

2,6-difluoropyridine $\mathrm{S}_{1}$

$\begin{array}{crrr}\mathrm{C} & -1.103893 & 0.000000 & -0.336229 \\ \mathrm{~N} & 0.000000 & 0.000000 & -1.100834 \\ \mathrm{C} & 1.103893 & 0.000000 & -0.336229 \\ \mathrm{C} & 1.196771 & 0.000000 & 1.072742 \\ \mathrm{C} & 0.000000 & 0.000000 & 1.846564 \\ \mathrm{C} & -1.196771 & 0.000000 & 1.072742 \\ \mathrm{~F} & 2.247435 & 0.000000 & -1.018323 \\ \mathrm{H} & 2.180417 & 0.000000 & 1.522185 \\ \mathrm{H} & 0.000000 & 0.000000 & 2.922810 \\ \mathrm{H} & -2.180417 & 0.000000 & 1.522185 \\ \mathrm{~F} & -2.247435 & 0.000000 & -1.018323\end{array}$

2-fluoropyridine $\mathrm{S}_{0}$

$\begin{array}{lrrr}\mathrm{C} & -1.315853 & 0.503722 & 0.000000 \\ \mathrm{~N} & -1.366748 & -0.802315 & 0.000000 \\ \mathrm{C} & -0.196489 & -1.454306 & 0.000000 \\ \mathrm{C} & 1.031560 & -0.809659 & 0.000000 \\ \mathrm{C} & 1.045457 & 0.584268 & 0.000000 \\ \mathrm{C} & -0.157408 & 1.272923 & 0.000000 \\ \mathrm{H} & -0.258754 & -2.536633 & 0.000000 \\ \mathrm{H} & 1.949878 & -1.381146 & 0.000000 \\ \mathrm{H} & 1.982074 & 1.127628 & 0.000000 \\ \mathrm{H} & -0.213370 & 2.352400 & 0.000000 \\ \mathrm{~F} & -2.500357 & 1.143110 & 0.000000\end{array}$

2-fluoropyridine $\mathrm{S}_{1}$

$\begin{array}{lrrr}\mathrm{C} & -1.321412 & 0.492354 & 0.000000 \\ \mathrm{~N} & -1.436379 & -0.843123 & 0.000000 \\ \mathrm{C} & -0.222770 & -1.469195 & 0.000000 \\ \mathrm{C} & 1.032234 & -0.795732 & 0.000000 \\ \mathrm{C} & 1.108078 & 0.618532 & 0.000000 \\ \mathrm{C} & -0.153099 & 1.286990 & 0.000000\end{array}$




$\begin{array}{rrrr}\mathrm{H} & -0.249096 & -2.551238 & 0.000000 \\ \mathrm{H} & 1.932017 & -1.397666 & 0.000000 \\ \mathrm{H} & 2.044535 & 1.151359 & 0.000000 \\ \mathrm{H} & -0.250928 & 2.363071 & 0.000000 \\ \mathrm{~F} & -2.483190 & 1.144640 & 0.000000\end{array}$

2-hydroxypyridine $\mathrm{S}_{0}$

$\begin{array}{crcc}\mathrm{C} & -0.979745 & 0.752737 & 0.000000 \\ \mathrm{C} & -0.971189 & -0.646252 & 0.000000 \\ \mathrm{~N} & 0.132525 & -1.378349 & 0.000000 \\ \mathrm{C} & 1.307882 & -0.736636 & 0.000000 \\ \mathrm{C} & 1.420295 & 0.644346 & 0.000000 \\ \mathrm{C} & 0.243771 & 1.397052 & 0.000000 \\ \mathrm{O} & -2.155791 & -1.302427 & 0.000000 \\ \mathrm{H} & 2.190061 & -1.367992 & 0.000000 \\ \mathrm{H} & 2.393261 & 1.117298 & 0.000000 \\ \mathrm{H} & 0.285225 & 2.479714 & 0.000000 \\ \mathrm{H} & -1.918681 & 1.289069 & 0.000000 \\ \mathrm{H} & -1.947613 & -2.248560 & 0.000000\end{array}$

2-hydroxypyridine $\mathrm{S}_{1}$

$\begin{array}{rrrr}\mathrm{C} & -1.003650 & 0.769010 & 0.000000 \\ \mathrm{C} & -0.968870 & -0.634010 & 0.000000 \\ \mathrm{~N} & 0.117070 & -1.450530 & 0.000000 \\ \mathrm{C} & 1.299510 & -0.773490 & 0.000000 \\ \mathrm{C} & 1.394870 & 0.643760 & 0.000000 \\ \mathrm{C} & 0.252200 & 1.462530 & 0.000000 \\ \mathrm{O} & -2.136490 & -1.297400 & 0.000000 \\ \mathrm{H} & 2.198250 & -1.376730 & 0.000000 \\ \mathrm{H} & 2.384980 & 1.083950 & 0.000000 \\ \mathrm{H} & 0.312160 & 2.538970 & 0.000000 \\ \mathrm{H} & -1.957780 & 1.276520 & 0.000000 \\ \mathrm{H} & -1.892250 & -2.242580 & 0.000000\end{array}$

2-pyridone $\mathrm{S}_{0}$

$\begin{array}{cccc}\mathrm{C} & -1.248580 & 0.717660 & 0.000000 \\ \mathrm{~N} & -1.136580 & -0.688950 & 0.000000 \\ \mathrm{C} & 0.033150 & -1.386450 & 0.000000 \\ \mathrm{C} & 1.224060 & -0.732630 & 0.000000 \\ \mathrm{C} & 1.206220 & 0.691100 & 0.000000 \\ \mathrm{C} & 0.037050 & 1.387730 & 0.000000 \\ \mathrm{H} & -2.021220 & -1.179140 & 0.000000 \\ \mathrm{H} & -0.054060 & -2.464810 & 0.000000\end{array}$




$\begin{array}{rrrr}\mathrm{H} & 2.151220 & -1.286170 & 0.000000 \\ \mathrm{H} & 2.147790 & 1.228840 & 0.000000 \\ \mathrm{H} & 0.013300 & 2.468720 & 0.000000 \\ \mathrm{O} & -2.352350 & 1.244110 & 0.000000\end{array}$

2-pyridone $\mathrm{S}_{1}$

$\begin{array}{rrrr}\mathrm{C} & -1.367970 & 0.102400 & 0.000000 \\ \mathrm{~N} & -0.732940 & -1.119770 & 0.000000 \\ \mathrm{C} & 0.634630 & -1.256190 & 0.000000 \\ \mathrm{C} & 1.420460 & -0.075740 & 0.000000 \\ \mathrm{C} & 0.832890 & 1.162830 & 0.000000 \\ \mathrm{C} & -0.616660 & 1.265490 & 0.000000 \\ \mathrm{H} & -1.348740 & -1.922210 & 0.000000 \\ \mathrm{H} & 1.036340 & -2.256140 & 0.000000 \\ \mathrm{H} & 2.497150 & -0.184600 & 0.000000 \\ \mathrm{H} & 1.436620 & 2.059480 & 0.000000 \\ \mathrm{H} & -1.132720 & 2.212420 & 0.000000 \\ \mathrm{O} & -2.659060 & 0.012020 & 0.000000\end{array}$

2-methylpyrimidine $\mathrm{S}_{0}$

$\begin{array}{lrrr}\mathrm{C} & 0.000134 & 1.965897 & 0.000000 \\ \mathrm{C} & -1.175835 & 1.226533 & 0.000000 \\ \mathrm{~N} & -1.182269 & -0.104064 & 0.000000 \\ \mathrm{C} & 0.010461 & -0.717842 & 0.000000 \\ \mathrm{~N} & 1.196513 & -0.096938 & 0.000000 \\ \mathrm{C} & 1.179438 & 1.236938 & 0.000000 \\ \mathrm{H} & -2.146217 & 1.713674 & 0.000000 \\ \mathrm{H} & 2.146662 & 1.729909 & 0.000000 \\ \mathrm{H} & -0.004483 & 3.047398 & 0.000000 \\ \mathrm{C} & 0.004978 & -2.217575 & 0.000000 \\ \mathrm{H} & 1.022884 & -2.600221 & 0.000000 \\ \mathrm{H} & -0.526138 & -2.591854 & 0.877751 \\ \mathrm{H} & -0.526138 & -2.591854 & -0.877751\end{array}$

2-methylpyrimidine $\mathrm{S}_{1}$

$\begin{array}{rrrr}\mathrm{C} & -0.013510 & 2.014240 & 0.000000 \\ \mathrm{C} & -1.170020 & 1.280130 & 0.000000 \\ \mathrm{~N} & -1.154980 & -0.093600 & 0.000000 \\ \mathrm{C} & 0.013780 & -0.784090 & 0.000000 \\ \mathrm{~N} & 1.092080 & -0.070360 & 0.000000 \\ \mathrm{C} & 1.207090 & 1.296040 & 0.000000 \\ \mathrm{H} & -2.148980 & 1.742930 & 0.000000 \\ \mathrm{H} & 2.193220 & 1.728900 & 0.000000\end{array}$




$\begin{array}{rrrr}\mathrm{H} & -0.025940 & 3.096510 & 0.000000 \\ \mathrm{C} & 0.012880 & -2.272160 & 0.000000 \\ \mathrm{H} & 1.030880 & -2.657990 & 0.000000 \\ \mathrm{H} & -0.518250 & -2.640280 & 0.881210 \\ \mathrm{H} & -0.518250 & -2.640280 & -0.881210\end{array}$

5-methylpyrimidine $\mathrm{S}_{0}$

$\begin{array}{lrrr}\mathrm{C} & 0.875259 & 0.852142 & 0.000000 \\ \mathrm{C} & -0.360891 & 0.210967 & 0.000000 \\ \mathrm{C} & -0.300073 & -1.183018 & 0.000000 \\ \mathrm{~N} & 0.845885 & -1.861596 & 0.000000 \\ \mathrm{C} & 1.962118 & -1.131811 & 0.000000 \\ \mathrm{~N} & 2.037392 & 0.196864 & 0.000000 \\ \mathrm{H} & 2.900451 & -1.675458 & 0.000000 \\ \mathrm{H} & -1.210932 & -1.776272 & 0.000000 \\ \mathrm{H} & 0.933628 & 1.937292 & 0.000000 \\ \mathrm{C} & -1.663399 & 0.960735 & 0.000000 \\ \mathrm{H} & -1.495038 & 2.038255 & 0.000000 \\ \mathrm{H} & -2.262200 & 0.715941 & -0.880517 \\ \mathrm{H} & -2.262200 & 0.715941 & 0.880517\end{array}$

5-methylpyrimidine $\mathrm{S}_{1}$

$\begin{array}{rrrr}\mathrm{C} & 0.846500 & 0.858370 & 0.000000 \\ \mathrm{C} & -0.377980 & 0.242810 & 0.000000 \\ \mathrm{C} & -0.349130 & -1.191800 & 0.000000 \\ \mathrm{~N} & 0.883900 & -1.765670 & 0.000000 \\ \mathrm{C} & 2.035390 & -1.182870 & 0.000000 \\ \mathrm{~N} & 2.034000 & 0.172120 & 0.000000 \\ \mathrm{H} & 2.962350 & -1.737440 & 0.000000 \\ \mathrm{H} & -1.218410 & -1.828680 & 0.000000 \\ \mathrm{H} & 0.933000 & 1.939050 & 0.000000 \\ \mathrm{C} & -1.679340 & 0.982080 & 0.000000 \\ \mathrm{H} & -1.519170 & 2.060820 & 0.000000 \\ \mathrm{H} & -2.275560 & 0.725610 & -0.881320 \\ \mathrm{H} & -2.275560 & 0.725610 & 0.881320\end{array}$

7-azaindole $\mathrm{S}_{0}$

$\begin{array}{rrrr}\mathrm{N} & -0.634970 & 1.846100 & 0.000000 \\ \mathrm{C} & -0.676370 & 0.472580 & 0.000000 \\ \mathrm{C} & 0.667750 & 0.006920 & 0.000000 \\ \mathrm{C} & 1.509400 & 1.166440 & 0.000000 \\ \mathrm{C} & 0.683460 & 2.256530 & 0.000000 \\ \mathrm{C} & 0.845260 & -1.378550 & 0.000000\end{array}$




$\begin{array}{rrrr}\mathrm{C} & -0.291770 & -2.174100 & 0.000000 \\ \mathrm{C} & -1.564960 & -1.583440 & 0.000000 \\ \mathrm{~N} & -1.777710 & -0.267220 & 0.000000 \\ \mathrm{H} & -2.452080 & -2.208010 & 0.000000 \\ \mathrm{H} & 1.835160 & -1.820180 & 0.000000 \\ \mathrm{H} & -0.210460 & -3.253650 & 0.000000 \\ \mathrm{H} & -1.448720 & 2.438020 & 0.000000 \\ \mathrm{H} & 2.587050 & 1.192430 & 0.000000 \\ \mathrm{H} & 0.928980 & 3.306130 & 0.000000\end{array}$

7-azaindole $\mathrm{S}_{1}$

$\begin{array}{rrrr}\mathrm{N} & -0.600874 & 1.877305 & 0.000000 \\ \mathrm{C} & -0.654691 & 0.442294 & 0.000000 \\ \mathrm{C} & 0.647573 & -0.011575 & 0.000000 \\ \mathrm{C} & 1.494489 & 1.157693 & 0.000000 \\ \mathrm{C} & 0.655160 & 2.313806 & 0.000000 \\ \mathrm{C} & 0.888831 & -1.413823 & 0.000000 \\ \mathrm{C} & -0.306364 & -2.187238 & 0.000000 \\ \mathrm{C} & -1.555062 & -1.598962 & 0.000000 \\ \mathrm{~N} & -1.809995 & -0.234370 & 0.000000 \\ \mathrm{H} & -2.441145 & -2.223042 & 0.000000 \\ \mathrm{H} & 1.876195 & -1.847621 & 0.000000 \\ \mathrm{H} & -0.249492 & -3.268751 & 0.000000 \\ \mathrm{H} & -1.434217 & 2.449421 & 0.000000 \\ \mathrm{H} & 2.572812 & 1.186955 & 0.000000 \\ \mathrm{H} & 0.916789 & 3.357928 & 0.000000\end{array}$

7-hydroxyquinoline, cis $\mathrm{S}_{0}$

$\begin{array}{lrrl}\mathrm{N} & 0.463212 & 1.972567 & 0.000000 \\ \mathrm{C} & 0.435680 & 0.610714 & 0.000000 \\ \mathrm{C} & -0.788385 & -0.125810 & 0.000000 \\ \mathrm{C} & -1.993589 & 0.607913 & 0.000000 \\ \mathrm{C} & -1.947687 & 1.978618 & 0.000000 \\ \mathrm{C} & -0.685515 & 2.611187 & 0.000000 \\ \mathrm{C} & 1.664141 & -0.089125 & 0.000000 \\ \mathrm{C} & 1.677260 & -1.462936 & 0.000000 \\ \mathrm{C} & 0.467731 & -2.199223 & 0.000000 \\ \mathrm{C} & -0.731647 & -1.542420 & 0.000000 \\ \mathrm{H} & -0.629875 & 3.696176 & 0.000000 \\ \mathrm{H} & -1.657675 & -2.105932 & 0.000000 \\ \mathrm{H} & 0.522076 & -3.279986 & 0.000000 \\ \mathrm{O} & 2.825296 & -2.202769 & 0.000000 \\ \mathrm{H} & 2.579248 & 0.492010 & 0.000000 \\ \mathrm{H} & -2.940507 & 0.079394 & 0.000000\end{array}$




$$
\begin{array}{rrrr}
H & -2.850440 & 2.575490 & 0.000000 \\
H & 3.590677 & -1.615858 & 0.000000
\end{array}
$$

7-hydroxyquinoline, cis $\mathrm{S}_{1}$

$$
\begin{array}{rrrr}
\mathrm{N} & 0.493129 & 1.973576 & 0.000000 \\
\mathrm{C} & 0.424420 & 0.632217 & 0.000000 \\
\mathrm{C} & -0.787155 & -0.132513 & 0.000000 \\
\mathrm{C} & -2.008391 & 0.599837 & 0.000000 \\
\mathrm{C} & -1.928527 & 2.003748 & 0.000000 \\
\mathrm{C} & -0.698486 & 2.643401 & 0.000000 \\
\mathrm{C} & 1.638730 & -0.096813 & 0.000000 \\
\mathrm{C} & 1.661854 & -1.523983 & 0.000000 \\
\mathrm{C} & 0.487107 & -2.268646 & 0.000000 \\
\mathrm{C} & -0.711881 & -1.550243 & 0.000000 \\
\mathrm{H} & -0.633518 & 3.723955 & 0.000000 \\
\mathrm{H} & -1.645161 & -2.102975 & 0.000000 \\
\mathrm{H} & 0.519372 & -3.347391 & 0.000000 \\
\mathrm{O} & 2.843084 & -2.168678 & 0.000000 \\
\mathrm{H} & 2.567698 & 0.464288 & 0.000000 \\
\mathrm{H} & -2.961138 & 0.088459 & 0.000000 \\
\mathrm{H} & -2.835372 & 2.596920 & 0.000000 \\
\mathrm{H} & 3.574235 & -1.535151 & 0.000000
\end{array}
$$

7-hydroxyquinoline, trans $\mathrm{S}_{0}$

$\begin{array}{rrrr}\mathrm{N} & 0.513612 & 2.044862 & 0.000000 \\ \mathrm{C} & 0.483463 & 0.681009 & 0.000000 \\ \mathrm{C} & -0.746073 & -0.049464 & 0.000000 \\ \mathrm{C} & -1.949929 & 0.689066 & 0.000000 \\ \mathrm{C} & -1.898376 & 2.058175 & 0.000000 \\ \mathrm{C} & -0.631762 & 2.686080 & 0.000000 \\ \mathrm{C} & 1.708642 & -0.018818 & 0.000000 \\ \mathrm{C} & 1.717999 & -1.392709 & 0.000000 \\ \mathrm{C} & 0.504371 & -2.122427 & 0.000000 \\ \mathrm{C} & -0.696620 & -1.463138 & 0.000000 \\ \mathrm{H} & -0.573027 & 3.771046 & 0.000000 \\ \mathrm{H} & -1.623580 & -2.025042 & 0.000000 \\ \mathrm{H} & 0.532433 & -3.207328 & 0.000000 \\ \mathrm{O} & 2.927117 & -2.030529 & 0.000000 \\ \mathrm{H} & 2.633591 & 0.541074 & 0.000000 \\ \mathrm{H} & -2.898796 & 0.163990 & 0.000000 \\ \mathrm{H} & -2.798330 & 2.659315 & 0.000000 \\ \mathrm{H} & 2.795245 & -2.985141 & 0.000000\end{array}$

7-hydroxyquinoline, trans $\mathrm{S}_{1}$ 


$\begin{array}{rrrr}\mathrm{N} & 0.374425 & 1.966977 & 0.000107 \\ \mathrm{C} & 0.522214 & 0.653656 & 0.000026 \\ \mathrm{C} & -0.756709 & -0.066200 & 0.000014 \\ \mathrm{C} & -1.950595 & 0.714155 & -0.000011 \\ \mathrm{C} & -1.920914 & 2.105646 & 0.000010 \\ \mathrm{C} & -0.697727 & 2.764388 & 0.000020 \\ \mathrm{C} & 1.739091 & -0.035915 & -0.000019 \\ \mathrm{C} & 1.726812 & -1.433810 & -0.000021 \\ \mathrm{C} & 0.537859 & -2.149485 & 0.000000 \\ \mathrm{C} & -0.684800 & -1.458839 & 0.000018 \\ \mathrm{H} & -0.556966 & 3.834453 & -0.000004 \\ \mathrm{H} & -1.614337 & -2.015066 & 0.000048 \\ \mathrm{H} & 0.550250 & -3.233088 & 0.000016 \\ \mathrm{O} & 2.950816 & -2.043061 & -0.000045 \\ \mathrm{H} & 2.676084 & 0.499874 & -0.000031 \\ \mathrm{H} & -2.901380 & 0.197142 & -0.000072 \\ \mathrm{H} & -2.825321 & 2.698768 & -0.000022 \\ \mathrm{H} & 2.831198 & -2.999613 & -0.000034\end{array}$

2-hydroxyquinoline, enol $\mathrm{S}_{0}$

$\begin{array}{rrrr}\mathrm{C} & 0.432526 & 1.905024 & 0.000000 \\ \mathrm{C} & 0.487973 & 0.492120 & 0.000000 \\ \mathrm{C} & -0.724642 & -0.259177 & 0.000000 \\ \mathrm{C} & -1.955863 & 0.436940 & 0.000000 \\ \mathrm{C} & -1.977975 & 1.810115 & 0.000000 \\ \mathrm{C} & -0.777659 & 2.553229 & 0.000000 \\ \mathrm{C} & 1.699019 & -0.242477 & 0.000000 \\ \mathrm{C} & 1.666814 & -1.607096 & 0.000000 \\ \mathrm{C} & 0.396947 & -2.251909 & 0.000000 \\ \mathrm{~N} & -0.739995 & -1.621145 & 0.000000 \\ \mathrm{O} & 0.315142 & -3.610786 & 0.000000 \\ \mathrm{H} & 1.359345 & 2.467868 & 0.000000 \\ \mathrm{H} & -0.816333 & 3.635372 & 0.000000 \\ \mathrm{H} & -2.926793 & 2.332878 & 0.000000 \\ \mathrm{H} & -2.866754 & -0.147319 & 0.000000 \\ \mathrm{H} & 2.645736 & 0.285706 & 0.000000 \\ \mathrm{H} & 2.581258 & -2.189230 & 0.000000 \\ \mathrm{H} & 1.201253 & -3.990112 & 0.000000\end{array}$

2-hydroxyquinoline, enol $\mathrm{S}_{1}$
$\begin{array}{llll}\text { C } & 0.425898 & 1.880509 & 0.000000\end{array}$
$\begin{array}{llll}\text { C } & 0.521875 & 0.485994 & 0.000000\end{array}$
$\begin{array}{llll}\text { C } & -0.710898 & -0.259207 & 0.000000\end{array}$ 


$\begin{array}{crrr}\mathrm{C} & -1.949244 & 0.444793 & 0.000000 \\ \mathrm{C} & -2.011955 & 1.851312 & 0.000000 \\ \mathrm{C} & -0.829088 & 2.561884 & 0.000000 \\ \mathrm{C} & 1.742155 & -0.232709 & 0.000000 \\ \mathrm{C} & 1.696365 & -1.646695 & 0.000000 \\ \mathrm{C} & 0.437175 & -2.248627 & 0.000000 \\ \mathrm{~N} & -0.748406 & -1.610408 & 0.000000 \\ \mathrm{O} & 0.292665 & -3.591098 & 0.000000 \\ \mathrm{H} & 1.335464 & 2.470679 & 0.000000 \\ \mathrm{H} & -0.833793 & 3.644580 & 0.000000 \\ \mathrm{H} & -2.967980 & 2.356199 & 0.000000 \\ \mathrm{H} & -2.851007 & -0.154336 & 0.000000 \\ \mathrm{H} & 2.685581 & 0.295425 & 0.000000 \\ \mathrm{H} & 2.599767 & -2.242334 & 0.000000 \\ \mathrm{H} & 1.165416 & -4.005941 & 0.000000\end{array}$

2-hydroxyquinoline, keto $\mathrm{S}_{0}$

$\begin{array}{rrrr}\mathrm{N} & 0.559580 & 1.707510 & 0.000000 \\ \mathrm{C} & 0.604000 & 0.328810 & 0.000000 \\ \mathrm{C} & -0.616210 & -0.383390 & 0.000000 \\ \mathrm{C} & -1.837040 & 0.377280 & 0.000000 \\ \mathrm{C} & -1.835800 & 1.727350 & 0.000000 \\ \mathrm{C} & -0.592950 & 2.494530 & 0.000000 \\ \mathrm{C} & 1.821660 & -0.364500 & 0.000000 \\ \mathrm{C} & 1.824330 & -1.747780 & 0.000000 \\ \mathrm{C} & 0.622780 & -2.467180 & 0.000000 \\ \mathrm{C} & -0.580280 & -1.786970 & 0.000000 \\ \mathrm{H} & -1.517450 & -2.331920 & 0.000000 \\ \mathrm{H} & 0.638610 & -3.549490 & 0.000000 \\ \mathrm{H} & 2.769320 & -2.277440 & 0.000000 \\ \mathrm{H} & 2.754700 & 0.187770 & 0.000000 \\ \mathrm{H} & -2.776740 & -0.165420 & 0.000000 \\ \mathrm{H} & -2.748940 & 2.306590 & 0.000000 \\ \mathrm{O} & -0.515180 & 3.714290 & 0.000000 \\ \mathrm{H} & 1.425630 & 2.229980 & 0.000000\end{array}$

2-hydroxyquinoline, keto $\mathrm{S}_{1}$
$\begin{array}{llll}\mathrm{N} & 0.557295 & 1.679745 & 0.000000\end{array}$
$\begin{array}{llll}\text { C } & 0.606723 & 0.320103 & 0.000000\end{array}$
$\begin{array}{llll}\text { C } & -0.666018 & -0.381303 & 0.000000\end{array}$
$\begin{array}{llll}\text { C } & -1.862653 & 0.366466 & 0.000000\end{array}$
$\begin{array}{llll}\text { C } & -1.860503 & 1.772967 & 0.000000\end{array}$
$\begin{array}{llll}\text { C } & -0.631591 & 2.473534 & 0.000000\end{array}$
$\begin{array}{llll}\text { C } & 1.834263 & -0.363953 & 0.000000\end{array}$ 


$\begin{array}{rrrr}\mathrm{C} & 1.860816 & -1.758543 & 0.000000 \\ \mathrm{C} & 0.642773 & -2.453594 & 0.000000 \\ \mathrm{C} & -0.588512 & -1.786036 & 0.000000 \\ \mathrm{H} & -1.506730 & -2.359696 & 0.000000 \\ \mathrm{H} & 0.649298 & -3.536814 & 0.000000 \\ \mathrm{H} & 2.801075 & -2.290980 & 0.000000 \\ \mathrm{H} & 2.755285 & 0.207812 & 0.000000 \\ \mathrm{H} & -2.802533 & -0.170102 & 0.000000 \\ \mathrm{H} & -2.767479 & 2.356084 & 0.000000 \\ \mathrm{O} & -0.430746 & 3.695973 & 0.000000 \\ \mathrm{H} & 1.409238 & 2.228347 & 0.000000\end{array}$

dibenzofuran $\mathrm{S}_{0}$

$\begin{array}{rrrr}\mathrm{C} & 1.721087 & 0.000000 & -1.358801 \\ \mathrm{C} & 0.724932 & 0.000000 & -0.381673 \\ \mathrm{C} & 1.099164 & 0.000000 & 0.971297 \\ \mathrm{C} & 2.415730 & 0.000000 & 1.395052 \\ \mathrm{C} & 3.392158 & 0.000000 & 0.403408 \\ \mathrm{C} & 3.050334 & 0.000000 & -0.954816 \\ \mathrm{C} & -0.724932 & 0.000000 & -0.381673 \\ \mathrm{C} & -1.099164 & 0.000000 & 0.971297 \\ \mathrm{O} & 0.000000 & 0.000000 & 1.799541 \\ \mathrm{C} & -1.721087 & 0.000000 & -1.358801 \\ \mathrm{C} & -3.050334 & 0.000000 & -0.954816 \\ \mathrm{C} & -3.392158 & 0.000000 & 0.403408 \\ \mathrm{C} & -2.415730 & 0.000000 & 1.395052 \\ \mathrm{H} & -2.666382 & 0.000000 & 2.447340 \\ \mathrm{H} & -4.436524 & 0.000000 & 0.689334 \\ \mathrm{H} & -3.836321 & 0.000000 & -1.699443 \\ \mathrm{H} & -1.465889 & 0.000000 & -2.411303 \\ \mathrm{H} & 1.465889 & 0.000000 & -2.411303 \\ \mathrm{H} & 3.836321 & 0.000000 & -1.699443 \\ \mathrm{H} & 4.436524 & 0.000000 & 0.689334 \\ \mathrm{H} & 2.666382 & 0.000000 & 2.447340\end{array}$

dibenzofuran $\mathrm{S}_{1}$

$\begin{array}{lrrr}\text { C } & 0.000000 & 1.735158 & -1.532005 \\ \mathrm{C} & 0.000000 & 0.712569 & -0.568164 \\ \mathrm{C} & 0.000000 & 1.108004 & 0.801527 \\ \mathrm{C} & 0.000000 & 2.412280 & 1.256919 \\ \mathrm{C} & 0.000000 & 3.427404 & 0.264658 \\ \mathrm{C} & 0.000000 & 3.079716 & -1.086254 \\ \mathrm{C} & 0.000000 & -0.712569 & -0.568164 \\ \mathrm{C} & 0.000000 & -1.108004 & 0.801527\end{array}$




$\begin{array}{lrrr}\mathrm{O} & 0.000000 & 0.000000 & 1.607013 \\ \mathrm{C} & 0.000000 & -1.735158 & -1.532005 \\ \mathrm{C} & 0.000000 & -3.079716 & -1.086254 \\ \mathrm{C} & 0.000000 & -3.427404 & 0.264658 \\ \mathrm{C} & 0.000000 & -2.412280 & 1.256919 \\ \mathrm{H} & 0.000000 & -2.637285 & 2.314292 \\ \mathrm{H} & 0.000000 & -4.467151 & 0.559150 \\ \mathrm{H} & 0.000000 & -3.867781 & -1.829560 \\ \mathrm{H} & 0.000000 & -1.513015 & -2.590358 \\ \mathrm{H} & 0.000000 & 1.513015 & -2.590358 \\ \mathrm{H} & 0.000000 & 3.867781 & -1.829560 \\ \mathrm{H} & 0.000000 & 4.467151 & 0.559150 \\ \mathrm{H} & 0.000000 & 2.637285 & 2.314292\end{array}$

pyrrolo[3,2-h]quinoline $\mathrm{S}_{0}$

$\begin{array}{lrrr}\mathrm{C} & 0.132211 & -0.688029 & 0.000000 \\ \mathrm{~N} & 0.062551 & -2.040871 & 0.000000 \\ \mathrm{C} & 1.198020 & -2.711778 & 0.000000 \\ \mathrm{C} & 2.466188 & -2.101586 & 0.000000 \\ \mathrm{C} & 2.542608 & -0.727647 & 0.000000 \\ \mathrm{C} & 1.361267 & 0.039596 & 0.000000 \\ \mathrm{H} & 1.117684 & -3.794682 & 0.000000 \\ \mathrm{H} & 3.358540 & -2.714137 & 0.000000 \\ \mathrm{H} & 3.504025 & -0.226030 & 0.000000 \\ \mathrm{C} & -1.054331 & 0.080678 & 0.000000 \\ \mathrm{C} & -1.058746 & 1.485548 & 0.000000 \\ \mathrm{C} & 0.180699 & 2.177830 & 0.000000 \\ \mathrm{C} & 1.350860 & 1.469633 & 0.000000 \\ \mathrm{H} & 0.196617 & 3.261126 & 0.000000 \\ \mathrm{H} & 2.302376 & 1.988556 & 0.000000 \\ \mathrm{C} & -2.429528 & 1.891669 & 0.000000 \\ \mathrm{C} & -3.187236 & 0.747945 & 0.000000 \\ \mathrm{~N} & -2.353214 & -0.346638 & 0.000000 \\ \mathrm{H} & -2.623502 & -1.316099 & 0.000000 \\ \mathrm{H} & -2.809512 & 2.900198 & 0.000000 \\ \mathrm{H} & -4.257565 & 0.624720 & 0.000000\end{array}$

pyrrolo[3,2-h]quinoline $\mathrm{S}_{1}$

$\begin{array}{lrrr}\mathrm{C} & 0.154831 & -0.699743 & 0.000000 \\ \mathrm{~N} & 0.011790 & -2.040886 & 0.000000 \\ \mathrm{C} & 1.203468 & -2.734803 & 0.000000 \\ \mathrm{C} & 2.443980 & -2.135667 & 0.000000 \\ \mathrm{C} & 2.574115 & -0.728896 & 0.000000 \\ \mathrm{C} & 1.357422 & 0.021956 & 0.000000\end{array}$




$\begin{array}{rrrr}\mathrm{H} & 1.113310 & -3.814359 & 0.000000 \\ \mathrm{H} & 3.328742 & -2.761299 & 0.000000 \\ \mathrm{H} & 3.537815 & -0.241030 & 0.000000 \\ \mathrm{C} & -1.029763 & 0.102235 & 0.000000 \\ \mathrm{C} & -1.067059 & 1.551347 & 0.000000 \\ \mathrm{C} & 0.143268 & 2.230976 & 0.000000 \\ \mathrm{C} & 1.314790 & 1.459779 & 0.000000 \\ \mathrm{H} & 0.194319 & 3.310977 & 0.000000 \\ \mathrm{H} & 2.266938 & 1.979280 & 0.000000 \\ \mathrm{C} & -2.459716 & 1.911279 & 0.000000 \\ \mathrm{C} & -3.175518 & 0.755088 & 0.000000 \\ \mathrm{~N} & -2.290400 & -0.340180 & 0.000000 \\ \mathrm{H} & -2.513596 & -1.325699 & 0.000000 \\ \mathrm{H} & -2.868637 & 2.907739 & 0.000000 \\ \mathrm{H} & -4.240110 & 0.591910 & 0.000000\end{array}$

carbazole $\mathrm{S}_{0}$

$\begin{array}{lrrr}\mathrm{C} & 1.131414 & 0.000000 & -0.899123 \\ \mathrm{~N} & 0.000000 & 0.000000 & -1.697447 \\ \mathrm{C} & -1.131414 & 0.000000 & -0.899123 \\ \mathrm{C} & -0.723937 & 0.000000 & 0.457912 \\ \mathrm{C} & 0.723937 & 0.000000 & 0.457912 \\ \mathrm{C} & -1.694804 & 0.000000 & 1.460909 \\ \mathrm{C} & -3.036212 & 0.000000 & 1.104934 \\ \mathrm{C} & -3.419430 & 0.000000 & -0.243509 \\ \mathrm{C} & -2.475717 & 0.000000 & -1.262040 \\ \mathrm{C} & 2.475717 & 0.000000 & -1.262040 \\ \mathrm{C} & 3.419430 & 0.000000 & -0.243509 \\ \mathrm{C} & 3.036212 & 0.000000 & 1.104934 \\ \mathrm{C} & 1.694804 & 0.000000 & 1.460909 \\ \mathrm{H} & -2.779047 & 0.000000 & -2.302156 \\ \mathrm{H} & -4.472335 & 0.000000 & -0.497445 \\ \mathrm{H} & -3.797580 & 0.000000 & 1.874708 \\ \mathrm{H} & -1.406314 & 0.000000 & 2.505214 \\ \mathrm{H} & 1.406314 & 0.000000 & 2.505214 \\ \mathrm{H} & 3.797580 & 0.000000 & 1.874708 \\ \mathrm{H} & 4.472335 & 0.000000 & -0.497445 \\ \mathrm{H} & 2.779047 & 0.000000 & -2.302156 \\ \mathrm{H} & 0.000000 & 0.000000 & -2.702320\end{array}$

carbazole $\mathrm{S}_{1}$

$\begin{array}{rrrr}\mathrm{C} & 1.144511 & 0.000000 & -0.836430 \\ \mathrm{~N} & 0.000000 & 0.000000 & -1.613816 \\ \mathrm{C} & -1.144511 & 0.000000 & -0.836430\end{array}$ 


$\begin{array}{lrrr}\mathrm{C} & -0.716813 & 0.000000 & 0.528938 \\ \mathrm{C} & 0.716813 & 0.000000 & 0.528938 \\ \mathrm{C} & -1.708787 & 0.000000 & 1.521762 \\ \mathrm{C} & -3.064785 & 0.000000 & 1.119765 \\ \mathrm{C} & -3.454684 & 0.000000 & -0.216484 \\ \mathrm{C} & -2.469182 & 0.000000 & -1.239805 \\ \mathrm{C} & 2.469182 & 0.000000 & -1.239805 \\ \mathrm{C} & 3.454684 & 0.000000 & -0.216484 \\ \mathrm{C} & 3.064785 & 0.000000 & 1.119765 \\ \mathrm{C} & 1.708787 & 0.000000 & 1.521762 \\ \mathrm{H} & -2.744040 & 0.000000 & -2.287029 \\ \mathrm{H} & -4.503396 & 0.000000 & -0.478114 \\ \mathrm{H} & -3.829729 & 0.000000 & 1.887404 \\ \mathrm{H} & -1.452596 & 0.000000 & 2.572406 \\ \mathrm{H} & 1.452596 & 0.000000 & 2.572406 \\ \mathrm{H} & 3.829729 & 0.000000 & 1.887404 \\ \mathrm{H} & 4.503396 & 0.000000 & -0.478114 \\ \mathrm{H} & 2.744040 & 0.000000 & -2.287029 \\ \mathrm{H} & 0.000000 & 0.000000 & -2.623638\end{array}$

tryptamine, A-ph $\mathrm{S}_{0}$

$\begin{array}{lrrr}\mathrm{C} & -2.127843 & 0.129408 & 0.886846 \\ \mathrm{~N} & -1.251512 & 0.122161 & 1.956721 \\ \mathrm{C} & 0.038582 & 0.045009 & 1.481507 \\ \mathrm{C} & -0.040221 & -0.003149 & 0.065371 \\ \mathrm{C} & -1.436296 & 0.052848 & -0.291117 \\ \mathrm{C} & 1.255996 & 0.018232 & 2.161125 \\ \mathrm{C} & 2.412852 & -0.056469 & 1.402100 \\ \mathrm{C} & 2.360381 & -0.100798 & -0.002278 \\ \mathrm{C} & 1.149583 & -0.074634 & -0.673489 \\ \mathrm{C} & -2.013016 & 0.004943 & -1.673152 \\ \mathrm{C} & -2.008473 & -1.399678 & -2.292335 \\ \mathrm{~N} & -2.507253 & -1.353384 & -3.670296 \\ \mathrm{H} & 1.299691 & 0.055574 & 3.243353 \\ \mathrm{H} & 3.374756 & -0.078942 & 1.899336 \\ \mathrm{H} & 3.284578 & -0.155009 & -0.564358 \\ \mathrm{H} & 1.124185 & -0.105641 & -1.756242 \\ \mathrm{H} & -1.515234 & 0.190573 & 2.923637 \\ \mathrm{H} & -3.191417 & 0.195273 & 1.053540 \\ \mathrm{H} & -1.459437 & 0.670660 & -2.341463 \\ \mathrm{H} & -3.042905 & 0.376815 & -1.651116 \\ \mathrm{H} & -2.576453 & -2.074845 & -1.635382 \\ \mathrm{H} & -0.983752 & -1.776649 & -2.319848 \\ \mathrm{H} & -3.489584 & -1.100443 & -3.688689 \\ \mathrm{H} & -2.424619 & -2.259475 & -4.116401\end{array}$


tryptamine, A-ph $\mathrm{S}_{1}$

$\begin{array}{rrrr}\mathrm{C} & -2.182810 & 0.168181 & 0.922039 \\ \mathrm{~N} & -1.294304 & 0.137456 & 1.922572 \\ \mathrm{C} & 0.036331 & 0.054503 & 1.436572 \\ \mathrm{C} & -0.039887 & 0.040993 & 0.050360 \\ \mathrm{C} & -1.442055 & 0.109966 & -0.300173 \\ \mathrm{C} & 1.233327 & -0.025787 & 2.173605 \\ \mathrm{C} & 2.415132 & -0.112372 & 1.361093 \\ \mathrm{C} & 2.369555 & -0.124587 & -0.016735 \\ \mathrm{C} & 1.138993 & -0.052440 & -0.739406 \\ \mathrm{C} & -2.026931 & 0.049791 & -1.654068 \\ \mathrm{C} & -1.992622 & -1.384658 & -2.252704 \\ \mathrm{~N} & -2.513705 & -1.369719 & -3.615031 \\ \mathrm{H} & 1.270171 & -0.016973 & 3.252359 \\ \mathrm{H} & 3.377465 & -0.170806 & 1.855919 \\ \mathrm{H} & 3.298692 & -0.190022 & -0.570199 \\ \mathrm{H} & 1.113519 & -0.020181 & -1.818440 \\ \mathrm{H} & -1.531416 & 0.187132 & 2.901964 \\ \mathrm{H} & -3.243711 & 0.255965 & 1.084317 \\ \mathrm{H} & -1.460661 & 0.688733 & -2.336168 \\ \mathrm{H} & -3.062296 & 0.402820 & -1.642522 \\ \mathrm{H} & -2.532682 & -2.059563 & -1.575274 \\ \mathrm{H} & -0.953844 & -1.716238 & -2.280258 \\ \mathrm{H} & -3.526301 & -1.341886 & -3.630738 \\ \mathrm{H} & -2.217392 & -2.187928 & -4.131754\end{array}$

tryptamine, A-py $\mathrm{S}_{0}$

$\begin{array}{crcc}\mathrm{C} & -2.120738 & 0.106325 & 0.863909 \\ \mathrm{~N} & -1.254967 & 0.113781 & 1.941846 \\ \mathrm{C} & 0.040205 & 0.046598 & 1.479969 \\ \mathrm{C} & -0.024171 & -0.010720 & 0.062983 \\ \mathrm{C} & -1.417284 & 0.028579 & -0.307069 \\ \mathrm{C} & 1.250672 & 0.035602 & 2.172291 \\ \mathrm{C} & 2.416375 & -0.031990 & 1.426310 \\ \mathrm{C} & 2.378867 & -0.085193 & 0.021808 \\ \mathrm{C} & 1.174725 & -0.075396 & -0.661718 \\ \mathrm{C} & -1.992270 & -0.025391 & -1.689981 \\ \mathrm{C} & -1.964531 & -1.425482 & -2.317554 \\ \mathrm{~N} & -2.556982 & -1.393417 & -3.658928 \\ \mathrm{H} & 1.282295 & 0.079507 & 3.254671 \\ \mathrm{H} & 3.373134 & -0.042097 & 1.933652 \\ \mathrm{H} & 3.309311 & -0.134738 & -0.530309 \\ \mathrm{H} & 1.162459 & -0.116124 & -1.744406\end{array}$




$\begin{array}{llll}\mathrm{H} & -1.529009 & 0.183506 & 2.905815 \\ \mathrm{H} & -3.186529 & 0.163049 & 1.018782 \\ \mathrm{H} & -1.442629 & 0.662530 & -2.343692 \\ \mathrm{H} & -3.027743 & 0.322592 & -1.681155 \\ \mathrm{H} & -2.564093 & -2.098789 & -1.700143 \\ \mathrm{H} & -0.935272 & -1.810740 & -2.297911 \\ \mathrm{H} & -1.983971 & -0.843258 & -4.290098 \\ \mathrm{H} & -2.617945 & -2.326347 & -4.049961\end{array}$

tryptamine, A-py $\mathrm{S}_{1}$

$\begin{array}{lrrr}\mathrm{C} & -2.177862 & 0.121775 & 0.886729 \\ \mathrm{~N} & -1.304927 & 0.103843 & 1.901648 \\ \mathrm{C} & 0.034128 & 0.047134 & 1.435871 \\ \mathrm{C} & -0.020770 & 0.035980 & 0.048555 \\ \mathrm{C} & -1.417142 & 0.082983 & -0.322853 \\ \mathrm{C} & 1.221873 & -0.012128 & 2.190159 \\ \mathrm{C} & 2.416586 & -0.076687 & 1.395355 \\ \mathrm{C} & 2.392512 & -0.086833 & 0.016773 \\ \mathrm{C} & 1.172017 & -0.033701 & -0.724052 \\ \mathrm{C} & -1.986471 & 0.022564 & -1.681704 \\ \mathrm{C} & -1.899557 & -1.406615 & -2.295079 \\ \mathrm{~N} & -2.528594 & -1.420042 & -3.607533 \\ \mathrm{H} & 1.242921 & -0.001904 & 3.269322 \\ \mathrm{H} & 3.372329 & -0.119344 & 1.904441 \\ \mathrm{H} & 3.331216 & -0.135486 & -0.522173 \\ \mathrm{H} & 1.162847 & 0.000494 & -1.803345 \\ \mathrm{H} & -1.558477 & 0.144799 & 2.877164 \\ \mathrm{H} & -3.243008 & 0.186459 & 1.031193 \\ \mathrm{H} & -1.433822 & 0.698591 & -2.342402 \\ \mathrm{H} & -3.034721 & 0.326692 & -1.686692 \\ \mathrm{H} & -2.443021 & -2.098190 & -1.647998 \\ \mathrm{H} & -0.846565 & -1.713208 & -2.291750 \\ \mathrm{H} & -1.943740 & -0.982894 & -4.309943 \\ \mathrm{H} & -2.737842 & -2.361901 & -3.912584\end{array}$

tryptamine, A-up $\mathrm{S}_{0}$

$\begin{array}{rrrc}\mathrm{C} & -2.120095 & 0.121139 & 0.875951 \\ \mathrm{~N} & -1.251604 & 0.100541 & 1.951468 \\ \mathrm{C} & 0.042075 & 0.036473 & 1.484935 \\ \mathrm{C} & -0.025713 & 0.010959 & 0.067393 \\ \mathrm{C} & -1.419433 & 0.068185 & -0.298422 \\ \mathrm{C} & 1.254071 & 0.002734 & 2.173815 \\ \mathrm{C} & 2.417354 & -0.056121 & 1.423426 \\ \mathrm{C} & 2.376284 & -0.078877 & 0.018228\end{array}$




$\begin{array}{rrrr}\mathrm{C} & 1.170735 & -0.045873 & -0.661954 \\ \mathrm{C} & -1.990720 & 0.031500 & -1.683129 \\ \mathrm{C} & -1.979496 & -1.372615 & -2.326567 \\ \mathrm{~N} & -2.497659 & -1.450177 & -3.691198 \\ \mathrm{H} & 1.288658 & 0.022429 & 3.256780 \\ \mathrm{H} & 3.375234 & -0.083844 & 1.927932 \\ \mathrm{H} & 3.304983 & -0.123393 & -0.537140 \\ \mathrm{H} & 1.155928 & -0.063985 & -1.745220 \\ \mathrm{H} & -1.522947 & 0.147114 & 2.917610 \\ \mathrm{H} & -3.185081 & 0.179338 & 1.035994 \\ \mathrm{H} & -1.434569 & 0.716401 & -2.333702 \\ \mathrm{H} & -3.022378 & 0.397627 & -1.664268 \\ \mathrm{H} & -2.563057 & -2.053397 & -1.701588 \\ \mathrm{H} & -0.959635 & -1.763878 & -2.330130 \\ \mathrm{H} & -1.954522 & -0.869588 & -4.320750 \\ \mathrm{H} & -3.457174 & -1.124782 & -3.736221\end{array}$

tryptamine, A-up $\mathrm{S}_{1}$

$\begin{array}{lrrr}\mathrm{C} & -2.173958 & 0.114759 & 0.891325 \\ \mathrm{~N} & -1.295565 & 0.072478 & 1.904747 \\ \mathrm{C} & 0.039676 & 0.030814 & 1.436140 \\ \mathrm{C} & -0.017669 & 0.056499 & 0.048354 \\ \mathrm{C} & -1.416070 & 0.123847 & -0.317029 \\ \mathrm{C} & 1.230240 & -0.044297 & 2.186059 \\ \mathrm{C} & 2.423249 & -0.088588 & 1.389734 \\ \mathrm{C} & 2.395781 & -0.066021 & 0.011298 \\ \mathrm{C} & 1.174869 & 0.004046 & -0.726270 \\ \mathrm{C} & -1.974830 & 0.101536 & -1.671123 \\ \mathrm{C} & -1.973467 & -1.372000 & -2.273901 \\ \mathrm{~N} & -2.477060 & -1.468491 & -3.617648 \\ \mathrm{H} & 1.252225 & -0.055010 & 3.265302 \\ \mathrm{H} & 3.380166 & -0.143533 & 1.895426 \\ \mathrm{H} & 3.333514 & -0.102735 & -0.530651 \\ \mathrm{H} & 1.165969 & 0.051802 & -1.805263 \\ \mathrm{H} & -1.546051 & 0.094846 & 2.881374 \\ \mathrm{H} & -3.237820 & 0.181379 & 1.044683 \\ \mathrm{H} & -1.372663 & 0.714986 & -2.345513 \\ \mathrm{H} & -3.003946 & 0.469081 & -1.688174 \\ \mathrm{H} & -2.574228 & -2.007401 & -1.623145 \\ \mathrm{H} & -0.947322 & -1.737971 & -2.243984 \\ \mathrm{H} & -1.929316 & -0.956940 & -4.297091 \\ \mathrm{H} & -3.454504 & -1.225165 & -3.711429\end{array}$

tryptamine, $\mathrm{Ph}$-out $\mathrm{S}_{0}$ 


$\begin{array}{lrrr}\mathrm{C} & 0.013860 & 0.003439 & 0.084204 \\ \mathrm{C} & 0.052172 & 0.068843 & 1.502061 \\ \mathrm{C} & 1.249915 & 0.087684 & 2.216436 \\ \mathrm{C} & 2.429407 & 0.044623 & 1.490952 \\ \mathrm{C} & 2.417255 & -0.017565 & 0.086047 \\ \mathrm{C} & 1.226961 & -0.037974 & -0.620978 \\ \mathrm{~N} & -1.251620 & 0.109380 & 1.942006 \\ \mathrm{H} & 1.261013 & 0.136494 & 3.299054 \\ \mathrm{H} & 3.377270 & 0.058837 & 2.014838 \\ \mathrm{H} & 3.358335 & -0.049278 & -0.449229 \\ \mathrm{H} & 1.228250 & -0.100602 & -1.701875 \\ \mathrm{C} & -1.374712 & 0.011906 & -0.309925 \\ \mathrm{C} & -2.098786 & 0.082218 & 0.850249 \\ \mathrm{H} & -1.542366 & 0.187466 & 2.900504 \\ \mathrm{H} & -3.167599 & 0.126152 & 0.988507 \\ \mathrm{C} & -1.935078 & -0.045533 & -1.702039 \\ \mathrm{H} & -1.296623 & 0.525030 & -2.382278 \\ \mathrm{C} & -2.099101 & -1.462555 & -2.271615 \\ \mathrm{H} & -2.917181 & 0.436484 & -1.711125 \\ \mathrm{H} & -2.571646 & -1.384670 & -3.254963 \\ \mathrm{~N} & -0.805058 & -2.123660 & -2.452461 \\ \mathrm{H} & -2.792015 & -2.021242 & -1.623388 \\ \mathrm{H} & -0.356953 & -2.285344 & -1.557056 \\ \mathrm{H} & -0.920729 & -3.026022 & -2.898715\end{array}$

tryptamine, $\mathrm{Ph}$-out $\mathrm{S}_{1}$

$\begin{array}{lrrr}\mathrm{C} & 0.009011 & 0.026555 & 0.079442 \\ \mathrm{C} & 0.039781 & 0.100260 & 1.466740 \\ \mathrm{C} & 1.211203 & 0.062997 & 2.246547 \\ \mathrm{C} & 2.422586 & -0.024708 & 1.479577 \\ \mathrm{C} & 2.424288 & -0.098222 & 0.103381 \\ \mathrm{C} & 1.218162 & -0.092172 & -0.663988 \\ \mathrm{~N} & -1.305257 & 0.177741 & 1.908057 \\ \mathrm{H} & 1.210714 & 0.138377 & 3.323395 \\ \mathrm{H} & 3.368147 & -0.036627 & 2.008823 \\ \mathrm{H} & 3.372744 & -0.164375 & -0.416120 \\ \mathrm{H} & 1.229616 & -0.084529 & -1.743162 \\ \mathrm{C} & -1.382799 & 0.056871 & -0.317511 \\ \mathrm{C} & -2.160874 & 0.151499 & 0.880469 \\ \mathrm{H} & -1.573506 & 0.264703 & 2.876719 \\ \mathrm{H} & -3.227664 & 0.229495 & 1.005528 \\ \mathrm{C} & -1.962337 & -0.052725 & -1.674183 \\ \mathrm{H} & -1.351673 & 0.500114 & -2.391872 \\ \mathrm{C} & -2.067241 & -1.516839 & -2.191516 \\ \mathrm{H} & -2.969631 & 0.372678 & -1.674784\end{array}$




$\begin{array}{llll}\mathrm{H} & -2.700105 & -1.496997 & -3.082334 \\ \mathrm{~N} & -0.765555 & -2.037295 & -2.574182 \\ \mathrm{H} & -2.595360 & -2.110325 & -1.430528 \\ \mathrm{H} & -0.119439 & -2.083651 & -1.791987 \\ \mathrm{H} & -0.839821 & -2.958705 & -2.987301\end{array}$

tryptamine, $\mathrm{Ph}$-up $\mathrm{S}_{0}$

$\begin{array}{lrrr}\mathrm{C} & -2.073203 & 0.016153 & 0.818151 \\ \mathrm{~N} & -1.239319 & 0.012750 & 1.920098 \\ \mathrm{C} & 0.069593 & 0.036792 & 1.496064 \\ \mathrm{C} & 0.048016 & 0.048045 & 0.076237 \\ \mathrm{C} & -1.334029 & 0.032573 & -0.335073 \\ \mathrm{C} & 1.258442 & 0.055265 & 2.225115 \\ \mathrm{C} & 2.446716 & 0.090326 & 1.513974 \\ \mathrm{C} & 2.452216 & 0.107909 & 0.107723 \\ \mathrm{C} & 1.270030 & 0.087257 & -0.612261 \\ \mathrm{C} & -1.869962 & 0.009207 & -1.736562 \\ \mathrm{C} & -2.138624 & -1.407310 & -2.295054 \\ \mathrm{~N} & -0.991846 & -2.302390 & -2.411813 \\ \mathrm{H} & 1.256133 & 0.044899 & 3.308778 \\ \mathrm{H} & 3.387759 & 0.106048 & 2.049722 \\ \mathrm{H} & 3.399413 & 0.139809 & -0.416513 \\ \mathrm{H} & 1.291002 & 0.103742 & -1.695284 \\ \mathrm{H} & -1.543571 & 0.021609 & 2.877642 \\ \mathrm{H} & -3.144216 & 0.013545 & 0.945877 \\ \mathrm{H} & -1.173181 & 0.526259 & -2.404533 \\ \mathrm{H} & -2.807791 & 0.573493 & -1.779599 \\ \mathrm{H} & -2.591640 & -1.311361 & -3.286325 \\ \mathrm{H} & -2.881875 & -1.899062 & -1.661018 \\ \mathrm{H} & -0.539317 & -2.430827 & -1.513585 \\ \mathrm{H} & -0.297100 & -1.925103 & -3.046702\end{array}$

tryptamine, Ph-up $\mathrm{S}_{1}$

$\begin{array}{rrrc}\mathrm{C} & -2.170745 & 0.170094 & 0.871959 \\ \mathrm{~N} & -1.313186 & 0.163400 & 1.908038 \\ \mathrm{C} & 0.025775 & 0.097134 & 1.465275 \\ \mathrm{C} & -0.006460 & 0.079348 & 0.073775 \\ \mathrm{C} & -1.395133 & 0.123313 & -0.317939 \\ \mathrm{C} & 1.199342 & 0.011091 & 2.238035 \\ \mathrm{C} & 2.409179 & -0.066198 & 1.471777 \\ \mathrm{C} & 2.411145 & -0.085794 & 0.093443 \\ \mathrm{C} & 1.207020 & -0.036308 & -0.674470 \\ \mathrm{C} & -1.949363 & 0.008260 & -1.666349 \\ \mathrm{C} & -1.987748 & -1.511694 & -2.191884\end{array}$




$\begin{array}{lrrr}\mathrm{N} & -0.722671 & -2.090223 & -2.498057 \\ \mathrm{H} & 1.199252 & 0.042441 & 3.317334 \\ \mathrm{H} & 3.354225 & -0.113934 & 2.000042 \\ \mathrm{H} & 3.360529 & -0.138100 & -0.426957 \\ \mathrm{H} & 1.235297 & 0.115902 & -1.744273 \\ \mathrm{H} & -1.581767 & 0.232595 & 2.877003 \\ \mathrm{H} & -3.237135 & 0.250178 & 1.001650 \\ \mathrm{H} & -1.365052 & 0.571714 & -2.396372 \\ \mathrm{H} & -2.983753 & 0.361119 & -1.697053 \\ \mathrm{H} & -2.609230 & -1.507296 & -3.089786 \\ \mathrm{H} & -2.491296 & -2.106092 & -1.430403 \\ \mathrm{H} & -0.063446 & -2.123261 & -1.726510 \\ \mathrm{H} & -0.271120 & -1.698081 & -3.313189\end{array}$

tryptamine, Py-out $\mathrm{S}_{0}$

$\begin{array}{lrrr}\mathrm{C} & 0.527079 & 0.455386 & 0.224129 \\ \mathrm{C} & 0.603413 & 0.533970 & 1.639613 \\ \mathrm{C} & 1.817945 & 0.499986 & 2.323928 \\ \mathrm{C} & 2.975828 & 0.386851 & 1.571122 \\ \mathrm{C} & 2.926650 & 0.312072 & 0.168068 \\ \mathrm{C} & 1.718417 & 0.345581 & -0.507853 \\ \mathrm{~N} & -0.686205 & 0.643967 & 2.109736 \\ \mathrm{H} & 1.858645 & 0.561507 & 3.405167 \\ \mathrm{H} & 3.935577 & 0.357626 & 2.072109 \\ \mathrm{H} & 3.851352 & 0.228046 & -0.389540 \\ \mathrm{H} & 1.697075 & 0.289343 & -1.589600 \\ \mathrm{C} & -0.867857 & 0.528400 & -0.136655 \\ \mathrm{C} & -1.560464 & 0.642054 & 1.038542 \\ \mathrm{H} & -0.950752 & 0.735691 & 3.074596 \\ \mathrm{H} & -2.623653 & 0.725657 & 1.197858 \\ \mathrm{C} & -1.452555 & 0.466256 & -1.516414 \\ \mathrm{H} & -0.891617 & 1.127560 & -2.184897 \\ \mathrm{C} & -1.463715 & -0.943622 & -2.126167 \\ \mathrm{H} & -2.481269 & 0.834027 & -1.492205 \\ \mathrm{H} & -0.428100 & -1.302328 & -2.221193 \\ \mathrm{~N} & -2.321686 & -1.838010 & -1.345603 \\ \mathrm{H} & -1.875254 & -0.880425 & -3.137140 \\ \mathrm{H} & -2.362306 & -2.758418 & -1.767671 \\ \mathrm{H} & -1.946527 & -1.951175 & -0.409911\end{array}$

tryptamine, Py-out $\mathrm{S}_{1}$
$\begin{array}{llll}\text { C } & 0.533090 & 0.491979 & 0.217325\end{array}$
$\begin{array}{llll}\mathrm{C} & 0.608856 & 0.552037 & 1.601861\end{array}$
$\begin{array}{llll}\text { C } & 1.805248 & 0.483736 & 2.342966\end{array}$ 


$\begin{array}{lrrr}\mathrm{C} & 2.986047 & 0.360840 & 1.534578 \\ \mathrm{C} & 2.941472 & 0.302910 & 0.157971 \\ \mathrm{C} & 1.711601 & 0.360530 & -0.568359 \\ \mathrm{~N} & -0.721786 & 0.658572 & 2.084786 \\ \mathrm{H} & 1.842657 & 0.543801 & 3.420043 \\ \mathrm{H} & 3.947782 & 0.310974 & 2.031625 \\ \mathrm{H} & 3.870496 & 0.210533 & -0.391893 \\ \mathrm{H} & 1.688289 & 0.357029 & -1.647828 \\ \mathrm{C} & -0.869711 & 0.561964 & -0.135551 \\ \mathrm{C} & -1.609576 & 0.661449 & 1.083476 \\ \mathrm{H} & -0.959818 & 0.730457 & 3.062522 \\ \mathrm{H} & -2.671837 & 0.742199 & 1.239424 \\ \mathrm{C} & -1.455916 & 0.485982 & -1.487556 \\ \mathrm{H} & -0.878449 & 1.123392 & -2.164761 \\ \mathrm{C} & -1.426632 & -0.952439 & -2.078807 \\ \mathrm{H} & -2.492226 & 0.829571 & -1.480810 \\ \mathrm{H} & -0.380098 & -1.278393 & -2.119747 \\ \mathrm{~N} & -2.300690 & -1.845146 & -1.327599 \\ \mathrm{H} & -1.800469 & -0.894704 & -3.102697 \\ \mathrm{H} & -2.474946 & -2.702093 & -1.838002 \\ \mathrm{H} & -1.893393 & -2.095190 & -0.432978\end{array}$

tryptamine, Py-up $\mathrm{S}_{0}$

$\begin{array}{lrrr}\mathrm{C} & -2.115612 & 0.241338 & 0.897573 \\ \mathrm{~N} & -1.238766 & 0.224781 & 1.966782 \\ \mathrm{C} & 0.047776 & 0.099400 & 1.492044 \\ \mathrm{C} & -0.033333 & 0.031151 & 0.076707 \\ \mathrm{C} & -1.427989 & 0.125524 & -0.280550 \\ \mathrm{C} & 1.263615 & 0.044154 & 2.172746 \\ \mathrm{C} & 2.417372 & -0.080686 & 1.415727 \\ \mathrm{C} & 2.363303 & -0.146466 & 0.012375 \\ \mathrm{C} & 1.153912 & -0.091860 & -0.659726 \\ \mathrm{C} & -2.009791 & 0.071141 & -1.659532 \\ \mathrm{C} & -2.025763 & -1.344721 & -2.282972 \\ \mathrm{~N} & -2.831437 & -2.346975 & -1.592787 \\ \mathrm{H} & 1.308232 & 0.097767 & 3.254221 \\ \mathrm{H} & 3.378023 & -0.126932 & 1.913667 \\ \mathrm{H} & 3.285029 & -0.241135 & -0.548337 \\ \mathrm{H} & 1.129067 & -0.141848 & -1.741719 \\ \mathrm{H} & -1.498854 & 0.315529 & 2.932925 \\ \mathrm{H} & -3.175938 & 0.348489 & 1.063097 \\ \mathrm{H} & -1.447266 & 0.735420 & -2.325846 \\ \mathrm{H} & -3.035468 & 0.454027 & -1.637753 \\ \mathrm{H} & -1.002889 & -1.724504 & -2.344256 \\ \mathrm{H} & -2.390155 & -1.269066 & -3.311297\end{array}$




$$
\begin{array}{llll}
H & -3.806346 & -2.068184 & -1.559834 \\
H & -2.518202 & -2.456725 & -0.634367
\end{array}
$$

tryptamine, Py-up $\mathrm{S}_{1}$

$\begin{array}{lrrr}\mathrm{C} & -2.159519 & 0.273977 & 0.972689 \\ \mathrm{~N} & -1.254966 & 0.269848 & 1.964144 \\ \mathrm{C} & 0.063839 & 0.137597 & 1.466369 \\ \mathrm{C} & -0.031146 & 0.067659 & 0.082027 \\ \mathrm{C} & -1.437987 & 0.158367 & -0.252497 \\ \mathrm{C} & 1.268082 & 0.054791 & 2.192117 \\ \mathrm{C} & 2.437379 & -0.099688 & 1.373456 \\ \mathrm{C} & 2.373342 & -0.169758 & -0.001624 \\ \mathrm{C} & 1.137168 & -0.094923 & -0.713724 \\ \mathrm{C} & -2.030035 & 0.086461 & -1.589625 \\ \mathrm{C} & -1.980200 & -1.366252 & -2.239581 \\ \mathrm{~N} & -2.847813 & -2.358835 & -1.673506 \\ \mathrm{H} & 1.317842 & 0.121227 & 3.268478 \\ \mathrm{H} & 3.403649 & -0.165714 & 1.859492 \\ \mathrm{H} & 3.293145 & -0.286410 & -0.562601 \\ \mathrm{H} & 1.104677 & -0.106944 & -1.793118 \\ \mathrm{H} & -1.476553 & 0.371032 & 2.942922 \\ \mathrm{H} & -3.214325 & 0.404064 & 1.146737 \\ \mathrm{H} & -1.458812 & 0.719357 & -2.277129 \\ \mathrm{H} & -3.068803 & 0.426305 & -1.590009 \\ \mathrm{H} & -0.948791 & -1.714395 & -2.181854 \\ \mathrm{H} & -2.235661 & -1.239187 & -3.293150 \\ \mathrm{H} & -3.832632 & -2.149511 & -1.768458 \\ \mathrm{H} & -2.633361 & -2.589439 & -0.712639\end{array}$

porphycene $\mathrm{S}_{0}$

$\begin{array}{lrrr}\mathrm{C} & -3.442066 & 1.919138 & 0.000000 \\ \mathrm{C} & -2.431354 & 0.909832 & 0.000000 \\ \mathrm{~N} & -1.230305 & 1.549126 & 0.000000 \\ \mathrm{C} & -1.398277 & 2.903302 & 0.000000 \\ \mathrm{C} & -2.810639 & 3.138971 & 0.000000 \\ \mathrm{C} & -0.367067 & 3.854952 & 0.000000 \\ \mathrm{C} & 1.014569 & 3.706048 & 0.000000 \\ \mathrm{C} & 1.838275 & 2.563995 & 0.000000 \\ \mathrm{H} & -0.271073 & 1.135135 & 0.000000 \\ \mathrm{~N} & 1.421343 & 1.270995 & 0.000000 \\ \mathrm{C} & 3.290391 & 2.604247 & 0.000000 \\ \mathrm{C} & 3.720097 & 1.312861 & 0.000000 \\ \mathrm{C} & 2.526054 & 0.495821 & 0.000000 \\ \mathrm{C} & 2.431354 & -0.909832 & 0.000000\end{array}$




$\begin{array}{lrrr}\mathrm{C} & 3.442066 & -1.919138 & 0.000000 \\ \mathrm{C} & 2.810639 & -3.138971 & 0.000000 \\ \mathrm{C} & 1.398277 & -2.903302 & 0.000000 \\ \mathrm{~N} & 1.230305 & -1.549126 & 0.000000 \\ \mathrm{C} & 0.367067 & -3.854952 & 0.000000 \\ \mathrm{C} & -1.014569 & -3.706048 & 0.000000 \\ \mathrm{C} & -1.838275 & -2.563995 & 0.000000 \\ \mathrm{C} & -3.290391 & -2.604247 & 0.000000 \\ \mathrm{C} & -3.720097 & -1.312861 & 0.000000 \\ \mathrm{C} & -2.526054 & -0.495821 & 0.000000 \\ \mathrm{~N} & -1.421343 & -1.270995 & 0.000000 \\ \mathrm{H} & 0.271073 & -1.135135 & 0.000000 \\ \mathrm{H} & 3.272364 & -4.113737 & 0.000000 \\ \mathrm{H} & 4.504292 & -1.735673 & 0.000000 \\ \mathrm{H} & 0.722814 & -4.879329 & 0.000000 \\ \mathrm{H} & -1.567268 & -4.639674 & 0.000000 \\ \mathrm{H} & -3.893197 & -3.499896 & 0.000000 \\ \mathrm{H} & -4.738198 & -0.956087 & 0.000000 \\ \mathrm{H} & -4.504292 & 1.735673 & 0.000000 \\ \mathrm{H} & -3.272364 & 4.113737 & 0.000000 \\ \mathrm{H} & -0.722814 & 4.879329 & 0.000000 \\ \mathrm{H} & 1.567268 & 4.639674 & 0.000000 \\ \mathrm{H} & 3.893197 & 3.499896 & 0.000000 \\ \mathrm{H} & 4.738198 & 0.956087 & 0.000000\end{array}$

porphycene $\mathrm{S}_{1}$

$\begin{array}{lrrr}\mathrm{C} & -3.464211 & 1.907721 & 0.000000 \\ \mathrm{C} & -2.458483 & 0.900268 & 0.000000 \\ \mathrm{~N} & -1.246401 & 1.544545 & 0.000000 \\ \mathrm{C} & -1.417904 & 2.893983 & 0.000000 \\ \mathrm{C} & -2.829331 & 3.130117 & 0.000000 \\ \mathrm{C} & -0.381666 & 3.858427 & 0.000000 \\ \mathrm{C} & 0.992265 & 3.718181 & 0.000000 \\ \mathrm{C} & 1.844686 & 2.576541 & 0.000000 \\ \mathrm{H} & -0.292992 & 1.129233 & 0.000000 \\ \mathrm{~N} & 1.431337 & 1.283867 & 0.000000 \\ \mathrm{C} & 3.285668 & 2.625234 & 0.000000 \\ \mathrm{C} & 3.730515 & 1.327340 & 0.000000 \\ \mathrm{C} & 2.559735 & 0.506164 & 0.000000 \\ \mathrm{C} & 2.458483 & -0.900268 & 0.000000 \\ \mathrm{C} & 3.464211 & -1.907721 & 0.000000 \\ \mathrm{C} & 2.829331 & -3.130117 & 0.000000 \\ \mathrm{C} & 1.417904 & -2.893983 & 0.000000 \\ \mathrm{~N} & 1.246401 & -1.544545 & 0.000000 \\ \mathrm{C} & 0.381666 & -3.858427 & 0.000000\end{array}$




$\begin{array}{lrrl}\mathrm{C} & -0.992265 & -3.718181 & 0.000000 \\ \mathrm{C} & -1.844686 & -2.576541 & 0.000000 \\ \mathrm{C} & -3.285668 & -2.625234 & 0.000000 \\ \mathrm{C} & -3.730515 & -1.327340 & 0.000000 \\ \mathrm{C} & -2.559735 & -0.506164 & 0.000000 \\ \mathrm{~N} & -1.431337 & -1.283867 & 0.000000 \\ \mathrm{H} & 0.292992 & -1.129233 & 0.000000 \\ \mathrm{H} & 3.289575 & -4.105574 & 0.000000 \\ \mathrm{H} & 4.526407 & -1.725050 & 0.000000 \\ \mathrm{H} & 0.745000 & -4.879788 & 0.000000 \\ \mathrm{H} & -1.538778 & -4.655871 & 0.000000 \\ \mathrm{H} & -3.884292 & -3.523687 & 0.000000 \\ \mathrm{H} & -4.752736 & -0.983555 & 0.000000 \\ \mathrm{H} & -4.526407 & 1.725050 & 0.000000 \\ \mathrm{H} & -3.289575 & 4.105574 & 0.000000 \\ \mathrm{H} & -0.745000 & 4.879788 & 0.000000 \\ \mathrm{H} & 1.538778 & 4.655871 & 0.000000 \\ \mathrm{H} & 3.884292 & 3.523687 & 0.000000 \\ \mathrm{H} & 4.752736 & 0.983555 & 0.000000\end{array}$

porphine $\mathrm{S}_{0}$

$\begin{array}{rrrr}\mathrm{N} & 0.000000 & -2.030709 & 0.000000 \\ \mathrm{~N} & 2.110481 & 0.000000 & 0.000000 \\ \mathrm{C} & 1.084273 & -2.851109 & 0.000000 \\ \mathrm{C} & 0.676043 & -4.250323 & 0.000000 \\ \mathrm{C} & 2.416609 & -2.435225 & 0.000000 \\ \mathrm{C} & 2.887581 & -1.127867 & 0.000000 \\ \mathrm{C} & 4.248970 & -0.683967 & 0.000000 \\ \mathrm{H} & 1.345099 & -5.097723 & 0.000000 \\ \mathrm{H} & 3.172808 & -3.210538 & 0.000000 \\ \mathrm{H} & 5.103904 & -1.341346 & 0.000000 \\ \mathrm{H} & 1.097850 & 0.000000 & 0.000000 \\ \mathrm{~N} & 0.000000 & 2.030709 & 0.000000 \\ \mathrm{~N} & -2.110481 & 0.000000 & 0.000000 \\ \mathrm{C} & 1.084273 & 2.851109 & 0.000000 \\ \mathrm{C} & -1.084273 & 2.851109 & 0.000000 \\ \mathrm{C} & -1.084273 & -2.851109 & 0.000000 \\ \mathrm{C} & 0.676043 & 4.250323 & 0.000000 \\ \mathrm{C} & -0.676043 & 4.250323 & 0.000000 \\ \mathrm{C} & -0.676043 & -4.250323 & 0.000000 \\ \mathrm{C} & 2.416609 & 2.435225 & 0.000000 \\ \mathrm{C} & -2.416609 & 2.435225 & 0.000000 \\ \mathrm{C} & -2.416609 & -2.435225 & 0.000000 \\ \mathrm{C} & 2.887581 & 1.127867 & 0.000000 \\ \mathrm{C} & -2.887581 & 1.127867 & 0.000000\end{array}$




$\begin{array}{lrcc}\mathrm{C} & -2.887581 & -1.127867 & 0.000000 \\ \mathrm{C} & 4.248970 & 0.683967 & 0.000000 \\ \mathrm{C} & -4.248970 & 0.683967 & 0.000000 \\ \mathrm{C} & -4.248970 & -0.683967 & 0.000000 \\ \mathrm{H} & 1.345099 & 5.097723 & 0.000000 \\ \mathrm{H} & -1.345099 & 5.097723 & 0.000000 \\ \mathrm{H} & -1.345099 & -5.097723 & 0.000000 \\ \mathrm{H} & 3.172808 & 3.210538 & 0.000000 \\ \mathrm{H} & -3.172808 & 3.210538 & 0.000000 \\ \mathrm{H} & -3.172808 & -3.210538 & 0.000000 \\ \mathrm{H} & 5.103904 & 1.341346 & 0.000000 \\ \mathrm{H} & -5.103904 & 1.341346 & 0.000000 \\ \mathrm{H} & -5.103904 & -1.341346 & 0.000000 \\ \mathrm{H} & -1.097850 & 0.000000 & 0.000000\end{array}$

porphine $\mathrm{S}_{1}$

$\begin{array}{lrrl}\mathrm{N} & 0.000000 & -2.051382 & 0.000000 \\ \mathrm{~N} & 2.108175 & 0.000000 & 0.000000 \\ \mathrm{C} & 1.088102 & -2.876572 & 0.000000 \\ \mathrm{C} & 0.679059 & -4.269031 & 0.000000 \\ \mathrm{C} & 2.422587 & -2.442733 & 0.000000 \\ \mathrm{C} & 2.888683 & -1.130453 & 0.000000 \\ \mathrm{C} & 4.249831 & -0.685479 & 0.000000 \\ \mathrm{H} & 1.346177 & -5.117615 & 0.000000 \\ \mathrm{H} & 3.184594 & -3.212359 & 0.000000 \\ \mathrm{H} & 5.105274 & -1.342006 & 0.000000 \\ \mathrm{H} & 1.097073 & 0.000000 & 0.000000 \\ \mathrm{~N} & 0.000000 & 2.051382 & 0.000000 \\ \mathrm{~N} & -2.108175 & 0.000000 & 0.000000 \\ \mathrm{C} & 1.088102 & 2.876572 & 0.000000 \\ \mathrm{C} & -1.088102 & 2.876572 & 0.000000 \\ \mathrm{C} & -1.088102 & -2.876572 & 0.000000 \\ \mathrm{C} & 0.679059 & 4.269031 & 0.000000 \\ \mathrm{C} & -0.679059 & 4.269031 & 0.000000 \\ \mathrm{C} & -0.679059 & -4.269031 & 0.000000 \\ \mathrm{C} & 2.422587 & 2.442733 & 0.000000 \\ \mathrm{C} & -2.422587 & 2.442733 & 0.000000 \\ \mathrm{C} & -2.422587 & -2.442733 & 0.000000 \\ \mathrm{C} & 2.888683 & 1.130453 & 0.000000 \\ \mathrm{C} & -2.888683 & 1.130453 & 0.000000 \\ \mathrm{C} & -2.888683 & -1.130453 & 0.000000 \\ \mathrm{C} & 4.249831 & 0.685479 & 0.000000 \\ \mathrm{C} & -4.249831 & 0.685479 & 0.000000 \\ \mathrm{C} & -4.249831 & -0.685479 & 0.000000 \\ \mathrm{H} & 1.346177 & 5.117615 & 0.000000\end{array}$




$\begin{array}{rrrr}\mathrm{H} & -1.346177 & 5.117615 & 0.000000 \\ \mathrm{H} & -1.346177 & -5.117615 & 0.000000 \\ \mathrm{H} & 3.184594 & 3.212359 & 0.000000 \\ \mathrm{H} & -3.184594 & 3.212359 & 0.000000 \\ \mathrm{H} & -3.184594 & -3.212359 & 0.000000 \\ \mathrm{H} & 5.105274 & 1.342006 & 0.000000 \\ \mathrm{H} & -5.105274 & 1.342006 & 0.000000 \\ \mathrm{H} & -5.105274 & -1.342006 & 0.000000 \\ \mathrm{H} & -1.097073 & 0.000000 & 0.000000\end{array}$

chlorin $\mathrm{S}_{0}$

$\begin{array}{lrrr}\mathrm{C} & 0.000000 & -4.241934 & 0.924571 \\ \mathrm{C} & 0.000000 & -2.887393 & 1.378189 \\ \mathrm{~N} & 0.000000 & -2.100583 & 0.245634 \\ \mathrm{C} & 0.000000 & -2.868583 & -0.881126 \\ \mathrm{C} & 0.000000 & -4.232159 & -0.444738 \\ \mathrm{C} & 0.000000 & -2.419200 & 2.680512 \\ \mathrm{C} & 0.000000 & -1.084507 & 3.103738 \\ \mathrm{~N} & 0.000000 & 0.000000 & 2.281976 \\ \mathrm{C} & 0.000000 & 1.084507 & 3.103738 \\ \mathrm{C} & 0.000000 & 0.678390 & 4.496335 \\ \mathrm{C} & 0.000000 & -0.678390 & 4.496335 \\ \mathrm{C} & 0.000000 & 2.419200 & 2.680512 \\ \mathrm{C} & 0.000000 & 2.887393 & 1.378189 \\ \mathrm{~N} & 0.000000 & 2.100583 & 0.245634 \\ \mathrm{C} & 0.000000 & 2.868583 & -0.881126 \\ \mathrm{C} & 0.000000 & 4.232159 & -0.444738 \\ \mathrm{C} & 0.000000 & 4.241934 & 0.924571 \\ \mathrm{C} & 0.000000 & 2.410053 & -2.202294 \\ \mathrm{C} & 0.000000 & 1.102495 & -2.657894 \\ \mathrm{C} & 0.000000 & 0.767686 & -4.144875 \\ \mathrm{C} & 0.000000 & -0.767686 & -4.144875 \\ \mathrm{C} & 0.000000 & -1.102495 & -2.657894 \\ \mathrm{~N} & 0.000000 & 0.000000 & -1.883136 \\ \mathrm{C} & 0.000000 & -2.410053 & -2.202294 \\ \mathrm{H} & -0.878261 & 1.189276 & -4.638165 \\ \mathrm{H} & 0.000000 & 3.187395 & -2.956936 \\ \mathrm{H} & 0.000000 & 5.082275 & -1.108217 \\ \mathrm{H} & 0.000000 & 1.346115 & 5.344854 \\ \mathrm{H} & 0.000000 & -1.346115 & 5.344854 \\ \mathrm{H} & -0.878261 & -1.189276 & -4.638165 \\ \mathrm{H} & 0.000000 & 3.176711 & 3.455028 \\ \mathrm{H} & 0.000000 & -3.176711 & 3.455028 \\ \mathrm{H} & 0.000000 & -3.187395 & -2.956936 \\ \mathrm{H} & 0.000000 & 5.102262 & 1.575077\end{array}$




$\begin{array}{rrrr}\mathrm{H} & 0.000000 & -5.102262 & 1.575077 \\ \mathrm{H} & 0.000000 & -5.082275 & -1.108217 \\ \mathrm{H} & 0.878261 & -1.189276 & -4.638165 \\ \mathrm{H} & 0.878261 & 1.189276 & -4.638165 \\ \mathrm{H} & 0.000000 & -1.090065 & 0.269046 \\ \mathrm{H} & 0.000000 & 1.090065 & 0.269046\end{array}$

chlorin $\mathrm{S}_{1}$

$\begin{array}{lrrr}\mathrm{C} & 0.000000 & -4.251183 & 0.925033 \\ \mathrm{C} & 0.000000 & -2.894544 & 1.375701 \\ \mathrm{~N} & 0.000000 & -2.108276 & 0.244158 \\ \mathrm{C} & 0.000000 & -2.883114 & -0.886642 \\ \mathrm{C} & 0.000000 & -4.243480 & -0.448987 \\ \mathrm{C} & 0.000000 & -2.424161 & 2.686709 \\ \mathrm{C} & 0.000000 & -1.087297 & 3.106004 \\ \mathrm{~N} & 0.000000 & 0.000000 & 2.281869 \\ \mathrm{C} & 0.000000 & 1.087297 & 3.106004 \\ \mathrm{C} & 0.000000 & 0.676738 & 4.504246 \\ \mathrm{C} & 0.000000 & -0.676738 & 4.504246 \\ \mathrm{C} & 0.000000 & 2.424161 & 2.686709 \\ \mathrm{C} & 0.000000 & 2.894544 & 1.375701 \\ \mathrm{~N} & 0.000000 & 2.108276 & 0.244158 \\ \mathrm{C} & 0.000000 & 2.883114 & -0.886642 \\ \mathrm{C} & 0.000000 & 4.243480 & -0.448987 \\ \mathrm{C} & 0.000000 & 4.251183 & 0.925033 \\ \mathrm{C} & 0.000000 & 2.419512 & -2.209796 \\ \mathrm{C} & 0.000000 & 1.104018 & -2.659419 \\ \mathrm{C} & 0.000000 & 0.766610 & -4.141058 \\ \mathrm{C} & 0.000000 & -0.766610 & -4.141058 \\ \mathrm{C} & 0.000000 & -1.104018 & -2.659419 \\ \mathrm{~N} & 0.000000 & 0.000000 & -1.876353 \\ \mathrm{C} & 0.000000 & -2.419512 & -2.209796 \\ \mathrm{H} & -0.877606 & 1.189211 & -4.636540 \\ \mathrm{H} & 0.000000 & 3.192141 & -2.968816 \\ \mathrm{H} & 0.000000 & 5.095193 & -1.110391 \\ \mathrm{H} & 0.000000 & 1.345726 & 5.351542 \\ \mathrm{H} & 0.000000 & -1.345726 & 5.351542 \\ \mathrm{H} & -0.877606 & -1.189211 & -4.636540 \\ \mathrm{H} & 0.000000 & 3.180674 & 3.461327 \\ \mathrm{H} & 0.000000 & -3.180674 & 3.461327 \\ \mathrm{H} & 0.000000 & -3.192141 & -2.968816 \\ \mathrm{H} & 0.000000 & 5.110026 & 1.577161 \\ \mathrm{H} & 0.000000 & -5.110026 & 1.577161 \\ \mathrm{H} & 0.000000 & -5.095193 & -1.110391 \\ \mathrm{H} & 0.877606 & -1.189211 & -4.636540\end{array}$




$$
\begin{array}{rrrr}
\mathrm{H} & 0.877606 & 1.189211 & -4.636540 \\
\mathrm{H} & 0.000000 & -1.097884 & 0.263558 \\
\mathrm{H} & 0.000000 & 1.097884 & 0.263558
\end{array}
$$

Zn-tetraphenylporphine $\mathrm{S}_{0}$

$\begin{array}{lrrr}\mathrm{C} & -3.779874 & 4.232037 & 1.158443 \\ \mathrm{C} & -3.501430 & 3.501412 & -0.000032 \\ \mathrm{C} & -4.232204 & 3.779989 & -1.158353 \\ \mathrm{C} & -5.215025 & 4.764690 & -1.159309 \\ \mathrm{C} & -5.484719 & 5.484714 & 0.000227 \\ \mathrm{C} & -4.764550 & 5.214861 & 1.159652 \\ \mathrm{C} & -2.441866 & 2.441839 & -0.000133 \\ \mathrm{C} & -1.104244 & 2.866313 & -0.010264 \\ \mathrm{~N} & 0.000000 & 2.051969 & 0.021629 \\ \mathrm{C} & 1.104244 & 2.866313 & -0.010264 \\ \mathrm{C} & 0.678537 & 4.243785 & -0.075062 \\ \mathrm{C} & -0.678537 & 4.243785 & -0.075062 \\ \mathrm{C} & 2.441866 & 2.441839 & -0.000133 \\ \mathrm{C} & 3.501430 & 3.501412 & -0.000032 \\ \mathrm{C} & 4.232204 & 3.779989 & -1.158353 \\ \mathrm{C} & 5.215025 & 4.764690 & -1.159309 \\ \mathrm{C} & 5.484719 & 5.484714 & 0.000227 \\ \mathrm{C} & 4.764550 & 5.214861 & 1.159652 \\ \mathrm{C} & 3.779874 & 4.232037 & 1.158443 \\ \mathrm{C} & -2.866376 & 1.104234 & 0.009992 \\ \mathrm{~N} & -2.052048 & 0.000000 & -0.022056 \\ \mathrm{C} & -2.866376 & -1.104234 & 0.009992 \\ \mathrm{C} & -4.243844 & -0.678537 & 0.075036 \\ \mathrm{C} & -4.243844 & 0.678537 & 0.075036 \\ \mathrm{C} & -2.441866 & -2.441839 & -0.000133 \\ \mathrm{C} & -3.501430 & -3.501412 & -0.000032 \\ \mathrm{C} & -4.232204 & -3.779989 & -1.158353 \\ \mathrm{C} & -5.215025 & -4.764690 & -1.159309 \\ \mathrm{C} & -5.484719 & -5.484714 & 0.000227 \\ \mathrm{C} & -4.764550 & -5.214861 & 1.159652 \\ \mathrm{C} & -3.779874 & -4.232037 & 1.158443 \\ \mathrm{C} & 2.866376 & 1.104234 & 0.009992 \\ \mathrm{~N} & 2.052048 & 0.000000 & -0.022056 \\ \mathrm{C} & 2.866376 & -1.104234 & 0.009992 \\ \mathrm{C} & 4.243844 & -0.678537 & 0.075036 \\ \mathrm{C} & 4.243844 & 0.678537 & 0.075036 \\ \mathrm{C} & 2.441866 & -2.441839 & -0.000133 \\ \mathrm{C} & 3.501430 & -3.501412 & -0.000032 \\ \mathrm{C} & 4.232204 & -3.779989 & -1.158353 \\ \mathrm{C} & 5.215025 & -4.764690 & -1.159309\end{array}$




$\begin{array}{rrrr}\mathrm{C} & 5.484719 & -5.484714 & 0.000227 \\ \mathrm{C} & 4.764550 & -5.214861 & 1.159652 \\ \mathrm{C} & 3.779874 & -4.232037 & 1.158443 \\ \mathrm{C} & 1.104244 & -2.866313 & -0.010264 \\ \mathrm{~N} & 0.000000 & -2.051969 & 0.021629 \\ \mathrm{C} & -1.104244 & -2.866313 & -0.010264 \\ \mathrm{C} & -0.678537 & -4.243785 & -0.075062 \\ \mathrm{C} & 0.678537 & -4.243785 & -0.075062 \\ \mathrm{H} & 1.335287 & -5.095916 & -0.126255 \\ \mathrm{H} & 5.095970 & -1.335287 & 0.126360 \\ \mathrm{H} & 1.335287 & 5.095916 & -0.126255 \\ \mathrm{H} & -1.335287 & 5.095916 & -0.126255 \\ \mathrm{H} & -1.335287 & -5.095916 & -0.126255 \\ \mathrm{H} & 5.095970 & 1.335287 & 0.126360 \\ \mathrm{H} & -5.095970 & 1.335287 & 0.126360 \\ \mathrm{H} & -5.095970 & -1.335287 & 0.126360 \\ \mathrm{H} & 4.023112 & 3.223126 & -2.063733 \\ \mathrm{H} & 5.768240 & 4.970454 & -2.067829 \\ \mathrm{H} & 6.250654 & 6.250662 & 0.000322 \\ \mathrm{H} & 4.970205 & 5.767968 & 2.068262 \\ \mathrm{H} & 3.222895 & 4.022804 & 2.063718 \\ \mathrm{H} & 4.023112 & -3.223126 & -2.063733 \\ \mathrm{H} & 5.768240 & -4.970454 & -2.067829 \\ \mathrm{H} & 6.250654 & -6.250662 & 0.000322 \\ \mathrm{H} & 4.970205 & -5.767968 & 2.068262 \\ \mathrm{H} & 3.222895 & -4.022804 & 2.063718 \\ \mathrm{H} & -4.023112 & -3.223126 & -2.063733 \\ \mathrm{H} & -5.768240 & -4.970454 & -2.067829 \\ \mathrm{H} & -6.250654 & -6.250662 & 0.000322 \\ \mathrm{H} & -4.970205 & -5.767968 & 2.068262 \\ \mathrm{H} & -3.222895 & -4.022804 & 2.063718 \\ \mathrm{H} & -4.023112 & 3.223126 & -2.063733 \\ \mathrm{H} & -5.768240 & 4.970454 & -2.067829 \\ \mathrm{H} & -6.250654 & 6.250662 & 0.000322 \\ \mathrm{H} & -4.970205 & 5.767968 & 2.068262 \\ \mathrm{H} & -3.222895 & 4.022804 & 2.063718 \\ \mathrm{Zn} & 0.000000 & 0.000000 & -0.000071\end{array}$

Zn-tetraphenylporphine $\mathrm{S}_{1}$

$\begin{array}{lrrr}\mathrm{C} & 3.616394 & 4.411814 & -1.059599 \\ \mathrm{C} & 3.501486 & 3.505601 & 0.001335 \\ \mathrm{C} & 4.400940 & 3.613777 & 1.069044 \\ \mathrm{C} & 5.378473 & 4.601946 & 1.079409 \\ \mathrm{C} & 5.483415 & 5.494853 & 0.016722 \\ \mathrm{C} & 4.600430 & 5.394358 & -1.053833\end{array}$




$\begin{array}{rrrr}\mathrm{C} & 2.449504 & 2.450309 & -0.004786 \\ \mathrm{C} & 1.106788 & 2.867221 & 0.025948 \\ \mathrm{~N} & 0.000000 & 2.052632 & -0.072020 \\ \mathrm{C} & -1.106788 & 2.867221 & 0.025948 \\ \mathrm{C} & -0.679327 & 4.232732 & 0.202276 \\ \mathrm{C} & 0.679327 & 4.232732 & 0.202276 \\ \mathrm{C} & -2.449504 & 2.450309 & -0.004786 \\ \mathrm{C} & -3.501486 & 3.505601 & 0.001335 \\ \mathrm{C} & -4.400940 & 3.613777 & 1.069044 \\ \mathrm{C} & -5.378473 & 4.601946 & 1.079409 \\ \mathrm{C} & -5.483415 & 5.494853 & 0.016722 \\ \mathrm{C} & -4.600430 & 5.394358 & -1.053833 \\ \mathrm{C} & -3.616394 & 4.411814 & -1.059599 \\ \mathrm{C} & 2.883747 & 1.104192 & -0.043847 \\ \mathrm{~N} & 2.070297 & 0.000000 & 0.055621 \\ \mathrm{C} & 2.883747 & -1.104192 & -0.043847 \\ \mathrm{C} & 4.245524 & -0.681983 & -0.220828 \\ \mathrm{C} & 4.245524 & 0.681983 & -0.220828 \\ \mathrm{C} & 2.449504 & -2.450309 & -0.004786 \\ \mathrm{C} & 3.501486 & -3.505601 & 0.001335 \\ \mathrm{C} & 4.400940 & -3.613777 & 1.069044 \\ \mathrm{C} & 5.378473 & -4.601946 & 1.079409 \\ \mathrm{C} & 5.483415 & -5.494853 & 0.016722 \\ \mathrm{C} & 4.600430 & -5.394358 & -1.053833 \\ \mathrm{C} & 3.616394 & -4.411814 & -1.059599 \\ \mathrm{C} & -2.883747 & 1.104192 & -0.043847 \\ \mathrm{~N} & -2.070297 & 0.000000 & 0.055621 \\ \mathrm{C} & -2.883747 & -1.104192 & -0.043847 \\ \mathrm{C} & -4.245524 & -0.681983 & -0.220828 \\ \mathrm{C} & -4.245524 & 0.681983 & -0.220828 \\ \mathrm{C} & -2.449504 & -2.450309 & -0.004786 \\ \mathrm{C} & -3.501486 & -3.505601 & 0.001335 \\ \mathrm{C} & -4.400940 & -3.613777 & 1.069044 \\ \mathrm{C} & -5.378473 & -4.601946 & 1.079409 \\ \mathrm{C} & -5.483415 & -5.494853 & 0.016722 \\ \mathrm{C} & -4.600430 & -5.394358 & -1.053833 \\ \mathrm{C} & -3.616394 & -4.411814 & -1.059599 \\ \mathrm{C} & -1.106788 & -2.867221 & 0.025948 \\ \mathrm{~N} & 0.000000 & -2.052632 & -0.072020 \\ \mathrm{C} & 1.106788 & -2.867221 & 0.025948 \\ \mathrm{C} & 0.679327 & -4.232732 & 0.202276 \\ \mathrm{C} & -0.679327 & -4.232732 & 0.202276 \\ \mathrm{H} & -1.333166 & -5.077310 & 0.337609 \\ \mathrm{H} & -5.089749 & -1.336527 & -0.353864 \\ \mathrm{H} & -1.333166 & 5.077310 & 0.337609 \\ \mathrm{H} & 1.333166 & 5.077310 & 0.337609\end{array}$




$\begin{array}{crrr}\mathrm{H} & 1.333166 & -5.077310 & 0.337609 \\ \mathrm{H} & -5.089749 & 1.336527 & -0.353864 \\ \mathrm{H} & 5.089749 & 1.336527 & -0.353864 \\ \mathrm{H} & 5.089749 & -1.336527 & -0.353864 \\ \mathrm{H} & -4.320647 & 2.924121 & 1.900163 \\ \mathrm{H} & -6.057008 & 4.676660 & 1.920706 \\ \mathrm{H} & -6.247682 & 6.262375 & 0.023018 \\ \mathrm{H} & -4.678941 & 6.079828 & -1.889142 \\ \mathrm{H} & -2.934987 & 4.333861 & -1.897683 \\ \mathrm{H} & -4.320647 & -2.924121 & 1.900163 \\ \mathrm{H} & -6.057008 & -4.676660 & 1.920706 \\ \mathrm{H} & -6.247682 & -6.262375 & 0.023018 \\ \mathrm{H} & -4.678941 & -6.079828 & -1.889142 \\ \mathrm{H} & -2.934987 & -4.333861 & -1.897683 \\ \mathrm{H} & 4.320647 & -2.924121 & 1.900163 \\ \mathrm{H} & 6.057008 & -4.676660 & 1.920706 \\ \mathrm{H} & 6.247682 & -6.262375 & 0.023018 \\ \mathrm{H} & 4.678941 & -6.079828 & -1.889142 \\ \mathrm{H} & 2.934987 & -4.333861 & -1.897683 \\ \mathrm{H} & 4.320647 & 2.924121 & 1.900163 \\ \mathrm{H} & 6.057008 & 4.676660 & 1.920706 \\ \mathrm{H} & 6.247682 & 6.262375 & 0.023018 \\ \mathrm{H} & 4.678941 & 6.079828 & -1.889142 \\ \mathrm{H} & 2.934987 & 4.333861 & -1.897683 \\ \mathrm{Zn} & 0.000000 & 0.000000 & -0.007261\end{array}$

tetraphenylporphine $\mathrm{S}_{0}$

$\begin{array}{lrrr}\mathrm{C} & -4.239366 & -0.684673 & 0.081020 \\ \mathrm{C} & -2.881471 & -1.131378 & 0.009403 \\ \mathrm{~N} & -2.102185 & -0.001001 & -0.028014 \\ \mathrm{C} & -2.882527 & 1.128664 & 0.009266 \\ \mathrm{C} & -4.240004 & 0.680696 & 0.080926 \\ \mathrm{C} & -2.434935 & 2.454487 & -0.008132 \\ \mathrm{C} & -1.088242 & 2.859020 & -0.030647 \\ \mathrm{~N} & -0.000944 & 2.037733 & 0.007909 \\ \mathrm{C} & 1.085574 & 2.860050 & -0.030519 \\ \mathrm{C} & 0.672867 & 4.255105 & -0.113982 \\ \mathrm{C} & -0.676864 & 4.254454 & -0.114090 \\ \mathrm{C} & 2.432658 & 2.456800 & -0.007929 \\ \mathrm{C} & 2.881471 & 1.131378 & 0.009403 \\ \mathrm{~N} & 2.102185 & 0.001001 & -0.028014 \\ \mathrm{C} & 2.882527 & -1.128664 & 0.009266 \\ \mathrm{C} & 4.240004 & -0.680696 & 0.080926 \\ \mathrm{C} & 4.239366 & 0.684673 & 0.081020 \\ \mathrm{C} & 2.434935 & -2.454487 & -0.008132\end{array}$




$\begin{array}{rrrc}\mathrm{C} & 1.088242 & -2.859020 & -0.030647 \\ \mathrm{~N} & 0.000944 & -2.037733 & 0.007909 \\ \mathrm{C} & -1.085574 & -2.860050 & -0.030519 \\ \mathrm{C} & -0.672867 & -4.255105 & -0.113982 \\ \mathrm{C} & 0.676864 & -4.254454 & -0.114090 \\ \mathrm{C} & -2.432658 & -2.456800 & -0.007929 \\ \mathrm{H} & 1.337741 & -5.102813 & -0.177685 \\ \mathrm{C} & 3.491488 & -3.516925 & 0.001231 \\ \mathrm{H} & 5.095029 & -1.332460 & 0.134787 \\ \mathrm{H} & 1.090174 & 0.000507 & -0.041562 \\ \mathrm{H} & 1.332899 & 5.104129 & -0.177496 \\ \mathrm{H} & -1.337741 & 5.102813 & -0.177685 \\ \mathrm{H} & -1.332899 & -5.104129 & -0.177496 \\ \mathrm{C} & 3.488222 & 3.520203 & 0.001600 \\ \mathrm{C} & -3.491488 & 3.516925 & 0.001231 \\ \mathrm{C} & -3.488222 & -3.520203 & 0.001600 \\ \mathrm{H} & 5.093743 & 1.337274 & 0.134980 \\ \mathrm{H} & -5.095029 & 1.332460 & 0.134787 \\ \mathrm{H} & -5.093743 & -1.337274 & 0.134980 \\ \mathrm{H} & -1.090174 & -0.000507 & -0.041562 \\ \mathrm{C} & 4.240004 & 3.791433 & -1.145042 \\ \mathrm{C} & 5.218833 & 4.780105 & -1.135750 \\ \mathrm{C} & 5.463836 & 5.510592 & 0.022688 \\ \mathrm{C} & 4.723008 & 5.247476 & 1.170651 \\ \mathrm{C} & 3.742287 & 4.260911 & 1.159368 \\ \mathrm{H} & 4.048567 & 3.227943 & -2.050258 \\ \mathrm{H} & 5.787605 & 4.981216 & -2.035649 \\ \mathrm{H} & 6.226360 & 6.279882 & 0.030690 \\ \mathrm{H} & 4.909032 & 5.809287 & 2.078121 \\ \mathrm{H} & 3.168199 & 4.057542 & 2.055160 \\ \mathrm{C} & 4.243133 & -3.787640 & -1.145605 \\ \mathrm{C} & 5.222821 & -4.775462 & -1.136481 \\ \mathrm{C} & 5.468822 & -5.505594 & 0.021969 \\ \mathrm{C} & 4.728135 & -5.242982 & 1.170137 \\ \mathrm{C} & 3.746554 & -4.257261 & 1.159024 \\ \mathrm{H} & 4.050923 & -3.224421 & -2.050827 \\ \mathrm{H} & 5.791480 & -4.976209 & -2.036533 \\ \mathrm{H} & 6.231988 & -6.274250 & 0.029796 \\ \mathrm{H} & 4.914917 & -5.804530 & 2.077612 \\ \mathrm{H} & 3.172559 & -4.054283 & 2.054964 \\ \mathrm{C} & -4.240004 & -3.791433 & -1.145042 \\ \mathrm{C} & -5.218833 & -4.780105 & -1.135750 \\ \mathrm{C} & -5.463836 & -5.510592 & 0.022688 \\ \mathrm{C} & -4.723008 & -5.247476 & 1.170651 \\ \mathrm{C} & -3.742287 & -4.260911 & 1.159368 \\ \mathrm{H} & -4.048567 & -3.227943 & -2.050258\end{array}$




$\begin{array}{lrrr}\mathrm{H} & -5.787605 & -4.981216 & -2.035649 \\ \mathrm{H} & -6.226360 & -6.279882 & 0.030690 \\ \mathrm{H} & -4.909032 & -5.809287 & 2.078121 \\ \mathrm{H} & -3.168199 & -4.057542 & 2.055160 \\ \mathrm{C} & -4.243133 & 3.787640 & -1.145605 \\ \mathrm{C} & -5.222821 & 4.775462 & -1.136481 \\ \mathrm{C} & -5.468822 & 5.505594 & 0.021969 \\ \mathrm{C} & -4.728135 & 5.242982 & 1.170137 \\ \mathrm{C} & -3.746554 & 4.257261 & 1.159024 \\ \mathrm{H} & -4.050923 & 3.224421 & -2.050827 \\ \mathrm{H} & -5.791480 & 4.976209 & -2.036533 \\ \mathrm{H} & -6.231988 & 6.274250 & 0.029796 \\ \mathrm{H} & -4.914917 & 5.804530 & 2.077612 \\ \mathrm{H} & -3.172559 & 4.054283 & 2.054964\end{array}$

tetraphenylporphine $\mathrm{S}_{1}$

$\begin{array}{lrrc}\mathrm{C} & 4.236136 & 0.681076 & 0.180332 \\ \mathrm{C} & 2.887081 & 1.131690 & 0.018359 \\ \mathrm{~N} & 2.107765 & -0.000990 & -0.070934 \\ \mathrm{C} & 2.885715 & -1.134736 & 0.016540 \\ \mathrm{C} & 4.235332 & -0.686056 & 0.178944 \\ \mathrm{C} & 2.441526 & -2.464363 & -0.029048 \\ \mathrm{C} & 1.088456 & -2.882327 & -0.095476 \\ \mathrm{~N} & -0.001137 & -2.058959 & -0.008775 \\ \mathrm{C} & -1.091718 & -2.881160 & -0.093785 \\ \mathrm{C} & -0.681376 & -4.258495 & -0.270363 \\ \mathrm{C} & 0.676324 & -4.259195 & -0.271723 \\ \mathrm{C} & -2.444298 & -2.461823 & -0.025830 \\ \mathrm{C} & -2.887081 & -1.131690 & 0.018359 \\ \mathrm{~N} & -2.107765 & 0.000990 & -0.070934 \\ \mathrm{C} & -2.885715 & 1.134736 & 0.016540 \\ \mathrm{C} & -4.235332 & 0.686056 & 0.178944 \\ \mathrm{C} & -4.236136 & -0.681076 & 0.180332 \\ \mathrm{C} & -2.441526 & 2.464363 & -0.029048 \\ \mathrm{C} & -1.088456 & 2.882327 & -0.095476 \\ \mathrm{~N} & 0.001137 & 2.058959 & -0.008775 \\ \mathrm{C} & 1.091718 & 2.881160 & -0.093785 \\ \mathrm{C} & 0.681376 & 4.258495 & -0.270363 \\ \mathrm{C} & -0.676324 & 4.259195 & -0.271723 \\ \mathrm{C} & 2.444298 & 2.461823 & -0.025830 \\ \mathrm{H} & -1.332587 & 5.103423 & -0.398956 \\ \mathrm{C} & -3.492067 & 3.522837 & -0.000963 \\ \mathrm{H} & -5.085100 & 1.335507 & 0.297570 \\ \mathrm{H} & -1.097587 & 0.000270 & -0.108890 \\ \mathrm{H} & -1.338844 & -5.101988 & -0.396347\end{array}$




$\begin{array}{rrrr}\mathrm{H} & 1.332587 & -5.103423 & -0.398956 \\ \mathrm{H} & 1.338844 & 5.101988 & -0.396347 \\ \mathrm{C} & -3.495407 & -3.519566 & 0.006560 \\ \mathrm{C} & 3.492067 & -3.522837 & -0.000963 \\ \mathrm{C} & 3.495407 & 3.519566 & 0.006560 \\ \mathrm{H} & -5.086751 & -1.329179 & 0.300168 \\ \mathrm{H} & 5.085100 & -1.335507 & 0.297570 \\ \mathrm{H} & 5.086751 & 1.329179 & 0.300168 \\ \mathrm{H} & 1.097587 & -0.000270 & -0.108890 \\ \mathrm{C} & -4.408095 & -3.662686 & -1.044514 \\ \mathrm{C} & -5.384496 & -4.652740 & -1.009092 \\ \mathrm{C} & -5.470533 & -5.512180 & 0.081455 \\ \mathrm{C} & -4.569877 & -5.378539 & 1.134351 \\ \mathrm{C} & -3.588436 & -4.395064 & 1.094822 \\ \mathrm{H} & -4.339949 & -3.000861 & -1.899152 \\ \mathrm{H} & -6.075700 & -4.754402 & -1.837124 \\ \mathrm{H} & -6.232635 & -6.281336 & 0.110467 \\ \mathrm{H} & -4.632668 & -6.039830 & 1.990191 \\ \mathrm{H} & -2.890570 & -4.293331 & 1.916621 \\ \mathrm{C} & -4.401241 & 3.664786 & -1.055227 \\ \mathrm{C} & -5.377438 & 4.655154 & -1.024521 \\ \mathrm{C} & -5.466914 & 5.516050 & 0.064587 \\ \mathrm{C} & -4.569778 & 5.383608 & 1.120656 \\ \mathrm{C} & -3.588436 & 4.399816 & 1.085815 \\ \mathrm{H} & -4.330538 & 3.001556 & -1.908559 \\ \mathrm{H} & -6.065914 & 4.755768 & -1.854951 \\ \mathrm{H} & -6.229003 & 6.285341 & 0.090097 \\ \mathrm{H} & -4.635319 & 6.046036 & 1.975407 \\ \mathrm{H} & -2.893433 & 4.298938 & 1.910145 \\ \mathrm{C} & 4.408095 & 3.662686 & -1.044514 \\ \mathrm{C} & 5.384496 & 4.652740 & -1.009092 \\ \mathrm{C} & 5.470533 & 5.512180 & 0.081455 \\ \mathrm{C} & 4.569877 & 5.378539 & 1.134351 \\ \mathrm{C} & 3.588436 & 4.395064 & 1.094822 \\ \mathrm{H} & 4.339949 & 3.000861 & -1.899152 \\ \mathrm{H} & 6.075700 & 4.754402 & -1.837124 \\ \mathrm{H} & 6.232635 & 6.281336 & 0.110467 \\ \mathrm{H} & 4.632668 & 6.039830 & 1.990191 \\ \mathrm{H} & 2.890570 & 4.293331 & 1.916621 \\ \mathrm{C} & 4.401241 & -3.664786 & -1.055227 \\ \mathrm{C} & 5.377438 & -4.655154 & -1.024521 \\ \mathrm{C} & 5.466914 & -5.516050 & 0.064587 \\ \mathrm{C} & 4.569778 & -5.383608 & 1.120656 \\ \mathrm{C} & 3.588436 & -4.399816 & 1.085815 \\ \mathrm{H} & 4.330538 & -3.001556 & -1.908559 \\ \mathrm{H} & 6.065914 & -4.755768 & -1.854951\end{array}$




$$
\begin{array}{llll}
\mathrm{H} & 6.229003 & -6.285341 & 0.090097 \\
\mathrm{H} & 4.635319 & -6.046036 & 1.975407 \\
\mathrm{H} & 2.893433 & -4.298938 & 1.910145
\end{array}
$$

\section{B3LYP/TZVP geometries - Group II}

tetrafluorobenzene $\mathrm{S}_{0}$

$\begin{array}{lrrr}\text { C } & -1.192398 & 0.694468 & 0.000000 \\ \text { C } & -1.192398 & -0.694468 & 0.000000 \\ \text { C } & 0.000000 & -1.398216 & 0.000000 \\ \text { C } & 1.192398 & -0.694468 & 0.000000 \\ \text { C } & 1.192398 & 0.694468 & 0.000000 \\ \text { C } & 0.000000 & 1.398216 & 0.000000 \\ \text { F } & -2.359921 & -1.355953 & 0.000000 \\ \text { H } & 0.000000 & -2.479402 & 0.000000 \\ \text { F } & 2.359921 & -1.355953 & 0.000000 \\ F & 2.359921 & 1.355953 & 0.000000 \\ \text { H } & 0.000000 & 2.479402 & 0.000000 \\ F & -2.359921 & 1.355953 & 0.000000\end{array}$

tetrafluorobenzene $\mathrm{S}_{1}$

$\begin{array}{lrrr}\text { C } & 1.168103 & -0.708304 & 0.108065 \\ \text { C } & 1.168103 & 0.708304 & 0.108065 \\ \text { C } & 0.000000 & 1.466015 & 0.294052 \\ \text { C } & -1.168103 & 0.708304 & 0.108065 \\ \text { C } & -1.168103 & -0.708304 & 0.108065 \\ \text { C } & 0.000000 & -1.466015 & 0.294052 \\ \text { F } & 2.319321 & 1.324056 & -0.202901 \\ \text { H } & 0.000000 & 2.506153 & 0.593745 \\ \text { F } & -2.319321 & 1.324056 & -0.202901 \\ \text { F } & -2.319321 & -1.324056 & -0.202901 \\ \text { H } & 0.000000 & -2.506153 & 0.593745 \\ \text { F } & 2.319321 & -1.324056 & -0.202901\end{array}$

benzonitrile $\mathrm{S}_{0}$

$\begin{array}{lrrr}\mathrm{C} & 0.000000 & 0.000000 & -1.059057 \\ \mathrm{C} & -1.212896 & 0.000000 & -0.359273 \\ \mathrm{C} & -1.206428 & 0.000000 & 1.028569 \\ \mathrm{C} & 0.000000 & 0.000000 & 1.723227 \\ \mathrm{C} & 1.206428 & 0.000000 & 1.028569 \\ \mathrm{C} & 1.212896 & 0.000000 & -0.359273 \\ \mathrm{H} & -2.146132 & 0.000000 & -0.906795\end{array}$




$\begin{array}{rrrr}\mathrm{H} & -2.144559 & 0.000000 & 1.568929 \\ \mathrm{H} & 0.000000 & 0.000000 & 2.806120 \\ \mathrm{H} & 2.144559 & 0.000000 & 1.568929 \\ \mathrm{H} & 2.146132 & 0.000000 & -0.906795 \\ \mathrm{C} & 0.000000 & 0.000000 & -2.489663 \\ \mathrm{~N} & 0.000000 & 0.000000 & -3.643479\end{array}$

benzonitrile $\mathrm{S}_{1}$

$\begin{array}{lrrr}\mathrm{C} & 0.000000 & 0.000000 & -1.112255 \\ \mathrm{C} & -1.227644 & 0.000000 & -0.374040 \\ \mathrm{C} & -1.214824 & 0.000000 & 1.048450 \\ \mathrm{C} & 0.000000 & 0.000000 & 1.766409 \\ \mathrm{C} & 1.214824 & 0.000000 & 1.048450 \\ \mathrm{C} & 1.227644 & 0.000000 & -0.374040 \\ \mathrm{H} & -2.167519 & 0.000000 & -0.907475 \\ \mathrm{H} & -2.158378 & 0.000000 & 1.577156 \\ \mathrm{H} & 0.000000 & 0.000000 & 2.846625 \\ \mathrm{H} & 2.158378 & 0.000000 & 1.577156 \\ \mathrm{H} & 2.167519 & 0.000000 & -0.907475 \\ \mathrm{C} & 0.000000 & 0.000000 & -2.511850 \\ \mathrm{~N} & 0.000000 & 0.000000 & -3.677113\end{array}$

$o$-fluorophenol, cis $\mathrm{S}_{0}$

$\begin{array}{lrrc}\mathrm{C} & 0.327530 & 1.454460 & 0.000000 \\ \mathrm{C} & -0.873209 & 0.776204 & 0.000000 \\ \mathrm{C} & -0.950353 & -0.614253 & 0.000000 \\ \mathrm{C} & 0.237851 & -1.337947 & 0.000000 \\ \mathrm{C} & 1.458694 & -0.671895 & 0.000000 \\ \mathrm{C} & 1.510513 & 0.719211 & 0.000000 \\ \mathrm{~F} & -2.053212 & 1.462092 & 0.000000 \\ \mathrm{O} & -2.144268 & -1.269846 & 0.000000 \\ \mathrm{H} & 0.184001 & -2.418884 & 0.000000 \\ \mathrm{H} & 2.375763 & -1.247111 & 0.000000 \\ \mathrm{H} & 2.462403 & 1.233417 & 0.000000 \\ \mathrm{H} & 0.325114 & 2.536619 & 0.000000 \\ \mathrm{H} & -2.860819 & -0.622086 & 0.000000\end{array}$

$o$-fluorophenol, cis $\mathrm{S}_{1}$
$\begin{array}{llll}\text { C } & 1.226623 & -0.248620 & -0.731540\end{array}$
$\begin{array}{llll}\text { C } & 0.003677 & -0.077853 & -0.095699\end{array}$
$\begin{array}{llll}\text { C } & -0.006181 & -0.028244 & 1.339440\end{array}$
$\begin{array}{llll}\text { C } & 1.178483 & 0.037109 & 2.089015\end{array}$
$\begin{array}{llll}\text { C } & 2.401595 & 0.103414 & 1.413309\end{array}$ 


$\begin{array}{crrr}\text { C } & 2.415655 & -0.024768 & 0.021881 \\ \mathrm{~F} & -1.003611 & 0.810513 & -0.703213 \\ \mathrm{O} & -1.174299 & -0.138285 & 1.990015 \\ \mathrm{H} & 1.126172 & -0.102752 & 3.162725 \\ \mathrm{H} & 3.326349 & 0.215018 & 1.961933 \\ \mathrm{H} & 3.352890 & 0.088821 & -0.514088 \\ \mathrm{H} & 1.284283 & -0.388740 & -1.803852 \\ \mathrm{H} & -1.876257 & -0.245614 & 1.320014\end{array}$

$o$-fluorophenol, trans $\mathrm{S}_{0}$

$\begin{array}{cccc}\mathrm{C} & 0.291050 & 1.262950 & 0.000000 \\ \mathrm{C} & -0.947280 & 0.617210 & 0.000000 \\ \mathrm{C} & -0.962540 & -0.775670 & 0.000000 \\ \mathrm{C} & 0.228060 & -1.498100 & 0.000000 \\ \mathrm{C} & 1.449790 & -0.836200 & 0.000000 \\ \mathrm{C} & 1.478050 & 0.557170 & 0.000000 \\ \mathrm{O} & -2.068640 & 1.392760 & 0.000000 \\ \mathrm{H} & -1.916020 & -1.294430 & 0.000000 \\ \mathrm{H} & 0.193150 & -2.580270 & 0.000000 \\ \mathrm{H} & 2.377450 & -1.393670 & 0.000000 \\ \mathrm{H} & 2.410180 & 1.107900 & 0.000000 \\ \mathrm{~F} & 0.316950 & 2.612150 & 0.000000 \\ \mathrm{H} & -2.850210 & 0.828200 & 0.000000\end{array}$

$o$-fluorophenol, trans $\mathrm{S}_{1}$

$\begin{array}{cccc}\mathrm{C} & 0.819463 & 1.038468 & 0.112157 \\ \mathrm{C} & -0.589079 & 0.974265 & 0.089922 \\ \mathrm{C} & -1.274712 & -0.287516 & 0.089429 \\ \mathrm{C} & -0.470036 & -1.427345 & -0.081862 \\ \mathrm{C} & 0.941536 & -1.339511 & -0.067194 \\ \mathrm{C} & 1.619066 & -0.128198 & 0.164170 \\ \mathrm{O} & -1.259802 & 2.126367 & -0.087680 \\ \mathrm{H} & -2.295093 & -0.361963 & 0.454740 \\ \mathrm{H} & -0.930016 & -2.401661 & -0.193087 \\ \mathrm{H} & 1.516885 & -2.247048 & -0.215461 \\ \mathrm{H} & 2.687751 & -0.068138 & 0.304761 \\ \mathrm{~F} & 1.402882 & 2.216372 & -0.199485 \\ \mathrm{H} & -2.168875 & 1.905909 & -0.370411\end{array}$

$m$-fluorophenol, cis $\mathrm{S}_{0}$
C $\quad 0.310040$
1.410270
0.000000
$\begin{array}{llll}\text { C } & -0.928180 & 0.788660 & 0.000000\end{array}$
$\begin{array}{llll}\text { C } & -0.967970 & -0.605260 & 0.000000\end{array}$ 


$\begin{array}{cccc}\mathrm{C} & 0.215220 & -1.344410 & 0.000000 \\ \mathrm{C} & 1.434810 & -0.681590 & 0.000000 \\ \mathrm{C} & 1.502810 & 0.710690 & 0.000000 \\ \mathrm{O} & -2.144130 & -1.300900 & 0.000000 \\ \mathrm{~F} & 0.341150 & 2.763970 & 0.000000 \\ \mathrm{H} & 0.159970 & -2.424760 & 0.000000 \\ \mathrm{H} & 2.351900 & -1.257670 & 0.000000 \\ \mathrm{H} & 2.444680 & 1.241940 & 0.000000 \\ \mathrm{H} & -1.830640 & 1.388580 & 0.000000 \\ \mathrm{H} & -2.889660 & -0.689520 & 0.000000\end{array}$

$m$-fluorophenol, cis $\mathrm{S}_{1}$

$\begin{array}{cccc}\mathrm{C} & 1.223835 & -0.054051 & -0.688316 \\ \mathrm{C} & -0.053977 & 0.026849 & -0.103688 \\ \mathrm{C} & -0.025102 & 0.039089 & 1.325686 \\ \mathrm{C} & 1.163040 & 0.031172 & 2.088986 \\ \mathrm{C} & 2.427202 & 0.104882 & 1.440042 \\ \mathrm{C} & 2.431608 & -0.037782 & 0.033235 \\ \mathrm{O} & -1.184698 & -0.021016 & 2.015626 \\ \mathrm{~F} & 1.300293 & -0.158986 & -2.033556 \\ \mathrm{H} & 1.078616 & -0.105651 & 3.159251 \\ \mathrm{H} & 3.347105 & 0.147523 & 2.000566 \\ \mathrm{H} & 3.357280 & -0.123665 & -0.522234 \\ \mathrm{H} & -0.905143 & 0.395368 & -0.667317 \\ \mathrm{H} & -1.904699 & -0.243732 & 1.401669\end{array}$

$m$-fluorophenol, trans $\mathrm{S}_{0}$

$\begin{array}{lrcc}\mathrm{C} & 0.268819 & 1.303777 & 0.000000 \\ \mathrm{C} & -0.950166 & 0.628236 & 0.000000 \\ \mathrm{C} & -0.984389 & -0.766467 & 0.000000 \\ \mathrm{C} & 0.208134 & -1.482428 & 0.000000 \\ \mathrm{C} & 1.436416 & -0.831346 & 0.000000 \\ \mathrm{C} & 1.429712 & 0.554668 & 0.000000 \\ \mathrm{O} & -2.080957 & 1.394757 & 0.000000 \\ \mathrm{H} & -1.935149 & -1.287904 & 0.000000 \\ \mathrm{H} & 0.178205 & -2.564703 & 0.000000 \\ \mathrm{H} & 2.373499 & -1.370145 & 0.000000 \\ \mathrm{~F} & 2.613243 & 1.210466 & 0.000000 \\ \mathrm{H} & 0.302520 & 2.384205 & 0.000000 \\ \mathrm{H} & -2.859888 & 0.826894 & 0.000000\end{array}$

$m$-fluorophenol, trans $\mathrm{S}_{1}$

$\begin{array}{llll}\text { C } & 1.085193 & -0.016489 & 0.000000\end{array}$ 


$\begin{array}{cccc}\mathrm{C} & 0.327151 & 1.183343 & 0.000000 \\ \mathrm{C} & -1.092029 & 1.215065 & 0.000000 \\ \mathrm{C} & -1.825305 & -0.008317 & 0.000000 \\ \mathrm{C} & -1.086606 & -1.221458 & 0.000000 \\ \mathrm{C} & 0.318361 & -1.200882 & 0.000000 \\ \mathrm{O} & 1.042726 & 2.331041 & 0.000000 \\ \mathrm{H} & -1.602956 & 2.170701 & 0.000000 \\ \mathrm{H} & -2.903387 & -0.015990 & 0.000000 \\ \mathrm{H} & -1.586575 & -2.180968 & 0.000000 \\ \mathrm{~F} & 0.973125 & -2.382200 & 0.000000 \\ \mathrm{H} & 2.162756 & -0.012217 & 0.000000 \\ \mathrm{H} & 0.446397 & 3.092519 & 0.000000\end{array}$

$p$-fluorophenol $\mathrm{S}_{0}$

$\begin{array}{rrrr}\mathrm{C} & 0.271848 & 1.316786 & 0.000000 \\ \mathrm{C} & -0.950929 & 0.647401 & 0.000000 \\ \mathrm{C} & -0.981116 & -0.745693 & 0.000000 \\ \mathrm{C} & 0.207032 & -1.471417 & 0.000000 \\ \mathrm{C} & 1.407987 & -0.787344 & 0.000000 \\ \mathrm{C} & 1.459176 & 0.596921 & 0.000000 \\ \mathrm{O} & -2.086605 & 1.414425 & 0.000000 \\ \mathrm{H} & -1.930099 & -1.271046 & 0.000000 \\ \mathrm{H} & 0.202442 & -2.553247 & 0.000000 \\ \mathrm{~F} & 2.568417 & -1.490678 & 0.000000 \\ \mathrm{H} & 2.417553 & 1.098966 & 0.000000 \\ \mathrm{H} & 0.279176 & 2.398645 & 0.000000 \\ \mathrm{H} & -2.864902 & 0.846270 & 0.000000\end{array}$

$p$-fluorophenol $\mathrm{S}_{1}$

$\begin{array}{rrrr}\text { C } & 1.266995 & 0.681414 & 0.000000 \\ \text { C } & 0.027239 & 1.360909 & 0.000000 \\ \text { C } & -1.236741 & 0.710964 & 0.000000 \\ \text { C } & -1.251157 & -0.708016 & 0.000000 \\ \text { C } & -0.006474 & -1.366420 & 0.000000 \\ \text { C } & 1.251811 & -0.738823 & 0.000000 \\ \mathrm{O} & 0.092905 & 2.709849 & 0.000000 \\ \text { H } & -2.152539 & 1.287568 & 0.000000 \\ \text { H } & -2.163574 & -1.284115 & 0.000000 \\ \text { F } & -0.026576 & -2.707767 & 0.000000 \\ \text { H } & 2.152147 & -1.333105 & 0.000000 \\ \text { H } & 2.183342 & 1.251768 & 0.000000 \\ \text { H } & -0.800682 & 3.081368 & 0.000000\end{array}$

phenylacetylene $S_{0}$ 


$\begin{array}{lrrr}\mathrm{C} & 1.208805 & 0.000000 & -0.014088 \\ \mathrm{C} & 0.000000 & 0.000000 & -0.726252 \\ \mathrm{C} & -1.208805 & 0.000000 & -0.014088 \\ \mathrm{C} & -1.204287 & 0.000000 & 1.374004 \\ \mathrm{C} & 0.000000 & 0.000000 & 2.072345 \\ \mathrm{C} & 1.204287 & 0.000000 & 1.374004 \\ \mathrm{C} & 0.000000 & 0.000000 & -2.152937 \\ \mathrm{C} & 0.000000 & 0.000000 & -3.356489 \\ \mathrm{H} & -2.143698 & 0.000000 & -0.559315 \\ \mathrm{H} & -2.143918 & 0.000000 & 1.912691 \\ \mathrm{H} & 0.000000 & 0.000000 & 3.155347 \\ \mathrm{H} & 2.143918 & 0.000000 & 1.912691 \\ \mathrm{H} & 2.143698 & 0.000000 & -0.559315 \\ \mathrm{H} & 0.000000 & 0.000000 & -4.418618\end{array}$

phenylacetylene $S_{1}$

$\begin{array}{lrrr}\mathrm{C} & 1.235902 & 0.000000 & -0.032094 \\ \mathrm{C} & 0.000000 & 0.000000 & -0.756783 \\ \mathrm{C} & -1.235902 & 0.000000 & -0.032094 \\ \mathrm{C} & -1.223681 & 0.000000 & 1.392543 \\ \mathrm{C} & 0.000000 & 0.000000 & 2.091188 \\ \mathrm{C} & 1.223681 & 0.000000 & 1.392543 \\ \mathrm{C} & 0.000000 & 0.000000 & -2.148788 \\ \mathrm{C} & 0.000000 & 0.000000 & -3.366302 \\ \mathrm{H} & -2.168808 & 0.000000 & -0.576644 \\ \mathrm{H} & -2.159397 & 0.000000 & 1.934138 \\ \mathrm{H} & 0.000000 & 0.000000 & 3.173262 \\ \mathrm{H} & 2.159397 & 0.000000 & 1.934138 \\ \mathrm{H} & 2.168808 & 0.000000 & -0.576644 \\ \mathrm{H} & 0.000000 & 0.000000 & -4.428461\end{array}$

aniline $\mathrm{S}_{0}$

$\begin{array}{rrrr}\mathrm{C} & 0.921128 & 0.026253 & 0.000000 \\ \mathrm{C} & 0.205060 & 0.034152 & 1.204062 \\ \mathrm{C} & -1.183441 & 0.043209 & 1.198540 \\ \mathrm{C} & -1.891316 & 0.048505 & 0.000000 \\ \mathrm{C} & -1.183441 & 0.043209 & -1.198540 \\ \mathrm{C} & 0.205060 & 0.034152 & -1.204062 \\ \mathrm{H} & 0.743388 & 0.034816 & 2.145661 \\ \mathrm{H} & -2.973607 & 0.055079 & 0.000000 \\ \mathrm{H} & -1.716115 & 0.046732 & 2.142191 \\ \mathrm{H} & -1.716115 & 0.046732 & -2.142191 \\ \mathrm{H} & 0.743388 & 0.034816 & -2.145661\end{array}$




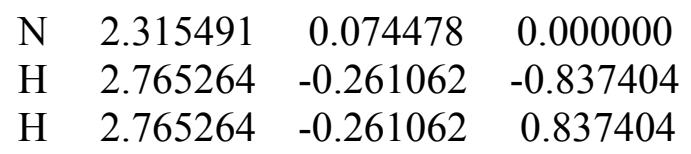

aniline $\mathrm{S}_{1}$

$\begin{array}{rrrr}\mathrm{C} & 0.891430 & -0.033620 & 0.000000 \\ \mathrm{C} & 0.225580 & -0.008070 & 1.246210 \\ \mathrm{C} & -1.201660 & 0.046090 & 1.236700 \\ \mathrm{C} & -1.871920 & 0.071230 & 0.000000 \\ \mathrm{C} & -1.201660 & 0.046090 & -1.236700 \\ \mathrm{C} & 0.225580 & -0.008070 & -1.246210 \\ \mathrm{H} & 0.791900 & -0.029740 & 2.168640 \\ \mathrm{H} & -2.955960 & 0.111990 & 0.000000 \\ \mathrm{H} & -1.759390 & 0.067210 & 2.161880 \\ \mathrm{H} & -1.759390 & 0.067210 & -2.161880 \\ \mathrm{H} & 0.791900 & -0.029740 & -2.168640 \\ \mathrm{~N} & 2.263040 & -0.086800 & 0.000000 \\ \mathrm{H} & 2.780270 & -0.106900 & -0.864400 \\ \mathrm{H} & 2.780270 & -0.106900 & 0.864400\end{array}$

catechol $\mathrm{S}_{0}$

$\begin{array}{rrrr}\mathrm{C} & 0.471928 & 1.279334 & 0.000000 \\ \mathrm{C} & -0.747855 & 0.620678 & 0.000000 \\ \mathrm{C} & -0.798623 & -0.778566 & 0.000000 \\ \mathrm{C} & 0.384195 & -1.504141 & 0.000000 \\ \mathrm{C} & 1.610060 & -0.843197 & 0.000000 \\ \mathrm{C} & 1.657371 & 0.545627 & 0.000000 \\ \mathrm{O} & -1.974916 & 1.250721 & 0.000000 \\ \mathrm{O} & -1.996699 & -1.430878 & 0.000000 \\ \mathrm{H} & 0.326755 & -2.585038 & 0.000000 \\ \mathrm{H} & 2.526678 & -1.419003 & 0.000000 \\ \mathrm{H} & 2.607687 & 1.063422 & 0.000000 \\ \mathrm{H} & 0.497166 & 2.364282 & 0.000000 \\ \mathrm{H} & -1.861325 & 2.206887 & 0.000000 \\ \mathrm{H} & -2.702420 & -0.770128 & 0.000000\end{array}$

catechol $\mathrm{S}_{1}$

$\begin{array}{rrrr}\mathrm{C} & 0.476371 & 1.347593 & 0.000000 \\ \mathrm{C} & -0.761018 & 0.626151 & 0.000000 \\ \mathrm{C} & -0.804866 & -0.783260 & 0.000000 \\ \mathrm{C} & 0.388807 & -1.572288 & 0.000000 \\ \mathrm{C} & 1.602468 & -0.858466 & 0.000000 \\ \mathrm{C} & 1.644492 & 0.557707 & 0.000000\end{array}$




$\begin{array}{rrrr}\mathrm{O} & -1.974226 & 1.257189 & 0.000000 \\ \mathrm{O} & -1.991875 & -1.432114 & 0.000000 \\ \mathrm{H} & 0.326724 & -2.648350 & 0.000000 \\ \mathrm{H} & 2.539601 & -1.399809 & 0.000000 \\ \mathrm{H} & 2.611458 & 1.044675 & 0.000000 \\ \mathrm{H} & 0.500593 & 2.427675 & -0.000001 \\ \mathrm{H} & -1.848797 & 2.213563 & 0.000001 \\ \mathrm{H} & -2.709731 & -0.780266 & 0.000000\end{array}$

resorcinol, isomer $1 \mathrm{~S}_{0}$

$\begin{array}{rrrr}\mathrm{C} & 0.074720 & 1.147540 & 0.000000 \\ \mathrm{C} & -1.139910 & 0.465360 & 0.000000 \\ \mathrm{C} & -1.170470 & -0.927470 & 0.000000 \\ \mathrm{C} & 0.033120 & -1.628670 & 0.000000 \\ \mathrm{C} & 1.253150 & -0.968820 & 0.000000 \\ \mathrm{C} & 1.264940 & 0.426710 & 0.000000 \\ \mathrm{O} & -2.276130 & 1.229440 & 0.000000 \\ \mathrm{H} & -2.117610 & -1.455950 & 0.000000 \\ \mathrm{H} & 0.014750 & -2.711880 & 0.000000 \\ \mathrm{H} & 2.191010 & -1.507540 & 0.000000 \\ \mathrm{O} & 2.485870 & 1.044010 & 0.000000 \\ \mathrm{H} & 0.069770 & 2.231870 & 0.000000 \\ \mathrm{H} & -3.050070 & 0.654650 & 0.000000 \\ \mathrm{H} & 2.366860 & 2.000740 & 0.000000\end{array}$

resorcinol, isomer $1 \mathrm{~S}_{1}$

$\begin{array}{lrrc}\mathrm{C} & -0.001111 & 1.067394 & 0.046271 \\ \mathrm{C} & -1.193576 & 0.306634 & -0.010189 \\ \mathrm{C} & -1.203649 & -1.108777 & -0.038098 \\ \mathrm{C} & 0.001304 & -1.849321 & 0.084556 \\ \mathrm{C} & 1.211282 & -1.109752 & 0.017523 \\ \mathrm{C} & 1.195807 & 0.302854 & 0.038991 \\ \mathrm{O} & -2.354369 & 1.014036 & -0.050779 \\ \mathrm{H} & -2.150493 & -1.622657 & -0.171684 \\ \mathrm{H} & -0.000240 & -2.927299 & 0.087124 \\ \mathrm{H} & 2.168665 & -1.604153 & -0.086404 \\ \mathrm{O} & 2.404083 & 0.923022 & -0.008545 \\ \mathrm{H} & -0.027035 & 2.100925 & 0.369783 \\ \mathrm{H} & -3.107659 & 0.410166 & -0.011387 \\ \mathrm{H} & 2.267984 & 1.853048 & -0.247513\end{array}$

resorcinol, isomer $2 \mathrm{~S}_{0}$

$\begin{array}{llll}\text { C } & 1.199870 & 0.000000 & -0.546160\end{array}$ 


$\begin{array}{rrrc}\mathrm{C} & 0.000000 & 0.000000 & -1.248540 \\ \mathrm{C} & -1.199870 & 0.000000 & -0.546160 \\ \mathrm{C} & -1.210110 & 0.000000 & 0.850420 \\ \mathrm{C} & 0.000000 & 0.000000 & 1.533780 \\ \mathrm{C} & 1.210110 & 0.000000 & 0.850420 \\ \mathrm{O} & -2.350850 & 0.000000 & -1.286440 \\ \mathrm{O} & 2.350850 & 0.000000 & -1.286440 \\ \mathrm{H} & -2.149430 & 0.000000 & 1.392680 \\ \mathrm{H} & 0.000000 & 0.000000 & 2.617170 \\ \mathrm{H} & 2.149430 & 0.000000 & 1.392680 \\ \mathrm{H} & 0.000000 & 0.000000 & -2.330050 \\ \mathrm{H} & -3.113540 & 0.000000 & -0.696920 \\ \mathrm{H} & 3.113540 & 0.000000 & -0.696920\end{array}$

resorcinol, isomer $2 \mathrm{~S}_{1}$

$\begin{array}{lrrr}\mathrm{C} & 1.198928 & 0.000000 & -0.328275 \\ \mathrm{C} & 0.000000 & 0.000000 & -1.080665 \\ \mathrm{C} & -1.198928 & 0.000000 & -0.328275 \\ \mathrm{C} & -1.215145 & 0.000000 & 1.088139 \\ \mathrm{C} & 0.000000 & 0.000000 & 1.828539 \\ \mathrm{C} & 1.215145 & 0.000000 & 1.088139 \\ \mathrm{O} & -2.357588 & 0.000000 & -1.042079 \\ \mathrm{O} & 2.357588 & 0.000000 & -1.042079 \\ \mathrm{H} & -2.168823 & 0.000000 & 1.604854 \\ \mathrm{H} & 0.000000 & 0.000000 & 2.906575 \\ \mathrm{H} & 2.168823 & 0.000000 & 1.604854 \\ \mathrm{H} & 0.000000 & 0.000000 & -2.158130 \\ \mathrm{H} & -3.112764 & 0.000000 & -0.440342 \\ \mathrm{H} & 3.112764 & 0.000000 & -0.440342\end{array}$

resorcinol, isomer $3 \mathrm{~S}_{0}$

$\begin{array}{cccc}\mathrm{C} & 1.205164 & 0.000000 & -0.288427 \\ \mathrm{C} & 0.000000 & 0.000000 & -0.990132 \\ \mathrm{C} & -1.205164 & 0.000000 & -0.288427 \\ \mathrm{C} & -1.212839 & 0.000000 & 1.103533 \\ \mathrm{C} & 0.000000 & 0.000000 & 1.781683 \\ \mathrm{C} & 1.212839 & 0.000000 & 1.103533 \\ \mathrm{O} & -2.416386 & 0.000000 & -0.926044 \\ \mathrm{O} & 2.416386 & 0.000000 & -0.926044 \\ \mathrm{H} & -2.158307 & 0.000000 & 1.628269 \\ \mathrm{H} & 0.000000 & 0.000000 & 2.864776 \\ \mathrm{H} & 2.158307 & 0.000000 & 1.628269 \\ \mathrm{H} & 0.000000 & 0.000000 & -2.076800 \\ \mathrm{H} & 2.285868 & 0.000000 & -1.880854\end{array}$


$\begin{array}{llll}\mathrm{H} & -2.285868 & 0.000000 & -1.880854\end{array}$

resorcinol, isomer $3 \mathrm{~S}_{1}$

$\begin{array}{rrrc}\mathrm{C} & -0.025730 & -0.302925 & 1.189319 \\ \mathrm{C} & -0.110620 & -1.076912 & 0.000000 \\ \mathrm{C} & -0.025730 & -0.302925 & -1.189319 \\ \mathrm{C} & 0.021034 & 1.110128 & -1.201902 \\ \mathrm{C} & -0.066715 & 1.849063 & 0.000000 \\ \mathrm{C} & 0.021034 & 1.110128 & 1.201902 \\ \mathrm{O} & 0.061516 & -0.919109 & -2.396383 \\ \mathrm{O} & 0.061516 & -0.919109 & 2.396383 \\ \mathrm{H} & 0.155231 & 1.600428 & -2.158206 \\ \mathrm{H} & -0.078858 & 2.927334 & 0.000000 \\ \mathrm{H} & 0.155231 & 1.600428 & 2.158206 \\ \mathrm{H} & -0.640368 & -2.031691 & 0.000000 \\ \mathrm{H} & 0.272436 & -1.855047 & 2.249631 \\ \mathrm{H} & 0.272436 & -1.855047 & -2.249631\end{array}$

hydroquinone, cis $\mathrm{S}_{0}$

$\begin{array}{rrrr}\mathrm{C} & -0.696953 & 0.000000 & 1.092428 \\ \mathrm{C} & 0.696953 & 0.000000 & 1.092428 \\ \mathrm{C} & 1.396255 & 0.000000 & -0.108957 \\ \mathrm{C} & 0.692887 & 0.000000 & -1.312779 \\ \mathrm{C} & -0.692887 & 0.000000 & -1.312779 \\ \mathrm{C} & -1.396255 & 0.000000 & -0.108957 \\ \mathrm{O} & 2.768757 & 0.000000 & -0.177005 \\ \mathrm{O} & -2.768757 & 0.000000 & -0.177005 \\ \mathrm{H} & 1.245549 & 0.000000 & -2.243246 \\ \mathrm{H} & -1.245549 & 0.000000 & -2.243246 \\ \mathrm{H} & -1.231506 & 0.000000 & 2.036617 \\ \mathrm{H} & 1.231506 & 0.000000 & 2.036617 \\ \mathrm{H} & -3.137003 & 0.000000 & 0.713232 \\ \mathrm{H} & 3.137003 & 0.000000 & 0.713232\end{array}$

hydroquinone, cis $\mathrm{S}_{1}$
$\begin{array}{llll}\text { C } & 0.708634 & 0.000000 & -1.131995\end{array}$
$\begin{array}{llll}\text { C } & -0.708634 & 0.000000 & -1.131995\end{array}$
$\begin{array}{llll}\text { C } & -1.375030 & 0.000000 & 0.119751\end{array}$
$\begin{array}{llll}\text { C } & -0.708799 & 0.000000 & 1.362428\end{array}$
$\begin{array}{llll}\text { C } & 0.708799 & 0.000000 & 1.362428\end{array}$
$\begin{array}{llll}\text { C } & 1.375030 & 0.000000 & 0.119751\end{array}$
$\begin{array}{llll}\mathrm{O} & -2.727422 & 0.000000 & 0.165749\end{array}$
$\begin{array}{llll}\mathrm{O} & 2.727422 & 0.000000 & 0.165749\end{array}$ 


$\begin{array}{rrrr}\mathrm{H} & -1.287896 & 0.000000 & 2.273269 \\ \mathrm{H} & 1.287896 & 0.000000 & 2.273269 \\ \mathrm{H} & 1.274725 & 0.000000 & -2.054647 \\ \mathrm{H} & -1.274725 & 0.000000 & -2.054647 \\ \mathrm{H} & 3.082155 & 0.000000 & -0.733787 \\ \mathrm{H} & -3.082155 & 0.000000 & -0.733787\end{array}$

hydroquinone, trans $\mathrm{S}_{0}$

$\begin{array}{rrrr}\mathrm{C} & 0.020550 & 1.391060 & 0.000000 \\ \mathrm{C} & -1.197370 & 0.718710 & 0.000000 \\ \mathrm{C} & -1.211860 & -0.674830 & 0.000000 \\ \mathrm{C} & -0.020550 & -1.391060 & 0.000000 \\ \mathrm{C} & 1.197370 & -0.718710 & 0.000000 \\ \mathrm{C} & 1.211860 & 0.674830 & 0.000000 \\ \mathrm{O} & -2.343130 & 1.478040 & 0.000000 \\ \mathrm{H} & -2.156550 & -1.209360 & 0.000000 \\ \mathrm{H} & -0.025930 & -2.473470 & 0.000000 \\ \mathrm{O} & 2.343130 & -1.478040 & 0.000000 \\ \mathrm{H} & 2.156550 & 1.209360 & 0.000000 \\ \mathrm{H} & 0.025930 & 2.473470 & 0.000000 \\ \mathrm{H} & -3.113960 & 0.899770 & 0.000000 \\ \mathrm{H} & 3.113960 & -0.899770 & 0.000000\end{array}$

hydroquinone, trans $\mathrm{S}_{1}$

$\begin{array}{rrrr}\mathrm{C} & 1.262903 & 0.679146 & 0.000000 \\ \mathrm{C} & 0.035137 & 1.374403 & 0.000000 \\ \mathrm{C} & -1.230899 & 0.737909 & 0.000000 \\ \mathrm{C} & -1.262903 & -0.679146 & 0.000000 \\ \mathrm{C} & -0.035137 & -1.374403 & 0.000000 \\ \mathrm{C} & 1.230899 & -0.737909 & 0.000000 \\ \mathrm{O} & 0.118582 & 2.724879 & 0.000000 \\ \mathrm{H} & -2.140739 & 1.324248 & 0.000000 \\ \mathrm{H} & -2.186410 & -1.238299 & 0.000000 \\ \mathrm{O} & -0.118582 & -2.724879 & 0.000000 \\ \mathrm{H} & 2.140739 & -1.324248 & 0.000000 \\ \mathrm{H} & 2.186410 & 1.238299 & 0.000000 \\ \mathrm{H} & -0.771428 & 3.103170 & 0.000000 \\ \mathrm{H} & 0.771428 & -3.103170 & 0.000000\end{array}$

salicylic acid $\mathrm{S}_{0}$
$\begin{array}{llll}\text { C } & 1.102298 & 1.599491 & 0.000000\end{array}$
$\begin{array}{llll}\mathrm{C} & 1.134246 & 0.200350 & 0.000000\end{array}$
$\begin{array}{llll}\text { C } & -0.085249 & -0.518596 & 0.000000\end{array}$ 


$\begin{array}{lrrr}\mathrm{C} & -1.300507 & 0.187187 & 0.000000 \\ \mathrm{C} & -1.318658 & 1.567037 & 0.000000 \\ \mathrm{C} & -0.107025 & 2.267998 & 0.000000 \\ \mathrm{O} & 2.335855 & -0.398219 & 0.000000 \\ \mathrm{C} & -0.044055 & -1.980565 & 0.000000 \\ \mathrm{O} & -1.251085 & -2.582019 & 0.000000 \\ \mathrm{O} & 0.986210 & -2.644565 & 0.000000 \\ \mathrm{H} & -2.224492 & -0.374359 & 0.000000 \\ \mathrm{H} & 2.043961 & 2.132349 & 0.000000 \\ \mathrm{H} & -2.259529 & 2.101067 & 0.000000 \\ \mathrm{H} & -0.112879 & 3.351415 & 0.000000 \\ \mathrm{H} & 2.189639 & -1.370531 & 0.000000 \\ \mathrm{H} & -1.088731 & -3.538040 & 0.000000\end{array}$

salicylic acid $\mathrm{S}_{1}$

$\begin{array}{rrrr}\mathrm{C} & 0.994817 & 1.911512 & 0.000000 \\ \mathrm{C} & 0.958670 & 0.506775 & 0.000000 \\ \mathrm{C} & -0.291123 & -0.222380 & 0.000000 \\ \mathrm{C} & -1.445925 & 0.539946 & 0.000000 \\ \mathrm{C} & -1.401626 & 1.978205 & 0.000000 \\ \mathrm{C} & -0.203963 & 2.662882 & 0.000000 \\ \mathrm{O} & 2.086995 & -0.176106 & 0.000000 \\ \mathrm{C} & -0.232739 & -1.664944 & 0.000000 \\ \mathrm{O} & -1.430006 & -2.315303 & 0.000000 \\ \mathrm{O} & 0.857445 & -2.321401 & 0.000000 \\ \mathrm{H} & -2.407450 & 0.044741 & 0.000000 \\ \mathrm{H} & 1.965709 & 2.389234 & 0.000000 \\ \mathrm{H} & -2.340181 & 2.517626 & 0.000000 \\ \mathrm{H} & -0.173814 & 3.742930 & 0.000000 \\ \mathrm{H} & 1.800014 & -1.195395 & 0.000000 \\ \mathrm{H} & -1.226245 & -3.261376 & 0.000000\end{array}$

$o$-cresol, cis $\mathrm{S}_{0}$

$\begin{array}{rrrr}\mathrm{C} & 1.965208 & 0.512912 & 0.000000 \\ \mathrm{C} & 0.730122 & 1.150860 & 0.000000 \\ \mathrm{C} & -0.466391 & 0.433086 & 0.000000 \\ \mathrm{C} & -0.389713 & -0.966856 & 0.000000 \\ \mathrm{C} & 0.840862 & -1.617667 & 0.000000 \\ \mathrm{C} & 2.015306 & -0.877710 & 0.000000 \\ \mathrm{C} & -1.802954 & 1.129994 & 0.000000 \\ \mathrm{O} & -1.509251 & -1.759793 & 0.000000 \\ \mathrm{H} & 0.856035 & -2.700017 & 0.000000 \\ \mathrm{H} & 2.969562 & -1.390047 & 0.000000 \\ \mathrm{H} & 2.877596 & 1.095491 & 0.000000\end{array}$




$\begin{array}{lrrr}\mathrm{H} & 0.686300 & 2.234342 & 0.000000 \\ \mathrm{H} & -2.301515 & -1.212019 & 0.000000 \\ \mathrm{H} & -2.398814 & 0.877808 & 0.884438 \\ \mathrm{H} & -1.673539 & 2.211817 & 0.000000 \\ \mathrm{H} & -2.398814 & 0.877808 & -0.884438\end{array}$

$o$-cresol, cis $\mathrm{S}_{1}$

$\begin{array}{lrrr}\mathrm{C} & 1.855686 & -0.032451 & -0.782592 \\ \mathrm{C} & 0.623916 & 0.127544 & -1.454438 \\ \mathrm{C} & -0.598108 & 0.096934 & -0.712651 \\ \mathrm{C} & -0.472701 & 0.077249 & 0.712939 \\ \mathrm{C} & 0.768792 & -0.003882 & 1.396309 \\ \mathrm{C} & 1.945256 & -0.106216 & 0.623416 \\ \mathrm{C} & -1.890890 & -0.094873 & -1.433079 \\ \mathrm{O} & -1.561907 & 0.112214 & 1.508562 \\ \mathrm{H} & 0.780771 & 0.101272 & 2.471502 \\ \mathrm{H} & 2.909804 & -0.210390 & 1.099991 \\ \mathrm{H} & 2.764479 & -0.104374 & -1.368860 \\ \mathrm{H} & 0.596145 & 0.189030 & -2.533431 \\ \mathrm{H} & -2.353065 & 0.287627 & 0.972675 \\ \mathrm{H} & -2.753156 & -0.260702 & -0.779306 \\ \mathrm{H} & -2.133895 & 0.765733 & -2.069642 \\ \mathrm{H} & -1.831707 & -0.964205 & -2.108985\end{array}$

o-cresol, trans $\mathrm{S}_{0}$

$\begin{array}{rrrr}\mathrm{C} & 2.418630 & 0.000505 & 0.029960 \\ \mathrm{C} & 1.243460 & 0.000078 & -0.718310 \\ \mathrm{C} & 0.028340 & 0.000172 & -0.018880 \\ \mathrm{C} & -0.000813 & -0.000243 & 1.372720 \\ \mathrm{C} & 1.189190 & -0.000381 & 2.094380 \\ \mathrm{C} & 2.405230 & 0.000320 & 1.423300 \\ \mathrm{C} & 1.251410 & -0.000447 & -2.222520 \\ \mathrm{O} & -1.119640 & 0.000780 & -0.772880 \\ \mathrm{H} & -0.954510 & -0.000254 & 1.892130 \\ \mathrm{H} & 1.158440 & -0.000760 & 3.177240 \\ \mathrm{H} & 3.336480 & 0.000417 & 1.976010 \\ \mathrm{H} & 3.367520 & 0.000915 & -0.495560 \\ \mathrm{H} & -1.887380 & 0.000865 & -0.190010 \\ \mathrm{H} & 2.273360 & -0.000449 & -2.603130 \\ \mathrm{H} & 0.734680 & -0.877050 & -2.621810 \\ \mathrm{H} & 0.734310 & 0.875530 & -2.622670\end{array}$

o-cresol, trans $\mathrm{S}_{1}$ 


$\begin{array}{lrrr}\mathrm{C} & 2.424982 & 0.254835 & -0.005114 \\ \mathrm{C} & 1.211059 & 0.116214 & -0.754587 \\ \mathrm{C} & 0.006667 & -0.009186 & -0.014758 \\ \mathrm{C} & -0.050399 & -0.094040 & 1.412951 \\ \mathrm{C} & 1.178705 & -0.072061 & 2.104950 \\ \mathrm{C} & 2.388998 & 0.089433 & 1.391588 \\ \mathrm{C} & 1.253067 & -0.017185 & -2.236648 \\ \mathrm{O} & -1.143306 & -0.109910 & -0.717826 \\ \mathrm{H} & -0.994119 & 0.053435 & 1.925251 \\ \mathrm{H} & 1.199822 & -0.141011 & 3.184140 \\ \mathrm{H} & 3.318949 & 0.100647 & 1.948256 \\ \mathrm{H} & 3.363081 & 0.399080 & -0.521724 \\ \mathrm{H} & -1.844915 & -0.418976 & -0.119741 \\ \mathrm{H} & 1.844189 & -0.896625 & -2.534699 \\ \mathrm{H} & 0.262260 & -0.102350 & -2.677244 \\ \mathrm{H} & 1.759648 & 0.847701 & -2.684816\end{array}$

$m$-cresol, cis $\mathrm{S}_{0}$

$\begin{array}{crcc}\mathrm{C} & 1.158489 & -0.004290 & 2.087516 \\ \mathrm{C} & 2.370500 & 0.010220 & 1.413993 \\ \mathrm{C} & 2.415801 & 0.020061 & 0.021277 \\ \mathrm{C} & 1.236154 & 0.013651 & -0.721538 \\ \mathrm{C} & 0.015619 & 0.000947 & -0.041062 \\ \mathrm{C} & -0.024413 & -0.008529 & 1.350245 \\ \mathrm{C} & 1.262830 & 0.002001 & -2.229252 \\ \mathrm{O} & -1.201918 & -0.019807 & 2.050796 \\ \mathrm{H} & 1.108498 & -0.009716 & 3.168366 \\ \mathrm{H} & 3.293454 & 0.016479 & 1.981341 \\ \mathrm{H} & 3.371655 & 0.033753 & -0.488375 \\ \mathrm{H} & -0.913771 & -0.000292 & -0.604225 \\ \mathrm{H} & -1.944535 & -0.021109 & 1.436371 \\ \mathrm{H} & 2.260706 & 0.225270 & -2.607879 \\ \mathrm{H} & 0.971576 & -0.976988 & -2.620839 \\ \mathrm{H} & 0.571655 & 0.737719 & -2.646785\end{array}$

$m$-cresol, cis $\mathrm{S}_{1}$

$\begin{array}{rrrr}\mathrm{C} & 1.162746 & 0.013464 & 2.125661 \\ \mathrm{C} & 2.407108 & 0.082778 & 1.447435 \\ \mathrm{C} & 2.408494 & 0.033544 & 0.035603 \\ \mathrm{C} & 1.221281 & -0.003846 & -0.745680 \\ \mathrm{C} & -0.032223 & 0.012296 & -0.070350 \\ \mathrm{C} & -0.019109 & 0.008932 & 1.353585 \\ \mathrm{C} & 1.266569 & -0.034077 & -2.235541 \\ \mathrm{O} & -1.184668 & -0.034294 & 2.036789\end{array}$




$\begin{array}{rrrr}\mathrm{H} & 1.089515 & -0.107562 & 3.197433 \\ \mathrm{H} & 3.336783 & 0.110967 & 1.995817 \\ \mathrm{H} & 3.359470 & 0.038157 & -0.485550 \\ \mathrm{H} & -0.950206 & 0.235323 & -0.604427 \\ \mathrm{H} & -1.909429 & -0.207239 & 1.415500 \\ \mathrm{H} & 2.285200 & 0.061918 & -2.613024 \\ \mathrm{H} & 0.845962 & -0.968431 & -2.634199 \\ \mathrm{H} & 0.664787 & 0.777440 & -2.669113\end{array}$

$m$-cresol, trans $\mathrm{S}_{0}$

$\begin{array}{rrrr}\mathrm{C} & -1.144261 & -1.134826 & 0.000000 \\ \mathrm{C} & -1.195511 & 0.261064 & 0.000000 \\ \mathrm{C} & 0.000000 & 0.979262 & 0.000000 \\ \mathrm{C} & 1.224155 & 0.318363 & 0.000000 \\ \mathrm{C} & 1.270983 & -1.075247 & 0.000000 \\ \mathrm{C} & 0.081102 & -1.792647 & 0.000000 \\ \mathrm{C} & -2.514960 & 0.991712 & 0.000000 \\ \mathrm{O} & 2.355256 & 1.092340 & 0.000000 \\ \mathrm{H} & 2.225630 & -1.591059 & 0.000000 \\ \mathrm{H} & 0.112604 & -2.875549 & 0.000000 \\ \mathrm{H} & -2.064127 & -1.706921 & 0.000000 \\ \mathrm{H} & -0.003090 & 2.062830 & 0.000000 \\ \mathrm{H} & 3.134720 & 0.525537 & 0.000000 \\ \mathrm{H} & -3.353406 & 0.294723 & 0.000000 \\ \mathrm{H} & -2.611713 & 1.632815 & 0.879954 \\ \mathrm{H} & -2.611713 & 1.632815 & -0.879954\end{array}$

$m$-cresol, trans $\mathrm{S}_{1}$

$\begin{array}{rrrr}\mathrm{C} & -1.204120 & -1.058551 & 0.000000 \\ \mathrm{C} & 0.203853 & -1.237453 & 0.000000 \\ \mathrm{C} & 1.025509 & -0.070949 & 0.000000 \\ \mathrm{C} & 0.396018 & 1.196629 & 0.000000 \\ \mathrm{C} & -1.012198 & 1.375042 & 0.000000 \\ \mathrm{C} & -1.827053 & 0.213748 & 0.000000 \\ \mathrm{C} & 0.826940 & -2.592109 & 0.000000 \\ \mathrm{O} & 1.220622 & 2.269440 & 0.000000 \\ \mathrm{H} & -1.436629 & 2.371587 & 0.000000 \\ \mathrm{H} & -2.904072 & 0.292831 & 0.000000 \\ \mathrm{H} & -1.833018 & -1.941672 & 0.000000 \\ \mathrm{H} & 2.104662 & -0.124270 & 0.000000 \\ \mathrm{H} & 0.697193 & 3.083058 & 0.000000 \\ \mathrm{H} & 0.074857 & -3.382350 & 0.000000 \\ \mathrm{H} & 1.471957 & -2.739982 & -0.877653 \\ \mathrm{H} & 1.471957 & -2.739982 & 0.877653\end{array}$


$p$-cresol $\mathrm{S}_{0}$

$\begin{array}{lrrr}\mathrm{C} & 0.710913 & 0.011299 & -1.189862 \\ \mathrm{C} & -0.676185 & 0.013268 & -1.198070 \\ \mathrm{C} & -1.374801 & 0.000369 & 0.007436 \\ \mathrm{C} & -0.672412 & -0.016639 & 1.208340 \\ \mathrm{C} & 0.720012 & -0.018389 & 1.197571 \\ \mathrm{C} & 1.439430 & -0.002586 & 0.004225 \\ \mathrm{O} & -2.744765 & 0.000620 & -0.053927 \\ \mathrm{C} & 2.947477 & 0.021659 & -0.004989 \\ \mathrm{H} & -1.207449 & -0.031666 & 2.152664 \\ \mathrm{H} & 1.251460 & -0.034277 & 2.142539 \\ \mathrm{H} & 1.240245 & 0.018830 & -2.136474 \\ \mathrm{H} & -1.231293 & 0.021584 & -2.127318 \\ \mathrm{H} & -3.108157 & -0.010510 & 0.838558 \\ \mathrm{H} & 3.353746 & -0.664109 & -0.751844 \\ \mathrm{H} & 3.329256 & 1.019909 & -0.239849 \\ \mathrm{H} & 3.354082 & -0.263986 & 0.966299\end{array}$

$p$-cresol $\mathrm{S}_{1}$

$\begin{array}{lrrr}\mathrm{C} & 0.732469 & -0.083614 & -1.235125 \\ \mathrm{C} & -0.685517 & -0.031740 & -1.243001 \\ \mathrm{C} & -1.355970 & -0.012778 & -0.000919 \\ \mathrm{C} & -0.685426 & -0.004286 & 1.251197 \\ \mathrm{C} & 0.731492 & -0.016384 & 1.238125 \\ \mathrm{C} & 1.431889 & -0.034016 & 0.001512 \\ \mathrm{O} & -2.706584 & 0.014531 & -0.047680 \\ \mathrm{C} & 2.919870 & 0.041662 & -0.000792 \\ \mathrm{H} & -1.247610 & -0.084991 & 2.173344 \\ \mathrm{H} & 1.288783 & -0.011797 & 2.164706 \\ \mathrm{H} & 1.287010 & -0.107211 & -2.162620 \\ \mathrm{H} & -1.261580 & 0.040555 & -2.154027 \\ \mathrm{H} & -3.057776 & 0.121545 & 0.848364 \\ \mathrm{H} & 3.343660 & -0.497566 & -0.852027 \\ \mathrm{H} & 3.250989 & 1.086110 & -0.089149 \\ \mathrm{H} & 3.345851 & -0.354642 & 0.923380\end{array}$

$o$-methoxyphenol $\mathrm{S}_{0}$
$\begin{array}{llll}\text { C } & 2.035437 & -0.092276 & 0.000000\end{array}$
$\begin{array}{llll}\text { C } & 0.881241 & 0.694589 & 0.000000\end{array}$
$\begin{array}{llll}\text { C } & -0.365491 & 0.085197 & 0.000000\end{array}$
$\begin{array}{llll}\text { C } & -0.466432 & -1.316651 & 0.000000\end{array}$
$\begin{array}{llll}\text { C } & 0.683410 & -2.089115 & 0.000000\end{array}$ 


$\begin{array}{rrrr}\mathrm{C} & 1.935560 & -1.475996 & 0.000000 \\ \mathrm{O} & -1.579963 & 0.729023 & 0.000000 \\ \mathrm{O} & -1.691002 & -1.916005 & 0.000000 \\ \mathrm{H} & 0.582734 & -3.166867 & 0.000000 \\ \mathrm{H} & 2.829141 & -2.087111 & 0.000000 \\ \mathrm{H} & 3.005634 & 0.387620 & 0.000000 \\ \mathrm{H} & 0.966035 & 1.772533 & 0.000000 \\ \mathrm{C} & -1.595613 & 2.149882 & 0.000000 \\ \mathrm{H} & -2.360328 & -1.217440 & 0.000000 \\ \mathrm{H} & -2.643882 & 2.439731 & 0.000000 \\ \mathrm{H} & -1.108240 & 2.551443 & 0.893210 \\ \mathrm{H} & -1.108240 & 2.551443 & -0.893210\end{array}$

$o$-methoxyphenol $\mathrm{S}_{1}$

$\begin{array}{rrrr}\mathrm{C} & 2.486015 & 0.054379 & 0.059853 \\ \mathrm{C} & 1.384672 & -0.285627 & -0.746620 \\ \mathrm{C} & 0.112572 & -0.284997 & -0.091524 \\ \mathrm{C} & 0.018815 & -0.153878 & 1.317564 \\ \mathrm{C} & 1.180249 & -0.039029 & 2.138143 \\ \mathrm{C} & 2.388593 & 0.177444 & 1.464234 \\ \mathrm{O} & -1.084036 & -0.127331 & -0.738215 \\ \mathrm{O} & -1.183879 & 0.063781 & 1.893189 \\ \mathrm{H} & 1.107512 & -0.148301 & 3.211002 \\ \mathrm{H} & 3.281982 & 0.429624 & 2.024856 \\ \mathrm{H} & 3.454152 & 0.212327 & -0.405441 \\ \mathrm{H} & 1.518457 & -0.652874 & -1.756430 \\ \mathrm{C} & -1.093311 & 0.135135 & -2.151952 \\ \mathrm{H} & -1.867003 & 0.028004 & 1.198247 \\ \mathrm{H} & -2.137470 & 0.293120 & -2.409576 \\ \mathrm{H} & -0.504316 & 1.022136 & -2.387964 \\ \mathrm{H} & -0.704446 & -0.723923 & -2.701286\end{array}$

$m$-methoxyphenol, isomer $1 \mathrm{~S}_{0}$

$\begin{array}{crcc}\mathrm{C} & 0.929644 & 1.446124 & 0.000000 \\ \mathrm{C} & -0.260246 & 0.714306 & 0.000000 \\ \mathrm{C} & -0.226388 & -0.680859 & 0.000000 \\ \mathrm{C} & 1.007853 & -1.336192 & 0.000000 \\ \mathrm{C} & 2.197494 & -0.618948 & 0.000000 \\ \mathrm{C} & 2.140625 & 0.771564 & 0.000000 \\ \mathrm{H} & -1.138787 & -1.263360 & 0.000000 \\ \mathrm{O} & 1.094213 & -2.703489 & 0.000000 \\ \mathrm{H} & 3.139147 & -1.150507 & 0.000000 \\ \mathrm{H} & 3.063189 & 1.339125 & 0.000000 \\ \mathrm{H} & 0.879607 & 2.526391 & 0.000000\end{array}$




$\begin{array}{rrrr}\mathrm{O} & -1.411252 & 1.447117 & 0.000000 \\ \mathrm{H} & 0.210218 & -3.086925 & 0.000000 \\ \mathrm{C} & -2.656193 & 0.765817 & 0.000000 \\ \mathrm{H} & -3.419697 & 1.540450 & 0.000000 \\ \mathrm{H} & -2.774713 & 0.144694 & -0.893589 \\ \mathrm{H} & -2.774713 & 0.144694 & 0.893589\end{array}$

$m$-methoxyphenol, isomer $1 \mathrm{~S}_{1}$

$\begin{array}{rrrr}\mathrm{C} & 1.167831 & -0.010217 & -0.620398 \\ \mathrm{C} & -0.029338 & -0.030484 & 0.131460 \\ \mathrm{C} & -0.047764 & -0.071810 & 1.554284 \\ \mathrm{C} & 1.243360 & -0.012275 & 2.151429 \\ \mathrm{C} & 2.449143 & 0.004756 & 1.416989 \\ \mathrm{C} & 2.435216 & -0.099463 & 0.008339 \\ \mathrm{H} & -0.877355 & -0.512982 & 2.099839 \\ \mathrm{O} & 1.354442 & 0.110024 & 3.500692 \\ \mathrm{H} & 3.372975 & 0.134371 & 1.967176 \\ \mathrm{H} & 3.347425 & -0.128311 & -0.565908 \\ \mathrm{H} & 1.078510 & 0.086497 & -1.695174 \\ \mathrm{O} & -1.158971 & 0.046395 & -0.618232 \\ \mathrm{H} & 0.492525 & 0.389109 & 3.853992 \\ \mathrm{C} & -2.433255 & 0.026382 & 0.022398 \\ \mathrm{H} & -3.153541 & 0.314258 & -0.739608 \\ \mathrm{H} & -2.669002 & -0.979105 & 0.381746 \\ \mathrm{H} & -2.463891 & 0.732856 & 0.853717\end{array}$

$m$-methoxyphenol, isomer $2 \mathrm{~S}_{0}$

$\begin{array}{lrrc}\mathrm{C} & 0.347796 & 2.163089 & 0.000000 \\ \mathrm{C} & -0.836005 & 1.447270 & 0.000000 \\ \mathrm{C} & -0.790768 & 0.047711 & 0.000000 \\ \mathrm{C} & 0.432510 & -0.616998 & 0.000000 \\ \mathrm{C} & 1.613189 & 0.127172 & 0.000000 \\ \mathrm{C} & 1.583759 & 1.516910 & 0.000000 \\ \mathrm{O} & -1.998871 & -0.583640 & 0.000000 \\ \mathrm{C} & -2.026238 & -2.004140 & 0.000000 \\ \mathrm{O} & 2.782680 & -0.587141 & 0.000000 \\ \mathrm{H} & 0.506706 & -1.694212 & 0.000000 \\ \mathrm{H} & 2.505758 & 2.087522 & 0.000000 \\ \mathrm{H} & 0.315423 & 3.245743 & 0.000000 \\ \mathrm{H} & -1.798460 & 1.940322 & 0.000000 \\ \mathrm{H} & -3.078066 & -2.281036 & 0.000000 \\ \mathrm{H} & -1.544870 & -2.414815 & -0.892797 \\ \mathrm{H} & -1.544870 & -2.414815 & 0.892797 \\ \mathrm{H} & 3.530317 & 0.021066 & 0.000000\end{array}$


$m$-methoxyphenol, isomer $2 \mathrm{~S}_{1}$

$\begin{array}{lrrr}\text { C } & 1.241283 & -0.075080 & -0.711957 \\ \mathrm{C} & 0.054054 & -0.023632 & 0.073641 \\ \mathrm{C} & 0.112578 & -0.039228 & 1.485656 \\ \mathrm{C} & 1.341796 & -0.028697 & 2.200602 \\ \mathrm{C} & 2.508722 & 0.010265 & 1.397540 \\ \mathrm{C} & 2.474334 & 0.031933 & -0.014829 \\ \mathrm{O} & -1.098636 & -0.026994 & 2.099724 \\ \mathrm{C} & -1.166154 & 0.028423 & 3.522117 \\ \mathrm{O} & 3.692052 & 0.054102 & 2.071625 \\ \mathrm{H} & 1.424482 & -0.202897 & 3.260804 \\ \mathrm{H} & 3.406296 & 0.137513 & -0.560936 \\ \mathrm{H} & 1.202316 & -0.050777 & -1.788959 \\ \mathrm{H} & -0.922595 & 0.036788 & -0.387929 \\ \mathrm{H} & -2.223322 & 0.104279 & 3.764379 \\ \mathrm{H} & -0.754198 & -0.879737 & 3.969426 \\ \mathrm{H} & -0.633087 & 0.900575 & 3.907248 \\ \mathrm{H} & 4.425211 & 0.023166 & 1.443747\end{array}$

$p$-methoxyphenol, cis $\mathrm{S}_{0}$

$\begin{array}{rrrr}\mathrm{C} & -0.313609 & 0.729314 & 0.000000 \\ \mathrm{C} & -1.547316 & 0.070448 & 0.000000 \\ \mathrm{C} & -1.609110 & -1.310920 & 0.000000 \\ \mathrm{C} & -0.434525 & -2.065180 & 0.000000 \\ \mathrm{C} & 0.792142 & -1.416661 & 0.000000 \\ \mathrm{C} & 0.858293 & -0.021317 & 0.000000 \\ \mathrm{O} & -0.561812 & -3.433472 & 0.000000 \\ \mathrm{O} & -0.366356 & 2.098137 & 0.000000 \\ \mathrm{C} & 0.856137 & 2.816505 & 0.000000 \\ \mathrm{H} & 1.828394 & 0.454696 & 0.000000 \\ \mathrm{H} & -2.452030 & 0.664558 & 0.000000 \\ \mathrm{H} & -2.562590 & -1.822977 & 0.000000 \\ \mathrm{H} & 1.712744 & -1.991259 & 0.000000 \\ \mathrm{H} & 0.584258 & 3.869826 & 0.000000 \\ \mathrm{H} & 1.451667 & 2.598747 & 0.892855 \\ \mathrm{H} & 1.451667 & 2.598747 & -0.892855 \\ \mathrm{H} & 0.312046 & -3.839172 & 0.000000\end{array}$

p-methoxyphenol, cis $\mathrm{S}_{1}$
$\begin{array}{llll}\text { C } & 1.329716 & -0.026500 & -0.612259\end{array}$
$\begin{array}{llll}\text { C } & 0.051279 & -0.049823 & 0.003310\end{array}$
$\begin{array}{llll}\text { C } & -0.017780 & -0.004341 & 1.417798\end{array}$ 


$\begin{array}{lrrc}\mathrm{C} & 1.186395 & -0.008001 & 2.143825 \\ \mathrm{C} & 2.468512 & -0.015483 & 1.535468 \\ \mathrm{C} & 2.540411 & -0.014042 & 0.118975 \\ \mathrm{O} & 1.082360 & 0.007168 & 3.493632 \\ \mathrm{O} & 1.290810 & -0.032892 & -1.961725 \\ \mathrm{C} & 2.495711 & 0.029328 & -2.724890 \\ \mathrm{H} & 3.497719 & -0.024696 & -0.377456 \\ \mathrm{H} & -0.829727 & -0.050626 & -0.619869 \\ \mathrm{H} & -0.955119 & 0.081043 & 1.947286 \\ \mathrm{H} & 3.356908 & -0.155286 & 2.138862 \\ \mathrm{H} & 2.183531 & 0.018050 & -3.765791 \\ \mathrm{H} & 3.043114 & 0.949070 & -2.510210 \\ \mathrm{H} & 3.128659 & -0.836379 & -2.519725 \\ \mathrm{H} & 1.961031 & 0.133393 & 3.879770\end{array}$

p-methoxyphenol, trans $\mathrm{S}_{0}$

$\begin{array}{cccc}\mathrm{C} & -0.657814 & 1.303408 & 0.000000 \\ \mathrm{C} & -1.891863 & 0.671487 & 0.000000 \\ \mathrm{C} & -1.968915 & -0.721596 & 0.000000 \\ \mathrm{C} & -0.799163 & -1.468937 & 0.000000 \\ \mathrm{C} & 0.442474 & -0.837450 & 0.000000 \\ \mathrm{C} & 0.520062 & 0.555130 & 0.000000 \\ \mathrm{O} & -3.163476 & -1.401444 & 0.000000 \\ \mathrm{H} & 1.335988 & -1.445472 & 0.000000 \\ \mathrm{O} & 1.686973 & 1.272984 & 0.000000 \\ \mathrm{H} & -0.588938 & 2.383485 & 0.000000 \\ \mathrm{H} & -2.798856 & 1.267443 & 0.000000 \\ \mathrm{H} & -3.892919 & -0.772189 & 0.000000 \\ \mathrm{H} & -0.864755 & -2.549240 & 0.000000 \\ \mathrm{C} & 2.912887 & 0.559998 & 0.000000 \\ \mathrm{H} & 3.697323 & 1.313743 & 0.000000 \\ \mathrm{H} & 3.015491 & -0.065685 & 0.892753 \\ \mathrm{H} & 3.015491 & -0.065685 & -0.892753\end{array}$

p-methoxyphenol, trans $\mathrm{S}_{1}$

$\begin{array}{rrrr}\mathrm{C} & 0.122542 & -0.030406 & -1.391944 \\ \mathrm{C} & -1.284650 & -0.017985 & -1.219491 \\ \mathrm{C} & -1.789010 & 0.016935 & 0.101626 \\ \mathrm{C} & -0.969427 & 0.056168 & 1.248261 \\ \mathrm{C} & 0.438898 & 0.027991 & 1.079329 \\ \mathrm{C} & 0.949497 & -0.000730 & -0.238686 \\ \mathrm{O} & -3.124487 & 0.026380 & 0.325452 \\ \mathrm{H} & 1.090491 & 0.057238 & 1.937674 \\ \mathrm{O} & 2.271131 & -0.012879 & -0.515082\end{array}$




$\begin{array}{rrrr}\mathrm{H} & 0.586493 & -0.064602 & -2.365898 \\ \mathrm{H} & -1.956913 & -0.139362 & -2.059533 \\ \mathrm{H} & -3.590337 & 0.079180 & -0.520594 \\ \mathrm{H} & -1.428032 & 0.141115 & 2.222139 \\ \mathrm{C} & 3.233109 & -0.022090 & 0.539914 \\ \mathrm{H} & 4.203484 & -0.035864 & 0.050783 \\ \mathrm{H} & 3.141025 & 0.875088 & 1.155171 \\ \mathrm{H} & 3.116975 & -0.911867 & 1.161580\end{array}$

1-naphthol, cis $\mathrm{S}_{0}$

$\begin{array}{lrrc}\mathrm{C} & 0.830778 & 1.518341 & 0.014542 \\ \mathrm{C} & 0.529571 & 0.123860 & -0.000199 \\ \mathrm{C} & -0.847439 & -0.268502 & -0.006766 \\ \mathrm{C} & -1.859102 & 0.721825 & -0.016029 \\ \mathrm{C} & -1.522355 & 2.051526 & -0.017084 \\ \mathrm{C} & -0.174763 & 2.455267 & 0.000476 \\ \mathrm{C} & 1.523619 & -0.886856 & -0.012039 \\ \mathrm{C} & 1.185870 & -2.216808 & -0.013905 \\ \mathrm{C} & -0.170471 & -2.604224 & -0.008687 \\ \mathrm{C} & -1.156959 & -1.652255 & -0.007627 \\ \mathrm{O} & 2.117817 & 1.981796 & 0.029571 \\ \mathrm{H} & -2.200061 & -1.947108 & -0.009261 \\ \mathrm{H} & -0.428012 & -3.656187 & -0.009614 \\ \mathrm{H} & 1.961785 & -2.972050 & -0.024400 \\ \mathrm{H} & 2.577294 & -0.628291 & -0.032130 \\ \mathrm{H} & -2.897260 & 0.413126 & -0.023237 \\ \mathrm{H} & -2.296625 & 2.808821 & -0.026122 \\ \mathrm{H} & 0.091239 & 3.504181 & 0.009520 \\ \mathrm{H} & 2.735083 & 1.253549 & 0.152987\end{array}$

1-naphthol, cis $\mathrm{S}_{1}$

$\begin{array}{lrrr}\mathrm{C} & 0.864608 & 1.470969 & 0.013458 \\ \mathrm{C} & 0.534546 & 0.086925 & -0.056302 \\ \mathrm{C} & -0.845334 & -0.246412 & 0.005272 \\ \mathrm{C} & -1.794141 & 0.809533 & -0.014731 \\ \mathrm{C} & -1.432097 & 2.174443 & -0.025156 \\ \mathrm{C} & -0.102825 & 2.515261 & -0.027172 \\ \mathrm{C} & 1.516220 & -0.935258 & -0.116428 \\ \mathrm{C} & 1.099757 & -2.297633 & -0.042571 \\ \mathrm{C} & -0.234674 & -2.619992 & 0.041898 \\ \mathrm{C} & -1.232474 & -1.610791 & 0.048636 \\ \mathrm{O} & 2.138702 & 1.865494 & 0.136227 \\ \mathrm{H} & -2.280902 & -1.869354 & 0.105337 \\ \mathrm{H} & -0.533615 & -3.659634 & 0.098148\end{array}$




$\begin{array}{rrrr}\mathrm{H} & 1.847410 & -3.080613 & -0.061772 \\ \mathrm{H} & 2.558969 & -0.715461 & -0.312879 \\ \mathrm{H} & -2.846108 & 0.548771 & -0.021136 \\ \mathrm{H} & -2.199114 & 2.936465 & -0.051165 \\ \mathrm{H} & 0.242726 & 3.539690 & -0.012795 \\ \mathrm{H} & 2.698346 & 1.087585 & 0.293109\end{array}$

1-naphthol, trans $\mathrm{S}_{0}$

$\begin{array}{lrrl}\mathrm{C} & -0.717152 & -0.541694 & 0.000000 \\ \mathrm{C} & -0.673678 & -1.958384 & 0.000000 \\ \mathrm{C} & 0.522475 & -2.630055 & 0.000000 \\ \mathrm{C} & 1.739860 & -1.916536 & 0.000000 \\ \mathrm{C} & 1.739205 & -0.543881 & 0.000000 \\ \mathrm{C} & 0.518983 & 0.173680 & 0.000000 \\ \mathrm{H} & -1.609108 & -2.506065 & 0.000000 \\ \mathrm{H} & 0.536186 & -3.713284 & 0.000000 \\ \mathrm{H} & 2.678349 & -2.457193 & 0.000000 \\ \mathrm{H} & 2.668902 & 0.008284 & 0.000000 \\ \mathrm{C} & -1.941334 & 0.174705 & 0.000000 \\ \mathrm{C} & -1.939122 & 1.544660 & 0.000000 \\ \mathrm{C} & -0.726207 & 2.265000 & 0.000000 \\ \mathrm{C} & 0.474939 & 1.596907 & 0.000000 \\ \mathrm{H} & -2.874085 & -0.376061 & 0.000000 \\ \mathrm{H} & -2.873790 & 2.091864 & 0.000000 \\ \mathrm{H} & -0.743247 & 3.350016 & 0.000000 \\ \mathrm{O} & 1.682081 & 2.242613 & 0.000000 \\ \mathrm{H} & 1.536755 & 3.195414 & 0.000000\end{array}$

1-naphthol, trans $\mathrm{S}_{1}$

$\begin{array}{lrrr}\mathrm{C} & -0.936455 & -0.392063 & 0.000000 \\ \mathrm{C} & -1.312111 & -1.754762 & 0.000000 \\ \mathrm{C} & -0.315076 & -2.771392 & 0.000000 \\ \mathrm{C} & 1.018152 & -2.434929 & 0.000000 \\ \mathrm{C} & 1.429474 & -1.071080 & 0.000000 \\ \mathrm{C} & 0.452588 & -0.049696 & 0.000000 \\ \mathrm{H} & -2.362215 & -2.016646 & 0.000000 \\ \mathrm{H} & -0.615482 & -3.811895 & 0.000000 \\ \mathrm{H} & 1.774591 & -3.210637 & 0.000000 \\ \mathrm{H} & 2.478437 & -0.815321 & 0.000000 \\ \mathrm{C} & -1.891254 & 0.653582 & 0.000000 \\ \mathrm{C} & -1.530588 & 2.023834 & 0.000000 \\ \mathrm{C} & -0.203644 & 2.369803 & 0.000000 \\ \mathrm{C} & 0.778540 & 1.332003 & 0.000000 \\ \mathrm{H} & -2.942992 & 0.391184 & 0.000000\end{array}$




$\begin{array}{rrrr}\mathrm{H} & -2.300887 & 2.783599 & 0.000000 \\ \mathrm{H} & 0.115039 & 3.406352 & 0.000000 \\ \mathrm{O} & 2.089649 & 1.653489 & 0.000000 \\ \mathrm{H} & 2.198555 & 2.613647 & 0.000000\end{array}$

2-naphthol, cis $\mathrm{S}_{0}$

$\begin{array}{rrrr}\mathrm{C} & 0.597839 & 2.011210 & 0.000000 \\ \mathrm{C} & 0.622792 & 0.595891 & 0.000000 \\ \mathrm{C} & -0.613032 & -0.121136 & 0.000000 \\ \mathrm{C} & -1.824052 & 0.615608 & 0.000000 \\ \mathrm{C} & -1.813310 & 1.988026 & 0.000000 \\ \mathrm{C} & -0.591825 & 2.695628 & 0.000000 \\ \mathrm{C} & 1.833260 & -0.143904 & 0.000000 \\ \mathrm{C} & 1.832148 & -1.511755 & 0.000000 \\ \mathrm{C} & 0.605567 & -2.215811 & 0.000000 \\ \mathrm{C} & -0.588950 & -1.537857 & 0.000000 \\ \mathrm{H} & -1.528796 & -2.081628 & 0.000000 \\ \mathrm{H} & 1.539310 & 2.549013 & 0.000000 \\ \mathrm{H} & -0.598242 & 3.778547 & 0.000000 \\ \mathrm{H} & -2.748132 & 2.535457 & 0.000000 \\ \mathrm{H} & -2.764996 & 0.077004 & 0.000000 \\ \mathrm{H} & 2.774509 & 0.393734 & 0.000000 \\ \mathrm{H} & 2.753574 & -2.079384 & 0.000000 \\ \mathrm{O} & 0.697996 & -3.581451 & 0.000000 \\ \mathrm{H} & -0.185668 & -3.967183 & 0.000000\end{array}$

2-naphthol, cis $\mathrm{S}_{1}$

$\begin{array}{rrrr}\mathrm{C} & 0.599340 & 1.996000 & 0.000000 \\ \mathrm{C} & 0.640930 & 0.586950 & 0.000000 \\ \mathrm{C} & -0.622680 & -0.115660 & 0.000000 \\ \mathrm{C} & -1.831380 & 0.609010 & 0.000000 \\ \mathrm{C} & -1.840250 & 2.026230 & 0.000000 \\ \mathrm{C} & -0.632100 & 2.698660 & 0.000000 \\ \mathrm{C} & 1.847950 & -0.152270 & 0.000000 \\ \mathrm{C} & 1.882370 & -1.560790 & 0.000000 \\ \mathrm{C} & 0.661080 & -2.225020 & 0.000000 \\ \mathrm{C} & -0.579000 & -1.525010 & 0.000000 \\ \mathrm{H} & -1.504450 & -2.093840 & 0.000000 \\ \mathrm{H} & 1.528660 & 2.552980 & 0.000000 \\ \mathrm{H} & -0.613420 & 3.782250 & 0.000000 \\ \mathrm{H} & -2.777320 & 2.566310 & 0.000000 \\ \mathrm{H} & -2.768360 & 0.063580 & 0.000000 \\ \mathrm{H} & 2.782780 & 0.396360 & 0.000000 \\ \mathrm{H} & 2.806760 & -2.118660 & 0.000000\end{array}$




$$
\begin{array}{rrrr}
\mathrm{O} & 0.663270 & -3.577140 & 0.000000 \\
\mathrm{H} & -0.244170 & -3.909930 & 0.000000
\end{array}
$$

2-naphthol, trans $\mathrm{S}_{0}$

$\begin{array}{lrrl}\mathrm{C} & 0.510481 & 2.013336 & 0.000000 \\ \mathrm{C} & 0.541332 & 0.596903 & 0.000000 \\ \mathrm{C} & -0.691805 & -0.127559 & 0.000000 \\ \mathrm{C} & -1.906779 & 0.605783 & 0.000000 \\ \mathrm{C} & -1.901205 & 1.977117 & 0.000000 \\ \mathrm{C} & -0.681766 & 2.691124 & 0.000000 \\ \mathrm{C} & 1.752654 & -0.135913 & 0.000000 \\ \mathrm{C} & 1.752115 & -1.506413 & 0.000000 \\ \mathrm{C} & 0.528680 & -2.216430 & 0.000000 \\ \mathrm{C} & -0.666894 & -1.541131 & 0.000000 \\ \mathrm{H} & -1.593131 & -2.102116 & 0.000000 \\ \mathrm{H} & 1.449484 & 2.555535 & 0.000000 \\ \mathrm{H} & -0.693806 & 3.774023 & 0.000000 \\ \mathrm{H} & -2.838069 & 2.521083 & 0.000000 \\ \mathrm{H} & -2.844581 & 0.062227 & 0.000000 \\ \mathrm{H} & 2.693149 & 0.402956 & 0.000000 \\ \mathrm{H} & 2.689260 & -2.054092 & 0.000000 \\ \mathrm{O} & 0.500490 & -3.586072 & 0.000000 \\ \mathrm{H} & 1.400400 & -3.930361 & 0.000000\end{array}$

2-naphthol, trans $\mathrm{S}_{1}$

$\begin{array}{lrrr}\mathrm{C} & 0.510978 & 2.000496 & 0.000000 \\ \mathrm{C} & 0.555084 & 0.591716 & 0.000000 \\ \mathrm{C} & -0.704368 & -0.116459 & 0.000000 \\ \mathrm{C} & -1.914086 & 0.604557 & 0.000000 \\ \mathrm{C} & -1.926332 & 2.022094 & 0.000000 \\ \mathrm{C} & -0.722187 & 2.699251 & 0.000000 \\ \mathrm{C} & 1.764613 & -0.141861 & 0.000000 \\ \mathrm{C} & 1.800770 & -1.550969 & 0.000000 \\ \mathrm{C} & 0.577000 & -2.217919 & 0.000000 \\ \mathrm{C} & -0.661407 & -1.525704 & 0.000000 \\ \mathrm{H} & -1.571243 & -2.112224 & 0.000000 \\ \mathrm{H} & 1.438737 & 2.559756 & 0.000000 \\ \mathrm{H} & -0.707517 & 3.782631 & 0.000000 \\ \mathrm{H} & -2.865289 & 2.558453 & 0.000000 \\ \mathrm{H} & -2.848753 & 0.056374 & 0.000000 \\ \mathrm{H} & 2.697795 & 0.408980 & 0.000000 \\ \mathrm{H} & 2.736985 & -2.093795 & 0.000000 \\ \mathrm{O} & 0.477959 & -3.566497 & 0.000000 \\ \mathrm{H} & 1.361262 & -3.958870 & 0.000000\end{array}$


5-methoxysalicylic acid $\mathrm{S}_{0}$

$\begin{array}{lrrc}\mathrm{C} & 2.279780 & -0.164394 & 0.000000 \\ \mathrm{C} & 1.565936 & -1.340646 & 0.000000 \\ \mathrm{C} & 0.160258 & -1.333561 & 0.000000 \\ \mathrm{C} & -0.509522 & -0.125925 & 0.000000 \\ \mathrm{C} & 0.211058 & 1.088164 & 0.000000 \\ \mathrm{C} & 1.619559 & 1.072602 & 0.000000 \\ \mathrm{O} & -0.435085 & -2.564133 & 0.000000 \\ \mathrm{C} & -1.853625 & -2.620977 & 0.000000 \\ \mathrm{C} & -0.477836 & 2.377093 & 0.000000 \\ \mathrm{O} & -1.826261 & 2.301338 & 0.000000 \\ \mathrm{O} & 2.375646 & 2.186101 & 0.000000 \\ \mathrm{O} & 0.085819 & 3.465456 & 0.000000 \\ \mathrm{H} & -2.110355 & -3.677785 & 0.000000 \\ \mathrm{H} & -2.274097 & -2.146960 & -0.892771 \\ \mathrm{H} & -2.274097 & -2.146960 & 0.892771 \\ \mathrm{H} & -1.587239 & -0.077631 & 0.000000 \\ \mathrm{H} & 3.361685 & -0.168032 & 0.000000 \\ \mathrm{H} & 2.075011 & -2.296120 & 0.000000 \\ \mathrm{H} & 1.770597 & 2.959688 & 0.000000 \\ \mathrm{H} & -2.157211 & 3.212682 & 0.000000\end{array}$

5-methoxysalicylic acid $\mathrm{S}_{1}$

$\begin{array}{lrrc}\mathrm{C} & 2.174052 & -0.136324 & 0.000000 \\ \mathrm{C} & 1.733492 & -1.459629 & 0.000000 \\ \mathrm{C} & 0.347569 & -1.685523 & 0.000000 \\ \mathrm{C} & -0.588475 & -0.610699 & 0.000000 \\ \mathrm{C} & -0.156310 & 0.713008 & 0.000000 \\ \mathrm{C} & 1.269670 & 0.934231 & 0.000000 \\ \mathrm{O} & -0.035848 & -2.974678 & 0.000000 \\ \mathrm{C} & -1.421059 & -3.336976 & 0.000000 \\ \mathrm{C} & -1.042153 & 1.851002 & 0.000000 \\ \mathrm{O} & -2.380359 & 1.557057 & 0.000000 \\ \mathrm{O} & 1.731254 & 2.179714 & 0.000000 \\ \mathrm{O} & -0.653398 & 3.051565 & 0.000000 \\ \mathrm{H} & -1.433628 & -4.423143 & 0.000000 \\ \mathrm{H} & -1.920998 & -2.961554 & -0.894437 \\ \mathrm{H} & -1.920998 & -2.961554 & 0.894437 \\ \mathrm{H} & -1.649771 & -0.807837 & 0.000000 \\ \mathrm{H} & 3.229848 & 0.100650 & 0.000000 \\ \mathrm{H} & 2.414730 & -2.296979 & 0.000000 \\ \mathrm{H} & 0.912808 & 2.788990 & 0.000000 \\ \mathrm{H} & -2.838375 & 2.409458 & 0.000000\end{array}$


$m$-dimethoxybenzene, trans $\mathrm{S}_{0}$

$\begin{array}{lrrr}\mathrm{C} & 0.000000 & -0.707606 & -0.169392 \\ \mathrm{C} & 0.000000 & 0.707606 & -0.169392 \\ \mathrm{C} & 0.000000 & 1.389445 & 1.040586 \\ \mathrm{C} & 0.000000 & 0.691067 & 2.251548 \\ \mathrm{C} & 0.000000 & -0.691067 & 2.251548 \\ \mathrm{C} & 0.000000 & -1.389445 & 1.040586 \\ \mathrm{O} & 0.000000 & 1.297647 & -1.396297 \\ \mathrm{H} & 0.000000 & 2.470089 & 1.052254 \\ \mathrm{H} & 0.000000 & 1.241980 & 3.183477 \\ \mathrm{H} & 0.000000 & -1.241980 & 3.183477 \\ \mathrm{H} & 0.000000 & -2.470089 & 1.052254 \\ \mathrm{O} & 0.000000 & -1.297647 & -1.396297 \\ \mathrm{C} & 0.000000 & -2.714599 & -1.460796 \\ \mathrm{H} & 0.000000 & -2.962496 & -2.519934 \\ \mathrm{H} & 0.892653 & -3.139859 & -0.990712 \\ \mathrm{H} & -0.892653 & -3.139859 & -0.990712 \\ \mathrm{C} & 0.000000 & 2.714599 & -1.460796 \\ \mathrm{H} & 0.000000 & 2.962496 & -2.519934 \\ \mathrm{H} & -0.892653 & 3.139859 & -0.990712 \\ \mathrm{H} & 0.892653 & 3.139859 & -0.990712\end{array}$

$m$-dimethoxybenzene, trans $\mathrm{S}_{1}$

$\begin{array}{lrrr}\mathrm{C} & -0.110813 & -1.073075 & -1.446202 \\ \mathrm{C} & -0.291626 & 0.146196 & -0.708836 \\ \mathrm{C} & -0.291626 & 0.146196 & 0.708836 \\ \mathrm{C} & -0.110813 & -1.073075 & 1.446202 \\ \mathrm{C} & 0.279095 & -2.198155 & 0.704635 \\ \mathrm{C} & 0.279095 & -2.198155 & -0.704635 \\ \mathrm{O} & -0.281915 & 1.367146 & -1.297859 \\ \mathrm{C} & 0.008156 & 1.471878 & -2.695261 \\ \mathrm{O} & -0.281915 & 1.367146 & 1.297859 \\ \mathrm{C} & 0.008156 & 1.471878 & 2.695261 \\ \mathrm{H} & -0.390750 & -1.158926 & 2.488528 \\ \mathrm{H} & 0.552504 & -3.111448 & 1.223580 \\ \mathrm{H} & 0.552504 & -3.111448 & -1.223580 \\ \mathrm{H} & -0.390750 & -1.158926 & -2.488528 \\ \mathrm{H} & 0.028690 & 2.538475 & -2.905018 \\ \mathrm{H} & 0.973509 & 1.022259 & -2.933628 \\ \mathrm{H} & -0.773762 & 0.998090 & -3.292747 \\ \mathrm{H} & 0.028690 & 2.538475 & 2.905018 \\ \mathrm{H} & -0.773762 & 0.998090 & 3.292747 \\ \mathrm{H} & 0.973509 & 1.022259 & 2.933628\end{array}$


3P-propionic acid, gauche $\mathrm{S}_{0}$

$\begin{array}{rrrr}\mathrm{C} & -1.039750 & 0.903712 & -1.170395 \\ \mathrm{C} & -0.392391 & 0.662737 & 0.044709 \\ \mathrm{C} & -1.058573 & -0.091079 & 1.014127 \\ \mathrm{C} & -2.334200 & -0.592474 & 0.773092 \\ \mathrm{C} & -2.966251 & -0.347747 & -0.441594 \\ \mathrm{C} & -2.314285 & 0.403665 & -1.414583 \\ \mathrm{C} & 1.001474 & 1.198140 & 0.289798 \\ \mathrm{C} & 2.117799 & 0.277196 & -0.226782 \\ \mathrm{C} & 2.336617 & -0.950610 & 0.626138 \\ \mathrm{O} & 2.056658 & -1.062116 & 1.793220 \\ \mathrm{O} & 2.944072 & -1.942186 & -0.071231 \\ \mathrm{H} & 1.936712 & -0.034553 & -1.256420 \\ \mathrm{H} & 3.073036 & 0.814279 & -0.230038 \\ \mathrm{H} & 1.153168 & 1.368405 & 1.356685 \\ \mathrm{H} & 1.107203 & 2.164455 & -0.208741 \\ \mathrm{H} & -0.568724 & -0.289117 & 1.959564 \\ \mathrm{H} & -2.835709 & -1.173042 & 1.538199 \\ \mathrm{H} & -3.960558 & -0.735248 & -0.627525 \\ \mathrm{H} & -2.800641 & 0.606121 & -2.361388 \\ \mathrm{H} & -0.544259 & 1.497488 & -1.931514 \\ \mathrm{H} & 3.088612 & -2.678046 & 0.544690\end{array}$

3P-propionic acid, gauche $\mathrm{S}_{1}$

$\begin{array}{lrrr}\mathrm{C} & -0.690643 & 0.240762 & -1.096931 \\ \mathrm{C} & -0.362209 & 0.550006 & 0.261196 \\ \mathrm{C} & -1.352555 & 0.372907 & 1.272814 \\ \mathrm{C} & -2.659640 & -0.058395 & 0.937907 \\ \mathrm{C} & -2.973858 & -0.347118 & -0.414274 \\ \mathrm{C} & -1.996786 & -0.195382 & -1.430900 \\ \mathrm{C} & 0.971853 & 1.111877 & 0.626118 \\ \mathrm{C} & 2.166265 & 0.500764 & -0.120053 \\ \mathrm{C} & 2.313616 & -0.976892 & 0.146697 \\ \mathrm{O} & 1.970962 & -1.549253 & 1.154833 \\ \mathrm{O} & 2.945703 & -1.616562 & -0.876087 \\ \mathrm{H} & 2.114584 & 0.673163 & -1.195143 \\ \mathrm{H} & 3.086290 & 0.990622 & 0.220142 \\ \mathrm{H} & 1.123774 & 1.001714 & 1.701223 \\ \mathrm{H} & 0.975254 & 2.195168 & 0.422024 \\ \mathrm{H} & -1.086025 & 0.552348 & 2.305596 \\ \mathrm{H} & -3.408399 & -0.180409 & 1.707813 \\ \mathrm{H} & -3.967637 & -0.687727 & -0.673864 \\ \mathrm{H} & -2.249532 & -0.413329 & -2.459293\end{array}$




$$
\begin{array}{lrrr}
\mathrm{H} & 0.042704 & 0.381225 & -1.877784 \\
\mathrm{H} & 3.036287 & -2.545498 & -0.612034
\end{array}
$$

3P-propionic acid, anti $\mathrm{S}_{0}$

$\begin{array}{rrrr}\mathrm{C} & 1.466946 & -0.447881 & 0.000000 \\ \mathrm{C} & 0.542642 & 0.775377 & 0.000000 \\ \mathrm{C} & 2.931929 & -0.085962 & 0.000000 \\ \mathrm{H} & 1.278300 & -1.082931 & 0.870291 \\ \mathrm{H} & 1.278300 & -1.082931 & -0.870291 \\ \mathrm{C} & -0.917669 & 0.385911 & 0.000000 \\ \mathrm{H} & 0.766819 & 1.390877 & 0.873201 \\ \mathrm{H} & 0.766819 & 1.390877 & -0.873201 \\ \mathrm{C} & -1.606526 & 0.188999 & 1.199093 \\ \mathrm{C} & -2.942566 & -0.198665 & 1.201922 \\ \mathrm{C} & -3.615141 & -0.395545 & 0.000000 \\ \mathrm{C} & -2.942566 & -0.198665 & -1.201922 \\ \mathrm{C} & -1.606526 & 0.188999 & -1.199093 \\ \mathrm{H} & -1.093529 & 0.349119 & 2.141390 \\ \mathrm{H} & -3.459931 & -0.341523 & 2.143081 \\ \mathrm{H} & -4.656375 & -0.693771 & 0.000000 \\ \mathrm{H} & -3.459931 & -0.341523 & -2.143081 \\ \mathrm{H} & -1.093529 & 0.349119 & -2.141390 \\ \mathrm{O} & 3.716274 & -1.194574 & 0.000000 \\ \mathrm{O} & 3.391039 & 1.027953 & 0.000000 \\ \mathrm{H} & 4.638057 & -0.891940 & 0.000000\end{array}$

3P-propionic acid, anti $\mathrm{S}_{1}$

$\begin{array}{lrrr}\mathrm{C} & 1.470453 & -0.434134 & 0.000000 \\ \mathrm{C} & 0.557256 & 0.809392 & 0.000000 \\ \mathrm{C} & 2.937461 & -0.083436 & 0.000000 \\ \mathrm{H} & 1.269040 & -1.062553 & 0.872001 \\ \mathrm{H} & 1.269040 & -1.062553 & -0.872001 \\ \mathrm{C} & -0.888972 & 0.444348 & 0.000000 \\ \mathrm{H} & 0.795644 & 1.414351 & 0.878706 \\ \mathrm{H} & 0.795644 & 1.414351 & -0.878706 \\ \mathrm{C} & -1.581965 & 0.235261 & 1.231940 \\ \mathrm{C} & -2.928978 & -0.215972 & 1.232329 \\ \mathrm{C} & -3.596845 & -0.437548 & 0.000000 \\ \mathrm{C} & -2.928978 & -0.215972 & -1.232329 \\ \mathrm{C} & -1.581965 & 0.235261 & -1.231940 \\ \mathrm{H} & -1.072387 & 0.427146 & 2.167215 \\ \mathrm{H} & -3.444376 & -0.389708 & 2.166608 \\ \mathrm{H} & -4.624447 & -0.776068 & 0.000000 \\ \mathrm{H} & -3.444376 & -0.389708 & -2.166608\end{array}$




$\begin{array}{rrrr}\mathrm{H} & -1.072387 & 0.427146 & -2.167215 \\ \mathrm{O} & 3.711669 & -1.199919 & 0.000000 \\ \mathrm{O} & 3.405673 & 1.027478 & 0.000000 \\ \mathrm{H} & 4.636422 & -0.906004 & 0.000000\end{array}$

fluorine $\mathrm{S}_{0}$

$\begin{array}{lrrr}\mathrm{C} & 0.000000 & 0.000000 & -1.793872 \\ \mathrm{C} & 0.000000 & 1.181917 & -0.850043 \\ \mathrm{C} & 0.000000 & 0.733675 & 0.483084 \\ \mathrm{C} & 0.000000 & -0.733675 & 0.483084 \\ \mathrm{C} & 0.000000 & 1.648576 & 1.532983 \\ \mathrm{C} & 0.000000 & 3.009378 & 1.240168 \\ \mathrm{C} & 0.000000 & 3.452371 & -0.082385 \\ \mathrm{C} & 0.000000 & 2.538054 & -1.135946 \\ \mathrm{H} & 0.000000 & 2.889427 & -2.161680 \\ \mathrm{H} & 0.000000 & 4.515033 & -0.292308 \\ \mathrm{H} & 0.000000 & 3.732389 & 2.046878 \\ \mathrm{H} & 0.000000 & 1.312945 & 2.563283 \\ \mathrm{C} & 0.000000 & -1.648576 & 1.532983 \\ \mathrm{C} & 0.000000 & -3.009378 & 1.240168 \\ \mathrm{C} & 0.000000 & -3.452371 & -0.082385 \\ \mathrm{C} & 0.000000 & -2.538054 & -1.135946 \\ \mathrm{C} & 0.000000 & -1.181917 & -0.850043 \\ \mathrm{H} & 0.000000 & -1.312945 & 2.563283 \\ \mathrm{H} & 0.000000 & -3.732389 & 2.046878 \\ \mathrm{H} & 0.000000 & -4.515033 & -0.292308 \\ \mathrm{H} & 0.000000 & -2.889427 & -2.161680 \\ \mathrm{H} & -0.878756 & 0.000000 & -2.447089 \\ \mathrm{H} & 0.878756 & 0.000000 & -2.447089\end{array}$

fluorine $\mathrm{S}_{1}$

$\begin{array}{lrrr}\mathrm{C} & 0.000000 & 0.000000 & -1.866771 \\ \mathrm{C} & 0.000000 & 1.182508 & -0.917552 \\ \mathrm{C} & 0.000000 & 0.700554 & 0.451385 \\ \mathrm{C} & 0.000000 & -0.700554 & 0.451385 \\ \mathrm{C} & 0.000000 & 1.642723 & 1.536167 \\ \mathrm{C} & 0.000000 & 2.985260 & 1.230840 \\ \mathrm{C} & 0.000000 & 3.442324 & -0.106494 \\ \mathrm{C} & 0.000000 & 2.526870 & -1.186759 \\ \mathrm{H} & 0.000000 & 2.894164 & -2.206442 \\ \mathrm{H} & 0.000000 & 4.506590 & -0.305009 \\ \mathrm{H} & 0.000000 & 3.717344 & 2.029915 \\ \mathrm{H} & 0.000000 & 1.299947 & 2.562744 \\ \mathrm{C} & 0.000000 & -1.642723 & 1.536167\end{array}$




$\begin{array}{lrrr}\mathrm{C} & 0.000000 & -2.985260 & 1.230840 \\ \mathrm{C} & 0.000000 & -3.442324 & -0.106494 \\ \mathrm{C} & 0.000000 & -2.526870 & -1.186759 \\ \mathrm{C} & 0.000000 & -1.182508 & -0.917552 \\ \mathrm{H} & 0.000000 & -1.299947 & 2.562744 \\ \mathrm{H} & 0.000000 & -3.717344 & 2.029915 \\ \mathrm{H} & 0.000000 & -4.506590 & -0.305009 \\ \mathrm{H} & 0.000000 & -2.894164 & -2.206442 \\ \mathrm{H} & -0.877962 & 0.000000 & -2.523247 \\ \mathrm{H} & 0.877962 & 0.000000 & -2.523247\end{array}$

phenanthrene $S_{0}$

$\begin{array}{lrrr}\mathrm{C} & 0.727341 & 0.000000 & -0.351684 \\ \mathrm{C} & -0.727341 & 0.000000 & -0.351684 \\ \mathrm{C} & -1.419262 & 0.000000 & 0.891197 \\ \mathrm{C} & -0.677222 & 0.000000 & 2.115797 \\ \mathrm{C} & 0.677222 & 0.000000 & 2.115797 \\ \mathrm{C} & 1.419262 & 0.000000 & 0.891197 \\ \mathrm{C} & -1.496301 & 0.000000 & -1.534662 \\ \mathrm{C} & -2.830169 & 0.000000 & 0.903080 \\ \mathrm{H} & -1.226910 & 0.000000 & 3.050105 \\ \mathrm{H} & 1.226910 & 0.000000 & 3.050105 \\ \mathrm{C} & 1.496301 & 0.000000 & -1.534662 \\ \mathrm{C} & 2.874044 & 0.000000 & -1.497775 \\ \mathrm{C} & 3.550999 & 0.000000 & -0.268753 \\ \mathrm{C} & 2.830169 & 0.000000 & 0.903080 \\ \mathrm{H} & 1.004654 & 0.000000 & -2.497663 \\ \mathrm{H} & 3.436197 & 0.000000 & -2.423647 \\ \mathrm{H} & 4.633768 & 0.000000 & -0.244989 \\ \mathrm{H} & 3.341408 & 0.000000 & 1.859083 \\ \mathrm{C} & -3.550999 & 0.000000 & -0.268753 \\ \mathrm{C} & -2.874044 & 0.000000 & -1.497775 \\ \mathrm{H} & -1.004654 & 0.000000 & -2.497663 \\ \mathrm{H} & -3.436197 & 0.000000 & -2.423647 \\ \mathrm{H} & -4.633768 & 0.000000 & -0.244989 \\ \mathrm{H} & -3.341408 & 0.000000 & 1.859083\end{array}$

phenanthrene $S_{1}$

$\begin{array}{lrrr}\mathrm{C} & 0.713217 & 0.000000 & -0.383114 \\ \mathrm{C} & -0.713217 & 0.000000 & -0.383114 \\ \mathrm{C} & -1.436114 & 0.000000 & 0.883912 \\ \mathrm{C} & -0.696837 & 0.000000 & 2.082271 \\ \mathrm{C} & 0.696837 & 0.000000 & 2.082271 \\ \mathrm{C} & 1.436114 & 0.000000 & 0.883912\end{array}$




$\begin{array}{lrrr}\mathrm{C} & -1.495344 & 0.000000 & -1.569583 \\ \mathrm{C} & -2.842263 & 0.000000 & 0.886818 \\ \mathrm{H} & -1.230773 & 0.000000 & 3.025350 \\ \mathrm{H} & 1.230773 & 0.000000 & 3.025350 \\ \mathrm{C} & 1.495344 & 0.000000 & -1.569583 \\ \mathrm{C} & 2.887605 & 0.000000 & -1.526705 \\ \mathrm{C} & 3.573540 & 0.000000 & -0.305314 \\ \mathrm{C} & 2.842263 & 0.000000 & 0.886818 \\ \mathrm{H} & 1.010172 & 0.000000 & -2.534943 \\ \mathrm{H} & 3.444545 & 0.000000 & -2.455649 \\ \mathrm{H} & 4.655053 & 0.000000 & -0.283683 \\ \mathrm{H} & 3.358557 & 0.000000 & 1.839679 \\ \mathrm{C} & -3.573540 & 0.000000 & -0.305314 \\ \mathrm{C} & -2.887605 & 0.000000 & -1.526705 \\ \mathrm{H} & -1.010172 & 0.000000 & -2.534943 \\ \mathrm{H} & -3.444545 & 0.000000 & -2.455649 \\ \mathrm{H} & -4.655053 & 0.000000 & -0.283683 \\ \mathrm{H} & -3.358557 & 0.000000 & 1.839679\end{array}$

2,4,6,8-decatetraene $\mathrm{S}_{0}$

$\begin{array}{rrrc}\text { C } & -2.590146 & -5.015583 & 0.000000 \\ \mathrm{C} & -2.472178 & -3.525678 & 0.000000 \\ \mathrm{C} & -1.318151 & -2.841467 & 0.000000 \\ \mathrm{C} & -1.216689 & -1.402081 & 0.000000 \\ \mathrm{C} & -0.052563 & -0.716434 & 0.000000 \\ \mathrm{C} & 0.052563 & 0.716434 & 0.000000 \\ \mathrm{C} & 1.216689 & 1.402081 & 0.000000 \\ \mathrm{C} & 1.318151 & 2.841467 & 0.000000 \\ \mathrm{C} & 2.472178 & 3.525678 & 0.000000 \\ \mathrm{C} & 2.590146 & 5.015583 & 0.000000 \\ \mathrm{H} & -0.380493 & -3.394393 & 0.000000 \\ \mathrm{H} & -2.151360 & -0.845092 & 0.000000 \\ \mathrm{H} & 0.880810 & -1.276260 & 0.000000 \\ \mathrm{H} & -0.880810 & 1.276260 & 0.000000 \\ \mathrm{H} & 2.151360 & 0.845092 & 0.000000 \\ \mathrm{H} & 0.380493 & 3.394393 & 0.000000 \\ \mathrm{H} & 3.405713 & 2.966697 & 0.000000 \\ \mathrm{H} & -3.405713 & -2.966697 & 0.000000 \\ \mathrm{H} & 1.611067 & 5.496329 & 0.000000 \\ \mathrm{H} & 3.144424 & 5.368422 & 0.876487 \\ \mathrm{H} & 3.144424 & 5.368422 & -0.876487 \\ \mathrm{H} & -1.611067 & -5.496329 & 0.000000 \\ \mathrm{H} & -3.144424 & -5.368422 & 0.876487 \\ \mathrm{H} & -3.144424 & -5.368422 & -0.876487\end{array}$


2,4,6,8-decatetraene $\mathrm{S}_{1}$

$\begin{array}{crcc}\mathrm{C} & -2.584166 & -5.029821 & 0.000000 \\ \mathrm{C} & -2.520962 & -3.545308 & 0.000000 \\ \mathrm{C} & -1.356446 & -2.816426 & 0.000000 \\ \mathrm{C} & -1.265625 & -1.409746 & 0.000000 \\ \mathrm{C} & -0.054368 & -0.696186 & 0.000000 \\ \mathrm{C} & 0.054368 & 0.696186 & 0.000000 \\ \mathrm{C} & 1.265625 & 1.409746 & 0.000000 \\ \mathrm{C} & 1.356446 & 2.816426 & 0.000000 \\ \mathrm{C} & 2.520962 & 3.545308 & 0.000000 \\ \mathrm{C} & 2.584166 & 5.029821 & 0.000000 \\ \mathrm{H} & -0.416770 & -3.367636 & 0.000000 \\ \mathrm{H} & -2.191338 & -0.838635 & 0.000000 \\ \mathrm{H} & 0.867045 & -1.275098 & 0.000000 \\ \mathrm{H} & -0.867045 & 1.275098 & 0.000000 \\ \mathrm{H} & 2.191338 & 0.838635 & 0.000000 \\ \mathrm{H} & 0.416770 & 3.367636 & 0.000000 \\ \mathrm{H} & 3.467531 & 3.009116 & 0.000000 \\ \mathrm{H} & -3.467531 & -3.009116 & 0.000000 \\ \mathrm{H} & 1.587863 & 5.476560 & 0.000000 \\ \mathrm{H} & 3.127439 & 5.415218 & 0.874700 \\ \mathrm{H} & 3.127439 & 5.415218 & -0.874700 \\ \mathrm{H} & -1.587863 & -5.476560 & 0.000000 \\ \mathrm{H} & -3.127439 & -5.415218 & 0.874700 \\ \mathrm{H} & -3.127439 & -5.415218 & -0.874700\end{array}$

tetracene $\mathrm{S}_{0}$

$\begin{array}{rrrr}\text { C } & 3.701544 & 1.405132 & 0.000000 \\ \mathrm{C} & 2.442975 & 0.724011 & 0.000000 \\ \mathrm{C} & 2.442975 & -0.724011 & 0.000000 \\ \mathrm{C} & 3.701544 & -1.405132 & 0.000000 \\ \mathrm{C} & 4.874449 & -0.713560 & 0.000000 \\ \mathrm{C} & 4.874449 & 0.713560 & 0.000000 \\ \mathrm{C} & 1.232042 & 1.402578 & 0.000000 \\ \mathrm{C} & 0.000000 & 0.724150 & 0.000000 \\ \mathrm{C} & 0.000000 & -0.724150 & 0.000000 \\ \mathrm{C} & 1.232042 & -1.402578 & 0.000000 \\ \mathrm{C} & -1.232042 & 1.402578 & 0.000000 \\ \mathrm{C} & -2.442975 & 0.724011 & 0.000000 \\ \mathrm{C} & -2.442975 & -0.724011 & 0.000000 \\ \mathrm{C} & -1.232042 & -1.402578 & 0.000000 \\ \mathrm{C} & -3.701544 & 1.405132 & 0.000000 \\ \mathrm{C} & -4.874449 & 0.713560 & 0.000000 \\ \mathrm{C} & -4.874449 & -0.713560 & 0.000000\end{array}$




$\begin{array}{rrrr}\mathrm{C} & -3.701544 & -1.405132 & 0.000000 \\ \mathrm{H} & -3.701580 & -2.489194 & 0.000000 \\ \mathrm{H} & -5.819069 & -1.243525 & 0.000000 \\ \mathrm{H} & -5.819069 & 1.243525 & 0.000000 \\ \mathrm{H} & -3.701580 & 2.489194 & 0.000000 \\ \mathrm{H} & -1.232320 & -2.487392 & 0.000000 \\ \mathrm{H} & -1.232320 & 2.487392 & 0.000000 \\ \mathrm{H} & 1.232320 & -2.487392 & 0.000000 \\ \mathrm{H} & 1.232320 & 2.487392 & 0.000000 \\ \mathrm{H} & 3.701580 & -2.489194 & 0.000000 \\ \mathrm{H} & 5.819069 & -1.243525 & 0.000000 \\ \mathrm{H} & 5.819069 & 1.243525 & 0.000000 \\ \mathrm{H} & 3.701580 & 2.489194 & 0.000000\end{array}$

tetracene $S_{1}$

$\begin{array}{rrrr}\mathrm{C} & 3.704126 & 1.396660 & 0.0000000 \\ \mathrm{C} & 2.470095 & 0.720289 & 0.000000 \\ \mathrm{C} & 2.470095 & -0.720289 & 0.000000 \\ \mathrm{C} & 3.704126 & -1.396660 & 0.000000 \\ \mathrm{C} & 4.908836 & -0.697940 & 0.000000 \\ \mathrm{C} & 4.908836 & 0.697940 & 0.000000 \\ \mathrm{C} & 1.230157 & 1.397218 & 0.000000 \\ \mathrm{C} & 0.000000 & 0.724565 & 0.000000 \\ \mathrm{C} & 0.000000 & -0.724565 & 0.000000 \\ \mathrm{C} & 1.230157 & -1.397218 & 0.000000 \\ \mathrm{C} & -1.230157 & 1.397218 & 0.000000 \\ \mathrm{C} & -2.470095 & 0.720289 & 0.000000 \\ \mathrm{C} & -2.470095 & -0.720289 & 0.000000 \\ \mathrm{C} & -1.230157 & -1.397218 & 0.000000 \\ \mathrm{C} & -3.704126 & 1.396660 & 0.000000 \\ \mathrm{C} & -4.908836 & 0.697940 & 0.000000 \\ \mathrm{C} & -4.908836 & -0.697940 & 0.000000 \\ \mathrm{C} & -3.704126 & -1.396660 & 0.000000 \\ \mathrm{H} & -3.707608 & -2.480602 & 0.000000 \\ \mathrm{H} & -5.845949 & -1.240319 & 0.000000 \\ \mathrm{H} & -5.845949 & 1.240319 & 0.000000 \\ \mathrm{H} & -3.707608 & 2.480602 & 0.000000 \\ \mathrm{H} & -1.231749 & -2.482172 & 0.000000 \\ \mathrm{H} & -1.231749 & 2.482172 & 0.000000 \\ \mathrm{H} & 1.231749 & -2.482172 & 0.000000 \\ \mathrm{H} & 1.231749 & 2.482172 & 0.000000 \\ \mathrm{H} & 3.707608 & -2.480602 & 0.000000 \\ \mathrm{H} & 5.845949 & -1.240319 & 0.000000 \\ \mathrm{H} & 5.845949 & 1.240319 & 0.000000 \\ \mathrm{H} & 3.707608 & 2.480602 & 0.000000\end{array}$


perylene $\mathrm{S}_{0}$

$\begin{array}{lrrr}\mathrm{C} & 0.020203 & 1.475614 & -2.420285 \\ \mathrm{C} & 0.010956 & 0.737097 & -1.246515 \\ \mathrm{C} & 0.014120 & 1.436211 & 0.000003 \\ \mathrm{C} & 0.034599 & 2.867787 & 0.000001 \\ \mathrm{C} & 0.043306 & 3.565836 & -1.230100 \\ \mathrm{C} & 0.036337 & 2.877860 & -2.415522 \\ \mathrm{C} & 0.044411 & 3.565824 & 1.230101 \\ \mathrm{C} & 0.038550 & 2.877835 & 2.415522 \\ \mathrm{C} & 0.022112 & 1.475592 & 2.420285 \\ \mathrm{C} & 0.011532 & 0.737089 & 1.246518 \\ \mathrm{C} & -0.010956 & -0.737097 & -1.246515 \\ \mathrm{C} & -0.014120 & -1.436211 & 0.000003 \\ \mathrm{C} & -0.011532 & -0.737089 & 1.246518 \\ \mathrm{C} & -0.034599 & -2.867787 & 0.000001 \\ \mathrm{C} & -0.044411 & -3.565824 & 1.230101 \\ \mathrm{C} & -0.038550 & -2.877835 & 2.415522 \\ \mathrm{C} & -0.022112 & -1.475592 & 2.420285 \\ \mathrm{C} & -0.020203 & -1.475614 & -2.420285 \\ \mathrm{C} & -0.036337 & -2.877860 & -2.415522 \\ \mathrm{C} & -0.043306 & -3.565836 & -1.230100 \\ \mathrm{H} & 0.057810 & 4.649271 & 1.217298 \\ \mathrm{H} & 0.046783 & 3.411504 & 3.358082 \\ \mathrm{H} & 0.019053 & 0.974748 & 3.377800 \\ \mathrm{H} & -0.057810 & -4.649271 & 1.217298 \\ \mathrm{H} & -0.046783 & -3.411504 & 3.358082 \\ \mathrm{H} & -0.019053 & -0.974748 & 3.377800 \\ \mathrm{H} & -0.056663 & -4.649285 & -1.217298 \\ \mathrm{H} & -0.043529 & -3.411540 & -3.358085 \\ \mathrm{H} & -0.016132 & -0.974781 & -3.377802 \\ \mathrm{H} & 0.056663 & 4.649285 & -1.217298 \\ \mathrm{H} & 0.043529 & 3.411540 & -3.358085 \\ \mathrm{H} & 0.016132 & 0.974781 & -3.377802\end{array}$

perylene $\mathrm{S}_{1}$

$\begin{array}{lrrc}\mathrm{C} & -0.006753 & 1.469981 & -2.449088 \\ \mathrm{C} & -0.006337 & 0.719704 & -1.241674 \\ \mathrm{C} & 0.010227 & 1.421963 & -0.000003 \\ \mathrm{C} & 0.008344 & 2.854711 & 0.000000 \\ \mathrm{C} & 0.009416 & 3.546975 & -1.230625 \\ \mathrm{C} & -0.001625 & 2.853779 & -2.434092 \\ \mathrm{C} & 0.009735 & 3.546971 & 1.230621 \\ \mathrm{C} & -0.000983 & 2.853769 & 2.434094\end{array}$




$\begin{array}{lrrr}\mathrm{C} & -0.006209 & 1.469976 & 2.449085 \\ \mathrm{C} & -0.006198 & 0.719702 & 1.241669 \\ \mathrm{C} & 0.006337 & -0.719704 & -1.241674 \\ \mathrm{C} & -0.010227 & -1.421963 & -0.000003 \\ \mathrm{C} & 0.006198 & -0.719702 & 1.241669 \\ \mathrm{C} & -0.008344 & -2.854711 & 0.000000 \\ \mathrm{C} & -0.009735 & -3.546971 & 1.230621 \\ \mathrm{C} & 0.000983 & -2.853769 & 2.434094 \\ \mathrm{C} & 0.006209 & -1.469976 & 2.449085 \\ \mathrm{C} & 0.006753 & -1.469981 & -2.449088 \\ \mathrm{C} & 0.001625 & -2.853779 & -2.434092 \\ \mathrm{C} & -0.009416 & -3.546975 & -1.230625 \\ \mathrm{H} & 0.014119 & 4.630425 & 1.222967 \\ \mathrm{H} & -0.004716 & 3.399248 & 3.369976 \\ \mathrm{H} & -0.029103 & 0.961720 & 3.401205 \\ \mathrm{H} & -0.014119 & -4.630425 & 1.222967 \\ \mathrm{H} & 0.004716 & -3.399248 & 3.369976 \\ \mathrm{H} & 0.029103 & -0.961720 & 3.401205 \\ \mathrm{H} & -0.013768 & -4.630429 & -1.222966 \\ \mathrm{H} & 0.005696 & -3.399257 & -3.369973 \\ \mathrm{H} & 0.030023 & -0.961732 & -3.401201 \\ \mathrm{H} & 0.013768 & 4.630429 & -1.222966 \\ \mathrm{H} & -0.005696 & 3.399257 & -3.369973 \\ \mathrm{H} & -0.030023 & 0.961732 & -3.401201\end{array}$

\section{PBE0/TZVP geometries - Group I}

pyrazine $\mathrm{S}_{0}$

$\begin{array}{lrrr}\mathrm{C} & 1.126134 & -0.694452 & 0.000000 \\ \mathrm{C} & 1.126134 & 0.694452 & 0.000000 \\ \mathrm{~N} & 0.000000 & 1.399102 & 0.000000 \\ \mathrm{C} & -1.126134 & 0.694452 & 0.000000 \\ \mathrm{C} & -1.126134 & -0.694452 & 0.000000 \\ \mathrm{~N} & 0.000000 & -1.399102 & 0.000000 \\ \mathrm{H} & -2.058237 & -1.251875 & 0.000000 \\ \mathrm{H} & 2.058237 & -1.251875 & 0.000000 \\ \mathrm{H} & 2.058237 & 1.251875 & 0.000000 \\ \mathrm{H} & -2.058237 & 1.251875 & 0.000000\end{array}$

pyrazine $S_{1}$
$\begin{array}{llll}\text { C } & -1.203528 & 0.671715 & 0.000000\end{array}$
$\begin{array}{llll}\text { C } & -1.112033 & -0.738801 & 0.000000\end{array}$
$\begin{array}{llll}\mathrm{N} & 0.022663 & -1.349694 & 0.000000\end{array}$ 


$\begin{array}{rrrr}\mathrm{C} & 1.203528 & -0.671715 & 0.000000 \\ \mathrm{C} & 1.112033 & 0.738801 & 0.000000 \\ \mathrm{~N} & -0.022663 & 1.349694 & 0.000000 \\ \mathrm{H} & 2.040861 & 1.307501 & 0.000000 \\ \mathrm{H} & -2.155920 & 1.178163 & 0.000000 \\ \mathrm{H} & -2.040861 & -1.307501 & 0.000000 \\ \mathrm{H} & 2.155920 & -1.178163 & 0.000000\end{array}$

2,6-difluoropyridine $\mathrm{S}_{0}$

$\begin{array}{lrrr}\mathrm{C} & 1.113061 & 0.000000 & -0.875147 \\ \mathrm{~N} & 0.000000 & 0.000000 & -1.564782 \\ \mathrm{C} & -1.113061 & 0.000000 & -0.875147 \\ \mathrm{C} & -1.200692 & 0.000000 & 0.506061 \\ \mathrm{C} & 0.000000 & 0.000000 & 1.199144 \\ \mathrm{C} & 1.200692 & 0.000000 & 0.506061 \\ \mathrm{~F} & -2.234451 & 0.000000 & -1.588469 \\ \mathrm{H} & -2.162993 & 0.000000 & 0.999120 \\ \mathrm{H} & 0.000000 & 0.000000 & 2.282929 \\ \mathrm{H} & 2.162993 & 0.000000 & 0.999120 \\ \mathrm{~F} & 2.234451 & 0.000000 & -1.588469\end{array}$

2,6-difluoropyridine $\mathrm{S}_{1}$

$\begin{array}{cccc}\mathrm{C} & -1.097439 & 0.000000 & -0.336019 \\ \mathrm{~N} & 0.000000 & 0.000000 & -1.102521 \\ \mathrm{C} & 1.097439 & 0.000000 & -0.336019 \\ \mathrm{C} & 1.189848 & 0.000000 & 1.070506 \\ \mathrm{C} & 0.000000 & 0.000000 & 1.845793 \\ \mathrm{C} & -1.189848 & 0.000000 & 1.070506 \\ \mathrm{~F} & 2.233651 & 0.000000 & -1.008800 \\ \mathrm{H} & 2.177089 & 0.000000 & 1.515674 \\ \mathrm{H} & 0.000000 & 0.000000 & 2.923302 \\ \mathrm{H} & -2.177089 & 0.000000 & 1.515674 \\ \mathrm{~F} & -2.233651 & 0.000000 & -1.008800\end{array}$

2-fluoropyridine $\mathrm{S}_{0}$
$\begin{array}{llll}\text { C } & -1.314008 & 0.502542 & 0.000000\end{array}$
$\begin{array}{llll}\mathrm{N} & -1.364532 & -0.800561 & 0.000000\end{array}$
$\begin{array}{llll}\text { C } & -0.198251 & -1.448323 & 0.000000\end{array}$
$\begin{array}{llll}\text { C } & 1.028277 & -0.807381 & 0.000000\end{array}$
$\begin{array}{llll}\text { C } & 1.042141 & 0.583012 & 0.000000\end{array}$
$\begin{array}{llll}\mathrm{C} & -0.157572 & 1.270181 & 0.000000\end{array}$
$\mathrm{H} \quad-0.260078 \quad-2.532245 \quad 0.000000$
H $\quad 1.946942 \quad-1.380429 \quad 0.000000$ 


$\begin{array}{lrrr}\mathrm{H} & 1.979956 & 1.127040 & 0.000000 \\ \mathrm{H} & -0.214360 & 2.350872 & 0.000000 \\ \mathrm{~F} & -2.488525 & 1.135282 & 0.000000\end{array}$

2-fluoropyridine $\mathrm{S}_{1}$

$\begin{array}{rrrr}\mathrm{C} & -1.318346 & 0.488620 & 0.000000 \\ \mathrm{~N} & -1.436568 & -0.842857 & 0.000000 \\ \mathrm{C} & -0.226257 & -1.460918 & 0.000000 \\ \mathrm{C} & 1.026949 & -0.790788 & 0.000000 \\ \mathrm{C} & 1.107126 & 0.618741 & 0.000000 \\ \mathrm{C} & -0.151828 & 1.281314 & 0.000000 \\ \mathrm{H} & -0.248483 & -2.544802 & 0.000000 \\ \mathrm{H} & 1.925450 & -1.397247 & 0.000000 \\ \mathrm{H} & 2.044818 & 1.151954 & 0.000000 \\ \mathrm{H} & -0.254254 & 2.358534 & 0.000000 \\ \mathrm{~F} & -2.468617 & 1.137438 & 0.000000\end{array}$

2-hydroxypyridine $\mathrm{S}_{0}$
$\begin{array}{llll}\text { C } & -0.978518 & 0.751041 & 0.000000\end{array}$
$\begin{array}{llll}\text { C } & -0.970229 & -0.644635 & 0.000000\end{array}$
$\begin{array}{llll}\mathrm{N} & 0.129491 & -1.375535 & 0.000000\end{array}$
$\begin{array}{llll}\text { C } & 1.299659 & -0.736047 & 0.000000\end{array}$
$\begin{array}{llll}\text { C } & 1.415214 & 0.641254 & 0.000000\end{array}$
$\begin{array}{llll}\text { C } & 0.242406 & 1.392599 & 0.000000\end{array}$
$\begin{array}{llll}\text { O } & -2.145579 & -1.297639 & 0.000000\end{array}$
$\begin{array}{llll}\mathrm{H} & 2.182513 & -1.368554 & 0.000000\end{array}$
$\begin{array}{llll}\mathrm{H} & 2.389726 & 1.112663 & 0.000000\end{array}$
$\begin{array}{llll}\mathrm{H} & 0.283878 & 2.476306 & 0.000000\end{array}$
$\mathrm{H} \quad-1.918340 \quad 1.287712 \quad 0.000000$
$\mathrm{H} \quad-1.930221 \quad-2.239164 \quad 0.000000$

2-hydroxypyridine $\mathrm{S}_{1}$

$\begin{array}{rrrc}\mathrm{C} & -0.999611 & 0.767337 & 0.000000 \\ \mathrm{C} & -0.965848 & -0.633713 & 0.000000 \\ \mathrm{~N} & 0.114671 & -1.450545 & 0.000000 \\ \mathrm{C} & 1.288954 & -0.773948 & 0.000000 \\ \mathrm{C} & 1.386970 & 0.640083 & 0.000000 \\ \mathrm{C} & 0.249752 & 1.460652 & 0.000000 \\ \mathrm{O} & -2.124548 & -1.289903 & 0.000000 \\ \mathrm{H} & 2.190760 & -1.375145 & 0.000000 \\ \mathrm{H} & 2.379812 & 1.076655 & 0.000000 \\ \mathrm{H} & 0.311076 & 2.538010 & 0.000000 \\ \mathrm{H} & -1.957009 & 1.271199 & 0.000000\end{array}$




$$
\begin{array}{llll}
\mathrm{H} & -1.874978 & -2.230683 & 0.000000
\end{array}
$$

2-pyridone $\mathrm{S}_{0}$

$\begin{array}{rrrr}\mathrm{C} & -1.244899 & 0.712984 & 0.000000 \\ \mathrm{~N} & -1.131631 & -0.684404 & 0.000000 \\ \mathrm{C} & 0.031385 & -1.379856 & 0.000000 \\ \mathrm{C} & 1.221392 & -0.730701 & 0.000000 \\ \mathrm{C} & 1.202855 & 0.688754 & 0.000000 \\ \mathrm{C} & 0.035841 & 1.382805 & 0.000000 \\ \mathrm{H} & -2.015551 & -1.172933 & 0.000000 \\ \mathrm{H} & -0.057463 & -2.459320 & 0.000000 \\ \mathrm{H} & 2.148266 & -1.286371 & 0.000000 \\ \mathrm{H} & 2.144832 & 1.227827 & 0.000000 \\ \mathrm{H} & 0.010319 & 2.464818 & 0.000000 \\ \mathrm{O} & -2.345346 & 1.236406 & 0.000000\end{array}$

2-pyridone $\mathrm{S}_{1}$

$\begin{array}{rrrr}\mathrm{C} & -1.367913 & 0.101128 & 0.000000 \\ \mathrm{~N} & -0.730170 & -1.121149 & 0.000000 \\ \mathrm{C} & 0.628223 & -1.252082 & 0.000000 \\ \mathrm{C} & 1.413183 & -0.076254 & 0.000000 \\ \mathrm{C} & 0.829114 & 1.163614 & 0.000000 \\ \mathrm{C} & -0.612721 & 1.262953 & 0.000000 \\ \mathrm{H} & -1.345900 & -1.921959 & 0.000000 \\ \mathrm{H} & 1.035558 & -2.251429 & 0.000000 \\ \mathrm{H} & 2.490498 & -0.187851 & 0.000000 \\ \mathrm{H} & 1.435481 & 2.059456 & 0.000000 \\ \mathrm{H} & -1.133377 & 2.208757 & 0.000000 \\ \mathrm{O} & -2.641975 & 0.014806 & 0.000000\end{array}$

2-methylpyrimidine $\mathrm{S}_{0}$

$\begin{array}{rrrr}\mathrm{C} & 0.000128 & 1.959302 & 0.000000 \\ \mathrm{C} & -1.171868 & 1.219899 & 0.000000 \\ \mathrm{~N} & -1.179285 & -0.106166 & 0.000000 \\ \mathrm{C} & 0.010343 & -0.715192 & 0.000000 \\ \mathrm{~N} & 1.193397 & -0.099013 & 0.000000 \\ \mathrm{C} & 1.175499 & 1.230223 & 0.000000 \\ \mathrm{H} & -2.143798 & 1.707184 & 0.000000 \\ \mathrm{H} & 2.144259 & 1.723390 & 0.000000 \\ \mathrm{H} & -0.004446 & 3.041855 & 0.000000 \\ \mathrm{C} & 0.005196 & -2.208431 & 0.000000 \\ \mathrm{H} & 1.024323 & -2.589825 & 0.000000 \\ \mathrm{H} & -0.526879 & -2.581613 & 0.878165\end{array}$


$\begin{array}{llll}\mathrm{H} & -0.526879 & -2.581613 & -0.878165\end{array}$

2-methylpyrimidine $\mathrm{S}_{1}$

$\begin{array}{rrrr}\mathrm{C} & -0.012877 & 2.007060 & 0.000000 \\ \mathrm{C} & -1.165366 & 1.272768 & 0.000000 \\ \mathrm{~N} & -1.148803 & -0.094803 & 0.000000 \\ \mathrm{C} & 0.012548 & -0.782303 & 0.000000 \\ \mathrm{~N} & 1.086460 & -0.068972 & 0.000000 \\ \mathrm{C} & 1.202574 & 1.289092 & 0.000000 \\ \mathrm{H} & -2.145505 & 1.735710 & 0.000000 \\ \mathrm{H} & 2.190170 & 1.722112 & 0.000000 \\ \mathrm{H} & -0.025973 & 3.090351 & 0.000000 \\ \mathrm{C} & 0.012907 & -2.263525 & 0.000000 \\ \mathrm{H} & 1.031771 & -2.647625 & 0.000000 \\ \mathrm{H} & -0.518952 & -2.629938 & 0.881268 \\ \mathrm{H} & -0.518952 & -2.629938 & -0.881268\end{array}$

5-methylpyrimidine $\mathrm{S}_{0}$

$\begin{array}{rrrr}\mathrm{C} & 0.872535 & 0.848742 & 0.000000 \\ \mathrm{C} & -0.361084 & 0.210516 & 0.000000 \\ \mathrm{C} & -0.298555 & -1.179770 & 0.000000 \\ \mathrm{~N} & 0.843233 & -1.856443 & 0.000000 \\ \mathrm{C} & 1.953973 & -1.126983 & 0.000000 \\ \mathrm{~N} & 2.031236 & 0.196873 & 0.000000 \\ \mathrm{H} & 2.893852 & -1.671279 & 0.000000 \\ \mathrm{H} & -1.210866 & -1.773578 & 0.000000 \\ \mathrm{H} & 0.929822 & 1.935565 & 0.000000 \\ \mathrm{C} & -1.656822 & 0.957165 & 0.000000 \\ \mathrm{H} & -1.486624 & 2.035083 & 0.000000 \\ \mathrm{H} & -2.255351 & 0.712044 & -0.881144 \\ \mathrm{H} & -2.255351 & 0.712044 & 0.881144\end{array}$

5-methylpyrimidine $\mathrm{S}_{1}$

$\begin{array}{rrrr}\mathrm{C} & 0.843962 & 0.854247 & 0.000000 \\ \mathrm{C} & -0.378215 & 0.241626 & 0.000000 \\ \mathrm{C} & -0.347752 & -1.187875 & 0.000000 \\ \mathrm{~N} & 0.879498 & -1.756034 & 0.000000 \\ \mathrm{C} & 2.028181 & -1.177102 & 0.000000 \\ \mathrm{~N} & 2.026096 & 0.170190 & 0.000000 \\ \mathrm{H} & 2.955693 & -1.733140 & 0.000000 \\ \mathrm{H} & -1.218281 & -1.825613 & 0.000000 \\ \mathrm{H} & 0.929290 & 1.936188 & 0.000000 \\ \mathrm{C} & -1.672554 & 0.978203 & 0.000000\end{array}$




$$
\begin{array}{lrrr}
\mathrm{H} & -1.510687 & 2.057067 & 0.000000 \\
\mathrm{H} & -2.267620 & 0.721127 & -0.881792 \\
\mathrm{H} & -2.267620 & 0.721127 & 0.881792
\end{array}
$$

7-azaindole $\mathrm{S}_{0}$

$\begin{array}{lrrr}\mathrm{N} & -0.632061 & 1.837346 & 0.000000 \\ \mathrm{C} & -0.672317 & 0.470767 & 0.000000 \\ \mathrm{C} & 0.667225 & 0.006998 & 0.000000 \\ \mathrm{C} & 1.505646 & 1.162983 & 0.000000 \\ \mathrm{C} & 0.678152 & 2.247926 & 0.000000 \\ \mathrm{C} & 0.843617 & -1.374964 & 0.000000 \\ \mathrm{C} & -0.291402 & -2.166367 & 0.000000 \\ \mathrm{C} & -1.559854 & -1.574491 & 0.000000 \\ \mathrm{~N} & -1.772450 & -0.263758 & 0.000000 \\ \mathrm{H} & -2.448261 & -2.199298 & 0.000000 \\ \mathrm{H} & 1.833985 & -1.817648 & 0.000000 \\ \mathrm{H} & -0.212552 & -3.246905 & 0.000000 \\ \mathrm{H} & -1.445658 & 2.427222 & 0.000000 \\ \mathrm{H} & 2.584115 & 1.191021 & 0.000000 \\ \mathrm{H} & 0.921835 & 3.299168 & 0.000000\end{array}$

7-azaindole $\mathrm{S}_{1}$

$\begin{array}{lrrr}\mathrm{N} & -0.599782 & 1.866400 & 0.000000 \\ \mathrm{C} & -0.652662 & 0.442854 & 0.000000 \\ \mathrm{C} & 0.650229 & -0.011677 & 0.000000 \\ \mathrm{C} & 1.489583 & 1.151316 & 0.000000 \\ \mathrm{C} & 0.649749 & 2.305007 & 0.000000 \\ \mathrm{C} & 0.889039 & -1.410118 & 0.000000 \\ \mathrm{C} & -0.304967 & -2.177539 & 0.000000 \\ \mathrm{C} & -1.549395 & -1.587266 & 0.000000 \\ \mathrm{~N} & -1.803354 & -0.228238 & 0.000000 \\ \mathrm{H} & -2.436448 & -2.212569 & 0.000000 \\ \mathrm{H} & 1.876877 & -1.846194 & 0.000000 \\ \mathrm{H} & -0.252863 & -3.260417 & 0.000000 \\ \mathrm{H} & -1.434454 & 2.435021 & 0.000000 \\ \mathrm{H} & 2.569464 & 1.182302 & 0.000000 \\ \mathrm{H} & 0.908994 & 3.351137 & 0.000000\end{array}$

7-hydroxyquinoline, cis $\mathrm{S}_{0}$
$\begin{array}{llll}\mathrm{N} & 0.464538 & 1.965196 & 0.000000\end{array}$
$\begin{array}{llll}\text { C } & 0.434314 & 0.608647 & 0.000000\end{array}$
$\begin{array}{llll}\text { C } & -0.784805 & -0.125478 & 0.000000\end{array}$
$\begin{array}{llll}\text { C } & -1.987607 & 0.605227 & 0.000000\end{array}$ 


$\begin{array}{lrrr}\mathrm{C} & -1.941318 & 1.972773 & 0.000000 \\ \mathrm{C} & -0.680605 & 2.601053 & 0.000000 \\ \mathrm{C} & 1.659848 & -0.088981 & 0.000000 \\ \mathrm{C} & 1.674431 & -1.460162 & 0.000000 \\ \mathrm{C} & 0.466783 & -2.193949 & 0.000000 \\ \mathrm{C} & -0.729528 & -1.538282 & 0.000000 \\ \mathrm{H} & -0.623773 & 3.687392 & 0.000000 \\ \mathrm{H} & -1.657477 & -2.101156 & 0.000000 \\ \mathrm{H} & 0.521346 & -3.276011 & 0.000000 \\ \mathrm{O} & 2.814421 & -2.193785 & 0.000000 \\ \mathrm{H} & 2.574724 & 0.495128 & 0.000000 \\ \mathrm{H} & -2.935055 & 0.074914 & 0.000000 \\ \mathrm{H} & -2.844170 & 2.571500 & 0.000000 \\ \mathrm{H} & 3.573935 & -1.604017 & 0.000000\end{array}$

7-hydroxyquinoline, cis $\mathrm{S}_{1}$

$\begin{array}{rrrr}\mathrm{N} & 0.493973 & 1.967057 & 0.000000 \\ \mathrm{C} & 0.423643 & 0.631098 & 0.000000 \\ \mathrm{C} & -0.784854 & -0.133670 & 0.000000 \\ \mathrm{C} & -2.000488 & 0.596029 & 0.000000 \\ \mathrm{C} & -1.919620 & 1.996816 & 0.000000 \\ \mathrm{C} & -0.690944 & 2.634014 & 0.000000 \\ \mathrm{C} & 1.632214 & -0.096210 & 0.000000 \\ \mathrm{C} & 1.657123 & -1.521071 & 0.000000 \\ \mathrm{C} & 0.485606 & -2.265522 & 0.000000 \\ \mathrm{C} & -0.709851 & -1.547783 & 0.000000 \\ \mathrm{H} & -0.626624 & 3.716048 & 0.000000 \\ \mathrm{H} & -1.645584 & -2.099068 & 0.000000 \\ \mathrm{H} & 0.518882 & -3.345433 & 0.000000 \\ \mathrm{O} & 2.831601 & -2.157163 & 0.000000 \\ \mathrm{H} & 2.561890 & 0.467103 & 0.000000 \\ \mathrm{H} & -2.954893 & 0.084688 & 0.000000 \\ \mathrm{H} & -2.826740 & 2.591711 & 0.000000 \\ \mathrm{H} & 3.554667 & -1.518634 & 0.000000\end{array}$

7-hydroxyquinoline, trans $\mathrm{S}_{0}$

$\begin{array}{rrrc}\mathrm{N} & 0.514148 & 2.037999 & 0.000000 \\ \mathrm{C} & 0.482015 & 0.679487 & 0.000000 \\ \mathrm{C} & -0.742270 & -0.049122 & 0.000000 \\ \mathrm{C} & -1.944151 & 0.685762 & 0.000000 \\ \mathrm{C} & -1.892797 & 2.051756 & 0.000000 \\ \mathrm{C} & -0.627963 & 2.675939 & 0.000000 \\ \mathrm{C} & 1.704749 & -0.017430 & 0.000000 \\ \mathrm{C} & 1.716278 & -1.388641 & 0.000000\end{array}$




$\begin{array}{rrrr}\mathrm{C} & 0.504805 & -2.116284 & 0.000000 \\ \mathrm{C} & -0.693550 & -1.458972 & 0.000000 \\ \mathrm{H} & -0.568545 & 3.762283 & 0.000000 \\ \mathrm{H} & -1.622088 & -2.020791 & 0.000000 \\ \mathrm{H} & 0.532522 & -3.202580 & 0.000000 \\ \mathrm{O} & 2.915662 & -2.023160 & 0.000000 \\ \mathrm{H} & 2.629693 & 0.545024 & 0.000000 \\ \mathrm{H} & -2.893306 & 0.158447 & 0.000000 \\ \mathrm{H} & -2.793134 & 2.654322 & 0.000000 \\ \mathrm{H} & 2.777912 & -2.974020 & 0.000000\end{array}$

7-hydroxyquinoline, trans $\mathrm{S}_{1}$

$\begin{array}{rrrr}\mathrm{N} & 0.370956 & 1.958655 & 0.000076 \\ \mathrm{C} & 0.520856 & 0.650847 & 0.000025 \\ \mathrm{C} & -0.752411 & -0.066193 & 0.000014 \\ \mathrm{C} & -1.945218 & 0.712047 & 0.000008 \\ \mathrm{C} & -1.914966 & 2.097565 & 0.000006 \\ \mathrm{C} & -0.693172 & 2.756874 & 0.000014 \\ \mathrm{C} & 1.734405 & -0.035926 & -0.000008 \\ \mathrm{C} & 1.725245 & -1.430463 & -0.000012 \\ \mathrm{C} & 0.537949 & -2.143938 & -0.000012 \\ \mathrm{C} & -0.679485 & -1.454098 & 0.000010 \\ \mathrm{H} & -0.552943 & 3.828434 & -0.000019 \\ \mathrm{H} & -1.611395 & -2.009322 & 0.000020 \\ \mathrm{H} & 0.549334 & -3.228850 & -0.000010 \\ \mathrm{O} & 2.939990 & -2.035338 & -0.000026 \\ \mathrm{H} & 2.671648 & 0.502019 & -0.000014 \\ \mathrm{H} & -2.896441 & 0.193197 & -0.000020 \\ \mathrm{H} & -2.819786 & 2.692801 & -0.000023 \\ \mathrm{H} & 2.815434 & -2.988331 & -0.000030\end{array}$

2-hydroxyquinoline, enol $\mathrm{S}_{0}$

$\begin{array}{rrrr}\mathrm{C} & 0.432072 & 1.898172 & 0.000000 \\ \mathrm{C} & 0.485431 & 0.489033 & 0.000000 \\ \mathrm{C} & -0.721913 & -0.260743 & 0.000000 \\ \mathrm{C} & -1.950148 & 0.434000 & 0.000000 \\ \mathrm{C} & -1.972162 & 1.804020 & 0.000000 \\ \mathrm{C} & -0.774932 & 2.545671 & 0.000000 \\ \mathrm{C} & 1.693968 & -0.242665 & 0.000000 \\ \mathrm{C} & 1.661154 & -1.604085 & 0.000000 \\ \mathrm{C} & 0.393401 & -2.246318 & 0.000000 \\ \mathrm{~N} & -0.740717 & -1.617050 & 0.000000 \\ \mathrm{O} & 0.313136 & -3.595203 & 0.000000 \\ \mathrm{H} & 1.361065 & 2.459935 & 0.000000\end{array}$




$\begin{array}{rrrr}\mathrm{H} & -0.813120 & 3.629033 & 0.000000 \\ \mathrm{H} & -2.922314 & 2.326983 & 0.000000 \\ \mathrm{H} & -2.861438 & -0.152137 & 0.000000 \\ \mathrm{H} & 2.641388 & 0.287024 & 0.000000 \\ \mathrm{H} & 2.576483 & -2.187357 & 0.000000 \\ \mathrm{H} & 1.198648 & -3.968314 & 0.000000\end{array}$

2-hydroxyquinoline, enol $\mathrm{S}_{1}$

$\begin{array}{rrrr}\mathrm{C} & 0.426409 & 1.873367 & 0.000000 \\ \mathrm{C} & 0.521945 & 0.482903 & 0.000000 \\ \mathrm{C} & -0.709971 & -0.260099 & 0.000000 \\ \mathrm{C} & -1.944789 & 0.441858 & 0.000000 \\ \mathrm{C} & -2.008043 & 1.844149 & 0.000000 \\ \mathrm{C} & -0.825213 & 2.551368 & 0.000000 \\ \mathrm{C} & 1.736989 & -0.233957 & 0.000000 \\ \mathrm{C} & 1.691955 & -1.642821 & 0.000000 \\ \mathrm{C} & 0.433297 & -2.241261 & 0.000000 \\ \mathrm{~N} & -0.748760 & -1.604606 & 0.000000 \\ \mathrm{O} & 0.289833 & -3.573340 & 0.000000 \\ \mathrm{H} & 1.337492 & 2.463686 & 0.000000 \\ \mathrm{H} & -0.829482 & 3.635547 & 0.000000 \\ \mathrm{H} & -2.964269 & 2.351090 & 0.000000 \\ \mathrm{H} & -2.846025 & -0.160767 & 0.000000 \\ \mathrm{H} & 2.681126 & 0.296059 & 0.000000 \\ \mathrm{H} & 2.595388 & -2.240708 & 0.000000 \\ \mathrm{H} & 1.162109 & -3.982448 & 0.000000\end{array}$

2-hydroxyquinoline, keto $\mathrm{S}_{0}$

$\begin{array}{rrrr}\mathrm{N} & 0.556279 & 1.700781 & 0.000000 \\ \mathrm{C} & 0.600286 & 0.329280 & 0.000000 \\ \mathrm{C} & -0.614419 & -0.382051 & 0.000000 \\ \mathrm{C} & -1.831951 & 0.375436 & 0.000000 \\ \mathrm{C} & -1.828947 & 1.722321 & 0.000000 \\ \mathrm{C} & -0.588306 & 2.485009 & 0.000000 \\ \mathrm{C} & 1.815325 & -0.360999 & 0.000000 \\ \mathrm{C} & 1.818440 & -1.740635 & 0.000000 \\ \mathrm{C} & 0.621019 & -2.459599 & 0.000000 \\ \mathrm{C} & -0.579017 & -1.781362 & 0.000000 \\ \mathrm{H} & -1.517673 & -2.325917 & 0.000000 \\ \mathrm{H} & 0.637545 & -3.542813 & 0.000000 \\ \mathrm{H} & 2.764769 & -2.269756 & 0.000000 \\ \mathrm{H} & 2.748705 & 0.192337 & 0.000000 \\ \mathrm{H} & -2.772363 & -0.167979 & 0.000000 \\ \mathrm{H} & -2.741691 & 2.303993 & 0.000000\end{array}$




$$
\begin{array}{rrrr}
\mathrm{O} & -0.509683 & 3.700185 & 0.000000 \\
\mathrm{H} & 1.421703 & 2.221790 & 0.000000
\end{array}
$$

2-hydroxyquinoline, keto $\mathrm{S}_{1}$

$\begin{array}{rrrr}\mathrm{N} & 0.554091 & 1.672357 & 0.000000 \\ \mathrm{C} & 0.603879 & 0.320373 & 0.000000 \\ \mathrm{C} & -0.665325 & -0.379588 & 0.000000 \\ \mathrm{C} & -1.857946 & 0.365740 & 0.000000 \\ \mathrm{C} & -1.854623 & 1.767674 & 0.000000 \\ \mathrm{C} & -0.626898 & 2.463772 & 0.000000 \\ \mathrm{C} & 1.829369 & -0.360511 & 0.000000 \\ \mathrm{C} & 1.856310 & -1.750927 & 0.000000 \\ \mathrm{C} & 0.640016 & -2.444852 & 0.000000 \\ \mathrm{C} & -0.587864 & -1.781187 & 0.000000 \\ \mathrm{H} & -1.507365 & -2.355232 & 0.000000 \\ \mathrm{H} & 0.647342 & -3.529441 & 0.000000 \\ \mathrm{H} & 2.797604 & -2.284142 & 0.000000 \\ \mathrm{H} & 2.750570 & 0.213376 & 0.000000 \\ \mathrm{H} & -2.798552 & -0.172362 & 0.000000 \\ \mathrm{H} & -2.761103 & 2.353998 & 0.000000 \\ \mathrm{O} & -0.424736 & 3.680607 & 0.000000 \\ \mathrm{H} & 1.405231 & 2.220354 & 0.000000\end{array}$

dibenzofuran $\mathrm{S}_{0}$

$\begin{array}{rrrr}\text { C } & 1.716742 & 0.000000 & -1.356753 \\ \mathrm{C} & 0.722429 & 0.000000 & -0.382976 \\ \mathrm{C} & 1.091932 & 0.000000 & 0.966963 \\ \mathrm{C} & 2.405193 & 0.000000 & 1.392365 \\ \mathrm{C} & 3.379770 & 0.000000 & 0.404307 \\ \mathrm{C} & 3.041597 & 0.000000 & -0.951130 \\ \mathrm{C} & -0.722429 & 0.000000 & -0.382976 \\ \mathrm{C} & -1.091932 & 0.000000 & 0.966963 \\ \mathrm{O} & 0.000000 & 0.000000 & 1.789095 \\ \mathrm{C} & -1.716742 & 0.000000 & -1.356753 \\ \mathrm{C} & -3.041597 & 0.000000 & -0.951130 \\ \mathrm{C} & -3.379770 & 0.000000 & 0.404307 \\ \mathrm{C} & -2.405193 & 0.000000 & 1.392365 \\ \mathrm{H} & -2.654661 & 0.000000 & 2.446191 \\ \mathrm{H} & -4.424971 & 0.000000 & 0.692235 \\ \mathrm{H} & -3.830059 & 0.000000 & -1.694934 \\ \mathrm{H} & -1.461996 & 0.000000 & -2.410650 \\ \mathrm{H} & 1.461996 & 0.000000 & -2.410650 \\ \mathrm{H} & 3.830059 & 0.000000 & -1.694934 \\ \mathrm{H} & 4.424971 & 0.000000 & 0.692235\end{array}$




\begin{tabular}{|c|c|c|c|}
\hline $\mathrm{H}$ & 2.654661 & 0.000000 & 2.446191 \\
\hline \multicolumn{4}{|c|}{ dibenzofuran $\mathrm{S}_{1}$} \\
\hline $\mathrm{C}$ & 0.000000 & 1.731144 & -1.530458 \\
\hline $\mathrm{C}$ & 0.000000 & 0.710166 & -0.571212 \\
\hline $\mathrm{C}$ & 0.000000 & 1.100487 & 0.796557 \\
\hline $\mathrm{C}$ & 0.000000 & & 53978 \\
\hline $\mathrm{C}$ & 0.000000 & 3.414620 & 0.266918 \\
\hline $\mathrm{C}$ & 0.000000 & 3.070340 & 1967 \\
\hline $\mathrm{C}$ & 0.000000 & -0.7 & 1212 \\
\hline $\mathrm{C}$ & 0.000000 & -1.100487 & 6557 \\
\hline $\mathrm{O}$ & 0.000000 & 0.000000 & 4841 \\
\hline C & 0.000000 & -1.731144 & 30458 \\
\hline $\mathrm{C}$ & 0.000000 & -3.070340 & 31967 \\
\hline $\mathrm{C}$ & 0.000000 & -3.414620 & 0.266918 \\
\hline $\mathrm{C}$ & 0.000000 & & \\
\hline $\mathrm{H}$ & 0.000000 & -2.624000 & 2.313199 \\
\hline $\mathrm{H}$ & 0.000000 & -4.455117 & 0.563421 \\
\hline $\mathrm{H}$ & 0.000000 & -3.861683 & -1.823687 \\
\hline $\mathrm{H}$ & 0.000000 & -1.510739 & -2.590459 \\
\hline $\mathrm{H}$ & 0.000000 & 1.510739 & -2.590459 \\
\hline $\mathrm{H}$ & 0.000000 & 3.861683 & -1.823687 \\
\hline $\mathrm{H}$ & 0.000000 & 4.455117 & 0.563421 \\
\hline $\mathrm{H}$ & 0.000000 & 2.624000 & 2.313199 \\
\hline
\end{tabular}

pyrrolo[3,2-h]quinoline $\mathrm{S}_{0}$

$\begin{array}{rrrr}\mathrm{C} & 0.132262 & -0.685787 & 0.000000 \\ \mathrm{~N} & 0.058712 & -2.033195 & 0.000000 \\ \mathrm{C} & 1.190291 & -2.702051 & 0.000000 \\ \mathrm{C} & 2.456675 & -2.096136 & 0.000000 \\ \mathrm{C} & 2.534168 & -0.725393 & 0.000000 \\ \mathrm{C} & 1.355789 & 0.039829 & 0.000000 \\ \mathrm{H} & 1.108523 & -3.786230 & 0.000000 \\ \mathrm{H} & 3.348899 & -2.710854 & 0.000000 \\ \mathrm{H} & 3.496589 & -0.222828 & 0.000000 \\ \mathrm{C} & -1.050989 & 0.082211 & 0.000000 \\ \mathrm{C} & -1.055754 & 1.482624 & 0.000000 \\ \mathrm{C} & 0.180617 & 2.173515 & 0.000000 \\ \mathrm{C} & 1.347313 & 1.465837 & 0.000000 \\ \mathrm{H} & 0.196948 & 3.257980 & 0.000000 \\ \mathrm{H} & 2.300952 & 1.983513 & 0.000000 \\ \mathrm{C} & -2.422733 & 1.885759 & 0.000000 \\ \mathrm{C} & -3.174775 & 0.741565 & 0.000000 \\ \mathrm{~N} & -2.342395 & -0.345190 & 0.000000\end{array}$




$$
\begin{array}{rrrr}
\mathrm{H} & -2.608849 & -1.314859 & 0.000000 \\
\mathrm{H} & -2.805950 & 2.894356 & 0.000000 \\
\mathrm{H} & -4.246281 & 0.615334 & 0.000000
\end{array}
$$

pyrrolo[3,2-h]quinoline $\mathrm{S}_{1}$

$\begin{array}{lrrr}\mathrm{C} & 0.154273 & -0.697847 & 0.000000 \\ \mathrm{~N} & 0.007041 & -2.030666 & 0.000000 \\ \mathrm{C} & 1.192461 & -2.724074 & 0.000000 \\ \mathrm{C} & 2.431590 & -2.129513 & 0.000000 \\ \mathrm{C} & 2.565112 & -0.725923 & 0.000000 \\ \mathrm{C} & 1.354855 & 0.023687 & 0.000000 \\ \mathrm{H} & 1.101370 & -3.804937 & 0.000000 \\ \mathrm{H} & 3.315183 & -2.758664 & 0.000000 \\ \mathrm{H} & 3.531183 & -0.239548 & 0.000000 \\ \mathrm{C} & -1.023919 & 0.105274 & 0.000000 \\ \mathrm{C} & -1.063802 & 1.550161 & 0.000000 \\ \mathrm{C} & 0.143141 & 2.228639 & 0.000000 \\ \mathrm{C} & 1.311267 & 1.455741 & 0.000000 \\ \mathrm{H} & 0.195518 & 3.309680 & 0.000000 \\ \mathrm{H} & 2.265790 & 1.974155 & 0.000000 \\ \mathrm{C} & -2.452893 & 1.904572 & 0.000000 \\ \mathrm{C} & -3.160784 & 0.745372 & 0.000000 \\ \mathrm{~N} & -2.278428 & -0.339397 & 0.000000 \\ \mathrm{H} & -2.494703 & -1.325879 & 0.000000 \\ \mathrm{H} & -2.867636 & 2.899868 & 0.000000 \\ \mathrm{H} & -4.226629 & 0.579298 & 0.000000\end{array}$

carbazole $\mathrm{S}_{0}$

$\begin{array}{crrr}\mathrm{C} & 1.125756 & 0.000000 & -0.895871 \\ \mathrm{~N} & 0.000000 & 0.000000 & -1.689701 \\ \mathrm{C} & -1.125756 & 0.000000 & -0.895871 \\ \mathrm{C} & -0.721481 & 0.000000 & 0.457399 \\ \mathrm{C} & 0.721481 & 0.000000 & 0.457399 \\ \mathrm{C} & -1.689966 & 0.000000 & 1.457939 \\ \mathrm{C} & -3.027505 & 0.000000 & 1.101472 \\ \mathrm{C} & -3.408143 & 0.000000 & -0.244145 \\ \mathrm{C} & -2.466814 & 0.000000 & -1.259850 \\ \mathrm{C} & 2.466814 & 0.000000 & -1.259850 \\ \mathrm{C} & 3.408143 & 0.000000 & -0.244145 \\ \mathrm{C} & 3.027505 & 0.000000 & 1.101472 \\ \mathrm{C} & 1.689966 & 0.000000 & 1.457939 \\ \mathrm{H} & -2.770016 & 0.000000 & -2.301178 \\ \mathrm{H} & -4.462063 & 0.000000 & -0.499275 \\ \mathrm{H} & -3.790568 & 0.000000 & 1.871238\end{array}$




$\begin{array}{rrrr}\mathrm{H} & -1.400844 & 0.000000 & 2.503375 \\ \mathrm{H} & 1.400844 & 0.000000 & 2.503375 \\ \mathrm{H} & 3.790568 & 0.000000 & 1.871238 \\ \mathrm{H} & 4.462063 & 0.000000 & -0.499275 \\ \mathrm{H} & 2.770016 & 0.000000 & -2.301178 \\ \mathrm{H} & 0.000000 & 0.000000 & -2.693468\end{array}$

carbazole $\mathrm{S}_{1}$

$\begin{array}{lrrr}\mathrm{C} & 1.139114 & 0.000000 & -0.831873 \\ \mathrm{~N} & 0.000000 & 0.000000 & -1.603322 \\ \mathrm{C} & -1.139114 & 0.000000 & -0.831873 \\ \mathrm{C} & -0.714360 & 0.000000 & 0.530385 \\ \mathrm{C} & 0.714360 & 0.000000 & 0.530385 \\ \mathrm{C} & -1.704621 & 0.000000 & 1.519105 \\ \mathrm{C} & -3.055770 & 0.000000 & 1.114956 \\ \mathrm{C} & -3.443561 & 0.000000 & -0.218894 \\ \mathrm{C} & -2.459922 & 0.000000 & -1.237526 \\ \mathrm{C} & 2.459922 & 0.000000 & -1.237526 \\ \mathrm{C} & 3.443561 & 0.000000 & -0.218894 \\ \mathrm{C} & 3.055770 & 0.000000 & 1.114956 \\ \mathrm{C} & 1.704621 & 0.000000 & 1.519105 \\ \mathrm{H} & -2.732883 & 0.000000 & -2.286530 \\ \mathrm{H} & -4.493291 & 0.000000 & -0.481592 \\ \mathrm{H} & -3.823120 & 0.000000 & 1.882039 \\ \mathrm{H} & -1.449482 & 0.000000 & 2.571365 \\ \mathrm{H} & 1.449482 & 0.000000 & 2.571365 \\ \mathrm{H} & 3.823120 & 0.000000 & 1.882039 \\ \mathrm{H} & 4.493291 & 0.000000 & -0.481592 \\ \mathrm{H} & 2.732883 & 0.000000 & -2.286530 \\ \mathrm{H} & 0.000000 & 0.000000 & -2.612175 \\ & & & \end{array}$

tryptamine, A-ph $\mathrm{S}_{0}$

$\begin{array}{lrrc}\mathrm{C} & -2.127038 & 0.135325 & 0.886958 \\ \mathrm{~N} & -1.251803 & 0.124520 & 1.947828 \\ \mathrm{C} & 0.030456 & 0.043190 & 1.472101 \\ \mathrm{C} & -0.050655 & -0.003552 & 0.060600 \\ \mathrm{C} & -1.442020 & 0.057749 & -0.291740 \\ \mathrm{C} & 1.246579 & 0.011221 & 2.148117 \\ \mathrm{C} & 2.397665 & -0.067318 & 1.387520 \\ \mathrm{C} & 2.342357 & -0.109850 & -0.013510 \\ \mathrm{C} & 1.133522 & -0.078368 & -0.680438 \\ \mathrm{C} & -2.016941 & 0.007658 & -1.666771 \\ \mathrm{C} & -1.990148 & -1.389573 & -2.280800 \\ \mathrm{~N} & -2.477186 & -1.351140 & -3.652127\end{array}$




$\begin{array}{rrrr}\mathrm{H} & 1.293381 & 0.047610 & 3.231375 \\ \mathrm{H} & 3.361783 & -0.094096 & 1.882925 \\ \mathrm{H} & 3.266297 & -0.167025 & -0.578001 \\ \mathrm{H} & 1.103829 & -0.107531 & -1.764561 \\ \mathrm{H} & -1.511652 & 0.191754 & 2.914818 \\ \mathrm{H} & -3.191568 & 0.203800 & 1.056179 \\ \mathrm{H} & -1.471270 & 0.682155 & -2.334721 \\ \mathrm{H} & -3.052257 & 0.366661 & -1.643471 \\ \mathrm{H} & -2.550666 & -2.071379 & -1.622022 \\ \mathrm{H} & -0.958065 & -1.751023 & -2.297494 \\ \mathrm{H} & -3.458289 & -1.099112 & -3.674725 \\ \mathrm{H} & -2.393721 & -2.259298 & -4.090669\end{array}$

tryptamine, A-ph $\mathrm{S}_{1}$

$\begin{array}{rrrr}\mathrm{C} & -2.184844 & 0.178720 & 0.923247 \\ \mathrm{~N} & -1.293311 & 0.141690 & 1.914289 \\ \mathrm{C} & 0.025099 & 0.054882 & 1.427634 \\ \mathrm{C} & -0.055422 & 0.047068 & 0.041929 \\ \mathrm{C} & -1.450302 & 0.120742 & -0.299486 \\ \mathrm{C} & 1.218965 & -0.037534 & 2.155866 \\ \mathrm{C} & 2.394659 & -0.130635 & 1.340202 \\ \mathrm{C} & 2.344354 & -0.136656 & -0.033963 \\ \mathrm{C} & 1.115952 & -0.050923 & -0.750027 \\ \mathrm{C} & -2.034698 & 0.052860 & -1.647287 \\ \mathrm{C} & -1.963795 & -1.371685 & -2.235936 \\ \mathrm{~N} & -2.466501 & -1.371568 & -3.595907 \\ \mathrm{H} & 1.260619 & -0.038925 & 3.235876 \\ \mathrm{H} & 3.359318 & -0.199429 & 1.831595 \\ \mathrm{H} & 3.272457 & -0.207054 & -0.590773 \\ \mathrm{H} & 1.086106 & -0.008520 & -1.830241 \\ \mathrm{H} & -1.524178 & 0.189892 & 2.894360 \\ \mathrm{H} & -3.245606 & 0.273034 & 1.091684 \\ \mathrm{H} & -1.479846 & 0.704261 & -2.329525 \\ \mathrm{H} & -3.077544 & 0.386030 & -1.634394 \\ \mathrm{H} & -2.492960 & -2.057320 & -1.557889 \\ \mathrm{H} & -0.915067 & -1.678155 & -2.248379 \\ \mathrm{H} & -3.477517 & -1.330102 & -3.617727 \\ \mathrm{H} & -2.183368 & -2.208291 & -4.087821\end{array}$

tryptamine, A-py $\mathrm{S}_{0}$
C $\quad-2.119623$
$0.112629 \quad 0.863156$
$\begin{array}{llll}\mathrm{N} & -1.255133 & 0.117369 & 1.932428\end{array}$
$\begin{array}{llll}\mathrm{C} & 0.032433 & 0.045153 & 1.470330\end{array}$
$\begin{array}{llll}\text { C } & -0.034037 & -0.012583 & 0.058115\end{array}$ 


$\begin{array}{lrrr}\mathrm{C} & -1.422408 & 0.032341 & -0.308280 \\ \mathrm{C} & 1.241568 & 0.029830 & 2.159292 \\ \mathrm{C} & 2.401657 & -0.042344 & 1.411968 \\ \mathrm{C} & 2.361553 & -0.095558 & 0.010836 \\ \mathrm{C} & 1.159378 & -0.081411 & -0.668561 \\ \mathrm{C} & -1.994954 & -0.024231 & -1.684317 \\ \mathrm{C} & -1.946645 & -1.417009 & -2.306068 \\ \mathrm{~N} & -2.535436 & -1.394539 & -3.637892 \\ \mathrm{H} & 1.276158 & 0.073911 & 3.242719 \\ \mathrm{H} & 3.360577 & -0.056213 & 1.917825 \\ \mathrm{H} & 3.291909 & -0.148511 & -0.543468 \\ \mathrm{H} & 1.142694 & -0.122046 & -1.752614 \\ \mathrm{H} & -1.525585 & 0.187236 & 2.896358 \\ \mathrm{H} & -3.186491 & 0.172491 & 1.020071 \\ \mathrm{H} & -1.452433 & 0.672028 & -2.336897 \\ \mathrm{H} & -3.035744 & 0.310907 & -1.675980 \\ \mathrm{H} & -2.535672 & -2.096707 & -1.683315 \\ \mathrm{H} & -0.910114 & -1.786793 & -2.280662 \\ \mathrm{H} & -1.974831 & -0.827683 & -4.263357 \\ \mathrm{H} & -2.568909 & -2.325878 & -4.032577\end{array}$

tryptamine, A-py $\mathrm{S}_{1}$

$\begin{array}{lrrr}\mathrm{C} & -2.179596 & 0.133778 & 0.886664 \\ \mathrm{~N} & -1.303854 & 0.109399 & 1.892315 \\ \mathrm{C} & 0.023401 & 0.047882 & 1.426050 \\ \mathrm{C} & -0.035491 & 0.042132 & 0.039346 \\ \mathrm{C} & -1.424752 & 0.094891 & -0.323366 \\ \mathrm{C} & 1.208152 & -0.024054 & 2.171949 \\ \mathrm{C} & 2.396866 & -0.096219 & 1.373988 \\ \mathrm{C} & 2.368319 & -0.100640 & -0.001036 \\ \mathrm{C} & 1.149892 & -0.033254 & -0.735526 \\ \mathrm{C} & -1.992550 & 0.026146 & -1.676535 \\ \mathrm{C} & -1.876700 & -1.394822 & -2.274700 \\ \mathrm{~N} & -2.498524 & -1.429032 & -3.580680 \\ \mathrm{H} & 1.234025 & -0.023584 & 3.252430 \\ \mathrm{H} & 3.355076 & -0.149716 & 1.879791 \\ \mathrm{H} & 3.306306 & -0.155055 & -0.542948 \\ \mathrm{H} & 1.136247 & 0.010318 & -1.816040 \\ \mathrm{H} & -1.551460 & 0.149305 & 2.868511 \\ \mathrm{H} & -3.244931 & 0.204925 & 1.036829 \\ \mathrm{H} & -1.447938 & 0.710691 & -2.337333 \\ \mathrm{H} & -3.046489 & 0.313798 & -1.683594 \\ \mathrm{H} & -2.408691 & -2.091348 & -1.620972 \\ \mathrm{H} & -0.815906 & -1.681276 & -2.260198 \\ \mathrm{H} & -1.928035 & -0.963298 & -4.275017\end{array}$




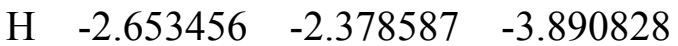

tryptamine, A-up $\mathrm{S}_{0}$

$\begin{array}{rrrr}\mathrm{C} & -2.118769 & 0.126410 & 0.874768 \\ \mathrm{~N} & -1.252009 & 0.103015 & 1.941887 \\ \mathrm{C} & 0.034266 & 0.035033 & 1.475485 \\ \mathrm{C} & -0.035055 & 0.009751 & 0.062655 \\ \mathrm{C} & -1.423895 & 0.071931 & -0.299879 \\ \mathrm{C} & 1.244657 & -0.002435 & 2.161370 \\ \mathrm{C} & 2.402615 & -0.064691 & 1.409955 \\ \mathrm{C} & 2.359504 & -0.086910 & 0.008093 \\ \mathrm{C} & 1.156199 & -0.050080 & -0.668328 \\ \mathrm{C} & -1.992389 & 0.032902 & -1.678010 \\ \mathrm{C} & -1.960934 & -1.363563 & -2.315382 \\ \mathrm{~N} & -2.470538 & -1.448919 & -3.672360 \\ \mathrm{H} & 1.281785 & 0.016904 & 3.245410 \\ \mathrm{H} & 3.362476 & -0.095767 & 1.913224 \\ \mathrm{H} & 3.288336 & -0.134022 & -0.549198 \\ \mathrm{H} & 1.137503 & -0.067574 & -1.752967 \\ \mathrm{H} & -1.520141 & 0.149027 & 2.907918 \\ \mathrm{H} & -3.184909 & 0.187009 & 1.036581 \\ \mathrm{H} & -1.442778 & 0.726100 & -2.327203 \\ \mathrm{H} & -3.029069 & 0.387496 & -1.659043 \\ \mathrm{H} & -2.535173 & -2.051557 & -1.687455 \\ \mathrm{H} & -0.934641 & -1.741928 & -2.311056 \\ \mathrm{H} & -1.934115 & -0.858178 & -4.296323 \\ \mathrm{H} & -3.431685 & -1.132043 & -3.716901\end{array}$

tryptamine, A-up $\mathrm{S}_{1}$

$\begin{array}{rrrr}\mathrm{C} & -2.175326 & 0.125327 & 0.890343 \\ \mathrm{~N} & -1.295596 & 0.076615 & 1.894494 \\ \mathrm{C} & 0.029234 & 0.031325 & 1.426742 \\ \mathrm{C} & -0.030504 & 0.063287 & 0.039985 \\ \mathrm{C} & -1.421543 & 0.135431 & -0.318601 \\ \mathrm{C} & 1.215795 & -0.054789 & 2.169655 \\ \mathrm{C} & 2.403619 & -0.104616 & 1.371045 \\ \mathrm{C} & 2.373304 & -0.075733 & -0.003640 \\ \mathrm{C} & 1.154779 & 0.007277 & -0.735926 \\ \mathrm{C} & -1.978141 & 0.102900 & -1.669202 \\ \mathrm{C} & -1.946844 & -1.358680 & -2.254953 \\ \mathrm{~N} & -2.440781 & -1.478601 & -3.594050 \\ \mathrm{H} & 1.241613 & -0.075621 & 3.250009 \\ \mathrm{H} & 3.362517 & -0.169290 & 1.874217 \\ \mathrm{H} & 3.310892 & -0.116788 & -0.547683\end{array}$




$\begin{array}{lrrr}\mathrm{H} & 1.142156 & 0.062759 & -1.816042 \\ \mathrm{H} & -1.541273 & 0.094531 & 2.871665 \\ \mathrm{H} & -3.239643 & 0.196058 & 1.048001 \\ \mathrm{H} & -1.381742 & 0.727237 & -2.341281 \\ \mathrm{H} & -3.012656 & 0.458389 & -1.686952 \\ \mathrm{H} & -2.534549 & -2.004056 & -1.599110 \\ \mathrm{H} & -0.912558 & -1.706846 & -2.214502 \\ \mathrm{H} & -1.902773 & -0.948125 & -4.265488 \\ \mathrm{H} & -3.418758 & -1.240071 & -3.685506\end{array}$

tryptamine, $\mathrm{Ph}$-out $\mathrm{S}_{0}$

$\begin{array}{lrrr}\mathrm{C} & 0.006192 & 0.001563 & 0.075229 \\ \mathrm{C} & 0.045212 & 0.060623 & 1.488777 \\ \mathrm{C} & 1.240979 & 0.073310 & 2.201076 \\ \mathrm{C} & 2.415768 & 0.030209 & 1.475205 \\ \mathrm{C} & 2.402467 & -0.025783 & 0.073301 \\ \mathrm{C} & 1.214839 & -0.039773 & -0.631046 \\ \mathrm{~N} & -1.251431 & 0.101814 & 1.928009 \\ \mathrm{H} & 1.253810 & 0.117301 & 3.284996 \\ \mathrm{H} & 3.365366 & 0.039457 & 1.998576 \\ \mathrm{H} & 3.344117 & -0.057919 & -0.463326 \\ \mathrm{H} & 1.212213 & -0.098665 & -1.713764 \\ \mathrm{C} & -1.377550 & 0.014612 & -0.316317 \\ \mathrm{C} & -2.096587 & 0.080400 & 0.844262 \\ \mathrm{H} & -1.539500 & 0.174157 & 2.886725 \\ \mathrm{H} & -3.166736 & 0.124275 & 0.983870 \\ \mathrm{C} & -1.935121 & -0.042366 & -1.701909 \\ \mathrm{H} & -1.296448 & 0.531343 & -2.381110 \\ \mathrm{C} & -2.082902 & -1.456069 & -2.260549 \\ \mathrm{H} & -2.920125 & 0.434978 & -1.712161 \\ \mathrm{H} & -2.547112 & -1.388951 & -3.249965 \\ \mathrm{~N} & -0.787918 & -2.098720 & -2.416247 \\ \mathrm{H} & -2.780600 & -2.013391 & -1.613916 \\ \mathrm{H} & -0.344047 & -2.220496 & -1.513188 \\ \mathrm{H} & -0.889912 & -3.017800 & -2.827317\end{array}$

tryptamine, $\mathrm{Ph}$-out $\mathrm{S}_{1}$

$\begin{array}{lrrr}\text { C } & 0.000351 & 0.030168 & 0.065029 \\ \mathrm{C} & 0.030876 & 0.089942 & 1.452752 \\ \mathrm{C} & 1.197674 & 0.042891 & 2.227377 \\ \mathrm{C} & 2.405896 & -0.043848 & 1.460823 \\ \mathrm{C} & 2.407495 & -0.103329 & 0.087401 \\ \mathrm{C} & 1.205321 & -0.083140 & -0.677600 \\ \mathrm{~N} & -1.303629 & 0.166935 & 1.891082\end{array}$




$\begin{array}{lrrr}\mathrm{H} & 1.198445 & 0.097451 & 3.306866 \\ \mathrm{H} & 3.352560 & -0.065611 & 1.990090 \\ \mathrm{H} & 3.357077 & -0.168077 & -0.432586 \\ \mathrm{H} & 1.215494 & -0.061998 & -1.758525 \\ \mathrm{C} & -1.383117 & 0.065815 & -0.327157 \\ \mathrm{C} & -2.159234 & 0.152119 & 0.869704 \\ \mathrm{H} & -1.568856 & 0.246556 & 2.860346 \\ \mathrm{H} & -3.226984 & 0.231492 & 0.997547 \\ \mathrm{C} & -1.959855 & -0.048626 & -1.678585 \\ \mathrm{H} & -1.345573 & 0.499082 & -2.399156 \\ \mathrm{C} & -2.051747 & -1.509975 & -2.171756 \\ \mathrm{H} & -2.968084 & 0.376292 & -1.683456 \\ \mathrm{H} & -2.682036 & -1.508163 & -3.066493 \\ \mathrm{~N} & -0.748408 & -2.016988 & -2.533972 \\ \mathrm{H} & -2.581195 & -2.096329 & -1.403723 \\ \mathrm{H} & -0.101908 & -2.005158 & -1.750733 \\ \mathrm{H} & -0.805571 & -2.963381 & -2.886065\end{array}$

tryptamine, $\mathrm{Ph}$-up $\mathrm{S}_{0}$

$\begin{array}{lrrr}\mathrm{C} & -2.073107 & 0.018643 & 0.813707 \\ \mathrm{~N} & -1.240183 & 0.010143 & 1.906798 \\ \mathrm{C} & 0.061424 & 0.030657 & 1.482439 \\ \mathrm{C} & 0.037797 & 0.046326 & 0.067344 \\ \mathrm{C} & -1.339867 & 0.037129 & -0.340193 \\ \mathrm{C} & 1.249161 & 0.041773 & 2.208036 \\ \mathrm{C} & 2.432224 & 0.073403 & 1.495400 \\ \mathrm{C} & 2.435252 & 0.095016 & 0.092466 \\ \mathrm{C} & 1.254767 & 0.082008 & -0.623302 \\ \mathrm{C} & -1.872759 & 0.011479 & -1.735219 \\ \mathrm{C} & -2.117131 & -1.404383 & -2.280144 \\ \mathrm{~N} & -0.962224 & -2.277196 & -2.365482 \\ \mathrm{H} & 1.249668 & 0.028213 & 3.292782 \\ \mathrm{H} & 3.375604 & 0.083260 & 2.029624 \\ \mathrm{H} & 3.382697 & 0.124261 & -0.433876 \\ \mathrm{H} & 1.271485 & 0.102053 & -1.707809 \\ \mathrm{H} & -1.540981 & 0.015351 & 2.864413 \\ \mathrm{H} & -3.145347 & 0.017583 & 0.943694 \\ \mathrm{H} & -1.177299 & 0.534535 & -2.401703 \\ \mathrm{H} & -2.815897 & 0.567826 & -1.780090 \\ \mathrm{H} & -2.559569 & -1.326783 & -3.278939 \\ \mathrm{H} & -2.865013 & -1.897293 & -1.650218 \\ \mathrm{H} & -0.519288 & -2.369617 & -1.458700 \\ \mathrm{H} & -0.267764 & -1.894758 & -2.995967\end{array}$

tryptamine, $\mathrm{Ph}$-up $\mathrm{S}_{1}$ 


$\begin{array}{lrrr}\mathrm{C} & -2.171070 & 0.178957 & 0.863687 \\ \mathrm{~N} & -1.313446 & 0.162608 & 1.891094 \\ \mathrm{C} & 0.016225 & 0.093813 & 1.449504 \\ \mathrm{C} & -0.017176 & 0.088657 & 0.059335 \\ \mathrm{C} & -1.399059 & 0.136084 & -0.326889 \\ \mathrm{C} & 1.185264 & -0.006802 & 2.216524 \\ \mathrm{C} & 2.389664 & -0.091604 & 1.448311 \\ \mathrm{C} & 2.390217 & -0.098251 & 0.073342 \\ \mathrm{C} & 1.189259 & -0.026262 & -0.690434 \\ \mathrm{C} & -1.951807 & 0.005582 & -1.670096 \\ \mathrm{C} & -1.960327 & -1.507566 & -2.161612 \\ \mathrm{~N} & -0.688735 & -2.070564 & -2.443589 \\ \mathrm{H} & 1.187621 & 0.004657 & 3.297479 \\ \mathrm{H} & 3.336156 & -0.154872 & 1.974687 \\ \mathrm{H} & 3.339783 & -0.156253 & -0.448303 \\ \mathrm{H} & 1.215307 & 0.139119 & -1.759881 \\ \mathrm{H} & -1.578016 & 0.225303 & 2.860832 \\ \mathrm{H} & -3.238279 & 0.261509 & 0.996552 \\ \mathrm{H} & -1.370198 & 0.570689 & -2.403040 \\ \mathrm{H} & -2.989810 & 0.350657 & -1.702033 \\ \mathrm{H} & -2.576497 & -1.533709 & -3.064815 \\ \mathrm{H} & -2.464562 & -2.097721 & -1.394582 \\ \mathrm{H} & -0.036609 & -2.053066 & -1.664884 \\ \mathrm{H} & -0.240246 & -1.671354 & -3.256096\end{array}$

tryptamine, Py-out $\mathrm{S}_{0}$

$\begin{array}{lrrr}\mathrm{C} & 0.516560 & 0.452942 & 0.216189 \\ \mathrm{C} & 0.591089 & 0.524095 & 1.627476 \\ \mathrm{C} & 1.802349 & 0.483149 & 2.311531 \\ \mathrm{C} & 2.956463 & 0.370393 & 1.559906 \\ \mathrm{C} & 2.908476 & 0.302817 & 0.159714 \\ \mathrm{C} & 1.704227 & 0.343411 & -0.515165 \\ \mathrm{~N} & -0.692036 & 0.635126 & 2.095070 \\ \mathrm{H} & 1.843160 & 0.539234 & 3.394173 \\ \mathrm{H} & 3.916990 & 0.335735 & 2.061695 \\ \mathrm{H} & 3.834519 & 0.218599 & -0.397897 \\ \mathrm{H} & 1.681215 & 0.292624 & -1.598532 \\ \mathrm{C} & -0.872498 & 0.532939 & -0.144301 \\ \mathrm{C} & -1.562180 & 0.640881 & 1.030313 \\ \mathrm{H} & -0.955559 & 0.721084 & 3.059699 \\ \mathrm{H} & -2.626902 & 0.726418 & 1.188997 \\ \mathrm{C} & -1.452544 & 0.471534 & -1.518367 \\ \mathrm{H} & -0.894996 & 1.137684 & -2.185880 \\ \mathrm{C} & -1.449583 & -0.935108 & -2.114065\end{array}$




$\begin{array}{rrrr}\mathrm{H} & -2.485390 & 0.830572 & -1.493849 \\ \mathrm{H} & -0.407606 & -1.279560 & -2.210744 \\ \mathrm{~N} & -2.285968 & -1.820471 & -1.319502 \\ \mathrm{H} & -1.865812 & -0.885750 & -3.125164 \\ \mathrm{H} & -2.286996 & -2.756616 & -1.704035 \\ \mathrm{H} & -1.916959 & -1.881734 & -0.377244\end{array}$

tryptamine, Py-out $\mathrm{S}_{1}$

$\begin{array}{lrrr}\mathrm{C} & 0.516702 & 0.492750 & 0.206090 \\ \mathrm{C} & 0.592536 & 0.543025 & 1.590858 \\ \mathrm{C} & 1.783418 & 0.461753 & 2.326833 \\ \mathrm{C} & 2.960767 & 0.335684 & 1.518657 \\ \mathrm{C} & 2.915655 & 0.287746 & 0.145242 \\ \mathrm{C} & 1.690228 & 0.359921 & -0.578408 \\ \mathrm{~N} & -0.727002 & 0.654524 & 2.070476 \\ \mathrm{H} & 1.822209 & 0.506489 & 3.405991 \\ \mathrm{H} & 3.923379 & 0.275507 & 2.015261 \\ \mathrm{H} & 3.845408 & 0.193001 & -0.405223 \\ \mathrm{H} & 1.665649 & 0.365857 & -1.659434 \\ \mathrm{C} & -0.877240 & 0.572662 & -0.142043 \\ \mathrm{C} & -1.614840 & 0.668843 & 1.075632 \\ \mathrm{H} & -0.961664 & 0.722714 & 3.048420 \\ \mathrm{H} & -2.677571 & 0.756553 & 1.234333 \\ \mathrm{C} & -1.459508 & 0.493016 & -1.489773 \\ \mathrm{H} & -0.887565 & 1.138663 & -2.165515 \\ \mathrm{C} & -1.403172 & -0.939726 & -2.062892 \\ \mathrm{H} & -2.501671 & 0.821715 & -1.483920 \\ \mathrm{H} & -0.347690 & -1.243716 & -2.099646 \\ \mathrm{~N} & -2.254933 & -1.829356 & -1.299891 \\ \mathrm{H} & -1.777526 & -0.902830 & -3.088982 \\ \mathrm{H} & -2.370728 & -2.715426 & -1.773853 \\ \mathrm{H} & -1.854850 & -2.019379 & -0.388222\end{array}$

tryptamine, Py-up $\mathrm{S}_{0}$

$\begin{array}{lrrr}\mathrm{C} & -2.117453 & 0.242093 & 0.888958 \\ \mathrm{~N} & -1.245278 & 0.219145 & 1.952039 \\ \mathrm{C} & 0.034949 & 0.092457 & 1.480546 \\ \mathrm{C} & -0.043716 & 0.030067 & 0.069432 \\ \mathrm{C} & -1.432262 & 0.130669 & -0.288081 \\ \mathrm{C} & 1.247151 & 0.031012 & 2.161474 \\ \mathrm{C} & 2.397434 & -0.094478 & 1.406248 \\ \mathrm{C} & 2.345184 & -0.154799 & 0.005859 \\ \mathrm{C} & 1.140151 & -0.093781 & -0.665728 \\ \mathrm{C} & -2.008804 & 0.075628 & -1.661727\end{array}$




$\begin{array}{lrrc}\mathrm{C} & -2.010026 & -1.337182 & -2.268754 \\ \mathrm{~N} & -2.788037 & -2.333449 & -1.558357 \\ \mathrm{H} & 1.291406 & 0.080435 & 3.244269 \\ \mathrm{H} & 3.358585 & -0.145764 & 1.905361 \\ \mathrm{H} & 3.268431 & -0.250612 & -0.554392 \\ \mathrm{H} & 1.114268 & -0.139541 & -1.749262 \\ \mathrm{H} & -1.504789 & 0.305096 & 2.917728 \\ \mathrm{H} & -3.179310 & 0.350814 & 1.053621 \\ \mathrm{H} & -1.449555 & 0.744711 & -2.327126 \\ \mathrm{H} & -3.038466 & 0.450339 & -1.640332 \\ \mathrm{H} & -0.980390 & -1.701418 & -2.336836 \\ \mathrm{H} & -2.383890 & -1.279555 & -3.295956 \\ \mathrm{H} & -3.763800 & -2.062469 & -1.518867 \\ \mathrm{H} & -2.463264 & -2.409800 & -0.601228\end{array}$

tryptamine, Py-up $\mathrm{S}_{1}$

$\begin{array}{lrrr}\mathrm{C} & -2.164526 & 0.280184 & 0.960026 \\ \mathrm{~N} & -1.262872 & 0.263987 & 1.946069 \\ \mathrm{C} & 0.047187 & 0.129405 & 1.454045 \\ \mathrm{C} & -0.044793 & 0.071462 & 0.069664 \\ \mathrm{C} & -1.442378 & 0.169404 & -0.263446 \\ \mathrm{C} & 1.244119 & 0.033804 & 2.177559 \\ \mathrm{C} & 2.411350 & -0.121371 & 1.360733 \\ \mathrm{C} & 2.349913 & -0.179027 & -0.011420 \\ \mathrm{C} & 1.119259 & -0.089301 & -0.723253 \\ \mathrm{C} & -2.028976 & 0.090319 & -1.599131 \\ \mathrm{C} & -1.958639 & -1.353466 & -2.220971 \\ \mathrm{~N} & -2.793526 & -2.351631 & -1.627252 \\ \mathrm{H} & 1.293051 & 0.083536 & 3.256195 \\ \mathrm{H} & 3.377386 & -0.197891 & 1.848132 \\ \mathrm{H} & 3.271452 & -0.296647 & -0.571401 \\ \mathrm{H} & 1.086996 & -0.091980 & -1.804258 \\ \mathrm{H} & -1.483484 & 0.356757 & 2.925150 \\ \mathrm{H} & -3.220131 & 0.414088 & 1.134549 \\ \mathrm{H} & -1.459806 & 0.731712 & -2.282606 \\ \mathrm{H} & -3.071211 & 0.423167 & -1.600804 \\ \mathrm{H} & -0.917754 & -1.681107 & -2.172702 \\ \mathrm{H} & -2.226612 & -1.253750 & -3.275972 \\ \mathrm{H} & -3.779626 & -2.141607 & -1.697272 \\ \mathrm{H} & -2.557858 & -2.540415 & -0.662721\end{array}$

porphycene $\mathrm{S}_{0}$
C -3.423792
$1.906944 \quad 0.000000$
C -2.407499
$0.908372 \quad 0.000000$ 


$\begin{array}{rrrr}\mathrm{N} & -1.214753 & 1.547662 & 0.000000 \\ \mathrm{C} & -1.390882 & 2.893911 & 0.000000 \\ \mathrm{C} & -2.799895 & 3.126509 & 0.000000 \\ \mathrm{C} & -0.367241 & 3.848742 & 0.000000 \\ \mathrm{C} & 1.010099 & 3.698220 & 0.000000 \\ \mathrm{C} & 1.824965 & 2.554115 & 0.000000 \\ \mathrm{H} & -0.243845 & 1.141892 & 0.000000 \\ \mathrm{~N} & 1.403542 & 1.268318 & 0.000000 \\ \mathrm{C} & 3.271531 & 2.592042 & 0.000000 \\ \mathrm{C} & 3.696145 & 1.301484 & 0.000000 \\ \mathrm{C} & 2.501962 & 0.494063 & 0.000000 \\ \mathrm{C} & 2.407499 & -0.908372 & 0.000000 \\ \mathrm{C} & 3.423792 & -1.906944 & 0.000000 \\ \mathrm{C} & 2.799895 & -3.126509 & 0.000000 \\ \mathrm{C} & 1.390882 & -2.893911 & 0.000000 \\ \mathrm{~N} & 1.214753 & -1.547662 & 0.000000 \\ \mathrm{C} & 0.367241 & -3.848742 & 0.000000 \\ \mathrm{C} & -1.010099 & -3.698220 & 0.000000 \\ \mathrm{C} & -1.824965 & -2.554115 & 0.000000 \\ \mathrm{C} & -3.271531 & -2.592042 & 0.000000 \\ \mathrm{C} & -3.696145 & -1.301484 & 0.000000 \\ \mathrm{C} & -2.501962 & -0.494063 & 0.000000 \\ \mathrm{~N} & -1.403542 & -1.268318 & 0.000000 \\ \mathrm{H} & 0.243845 & -1.141892 & 0.000000 \\ \mathrm{H} & 3.264724 & -4.101300 & 0.000000 \\ \mathrm{H} & 4.485888 & -1.715051 & 0.000000 \\ \mathrm{H} & 0.726390 & -4.873137 & 0.000000 \\ \mathrm{H} & -1.568963 & -4.629549 & 0.000000 \\ \mathrm{H} & -3.876448 & -3.487833 & 0.000000 \\ \mathrm{H} & -4.713554 & -0.938627 & 0.000000 \\ \mathrm{H} & -4.485888 & 1.715051 & 0.000000 \\ \mathrm{H} & -3.264724 & 4.101300 & 0.000000 \\ \mathrm{H} & -0.726390 & 4.873137 & 0.000000 \\ \mathrm{H} & 1.568963 & 4.629549 & 0.000000 \\ \mathrm{H} & 3.876448 & 3.487833 & 0.000000 \\ \mathrm{H} & 4.713554 & 0.938627 & 0.000000\end{array}$

porphycene $S_{1}$

$\begin{array}{lrrr}\mathrm{C} & -3.445589 & 1.894645 & 0.000000 \\ \mathrm{C} & -2.433354 & 0.898415 & 0.000000 \\ \mathrm{~N} & -1.229934 & 1.544740 & 0.000000 \\ \mathrm{C} & -1.411247 & 2.885191 & 0.000000 \\ \mathrm{C} & -2.819483 & 3.117317 & 0.000000 \\ \mathrm{C} & -0.381315 & 3.854182 & 0.000000 \\ \mathrm{C} & 0.986529 & 3.710887 & 0.000000\end{array}$




$\begin{array}{rrrr}\text { C } & 1.829189 & 2.563958 & 0.000000 \\ \mathrm{H} & -0.264090 & 1.138806 & 0.000000 \\ \mathrm{~N} & 1.411039 & 1.281163 & 0.000000 \\ \mathrm{C} & 3.266870 & 2.610725 & 0.000000 \\ \mathrm{C} & 3.705644 & 1.315253 & 0.000000 \\ \mathrm{C} & 2.531935 & 0.503813 & 0.000000 \\ \mathrm{C} & 2.433354 & -0.898415 & 0.000000 \\ \mathrm{C} & 3.445589 & -1.894645 & 0.000000 \\ \mathrm{C} & 2.819483 & -3.117317 & 0.000000 \\ \mathrm{C} & 1.411247 & -2.885191 & 0.000000 \\ \mathrm{~N} & 1.229934 & -1.544740 & 0.000000 \\ \mathrm{C} & 0.381315 & -3.854182 & 0.000000 \\ \mathrm{C} & -0.986529 & -3.710887 & 0.000000 \\ \mathrm{C} & -1.829189 & -2.563958 & 0.000000 \\ \mathrm{C} & -3.266870 & -2.610725 & 0.000000 \\ \mathrm{C} & -3.705644 & -1.315253 & 0.000000 \\ \mathrm{C} & -2.531935 & -0.503813 & 0.000000 \\ \mathrm{~N} & -1.411039 & -1.281163 & 0.000000 \\ \mathrm{H} & 0.264090 & -1.138806 & 0.000000 \\ \mathrm{H} & 3.283420 & -4.092492 & 0.000000 \\ \mathrm{H} & 4.507500 & -1.702683 & 0.000000 \\ \mathrm{H} & 0.747729 & -4.875621 & 0.000000 \\ \mathrm{H} & -1.540379 & -4.645648 & 0.000000 \\ \mathrm{H} & -3.867112 & -3.509689 & 0.000000 \\ \mathrm{H} & -4.726730 & -0.964217 & 0.000000 \\ \mathrm{H} & -4.507500 & 1.702683 & 0.000000 \\ \mathrm{H} & -3.283420 & 4.092492 & 0.000000 \\ \mathrm{H} & -0.747729 & 4.875621 & 0.000000 \\ \mathrm{H} & 1.540379 & 4.645648 & 0.000000 \\ \mathrm{H} & 3.867112 & 3.509689 & 0.000000 \\ \mathrm{H} & 4.726730 & 0.964217 & 0.000000\end{array}$

porphine $\mathrm{S}_{0}$

$\begin{array}{rrrr}\mathrm{N} & 0.000000 & -2.016480 & 0.000000 \\ \mathrm{~N} & 2.103984 & 0.000000 & 0.000000 \\ \mathrm{C} & 1.077905 & -2.836149 & 0.000000 \\ \mathrm{C} & 0.674788 & -4.231517 & 0.000000 \\ \mathrm{C} & 2.408516 & -2.425812 & 0.000000 \\ \mathrm{C} & 2.877898 & -1.121493 & 0.000000 \\ \mathrm{C} & 4.236666 & -0.682365 & 0.000000 \\ \mathrm{H} & 1.346608 & -5.078384 & 0.000000 \\ \mathrm{H} & 3.164256 & -3.203168 & 0.000000 \\ \mathrm{H} & 5.091158 & -1.342467 & 0.000000 \\ \mathrm{H} & 1.090982 & 0.000000 & 0.000000 \\ \mathrm{~N} & 0.000000 & 2.016480 & 0.000000\end{array}$




$\begin{array}{rrrr}\mathrm{N} & -2.103984 & 0.000000 & 0.000000 \\ \mathrm{C} & 1.077905 & 2.836149 & 0.000000 \\ \mathrm{C} & -1.077905 & 2.836149 & 0.000000 \\ \mathrm{C} & -1.077905 & -2.836149 & 0.000000 \\ \mathrm{C} & 0.674788 & 4.231517 & 0.000000 \\ \mathrm{C} & -0.674788 & 4.231517 & 0.000000 \\ \mathrm{C} & -0.674788 & -4.231517 & 0.000000 \\ \mathrm{C} & 2.408516 & 2.425812 & 0.000000 \\ \mathrm{C} & -2.408516 & 2.425812 & 0.000000 \\ \mathrm{C} & -2.408516 & -2.425812 & 0.000000 \\ \mathrm{C} & 2.877898 & 1.121493 & 0.000000 \\ \mathrm{C} & -2.877898 & 1.121493 & 0.000000 \\ \mathrm{C} & -2.877898 & -1.121493 & 0.000000 \\ \mathrm{C} & 4.236666 & 0.682365 & 0.000000 \\ \mathrm{C} & -4.236666 & 0.682365 & 0.000000 \\ \mathrm{C} & -4.236666 & -0.682365 & 0.000000 \\ \mathrm{H} & 1.346608 & 5.078384 & 0.000000 \\ \mathrm{H} & -1.346608 & 5.078384 & 0.000000 \\ \mathrm{H} & -1.346608 & -5.078384 & 0.000000 \\ \mathrm{H} & 3.164256 & 3.203168 & 0.000000 \\ \mathrm{H} & -3.164256 & 3.203168 & 0.000000 \\ \mathrm{H} & -3.164256 & -3.203168 & 0.000000 \\ \mathrm{H} & 5.091158 & 1.342467 & 0.000000 \\ \mathrm{H} & -5.091158 & 1.342467 & 0.000000 \\ \mathrm{H} & -5.091158 & -1.342467 & 0.000000 \\ \mathrm{H} & -1.090982 & 0.000000 & 0.000000\end{array}$

porphine $\mathrm{S}_{1}$

$\begin{array}{rrrr}\mathrm{N} & 0.000000 & -2.037392 & 0.000000 \\ \mathrm{~N} & 2.101308 & 0.000000 & 0.000000 \\ \mathrm{C} & 1.081358 & -2.861417 & 0.000000 \\ \mathrm{C} & 0.677408 & -4.250920 & 0.000000 \\ \mathrm{C} & 2.413961 & -2.433311 & 0.000000 \\ \mathrm{C} & 2.878604 & -1.123997 & 0.000000 \\ \mathrm{C} & 4.237106 & -0.683835 & 0.000000 \\ \mathrm{H} & 1.347327 & -5.098959 & 0.000000 \\ \mathrm{H} & 3.175616 & -3.204897 & 0.000000 \\ \mathrm{H} & 5.092100 & -1.343107 & 0.000000 \\ \mathrm{H} & 1.089910 & 0.000000 & 0.000000 \\ \mathrm{~N} & 0.000000 & 2.037392 & 0.000000 \\ \mathrm{~N} & -2.101308 & 0.000000 & 0.000000 \\ \mathrm{C} & 1.081358 & 2.861417 & 0.000000 \\ \mathrm{C} & -1.081358 & 2.861417 & 0.000000 \\ \mathrm{C} & -1.081358 & -2.861417 & 0.000000 \\ \mathrm{C} & 0.677408 & 4.250920 & 0.000000\end{array}$




$\begin{array}{rrrr}\mathrm{C} & -0.677408 & 4.250920 & 0.000000 \\ \mathrm{C} & -0.677408 & -4.250920 & 0.000000 \\ \mathrm{C} & 2.413961 & 2.433311 & 0.000000 \\ \mathrm{C} & -2.413961 & 2.433311 & 0.000000 \\ \mathrm{C} & -2.413961 & -2.433311 & 0.000000 \\ \mathrm{C} & 2.878604 & 1.123997 & 0.000000 \\ \mathrm{C} & -2.878604 & 1.123997 & 0.000000 \\ \mathrm{C} & -2.878604 & -1.123997 & 0.000000 \\ \mathrm{C} & 4.237106 & 0.683835 & 0.000000 \\ \mathrm{C} & -4.237106 & 0.683835 & 0.000000 \\ \mathrm{C} & -4.237106 & -0.683835 & 0.000000 \\ \mathrm{H} & 1.347327 & 5.098959 & 0.000000 \\ \mathrm{H} & -1.347327 & 5.098959 & 0.000000 \\ \mathrm{H} & -1.347327 & -5.098959 & 0.000000 \\ \mathrm{H} & 3.175616 & 3.204897 & 0.000000 \\ \mathrm{H} & -3.175616 & 3.204897 & 0.000000 \\ \mathrm{H} & -3.175616 & -3.204897 & 0.000000 \\ \mathrm{H} & 5.092100 & 1.343107 & 0.000000 \\ \mathrm{H} & -5.092100 & 1.343107 & 0.000000 \\ \mathrm{H} & -5.092100 & -1.343107 & 0.000000 \\ \mathrm{H} & -1.089910 & 0.000000 & 0.000000\end{array}$

chlorin $\mathrm{S}_{0}$

$\begin{array}{rrrr}\mathrm{C} & 0.000000 & -4.229941 & 0.921136 \\ \mathrm{C} & 0.000000 & -2.877953 & 1.370182 \\ \mathrm{~N} & 0.000000 & -2.094379 & 0.244080 \\ \mathrm{C} & 0.000000 & -2.858814 & -0.876594 \\ \mathrm{C} & 0.000000 & -4.219962 & -0.444860 \\ \mathrm{C} & 0.000000 & -2.411340 & 2.669160 \\ \mathrm{C} & 0.000000 & -1.078173 & 3.087409 \\ \mathrm{~N} & 0.000000 & 0.000000 & 2.266546 \\ \mathrm{C} & 0.000000 & 1.078173 & 3.087409 \\ \mathrm{C} & 0.000000 & 0.677231 & 4.475939 \\ \mathrm{C} & 0.000000 & -0.677231 & 4.475939 \\ \mathrm{C} & 0.000000 & 2.411340 & 2.669160 \\ \mathrm{C} & 0.000000 & 2.877953 & 1.370182 \\ \mathrm{~N} & 0.000000 & 2.094379 & 0.244080 \\ \mathrm{C} & 0.000000 & 2.858814 & -0.876594 \\ \mathrm{C} & 0.000000 & 4.219962 & -0.444860 \\ \mathrm{C} & 0.000000 & 4.229941 & 0.921136 \\ \mathrm{C} & 0.000000 & 2.402247 & -2.194781 \\ \mathrm{C} & 0.000000 & 1.096064 & -2.644952 \\ \mathrm{C} & 0.000000 & 0.763222 & -4.123749 \\ \mathrm{C} & 0.000000 & -0.763222 & -4.123749 \\ \mathrm{C} & 0.000000 & -1.096064 & -2.644952\end{array}$




$\begin{array}{rrrr}\mathrm{N} & 0.000000 & 0.000000 & -1.868955 \\ \mathrm{C} & 0.000000 & -2.402247 & -2.194781 \\ \mathrm{H} & -0.878961 & 1.187160 & -4.615607 \\ \mathrm{H} & 0.000000 & 3.179153 & -2.951417 \\ \mathrm{H} & 0.000000 & 5.069475 & -1.111223 \\ \mathrm{H} & 0.000000 & 1.347730 & 5.323915 \\ \mathrm{H} & 0.000000 & -1.347730 & 5.323915 \\ \mathrm{H} & -0.878961 & -1.187160 & -4.615607 \\ \mathrm{H} & 0.000000 & 3.168545 & 3.445606 \\ \mathrm{H} & 0.000000 & -3.168545 & 3.445606 \\ \mathrm{H} & 0.000000 & -3.179153 & -2.951417 \\ \mathrm{H} & 0.000000 & 5.089927 & 1.574267 \\ \mathrm{H} & 0.000000 & -5.089927 & 1.574267 \\ \mathrm{H} & 0.000000 & -5.069475 & -1.111223 \\ \mathrm{H} & 0.878961 & -1.187160 & -4.615607 \\ \mathrm{H} & 0.878961 & 1.187160 & -4.615607 \\ \mathrm{H} & 0.000000 & -1.083636 & 0.268296 \\ \mathrm{H} & 0.000000 & 1.083636 & 0.268296\end{array}$

chlorin $\mathrm{S}_{1}$

$\begin{array}{lrrr}\mathrm{C} & 0.000000 & -4.239184 & 0.921465 \\ \mathrm{C} & 0.000000 & -2.885201 & 1.367273 \\ \mathrm{~N} & 0.000000 & -2.102457 & 0.242318 \\ \mathrm{C} & 0.000000 & -2.873779 & -0.882447 \\ \mathrm{C} & 0.000000 & -4.231592 & -0.449217 \\ \mathrm{C} & 0.000000 & -2.415830 & 2.675329 \\ \mathrm{C} & 0.000000 & -1.080958 & 3.088295 \\ \mathrm{~N} & 0.000000 & 0.000000 & 2.265272 \\ \mathrm{C} & 0.000000 & 1.080958 & 3.088295 \\ \mathrm{C} & 0.000000 & 0.675223 & 4.483261 \\ \mathrm{C} & 0.000000 & -0.675223 & 4.483261 \\ \mathrm{C} & 0.000000 & 2.415830 & 2.675329 \\ \mathrm{C} & 0.000000 & 2.885201 & 1.367273 \\ \mathrm{~N} & 0.000000 & 2.102457 & 0.242318 \\ \mathrm{C} & 0.000000 & 2.873779 & -0.882447 \\ \mathrm{C} & 0.000000 & 4.231592 & -0.449217 \\ \mathrm{C} & 0.000000 & 4.239184 & 0.921465 \\ \mathrm{C} & 0.000000 & 2.411874 & -2.202303 \\ \mathrm{C} & 0.000000 & 1.097482 & -2.645443 \\ \mathrm{C} & 0.000000 & 0.762012 & -4.118867 \\ \mathrm{C} & 0.000000 & -0.762012 & -4.118867 \\ \mathrm{C} & 0.000000 & -1.097482 & -2.645443 \\ \mathrm{~N} & 0.000000 & 0.000000 & -1.861130 \\ \mathrm{C} & 0.000000 & -2.411874 & -2.202303 \\ \mathrm{H} & -0.878271 & 1.187276 & -4.612777\end{array}$




$\begin{array}{rrrc}\mathrm{H} & 0.000000 & 3.183568 & -2.963820 \\ \mathrm{H} & 0.000000 & 5.082846 & -1.113333 \\ \mathrm{H} & 0.000000 & 1.347007 & 5.329999 \\ \mathrm{H} & 0.000000 & -1.347007 & 5.329999 \\ \mathrm{H} & -0.878271 & -1.187276 & -4.612777 \\ \mathrm{H} & 0.000000 & 3.171603 & 3.452238 \\ \mathrm{H} & 0.000000 & -3.171603 & 3.452238 \\ \mathrm{H} & 0.000000 & -3.183568 & -2.963820 \\ \mathrm{H} & 0.000000 & 5.097523 & 1.576430 \\ \mathrm{H} & 0.000000 & -5.097523 & 1.576430 \\ \mathrm{H} & 0.000000 & -5.082846 & -1.113333 \\ \mathrm{H} & 0.878271 & -1.187276 & -4.612777 \\ \mathrm{H} & 0.878271 & 1.187276 & -4.612777 \\ \mathrm{H} & 0.000000 & -1.091797 & 0.262315 \\ \mathrm{H} & 0.000000 & 1.091797 & 0.262315\end{array}$

Zn-tetraphenylporphine $\mathrm{S}_{0}$

$\begin{array}{lrrr}\text { C } & -3.681346 & 4.292963 & 1.118720 \\ \mathrm{C} & -3.484166 & 3.484174 & -0.000006 \\ \mathrm{C} & -4.292941 & 3.681354 & -1.118743 \\ \mathrm{C} & -5.272900 & 4.663784 & -1.119857 \\ \mathrm{C} & -5.461961 & 5.461987 & -0.000023 \\ \mathrm{C} & -4.663767 & 5.272930 & 1.119818 \\ \mathrm{C} & -2.431481 & 2.431483 & 0.000000 \\ \mathrm{C} & -1.096873 & 2.853082 & -0.007404 \\ \mathrm{~N} & 0.000000 & 2.040898 & 0.033415 \\ \mathrm{C} & 1.096873 & 2.853082 & -0.007404 \\ \mathrm{C} & 0.677417 & 4.226549 & -0.088676 \\ \mathrm{C} & -0.677417 & 4.226549 & -0.088676 \\ \mathrm{C} & 2.431481 & 2.431483 & 0.000000 \\ \mathrm{C} & 3.484166 & 3.484174 & -0.000006 \\ \mathrm{C} & 4.292941 & 3.681354 & -1.118743 \\ \mathrm{C} & 5.272900 & 4.663784 & -1.119857 \\ \mathrm{C} & 5.461961 & 5.461987 & -0.000023 \\ \mathrm{C} & 4.663767 & 5.272930 & 1.119818 \\ \mathrm{C} & 3.681346 & 4.292963 & 1.118720 \\ \mathrm{C} & -2.853083 & 1.096875 & 0.007416 \\ \mathrm{~N} & -2.040899 & 0.000000 & -0.033378 \\ \mathrm{C} & -2.853083 & -1.096875 & 0.007416 \\ \mathrm{C} & -4.226548 & -0.677417 & 0.088704 \\ \mathrm{C} & -4.226548 & 0.677417 & 0.088704 \\ \mathrm{C} & -2.431481 & -2.431483 & 0.000000 \\ \mathrm{C} & -3.484166 & -3.484174 & -0.000006 \\ \mathrm{C} & -4.292941 & -3.681354 & -1.118743 \\ \mathrm{C} & -5.272900 & -4.663784 & -1.119857\end{array}$




$\begin{array}{rrrr}\mathrm{C} & -5.461961 & -5.461987 & -0.000023 \\ \mathrm{C} & -4.663767 & -5.272930 & 1.119818 \\ \mathrm{C} & -3.681346 & -4.292963 & 1.118720 \\ \mathrm{C} & 2.853083 & 1.096875 & 0.007416 \\ \mathrm{~N} & 2.040899 & 0.000000 & -0.033378 \\ \mathrm{C} & 2.853083 & -1.096875 & 0.007416 \\ \mathrm{C} & 4.226548 & -0.677417 & 0.088704 \\ \mathrm{C} & 4.226548 & 0.677417 & 0.088704 \\ \mathrm{C} & 2.431481 & -2.431483 & 0.000000 \\ \mathrm{C} & 3.484166 & -3.484174 & -0.000006 \\ \mathrm{C} & 4.292941 & -3.681354 & -1.118743 \\ \mathrm{C} & 5.272900 & -4.663784 & -1.119857 \\ \mathrm{C} & 5.461961 & -5.461987 & -0.000023 \\ \mathrm{C} & 4.663767 & -5.272930 & 1.119818 \\ \mathrm{C} & 3.681346 & -4.292963 & 1.118720 \\ \mathrm{C} & 1.096873 & -2.853082 & -0.007404 \\ \mathrm{~N} & 0.000000 & -2.040898 & 0.033415 \\ \mathrm{C} & -1.096873 & -2.853082 & -0.007404 \\ \mathrm{C} & -0.677417 & -4.226549 & -0.088676 \\ \mathrm{C} & 0.677417 & -4.226549 & -0.088676 \\ \mathrm{H} & 1.338948 & -5.076009 & -0.155651 \\ \mathrm{H} & 5.076008 & -1.338947 & 0.155686 \\ \mathrm{H} & 1.338948 & 5.076009 & -0.155651 \\ \mathrm{H} & -1.338948 & 5.076009 & -0.155651 \\ \mathrm{H} & -1.338948 & -5.076009 & -0.155651 \\ \mathrm{H} & 5.076008 & 1.338947 & 0.155686 \\ \mathrm{H} & -5.076008 & 1.338947 & 0.155686 \\ \mathrm{H} & -5.076008 & -1.338947 & 0.155686 \\ \mathrm{H} & 4.142833 & 3.061031 & -1.995761 \\ \mathrm{H} & 5.888845 & 4.807862 & -2.000882 \\ \mathrm{H} & 6.228741 & 6.228777 & -0.000029 \\ \mathrm{H} & 4.807844 & 5.888885 & 2.000837 \\ \mathrm{H} & 3.061028 & 4.142858 & 1.995745 \\ \mathrm{H} & 4.142833 & -3.061031 & -1.995761 \\ \mathrm{H} & 5.888845 & -4.807862 & -2.000882 \\ \mathrm{H} & 6.228741 & -6.228777 & -0.000029 \\ \mathrm{H} & 4.807844 & -5.888885 & 2.000837 \\ \mathrm{H} & 3.061028 & -4.142858 & 1.995745 \\ \mathrm{H} & -4.142833 & -3.061031 & -1.995761 \\ \mathrm{H} & -5.888845 & -4.807862 & -2.000882 \\ \mathrm{H} & -6.228741 & -6.228777 & -0.000029 \\ \mathrm{H} & -4.807844 & -5.888885 & 2.000837 \\ \mathrm{H} & -3.061028 & -4.142858 & 1.995745 \\ \mathrm{H} & -4.142833 & 3.061031 & -1.995761 \\ \mathrm{H} & -5.888845 & 4.807862 & -2.000882 \\ \mathrm{H} & -6.228741 & 6.228777 & -0.000029\end{array}$




$\begin{array}{cccc}\mathrm{H} & -4.807844 & 5.888885 & 2.000837 \\ \mathrm{H} & -3.061028 & 4.142858 & 1.995745 \\ \mathrm{Zn} & 0.000000 & 0.000000 & 0.000030\end{array}$

Zn-tetraphenylporphine $\mathrm{S}_{1}$

$\begin{array}{lrrr}\mathrm{C} & -3.564123 & 4.462570 & 0.991647 \\ \mathrm{C} & -3.486248 & 3.484512 & -0.002615 \\ \mathrm{C} & -4.424414 & 3.511822 & -1.037936 \\ \mathrm{C} & -5.403680 & 4.491701 & -1.082644 \\ \mathrm{C} & -5.471879 & 5.457077 & -0.086404 \\ \mathrm{C} & -4.550399 & 5.437130 & 0.951758 \\ \mathrm{C} & -2.437866 & 2.440249 & 0.031873 \\ \mathrm{C} & -1.099216 & 2.854589 & -0.002606 \\ \mathrm{~N} & 0.000000 & 2.044292 & 0.115216 \\ \mathrm{C} & 1.099216 & 2.854589 & -0.002606 \\ \mathrm{C} & 0.678054 & 4.212426 & -0.212969 \\ \mathrm{C} & -0.678054 & 4.212426 & -0.212969 \\ \mathrm{C} & 2.437866 & 2.440249 & 0.031873 \\ \mathrm{C} & 3.486248 & 3.484512 & -0.002615 \\ \mathrm{C} & 4.424414 & 3.511822 & -1.037936 \\ \mathrm{C} & 5.403680 & 4.491701 & -1.082644 \\ \mathrm{C} & 5.471879 & 5.457077 & -0.086404 \\ \mathrm{C} & 4.550399 & 5.437130 & 0.951758 \\ \mathrm{C} & 3.564123 & 4.462570 & 0.991647 \\ \mathrm{C} & -2.867337 & 1.096573 & 0.087674 \\ \mathrm{~N} & -2.056954 & 0.000000 & -0.021416 \\ \mathrm{C} & -2.867337 & -1.096573 & 0.087674 \\ \mathrm{C} & -4.222064 & -0.681107 & 0.287648 \\ \mathrm{C} & -4.222064 & 0.681107 & 0.287648 \\ \mathrm{C} & -2.437866 & -2.440249 & 0.031873 \\ \mathrm{C} & -3.486248 & -3.484512 & -0.002615 \\ \mathrm{C} & -4.424414 & -3.511822 & -1.037936 \\ \mathrm{C} & -5.403680 & -4.491701 & -1.082644 \\ \mathrm{C} & -5.471879 & -5.457077 & -0.086404 \\ \mathrm{C} & -4.550399 & -5.437130 & 0.951758 \\ \mathrm{C} & -3.564123 & -4.462570 & 0.991647 \\ \mathrm{C} & 2.867337 & 1.096573 & 0.087674 \\ \mathrm{~N} & 2.056954 & 0.000000 & -0.021416 \\ \mathrm{C} & 2.867337 & -1.096573 & 0.087674 \\ \mathrm{C} & 4.222064 & -0.681107 & 0.287648 \\ \mathrm{C} & 4.222064 & 0.681107 & 0.287648 \\ \mathrm{C} & 2.437866 & -2.440249 & 0.031873 \\ \mathrm{C} & 3.486248 & -3.484512 & -0.002615 \\ \mathrm{C} & 4.424414 & -3.511822 & -1.037936 \\ \mathrm{C} & 5.403680 & -4.491701 & -1.082644\end{array}$




$\begin{array}{rrrr}\mathrm{C} & 5.471879 & -5.457077 & -0.086404 \\ \mathrm{C} & 4.550399 & -5.437130 & 0.951758 \\ \mathrm{C} & 3.564123 & -4.462570 & 0.991647 \\ \mathrm{C} & 1.099216 & -2.854589 & -0.002606 \\ \mathrm{~N} & 0.000000 & -2.044292 & 0.115216 \\ \mathrm{C} & -1.099216 & -2.854589 & -0.002606 \\ \mathrm{C} & -0.678054 & -4.212426 & -0.212969 \\ \mathrm{C} & 0.678054 & -4.212426 & -0.212969 \\ \mathrm{H} & 1.336932 & -5.050521 & -0.375342 \\ \mathrm{H} & 5.061410 & -1.340873 & 0.438661 \\ \mathrm{H} & 1.336932 & 5.050521 & -0.375342 \\ \mathrm{H} & -1.336932 & 5.050521 & -0.375342 \\ \mathrm{H} & -1.336932 & -5.050521 & -0.375342 \\ \mathrm{H} & 5.061410 & 1.340873 & 0.438661 \\ \mathrm{H} & -5.061410 & 1.340873 & 0.438661 \\ \mathrm{H} & -5.061410 & -1.340873 & 0.438661 \\ \mathrm{H} & 4.368227 & 2.762699 & -1.819949 \\ \mathrm{H} & 6.114427 & 4.504316 & -1.901789 \\ \mathrm{H} & 6.240563 & 6.221223 & -0.118819 \\ \mathrm{H} & 4.601812 & 6.181534 & 1.738881 \\ \mathrm{H} & 2.850846 & 4.442477 & 1.808186 \\ \mathrm{H} & 4.368227 & -2.762699 & -1.819949 \\ \mathrm{H} & 6.114427 & -4.504316 & -1.901789 \\ \mathrm{H} & 6.240563 & -6.221223 & -0.118819 \\ \mathrm{H} & 4.601812 & -6.181534 & 1.738881 \\ \mathrm{H} & 2.850846 & -4.442477 & 1.808186 \\ \mathrm{H} & -4.368227 & -2.762699 & -1.819949 \\ \mathrm{H} & -6.114427 & -4.504316 & -1.901789 \\ \mathrm{H} & -6.240563 & -6.221223 & -0.118819 \\ \mathrm{H} & -4.601812 & -6.181534 & 1.738881 \\ \mathrm{H} & -2.850846 & -4.442477 & 1.808186 \\ \mathrm{H} & -4.368227 & 2.762699 & -1.819949 \\ \mathrm{H} & -6.114427 & 4.504316 & -1.901789 \\ \mathrm{H} & -6.240563 & 6.221223 & -0.118819 \\ \mathrm{H} & -4.601812 & 6.181534 & 1.738881 \\ \mathrm{H} & -2.850846 & 4.442477 & 1.808186 \\ \mathrm{Zn} & 0.000000 & 0.000000 & 0.046575\end{array}$

tetraphenylporphine $\mathrm{S}_{0}$

$\begin{array}{lrrr}\mathrm{C} & -0.681404 & 4.226342 & 0.089943 \\ \mathrm{C} & -1.122883 & 2.872292 & 0.000276 \\ \mathrm{~N} & 0.000000 & 2.096302 & -0.047398 \\ \mathrm{C} & 1.122883 & 2.872292 & 0.000277 \\ \mathrm{C} & 0.681405 & 4.226342 & 0.089944 \\ \mathrm{C} & 2.444926 & 2.424435 & -0.016160\end{array}$




$\begin{array}{lrrr}\mathrm{C} & 2.844275 & 1.079839 & -0.041290 \\ \mathrm{~N} & 2.023673 & 0.000000 & 0.004583 \\ \mathrm{C} & 2.844275 & -1.079839 & -0.041291 \\ \mathrm{C} & 4.234579 & -0.673908 & -0.142343 \\ \mathrm{C} & 4.234579 & 0.673908 & -0.142341 \\ \mathrm{C} & 2.444926 & -2.424435 & -0.016161 \\ \mathrm{C} & 1.122883 & -2.872292 & 0.000276 \\ \mathrm{~N} & 0.000000 & -2.096302 & -0.047398 \\ \mathrm{C} & -1.122883 & -2.872292 & 0.000277 \\ \mathrm{C} & -0.681405 & -4.226342 & 0.089944 \\ \mathrm{C} & 0.681404 & -4.226342 & 0.089943 \\ \mathrm{C} & -2.444926 & -2.424435 & -0.016160 \\ \mathrm{C} & -2.844275 & -1.079839 & -0.041290 \\ \mathrm{~N} & -2.023673 & 0.000000 & 0.004583 \\ \mathrm{C} & -2.844275 & 1.079839 & -0.041291 \\ \mathrm{C} & -4.234579 & 0.673908 & -0.142343 \\ \mathrm{C} & -4.234579 & -0.673908 & -0.142341 \\ \mathrm{C} & -2.444926 & 2.424435 & -0.016161 \\ \mathrm{H} & -5.080640 & -1.338675 & -0.222302 \\ \mathrm{C} & -3.502579 & -3.471939 & 0.001203 \\ \mathrm{H} & -1.338427 & -5.078334 & 0.159853 \\ \mathrm{H} & 0.000000 & -1.083915 & -0.062643 \\ \mathrm{H} & 5.080639 & -1.338675 & -0.222306 \\ \mathrm{H} & 5.080640 & 1.338675 & -0.222302 \\ \mathrm{H} & -5.080639 & 1.338675 & -0.222306 \\ \mathrm{C} & 3.502579 & -3.471940 & 0.001200 \\ \mathrm{C} & 3.502579 & 3.471939 & 0.001203 \\ \mathrm{C} & -3.502579 & 3.471940 & 0.001200 \\ \mathrm{H} & 1.338427 & -5.078334 & 0.159852 \\ \mathrm{H} & 1.338427 & 5.078334 & 0.159853 \\ \mathrm{H} & -1.338427 & 5.078334 & 0.159852 \\ \mathrm{H} & 0.000000 & 1.083915 & -0.062643 \\ \mathrm{C} & 3.700163 & -4.305404 & -1.099209 \\ \mathrm{C} & 4.687840 & -5.280047 & -1.080655 \\ \mathrm{C} & 5.490036 & -5.439036 & 0.040920 \\ \mathrm{C} & 5.300137 & -4.616168 & 1.142724 \\ \mathrm{C} & 4.315090 & -3.639288 & 1.122192 \\ \mathrm{H} & 3.078642 & -4.176939 & -1.978876 \\ \mathrm{H} & 4.833446 & -5.914587 & -1.948115 \\ \mathrm{H} & 5.261190 & -6.201269 & 0.056055 \\ \mathrm{C} & -4.736255 & 2.024658 \\ \mathrm{C} & -2.998475 & 1.984215 \\ \mathrm{H} & -4.305404 & -1.099205 \\ \mathrm{H} & -5.280048 & -1.080649 \\ \mathrm{H} & -4.616165 & 1.142729\end{array}$




$\begin{array}{lrrr}\mathrm{C} & -4.315090 & -3.639286 & 1.122195 \\ \mathrm{H} & -3.078644 & -4.176941 & -1.978873 \\ \mathrm{H} & -4.833448 & -5.914588 & -1.948109 \\ \mathrm{H} & -6.261191 & -6.201267 & 0.056063 \\ \mathrm{H} & -5.919869 & -4.736251 & 2.024663 \\ \mathrm{H} & -4.164804 & -2.998472 & 1.984217 \\ \mathrm{C} & -3.700163 & 4.305404 & -1.099209 \\ \mathrm{C} & -4.687840 & 5.280047 & -1.080655 \\ \mathrm{C} & -5.490036 & 5.439036 & 0.040920 \\ \mathrm{C} & -5.300137 & 4.616168 & 1.142724 \\ \mathrm{C} & -4.315090 & 3.639288 & 1.122192 \\ \mathrm{H} & -3.078642 & 4.176939 & -1.978876 \\ \mathrm{H} & -4.833446 & 5.914587 & -1.948115 \\ \mathrm{H} & -6.261190 & 6.201269 & 0.056055 \\ \mathrm{H} & -5.919869 & 4.736255 & 2.024658 \\ \mathrm{H} & -4.164805 & 2.998475 & 1.984215 \\ \mathrm{C} & 3.700164 & 4.305404 & -1.099205 \\ \mathrm{C} & 4.687842 & 5.280048 & -1.080649 \\ \mathrm{C} & 5.490037 & 5.439035 & 0.040927 \\ \mathrm{C} & 5.300137 & 4.616165 & 1.142729 \\ \mathrm{C} & 4.315090 & 3.639286 & 1.122195 \\ \mathrm{H} & 3.078644 & 4.176941 & -1.978873 \\ \mathrm{H} & 4.833448 & 5.914588 & -1.948109 \\ \mathrm{H} & 6.261191 & 6.201267 & 0.056063 \\ \mathrm{H} & 5.919869 & 4.736251 & 2.024663 \\ \mathrm{H} & 4.164804 & 2.998472 & 1.984217\end{array}$

tetraphenylporphine $\mathrm{S}_{1}$

$\begin{array}{rrrr}\mathrm{C} & -4.218748 & -0.682299 & 0.192192 \\ \mathrm{C} & -2.877588 & -1.126033 & -0.011443 \\ \mathrm{~N} & -2.105613 & -0.000007 & -0.124412 \\ \mathrm{C} & -2.877588 & 1.126011 & -0.011392 \\ \mathrm{C} & -4.218740 & 0.682262 & 0.192262 \\ \mathrm{C} & -2.433204 & 2.451361 & -0.064121 \\ \mathrm{C} & -1.082549 & 2.862193 & -0.155256 \\ \mathrm{~N} & 0.000001 & 2.042059 & -0.058374 \\ \mathrm{C} & 1.082543 & 2.862211 & -0.155306 \\ \mathrm{C} & 0.677734 & 4.231980 & -0.360725 \\ \mathrm{C} & -0.677782 & 4.231972 & -0.360671 \\ \mathrm{C} & 2.433193 & 2.451377 & -0.064183 \\ \mathrm{C} & 2.877588 & 1.126033 & -0.011443 \\ \mathrm{~N} & 2.105613 & 0.000007 & -0.124412 \\ \mathrm{C} & 2.877588 & -1.126011 & -0.011392 \\ \mathrm{C} & 4.218740 & -0.682262 & 0.192262 \\ \mathrm{C} & 4.218748 & 0.682299 & 0.192192\end{array}$




$\begin{array}{rrrr}\mathrm{C} & 2.433204 & -2.451361 & -0.064121 \\ \mathrm{C} & 1.082549 & -2.862193 & -0.155256 \\ \mathrm{~N} & -0.000001 & -2.042059 & -0.058374 \\ \mathrm{C} & -1.082543 & -2.862211 & -0.155306 \\ \mathrm{C} & -0.677734 & -4.231980 & -0.360725 \\ \mathrm{C} & 0.677782 & -4.231972 & -0.360671 \\ \mathrm{C} & -2.433193 & -2.451377 & -0.064183 \\ \mathrm{H} & 1.339602 & -5.070600 & -0.509117 \\ \mathrm{C} & 3.469565 & -3.507959 & 0.000601 \\ \mathrm{H} & 5.062405 & -1.335863 & 0.343188 \\ \mathrm{H} & 1.095688 & 0.000009 & -0.177372 \\ \mathrm{H} & 1.339532 & 5.070619 & -0.509198 \\ \mathrm{H} & -1.339602 & 5.070600 & -0.509117 \\ \mathrm{H} & -1.339532 & -5.070619 & -0.509198 \\ \mathrm{C} & 3.469573 & 3.507969 & 0.000440 \\ \mathrm{C} & -3.469565 & 3.507959 & 0.000601 \\ \mathrm{C} & -3.469573 & -3.507969 & 0.000440 \\ \mathrm{H} & 5.062426 & 1.335899 & 0.343042 \\ \mathrm{H} & -5.062405 & 1.335863 & 0.343188 \\ \mathrm{H} & -5.062426 & -1.335899 & 0.343042 \\ \mathrm{H} & -1.095688 & -0.000009 & -0.177372 \\ \mathrm{C} & 4.464755 & 3.609983 & -0.973578 \\ \mathrm{C} & 5.429168 & 4.604717 & -0.899198 \\ \mathrm{C} & 5.420163 & 5.509291 & 0.153625 \\ \mathrm{C} & 4.436489 & 5.416534 & 1.129775 \\ \mathrm{C} & 3.467348 & 4.428903 & 1.050975 \\ \mathrm{H} & 4.466101 & 2.911595 & -1.803297 \\ \mathrm{H} & 6.187574 & 4.676122 & -1.671237 \\ \mathrm{H} & 6.175834 & 6.284754 & 0.212595 \\ \mathrm{H} & 4.425810 & 6.115046 & 1.959365 \\ \mathrm{H} & 2.702103 & 4.354364 & 1.815579 \\ \mathrm{C} & 4.464752 & -3.610048 & -0.973408 \\ \mathrm{C} & 5.429168 & -4.604773 & -0.898952 \\ \mathrm{C} & 5.420181 & -5.509253 & 0.153955 \\ \mathrm{C} & 4.436513 & -5.416411 & 1.130103 \\ \mathrm{C} & 3.467363 & -4.428795 & 1.051219 \\ \mathrm{H} & 4.466098 & -2.911695 & -1.803155 \\ \mathrm{H} & 6.187584 & -4.676221 & -1.670977 \\ \mathrm{H} & 6.175866 & -6.284696 & 0.213006 \\ \mathrm{H} & 4.425850 & -6.114844 & 1.959758 \\ \mathrm{H} & 2.702133 & -4.354191 & 1.815833 \\ \mathrm{C} & -4.464755 & -3.609983 & -0.973578 \\ \mathrm{C} & -5.429168 & -4.604717 & -0.899198 \\ \mathrm{C} & -5.420163 & -5.509291 & 0.153625 \\ \mathrm{C} & -4.436489 & -5.416534 & 1.129775 \\ \mathrm{C} & -3.467348 & -4.428903 & 1.050975\end{array}$




$\begin{array}{lllr}\mathrm{H} & -4.466101 & -2.911595 & -1.803297 \\ \mathrm{H} & -6.187574 & -4.676122 & -1.671237 \\ \mathrm{H} & -6.175834 & -6.284754 & 0.212595 \\ \mathrm{H} & -4.425810 & -6.115046 & 1.959365 \\ \mathrm{H} & -2.702103 & -4.354364 & 1.815579 \\ \mathrm{C} & -4.464752 & 3.610048 & -0.973408 \\ \mathrm{C} & -5.429168 & 4.604773 & -0.898952 \\ \mathrm{C} & -5.420181 & 5.509253 & 0.153955 \\ \mathrm{C} & -4.436513 & 5.416411 & 1.130103 \\ \mathrm{C} & -3.467363 & 4.428795 & 1.051219 \\ \mathrm{H} & -4.466098 & 2.911695 & -1.803155 \\ \mathrm{H} & -6.187584 & 4.676221 & -1.670977 \\ \mathrm{H} & -6.175866 & 6.284696 & 0.213006 \\ \mathrm{H} & -4.425850 & 6.114844 & 1.959758 \\ \mathrm{H} & -2.702133 & 4.354191 & 1.815833\end{array}$

\section{PBE0/TZVP geometries - Group II}

tetrafluorobenzene $\mathrm{S}_{0}$

$\begin{array}{lrrr}\text { C } & -1.190569 & 0.693080 & 0.000000 \\ \text { C } & -1.190569 & -0.693080 & 0.000000 \\ \text { C } & 0.000000 & -1.393702 & 0.000000 \\ \text { C } & 1.190569 & -0.693080 & 0.000000 \\ \text { C } & 1.190569 & 0.693080 & 0.000000 \\ \text { C } & 0.000000 & 1.393702 & 0.000000 \\ \text { F } & -2.349344 & -1.348845 & 0.000000 \\ \text { H } & 0.000000 & -2.476201 & 0.000000 \\ \text { F } & 2.349344 & -1.348845 & 0.000000 \\ \text { F } & 2.349344 & 1.348845 & 0.000000 \\ \text { H } & 0.000000 & 2.476201 & 0.000000 \\ \text { F } & -2.349344 & 1.348845 & 0.000000\end{array}$

tetrafluorobenzene $\mathrm{S}_{1}$

$\begin{array}{lrrr}\text { C } & 1.161456 & -0.707271 & 0.105386 \\ \text { C } & 1.161456 & 0.707271 & 0.105386 \\ \text { C } & 0.000000 & 1.462488 & 0.314329 \\ \text { C } & -1.161456 & 0.707271 & 0.105386 \\ \text { C } & -1.161456 & -0.707271 & 0.105386 \\ \text { C } & 0.000000 & -1.462488 & 0.314329 \\ \text { F } & 2.300105 & 1.314967 & -0.219340 \\ \text { H } & 0.000000 & 2.504076 & 0.611703 \\ \text { F } & -2.300105 & 1.314967 & -0.219340 \\ \text { F } & -2.300105 & -1.314967 & -0.219340\end{array}$




$$
\begin{array}{lllr}
\mathrm{H} & 0.000000 & -2.504076 & 0.611703 \\
\mathrm{~F} & 2.300105 & -1.314967 & -0.219340
\end{array}
$$

benzonitrile $\mathrm{S}_{0}$

$\begin{array}{crrr}\mathrm{C} & 0.000000 & 0.000000 & -1.054789 \\ \mathrm{C} & -1.210328 & 0.000000 & -0.358801 \\ \mathrm{C} & -1.203810 & 0.000000 & 1.025861 \\ \mathrm{C} & 0.000000 & 0.000000 & 1.718460 \\ \mathrm{C} & 1.203810 & 0.000000 & 1.025861 \\ \mathrm{C} & 1.210328 & 0.000000 & -0.358801 \\ \mathrm{H} & -2.143839 & 0.000000 & -0.908514 \\ \mathrm{H} & -2.142957 & 0.000000 & 1.566939 \\ \mathrm{H} & 0.000000 & 0.000000 & 2.802614 \\ \mathrm{H} & 2.142957 & 0.000000 & 1.566939 \\ \mathrm{H} & 2.143839 & 0.000000 & -0.908514 \\ \mathrm{C} & 0.000000 & 0.000000 & -2.482239 \\ \mathrm{~N} & 0.000000 & 0.000000 & -3.635007\end{array}$

benzonitrile $S_{1}$

$\begin{array}{lrrr}\mathrm{C} & 0.000000 & 0.000000 & -1.107956 \\ \mathrm{C} & -1.223762 & 0.000000 & -0.373179 \\ \mathrm{C} & -1.211418 & 0.000000 & 1.045377 \\ \mathrm{C} & 0.000000 & 0.000000 & 1.762912 \\ \mathrm{C} & 1.211418 & 0.000000 & 1.045377 \\ \mathrm{C} & 1.223762 & 0.000000 & -0.373179 \\ \mathrm{H} & -2.164485 & 0.000000 & -0.908237 \\ \mathrm{H} & -2.156736 & 0.000000 & 1.573858 \\ \mathrm{H} & 0.000000 & 0.000000 & 2.844369 \\ \mathrm{H} & 2.156736 & 0.000000 & 1.573858 \\ \mathrm{H} & 2.164485 & 0.000000 & -0.908237 \\ \mathrm{C} & 0.000000 & 0.000000 & -2.505761 \\ \mathrm{~N} & 0.000000 & 0.000000 & -3.669200\end{array}$

$o$-fluorophenol, cis $\mathrm{S}_{0}$

$\begin{array}{lrrc}\mathrm{C} & 0.324607 & 1.450080 & 0.000000 \\ \mathrm{C} & -0.874044 & 0.773986 & 0.000000 \\ \mathrm{C} & -0.950202 & -0.614542 & 0.000000 \\ \mathrm{C} & 0.236138 & -1.334966 & 0.000000 \\ \mathrm{C} & 1.453711 & -0.670123 & 0.000000 \\ \mathrm{C} & 1.505014 & 0.717384 & 0.000000 \\ \mathrm{~F} & -2.044628 & 1.452179 & 0.000000 \\ \mathrm{O} & -2.136738 & -1.263738 & 0.000000 \\ \mathrm{H} & 0.182366 & -2.417224 & 0.000000\end{array}$




$$
\begin{array}{cccc}
\mathrm{H} & 2.372013 & -1.245725 & 0.000000 \\
\mathrm{H} & 2.457812 & 1.232544 & 0.000000 \\
\mathrm{H} & 0.321199 & 2.533552 & 0.000000 \\
\mathrm{H} & -2.847238 & -0.613427 & 0.000000
\end{array}
$$

$o$-fluorophenol, cis $\mathrm{S}_{1}$

$\begin{array}{crcc}\mathrm{C} & 1.228719 & -0.254498 & -0.733628 \\ \mathrm{C} & 0.002845 & -0.062411 & -0.092903 \\ \mathrm{C} & -0.008573 & -0.025175 & 1.344372 \\ \mathrm{C} & 1.173559 & 0.029728 & 2.090224 \\ \mathrm{C} & 2.389748 & 0.109779 & 1.410100 \\ \mathrm{C} & 2.403078 & -0.016180 & 0.017596 \\ \mathrm{~F} & -0.963397 & 0.789098 & -0.689034 \\ \mathrm{O} & -1.172376 & -0.113667 & 1.987729 \\ \mathrm{H} & 1.124792 & -0.131938 & 3.162432 \\ \mathrm{H} & 3.318151 & 0.221958 & 1.955197 \\ \mathrm{H} & 3.343297 & 0.103951 & -0.515362 \\ \mathrm{H} & 1.282738 & -0.406396 & -1.805215 \\ \mathrm{H} & -1.867201 & -0.244248 & 1.318432\end{array}$

$o$-fluorophenol, trans $\mathrm{S}_{0}$

$\begin{array}{cccc}\mathrm{C} & 0.289693 & 1.262096 & 0.000000 \\ \mathrm{C} & -0.945994 & 0.618329 & 0.000000 \\ \mathrm{C} & -0.960431 & -0.770800 & 0.000000 \\ \mathrm{C} & 0.226093 & -1.492271 & 0.000000 \\ \mathrm{C} & 1.444554 & -0.832947 & 0.000000 \\ \mathrm{C} & 1.472827 & 0.556509 & 0.000000 \\ \mathrm{O} & -2.060146 & 1.387671 & 0.000000 \\ \mathrm{H} & -1.914966 & -1.289869 & 0.000000 \\ \mathrm{H} & 0.190456 & -2.575420 & 0.000000 \\ \mathrm{H} & 2.372849 & -1.391185 & 0.000000 \\ \mathrm{H} & 2.405689 & 1.107811 & 0.000000 \\ \mathrm{~F} & 0.314831 & 2.600190 & 0.000000 \\ \mathrm{H} & -2.835466 & 0.819886 & 0.000000\end{array}$

$o$-fluorophenol, trans $\mathrm{S}_{1}$
$\begin{array}{llll}\text { C } & 0.818909 & 1.036675 & 0.110395\end{array}$
$\begin{array}{llll}\text { C } & -0.587028 & 0.973139 & 0.090501\end{array}$
$\begin{array}{llll}\text { C } & -1.273182 & -0.284027 & 0.106787\end{array}$
$\begin{array}{llll}\text { C } & -0.470979 & -1.416661 & -0.083271\end{array}$
$\begin{array}{llll}\text { C } & 0.937503 & -1.329545 & -0.071157\end{array}$
$\begin{array}{llll}\text { C } & 1.615875 & -0.127053 & 0.181898\end{array}$
$\begin{array}{llll}\mathrm{O} & -1.251475 & 2.115149 & -0.110315\end{array}$ 


$$
\begin{array}{cccc}
\mathrm{H} & -2.292380 & -0.357286 & 0.479847 \\
\mathrm{H} & -0.929883 & -2.391614 & -0.208625 \\
\mathrm{H} & 1.511076 & -2.236714 & -0.239263 \\
\mathrm{H} & 2.685539 & -0.068156 & 0.323856 \\
\mathrm{~F} & 1.394878 & 2.202367 & -0.206901 \\
\mathrm{H} & -2.158882 & 1.883726 & -0.373752
\end{array}
$$

$m$-fluorophenol, cis $\mathrm{S}_{0}$

$\begin{array}{cccc}\mathrm{C} & 0.307724 & 1.408000 & 0.000000 \\ \mathrm{C} & -0.926696 & 0.785370 & 0.000000 \\ \mathrm{C} & -0.967108 & -0.605169 & 0.000000 \\ \mathrm{C} & 0.214013 & -1.341420 & 0.000000 \\ \mathrm{C} & 1.429397 & -0.679127 & 0.000000 \\ \mathrm{C} & 1.497568 & 0.709137 & 0.000000 \\ \mathrm{O} & -2.134665 & -1.294653 & 0.000000 \\ \mathrm{~F} & 0.337570 & 2.749536 & 0.000000 \\ \mathrm{H} & 0.158709 & -2.422760 & 0.000000 \\ \mathrm{H} & 2.347636 & -1.255306 & 0.000000 \\ \mathrm{H} & 2.439942 & 1.241204 & 0.000000 \\ \mathrm{H} & -1.829884 & 1.386241 & 0.000000 \\ \mathrm{H} & -2.874206 & -0.681053 & 0.000000\end{array}$

$m$-fluorophenol, cis $\mathrm{S}_{1}$

$\begin{array}{lrrr}\mathrm{C} & 1.218995 & -0.054117 & -0.683368 \\ \mathrm{C} & -0.053805 & 0.043026 & -0.101164 \\ \mathrm{C} & -0.022790 & 0.038286 & 1.323866 \\ \mathrm{C} & 1.163971 & 0.029834 & 2.083714 \\ \mathrm{C} & 2.423758 & 0.128900 & 1.438894 \\ \mathrm{C} & 2.423956 & -0.038833 & 0.037735 \\ \mathrm{O} & -1.172759 & -0.039254 & 2.009260 \\ \mathrm{~F} & 1.296074 & -0.173842 & -2.015505 \\ \mathrm{H} & 1.078534 & -0.132803 & 3.152105 \\ \mathrm{H} & 3.345234 & 0.175316 & 1.998770 \\ \mathrm{H} & 3.348238 & -0.152098 & -0.518560 \\ \mathrm{H} & -0.906148 & 0.414371 & -0.663519 \\ \mathrm{H} & -1.887899 & -0.238786 & 1.387721\end{array}$

$m$-fluorophenol, trans $\mathrm{S}_{0}$
$\begin{array}{llll}\text { C } & 0.268213 & 1.301922 & 0.000000\end{array}$
$\begin{array}{llll}\text { C } & -0.948484 & 0.629029 & 0.000000\end{array}$
$\begin{array}{llll}\text { C } & -0.982723 & -0.762306 & 0.000000\end{array}$
$\begin{array}{llll}\text { C } & 0.206536 & -1.476353 & 0.000000\end{array}$
$\begin{array}{llll}\text { C } & 1.432261 & -0.828478 & 0.000000\end{array}$ 


$\begin{array}{crcc}\mathrm{C} & 1.426676 & 0.554548 & 0.000000 \\ \mathrm{O} & -2.072228 & 1.388928 & 0.000000 \\ \mathrm{H} & -1.934652 & -1.284400 & 0.000000 \\ \mathrm{H} & 0.175850 & -2.559975 & 0.000000 \\ \mathrm{H} & 2.370290 & -1.368094 & 0.000000 \\ \mathrm{~F} & 2.600730 & 1.204297 & 0.000000 \\ \mathrm{H} & 0.302297 & 2.383554 & 0.000000 \\ \mathrm{H} & -2.844766 & 0.817337 & 0.000000\end{array}$

$m$-fluorophenol, trans $\mathrm{S}_{1}$

$\begin{array}{cccc}\mathrm{C} & 1.084426 & -0.016187 & 0.000000 \\ \mathrm{C} & 0.328299 & 1.180033 & 0.000000 \\ \mathrm{C} & -1.088071 & 1.211666 & 0.000000 \\ \mathrm{C} & -1.821809 & -0.005646 & 0.000000 \\ \mathrm{C} & -1.083179 & -1.214371 & 0.000000 \\ \mathrm{C} & 0.319473 & -1.196692 & 0.000000 \\ \mathrm{O} & 1.036264 & 2.319997 & 0.000000 \\ \mathrm{H} & -1.597033 & 2.170127 & 0.000000 \\ \mathrm{H} & -2.901137 & -0.013174 & 0.000000 \\ \mathrm{H} & -1.582668 & -2.175818 & 0.000000 \\ \mathrm{~F} & 0.965971 & -2.368793 & 0.000000 \\ \mathrm{H} & 2.163177 & -0.011061 & 0.000000 \\ \mathrm{H} & 0.435137 & 3.074067 & 0.000000\end{array}$

$p$-fluorophenol $\mathrm{S}_{0}$

$\begin{array}{cccc}\mathrm{C} & 0.271157 & 1.313721 & 0.000000 \\ \mathrm{C} & -0.950172 & 0.647862 & 0.000000 \\ \mathrm{C} & -0.978920 & -0.742249 & 0.000000 \\ \mathrm{C} & 0.205511 & -1.466706 & 0.000000 \\ \mathrm{C} & 1.405772 & -0.786464 & 0.000000 \\ \mathrm{C} & 1.454938 & 0.595357 & 0.000000 \\ \mathrm{O} & -2.077926 & 1.408414 & 0.000000 \\ \mathrm{H} & -1.928974 & -1.268625 & 0.000000 \\ \mathrm{H} & 0.199990 & -2.549883 & 0.000000 \\ \mathrm{~F} & 2.555770 & -1.483790 & 0.000000 \\ \mathrm{H} & 2.414623 & 1.097870 & 0.000000 \\ \mathrm{H} & 0.278808 & 2.396937 & 0.000000 \\ \mathrm{H} & -2.850597 & 0.837547 & 0.000000\end{array}$

p-fluorophenol $\mathrm{S}_{1}$
$\begin{array}{llll}\text { C } & 1.264933 & 0.679778 & 0.000000\end{array}$
$\begin{array}{llll}\text { C } & 0.028084 & 1.357868 & 0.000000\end{array}$
$\begin{array}{llll}\text { C } & -1.233485 & 0.710932 & 0.000000\end{array}$ 


$\begin{array}{rrrr}\text { C } & -1.248535 & -0.703085 & 0.000000 \\ \text { C } & -0.006662 & -1.360396 & 0.000000 \\ \text { C } & 1.249590 & -0.735524 & 0.000000 \\ \text { O } & 0.092157 & 2.696837 & 0.000000 \\ \text { H } & -2.149900 & 1.290008 & 0.000000 \\ \text { H } & -2.161337 & -1.281175 & 0.000000 \\ \text { F } & -0.027693 & -2.690680 & 0.000000 \\ \text { H } & 2.149671 & -1.332670 & 0.000000 \\ \text { H } & 2.181413 & 1.252743 & 0.000000 \\ \text { H } & -0.801538 & 3.060958 & 0.000000\end{array}$

phenylacetylene $S_{0}$

$\begin{array}{rrrr}\mathrm{C} & 1.206133 & 0.000000 & -0.013854 \\ \mathrm{C} & 0.000000 & 0.000000 & -0.722235 \\ \mathrm{C} & -1.206133 & 0.000000 & -0.013854 \\ \mathrm{C} & -1.201625 & 0.000000 & 1.371055 \\ \mathrm{C} & 0.000000 & 0.000000 & 2.067412 \\ \mathrm{C} & 1.201625 & 0.000000 & 1.371055 \\ \mathrm{C} & 0.000000 & 0.000000 & -2.146073 \\ \mathrm{C} & 0.000000 & 0.000000 & -3.349504 \\ \mathrm{H} & -2.141170 & 0.000000 & -0.561429 \\ \mathrm{H} & -2.142280 & 0.000000 & 1.910379 \\ \mathrm{H} & 0.000000 & 0.000000 & 3.151640 \\ \mathrm{H} & 2.142280 & 0.000000 & 1.910379 \\ \mathrm{H} & 2.141170 & 0.000000 & -0.561429 \\ \mathrm{H} & 0.000000 & 0.000000 & -4.413561\end{array}$

phenylacetylene $S_{1}$

$\begin{array}{rrrr}\mathrm{C} & 1.232251 & 0.000000 & -0.031173 \\ \mathrm{C} & 0.000000 & 0.000000 & -0.752683 \\ \mathrm{C} & -1.232251 & 0.000000 & -0.031173 \\ \mathrm{C} & -1.220655 & 0.000000 & 1.389054 \\ \mathrm{C} & 0.000000 & 0.000000 & 2.087415 \\ \mathrm{C} & 1.220655 & 0.000000 & 1.389054 \\ \mathrm{C} & 0.000000 & 0.000000 & -2.143191 \\ \mathrm{C} & 0.000000 & 0.000000 & -3.359681 \\ \mathrm{H} & -2.165603 & 0.000000 & -0.577910 \\ \mathrm{H} & -2.157950 & 0.000000 & 1.930660 \\ \mathrm{H} & 0.000000 & 0.000000 & 3.170663 \\ \mathrm{H} & 2.157950 & 0.000000 & 1.930660 \\ \mathrm{H} & 2.165603 & 0.000000 & -0.577910 \\ \mathrm{H} & 0.000000 & 0.000000 & -4.423788\end{array}$

aniline $\mathrm{S}_{0}$ 


$\begin{array}{lrrr}\mathrm{C} & 0.920600 & 0.027617 & 0.000000 \\ \mathrm{C} & 0.205937 & 0.035555 & 1.200842 \\ \mathrm{C} & -1.179209 & 0.042901 & 1.195237 \\ \mathrm{C} & -1.885834 & 0.047624 & 0.000000 \\ \mathrm{C} & -1.179209 & 0.042901 & -1.195237 \\ \mathrm{C} & 0.205937 & 0.035555 & -1.200842 \\ \mathrm{H} & 0.744895 & 0.036602 & 2.143446 \\ \mathrm{H} & -2.969291 & 0.052977 & 0.000000 \\ \mathrm{H} & -1.712183 & 0.046105 & 2.140145 \\ \mathrm{H} & -1.712183 & 0.046105 & -2.140145 \\ \mathrm{H} & 0.744895 & 0.036602 & -2.143446 \\ \mathrm{~N} & 2.307859 & 0.075778 & 0.000000 \\ \mathrm{H} & 2.753898 & -0.263155 & -0.836679 \\ \mathrm{H} & 2.753898 & -0.263155 & 0.836679\end{array}$

aniline $\mathrm{S}_{1}$

$\begin{array}{lrrr}\mathrm{C} & 0.889519 & -0.033948 & 0.000000 \\ \mathrm{C} & 0.226010 & -0.008578 & 1.244196 \\ \mathrm{C} & -1.195501 & 0.045719 & 1.234415 \\ \mathrm{C} & -1.863138 & 0.071172 & 0.000000 \\ \mathrm{C} & -1.195501 & 0.045719 & -1.234415 \\ \mathrm{C} & 0.226010 & -0.008578 & -1.244196 \\ \mathrm{H} & 0.794446 & -0.030353 & 2.166392 \\ \mathrm{H} & -2.948159 & 0.112516 & 0.000000 \\ \mathrm{H} & -1.754914 & 0.067063 & 2.159703 \\ \mathrm{H} & -1.754914 & 0.067063 & -2.159703 \\ \mathrm{H} & 0.794446 & -0.030353 & -2.166392 \\ \mathrm{~N} & 2.250658 & -0.086059 & 0.000000 \\ \mathrm{H} & 2.765514 & -0.105701 & -0.864341 \\ \mathrm{H} & 2.765514 & -0.105701 & 0.864341\end{array}$

catechol $\mathrm{S}_{0}$

$\begin{array}{rrrr}\mathrm{C} & 0.467969 & 1.275646 & 0.000000 \\ \mathrm{C} & -0.748718 & 0.618218 & 0.000000 \\ \mathrm{C} & -0.798854 & -0.778580 & 0.000000 \\ \mathrm{C} & 0.382068 & -1.501011 & 0.000000 \\ \mathrm{C} & 1.604518 & -0.840770 & 0.000000 \\ \mathrm{C} & 1.651034 & 0.544542 & 0.000000 \\ \mathrm{O} & -1.968069 & 1.240578 & 0.000000 \\ \mathrm{O} & -1.990478 & -1.422100 & 0.000000 \\ \mathrm{H} & 0.324838 & -2.583205 & 0.000000 \\ \mathrm{H} & 2.522614 & -1.416530 & 0.000000 \\ \mathrm{H} & 2.602130 & 1.063455 & 0.000000\end{array}$




$\begin{array}{rrrr}\mathrm{H} & 0.492555 & 2.361930 & 0.000000 \\ \mathrm{H} & -1.853845 & 2.193774 & 0.000000 \\ \mathrm{H} & -2.687762 & -0.755947 & 0.000000\end{array}$

catechol $\mathrm{S}_{1}$

$\begin{array}{rrrr}\mathrm{C} & 0.471773 & 1.346249 & 0.000000 \\ \mathrm{C} & -0.759807 & 0.623577 & 0.000000 \\ \mathrm{C} & -0.803054 & -0.783328 & 0.000000 \\ \mathrm{C} & 0.386542 & -1.571456 & 0.000000 \\ \mathrm{C} & 1.594070 & -0.856238 & 0.000000 \\ \mathrm{C} & 1.635078 & 0.556840 & 0.000000 \\ \mathrm{O} & -1.966091 & 1.245537 & 0.000000 \\ \mathrm{O} & -1.983891 & -1.422210 & 0.000000 \\ \mathrm{H} & 0.324565 & -2.648786 & 0.000000 \\ \mathrm{H} & 2.534120 & -1.395336 & 0.000000 \\ \mathrm{H} & 2.604097 & 1.042854 & 0.000000 \\ \mathrm{H} & 0.495493 & 2.427660 & 0.000000 \\ \mathrm{H} & -1.839944 & 2.199034 & 0.000000 \\ \mathrm{H} & -2.692951 & -0.764397 & 0.000000\end{array}$

resorcinol, isomer $1 \mathrm{~S}_{0}$

$\begin{array}{rrrr}\mathrm{C} & 0.075855 & 1.145705 & 0.000000 \\ \mathrm{C} & -1.136511 & 0.466702 & 0.000000 \\ \mathrm{C} & -1.167597 & -0.922489 & 0.000000 \\ \mathrm{C} & 0.032205 & -1.622083 & 0.000000 \\ \mathrm{C} & 1.249515 & -0.965713 & 0.000000 \\ \mathrm{C} & 1.262670 & 0.426331 & 0.000000 \\ \mathrm{O} & -2.264811 & 1.224742 & 0.000000 \\ \mathrm{H} & -2.115703 & -1.451159 & 0.000000 \\ \mathrm{H} & 0.013024 & -2.706317 & 0.000000 \\ \mathrm{H} & 2.188049 & -1.505107 & 0.000000 \\ \mathrm{O} & 2.474682 & 1.039293 & 0.000000 \\ \mathrm{H} & 0.070721 & 2.230941 & 0.000000 \\ \mathrm{H} & -3.032582 & 0.646926 & 0.000000 \\ \mathrm{H} & 2.350482 & 1.992216 & 0.000000\end{array}$

resorcinol, isomer $1 \mathrm{~S}_{1}$
$\begin{array}{llll}\text { C } & 0.000339 & 1.066031 & 0.066045\end{array}$
$\begin{array}{llll}\text { C } & -1.188015 & 0.309232 & -0.003906\end{array}$
$\begin{array}{llll}\text { C } & -1.197212 & -1.103236 & -0.034600\end{array}$
$\begin{array}{llll}\text { C } & -0.000515 & -1.845977 & 0.117007\end{array}$
$\begin{array}{lrrr}\text { C } & 1.204250 & -1.107477 & 0.012815\end{array}$
$\begin{array}{llll}\text { C } & 1.191412 & 0.301851 & 0.034655\end{array}$ 


$\begin{array}{rrrr}\mathrm{O} & -2.341042 & 1.008679 & -0.057537 \\ \mathrm{H} & -2.142480 & -1.614204 & -0.202277 \\ \mathrm{H} & -0.003701 & -2.925057 & 0.123081 \\ \mathrm{H} & 2.159816 & -1.600067 & -0.128527 \\ \mathrm{O} & 2.391196 & 0.915278 & -0.030401 \\ \mathrm{H} & -0.022277 & 2.102349 & 0.384614 \\ \mathrm{H} & -3.087311 & 0.400107 & -0.029161 \\ \mathrm{H} & 2.246532 & 1.848610 & -0.232158\end{array}$

resorcinol, isomer $2 \mathrm{~S}_{0}$

$\begin{array}{lrcc}\mathrm{C} & 1.197141 & 0.000000 & -0.547096 \\ \mathrm{C} & 0.000000 & 0.000000 & -1.247071 \\ \mathrm{C} & -1.197141 & 0.000000 & -0.547096 \\ \mathrm{C} & -1.207061 & 0.000000 & 0.845809 \\ \mathrm{C} & 0.000000 & 0.000000 & 1.526388 \\ \mathrm{C} & 1.207061 & 0.000000 & 0.845809 \\ \mathrm{O} & -2.340664 & 0.000000 & -1.280616 \\ \mathrm{O} & 2.340664 & 0.000000 & -1.280616 \\ \mathrm{H} & -2.146894 & 0.000000 & 1.389053 \\ \mathrm{H} & 0.000000 & 0.000000 & 2.610732 \\ \mathrm{H} & 2.146894 & 0.000000 & 1.389053 \\ \mathrm{H} & 0.000000 & 0.000000 & -2.329451 \\ \mathrm{H} & -3.096721 & 0.000000 & -0.687688 \\ \mathrm{H} & 3.096721 & 0.000000 & -0.687688\end{array}$

resorcinol, isomer $2 \mathrm{~S}_{1}$

$\begin{array}{lrrr}\mathrm{C} & 1.194403 & 0.000000 & -0.330223 \\ \mathrm{C} & 0.000000 & 0.000000 & -1.081365 \\ \mathrm{C} & -1.194403 & 0.000000 & -0.330223 \\ \mathrm{C} & -1.210070 & 0.000000 & 1.083197 \\ \mathrm{C} & 0.000000 & 0.000000 & 1.823451 \\ \mathrm{C} & 1.210070 & 0.000000 & 1.083197 \\ \mathrm{O} & -2.346487 & 0.000000 & -1.035591 \\ \mathrm{O} & 2.346487 & 0.000000 & -1.035591 \\ \mathrm{H} & -2.165902 & 0.000000 & 1.599323 \\ \mathrm{H} & 0.000000 & 0.000000 & 2.902859 \\ \mathrm{H} & 2.165902 & 0.000000 & 1.599323 \\ \mathrm{H} & 0.000000 & 0.000000 & -2.160254 \\ \mathrm{H} & -3.093829 & 0.000000 & -0.428596 \\ \mathrm{H} & 3.093829 & 0.000000 & -0.428596\end{array}$

resorcinol, isomer $3 \mathrm{~S}_{0}$

$\begin{array}{llll}\text { C } & 1.202655 & 0.000000 & -0.288374\end{array}$ 


$\begin{array}{cccc}\mathrm{C} & 0.000000 & 0.000000 & -0.987787 \\ \mathrm{C} & -1.202655 & 0.000000 & -0.288374 \\ \mathrm{C} & -1.209998 & 0.000000 & 1.100373 \\ \mathrm{C} & 0.000000 & 0.000000 & 1.776090 \\ \mathrm{C} & 1.209998 & 0.000000 & 1.100373 \\ \mathrm{O} & -2.404401 & 0.000000 & -0.923328 \\ \mathrm{O} & 2.404401 & 0.000000 & -0.923328 \\ \mathrm{H} & -2.156786 & 0.000000 & 1.625191 \\ \mathrm{H} & 0.000000 & 0.000000 & 2.860537 \\ \mathrm{H} & 2.156786 & 0.000000 & 1.625191 \\ \mathrm{H} & 0.000000 & 0.000000 & -2.075816 \\ \mathrm{H} & 2.267230 & 0.000000 & -1.874135 \\ \mathrm{H} & -2.267230 & 0.000000 & -1.874135\end{array}$

resorcinol, isomer $3 \mathrm{~S}_{1}$

$\begin{array}{lrrc}\mathrm{C} & -0.025230 & -0.303377 & 1.184060 \\ \mathrm{C} & -0.125095 & -1.076036 & 0.000000 \\ \mathrm{C} & -0.025230 & -0.303377 & -1.184060 \\ \mathrm{C} & 0.024883 & 1.106292 & -1.195806 \\ \mathrm{C} & -0.083305 & 1.846572 & 0.000000 \\ \mathrm{C} & 0.024883 & 1.106292 & 1.195806 \\ \mathrm{O} & 0.071148 & -0.915065 & -2.381934 \\ \mathrm{O} & 0.071148 & -0.915065 & 2.381934 \\ \mathrm{H} & 0.184290 & 1.593224 & -2.151929 \\ \mathrm{H} & -0.091240 & 2.925952 & 0.000000 \\ \mathrm{H} & 0.184290 & 1.593224 & 2.151929 \\ \mathrm{H} & -0.666938 & -2.025965 & 0.000000 \\ \mathrm{H} & 0.252898 & -1.851799 & 2.226500 \\ \mathrm{H} & 0.252898 & -1.851799 & -2.226500\end{array}$

hydroquinone, cis $\mathrm{S}_{0}$

$\begin{array}{lrrr}\mathrm{C} & -0.695123 & 0.000000 & 1.088014 \\ \mathrm{C} & 0.695123 & 0.000000 & 1.088014 \\ \mathrm{C} & 1.394034 & 0.000000 & -0.109667 \\ \mathrm{C} & 0.691135 & 0.000000 & -1.309866 \\ \mathrm{C} & -0.691135 & 0.000000 & -1.309866 \\ \mathrm{C} & -1.394034 & 0.000000 & -0.109667 \\ \mathrm{O} & 2.756974 & 0.000000 & -0.175391 \\ \mathrm{O} & -2.756974 & 0.000000 & -0.175391 \\ \mathrm{H} & 1.244772 & 0.000000 & -2.241258 \\ \mathrm{H} & -1.244772 & 0.000000 & -2.241258 \\ \mathrm{H} & -1.229758 & 0.000000 & 2.033710 \\ \mathrm{H} & 1.229758 & 0.000000 & 2.033710 \\ \mathrm{H} & -3.117541 & 0.000000 & 0.714748\end{array}$




\section{H $\quad 3.117541 \quad 0.000000 \quad 0.714748$}

hydroquinone, cis $\mathrm{S}_{1}$

$\begin{array}{rrrr}\mathrm{C} & 0.706134 & 0.000000 & -1.128515 \\ \mathrm{C} & -0.706134 & 0.000000 & -1.128515 \\ \mathrm{C} & -1.370313 & 0.000000 & 0.120519 \\ \mathrm{C} & -0.706389 & 0.000000 & 1.360853 \\ \mathrm{C} & 0.706389 & 0.000000 & 1.360853 \\ \mathrm{C} & 1.370313 & 0.000000 & 0.120519 \\ \mathrm{O} & -2.712924 & 0.000000 & 0.164078 \\ \mathrm{O} & 2.712924 & 0.000000 & 0.164078 \\ \mathrm{H} & -1.288244 & 0.000000 & 2.271539 \\ \mathrm{H} & 1.288244 & 0.000000 & 2.271539 \\ \mathrm{H} & 1.273921 & 0.000000 & -2.051975 \\ \mathrm{H} & -1.273921 & 0.000000 & -2.051975 \\ \mathrm{H} & 3.059081 & 0.000000 & -0.735728 \\ \mathrm{H} & -3.059081 & 0.000000 & -0.735728\end{array}$

hydroquinone, trans $\mathrm{S}_{0}$

$\begin{array}{rrrr}\mathrm{C} & 0.021432 & 1.386731 & 0.000000 \\ \mathrm{C} & -1.194634 & 0.718528 & 0.000000 \\ \mathrm{C} & -1.208690 & -0.671299 & 0.000000 \\ \mathrm{C} & -0.021432 & -1.386731 & 0.000000 \\ \mathrm{C} & 1.194634 & -0.718528 & 0.000000 \\ \mathrm{C} & 1.208690 & 0.671299 & 0.000000 \\ \mathrm{O} & -2.332575 & 1.471642 & 0.000000 \\ \mathrm{H} & -2.154571 & -1.205959 & 0.000000 \\ \mathrm{H} & -0.027501 & -2.470129 & 0.000000 \\ \mathrm{O} & 2.332575 & -1.471642 & 0.000000 \\ \mathrm{H} & 2.154571 & 1.205959 & 0.000000 \\ \mathrm{H} & 0.027501 & 2.470129 & 0.000000 \\ \mathrm{H} & -3.096896 & 0.890104 & 0.000000 \\ \mathrm{H} & 3.096896 & -0.890104 & 0.000000\end{array}$

hydroquinone, trans $\mathrm{S}_{1}$

$\begin{array}{rrrr}\mathrm{C} & 1.260738 & 0.675264 & 0.000000 \\ \mathrm{C} & 0.036115 & 1.369834 & 0.000000 \\ \mathrm{C} & -1.227968 & 0.736942 & 0.000000 \\ \mathrm{C} & -1.260738 & -0.675264 & 0.000000 \\ \mathrm{C} & -0.036115 & -1.369834 & 0.000000 \\ \mathrm{C} & 1.227968 & -0.736942 & 0.000000 \\ \mathrm{O} & 0.118586 & 2.710597 & 0.000000 \\ \mathrm{H} & -2.138012 & 1.325861 & 0.000000\end{array}$




$\begin{array}{rrrr}\mathrm{H} & -2.184631 & -1.236442 & 0.000000 \\ \mathrm{O} & -0.118586 & -2.710597 & 0.000000 \\ \mathrm{H} & 2.138012 & -1.325861 & 0.000000 \\ \mathrm{H} & 2.184631 & 1.236442 & 0.000000 \\ \mathrm{H} & -0.771487 & 3.081835 & 0.000000 \\ \mathrm{H} & 0.771487 & -3.081835 & 0.000000\end{array}$

salicylic acid $\mathrm{S}_{0}$

$\begin{array}{lrrl}\mathrm{C} & 1.101102 & 1.593830 & 0.000000 \\ \mathrm{C} & 1.132280 & 0.197228 & 0.000000 \\ \mathrm{C} & -0.085953 & -0.515232 & 0.000000 \\ \mathrm{C} & -1.298201 & 0.186945 & 0.000000 \\ \mathrm{C} & -1.315833 & 1.563718 & 0.000000 \\ \mathrm{C} & -0.105434 & 2.260709 & 0.000000 \\ \mathrm{O} & 2.320976 & -0.405299 & 0.000000 \\ \mathrm{C} & -0.042154 & -1.972912 & 0.000000 \\ \mathrm{O} & -1.237846 & -2.573655 & 0.000000 \\ \mathrm{O} & 0.989200 & -2.629085 & 0.000000 \\ \mathrm{H} & -2.222562 & -0.377261 & 0.000000 \\ \mathrm{H} & 2.044270 & 2.126589 & 0.000000 \\ \mathrm{H} & -2.256955 & 2.099644 & 0.000000 \\ \mathrm{H} & -0.109969 & 3.345463 & 0.000000 \\ \mathrm{H} & 2.156755 & -1.374860 & 0.000000 \\ \mathrm{H} & -1.069676 & -3.525820 & 0.000000\end{array}$

salicylic acid $\mathrm{S}_{1}$

$\begin{array}{lrrr}\mathrm{C} & 0.994460 & 1.906665 & 0.000000 \\ \mathrm{C} & 0.958581 & 0.505006 & 0.000000 \\ \mathrm{C} & -0.294461 & -0.216671 & 0.000000 \\ \mathrm{C} & -1.447605 & 0.542775 & 0.000000 \\ \mathrm{C} & -1.398773 & 1.974635 & 0.000000 \\ \mathrm{C} & -0.201026 & 2.656410 & 0.000000 \\ \mathrm{O} & 2.066833 & -0.186203 & 0.000000 \\ \mathrm{C} & -0.230925 & -1.650542 & 0.000000 \\ \mathrm{O} & -1.406117 & -2.311589 & 0.000000 \\ \mathrm{O} & 0.864947 & -2.294182 & 0.000000 \\ \mathrm{H} & -2.410643 & 0.046808 & 0.000000 \\ \mathrm{H} & 1.967397 & 2.383505 & 0.000000 \\ \mathrm{H} & -2.336449 & 2.518470 & 0.000000 \\ \mathrm{H} & -0.171123 & 3.737760 & 0.000000 \\ \mathrm{H} & 1.743971 & -1.224004 & 0.000000 \\ \mathrm{H} & -1.188488 & -3.251897 & 0.000000\end{array}$

$o$-cresol, cis $\mathrm{S}_{0}$ 


$\begin{array}{rrrr}\mathrm{C} & 1.959621 & 0.511754 & 0.000000 \\ \mathrm{C} & 0.727346 & 1.147950 & 0.000000 \\ \mathrm{C} & -0.465295 & 0.431383 & 0.000000 \\ \mathrm{C} & -0.390875 & -0.965507 & 0.000000 \\ \mathrm{C} & 0.837263 & -1.614378 & 0.000000 \\ \mathrm{C} & 2.008632 & -0.875659 & 0.000000 \\ \mathrm{C} & -1.797443 & 1.120498 & 0.000000 \\ \mathrm{O} & -1.505925 & -1.746022 & 0.000000 \\ \mathrm{H} & 0.852184 & -2.698004 & 0.000000 \\ \mathrm{H} & 2.963865 & -1.388842 & 0.000000 \\ \mathrm{H} & 2.873234 & 1.094562 & 0.000000 \\ \mathrm{H} & 0.681700 & 2.232758 & 0.000000 \\ \mathrm{H} & -2.288207 & -1.187959 & 0.000000 \\ \mathrm{H} & -2.392471 & 0.867014 & 0.885611 \\ \mathrm{H} & -1.671158 & 2.203450 & 0.000000 \\ \mathrm{H} & -2.392471 & 0.867014 & -0.885611\end{array}$

$o$-cresol, cis $\mathrm{S}_{1}$

$\begin{array}{rrrr}\mathrm{C} & 1.851122 & -0.044231 & -0.779357 \\ \mathrm{C} & 0.622869 & 0.096372 & -1.454749 \\ \mathrm{C} & -0.593182 & 0.064017 & -0.711754 \\ \mathrm{C} & -0.470256 & 0.075622 & 0.709446 \\ \mathrm{C} & 0.767442 & 0.016253 & 1.395704 \\ \mathrm{C} & 1.941025 & -0.090966 & 0.625251 \\ \mathrm{C} & -1.888543 & -0.080322 & -1.422385 \\ \mathrm{O} & -1.556226 & 0.120883 & 1.492031 \\ \mathrm{H} & 0.774522 & 0.123344 & 2.471762 \\ \mathrm{H} & 2.908090 & -0.180481 & 1.102583 \\ \mathrm{H} & 2.762708 & -0.119435 & -1.363287 \\ \mathrm{H} & 0.593366 & 0.132245 & -2.536085 \\ \mathrm{H} & -2.340580 & 0.247527 & 0.939435 \\ \mathrm{H} & -2.742361 & -0.308431 & -0.774134 \\ \mathrm{H} & -2.149472 & 0.823809 & -1.991692 \\ \mathrm{H} & -1.831103 & -0.895695 & -2.160358\end{array}$

$o$-cresol, trans $\mathrm{S}_{0}$

$\begin{array}{lrrc}\mathrm{C} & 2.414841 & 0.000015 & 0.029394 \\ \mathrm{C} & 1.243567 & -0.000039 & -0.717390 \\ \mathrm{C} & 0.030124 & -0.000011 & -0.022245 \\ \mathrm{C} & 0.001158 & 0.000065 & 1.366295 \\ \mathrm{C} & 1.187341 & 0.000114 & 2.086952 \\ \mathrm{C} & 2.400976 & 0.000087 & 1.419033 \\ \mathrm{C} & 1.249248 & -0.000110 & -2.213767\end{array}$




$\begin{array}{rrrc}\mathrm{O} & -1.107489 & -0.000067 & -0.771915 \\ \mathrm{H} & -0.953831 & 0.000094 & 1.885486 \\ \mathrm{H} & 1.155768 & 0.000173 & 3.170741 \\ \mathrm{H} & 3.332578 & 0.000134 & 1.972742 \\ \mathrm{H} & 3.364048 & -0.000010 & -0.497627 \\ \mathrm{H} & -1.871045 & -0.000057 & -0.188643 \\ \mathrm{H} & 2.270823 & -0.000058 & -2.596311 \\ \mathrm{H} & 0.730360 & -0.876631 & -2.611348 \\ \mathrm{H} & 0.730239 & 0.876301 & -2.611427\end{array}$

$o$-cresol, trans $\mathrm{S}_{1}$

$\begin{array}{rrrr}\mathrm{C} & 2.421052 & 0.267766 & -0.006760 \\ \mathrm{C} & 1.211808 & 0.117698 & -0.753138 \\ \mathrm{C} & 0.009411 & -0.008873 & -0.016790 \\ \mathrm{C} & -0.049898 & -0.092488 & 1.406932 \\ \mathrm{C} & 1.175994 & -0.074690 & 2.096170 \\ \mathrm{C} & 2.383391 & 0.090023 & 1.385088 \\ \mathrm{C} & 1.251415 & -0.022034 & -2.227484 \\ \mathrm{O} & -1.130423 & -0.110947 & -0.716995 \\ \mathrm{H} & -0.996703 & 0.048633 & 1.918688 \\ \mathrm{H} & 1.198915 & -0.151725 & 3.176147 \\ \mathrm{H} & 3.314227 & 0.092012 & 1.943188 \\ \mathrm{H} & 3.360205 & 0.412581 & -0.524000 \\ \mathrm{H} & -1.831262 & -0.397641 & -0.113532 \\ \mathrm{H} & 1.847933 & -0.899410 & -2.521932 \\ \mathrm{H} & 0.259230 & -0.115204 & -2.665917 \\ \mathrm{H} & 1.753393 & 0.844298 & -2.679687\end{array}$

$m$-cresol, cis $\mathrm{S}_{0}$

$\begin{array}{rrrr}\mathrm{C} & 1.157568 & -0.004873 & 2.082239 \\ \mathrm{C} & 2.366392 & 0.009183 & 1.409943 \\ \mathrm{C} & 2.411927 & 0.019591 & 0.020810 \\ \mathrm{C} & 1.234573 & 0.014027 & -0.719122 \\ \mathrm{C} & 0.017381 & 0.002082 & -0.040455 \\ \mathrm{C} & -0.023838 & -0.008101 & 1.347942 \\ \mathrm{C} & 1.260582 & 0.002126 & -2.219355 \\ \mathrm{O} & -1.193170 & -0.018861 & 2.041961 \\ \mathrm{H} & 1.107574 & -0.010680 & 3.164338 \\ \mathrm{H} & 3.290268 & 0.014775 & 1.978157 \\ \mathrm{H} & 3.368054 & 0.032952 & -0.491052 \\ \mathrm{H} & -0.912382 & 0.001775 & -0.605704 \\ \mathrm{H} & -1.929440 & -0.019430 & 1.424400 \\ \mathrm{H} & 2.257607 & 0.231990 & -2.598200 \\ \mathrm{H} & 0.975908 & -0.980047 & -2.609182\end{array}$




\begin{tabular}{|c|c|c|c|}
\hline $\mathrm{H}$ & 0.563296 & 0.732862 & -2.636771 \\
\hline \multicolumn{4}{|c|}{$m$-cresol, cis $\mathrm{S}_{1}$} \\
\hline $\mathrm{C}$ & 1.163158 & 0.009973 & 2.120456 \\
\hline $\mathrm{C}$ & 2.403789 & 0.085993 & 1.444649 \\
\hline $\mathrm{C}$ & 2.402340 & 0.035007 & 0.035989 \\
\hline $\mathrm{C}$ & 1.218170 & -0.003395 & -0.742735 \\
\hline $\mathrm{C}$ & -0.031048 & 0.011026 & -0.0 \\
\hline $\mathrm{C}$ & -0.016704 & 0.005393 & 1.350320 \\
\hline $\mathrm{C}$ & 1.264984 & -0.032997 & 25942 \\
\hline $\mathrm{O}$ & -1.173442 & -0.038564 & 2.028302 \\
\hline $\mathrm{H}$ & 1.087502 & -0.111172 & 3.193414 \\
\hline $\mathrm{H}$ & 3.335345 & 0.114699 & 1.992169 \\
\hline $\mathrm{H}$ & 3.353254 & 0.037345 & -0.488512 \\
\hline $\mathrm{H}$ & -0.953208 & 0.220627 & -0.605051 \\
\hline $\mathrm{H}$ & -1.894297 & -0.189336 & 1.402252 \\
\hline $\mathrm{H}$ & 2.285223 & 0.060278 & -2.601740 \\
\hline $\mathrm{H}$ & 0.841611 & -0.966457 & -2.624608 \\
\hline $\mathrm{H}$ & 0.665602 & 0.780950 & -2.659118 \\
\hline
\end{tabular}

$m$-cresol, trans $\mathrm{S}_{0}$

$\begin{array}{rrrr}\mathrm{C} & -1.188078 & -1.096578 & 0.000000 \\ \mathrm{C} & 0.200779 & -1.208254 & 0.000000 \\ \mathrm{C} & 0.969468 & -0.048996 & 0.000000 \\ \mathrm{C} & 0.365746 & 1.201077 & 0.000000 \\ \mathrm{C} & -1.021798 & 1.307711 & 0.000000 \\ \mathrm{C} & -1.789148 & 0.153640 & 0.000000 \\ \mathrm{C} & 0.869038 & -2.551977 & 0.000000 \\ \mathrm{O} & 1.181302 & 2.289686 & 0.000000 \\ \mathrm{H} & -1.496131 & 2.285119 & 0.000000 \\ \mathrm{H} & -2.870787 & 0.233424 & 0.000000 \\ \mathrm{H} & -1.799684 & -1.992222 & 0.000000 \\ \mathrm{H} & 2.053091 & -0.100832 & 0.000000 \\ \mathrm{H} & 0.645504 & 3.087323 & 0.000000 \\ \mathrm{H} & 0.134738 & -3.358828 & 0.000000 \\ \mathrm{H} & 1.505523 & -2.675884 & -0.880536 \\ \mathrm{H} & 1.505523 & -2.675884 & 0.880536\end{array}$

$m$-cresol, trans $\mathrm{S}_{1}$
$\begin{array}{llll}\text { C } & -1.198090 & -1.054429 & 0.000000\end{array}$
$\begin{array}{llll}\mathrm{C} & 0.205867 & -1.233725 & 0.000000\end{array}$
$\begin{array}{llll}\text { C } & 1.025711 & -0.072357 & 0.000000\end{array}$
$\begin{array}{llll}\text { C } & 0.397165 & 1.191824 & 0.000000\end{array}$ 


$\begin{array}{rrrr}\mathrm{C} & -1.007904 & 1.370706 & 0.000000 \\ \mathrm{C} & -1.821084 & 0.213845 & 0.000000 \\ \mathrm{C} & 0.823348 & -2.583893 & 0.000000 \\ \mathrm{O} & 1.213423 & 2.258015 & 0.000000 \\ \mathrm{H} & -1.431538 & 2.369288 & 0.000000 \\ \mathrm{H} & -2.899506 & 0.292122 & 0.000000 \\ \mathrm{H} & -1.826372 & -1.939914 & 0.000000 \\ \mathrm{H} & 2.106283 & -0.125088 & 0.000000 \\ \mathrm{H} & 0.685327 & 3.065253 & 0.000000 \\ \mathrm{H} & 0.068218 & -3.372541 & 0.000000 \\ \mathrm{H} & 1.467816 & -2.732045 & -0.878122 \\ \mathrm{H} & 1.467816 & -2.732045 & 0.878122\end{array}$

$p$-cresol $\mathrm{S}_{0}$

$\begin{array}{lrrr}\mathrm{C} & 0.710248 & 0.013307 & -1.186798 \\ \mathrm{C} & -0.673290 & 0.015142 & -1.195583 \\ \mathrm{C} & -1.372119 & 0.000524 & 0.006535 \\ \mathrm{C} & -0.670167 & -0.017896 & 1.204429 \\ \mathrm{C} & 0.718600 & -0.019475 & 1.193950 \\ \mathrm{C} & 1.436954 & -0.001970 & 0.004160 \\ \mathrm{O} & -2.731774 & 0.000692 & -0.053137 \\ \mathrm{C} & 2.937503 & 0.021241 & -0.004313 \\ \mathrm{H} & -1.205588 & -0.034234 & 2.150044 \\ \mathrm{H} & 1.251331 & -0.036517 & 2.139727 \\ \mathrm{H} & 1.241144 & 0.022226 & -2.134026 \\ \mathrm{H} & -1.228967 & 0.024646 & -2.125945 \\ \mathrm{H} & -3.088349 & -0.012243 & 0.838971 \\ \mathrm{H} & 3.343735 & -0.672822 & -0.744353 \\ \mathrm{H} & 3.319330 & 1.017502 & -0.249307 \\ \mathrm{H} & 3.342969 & -0.254747 & 0.970947\end{array}$

$p$-cresol $\mathrm{S}_{1}$
$\begin{array}{llll}\text { C } & 0.731845 & -0.083731 & -1.233612\end{array}$
$\begin{array}{llll}\text { C } & -0.681302 & -0.028374 & -1.242110\end{array}$
$\begin{array}{llll}\text { C } & -1.350002 & -0.008978 & -0.002108\end{array}$
$\begin{array}{llll}\text { C } & -0.681891 & -0.000870 & 1.247695\end{array}$
$\begin{array}{llll}\text { C } & 0.730317 & -0.021672 & 1.235705\end{array}$
$\begin{array}{llll}\text { C } & 1.427054 & -0.037251 & 0.001025\end{array}$
$\begin{array}{llll}\text { O } & -2.690141 & 0.019732 & -0.047496\end{array}$
$\begin{array}{llll}\text { C } & 2.907700 & 0.039762 & -0.000195\end{array}$
$\begin{array}{llll}\mathrm{H} & -1.247694 & -0.057203 & 2.171168\end{array}$
$\begin{array}{llll}\mathrm{H} & 1.290968 & -0.016142 & 2.161911\end{array}$
H $\quad 1.290274 \quad-0.107357 \quad-2.160438$
$\begin{array}{llll}\mathrm{H} & -1.260777 & 0.032972 & -2.153263\end{array}$ 


$$
\begin{array}{rrrr}
\mathrm{H} & -3.035088 & 0.100649 & 0.850214 \\
\mathrm{H} & 3.332275 & -0.486286 & -0.859882 \\
\mathrm{H} & 3.234557 & 1.087299 & -0.073706 \\
\mathrm{H} & 3.333455 & -0.367169 & 0.920384
\end{array}
$$

$o$-methoxyphenol $\mathrm{S}_{0}$

$\begin{array}{rrrr}\mathrm{C} & 2.023520 & -0.086516 & 0.000000 \\ \mathrm{C} & 0.870458 & 0.695927 & 0.000000 \\ \mathrm{C} & -0.371361 & 0.084796 & 0.000000 \\ \mathrm{C} & -0.470370 & -1.314323 & 0.000000 \\ \mathrm{C} & 0.678997 & -2.081870 & 0.000000 \\ \mathrm{C} & 1.926594 & -1.467002 & 0.000000 \\ \mathrm{O} & -1.578508 & 0.721432 & 0.000000 \\ \mathrm{O} & -1.687452 & -1.906644 & 0.000000 \\ \mathrm{H} & 0.580331 & -3.161079 & 0.000000 \\ \mathrm{H} & 2.822638 & -2.076709 & 0.000000 \\ \mathrm{H} & 2.993840 & 0.395841 & 0.000000 \\ \mathrm{H} & 0.951599 & 1.775805 & 0.000000 \\ \mathrm{C} & -1.582249 & 2.130933 & 0.000000 \\ \mathrm{H} & -2.349627 & -1.204440 & 0.000000 \\ \mathrm{H} & -2.627683 & 2.434451 & 0.000000 \\ \mathrm{H} & -1.090363 & 2.529699 & 0.893500 \\ \mathrm{H} & -1.090363 & 2.529699 & -0.893500\end{array}$

$o$-methoxyphenol $\mathrm{S}_{1}$

$\begin{array}{lrcc}\mathrm{C} & 2.466707 & 0.069305 & 0.052764 \\ \mathrm{C} & 1.374233 & -0.294477 & -0.748976 \\ \mathrm{C} & 0.108154 & -0.281123 & -0.091233 \\ \mathrm{C} & 0.016898 & -0.163905 & 1.315882 \\ \mathrm{C} & 1.177765 & -0.068342 & 2.133253 \\ \mathrm{C} & 2.373283 & 0.179211 & 1.455668 \\ \mathrm{O} & -1.079619 & -0.089335 & -0.725952 \\ \mathrm{O} & -1.176824 & 0.060962 & 1.884453 \\ \mathrm{H} & 1.108441 & -0.183116 & 3.206698 \\ \mathrm{H} & 3.266099 & 0.446982 & 2.012917 \\ \mathrm{H} & 3.430213 & 0.257872 & -0.414589 \\ \mathrm{H} & 1.506635 & -0.659418 & -1.760870 \\ \mathrm{C} & -1.077325 & 0.130508 & -2.131265 \\ \mathrm{H} & -1.849962 & 0.043173 & 1.183249 \\ \mathrm{H} & -2.112666 & 0.328486 & -2.401520 \\ \mathrm{H} & -0.447675 & 0.983334 & -2.393858 \\ \mathrm{H} & -0.725797 & -0.760125 & -2.658540\end{array}$

m-methoxyphenol, isomer $1 \mathrm{~S}_{0}$ 


$\begin{array}{lrrc}\mathrm{C} & 0.926270 & 1.445577 & 0.000000 \\ \mathrm{C} & -0.262269 & 0.717863 & 0.000000 \\ \mathrm{C} & -0.230991 & -0.673952 & 0.000000 \\ \mathrm{C} & 0.998574 & -1.331680 & 0.000000 \\ \mathrm{C} & 2.186875 & -0.617579 & 0.000000 \\ \mathrm{C} & 2.132421 & 0.769490 & 0.000000 \\ \mathrm{H} & -1.146685 & -1.254464 & 0.000000 \\ \mathrm{O} & 1.078881 & -2.689066 & 0.000000 \\ \mathrm{H} & 3.128246 & -1.152105 & 0.000000 \\ \mathrm{H} & 3.057349 & 1.335729 & 0.000000 \\ \mathrm{H} & 0.877764 & 2.527153 & 0.000000 \\ \mathrm{O} & -1.405610 & 1.445049 & 0.000000 \\ \mathrm{H} & 0.194247 & -3.063638 & 0.000000 \\ \mathrm{C} & -2.633193 & 0.756311 & 0.000000 \\ \mathrm{H} & -3.408808 & 1.520301 & 0.000000 \\ \mathrm{H} & -2.746536 & 0.132505 & -0.893927 \\ \mathrm{H} & -2.746536 & 0.132505 & 0.893927\end{array}$

$m$-methoxyphenol, isomer $1 \mathrm{~S}_{1}$

$\begin{array}{lrrr}\mathrm{C} & 1.165844 & -0.018485 & -0.616203 \\ \mathrm{C} & -0.030791 & -0.039903 & 0.130340 \\ \mathrm{C} & -0.054659 & -0.090578 & 1.548203 \\ \mathrm{C} & 1.231657 & -0.003221 & 2.142410 \\ \mathrm{C} & 2.436114 & 0.014451 & 1.412643 \\ \mathrm{C} & 2.429407 & -0.124933 & 0.009374 \\ \mathrm{H} & -0.877827 & -0.549152 & 2.093575 \\ \mathrm{O} & 1.338472 & 0.136279 & 3.480525 \\ \mathrm{H} & 3.355026 & 0.180798 & 1.964628 \\ \mathrm{H} & 3.343750 & -0.152400 & -0.563503 \\ \mathrm{H} & 1.076021 & 0.101867 & -1.690440 \\ \mathrm{O} & -1.151087 & 0.046771 & -0.615352 \\ \mathrm{H} & 0.464925 & 0.367830 & 3.826327 \\ \mathrm{C} & -2.407306 & 0.033984 & 0.033298 \\ \mathrm{H} & -3.137490 & 0.336653 & -0.715043 \\ \mathrm{H} & -2.652521 & -0.972370 & 0.387954 \\ \mathrm{H} & -2.421224 & 0.732407 & 0.874003\end{array}$

$m$-methoxyphenol, isomer $2 \mathrm{~S}_{0}$
$\begin{array}{llll}\text { C } & 0.346206 & 2.154346 & 0.000000\end{array}$
$\begin{array}{llll}\text { C } & -0.836910 & 1.444142 & 0.000000\end{array}$
$\begin{array}{llll}\text { C } & -0.795438 & 0.047674 & 0.000000\end{array}$
$\begin{array}{llll}\text { C } & 0.423126 & -0.618551 & 0.000000\end{array}$
$\begin{array}{llll}\text { C } & 1.603972 & 0.119876 & 0.000000\end{array}$ 


$\begin{array}{rrrc}\mathrm{C} & 1.577426 & 1.506888 & 0.000000 \\ \mathrm{O} & -1.995342 & -0.578889 & 0.000000 \\ \mathrm{C} & -2.007939 & -1.988114 & 0.000000 \\ \mathrm{O} & 2.763519 & -0.590894 & 0.000000 \\ \mathrm{H} & 0.494694 & -1.697559 & 0.000000 \\ \mathrm{H} & 2.501898 & 2.076003 & 0.000000 \\ \mathrm{H} & 0.316758 & 3.238389 & 0.000000 \\ \mathrm{H} & -1.799472 & 1.939724 & 0.000000 \\ \mathrm{H} & -3.056677 & -2.280341 & 0.000000 \\ \mathrm{H} & -1.521341 & -2.395343 & -0.892988 \\ \mathrm{H} & -1.521341 & -2.395343 & 0.892988 \\ \mathrm{H} & 3.506853 & 0.018003 & 0.000000\end{array}$

$m$-methoxyphenol, isomer $2 \mathrm{~S}_{1}$

$\begin{array}{cccc}\mathrm{C} & 1.241534 & -0.129749 & -0.704470 \\ \mathrm{C} & 0.059095 & -0.029530 & 0.077431 \\ \mathrm{C} & 0.111050 & -0.057788 & 1.486166 \\ \mathrm{C} & 1.332013 & -0.092725 & 2.203247 \\ \mathrm{C} & 2.493784 & 0.001174 & 1.407176 \\ \mathrm{C} & 2.461468 & 0.037808 & -0.002423 \\ \mathrm{O} & -1.091926 & -0.007454 & 2.092611 \\ \mathrm{C} & -1.143893 & 0.043345 & 3.503861 \\ \mathrm{O} & 3.665787 & 0.081762 & 2.075408 \\ \mathrm{H} & 1.412648 & -0.296668 & 3.259957 \\ \mathrm{H} & 3.388422 & 0.218977 & -0.541588 \\ \mathrm{H} & 1.206336 & -0.120086 & -1.782974 \\ \mathrm{H} & -0.913843 & 0.098325 & -0.383364 \\ \mathrm{H} & -2.195258 & 0.156919 & 3.760991 \\ \mathrm{H} & -0.761341 & -0.881587 & 3.946148 \\ \mathrm{H} & -0.573957 & 0.893432 & 3.889509 \\ \mathrm{H} & 4.393214 & 0.083848 & 1.444215\end{array}$

p-methoxyphenol, cis $\mathrm{S}_{0}$

$\begin{array}{lrrr}\mathrm{C} & -0.317387 & 0.733443 & 0.000000 \\ \mathrm{C} & -1.546278 & 0.072613 & 0.000000 \\ \mathrm{C} & -1.605102 & -1.305397 & 0.000000 \\ \mathrm{C} & -0.432279 & -2.057090 & 0.000000 \\ \mathrm{C} & 0.789493 & -1.405427 & 0.000000 \\ \mathrm{C} & 0.852705 & -0.013625 & 0.000000 \\ \mathrm{O} & -0.553797 & -3.415554 & 0.000000 \\ \mathrm{O} & -0.369568 & 2.092695 & 0.000000 \\ \mathrm{C} & 0.850545 & 2.791826 & 0.000000 \\ \mathrm{H} & 1.823137 & 0.465559 & 0.000000 \\ \mathrm{H} & -2.453171 & 0.665882 & 0.000000\end{array}$




$\begin{array}{rrrr}\mathrm{H} & -2.558596 & -1.820201 & 0.000000 \\ \mathrm{H} & 1.712951 & -1.978063 & 0.000000 \\ \mathrm{H} & 0.594978 & 3.850307 & 0.000000 \\ \mathrm{H} & 1.445834 & 2.567948 & 0.893151 \\ \mathrm{H} & 1.445834 & 2.567948 & -0.893151 \\ \mathrm{H} & 0.320698 & -3.812845 & 0.000000\end{array}$

$p$-methoxyphenol, cis $\mathrm{S}_{1}$

$\begin{array}{lrrr}\mathrm{C} & 1.326589 & -0.022174 & -0.614479 \\ \mathrm{C} & 0.051925 & -0.043089 & 0.001166 \\ \mathrm{C} & -0.015443 & 0.000354 & 1.411142 \\ \mathrm{C} & 1.188267 & -0.004963 & 2.133200 \\ \mathrm{C} & 2.466254 & -0.010612 & 1.523699 \\ \mathrm{C} & 2.536848 & -0.011958 & 0.112110 \\ \mathrm{O} & 1.088911 & 0.008213 & 3.473061 \\ \mathrm{O} & 1.287494 & -0.027650 & -1.953885 \\ \mathrm{C} & 2.489383 & 0.023330 & -2.699229 \\ \mathrm{H} & 3.493979 & -0.017282 & -0.388191 \\ \mathrm{H} & -0.829539 & -0.043935 & -0.623791 \\ \mathrm{H} & -0.952575 & 0.069490 & 1.945728 \\ \mathrm{H} & 3.358545 & -0.123911 & 2.129492 \\ \mathrm{H} & 2.190845 & 0.014134 & -3.745266 \\ \mathrm{H} & 3.045006 & 0.939008 & -2.481168 \\ \mathrm{H} & 3.115627 & -0.847636 & -2.488404 \\ \mathrm{H} & 1.971413 & 0.098666 & 3.851818\end{array}$

$p$-methoxyphenol, trans $\mathrm{S}_{0}$

$\begin{array}{crcc}\mathrm{C} & -0.655728 & 1.302548 & 0.000000 \\ \mathrm{C} & -1.884967 & 0.669004 & 0.000000 \\ \mathrm{C} & -1.960068 & -0.721223 & 0.000000 \\ \mathrm{C} & -0.789607 & -1.462251 & 0.000000 \\ \mathrm{C} & 0.447191 & -0.829254 & 0.000000 \\ \mathrm{C} & 0.521894 & 0.560095 & 0.000000 \\ \mathrm{O} & -3.144768 & -1.397536 & 0.000000 \\ \mathrm{H} & 1.343842 & -1.435618 & 0.000000 \\ \mathrm{O} & 1.680282 & 1.273610 & 0.000000 \\ \mathrm{H} & -0.589219 & 2.384119 & 0.000000 \\ \mathrm{H} & -2.794231 & 1.264073 & 0.000000 \\ \mathrm{H} & -3.869869 & -0.767759 & 0.000000 \\ \mathrm{H} & -0.852622 & -2.544005 & 0.000000 \\ \mathrm{C} & 2.889150 & 0.554394 & 0.000000 \\ \mathrm{H} & 3.684970 & 1.297614 & 0.000000 \\ \mathrm{H} & 2.986870 & -0.073915 & 0.893027 \\ \mathrm{H} & 2.986870 & -0.073915 & -0.893027\end{array}$


p-methoxyphenol, trans $\mathrm{S}_{1}$

$\begin{array}{lrrc}\mathrm{C} & 0.123794 & -0.045683 & -1.390538 \\ \mathrm{C} & -1.277616 & -0.011043 & -1.215602 \\ \mathrm{C} & -1.777954 & 0.022239 & 0.103582 \\ \mathrm{C} & -0.957280 & 0.055058 & 1.246877 \\ \mathrm{C} & 0.445794 & 0.028933 & 1.075042 \\ \mathrm{C} & 0.951064 & -0.010647 & -0.241529 \\ \mathrm{O} & -3.102712 & 0.037495 & 0.327937 \\ \mathrm{H} & 1.102181 & 0.060641 & 1.931703 \\ \mathrm{O} & 2.262430 & -0.029791 & -0.517182 \\ \mathrm{H} & 0.588368 & -0.083944 & -2.365557 \\ \mathrm{H} & -1.955890 & -0.086337 & -2.057903 \\ \mathrm{H} & -3.564619 & 0.054910 & -0.518145 \\ \mathrm{H} & -1.417003 & 0.113830 & 2.223501 \\ \mathrm{C} & 3.207221 & -0.017498 & 0.536519 \\ \mathrm{H} & 4.184599 & -0.042234 & 0.059530 \\ \mathrm{H} & 3.111690 & 0.892631 & 1.134399 \\ \mathrm{H} & 3.086723 & -0.894249 & 1.178068\end{array}$

1-naphthol, cis $\mathrm{S}_{0}$

$\begin{array}{lrrl}\mathrm{C} & 0.830530 & 1.515713 & 0.016065 \\ \mathrm{C} & 0.527547 & 0.125192 & 0.001400 \\ \mathrm{C} & -0.843446 & -0.266021 & -0.007509 \\ \mathrm{C} & -1.854136 & 0.719773 & -0.016254 \\ \mathrm{C} & -1.517993 & 2.046444 & -0.014625 \\ \mathrm{C} & -0.174047 & 2.449825 & 0.003035 \\ \mathrm{C} & 1.520280 & -0.881405 & -0.006860 \\ \mathrm{C} & 1.184534 & -2.208601 & -0.010636 \\ \mathrm{C} & -0.168405 & -2.595719 & -0.010042 \\ \mathrm{C} & -1.152661 & -1.645978 & -0.010523 \\ \mathrm{O} & 2.108755 & 1.970926 & 0.033768 \\ \mathrm{H} & -2.197357 & -1.939888 & -0.014680 \\ \mathrm{H} & -0.426217 & -3.648837 & -0.012522 \\ \mathrm{H} & 1.962094 & -2.963876 & -0.018209 \\ \mathrm{H} & 2.576261 & -0.624707 & -0.020276 \\ \mathrm{H} & -2.892879 & 0.408833 & -0.024742 \\ \mathrm{H} & -2.293317 & 2.804432 & -0.022992 \\ \mathrm{H} & 0.092677 & 3.499835 & 0.012288 \\ \mathrm{H} & 2.717792 & 1.234069 & 0.123311\end{array}$

1-naphthol, cis $\mathrm{S}_{1}$

$\begin{array}{llll}\text { C } & 0.862804 & 1.466575 & 0.017341\end{array}$ 


$\begin{array}{rrrr}\mathrm{C} & 0.532170 & 0.087939 & -0.063223 \\ \mathrm{C} & -0.843219 & -0.245548 & 0.005986 \\ \mathrm{C} & -1.788079 & 0.807624 & -0.018949 \\ \mathrm{C} & -1.426621 & 2.170073 & -0.028562 \\ \mathrm{C} & -0.100970 & 2.510332 & -0.028020 \\ \mathrm{C} & 1.512532 & -0.927358 & -0.127970 \\ \mathrm{C} & 1.099990 & -2.286678 & -0.045707 \\ \mathrm{C} & -0.230355 & -2.609800 & 0.047177 \\ \mathrm{C} & -1.227294 & -1.604525 & 0.053956 \\ \mathrm{O} & 2.129150 & 1.848470 & 0.154545 \\ \mathrm{H} & -2.276577 & -1.863775 & 0.117962 \\ \mathrm{H} & -0.527261 & -3.650862 & 0.110074 \\ \mathrm{H} & 1.849061 & -3.069956 & -0.065556 \\ \mathrm{H} & 2.553581 & -0.707080 & -0.342804 \\ \mathrm{H} & -2.841285 & 0.545900 & -0.029331 \\ \mathrm{H} & -2.194592 & 2.932799 & -0.057986 \\ \mathrm{H} & 0.246084 & 3.535652 & -0.011563 \\ \mathrm{H} & 2.670881 & 1.060207 & 0.312609\end{array}$

1-naphthol, trans $\mathrm{S}_{0}$

$\begin{array}{lrrl}\mathrm{C} & -0.714631 & -0.539245 & 0.000000 \\ \mathrm{C} & -0.672094 & -1.952245 & 0.000000 \\ \mathrm{C} & 0.521512 & -2.622310 & 0.000000 \\ \mathrm{C} & 1.736007 & -1.910475 & 0.000000 \\ \mathrm{C} & 1.734565 & -0.540896 & 0.000000 \\ \mathrm{C} & 0.516620 & 0.172710 & 0.000000 \\ \mathrm{H} & -1.609245 & -2.499529 & 0.000000 \\ \mathrm{H} & 0.535112 & -3.706757 & 0.000000 \\ \mathrm{H} & 2.675866 & -2.451187 & 0.000000 \\ \mathrm{H} & 2.664165 & 0.014554 & 0.000000 \\ \mathrm{C} & -1.936158 & 0.174213 & 0.000000 \\ \mathrm{C} & -1.932566 & 1.541100 & 0.000000 \\ \mathrm{C} & -0.722451 & 2.259306 & 0.000000 \\ \mathrm{C} & 0.476503 & 1.592688 & 0.000000 \\ \mathrm{H} & -2.869602 & -0.377807 & 0.000000 \\ \mathrm{H} & -2.868127 & 2.089275 & 0.000000 \\ \mathrm{H} & -0.739225 & 3.345613 & 0.000000 \\ \mathrm{O} & 1.675578 & 2.230696 & 0.000000 \\ \mathrm{H} & 1.528182 & 3.180285 & 0.000000\end{array}$

1-naphthol, trans $\mathrm{S}_{1}$
$\begin{array}{llll}\text { C } & -0.933731 & -0.391293 & 0.000000\end{array}$
$\begin{array}{llll}\text { C } & -1.307945 & -1.748668 & 0.000000\end{array}$
$\begin{array}{llll}\text { C } & -0.314291 & -2.762966 & 0.000000\end{array}$ 


$\begin{array}{lrrr}\mathrm{C} & 1.015358 & -2.426910 & 0.000000 \\ \mathrm{C} & 1.424705 & -1.066608 & 0.000000 \\ \mathrm{C} & 0.450939 & -0.050085 & 0.000000 \\ \mathrm{H} & -2.359523 & -2.009649 & 0.000000 \\ \mathrm{H} & -0.614509 & -3.804445 & 0.000000 \\ \mathrm{H} & 1.772630 & -3.203033 & 0.000000 \\ \mathrm{H} & 2.474527 & -0.808333 & 0.000000 \\ \mathrm{C} & -1.885124 & 0.651168 & 0.000000 \\ \mathrm{C} & -1.524854 & 2.018286 & 0.000000 \\ \mathrm{C} & -0.201442 & 2.362813 & 0.000000 \\ \mathrm{C} & 0.778014 & 1.326604 & 0.000000 \\ \mathrm{H} & -2.937700 & 0.387382 & 0.000000 \\ \mathrm{H} & -2.295605 & 2.779095 & 0.000000 \\ \mathrm{H} & 0.117716 & 3.400578 & 0.000000 \\ \mathrm{O} & 2.080276 & 1.643784 & 0.000000 \\ \mathrm{H} & 2.184880 & 2.601351 & 0.000000\end{array}$

2-naphthol, cis $\mathrm{S}_{0}$

$\begin{array}{lrrl}\mathrm{C} & 0.596761 & 2.004599 & 0.000000 \\ \mathrm{C} & 0.620281 & 0.593076 & 0.000000 \\ \mathrm{C} & -0.610164 & -0.121713 & 0.000000 \\ \mathrm{C} & -1.818691 & 0.611973 & 0.000000 \\ \mathrm{C} & -1.808331 & 1.981300 & 0.000000 \\ \mathrm{C} & -0.590053 & 2.687777 & 0.000000 \\ \mathrm{C} & 1.828301 & -0.143551 & 0.000000 \\ \mathrm{C} & 1.827577 & -1.508142 & 0.000000 \\ \mathrm{C} & 0.604157 & -2.212232 & 0.000000 \\ \mathrm{C} & -0.587498 & -1.534475 & 0.000000 \\ \mathrm{H} & -1.529517 & -2.077127 & 0.000000 \\ \mathrm{H} & 1.540231 & 2.541535 & 0.000000 \\ \mathrm{H} & -0.596365 & 3.771883 & 0.000000 \\ \mathrm{H} & -2.744572 & 2.528740 & 0.000000 \\ \mathrm{H} & -2.760096 & 0.071620 & 0.000000 \\ \mathrm{H} & 2.769920 & 0.396096 & 0.000000 \\ \mathrm{H} & 2.749954 & -2.076725 & 0.000000 \\ \mathrm{O} & 0.695591 & -3.567443 & 0.000000 \\ \mathrm{H} & -0.187498 & -3.947182 & 0.000000\end{array}$

2-naphthol, cis $\mathrm{S}_{1}$
C $\quad 0.598017$
$1.987444 \quad 0.000000$
$\begin{array}{llll}\text { C } & 0.639236 & 0.583307 & 0.000000\end{array}$
$\begin{array}{llll}\text { C } & -0.621076 & -0.115669 & 0.000000\end{array}$
$\begin{array}{llll}\text { C } & -1.826468 & 0.605818 & 0.000000\end{array}$
$\begin{array}{llll}\text { C } & -1.836369 & 2.018378 & 0.000000\end{array}$ 


$\begin{array}{lrrr}\mathrm{C} & -0.629747 & 2.687844 & 0.000000 \\ \mathrm{C} & 1.842845 & -0.152989 & 0.000000 \\ \mathrm{C} & 1.879115 & -1.556806 & 0.000000 \\ \mathrm{C} & 0.660225 & -2.219185 & 0.000000 \\ \mathrm{C} & -0.576907 & -1.519805 & 0.000000 \\ \mathrm{H} & -1.503729 & -2.088950 & 0.000000 \\ \mathrm{H} & 1.528710 & 2.544380 & 0.000000 \\ \mathrm{H} & -0.610448 & 3.772489 & 0.000000 \\ \mathrm{H} & -2.773654 & 2.559672 & 0.000000 \\ \mathrm{H} & -2.763075 & 0.057691 & 0.000000 \\ \mathrm{H} & 2.777116 & 0.398698 & 0.000000 \\ \mathrm{H} & 2.803865 & -2.115689 & 0.000000 \\ \mathrm{O} & 0.659736 & -3.560903 & 0.000000 \\ \mathrm{H} & -0.247379 & -3.885714 & 0.000000\end{array}$

2-naphthol, trans $\mathrm{S}_{0}$

$\begin{array}{lrrl}\mathrm{C} & 0.509987 & 2.006505 & 0.000000 \\ \mathrm{C} & 0.538739 & 0.593827 & 0.000000 \\ \mathrm{C} & -0.689344 & -0.127863 & 0.000000 \\ \mathrm{C} & -1.901512 & 0.602940 & 0.000000 \\ \mathrm{C} & -1.895721 & 1.971186 & 0.000000 \\ \mathrm{C} & -0.679138 & 2.683510 & 0.000000 \\ \mathrm{C} & 1.747188 & -0.136401 & 0.000000 \\ \mathrm{C} & 1.746262 & -1.503652 & 0.000000 \\ \mathrm{C} & 0.525833 & -2.213230 & 0.000000 \\ \mathrm{C} & -0.666558 & -1.537530 & 0.000000 \\ \mathrm{H} & -1.594689 & -2.097858 & 0.000000 \\ \mathrm{H} & 1.451158 & 2.547552 & 0.000000 \\ \mathrm{H} & -0.690585 & 3.767605 & 0.000000 \\ \mathrm{H} & -2.833764 & 2.515569 & 0.000000 \\ \mathrm{H} & -2.839916 & 0.057873 & 0.000000 \\ \mathrm{H} & 2.688346 & 0.403966 & 0.000000 \\ \mathrm{H} & 2.684850 & -2.051604 & 0.000000 \\ \mathrm{O} & 0.499715 & -3.572526 & 0.000000 \\ \mathrm{H} & 1.399161 & -3.909868 & 0.000000\end{array}$

2-naphthol, trans $\mathrm{S}_{1}$
$\begin{array}{llll}\text { C } & 0.510247 & 1.992357 & 0.000000\end{array}$
$\begin{array}{llll}\text { C } & 0.553455 & 0.588134 & 0.000000\end{array}$
$\begin{array}{llll}\text { C } & -0.703263 & -0.116030 & 0.000000\end{array}$
$\begin{array}{llll}\text { C } & -1.909574 & 0.602537 & 0.000000\end{array}$
$\begin{array}{llll}\text { C } & -1.922372 & 2.015841 & 0.000000\end{array}$
$\begin{array}{llll}\text { C } & -0.719303 & 2.689624 & 0.000000\end{array}$
$\begin{array}{llll}\text { C } & 1.759567 & -0.143302 & 0.000000\end{array}$ 


$\begin{array}{lrrl}\mathrm{C} & 1.796813 & -1.548205 & 0.000000 \\ \mathrm{C} & 0.574883 & -2.212845 & 0.000000 \\ \mathrm{C} & -0.660619 & -1.520531 & 0.000000 \\ \mathrm{H} & -1.571559 & -2.108072 & 0.000000 \\ \mathrm{H} & 1.439814 & 2.551308 & 0.000000 \\ \mathrm{H} & -0.703571 & 3.774314 & 0.000000 \\ \mathrm{H} & -2.861594 & 2.554036 & 0.000000 \\ \mathrm{H} & -2.844309 & 0.051719 & 0.000000 \\ \mathrm{H} & 2.692737 & 0.410341 & 0.000000 \\ \mathrm{H} & 2.733943 & -2.092015 & 0.000000 \\ \mathrm{O} & 0.475997 & -3.551259 & 0.000000 \\ \mathrm{H} & 1.358709 & -3.937944 & 0.000000\end{array}$

5-methoxysalicylic acid $\mathrm{S}_{0}$

$\begin{array}{lrrc}\mathrm{C} & 2.273824 & -0.164666 & 0.000000 \\ \mathrm{C} & 1.562979 & -1.338928 & 0.000000 \\ \mathrm{C} & 0.160302 & -1.336593 & 0.000000 \\ \mathrm{C} & -0.509798 & -0.132650 & 0.000000 \\ \mathrm{C} & 0.208524 & 1.077304 & 0.000000 \\ \mathrm{C} & 1.612952 & 1.069055 & 0.000000 \\ \mathrm{O} & -0.430768 & -2.558853 & 0.000000 \\ \mathrm{C} & -1.838084 & -2.598683 & 0.000000 \\ \mathrm{C} & -0.478336 & 2.362548 & 0.000000 \\ \mathrm{O} & -1.816244 & 2.289227 & 0.000000 \\ \mathrm{O} & 2.354652 & 2.180265 & 0.000000 \\ \mathrm{O} & 0.087951 & 3.445890 & 0.000000 \\ \mathrm{H} & -2.112394 & -3.652158 & 0.000000 \\ \mathrm{H} & -2.255013 & -2.118798 & -0.893017 \\ \mathrm{H} & -2.255013 & -2.118798 & 0.893017 \\ \mathrm{H} & -1.589602 & -0.084132 & 0.000000 \\ \mathrm{H} & 3.357045 & -0.166672 & 0.000000 \\ \mathrm{H} & 2.074116 & -2.294949 & 0.000000 \\ \mathrm{H} & 1.734582 & 2.941981 & 0.000000 \\ \mathrm{H} & -2.141655 & 3.199611 & 0.000000\end{array}$

5-methoxysalicylic acid $\mathrm{S}_{1}$

$\begin{array}{lrrc}\text { C } & 2.168051 & -0.137447 & 0.000000 \\ \mathrm{C} & 1.729513 & -1.458555 & 0.000000 \\ \mathrm{C} & 0.345742 & -1.686637 & 0.000000 \\ \mathrm{C} & -0.590941 & -0.618702 & 0.000000 \\ \mathrm{C} & -0.159878 & 0.702005 & 0.000000 \\ \mathrm{C} & 1.263706 & 0.929182 & 0.000000 \\ \mathrm{O} & -0.031919 & -2.967846 & 0.000000 \\ \mathrm{C} & -1.406968 & -3.315279 & 0.000000\end{array}$




$\begin{array}{rrrr}\mathrm{C} & -1.037212 & 1.837236 & 0.000000 \\ \mathrm{O} & -2.364444 & 1.557457 & 0.000000 \\ \mathrm{O} & 1.707169 & 2.169056 & 0.000000 \\ \mathrm{O} & -0.640007 & 3.030964 & 0.000000 \\ \mathrm{H} & -1.434029 & -4.402367 & 0.000000 \\ \mathrm{H} & -1.907399 & -2.936250 & -0.894488 \\ \mathrm{H} & -1.907399 & -2.936250 & 0.894488 \\ \mathrm{H} & -1.653970 & -0.817462 & 0.000000 \\ \mathrm{H} & 3.224502 & 0.102590 & 0.000000 \\ \mathrm{H} & 2.411052 & -2.297343 & 0.000000 \\ \mathrm{H} & 0.868843 & 2.764208 & 0.000000 \\ \mathrm{H} & -2.812358 & 2.412216 & 0.000000\end{array}$

$m$-dimethoxybenzene, trans $\mathrm{S}_{0}$

$\begin{array}{rrrr}\mathrm{C} & 0.000000 & -0.705967 & -0.179561 \\ \mathrm{C} & 0.000000 & 0.705967 & -0.179561 \\ \mathrm{C} & 0.000000 & 1.386159 & 1.027173 \\ \mathrm{C} & 0.000000 & 0.689390 & 2.235185 \\ \mathrm{C} & 0.000000 & -0.689390 & 2.235185 \\ \mathrm{C} & 0.000000 & -1.386159 & 1.027173 \\ \mathrm{O} & 0.000000 & 1.292295 & -1.398009 \\ \mathrm{H} & 0.000000 & 2.468469 & 1.037540 \\ \mathrm{H} & 0.000000 & 1.240764 & 3.168243 \\ \mathrm{H} & 0.000000 & -1.240764 & 3.168243 \\ \mathrm{H} & 0.000000 & -2.468469 & 1.037540 \\ \mathrm{O} & 0.000000 & -1.292295 & -1.398009 \\ \mathrm{C} & 0.000000 & -2.698578 & -1.446479 \\ \mathrm{H} & 0.000000 & -2.963242 & -2.502607 \\ \mathrm{H} & 0.892946 & -3.120100 & -0.970733 \\ \mathrm{H} & -0.892946 & -3.120100 & -0.970733 \\ \mathrm{C} & 0.000000 & 2.698578 & -1.446479 \\ \mathrm{H} & 0.000000 & 2.963242 & -2.502607 \\ \mathrm{H} & -0.892946 & 3.120100 & -0.970733 \\ \mathrm{H} & 0.892946 & 3.120100 & -0.970733\end{array}$

$m$-dimethoxybenzene, trans $\mathrm{S}_{1}$
C $\quad-0.131453 \quad-1.060624 \quad-1.442117$
$\begin{array}{llll}\text { C } & -0.294373 & 0.157231 & -0.707340\end{array}$
$\begin{array}{llll}\text { C } & -0.294373 & 0.157231 & 0.707340\end{array}$
$\begin{array}{llll}\text { C } & -0.131453 & -1.060624 & 1.442117\end{array}$
$\begin{array}{llll}\text { C } & 0.291495 & -2.170585 & 0.703328\end{array}$
$\begin{array}{llll}\text { C } & 0.291495 & -2.170585 & -0.703328\end{array}$
$\begin{array}{llll}\mathrm{O} & -0.252116 & 1.370736 & -1.289008\end{array}$
$\begin{array}{llll}\text { C } & 0.003818 & 1.451832 & -2.681004\end{array}$ 


$\begin{array}{crcc}\mathrm{O} & -0.252116 & 1.370736 & 1.289008 \\ \mathrm{C} & 0.003818 & 1.451832 & 2.681004 \\ \mathrm{H} & -0.411610 & -1.148198 & 2.485165 \\ \mathrm{H} & 0.592052 & -3.077737 & 1.221337 \\ \mathrm{H} & 0.592052 & -3.077737 & -1.221337 \\ \mathrm{H} & -0.411610 & -1.148198 & -2.485165 \\ \mathrm{H} & 0.062947 & 2.514976 & -2.906122 \\ \mathrm{H} & 0.943987 & 0.960020 & -2.942095 \\ \mathrm{H} & -0.811656 & 1.004788 & -3.256813 \\ \mathrm{H} & 0.062947 & 2.514976 & 2.906122 \\ \mathrm{H} & -0.811656 & 1.004788 & 3.256813 \\ \mathrm{H} & 0.943987 & 0.960020 & 2.942095\end{array}$

3P-propionic acid, gauche $\mathrm{S}_{0}$

$\begin{array}{lrrr}\mathrm{C} & -1.028387 & 0.902608 & -1.164992 \\ \mathrm{C} & -0.384109 & 0.664720 & 0.048129 \\ \mathrm{C} & -1.045378 & -0.090545 & 1.014408 \\ \mathrm{C} & -2.315417 & -0.596202 & 0.772122 \\ \mathrm{C} & -2.945486 & -0.354185 & -0.440371 \\ \mathrm{C} & -2.297278 & 0.398372 & -1.410374 \\ \mathrm{C} & 1.002303 & 1.199137 & 0.291506 \\ \mathrm{C} & 2.102621 & 0.279064 & -0.231840 \\ \mathrm{C} & 2.310009 & -0.944496 & 0.617351 \\ \mathrm{O} & 2.019190 & -1.052418 & 1.779276 \\ \mathrm{O} & 2.915454 & -1.930983 & -0.068682 \\ \mathrm{H} & 1.913064 & -0.032013 & -1.261470 \\ \mathrm{H} & 3.064908 & 0.805061 & -0.240084 \\ \mathrm{H} & 1.157761 & 1.359882 & 1.360631 \\ \mathrm{H} & 1.105848 & 2.169554 & -0.201398 \\ \mathrm{H} & -0.551984 & -0.286901 & 1.960100 \\ \mathrm{H} & -2.815915 & -1.180064 & 1.537094 \\ \mathrm{H} & -3.938993 & -0.746354 & -0.627772 \\ \mathrm{H} & -2.784032 & 0.598957 & -2.358738 \\ \mathrm{H} & -0.532145 & 1.499132 & -1.925452 \\ \mathrm{H} & 3.047976 & -2.662348 & 0.550568\end{array}$

3P-propionic acid, gauche $\mathrm{S}_{1}$
C $\quad-0.679614$
$0.242153-1.094323$
C -0.355569
$0.549002 \quad 0.260421$
$\begin{array}{llll}\text { C } & -1.341730 & 0.369715 & 1.269680\end{array}$
$\begin{array}{llll}\text { C } & -2.646055 & -0.062184 & 0.934883\end{array}$
$\begin{array}{llll}\text { C } & -2.956757 & -0.350571 & -0.414403\end{array}$
$\begin{array}{llll}\text { C } & -1.982983 & -0.194560 & -1.428616\end{array}$
$\begin{array}{llll}\text { C } & 0.972279 & 1.108698 & 0.626256\end{array}$ 


$\begin{array}{lrrr}\mathrm{C} & 2.151981 & 0.502786 & -0.126130 \\ \mathrm{C} & 2.296170 & -0.968412 & 0.146051 \\ \mathrm{O} & 1.948154 & -1.530476 & 1.152118 \\ \mathrm{O} & 2.920088 & -1.609010 & -0.864244 \\ \mathrm{H} & 2.090772 & 0.668486 & -1.202781 \\ \mathrm{H} & 3.080218 & 0.986167 & 0.201649 \\ \mathrm{H} & 1.126618 & 0.985928 & 1.701164 \\ \mathrm{H} & 0.973170 & 2.194695 & 0.433882 \\ \mathrm{H} & -1.072892 & 0.548272 & 2.303645 \\ \mathrm{H} & -3.395898 & -0.186762 & 1.705105 \\ \mathrm{H} & -3.950419 & -0.694922 & -0.675326 \\ \mathrm{H} & -2.236502 & -0.409584 & -2.458827 \\ \mathrm{H} & 0.055740 & 0.384826 & -1.875220 \\ \mathrm{H} & 3.003239 & -2.534257 & -0.594983\end{array}$

3P-propionic acid, anti $\mathrm{S}_{0}$

$\begin{array}{lrrr}\mathrm{C} & 1.453460 & -0.447142 & 0.000000 \\ \mathrm{C} & 0.542608 & 0.774464 & 0.000000 \\ \mathrm{C} & 2.912102 & -0.090008 & 0.000000 \\ \mathrm{H} & 1.262138 & -1.082744 & 0.870596 \\ \mathrm{H} & 1.262138 & -1.082744 & -0.870596 \\ \mathrm{C} & -0.910640 & 0.388462 & 0.000000 \\ \mathrm{H} & 0.769602 & 1.389721 & 0.874033 \\ \mathrm{H} & 0.769602 & 1.389721 & -0.874033 \\ \mathrm{C} & -1.596512 & 0.190022 & 1.196301 \\ \mathrm{C} & -2.928469 & -0.199501 & 1.199136 \\ \mathrm{C} & -3.598905 & -0.397069 & 0.000000 \\ \mathrm{C} & -2.928469 & -0.199501 & -1.199136 \\ \mathrm{C} & -1.596512 & 0.190022 & -1.196301 \\ \mathrm{H} & -1.081441 & 0.351372 & 2.138828 \\ \mathrm{H} & -3.446190 & -0.343781 & 2.141267 \\ \mathrm{H} & -4.640666 & -0.697789 & 0.000000 \\ \mathrm{H} & -3.446190 & -0.343781 & -2.141267 \\ \mathrm{H} & -1.081441 & 0.351372 & -2.138828 \\ \mathrm{O} & 3.691011 & -1.189709 & 0.000000 \\ \mathrm{O} & 3.367499 & 1.022353 & 0.000000 \\ \mathrm{H} & 4.608111 & -0.882419 & 0.000000\end{array}$

3P-propionic acid, anti $\mathrm{S}_{1}$

$\begin{array}{lrrr}\mathrm{C} & 1.452878 & -0.431716 & 0.000000 \\ \mathrm{C} & 0.557474 & 0.811826 & 0.000000 \\ \mathrm{C} & 2.914922 & -0.090205 & 0.000000 \\ \mathrm{H} & 1.247521 & -1.060107 & 0.872382 \\ \mathrm{H} & 1.247521 & -1.060107 & -0.872382\end{array}$




$\begin{array}{lrrr}\mathrm{C} & -0.883604 & 0.452221 & 0.000000 \\ \mathrm{H} & 0.799114 & 1.415945 & 0.879808 \\ \mathrm{H} & 0.799114 & 1.415945 & -0.879808 \\ \mathrm{C} & -1.571586 & 0.241718 & 1.229238 \\ \mathrm{C} & -2.910914 & -0.220266 & 1.229838 \\ \mathrm{C} & -3.575319 & -0.442595 & 0.000000 \\ \mathrm{C} & -2.910914 & -0.220266 & -1.229838 \\ \mathrm{C} & -1.571586 & 0.241718 & -1.229238 \\ \mathrm{H} & -1.060515 & 0.439000 & 2.164337 \\ \mathrm{H} & -3.425765 & -0.400370 & 2.164726 \\ \mathrm{H} & -4.603371 & -0.784290 & 0.000000 \\ \mathrm{H} & -3.425765 & -0.400370 & -2.164726 \\ \mathrm{H} & -1.060515 & 0.439000 & -2.164337 \\ \mathrm{O} & 3.680254 & -1.199569 & 0.000000 \\ \mathrm{O} & 3.382352 & 1.017617 & 0.000000 \\ \mathrm{H} & 4.601330 & -0.903974 & 0.000000\end{array}$

fluorine $\mathrm{S}_{0}$

$\begin{array}{lrrr}\text { C } & 0.000000 & 0.000000 & -1.785581 \\ \mathrm{C} & 0.000000 & 1.176468 & -0.847418 \\ \mathrm{C} & 0.000000 & 0.731139 & 0.482365 \\ \mathrm{C} & 0.000000 & -0.731139 & 0.482365 \\ \mathrm{C} & 0.000000 & 1.643626 & 1.529811 \\ \mathrm{C} & 0.000000 & 3.000640 & 1.236130 \\ \mathrm{C} & 0.000000 & 3.441491 & -0.083719 \\ \mathrm{C} & 0.000000 & 2.529496 & -1.134405 \\ \mathrm{H} & 0.000000 & 2.880168 & -2.161609 \\ \mathrm{H} & 0.000000 & 4.505283 & -0.294328 \\ \mathrm{H} & 0.000000 & 3.725336 & 2.042961 \\ \mathrm{H} & 0.000000 & 1.307338 & 2.561251 \\ \mathrm{C} & 0.000000 & -1.643626 & 1.529811 \\ \mathrm{C} & 0.000000 & -3.000640 & 1.236130 \\ \mathrm{C} & 0.000000 & -3.441491 & -0.083719 \\ \mathrm{C} & 0.000000 & -2.529496 & -1.134405 \\ \mathrm{C} & 0.000000 & -1.176468 & -0.847418 \\ \mathrm{H} & 0.000000 & -1.307338 & 2.561251 \\ \mathrm{H} & 0.000000 & -3.725336 & 2.042961 \\ \mathrm{H} & 0.000000 & -4.505283 & -0.294328 \\ \mathrm{H} & 0.000000 & -2.880168 & -2.161609 \\ \mathrm{H} & -0.879997 & 0.000000 & -2.438238 \\ \mathrm{H} & 0.879997 & 0.000000 & -2.438238\end{array}$

fluorine $\mathrm{S}_{1}$

$\begin{array}{llll}\text { C } & 0.000000 & 0.000000 & -1.858368\end{array}$ 


$\begin{array}{rrrr}\mathrm{C} & 0.000000 & 1.176864 & -0.914642 \\ \mathrm{C} & 0.000000 & 0.698166 & 0.450535 \\ \mathrm{C} & 0.000000 & -0.698166 & 0.450535 \\ \mathrm{C} & 0.000000 & 1.637271 & 1.532832 \\ \mathrm{C} & 0.000000 & 2.976066 & 1.226570 \\ \mathrm{C} & 0.000000 & 3.430811 & -0.107794 \\ \mathrm{C} & 0.000000 & 2.518069 & -1.185053 \\ \mathrm{H} & 0.000000 & 2.884379 & -2.206316 \\ \mathrm{H} & 0.000000 & 4.496356 & -0.306779 \\ \mathrm{H} & 0.000000 & 3.709817 & 2.025792 \\ \mathrm{H} & 0.000000 & 1.293785 & 2.560593 \\ \mathrm{C} & 0.000000 & -1.637271 & 1.532832 \\ \mathrm{C} & 0.000000 & -2.976066 & 1.226570 \\ \mathrm{C} & 0.000000 & -3.430811 & -0.107794 \\ \mathrm{C} & 0.000000 & -2.518069 & -1.185053 \\ \mathrm{C} & 0.000000 & -1.176864 & -0.914642 \\ \mathrm{H} & 0.000000 & -1.293785 & 2.560593 \\ \mathrm{H} & 0.000000 & -3.709817 & 2.025792 \\ \mathrm{H} & 0.000000 & -4.496356 & -0.306779 \\ \mathrm{H} & 0.000000 & -2.884379 & -2.206316 \\ \mathrm{H} & -0.879111 & 0.000000 & -2.514390 \\ \mathrm{H} & 0.879111 & 0.000000 & -2.514390\end{array}$

phenanthrene $\mathrm{S}_{0}$

$\begin{array}{rrrr}\mathrm{C} & 0.724812 & 0.000000 & -0.349696 \\ \mathrm{C} & -0.724812 & 0.000000 & -0.349696 \\ \mathrm{C} & -1.414316 & 0.000000 & 0.888276 \\ \mathrm{C} & -0.675822 & 0.000000 & 2.110785 \\ \mathrm{C} & 0.675822 & 0.000000 & 2.110785 \\ \mathrm{C} & 1.414316 & 0.000000 & 0.888276 \\ \mathrm{C} & -1.489756 & 0.000000 & -1.530481 \\ \mathrm{C} & -2.821386 & 0.000000 & 0.900885 \\ \mathrm{H} & -1.227590 & 0.000000 & 3.045355 \\ \mathrm{H} & 1.227590 & 0.000000 & 3.045355 \\ \mathrm{C} & 1.489756 & 0.000000 & -1.530481 \\ \mathrm{C} & 2.864404 & 0.000000 & -1.494380 \\ \mathrm{C} & 3.540385 & 0.000000 & -0.268525 \\ \mathrm{C} & 2.821386 & 0.000000 & 0.900885 \\ \mathrm{H} & 0.995895 & 0.000000 & -2.494084 \\ \mathrm{H} & 3.426528 & 0.000000 & -2.421674 \\ \mathrm{H} & 4.624344 & 0.000000 & -0.244990 \\ \mathrm{H} & 3.332162 & 0.000000 & 1.858622 \\ \mathrm{C} & -3.540385 & 0.000000 & -0.268525 \\ \mathrm{C} & -2.864404 & 0.000000 & -1.494380 \\ \mathrm{H} & -0.995895 & 0.000000 & -2.494084\end{array}$




$$
\begin{array}{cccc}
H & -3.426528 & 0.000000 & -2.421674 \\
H & -4.624344 & 0.000000 & -0.244990 \\
H & -3.332162 & 0.000000 & 1.858622
\end{array}
$$

phenanthrene $\mathrm{S}_{1}$

$\begin{array}{lrrr}\mathrm{C} & 0.710975 & 0.000000 & -0.382214 \\ \mathrm{C} & -0.710975 & 0.000000 & -0.382214 \\ \mathrm{C} & -1.431580 & 0.000000 & 0.880302 \\ \mathrm{C} & -0.695014 & 0.000000 & 2.076191 \\ \mathrm{C} & 0.695014 & 0.000000 & 2.076191 \\ \mathrm{C} & 1.431580 & 0.000000 & 0.880302 \\ \mathrm{C} & -1.489606 & 0.000000 & -1.565260 \\ \mathrm{C} & -2.833840 & 0.000000 & 0.884374 \\ \mathrm{H} & -1.231098 & 0.000000 & 3.019631 \\ \mathrm{H} & 1.231098 & 0.000000 & 3.019631 \\ \mathrm{C} & 1.489606 & 0.000000 & -1.565260 \\ \mathrm{C} & 2.878229 & 0.000000 & -1.522806 \\ \mathrm{C} & 3.563400 & 0.000000 & -0.303734 \\ \mathrm{C} & 2.833840 & 0.000000 & 0.884374 \\ \mathrm{H} & 1.002982 & 0.000000 & -2.531649 \\ \mathrm{H} & 3.435537 & 0.000000 & -2.452984 \\ \mathrm{H} & 4.646156 & 0.000000 & -0.282261 \\ \mathrm{H} & 3.348954 & 0.000000 & 1.839448 \\ \mathrm{C} & -3.563400 & 0.000000 & -0.303734 \\ \mathrm{C} & -2.878229 & 0.000000 & -1.522806 \\ \mathrm{H} & -1.002982 & 0.000000 & -2.531649 \\ \mathrm{H} & -3.435537 & 0.000000 & -2.452984 \\ \mathrm{H} & -4.646156 & 0.000000 & -0.282261 \\ \mathrm{H} & -3.348954 & 0.000000 & 1.839448\end{array}$

\section{2,4,6,8-decatetraene $\mathrm{S}_{0}$}

$\begin{array}{lrrr}\mathrm{C} & -2.582387 & -4.997683 & 0.000000 \\ \mathrm{C} & -2.464962 & -3.514345 & 0.000000 \\ \mathrm{C} & -1.311692 & -2.834007 & 0.000000 \\ \mathrm{C} & -1.212712 & -1.397908 & 0.000000 \\ \mathrm{C} & -0.050358 & -0.715216 & 0.000000 \\ \mathrm{C} & 0.050358 & 0.715216 & 0.000000 \\ \mathrm{C} & 1.212712 & 1.397908 & 0.000000 \\ \mathrm{C} & 1.311692 & 2.834007 & 0.000000 \\ \mathrm{C} & 2.464962 & 3.514345 & 0.000000 \\ \mathrm{C} & 2.582387 & 4.997683 & 0.000000 \\ \mathrm{H} & -0.373717 & -3.388677 & 0.000000 \\ \mathrm{H} & -2.148252 & -0.839996 & 0.000000 \\ \mathrm{H} & 0.884245 & -1.274966 & 0.000000\end{array}$




$\begin{array}{crcc}\mathrm{H} & -0.884245 & 1.274966 & 0.000000 \\ \mathrm{H} & 2.148252 & 0.839996 & 0.000000 \\ \mathrm{H} & 0.373717 & 3.388677 & 0.000000 \\ \mathrm{H} & 3.397863 & 2.951895 & 0.000000 \\ \mathrm{H} & -3.397863 & -2.951895 & 0.000000 \\ \mathrm{H} & 1.601374 & 5.477255 & 0.000000 \\ \mathrm{H} & 3.135382 & 5.350772 & 0.877318 \\ \mathrm{H} & 3.135382 & 5.350772 & -0.877318 \\ \mathrm{H} & -1.601374 & -5.477255 & 0.000000 \\ \mathrm{H} & -3.135382 & -5.350772 & 0.877318 \\ \mathrm{H} & -3.135382 & -5.350772 & -0.877318\end{array}$

\section{2,4,6,8-decatetraene $S_{1}$}

$\begin{array}{crcc}\mathrm{C} & -2.574353 & -5.011548 & 0.000000 \\ \mathrm{C} & -2.514422 & -3.533284 & 0.000000 \\ \mathrm{C} & -1.351843 & -2.806801 & 0.000000 \\ \mathrm{C} & -1.263537 & -1.403885 & 0.000000 \\ \mathrm{C} & -0.053591 & -0.694160 & 0.000000 \\ \mathrm{C} & 0.053591 & 0.694160 & 0.000000 \\ \mathrm{C} & 1.263537 & 1.403885 & 0.000000 \\ \mathrm{C} & 1.351843 & 2.806801 & 0.000000 \\ \mathrm{C} & 2.514422 & 3.533284 & 0.000000 \\ \mathrm{C} & 2.574353 & 5.011548 & 0.000000 \\ \mathrm{H} & -0.411020 & -3.358591 & 0.000000 \\ \mathrm{H} & -2.190152 & -0.831870 & 0.000000 \\ \mathrm{H} & 0.868509 & -1.274334 & 0.000000 \\ \mathrm{H} & -0.868509 & 1.274334 & 0.000000 \\ \mathrm{H} & 2.190152 & 0.831870 & 0.000000 \\ \mathrm{H} & 0.411020 & 3.358591 & 0.000000 \\ \mathrm{H} & 3.461429 & 2.995339 & 0.000000 \\ \mathrm{H} & -3.461429 & -2.995339 & 0.000000 \\ \mathrm{H} & 1.575814 & 5.454950 & 0.000000 \\ \mathrm{H} & 3.117023 & 5.397594 & 0.875124 \\ \mathrm{H} & 3.117023 & 5.397594 & -0.875124 \\ \mathrm{H} & -1.575814 & -5.454950 & 0.000000 \\ \mathrm{H} & -3.117023 & -5.397594 & 0.875124 \\ \mathrm{H} & -3.117023 & -5.397594 & -0.875124\end{array}$

tetracene $\mathrm{S}_{0}$
C 3.690030
1.401692
0.000000
$\begin{array}{llll}\text { C } & 2.435629 & 0.721136 & 0.000000\end{array}$
$\begin{array}{llll}\text { C } & 2.435629 & -0.721136 & 0.000000\end{array}$
$\begin{array}{llll}\text { C } & 3.690030 & -1.401692 & 0.000000\end{array}$
$\begin{array}{llll}\text { C } & 4.860410 & -0.711836 & 0.000000\end{array}$ 


$\begin{array}{rrrr}\mathrm{C} & 4.860410 & 0.711836 & 0.000000 \\ \mathrm{C} & 1.228324 & 1.399020 & 0.000000 \\ \mathrm{C} & 0.000000 & 0.721282 & 0.000000 \\ \mathrm{C} & 0.000000 & -0.721282 & 0.000000 \\ \mathrm{C} & 1.228324 & -1.399020 & 0.000000 \\ \mathrm{C} & -1.228324 & 1.399020 & 0.000000 \\ \mathrm{C} & -2.435629 & 0.721136 & 0.000000 \\ \mathrm{C} & -2.435629 & -0.721136 & 0.000000 \\ \mathrm{C} & -1.228324 & -1.399020 & 0.000000 \\ \mathrm{C} & -3.690030 & 1.401692 & 0.000000 \\ \mathrm{C} & -4.860410 & 0.711836 & 0.000000 \\ \mathrm{C} & -4.860410 & -0.711836 & 0.000000 \\ \mathrm{C} & -3.690030 & -1.401692 & 0.000000 \\ \mathrm{H} & -3.688642 & -2.487012 & 0.000000 \\ \mathrm{H} & -5.805864 & -1.242789 & 0.000000 \\ \mathrm{H} & -5.805864 & 1.242789 & 0.000000 \\ \mathrm{H} & -3.688642 & 2.487012 & 0.000000 \\ \mathrm{H} & -1.228695 & -2.485176 & 0.000000 \\ \mathrm{H} & -1.228695 & 2.485176 & 0.000000 \\ \mathrm{H} & 1.228695 & -2.485176 & 0.000000 \\ \mathrm{H} & 1.228695 & 2.485176 & 0.000000 \\ \mathrm{H} & 3.688642 & -2.487012 & 0.000000 \\ \mathrm{H} & 5.805864 & -1.242789 & 0.000000 \\ \mathrm{H} & 5.805864 & 1.242789 & 0.000000 \\ \mathrm{H} & 3.688642 & 2.487012 & 0.000000\end{array}$

tetracene $\mathrm{S}_{1}$

$\begin{array}{rrrr}\mathrm{C} & 3.692823 & 1.392958 & 0.000000 \\ \mathrm{C} & 2.463023 & 0.717487 & 0.000000 \\ \mathrm{C} & 2.463023 & -0.717487 & 0.000000 \\ \mathrm{C} & 3.692823 & -1.392958 & 0.000000 \\ \mathrm{C} & 4.894664 & -0.696318 & 0.000000 \\ \mathrm{C} & 4.894664 & 0.696318 & 0.000000 \\ \mathrm{C} & 1.226338 & 1.393520 & 0.000000 \\ \mathrm{C} & 0.000000 & 0.722150 & 0.000000 \\ \mathrm{C} & 0.000000 & -0.722150 & 0.000000 \\ \mathrm{C} & 1.226338 & -1.393520 & 0.000000 \\ \mathrm{C} & -1.226338 & 1.393520 & 0.000000 \\ \mathrm{C} & -2.463023 & 0.717487 & 0.000000 \\ \mathrm{C} & -2.463023 & -0.717487 & 0.000000 \\ \mathrm{C} & -1.226338 & -1.393520 & 0.000000 \\ \mathrm{C} & -3.692823 & 1.392958 & 0.000000 \\ \mathrm{C} & -4.894664 & 0.696318 & 0.000000 \\ \mathrm{C} & -4.894664 & -0.696318 & 0.000000 \\ \mathrm{C} & -3.692823 & -1.392958 & 0.000000\end{array}$




$\begin{array}{rrrr}\mathrm{H} & -3.694615 & -2.478223 & 0.000000 \\ \mathrm{H} & -5.832829 & -1.239354 & 0.000000 \\ \mathrm{H} & -5.832829 & 1.239354 & 0.000000 \\ \mathrm{H} & -3.694615 & 2.478223 & 0.000000 \\ \mathrm{H} & -1.227913 & -2.479867 & 0.000000 \\ \mathrm{H} & -1.227913 & 2.479867 & 0.000000 \\ \mathrm{H} & 1.227913 & -2.479867 & 0.000000 \\ \mathrm{H} & 1.227913 & 2.479867 & 0.000000 \\ \mathrm{H} & 3.694615 & -2.478223 & 0.000000 \\ \mathrm{H} & 5.832829 & -1.239354 & 0.000000 \\ \mathrm{H} & 5.832829 & 1.239354 & 0.000000 \\ \mathrm{H} & 3.694615 & 2.478223 & 0.000000\end{array}$

perylene $\mathrm{S}_{0}$

$\begin{array}{lrrc}\text { C } & 0.022671 & 1.469409 & -2.413284 \\ \mathrm{C} & 0.012315 & 0.734375 & -1.242116 \\ \mathrm{C} & 0.010789 & 1.431528 & 0.000008 \\ \mathrm{C} & 0.033183 & 2.857320 & -0.000001 \\ \mathrm{C} & 0.042433 & 3.554770 & -1.226204 \\ \mathrm{C} & 0.037709 & 2.868647 & -2.409226 \\ \mathrm{C} & 0.042751 & 3.554780 & 1.226193 \\ \mathrm{C} & 0.038312 & 2.868670 & 2.409221 \\ \mathrm{C} & 0.023166 & 1.469431 & 2.413297 \\ \mathrm{C} & 0.012455 & 0.734379 & 1.242139 \\ \mathrm{C} & -0.012315 & -0.734375 & -1.242116 \\ \mathrm{C} & -0.010789 & -1.431528 & 0.000008 \\ \mathrm{C} & -0.012455 & -0.734379 & 1.242139 \\ \mathrm{C} & -0.033183 & -2.857320 & -0.000001 \\ \mathrm{C} & -0.042751 & -3.554780 & 1.226193 \\ \mathrm{C} & -0.038312 & -2.868670 & 2.409221 \\ \mathrm{C} & -0.023166 & -1.469431 & 2.413297 \\ \mathrm{C} & -0.022671 & -1.469409 & -2.413284 \\ \mathrm{C} & -0.037709 & -2.868647 & -2.409226 \\ \mathrm{C} & -0.042433 & -3.554770 & -1.226204 \\ \mathrm{H} & 0.055933 & 4.639490 & 1.211538 \\ \mathrm{H} & 0.046916 & 3.402964 & 3.352737 \\ \mathrm{H} & 0.022062 & 0.966190 & 3.371354 \\ \mathrm{H} & -0.055933 & -4.639490 & 1.211538 \\ \mathrm{H} & -0.046916 & -3.402964 & 3.352737 \\ \mathrm{H} & -0.022062 & -0.966190 & 3.371354 \\ \mathrm{H} & -0.055582 & -4.639480 & -1.211565 \\ \mathrm{H} & -0.046005 & -3.402929 & -3.352751 \\ \mathrm{H} & -0.021354 & -0.966153 & -3.371337 \\ \mathrm{H} & 0.055582 & 4.639480 & -1.211565 \\ \mathrm{H} & 0.046005 & 3.402929 & -3.352751\end{array}$




\section{$\begin{array}{llll}\mathrm{H} & 0.021354 & 0.966153 & -3.371337\end{array}$}

perylene $S_{1}$

$\begin{array}{lrrc}\mathrm{C} & -0.006669 & 1.463734 & -2.441977 \\ \mathrm{C} & -0.006126 & 0.717289 & -1.237131 \\ \mathrm{C} & 0.011073 & 1.416938 & 0.000002 \\ \mathrm{C} & 0.008161 & 2.844707 & -0.000001 \\ \mathrm{C} & 0.009487 & 3.535718 & -1.226603 \\ \mathrm{C} & -0.001534 & 2.844327 & -2.427655 \\ \mathrm{C} & 0.009733 & 3.535724 & 1.226592 \\ \mathrm{C} & -0.001032 & 2.844336 & 2.427652 \\ \mathrm{C} & -0.006239 & 1.463746 & 2.441981 \\ \mathrm{C} & -0.006018 & 0.717291 & 1.237138 \\ \mathrm{C} & 0.006126 & -0.717289 & -1.237131 \\ \mathrm{C} & -0.011073 & -1.416938 & 0.000002 \\ \mathrm{C} & 0.006018 & -0.717291 & 1.237138 \\ \mathrm{C} & -0.008161 & -2.844707 & -0.000001 \\ \mathrm{C} & -0.009733 & -3.535724 & 1.226592 \\ \mathrm{C} & 0.001032 & -2.844336 & 2.427652 \\ \mathrm{C} & 0.006239 & -1.463746 & 2.441981 \\ \mathrm{C} & 0.006669 & -1.463734 & -2.441977 \\ \mathrm{C} & 0.001534 & -2.844327 & -2.427655 \\ \mathrm{C} & -0.009487 & -3.535718 & -1.226603 \\ \mathrm{H} & 0.013838 & 4.620522 & 1.217455 \\ \mathrm{H} & -0.004733 & 3.390374 & 3.364582 \\ \mathrm{H} & -0.029043 & 0.953505 & 3.394895 \\ \mathrm{H} & -0.013838 & -4.620522 & 1.217455 \\ \mathrm{H} & 0.004733 & -3.390374 & 3.364582 \\ \mathrm{H} & 0.029043 & -0.953505 & 3.394895 \\ \mathrm{H} & -0.013570 & -4.620516 & -1.217469 \\ \mathrm{H} & 0.005501 & -3.390358 & -3.364588 \\ \mathrm{H} & 0.029777 & -0.953482 & -3.394879 \\ \mathrm{H} & 0.013570 & 4.620516 & -1.217469 \\ \mathrm{H} & -0.005501 & 3.390358 & -3.364588 \\ \mathrm{H} & -0.029777 & 0.953482 & -3.394879\end{array}$

M06-2X/TZVP geometries - Group I

pyrazine $S_{0}$
$\begin{array}{llll}\text { C } & 1.127391 & -0.694999 & 0.000000\end{array}$
$\begin{array}{llll}\mathrm{C} & 1.127391 & 0.694999 & 0.000000\end{array}$
$\begin{array}{llll}\mathrm{N} & 0.000000 & 1.398730 & 0.000000\end{array}$
$\begin{array}{llll}\text { C } & -1.127391 & 0.694999 & 0.000000\end{array}$ 


$\begin{array}{rrrr}\mathrm{C} & -1.127391 & -0.694999 & 0.000000 \\ \mathrm{~N} & 0.000000 & -1.398730 & 0.000000 \\ \mathrm{H} & -2.057378 & -1.251526 & 0.000000 \\ \mathrm{H} & 2.057378 & -1.251526 & 0.000000 \\ \mathrm{H} & 2.057378 & 1.251526 & 0.000000 \\ \mathrm{H} & -2.057378 & 1.251526 & 0.000000\end{array}$

pyrazine $\mathrm{S}_{1}$

$\begin{array}{rrrr}\mathrm{C} & -1.181751 & 0.655882 & 0.000000 \\ \mathrm{C} & -1.135290 & -0.733913 & 0.000000 \\ \mathrm{~N} & 0.045942 & -1.360963 & 0.000000 \\ \mathrm{C} & 1.181751 & -0.655882 & 0.000000 \\ \mathrm{C} & 1.135290 & 0.733913 & 0.000000 \\ \mathrm{~N} & -0.045942 & 1.360963 & 0.000000 \\ \mathrm{H} & 2.060333 & 1.295159 & 0.000000 \\ \mathrm{H} & -2.142420 & 1.153801 & 0.000000 \\ \mathrm{H} & -2.060333 & -1.295159 & 0.000000 \\ \mathrm{H} & 2.142420 & -1.153801 & 0.000000\end{array}$

2,6-difluoropyridine $\mathrm{S}_{0}$

$\begin{array}{lrrr}\mathrm{C} & 1.112162 & 0.000000 & -0.874080 \\ \mathrm{~N} & 0.000000 & 0.000000 & -1.566005 \\ \mathrm{C} & -1.112162 & 0.000000 & -0.874080 \\ \mathrm{C} & -1.200823 & 0.000000 & 0.506827 \\ \mathrm{C} & 0.000000 & 0.000000 & 1.200616 \\ \mathrm{C} & 1.200823 & 0.000000 & 0.506827 \\ \mathrm{~F} & -2.235972 & 0.000000 & -1.586179 \\ \mathrm{H} & -2.163574 & 0.000000 & 0.995019 \\ \mathrm{H} & 0.000000 & 0.000000 & 2.282636 \\ \mathrm{H} & 2.163574 & 0.000000 & 0.995019 \\ \mathrm{~F} & 2.235972 & 0.000000 & -1.586179\end{array}$

2,6-difluoropyridine $\mathrm{S}_{1}$

$\begin{array}{crcc}\mathrm{C} & -1.096807 & 0.000000 & -0.337179 \\ \mathrm{~N} & 0.000000 & 0.000000 & -1.105924 \\ \mathrm{C} & 1.096807 & 0.000000 & -0.337179 \\ \mathrm{C} & 1.188961 & 0.000000 & 1.072628 \\ \mathrm{C} & 0.000000 & 0.000000 & 1.848425 \\ \mathrm{C} & -1.188961 & 0.000000 & 1.072628 \\ \mathrm{~F} & 2.233534 & 0.000000 & -1.005160 \\ \mathrm{H} & 2.177728 & 0.000000 & 1.511010 \\ \mathrm{H} & 0.000000 & 0.000000 & 2.924193 \\ \mathrm{H} & -2.177728 & 0.000000 & 1.511010\end{array}$




$$
\begin{array}{llll}
\text { F } & -2.233534 & 0.000000 & -1.005160
\end{array}
$$

2-fluoropyridine $\mathrm{S}_{0}$

$\begin{array}{crrr}\mathrm{C} & -1.312961 & 0.501983 & 0.000000 \\ \mathrm{~N} & -1.365191 & -0.800542 & 0.000000 \\ \mathrm{C} & -0.197482 & -1.449668 & 0.000000 \\ \mathrm{C} & 1.028737 & -0.807649 & 0.000000 \\ \mathrm{C} & 1.043236 & 0.583845 & 0.000000 \\ \mathrm{C} & -0.156191 & 1.270854 & 0.000000 \\ \mathrm{H} & -0.259873 & -2.531597 & 0.000000 \\ \mathrm{H} & 1.945430 & -1.380308 & 0.000000 \\ \mathrm{H} & 1.980033 & 1.126341 & 0.000000 \\ \mathrm{H} & -0.218347 & 2.349480 & 0.000000 \\ \mathrm{~F} & -2.487400 & 1.137251 & 0.000000\end{array}$

2-fluoropyridine $\mathrm{S}_{1}$

$\begin{array}{rrrr}\mathrm{C} & -1.319221 & 0.488488 & 0.000000 \\ \mathrm{~N} & -1.438034 & -0.844579 & 0.000000 \\ \mathrm{C} & -0.227773 & -1.461034 & 0.000000 \\ \mathrm{C} & 1.028607 & -0.790286 & 0.000000 \\ \mathrm{C} & 1.109098 & 0.620571 & 0.000000 \\ \mathrm{C} & -0.149420 & 1.280385 & 0.000000 \\ \mathrm{H} & -0.248339 & -2.543485 & 0.000000 \\ \mathrm{H} & 1.924159 & -1.398266 & 0.000000 \\ \mathrm{H} & 2.045172 & 1.153354 & 0.000000 \\ \mathrm{H} & -0.258035 & 2.355537 & 0.000000 \\ \mathrm{~F} & -2.466221 & 1.139304 & 0.000000\end{array}$

2-hydroxypyridine $\mathrm{S}_{0}$

$\begin{array}{rrrr}\mathrm{C} & -0.977209 & 0.751209 & 0.000000 \\ \mathrm{C} & -0.966703 & -0.644774 & 0.000000 \\ \mathrm{~N} & 0.131589 & -1.375726 & 0.000000 \\ \mathrm{C} & 1.303389 & -0.734930 & 0.000000 \\ \mathrm{C} & 1.416807 & 0.642527 & 0.000000 \\ \mathrm{C} & 0.242897 & 1.394003 & 0.000000 \\ \mathrm{O} & -2.147225 & -1.296303 & 0.000000 \\ \mathrm{H} & 2.184783 & -1.366074 & 0.000000 \\ \mathrm{H} & 2.389360 & 1.113471 & 0.000000 \\ \mathrm{H} & 0.284090 & 2.476017 & 0.000000 \\ \mathrm{H} & -1.918430 & 1.281678 & 0.000000 \\ \mathrm{H} & -1.943349 & -2.241098 & 0.000000\end{array}$

2-hydroxypyridine $\mathrm{S}_{1}$ 


$\begin{array}{crrl}\mathrm{C} & -0.993774 & 0.767248 & 0.000000 \\ \mathrm{C} & -0.964841 & -0.640011 & 0.000000 \\ \mathrm{~N} & 0.123840 & -1.457542 & 0.000000 \\ \mathrm{C} & 1.290719 & -0.774495 & 0.000000 \\ \mathrm{C} & 1.390117 & 0.643604 & 0.000000 \\ \mathrm{C} & 0.247835 & 1.465056 & 0.000000 \\ \mathrm{O} & -2.122390 & -1.284068 & 0.000000 \\ \mathrm{H} & 2.195021 & -1.369665 & 0.000000 \\ \mathrm{H} & 2.382202 & 1.077548 & 0.000000 \\ \mathrm{H} & 0.306131 & 2.540805 & 0.000000 \\ \mathrm{H} & -1.954219 & 1.262324 & 0.000000 \\ \mathrm{H} & -1.900640 & -2.230803 & 0.000000\end{array}$

2-pyridone $\mathrm{S}_{0}$

$\begin{array}{cccc}\mathrm{C} & -1.246033 & 0.711123 & 0.000000 \\ \mathrm{~N} & -1.134126 & -0.685093 & 0.000000 \\ \mathrm{C} & 0.034022 & -1.381753 & 0.000000 \\ \mathrm{C} & 1.221577 & -0.733679 & 0.000000 \\ \mathrm{C} & 1.202709 & 0.692197 & 0.000000 \\ \mathrm{C} & 0.039389 & 1.385955 & 0.000000 \\ \mathrm{H} & -2.019260 & -1.173433 & 0.000000 \\ \mathrm{H} & -0.056170 & -2.459492 & 0.000000 \\ \mathrm{H} & 2.147134 & -1.287570 & 0.000000 \\ \mathrm{H} & 2.143977 & 1.229093 & 0.000000 \\ \mathrm{H} & 0.009622 & 2.466280 & 0.000000 \\ \mathrm{O} & -2.342840 & 1.236380 & 0.000000\end{array}$

2-pyridone $\mathrm{S}_{1}$

$\begin{array}{rrrr}\mathrm{C} & -1.393354 & 0.112286 & 0.000000 \\ \mathrm{~N} & -0.727810 & -1.147519 & 0.000000 \\ \mathrm{C} & 0.620864 & -1.253574 & 0.000000 \\ \mathrm{C} & 1.408569 & -0.077236 & 0.000000 \\ \mathrm{C} & 0.827769 & 1.176577 & 0.000000 \\ \mathrm{C} & -0.605235 & 1.270266 & 0.000000 \\ \mathrm{H} & -1.340569 & -1.951477 & 0.000000 \\ \mathrm{H} & 1.047398 & -2.245022 & 0.000000 \\ \mathrm{H} & 2.483208 & -0.197634 & 0.000000 \\ \mathrm{H} & 1.436294 & 2.067981 & 0.000000 \\ \mathrm{H} & -1.125835 & 2.215624 & 0.000000 \\ \mathrm{O} & -2.631299 & 0.029719 & 0.000000\end{array}$

2-methylpyrimidine $\mathrm{S}_{0}$ 


$\begin{array}{crrr}\mathrm{C} & 0.000008 & 1.960750 & 0.000000 \\ \mathrm{C} & -1.172734 & 1.220868 & 0.000000 \\ \mathrm{~N} & -1.177603 & -0.105789 & 0.000000 \\ \mathrm{C} & 0.011914 & -0.715621 & 0.000000 \\ \mathrm{~N} & 1.193856 & -0.097448 & 0.000000 \\ \mathrm{C} & 1.176731 & 1.232610 & 0.000000 \\ \mathrm{H} & -2.143477 & 1.706264 & 0.000000 \\ \mathrm{H} & 2.143741 & 1.725054 & 0.000000 \\ \mathrm{H} & -0.005198 & 3.041108 & 0.000000 \\ \mathrm{C} & 0.004785 & -2.213213 & 0.000000 \\ \mathrm{H} & 1.022592 & -2.593395 & 0.000000 \\ \mathrm{H} & -0.527313 & -2.580594 & 0.878306 \\ \mathrm{H} & -0.527313 & -2.580594 & -0.878306\end{array}$

2-methylpyrimidine $\mathrm{S}_{1}$

$\begin{array}{crcc}\mathrm{C} & -0.005255 & 2.007870 & 0.000000 \\ \mathrm{C} & -1.180410 & 1.288758 & 0.000000 \\ \mathrm{~N} & -1.116084 & -0.087134 & 0.000000 \\ \mathrm{C} & 0.009788 & -0.786637 & 0.000000 \\ \mathrm{~N} & 1.107501 & -0.078016 & 0.000000 \\ \mathrm{C} & 1.191323 & 1.290691 & 0.000000 \\ \mathrm{H} & -2.162476 & 1.735891 & 0.000000 \\ \mathrm{H} & 2.172756 & 1.735949 & 0.000000 \\ \mathrm{H} & -0.009767 & 3.090014 & 0.000000 \\ \mathrm{C} & 0.006361 & -2.274308 & 0.000000 \\ \mathrm{H} & 1.027838 & -2.646119 & 0.000000 \\ \mathrm{H} & -0.520787 & -2.638485 & 0.882106 \\ \mathrm{H} & -0.520787 & -2.638485 & -0.882106\end{array}$

5-methylpyrimidine $\mathrm{S}_{0}$

$\begin{array}{rrrr}\mathrm{C} & 0.873726 & 0.850587 & 0.000000 \\ \mathrm{C} & -0.359111 & 0.211351 & 0.000000 \\ \mathrm{C} & -0.299502 & -1.178907 & 0.000000 \\ \mathrm{~N} & 0.842529 & -1.855963 & 0.000000 \\ \mathrm{C} & 1.955864 & -1.128521 & 0.000000 \\ \mathrm{~N} & 2.031875 & 0.196231 & 0.000000 \\ \mathrm{H} & 2.893517 & -1.672406 & 0.000000 \\ \mathrm{H} & -1.212246 & -1.769298 & 0.000000 \\ \mathrm{H} & 0.930940 & 1.935756 & 0.000000 \\ \mathrm{C} & -1.660120 & 0.957814 & 0.000000 \\ \mathrm{H} & -1.489415 & 2.033896 & 0.000000 \\ \mathrm{H} & -2.254028 & 0.709719 & -0.881023 \\ \mathrm{H} & -2.254028 & 0.709719 & 0.881023\end{array}$


5-methylpyrimidine $\mathrm{S}_{1}$

$\begin{array}{rrrr}\mathrm{C} & 0.839453 & 0.871874 & 0.000000 \\ \mathrm{C} & -0.377645 & 0.234694 & 0.000000 \\ \mathrm{C} & -0.344698 & -1.178206 & 0.000000 \\ \mathrm{~N} & 0.879421 & -1.774462 & 0.000000 \\ \mathrm{C} & 2.035877 & -1.178505 & 0.000000 \\ \mathrm{~N} & 2.011756 & 0.146848 & 0.000000 \\ \mathrm{H} & 2.965807 & -1.728151 & 0.000000 \\ \mathrm{H} & -1.223156 & -1.803794 & 0.000000 \\ \mathrm{H} & 0.935825 & 1.948323 & 0.000000 \\ \mathrm{C} & -1.678097 & 0.975759 & 0.000000 \\ \mathrm{H} & -1.512384 & 2.052186 & 0.000000 \\ \mathrm{H} & -2.266084 & 0.716723 & -0.882272 \\ \mathrm{H} & -2.266084 & 0.716723 & 0.882272\end{array}$

7-azaindole $\mathrm{S}_{0}$

$\begin{array}{rrrr}\mathrm{N} & -0.632533 & 1.839626 & 0.000000 \\ \mathrm{C} & -0.671206 & 0.470376 & 0.000000 \\ \mathrm{C} & 0.666089 & 0.006259 & 0.000000 \\ \mathrm{C} & 1.507612 & 1.166164 & 0.000000 \\ \mathrm{C} & 0.680964 & 2.249698 & 0.000000 \\ \mathrm{C} & 0.843070 & -1.376845 & 0.000000 \\ \mathrm{C} & -0.290525 & -2.168763 & 0.000000 \\ \mathrm{C} & -1.561593 & -1.575556 & 0.000000 \\ \mathrm{~N} & -1.773408 & -0.266002 & 0.000000 \\ \mathrm{H} & -2.448329 & -2.199589 & 0.000000 \\ \mathrm{H} & 1.832791 & -1.817338 & 0.000000 \\ \mathrm{H} & -0.212649 & -3.247379 & 0.000000 \\ \mathrm{H} & -1.448508 & 2.427663 & 0.000000 \\ \mathrm{H} & 2.584153 & 1.192289 & 0.000000 \\ \mathrm{H} & 0.924092 & 3.299398 & 0.000000\end{array}$

7-azaindole $\mathrm{S}_{1}$

$\begin{array}{rrrr}\mathrm{N} & -0.604511 & 1.870716 & 0.000000 \\ \mathrm{C} & -0.659319 & 0.447950 & 0.000000 \\ \mathrm{C} & 0.660529 & -0.006058 & 0.000000 \\ \mathrm{C} & 1.490428 & 1.141442 & 0.000000 \\ \mathrm{C} & 0.647749 & 2.305667 & 0.000000 \\ \mathrm{C} & 0.887557 & -1.408835 & 0.000000 \\ \mathrm{C} & -0.304031 & -2.179991 & 0.000000 \\ \mathrm{C} & -1.547869 & -1.594960 & 0.000000 \\ \mathrm{~N} & -1.793856 & -0.221135 & 0.000000 \\ \mathrm{H} & -2.438127 & -2.210534 & 0.000000\end{array}$




$\begin{array}{rrrr}\mathrm{H} & 1.873627 & -1.845568 & 0.000000 \\ \mathrm{H} & -0.247573 & -3.260569 & 0.000000 \\ \mathrm{H} & -1.440952 & 2.438583 & 0.000000 \\ \mathrm{H} & 2.568608 & 1.173149 & 0.000000 \\ \mathrm{H} & 0.907748 & 3.350163 & 0.000000\end{array}$

7-hydroxyquinoline, cis $\mathrm{S}_{0}$

$\begin{array}{rrrl}\mathrm{N} & 0.462333 & 1.967683 & 0.000000 \\ \mathrm{C} & 0.431775 & 0.607750 & 0.000000 \\ \mathrm{C} & -0.783492 & -0.123740 & 0.000000 \\ \mathrm{C} & -1.989985 & 0.607175 & 0.000000 \\ \mathrm{C} & -1.944727 & 1.972781 & 0.000000 \\ \mathrm{C} & -0.680658 & 2.604009 & 0.000000 \\ \mathrm{C} & 1.662211 & -0.089005 & 0.000000 \\ \mathrm{C} & 1.674461 & -1.457049 & 0.000000 \\ \mathrm{C} & 0.464921 & -2.194887 & 0.000000 \\ \mathrm{C} & -0.730033 & -1.540492 & 0.000000 \\ \mathrm{H} & -0.624603 & 3.688427 & 0.000000 \\ \mathrm{H} & -1.658228 & -2.100070 & 0.000000 \\ \mathrm{H} & 0.524473 & -3.275167 & 0.000000 \\ \mathrm{O} & 2.815353 & -2.195929 & 0.000000 \\ \mathrm{H} & 2.575755 & 0.494375 & 0.000000 \\ \mathrm{H} & -2.934564 & 0.074731 & 0.000000 \\ \mathrm{H} & -2.845472 & 2.571247 & 0.000000 \\ \mathrm{H} & 3.580480 & -1.611830 & 0.000000\end{array}$

7-hydroxyquinoline, cis $\mathrm{S}_{1}$

$\begin{array}{lrrl}\mathrm{N} & 0.490063 & 1.974270 & 0.000000 \\ \mathrm{C} & 0.435927 & 0.634351 & 0.000000 \\ \mathrm{C} & -0.788356 & -0.140460 & 0.000000 \\ \mathrm{C} & -1.993533 & 0.591895 & 0.000000 \\ \mathrm{C} & -1.918972 & 1.993372 & 0.000000 \\ \mathrm{C} & -0.685454 & 2.640028 & 0.000000 \\ \mathrm{C} & 1.636030 & -0.083925 & 0.000000 \\ \mathrm{C} & 1.652056 & -1.512985 & 0.000000 \\ \mathrm{C} & 0.480310 & -2.264805 & 0.000000 \\ \mathrm{C} & -0.720984 & -1.556502 & 0.000000 \\ \mathrm{H} & -0.625768 & 3.720265 & 0.000000 \\ \mathrm{H} & -1.656437 & -2.104298 & 0.000000 \\ \mathrm{H} & 0.527056 & -3.342603 & 0.000000 \\ \mathrm{O} & 2.820960 & -2.156579 & 0.000000 \\ \mathrm{H} & 2.568385 & 0.471457 & 0.000000 \\ \mathrm{H} & -2.947510 & 0.081881 & 0.000000 \\ \mathrm{H} & -2.827511 & 2.582647 & 0.000000\end{array}$


$\begin{array}{llll}\mathrm{H} & 3.553738 & -1.527998 & 0.000000\end{array}$

7-hydroxyquinoline, trans $\mathrm{S}_{0}$

$\begin{array}{rrrl}\mathrm{N} & 0.512504 & 2.040395 & 0.000000 \\ \mathrm{C} & 0.479797 & 0.678037 & 0.000000 \\ \mathrm{C} & -0.740811 & -0.047621 & 0.000000 \\ \mathrm{C} & -1.946681 & 0.687965 & 0.000000 \\ \mathrm{C} & -1.895870 & 2.051735 & 0.000000 \\ \mathrm{C} & -0.627204 & 2.678714 & 0.000000 \\ \mathrm{C} & 1.706621 & -0.018424 & 0.000000 \\ \mathrm{C} & 1.715574 & -1.386872 & 0.000000 \\ \mathrm{C} & 0.502569 & -2.117799 & 0.000000 \\ \mathrm{C} & -0.694275 & -1.460783 & 0.000000 \\ \mathrm{H} & -0.568613 & 3.763145 & 0.000000 \\ \mathrm{H} & -1.623121 & -2.019102 & 0.000000 \\ \mathrm{H} & 0.533612 & -3.202159 & 0.000000 \\ \mathrm{O} & 2.917287 & -2.025067 & 0.000000 \\ \mathrm{H} & 2.630916 & 0.542423 & 0.000000 \\ \mathrm{H} & -2.893086 & 0.158667 & 0.000000 \\ \mathrm{H} & -2.793961 & 2.654372 & 0.000000 \\ \mathrm{H} & 2.784723 & -2.977605 & 0.000000\end{array}$

7-hydroxyquinoline, trans $\mathrm{S}_{1}$

$\begin{array}{rrrr}\mathrm{N} & 0.533166 & 2.049502 & 0.000000 \\ \mathrm{C} & 0.478227 & 0.711859 & 0.000000 \\ \mathrm{C} & -0.747272 & -0.057574 & 0.000001 \\ \mathrm{C} & -1.951784 & 0.675102 & 0.000001 \\ \mathrm{C} & -1.875446 & 2.077544 & 0.000000 \\ \mathrm{C} & -0.643138 & 2.718866 & 0.000000 \\ \mathrm{C} & 1.680157 & -0.008988 & 0.000001 \\ \mathrm{C} & 1.688997 & -1.435041 & 0.000000 \\ \mathrm{C} & 0.514602 & -2.185844 & 0.000000 \\ \mathrm{C} & -0.686086 & -1.472565 & 0.000000 \\ \mathrm{H} & -0.579174 & 3.798821 & -0.000001 \\ \mathrm{H} & -1.623273 & -2.017663 & -0.000001 \\ \mathrm{H} & 0.538730 & -3.266977 & -0.000001 \\ \mathrm{O} & 2.903574 & -1.989002 & 0.000000 \\ \mathrm{H} & 2.624206 & 0.519664 & 0.000001 \\ \mathrm{H} & -2.906243 & 0.165796 & 0.000001 \\ \mathrm{H} & -2.783030 & 2.668336 & 0.000000 \\ \mathrm{H} & 2.833768 & -2.951815 & 0.000000\end{array}$

2-hydroxyquinoline, enol $\mathrm{S}_{0}$ 


$\begin{array}{rrrr}\mathrm{C} & 0.431488 & 1.900879 & 0.000000 \\ \mathrm{C} & 0.483499 & 0.488742 & 0.000000 \\ \mathrm{C} & -0.720456 & -0.257484 & 0.000000 \\ \mathrm{C} & -1.952613 & 0.436087 & 0.000000 \\ \mathrm{C} & -1.975010 & 1.804414 & 0.000000 \\ \mathrm{C} & -0.774024 & 2.547827 & 0.000000 \\ \mathrm{C} & 1.696635 & -0.243171 & 0.000000 \\ \mathrm{C} & 1.664036 & -1.602332 & 0.000000 \\ \mathrm{C} & 0.391729 & -2.245755 & 0.000000 \\ \mathrm{~N} & -0.740332 & -1.618708 & 0.000000 \\ \mathrm{O} & 0.315669 & -3.597519 & 0.000000 \\ \mathrm{H} & 1.360561 & 2.459544 & 0.000000 \\ \mathrm{H} & -0.812645 & 3.629393 & 0.000000 \\ \mathrm{H} & -2.922996 & 2.327698 & 0.000000 \\ \mathrm{H} & -2.860743 & -0.152374 & 0.000000 \\ \mathrm{H} & 2.641189 & 0.288748 & 0.000000 \\ \mathrm{H} & 2.574605 & -2.189308 & 0.000000 \\ \mathrm{H} & 1.199408 & -3.976679 & 0.000000\end{array}$

2-hydroxyquinoline, enol $\mathrm{S}_{1}$

$\begin{array}{lrrl}\mathrm{C} & 0.437929 & 1.878281 & 0.000000 \\ \mathrm{C} & 0.528434 & 0.487546 & 0.000000 \\ \mathrm{C} & -0.726896 & -0.261672 & 0.000000 \\ \mathrm{C} & -1.961060 & 0.438646 & 0.000000 \\ \mathrm{C} & -2.012433 & 1.836154 & 0.000000 \\ \mathrm{C} & -0.813834 & 2.541587 & 0.000000 \\ \mathrm{C} & 1.734506 & -0.235151 & 0.000000 \\ \mathrm{C} & 1.695994 & -1.637365 & 0.000000 \\ \mathrm{C} & 0.430175 & -2.238792 & 0.000000 \\ \mathrm{~N} & -0.752396 & -1.602704 & 0.000000 \\ \mathrm{O} & 0.296373 & -3.568662 & 0.000000 \\ \mathrm{H} & 1.346996 & 2.468065 & 0.000000 \\ \mathrm{H} & -0.823289 & 3.624692 & 0.000000 \\ \mathrm{H} & -2.959474 & 2.355618 & 0.000000 \\ \mathrm{H} & -2.860772 & -0.163167 & 0.000000 \\ \mathrm{H} & 2.676825 & 0.296539 & 0.000000 \\ \mathrm{H} & 2.595543 & -2.237152 & 0.000000 \\ \mathrm{H} & 1.167370 & -3.982442 & 0.000000\end{array}$

2-hydroxyquinoline, keto $\mathrm{S}_{0}$
$\begin{array}{llll}\mathrm{N} & 0.559205 & 1.703882 & 0.000000\end{array}$
$\begin{array}{llll}\text { C } & 0.600811 & 0.326474 & 0.000000\end{array}$
$\begin{array}{llll}\text { C } & -0.611389 & -0.382654 & 0.000000\end{array}$
$\begin{array}{llll}\text { C } & -1.836508 & 0.377514 & 0.000000\end{array}$ 


$\begin{array}{rrrr}\mathrm{C} & -1.834894 & 1.720661 & 0.000000 \\ \mathrm{C} & -0.586162 & 2.486276 & 0.000000 \\ \mathrm{C} & 1.817542 & -0.362580 & 0.000000 \\ \mathrm{C} & 1.820293 & -1.742009 & 0.000000 \\ \mathrm{C} & 0.621004 & -2.461353 & 0.000000 \\ \mathrm{C} & -0.578717 & -1.782145 & 0.000000 \\ \mathrm{H} & -1.517479 & -2.323801 & 0.000000 \\ \mathrm{H} & 0.637187 & -3.542752 & 0.000000 \\ \mathrm{H} & 2.764753 & -2.271080 & 0.000000 \\ \mathrm{H} & 2.747902 & 0.192768 & 0.000000 \\ \mathrm{H} & -2.773608 & -0.168685 & 0.000000 \\ \mathrm{H} & -2.743474 & 2.306019 & 0.000000 \\ \mathrm{O} & -0.512475 & 3.698567 & 0.000000 \\ \mathrm{H} & 1.426029 & 2.224918 & 0.000000\end{array}$

2-hydroxyquinoline, keto $\mathrm{S}_{1}$

$\begin{array}{rrrr}\mathrm{N} & 0.564140 & 1.672038 & 0.000000 \\ \mathrm{C} & 0.613663 & 0.327322 & 0.000000 \\ \mathrm{C} & -0.665335 & -0.380310 & 0.000000 \\ \mathrm{C} & -1.856872 & 0.365296 & 0.000000 \\ \mathrm{C} & -1.857994 & 1.763547 & 0.000000 \\ \mathrm{C} & -0.634509 & 2.475236 & 0.000000 \\ \mathrm{C} & 1.839136 & -0.361529 & 0.000000 \\ \mathrm{C} & 1.856322 & -1.748258 & 0.000000 \\ \mathrm{C} & 0.632319 & -2.446083 & 0.000000 \\ \mathrm{C} & -0.591179 & -1.784933 & 0.000000 \\ \mathrm{H} & -1.512784 & -2.352777 & 0.000000 \\ \mathrm{H} & 0.643595 & -3.528962 & 0.000000 \\ \mathrm{H} & 2.792677 & -2.286940 & 0.000000 \\ \mathrm{H} & 2.759836 & 0.209997 & 0.000000 \\ \mathrm{H} & -2.794894 & -0.174748 & 0.000000 \\ \mathrm{H} & -2.764923 & 2.346867 & 0.000000 \\ \mathrm{O} & -0.439083 & 3.682532 & 0.000000 \\ \mathrm{H} & 1.415883 & 2.221713 & 0.000000\end{array}$

dibenzofuran $\mathrm{S}_{0}$

$\begin{array}{lrrr}\text { C } & 1.717214 & 0.000000 & -1.356906 \\ \mathrm{C} & 0.725228 & 0.000000 & -0.380878 \\ \mathrm{C} & 1.094118 & 0.000000 & 0.967946 \\ \mathrm{C} & 2.408881 & 0.000000 & 1.392985 \\ \mathrm{C} & 3.382166 & 0.000000 & 0.403716 \\ \mathrm{C} & 3.042981 & 0.000000 & -0.952957 \\ \mathrm{C} & -0.725228 & 0.000000 & -0.380878 \\ \mathrm{C} & -1.094118 & 0.000000 & 0.967946\end{array}$




$\begin{array}{rrrr}\mathrm{O} & 0.000000 & 0.000000 & 1.790090 \\ \mathrm{C} & -1.717214 & 0.000000 & -1.356906 \\ \mathrm{C} & -3.042981 & 0.000000 & -0.952957 \\ \mathrm{C} & -3.382166 & 0.000000 & 0.403716 \\ \mathrm{C} & -2.408881 & 0.000000 & 1.392985 \\ \mathrm{H} & -2.656156 & 0.000000 & 2.445654 \\ \mathrm{H} & -4.426122 & 0.000000 & 0.690060 \\ \mathrm{H} & -3.829672 & 0.000000 & -1.695999 \\ \mathrm{H} & -1.458993 & 0.000000 & -2.408500 \\ \mathrm{H} & 1.458993 & 0.000000 & -2.408500 \\ \mathrm{H} & 3.829672 & 0.000000 & -1.695999 \\ \mathrm{H} & 4.426122 & 0.000000 & 0.690060 \\ \mathrm{H} & 2.656156 & 0.000000 & 2.445654\end{array}$

dibenzofuran $\mathrm{S}_{1}$

$\begin{array}{rrrr}\mathrm{C} & 0.000000 & 1.727845 & -1.531333 \\ \mathrm{C} & 0.000000 & 0.710703 & -0.570267 \\ \mathrm{C} & 0.000000 & 1.101399 & 0.804143 \\ \mathrm{C} & 0.000000 & 2.406184 & 1.256139 \\ \mathrm{C} & 0.000000 & 3.413615 & 0.266865 \\ \mathrm{C} & 0.000000 & 3.067854 & -1.087004 \\ \mathrm{C} & 0.000000 & -0.710703 & -0.570267 \\ \mathrm{C} & 0.000000 & -1.101399 & 0.804143 \\ \mathrm{O} & 0.000000 & 0.000000 & 1.598122 \\ \mathrm{C} & 0.000000 & -1.727845 & -1.531333 \\ \mathrm{C} & 0.000000 & -3.067854 & -1.087004 \\ \mathrm{C} & 0.000000 & -3.413615 & 0.266865 \\ \mathrm{C} & 0.000000 & -2.406184 & 1.256139 \\ \mathrm{H} & 0.000000 & -2.630859 & 2.313396 \\ \mathrm{H} & 0.000000 & -4.453665 & 0.559446 \\ \mathrm{H} & 0.000000 & -3.857660 & -1.827832 \\ \mathrm{H} & 0.000000 & -1.502480 & -2.588904 \\ \mathrm{H} & 0.000000 & 1.502480 & -2.588904 \\ \mathrm{H} & 0.000000 & 3.857660 & -1.827832 \\ \mathrm{H} & 0.000000 & 4.453665 & 0.559446 \\ \mathrm{H} & 0.000000 & 2.630859 & 2.313396\end{array}$

pyrrolo[3,2-h]quinoline $\mathrm{S}_{0}$

$\begin{array}{lrrr}\mathrm{C} & 0.136122 & -0.683353 & 0.000000 \\ \mathrm{~N} & 0.058309 & -2.032238 & 0.000000 \\ \mathrm{C} & 1.187500 & -2.703243 & 0.000000 \\ \mathrm{C} & 2.457494 & -2.096861 & 0.000000 \\ \mathrm{C} & 2.536841 & -0.727395 & 0.000000 \\ \mathrm{C} & 1.356449 & 0.038707 & 0.000000\end{array}$




$\begin{array}{rrrr}\mathrm{H} & 1.105088 & -3.785457 & 0.000000 \\ \mathrm{H} & 3.346426 & -2.713068 & 0.000000 \\ \mathrm{H} & 3.497551 & -0.224674 & 0.000000 \\ \mathrm{C} & -1.051410 & 0.086428 & 0.000000 \\ \mathrm{C} & -1.056786 & 1.483043 & 0.000000 \\ \mathrm{C} & 0.184373 & 2.176541 & 0.000000 \\ \mathrm{C} & 1.349130 & 1.470341 & 0.000000 \\ \mathrm{H} & 0.198973 & 3.259378 & 0.000000 \\ \mathrm{H} & 2.302839 & 1.984388 & 0.000000 \\ \mathrm{C} & -2.427568 & 1.886134 & 0.000000 \\ \mathrm{C} & -3.177697 & 0.741375 & 0.000000 \\ \mathrm{~N} & -2.342222 & -0.346784 & 0.000000 \\ \mathrm{H} & -2.603950 & -1.319147 & 0.000000 \\ \mathrm{H} & -2.810247 & 2.892935 & 0.000000 \\ \mathrm{H} & -4.247206 & 0.612949 & 0.000000\end{array}$

pyrrolo[3,2-h]quinoline $\mathrm{S}_{1}$

$\begin{array}{rrrr}\mathrm{C} & 0.152257 & -0.701518 & 0.000000 \\ \mathrm{~N} & 0.012463 & -2.022941 & 0.000000 \\ \mathrm{C} & 1.197388 & -2.729807 & 0.000000 \\ \mathrm{C} & 2.432505 & -2.134150 & 0.000000 \\ \mathrm{C} & 2.561014 & -0.725703 & 0.000000 \\ \mathrm{C} & 1.362571 & 0.029102 & 0.000000 \\ \mathrm{H} & 1.098708 & -3.807263 & 0.000000 \\ \mathrm{H} & 3.317692 & -2.757569 & 0.000000 \\ \mathrm{H} & 3.526890 & -0.241381 & 0.000000 \\ \mathrm{C} & -1.024474 & 0.104096 & 0.000000 \\ \mathrm{C} & -1.061151 & 1.552713 & 0.000000 \\ \mathrm{C} & 0.140847 & 2.231191 & 0.000000 \\ \mathrm{C} & 1.319966 & 1.454688 & 0.000000 \\ \mathrm{H} & 0.190959 & 3.310554 & 0.000000 \\ \mathrm{H} & 2.272410 & 1.973758 & 0.000000 \\ \mathrm{C} & -2.457347 & 1.905743 & 0.000000 \\ \mathrm{C} & -3.162725 & 0.746869 & 0.000000 \\ \mathrm{~N} & -2.281566 & -0.340330 & 0.000000 \\ \mathrm{H} & -2.499446 & -1.327756 & 0.000000 \\ \mathrm{H} & -2.871973 & 2.899174 & 0.000000 \\ \mathrm{H} & -4.226999 & 0.580528 & 0.000000\end{array}$

carbazole $\mathrm{S}_{0}$
$\begin{array}{llll}\text { C } & 1.127714 & 0.000000 & -0.895809\end{array}$
$\begin{array}{llll}\mathrm{N} & 0.000000 & 0.000000 & -1.693215\end{array}$
$\begin{array}{llll}\mathrm{C} & -1.127714 & 0.000000 & -0.895809\end{array}$
$\begin{array}{llll}\text { C } & -0.723829 & 0.000000 & 0.455919\end{array}$ 


$\begin{array}{rrrr}\mathrm{C} & 0.723829 & 0.000000 & 0.455919 \\ \mathrm{C} & -1.690760 & 0.000000 & 1.458539 \\ \mathrm{C} & -3.028767 & 0.000000 & 1.103429 \\ \mathrm{C} & -3.410314 & 0.000000 & -0.243656 \\ \mathrm{C} & -2.470297 & 0.000000 & -1.259971 \\ \mathrm{C} & 2.470297 & 0.000000 & -1.259971 \\ \mathrm{C} & 3.410314 & 0.000000 & -0.243656 \\ \mathrm{C} & 3.028767 & 0.000000 & 1.103429 \\ \mathrm{C} & 1.690760 & 0.000000 & 1.458539 \\ \mathrm{H} & -2.771454 & 0.000000 & -2.300146 \\ \mathrm{H} & -4.462885 & 0.000000 & -0.497435 \\ \mathrm{H} & -3.790111 & 0.000000 & 1.872371 \\ \mathrm{H} & -1.398678 & 0.000000 & 2.501791 \\ \mathrm{H} & 1.398678 & 0.000000 & 2.501791 \\ \mathrm{H} & 3.790111 & 0.000000 & 1.872371 \\ \mathrm{H} & 4.462885 & 0.000000 & -0.497435 \\ \mathrm{H} & 2.771454 & 0.000000 & -2.300146 \\ \mathrm{H} & 0.000000 & 0.000000 & -2.697809\end{array}$

carbazole $\mathrm{S}_{1}$

$\begin{array}{rrrr}\mathrm{C} & 1.140401 & 0.000000 & -0.836026 \\ \mathrm{~N} & 0.000000 & 0.000000 & -1.605871 \\ \mathrm{C} & -1.140401 & 0.000000 & -0.836026 \\ \mathrm{C} & -0.714381 & 0.000000 & 0.532861 \\ \mathrm{C} & 0.714381 & 0.000000 & 0.532861 \\ \mathrm{C} & -1.703002 & 0.000000 & 1.520599 \\ \mathrm{C} & -3.055201 & 0.000000 & 1.117357 \\ \mathrm{C} & -3.444146 & 0.000000 & -0.219726 \\ \mathrm{C} & -2.462840 & 0.000000 & -1.238663 \\ \mathrm{C} & 2.462840 & 0.000000 & -1.238663 \\ \mathrm{C} & 3.444146 & 0.000000 & -0.219726 \\ \mathrm{C} & 3.055201 & 0.000000 & 1.117357 \\ \mathrm{C} & 1.703002 & 0.000000 & 1.520599 \\ \mathrm{H} & -2.735122 & 0.000000 & -2.286263 \\ \mathrm{H} & -4.492581 & 0.000000 & -0.480498 \\ \mathrm{H} & -3.821059 & 0.000000 & 1.883642 \\ \mathrm{H} & -1.446140 & 0.000000 & 2.571074 \\ \mathrm{H} & 1.446140 & 0.000000 & 2.571074 \\ \mathrm{H} & 3.821059 & 0.000000 & 1.883642 \\ \mathrm{H} & 4.492581 & 0.000000 & -0.480498 \\ \mathrm{H} & 2.735122 & 0.000000 & -2.286263 \\ \mathrm{H} & 0.000000 & 0.000000 & -2.615472\end{array}$

tryptamine, A-ph $\mathrm{S}_{0}$ 


$\begin{array}{lrrc}\mathrm{C} & -2.144586 & 0.161512 & 0.904482 \\ \mathrm{~N} & -1.258530 & 0.142527 & 1.961880 \\ \mathrm{C} & 0.021277 & 0.046120 & 1.472629 \\ \mathrm{C} & -0.073166 & -0.001486 & 0.064896 \\ \mathrm{C} & -1.469330 & 0.076023 & -0.276058 \\ \mathrm{C} & 1.247172 & 0.000206 & 2.136302 \\ \mathrm{C} & 2.386462 & -0.093370 & 1.361965 \\ \mathrm{C} & 2.315869 & -0.136738 & -0.041287 \\ \mathrm{C} & 1.100408 & -0.091023 & -0.693503 \\ \mathrm{C} & -2.038864 & 0.021426 & -1.656361 \\ \mathrm{C} & -1.949354 & -1.377034 & -2.265913 \\ \mathrm{~N} & -2.423464 & -1.352783 & -3.647744 \\ \mathrm{H} & 1.305362 & 0.036911 & 3.217186 \\ \mathrm{H} & 3.354821 & -0.131054 & 1.844721 \\ \mathrm{H} & 3.231964 & -0.204893 & -0.613724 \\ \mathrm{H} & 1.052070 & -0.118109 & -1.776001 \\ \mathrm{H} & -1.508751 & 0.212759 & 2.932022 \\ \mathrm{H} & -3.205689 & 0.239940 & 1.080956 \\ \mathrm{H} & -1.509289 & 0.714244 & -2.315779 \\ \mathrm{H} & -3.085627 & 0.339636 & -1.637278 \\ \mathrm{H} & -2.493589 & -2.077145 & -1.618020 \\ \mathrm{H} & -0.904719 & -1.695359 & -2.270864 \\ \mathrm{H} & -3.412696 & -1.133249 & -3.678954 \\ \mathrm{H} & -2.305160 & -2.256681 & -4.088182\end{array}$

tryptamine, A-ph $\mathrm{S}_{1}$

$\begin{array}{lrrr}\mathrm{C} & -2.195016 & 0.185697 & 0.919293 \\ \mathrm{~N} & -1.303614 & 0.136517 & 1.915100 \\ \mathrm{C} & 0.015101 & 0.058104 & 1.430782 \\ \mathrm{C} & -0.072081 & 0.076751 & 0.032729 \\ \mathrm{C} & -1.449589 & 0.150324 & -0.306003 \\ \mathrm{C} & 1.200050 & -0.065705 & 2.141729 \\ \mathrm{C} & 2.388911 & -0.153474 & 1.322995 \\ \mathrm{C} & 2.333939 & -0.133875 & -0.047871 \\ \mathrm{C} & 1.103250 & -0.019457 & -0.756831 \\ \mathrm{C} & -2.024579 & 0.070100 & -1.665335 \\ \mathrm{C} & -1.925859 & -1.364151 & -2.215055 \\ \mathrm{~N} & -2.444698 & -1.403286 & -3.576529 \\ \mathrm{H} & 1.242858 & -0.100375 & 3.219893 \\ \mathrm{H} & 3.348793 & -0.238242 & 1.816098 \\ \mathrm{H} & 3.256517 & -0.201489 & -0.610486 \\ \mathrm{H} & 1.069647 & 0.044344 & -1.835082 \\ \mathrm{H} & -1.536149 & 0.169959 & 2.896590 \\ \mathrm{H} & -3.253966 & 0.286843 & 1.086585 \\ \mathrm{H} & -1.472982 & 0.721224 & -2.348053\end{array}$




$\begin{array}{llll}\mathrm{H} & -3.070683 & 0.388605 & -1.662366 \\ \mathrm{H} & -2.434837 & -2.044804 & -1.519964 \\ \mathrm{H} & -0.871185 & -1.646434 & -2.233061 \\ \mathrm{H} & -3.453919 & -1.313060 & -3.582791 \\ \mathrm{H} & -2.217338 & -2.281737 & -4.025038\end{array}$

tryptamine, A-py $\mathrm{S}_{0}$

$\begin{array}{lrrc}\mathrm{C} & -2.138916 & 0.135367 & 0.883051 \\ \mathrm{~N} & -1.261393 & 0.133043 & 1.947326 \\ \mathrm{C} & 0.023258 & 0.047825 & 1.469288 \\ \mathrm{C} & -0.059361 & -0.010458 & 0.060920 \\ \mathrm{C} & -1.453487 & 0.048702 & -0.291620 \\ \mathrm{C} & 1.243984 & 0.020108 & 2.143410 \\ \mathrm{C} & 2.391036 & -0.065609 & 1.379765 \\ \mathrm{C} & 2.332736 & -0.119992 & -0.023633 \\ \mathrm{C} & 1.122090 & -0.093200 & -0.685758 \\ \mathrm{C} & -2.021114 & -0.013600 & -1.672572 \\ \mathrm{C} & -1.916011 & -1.409620 & -2.284528 \\ \mathrm{~N} & -2.460217 & -1.400369 & -3.640846 \\ \mathrm{H} & 1.292273 & 0.064159 & 3.224522 \\ \mathrm{H} & 3.355522 & -0.089168 & 1.871048 \\ \mathrm{H} & 3.254296 & -0.183055 & -0.587842 \\ \mathrm{H} & 1.084230 & -0.132523 & -1.768348 \\ \mathrm{H} & -1.520562 & 0.204805 & 2.915026 \\ \mathrm{H} & -3.202311 & 0.203400 & 1.049438 \\ \mathrm{H} & -1.491605 & 0.695454 & -2.319156 \\ \mathrm{H} & -3.070608 & 0.286770 & -1.669912 \\ \mathrm{H} & -2.509866 & -2.099510 & -1.681571 \\ \mathrm{H} & -0.873187 & -1.749185 & -2.225684 \\ \mathrm{H} & -1.890588 & -0.819118 & -4.245496 \\ \mathrm{H} & -2.460289 & -2.331834 & -4.037720\end{array}$

tryptamine, A-py $\mathrm{S}_{1}$

$\begin{array}{rrrr}\mathrm{C} & -2.189966 & 0.145532 & 0.898130 \\ \mathrm{~N} & -1.308038 & 0.113327 & 1.902000 \\ \mathrm{C} & 0.016893 & 0.054833 & 1.429704 \\ \mathrm{C} & -0.057993 & 0.065833 & 0.031530 \\ \mathrm{C} & -1.432977 & 0.118244 & -0.319899 \\ \mathrm{C} & 1.198281 & -0.043998 & 2.151950 \\ \mathrm{C} & 2.394116 & -0.116534 & 1.343074 \\ \mathrm{C} & 2.351659 & -0.104619 & -0.028623 \\ \mathrm{C} & 1.125373 & -0.013987 & -0.748645 \\ \mathrm{C} & -1.998863 & 0.034406 & -1.681839 \\ \mathrm{C} & -1.876106 & -1.397149 & -2.237990\end{array}$




$\begin{array}{lrrr}\mathrm{N} & -2.455696 & -1.449257 & -3.573426 \\ \mathrm{H} & 1.233293 & -0.068805 & 3.230578 \\ \mathrm{H} & 3.351230 & -0.183118 & 1.844457 \\ \mathrm{H} & 3.280650 & -0.160710 & -0.581844 \\ \mathrm{H} & 1.099640 & 0.040384 & -1.827594 \\ \mathrm{H} & -1.550545 & 0.144723 & 2.881102 \\ \mathrm{H} & -3.252617 & 0.224829 & 1.053497 \\ \mathrm{H} & -1.447199 & 0.707320 & -2.346588 \\ \mathrm{H} & -3.050192 & 0.326015 & -1.698015 \\ \mathrm{H} & -2.440805 & -2.071903 & -1.591208 \\ \mathrm{H} & -0.821225 & -1.695592 & -2.185518 \\ \mathrm{H} & -1.877293 & -0.942866 & -4.233953 \\ \mathrm{H} & -2.521711 & -2.404528 & -3.901780\end{array}$

tryptamine, A-up $\mathrm{S}_{0}$

$\begin{array}{rrrr}\mathrm{C} & -2.137423 & 0.149943 & 0.893941 \\ \mathrm{~N} & -1.258946 & 0.120244 & 1.956818 \\ \mathrm{C} & 0.024809 & 0.038514 & 1.475668 \\ \mathrm{C} & -0.059034 & 0.010981 & 0.066617 \\ \mathrm{C} & -1.453129 & 0.087309 & -0.283196 \\ \mathrm{C} & 1.245750 & -0.010869 & 2.148071 \\ \mathrm{C} & 2.391500 & -0.087847 & 1.381657 \\ \mathrm{C} & 2.331879 & -0.112700 & -0.022490 \\ \mathrm{C} & 1.121145 & -0.063653 & -0.682991 \\ \mathrm{C} & -2.016608 & 0.043834 & -1.666406 \\ \mathrm{C} & -1.917469 & -1.352486 & -2.298674 \\ \mathrm{~N} & -2.395878 & -1.451759 & -3.671866 \\ \mathrm{H} & 1.295148 & 0.009890 & 3.229795 \\ \mathrm{H} & 3.356228 & -0.128199 & 1.871324 \\ \mathrm{H} & 3.252463 & -0.170931 & -0.588711 \\ \mathrm{H} & 1.082790 & -0.081074 & -1.766086 \\ \mathrm{H} & -1.516848 & 0.169668 & 2.926290 \\ \mathrm{H} & -3.200207 & 0.219042 & 1.063992 \\ \mathrm{H} & -1.485657 & 0.757324 & -2.306254 \\ \mathrm{H} & -3.065216 & 0.355213 & -1.651017 \\ \mathrm{H} & -2.480443 & -2.059353 & -1.685312 \\ \mathrm{H} & -0.878317 & -1.688188 & -2.273260 \\ \mathrm{H} & -1.867842 & -0.836951 & -4.280415 \\ \mathrm{H} & -3.367458 & -1.170040 & -3.734254\end{array}$

tryptamine, A-up $\mathrm{S}_{1}$
$\begin{array}{llll}\text { C } & -2.188567 & 0.151684 & 0.904333\end{array}$
$\begin{array}{llll}\mathrm{N} & -1.304045 & 0.091257 & 1.906027\end{array}$
$\begin{array}{llll}\text { C } & 0.019718 & 0.043439 & 1.432548\end{array}$ 


$\begin{array}{rrrr}\mathrm{C} & -0.055316 & 0.093591 & 0.035117 \\ \mathrm{C} & -1.431462 & 0.164447 & -0.313958 \\ \mathrm{C} & 1.201118 & -0.077208 & 2.151223 \\ \mathrm{C} & 2.396507 & -0.133811 & 1.341351 \\ \mathrm{C} & 2.353089 & -0.086823 & -0.029171 \\ \mathrm{C} & 1.126510 & 0.028114 & -0.746189 \\ \mathrm{C} & -1.988179 & 0.103167 & -1.676262 \\ \mathrm{C} & -1.910038 & -1.351445 & -2.228719 \\ \mathrm{~N} & -2.389274 & -1.514756 & -3.584845 \\ \mathrm{H} & 1.235658 & -0.131562 & 3.228822 \\ \mathrm{H} & 3.353303 & -0.218708 & 1.840412 \\ \mathrm{H} & 3.281004 & -0.134427 & -0.585002 \\ \mathrm{H} & 1.101739 & 0.102503 & -1.824021 \\ \mathrm{H} & -1.545425 & 0.097012 & 2.885947 \\ \mathrm{H} & -3.250045 & 0.233997 & 1.065860 \\ \mathrm{H} & -1.406623 & 0.745550 & -2.343907 \\ \mathrm{H} & -3.028684 & 0.437384 & -1.694792 \\ \mathrm{H} & -2.485329 & -2.006510 & -1.571978 \\ \mathrm{H} & -0.869078 & -1.677188 & -2.175222 \\ \mathrm{H} & -1.852246 & -0.959182 & -4.239141 \\ \mathrm{H} & -3.363113 & -1.252605 & -3.675213\end{array}$

tryptamine, $\mathrm{Ph}$-out $\mathrm{S}_{0}$

$\begin{array}{lrrr}\mathrm{C} & -0.006446 & 0.003984 & 0.068710 \\ \mathrm{C} & 0.038745 & 0.054388 & 1.479295 \\ \mathrm{C} & 1.241037 & 0.055792 & 2.186593 \\ \mathrm{C} & 2.409456 & 0.009845 & 1.453035 \\ \mathrm{C} & 2.388423 & -0.039061 & 0.047769 \\ \mathrm{C} & 1.197407 & -0.042731 & -0.649010 \\ \mathrm{~N} & -1.258354 & 0.098883 & 1.927085 \\ \mathrm{H} & 1.258409 & 0.092333 & 3.268921 \\ \mathrm{H} & 3.361439 & 0.010773 & 1.968611 \\ \mathrm{H} & 3.325506 & -0.074655 & -0.492960 \\ \mathrm{H} & 1.181428 & -0.099654 & -1.730802 \\ \mathrm{C} & -1.394754 & 0.026099 & -0.317496 \\ \mathrm{C} & -2.109843 & 0.086072 & 0.842849 \\ \mathrm{H} & -1.541401 & 0.162099 & 2.888757 \\ \mathrm{H} & -3.178086 & 0.134018 & 0.985242 \\ \mathrm{C} & -1.947033 & -0.026809 & -1.708925 \\ \mathrm{H} & -1.301899 & 0.544738 & -2.381048 \\ \mathrm{C} & -2.068798 & -1.450552 & -2.252780 \\ \mathrm{H} & -2.933975 & 0.441969 & -1.724442 \\ \mathrm{H} & -2.498131 & -1.409092 & -3.256443 \\ \mathrm{~N} & -0.750704 & -2.070880 & -2.353437 \\ \mathrm{H} & -2.768559 & -2.008770 & -1.615103\end{array}$




$$
\begin{array}{llll}
\mathrm{H} & -0.342165 & -2.180777 & -1.430897 \\
\mathrm{H} & -0.816733 & -2.993901 & -2.764311
\end{array}
$$

tryptamine, $\mathrm{Ph}$-out $\mathrm{S}_{1}$

$\begin{array}{rrrr}\mathrm{C} & -0.000758 & 0.029305 & 0.049116 \\ \mathrm{C} & 0.034864 & 0.065821 & 1.451391 \\ \mathrm{C} & 1.195778 & 0.032340 & 2.209430 \\ \mathrm{C} & 2.420667 & -0.014344 & 1.441924 \\ \mathrm{C} & 2.417582 & -0.053994 & 0.070717 \\ \mathrm{C} & 1.212088 & -0.046970 & -0.688291 \\ \mathrm{~N} & -1.300307 & 0.114351 & 1.885847 \\ \mathrm{H} & 1.196678 & 0.056162 & 3.288935 \\ \mathrm{H} & 3.363060 & -0.021062 & 1.974147 \\ \mathrm{H} & 3.362995 & -0.091330 & -0.455732 \\ \mathrm{H} & 1.215016 & -0.065617 & -1.768592 \\ \mathrm{C} & -1.366781 & 0.060548 & -0.341215 \\ \mathrm{C} & -2.156329 & 0.104943 & 0.858484 \\ \mathrm{H} & -1.568936 & 0.164054 & 2.857360 \\ \mathrm{H} & -3.223824 & 0.168456 & 0.985641 \\ \mathrm{C} & -1.932247 & -0.021464 & -1.707430 \\ \mathrm{H} & -1.276455 & 0.492350 & -2.413582 \\ \mathrm{C} & -2.078584 & -1.482297 & -2.177956 \\ \mathrm{H} & -2.915701 & 0.455079 & -1.726978 \\ \mathrm{H} & -2.643131 & -1.478092 & -3.112780 \\ \mathrm{~N} & -0.768928 & -2.055015 & -2.438234 \\ \mathrm{H} & -2.674652 & -2.028142 & -1.432917 \\ \mathrm{H} & -0.183912 & -2.050103 & -1.607654 \\ \mathrm{H} & -0.843193 & -3.010859 & -2.762419\end{array}$

tryptamine, $\mathrm{Ph}$-up $\mathrm{S}_{0}$

$\begin{array}{lrrr}\mathrm{C} & -2.097227 & 0.054330 & 0.822079 \\ \mathrm{~N} & -1.254562 & 0.040293 & 1.912837 \\ \mathrm{C} & 0.046156 & 0.034402 & 1.475324 \\ \mathrm{C} & 0.011782 & 0.042234 & 0.063369 \\ \mathrm{C} & -1.372139 & 0.053621 & -0.333912 \\ \mathrm{C} & 1.242777 & 0.025505 & 2.191867 \\ \mathrm{C} & 2.418133 & 0.027796 & 1.468332 \\ \mathrm{C} & 2.409570 & 0.041305 & 0.062181 \\ \mathrm{C} & 1.223107 & 0.048184 & -0.641728 \\ \mathrm{C} & -1.908349 & 0.016727 & -1.730802 \\ \mathrm{C} & -2.073588 & -1.414637 & -2.269048 \\ \mathrm{~N} & -0.857511 & -2.212580 & -2.334275 \\ \mathrm{H} & 1.250643 & 0.016502 & 3.274875 \\ \mathrm{H} & 3.365482 & 0.020577 & 1.992015\end{array}$




$\begin{array}{lrrr}\mathrm{H} & 3.351207 & 0.047523 & -0.471559 \\ \mathrm{H} & 1.225600 & 0.061766 & -1.725451 \\ \mathrm{H} & -1.546755 & 0.052069 & 2.873852 \\ \mathrm{H} & -3.167195 & 0.072290 & 0.958061 \\ \mathrm{H} & -1.237161 & 0.572384 & -2.393627 \\ \mathrm{H} & -2.876662 & 0.523877 & -1.767015 \\ \mathrm{H} & -2.507024 & -1.374169 & -3.271215 \\ \mathrm{H} & -2.788373 & -1.946371 & -1.636067 \\ \mathrm{H} & -0.422982 & -2.270622 & -1.419409 \\ \mathrm{H} & -0.181281 & -1.783376 & -2.955624\end{array}$

tryptamine, $\mathrm{Ph}$-up $\mathrm{S}_{1}$

$\begin{array}{lrrr}\mathrm{C} & -2.162293 & 0.126905 & 0.852581 \\ \mathrm{~N} & -1.306948 & 0.098811 & 1.880455 \\ \mathrm{C} & 0.028664 & 0.063706 & 1.444188 \\ \mathrm{C} & -0.007543 & 0.087011 & 0.043166 \\ \mathrm{C} & -1.374006 & 0.122836 & -0.345418 \\ \mathrm{C} & 1.190831 & -0.016140 & 2.199723 \\ \mathrm{C} & 2.412014 & -0.045033 & 1.429403 \\ \mathrm{C} & 2.410196 & -0.020995 & 0.057438 \\ \mathrm{C} & 1.204398 & 0.038235 & -0.699622 \\ \mathrm{C} & -1.919582 & 0.024296 & -1.708298 \\ \mathrm{C} & -2.012274 & -1.469680 & -2.172450 \\ \mathrm{~N} & -0.756914 & -2.153185 & -2.348527 \\ \mathrm{H} & 1.192306 & -0.046423 & 3.278949 \\ \mathrm{H} & 3.354922 & -0.088798 & 1.959136 \\ \mathrm{H} & 3.356249 & -0.041856 & -0.468802 \\ \mathrm{H} & 1.214216 & 0.125897 & -1.776554 \\ \mathrm{H} & -1.575746 & 0.119888 & 2.852762 \\ \mathrm{H} & -3.229841 & 0.186628 & 0.982135 \\ \mathrm{H} & -1.278184 & 0.558237 & -2.413589 \\ \mathrm{H} & -2.922918 & 0.456768 & -1.751760 \\ \mathrm{H} & -2.556207 & -1.483553 & -3.119193 \\ \mathrm{H} & -2.614739 & -2.016673 & -1.444823 \\ \mathrm{H} & -0.192261 & -2.147476 & -1.504659 \\ \mathrm{H} & -0.200679 & -1.729795 & -3.081151\end{array}$

tryptamine, Py-out $\mathrm{S}_{0}$

$\begin{array}{llll}\mathrm{C} & 0.499273 & 0.462319 & 0.211118 \\ \mathrm{C} & 0.576419 & 0.518790 & 1.620059 \\ \mathrm{C} & 1.791766 & 0.458137 & 2.301203 \\ \mathrm{C} & 2.940319 & 0.339759 & 1.543971 \\ \mathrm{C} & 2.887809 & 0.285323 & 0.140441 \\ \mathrm{C} & 1.682487 & 0.346230 & -0.529122\end{array}$




$\begin{array}{rrrr}\mathrm{N} & -0.707628 & 0.637120 & 2.092914 \\ \mathrm{H} & 1.834653 & 0.503539 & 3.382396 \\ \mathrm{H} & 3.901232 & 0.290560 & 2.040326 \\ \mathrm{H} & 3.810090 & 0.196861 & -0.419107 \\ \mathrm{H} & 1.650419 & 0.307693 & -1.611681 \\ \mathrm{C} & -0.891824 & 0.559589 & -0.147279 \\ \mathrm{C} & -1.580857 & 0.659489 & 1.025085 \\ \mathrm{H} & -0.968583 & 0.711476 & 3.059999 \\ \mathrm{H} & -2.644231 & 0.746014 & 1.183215 \\ \mathrm{C} & -1.460175 & 0.492558 & -1.528836 \\ \mathrm{H} & -0.906057 & 1.164323 & -2.190579 \\ \mathrm{C} & -1.413843 & -0.925284 & -2.101434 \\ \mathrm{H} & -2.500188 & 0.824991 & -1.513819 \\ \mathrm{H} & -0.365111 & -1.244863 & -2.176949 \\ \mathrm{~N} & -2.232659 & -1.813163 & -1.281140 \\ \mathrm{H} & -1.828076 & -0.914184 & -3.111775 \\ \mathrm{H} & -2.220977 & -2.756643 & -1.648520 \\ \mathrm{H} & -1.854240 & -1.850633 & -0.340466\end{array}$

tryptamine, Py-out $\mathrm{S}_{1}$

$\begin{array}{lrrr}\mathrm{C} & 0.494475 & 0.504329 & 0.186270 \\ \mathrm{C} & 0.570733 & 0.523906 & 1.584101 \\ \mathrm{C} & 1.749964 & 0.414044 & 2.309509 \\ \mathrm{C} & 2.943643 & 0.299136 & 1.502149 \\ \mathrm{C} & 2.900180 & 0.281971 & 0.130987 \\ \mathrm{C} & 1.674981 & 0.379336 & -0.591732 \\ \mathrm{~N} & -0.750722 & 0.634575 & 2.056937 \\ \mathrm{H} & 1.784791 & 0.423033 & 3.388412 \\ \mathrm{H} & 3.899654 & 0.225043 & 2.004516 \\ \mathrm{H} & 3.827206 & 0.195969 & -0.421679 \\ \mathrm{H} & 1.650419 & 0.401854 & -1.671633 \\ \mathrm{C} & -0.880442 & 0.601919 & -0.164459 \\ \mathrm{C} & -1.633212 & 0.675970 & 1.054378 \\ \mathrm{H} & -0.990467 & 0.681026 & 3.036127 \\ \mathrm{H} & -2.695898 & 0.761316 & 1.205804 \\ \mathrm{C} & -1.453491 & 0.518935 & -1.524852 \\ \mathrm{H} & -0.879369 & 1.161664 & -2.198563 \\ \mathrm{C} & -1.383041 & -0.926031 & -2.053194 \\ \mathrm{H} & -2.495824 & 0.842375 & -1.524354 \\ \mathrm{H} & -0.326583 & -1.217337 & -2.109081 \\ \mathrm{~N} & -2.191588 & -1.800150 & -1.210541 \\ \mathrm{H} & -1.795706 & -0.944290 & -3.062791 \\ \mathrm{H} & -2.269402 & -2.721928 & -1.622650 \\ \mathrm{H} & -1.750311 & -1.916673 & -0.303671\end{array}$


tryptamine, Py-up $\mathrm{S}_{0}$

$\begin{array}{rrrr}\mathrm{C} & -2.137729 & 0.266505 & 0.887946 \\ \mathrm{~N} & -1.261434 & 0.230409 & 1.953013 \\ \mathrm{C} & 0.018711 & 0.091070 & 1.475233 \\ \mathrm{C} & -0.064434 & 0.036030 & 0.067007 \\ \mathrm{C} & -1.454980 & 0.154567 & -0.287093 \\ \mathrm{C} & 1.235711 & 0.011181 & 2.151719 \\ \mathrm{C} & 2.378864 & -0.125305 & 1.389738 \\ \mathrm{C} & 2.320157 & -0.179218 & -0.013639 \\ \mathrm{C} & 1.113502 & -0.099632 & -0.678310 \\ \mathrm{C} & -2.020288 & 0.094987 & -1.668384 \\ \mathrm{C} & -1.968163 & -1.324666 & -2.260239 \\ \mathrm{~N} & -2.719334 & -2.332709 & -1.526093 \\ \mathrm{H} & 1.283676 & 0.055260 & 3.232717 \\ \mathrm{H} & 3.340780 & -0.190348 & 1.882302 \\ \mathrm{H} & 3.238648 & -0.283526 & -0.576560 \\ \mathrm{H} & 1.076778 & -0.138609 & -1.760705 \\ \mathrm{H} & -1.517120 & 0.310801 & 2.921000 \\ \mathrm{H} & -3.197585 & 0.380477 & 1.053040 \\ \mathrm{H} & -1.469588 & 0.776647 & -2.324748 \\ \mathrm{H} & -3.059718 & 0.436556 & -1.654536 \\ \mathrm{H} & -0.928120 & -1.655925 & -2.309304 \\ \mathrm{H} & -2.340529 & -1.300548 & -3.286745 \\ \mathrm{H} & -3.699764 & -2.077630 & -1.476690 \\ \mathrm{H} & -2.379521 & -2.386751 & -0.571778\end{array}$

tryptamine, Py-up $\mathrm{S}_{1}$

$\begin{array}{rrrr}\mathrm{C} & -2.190972 & 0.287049 & 0.919975 \\ \mathrm{~N} & -1.301353 & 0.238725 & 1.918656 \\ \mathrm{C} & 0.014224 & 0.107154 & 1.439997 \\ \mathrm{C} & -0.070137 & 0.088492 & 0.041318 \\ \mathrm{C} & -1.445477 & 0.200314 & -0.302779 \\ \mathrm{C} & 1.193435 & -0.026554 & 2.158034 \\ \mathrm{C} & 2.382571 & -0.168476 & 1.347068 \\ \mathrm{C} & 2.331022 & -0.185555 & -0.023255 \\ \mathrm{C} & 1.104784 & -0.060758 & -0.739376 \\ \mathrm{C} & -2.019228 & 0.115297 & -1.658853 \\ \mathrm{C} & -1.936497 & -1.334336 & -2.214993 \\ \mathrm{~N} & -2.691390 & -2.333892 & -1.487113 \\ \mathrm{H} & 1.232295 & -0.024105 & 3.236945 \\ \mathrm{H} & 3.338256 & -0.265252 & 1.845809 \\ \mathrm{H} & 3.252273 & -0.293618 & -0.581630 \\ \mathrm{H} & 1.077523 & -0.039003 & -1.819349 \\ \mathrm{H} & -1.533620 & 0.300671 & 2.898853\end{array}$




$$
\begin{array}{cccc}
\mathrm{H} & -3.246718 & 0.424709 & 1.081556 \\
\mathrm{H} & -1.453091 & 0.765869 & -2.333363 \\
\mathrm{H} & -3.062218 & 0.443390 & -1.660538 \\
\mathrm{H} & -0.887282 & -1.636267 & -2.223776 \\
\mathrm{H} & -2.283583 & -1.320645 & -3.249645 \\
\mathrm{H} & -3.674022 & -2.093624 & -1.432964 \\
\mathrm{H} & -2.342273 & -2.439955 & -0.541663
\end{array}
$$

porphycene $\mathrm{S}_{0}$

$\begin{array}{lrrr}\mathrm{C} & -3.433333 & 1.913816 & 0.000000 \\ \mathrm{C} & -2.420290 & 0.908273 & 0.000000 \\ \mathrm{~N} & -1.223806 & 1.540969 & 0.000000 \\ \mathrm{C} & -1.390824 & 2.889270 & 0.000000 \\ \mathrm{C} & -2.802337 & 3.128491 & 0.000000 \\ \mathrm{C} & -0.367084 & 3.844262 & 0.000000 \\ \mathrm{C} & 1.010123 & 3.693444 & 0.000000 \\ \mathrm{C} & 1.824149 & 2.548078 & 0.000000 \\ \mathrm{H} & -0.265910 & 1.120234 & 0.000000 \\ \mathrm{~N} & 1.410237 & 1.260317 & 0.000000 \\ \mathrm{C} & 3.276580 & 2.593004 & 0.000000 \\ \mathrm{C} & 3.707534 & 1.307155 & 0.000000 \\ \mathrm{C} & 2.512574 & 0.492982 & 0.000000 \\ \mathrm{C} & 2.420290 & -0.908273 & 0.000000 \\ \mathrm{C} & 3.433333 & -1.913816 & 0.000000 \\ \mathrm{C} & 2.802337 & -3.128491 & 0.000000 \\ \mathrm{C} & 1.390824 & -2.889270 & 0.000000 \\ \mathrm{~N} & 1.223806 & -1.540969 & 0.000000 \\ \mathrm{C} & 0.367084 & -3.844262 & 0.000000 \\ \mathrm{C} & -1.010123 & -3.693444 & 0.000000 \\ \mathrm{C} & -1.824149 & -2.548078 & 0.000000 \\ \mathrm{C} & -3.276580 & -2.593004 & 0.000000 \\ \mathrm{C} & -3.707534 & -1.307155 & 0.000000 \\ \mathrm{C} & -2.512574 & -0.492982 & 0.000000 \\ \mathrm{~N} & -1.410237 & -1.260317 & 0.000000 \\ \mathrm{H} & 0.265910 & -1.120234 & 0.000000 \\ \mathrm{H} & 3.256916 & -4.106444 & 0.000000 \\ \mathrm{H} & 4.494354 & -1.723979 & 0.000000 \\ \mathrm{H} & 0.727771 & -4.866654 & 0.000000 \\ \mathrm{H} & -1.569758 & -4.622690 & 0.000000 \\ \mathrm{H} & -3.871870 & -3.493485 & 0.000000 \\ \mathrm{H} & -4.723249 & -0.944237 & 0.000000 \\ \mathrm{H} & -4.494354 & 1.723979 & 0.000000 \\ \mathrm{H} & -3.256916 & 4.106444 & 0.000000 \\ \mathrm{H} & -0.727771 & 4.866654 & 0.000000 \\ \mathrm{H} & 1.569758 & 4.622690 & 0.000000\end{array}$




$$
\begin{array}{llll}
\mathrm{H} & 3.871870 & 3.493485 & 0.000000 \\
\mathrm{H} & 4.723249 & 0.944237 & 0.000000
\end{array}
$$

porphycene $\mathrm{S}_{1}$

$\begin{array}{lrrr}\mathrm{C} & -3.455786 & 1.901181 & 0.000000 \\ \mathrm{C} & -2.447377 & 0.897748 & 0.000000 \\ \mathrm{~N} & -1.237958 & 1.540209 & 0.000000 \\ \mathrm{C} & -1.412807 & 2.879268 & 0.000000 \\ \mathrm{C} & -2.822197 & 3.119455 & 0.000000 \\ \mathrm{C} & -0.378883 & 3.853386 & 0.000000 \\ \mathrm{C} & 0.983903 & 3.707359 & 0.000000 \\ \mathrm{C} & 1.826698 & 2.552470 & 0.000000 \\ \mathrm{H} & -0.283232 & 1.119511 & 0.000000 \\ \mathrm{~N} & 1.416307 & 1.273212 & 0.000000 \\ \mathrm{C} & 3.270740 & 2.610415 & 0.000000 \\ \mathrm{C} & 3.716906 & 1.321794 & 0.000000 \\ \mathrm{C} & 2.541464 & 0.501306 & 0.000000 \\ \mathrm{C} & 2.447377 & -0.897748 & 0.000000 \\ \mathrm{C} & 3.455786 & -1.901181 & 0.000000 \\ \mathrm{C} & 2.822197 & -3.119455 & 0.000000 \\ \mathrm{C} & 1.412807 & -2.879268 & 0.000000 \\ \mathrm{~N} & 1.237958 & -1.540209 & 0.000000 \\ \mathrm{C} & 0.378883 & -3.853386 & 0.000000 \\ \mathrm{C} & -0.983903 & -3.707359 & 0.000000 \\ \mathrm{C} & -1.826698 & -2.552470 & 0.000000 \\ \mathrm{C} & -3.270740 & -2.610415 & 0.000000 \\ \mathrm{C} & -3.716906 & -1.321794 & 0.000000 \\ \mathrm{C} & -2.541464 & -0.501306 & 0.000000 \\ \mathrm{~N} & -1.416307 & -1.273212 & 0.000000 \\ \mathrm{H} & 0.283232 & -1.119511 & 0.000000 \\ \mathrm{H} & 3.275203 & -4.098065 & 0.000000 \\ \mathrm{H} & 4.516598 & -1.710965 & 0.000000 \\ \mathrm{H} & 0.747861 & -4.872347 & 0.000000 \\ \mathrm{H} & -1.542591 & -4.637747 & 0.000000 \\ \mathrm{H} & -3.859239 & -3.515400 & 0.000000 \\ \mathrm{H} & -4.736386 & -0.970805 & 0.000000 \\ \mathrm{H} & -4.516598 & 1.710965 & 0.000000 \\ \mathrm{H} & -3.275203 & 4.098065 & 0.000000 \\ \mathrm{H} & -0.747861 & 4.872347 & 0.000000 \\ \mathrm{H} & 1.542591 & 4.637747 & 0.000000 \\ & 3.859239 & 3.515400 & 0.000000 \\ & 4.736386 & 0.970805 & 0.000000\end{array}$

porphine $\mathrm{S}_{0}$ 


$\begin{array}{lrrr}\mathrm{N} & 0.000000 & -2.013446 & 0.000000 \\ \mathrm{~N} & 2.095889 & 0.000000 & 0.000000 \\ \mathrm{C} & 1.077887 & -2.833278 & 0.000000 \\ \mathrm{C} & 0.673628 & -4.233410 & 0.000000 \\ \mathrm{C} & 2.409025 & -2.427440 & 0.000000 \\ \mathrm{C} & 2.872370 & -1.121639 & 0.000000 \\ \mathrm{C} & 4.234477 & -0.681414 & 0.000000 \\ \mathrm{H} & 1.348145 & -5.076011 & 0.000000 \\ \mathrm{H} & 3.164881 & -3.203162 & 0.000000 \\ \mathrm{H} & 5.085583 & -1.343541 & 0.000000 \\ \mathrm{H} & 1.081891 & 0.000000 & 0.000000 \\ \mathrm{~N} & 0.000000 & 2.013446 & 0.000000 \\ \mathrm{~N} & -2.095889 & 0.000000 & 0.000000 \\ \mathrm{C} & 1.077887 & 2.833278 & 0.000000 \\ \mathrm{C} & -1.077887 & 2.833278 & 0.000000 \\ \mathrm{C} & -1.077887 & -2.833278 & 0.000000 \\ \mathrm{C} & 0.673628 & 4.233410 & 0.000000 \\ \mathrm{C} & -0.673628 & 4.233410 & 0.000000 \\ \mathrm{C} & -0.673628 & -4.233410 & 0.000000 \\ \mathrm{C} & 2.409025 & 2.427440 & 0.000000 \\ \mathrm{C} & -2.409025 & 2.427440 & 0.000000 \\ \mathrm{C} & -2.409025 & -2.427440 & 0.000000 \\ \mathrm{C} & 2.872370 & 1.121639 & 0.000000 \\ \mathrm{C} & -2.872370 & 1.121639 & 0.000000 \\ \mathrm{C} & -2.872370 & -1.121639 & 0.000000 \\ \mathrm{C} & 4.234477 & 0.681414 & 0.000000 \\ \mathrm{C} & -4.234477 & 0.681414 & 0.000000 \\ \mathrm{C} & -4.234477 & -0.681414 & 0.000000 \\ \mathrm{H} & 1.348145 & 5.076011 & 0.000000 \\ \mathrm{H} & -1.348145 & 5.076011 & 0.000000 \\ \mathrm{H} & -1.348145 & -5.076011 & 0.000000 \\ \mathrm{H} & 3.164881 & 3.203162 & 0.000000 \\ \mathrm{H} & -3.164881 & 3.203162 & 0.000000 \\ \mathrm{H} & -3.164881 & -3.203162 & 0.000000 \\ \mathrm{H} & 5.085583 & 1.343541 & 0.000000 \\ \mathrm{H} & -5.085583 & 1.343541 & 0.000000 \\ \mathrm{H} & -5.085583 & -1.343541 & 0.000000 \\ \mathrm{H} & -1.081891 & 0.000000 & 0.000000\end{array}$

porphine $\mathrm{S}_{1}$
$\begin{array}{llll}\mathrm{N} & 0.000000 & -2.033010 & 0.000000\end{array}$
$\begin{array}{llll}\mathrm{N} & 2.093361 & 0.000000 & 0.000000\end{array}$
$\begin{array}{llll}\text { C } & 1.080936 & -2.856154 & 0.000000\end{array}$
$\begin{array}{llll}\text { C } & 0.674877 & -4.253096 & 0.000000\end{array}$
$\begin{array}{llll}\text { C } & 2.414150 & -2.435992 & 0.000000\end{array}$ 


$\begin{array}{rrrr}\mathrm{C} & 2.873990 & -1.124535 & 0.000000 \\ \mathrm{C} & 4.235048 & -0.683140 & 0.000000 \\ \mathrm{H} & 1.347605 & -5.096822 & 0.000000 \\ \mathrm{H} & 3.174768 & -3.207020 & 0.000000 \\ \mathrm{H} & 5.086885 & -1.344165 & 0.000000 \\ \mathrm{H} & 1.081039 & 0.000000 & 0.000000 \\ \mathrm{~N} & 0.000000 & 2.033010 & 0.000000 \\ \mathrm{~N} & -2.093361 & 0.000000 & 0.000000 \\ \mathrm{C} & 1.080936 & 2.856154 & 0.000000 \\ \mathrm{C} & -1.080936 & 2.856154 & 0.000000 \\ \mathrm{C} & -1.080936 & -2.856154 & 0.000000 \\ \mathrm{C} & 0.674877 & 4.253096 & 0.000000 \\ \mathrm{C} & -0.674877 & 4.253096 & 0.000000 \\ \mathrm{C} & -0.674877 & -4.253096 & 0.000000 \\ \mathrm{C} & 2.414150 & 2.435992 & 0.000000 \\ \mathrm{C} & -2.414150 & 2.435992 & 0.000000 \\ \mathrm{C} & -2.414150 & -2.435992 & 0.000000 \\ \mathrm{C} & 2.873990 & 1.124535 & 0.000000 \\ \mathrm{C} & -2.873990 & 1.124535 & 0.000000 \\ \mathrm{C} & -2.873990 & -1.124535 & 0.000000 \\ \mathrm{C} & 4.235048 & 0.683140 & 0.000000 \\ \mathrm{C} & -4.235048 & 0.683140 & 0.000000 \\ \mathrm{C} & -4.235048 & -0.683140 & 0.000000 \\ \mathrm{H} & 1.347605 & 5.096822 & 0.000000 \\ \mathrm{H} & -1.347605 & 5.096822 & 0.000000 \\ \mathrm{H} & -1.347605 & -5.096822 & 0.000000 \\ \mathrm{H} & 3.174768 & 3.207020 & 0.000000 \\ \mathrm{H} & -3.174768 & 3.207020 & 0.000000 \\ \mathrm{H} & -3.174768 & -3.207020 & 0.000000 \\ \mathrm{H} & 5.086885 & 1.344165 & 0.000000 \\ \mathrm{H} & -5.086885 & 1.344165 & 0.000000 \\ \mathrm{H} & -5.086885 & -1.344165 & 0.00000 \\ \mathrm{H} & -1.081039 & 0.000000 & 0.000000 \\ & & & \end{array}$

chlorin $\mathrm{S}_{0}$

$\begin{array}{rrrr}\mathrm{C} & 0.000000 & -4.226886 & 0.920417 \\ \mathrm{C} & 0.000000 & -2.871262 & 1.370412 \\ \mathrm{~N} & 0.000000 & -2.085722 & 0.240222 \\ \mathrm{C} & 0.000000 & -2.853678 & -0.876917 \\ \mathrm{C} & 0.000000 & -4.218745 & -0.443109 \\ \mathrm{C} & 0.000000 & -2.412120 & 2.668714 \\ \mathrm{C} & 0.000000 & -1.077323 & 3.087317 \\ \mathrm{~N} & 0.000000 & 0.000000 & 2.266333 \\ \mathrm{C} & 0.000000 & 1.077323 & 3.087317 \\ \mathrm{C} & 0.000000 & 0.677036 & 4.478221\end{array}$




$\begin{array}{lrrr}\mathrm{C} & 0.000000 & -0.677036 & 4.478221 \\ \mathrm{C} & 0.000000 & 2.412120 & 2.668714 \\ \mathrm{C} & 0.000000 & 2.871262 & 1.370412 \\ \mathrm{~N} & 0.000000 & 2.085722 & 0.240222 \\ \mathrm{C} & 0.000000 & 2.853678 & -0.876917 \\ \mathrm{C} & 0.000000 & 4.218745 & -0.443109 \\ \mathrm{C} & 0.000000 & 4.226886 & 0.920417 \\ \mathrm{C} & 0.000000 & 2.403523 & -2.198120 \\ \mathrm{C} & 0.000000 & 1.096554 & -2.640811 \\ \mathrm{C} & 0.000000 & 0.764514 & -4.124411 \\ \mathrm{C} & 0.000000 & -0.764514 & -4.124411 \\ \mathrm{C} & 0.000000 & -1.096554 & -2.640811 \\ \mathrm{~N} & 0.000000 & 0.000000 & -1.861882 \\ \mathrm{C} & 0.000000 & -2.403523 & -2.198120 \\ \mathrm{H} & -0.879634 & 1.188835 & -4.609719 \\ \mathrm{H} & 0.000000 & 3.179405 & -2.953819 \\ \mathrm{H} & 0.000000 & 5.065173 & -1.110872 \\ \mathrm{H} & 0.000000 & 1.349836 & 5.322229 \\ \mathrm{H} & 0.000000 & -1.349836 & 5.322229 \\ \mathrm{H} & -0.879634 & -1.188835 & -4.609719 \\ \mathrm{H} & 0.000000 & 3.170864 & 3.442466 \\ \mathrm{H} & 0.000000 & -3.170864 & 3.442466 \\ \mathrm{H} & 0.000000 & -3.179405 & -2.953819 \\ \mathrm{H} & 0.000000 & 5.083065 & 1.576353 \\ \mathrm{H} & 0.000000 & -5.083065 & 1.576353 \\ \mathrm{H} & 0.000000 & -5.065173 & -1.110872 \\ \mathrm{H} & 0.879634 & -1.188835 & -4.609719 \\ \mathrm{H} & 0.879634 & 1.188835 & -4.609719 \\ \mathrm{H} & 0.000000 & -1.073781 & 0.258913 \\ \mathrm{H} & 0.000000 & 1.073781 & 0.258913\end{array}$

chlorin $\mathrm{S}_{1}$

$\begin{array}{lrrr}\mathrm{C} & 0.000000 & -4.233899 & 0.921079 \\ \mathrm{C} & 0.000000 & -2.877176 & 1.366770 \\ \mathrm{~N} & 0.000000 & -2.092434 & 0.239738 \\ \mathrm{C} & 0.000000 & -2.868189 & -0.884135 \\ \mathrm{C} & 0.000000 & -4.228433 & -0.447848 \\ \mathrm{C} & 0.000000 & -2.414990 & 2.677644 \\ \mathrm{C} & 0.000000 & -1.080911 & 3.087100 \\ \mathrm{~N} & 0.000000 & 0.000000 & 2.264815 \\ \mathrm{C} & 0.000000 & 1.080911 & 3.087100 \\ \mathrm{C} & 0.000000 & 0.673325 & 4.488653 \\ \mathrm{C} & 0.000000 & -0.673325 & 4.488653 \\ \mathrm{C} & 0.000000 & 2.414990 & 2.677644 \\ \mathrm{C} & 0.000000 & 2.877176 & 1.366770\end{array}$




$\begin{array}{rrrr}\mathrm{N} & 0.000000 & 2.092434 & 0.239738 \\ \mathrm{C} & 0.000000 & 2.868189 & -0.884135 \\ \mathrm{C} & 0.000000 & 4.228433 & -0.447848 \\ \mathrm{C} & 0.000000 & 4.233899 & 0.921079 \\ \mathrm{C} & 0.000000 & 2.413002 & -2.206250 \\ \mathrm{C} & 0.000000 & 1.097903 & -2.643487 \\ \mathrm{C} & 0.000000 & 0.763560 & -4.122366 \\ \mathrm{C} & 0.000000 & -0.763560 & -4.122366 \\ \mathrm{C} & 0.000000 & -1.097903 & -2.643487 \\ \mathrm{~N} & 0.000000 & 0.000000 & -1.856762 \\ \mathrm{C} & 0.000000 & -2.413002 & -2.206250 \\ \mathrm{H} & -0.879225 & 1.189045 & -4.609071 \\ \mathrm{H} & 0.000000 & 3.183972 & -2.966682 \\ \mathrm{H} & 0.000000 & 5.077296 & -1.112624 \\ \mathrm{H} & 0.000000 & 1.347797 & 5.331144 \\ \mathrm{H} & 0.000000 & -1.347797 & 5.331144 \\ \mathrm{H} & -0.879225 & -1.189045 & -4.609071 \\ \mathrm{H} & 0.000000 & 3.171957 & 3.451804 \\ \mathrm{H} & 0.000000 & -3.171957 & 3.451804 \\ \mathrm{H} & 0.000000 & -3.183972 & -2.966682 \\ \mathrm{H} & 0.000000 & 5.088211 & 1.579052 \\ \mathrm{H} & 0.000000 & -5.088211 & 1.579052 \\ \mathrm{H} & 0.000000 & -5.077296 & -1.112624 \\ \mathrm{H} & 0.879225 & -1.189045 & -4.609071 \\ \mathrm{H} & 0.879225 & 1.189045 & -4.609071 \\ \mathrm{H} & 0.000000 & -1.080827 & 0.254533 \\ \mathrm{H} & 0.000000 & 1.080827 & 0.254533\end{array}$

Zn-tetraphenylporphine $\mathrm{S}_{0}$

$\begin{array}{lrrr}\mathrm{C} & -3.667264 & 4.424181 & 1.007125 \\ \mathrm{C} & -3.493715 & 3.480094 & -0.004287 \\ \mathrm{C} & -4.333529 & 3.524952 & -1.117506 \\ \mathrm{C} & -5.325062 & 4.490148 & -1.218469 \\ \mathrm{C} & -5.491435 & 5.425607 & -0.205055 \\ \mathrm{C} & -4.660280 & 5.390070 & 0.907342 \\ \mathrm{C} & -2.433582 & 2.434997 & 0.082521 \\ \mathrm{C} & -1.099057 & 2.861557 & 0.072765 \\ \mathrm{~N} & 0.000000 & 2.055512 & 0.151298 \\ \mathrm{C} & 1.099057 & 2.861557 & 0.072765 \\ \mathrm{C} & 0.676854 & 4.235530 & -0.070357 \\ \mathrm{C} & -0.676854 & 4.235530 & -0.070357 \\ \mathrm{C} & 2.433582 & 2.434997 & 0.082521 \\ \mathrm{C} & 3.493715 & 3.480094 & -0.004287 \\ \mathrm{C} & 4.333529 & 3.524952 & -1.117506 \\ \mathrm{C} & 5.325062 & 4.490148 & -1.218469\end{array}$




$\begin{array}{rrrr}\mathrm{C} & 5.491435 & 5.425607 & -0.205055 \\ \mathrm{C} & 4.660280 & 5.390070 & 0.907342 \\ \mathrm{C} & 3.667264 & 4.424181 & 1.007125 \\ \mathrm{C} & -2.855935 & 1.099237 & 0.125295 \\ \mathrm{~N} & -2.047577 & 0.000000 & 0.091609 \\ \mathrm{C} & -2.855935 & -1.099237 & 0.125295 \\ \mathrm{C} & -4.234367 & -0.676849 & 0.213964 \\ \mathrm{C} & -4.234367 & 0.676849 & 0.213964 \\ \mathrm{C} & -2.433582 & -2.434997 & 0.082521 \\ \mathrm{C} & -3.493715 & -3.480094 & -0.004287 \\ \mathrm{C} & -4.333529 & -3.524952 & -1.117506 \\ \mathrm{C} & -5.325062 & -4.490148 & -1.218469 \\ \mathrm{C} & -5.491435 & -5.425607 & -0.205055 \\ \mathrm{C} & -4.660280 & -5.390070 & 0.907342 \\ \mathrm{C} & -3.667264 & -4.424181 & 1.007125 \\ \mathrm{C} & 2.855935 & 1.099237 & 0.125295 \\ \mathrm{~N} & 2.047577 & 0.000000 & 0.091609 \\ \mathrm{C} & 2.855935 & -1.099237 & 0.125295 \\ \mathrm{C} & 4.234367 & -0.676849 & 0.213964 \\ \mathrm{C} & 4.234367 & 0.676849 & 0.213964 \\ \mathrm{C} & 2.433582 & -2.434997 & 0.082521 \\ \mathrm{C} & 3.493715 & -3.480094 & -0.004287 \\ \mathrm{C} & 4.333529 & -3.524952 & -1.117506 \\ \mathrm{C} & 5.325062 & -4.490148 & -1.218469 \\ \mathrm{C} & 5.491435 & -5.425607 & -0.205055 \\ \mathrm{C} & 4.660280 & -5.390070 & 0.907342 \\ \mathrm{C} & 3.667264 & -4.424181 & 1.007125 \\ \mathrm{C} & 1.099057 & -2.861557 & 0.072765 \\ \mathrm{~N} & 0.000000 & -2.055512 & 0.151298 \\ \mathrm{C} & -1.099057 & -2.861557 & 0.072765 \\ \mathrm{C} & -0.676854 & -4.235530 & -0.070357 \\ \mathrm{C} & 0.676854 & -4.235530 & -0.070357 \\ \mathrm{H} & 1.337689 & -5.079198 & -0.182585 \\ \mathrm{H} & 5.082415 & -1.337644 & 0.287044 \\ \mathrm{H} & 1.337689 & 5.079198 & -0.182585 \\ \mathrm{H} & -1.337689 & 5.079198 & -0.182585 \\ \mathrm{H} & -1.337689 & -5.079198 & -0.182585 \\ \mathrm{H} & 5.082415 & 1.337644 & 0.287044 \\ \mathrm{H} & -5.082415 & 1.337644 & 0.287044 \\ \mathrm{H} & -5.082415 & -1.337644 & 0.287044 \\ \mathrm{H} & 4.198163 & 2.795265 & -1.907034 \\ \mathrm{H} & 5.965042 & 4.514120 & -2.091661 \\ \mathrm{H} & 6.264766 & 6.179394 & -0.282813 \\ \mathrm{H} & 4.787827 & 6.113857 & 1.702564 \\ \mathrm{H} & 3.021256 & 4.391901 & 1.876640 \\ \mathrm{H} & 4.198163 & -2.795265 & -1.907034\end{array}$




$\begin{array}{rrrr}\mathrm{H} & 5.965042 & -4.514120 & -2.091661 \\ \mathrm{H} & 6.264766 & -6.179394 & -0.282813 \\ \mathrm{H} & 4.787827 & -6.113857 & 1.702564 \\ \mathrm{H} & 3.021256 & -4.391901 & 1.876640 \\ \mathrm{H} & -4.198163 & -2.795265 & -1.907034 \\ \mathrm{H} & -5.965042 & -4.514120 & -2.091661 \\ \mathrm{H} & -6.264766 & -6.179394 & -0.282813 \\ \mathrm{H} & -4.787827 & -6.113857 & 1.702564 \\ \mathrm{H} & -3.021256 & -4.391901 & 1.876640 \\ \mathrm{H} & -4.198163 & 2.795265 & -1.907034 \\ \mathrm{H} & -5.965042 & 4.514120 & -2.091661 \\ \mathrm{H} & -6.264766 & 6.179394 & -0.282813 \\ \mathrm{H} & -4.787827 & 6.113857 & 1.702564 \\ \mathrm{H} & -3.021256 & 4.391901 & 1.876640 \\ \mathrm{Zn} & 0.000000 & 0.000000 & 0.131686\end{array}$

Zn-tetraphenylporphine $\mathrm{S}_{1}$

$\begin{array}{lrrr}\mathrm{C} & -3.622151 & 4.485861 & 0.956037 \\ \mathrm{C} & -3.496402 & 3.481808 & -0.005327 \\ \mathrm{C} & -4.388739 & 3.466140 & -1.079866 \\ \mathrm{C} & -5.377714 & 4.431286 & -1.194167 \\ \mathrm{C} & -5.495694 & 5.425202 & -0.230286 \\ \mathrm{C} & -4.616692 & 5.448470 & 0.845002 \\ \mathrm{C} & -2.440101 & 2.442241 & 0.090487 \\ \mathrm{C} & -1.101580 & 2.864246 & 0.078588 \\ \mathrm{~N} & 0.000000 & 2.056420 & 0.194078 \\ \mathrm{C} & 1.101580 & 2.864246 & 0.078588 \\ \mathrm{C} & 0.677839 & 4.229165 & -0.121347 \\ \mathrm{C} & -0.677839 & 4.229165 & -0.121347 \\ \mathrm{C} & 2.440101 & 2.442241 & 0.090487 \\ \mathrm{C} & 3.496402 & 3.481808 & -0.005327 \\ \mathrm{C} & 4.388739 & 3.466140 & -1.079866 \\ \mathrm{C} & 5.377714 & 4.431286 & -1.194167 \\ \mathrm{C} & 5.495694 & 5.425202 & -0.230286 \\ \mathrm{C} & 4.616692 & 5.448470 & 0.845002 \\ \mathrm{C} & 3.622151 & 4.485861 & 0.956037 \\ \mathrm{C} & -2.871809 & 1.098597 & 0.144835 \\ \mathrm{~N} & -2.062530 & 0.000000 & 0.070239 \\ \mathrm{C} & -2.871809 & -1.098597 & 0.144835 \\ \mathrm{C} & -4.239895 & -0.679745 & 0.295897 \\ \mathrm{C} & -4.239895 & 0.679745 & 0.295897 \\ \mathrm{C} & -2.440101 & -2.442241 & 0.090487 \\ \mathrm{C} & -3.496402 & -3.481808 & -0.005327 \\ \mathrm{C} & -4.388739 & -3.466140 & -1.079866 \\ \mathrm{C} & -5.377714 & -4.431286 & -1.194167\end{array}$




$\begin{array}{rrrr}\mathrm{C} & -5.495694 & -5.425202 & -0.230286 \\ \mathrm{C} & -4.616692 & -5.448470 & 0.845002 \\ \mathrm{C} & -3.622151 & -4.485861 & 0.956037 \\ \mathrm{C} & 2.871809 & 1.098597 & 0.144835 \\ \mathrm{~N} & 2.062530 & 0.000000 & 0.070239 \\ \mathrm{C} & 2.871809 & -1.098597 & 0.144835 \\ \mathrm{C} & 4.239895 & -0.679745 & 0.295897 \\ \mathrm{C} & 4.239895 & 0.679745 & 0.295897 \\ \mathrm{C} & 2.440101 & -2.442241 & 0.090487 \\ \mathrm{C} & 3.496402 & -3.481808 & -0.005327 \\ \mathrm{C} & 4.388739 & -3.466140 & -1.079866 \\ \mathrm{C} & 5.377714 & -4.431286 & -1.194167 \\ \mathrm{C} & 5.495694 & -5.425202 & -0.230286 \\ \mathrm{C} & 4.616692 & -5.448470 & 0.845002 \\ \mathrm{C} & 3.622151 & -4.485861 & 0.956037 \\ \mathrm{C} & 1.101580 & -2.864246 & 0.078588 \\ \mathrm{~N} & 0.000000 & -2.056420 & 0.194078 \\ \mathrm{C} & -1.101580 & -2.864246 & 0.078588 \\ \mathrm{C} & -0.677839 & -4.229165 & -0.121347 \\ \mathrm{C} & 0.677839 & -4.229165 & -0.121347 \\ \mathrm{H} & 1.336684 & -5.066851 & -0.278862 \\ \mathrm{H} & 5.082274 & -1.340639 & 0.414300 \\ \mathrm{H} & 1.336684 & 5.066851 & -0.278862 \\ \mathrm{H} & -1.336684 & 5.066851 & -0.278862 \\ \mathrm{H} & -1.336684 & -5.066851 & -0.278862 \\ \mathrm{H} & 5.082274 & 1.340639 & 0.414300 \\ \mathrm{H} & -5.082274 & 1.340639 & 0.414300 \\ \mathrm{H} & -5.082274 & -1.340639 & 0.414300 \\ \mathrm{H} & 4.290338 & 2.692922 & -1.832478 \\ \mathrm{H} & 6.053759 & 4.410743 & -2.039680 \\ \mathrm{H} & 6.268881 & 6.178061 & -0.317569 \\ \mathrm{H} & 4.708877 & 6.215406 & 1.603919 \\ \mathrm{H} & 2.940578 & 4.498114 & 1.798193 \\ \mathrm{H} & 4.290338 & -2.692922 & -1.832478 \\ \mathrm{H} & 6.053759 & -4.410743 & -2.039680 \\ \mathrm{H} & 6.268881 & -6.178061 & -0.317569 \\ \mathrm{H} & 4.708877 & -6.215406 & 1.603919 \\ \mathrm{H} & 2.940578 & -4.498114 & 1.798193 \\ \mathrm{H} & -4.290338 & -2.692922 & -1.832478 \\ \mathrm{H} & -6.053759 & -4.410743 & -2.039680 \\ \mathrm{H} & -6.268881 & -6.178061 & -0.317569 \\ \mathrm{H} & -4.708877 & -6.215406 & 1.603919 \\ \mathrm{H} & -2.940578 & -4.498114 & 1.798193 \\ \mathrm{H} & -4.290338 & 2.692922 & -1.832478 \\ \mathrm{H} & -6.053759 & 4.410743 & -2.039680 \\ \mathrm{H} & -6.268881 & 6.178061 & -0.317569\end{array}$




$\begin{array}{rrrr}\mathrm{H} & -4.708877 & 6.215406 & 1.603919 \\ \mathrm{H} & -2.940578 & 4.498114 & 1.798193 \\ \mathrm{Zn} & 0.000000 & 0.000000 & 0.139726\end{array}$

tetraphenylporphine $\mathrm{S}_{0}$

$\begin{array}{lrrr}\mathrm{C} & 4.221725 & 0.689507 & 0.086161 \\ \mathrm{C} & 2.869112 & 1.129263 & -0.070713 \\ \mathrm{~N} & 2.096976 & 0.004297 & -0.157282 \\ \mathrm{C} & 2.873758 & -1.117369 & -0.069408 \\ \mathrm{C} & 4.224497 & -0.671904 & 0.087057 \\ \mathrm{C} & 2.429345 & -2.439308 & -0.099108 \\ \mathrm{C} & 1.085957 & -2.834926 & -0.169104 \\ \mathrm{~N} & 0.004184 & -2.017328 & -0.121052 \\ \mathrm{C} & -1.074148 & -2.839378 & -0.170421 \\ \mathrm{C} & -0.663939 & -4.232424 & -0.298452 \\ \mathrm{C} & 0.681654 & -4.229664 & -0.297569 \\ \mathrm{C} & -2.419225 & -2.449351 & -0.101328 \\ \mathrm{C} & -2.869112 & -1.129263 & -0.070713 \\ \mathrm{~N} & -2.096976 & -0.004297 & -0.157282 \\ \mathrm{C} & -2.873758 & 1.117369 & -0.069408 \\ \mathrm{C} & -4.224497 & 0.671904 & 0.087057 \\ \mathrm{C} & -4.221725 & -0.689507 & 0.086161 \\ \mathrm{C} & -2.429345 & 2.439308 & -0.099108 \\ \mathrm{C} & -1.085957 & 2.834926 & -0.169104 \\ \mathrm{~N} & -0.004184 & 2.017328 & -0.121052 \\ \mathrm{C} & 1.074148 & 2.839378 & -0.170421 \\ \mathrm{C} & 0.663939 & 4.232424 & -0.298452 \\ \mathrm{C} & -0.681654 & 4.229664 & -0.297569 \\ \mathrm{C} & 2.419225 & 2.449351 & -0.101328 \\ \mathrm{H} & -1.349957 & 5.069251 & -0.396553 \\ \mathrm{C} & -3.469767 & 3.502441 & -0.002434 \\ \mathrm{H} & -5.070527 & 1.327813 & 0.205441 \\ \mathrm{H} & -1.084379 & -0.002044 & -0.206101 \\ \mathrm{H} & -1.328635 & -5.074758 & -0.398338 \\ \mathrm{H} & 1.349957 & -5.069251 & -0.396533 \\ \mathrm{H} & 1.328635 & 5.074758 & -0.398338 \\ \mathrm{C} & -3.455367 & -3.516844 & -0.006399 \\ \mathrm{C} & 3.469767 & -3.502441 & -0.002434 \\ \mathrm{C} & 3.455367 & 3.516844 & -0.006399 \\ \mathrm{H} & -5.065102 & -1.348970 & 0.203649 \\ \mathrm{H} & 5.070527 & -1.327813 & 0.205441 \\ \mathrm{H} & 5.065102 & 1.348970 & 0.203649 \\ \mathrm{H} & 1.084379 & 0.002044 & -0.206101 \\ \mathrm{C} & -4.421324 & -3.683232 & -0.998247 \\ \mathrm{C} & -5.379289 & -4.682932 & -0.888771\end{array}$




$\begin{array}{lrrr}\mathrm{C} & -5.384678 & -5.527470 & 0.213948 \\ \mathrm{C} & -4.426896 & -5.368390 & 1.207604 \\ \mathrm{C} & -3.469767 & -4.370173 & 1.097325 \\ \mathrm{H} & -4.410186 & -3.029183 & -1.862278 \\ \mathrm{H} & -6.119311 & -4.806049 & -1.669671 \\ \mathrm{H} & -6.132088 & -6.306161 & 0.299192 \\ \mathrm{H} & -4.427534 & -6.019312 & 2.072907 \\ \mathrm{H} & -2.722501 & -4.240243 & 1.871117 \\ \mathrm{C} & -4.437090 & 3.665777 & -0.993421 \\ \mathrm{C} & -5.398982 & 4.661520 & -0.882420 \\ \mathrm{C} & -5.406975 & 5.505115 & 0.221000 \\ \mathrm{C} & -4.447857 & 5.349053 & 1.213859 \\ \mathrm{C} & -3.486797 & 4.354766 & 1.102068 \\ \mathrm{H} & -4.423945 & 3.012479 & -1.858000 \\ \mathrm{H} & -6.139988 & 4.782368 & -1.662742 \\ \mathrm{H} & -6.157444 & 6.280727 & 0.307430 \\ \mathrm{H} & -4.450486 & 5.999265 & 2.079699 \\ \mathrm{H} & -2.738490 & 4.227158 & 1.875239 \\ \mathrm{C} & 4.421324 & 3.683232 & -0.998247 \\ \mathrm{C} & 5.379289 & 4.682932 & -0.888771 \\ \mathrm{C} & 5.384678 & 5.527470 & 0.213948 \\ \mathrm{C} & 4.426896 & 5.368390 & 1.207604 \\ \mathrm{C} & 3.469767 & 4.370173 & 1.097325 \\ \mathrm{H} & 4.410186 & 3.029183 & -1.862278 \\ \mathrm{H} & 6.119311 & 4.806049 & -1.669671 \\ \mathrm{H} & 6.132088 & 6.306161 & 0.299192 \\ \mathrm{H} & 4.427534 & 6.019312 & 2.072907 \\ \mathrm{H} & 2.722501 & 4.240243 & 1.871117 \\ \mathrm{C} & 4.437090 & -3.665777 & -0.993421 \\ \mathrm{C} & 5.398982 & -4.661520 & -0.882420 \\ \mathrm{C} & 5.406975 & -5.505115 & 0.221000 \\ \mathrm{C} & 4.447857 & -5.349053 & 1.213859 \\ \mathrm{C} & 3.486797 & -4.354766 & 1.102068 \\ \mathrm{H} & 4.423945 & -3.012479 & -1.858000 \\ \mathrm{H} & 6.139988 & -4.782368 & -1.662742 \\ \mathrm{H} & 6.157444 & -6.280727 & 0.307430 \\ \mathrm{H} & 4.450486 & -5.999265 & 2.079699 \\ \mathrm{H} & 2.738490 & -4.227158 & 1.875239 \\ & & & \end{array}$

tetraphenylporphine $\mathrm{S}_{1}$
C 4.218518
0.700379
0.139337
$\begin{array}{llll}\text { C } & 2.871518 & 1.139110 & -0.064282\end{array}$
$\begin{array}{llll}\mathrm{N} & 2.101825 & 0.008845 & -0.177785\end{array}$
$\begin{array}{llll}\text { C } & 2.881410 & -1.114301 & -0.060655 \\ \text { C } & 4.224426 & -0.663234 & 0.141769\end{array}$
$\begin{array}{llll}\text { C } & 4.224426 & -0.663234 & 0.141769\end{array}$ 


$\begin{array}{rrrr}\mathrm{C} & 2.442647 & -2.440740 & -0.100840 \\ \mathrm{C} & 1.094769 & -2.852942 & -0.194380 \\ \mathrm{~N} & 0.008905 & -2.036838 & -0.116534 \\ \mathrm{C} & -1.069698 & -2.862182 & -0.198039 \\ \mathrm{C} & -0.656940 & -4.241343 & -0.378867 \\ \mathrm{C} & 0.694387 & -4.235635 & -0.376284 \\ \mathrm{C} & -2.421281 & -2.461635 & -0.107224 \\ \mathrm{C} & -2.871518 & -1.139110 & -0.064282 \\ \mathrm{~N} & -2.101825 & -0.008845 & -0.177785 \\ \mathrm{C} & -2.881410 & 1.114301 & -0.060655 \\ \mathrm{C} & -4.224426 & 0.663234 & 0.141769 \\ \mathrm{C} & -4.218518 & -0.700379 & 0.139337 \\ \mathrm{C} & -2.442647 & 2.440740 & -0.100840 \\ \mathrm{C} & -1.094769 & 2.852942 & -0.194380 \\ \mathrm{~N} & -0.008905 & 2.036838 & -0.116534 \\ \mathrm{C} & 1.069698 & 2.862182 & -0.198039 \\ \mathrm{C} & 0.656940 & 4.241343 & -0.378867 \\ \mathrm{C} & -0.694387 & 4.235635 & -0.376284 \\ \mathrm{C} & 2.421281 & 2.461635 & -0.107224 \\ \mathrm{H} & -1.363509 & 5.069671 & -0.508615 \\ \mathrm{C} & -3.480746 & 3.498608 & 0.003649 \\ \mathrm{H} & -5.067910 & 1.314704 & 0.295893 \\ \mathrm{H} & -1.091086 & -0.004165 & -0.235689 \\ \mathrm{H} & -1.318451 & -5.081035 & -0.513699 \\ \mathrm{H} & 1.363509 & -5.069671 & -0.508615 \\ \mathrm{H} & 1.318451 & 5.081035 & -0.513699 \\ \mathrm{C} & -3.450861 & -3.528320 & -0.008116 \\ \mathrm{C} & 3.480746 & -3.498608 & 0.003649 \\ \mathrm{C} & 3.450861 & 3.528320 & -0.008116 \\ \mathrm{H} & -5.056337 & -1.359658 & 0.291095 \\ \mathrm{H} & 5.067910 & -1.314704 & 0.295893 \\ \mathrm{H} & 5.056337 & 1.359658 & 0.291095 \\ \mathrm{H} & 1.091086 & 0.004165 & -0.235689 \\ \mathrm{C} & -4.465347 & -3.653604 & -0.957886 \\ \mathrm{C} & -5.417958 & -4.657609 & -0.843984 \\ \mathrm{C} & -5.372863 & -5.545685 & 0.222869 \\ \mathrm{C} & -4.367398 & -5.428037 & 1.174834 \\ \mathrm{C} & -3.412345 & -4.429539 & 1.057866 \\ \mathrm{H} & -4.493114 & -2.966165 & -1.794985 \\ \mathrm{H} & -6.193216 & -4.750148 & -1.594309 \\ \mathrm{H} & -6.117432 & -6.326579 & 0.312425 \\ \mathrm{H} & -4.329261 & -6.112772 & 2.012678 \\ \mathrm{H} & -2.628579 & -4.332265 & 1.799541 \\ \mathrm{C} & -4.498247 & 3.618918 & -0.943514 \\ \mathrm{C} & -5.458431 & 4.615095 & -0.824323 \\ \mathrm{C} & -5.417958 & 5.500091 & 0.245279\end{array}$




$\begin{array}{lrrr}\mathrm{C} & -4.409532 & 5.387304 & 1.194679 \\ \mathrm{C} & -3.446921 & 4.396726 & 1.072405 \\ \mathrm{H} & -4.522371 & 2.934033 & -1.782813 \\ \mathrm{H} & -6.236018 & 4.703971 & -1.572677 \\ \mathrm{H} & -6.168440 & 6.274827 & 0.338974 \\ \mathrm{H} & -4.374962 & 6.069592 & 2.034669 \\ \mathrm{H} & -2.660723 & 4.303281 & 1.811994 \\ \mathrm{C} & 4.465347 & 3.653604 & -0.957886 \\ \mathrm{C} & 5.417958 & 4.657609 & -0.843984 \\ \mathrm{C} & 5.372863 & 5.545685 & 0.222869 \\ \mathrm{C} & 4.367398 & 5.428037 & 1.174834 \\ \mathrm{C} & 3.412345 & 4.429539 & 1.057866 \\ \mathrm{H} & 4.493114 & 2.966165 & -1.794985 \\ \mathrm{H} & 6.193216 & 4.750148 & -1.594309 \\ \mathrm{H} & 6.117432 & 6.326579 & 0.312425 \\ \mathrm{H} & 4.329261 & 6.112772 & 2.012678 \\ \mathrm{H} & 2.628579 & 4.332265 & 1.799541 \\ \mathrm{C} & 4.498247 & -3.618918 & -0.943514 \\ \mathrm{C} & 5.458431 & -4.615095 & -0.824323 \\ \mathrm{C} & 5.417958 & -5.500091 & 0.245279 \\ \mathrm{C} & 4.409532 & -5.387304 & 1.194679 \\ \mathrm{C} & 3.446921 & -4.396726 & 1.072405 \\ \mathrm{H} & 4.522371 & -2.934033 & -1.782813 \\ \mathrm{H} & 6.236018 & -4.703971 & -1.572677 \\ \mathrm{H} & 6.168440 & -6.274827 & 0.338974 \\ \mathrm{H} & 4.374962 & -6.069592 & 2.034669 \\ \mathrm{H} & 2.660723 & -4.303281 & 1.811994\end{array}$

\section{M06-2X/TZVP geometries - Group II}

tetrafluorobenzene $\mathrm{S}_{0}$

$\begin{array}{lrrr}\text { C } & -1.188886 & 0.692623 & 0.000000 \\ \text { C } & -1.188886 & -0.692623 & 0.000000 \\ \text { C } & 0.000000 & -1.395870 & 0.000000 \\ \text { C } & 1.188886 & -0.692623 & 0.000000 \\ \text { C } & 1.188886 & 0.692623 & 0.000000 \\ \text { C } & 0.000000 & 1.395870 & 0.000000 \\ \text { F } & -2.350202 & -1.346075 & 0.000000 \\ \text { H } & 0.000000 & -2.476914 & 0.000000 \\ \text { F } & 2.350202 & -1.346075 & 0.000000 \\ \text { F } & 2.350202 & 1.346075 & 0.000000 \\ \text { H } & 0.000000 & 2.476914 & 0.000000 \\ \text { F } & -2.350202 & 1.346075 & 0.000000\end{array}$


tetrafluorobenzene $S_{1}$

$\begin{array}{lrrr}\text { C } & 1.158490 & -0.708283 & 0.107844 \\ \text { C } & 1.158490 & 0.708283 & 0.107844 \\ \text { C } & 0.000000 & 1.464190 & 0.338466 \\ \text { C } & -1.158490 & 0.708283 & 0.107844 \\ \text { C } & -1.158490 & -0.708283 & 0.107844 \\ \text { C } & 0.000000 & -1.464190 & 0.338466 \\ \text { F } & 2.293934 & 1.310715 & -0.229603 \\ \text { H } & 0.000000 & 2.510884 & 0.603175 \\ \text { F } & -2.293934 & 1.310715 & -0.229603 \\ \text { F } & -2.293934 & -1.310715 & -0.229603 \\ \text { H } & 0.000000 & -2.510884 & 0.603175 \\ \text { F } & 2.293934 & -1.310715 & -0.229603\end{array}$

benzonitrile $\mathrm{S}_{0}$

$\begin{array}{lrrr}\mathrm{C} & 0.000000 & 0.000000 & -1.050902 \\ \mathrm{C} & -1.210762 & 0.000000 & -0.358628 \\ \mathrm{C} & -1.204758 & 0.000000 & 1.027084 \\ \mathrm{C} & 0.000000 & 0.000000 & 1.719255 \\ \mathrm{C} & 1.204758 & 0.000000 & 1.027084 \\ \mathrm{C} & 1.210762 & 0.000000 & -0.358628 \\ \mathrm{H} & -2.141603 & 0.000000 & -0.910132 \\ \mathrm{H} & -2.142119 & 0.000000 & 1.567739 \\ \mathrm{H} & 0.000000 & 0.000000 & 2.801662 \\ \mathrm{H} & 2.142119 & 0.000000 & 1.567739 \\ \mathrm{H} & 2.141603 & 0.000000 & -0.910132 \\ \mathrm{C} & 0.000000 & 0.000000 & -2.486946 \\ \mathrm{~N} & 0.000000 & 0.000000 & -3.635185\end{array}$

benzonitrile $S_{1}$

$\begin{array}{lrrr}\mathrm{C} & 0.000000 & 0.000000 & -1.101751 \\ \mathrm{C} & -1.225520 & 0.000000 & -0.372970 \\ \mathrm{C} & -1.215189 & 0.000000 & 1.045782 \\ \mathrm{C} & 0.000000 & 0.000000 & 1.764551 \\ \mathrm{C} & 1.215189 & 0.000000 & 1.045782 \\ \mathrm{C} & 1.225520 & 0.000000 & -0.372970 \\ \mathrm{H} & -2.163247 & 0.000000 & -0.910909 \\ \mathrm{H} & -2.159027 & 0.000000 & 1.573635 \\ \mathrm{H} & 0.000000 & 0.000000 & 2.844180 \\ \mathrm{H} & 2.159027 & 0.000000 & 1.573635 \\ \mathrm{H} & 2.163247 & 0.000000 & -0.910909 \\ \mathrm{C} & 0.000000 & 0.000000 & -2.511353 \\ \mathrm{~N} & 0.000000 & 0.000000 & -3.666702\end{array}$




\begin{tabular}{lrrr}
$o$-fluorophenol, cis $\mathrm{S}_{0}$ \\
\multicolumn{4}{l}{} \\
$\mathrm{C}$ & 0.325793 & 1.451406 & 0.000000 \\
$\mathrm{C}$ & -0.871368 & 0.773115 & 0.000000 \\
$\mathrm{C}$ & -0.947025 & -0.613599 & 0.000000 \\
$\mathrm{C}$ & 0.237366 & -1.336051 & 0.000000 \\
$\mathrm{C}$ & 1.455452 & -0.670447 & 0.000000 \\
$\mathrm{C}$ & 1.506366 & 0.717737 & 0.000000 \\
$\mathrm{~F}$ & -2.044486 & 1.448725 & 0.000000 \\
$\mathrm{O}$ & -2.138453 & -1.261468 & 0.000000 \\
$\mathrm{H}$ & 0.180175 & -2.416423 & 0.000000 \\
$\mathrm{H}$ & 2.372551 & -1.244615 & 0.000000 \\
$\mathrm{H}$ & 2.457509 & 1.232062 & 0.000000 \\
$\mathrm{H}$ & 0.318907 & 2.533139 & 0.000000 \\
$\mathrm{H}$ & -2.852776 & -0.613601 & 0.000000
\end{tabular}

$o$-fluorophenol, cis $\mathrm{S}_{1}$

$\begin{array}{lrrr}\mathrm{C} & 1.222559 & -0.274561 & -0.740053 \\ \mathrm{C} & 0.001514 & -0.012885 & -0.095050 \\ \mathrm{C} & -0.020633 & -0.024397 & 1.353203 \\ \mathrm{C} & 1.161453 & 0.077335 & 2.090359 \\ \mathrm{C} & 2.366984 & 0.133277 & 1.402190 \\ \mathrm{C} & 2.385897 & -0.043734 & 0.003662 \\ \mathrm{~F} & -0.847798 & 0.961788 & -0.651114 \\ \mathrm{O} & -1.175387 & -0.210356 & 1.990338 \\ \mathrm{H} & 1.118991 & -0.046756 & 3.166168 \\ \mathrm{H} & 3.298606 & 0.235352 & 1.940970 \\ \mathrm{H} & 3.333072 & 0.027751 & -0.521516 \\ \mathrm{H} & 1.269712 & -0.433671 & -1.808941 \\ \mathrm{H} & -1.859591 & -0.389144 & 1.319725\end{array}$

$o$-fluorophenol, trans $\mathrm{S}_{0}$

$\begin{array}{rrrr}\mathrm{C} & 0.289912 & 1.258725 & 0.000000 \\ \mathrm{C} & -0.944181 & 0.614538 & 0.000000 \\ \mathrm{C} & -0.961013 & -0.773667 & 0.000000 \\ \mathrm{C} & 0.227665 & -1.494068 & 0.000000 \\ \mathrm{C} & 1.446198 & -0.833904 & 0.000000 \\ \mathrm{C} & 1.474727 & 0.556663 & 0.000000 \\ \mathrm{O} & -2.057879 & 1.390018 & 0.000000 \\ \mathrm{H} & -1.914707 & -1.290261 & 0.000000 \\ \mathrm{H} & 0.193269 & -2.575366 & 0.000000 \\ \mathrm{H} & 2.372655 & -1.391532 & 0.000000 \\ \mathrm{H} & 2.404022 & 1.110790 & 0.000000\end{array}$




$$
\begin{array}{cccc}
\mathrm{F} & 0.309835 & 2.597855 & 0.000000 \\
\mathrm{H} & -2.840514 & 0.830208 & 0.000000
\end{array}
$$

$o$-fluorophenol, trans $\mathrm{S}_{1}$

$\begin{array}{lrrr}\mathrm{C} & 0.819948 & 1.031578 & 0.113171 \\ \mathrm{C} & -0.588297 & 0.970582 & 0.095057 \\ \mathrm{C} & -1.274893 & -0.289159 & 0.141528 \\ \mathrm{C} & -0.474285 & -1.415834 & -0.077157 \\ \mathrm{C} & 0.938065 & -1.327824 & -0.076923 \\ \mathrm{C} & 1.618264 & -0.127307 & 0.200538 \\ \mathrm{O} & -1.244750 & 2.109054 & -0.136034 \\ \mathrm{H} & -2.295791 & -0.355520 & 0.498896 \\ \mathrm{H} & -0.930437 & -2.388885 & -0.213064 \\ \mathrm{H} & 1.508862 & -2.228020 & -0.276419 \\ \mathrm{H} & 2.687296 & -0.063966 & 0.327518 \\ \mathrm{~F} & 1.389750 & 2.200184 & -0.195707 \\ \mathrm{H} & -2.153761 & 1.885118 & -0.401406\end{array}$

$m$-fluorophenol, cis $\mathrm{S}_{0}$

$\begin{array}{cccc}\text { C } & 0.308886 & 1.405738 & 0.000000 \\ \mathrm{C} & -0.926973 & 0.786679 & 0.000000 \\ \mathrm{C} & -0.964700 & -0.602908 & 0.000000 \\ \mathrm{C} & 0.214999 & -1.341040 & 0.000000 \\ \mathrm{C} & 1.431810 & -0.680203 & 0.000000 \\ \mathrm{C} & 1.499178 & 0.709006 & 0.000000 \\ \mathrm{O} & -2.135166 & -1.295236 & 0.000000 \\ \mathrm{~F} & 0.339047 & 2.749223 & 0.000000 \\ \mathrm{H} & 0.155071 & -2.420438 & 0.000000 \\ \mathrm{H} & 2.348275 & -1.255923 & 0.000000 \\ \mathrm{H} & 2.437867 & 1.244089 & 0.000000 \\ \mathrm{H} & -1.828187 & 1.387500 & 0.000000 \\ \mathrm{H} & -2.880107 & -0.686486 & 0.000000\end{array}$

$m$-fluorophenol, cis $\mathrm{S}_{1}$

$\begin{array}{lccc}\text { C } & 1.217941 & -0.039379 & -0.679726 \\ \text { C } & -0.047806 & 0.106430 & -0.101998 \\ \text { C } & -0.023088 & 0.044973 & 1.321519 \\ \text { C } & 1.169554 & 0.024799 & 2.079241 \\ \text { C } & 2.426354 & 0.187334 & 1.439849 \\ \text { C } & 2.425500 & -0.041964 & 0.041596 \\ \text { O } & -1.167460 & -0.069543 & 2.007522 \\ \text { F } & 1.293770 & -0.188800 & -2.007827 \\ \text { H } & 1.084069 & -0.199019 & 3.135607\end{array}$




$$
\begin{array}{rrrr}
\mathrm{H} & 3.345405 & 0.237776 & 1.999502 \\
\mathrm{H} & 3.339295 & -0.240226 & -0.506129 \\
\mathrm{H} & -0.913777 & 0.416946 & -0.670629 \\
\mathrm{H} & -1.894397 & -0.239327 & 1.391422
\end{array}
$$

$m$-fluorophenol, trans $\mathrm{S}_{0}$

$\begin{array}{cccc}\mathrm{C} & 0.268395 & 1.301996 & 0.000000 \\ \mathrm{C} & -0.946779 & 0.626886 & 0.000000 \\ \mathrm{C} & -0.982446 & -0.764026 & 0.000000 \\ \mathrm{C} & 0.207536 & -1.478682 & 0.000000 \\ \mathrm{C} & 1.433201 & -0.829550 & 0.000000 \\ \mathrm{C} & 1.426201 & 0.553698 & 0.000000 \\ \mathrm{O} & -2.072851 & 1.389211 & 0.000000 \\ \mathrm{H} & -1.933886 & -1.282969 & 0.000000 \\ \mathrm{H} & 0.177115 & -2.560413 & 0.000000 \\ \mathrm{H} & 2.371508 & -1.365313 & 0.000000 \\ \mathrm{~F} & 2.601301 & 1.203926 & 0.000000 \\ \mathrm{H} & 0.301573 & 2.382114 & 0.000000 \\ \mathrm{H} & -2.850867 & 0.823130 & 0.000000\end{array}$

$m$-fluorophenol, trans $\mathrm{S}_{1}$

$\begin{array}{cccc}\mathrm{C} & 1.086258 & -0.016819 & 0.000000 \\ \mathrm{C} & 0.328819 & 1.180418 & 0.000000 \\ \mathrm{C} & -1.091223 & 1.209931 & 0.000000 \\ \mathrm{C} & -1.824376 & -0.005771 & 0.000000 \\ \mathrm{C} & -1.085596 & -1.216876 & 0.000000 \\ \mathrm{C} & 0.319920 & -1.194190 & 0.000000 \\ \mathrm{O} & 1.035015 & 2.319614 & 0.000000 \\ \mathrm{H} & -1.597994 & 2.167904 & 0.000000 \\ \mathrm{H} & -2.901980 & -0.012320 & 0.000000 \\ \mathrm{H} & -1.580394 & -2.179086 & 0.000000 \\ \mathrm{~F} & 0.964773 & -2.367183 & 0.000000 \\ \mathrm{H} & 2.163705 & -0.012170 & 0.000000 \\ \mathrm{H} & 0.441924 & 3.080694 & 0.000000\end{array}$

$p$-fluorophenol $\mathrm{S}_{0}$

$\begin{array}{rrrr}\text { C } & 0.271685 & 1.314121 & 0.000000 \\ \text { C } & -0.947258 & 0.644972 & 0.000000 \\ \text { C } & -0.979382 & -0.744058 & 0.000000 \\ \text { C } & 0.206274 & -1.468494 & 0.000000 \\ \text { C } & 1.403763 & -0.785117 & 0.000000 \\ \text { C } & 1.456515 & 0.596206 & 0.000000 \\ \text { O } & -2.078001 & 1.408743 & 0.000000\end{array}$




$\begin{array}{lccc}\mathrm{H} & -1.929645 & -1.266079 & 0.000000 \\ \mathrm{H} & 0.206045 & -2.550018 & 0.000000 \\ \mathrm{~F} & 2.555569 & -1.483368 & 0.000000 \\ \mathrm{H} & 2.416891 & 1.093799 & 0.000000 \\ \mathrm{H} & 0.273638 & 2.395681 & 0.000000 \\ \mathrm{H} & -2.856114 & 0.843600 & 0.000000\end{array}$

p-fluorophenol $\mathrm{S}_{1}$

$\begin{array}{lrrl}\mathrm{C} & 1.266286 & 0.678345 & 0.000000 \\ \mathrm{C} & 0.026471 & 1.358842 & 0.000000 \\ \mathrm{C} & -1.238185 & 0.708541 & 0.000000 \\ \mathrm{C} & -1.250999 & -0.703544 & 0.000000 \\ \mathrm{C} & -0.006217 & -1.361186 & 0.000000 \\ \mathrm{C} & 1.252333 & -0.735156 & 0.000000 \\ \mathrm{O} & 0.093103 & 2.694367 & 0.000000 \\ \mathrm{H} & -2.151810 & 1.288626 & 0.000000 \\ \mathrm{H} & -2.159753 & -1.285212 & 0.000000 \\ \mathrm{~F} & -0.026790 & -2.690557 & 0.000000 \\ \mathrm{H} & 2.149102 & -1.334428 & 0.000000 \\ \mathrm{H} & 2.178594 & 1.255028 & 0.000000 \\ \mathrm{H} & -0.795437 & 3.071929 & 0.000000\end{array}$

phenylacetylene $\mathrm{S}_{0}$

$\begin{array}{lrrr}\mathrm{C} & 1.206522 & 0.000000 & -0.013560 \\ \mathrm{C} & 0.000000 & 0.000000 & -0.718213 \\ \mathrm{C} & -1.206522 & 0.000000 & -0.013560 \\ \mathrm{C} & -1.202631 & 0.000000 & 1.372388 \\ \mathrm{C} & 0.000000 & 0.000000 & 2.068166 \\ \mathrm{C} & 1.202631 & 0.000000 & 1.372388 \\ \mathrm{C} & 0.000000 & 0.000000 & -2.150544 \\ \mathrm{C} & 0.000000 & 0.000000 & -3.350600 \\ \mathrm{H} & -2.138813 & 0.000000 & -0.563034 \\ \mathrm{H} & -2.141391 & 0.000000 & 1.911408 \\ \mathrm{H} & 0.000000 & 0.000000 & 3.150604 \\ \mathrm{H} & 2.141391 & 0.000000 & 1.911408 \\ \mathrm{H} & 2.138813 & 0.000000 & -0.563034 \\ \mathrm{H} & 0.000000 & 0.000000 & -4.413835\end{array}$

phenylacetylene $S_{1}$
$\begin{array}{llll}\text { C } & 1.231734 & 0.000000 & -0.029424\end{array}$
$\begin{array}{llll}\text { C } & 0.000000 & 0.000000 & -0.751325\end{array}$
$\begin{array}{llll}\text { C } & -1.231734 & 0.000000 & -0.029424\end{array}$
$\begin{array}{llll}\text { C } & -1.222606 & 0.000000 & 1.389903\end{array}$ 


$\begin{array}{lrrr}\mathrm{C} & 0.000000 & 0.000000 & 2.094189 \\ \mathrm{C} & 1.222606 & 0.000000 & 1.389903 \\ \mathrm{C} & 0.000000 & 0.000000 & -2.153939 \\ \mathrm{C} & 0.000000 & 0.000000 & -3.362725 \\ \mathrm{H} & -2.163128 & 0.000000 & -0.577163 \\ \mathrm{H} & -2.159594 & 0.000000 & 1.928889 \\ \mathrm{H} & 0.000000 & 0.000000 & 3.175333 \\ \mathrm{H} & 2.159594 & 0.000000 & 1.928889 \\ \mathrm{H} & 2.163128 & 0.000000 & -0.577163 \\ \mathrm{H} & 0.000000 & 0.000000 & -4.425943\end{array}$

aniline $\mathrm{S}_{0}$

$\begin{array}{lrrr}\mathrm{C} & 0.917225 & 0.029270 & 0.000000 \\ \mathrm{C} & 0.205722 & 0.037335 & 1.201817 \\ \mathrm{C} & -1.180271 & 0.042490 & 1.196547 \\ \mathrm{C} & -1.886026 & 0.046138 & 0.000000 \\ \mathrm{C} & -1.180271 & 0.042490 & -1.196547 \\ \mathrm{C} & 0.205722 & 0.037335 & -1.201817 \\ \mathrm{H} & 0.747724 & 0.040129 & 2.140657 \\ \mathrm{H} & -2.967613 & 0.050034 & 0.000000 \\ \mathrm{H} & -1.713366 & 0.045896 & 2.139312 \\ \mathrm{H} & -1.713366 & 0.045896 & -2.139312 \\ \mathrm{H} & 0.747724 & 0.040129 & -2.140657 \\ \mathrm{~N} & 2.310885 & 0.079876 & 0.000000 \\ \mathrm{H} & 2.752961 & -0.268503 & -0.836186 \\ \mathrm{H} & 2.752961 & -0.268503 & 0.836186\end{array}$

aniline $S_{1}$

$\begin{array}{rrrr}\mathrm{C} & 0.894975 & -0.034134 & 0.000000 \\ \mathrm{C} & 0.224229 & -0.008473 & 1.248952 \\ \mathrm{C} & -1.192689 & 0.045615 & 1.237701 \\ \mathrm{C} & -1.864098 & 0.071182 & 0.000000 \\ \mathrm{C} & -1.192689 & 0.045615 & -1.237701 \\ \mathrm{C} & 0.224229 & -0.008473 & -1.248952 \\ \mathrm{H} & 0.794101 & -0.030281 & 2.167952 \\ \mathrm{H} & -2.947249 & 0.112419 & 0.000000 \\ \mathrm{H} & -1.752629 & 0.066976 & 2.160567 \\ \mathrm{H} & -1.752629 & 0.066976 & -2.160567 \\ \mathrm{H} & 0.794101 & -0.030281 & -2.167952 \\ \mathrm{~N} & 2.246717 & -0.085949 & 0.000000 \\ \mathrm{H} & 2.761809 & -0.105606 & -0.864696 \\ \mathrm{H} & 2.761809 & -0.105606 & 0.864696\end{array}$

catechol $\mathrm{S}_{0}$ 


$\begin{array}{rrrr}\mathrm{C} & 0.469923 & 1.276737 & 0.000000 \\ \mathrm{C} & -0.745083 & 0.617803 & 0.000000 \\ \mathrm{C} & -0.795256 & -0.777346 & 0.000000 \\ \mathrm{C} & 0.383385 & -1.502156 & 0.000000 \\ \mathrm{C} & 1.606569 & -0.841482 & 0.000000 \\ \mathrm{C} & 1.653120 & 0.544194 & 0.000000 \\ \mathrm{O} & -1.968689 & 1.240196 & 0.000000 \\ \mathrm{O} & -1.990975 & -1.420388 & 0.000000 \\ \mathrm{H} & 0.322363 & -2.582445 & 0.000000 \\ \mathrm{H} & 2.523216 & -1.416143 & 0.000000 \\ \mathrm{H} & 2.602618 & 1.062125 & 0.000000 \\ \mathrm{H} & 0.491837 & 2.361149 & 0.000000 \\ \mathrm{H} & -1.860081 & 2.195381 & 0.000000 \\ \mathrm{H} & -2.692948 & -0.757627 & 0.000000\end{array}$

catechol $\mathrm{S}_{1}$

$\begin{array}{rrrr}\mathrm{C} & 0.473891 & 1.349304 & 0.000000 \\ \mathrm{C} & -0.755447 & 0.625197 & 0.000000 \\ \mathrm{C} & -0.800532 & -0.784571 & 0.000000 \\ \mathrm{C} & 0.388091 & -1.575170 & 0.000000 \\ \mathrm{C} & 1.595596 & -0.859218 & 0.000000 \\ \mathrm{C} & 1.637594 & 0.557971 & 0.000000 \\ \mathrm{O} & -1.965725 & 1.239744 & 0.000000 \\ \mathrm{O} & -1.983386 & -1.414853 & 0.000000 \\ \mathrm{H} & 0.322394 & -2.650461 & 0.000000 \\ \mathrm{H} & 2.534896 & -1.396273 & 0.000000 \\ \mathrm{H} & 2.605928 & 1.041733 & 0.000000 \\ \mathrm{H} & 0.494718 & 2.428761 & 0.000000 \\ \mathrm{H} & -1.853353 & 2.196131 & 0.000000 \\ \mathrm{H} & -2.694663 & -0.758296 & 0.000000\end{array}$

resorcinol, isomer $1 \mathrm{~S}_{0}$

$\begin{array}{rrrr}\mathrm{C} & 0.075029 & 1.145527 & 0.000000 \\ \mathrm{C} & -1.135818 & 0.463947 & 0.000000 \\ \mathrm{C} & -1.167908 & -0.924679 & 0.000000 \\ \mathrm{C} & 0.032958 & -1.624709 & 0.000000 \\ \mathrm{C} & 1.249819 & -0.966653 & 0.000000 \\ \mathrm{C} & 1.260820 & 0.425491 & 0.000000 \\ \mathrm{O} & -2.266202 & 1.224640 & 0.000000 \\ \mathrm{H} & -2.115324 & -1.450557 & 0.000000 \\ \mathrm{H} & 0.014422 & -2.707086 & 0.000000 \\ \mathrm{H} & 2.188963 & -1.501538 & 0.000000 \\ \mathrm{O} & 2.476053 & 1.039477 & 0.000000\end{array}$




$\begin{array}{cccc}\mathrm{H} & 0.068233 & 2.228976 & 0.000000 \\ \mathrm{H} & -3.039946 & 0.652763 & 0.000000 \\ \mathrm{H} & 2.358900 & 1.994391 & 0.000000\end{array}$

resorcinol, isomer $1 \mathrm{~S}_{1}$

$\begin{array}{rrrc}\mathrm{C} & 0.000585 & 1.046867 & 0.253653 \\ \mathrm{C} & -1.174005 & 0.310408 & 0.055097 \\ \mathrm{C} & -1.164309 & -1.103931 & -0.023298 \\ \mathrm{C} & -0.001622 & -1.840911 & 0.355291 \\ \mathrm{C} & 1.168966 & -1.108586 & -0.013132 \\ \mathrm{C} & 1.176092 & 0.301499 & 0.049376 \\ \mathrm{O} & -2.319463 & 1.003229 & -0.093773 \\ \mathrm{H} & -2.028717 & -1.602768 & -0.458047 \\ \mathrm{H} & -0.005048 & -2.918573 & 0.358572 \\ \mathrm{H} & 2.043956 & -1.595700 & -0.431765 \\ \mathrm{O} & 2.365379 & 0.905243 & -0.135948 \\ \mathrm{H} & -0.014920 & 2.105990 & 0.463682 \\ \mathrm{H} & -3.067277 & 0.396193 & -0.140527 \\ \mathrm{H} & 2.231376 & 1.857160 & -0.219530\end{array}$

resorcinol, isomer $2 \mathrm{~S}_{0}$

$\begin{array}{rrrr}\mathrm{C} & 1.196044 & 0.000000 & -0.544789 \\ \mathrm{C} & 0.000000 & 0.000000 & -1.246160 \\ \mathrm{C} & -1.196044 & 0.000000 & -0.544789 \\ \mathrm{C} & -1.207489 & 0.000000 & 0.847904 \\ \mathrm{C} & 0.000000 & 0.000000 & 1.529222 \\ \mathrm{C} & 1.207489 & 0.000000 & 0.847904 \\ \mathrm{O} & -2.341561 & 0.000000 & -1.281084 \\ \mathrm{O} & 2.341561 & 0.000000 & -1.281084 \\ \mathrm{H} & -2.146871 & 0.000000 & 1.387995 \\ \mathrm{H} & 0.000000 & 0.000000 & 2.611674 \\ \mathrm{H} & 2.146871 & 0.000000 & 1.387995 \\ \mathrm{H} & 0.000000 & 0.000000 & -2.326959 \\ \mathrm{H} & -3.103773 & 0.000000 & -0.694154 \\ \mathrm{H} & 3.103773 & 0.000000 & -0.694154\end{array}$

resorcinol, isomer $2 \mathrm{~S}_{1}$
$\begin{array}{llll}\text { C } & 1.193673 & 0.000000 & -0.330419\end{array}$
$\begin{array}{llll}\text { C } & 0.000000 & 0.000000 & -1.082271\end{array}$
$\begin{array}{llll}\text { C } & -1.193673 & 0.000000 & -0.330419\end{array}$
$\begin{array}{llll}\text { C } & -1.210541 & 0.000000 & 1.086801\end{array}$
$\begin{array}{llll}\text { C } & 0.000000 & 0.000000 & 1.826971\end{array}$
$\begin{array}{llll}\text { C } & 1.210541 & 0.000000 & 1.086801\end{array}$ 


$\begin{array}{rrrr}\mathrm{O} & -2.344905 & 0.000000 & -1.034772 \\ \mathrm{O} & 2.344905 & 0.000000 & -1.034772 \\ \mathrm{H} & -2.166328 & 0.000000 & 1.599331 \\ \mathrm{H} & 0.000000 & 0.000000 & 2.904498 \\ \mathrm{H} & 2.166328 & 0.000000 & 1.599331 \\ \mathrm{H} & 0.000000 & 0.000000 & -2.159437 \\ \mathrm{H} & -3.099398 & 0.000000 & -0.435363 \\ \mathrm{H} & 3.099398 & 0.000000 & -0.435363\end{array}$

resorcinol, isomer $3 \mathrm{~S}_{0}$

$\begin{array}{lrrr}\mathrm{C} & 1.201167 & 0.000000 & -0.287104 \\ \mathrm{C} & 0.000000 & 0.000000 & -0.988394 \\ \mathrm{C} & -1.201167 & 0.000000 & -0.287104 \\ \mathrm{C} & -1.210122 & 0.000000 & 1.101387 \\ \mathrm{C} & 0.000000 & 0.000000 & 1.778471 \\ \mathrm{C} & 1.210122 & 0.000000 & 1.101387 \\ \mathrm{O} & -2.406400 & 0.000000 & -0.922747 \\ \mathrm{O} & 2.406400 & 0.000000 & -0.922747 \\ \mathrm{H} & -2.157250 & 0.000000 & 1.622053 \\ \mathrm{H} & 0.000000 & 0.000000 & 2.861055 \\ \mathrm{H} & 2.157250 & 0.000000 & 1.622053 \\ \mathrm{H} & 0.000000 & 0.000000 & -2.074397 \\ \mathrm{H} & 2.276884 & 0.000000 & -1.875717 \\ \mathrm{H} & -2.276884 & 0.000000 & -1.875717\end{array}$

resorcinol, isomer $3 \mathrm{~S}_{1}$

$\begin{array}{lrrc}\mathrm{C} & -0.024806 & -0.304653 & 1.183381 \\ \mathrm{C} & -0.148249 & -1.073242 & 0.000000 \\ \mathrm{C} & -0.024806 & -0.304653 & -1.183381 \\ \mathrm{C} & 0.035974 & 1.106820 & -1.193952 \\ \mathrm{C} & -0.110992 & 1.849466 & 0.000000 \\ \mathrm{C} & 0.035974 & 1.106820 & 1.193952 \\ \mathrm{O} & 0.075829 & -0.914696 & -2.380728 \\ \mathrm{O} & 0.075829 & -0.914696 & 2.380728 \\ \mathrm{H} & 0.241601 & 1.586506 & -2.143542 \\ \mathrm{H} & -0.120687 & 2.926725 & 0.000000 \\ \mathrm{H} & 0.241601 & 1.586506 & 2.143542 \\ \mathrm{H} & -0.650475 & -2.037488 & 0.000000 \\ \mathrm{H} & 0.248060 & -1.855227 & 2.236013 \\ \mathrm{H} & 0.248060 & -1.855227 & -2.236013\end{array}$

hydroquinone, cis $\mathrm{S}_{0}$

$\begin{array}{llll}\text { C } & -0.695682 & 0.000000 & 1.090043\end{array}$ 


$\begin{array}{lrrr}\mathrm{C} & 0.695682 & 0.000000 & 1.090043 \\ \mathrm{C} & 1.391412 & 0.000000 & -0.108597 \\ \mathrm{C} & 0.691286 & 0.000000 & -1.310594 \\ \mathrm{C} & -0.691286 & 0.000000 & -1.310594 \\ \mathrm{C} & -1.391412 & 0.000000 & -0.108597 \\ \mathrm{O} & 2.757755 & 0.000000 & -0.176175 \\ \mathrm{O} & -2.757755 & 0.000000 & -0.176175 \\ \mathrm{H} & 1.248242 & 0.000000 & -2.238106 \\ \mathrm{H} & -1.248242 & 0.000000 & -2.238106 \\ \mathrm{H} & -1.232727 & 0.000000 & 2.032196 \\ \mathrm{H} & 1.232727 & 0.000000 & 2.032196 \\ \mathrm{H} & -3.127097 & 0.000000 & 0.711523 \\ \mathrm{H} & 3.127097 & 0.000000 & 0.711523\end{array}$

hydroquinone, cis $\mathrm{S}_{1}$

$\begin{array}{lrrr}\mathrm{C} & 0.705035 & 0.000000 & -1.133317 \\ \mathrm{C} & -0.705035 & 0.000000 & -1.133317 \\ \mathrm{C} & -1.371356 & 0.000000 & 0.119358 \\ \mathrm{C} & -0.705480 & 0.000000 & 1.362272 \\ \mathrm{C} & 0.705480 & 0.000000 & 1.362272 \\ \mathrm{C} & 1.371356 & 0.000000 & 0.119358 \\ \mathrm{O} & -2.711530 & 0.000000 & 0.165798 \\ \mathrm{O} & 2.711530 & 0.000000 & 0.165798 \\ \mathrm{H} & -1.290473 & 0.000000 & 2.268949 \\ \mathrm{H} & 1.290473 & 0.000000 & 2.268949 \\ \mathrm{H} & 1.274671 & 0.000000 & -2.053330 \\ \mathrm{H} & -1.274671 & 0.000000 & -2.053330 \\ \mathrm{H} & 3.071717 & 0.000000 & -0.728961 \\ \mathrm{H} & -3.071717 & 0.000000 & -0.728961\end{array}$

hydroquinone, trans $\mathrm{S}_{0}$

$\begin{array}{lrrr}\mathrm{C} & 0.020931 & 1.387773 & 0.000000 \\ \mathrm{C} & -1.192935 & 0.716276 & 0.000000 \\ \mathrm{C} & -1.209547 & -0.673224 & 0.000000 \\ \mathrm{C} & -0.020931 & -1.387773 & 0.000000 \\ \mathrm{C} & 1.192935 & -0.716276 & 0.000000 \\ \mathrm{C} & 1.209547 & 0.673224 & 0.000000 \\ \mathrm{O} & -2.333329 & 1.472042 & 0.000000 \\ \mathrm{H} & -2.155314 & -1.204277 & 0.000000 \\ \mathrm{H} & -0.021455 & -2.469538 & 0.000000 \\ \mathrm{O} & 2.333329 & -1.472042 & 0.000000 \\ \mathrm{H} & 2.155314 & 1.204277 & 0.000000 \\ \mathrm{H} & 0.021455 & 2.469538 & 0.000000 \\ \mathrm{H} & -3.103720 & 0.896752 & 0.000000\end{array}$


$\begin{array}{llll}\mathrm{H} & 3.103720 & -0.896752 & 0.000000\end{array}$

hydroquinone, trans $\mathrm{S}_{1}$

$\begin{array}{rrrr}\mathrm{C} & 1.262363 & 0.674889 & 0.000000 \\ \mathrm{C} & 0.034305 & 1.370854 & 0.000000 \\ \mathrm{C} & -1.232585 & 0.735546 & 0.000000 \\ \mathrm{C} & -1.262363 & -0.674889 & 0.000000 \\ \mathrm{C} & -0.034305 & -1.370854 & 0.000000 \\ \mathrm{C} & 1.232585 & -0.735546 & 0.000000 \\ \mathrm{O} & 0.118824 & 2.709442 & 0.000000 \\ \mathrm{H} & -2.140060 & 1.324951 & 0.000000 \\ \mathrm{H} & -2.181588 & -1.240524 & 0.000000 \\ \mathrm{O} & -0.118824 & -2.709442 & 0.000000 \\ \mathrm{H} & 2.140060 & -1.324951 & 0.000000 \\ \mathrm{H} & 2.181588 & 1.240524 & 0.000000 \\ \mathrm{H} & -0.766867 & 3.092717 & 0.000000 \\ \mathrm{H} & 0.766867 & -3.092717 & 0.000000\end{array}$

salicylic acid $\mathrm{S}_{0}$

$\begin{array}{lrrr}\mathrm{C} & 1.099566 & 1.595767 & 0.000000 \\ \mathrm{C} & 1.132728 & 0.198811 & 0.000000 \\ \mathrm{C} & -0.080507 & -0.514006 & 0.000000 \\ \mathrm{C} & -1.295650 & 0.182790 & 0.000000 \\ \mathrm{C} & -1.317386 & 1.560044 & 0.000000 \\ \mathrm{C} & -0.108239 & 2.260764 & 0.000000 \\ \mathrm{O} & 2.334279 & -0.391055 & 0.000000 \\ \mathrm{C} & -0.045483 & -1.980241 & 0.000000 \\ \mathrm{O} & -1.250638 & -2.564096 & 0.000000 \\ \mathrm{O} & 0.972996 & -2.645561 & 0.000000 \\ \mathrm{H} & -2.216144 & -0.385157 & 0.000000 \\ \mathrm{H} & 2.042336 & 2.126002 & 0.000000 \\ \mathrm{H} & -2.258473 & 2.092255 & 0.000000 \\ \mathrm{H} & -0.115581 & 3.343754 & 0.000000 \\ \mathrm{H} & 2.209088 & -1.359339 & 0.000000 \\ \mathrm{H} & -1.102895 & -3.520732 & 0.000000\end{array}$

salicylic acid $\mathrm{S}_{1}$
$\begin{array}{llll}\text { C } & 1.003868 & 1.913647 & 0.000000\end{array}$
$\begin{array}{llll}\text { C } & 0.968847 & 0.510301 & 0.000000\end{array}$
$\begin{array}{llll}\text { C } & -0.300341 & -0.222569 & 0.000000\end{array}$
$\begin{array}{llll}\text { C } & -1.460435 & 0.537211 & 0.000000\end{array}$
$\begin{array}{llll}\text { C } & -1.400979 & 1.959069 & 0.000000\end{array}$
$\begin{array}{llll}\text { C } & -0.191979 & 2.652348 & 0.000000\end{array}$ 


$\begin{array}{rrrr}\mathrm{O} & 2.068611 & -0.182352 & 0.000000 \\ \mathrm{C} & -0.234107 & -1.646038 & 0.000000 \\ \mathrm{O} & -1.409769 & -2.304113 & 0.000000 \\ \mathrm{O} & 0.851830 & -2.293226 & 0.000000 \\ \mathrm{H} & -2.420954 & 0.039983 & 0.000000 \\ \mathrm{H} & 1.975900 & 2.388818 & 0.000000 \\ \mathrm{H} & -2.333104 & 2.509963 & 0.000000 \\ \mathrm{H} & -0.173563 & 3.731944 & 0.000000 \\ \mathrm{H} & 1.767297 & -1.210267 & 0.000000 \\ \mathrm{H} & -1.200542 & -3.247773 & 0.000000\end{array}$

$o$-cresol, cis $\mathrm{S}_{0}$

$\begin{array}{rrrr}\mathrm{C} & 1.961540 & 0.512445 & 0.000000 \\ \mathrm{C} & 0.729564 & 1.150265 & 0.000000 \\ \mathrm{C} & -0.463130 & 0.433302 & 0.000000 \\ \mathrm{C} & -0.388533 & -0.961838 & 0.000000 \\ \mathrm{C} & 0.837343 & -1.613782 & 0.000000 \\ \mathrm{C} & 2.010077 & -0.875722 & 0.000000 \\ \mathrm{C} & -1.800173 & 1.122679 & 0.000000 \\ \mathrm{O} & -1.506955 & -1.745269 & 0.000000 \\ \mathrm{H} & 0.847324 & -2.695733 & 0.000000 \\ \mathrm{H} & 2.963409 & -1.388626 & 0.000000 \\ \mathrm{H} & 2.874048 & 1.093665 & 0.000000 \\ \mathrm{H} & 0.684104 & 2.233433 & 0.000000 \\ \mathrm{H} & -2.296420 & -1.195682 & 0.000000 \\ \mathrm{H} & -2.389531 & 0.863583 & 0.884903 \\ \mathrm{H} & -1.673136 & 2.203706 & 0.000000 \\ \mathrm{H} & -2.389531 & 0.863583 & -0.884903\end{array}$

$o$-cresol, cis $\mathrm{S}_{1}$

$\begin{array}{rrrr}\mathrm{C} & 1.853709 & -0.057531 & -0.780753 \\ \mathrm{C} & 0.625805 & 0.102051 & -1.458478 \\ \mathrm{C} & -0.587821 & 0.052731 & -0.716501 \\ \mathrm{C} & -0.471880 & 0.090273 & 0.706676 \\ \mathrm{C} & 0.766482 & 0.028374 & 1.397278 \\ \mathrm{C} & 1.938155 & -0.102180 & 0.628420 \\ \mathrm{C} & -1.891923 & -0.096011 & -1.426252 \\ \mathrm{O} & -1.558643 & 0.155157 & 1.485625 \\ \mathrm{H} & 0.769787 & 0.146029 & 2.470440 \\ \mathrm{H} & 2.902484 & -0.201073 & 1.106040 \\ \mathrm{H} & 2.764374 & -0.150253 & -1.360689 \\ \mathrm{H} & 0.596297 & 0.136475 & -2.538312 \\ \mathrm{H} & -2.345408 & 0.308581 & 0.944484 \\ \mathrm{H} & -2.705028 & -0.450401 & -0.786698\end{array}$




$$
\begin{array}{llll}
\mathrm{H} & -2.218227 & 0.843144 & -1.890918 \\
\mathrm{H} & -1.788745 & -0.824856 & -2.237952
\end{array}
$$

o-cresol, trans $\mathrm{S}_{0}$

$\begin{array}{rrrc}\mathrm{C} & 2.421293 & 0.000019 & 0.031229 \\ \mathrm{C} & 1.249430 & -0.000042 & -0.714024 \\ \mathrm{C} & 0.036519 & -0.000020 & -0.020748 \\ \mathrm{C} & 0.001391 & 0.000060 & 1.366631 \\ \mathrm{C} & 1.188602 & 0.000110 & 2.088394 \\ \mathrm{C} & 2.403617 & 0.000093 & 1.422034 \\ \mathrm{C} & 1.243446 & -0.000109 & -2.214900 \\ \mathrm{O} & -1.099292 & -0.000074 & -0.780266 \\ \mathrm{H} & -0.953638 & 0.000089 & 1.881705 \\ \mathrm{H} & 1.156711 & 0.000178 & 3.170413 \\ \mathrm{H} & 3.332184 & 0.000143 & 1.977479 \\ \mathrm{H} & 3.369250 & -0.000003 & -0.494785 \\ \mathrm{H} & -1.871971 & -0.000070 & -0.207208 \\ \mathrm{H} & 2.261025 & -0.000087 & -2.603019 \\ \mathrm{H} & 0.720102 & -0.876490 & -2.601448 \\ \mathrm{H} & 0.720037 & 0.876201 & -2.601517\end{array}$

o-cresol, trans $\mathrm{S}_{1}$

$\begin{array}{cccc}\mathrm{C} & 2.423038 & 0.283546 & -0.007775 \\ \mathrm{C} & 1.215588 & 0.119067 & -0.748377 \\ \mathrm{C} & 0.007583 & 0.002921 & -0.015880 \\ \mathrm{C} & -0.053057 & -0.069310 & 1.411602 \\ \mathrm{C} & 1.174426 & -0.076940 & 2.095173 \\ \mathrm{C} & 2.387052 & 0.078554 & 1.384354 \\ \mathrm{C} & 1.252454 & -0.029093 & -2.228630 \\ \mathrm{O} & -1.127506 & -0.100473 & -0.720359 \\ \mathrm{H} & -0.998080 & 0.067844 & 1.921739 \\ \mathrm{H} & 1.199194 & -0.161843 & 3.173047 \\ \mathrm{H} & 3.317315 & 0.049427 & 1.939395 \\ \mathrm{H} & 3.359808 & 0.423457 & -0.527298 \\ \mathrm{H} & -1.835050 & -0.397209 & -0.128561 \\ \mathrm{H} & 1.893739 & -0.873126 & -2.511045 \\ \mathrm{H} & 0.263136 & -0.179357 & -2.653470 \\ \mathrm{H} & 1.699050 & 0.862537 & -2.683935\end{array}$

$m$-cresol, cis $\mathrm{S}_{0}$
$\begin{array}{llll}\text { C } & 1.157957 & -0.012057 & 2.081904\end{array}$
$\begin{array}{llll}\text { C } & 2.368191 & -0.008994 & 1.409897\end{array}$
$\begin{array}{llll}\text { C } & 2.412985 & 0.006830 & 0.020198\end{array}$ 


$\begin{array}{rrrr}\mathrm{C} & 1.234834 & 0.017321 & -0.718466 \\ \mathrm{C} & 0.016637 & 0.016991 & -0.041655 \\ \mathrm{C} & -0.022083 & 0.001542 & 1.346365 \\ \mathrm{C} & 1.261414 & 0.001451 & -2.223327 \\ \mathrm{O} & -1.193946 & 0.002231 & 2.043629 \\ \mathrm{H} & 1.103670 & -0.021413 & 3.162160 \\ \mathrm{H} & 3.290588 & -0.015915 & 1.977185 \\ \mathrm{H} & 3.367085 & 0.011938 & -0.492535 \\ \mathrm{H} & -0.911959 & 0.030284 & -0.605445 \\ \mathrm{H} & -1.935864 & 0.013898 & 1.431366 \\ \mathrm{H} & 2.221888 & 0.350509 & -2.601065 \\ \mathrm{H} & 1.102485 & -1.011431 & -2.600546 \\ \mathrm{H} & 0.478418 & 0.636186 & -2.639716\end{array}$

m-cresol, cis $\mathrm{S}_{1}$

$\begin{array}{rrrr}\mathrm{C} & 1.165367 & 0.002444 & 2.118496 \\ \mathrm{C} & 2.407852 & 0.089500 & 1.447759 \\ \mathrm{C} & 2.406903 & 0.029874 & 0.035084 \\ \mathrm{C} & 1.218366 & 0.005609 & -0.740617 \\ \mathrm{C} & -0.030746 & 0.029622 & -0.073974 \\ \mathrm{C} & -0.018681 & 0.008731 & 1.348563 \\ \mathrm{C} & 1.264610 & -0.029821 & -2.230332 \\ \mathrm{O} & -1.172391 & -0.035969 & 2.028337 \\ \mathrm{H} & 1.083753 & -0.119107 & 3.189489 \\ \mathrm{H} & 3.336940 & 0.114759 & 1.995825 \\ \mathrm{H} & 3.355219 & 0.011017 & -0.490846 \\ \mathrm{H} & -0.954246 & 0.212388 & -0.611226 \\ \mathrm{H} & -1.902462 & -0.176979 & 1.410577 \\ \mathrm{H} & 2.279730 & 0.098019 & -2.604703 \\ \mathrm{H} & 0.875682 & -0.980558 & -2.614421 \\ \mathrm{H} & 0.636383 & 0.759840 & -2.658072\end{array}$

$m$-cresol, trans $\mathrm{S}_{0}$

$\begin{array}{rrrr}\mathrm{C} & -1.191806 & -1.097691 & 0.000000 \\ \mathrm{C} & 0.197022 & -1.209012 & 0.000000 \\ \mathrm{C} & 0.967729 & -0.051290 & 0.000000 \\ \mathrm{C} & 0.363387 & 1.197954 & 0.000000 \\ \mathrm{C} & -1.023429 & 1.307623 & 0.000000 \\ \mathrm{C} & -1.792413 & 0.153433 & 0.000000 \\ \mathrm{C} & 0.872062 & -2.554823 & 0.000000 \\ \mathrm{O} & 1.183144 & 2.287851 & 0.000000 \\ \mathrm{H} & -1.493884 & 2.284805 & 0.000000 \\ \mathrm{H} & -2.872202 & 0.234058 & 0.000000 \\ \mathrm{H} & -1.802424 & -1.992099 & 0.000000\end{array}$




$\begin{array}{lrrr}\mathrm{H} & 2.050035 & -0.102640 & 0.000000 \\ \mathrm{H} & 0.653648 & 3.091032 & 0.000000 \\ \mathrm{H} & 0.140531 & -3.361865 & 0.000000 \\ \mathrm{H} & 1.506842 & -2.669405 & -0.880424 \\ \mathrm{H} & 1.506842 & -2.669405 & 0.880424\end{array}$

$m$-cresol, trans $\mathrm{S}_{1}$

$\begin{array}{rrrr}\mathrm{C} & -1.203515 & -1.057536 & 0.000000 \\ \mathrm{C} & 0.203502 & -1.232575 & 0.000000 \\ \mathrm{C} & 1.024943 & -0.075641 & 0.000000 \\ \mathrm{C} & 0.397843 & 1.191829 & 0.000000 \\ \mathrm{C} & -1.011274 & 1.369653 & 0.000000 \\ \mathrm{C} & -1.826302 & 0.214916 & 0.000000 \\ \mathrm{C} & 0.826918 & -2.586769 & 0.000000 \\ \mathrm{O} & 1.214583 & 2.255223 & 0.000000 \\ \mathrm{H} & -1.431119 & 2.367771 & 0.000000 \\ \mathrm{H} & -2.902702 & 0.294523 & 0.000000 \\ \mathrm{H} & -1.829597 & -1.942754 & 0.000000 \\ \mathrm{H} & 2.104024 & -0.129028 & 0.000000 \\ \mathrm{H} & 0.696847 & 3.069699 & 0.000000 \\ \mathrm{H} & 0.075165 & -3.375895 & 0.000000 \\ \mathrm{H} & 1.468582 & -2.724200 & -0.877885 \\ \mathrm{H} & 1.468582 & -2.724200 & 0.877885\end{array}$

$p$-cresol $\mathrm{S}_{0}$

$\begin{array}{cccc}\mathrm{C} & 0.712141 & 0.009766 & -1.187480 \\ \mathrm{C} & -0.671861 & 0.011732 & -1.196696 \\ \mathrm{C} & -1.368025 & 0.000169 & 0.007031 \\ \mathrm{C} & -0.670242 & -0.015183 & 1.206492 \\ \mathrm{C} & 0.719686 & -0.016702 & 1.196574 \\ \mathrm{C} & 1.435679 & -0.002602 & 0.005697 \\ \mathrm{O} & -2.731559 & 0.000427 & -0.055073 \\ \mathrm{C} & 2.941092 & 0.021653 & -0.004577 \\ \mathrm{H} & -1.208930 & -0.028749 & 2.148146 \\ \mathrm{H} & 1.253470 & -0.031295 & 2.140019 \\ \mathrm{H} & 1.246212 & 0.016285 & -2.131247 \\ \mathrm{H} & -1.231357 & 0.019093 & -2.122889 \\ \mathrm{H} & -3.096328 & -0.010547 & 0.834804 \\ \mathrm{H} & 3.340672 & -0.653968 & -0.762153 \\ \mathrm{H} & 3.315953 & 1.023654 & -0.225675 \\ \mathrm{H} & 3.344956 & -0.278356 & 0.962327\end{array}$

$p$-cresol $\mathrm{S}_{1}$ 


$\begin{array}{lrrr}\mathrm{C} & 0.731528 & -0.078951 & -1.236715 \\ \mathrm{C} & -0.680169 & -0.022970 & -1.244396 \\ \mathrm{C} & -1.349779 & -0.002752 & -0.000929 \\ \mathrm{C} & -0.679126 & 0.003934 & 1.251131 \\ \mathrm{C} & 0.731348 & -0.037282 & 1.238290 \\ \mathrm{C} & 1.427211 & -0.043820 & -0.000186 \\ \mathrm{O} & -2.687684 & 0.027515 & -0.048660 \\ \mathrm{C} & 2.912912 & 0.039925 & -0.000882 \\ \mathrm{H} & -1.247140 & 0.006919 & 2.172639 \\ \mathrm{H} & 1.294368 & -0.037090 & 2.161295 \\ \mathrm{H} & 1.291036 & -0.100705 & -2.161239 \\ \mathrm{H} & -1.265149 & 0.006017 & -2.151521 \\ \mathrm{H} & -3.046613 & 0.068310 & 0.846360 \\ \mathrm{H} & 3.335209 & -0.462806 & -0.872752 \\ \mathrm{H} & 3.228012 & 1.090195 & -0.045116 \\ \mathrm{H} & 3.335586 & -0.391059 & 0.907970\end{array}$

$o$-methoxyphenol $\mathrm{S}_{0}$

$\begin{array}{rrrr}\mathrm{C} & 2.021551 & -0.083663 & 0.000000 \\ \mathrm{C} & 0.866580 & 0.698786 & 0.000000 \\ \mathrm{C} & -0.373359 & 0.084229 & 0.000000 \\ \mathrm{C} & -0.468858 & -1.314408 & 0.000000 \\ \mathrm{C} & 0.679610 & -2.081761 & 0.000000 \\ \mathrm{C} & 1.927282 & -1.464285 & 0.000000 \\ \mathrm{O} & -1.583953 & 0.720701 & 0.000000 \\ \mathrm{O} & -1.687678 & -1.910545 & 0.000000 \\ \mathrm{H} & 0.578686 & -3.159107 & 0.000000 \\ \mathrm{H} & 2.823220 & -2.071068 & 0.000000 \\ \mathrm{H} & 2.989453 & 0.399435 & 0.000000 \\ \mathrm{H} & 0.946647 & 1.777098 & 0.000000 \\ \mathrm{C} & -1.578491 & 2.133274 & 0.000000 \\ \mathrm{H} & -2.358242 & -1.215636 & 0.000000 \\ \mathrm{H} & -2.619917 & 2.443501 & 0.000000 \\ \mathrm{H} & -1.081265 & 2.521725 & 0.892835 \\ \mathrm{H} & -1.081265 & 2.521725 & -0.892835\end{array}$

$o$-methoxyphenol $\mathrm{S}_{1}$
$\begin{array}{llll}\text { C } & 2.465364 & 0.089281 & 0.053523\end{array}$
$\begin{array}{llll}\text { C } & 1.378435 & -0.290631 & -0.750655\end{array}$
$\begin{array}{llll}\text { C } & 0.108819 & -0.236517 & -0.095308\end{array}$
$\begin{array}{llll}\text { C } & 0.018697 & -0.165820 & 1.317550\end{array}$
$\begin{array}{llll}\text { C } & 1.182335 & -0.121809 & 2.136398\end{array}$
$\begin{array}{llll}\text { C } & 2.374714 & 0.157018 & 1.464090\end{array}$
O $\quad-1.067459 \quad 0.042246 \quad-0.719221$ 


$\begin{array}{rrrr}\mathrm{O} & -1.171806 & 0.049556 & 1.891461 \\ \mathrm{H} & 1.103699 & -0.237402 & 3.206374 \\ \mathrm{H} & 3.264197 & 0.421001 & 2.024477 \\ \mathrm{H} & 3.417052 & 0.326766 & -0.411256 \\ \mathrm{H} & 1.496813 & -0.586925 & -1.781981 \\ \mathrm{C} & -1.076119 & 0.109968 & -2.138397 \\ \mathrm{H} & -1.852671 & 0.062428 & 1.198686 \\ \mathrm{H} & -2.099957 & 0.338469 & -2.420567 \\ \mathrm{H} & -0.403970 & 0.892926 & -2.492037 \\ \mathrm{H} & -0.779584 & -0.850565 & -2.565057\end{array}$

$m$-methoxyphenol, isomer $1 \mathrm{~S}_{0}$

$\begin{array}{rrrr}\mathrm{C} & 0.927167 & 1.446899 & 0.000000 \\ \mathrm{C} & -0.261760 & 0.719460 & 0.000000 \\ \mathrm{C} & -0.234294 & -0.672492 & 0.000000 \\ \mathrm{C} & 0.996503 & -1.327782 & 0.000000 \\ \mathrm{C} & 2.185918 & -0.617016 & 0.000000 \\ \mathrm{C} & 2.133979 & 0.771264 & 0.000000 \\ \mathrm{H} & -1.146558 & -1.255148 & 0.000000 \\ \mathrm{O} & 1.077612 & -2.688837 & 0.000000 \\ \mathrm{H} & 3.122786 & -1.155987 & 0.000000 \\ \mathrm{H} & 3.057640 & 1.336170 & 0.000000 \\ \mathrm{H} & 0.875040 & 2.526624 & 0.000000 \\ \mathrm{O} & -1.406452 & 1.449858 & 0.000000 \\ \mathrm{H} & 0.195107 & -3.070817 & 0.000000 \\ \mathrm{C} & -2.634635 & 0.756000 & 0.000000 \\ \mathrm{H} & -3.411519 & 1.515949 & 0.000000 \\ \mathrm{H} & -2.738266 & 0.132928 & -0.893238 \\ \mathrm{H} & -2.738266 & 0.132928 & 0.893238\end{array}$

$m$-methoxyphenol, isomer $1 \mathrm{~S}_{1}$

$\begin{array}{rrrr}\mathrm{C} & 1.173552 & -0.010020 & -0.618239 \\ \mathrm{C} & -0.029376 & -0.052058 & 0.122739 \\ \mathrm{C} & -0.049756 & -0.173889 & 1.534695 \\ \mathrm{C} & 1.224293 & -0.021655 & 2.134973 \\ \mathrm{C} & 2.431349 & 0.018251 & 1.405457 \\ \mathrm{C} & 2.433336 & -0.198716 & 0.006651 \\ \mathrm{H} & -0.894064 & -0.558062 & 2.091232 \\ \mathrm{O} & 1.326481 & 0.143657 & 3.469531 \\ \mathrm{H} & 3.331462 & 0.280606 & 1.949434 \\ \mathrm{H} & 3.347862 & -0.224437 & -0.562192 \\ \mathrm{H} & 1.089751 & 0.211762 & -1.675927 \\ \mathrm{O} & -1.147324 & 0.070968 & -0.616465 \\ \mathrm{H} & 0.447155 & 0.325231 & 3.828046\end{array}$




$\begin{array}{rrrr}\mathrm{C} & -2.400349 & 0.050421 & 0.044524 \\ \mathrm{H} & -3.146065 & 0.250498 & -0.719647 \\ \mathrm{H} & -2.588261 & -0.930310 & 0.487685 \\ \mathrm{H} & -2.441736 & 0.817754 & 0.820242\end{array}$

$m$-methoxyphenol, isomer $2 \mathrm{~S}_{0}$

$\begin{array}{lrrc}\mathrm{C} & 0.344833 & 2.156580 & 0.000000 \\ \mathrm{C} & -0.838125 & 1.446054 & 0.000000 \\ \mathrm{C} & -0.796750 & 0.049057 & 0.000000 \\ \mathrm{C} & 0.420066 & -0.619637 & 0.000000 \\ \mathrm{C} & 1.600057 & 0.121339 & 0.000000 \\ \mathrm{C} & 1.576544 & 1.507202 & 0.000000 \\ \mathrm{O} & -1.999889 & -0.577500 & 0.000000 \\ \mathrm{C} & -2.006955 & -1.989624 & 0.000000 \\ \mathrm{O} & 2.761340 & -0.593406 & 0.000000 \\ \mathrm{H} & 0.495814 & -1.696883 & 0.000000 \\ \mathrm{H} & 2.501298 & 2.072138 & 0.000000 \\ \mathrm{H} & 0.316205 & 3.238821 & 0.000000 \\ \mathrm{H} & -1.800794 & 1.937803 & 0.000000 \\ \mathrm{H} & -3.052463 & -2.286017 & 0.000000 \\ \mathrm{H} & -1.515939 & -2.387881 & -0.892319 \\ \mathrm{H} & -1.515939 & -2.387881 & 0.892319 \\ \mathrm{H} & 3.510688 & 0.009845 & 0.000000\end{array}$

$m$-methoxyphenol, isomer $2 \mathrm{~S}_{1}$

$\begin{array}{lrrr}\mathrm{C} & 1.249912 & -0.380815 & -0.696613 \\ \mathrm{C} & 0.096209 & -0.025850 & 0.072360 \\ \mathrm{C} & 0.118667 & -0.102292 & 1.484091 \\ \mathrm{C} & 1.322983 & -0.285732 & 2.191242 \\ \mathrm{C} & 2.466387 & -0.043787 & 1.418627 \\ \mathrm{C} & 2.415753 & 0.048495 & 0.006972 \\ \mathrm{O} & -1.073466 & 0.051745 & 2.081086 \\ \mathrm{C} & -1.117988 & 0.072212 & 3.498264 \\ \mathrm{O} & 3.625606 & 0.141715 & 2.081037 \\ \mathrm{H} & 1.399666 & -0.469716 & 3.249435 \\ \mathrm{H} & 3.244447 & 0.530056 & -0.510055 \\ \mathrm{H} & 1.220760 & -0.372657 & -1.774164 \\ \mathrm{H} & -0.797228 & 0.383374 & -0.389104 \\ \mathrm{H} & -2.156087 & 0.253583 & 3.761691 \\ \mathrm{H} & -0.797175 & -0.886541 & 3.910772 \\ \mathrm{H} & -0.487032 & 0.871359 & 3.893111 \\ \mathrm{H} & 4.353716 & 0.214850 & 1.453147\end{array}$

$p$-methoxyphenol, cis $\mathrm{S}_{0}$ 


$\begin{array}{crrr}\mathrm{C} & -0.318630 & 0.733390 & 0.000000 \\ \mathrm{C} & -1.548592 & 0.073384 & 0.000000 \\ \mathrm{C} & -1.606193 & -1.304612 & 0.000000 \\ \mathrm{C} & -0.430169 & -2.052215 & 0.000000 \\ \mathrm{C} & 0.791156 & -1.402999 & 0.000000 \\ \mathrm{C} & 0.853625 & -0.009531 & 0.000000 \\ \mathrm{O} & -0.552187 & -3.414556 & 0.000000 \\ \mathrm{O} & -0.373078 & 2.095851 & 0.000000 \\ \mathrm{C} & 0.852165 & 2.792356 & 0.000000 \\ \mathrm{H} & 1.822289 & 0.469354 & 0.000000 \\ \mathrm{H} & -2.451992 & 0.669159 & 0.000000 \\ \mathrm{H} & -2.555171 & -1.824352 & 0.000000 \\ \mathrm{H} & 1.711514 & -1.977044 & 0.000000 \\ \mathrm{H} & 0.601460 & 3.849868 & 0.000000 \\ \mathrm{H} & 1.441553 & 2.559871 & 0.892219 \\ \mathrm{H} & 1.441553 & 2.559871 & -0.892219 \\ \mathrm{H} & 0.320698 & -3.817775 & 0.000000\end{array}$

p-methoxyphenol, cis $\mathrm{S}_{1}$

$\begin{array}{lrrc}\mathrm{C} & 1.322692 & -0.017717 & -0.619795 \\ \mathrm{C} & 0.048241 & -0.046871 & 0.002123 \\ \mathrm{C} & -0.018254 & 0.023127 & 1.409178 \\ \mathrm{C} & 1.189396 & 0.000128 & 2.131217 \\ \mathrm{C} & 2.468005 & -0.051697 & 1.516532 \\ \mathrm{C} & 2.539780 & 0.013432 & 0.108859 \\ \mathrm{O} & 1.090059 & 0.025402 & 3.469194 \\ \mathrm{O} & 1.282973 & -0.019785 & -1.955281 \\ \mathrm{C} & 2.491505 & 0.015529 & -2.697456 \\ \mathrm{H} & 3.494564 & 0.041316 & -0.391212 \\ \mathrm{H} & -0.830178 & -0.077386 & -0.623916 \\ \mathrm{H} & -0.950816 & 0.090579 & 1.948421 \\ \mathrm{H} & 3.360856 & -0.119485 & 2.124658 \\ \mathrm{H} & 2.197702 & 0.009713 & -3.742855 \\ \mathrm{H} & 3.053040 & 0.924484 & -2.474515 \\ \mathrm{H} & 3.102377 & -0.862120 & -2.478676 \\ \mathrm{H} & 1.971587 & 0.051332 & 3.860523\end{array}$

p-methoxyphenol, trans $\mathrm{S}_{0}$
C $\quad-0.655664$
1.304635
0.000000
$\begin{array}{llll}\text { C } & -1.885173 & 0.671141 & 0.000000\end{array}$
$\begin{array}{llll}\text { C } & -1.955945 & -0.719105 & 0.000000\end{array}$
$\begin{array}{llll}\text { C } & -0.787393 & -1.461639 & 0.000000\end{array}$
$\begin{array}{llll}\text { C } & 0.450647 & -0.828654 & 0.000000\end{array}$ 


$\begin{array}{rrrc}\mathrm{C} & 0.521378 & 0.560846 & 0.000000 \\ \mathrm{O} & -3.143124 & -1.399013 & 0.000000 \\ \mathrm{H} & 1.345983 & -1.434181 & 0.000000 \\ \mathrm{O} & 1.681441 & 1.277674 & 0.000000 \\ \mathrm{H} & -0.584844 & 2.384308 & 0.000000 \\ \mathrm{H} & -2.795178 & 1.261736 & 0.000000 \\ \mathrm{H} & -3.873788 & -0.774092 & 0.000000 \\ \mathrm{H} & -0.854583 & -2.541486 & 0.000000 \\ \mathrm{C} & 2.890923 & 0.553248 & 0.000000 \\ \mathrm{H} & 3.687889 & 1.292369 & 0.000000 \\ \mathrm{H} & 2.978711 & -0.073904 & 0.892347 \\ \mathrm{H} & 2.978711 & -0.073904 & -0.892347\end{array}$

p-methoxyphenol, trans $\mathrm{S}_{1}$

$\begin{array}{rrrr}\mathrm{C} & 0.122765 & -0.028834 & -1.394510 \\ \mathrm{C} & -1.276746 & -0.025958 & -1.220436 \\ \mathrm{C} & -1.776623 & 0.019368 & 0.102227 \\ \mathrm{C} & -0.951804 & 0.065594 & 1.247129 \\ \mathrm{C} & 0.449018 & 0.017729 & 1.078816 \\ \mathrm{C} & 0.955258 & -0.005615 & -0.244749 \\ \mathrm{O} & -3.099014 & 0.032425 & 0.329981 \\ \mathrm{H} & 1.104444 & 0.039616 & 1.934278 \\ \mathrm{O} & 2.262540 & -0.019144 & -0.520525 \\ \mathrm{H} & 0.590916 & -0.057694 & -2.366486 \\ \mathrm{H} & -1.956196 & -0.111564 & -2.058246 \\ \mathrm{H} & -3.571840 & 0.052349 & -0.510790 \\ \mathrm{H} & -1.415347 & 0.137320 & 2.219479 \\ \mathrm{C} & 3.205957 & -0.017719 & 0.538959 \\ \mathrm{H} & 4.182550 & -0.058390 & 0.065612 \\ \mathrm{H} & 3.118115 & 0.895333 & 1.130656 \\ \mathrm{H} & 3.066797 & -0.890507 & 1.179303\end{array}$

1-naphthol, cis $\mathrm{S}_{0}$
$\begin{array}{llll}\text { C } & 0.828598 & 1.513983 & 0.007829\end{array}$
$\begin{array}{llll}\text { C } & 0.525184 & 0.121669 & -0.007793\end{array}$
$\begin{array}{llll}\text { C } & -0.841812 & -0.267183 & -0.000307\end{array}$
$\begin{array}{llll}\text { C } & -1.854754 & 0.721569 & -0.013765\end{array}$
$\begin{array}{llll}\text { C } & -1.519421 & 2.046271 & -0.030117\end{array}$
$\begin{array}{llll}\text { C } & -0.170162 & 2.449090 & -0.015091\end{array}$
$\begin{array}{llll}\text { C } & 1.522697 & -0.884767 & -0.038309\end{array}$
$\begin{array}{llll}\text { C } & 1.185523 & -2.209789 & -0.030908\end{array}$
$\begin{array}{llll}\text { C } & -0.171055 & -2.598041 & -0.000404\end{array}$
$\begin{array}{llll}\text { C } & -1.154532 & -1.649692 & 0.009386\end{array}$
$\begin{array}{llll}\text { O } & 2.115333 & 1.962751 & 0.015853\end{array}$ 


$\begin{array}{rrrr}\mathrm{H} & -2.198589 & -1.940718 & 0.021113 \\ \mathrm{H} & -0.427332 & -3.649889 & 0.006312 \\ \mathrm{H} & 1.959876 & -2.965569 & -0.059827 \\ \mathrm{H} & 2.573131 & -0.621843 & -0.093208 \\ \mathrm{H} & -2.891792 & 0.409319 & -0.013016 \\ \mathrm{H} & -2.292617 & 2.803945 & -0.045348 \\ \mathrm{H} & 0.100867 & 3.496488 & -0.008345 \\ \mathrm{H} & 2.710868 & 1.262416 & 0.295942\end{array}$

1-naphthol, cis $\mathrm{S}_{1}$

$\begin{array}{rrrr}\mathrm{C} & 0.867958 & 1.463911 & 0.019731 \\ \mathrm{C} & 0.537260 & 0.087854 & -0.062570 \\ \mathrm{C} & -0.849255 & -0.247147 & 0.016903 \\ \mathrm{C} & -1.791845 & 0.799903 & -0.016643 \\ \mathrm{C} & -1.423553 & 2.170717 & -0.034494 \\ \mathrm{C} & -0.102906 & 2.510400 & -0.038978 \\ \mathrm{C} & 1.503655 & -0.928575 & -0.141151 \\ \mathrm{C} & 1.095444 & -2.294056 & -0.060281 \\ \mathrm{C} & -0.231199 & -2.616525 & 0.044961 \\ \mathrm{C} & -1.224796 & -1.602420 & 0.064987 \\ \mathrm{O} & 2.134000 & 1.856753 & 0.164043 \\ \mathrm{H} & -2.272499 & -1.860308 & 0.141472 \\ \mathrm{H} & -0.533335 & -3.654085 & 0.106220 \\ \mathrm{H} & 1.846451 & -3.072450 & -0.091016 \\ \mathrm{H} & 2.544214 & -0.707947 & -0.347365 \\ \mathrm{H} & -2.844215 & 0.540850 & -0.025380 \\ \mathrm{H} & -2.190601 & 2.932005 & -0.070398 \\ \mathrm{H} & 0.246835 & 3.533361 & -0.033782 \\ \mathrm{H} & 2.688387 & 1.087749 & 0.363724\end{array}$

1-naphthol, trans $\mathrm{S}_{0}$

$\begin{array}{lrrc}\mathrm{C} & -0.714991 & -0.539554 & 0.000000 \\ \mathrm{C} & -0.672681 & -1.955648 & 0.000000 \\ \mathrm{C} & 0.520971 & -2.622728 & 0.000000 \\ \mathrm{C} & 1.738111 & -1.907395 & 0.000000 \\ \mathrm{C} & 1.735386 & -0.539303 & 0.000000 \\ \mathrm{C} & 0.511860 & 0.170536 & 0.000000 \\ \mathrm{H} & -1.608673 & -2.501989 & 0.000000 \\ \mathrm{H} & 0.537330 & -3.705362 & 0.000000 \\ \mathrm{H} & 2.676565 & -2.446990 & 0.000000 \\ \mathrm{H} & 2.660735 & 0.020746 & 0.000000 \\ \mathrm{C} & -1.939993 & 0.175416 & 0.000000 \\ \mathrm{C} & -1.936661 & 1.540427 & 0.000000 \\ \mathrm{C} & -0.722689 & 2.261327 & 0.000000\end{array}$




$\begin{array}{cccc}\mathrm{C} & 0.471605 & 1.592805 & 0.000000 \\ \mathrm{H} & -2.870996 & -0.377760 & 0.000000 \\ \mathrm{H} & -2.870654 & 2.087825 & 0.000000 \\ \mathrm{H} & -0.737140 & 3.345683 & 0.000000 \\ \mathrm{O} & 1.678041 & 2.224641 & 0.000000 \\ \mathrm{H} & 1.543885 & 3.177313 & 0.000000\end{array}$

1-naphthol, trans $\mathrm{S}_{1}$

$\begin{array}{lrrr}\mathrm{C} & -0.943309 & -0.393425 & 0.000000 \\ \mathrm{C} & -1.306500 & -1.748989 & 0.000000 \\ \mathrm{C} & -0.311801 & -2.767195 & 0.000000 \\ \mathrm{C} & 1.014487 & -2.426976 & 0.000000 \\ \mathrm{C} & 1.417365 & -1.059560 & 0.000000 \\ \mathrm{C} & 0.448994 & -0.047081 & 0.000000 \\ \mathrm{H} & -2.356273 & -2.012534 & 0.000000 \\ \mathrm{H} & -0.611802 & -3.806529 & 0.000000 \\ \mathrm{H} & 1.775998 & -3.196254 & 0.000000 \\ \mathrm{H} & 2.465568 & -0.798268 & 0.000000 \\ \mathrm{C} & -1.892297 & 0.645738 & 0.000000 \\ \mathrm{C} & -1.522333 & 2.018813 & 0.000000 \\ \mathrm{C} & -0.203600 & 2.366648 & 0.000000 \\ \mathrm{C} & 0.780013 & 1.326086 & 0.000000 \\ \mathrm{H} & -2.944344 & 0.386770 & 0.000000 \\ \mathrm{H} & -2.292427 & 2.778252 & 0.000000 \\ \mathrm{H} & 0.115400 & 3.402443 & 0.000000 \\ \mathrm{O} & 2.086473 & 1.637251 & 0.000000 \\ \mathrm{H} & 2.204710 & 2.593882 & 0.000000\end{array}$

2-naphthol, cis $\mathrm{S}_{0}$

$\begin{array}{lrrr}\mathrm{C} & 0.596714 & 2.007293 & 0.000000 \\ \mathrm{C} & 0.618399 & 0.592625 & 0.000000 \\ \mathrm{C} & -0.608687 & -0.119413 & 0.000000 \\ \mathrm{C} & -1.820724 & 0.614040 & 0.000000 \\ \mathrm{C} & -1.810608 & 1.981736 & 0.000000 \\ \mathrm{C} & -0.588577 & 2.689912 & 0.000000 \\ \mathrm{C} & 1.830941 & -0.143960 & 0.000000 \\ \mathrm{C} & 1.829565 & -1.506766 & 0.000000 \\ \mathrm{C} & 0.601522 & -2.210104 & 0.000000 \\ \mathrm{C} & -0.588609 & -1.535848 & 0.000000 \\ \mathrm{H} & -1.529099 & -2.077990 & 0.000000 \\ \mathrm{H} & 1.540161 & 2.541378 & 0.000000 \\ \mathrm{H} & -0.595820 & 3.772239 & 0.000000 \\ \mathrm{H} & -2.744832 & 2.529187 & 0.000000 \\ \mathrm{H} & -2.759488 & 0.072062 & 0.000000\end{array}$




$$
\begin{array}{cccc}
\mathrm{H} & 2.769982 & 0.397347 & 0.000000 \\
\mathrm{H} & 2.747535 & -2.079542 & 0.000000 \\
\mathrm{O} & 0.696342 & -3.568721 & 0.000000 \\
\mathrm{H} & -0.184726 & -3.955465 & 0.000000
\end{array}
$$

2-naphthol, cis $\mathrm{S}_{1}$

$\begin{array}{lrrl}\mathrm{C} & 0.601906 & 1.985924 & 0.000000 \\ \mathrm{C} & 0.645183 & 0.584053 & 0.000000 \\ \mathrm{C} & -0.629423 & -0.117208 & 0.000000 \\ \mathrm{C} & -1.833042 & 0.607290 & 0.000000 \\ \mathrm{C} & -1.841918 & 2.016472 & 0.000000 \\ \mathrm{C} & -0.625883 & 2.682736 & 0.000000 \\ \mathrm{C} & 1.848890 & -0.150934 & 0.000000 \\ \mathrm{C} & 1.881205 & -1.553156 & 0.000000 \\ \mathrm{C} & 0.656720 & -2.213284 & 0.000000 \\ \mathrm{C} & -0.584741 & -1.517290 & 0.000000 \\ \mathrm{H} & -1.507660 & -2.089621 & 0.000000 \\ \mathrm{H} & 1.530366 & 2.543797 & 0.000000 \\ \mathrm{H} & -0.605032 & 3.765984 & 0.000000 \\ \mathrm{H} & -2.774137 & 2.561987 & 0.000000 \\ \mathrm{H} & -2.767522 & 0.058261 & 0.000000 \\ \mathrm{H} & 2.780877 & 0.401686 & 0.000000 \\ \mathrm{H} & 2.799327 & -2.119432 & 0.000000 \\ \mathrm{O} & 0.663821 & -3.554173 & 0.000000 \\ \mathrm{H} & -0.238927 & -3.893082 & 0.000000\end{array}$

2-naphthol, trans $\mathrm{S}_{0}$

$\begin{array}{lrrr}\mathrm{C} & 0.509622 & 2.009159 & 0.000000 \\ \mathrm{C} & 0.537171 & 0.593464 & 0.000000 \\ \mathrm{C} & -0.687950 & -0.125981 & 0.000000 \\ \mathrm{C} & -1.903402 & 0.604612 & 0.000000 \\ \mathrm{C} & -1.898034 & 1.971153 & 0.000000 \\ \mathrm{C} & -0.677999 & 2.685314 & 0.000000 \\ \mathrm{C} & 1.749068 & -0.136290 & 0.000000 \\ \mathrm{C} & 1.748906 & -1.502230 & 0.000000 \\ \mathrm{C} & 0.525078 & -2.210618 & 0.000000 \\ \mathrm{C} & -0.666198 & -1.538446 & 0.000000 \\ \mathrm{H} & -1.592011 & -2.099939 & 0.000000 \\ \mathrm{H} & 1.450361 & 2.548045 & 0.000000 \\ \mathrm{H} & -0.689938 & 3.767599 & 0.000000 \\ \mathrm{H} & -2.834310 & 2.515060 & 0.000000 \\ \mathrm{H} & -2.839358 & 0.058277 & 0.000000 \\ \mathrm{H} & 2.687825 & 0.405392 & 0.000000 \\ \mathrm{H} & 2.684289 & -2.051995 & 0.000000\end{array}$




$$
\begin{array}{llll}
\mathrm{O} & 0.499472 & -3.574109 & 0.000000 \\
\mathrm{H} & 1.397419 & -3.918466 & 0.000000
\end{array}
$$

2-naphthol, trans $\mathrm{S}_{1}$

$\begin{array}{lrrl}\mathrm{C} & 0.513447 & 1.990079 & 0.000000 \\ \mathrm{C} & 0.559180 & 0.588591 & 0.000000 \\ \mathrm{C} & -0.710387 & -0.117318 & 0.000000 \\ \mathrm{C} & -1.914826 & 0.603320 & 0.000000 \\ \mathrm{C} & -1.927905 & 2.014395 & 0.000000 \\ \mathrm{C} & -0.717083 & 2.684511 & 0.000000 \\ \mathrm{C} & 1.765527 & -0.141249 & 0.000000 \\ \mathrm{C} & 1.800079 & -1.545647 & 0.000000 \\ \mathrm{C} & 0.573644 & -2.207843 & 0.000000 \\ \mathrm{C} & -0.666296 & -1.518167 & 0.000000 \\ \mathrm{H} & -1.572971 & -2.109781 & 0.000000 \\ \mathrm{H} & 1.440570 & 2.550349 & 0.000000 \\ \mathrm{H} & -0.699327 & 3.767781 & 0.000000 \\ \mathrm{H} & -2.862655 & 2.555817 & 0.000000 \\ \mathrm{H} & -2.847239 & 0.051172 & 0.000000 \\ \mathrm{H} & 2.696342 & 0.413392 & 0.000000 \\ \mathrm{H} & 2.731875 & -2.094621 & 0.000000 \\ \mathrm{O} & 0.478570 & -3.546100 & 0.000000 \\ \mathrm{H} & 1.359456 & -3.938672 & 0.000000\end{array}$

5-methoxysalicylic acid $\mathrm{S}_{0}$

$\begin{array}{lrrc}\mathrm{C} & 2.275687 & -0.167461 & 0.000000 \\ \mathrm{C} & 1.565620 & -1.341591 & 0.000000 \\ \mathrm{C} & 0.162329 & -1.335984 & 0.000000 \\ \mathrm{C} & -0.507991 & -0.132687 & 0.000000 \\ \mathrm{C} & 0.213900 & 1.075749 & 0.000000 \\ \mathrm{C} & 1.612973 & 1.066807 & 0.000000 \\ \mathrm{O} & -0.432202 & -2.559967 & 0.000000 \\ \mathrm{C} & -1.842491 & -2.590019 & 0.000000 \\ \mathrm{C} & -0.487518 & 2.363211 & 0.000000 \\ \mathrm{O} & -1.824786 & 2.267287 & 0.000000 \\ \mathrm{O} & 2.371346 & 2.174202 & 0.000000 \\ \mathrm{O} & 0.060888 & 3.448705 & 0.000000 \\ \mathrm{H} & -2.124241 & -3.639473 & 0.000000 \\ \mathrm{H} & -2.248906 & -2.104406 & -0.892356 \\ \mathrm{H} & -2.248906 & -2.104406 & 0.892356 \\ \mathrm{H} & -1.586397 & -0.080822 & 0.000000 \\ \mathrm{H} & 3.357332 & -0.164390 & 0.000000 \\ \mathrm{H} & 2.072193 & -2.298307 & 0.000000 \\ \mathrm{H} & 1.784970 & 2.953568 & 0.000000\end{array}$




\section{$\begin{array}{llll}\mathrm{H} & -2.173780 & 3.169984 & 0.000000\end{array}$}

5-methoxysalicylic acid $\mathrm{S}_{1}$

$\begin{array}{crcc}\mathrm{C} & 2.176735 & -0.136544 & 0.000000 \\ \mathrm{C} & 1.729844 & -1.455393 & 0.000000 \\ \mathrm{C} & 0.338247 & -1.684782 & 0.000000 \\ \mathrm{C} & -0.604226 & -0.623280 & 0.000000 \\ \mathrm{C} & -0.167544 & 0.698222 & 0.000000 \\ \mathrm{C} & 1.270112 & 0.929518 & 0.000000 \\ \mathrm{O} & -0.027210 & -2.967146 & 0.000000 \\ \mathrm{C} & -1.405420 & -3.313625 & 0.000000 \\ \mathrm{C} & -1.035349 & 1.831961 & 0.000000 \\ \mathrm{O} & -2.361017 & 1.556523 & 0.000000 \\ \mathrm{O} & 1.706091 & 2.166065 & 0.000000 \\ \mathrm{O} & -0.640658 & 3.022083 & 0.000000 \\ \mathrm{H} & -1.434681 & -4.398852 & 0.000000 \\ \mathrm{H} & -1.899884 & -2.929888 & -0.894014 \\ \mathrm{H} & -1.899884 & -2.929888 & 0.894014 \\ \mathrm{H} & -1.666116 & -0.820153 & 0.000000 \\ \mathrm{H} & 3.231084 & 0.104866 & 0.000000 \\ \mathrm{H} & 2.401774 & -2.299795 & 0.000000 \\ \mathrm{H} & 0.875828 & 2.771409 & 0.000000 \\ \mathrm{H} & -2.815677 & 2.409475 & 0.000000\end{array}$

$m$-dimethoxybenzene, trans $\mathrm{S}_{0}$

$\begin{array}{rrrr}\mathrm{C} & 0.000000 & -0.705904 & -0.178327 \\ \mathrm{C} & 0.000000 & 0.705904 & -0.178327 \\ \mathrm{C} & 0.000000 & 1.388475 & 1.025937 \\ \mathrm{C} & 0.000000 & 0.689114 & 2.234937 \\ \mathrm{C} & 0.000000 & -0.689114 & 2.234937 \\ \mathrm{C} & 0.000000 & -1.388475 & 1.025937 \\ \mathrm{O} & 0.000000 & 1.288726 & -1.401205 \\ \mathrm{H} & 0.000000 & 2.469030 & 1.037566 \\ \mathrm{H} & 0.000000 & 1.239215 & 3.166559 \\ \mathrm{H} & 0.000000 & -1.239215 & 3.166559 \\ \mathrm{H} & 0.000000 & -2.469030 & 1.037566 \\ \mathrm{O} & 0.000000 & -1.288726 & -1.401205 \\ \mathrm{C} & 0.000000 & -2.697936 & -1.447573 \\ \mathrm{H} & 0.000000 & -2.964203 & -2.501188 \\ \mathrm{H} & 0.892269 & -3.111800 & -0.968343 \\ \mathrm{H} & -0.892269 & -3.111800 & -0.968343 \\ \mathrm{C} & 0.000000 & 2.697936 & -1.447573 \\ \mathrm{H} & 0.000000 & 2.964203 & -2.501188 \\ \mathrm{H} & -0.892269 & 3.111800 & -0.968343\end{array}$




\section{H $\quad 0.892269 \quad 3.111800 \quad-0.968343$}

$m$-dimethoxybenzene, trans $\mathrm{S}_{1}$

$\begin{array}{rrrr}\mathrm{C} & -0.157917 & -1.063951 & -1.443138 \\ \mathrm{C} & -0.264073 & 0.163396 & -0.708687 \\ \mathrm{C} & -0.264073 & 0.163396 & 0.708687 \\ \mathrm{C} & -0.157917 & -1.063951 & 1.443138 \\ \mathrm{C} & 0.288905 & -2.167027 & 0.705994 \\ \mathrm{C} & 0.288905 & -2.167027 & -0.705994 \\ \mathrm{O} & -0.173493 & 1.374560 & -1.282961 \\ \mathrm{C} & -0.009584 & 1.445422 & -2.690574 \\ \mathrm{O} & -0.173493 & 1.374560 & 1.282961 \\ \mathrm{C} & -0.009584 & 1.445422 & 2.690574 \\ \mathrm{H} & -0.392023 & -1.132513 & 2.494468 \\ \mathrm{H} & 0.612819 & -3.064758 & 1.221911 \\ \mathrm{H} & 0.612819 & -3.064758 & -1.221911 \\ \mathrm{H} & -0.392023 & -1.132513 & -2.494468 \\ \mathrm{H} & 0.071358 & 2.503370 & -2.923629 \\ \mathrm{H} & 0.893776 & 0.921218 & -3.006487 \\ \mathrm{H} & -0.876678 & 1.022723 & -3.202775 \\ \mathrm{H} & 0.071358 & 2.503370 & 2.923629 \\ \mathrm{H} & -0.876678 & 1.022723 & 3.202775 \\ \mathrm{H} & 0.893776 & 0.921218 & 3.006487\end{array}$

3P-propionic acid, gauche $\mathrm{S}_{0}$

$\begin{array}{lrrr}\mathrm{C} & -0.997840 & 0.880304 & -1.161539 \\ \mathrm{C} & -0.372401 & 0.679093 & 0.068110 \\ \mathrm{C} & -1.036328 & -0.062196 & 1.042806 \\ \mathrm{C} & -2.297300 & -0.589291 & 0.792376 \\ \mathrm{C} & -2.911169 & -0.382692 & -0.435560 \\ \mathrm{C} & -2.256921 & 0.354597 & -1.414751 \\ \mathrm{C} & 1.010750 & 1.230581 & 0.312825 \\ \mathrm{C} & 2.100357 & 0.310350 & -0.238048 \\ \mathrm{C} & 2.253317 & -0.944310 & 0.582379 \\ \mathrm{O} & 1.918934 & -1.076680 & 1.727737 \\ \mathrm{O} & 2.857624 & -1.928485 & -0.108638 \\ \mathrm{H} & 1.902360 & 0.029054 & -1.273352 \\ \mathrm{H} & 3.075251 & 0.807218 & -0.223610 \\ \mathrm{H} & 1.173703 & 1.370834 & 1.382050 \\ \mathrm{H} & 1.104091 & 2.205318 & -0.169354 \\ \mathrm{H} & -0.552916 & -0.231732 & 1.997377 \\ \mathrm{H} & -2.801675 & -1.161241 & 1.561313 \\ \mathrm{H} & -3.895010 & -0.791112 & -0.628948 \\ \mathrm{H} & -2.729608 & 0.524252 & -2.374250\end{array}$




$\begin{array}{rrrr}H & -0.493906 & 1.461926 & -1.926763 \\ H & 2.948698 & -2.685809 & 0.487849\end{array}$

3P-propionic acid, gauche $\mathrm{S}_{1}$

$\begin{array}{lrrr}\mathrm{C} & -0.998415 & 0.967587 & -1.181219 \\ \mathrm{C} & -0.356855 & 0.712466 & 0.064880 \\ \mathrm{C} & -1.026656 & -0.067735 & 1.050792 \\ \mathrm{C} & -2.309219 & -0.611581 & 0.785300 \\ \mathrm{C} & -2.931833 & -0.362278 & -0.462276 \\ \mathrm{C} & -2.274534 & 0.412371 & -1.451342 \\ \mathrm{C} & 1.028058 & 1.219093 & 0.299448 \\ \mathrm{C} & 2.097168 & 0.257788 & -0.242023 \\ \mathrm{C} & 2.255884 & -0.962443 & 0.627865 \\ \mathrm{O} & 1.950101 & -1.042845 & 1.786032 \\ \mathrm{O} & 2.836449 & -1.979157 & -0.035241 \\ \mathrm{H} & 1.869596 & -0.058912 & -1.260462 \\ \mathrm{H} & 3.076009 & 0.746989 & -0.268837 \\ \mathrm{H} & 1.202331 & 1.365349 & 1.367175 \\ \mathrm{H} & 1.150772 & 2.181490 & -0.204332 \\ \mathrm{H} & -0.531912 & -0.255225 & 1.994367 \\ \mathrm{H} & -2.807911 & -1.213766 & 1.531287 \\ \mathrm{H} & -3.914002 & -0.767304 & -0.665404 \\ \mathrm{H} & -2.750704 & 0.584236 & -2.406622 \\ \mathrm{H} & -0.503067 & 1.584733 & -1.920069 \\ \mathrm{H} & 2.938749 & -2.710865 & 0.590682\end{array}$

3P-propionic acid, anti $\mathrm{S}_{0}$

$\begin{array}{lrrr}\mathrm{C} & 1.439005 & -0.447292 & 0.000000 \\ \mathrm{C} & 0.544662 & 0.789787 & 0.000000 \\ \mathrm{C} & 2.901904 & -0.095854 & 0.000000 \\ \mathrm{H} & 1.242752 & -1.076223 & 0.872427 \\ \mathrm{H} & 1.242752 & -1.076223 & -0.872427 \\ \mathrm{C} & -0.911487 & 0.402304 & 0.000000 \\ \mathrm{H} & 0.776342 & 1.399114 & 0.874999 \\ \mathrm{H} & 0.776342 & 1.399114 & -0.874999 \\ \mathrm{C} & -1.592227 & 0.196944 & 1.197729 \\ \mathrm{C} & -2.921575 & -0.204102 & 1.200509 \\ \mathrm{C} & -3.589915 & -0.406432 & 0.000000 \\ \mathrm{C} & -2.921575 & -0.204102 & -1.200509 \\ \mathrm{C} & -1.592227 & 0.196944 & -1.197729 \\ \mathrm{H} & -1.075272 & 0.360699 & 2.137257 \\ \mathrm{H} & -3.437886 & -0.354377 & 2.140427 \\ \mathrm{H} & -4.627311 & -0.715572 & 0.000000 \\ \mathrm{H} & -3.437886 & -0.354377 & -2.140427\end{array}$




$$
\begin{array}{rrrr}
\mathrm{H} & -1.075272 & 0.360699 & -2.137257 \\
\mathrm{O} & 3.681116 & -1.195992 & 0.000000 \\
\mathrm{O} & 3.358570 & 1.013512 & 0.000000 \\
\mathrm{H} & 4.602026 & -0.897249 & 0.000000
\end{array}
$$

3P-propionic acid, anti $\mathrm{S}_{1}$

$\begin{array}{lrrr}\mathrm{C} & 1.436562 & -0.431580 & 0.000000 \\ \mathrm{C} & 0.560925 & 0.828388 & 0.000000 \\ \mathrm{C} & 2.903748 & -0.098190 & 0.000000 \\ \mathrm{H} & 1.227411 & -1.053490 & 0.873892 \\ \mathrm{H} & 1.227411 & -1.053490 & -0.873892 \\ \mathrm{C} & -0.886379 & 0.470885 & 0.000000 \\ \mathrm{H} & 0.806767 & 1.427179 & 0.879838 \\ \mathrm{H} & 0.806767 & 1.427179 & -0.879838 \\ \mathrm{C} & -1.568776 & 0.254141 & 1.230763 \\ \mathrm{C} & -2.901112 & -0.229439 & 1.231819 \\ \mathrm{C} & -3.564597 & -0.454085 & 0.000000 \\ \mathrm{C} & -2.901112 & -0.229439 & -1.231819 \\ \mathrm{C} & -1.568776 & 0.254141 & -1.230763 \\ \mathrm{H} & -1.057168 & 0.457034 & 2.162935 \\ \mathrm{H} & -3.412696 & -0.419684 & 2.164550 \\ \mathrm{H} & -4.589893 & -0.799153 & 0.000000 \\ \mathrm{H} & -3.412696 & -0.419684 & -2.164550 \\ \mathrm{H} & -1.057168 & 0.457034 & -2.162935 \\ \mathrm{O} & 3.667277 & -1.208829 & 0.000000 \\ \mathrm{O} & 3.373606 & 1.005634 & 0.000000 \\ \mathrm{H} & 4.592525 & -0.923395 & 0.000000\end{array}$

fluorine $\mathrm{S}_{0}$

$\begin{array}{lrrr}\mathrm{C} & 0.000000 & 0.000000 & -1.791376 \\ \mathrm{C} & 0.000000 & 1.179027 & -0.847741 \\ \mathrm{C} & 0.000000 & 0.734265 & 0.481192 \\ \mathrm{C} & 0.000000 & -0.734265 & 0.481192 \\ \mathrm{C} & 0.000000 & 1.643662 & 1.530235 \\ \mathrm{C} & 0.000000 & 3.002242 & 1.237652 \\ \mathrm{C} & 0.000000 & 3.444036 & -0.082504 \\ \mathrm{C} & 0.000000 & 2.532527 & -1.134573 \\ \mathrm{H} & 0.000000 & 2.881817 & -2.160559 \\ \mathrm{H} & 0.000000 & 4.506301 & -0.291752 \\ \mathrm{H} & 0.000000 & 3.725001 & 2.043818 \\ \mathrm{H} & 0.000000 & 1.304100 & 2.559144 \\ \mathrm{C} & 0.000000 & -1.643662 & 1.530235 \\ \mathrm{C} & 0.000000 & -3.002242 & 1.237652 \\ \mathrm{C} & 0.000000 & -3.444036 & -0.082504\end{array}$




$\begin{array}{rrrr}\mathrm{C} & 0.000000 & -2.532527 & -1.134573 \\ \mathrm{C} & 0.000000 & -1.179027 & -0.847741 \\ \mathrm{H} & 0.000000 & -1.304100 & 2.559144 \\ \mathrm{H} & 0.000000 & -3.725001 & 2.043818 \\ \mathrm{H} & 0.000000 & -4.506301 & -0.291752 \\ \mathrm{H} & 0.000000 & -2.881817 & -2.160559 \\ \mathrm{H} & -0.880767 & 0.000000 & -2.439214 \\ \mathrm{H} & 0.880767 & 0.000000 & -2.439214\end{array}$

fluorine $\mathrm{S}_{1}$

$\begin{array}{rrrr}\mathrm{C} & 0.000000 & 0.000000 & -1.868667 \\ \mathrm{C} & 0.000000 & 1.178727 & -0.918744 \\ \mathrm{C} & 0.000000 & 0.695379 & 0.449810 \\ \mathrm{C} & 0.000000 & -0.695379 & 0.449810 \\ \mathrm{C} & 0.000000 & 1.635475 & 1.536005 \\ \mathrm{C} & 0.000000 & 2.973334 & 1.229564 \\ \mathrm{C} & 0.000000 & 3.429703 & -0.106011 \\ \mathrm{C} & 0.000000 & 2.519286 & -1.185822 \\ \mathrm{H} & 0.000000 & 2.886008 & -2.205166 \\ \mathrm{H} & 0.000000 & 4.493837 & -0.302822 \\ \mathrm{H} & 0.000000 & 3.706012 & 2.027474 \\ \mathrm{H} & 0.000000 & 1.289421 & 2.561205 \\ \mathrm{C} & 0.000000 & -1.635475 & 1.536005 \\ \mathrm{C} & 0.000000 & -2.973334 & 1.229564 \\ \mathrm{C} & 0.000000 & -3.429703 & -0.106011 \\ \mathrm{C} & 0.000000 & -2.519286 & -1.185822 \\ \mathrm{C} & 0.000000 & -1.178727 & -0.918744 \\ \mathrm{H} & 0.000000 & -1.289421 & 2.561205 \\ \mathrm{H} & 0.000000 & -3.706012 & 2.027474 \\ \mathrm{H} & 0.000000 & -4.493837 & -0.302822 \\ \mathrm{H} & 0.000000 & -2.886008 & -2.205166 \\ \mathrm{H} & -0.880085 & 0.000000 & -2.518997 \\ \mathrm{H} & 0.880085 & 0.000000 & -2.518997\end{array}$

phenanthrene $\mathrm{S}_{0}$

$\begin{array}{lrrc}\mathrm{C} & 0.727724 & 0.000000 & -0.349062 \\ \mathrm{C} & -0.727724 & 0.000000 & -0.349062 \\ \mathrm{C} & -1.414694 & 0.000000 & 0.885521 \\ \mathrm{C} & -0.674288 & 0.000000 & 2.114809 \\ \mathrm{C} & 0.674288 & 0.000000 & 2.114809 \\ \mathrm{C} & 1.414694 & 0.000000 & 0.885521 \\ \mathrm{C} & -1.491776 & 0.000000 & -1.532936 \\ \mathrm{C} & -2.823028 & 0.000000 & 0.901048 \\ \mathrm{H} & -1.228489 & 0.000000 & 3.046186\end{array}$




$\begin{array}{lrrr}\mathrm{H} & 1.228489 & 0.000000 & 3.046186 \\ \mathrm{C} & 1.491776 & 0.000000 & -1.532936 \\ \mathrm{C} & 2.866019 & 0.000000 & -1.495818 \\ \mathrm{C} & 3.542326 & 0.000000 & -0.267772 \\ \mathrm{C} & 2.823028 & 0.000000 & 0.901048 \\ \mathrm{H} & 0.999092 & 0.000000 & -2.495161 \\ \mathrm{H} & 3.428517 & 0.000000 & -2.420841 \\ \mathrm{H} & 4.624491 & 0.000000 & -0.244339 \\ \mathrm{H} & 3.331325 & 0.000000 & 1.858456 \\ \mathrm{C} & -3.542326 & 0.000000 & -0.267772 \\ \mathrm{C} & -2.866019 & 0.000000 & -1.495818 \\ \mathrm{H} & -0.999092 & 0.000000 & -2.495161 \\ \mathrm{H} & -3.428517 & 0.000000 & -2.420841 \\ \mathrm{H} & -4.624491 & 0.000000 & -0.244339 \\ \mathrm{H} & -3.331325 & 0.000000 & 1.858456\end{array}$

phenanthrene $S_{1}$

$\begin{array}{rrrr}\mathrm{C} & 0.710344 & 0.000000 & -0.388347 \\ \mathrm{C} & -0.710344 & 0.000000 & -0.388347 \\ \mathrm{C} & -1.431911 & 0.000000 & 0.883092 \\ \mathrm{C} & -0.694399 & 0.000000 & 2.079910 \\ \mathrm{C} & 0.694399 & 0.000000 & 2.079910 \\ \mathrm{C} & 1.431911 & 0.000000 & 0.883092 \\ \mathrm{C} & -1.490768 & 0.000000 & -1.570032 \\ \mathrm{C} & -2.836045 & 0.000000 & 0.885631 \\ \mathrm{H} & -1.232050 & 0.000000 & 3.020840 \\ \mathrm{H} & 1.232050 & 0.000000 & 3.020840 \\ \mathrm{C} & 1.490768 & 0.000000 & -1.570032 \\ \mathrm{C} & 2.877666 & 0.000000 & -1.525457 \\ \mathrm{C} & 3.563064 & 0.000000 & -0.299379 \\ \mathrm{C} & 2.836045 & 0.000000 & 0.885631 \\ \mathrm{H} & 1.006799 & 0.000000 & -2.536223 \\ \mathrm{H} & 3.437171 & 0.000000 & -2.452172 \\ \mathrm{H} & 4.644126 & 0.000000 & -0.278738 \\ \mathrm{H} & 3.349864 & 0.000000 & 1.839914 \\ \mathrm{C} & -3.563064 & 0.000000 & -0.299379 \\ \mathrm{C} & -2.877666 & 0.000000 & -1.525457 \\ \mathrm{H} & -1.006799 & 0.000000 & -2.536223 \\ \mathrm{H} & -3.437171 & 0.000000 & -2.452172 \\ \mathrm{H} & -4.644126 & 0.000000 & -0.278738 \\ \mathrm{H} & -3.349864 & 0.000000 & 1.839914\end{array}$

2,4,6,8-decatetraene $\mathrm{S}_{0}$

$\begin{array}{llll}\text { C } & -2.584955 & -5.002335 & 0.000000\end{array}$ 


$\begin{array}{crrc}\mathrm{C} & -2.464127 & -3.512769 & 0.000000 \\ \mathrm{C} & -1.310516 & -2.841749 & 0.000000 \\ \mathrm{C} & -1.210360 & -1.394935 & 0.000000 \\ \mathrm{C} & -0.050151 & -0.721087 & 0.000000 \\ \mathrm{C} & 0.050151 & 0.721087 & 0.000000 \\ \mathrm{C} & 1.210360 & 1.394935 & 0.000000 \\ \mathrm{C} & 1.310516 & 2.841749 & 0.000000 \\ \mathrm{C} & 2.464127 & 3.512769 & 0.000000 \\ \mathrm{C} & 2.584955 & 5.002335 & 0.000000 \\ \mathrm{H} & -0.373916 & -3.395720 & 0.000000 \\ \mathrm{H} & -2.144922 & -0.838091 & 0.000000 \\ \mathrm{H} & 0.883975 & -1.278617 & 0.000000 \\ \mathrm{H} & -0.883975 & 1.278617 & 0.000000 \\ \mathrm{H} & 2.144922 & 0.838091 & 0.000000 \\ \mathrm{H} & 0.373916 & 3.395720 & 0.000000 \\ \mathrm{H} & 3.393361 & 2.947039 & 0.000000 \\ \mathrm{H} & -3.393361 & -2.947039 & 0.000000 \\ \mathrm{H} & 1.603539 & 5.477098 & 0.000000 \\ \mathrm{H} & 3.134790 & 5.350760 & 0.877959 \\ \mathrm{H} & 3.134790 & 5.350760 & -0.877959 \\ \mathrm{H} & -1.603539 & -5.477098 & 0.000000 \\ \mathrm{H} & -3.134790 & -5.350760 & 0.877959 \\ \mathrm{H} & -3.134790 & -5.350760 & -0.877959\end{array}$

2,4,6,8-decatetraene $\mathrm{S}_{1}$

$\begin{array}{crcc}\mathrm{C} & -2.572276 & -5.011606 & 0.000000 \\ \mathrm{C} & -2.513884 & -3.527221 & 0.000000 \\ \mathrm{C} & -1.352296 & -2.804705 & 0.000000 \\ \mathrm{C} & -1.267317 & -1.400403 & 0.000000 \\ \mathrm{C} & -0.052825 & -0.692749 & 0.000000 \\ \mathrm{C} & 0.052825 & 0.692749 & 0.000000 \\ \mathrm{C} & 1.267317 & 1.400403 & 0.000000 \\ \mathrm{C} & 1.352296 & 2.804705 & 0.000000 \\ \mathrm{C} & 2.513884 & 3.527221 & 0.000000 \\ \mathrm{C} & 2.572276 & 5.011606 & 0.000000 \\ \mathrm{H} & -0.412250 & -3.354990 & 0.000000 \\ \mathrm{H} & -2.192357 & -0.828848 & 0.000000 \\ \mathrm{H} & 0.866965 & -1.273841 & 0.000000 \\ \mathrm{H} & -0.866965 & 1.273841 & 0.000000 \\ \mathrm{H} & 2.192357 & 0.828848 & 0.000000 \\ \mathrm{H} & 0.412250 & 3.354990 & 0.000000 \\ \mathrm{H} & 3.458773 & 2.989014 & 0.000000 \\ \mathrm{H} & -3.458773 & -2.989014 & 0.000000 \\ \mathrm{H} & 1.572654 & 5.447873 & 0.000000 \\ \mathrm{H} & 3.111192 & 5.393845 & 0.875505\end{array}$




$$
\begin{array}{lrrr}
\mathrm{H} & 3.111192 & 5.393845 & -0.875505 \\
\mathrm{H} & -1.572654 & -5.447873 & 0.000000 \\
\mathrm{H} & -3.111192 & -5.393845 & 0.875505 \\
\mathrm{H} & -3.111192 & -5.393845 & -0.875505
\end{array}
$$

tetracene $\mathrm{S}_{0}$

$\begin{array}{lrrr}\mathrm{C} & 3.694634 & 1.404410 & 0.000000 \\ \mathrm{C} & 2.433944 & 0.720705 & 0.000000 \\ \mathrm{C} & 2.433944 & -0.720705 & 0.000000 \\ \mathrm{C} & 3.694634 & -1.404410 & 0.000000 \\ \mathrm{C} & 4.861350 & -0.715780 & 0.000000 \\ \mathrm{C} & 4.861350 & 0.715780 & 0.000000 \\ \mathrm{C} & 1.229932 & 1.400020 & 0.000000 \\ \mathrm{C} & 0.000000 & 0.719880 & 0.000000 \\ \mathrm{C} & 0.000000 & -0.719880 & 0.000000 \\ \mathrm{C} & 1.229932 & -1.400020 & 0.000000 \\ \mathrm{C} & -1.229932 & 1.400020 & 0.000000 \\ \mathrm{C} & -2.433944 & 0.720705 & 0.000000 \\ \mathrm{C} & -2.433944 & -0.720705 & 0.000000 \\ \mathrm{C} & -1.229932 & -1.400020 & 0.000000 \\ \mathrm{C} & -3.694634 & 1.404410 & 0.000000 \\ \mathrm{C} & -4.861350 & 0.715780 & 0.000000 \\ \mathrm{C} & -4.861350 & -0.715780 & 0.000000 \\ \mathrm{C} & -3.694634 & -1.404410 & 0.000000 \\ \mathrm{H} & -3.690599 & -2.488189 & 0.000000 \\ \mathrm{H} & -5.806109 & -1.244361 & 0.000000 \\ \mathrm{H} & -5.806109 & 1.244361 & 0.000000 \\ \mathrm{H} & -3.690599 & 2.488189 & 0.000000 \\ \mathrm{H} & -1.230040 & -2.484929 & 0.000000 \\ \mathrm{H} & -1.230040 & 2.484929 & 0.000000 \\ \mathrm{H} & 1.230040 & -2.484929 & 0.000000 \\ \mathrm{H} & 1.230040 & 2.484929 & 0.000000 \\ \mathrm{H} & 3.690599 & -2.488189 & 0.000000 \\ \mathrm{H} & 5.806109 & -1.244361 & 0.000000 \\ \mathrm{H} & 5.806109 & 1.244361 & 0.000000 \\ \mathrm{H} & 3.690599 & 2.488189 & 0.000000\end{array}$

tetracene $\mathrm{S}_{1}$

$\begin{array}{rrrr}\mathrm{C} & 3.693373 & 1.393406 & 0.000000 \\ \mathrm{C} & 2.464776 & 0.717157 & 0.000000 \\ \mathrm{C} & 2.464776 & -0.717157 & 0.000000 \\ \mathrm{C} & 3.693373 & -1.393406 & 0.000000 \\ \mathrm{C} & 4.894888 & -0.696899 & 0.000000 \\ \mathrm{C} & 4.894888 & 0.696899 & 0.000000\end{array}$




$\begin{array}{rrrr}\mathrm{C} & 1.225179 & 1.396347 & 0.000000 \\ \mathrm{C} & 0.000000 & 0.725031 & 0.000000 \\ \mathrm{C} & 0.000000 & -0.725031 & 0.000000 \\ \mathrm{C} & 1.225179 & -1.396347 & 0.000000 \\ \mathrm{C} & -1.225179 & 1.396347 & 0.000000 \\ \mathrm{C} & -2.464776 & 0.717157 & 0.000000 \\ \mathrm{C} & -2.464776 & -0.717157 & 0.000000 \\ \mathrm{C} & -1.225179 & -1.396347 & 0.000000 \\ \mathrm{C} & -3.693373 & 1.393406 & 0.000000 \\ \mathrm{C} & -4.894888 & 0.696899 & 0.000000 \\ \mathrm{C} & -4.894888 & -0.696899 & 0.000000 \\ \mathrm{C} & -3.693373 & -1.393406 & 0.000000 \\ \mathrm{H} & -3.693528 & -2.477165 & 0.000000 \\ \mathrm{H} & -5.831498 & -1.239099 & 0.000000 \\ \mathrm{H} & -5.831498 & 1.239099 & 0.000000 \\ \mathrm{H} & -3.693528 & 2.477165 & 0.000000 \\ \mathrm{H} & -1.227389 & -2.481379 & 0.000000 \\ \mathrm{H} & -1.227389 & 2.481379 & 0.000000 \\ \mathrm{H} & 1.227389 & -2.481379 & 0.000000 \\ \mathrm{H} & 1.227389 & 2.481379 & 0.000000 \\ \mathrm{H} & 3.693528 & -2.477165 & 0.000000 \\ \mathrm{H} & 5.831498 & -1.239099 & 0.000000 \\ \mathrm{H} & 5.831498 & 1.239099 & 0.000000 \\ \mathrm{H} & 3.693528 & 2.477165 & 0.000000\end{array}$

perylene $\mathrm{S}_{0}$

$\begin{array}{lrrr}\text { C } & 0.115457 & 1.467257 & -2.412133 \\ \mathrm{C} & 0.038179 & 0.737575 & -1.244392 \\ \mathrm{C} & 0.010959 & 1.435762 & -0.000150 \\ \mathrm{C} & 0.034642 & 2.857817 & -0.000149 \\ \mathrm{C} & 0.098305 & 3.556880 & -1.227557 \\ \mathrm{C} & 0.146570 & 2.871319 & -2.407612 \\ \mathrm{C} & -0.010561 & 3.558624 & 1.227010 \\ \mathrm{C} & -0.069069 & 2.874620 & 2.407548 \\ \mathrm{C} & -0.070016 & 1.470252 & 2.412429 \\ \mathrm{C} & -0.013213 & 0.738519 & 1.244688 \\ \mathrm{C} & -0.038179 & -0.737575 & -1.244392 \\ \mathrm{C} & -0.010959 & -1.435762 & -0.000150 \\ \mathrm{C} & 0.013213 & -0.738519 & 1.244688 \\ \mathrm{C} & -0.034642 & -2.857817 & -0.000149 \\ \mathrm{C} & 0.010561 & -3.558624 & 1.227010 \\ \mathrm{C} & 0.069069 & -2.874620 & 2.407548 \\ \mathrm{C} & 0.070016 & -1.470252 & 2.412429 \\ \mathrm{C} & -0.115457 & -1.467257 & -2.412133 \\ \mathrm{C} & -0.146570 & -2.871319 & -2.407612\end{array}$




$\begin{array}{rrrr}\mathrm{C} & -0.098305 & -3.556880 & -1.227557 \\ \mathrm{H} & 0.000777 & 4.641838 & 1.210124 \\ \mathrm{H} & -0.112105 & 3.408032 & 3.348643 \\ \mathrm{H} & -0.116885 & 0.969642 & 3.368783 \\ \mathrm{H} & -0.000777 & -4.641838 & 1.210124 \\ \mathrm{H} & 0.112105 & -3.408032 & 3.348643 \\ \mathrm{H} & 0.116885 & -0.969642 & 3.368783 \\ \mathrm{H} & -0.114420 & -4.640038 & -1.210905 \\ \mathrm{H} & -0.206340 & -3.403302 & -3.348607 \\ \mathrm{H} & -0.159528 & -0.964853 & -3.367718 \\ \mathrm{H} & 0.114420 & 4.640038 & -1.210905 \\ \mathrm{H} & 0.206340 & 3.403302 & -3.348607 \\ \mathrm{H} & 0.159528 & 0.964853 & -3.367718\end{array}$

perylene $S_{1}$

$\begin{array}{rrrr}\mathrm{C} & -0.006287 & 1.465712 & -2.444722 \\ \mathrm{C} & -0.006683 & 0.718028 & -1.237487 \\ \mathrm{C} & 0.013028 & 1.416432 & 0.000001 \\ \mathrm{C} & 0.007203 & 2.846547 & -0.000001 \\ \mathrm{C} & 0.009291 & 3.538081 & -1.226442 \\ \mathrm{C} & -0.001093 & 2.846623 & -2.428991 \\ \mathrm{C} & 0.009504 & 3.538086 & 1.226433 \\ \mathrm{C} & -0.000649 & 2.846630 & 2.428988 \\ \mathrm{C} & -0.005898 & 1.465721 & 2.444725 \\ \mathrm{C} & -0.006587 & 0.718029 & 1.237492 \\ \mathrm{C} & 0.006683 & -0.718028 & -1.237487 \\ \mathrm{C} & -0.013028 & -1.416432 & 0.000001 \\ \mathrm{C} & 0.006587 & -0.718029 & 1.237492 \\ \mathrm{C} & -0.007203 & -2.846547 & -0.000001 \\ \mathrm{C} & -0.009504 & -3.538086 & 1.226433 \\ \mathrm{C} & 0.000649 & -2.846630 & 2.428988 \\ \mathrm{C} & 0.005898 & -1.465721 & 2.444725 \\ \mathrm{C} & 0.006287 & -1.465712 & -2.444722 \\ \mathrm{C} & 0.001093 & -2.846623 & -2.428991 \\ \mathrm{C} & -0.009291 & -3.538081 & -1.226442 \\ \mathrm{H} & 0.013013 & 4.621365 & 1.215871 \\ \mathrm{H} & -0.004062 & 3.392670 & 3.363849 \\ \mathrm{H} & -0.028469 & 0.958906 & 3.397291 \\ \mathrm{H} & -0.013013 & -4.621365 & 1.215871 \\ \mathrm{H} & 0.004062 & -3.392670 & 3.363849 \\ \mathrm{H} & 0.028469 & -0.958906 & 3.397291 \\ \mathrm{H} & -0.012785 & -4.621361 & -1.215882 \\ \mathrm{H} & 0.004743 & -3.392658 & -3.363854 \\ \mathrm{H} & 0.029140 & -0.958888 & -3.397278 \\ \mathrm{H} & 0.012785 & 4.621361 & -1.215882\end{array}$



H -0.004743
$3.392658 \quad-3.363854$
$\mathrm{H} \quad-0.029140 \quad 0.958888 \quad-3.397278$

M06-HF/TZVP geometries - Group I

pyrazine $\mathrm{S}_{0}$

$\begin{array}{lrrr}\mathrm{C} & 1.127486 & -0.695198 & 0.000000 \\ \mathrm{C} & 1.127486 & 0.695198 & 0.000000 \\ \mathrm{~N} & 0.000000 & 1.392107 & 0.000000 \\ \mathrm{C} & -1.127486 & 0.695198 & 0.000000 \\ \mathrm{C} & -1.127486 & -0.695198 & 0.000000 \\ \mathrm{~N} & 0.000000 & -1.392107 & 0.000000 \\ \mathrm{H} & -2.053929 & -1.250428 & 0.000000 \\ \mathrm{H} & 2.053929 & -1.250428 & 0.000000 \\ \mathrm{H} & 2.053929 & 1.250428 & 0.000000 \\ \mathrm{H} & -2.053929 & 1.250428 & 0.000000\end{array}$

pyrazine $\mathrm{S}_{1}$

$\begin{array}{lrrr}\mathrm{C} & -1.185347 & 0.656584 & 0.000000 \\ \mathrm{C} & -1.138475 & -0.735169 & 0.000000 \\ \mathrm{~N} & 0.045667 & -1.355194 & 0.000000 \\ \mathrm{C} & 1.185347 & -0.656584 & 0.000000 \\ \mathrm{C} & 1.138475 & 0.735169 & 0.000000 \\ \mathrm{~N} & -0.045667 & 1.355194 & 0.000000 \\ \mathrm{H} & 2.058022 & 1.298798 & 0.000000 \\ \mathrm{H} & -2.140579 & 1.157580 & 0.000000 \\ \mathrm{H} & -2.058022 & -1.298798 & 0.000000 \\ \mathrm{H} & 2.140579 & -1.157580 & 0.000000\end{array}$

2,6-difluoropyridine $\mathrm{S}_{0}$

$\begin{array}{lrrr}\mathrm{C} & 1.110202 & 0.000000 & -0.871840 \\ \mathrm{~N} & 0.000000 & 0.000000 & -1.558535 \\ \mathrm{C} & -1.110202 & 0.000000 & -0.871840 \\ \mathrm{C} & -1.201421 & 0.000000 & 0.507674 \\ \mathrm{C} & 0.000000 & 0.000000 & 1.200869 \\ \mathrm{C} & 1.201421 & 0.000000 & 0.507674 \\ \mathrm{~F} & -2.237282 & 0.000000 & -1.588490 \\ \mathrm{H} & -2.163829 & 0.000000 & 0.991274 \\ \mathrm{H} & 0.000000 & 0.000000 & 2.280850 \\ \mathrm{H} & 2.163829 & 0.000000 & 0.991274 \\ \mathrm{~F} & 2.237282 & 0.000000 & -1.588490\end{array}$


2,6-difluoropyridine $\mathrm{S}_{1}$

$\begin{array}{lrrr}\mathrm{C} & -1.091712 & 0.000000 & -0.337381 \\ \mathrm{~N} & 0.000000 & 0.000000 & -1.101717 \\ \mathrm{C} & 1.091712 & 0.000000 & -0.337381 \\ \mathrm{C} & 1.184885 & 0.000000 & 1.073822 \\ \mathrm{C} & 0.000000 & 0.000000 & 1.851200 \\ \mathrm{C} & -1.184885 & 0.000000 & 1.073822 \\ \mathrm{~F} & 2.234066 & 0.000000 & -1.002373 \\ \mathrm{H} & 2.174930 & 0.000000 & 1.503288 \\ \mathrm{H} & 0.000000 & 0.000000 & 2.925098 \\ \mathrm{H} & -2.174930 & 0.000000 & 1.503288 \\ \mathrm{~F} & -2.234066 & 0.000000 & -1.002373\end{array}$

2-fluoropyridine $\mathrm{S}_{0}$

$\begin{array}{lrrr}\mathrm{C} & -1.309857 & 0.499947 & 0.000000 \\ \mathrm{~N} & -1.359221 & -0.796992 & 0.000000 \\ \mathrm{C} & -0.196411 & -1.450472 & 0.000000 \\ \mathrm{C} & 1.029466 & -0.808421 & 0.000000 \\ \mathrm{C} & 1.043389 & 0.583705 & 0.000000 \\ \mathrm{C} & -0.155427 & 1.271064 & 0.000000 \\ \mathrm{H} & -0.262311 & -2.528526 & 0.000000 \\ \mathrm{H} & 1.943590 & -1.381243 & 0.000000 \\ \mathrm{H} & 1.978039 & 1.125820 & 0.000000 \\ \mathrm{H} & -0.221607 & 2.347074 & 0.000000 \\ \mathrm{~F} & -2.489660 & 1.138033 & 0.000000\end{array}$

2-fluoropyridine $\mathrm{S}_{1}$
$\begin{array}{llll}\text { C } & -1.317280 & 0.482677 & 0.000000\end{array}$
$\begin{array}{llll}\mathrm{N} & -1.435122 & -0.842825 & 0.000000\end{array}$
$\begin{array}{llll}\text { C } & -0.230846 & -1.458279 & 0.000000\end{array}$
$\begin{array}{llll}\text { C } & 1.028282 & -0.786393 & 0.000000\end{array}$
$\begin{array}{llll}\text { C } & 1.112842 & 0.622232 & 0.000000\end{array}$
$\begin{array}{llll}\mathrm{C} & -0.146483 & 1.274544 & 0.000000\end{array}$
$\begin{array}{llll}\mathrm{H} & -0.248036 & -2.537835 & 0.000000\end{array}$
$\begin{array}{llll}\mathrm{H} & 1.918018 & -1.399425 & 0.000000\end{array}$
$\begin{array}{llll}\mathrm{H} & 2.046093 & 1.156239 & 0.000000\end{array}$
$\begin{array}{llll}\mathrm{H} & -0.263196 & 2.346959 & 0.000000\end{array}$
$\begin{array}{llll}\text { F } & -2.464281 & 1.142098 & 0.000000\end{array}$

2-hydroxypyridine $\mathrm{S}_{0}$
$\begin{array}{llll}\text { C } & -0.975687 & 0.751186 & 0.000000\end{array}$
$\begin{array}{llll}\text { C } & -0.962731 & -0.645014 & 0.000000\end{array}$ 


$\begin{array}{rrrc}\mathrm{N} & 0.133336 & -1.368099 & 0.000000 \\ \mathrm{C} & 1.306040 & -0.733350 & 0.000000 \\ \mathrm{C} & 1.418797 & 0.643756 & 0.000000 \\ \mathrm{C} & 0.243393 & 1.394560 & 0.000000 \\ \mathrm{O} & -2.141531 & -1.298283 & 0.000000 \\ \mathrm{H} & 2.182675 & -1.364877 & 0.000000 \\ \mathrm{H} & 2.389629 & 1.113479 & 0.000000 \\ \mathrm{H} & 0.283319 & 2.474409 & 0.000000 \\ \mathrm{H} & -1.917619 & 1.275657 & 0.000000 \\ \mathrm{H} & -1.959620 & -2.243423 & 0.000000\end{array}$

2-hydroxypyridine $\mathrm{S}_{1}$

$\begin{array}{crrc}\mathrm{C} & -0.986545 & 0.767540 & 0.000000 \\ \mathrm{C} & -0.961413 & -0.643178 & 0.000000 \\ \mathrm{~N} & 0.129621 & -1.456799 & 0.000000 \\ \mathrm{C} & 1.287553 & -0.775853 & 0.000000 \\ \mathrm{C} & 1.388126 & 0.644978 & 0.000000 \\ \mathrm{C} & 0.245890 & 1.469600 & 0.000000 \\ \mathrm{O} & -2.111290 & -1.281237 & 0.000000 \\ \mathrm{H} & 2.192841 & -1.364536 & 0.000000 \\ \mathrm{H} & 2.381227 & 1.071718 & 0.000000 \\ \mathrm{H} & 0.300947 & 2.543450 & 0.000000 \\ \mathrm{H} & -1.949793 & 1.253454 & 0.000000 \\ \mathrm{H} & -1.917165 & -2.229138 & 0.000000\end{array}$

2-pyridone $\mathrm{S}_{0}$

$\begin{array}{crrl}\mathrm{C} & -1.243772 & 0.701331 & 0.000000 \\ \mathrm{~N} & -1.135171 & -0.682465 & 0.000000 \\ \mathrm{C} & 0.034616 & -1.379434 & 0.000000 \\ \mathrm{C} & 1.220460 & -0.737487 & 0.000000 \\ \mathrm{C} & 1.202047 & 0.696536 & 0.000000 \\ \mathrm{C} & 0.043326 & 1.388730 & 0.000000 \\ \mathrm{H} & -2.018475 & -1.171035 & 0.000000 \\ \mathrm{H} & -0.060459 & -2.454280 & 0.000000 \\ \mathrm{H} & 2.144516 & -1.289624 & 0.000000 \\ \mathrm{H} & 2.141903 & 1.230669 & 0.000000 \\ \mathrm{H} & 0.005239 & 2.466566 & 0.000000 \\ \mathrm{O} & -2.334229 & 1.230503 & 0.000000\end{array}$

2-pyridone $\mathrm{S}_{1}$
$\begin{array}{llll}\text { C } & -1.402447 & 0.118803 & 0.000000\end{array}$
$\begin{array}{llll}\mathrm{N} & -0.725714 & -1.164466 & 0.000000\end{array}$
$\begin{array}{llll}\text { C } & 0.610936 & -1.252247 & 0.000000\end{array}$ 


$\begin{array}{rrrc}\mathrm{C} & 1.403785 & -0.077776 & 0.000000 \\ \mathrm{C} & 0.826328 & 1.185387 & 0.000000 \\ \mathrm{C} & -0.597927 & 1.275682 & 0.000000 \\ \mathrm{H} & -1.333874 & -1.969733 & 0.000000 \\ \mathrm{H} & 1.048706 & -2.237623 & 0.000000 \\ \mathrm{H} & 2.475659 & -0.205085 & 0.000000 \\ \mathrm{H} & 1.434449 & 2.073579 & 0.000000 \\ \mathrm{H} & -1.119901 & 2.218540 & 0.000000 \\ \mathrm{O} & -2.620001 & 0.034928 & 0.000000\end{array}$

2-methylpyrimidine $\mathrm{S}_{0}$

$\begin{array}{rrrr}\mathrm{C} & 0.000076 & 1.961887 & 0.000000 \\ \mathrm{C} & -1.172683 & 1.221294 & 0.000000 \\ \mathrm{~N} & -1.169707 & -0.102282 & 0.000000 \\ \mathrm{C} & 0.012093 & -0.716181 & 0.000000 \\ \mathrm{~N} & 1.185937 & -0.094102 & 0.000000 \\ \mathrm{C} & 1.176496 & 1.233167 & 0.000000 \\ \mathrm{H} & -2.141944 & 1.701118 & 0.000000 \\ \mathrm{H} & 2.142078 & 1.720047 & 0.000000 \\ \mathrm{H} & -0.005128 & 3.039897 & 0.000000 \\ \mathrm{C} & 0.003620 & -2.222499 & 0.000000 \\ \mathrm{H} & 1.024215 & -2.589927 & 0.000000 \\ \mathrm{H} & -0.527531 & -2.576210 & 0.882195 \\ \mathrm{H} & -0.527531 & -2.576210 & -0.882195\end{array}$

2-methylpyrimidine $\mathrm{S}_{1}$

$\begin{array}{rrrr}\mathrm{C} & -0.001258 & 2.007760 & 0.000000 \\ \mathrm{C} & -1.193958 & 1.296092 & 0.000000 \\ \mathrm{~N} & -1.090295 & -0.068343 & 0.000000 \\ \mathrm{C} & 0.017740 & -0.792386 & 0.000000 \\ \mathrm{~N} & 1.112808 & -0.075679 & 0.000000 \\ \mathrm{C} & 1.186870 & 1.293055 & 0.000000 \\ \mathrm{H} & -2.175044 & 1.734641 & 0.000000 \\ \mathrm{H} & 2.164547 & 1.739551 & 0.000000 \\ \mathrm{H} & -0.003299 & 3.087770 & 0.000000 \\ \mathrm{C} & 0.001610 & -2.289765 & 0.000000 \\ \mathrm{H} & 1.026074 & -2.648818 & 0.000000 \\ \mathrm{H} & -0.522897 & -2.641944 & 0.886322 \\ \mathrm{H} & -0.522897 & -2.641944 & -0.886322\end{array}$

5-methylpyrimidine $\mathrm{S}_{0}$
$\begin{array}{llll}\text { C } & 0.873794 & 0.851131 & 0.000000\end{array}$
$\begin{array}{llll}\text { C } & -0.357762 & 0.212317 & 0.000000\end{array}$ 


$\begin{array}{lrrr}\mathrm{C} & -0.299766 & -1.176935 & 0.000000 \\ \mathrm{~N} & 0.842120 & -1.847683 & 0.000000 \\ \mathrm{C} & 1.957389 & -1.129814 & 0.000000 \\ \mathrm{~N} & 2.025671 & 0.191499 & 0.000000 \\ \mathrm{H} & 2.891394 & -1.672383 & 0.000000 \\ \mathrm{H} & -1.207972 & -1.767531 & 0.000000 \\ \mathrm{H} & 0.936974 & 1.931936 & 0.000000 \\ \mathrm{C} & -1.669339 & 0.961179 & 0.000000 \\ \mathrm{H} & -1.490479 & 2.034249 & 0.000000 \\ \mathrm{H} & -2.251012 & 0.706008 & -0.884518 \\ \mathrm{H} & -2.251012 & 0.706008 & 0.884518\end{array}$

5-methylpyrimidine $\mathrm{S}_{1}$

$\begin{array}{rrrr}\mathrm{C} & 0.839266 & 0.882943 & 0.000000 \\ \mathrm{C} & -0.375734 & 0.228881 & 0.000000 \\ \mathrm{C} & -0.348022 & -1.175105 & 0.000000 \\ \mathrm{~N} & 0.872831 & -1.772564 & 0.000000 \\ \mathrm{C} & 2.045437 & -1.178573 & 0.000000 \\ \mathrm{~N} & 1.993157 & 0.134124 & 0.000000 \\ \mathrm{H} & 2.972794 & -1.725304 & 0.000000 \\ \mathrm{H} & -1.226393 & -1.795986 & 0.000000 \\ \mathrm{H} & 0.944279 & 1.953595 & 0.000000 \\ \mathrm{C} & -1.686245 & 0.976324 & 0.000000 \\ \mathrm{H} & -1.508508 & 2.048938 & 0.000000 \\ \mathrm{H} & -2.261436 & 0.711369 & -0.885928 \\ \mathrm{H} & -2.261436 & 0.711369 & 0.885928\end{array}$

7-azaindole $\mathrm{S}_{0}$

$\begin{array}{rrrr}\mathrm{N} & -0.631108 & 1.836233 & 0.000000 \\ \mathrm{C} & -0.666354 & 0.470818 & 0.000000 \\ \mathrm{C} & 0.665438 & 0.005274 & 0.000000 \\ \mathrm{C} & 1.509771 & 1.170079 & 0.000000 \\ \mathrm{C} & 0.679681 & 2.248188 & 0.000000 \\ \mathrm{C} & 0.842241 & -1.379115 & 0.000000 \\ \mathrm{C} & -0.289734 & -2.169150 & 0.000000 \\ \mathrm{C} & -1.563004 & -1.571794 & 0.000000 \\ \mathrm{~N} & -1.766189 & -0.268000 & 0.000000 \\ \mathrm{H} & -2.449438 & -2.191270 & 0.000000 \\ \mathrm{H} & 1.830666 & -1.817642 & 0.000000 \\ \mathrm{H} & -0.215333 & -3.245378 & 0.000000 \\ \mathrm{H} & -1.449895 & 2.419323 & 0.000000 \\ \mathrm{H} & 2.584189 & 1.195969 & 0.000000 \\ \mathrm{H} & 0.919089 & 3.296466 & 0.000000\end{array}$


7-azaindole $\mathrm{S}_{1}$

$\begin{array}{rrrr}\mathrm{N} & -0.610148 & 1.872917 & 0.000000 \\ \mathrm{C} & -0.666077 & 0.456778 & 0.000000 \\ \mathrm{C} & 0.675287 & 0.000458 & 0.000000 \\ \mathrm{C} & 1.492413 & 1.130545 & 0.000000 \\ \mathrm{C} & 0.640304 & 2.303436 & 0.000000 \\ \mathrm{C} & 0.885789 & -1.410970 & 0.000000 \\ \mathrm{C} & -0.302458 & -2.180343 & 0.000000 \\ \mathrm{C} & -1.545215 & -1.599439 & 0.000000 \\ \mathrm{~N} & -1.773472 & -0.213526 & 0.000000 \\ \mathrm{H} & -2.440072 & -2.200655 & 0.000000 \\ \mathrm{H} & 1.867877 & -1.851325 & 0.000000 \\ \mathrm{H} & -0.242894 & -3.258289 & 0.000000 \\ \mathrm{H} & -1.447276 & 2.438517 & 0.000000 \\ \mathrm{H} & 2.567906 & 1.165395 & 0.000000 \\ \mathrm{H} & 0.898045 & 3.346520 & 0.000000\end{array}$

7-hydroxyquinoline, cis $\mathrm{S}_{0}$

$\begin{array}{rrrr}\mathrm{N} & 0.455372 & 1.963394 & 0.000000 \\ \mathrm{C} & 0.425691 & 0.604797 & 0.000000 \\ \mathrm{C} & -0.781707 & -0.121744 & 0.000000 \\ \mathrm{C} & -1.992416 & 0.608963 & 0.000000 \\ \mathrm{C} & -1.946648 & 1.971155 & 0.000000 \\ \mathrm{C} & -0.677734 & 2.604210 & 0.000000 \\ \mathrm{C} & 1.662807 & -0.088129 & 0.000000 \\ \mathrm{C} & 1.674356 & -1.450928 & 0.000000 \\ \mathrm{C} & 0.462263 & -2.193124 & 0.000000 \\ \mathrm{C} & -0.730764 & -1.542284 & 0.000000 \\ \mathrm{H} & -0.619901 & 3.685065 & 0.000000 \\ \mathrm{H} & -1.657322 & -2.100517 & 0.000000 \\ \mathrm{H} & 0.528161 & -3.270474 & 0.000000 \\ \mathrm{O} & 2.815101 & -2.191338 & 0.000000 \\ \mathrm{H} & 2.569311 & 0.501936 & 0.000000 \\ \mathrm{H} & -2.934861 & 0.077423 & 0.000000 \\ \mathrm{H} & -2.843927 & 2.570741 & 0.000000 \\ \mathrm{H} & 3.592219 & -1.629136 & 0.000000\end{array}$

7-hydroxyquinoline, cis $\mathrm{S}_{1}$
$\mathrm{N} \quad 0.482534$
1.979333
0.000000
$\begin{array}{llll}\text { C } & 0.450747 & 0.637665 & 0.000000\end{array}$
$\begin{array}{llll}\text { C } & -0.794141 & -0.148869 & 0.000000\end{array}$
$\begin{array}{llll}\text { C } & -1.991154 & 0.587208 & 0.000000\end{array}$
$\begin{array}{llll}\text { C } & -1.919714 & 1.984148 & 0.000000\end{array}$ 


$\begin{array}{rrrr}\mathrm{C} & -0.676040 & 2.639802 & 0.000000 \\ \mathrm{C} & 1.644581 & -0.071194 & 0.000000 \\ \mathrm{C} & 1.647608 & -1.497579 & 0.000000 \\ \mathrm{C} & 0.473773 & -2.256769 & 0.000000 \\ \mathrm{C} & -0.733677 & -1.561987 & 0.000000 \\ \mathrm{H} & -0.623005 & 3.717485 & 0.000000 \\ \mathrm{H} & -1.666192 & -2.109564 & 0.000000 \\ \mathrm{H} & 0.535397 & -3.331671 & 0.000000 \\ \mathrm{O} & 2.807498 & -2.151880 & 0.000000 \\ \mathrm{H} & 2.574294 & 0.482147 & 0.000000 \\ \mathrm{H} & -2.944969 & 0.080529 & 0.000000 \\ \mathrm{H} & -2.825456 & 2.573003 & 0.000000 \\ \mathrm{H} & 3.557917 & -1.551797 & 0.000000\end{array}$

7-hydroxyquinoline, trans $\mathrm{S}_{0}$

$\begin{array}{rrrl}\mathrm{N} & 0.506139 & 2.036308 & 0.000000 \\ \mathrm{C} & 0.474210 & 0.674731 & 0.000000 \\ \mathrm{C} & -0.738353 & -0.045423 & 0.000000 \\ \mathrm{C} & -1.949108 & 0.690145 & 0.000000 \\ \mathrm{C} & -1.897562 & 2.050192 & 0.000000 \\ \mathrm{C} & -0.623609 & 2.679077 & 0.000000 \\ \mathrm{C} & 1.705895 & -0.019631 & 0.000000 \\ \mathrm{C} & 1.714017 & -1.383088 & 0.000000 \\ \mathrm{C} & 0.498944 & -2.117889 & 0.000000 \\ \mathrm{C} & -0.695222 & -1.461962 & 0.000000 \\ \mathrm{H} & -0.561947 & 3.759959 & 0.000000 \\ \mathrm{H} & -1.622917 & -2.017471 & 0.000000 \\ \mathrm{H} & 0.528698 & -3.199760 & 0.000000 \\ \mathrm{O} & 2.914741 & -2.025022 & 0.000000 \\ \mathrm{H} & 2.627783 & 0.540825 & 0.000000 \\ \mathrm{H} & -2.893094 & 0.161956 & 0.000000 \\ \mathrm{H} & -2.792570 & 2.653813 & 0.000000 \\ \mathrm{H} & 2.803934 & -2.976739 & 0.000000\end{array}$

7-hydroxyquinoline, trans $\mathrm{S}_{1}$

$\begin{array}{rrrr}\mathrm{N} & 0.526124 & 2.053529 & 0.000000 \\ \mathrm{C} & 0.491233 & 0.715078 & 0.000000 \\ \mathrm{C} & -0.751712 & -0.066585 & 0.000000 \\ \mathrm{C} & -1.947063 & 0.670137 & 0.000000 \\ \mathrm{C} & -1.873171 & 2.068916 & 0.000000 \\ \mathrm{C} & -0.631959 & 2.720373 & 0.000000 \\ \mathrm{C} & 1.684793 & 0.000919 & 0.000000 \\ \mathrm{C} & 1.683423 & -1.422085 & 0.000000 \\ \mathrm{C} & 0.508031 & -2.183190 & 0.000000\end{array}$




$\begin{array}{rrrr}\mathrm{C} & -0.697061 & -1.479858 & 0.000000 \\ \mathrm{H} & -0.573518 & 3.797558 & 0.000000 \\ \mathrm{H} & -1.632012 & -2.023473 & 0.000000 \\ \mathrm{H} & 0.539040 & -3.261518 & 0.000000 \\ \mathrm{O} & 2.888994 & -1.988573 & 0.000000 \\ \mathrm{H} & 2.629303 & 0.522587 & 0.000000 \\ \mathrm{H} & -2.902173 & 0.165827 & 0.000000 \\ \mathrm{H} & -2.778813 & 2.658082 & 0.000000 \\ \mathrm{H} & 2.836521 & -2.947702 & 0.000000\end{array}$

2-hydroxyquinoline, enol $\mathrm{S}_{0}$

$\begin{array}{rrrl}\mathrm{C} & 0.431293 & 1.904228 & 0.000000 \\ \mathrm{C} & 0.480020 & 0.488863 & 0.000000 \\ \mathrm{C} & -0.715923 & -0.252862 & 0.000000 \\ \mathrm{C} & -1.952966 & 0.436632 & 0.000000 \\ \mathrm{C} & -1.976905 & 1.801900 & 0.000000 \\ \mathrm{C} & -0.772273 & 2.548552 & 0.000000 \\ \mathrm{C} & 1.697943 & -0.243570 & 0.000000 \\ \mathrm{C} & 1.665472 & -1.598886 & 0.000000 \\ \mathrm{C} & 0.387327 & -2.243992 & 0.000000 \\ \mathrm{~N} & -0.734057 & -1.615002 & 0.000000 \\ \mathrm{O} & 0.318799 & -3.594212 & 0.000000 \\ \mathrm{H} & 1.359217 & 2.460284 & 0.000000 \\ \mathrm{H} & -0.813532 & 3.627946 & 0.000000 \\ \mathrm{H} & -2.922389 & 2.324239 & 0.000000 \\ \mathrm{H} & -2.856411 & -0.155397 & 0.000000 \\ \mathrm{H} & 2.640629 & 0.286885 & 0.000000 \\ \mathrm{H} & 2.573014 & -2.185320 & 0.000000 \\ \mathrm{H} & 1.190741 & -3.990287 & 0.000000\end{array}$

2-hydroxyquinoline, enol $\mathrm{S}_{1}$

$\begin{array}{rrrr}\mathrm{C} & 0.445717 & 1.885478 & 0.000000 \\ \mathrm{C} & 0.533764 & 0.492319 & 0.000000 \\ \mathrm{C} & -0.741595 & -0.265830 & 0.000000 \\ \mathrm{C} & -1.971781 & 0.433681 & 0.000000 \\ \mathrm{C} & -2.012396 & 1.827034 & 0.000000 \\ \mathrm{C} & -0.802471 & 2.535286 & 0.000000 \\ \mathrm{C} & 1.732993 & -0.234797 & 0.000000 \\ \mathrm{C} & 1.694324 & -1.630590 & 0.000000 \\ \mathrm{C} & 0.423697 & -2.236572 & 0.000000 \\ \mathrm{~N} & -0.750995 & -1.598646 & 0.000000 \\ \mathrm{O} & 0.305528 & -3.561545 & 0.000000 \\ \mathrm{H} & 1.352630 & 2.474070 & 0.000000 \\ \mathrm{H} & -0.819594 & 3.616431 & 0.000000\end{array}$




$\begin{array}{rrrr}\mathrm{H} & -2.952879 & 2.353335 & 0.000000 \\ \mathrm{H} & -2.870464 & -0.165476 & 0.000000 \\ \mathrm{H} & 2.675797 & 0.292036 & 0.000000 \\ \mathrm{H} & 2.592699 & -2.227845 & 0.000000 \\ \mathrm{H} & 1.165016 & -3.988349 & 0.000000\end{array}$

2-hydroxyquinoline, keto $\mathrm{S}_{0}$

$\begin{array}{rrrr}\mathrm{N} & 0.560440 & 1.704684 & 0.000000 \\ \mathrm{C} & 0.598640 & 0.325399 & 0.000000 \\ \mathrm{C} & -0.606948 & -0.382287 & 0.000000 \\ \mathrm{C} & -1.842355 & 0.379892 & 0.000000 \\ \mathrm{C} & -1.840092 & 1.717458 & 0.000000 \\ \mathrm{C} & -0.577941 & 2.478447 & 0.000000 \\ \mathrm{C} & 1.817679 & -0.361213 & 0.000000 \\ \mathrm{C} & 1.820475 & -1.739556 & 0.000000 \\ \mathrm{C} & 0.620535 & -2.460626 & 0.000000 \\ \mathrm{C} & -0.578282 & -1.781416 & 0.000000 \\ \mathrm{H} & -1.515624 & -2.321245 & 0.000000 \\ \mathrm{H} & 0.637934 & -3.539932 & 0.000000 \\ \mathrm{H} & 2.763065 & -2.266909 & 0.000000 \\ \mathrm{H} & 2.746356 & 0.192863 & 0.000000 \\ \mathrm{H} & -2.776549 & -0.166889 & 0.000000 \\ \mathrm{H} & -2.741079 & 2.310193 & 0.000000 \\ \mathrm{O} & -0.512284 & 3.686198 & 0.000000 \\ \mathrm{H} & 1.426051 & 2.224958 & 0.000000\end{array}$

2-hydroxyquinoline, keto $\mathrm{S}_{1}$

$\begin{array}{rrrr}\mathrm{N} & 0.571365 & 1.669699 & 0.000000 \\ \mathrm{C} & 0.618791 & 0.336570 & 0.000000 \\ \mathrm{C} & -0.668014 & -0.379307 & 0.000000 \\ \mathrm{C} & -1.856233 & 0.361545 & 0.000000 \\ \mathrm{C} & -1.857857 & 1.759836 & 0.000000 \\ \mathrm{C} & -0.635514 & 2.472131 & 0.000000 \\ \mathrm{C} & 1.845019 & -0.358476 & 0.000000 \\ \mathrm{C} & 1.853022 & -1.738942 & 0.000000 \\ \mathrm{C} & 0.623190 & -2.443841 & 0.000000 \\ \mathrm{C} & -0.593786 & -1.789635 & 0.000000 \\ \mathrm{H} & -1.515339 & -2.353710 & 0.000000 \\ \mathrm{H} & 0.641265 & -3.524547 & 0.000000 \\ \mathrm{H} & 2.785615 & -2.280129 & 0.000000 \\ \mathrm{H} & 2.766561 & 0.207556 & 0.000000 \\ \mathrm{H} & -2.794030 & -0.174409 & 0.000000 \\ \mathrm{H} & -2.763304 & 2.341652 & 0.000000 \\ \mathrm{O} & -0.440564 & 3.671644 & 0.000000\end{array}$




$$
\begin{array}{llll}
\mathrm{H} & 1.419814 & 2.222374 & 0.000000 \\
\text { dibenzofuran } \mathrm{S}_{0} &
\end{array}
$$

$\begin{array}{rrrr}\mathrm{C} & 1.721547 & 0.000000 & -1.359186 \\ \mathrm{C} & 0.725033 & 0.000000 & -0.382029 \\ \mathrm{C} & 1.099430 & 0.000000 & 0.971129 \\ \mathrm{C} & 2.416241 & 0.000000 & 1.395372 \\ \mathrm{C} & 3.393078 & 0.000000 & 0.403744 \\ \mathrm{C} & 3.051117 & 0.000000 & -0.954809 \\ \mathrm{C} & -0.725033 & 0.000000 & -0.382029 \\ \mathrm{C} & -1.099430 & 0.000000 & 0.971129 \\ \mathrm{O} & 0.000000 & 0.000000 & 1.799285 \\ \mathrm{C} & -1.721547 & 0.000000 & -1.359186 \\ \mathrm{C} & -3.051117 & 0.000000 & -0.954809 \\ \mathrm{C} & -3.393078 & 0.000000 & 0.403744 \\ \mathrm{C} & -2.416241 & 0.000000 & 1.395372 \\ \mathrm{H} & -2.666677 & 0.000000 & 2.448186 \\ \mathrm{H} & -4.437585 & 0.000000 & 0.689760 \\ \mathrm{H} & -3.837606 & 0.000000 & -1.699501 \\ \mathrm{H} & -1.466221 & 0.000000 & -2.412142 \\ \mathrm{H} & 1.466221 & 0.000000 & -2.412142 \\ \mathrm{H} & 3.837606 & 0.000000 & -1.699501 \\ \mathrm{H} & 4.437585 & 0.000000 & 0.689760 \\ \mathrm{H} & 2.666677 & 0.000000 & 2.448186\end{array}$

dibenzofuran $\mathrm{S}_{1}$

$\begin{array}{lrrr}\mathrm{C} & 0.000000 & 1.728507 & -1.533998 \\ \mathrm{C} & 0.000000 & 0.712933 & -0.574828 \\ \mathrm{C} & 0.000000 & 1.099268 & 0.802872 \\ \mathrm{C} & 0.000000 & 2.405417 & 1.257844 \\ \mathrm{C} & 0.000000 & 3.409831 & 0.271387 \\ \mathrm{C} & 0.000000 & 3.066749 & -1.086696 \\ \mathrm{C} & 0.000000 & -0.712933 & -0.574828 \\ \mathrm{C} & 0.000000 & -1.099268 & 0.802872 \\ \mathrm{O} & 0.000000 & 0.000000 & 1.585538 \\ \mathrm{C} & 0.000000 & -1.728507 & -1.533998 \\ \mathrm{C} & 0.000000 & -3.066749 & -1.086696 \\ \mathrm{C} & 0.000000 & -3.409831 & 0.271387 \\ \mathrm{C} & 0.000000 & -2.405417 & 1.257844 \\ \mathrm{H} & 0.000000 & -2.625072 & 2.314161 \\ \mathrm{H} & 0.000000 & -4.448327 & 0.563263 \\ \mathrm{H} & 0.000000 & -3.857232 & -1.823341 \\ \mathrm{H} & 0.000000 & -1.504175 & -2.589722 \\ \mathrm{H} & 0.000000 & 1.504175 & -2.589722\end{array}$




$$
\begin{array}{lrrr}
\mathrm{H} & 0.000000 & 3.857232 & -1.823341 \\
\mathrm{H} & 0.000000 & 4.448327 & 0.563263 \\
\mathrm{H} & 0.000000 & 2.625072 & 2.314161
\end{array}
$$

pyrrolo[3,2-h]quinoline $\mathrm{S}_{0}$

$\begin{array}{lrrr}\mathrm{C} & 0.140370 & -0.681141 & 0.000000 \\ \mathrm{~N} & 0.063150 & -2.026925 & 0.000000 \\ \mathrm{C} & 1.183087 & -2.703236 & 0.000000 \\ \mathrm{C} & 2.456493 & -2.096564 & 0.000000 \\ \mathrm{C} & 2.536034 & -0.729287 & 0.000000 \\ \mathrm{C} & 1.352513 & 0.035976 & 0.000000 \\ \mathrm{H} & 1.096759 & -3.781451 & 0.000000 \\ \mathrm{H} & 3.342201 & -2.713911 & 0.000000 \\ \mathrm{H} & 3.494072 & -0.226854 & 0.000000 \\ \mathrm{C} & -1.053286 & 0.090159 & 0.000000 \\ \mathrm{C} & -1.056648 & 1.480845 & 0.000000 \\ \mathrm{C} & 0.188912 & 2.178305 & 0.000000 \\ \mathrm{C} & 1.349875 & 1.474660 & 0.000000 \\ \mathrm{H} & 0.200858 & 3.258907 & 0.000000 \\ \mathrm{H} & 2.302296 & 1.985564 & 0.000000 \\ \mathrm{C} & -2.430679 & 1.885091 & 0.000000 \\ \mathrm{C} & -3.175483 & 0.737755 & 0.000000 \\ \mathrm{~N} & -2.338749 & -0.345491 & 0.000000 \\ \mathrm{H} & -2.596119 & -1.318351 & 0.000000 \\ \mathrm{H} & -2.812898 & 2.890023 & 0.000000 \\ \mathrm{H} & -4.242747 & 0.605925 & 0.000000\end{array}$

pyrrolo[3,2-h]quinoline $S_{1}$

$\begin{array}{lrrr}\mathrm{C} & 0.152329 & -0.709155 & 0.000000 \\ \mathrm{~N} & 0.031080 & -2.009002 & 0.000000 \\ \mathrm{C} & 1.204998 & -2.737535 & 0.000000 \\ \mathrm{C} & 2.435356 & -2.138672 & 0.000000 \\ \mathrm{C} & 2.556142 & -0.730474 & 0.000000 \\ \mathrm{C} & 1.371224 & 0.037178 & 0.000000 \\ \mathrm{H} & 1.090895 & -3.808762 & 0.000000 \\ \mathrm{H} & 3.324141 & -2.752992 & 0.000000 \\ \mathrm{H} & 3.523763 & -0.253412 & 0.000000 \\ \mathrm{C} & -1.029196 & 0.107188 & 0.000000 \\ \mathrm{C} & -1.058974 & 1.553395 & 0.000000 \\ \mathrm{C} & 0.132538 & 2.233230 & 0.000000 \\ \mathrm{C} & 1.330817 & 1.448060 & 0.000000 \\ \mathrm{H} & 0.183919 & 3.309919 & 0.000000 \\ \mathrm{H} & 2.277358 & 1.971155 & 0.000000 \\ \mathrm{C} & -2.467742 & 1.907296 & 0.000000\end{array}$




$\begin{array}{cccc}\mathrm{C} & -3.165479 & 0.748577 & 0.000000 \\ \mathrm{~N} & -2.282123 & -0.334057 & 0.000000 \\ \mathrm{H} & -2.502779 & -1.320086 & 0.000000 \\ \mathrm{H} & -2.881031 & 2.899633 & 0.000000 \\ \mathrm{H} & -4.227245 & 0.578515 & 0.000000\end{array}$

carbazole $\mathrm{S}_{0}$

$\begin{array}{rrrr}\mathrm{C} & 1.125490 & 0.000000 & -0.893408 \\ \mathrm{~N} & 0.000000 & 0.000000 & -1.690493 \\ \mathrm{C} & -1.125490 & 0.000000 & -0.893408 \\ \mathrm{C} & -0.727123 & 0.000000 & 0.455179 \\ \mathrm{C} & 0.727123 & 0.000000 & 0.455179 \\ \mathrm{C} & -1.692698 & 0.000000 & 1.460054 \\ \mathrm{C} & -3.028374 & 0.000000 & 1.104585 \\ \mathrm{C} & -3.408434 & 0.000000 & -0.246079 \\ \mathrm{C} & -2.470603 & 0.000000 & -1.260637 \\ \mathrm{C} & 2.470603 & 0.000000 & -1.260637 \\ \mathrm{C} & 3.408434 & 0.000000 & -0.246079 \\ \mathrm{C} & 3.028374 & 0.000000 & 1.104585 \\ \mathrm{C} & 1.692698 & 0.000000 & 1.460054 \\ \mathrm{H} & -2.769098 & 0.000000 & -2.299559 \\ \mathrm{H} & -4.459225 & 0.000000 & -0.499218 \\ \mathrm{H} & -3.789546 & 0.000000 & 1.870152 \\ \mathrm{H} & -1.399284 & 0.000000 & 2.500825 \\ \mathrm{H} & 1.399284 & 0.000000 & 2.500825 \\ \mathrm{H} & 3.789546 & 0.000000 & 1.870152 \\ \mathrm{H} & 4.459225 & 0.000000 & -0.499218 \\ \mathrm{H} & 2.769098 & 0.000000 & -2.299559 \\ \mathrm{H} & 0.000000 & 0.000000 & -2.694254\end{array}$

carbazole $\mathrm{S}_{1}$

$\begin{array}{lrrr}\mathrm{C} & 1.137857 & 0.000000 & -0.836071 \\ \mathrm{~N} & 0.00000 & 0.000000 & -1.598979 \\ \mathrm{C} & -1.137857 & 0.000000 & -0.836071 \\ \mathrm{C} & -0.715014 & 0.000000 & 0.538980 \\ \mathrm{C} & 0.715014 & 0.000000 & 0.538980 \\ \mathrm{C} & -1.702782 & 0.000000 & 1.523142 \\ \mathrm{C} & -3.054106 & 0.000000 & 1.116040 \\ \mathrm{C} & -3.440936 & 0.000000 & -0.224423 \\ \mathrm{C} & -2.461764 & 0.000000 & -1.240456 \\ \mathrm{C} & 2.461764 & 0.000000 & -1.240456 \\ \mathrm{C} & 3.440936 & 0.000000 & -0.224423 \\ \mathrm{C} & 3.054106 & 0.000000 & 1.116040 \\ \mathrm{C} & 1.702782 & 0.000000 & 1.523142\end{array}$




$\begin{array}{rrrr}\mathrm{H} & -2.731791 & 0.000000 & -2.286859 \\ \mathrm{H} & -4.487641 & 0.000000 & -0.484422 \\ \mathrm{H} & -3.820346 & 0.000000 & 1.878620 \\ \mathrm{H} & -1.449143 & 0.000000 & 2.572489 \\ \mathrm{H} & 1.449143 & 0.000000 & 2.572489 \\ \mathrm{H} & 3.820346 & 0.000000 & 1.878620 \\ \mathrm{H} & 4.487641 & 0.000000 & -0.484422 \\ \mathrm{H} & 2.731791 & 0.000000 & -2.286859 \\ \mathrm{H} & 0.000000 & 0.000000 & -2.607728\end{array}$

tryptamine, A-ph $\mathrm{S}_{0}$

$\begin{array}{lrrr}\mathrm{C} & -2.133535 & 0.146251 & 0.893353 \\ \mathrm{~N} & -1.252649 & 0.119945 & 1.951318 \\ \mathrm{C} & 0.026431 & 0.038109 & 1.465606 \\ \mathrm{C} & -0.057798 & 0.007899 & 0.061297 \\ \mathrm{C} & -1.457620 & 0.078822 & -0.285412 \\ \mathrm{C} & 1.250646 & -0.007419 & 2.140328 \\ \mathrm{C} & 2.392315 & -0.082122 & 1.373946 \\ \mathrm{C} & 2.331588 & -0.107438 & -0.033924 \\ \mathrm{C} & 1.122776 & -0.062218 & -0.692831 \\ \mathrm{C} & -2.030481 & 0.029724 & -1.672921 \\ \mathrm{C} & -1.990869 & -1.394526 & -2.246282 \\ \mathrm{~N} & -2.441768 & -1.381203 & -3.637778 \\ \mathrm{H} & 1.298840 & 0.015492 & 3.219709 \\ \mathrm{H} & 3.356495 & -0.118933 & 1.860355 \\ \mathrm{H} & 3.250509 & -0.159624 & -0.598737 \\ \mathrm{H} & 1.081068 & -0.076106 & -1.773108 \\ \mathrm{H} & -1.505148 & 0.176092 & 2.920741 \\ \mathrm{H} & -3.193777 & 0.216510 & 1.068075 \\ \mathrm{H} & -1.465605 & 0.681565 & -2.338349 \\ \mathrm{H} & -3.063474 & 0.380858 & -1.654136 \\ \mathrm{H} & -2.584980 & -2.044766 & -1.598254 \\ \mathrm{H} & -0.962071 & -1.748729 & -2.227832 \\ \mathrm{H} & -3.411354 & -1.105635 & -3.714219 \\ \mathrm{H} & -2.326947 & -2.280168 & -4.083575\end{array}$

tryptamine, A-ph $\mathrm{S}_{1}$

$\begin{array}{lrrr}\mathrm{C} & -2.182740 & 0.167636 & 0.886616 \\ \mathrm{~N} & -1.307516 & 0.104164 & 1.891887 \\ \mathrm{C} & 0.015063 & 0.056578 & 1.427581 \\ \mathrm{C} & -0.059449 & 0.129327 & 0.014945 \\ \mathrm{C} & -1.416004 & 0.183192 & -0.336543 \\ \mathrm{C} & 1.178571 & -0.112452 & 2.133484 \\ \mathrm{C} & 2.397029 & -0.156513 & 1.332186\end{array}$




$\begin{array}{rrrr}\mathrm{C} & 2.357152 & -0.086181 & -0.033370 \\ \mathrm{C} & 1.136814 & 0.056931 & -0.751445 \\ \mathrm{C} & -1.988381 & 0.091822 & -1.707037 \\ \mathrm{C} & -1.952427 & -1.377983 & -2.181802 \\ \mathrm{~N} & -2.497867 & -1.464910 & -3.533357 \\ \mathrm{H} & 1.205953 & -0.213568 & 3.206465 \\ \mathrm{H} & 3.343597 & -0.252826 & 1.841369 \\ \mathrm{H} & 3.282611 & -0.125328 & -0.589138 \\ \mathrm{H} & 1.124153 & 0.163487 & -1.824352 \\ \mathrm{H} & -1.554394 & 0.110911 & 2.869383 \\ \mathrm{H} & -3.241999 & 0.267115 & 1.043945 \\ \mathrm{H} & -1.400311 & 0.683720 & -2.406219 \\ \mathrm{H} & -3.018259 & 0.451213 & -1.714780 \\ \mathrm{H} & -2.482479 & -1.995812 & -1.451365 \\ \mathrm{H} & -0.910355 & -1.691586 & -2.205488 \\ \mathrm{H} & -3.496758 & -1.311510 & -3.548602 \\ \mathrm{H} & -2.299432 & -2.355049 & -3.967036\end{array}$

tryptamine, A-py $\mathrm{S}_{0}$

$\begin{array}{lrrr}\mathrm{C} & -2.125886 & 0.121553 & 0.864618 \\ \mathrm{~N} & -1.257370 & 0.109687 & 1.932450 \\ \mathrm{C} & 0.027908 & 0.039305 & 1.462291 \\ \mathrm{C} & -0.039354 & 0.000889 & 0.056777 \\ \mathrm{C} & -1.436101 & 0.054420 & -0.306266 \\ \mathrm{C} & 1.243929 & 0.009575 & 2.152476 \\ \mathrm{C} & 2.396223 & -0.057204 & 1.401466 \\ \mathrm{C} & 2.353329 & -0.090849 & -0.006736 \\ \mathrm{C} & 1.152143 & -0.062043 & -0.680543 \\ \mathrm{C} & -2.006698 & 0.000691 & -1.695008 \\ \mathrm{C} & -1.939007 & -1.418098 & -2.277995 \\ \mathrm{~N} & -2.483721 & -1.417892 & -3.636331 \\ \mathrm{H} & 1.277937 & 0.038009 & 3.232301 \\ \mathrm{H} & 3.354349 & -0.081833 & 1.900457 \\ \mathrm{H} & 3.279626 & -0.137919 & -0.559868 \\ \mathrm{H} & 1.126223 & -0.085138 & -1.761048 \\ \mathrm{H} & -1.521786 & 0.167073 & 2.898635 \\ \mathrm{H} & -3.188955 & 0.181859 & 1.025209 \\ \mathrm{H} & -1.450900 & 0.682449 & -2.341995 \\ \mathrm{H} & -3.047694 & 0.318835 & -1.688860 \\ \mathrm{H} & -2.559989 & -2.066064 & -1.662456 \\ \mathrm{H} & -0.909233 & -1.779758 & -2.220599 \\ \mathrm{H} & -1.924012 & -0.854655 & -4.262048 \\ \mathrm{H} & -2.551050 & -2.350503 & -4.017818\end{array}$

tryptamine, A-py $\mathrm{S}_{1}$ 


$\begin{array}{lrrr}\mathrm{C} & -2.179028 & 0.135296 & 0.867832 \\ \mathrm{~N} & -1.311641 & 0.081855 & 1.879283 \\ \mathrm{C} & 0.015480 & 0.054397 & 1.424919 \\ \mathrm{C} & -0.048988 & 0.129965 & 0.012515 \\ \mathrm{C} & -1.403301 & 0.164483 & -0.349136 \\ \mathrm{C} & 1.175854 & -0.102948 & 2.139967 \\ \mathrm{C} & 2.399721 & -0.131468 & 1.347152 \\ \mathrm{C} & 2.370279 & -0.057653 & -0.018746 \\ \mathrm{C} & 1.154055 & 0.073800 & -0.745805 \\ \mathrm{C} & -1.967763 & 0.066464 & -1.721976 \\ \mathrm{C} & -1.897003 & -1.401761 & -2.199346 \\ \mathrm{~N} & -2.478387 & -1.505745 & -3.534695 \\ \mathrm{H} & 1.196582 & -0.206476 & 3.212725 \\ \mathrm{H} & 3.343611 & -0.218773 & 1.863056 \\ \mathrm{H} & 3.300905 & -0.085559 & -0.566520 \\ \mathrm{H} & 1.149050 & 0.184706 & -1.818156 \\ \mathrm{H} & -1.566349 & 0.082268 & 2.854877 \\ \mathrm{H} & -3.241320 & 0.215706 & 1.015365 \\ \mathrm{H} & -1.382722 & 0.687139 & -2.402188 \\ \mathrm{H} & -3.006762 & 0.389403 & -1.748036 \\ \mathrm{H} & -2.492372 & -2.009153 & -1.519069 \\ \mathrm{H} & -0.856062 & -1.728820 & -2.137348 \\ \mathrm{H} & -1.915475 & -1.027162 & -4.225059 \\ \mathrm{H} & -2.588455 & -2.467586 & -3.822510\end{array}$

tryptamine, A-up $\mathrm{S}_{0}$

$\begin{array}{rrrc}\mathrm{C} & -2.124916 & 0.139114 & 0.880848 \\ \mathrm{~N} & -1.253182 & 0.100251 & 1.945235 \\ \mathrm{C} & 0.030079 & 0.030390 & 1.469490 \\ \mathrm{C} & -0.041641 & 0.020808 & 0.063956 \\ \mathrm{C} & -1.438893 & 0.093167 & -0.293492 \\ \mathrm{C} & 1.247854 & -0.021463 & 2.155179 \\ \mathrm{C} & 2.397129 & -0.081776 & 1.399233 \\ \mathrm{C} & 2.349691 & -0.087605 & -0.009266 \\ \mathrm{C} & 1.146986 & -0.036210 & -0.678607 \\ \mathrm{C} & -2.007970 & 0.060499 & -1.683214 \\ \mathrm{C} & -1.949871 & -1.357573 & -2.289856 \\ \mathrm{~N} & -2.433696 & -1.456780 & -3.662622 \\ \mathrm{H} & 1.285211 & -0.014781 & 3.235201 \\ \mathrm{H} & 3.356644 & -0.123594 & 1.894268 \\ \mathrm{H} & 3.273852 & -0.131617 & -0.566118 \\ \mathrm{H} & 1.117975 & -0.038997 & -1.759237 \\ \mathrm{H} & -1.514354 & 0.136903 & 2.913345 \\ \mathrm{H} & -3.186889 & 0.202359 & 1.047531\end{array}$




$\begin{array}{llll}\mathrm{H} & -1.450537 & 0.745882 & -2.324380 \\ \mathrm{H} & -3.046235 & 0.394492 & -1.661588 \\ \mathrm{H} & -2.542287 & -2.026188 & -1.667545 \\ \mathrm{H} & -0.923482 & -1.718766 & -2.262197 \\ \mathrm{H} & -1.886275 & -0.894423 & -4.299148 \\ \mathrm{H} & -3.403954 & -1.186179 & -3.743773\end{array}$

tryptamine, A-up $\mathrm{S}_{1}$

$\begin{array}{lrrr}\mathrm{C} & -2.175807 & 0.151358 & 0.877718 \\ \mathrm{~N} & -1.306217 & 0.067038 & 1.886118 \\ \mathrm{C} & 0.019926 & 0.042585 & 1.430515 \\ \mathrm{C} & -0.045084 & 0.152210 & 0.020140 \\ \mathrm{C} & -1.400561 & 0.207926 & -0.339198 \\ \mathrm{C} & 1.180322 & -0.132515 & 2.140683 \\ \mathrm{C} & 2.403637 & -0.149598 & 1.347253 \\ \mathrm{C} & 2.373102 & -0.044590 & -0.015971 \\ \mathrm{C} & 1.155966 & 0.109219 & -0.738703 \\ \mathrm{C} & -1.961164 & 0.131724 & -1.712257 \\ \mathrm{C} & -1.935012 & -1.351091 & -2.193090 \\ \mathrm{~N} & -2.441751 & -1.561251 & -3.537615 \\ \mathrm{H} & 1.201201 & -0.259814 & 3.210999 \\ \mathrm{H} & 3.347096 & -0.255260 & 1.860295 \\ \mathrm{H} & 3.302437 & -0.065834 & -0.566205 \\ \mathrm{H} & 1.151303 & 0.238884 & -1.809015 \\ \mathrm{H} & -1.559775 & 0.043514 & 2.861720 \\ \mathrm{H} & -3.236910 & 0.235951 & 1.030981 \\ \mathrm{H} & -1.354901 & 0.728187 & -2.393979 \\ \mathrm{H} & -2.987987 & 0.497833 & -1.733166 \\ \mathrm{H} & -2.524327 & -1.950278 & -1.501106 \\ \mathrm{H} & -0.905701 & -1.701779 & -2.143502 \\ \mathrm{H} & -1.888306 & -1.092745 & -4.240628 \\ \mathrm{H} & -3.410266 & -1.293755 & -3.638768\end{array}$

tryptamine, $\mathrm{Ph}$-out $\mathrm{S}_{0}$

$\begin{array}{crrr}\mathrm{C} & -0.005760 & 0.003631 & 0.066715 \\ \mathrm{C} & 0.037600 & 0.053621 & 1.472724 \\ \mathrm{C} & 1.242495 & 0.054855 & 2.183771 \\ \mathrm{C} & 2.407210 & 0.007073 & 1.451312 \\ \mathrm{C} & 2.387099 & -0.043653 & 0.042198 \\ \mathrm{C} & 1.198942 & -0.046633 & -0.654057 \\ \mathrm{~N} & -1.256346 & 0.099995 & 1.922296 \\ \mathrm{H} & 1.257560 & 0.091755 & 3.263808 \\ \mathrm{H} & 3.357735 & 0.006934 & 1.964749 \\ \mathrm{H} & 3.322902 & -0.082492 & -0.495543\end{array}$




$\begin{array}{lrrr}\mathrm{H} & 1.180458 & -0.108181 & -1.732879 \\ \mathrm{C} & -1.398496 & 0.026483 & -0.319345 \\ \mathrm{C} & -2.107816 & 0.087418 & 0.841385 \\ \mathrm{H} & -1.537618 & 0.150732 & 2.883971 \\ \mathrm{H} & -3.174255 & 0.136854 & 0.986088 \\ \mathrm{C} & -1.959083 & -0.018281 & -1.715529 \\ \mathrm{H} & -1.311733 & 0.547811 & -2.384067 \\ \mathrm{C} & -2.076074 & -1.452732 & -2.252224 \\ \mathrm{H} & -2.946920 & 0.442820 & -1.717020 \\ \mathrm{H} & -2.478866 & -1.413573 & -3.263432 \\ \mathrm{~N} & -0.752566 & -2.068068 & -2.319818 \\ \mathrm{H} & -2.774931 & -2.004633 & -1.616810 \\ \mathrm{H} & -0.349635 & -2.176723 & -1.397296 \\ \mathrm{H} & -0.776931 & -2.970902 & -2.771789\end{array}$

tryptamine, $\mathrm{Ph}$-out $\mathrm{S}_{1}$

$\begin{array}{lrrr}\mathrm{C} & -0.001605 & 0.026423 & 0.033260 \\ \mathrm{C} & 0.037110 & 0.046965 & 1.451390 \\ \mathrm{C} & 1.187803 & 0.019418 & 2.195502 \\ \mathrm{C} & 2.431591 & 0.002123 & 1.431985 \\ \mathrm{C} & 2.427125 & -0.025882 & 0.064972 \\ \mathrm{C} & 1.222823 & -0.029931 & -0.692985 \\ \mathrm{~N} & -1.296885 & 0.079933 & 1.878896 \\ \mathrm{H} & 1.190052 & 0.029234 & 3.273861 \\ \mathrm{H} & 3.366148 & 0.006330 & 1.971356 \\ \mathrm{H} & 3.368769 & -0.045326 & -0.464280 \\ \mathrm{H} & 1.231296 & -0.065466 & -1.770321 \\ \mathrm{C} & -1.349621 & 0.062973 & -0.355485 \\ \mathrm{C} & -2.147141 & 0.082366 & 0.849959 \\ \mathrm{H} & -1.569866 & 0.104346 & 2.848769 \\ \mathrm{H} & -3.214296 & 0.127535 & 0.974947 \\ \mathrm{C} & -1.923036 & -0.000090 & -1.729784 \\ \mathrm{H} & -1.251816 & 0.488815 & -2.433376 \\ \mathrm{C} & -2.101563 & -1.468659 & -2.179461 \\ \mathrm{H} & -2.892814 & 0.499150 & -1.739852 \\ \mathrm{H} & -2.614613 & -1.465037 & -3.139666 \\ \mathrm{~N} & -0.792916 & -2.078734 & -2.363935 \\ \mathrm{H} & -2.731575 & -1.981914 & -1.446312 \\ \mathrm{H} & -0.235492 & -2.062771 & -1.517165 \\ \mathrm{H} & -0.854487 & -3.027680 & -2.703063\end{array}$

tryptamine, $\mathrm{Ph}$-up $\mathrm{S}_{0}$

$\begin{array}{cccc}\mathrm{C} & -2.084656 & 0.031084 & 0.805550 \\ \mathrm{~N} & -1.247434 & 0.009062 & 1.896836\end{array}$ 


$\begin{array}{lrrc}\mathrm{C} & 0.052259 & 0.024052 & 1.463896 \\ \mathrm{C} & 0.026553 & 0.054470 & 0.056331 \\ \mathrm{C} & -1.359678 & 0.056198 & -0.347360 \\ \mathrm{C} & 1.247750 & 0.015720 & 2.190503 \\ \mathrm{C} & 2.423078 & 0.041364 & 1.474652 \\ \mathrm{C} & 2.422515 & 0.077769 & 0.064808 \\ \mathrm{C} & 1.242651 & 0.084833 & -0.644413 \\ \mathrm{C} & -1.900505 & 0.035969 & -1.751016 \\ \mathrm{C} & -2.101352 & -1.405814 & -2.269498 \\ \mathrm{~N} & -0.902287 & -2.232299 & -2.280908 \\ \mathrm{H} & 1.247605 & -0.010968 & 3.271204 \\ \mathrm{H} & 3.366240 & 0.034229 & 2.001129 \\ \mathrm{H} & 3.365852 & 0.101960 & -0.460312 \\ \mathrm{H} & 1.250613 & 0.115830 & -1.724930 \\ \mathrm{H} & -1.541380 & -0.002786 & 2.856040 \\ \mathrm{H} & -3.153807 & 0.033591 & 0.937559 \\ \mathrm{H} & -1.211352 & 0.564603 & -2.410883 \\ \mathrm{H} & -2.856187 & 0.561235 & -1.779301 \\ \mathrm{H} & -2.499127 & -1.370281 & -3.283201 \\ \mathrm{H} & -2.840713 & -1.898317 & -1.639077 \\ \mathrm{H} & -0.486897 & -2.292684 & -1.359812 \\ \mathrm{H} & -0.206093 & -1.879188 & -2.922739\end{array}$

tryptamine, $\mathrm{Ph}$-up $\mathrm{S}_{1}$

$\begin{array}{rrrr}\mathrm{C} & -2.150559 & 0.108316 & 0.841430 \\ \mathrm{~N} & -1.303325 & 0.068292 & 1.870514 \\ \mathrm{C} & 0.032051 & 0.050934 & 1.443746 \\ \mathrm{C} & -0.004438 & 0.097913 & 0.027400 \\ \mathrm{C} & -1.352137 & 0.129307 & -0.361875 \\ \mathrm{C} & 1.181137 & -0.036981 & 2.187694 \\ \mathrm{C} & 2.423617 & -0.033754 & 1.424641 \\ \mathrm{C} & 2.423698 & 0.015266 & 0.058098 \\ \mathrm{C} & 1.220714 & 0.078120 & -0.700406 \\ \mathrm{C} & -1.911168 & 0.041551 & -1.734421 \\ \mathrm{C} & -2.040854 & -1.456923 & -2.164099 \\ \mathrm{~N} & -0.787806 & -2.168927 & -2.292323 \\ \mathrm{H} & 1.181212 & -0.095788 & 3.264505 \\ \mathrm{H} & 3.357492 & -0.074451 & 1.963997 \\ \mathrm{H} & 3.367379 & 0.015863 & -0.467757 \\ \mathrm{H} & 1.238116 & 0.160793 & -1.775047 \\ \mathrm{H} & -1.577608 & 0.065455 & 2.840323 \\ \mathrm{H} & -3.217825 & 0.154475 & 0.966202 \\ \mathrm{H} & -1.254372 & 0.552195 & -2.437744 \\ \mathrm{H} & -2.899042 & 0.504415 & -1.764909 \\ \mathrm{H} & -2.557416 & -1.488858 & -3.122197\end{array}$




$$
\begin{array}{llll}
\mathrm{H} & -2.660207 & -1.970481 & -1.430629 \\
\mathrm{H} & -0.235922 & -2.150569 & -1.442722 \\
\mathrm{H} & -0.219076 & -1.816554 & -3.049329
\end{array}
$$

tryptamine, Py-out $\mathrm{S}_{0}$

$\begin{array}{lrrr}\mathrm{C} & 0.512748 & 0.465069 & 0.205299 \\ \mathrm{C} & 0.578994 & 0.510782 & 1.610798 \\ \mathrm{C} & 1.791835 & 0.451948 & 2.303932 \\ \mathrm{C} & 2.943776 & 0.348503 & 1.555890 \\ \mathrm{C} & 2.901903 & 0.306073 & 0.147789 \\ \mathrm{C} & 1.703717 & 0.364696 & -0.529296 \\ \mathrm{~N} & -0.704871 & 0.618277 & 2.079285 \\ \mathrm{H} & 1.823386 & 0.488060 & 3.383692 \\ \mathrm{H} & 3.899980 & 0.301221 & 2.056697 \\ \mathrm{H} & 3.827181 & 0.229315 & -0.403523 \\ \mathrm{H} & 1.679815 & 0.335694 & -1.609714 \\ \mathrm{C} & -0.881723 & 0.556564 & -0.159737 \\ \mathrm{C} & -1.571866 & 0.644508 & 1.010186 \\ \mathrm{H} & -0.968489 & 0.675480 & 3.045530 \\ \mathrm{H} & -2.635224 & 0.721491 & 1.163238 \\ \mathrm{C} & -1.455693 & 0.497529 & -1.547102 \\ \mathrm{H} & -0.890757 & 1.157880 & -2.205839 \\ \mathrm{C} & -1.423724 & -0.934512 & -2.105857 \\ \mathrm{H} & -2.492492 & 0.829168 & -1.523966 \\ \mathrm{H} & -0.383741 & -1.265119 & -2.179210 \\ \mathrm{~N} & -2.239388 & -1.799457 & -1.256893 \\ \mathrm{H} & -1.859144 & -0.928918 & -3.103833 \\ \mathrm{H} & -2.295249 & -2.741062 & -1.618523 \\ \mathrm{H} & -1.860956 & -1.833191 & -0.318823\end{array}$

tryptamine, Py-out $\mathrm{S}_{1}$

$\begin{array}{lrrr}\mathrm{C} & 0.503946 & 0.494692 & 0.173945 \\ \mathrm{C} & 0.574734 & 0.502680 & 1.587905 \\ \mathrm{C} & 1.739762 & 0.406730 & 2.307622 \\ \mathrm{C} & 2.959926 & 0.326366 & 1.510536 \\ \mathrm{C} & 2.924435 & 0.324695 & 0.143507 \\ \mathrm{C} & 1.704119 & 0.405122 & -0.585727 \\ \mathrm{~N} & -0.748148 & 0.594584 & 2.046429 \\ \mathrm{H} & 1.767923 & 0.402251 & 3.385402 \\ \mathrm{H} & 3.906333 & 0.268540 & 2.025763 \\ \mathrm{H} & 3.852660 & 0.265821 & -0.405574 \\ \mathrm{H} & 1.692885 & 0.424648 & -1.663489 \\ \mathrm{C} & -0.850271 & 0.587521 & -0.183359 \\ \mathrm{C} & -1.618544 & 0.640443 & 1.037784\end{array}$




$\begin{array}{lrrr}\mathrm{H} & -0.997027 & 0.622414 & 3.022678 \\ \mathrm{H} & -2.682803 & 0.702192 & 1.179320 \\ \mathrm{C} & -1.426423 & 0.520477 & -1.555080 \\ \mathrm{H} & -0.827856 & 1.138335 & -2.225152 \\ \mathrm{C} & -1.406309 & -0.939174 & -2.059675 \\ \mathrm{H} & -2.457190 & 0.870534 & -1.549097 \\ \mathrm{H} & -0.366019 & -1.268315 & -2.115955 \\ \mathrm{~N} & -2.228472 & -1.768823 & -1.182188 \\ \mathrm{H} & -1.842589 & -0.961877 & -3.056098 \\ \mathrm{H} & -2.376576 & -2.690364 & -1.568471 \\ \mathrm{H} & -1.798506 & -1.869502 & -0.271035\end{array}$

tryptamine, Py-up $\mathrm{S}_{0}$

$\begin{array}{lrrr}\mathrm{C} & -2.130818 & 0.259734 & 0.874176 \\ \mathrm{~N} & -1.260024 & 0.218324 & 1.939830 \\ \mathrm{C} & 0.019151 & 0.084282 & 1.465581 \\ \mathrm{C} & -0.054217 & 0.037834 & 0.060985 \\ \mathrm{C} & -1.448248 & 0.154621 & -0.298822 \\ \mathrm{C} & 1.233904 & 0.003606 & 2.153527 \\ \mathrm{C} & 2.379846 & -0.122529 & 1.400179 \\ \mathrm{C} & 2.330858 & -0.166207 & -0.007697 \\ \mathrm{C} & 1.131174 & -0.086334 & -0.679291 \\ \mathrm{C} & -2.019838 & 0.104012 & -1.685837 \\ \mathrm{C} & -1.981695 & -1.330206 & -2.263362 \\ \mathrm{~N} & -2.734959 & -2.311904 & -1.495575 \\ \mathrm{H} & 1.271171 & 0.040107 & 3.232981 \\ \mathrm{H} & 3.337336 & -0.187692 & 1.896632 \\ \mathrm{H} & 3.251997 & -0.261766 & -0.562955 \\ \mathrm{H} & 1.102105 & -0.116959 & -1.759469 \\ \mathrm{H} & -1.517624 & 0.283653 & 2.907146 \\ \mathrm{H} & -3.190057 & 0.369051 & 1.035883 \\ \mathrm{H} & -1.457935 & 0.773487 & -2.339504 \\ \mathrm{H} & -3.055083 & 0.447754 & -1.662845 \\ \mathrm{H} & -0.948334 & -1.669898 & -2.311244 \\ \mathrm{H} & -2.374414 & -1.316681 & -3.279221 \\ \mathrm{H} & -3.717664 & -2.077220 & -1.450929 \\ \mathrm{H} & -2.378110 & -2.379448 & -0.551280\end{array}$

tryptamine, Py-up $\mathrm{S}_{1}$
$\begin{array}{llll}\text { C } & -2.188574 & 0.278984 & 0.896853\end{array}$
$\begin{array}{llll}\mathrm{N} & -1.312448 & 0.215310 & 1.902073\end{array}$
$\begin{array}{llll}\text { C } & 0.004209 & 0.089769 & 1.437209\end{array}$
$\begin{array}{llll}\text { C } & -0.074049 & 0.091397 & 0.022639\end{array}$
$\begin{array}{llll}\text { C } & -1.428482 & 0.205873 & -0.328154\end{array}$ 


$\begin{array}{lrrr}\mathrm{C} & 1.166918 & -0.056675 & 2.149311 \\ \mathrm{C} & 2.382266 & -0.174173 & 1.348572 \\ \mathrm{C} & 2.339628 & -0.166297 & -0.017754 \\ \mathrm{C} & 1.119860 & -0.034457 & -0.740022 \\ \mathrm{C} & -2.006418 & 0.127177 & -1.696187 \\ \mathrm{C} & -1.949061 & -1.339389 & -2.214474 \\ \mathrm{~N} & -2.685080 & -2.302207 & -1.412996 \\ \mathrm{H} & 1.197732 & -0.080437 & 3.226682 \\ \mathrm{H} & 3.327372 & -0.270266 & 1.860239 \\ \mathrm{H} & 3.261819 & -0.254655 & -0.573100 \\ \mathrm{H} & 1.107646 & 0.002758 & -1.817323 \\ \mathrm{H} & -1.554012 & 0.262095 & 2.879403 \\ \mathrm{H} & -3.245146 & 0.408702 & 1.049896 \\ \mathrm{H} & -1.427916 & 0.761046 & -2.370629 \\ \mathrm{H} & -3.042304 & 0.468259 & -1.689446 \\ \mathrm{H} & -0.906497 & -1.651192 & -2.241206 \\ \mathrm{H} & -2.340086 & -1.362384 & -3.230050 \\ \mathrm{H} & -3.666288 & -2.072940 & -1.336814 \\ \mathrm{H} & -2.292570 & -2.396668 & -0.485808\end{array}$

porphycene $\mathrm{S}_{0}$

$\begin{array}{lrrr}\mathrm{C} & -3.413519 & 1.867761 & 0.000047 \\ \mathrm{C} & -2.356483 & 0.883850 & 0.000012 \\ \mathrm{~N} & -1.183131 & 1.541563 & -0.000043 \\ \mathrm{C} & -1.391332 & 2.881472 & -0.000010 \\ \mathrm{C} & -2.822048 & 3.090255 & 0.000026 \\ \mathrm{C} & -0.408330 & 3.864715 & -0.000014 \\ \mathrm{C} & 0.978451 & 3.727715 & -0.000026 \\ \mathrm{C} & 1.776054 & 2.584130 & -0.000013 \\ \mathrm{H} & -0.151880 & 1.164188 & -0.000114 \\ \mathrm{~N} & 1.359084 & 1.293574 & 0.000011 \\ \mathrm{C} & 3.234349 & 2.610694 & -0.000018 \\ \mathrm{C} & 3.648464 & 1.324554 & 0.000004 \\ \mathrm{C} & 2.440209 & 0.514834 & 0.000008 \\ \mathrm{C} & 2.360707 & -0.889714 & 0.000019 \\ \mathrm{C} & 3.409025 & -1.862630 & 0.000075 \\ \mathrm{C} & 2.813191 & -3.093811 & 0.000062 \\ \mathrm{C} & 1.395780 & -2.883277 & -0.000011 \\ \mathrm{~N} & 1.185220 & -1.548072 & -0.000046 \\ \mathrm{C} & 0.395147 & -3.871706 & -0.000046 \\ \mathrm{C} & -0.975765 & -3.735694 & -0.000050 \\ \mathrm{C} & -1.781657 & -2.576208 & -0.000021 \\ \mathrm{C} & -3.236654 & -2.605965 & 0.000010 \\ \mathrm{C} & -3.652945 & -1.317120 & 0.000039 \\ \mathrm{C} & -2.449421 & -0.510643 & 0.000012\end{array}$




$\begin{array}{lrrr}\mathrm{N} & -1.361904 & -1.300700 & -0.000009 \\ \mathrm{H} & 0.158748 & -1.171501 & -0.000132 \\ \mathrm{H} & 3.291886 & -4.057591 & 0.000098 \\ \mathrm{H} & 4.462329 & -1.643152 & 0.000124 \\ \mathrm{H} & 0.775748 & -4.884086 & -0.000062 \\ \mathrm{H} & -1.537458 & -4.660336 & -0.000073 \\ \mathrm{H} & -3.837318 & -3.500418 & 0.000011 \\ \mathrm{H} & -4.662198 & -0.943787 & 0.000064 \\ \mathrm{H} & -4.466025 & 1.644226 & 0.000076 \\ \mathrm{H} & -3.298393 & 4.055446 & 0.000039 \\ \mathrm{H} & -0.787068 & 4.877432 & 0.000012 \\ \mathrm{H} & 1.534789 & 4.655642 & -0.000039 \\ \mathrm{H} & 3.834926 & 3.505376 & -0.000032 \\ \mathrm{H} & 4.656326 & 0.947890 & 0.000009\end{array}$

porphycene $\mathrm{S}_{1}$

$\begin{array}{lrrr}\mathrm{C} & -3.429958 & 1.868229 & 0.000045 \\ \mathrm{C} & -2.392145 & 0.886792 & 0.000008 \\ \mathrm{~N} & -1.196877 & 1.554197 & -0.000050 \\ \mathrm{C} & -1.408758 & 2.876652 & -0.000013 \\ \mathrm{C} & -2.824377 & 3.096991 & 0.000025 \\ \mathrm{C} & -0.391677 & 3.884782 & -0.000012 \\ \mathrm{C} & 0.961782 & 3.744376 & -0.000025 \\ \mathrm{C} & 1.789474 & 2.566892 & -0.000012 \\ \mathrm{H} & -0.181363 & 1.170847 & -0.000120 \\ \mathrm{~N} & 1.370440 & 1.301903 & 0.000010 \\ \mathrm{C} & 3.237214 & 2.609599 & -0.000017 \\ \mathrm{C} & 3.666754 & 1.318916 & 0.000008 \\ \mathrm{C} & 2.478033 & 0.506643 & 0.000008 \\ \mathrm{C} & 2.388737 & -0.887833 & 0.000015 \\ \mathrm{C} & 3.426570 & -1.869300 & 0.000072 \\ \mathrm{C} & 2.821026 & -3.097979 & 0.000057 \\ \mathrm{C} & 1.405387 & -2.877620 & -0.000013 \\ \mathrm{~N} & 1.193415 & -1.555295 & -0.000050 \\ \mathrm{C} & 0.388385 & -3.886109 & -0.000044 \\ \mathrm{C} & -0.964987 & -3.745633 & -0.000049 \\ \mathrm{C} & -1.792705 & -2.568033 & -0.000023 \\ \mathrm{C} & -3.240345 & -2.610670 & 0.000011 \\ \mathrm{C} & -3.669909 & -1.319957 & 0.000040 \\ \mathrm{C} & -2.481282 & -0.507722 & 0.000009 \\ \mathrm{~N} & -1.373655 & -1.302990 & -0.000012 \\ \mathrm{H} & 0.177141 & -1.172136 & -0.000133 \\ \mathrm{H} & 3.291049 & -4.066016 & 0.000094 \\ \mathrm{H} & 4.480920 & -1.655726 & 0.000121 \\ \mathrm{H} & 0.774651 & -4.895554 & -0.000053\end{array}$




$\begin{array}{rrrr}H & -1.533632 & -4.666601 & -0.000066 \\ H & -3.834697 & -3.509405 & 0.000015 \\ H & -4.681988 & -0.955051 & 0.000068 \\ H & -4.484306 & 1.654645 & 0.000075 \\ H & -3.294314 & 4.065063 & 0.000040 \\ H & -0.777868 & 4.894288 & 0.000019 \\ H & 1.530389 & 4.665367 & -0.000038 \\ H & 3.831553 & 3.508343 & -0.000030 \\ H & 4.678829 & 0.954008 & 0.000015\end{array}$

porphine $\mathrm{S}_{0}$

$\begin{array}{rrrr}\mathrm{N} & -0.000191 & 2.016498 & -0.025205 \\ \mathrm{~N} & -0.000086 & -0.000001 & 2.087514 \\ \mathrm{C} & -0.000144 & 2.837133 & 1.078293 \\ \mathrm{C} & -0.000180 & 4.241636 & 0.662319 \\ \mathrm{C} & 0.000011 & 2.439249 & 2.383744 \\ \mathrm{C} & 0.000066 & 1.112174 & 2.862532 \\ \mathrm{C} & 0.000283 & 0.689289 & 4.211807 \\ \mathrm{H} & -0.000135 & 5.084155 & 1.333913 \\ \mathrm{H} & 0.000108 & 3.210728 & 3.141600 \\ \mathrm{H} & 0.000439 & 1.350206 & 5.061409 \\ \mathrm{H} & -0.000256 & -0.000001 & 1.072695 \\ \mathrm{~N} & -0.000017 & -2.016503 & -0.025206 \\ \mathrm{~N} & 0.000127 & -0.000001 & -2.099811 \\ \mathrm{C} & -0.000111 & -2.837132 & 1.078296 \\ \mathrm{C} & -0.000067 & -2.818254 & -1.076544 \\ \mathrm{C} & -0.000226 & 2.818251 & -1.076541 \\ \mathrm{C} & -0.000218 & -4.241635 & 0.662320 \\ \mathrm{C} & -0.000208 & -4.233291 & -0.679558 \\ \mathrm{C} & -0.000246 & 4.233289 & -0.679558 \\ \mathrm{C} & -0.000085 & -2.439248 & 2.383747 \\ \mathrm{C} & 0.000060 & -2.415655 & -2.436185 \\ \mathrm{C} & -0.000080 & 2.415654 & -2.436180 \\ \mathrm{C} & 0.000023 & -1.112174 & 2.862533 \\ \mathrm{C} & 0.000139 & -1.123989 & -2.871768 \\ \mathrm{C} & 0.000074 & 1.123989 & -2.871766 \\ \mathrm{C} & 0.000258 & -0.689288 & 4.211808 \\ \mathrm{C} & 0.000271 & -0.672087 & -4.260059 \\ \mathrm{C} & 0.000232 & 0.672090 & -4.260058 \\ \mathrm{H} & -0.000293 & -5.084153 & 1.333914 \\ \mathrm{H} & -0.000266 & -5.066548 & -1.361994 \\ \mathrm{H} & -0.000265 & 5.066546 & -1.361996 \\ \mathrm{H} & -0.000157 & -3.210727 & 3.141604 \\ \mathrm{H} & 0.000093 & -3.191936 & -3.186405 \\ \mathrm{H} & -0.000094 & 3.191937 & -3.186399\end{array}$




$\begin{array}{rrrr}\mathrm{H} & 0.000387 & -1.350204 & 5.061411 \\ \mathrm{H} & 0.000376 & -1.339747 & -5.104912 \\ \mathrm{H} & 0.000297 & 1.339751 & -5.104910 \\ \mathrm{H} & 0.000083 & -0.000002 & -1.086400\end{array}$

porphine $\mathrm{S}_{1}$

$\begin{array}{lrrr}\mathrm{N} & 0.000000 & -2.035396 & 0.000000 \\ \mathrm{~N} & 2.092271 & 0.000000 & 0.000000 \\ \mathrm{C} & 1.080167 & -2.846937 & 0.000000 \\ \mathrm{C} & 0.671393 & -4.256214 & 0.000000 \\ \mathrm{C} & 2.413511 & -2.436162 & 0.000000 \\ \mathrm{C} & 2.869708 & -1.121438 & 0.000000 \\ \mathrm{C} & 4.236096 & -0.682398 & 0.000000 \\ \mathrm{H} & 1.347115 & -5.094993 & 0.000000 \\ \mathrm{H} & 3.171019 & -3.206456 & 0.000000 \\ \mathrm{H} & 5.084544 & -1.345019 & 0.000000 \\ \mathrm{H} & 1.079961 & 0.000000 & 0.000000 \\ \mathrm{~N} & 0.000000 & 2.035396 & 0.000000 \\ \mathrm{~N} & -2.092271 & 0.000000 & 0.000000 \\ \mathrm{C} & 1.080167 & 2.846937 & 0.000000 \\ \mathrm{C} & -1.080167 & 2.846937 & 0.000000 \\ \mathrm{C} & -1.080167 & -2.846937 & 0.000000 \\ \mathrm{C} & 0.671393 & 4.256214 & 0.000000 \\ \mathrm{C} & -0.671393 & 4.256214 & 0.000000 \\ \mathrm{C} & -0.671393 & -4.256214 & 0.000000 \\ \mathrm{C} & 2.413511 & 2.436162 & 0.000000 \\ \mathrm{C} & -2.413511 & 2.436162 & 0.000000 \\ \mathrm{C} & -2.413511 & -2.436162 & 0.000000 \\ \mathrm{C} & 2.869708 & 1.121438 & 0.000000 \\ \mathrm{C} & -2.869708 & 1.121438 & 0.000000 \\ \mathrm{C} & -2.869708 & -1.121438 & 0.000000 \\ \mathrm{C} & 4.236096 & 0.682398 & 0.000000 \\ \mathrm{C} & -4.236096 & 0.682398 & 0.000000 \\ \mathrm{C} & -4.236096 & -0.682398 & 0.000000 \\ \mathrm{H} & 1.347115 & 5.094993 & 0.000000 \\ \mathrm{H} & -1.347115 & 5.094993 & 0.000000 \\ \mathrm{H} & -1.347115 & -5.094993 & 0.000000 \\ \mathrm{H} & 3.171019 & 3.206456 & 0.000000 \\ \mathrm{H} & -3.171019 & 3.206456 & 0.000000 \\ \mathrm{H} & -3.171019 & -3.206456 & 0.000000 \\ \mathrm{H} & 5.084544 & 1.345019 & 0.000000 \\ \mathrm{H} & -5.084544 & 1.345019 & 0.000000 \\ \mathrm{H} & -5.084544 & -1.345019 & 0.000000 \\ \mathrm{H} & -1.079961 & 0.000000 & 0.000000\end{array}$


chlorin $\mathrm{S}_{0}$

$\begin{array}{lrrr}\mathrm{C} & 0.000000 & 4.205509 & 0.763144 \\ \mathrm{C} & 0.000000 & 2.863143 & 1.185260 \\ \mathrm{~N} & 0.000000 & 2.087387 & 0.057805 \\ \mathrm{C} & 0.000000 & 2.862090 & -1.042643 \\ \mathrm{C} & 0.000000 & 4.207214 & -0.620493 \\ \mathrm{C} & 0.000000 & 2.385067 & 2.504382 \\ \mathrm{C} & 0.000000 & 1.081154 & 2.920852 \\ \mathrm{~N} & 0.000000 & -0.028836 & 2.103702 \\ \mathrm{C} & 0.000000 & -1.072410 & 2.910563 \\ \mathrm{C} & 0.000000 & -0.675231 & 4.314104 \\ \mathrm{C} & 0.000000 & 0.673537 & 4.316559 \\ \mathrm{C} & 0.000000 & -2.442984 & 2.493667 \\ \mathrm{C} & 0.000000 & -2.868006 & 1.211460 \\ \mathrm{~N} & 0.000000 & -2.082664 & 0.079541 \\ \mathrm{C} & 0.000000 & -2.841122 & -1.042473 \\ \mathrm{C} & 0.000000 & -4.239179 & -0.599265 \\ \mathrm{C} & 0.000000 & -4.252594 & 0.741622 \\ \mathrm{C} & 0.000000 & -2.421220 & -2.342810 \\ \mathrm{C} & 0.000000 & -1.075733 & -2.785932 \\ \mathrm{C} & 0.000000 & -0.781007 & -4.283443 \\ \mathrm{C} & 0.000000 & 0.755819 & -4.316351 \\ \mathrm{C} & 0.000000 & 1.119603 & -2.831362 \\ \mathrm{~N} & 0.000000 & -0.012166 & -2.032111 \\ \mathrm{C} & 0.000000 & 2.394776 & -2.396968 \\ \mathrm{H} & 0.884300 & -1.215895 & -4.744004 \\ \mathrm{H} & 0.000000 & -3.189192 & -3.101436 \\ \mathrm{H} & 0.000000 & -5.076606 & -1.275732 \\ \mathrm{H} & 0.000000 & -1.351354 & 5.152192 \\ \mathrm{H} & 0.000000 & 1.344791 & 5.159839 \\ \mathrm{H} & 0.884820 & 1.156988 & -4.802775 \\ \mathrm{H} & 0.000000 & -3.196395 & 3.267551 \\ \mathrm{H} & 0.000000 & 3.147154 & 3.272826 \\ \mathrm{H} & 0.000000 & 3.176242 & -3.144336 \\ \mathrm{H} & 0.000000 & -5.106034 & 1.398826 \\ \mathrm{H} & 0.000000 & 5.055794 & 1.423240 \\ \mathrm{H} & 0.000000 & 5.057457 & -1.279815 \\ \mathrm{H} & -0.884820 & 1.156988 & -4.802775 \\ \mathrm{H} & -0.884300 & -1.215895 & -4.744004 \\ & 0.000000 & 1.075276 & 0.057360 \\ & 0.000000 & -1.071469 & 0.104362 \\ & & & \end{array}$

chlorin $\mathrm{S}_{1}$

$\begin{array}{llll}\text { C } & 0.000000 & -4.230498 & 0.920483\end{array}$ 


$\begin{array}{lrrr}\mathrm{C} & 0.000000 & -2.868441 & 1.362366 \\ \mathrm{~N} & 0.000000 & -2.087654 & 0.240946 \\ \mathrm{C} & 0.000000 & -2.860523 & -0.881622 \\ \mathrm{C} & 0.000000 & -4.225675 & -0.446711 \\ \mathrm{C} & 0.000000 & -2.411882 & 2.679153 \\ \mathrm{C} & 0.000000 & -1.080249 & 3.080030 \\ \mathrm{~N} & 0.000000 & 0.000000 & 2.268704 \\ \mathrm{C} & 0.000000 & 1.080249 & 3.080030 \\ \mathrm{C} & 0.000000 & 0.669517 & 4.494715 \\ \mathrm{C} & 0.000000 & -0.669517 & 4.494715 \\ \mathrm{C} & 0.000000 & 2.411882 & 2.679153 \\ \mathrm{C} & 0.000000 & 2.868441 & 1.362366 \\ \mathrm{~N} & 0.000000 & 2.087654 & 0.240946 \\ \mathrm{C} & 0.000000 & 2.860523 & -0.881622 \\ \mathrm{C} & 0.000000 & 4.225675 & -0.446711 \\ \mathrm{C} & 0.000000 & 4.230498 & 0.920483 \\ \mathrm{C} & 0.000000 & 2.412025 & -2.205502 \\ \mathrm{C} & 0.000000 & 1.097174 & -2.643266 \\ \mathrm{C} & 0.000000 & 0.768098 & -4.132633 \\ \mathrm{C} & 0.000000 & -0.768098 & -4.132633 \\ \mathrm{C} & 0.000000 & -1.097174 & -2.643266 \\ \mathrm{~N} & 0.000000 & 0.000000 & -1.865410 \\ \mathrm{C} & 0.000000 & -2.412025 & -2.205502 \\ \mathrm{H} & -0.884550 & 1.186924 & -4.607050 \\ \mathrm{H} & 0.000000 & 3.183174 & -2.962283 \\ \mathrm{H} & 0.000000 & 5.071868 & -1.112073 \\ \mathrm{H} & 0.000000 & 1.346343 & 5.332701 \\ \mathrm{H} & 0.000000 & -1.346343 & 5.332701 \\ \mathrm{H} & -0.884550 & -1.186924 & -4.607050 \\ \mathrm{H} & 0.000000 & 3.167360 & 3.450840 \\ \mathrm{H} & 0.000000 & -3.167360 & 3.450840 \\ \mathrm{H} & 0.000000 & -3.183174 & -2.962283 \\ \mathrm{H} & 0.000000 & 5.081115 & 1.580446 \\ \mathrm{H} & 0.000000 & -5.081115 & 1.580446 \\ \mathrm{H} & 0.000000 & -5.071868 & -1.112073 \\ \mathrm{H} & 0.884550 & -1.186924 & -4.607050 \\ \mathrm{H} & 0.884550 & 1.186924 & -4.607050 \\ \mathrm{H} & 0.000000 & -1.076341 & 0.254873 \\ & 0.000000 & 1.076341 & 0.254873\end{array}$

Zn-tetraphenylporphine $\mathrm{S}_{0}$
C $\quad-3.856795$
$4.103898 \quad 1.189685$
$\begin{array}{llll}\text { C } & -3.485260 & 3.485269 & -0.000026\end{array}$
C $\quad-4.103602 \quad 3.856555 \quad-1.189964$
$\begin{array}{llll}\text { C } & -5.085799 & 4.838828 & -1.190292\end{array}$ 


$\begin{array}{rrrr}\mathrm{C} & -5.454334 & 5.454424 & -0.000483 \\ \mathrm{C} & -4.839030 & 5.086135 & 1.189555 \\ \mathrm{C} & -2.425282 & 2.425282 & 0.000201 \\ \mathrm{C} & -1.095438 & 2.850630 & 0.004806 \\ \mathrm{~N} & 0.000000 & 2.049910 & 0.017752 \\ \mathrm{C} & 1.095438 & 2.850630 & 0.004806 \\ \mathrm{C} & 0.675787 & 4.241322 & -0.020094 \\ \mathrm{C} & -0.675787 & 4.241322 & -0.020094 \\ \mathrm{C} & 2.425282 & 2.425282 & 0.000201 \\ \mathrm{C} & 3.485260 & 3.485269 & -0.000026 \\ \mathrm{C} & 4.103602 & 3.856555 & -1.189964 \\ \mathrm{C} & 5.085799 & 4.838828 & -1.190292 \\ \mathrm{C} & 5.454334 & 5.454424 & -0.000483 \\ \mathrm{C} & 4.839030 & 5.086135 & 1.189555 \\ \mathrm{C} & 3.856795 & 4.103898 & 1.189685 \\ \mathrm{C} & -2.850632 & 1.095439 & -0.004348 \\ \mathrm{~N} & -2.049910 & 0.000000 & -0.017165 \\ \mathrm{C} & -2.850632 & -1.095439 & -0.004348 \\ \mathrm{C} & -4.241327 & -0.675787 & 0.020391 \\ \mathrm{C} & -4.241327 & 0.675787 & 0.020391 \\ \mathrm{C} & -2.425282 & -2.425282 & 0.000201 \\ \mathrm{C} & -3.485260 & -3.485269 & -0.000026 \\ \mathrm{C} & -4.103602 & -3.856555 & -1.189964 \\ \mathrm{C} & -5.085799 & -4.838828 & -1.190292 \\ \mathrm{C} & -5.454334 & -5.454424 & -0.000483 \\ \mathrm{C} & -4.839030 & -5.086135 & 1.189555 \\ \mathrm{C} & -3.856795 & -4.103898 & 1.189685 \\ \mathrm{C} & 2.850632 & 1.095439 & -0.004348 \\ \mathrm{~N} & 2.049910 & 0.000000 & -0.017165 \\ \mathrm{C} & 2.850632 & -1.095439 & -0.004348 \\ \mathrm{C} & 4.241327 & -0.675787 & 0.020391 \\ \mathrm{C} & 4.241327 & 0.675787 & 0.020391 \\ \mathrm{C} & 2.425282 & -2.425282 & 0.000201 \\ \mathrm{C} & 3.485260 & -3.485269 & -0.000026 \\ \mathrm{C} & 4.103602 & -3.856555 & -1.189964 \\ \mathrm{C} & 5.085799 & -4.838828 & -1.190292 \\ \mathrm{C} & 5.454334 & -5.454424 & -0.000483 \\ \mathrm{C} & 4.839030 & -5.086135 & 1.189555 \\ \mathrm{C} & 3.856795 & -4.103898 & 1.189685 \\ \mathrm{C} & 1.095438 & -2.850630 & 0.004806 \\ \mathrm{~N} & 0.000000 & -2.049910 & 0.017752 \\ \mathrm{C} & -1.095438 & -2.850630 & 0.004806 \\ \mathrm{C} & -0.675787 & -4.241322 & -0.020094 \\ \mathrm{C} & 0.675787 & -4.241322 & -0.020094 \\ \mathrm{H} & 1.346670 & -5.083013 & -0.042989 \\ \mathrm{H} & 5.083022 & -1.346670 & 0.043150\end{array}$




$\begin{array}{rrrr}\mathrm{H} & 1.346670 & 5.083013 & -0.042989 \\ \mathrm{H} & -1.346670 & 5.083013 & -0.042989 \\ \mathrm{H} & -1.346670 & -5.083013 & -0.042989 \\ \mathrm{H} & 5.083022 & 1.346670 & 0.043150 \\ \mathrm{H} & -5.083022 & 1.346670 & 0.043150 \\ \mathrm{H} & -5.083022 & -1.346670 & 0.043150 \\ \mathrm{H} & 3.807976 & 3.371280 & -2.109758 \\ \mathrm{H} & 5.560956 & 5.123909 & -2.117661 \\ \mathrm{H} & 6.218412 & 6.218538 & -0.000658 \\ \mathrm{H} & 5.124300 & 5.561527 & 2.116745 \\ \mathrm{H} & 3.371737 & 3.808476 & 2.109660 \\ \mathrm{H} & 3.807976 & -3.371280 & -2.109758 \\ \mathrm{H} & 5.560956 & -5.123909 & -2.117661 \\ \mathrm{H} & 6.218412 & -6.218538 & -0.000658 \\ \mathrm{H} & 5.124300 & -5.561527 & 2.116745 \\ \mathrm{H} & 3.371737 & -3.808476 & 2.109660 \\ \mathrm{H} & -3.807976 & -3.371280 & -2.109758 \\ \mathrm{H} & -5.560956 & -5.123909 & -2.117661 \\ \mathrm{H} & -6.218412 & -6.218538 & -0.000658 \\ \mathrm{H} & -5.124300 & -5.561527 & 2.116745 \\ \mathrm{H} & -3.371737 & -3.808476 & 2.109660 \\ \mathrm{H} & -3.807976 & 3.371280 & -2.109758 \\ \mathrm{H} & -5.560956 & 5.123909 & -2.117661 \\ \mathrm{H} & -6.218412 & 6.218538 & -0.000658 \\ \mathrm{H} & -5.124300 & 5.561527 & 2.116745 \\ \mathrm{H} & -3.371737 & 3.808476 & 2.109660 \\ \mathrm{Zn} & 0.000000 & 0.000000 & 0.000382\end{array}$

Zn-tetraphenylporphine $\mathrm{S}_{1}$

$\begin{array}{lrrr}\mathrm{C} & -3.691586 & 4.268525 & 1.137066 \\ \mathrm{C} & -3.491377 & 3.489164 & 0.000549 \\ \mathrm{C} & -4.281309 & 3.705387 & -1.125556 \\ \mathrm{C} & -5.260389 & 4.690422 & -1.115316 \\ \mathrm{C} & -5.457153 & 5.464499 & 0.021388 \\ \mathrm{C} & -4.671615 & 5.252286 & 1.147576 \\ \mathrm{C} & -2.438728 & 2.429103 & -0.008318 \\ \mathrm{C} & -1.098803 & 2.850930 & -0.003421 \\ \mathrm{~N} & 0.000000 & 2.044536 & 0.036589 \\ \mathrm{C} & 1.098803 & 2.850930 & -0.003421 \\ \mathrm{C} & 0.678328 & 4.234460 & -0.076657 \\ \mathrm{C} & -0.678328 & 4.234460 & -0.076657 \\ \mathrm{C} & 2.438728 & 2.429103 & -0.008318 \\ \mathrm{C} & 3.491377 & 3.489164 & 0.000549 \\ \mathrm{C} & 4.281309 & 3.705387 & -1.125556 \\ \mathrm{C} & 5.260389 & 4.690422 & -1.115316\end{array}$




$\begin{array}{rrrr}\mathrm{C} & 5.457153 & 5.464499 & 0.021388 \\ \mathrm{C} & 4.671615 & 5.252286 & 1.147576 \\ \mathrm{C} & 3.691586 & 4.268525 & 1.137066 \\ \mathrm{C} & -2.870696 & 1.096768 & -0.017900 \\ \mathrm{~N} & -2.069685 & 0.000000 & -0.062005 \\ \mathrm{C} & -2.870696 & -1.096768 & -0.017900 \\ \mathrm{C} & -4.262716 & -0.675163 & 0.060424 \\ \mathrm{C} & -4.262716 & 0.675163 & 0.060424 \\ \mathrm{C} & -2.438728 & -2.429103 & -0.008318 \\ \mathrm{C} & -3.491377 & -3.489164 & 0.000549 \\ \mathrm{C} & -4.281309 & -3.705387 & -1.125556 \\ \mathrm{C} & -5.260389 & -4.690422 & -1.115316 \\ \mathrm{C} & -5.457153 & -5.464499 & 0.021388 \\ \mathrm{C} & -4.671615 & -5.252286 & 1.147576 \\ \mathrm{C} & -3.691586 & -4.268525 & 1.137066 \\ \mathrm{C} & 2.870696 & 1.096768 & -0.017900 \\ \mathrm{~N} & 2.069685 & 0.000000 & -0.062005 \\ \mathrm{C} & 2.870696 & -1.096768 & -0.017900 \\ \mathrm{C} & 4.262716 & -0.675163 & 0.060424 \\ \mathrm{C} & 4.262716 & 0.675163 & 0.060424 \\ \mathrm{C} & 2.438728 & -2.429103 & -0.008318 \\ \mathrm{C} & 3.491377 & -3.489164 & 0.000549 \\ \mathrm{C} & 4.281309 & -3.705387 & -1.125556 \\ \mathrm{C} & 5.260389 & -4.690422 & -1.115316 \\ \mathrm{C} & 5.457153 & -5.464499 & 0.021388 \\ \mathrm{C} & 4.671615 & -5.252286 & 1.147576 \\ \mathrm{C} & 3.691586 & -4.268525 & 1.137066 \\ \mathrm{C} & 1.098803 & -2.850930 & -0.003421 \\ \mathrm{~N} & 0.000000 & -2.044536 & 0.036589 \\ \mathrm{C} & -1.098803 & -2.850930 & -0.003421 \\ \mathrm{C} & -0.678328 & -4.234460 & -0.076657 \\ \mathrm{C} & 0.678328 & -4.234460 & -0.076657 \\ \mathrm{H} & 1.345920 & -5.076095 & -0.141518 \\ \mathrm{H} & 5.101707 & -1.345659 & 0.128676 \\ \mathrm{H} & 1.345920 & 5.076095 & -0.141518 \\ \mathrm{H} & -1.345920 & 5.076095 & -0.141518 \\ \mathrm{H} & -1.345920 & -5.076095 & -0.141518 \\ \mathrm{H} & 5.101707 & 1.345659 & 0.128676 \\ \mathrm{H} & -5.101707 & 1.345659 & 0.128676 \\ \mathrm{H} & -5.101707 & -1.345659 & 0.128676 \\ \mathrm{H} & 4.115834 & 3.102260 & -2.007935 \\ \mathrm{H} & 5.863945 & 4.857256 & -1.995971 \\ \mathrm{H} & 6.219224 & 6.230531 & 0.029626 \\ \mathrm{H} & 4.825315 & 5.847498 & 2.036212 \\ \mathrm{H} & 3.077621 & 4.091954 & 2.009708 \\ \mathrm{H} & 4.115834 & -3.102260 & -2.007935\end{array}$




$\begin{array}{crrr}\mathrm{H} & 5.863945 & -4.857256 & -1.995971 \\ \mathrm{H} & 6.219224 & -6.230531 & 0.029626 \\ \mathrm{H} & 4.825315 & -5.847498 & 2.036212 \\ \mathrm{H} & 3.077621 & -4.091954 & 2.009708 \\ \mathrm{H} & -4.115834 & -3.102260 & -2.007935 \\ \mathrm{H} & -5.863945 & -4.857256 & -1.995971 \\ \mathrm{H} & -6.219224 & -6.230531 & 0.029626 \\ \mathrm{H} & -4.825315 & -5.847498 & 2.036212 \\ \mathrm{H} & -3.077621 & -4.091954 & 2.009708 \\ \mathrm{H} & -4.115834 & 3.102260 & -2.007935 \\ \mathrm{H} & -5.863945 & 4.857256 & -1.995971 \\ \mathrm{H} & -6.219224 & 6.230531 & 0.029626 \\ \mathrm{H} & -4.825315 & 5.847498 & 2.036212 \\ \mathrm{H} & -3.077621 & 4.091954 & 2.009708 \\ \mathrm{Zn} & 0.000000 & 0.000000 & -0.011848\end{array}$

tetraphenylporphine $\mathrm{S}_{0}$

$\begin{array}{lrrr}\mathrm{C} & 4.229068 & 0.679556 & 0.023542 \\ \mathrm{C} & 2.859074 & 1.116974 & -0.000250 \\ \mathrm{~N} & 2.083481 & 0.000005 & -0.012110 \\ \mathrm{C} & 2.859070 & -1.116968 & -0.000296 \\ \mathrm{C} & 4.229063 & -0.679556 & 0.023515 \\ \mathrm{C} & 2.415167 & -2.434199 & -0.001191 \\ \mathrm{C} & 1.076577 & -2.829993 & -0.002907 \\ \mathrm{~N} & 0.000002 & -2.016999 & 0.011808 \\ \mathrm{C} & -1.076574 & -2.829991 & -0.002847 \\ \mathrm{C} & -0.671456 & -4.238595 & -0.034589 \\ \mathrm{C} & 0.671458 & -4.238600 & -0.034599 \\ \mathrm{C} & -2.415167 & -2.434203 & -0.001096 \\ \mathrm{C} & -2.859074 & -1.116974 & -0.000250 \\ \mathrm{~N} & -2.083481 & -0.000005 & -0.012110 \\ \mathrm{C} & -2.859070 & 1.116968 & -0.000296 \\ \mathrm{C} & -4.229063 & 0.679556 & 0.023515 \\ \mathrm{C} & -4.229068 & -0.679556 & 0.023542 \\ \mathrm{C} & -2.415167 & 2.434199 & -0.001191 \\ \mathrm{C} & -1.076577 & 2.829993 & -0.002907 \\ \mathrm{~N} & -0.000002 & 2.016999 & 0.011808 \\ \mathrm{C} & 1.076574 & 2.829991 & -0.002847 \\ \mathrm{C} & 0.671456 & 4.238595 & -0.034589 \\ \mathrm{C} & -0.671458 & 4.238600 & -0.034599 \\ \mathrm{C} & 2.415167 & 2.434203 & -0.001096 \\ \mathrm{H} & -1.348221 & 5.075470 & -0.061534 \\ \mathrm{C} & -3.471124 & 3.497866 & 0.000598 \\ \mathrm{H} & -5.072958 & 1.347225 & 0.043902 \\ \mathrm{H} & -1.069131 & -0.000003 & -0.014688\end{array}$




$\begin{array}{rrrr}\mathrm{H} & -1.348226 & -5.075462 & -0.061501 \\ \mathrm{H} & 1.348221 & -5.075470 & -0.061534 \\ \mathrm{H} & 1.348226 & 5.075462 & -0.061501 \\ \mathrm{C} & -3.471123 & -3.497870 & 0.000713 \\ \mathrm{C} & 3.471124 & -3.497866 & 0.000598 \\ \mathrm{C} & 3.471123 & 3.497870 & 0.000713 \\ \mathrm{H} & -5.072968 & -1.347216 & 0.043960 \\ \mathrm{H} & 5.072958 & -1.347225 & 0.043902 \\ \mathrm{H} & 5.072968 & 1.347216 & 0.043960 \\ \mathrm{H} & 1.069131 & 0.000003 & -0.014688 \\ \mathrm{C} & -4.086319 & -3.875423 & -1.188813 \\ \mathrm{C} & -5.064402 & -4.861868 & -1.186719 \\ \mathrm{C} & -5.431696 & -5.474506 & 0.004978 \\ \mathrm{C} & -4.819677 & -5.099282 & 1.194599 \\ \mathrm{C} & -3.841758 & -4.112803 & 1.192601 \\ \mathrm{H} & -3.789754 & -3.393739 & -2.110238 \\ \mathrm{H} & -5.536583 & -5.153047 & -2.113723 \\ \mathrm{H} & -6.191920 & -6.242466 & 0.006575 \\ \mathrm{H} & -5.103583 & -5.573159 & 2.122958 \\ \mathrm{H} & -3.357673 & -3.812789 & 2.11617 \\ \mathrm{C} & -4.086297 & 3.875395 & -1.188951 \\ \mathrm{C} & -5.064400 & 4.861822 & -1.186893 \\ \mathrm{C} & -5.431735 & 5.474465 & 0.004787 \\ \mathrm{C} & -4.819733 & 5.099268 & 1.194427 \\ \mathrm{C} & -3.841797 & 4.112808 & 1.192465 \\ \mathrm{H} & -3.789702 & 3.393699 & -2.110361 \\ \mathrm{H} & -5.536563 & 5.152980 & -2.113913 \\ \mathrm{H} & -6.191977 & 6.242406 & 0.006355 \\ \mathrm{H} & -5.103669 & 5.573155 & 2.122772 \\ \mathrm{H} & -3.357731 & 3.812815 & 2.111498 \\ \mathrm{C} & 4.086319 & 3.875423 & -1.188813 \\ \mathrm{C} & 5.064402 & 4.861868 & -1.186719 \\ \mathrm{C} & 5.431696 & 5.474506 & 0.004978 \\ \mathrm{C} & 4.819677 & 5.099282 & 1.194599 \\ \mathrm{C} & 3.841758 & 4.112803 & 1.192601 \\ \mathrm{H} & 3.789754 & 3.393739 & -2.110238 \\ \mathrm{H} & 5.536583 & 5.153047 & -2.113723 \\ \mathrm{H} & 6.191920 & 6.242466 & 0.006575 \\ \mathrm{H} & 5.103583 & 5.573159 & 2.122958 \\ \mathrm{H} & 3.357673 & 3.812789 & 2.111617 \\ \mathrm{C} & 4.086297 & -3.875395 & -1.188951 \\ \mathrm{C} & 5.064400 & -4.861822 & -1.186893 \\ \mathrm{C} & 5.431735 & -5.474465 & 0.004787 \\ \mathrm{C} & 4.819733 & -5.099268 & 1.194427 \\ \mathrm{C} & 3.841797 & -4.112808 & 1.192465 \\ \mathrm{H} & 3.789702 & -3.393699 & -2.110361\end{array}$




$\begin{array}{llll}\mathrm{H} & 5.536563 & -5.152980 & -2.113913 \\ \mathrm{H} & 6.191977 & -6.242406 & 0.006355 \\ \mathrm{H} & 5.103669 & -5.573155 & 2.122772 \\ \mathrm{H} & 3.357731 & -3.812815 & 2.111498\end{array}$

tetraphenylporphine $\mathrm{S}_{1}$

$\begin{array}{rrrr}\mathrm{C} & -0.922479 & -4.192012 & 0.162133 \\ \mathrm{C} & -0.011996 & -3.082845 & 0.064026 \\ \mathrm{~N} & -0.767150 & -1.951787 & -0.017893 \\ \mathrm{C} & -2.092570 & -2.263536 & 0.004044 \\ \mathrm{C} & -2.188384 & -3.693600 & 0.122114 \\ \mathrm{C} & -3.159861 & -1.362211 & -0.049968 \\ \mathrm{C} & -3.036593 & 0.033757 & -0.059297 \\ \mathrm{~N} & -1.882314 & 0.738080 & 0.032464 \\ \mathrm{C} & -2.244395 & 2.041886 & 0.000912 \\ \mathrm{C} & -3.693719 & 2.180156 & -0.140218 \\ \mathrm{C} & -4.187067 & 0.928204 & -0.181888 \\ \mathrm{C} & -1.382989 & 3.149027 & 0.057283 \\ \mathrm{C} & 0.011996 & 3.082846 & 0.064023 \\ \mathrm{~N} & 0.767150 & 1.951788 & -0.017893 \\ \mathrm{C} & 2.092570 & 2.263537 & 0.004045 \\ \mathrm{C} & 2.188384 & 3.693601 & 0.122113 \\ \mathrm{C} & 0.922479 & 4.192013 & 0.162130 \\ \mathrm{C} & 3.159861 & 1.362212 & -0.049968 \\ \mathrm{C} & 3.036593 & -0.033756 & -0.059297 \\ \mathrm{~N} & 1.882314 & -0.738080 & 0.032468 \\ \mathrm{C} & 2.244395 & -2.041885 & 0.000911 \\ \mathrm{C} & 3.693718 & -2.180155 & -0.140227 \\ \mathrm{C} & 4.187066 & -0.928203 & -0.181896 \\ \mathrm{C} & 1.382989 & -3.149026 & 0.057284 \\ \mathrm{H} & 5.207769 & -0.611177 & -0.305006 \\ \mathrm{C} & 4.527980 & 1.956965 & -0.098946 \\ \mathrm{H} & 3.118034 & 4.230446 & 0.187552 \\ \mathrm{H} & 0.394603 & 1.008299 & -0.048604 \\ \mathrm{H} & -4.221286 & 3.114426 & -0.222191 \\ \mathrm{H} & -5.207771 & 0.611179 & -0.304993 \\ \mathrm{H} & 4.221285 & -3.114425 & -0.222204 \\ \mathrm{C} & -1.989409 & 4.511617 & 0.110124 \\ \mathrm{C} & -4.527980 & -1.956965 & -0.098946 \\ \mathrm{C} & 1.989409 & -4.511616 & 0.110124 \\ \mathrm{H} & 0.613388 & 5.216546 & 0.267544 \\ \mathrm{H} & -3.118034 & -4.230445 & 0.187554 \\ \mathrm{H} & -0.613388 & -5.216545 & 0.267549 \\ \mathrm{H} & -0.394603 & -1.008298 & -0.048605 \\ \mathrm{C} & -1.845515 & 5.394214 & -0.957880\end{array}$




$\begin{array}{lrrr}\mathrm{C} & -2.414500 & 6.660012 & -0.901447 \\ \mathrm{C} & -3.128766 & 7.053014 & 0.223845 \\ \mathrm{C} & -3.275172 & 6.175801 & 1.291721 \\ \mathrm{C} & -2.708093 & 4.909819 & 1.235488 \\ \mathrm{H} & -1.295360 & 5.077069 & -1.833460 \\ \mathrm{H} & -2.304709 & 7.336330 & -1.736820 \\ \mathrm{H} & -3.571034 & 8.037675 & 0.268633 \\ \mathrm{H} & -3.826885 & 6.478031 & 2.170430 \\ \mathrm{H} & -2.814542 & 4.219660 & 2.060942 \\ \mathrm{C} & 4.938787 & 2.670556 & -1.222871 \\ \mathrm{C} & 6.210978 & 3.224454 & -1.274550 \\ \mathrm{C} & 7.081079 & 3.070619 & -0.202096 \\ \mathrm{C} & 6.675095 & 2.361752 & 0.922072 \\ \mathrm{C} & 5.403373 & 1.805852 & 0.973836 \\ \mathrm{H} & 4.256559 & 2.778200 & -2.054935 \\ \mathrm{H} & 6.523818 & 3.769331 & -2.153751 \\ \mathrm{H} & 8.071124 & 3.501043 & -0.243082 \\ \mathrm{H} & 7.347285 & 2.243767 & 1.759651 \\ \mathrm{H} & 5.077940 & 1.254324 & 1.845309 \\ \mathrm{C} & 1.845520 & -5.394212 & -0.957881 \\ \mathrm{C} & 2.414504 & -6.660010 & -0.901448 \\ \mathrm{C} & 3.128765 & -7.053014 & 0.223847 \\ \mathrm{C} & 3.275167 & -6.175802 & 1.291725 \\ \mathrm{C} & 2.708089 & -4.909819 & 1.235491 \\ \mathrm{H} & 1.295368 & -5.077066 & -1.833463 \\ \mathrm{H} & 2.304717 & -7.336326 & -1.736823 \\ \mathrm{H} & 3.571032 & -8.037675 & 0.268636 \\ \mathrm{H} & 3.826876 & -6.478034 & 2.170436 \\ \mathrm{H} & 2.814534 & -4.219662 & 2.060946 \\ \mathrm{C} & -4.938786 & -2.670556 & -1.222871 \\ \mathrm{C} & -6.210976 & -3.224455 & -1.274552 \\ \mathrm{C} & -7.081077 & -3.070622 & -0.202096 \\ \mathrm{C} & -6.675094 & -2.361757 & 0.922072 \\ \mathrm{C} & -5.403373 & -1.805854 & 0.973836 \\ \mathrm{H} & -4.256559 & -2.778198 & -2.054936 \\ \mathrm{H} & -6.523816 & -3.769332 & -2.153753 \\ \mathrm{H} & -8.071121 & -3.501049 & -0.243083 \\ \mathrm{H} & -7.347284 & -2.243773 & 1.759651 \\ \mathrm{H} & -5.077940 & -1.254327 & 1.845310\end{array}$

\section{M06-HF/TZVP geometries - Group II}

tetrafluorobenzene $\mathrm{S}_{0}$
$\begin{array}{llll}\text { C } & -1.186994 & 0.691194 & 0.000000\end{array}$ 


$\begin{array}{lrrr}\text { C } & -1.186994 & -0.691194 & 0.000000 \\ \text { C } & 0.000000 & -1.394354 & 0.000000 \\ \text { C } & 1.186994 & -0.691194 & 0.000000 \\ \text { C } & 1.186994 & 0.691194 & 0.000000 \\ \text { C } & 0.000000 & 1.394354 & 0.000000 \\ \text { F } & -2.350057 & -1.351609 & 0.000000 \\ \text { H } & 0.000000 & -2.474113 & 0.000000 \\ \text { F } & 2.350057 & -1.351609 & 0.000000 \\ \text { F } & 2.350057 & 1.351609 & 0.000000 \\ \text { H } & 0.000000 & 2.474113 & 0.000000 \\ \text { F } & -2.350057 & 1.351609 & 0.000000\end{array}$

tetrafluorobenzene $\mathrm{S}_{1}$

$\begin{array}{lrrr}\text { C } & 1.144987 & -0.708154 & 0.105255 \\ \text { C } & 1.144987 & 0.708154 & 0.105255 \\ \text { C } & 0.000000 & 1.458485 & 0.400006 \\ \text { C } & -1.144987 & 0.708154 & 0.105255 \\ \text { C } & -1.144987 & -0.708154 & 0.105255 \\ \text { C } & 0.000000 & -1.458485 & 0.400006 \\ \text { F } & 2.267585 & 1.316119 & -0.281158 \\ \text { H } & 0.000000 & 2.505655 & 0.649924 \\ \text { F } & -2.267585 & 1.316119 & -0.281158 \\ \text { F } & -2.267585 & -1.316119 & -0.281158 \\ \text { H } & 0.000000 & -2.505655 & 0.649924 \\ \text { F } & 2.267585 & -1.316119 & -0.281158\end{array}$

benzonitrile $\mathrm{S}_{0}$

$\begin{array}{rrrr}\mathrm{C} & 0.000000 & 0.000000 & -1.043494 \\ \mathrm{C} & -1.211848 & 0.000000 & -0.359468 \\ \mathrm{C} & -1.205815 & 0.000000 & 1.027150 \\ \mathrm{C} & 0.000000 & 0.000000 & 1.717963 \\ \mathrm{C} & 1.205815 & 0.000000 & 1.027150 \\ \mathrm{C} & 1.211848 & 0.000000 & -0.359468 \\ \mathrm{H} & -2.138965 & 0.000000 & -0.912885 \\ \mathrm{H} & -2.140837 & 0.000000 & 1.567084 \\ \mathrm{H} & 0.000000 & 0.000000 & 2.798337 \\ \mathrm{H} & 2.140837 & 0.000000 & 1.567084 \\ \mathrm{H} & 2.138965 & 0.000000 & -0.912885 \\ \mathrm{C} & 0.000000 & 0.000000 & -2.489284 \\ \mathrm{~N} & 0.000000 & 0.000000 & -3.627277\end{array}$

benzonitrile $\mathrm{S}_{1}$

$\begin{array}{llll}\text { C } & 0.000000 & 0.000000 & -1.089630\end{array}$ 


$\begin{array}{rrrr}\mathrm{C} & -1.219457 & 0.000000 & -0.371095 \\ \mathrm{C} & -1.211706 & 0.000000 & 1.039983 \\ \mathrm{C} & 0.000000 & 0.000000 & 1.753539 \\ \mathrm{C} & 1.211706 & 0.000000 & 1.039983 \\ \mathrm{C} & 1.219457 & 0.000000 & -0.371095 \\ \mathrm{H} & -2.150596 & 0.000000 & -0.901941 \\ \mathrm{H} & -2.146503 & 0.000000 & 1.565245 \\ \mathrm{H} & 0.000000 & 0.000000 & 2.824748 \\ \mathrm{H} & 2.146503 & 0.000000 & 1.565245 \\ \mathrm{H} & 2.150596 & 0.000000 & -0.901941 \\ \mathrm{C} & 0.000000 & 0.000000 & -2.510024 \\ \mathrm{~N} & 0.000000 & 0.000000 & -3.643018\end{array}$

$o$-fluorophenol, cis $\mathrm{S}_{0}$

$\begin{array}{cccc}\mathrm{C} & 0.328414 & 1.451261 & 0.000000 \\ \mathrm{C} & -0.868137 & 0.773341 & 0.000000 \\ \mathrm{C} & -0.945459 & -0.610922 & 0.000000 \\ \mathrm{C} & 0.237501 & -1.333579 & 0.000000 \\ \mathrm{C} & 1.456943 & -0.670301 & 0.000000 \\ \mathrm{C} & 1.508860 & 0.718085 & 0.000000 \\ \mathrm{~F} & -2.040613 & 1.459556 & 0.000000 \\ \mathrm{O} & -2.136516 & -1.259917 & 0.000000 \\ \mathrm{H} & 0.175619 & -2.411715 & 0.000000 \\ \mathrm{H} & 2.371626 & -1.244208 & 0.000000 \\ \mathrm{H} & 2.457839 & 1.231069 & 0.000000 \\ \mathrm{H} & 0.318931 & 2.531081 & 0.000000 \\ \mathrm{H} & -2.864996 & -0.633770 & 0.000000\end{array}$

$o$-fluorophenol, cis $\mathrm{S}_{1}$

$\begin{array}{cccc}\mathrm{C} & 0.321191 & 1.519085 & 0.000000 \\ \mathrm{C} & -0.881973 & 0.792425 & 0.000000 \\ \mathrm{C} & -0.941019 & -0.617874 & 0.000000 \\ \mathrm{C} & 0.244042 & -1.408490 & 0.000000 \\ \mathrm{C} & 1.458110 & -0.696406 & 0.000000 \\ \mathrm{C} & 1.491026 & 0.722884 & 0.000000 \\ \mathrm{~F} & -2.067372 & 1.442081 & 0.000000 \\ \mathrm{O} & -2.116838 & -1.237349 & 0.000000 \\ \mathrm{H} & 0.166931 & -2.481137 & 0.000000 \\ \mathrm{H} & 2.395431 & -1.230285 & 0.000000 \\ \mathrm{H} & 2.453913 & 1.212536 & 0.000000 \\ \mathrm{H} & 0.326117 & 2.594508 & 0.000000 \\ \mathrm{H} & -2.849546 & -0.611997 & 0.000000\end{array}$

$o$-fluorophenol, trans $\mathrm{S}_{0}$ 


$\begin{array}{cccc}\mathrm{C} & 0.289187 & 1.254050 & 0.000000 \\ \mathrm{C} & -0.943095 & 0.612056 & 0.000000 \\ \mathrm{C} & -0.960426 & -0.775081 & 0.000000 \\ \mathrm{C} & 0.229413 & -1.494591 & 0.000000 \\ \mathrm{C} & 1.447833 & -0.834647 & 0.000000 \\ \mathrm{C} & 1.474938 & 0.556044 & 0.000000 \\ \mathrm{O} & -2.058841 & 1.384697 & 0.000000 \\ \mathrm{H} & -1.910176 & -1.292994 & 0.000000 \\ \mathrm{H} & 0.194796 & -2.573799 & 0.000000 \\ \mathrm{H} & 2.372852 & -1.390189 & 0.000000 \\ \mathrm{H} & 2.400875 & 1.111768 & 0.000000 \\ \mathrm{~F} & 0.313649 & 2.598230 & 0.000000 \\ \mathrm{H} & -2.851015 & 0.844457 & 0.000000\end{array}$

$o$-fluorophenol, trans $\mathrm{S}_{1}$

$\begin{array}{cccc}\mathrm{C} & 0.823196 & 0.999390 & 0.095773 \\ \mathrm{C} & -0.586331 & 0.956505 & 0.096890 \\ \mathrm{C} & -1.264115 & -0.294918 & 0.231727 \\ \mathrm{C} & -0.474163 & -1.401079 & -0.056051 \\ \mathrm{C} & 0.939571 & -1.293512 & -0.094369 \\ \mathrm{C} & 1.610756 & -0.138204 & 0.379289 \\ \mathrm{O} & -1.238269 & 2.083983 & -0.167946 \\ \mathrm{H} & -2.305127 & -0.353918 & 0.508559 \\ \mathrm{H} & -0.923508 & -2.369480 & -0.232900 \\ \mathrm{H} & 1.511202 & -2.122303 & -0.494477 \\ \mathrm{H} & 2.675348 & -0.074045 & 0.514830 \\ \mathrm{~F} & 1.393764 & 2.111205 & -0.409658 \\ \mathrm{H} & -2.162354 & 1.896375 & -0.371668\end{array}$

$m$-fluorophenol, cis $\mathrm{S}_{0}$

$\begin{array}{cccc}\mathrm{C} & 0.310641 & 1.401969 & 0.000000 \\ \mathrm{C} & -0.925878 & 0.787309 & 0.000000 \\ \mathrm{C} & -0.962567 & -0.600799 & 0.000000 \\ \mathrm{C} & 0.215386 & -1.339587 & 0.000000 \\ \mathrm{C} & 1.433367 & -0.680646 & 0.000000 \\ \mathrm{C} & 1.500984 & 0.709024 & 0.000000 \\ \mathrm{O} & -2.134841 & -1.291399 & 0.000000 \\ \mathrm{~F} & 0.340570 & 2.751765 & 0.000000 \\ \mathrm{H} & 0.151072 & -2.416764 & 0.000000 \\ \mathrm{H} & 2.347812 & -1.255511 & 0.000000 \\ \mathrm{H} & 2.436702 & 1.245021 & 0.000000 \\ \mathrm{H} & -1.822521 & 1.391473 & 0.000000 \\ \mathrm{H} & -2.890728 & -0.701854 & 0.000000\end{array}$


$m$-fluorophenol, cis $\mathrm{S}_{1}$

$\begin{array}{cccc}\mathrm{C} & 0.286167 & 1.411127 & 0.000000 \\ \mathrm{C} & -0.986880 & 0.832625 & 0.000000 \\ \mathrm{C} & -0.961342 & -0.592470 & 0.000000 \\ \mathrm{C} & 0.227839 & -1.360436 & 0.000000 \\ \mathrm{C} & 1.493116 & -0.723018 & 0.000000 \\ \mathrm{C} & 1.495918 & 0.693189 & 0.000000 \\ \mathrm{O} & -2.104376 & -1.279289 & 0.000000 \\ \mathrm{~F} & 0.364145 & 2.752669 & 0.000000 \\ \mathrm{H} & 0.119072 & -2.434089 & 0.000000 \\ \mathrm{H} & 2.410421 & -1.284013 & 0.000000 \\ \mathrm{H} & 2.414671 & 1.261581 & 0.000000 \\ \mathrm{H} & -1.889392 & 1.419968 & 0.000000 \\ \mathrm{H} & -2.869360 & -0.697845 & 0.000000\end{array}$

$m$-fluorophenol, trans $\mathrm{S}_{0}$

$\begin{array}{lrrr}\mathrm{C} & 0.267838 & 1.298988 & 0.000000 \\ \mathrm{C} & -0.945438 & 0.623559 & 0.000000 \\ \mathrm{C} & -0.982042 & -0.766068 & 0.000000 \\ \mathrm{C} & 0.209232 & -1.479875 & 0.000000 \\ \mathrm{C} & 1.435016 & -0.830786 & 0.000000 \\ \mathrm{C} & 1.423881 & 0.550955 & 0.000000 \\ \mathrm{O} & -2.072285 & 1.386018 & 0.000000 \\ \mathrm{H} & -1.929788 & -1.286346 & 0.000000 \\ \mathrm{H} & 0.179231 & -2.559515 & 0.000000 \\ \mathrm{H} & 2.372835 & -1.362877 & 0.000000 \\ \mathrm{~F} & 2.602909 & 1.207170 & 0.000000 \\ \mathrm{H} & 0.299974 & 2.377353 & 0.000000 \\ \mathrm{H} & -2.861365 & 0.841432 & 0.000000\end{array}$

$m$-fluorophenol, trans $\mathrm{S}_{1}$

$\begin{array}{lrrr}\mathrm{C} & 1.087635 & -0.017492 & 0.000000 \\ \mathrm{C} & 0.327143 & 1.177857 & 0.000000 \\ \mathrm{C} & -1.095296 & 1.207732 & 0.000000 \\ \mathrm{C} & -1.826986 & -0.004616 & 0.000000 \\ \mathrm{C} & -1.085872 & -1.214713 & 0.000000 \\ \mathrm{C} & 0.319356 & -1.188595 & 0.000000 \\ \mathrm{O} & 1.025980 & 2.313252 & 0.000000 \\ \mathrm{H} & -1.600245 & 2.163331 & 0.000000 \\ \mathrm{H} & -2.902609 & -0.012925 & 0.000000 \\ \mathrm{H} & -1.574753 & -2.177733 & 0.000000 \\ \mathrm{~F} & 0.964832 & -2.368030 & 0.000000 \\ \mathrm{H} & 2.162656 & -0.011379 & 0.000000\end{array}$




$\begin{array}{lccc}\mathrm{H} & 0.457010 & 3.087458 & 0.000000 \\ p \text {-fluorophenol } \mathrm{S}_{0} & & \\ \mathrm{C} & 0.271360 & 1.312866 & 0.000000 \\ \mathrm{C} & -0.945283 & 0.642114 & 0.000000 \\ \mathrm{C} & -0.979116 & -0.745627 & 0.000000 \\ \mathrm{C} & 0.207562 & -1.469232 & 0.000000 \\ \mathrm{C} & 1.401748 & -0.783529 & 0.000000 \\ \mathrm{C} & 1.457163 & 0.596440 & 0.000000 \\ \mathrm{O} & -2.077476 & 1.404838 & 0.000000 \\ \mathrm{H} & -1.925637 & -1.268774 & 0.000000 \\ \mathrm{H} & 0.209958 & -2.548798 & 0.000000 \\ \mathrm{~F} & 2.558813 & -1.484491 & 0.000000 \\ \mathrm{H} & 2.416371 & 1.091888 & 0.000000 \\ \mathrm{H} & 0.269956 & 2.392227 & 0.000000 \\ \mathrm{H} & -2.865439 & 0.860068 & 0.000000\end{array}$

$p$-fluorophenol $\mathrm{S}_{1}$

$\begin{array}{lrrr}\mathrm{C} & 1.266010 & 0.676195 & 0.000000 \\ \mathrm{C} & 0.025115 & 1.356622 & 0.000000 \\ \mathrm{C} & -1.243113 & 0.707167 & 0.000000 \\ \mathrm{C} & -1.251328 & -0.701576 & 0.000000 \\ \mathrm{C} & -0.005271 & -1.355944 & 0.000000 \\ \mathrm{C} & 1.254515 & -0.734052 & 0.000000 \\ \mathrm{O} & 0.089784 & 2.685143 & 0.000000 \\ \mathrm{H} & -2.156196 & 1.282793 & 0.000000 \\ \mathrm{H} & -2.155974 & -1.286255 & 0.000000 \\ \mathrm{~F} & -0.026231 & -2.690650 & 0.000000 \\ \mathrm{H} & 2.148632 & -1.334058 & 0.000000 \\ \mathrm{H} & 2.174118 & 1.255407 & 0.000000 \\ \mathrm{H} & -0.783363 & 3.084801 & 0.000000\end{array}$

phenylacetylene $S_{0}$

$\begin{array}{rrrr}\mathrm{C} & 1.207062 & 0.000000 & -0.013745 \\ \mathrm{C} & 0.000000 & 0.000000 & -0.711790 \\ \mathrm{C} & -1.207062 & 0.000000 & -0.013745 \\ \mathrm{C} & -1.203603 & 0.000000 & 1.373043 \\ \mathrm{C} & 0.000000 & 0.000000 & 2.067734 \\ \mathrm{C} & 1.203603 & 0.000000 & 1.373043 \\ \mathrm{C} & 0.000000 & 0.000000 & -2.153422 \\ \mathrm{C} & 0.000000 & 0.000000 & -3.349020 \\ \mathrm{H} & -2.135639 & 0.000000 & -0.564706 \\ \mathrm{H} & -2.140126 & 0.000000 & 1.910803 \\ \mathrm{H} & 0.000000 & 0.000000 & 3.147979\end{array}$




$\begin{array}{cccc}\mathrm{H} & 2.140126 & 0.000000 & 1.910803 \\ \mathrm{H} & 2.135639 & 0.000000 & -0.564706 \\ \mathrm{H} & 0.000000 & 0.000000 & -4.412289\end{array}$

phenylacetylene $\mathrm{S}_{1}$

$\begin{array}{rrrr}\mathrm{C} & 1.229743 & 0.000000 & -0.028561 \\ \mathrm{C} & 0.000000 & 0.000000 & -0.746307 \\ \mathrm{C} & -1.229743 & 0.000000 & -0.028561 \\ \mathrm{C} & -1.222464 & 0.000000 & 1.389718 \\ \mathrm{C} & 0.000000 & 0.000000 & 2.098721 \\ \mathrm{C} & 1.222464 & 0.000000 & 1.389718 \\ \mathrm{C} & 0.000000 & 0.000000 & -2.161832 \\ \mathrm{C} & 0.000000 & 0.000000 & -3.363354 \\ \mathrm{H} & -2.159071 & 0.000000 & -0.575652 \\ \mathrm{H} & -2.158897 & 0.000000 & 1.925428 \\ \mathrm{H} & 0.000000 & 0.000000 & 3.177426 \\ \mathrm{H} & 2.158897 & 0.000000 & 1.925428 \\ \mathrm{H} & 2.159071 & 0.000000 & -0.575652 \\ \mathrm{H} & 0.000000 & 0.000000 & -4.426538\end{array}$
aniline $\mathrm{S}_{0}$

$\begin{array}{rrrr}\mathrm{C} & 0.914561 & 0.024085 & 0.000000 \\ \mathrm{C} & 0.204724 & 0.036391 & 1.201852 \\ \mathrm{C} & -1.181755 & 0.042339 & 1.196407 \\ \mathrm{C} & -1.887729 & 0.046214 & 0.000000 \\ \mathrm{C} & -1.181755 & 0.042339 & -1.196407 \\ \mathrm{C} & 0.204724 & 0.036391 & -1.201852 \\ \mathrm{H} & 0.744363 & 0.040891 & 2.138832 \\ \mathrm{H} & -2.967095 & 0.051272 & 0.000000 \\ \mathrm{H} & -1.713162 & 0.048235 & 2.137243 \\ \mathrm{H} & -1.713162 & 0.048235 & -2.137243 \\ \mathrm{H} & 0.744363 & 0.040891 & -2.138832 \\ \mathrm{~N} & 2.308290 & 0.056299 & 0.000000 \\ \mathrm{H} & 2.761823 & -0.256785 & -0.840786 \\ \mathrm{H} & 2.761823 & -0.256785 & 0.840786\end{array}$

aniline $S_{1}$
$\begin{array}{llll}\text { C } & 0.898598 & -0.034293 & 0.000000\end{array}$
$\begin{array}{llll}\text { C } & 0.223586 & -0.008487 & 1.253735\end{array}$
$\begin{array}{llll}\text { C } & -1.187372 & 0.045392 & 1.239641\end{array}$
$\begin{array}{llll}\text { C } & -1.860262 & 0.071067 & 0.000000\end{array}$
$\begin{array}{llll}\text { C } & -1.187372 & 0.045392 & -1.239641\end{array}$
$\begin{array}{llll}\text { C } & 0.223586 & -0.008487 & -1.253735\end{array}$
H $\quad 0.791923 \quad-0.030235 \quad 2.170790$ 


$\begin{array}{rrrr}\mathrm{H} & -2.941239 & 0.112270 & 0.000000 \\ \mathrm{H} & -1.748011 & 0.066789 & 2.159721 \\ \mathrm{H} & -1.748011 & 0.066789 & -2.159721 \\ \mathrm{H} & 0.791923 & -0.030235 & -2.170790 \\ \mathrm{~N} & 2.238521 & -0.085617 & 0.000000 \\ \mathrm{H} & 2.752060 & -0.105183 & -0.864005 \\ \mathrm{H} & 2.752060 & -0.105183 & 0.864005\end{array}$

catechol $\mathrm{S}_{0}$

$\begin{array}{lrrr}\mathrm{C} & 0.472184 & 1.276257 & 0.000000 \\ \mathrm{C} & -0.741905 & 0.617133 & 0.000000 \\ \mathrm{C} & -0.791893 & -0.775701 & 0.000000 \\ \mathrm{C} & 0.384563 & -1.501694 & 0.000000 \\ \mathrm{C} & 1.608655 & -0.842343 & 0.000000 \\ \mathrm{C} & 1.655275 & 0.543352 & 0.000000 \\ \mathrm{O} & -1.963724 & 1.242783 & 0.000000 \\ \mathrm{O} & -1.989187 & -1.414551 & 0.000000 \\ \mathrm{H} & 0.319155 & -2.579710 & 0.000000 \\ \mathrm{H} & 2.523341 & -1.415999 & 0.000000 \\ \mathrm{H} & 2.602522 & 1.060096 & 0.000000 \\ \mathrm{H} & 0.496056 & 2.358212 & 0.000000 \\ \mathrm{H} & -1.876160 & 2.197088 & 0.000000 \\ \mathrm{H} & -2.698881 & -0.764922 & 0.000000\end{array}$

catechol $\mathrm{S}_{1}$

$\begin{array}{lrrr}\mathrm{C} & 0.475165 & 1.354839 & 0.000000 \\ \mathrm{C} & -0.748634 & 0.626173 & 0.000000 \\ \mathrm{C} & -0.794191 & -0.784382 & 0.000000 \\ \mathrm{C} & 0.389943 & -1.581155 & 0.000000 \\ \mathrm{C} & 1.592787 & -0.862209 & 0.000000 \\ \mathrm{C} & 1.634741 & 0.557762 & 0.000000 \\ \mathrm{O} & -1.956992 & 1.234610 & 0.000000 \\ \mathrm{O} & -1.976616 & -1.400053 & 0.000000 \\ \mathrm{H} & 0.318460 & -2.653696 & 0.000000 \\ \mathrm{H} & 2.532986 & -1.393254 & 0.000000 \\ \mathrm{H} & 2.603470 & 1.035687 & 0.000000 \\ \mathrm{H} & 0.497873 & 2.431582 & 0.000000 \\ \mathrm{H} & -1.873985 & 2.190750 & 0.000000 \\ \mathrm{H} & -2.695009 & -0.756653 & 0.000000\end{array}$

resorcinol, isomer $1 \mathrm{~S}_{0}$
C $\quad 0.073420$
1.142692
0.000000
C -1.135296
0.459483
0.000000 


$\begin{array}{rrrr}\mathrm{C} & -1.168351 & -0.927598 & 0.000000 \\ \mathrm{C} & 0.034294 & -1.626015 & 0.000000 \\ \mathrm{C} & 1.250241 & -0.966486 & 0.000000 \\ \mathrm{C} & 1.258611 & 0.424448 & 0.000000 \\ \mathrm{O} & -2.266248 & 1.220661 & 0.000000 \\ \mathrm{H} & -2.111578 & -1.455266 & 0.000000 \\ \mathrm{H} & 0.016799 & -2.706284 & 0.000000 \\ \mathrm{H} & 2.189431 & -1.496461 & 0.000000 \\ \mathrm{O} & 2.472941 & 1.041455 & 0.000000 \\ \mathrm{H} & 0.059804 & 2.223699 & 0.000000 \\ \mathrm{H} & -3.051344 & 0.670436 & 0.000000 \\ \mathrm{H} & 2.377276 & 1.995225 & 0.000000\end{array}$

resorcinol, isomer $1 \mathrm{~S}_{1}$

$\begin{array}{lrrr}\mathrm{C} & 0.074135 & 1.222807 & 0.000000 \\ \mathrm{C} & -1.124495 & 0.483224 & 0.000000 \\ \mathrm{C} & -1.165639 & -0.935594 & 0.000000 \\ \mathrm{C} & 0.026482 & -1.699169 & 0.000000 \\ \mathrm{C} & 1.245439 & -0.974437 & 0.000000 \\ \mathrm{C} & 1.252072 & 0.438870 & 0.000000 \\ \mathrm{O} & -2.262643 & 1.198344 & 0.000000 \\ \mathrm{H} & -2.130983 & -1.424582 & 0.000000 \\ \mathrm{H} & 0.008714 & -2.774300 & 0.000000 \\ \mathrm{H} & 2.203790 & -1.471429 & 0.000000 \\ \mathrm{O} & 2.462696 & 1.023744 & 0.000000 \\ \mathrm{H} & 0.064314 & 2.299195 & 0.000000 \\ \mathrm{H} & -3.038483 & 0.632868 & 0.000000 \\ \mathrm{H} & 2.384599 & 1.980448 & 0.000000\end{array}$

resorcinol, isomer $2 \mathrm{~S}_{0}$

$\begin{array}{lrrr}\mathrm{C} & 1.195051 & 0.000000 & -0.541042 \\ \mathrm{C} & 0.000000 & 0.000000 & -1.241395 \\ \mathrm{C} & -1.195051 & 0.000000 & -0.541042 \\ \mathrm{C} & -1.208412 & 0.000000 & 0.850427 \\ \mathrm{C} & 0.000000 & 0.000000 & 1.530774 \\ \mathrm{C} & 1.208412 & 0.000000 & 0.850427 \\ \mathrm{O} & -2.340571 & 0.000000 & -1.278260 \\ \mathrm{O} & 2.340571 & 0.000000 & -1.278260 \\ \mathrm{H} & -2.144061 & 0.000000 & 1.391553 \\ \mathrm{H} & 0.000000 & 0.000000 & 2.611121 \\ \mathrm{H} & 2.144061 & 0.000000 & 1.391553 \\ \mathrm{H} & 0.000000 & 0.000000 & -2.320189 \\ \mathrm{H} & -3.114748 & 0.000000 & -0.713072 \\ \mathrm{H} & 3.114748 & 0.000000 & -0.713072\end{array}$


resorcinol, isomer $2 \mathrm{~S}_{1}$

$\begin{array}{lrrr}\mathrm{C} & 1.190010 & 0.000000 & -0.329410 \\ \mathrm{C} & 0.000000 & 0.000000 & -1.082744 \\ \mathrm{C} & -1.190010 & 0.000000 & -0.329410 \\ \mathrm{C} & -1.208955 & 0.000000 & 1.089763 \\ \mathrm{C} & 0.000000 & 0.000000 & 1.829997 \\ \mathrm{C} & 1.208955 & 0.000000 & 1.089763 \\ \mathrm{O} & -2.339786 & 0.000000 & -1.027722 \\ \mathrm{O} & 2.339786 & 0.000000 & -1.027722 \\ \mathrm{H} & -2.162232 & 0.000000 & 1.601075 \\ \mathrm{H} & 0.000000 & 0.000000 & 2.905537 \\ \mathrm{H} & 2.162232 & 0.000000 & 1.601075 \\ \mathrm{H} & 0.000000 & 0.000000 & -2.157442 \\ \mathrm{H} & -3.106631 & 0.000000 & -0.450922 \\ \mathrm{H} & 3.106631 & 0.000000 & -0.450922\end{array}$

resorcinol, isomer $3 \mathrm{~S}_{0}$

$\begin{array}{rrrr}\mathrm{C} & 1.199400 & 0.000000 & -0.285380 \\ \mathrm{C} & 0.000000 & 0.000000 & -0.987531 \\ \mathrm{C} & -1.199400 & 0.000000 & -0.285380 \\ \mathrm{C} & -1.209989 & 0.000000 & 1.101546 \\ \mathrm{C} & 0.000000 & 0.000000 & 1.779426 \\ \mathrm{C} & 1.209989 & 0.000000 & 1.101546 \\ \mathrm{O} & -2.404353 & 0.000000 & -0.923136 \\ \mathrm{O} & 2.404353 & 0.000000 & -0.923136 \\ \mathrm{H} & -2.156599 & 0.000000 & 1.618091 \\ \mathrm{H} & 0.000000 & 0.000000 & 2.859851 \\ \mathrm{H} & 2.156599 & 0.000000 & 1.618091 \\ \mathrm{H} & 0.000000 & 0.000000 & -2.070779 \\ \mathrm{H} & 2.297444 & 0.000000 & -1.875365 \\ \mathrm{H} & -2.297444 & 0.000000 & -1.875365\end{array}$

resorcinol, isomer $3 \mathrm{~S}_{1}$

$\begin{array}{rrrc}\text { C } & -0.034229 & -0.310281 & 1.161268 \\ \mathrm{C} & -0.320141 & -1.039916 & 0.000000 \\ \mathrm{C} & -0.034229 & -0.310281 & -1.161268 \\ \mathrm{C} & 0.139227 & 1.091108 & -1.113842 \\ \mathrm{C} & -0.361725 & 1.842099 & 0.000000 \\ \mathrm{C} & 0.139227 & 1.091108 & 1.113842 \\ \mathrm{O} & 0.129883 & -0.902445 & -2.355812 \\ \mathrm{O} & 0.129883 & -0.902445 & 2.355812 \\ \mathrm{H} & 0.697982 & 1.560715 & -1.913780\end{array}$




$\begin{array}{crrc}\mathrm{H} & -0.331707 & 2.917381 & 0.000000 \\ \mathrm{H} & 0.697982 & 1.560715 & 1.913780 \\ \mathrm{H} & -0.650956 & -2.067307 & 0.000000 \\ \mathrm{H} & 0.169897 & -1.857698 & 2.263527 \\ \mathrm{H} & 0.169897 & -1.857698 & -2.263527\end{array}$

hydroquinone, cis $\mathrm{S}_{0}$

$\begin{array}{rrrr}\mathrm{C} & -0.696067 & 0.000000 & 1.090964 \\ \mathrm{C} & 0.696067 & 0.000000 & 1.090964 \\ \mathrm{C} & 1.389431 & 0.000000 & -0.107292 \\ \mathrm{C} & 0.691231 & 0.000000 & -1.309307 \\ \mathrm{C} & -0.691231 & 0.000000 & -1.309307 \\ \mathrm{C} & -1.389431 & 0.000000 & -0.107292 \\ \mathrm{O} & 2.756362 & 0.000000 & -0.173859 \\ \mathrm{O} & -2.756362 & 0.000000 & -0.173859 \\ \mathrm{H} & 1.249356 & 0.000000 & -2.233467 \\ \mathrm{H} & -1.249356 & 0.000000 & -2.233467 \\ \mathrm{H} & -1.229821 & 0.000000 & 2.031861 \\ \mathrm{H} & 1.229821 & 0.000000 & 2.031861 \\ \mathrm{H} & -3.145082 & 0.000000 & 0.701390 \\ \mathrm{H} & 3.145082 & 0.000000 & 0.701390\end{array}$

hydroquinone, cis $\mathrm{S}_{1}$

$\begin{array}{rrrr}\mathrm{C} & 0.703387 & 0.000000 & -1.136950 \\ \mathrm{C} & -0.703387 & 0.000000 & -1.136950 \\ \mathrm{C} & -1.369521 & 0.000000 & 0.118964 \\ \mathrm{C} & -0.703904 & 0.000000 & 1.362472 \\ \mathrm{C} & 0.703904 & 0.000000 & 1.362472 \\ \mathrm{C} & 1.369521 & 0.000000 & 0.118964 \\ \mathrm{O} & -2.703961 & 0.000000 & 0.163166 \\ \mathrm{O} & 2.703961 & 0.000000 & 0.163166 \\ \mathrm{H} & -1.290452 & 0.000000 & 2.265670 \\ \mathrm{H} & 1.290452 & 0.000000 & 2.265670 \\ \mathrm{H} & 1.269924 & 0.000000 & -2.055619 \\ \mathrm{H} & -1.269924 & 0.000000 & -2.055619 \\ \mathrm{H} & 3.086484 & 0.000000 & -0.716932 \\ \mathrm{H} & -3.086484 & 0.000000 & -0.716932\end{array}$

hydroquinone, trans $\mathrm{S}_{0}$
$\begin{array}{llll}\text { C } & 0.019271 & 1.386852 & 0.000000\end{array}$
$\begin{array}{llll}\text { C } & -1.192066 & 0.713835 & 0.000000\end{array}$
$\begin{array}{llll}\text { C } & -1.209658 & -0.674625 & 0.000000\end{array}$
$\begin{array}{llll}\text { C } & -0.019271 & -1.386852 & 0.000000\end{array}$ 


$\begin{array}{rrrr}\mathrm{C} & 1.192066 & -0.713835 & 0.000000 \\ \mathrm{C} & 1.209658 & 0.674625 & 0.000000 \\ \mathrm{O} & -2.334010 & 1.468244 & 0.000000 \\ \mathrm{H} & -2.151341 & -1.207332 & 0.000000 \\ \mathrm{H} & -0.015943 & -2.466365 & 0.000000 \\ \mathrm{O} & 2.334010 & -1.468244 & 0.000000 \\ \mathrm{H} & 2.151341 & 1.207332 & 0.000000 \\ \mathrm{H} & 0.015943 & 2.466365 & 0.000000 \\ \mathrm{H} & -3.114364 & 0.913001 & 0.000000 \\ \mathrm{H} & 3.114364 & -0.913001 & 0.000000\end{array}$

hydroquinone, trans $\mathrm{S}_{1}$

$\begin{array}{rrrr}\mathrm{C} & 1.261307 & 0.672896 & 0.000000 \\ \mathrm{C} & 0.031988 & 1.368576 & 0.000000 \\ \mathrm{C} & -1.237695 & 0.734064 & 0.000000 \\ \mathrm{C} & -1.261307 & -0.672896 & 0.000000 \\ \mathrm{C} & -0.031988 & -1.368576 & 0.000000 \\ \mathrm{C} & 1.237695 & -0.734064 & 0.000000 \\ \mathrm{O} & 0.115093 & 2.701013 & 0.000000 \\ \mathrm{H} & -2.145092 & 1.318682 & 0.000000 \\ \mathrm{H} & -2.175573 & -1.242350 & 0.000000 \\ \mathrm{O} & -0.115093 & -2.701013 & 0.000000 \\ \mathrm{H} & 2.145092 & -1.318682 & 0.000000 \\ \mathrm{H} & 2.175573 & 1.242350 & 0.000000 \\ \mathrm{H} & -0.754990 & 3.106706 & 0.000000 \\ \mathrm{H} & 0.754990 & -3.106706 & 0.000000\end{array}$

salicylic acid $\mathrm{S}_{0}$

$\begin{array}{rrrl}\mathrm{C} & 1.100808 & 1.596628 & 0.000000 \\ \mathrm{C} & 1.129177 & 0.199573 & 0.000000 \\ \mathrm{C} & -0.082945 & -0.503363 & 0.000000 \\ \mathrm{C} & -1.300259 & 0.185631 & 0.000000 \\ \mathrm{C} & -1.318819 & 1.562782 & 0.000000 \\ \mathrm{C} & -0.106953 & 2.261105 & 0.000000 \\ \mathrm{O} & 2.322460 & -0.404743 & 0.000000 \\ \mathrm{C} & -0.043117 & -1.975601 & 0.000000 \\ \mathrm{O} & -1.231811 & -2.573351 & 0.000000 \\ \mathrm{O} & 0.977760 & -2.625692 & 0.000000 \\ \mathrm{H} & -2.218682 & -0.382390 & 0.000000 \\ \mathrm{H} & 2.043747 & 2.121724 & 0.000000 \\ \mathrm{H} & -2.256284 & 2.096349 & 0.000000 \\ \mathrm{H} & -0.114136 & 3.342098 & 0.000000 \\ \mathrm{H} & 2.195372 & -1.372126 & 0.000000 \\ \mathrm{H} & -1.096318 & -3.528625 & 0.000000\end{array}$


salicylic acid $\mathrm{S}_{1}$

$\begin{array}{rrrl}\mathrm{C} & 1.013546 & 1.921051 & 0.000000 \\ \mathrm{C} & 0.978798 & 0.516927 & 0.000000 \\ \mathrm{C} & -0.313935 & -0.224543 & 0.000000 \\ \mathrm{C} & -1.476289 & 0.540282 & 0.000000 \\ \mathrm{C} & -1.402176 & 1.948848 & 0.000000 \\ \mathrm{C} & -0.179336 & 2.647041 & 0.000000 \\ \mathrm{O} & 2.053489 & -0.185720 & 0.000000 \\ \mathrm{C} & -0.239731 & -1.634989 & 0.000000 \\ \mathrm{O} & -1.390687 & -2.317851 & 0.000000 \\ \mathrm{O} & 0.852170 & -2.269869 & 0.000000 \\ \mathrm{H} & -2.437291 & 0.049144 & 0.000000 \\ \mathrm{H} & 1.983914 & 2.394475 & 0.000000 \\ \mathrm{H} & -2.326201 & 2.509044 & 0.000000 \\ \mathrm{H} & -0.168271 & 3.725169 & 0.000000 \\ \mathrm{H} & 1.744497 & -1.223679 & 0.000000 \\ \mathrm{H} & -1.181919 & -3.258385 & 0.000000\end{array}$

$o$-cresol, cis $\mathrm{S}_{0}$

$\begin{array}{rrrr}\mathrm{C} & 1.962167 & 0.513364 & 0.000000 \\ \mathrm{C} & 0.729919 & 1.150781 & 0.000000 \\ \mathrm{C} & -0.461612 & 0.432774 & 0.000000 \\ \mathrm{C} & -0.386812 & -0.959491 & 0.000000 \\ \mathrm{C} & 0.837226 & -1.612360 & 0.000000 \\ \mathrm{C} & 2.010639 & -0.875197 & 0.000000 \\ \mathrm{C} & -1.807483 & 1.126567 & 0.000000 \\ \mathrm{O} & -1.506506 & -1.740882 & 0.000000 \\ \mathrm{H} & 0.843980 & -2.692077 & 0.000000 \\ \mathrm{H} & 2.961433 & -1.387149 & 0.000000 \\ \mathrm{H} & 2.872912 & 1.093308 & 0.000000 \\ \mathrm{H} & 0.684990 & 2.231619 & 0.000000 \\ \mathrm{H} & -2.304452 & -1.210173 & 0.000000 \\ \mathrm{H} & -2.384419 & 0.862287 & 0.888635 \\ \mathrm{H} & -1.667560 & 2.204351 & 0.000000 \\ \mathrm{H} & -2.384419 & 0.862287 & -0.888635\end{array}$

o-cresol, cis $\mathrm{S}_{1}$
$\begin{array}{llll}\text { C } & 1.957979 & 0.504525 & 0.000000\end{array}$
$\begin{array}{llll}\text { C } & 0.722711 & 1.193722 & 0.000000\end{array}$
$\begin{array}{llll}\text { C } & -0.487945 & 0.456384 & 0.000000\end{array}$
$\begin{array}{llll}\text { C } & -0.377863 & -0.965361 & 0.000000\end{array}$
$\begin{array}{llll}\text { C } & 0.854973 & -1.670611 & 0.000000\end{array}$ 


$\begin{array}{rrrr}\mathrm{C} & 2.041502 & -0.908899 & 0.000000 \\ \mathrm{C} & -1.835904 & 1.129496 & 0.000000 \\ \mathrm{O} & -1.478533 & -1.721399 & 0.000000 \\ \mathrm{H} & 0.835799 & -2.747135 & 0.000000 \\ \mathrm{H} & 3.006746 & -1.389081 & 0.000000 \\ \mathrm{H} & 2.873611 & 1.079125 & 0.000000 \\ \mathrm{H} & 0.703470 & 2.272182 & 0.000000 \\ \mathrm{H} & -2.278957 & -1.189524 & 0.000000 \\ \mathrm{H} & -2.420826 & 0.874610 & 0.889771 \\ \mathrm{H} & -1.695935 & 2.207365 & 0.000000 \\ \mathrm{H} & -2.420826 & 0.874610 & -0.889771\end{array}$

o-cresol, trans $\mathrm{S}_{0}$

$\begin{array}{cccc}\mathrm{C} & 2.421866 & 0.000038 & 0.030735 \\ \mathrm{C} & 1.248909 & -0.000036 & -0.711654 \\ \mathrm{C} & 0.038599 & -0.000023 & -0.019217 \\ \mathrm{C} & 0.002108 & 0.000038 & 1.366930 \\ \mathrm{C} & 1.190380 & 0.000103 & 2.087935 \\ \mathrm{C} & 2.405269 & 0.000088 & 1.421525 \\ \mathrm{C} & 1.242207 & -0.000117 & -2.222465 \\ \mathrm{O} & -1.100314 & -0.000036 & -0.775268 \\ \mathrm{H} & -0.948868 & 0.000067 & 1.884088 \\ \mathrm{H} & 1.158279 & 0.000151 & 3.167241 \\ \mathrm{H} & 3.332176 & 0.000151 & 1.975820 \\ \mathrm{H} & 3.367385 & 0.000029 & -0.494649 \\ \mathrm{H} & -1.882737 & -0.000024 & -0.222024 \\ \mathrm{H} & 2.262875 & -0.000095 & -2.598104 \\ \mathrm{H} & 0.720329 & -0.880227 & -2.595403 \\ \mathrm{H} & 0.720244 & 0.879890 & -2.595519\end{array}$

$o$-cresol, trans $\mathrm{S}_{1}$

$\begin{array}{lrrc}\mathrm{C} & 2.468043 & -0.000105 & 0.003878 \\ \mathrm{C} & 1.258198 & -0.000095 & -0.741876 \\ \mathrm{C} & 0.039891 & -0.000113 & -0.019562 \\ \mathrm{C} & -0.049725 & 0.000268 & 1.408010 \\ \mathrm{C} & 1.166805 & 0.000069 & 2.115198 \\ \mathrm{C} & 2.398331 & 0.000144 & 1.413080 \\ \mathrm{C} & 1.239917 & -0.000088 & -2.244606 \\ \mathrm{O} & -1.079401 & -0.000289 & -0.751231 \\ \mathrm{H} & -1.009002 & 0.000095 & 1.901616 \\ \mathrm{H} & 1.171887 & 0.000070 & 3.193865 \\ \mathrm{H} & 3.315201 & 0.000278 & 1.986155 \\ \mathrm{H} & 3.419190 & -0.000094 & -0.502653 \\ \mathrm{H} & -1.860369 & 0.000122 & -0.191939\end{array}$




$\begin{array}{rrrr}\mathrm{H} & 2.260368 & 0.000027 & -2.619911 \\ \mathrm{H} & 0.719786 & -0.880927 & -2.625027 \\ \mathrm{H} & 0.719586 & 0.880634 & -2.625026\end{array}$

$m$-cresol, cis $\mathrm{S}_{0}$

$\begin{array}{crrr}\mathrm{C} & 1.158209 & -0.009297 & 2.080129 \\ \mathrm{C} & 2.369359 & -0.003194 & 1.409697 \\ \mathrm{C} & 2.414843 & 0.010608 & 0.019479 \\ \mathrm{C} & 1.237388 & 0.016256 & -0.717245 \\ \mathrm{C} & 0.018306 & 0.012247 & -0.043192 \\ \mathrm{C} & -0.019539 & -0.001453 & 1.343167 \\ \mathrm{C} & 1.262063 & 0.000991 & -2.232014 \\ \mathrm{O} & -1.193550 & -0.004759 & 2.039241 \\ \mathrm{H} & 1.100080 & -0.017706 & 3.157487 \\ \mathrm{H} & 3.289250 & -0.006696 & 1.976930 \\ \mathrm{H} & 3.367318 & 0.017404 & -0.491503 \\ \mathrm{H} & -0.906322 & 0.020548 & -0.608819 \\ \mathrm{H} & -1.947005 & 0.003973 & 1.447261 \\ \mathrm{H} & 2.235797 & 0.316792 & -2.599435 \\ \mathrm{H} & 1.063178 & -1.006499 & -2.597712 \\ \mathrm{H} & 0.502926 & 0.670155 & -2.633521\end{array}$

$m$-cresol, cis $\mathrm{S}_{1}$

$\begin{array}{rrrr}\mathrm{C} & 1.167115 & 0.009593 & 2.128469 \\ \mathrm{C} & 2.412882 & 0.063543 & 1.452907 \\ \mathrm{C} & 2.411888 & 0.040750 & 0.034009 \\ \mathrm{C} & 1.219368 & 0.003465 & -0.743906 \\ \mathrm{C} & -0.030678 & 0.001888 & -0.072560 \\ \mathrm{C} & -0.022230 & -0.013493 & 1.354465 \\ \mathrm{C} & 1.263999 & -0.009653 & -2.247788 \\ \mathrm{O} & -1.168203 & -0.059801 & 2.024981 \\ \mathrm{H} & 1.077197 & -0.035438 & 3.209374 \\ \mathrm{H} & 3.348078 & 0.095857 & 2.002382 \\ \mathrm{H} & 3.364786 & 0.052827 & -0.494897 \\ \mathrm{H} & -0.971877 & 0.035885 & -0.618335 \\ \mathrm{H} & -1.913563 & -0.127242 & 1.418555 \\ \mathrm{H} & 2.297929 & 0.012549 & -2.610179 \\ \mathrm{H} & 0.769992 & -0.914405 & -2.636351 \\ \mathrm{H} & 0.725619 & 0.863047 & -2.651176\end{array}$

$m$-cresol, trans $\mathrm{S}_{0}$

$\begin{array}{lrrr}\text { C } & -1.193136 & -1.100141 & 0.000000 \\ \text { C } & 0.194601 & -1.208803 & 0.000000\end{array}$ 


$\begin{array}{rrrr}\mathrm{C} & 0.964801 & -0.052196 & 0.000000 \\ \mathrm{C} & 0.360369 & 1.195763 & 0.000000 \\ \mathrm{C} & -1.025212 & 1.306714 & 0.000000 \\ \mathrm{C} & -1.793532 & 0.151353 & 0.000000 \\ \mathrm{C} & 0.877388 & -2.562070 & 0.000000 \\ \mathrm{O} & 1.180652 & 2.286560 & 0.000000 \\ \mathrm{H} & -1.497288 & 2.279947 & 0.000000 \\ \mathrm{H} & -2.871207 & 0.232153 & 0.000000 \\ \mathrm{H} & -1.802758 & -1.992347 & 0.000000 \\ \mathrm{H} & 2.045150 & -0.100697 & 0.000000 \\ \mathrm{H} & 0.673311 & 3.099921 & 0.000000 \\ \mathrm{H} & 0.140616 & -3.361990 & 0.000000 \\ \mathrm{H} & 1.505665 & -2.662822 & -0.884348 \\ \mathrm{H} & 1.505665 & -2.662822 & 0.884348\end{array}$

$m$-cresol, trans $\mathrm{S}_{1}$

$\begin{array}{rrrr}\mathrm{C} & -1.203000 & -1.057300 & 0.000000 \\ \mathrm{C} & 0.203986 & -1.230391 & 0.000000 \\ \mathrm{C} & 1.024177 & -0.078296 & 0.000000 \\ \mathrm{C} & 0.395340 & 1.188623 & 0.000000 \\ \mathrm{C} & -1.015579 & 1.370013 & 0.000000 \\ \mathrm{C} & -1.827647 & 0.216337 & 0.000000 \\ \mathrm{C} & 0.832885 & -2.594744 & 0.000000 \\ \mathrm{O} & 1.207184 & 2.248268 & 0.000000 \\ \mathrm{H} & -1.435098 & 2.364915 & 0.000000 \\ \mathrm{H} & -2.902157 & 0.293556 & 0.000000 \\ \mathrm{H} & -1.827636 & -1.940816 & 0.000000 \\ \mathrm{H} & 2.101263 & -0.127425 & 0.000000 \\ \mathrm{H} & 0.714041 & 3.072663 & 0.000000 \\ \mathrm{H} & 0.075640 & -3.376033 & 0.000000 \\ \mathrm{H} & 1.466541 & -2.717178 & -0.882277 \\ \mathrm{H} & 1.466541 & -2.717178 & 0.882277\end{array}$

$p$-cresol $\mathrm{S}_{0}$

$\begin{array}{lrcc}\mathrm{C} & 0.713916 & 0.025152 & -1.185945 \\ \mathrm{C} & -0.669967 & 0.025560 & -1.194536 \\ \mathrm{C} & -1.364018 & 0.000021 & 0.009464 \\ \mathrm{C} & -0.669599 & -0.026592 & 1.208428 \\ \mathrm{C} & 0.721931 & -0.026348 & 1.198116 \\ \mathrm{C} & 1.434937 & 0.000568 & 0.008051 \\ \mathrm{O} & -2.728523 & -0.000194 & -0.052371 \\ \mathrm{C} & 2.950754 & 0.020132 & -0.007309 \\ \mathrm{H} & -1.204959 & -0.049234 & 2.148653 \\ \mathrm{H} & 1.253310 & -0.048736 & 2.139622\end{array}$




$\begin{array}{rrrr}\mathrm{H} & 1.247498 & 0.043479 & -2.127146 \\ \mathrm{H} & -1.231318 & 0.043186 & -2.116699 \\ \mathrm{H} & -3.112911 & -0.020020 & 0.824835 \\ \mathrm{H} & 3.332656 & -0.723116 & -0.705748 \\ \mathrm{H} & 3.312917 & 1.000930 & -0.314832 \\ \mathrm{H} & 3.344938 & -0.199413 & 0.982716\end{array}$

$p$-cresol $\mathrm{S}_{1}$

$\begin{array}{lrrr}\mathrm{C} & 0.723583 & -0.563234 & -1.211302 \\ \mathrm{C} & -0.554310 & 0.054567 & -1.171513 \\ \mathrm{C} & -1.305432 & 0.008375 & 0.022400 \\ \mathrm{C} & -0.698846 & -0.308758 & 1.265444 \\ \mathrm{C} & 0.689439 & -0.238398 & 1.244319 \\ \mathrm{C} & 1.390821 & -0.207963 & 0.020501 \\ \mathrm{O} & -2.598335 & 0.338061 & -0.056104 \\ \mathrm{C} & 2.829289 & 0.235370 & -0.052814 \\ \mathrm{H} & -1.273785 & -0.474904 & 2.162611 \\ \mathrm{H} & 1.254673 & -0.212840 & 2.167073 \\ \mathrm{H} & 1.283153 & -0.602421 & -2.131572 \\ \mathrm{H} & -0.974900 & 0.582267 & -2.016888 \\ \mathrm{H} & -2.987559 & 0.415017 & 0.820052 \\ \mathrm{H} & 3.395542 & -0.477739 & -0.654915 \\ \mathrm{H} & 2.892764 & 1.213126 & -0.533718 \\ \mathrm{H} & 3.265453 & 0.304854 & 0.941718\end{array}$

$o$-methoxyphenol $\mathrm{S}_{0}$

$\begin{array}{rrrr}\mathrm{C} & 2.021841 & -0.083708 & 0.000000 \\ \mathrm{C} & 0.866027 & 0.698937 & 0.000000 \\ \mathrm{C} & -0.372173 & 0.083852 & 0.000000 \\ \mathrm{C} & -0.465920 & -1.313008 & 0.000000 \\ \mathrm{C} & 0.679854 & -2.081063 & 0.000000 \\ \mathrm{C} & 1.928292 & -1.463840 & 0.000000 \\ \mathrm{O} & -1.578582 & 0.723037 & 0.000000 \\ \mathrm{O} & -1.687103 & -1.902922 & 0.000000 \\ \mathrm{H} & 0.575028 & -3.155982 & 0.000000 \\ \mathrm{H} & 2.823106 & -2.068834 & 0.000000 \\ \mathrm{H} & 2.986991 & 0.399095 & 0.000000 \\ \mathrm{H} & 0.947450 & 1.775233 & 0.000000 \\ \mathrm{C} & -1.577323 & 2.139230 & 0.000000 \\ \mathrm{H} & -2.364138 & -1.218731 & 0.000000 \\ \mathrm{H} & -2.620402 & 2.437968 & 0.000000 \\ \mathrm{H} & -1.081474 & 2.515369 & 0.895437 \\ \mathrm{H} & -1.081474 & 2.515369 & -0.895437\end{array}$


$o$-methoxyphenol $\mathrm{S}_{1}$

$\begin{array}{rrrr}\mathrm{C} & 2.438007 & 0.136992 & 0.051997 \\ \mathrm{C} & 1.410532 & -0.408798 & -0.741295 \\ \mathrm{C} & 0.137410 & -0.187501 & -0.106392 \\ \mathrm{C} & 0.027545 & -0.187563 & 1.308387 \\ \mathrm{C} & 1.181136 & -0.194998 & 2.129105 \\ \mathrm{C} & 2.357659 & 0.143296 & 1.469000 \\ \mathrm{O} & -0.968381 & 0.301525 & -0.723743 \\ \mathrm{O} & -1.170072 & -0.013302 & 1.866603 \\ \mathrm{H} & 1.093467 & -0.318021 & 3.194996 \\ \mathrm{H} & 3.238607 & 0.422570 & 2.032309 \\ \mathrm{H} & 3.324287 & 0.552247 & -0.412741 \\ \mathrm{H} & 1.523133 & -0.628359 & -1.787451 \\ \mathrm{C} & -1.070183 & 0.111647 & -2.129694 \\ \mathrm{H} & -1.840541 & 0.059225 & 1.174208 \\ \mathrm{H} & -2.062793 & 0.451574 & -2.403879 \\ \mathrm{H} & -0.313177 & 0.705180 & -2.638767 \\ \mathrm{H} & -0.948076 & -0.945726 & -2.364563\end{array}$

$m$-methoxyphenol, isomer $1 \mathrm{~S}_{0}$

$\begin{array}{rrrr}\mathrm{C} & 0.928341 & 1.449182 & 0.000000 \\ \mathrm{C} & -0.260395 & 0.723156 & 0.000000 \\ \mathrm{C} & -0.236281 & -0.667501 & 0.000000 \\ \mathrm{C} & 0.993486 & -1.322675 & 0.000000 \\ \mathrm{C} & 2.183144 & -0.616442 & 0.000000 \\ \mathrm{C} & 2.134515 & 0.772797 & 0.000000 \\ \mathrm{H} & -1.148400 & -1.246224 & 0.000000 \\ \mathrm{O} & 1.070381 & -2.684852 & 0.000000 \\ \mathrm{H} & 3.115569 & -1.158368 & 0.000000 \\ \mathrm{H} & 3.057499 & 1.334462 & 0.000000 \\ \mathrm{H} & 0.874252 & 2.526753 & 0.000000 \\ \mathrm{O} & -1.407861 & 1.446560 & 0.000000 \\ \mathrm{H} & 0.199791 & -3.083812 & 0.000000 \\ \mathrm{C} & -2.637737 & 0.749610 & 0.000000 \\ \mathrm{H} & -3.409511 & 1.512104 & 0.000000 \\ \mathrm{H} & -2.728396 & 0.132625 & -0.895862 \\ \mathrm{H} & -2.728396 & 0.132625 & 0.895862\end{array}$

$m$-methoxyphenol, isomer $1 \mathrm{~S}_{1}$
$\begin{array}{llll}\text { C } & 1.209360 & 0.030074 & -0.542021\end{array}$
$\begin{array}{llll}\text { C } & -0.025979 & -0.101586 & 0.140111\end{array}$
$\begin{array}{llll}\text { C } & -0.043058 & -0.325841 & 1.524334\end{array}$
$\begin{array}{llll}\text { C } & 1.194838 & -0.038528 & 2.111887\end{array}$ 


$\begin{array}{rrrr}\mathrm{C} & 2.361894 & 0.086244 & 1.325517 \\ \mathrm{C} & 2.415369 & -0.510187 & 0.021904 \\ \mathrm{H} & -0.917744 & -0.584442 & 2.097413 \\ \mathrm{O} & 1.326784 & 0.181004 & 3.431832 \\ \mathrm{H} & 3.184198 & 0.667802 & 1.723030 \\ \mathrm{H} & 3.332045 & -0.515993 & -0.541613 \\ \mathrm{H} & 1.214242 & 0.569672 & -1.480323 \\ \mathrm{O} & -1.124680 & 0.050825 & -0.603886 \\ \mathrm{H} & 0.468307 & 0.242017 & 3.857341 \\ \mathrm{C} & -2.384766 & 0.059052 & 0.050618 \\ \mathrm{H} & -3.117115 & 0.253812 & -0.724885 \\ \mathrm{H} & -2.576607 & -0.911188 & 0.507940 \\ \mathrm{H} & -2.408779 & 0.847263 & 0.803540\end{array}$

$m$-methoxyphenol, isomer $2 \mathrm{~S}_{0}$

$\begin{array}{rrrr}\mathrm{C} & 0.342612 & 2.157583 & 0.000000 \\ \mathrm{C} & -0.840631 & 1.448411 & 0.000000 \\ \mathrm{C} & -0.799877 & 0.051960 & 0.000000 \\ \mathrm{C} & 0.415259 & -0.616306 & 0.000000 \\ \mathrm{C} & 1.595032 & 0.123811 & 0.000000 \\ \mathrm{C} & 1.575191 & 1.507656 & 0.000000 \\ \mathrm{O} & -1.997546 & -0.581075 & 0.000000 \\ \mathrm{C} & -2.002198 & -1.996145 & 0.000000 \\ \mathrm{O} & 2.755764 & -0.593165 & 0.000000 \\ \mathrm{H} & 0.496191 & -1.691340 & 0.000000 \\ \mathrm{H} & 2.496994 & 2.072476 & 0.000000 \\ \mathrm{H} & 0.315173 & 3.237763 & 0.000000 \\ \mathrm{H} & -1.801807 & 1.937452 & 0.000000 \\ \mathrm{H} & -3.047532 & -2.286990 & 0.000000 \\ \mathrm{H} & -1.510201 & -2.380196 & -0.894629 \\ \mathrm{H} & -1.510201 & -2.380196 & 0.894629 \\ \mathrm{H} & 3.517766 & -0.011689 & 0.000000\end{array}$

$m$-methoxyphenol, isomer $2 \mathrm{~S}_{1}$

$\begin{array}{rrrr}\text { C } & 0.348951 & 2.227701 & 0.000000 \\ \mathrm{C} & -0.832789 & 1.441545 & 0.000000 \\ \mathrm{C} & -0.781155 & 0.026904 & 0.000001 \\ \mathrm{C} & 0.439800 & -0.698230 & 0.000001 \\ \mathrm{C} & 1.598089 & 0.104824 & 0.000000 \\ \mathrm{C} & 1.574811 & 1.522818 & 0.000000 \\ \mathrm{O} & -1.974479 & -0.577839 & 0.000001 \\ \mathrm{C} & -2.027724 & -1.996512 & 0.000000 \\ \mathrm{O} & 2.771196 & -0.554886 & 0.000000 \\ \mathrm{H} & 0.527739 & -1.768354 & 0.000001\end{array}$




$\begin{array}{rrrr}\mathrm{H} & 2.516818 & 2.055641 & 0.000000 \\ \mathrm{H} & 0.313150 & 3.302433 & -0.000001 \\ \mathrm{H} & -1.814288 & 1.890977 & 0.000000 \\ \mathrm{H} & -3.082897 & -2.247072 & 0.000000 \\ \mathrm{H} & -1.547095 & -2.389477 & -0.895144 \\ \mathrm{H} & -1.547094 & -2.389479 & 0.895142 \\ \mathrm{H} & 3.516956 & 0.049016 & 0.000000\end{array}$

$p$-methoxyphenol, cis $\mathrm{S}_{0}$

$\begin{array}{rrrr}\mathrm{C} & -0.321411 & 0.734926 & 0.000000 \\ \mathrm{C} & -1.550299 & 0.074065 & 0.000000 \\ \mathrm{C} & -1.605427 & -1.303686 & 0.000000 \\ \mathrm{C} & -0.427843 & -2.047878 & 0.000000 \\ \mathrm{C} & 0.791371 & -1.399333 & 0.000000 \\ \mathrm{C} & 0.850826 & -0.004617 & 0.000000 \\ \mathrm{O} & -0.547061 & -3.411218 & 0.000000 \\ \mathrm{O} & -0.369153 & 2.096085 & 0.000000 \\ \mathrm{C} & 0.859731 & 2.792963 & 0.000000 \\ \mathrm{H} & 1.817285 & 0.473157 & 0.000000 \\ \mathrm{H} & -2.451733 & 0.669145 & 0.000000 \\ \mathrm{H} & -2.549694 & -1.826914 & 0.000000 \\ \mathrm{H} & 1.712133 & -1.967898 & 0.000000 \\ \mathrm{H} & 0.603901 & 3.847490 & 0.000000 \\ \mathrm{H} & 1.436893 & 2.552509 & 0.894574 \\ \mathrm{H} & 1.436893 & 2.552509 & -0.894574 \\ \mathrm{H} & 0.313589 & -3.831284 & 0.000000\end{array}$

$p$-methoxyphenol, cis $\mathrm{S}_{1}$

$\begin{array}{lrrr}\mathrm{C} & 1.319260 & -0.052423 & -0.618871 \\ \mathrm{C} & 0.050133 & -0.154133 & 0.013164 \\ \mathrm{C} & -0.009998 & 0.078640 & 1.395981 \\ \mathrm{C} & 1.189371 & 0.001503 & 2.124588 \\ \mathrm{C} & 2.461247 & -0.179053 & 1.503260 \\ \mathrm{C} & 2.532651 & 0.041699 & 0.116720 \\ \mathrm{O} & 1.103860 & 0.106879 & 3.454624 \\ \mathrm{O} & 1.288368 & -0.050879 & -1.946308 \\ \mathrm{C} & 2.496912 & 0.052257 & -2.688327 \\ \mathrm{H} & 3.483085 & 0.151486 & -0.377364 \\ \mathrm{H} & -0.824665 & -0.255090 & -0.606705 \\ \mathrm{H} & -0.929771 & 0.277913 & 1.921363 \\ \mathrm{H} & 3.336597 & -0.403035 & 2.094856 \\ \mathrm{H} & 2.198120 & 0.025071 & -3.730234 \\ \mathrm{H} & 2.993538 & 0.994147 & -2.462024 \\ \mathrm{H} & 3.146884 & -0.791325 & -2.459919\end{array}$




\section{H $\quad 1.977939 \quad 0.156327 \quad 3.852196$}

p-methoxyphenol, trans $\mathrm{S}_{0}$

$\begin{array}{crcc}\mathrm{C} & -0.655224 & 1.306281 & 0.000000 \\ \mathrm{C} & -1.884792 & 0.673225 & 0.000000 \\ \mathrm{C} & -1.952272 & -0.716555 & 0.000000 \\ \mathrm{C} & -0.785141 & -1.457564 & 0.000000 \\ \mathrm{C} & 0.453196 & -0.824392 & 0.000000 \\ \mathrm{C} & 0.521708 & 0.563864 & 0.000000 \\ \mathrm{O} & -3.139967 & -1.397071 & 0.000000 \\ \mathrm{H} & 1.346792 & -1.429362 & 0.000000 \\ \mathrm{O} & 1.683957 & 1.274224 & 0.000000 \\ \mathrm{H} & -0.581897 & 2.383781 & 0.000000 \\ \mathrm{H} & -2.792291 & 1.263348 & 0.000000 \\ \mathrm{H} & -3.882715 & -0.792005 & 0.000000 \\ \mathrm{H} & -0.854824 & -2.535253 & 0.000000 \\ \mathrm{C} & 2.895576 & 0.546618 & 0.000000 \\ \mathrm{H} & 3.687316 & 1.288546 & 0.000000 \\ \mathrm{H} & 2.970283 & -0.073851 & 0.894969 \\ \mathrm{H} & 2.970283 & -0.073851 & -0.894969\end{array}$

p-methoxyphenol, trans $\mathrm{S}_{1}$

$\begin{array}{crrr}\mathrm{C} & 0.106818 & 0.053738 & -1.385488 \\ \mathrm{C} & -1.266538 & -0.185978 & -1.209914 \\ \mathrm{C} & -1.770269 & 0.013454 & 0.102067 \\ \mathrm{C} & -0.934503 & 0.145099 & 1.231760 \\ \mathrm{C} & 0.445466 & -0.074454 & 1.080399 \\ \mathrm{C} & 0.953560 & 0.009843 & -0.246655 \\ \mathrm{O} & -3.086824 & 0.095818 & 0.321317 \\ \mathrm{H} & 1.094333 & -0.141578 & 1.936001 \\ \mathrm{O} & 2.253512 & 0.065097 & -0.513510 \\ \mathrm{H} & 0.569521 & 0.146129 & -2.354794 \\ \mathrm{H} & -1.925446 & -0.424076 & -2.030737 \\ \mathrm{H} & -3.570676 & 0.117267 & -0.509307 \\ \mathrm{H} & -1.388745 & 0.370276 & 2.183466 \\ \mathrm{C} & 3.200375 & -0.021567 & 0.544152 \\ \mathrm{H} & 4.171499 & 0.026301 & 0.065022 \\ \mathrm{H} & 3.075661 & 0.816867 & 1.227736 \\ \mathrm{H} & 3.083045 & -0.967927 & 1.069183\end{array}$

1-naphthol, cis $\mathrm{S}_{0}$
C 0.829069
$\begin{array}{ll}1.507680 & -0.008087\end{array}$
C 0.520578
$0.114493-0.021455$ 


$\begin{array}{rrrr}\mathrm{C} & -0.838013 & -0.269189 & 0.004086 \\ \mathrm{C} & -1.853672 & 0.722766 & -0.010675 \\ \mathrm{C} & -1.517723 & 2.042833 & -0.042709 \\ \mathrm{C} & -0.160594 & 2.443224 & -0.033763 \\ \mathrm{C} & 1.524041 & -0.889651 & -0.073947 \\ \mathrm{C} & 1.184818 & -2.210759 & -0.053687 \\ \mathrm{C} & -0.175565 & -2.599732 & 0.008569 \\ \mathrm{C} & -1.157289 & -1.653683 & 0.028390 \\ \mathrm{O} & 2.127665 & 1.935659 & -0.007230 \\ \mathrm{H} & -2.199402 & -1.942484 & 0.058875 \\ \mathrm{H} & -0.429811 & -3.650023 & 0.027194 \\ \mathrm{H} & 1.954190 & -2.967716 & -0.100642 \\ \mathrm{H} & 2.565679 & -0.615780 & -0.164979 \\ \mathrm{H} & -2.889171 & 0.411853 & -0.000049 \\ \mathrm{H} & -2.286515 & 2.801558 & -0.061954 \\ \mathrm{H} & 0.114549 & 3.487694 & -0.030976 \\ \mathrm{H} & 2.687177 & 1.331267 & 0.483036\end{array}$

1-naphthol, cis $\mathrm{S}_{1}$

$\begin{array}{rrrr}\text { C } & 0.872081 & 1.459243 & 0.023968 \\ \mathrm{C} & 0.540929 & 0.087808 & -0.069471 \\ \mathrm{C} & -0.854694 & -0.247439 & 0.029434 \\ \mathrm{C} & -1.793236 & 0.792888 & -0.015889 \\ \mathrm{C} & -1.417034 & 2.172199 & -0.049647 \\ \mathrm{C} & -0.102789 & 2.510531 & -0.058516 \\ \mathrm{C} & 1.492736 & -0.926508 & -0.176476 \\ \mathrm{C} & 1.091476 & -2.298735 & -0.087208 \\ \mathrm{C} & -0.229006 & -2.620405 & 0.046315 \\ \mathrm{C} & -1.218654 & -1.598945 & 0.088442 \\ \mathrm{O} & 2.133377 & 1.849459 & 0.202428 \\ \mathrm{H} & -2.260622 & -1.860552 & 0.194570 \\ \mathrm{H} & -0.535653 & -3.653298 & 0.116753 \\ \mathrm{H} & 1.844974 & -3.070397 & -0.133558 \\ \mathrm{H} & 2.527184 & -0.704639 & -0.399701 \\ \mathrm{H} & -2.844324 & 0.540144 & -0.024440 \\ \mathrm{H} & -2.183745 & 2.930409 & -0.096436 \\ \mathrm{H} & 0.251199 & 3.529570 & -0.070342 \\ \mathrm{H} & 2.685801 & 1.108657 & 0.479754\end{array}$

1-naphthol, trans $\mathrm{S}_{0}$
$\begin{array}{llll}\text { C } & -0.713324 & -0.538763 & 0.000000\end{array}$
$\begin{array}{llll}\text { C } & -0.673600 & -1.957912 & 0.000000\end{array}$
$\begin{array}{llll}\text { C } & 0.518808 & -2.621590 & 0.000000\end{array}$
$\begin{array}{llll}\text { C } & 1.738982 & -1.901758 & 0.000000\end{array}$ 


$\begin{array}{crcc}\mathrm{C} & 1.734998 & -0.536864 & 0.000000 \\ \mathrm{C} & 0.505080 & 0.168101 & 0.000000 \\ \mathrm{H} & -1.607047 & -2.503994 & 0.000000 \\ \mathrm{H} & 0.537612 & -3.701818 & 0.000000 \\ \mathrm{H} & 2.675318 & -2.440158 & 0.000000 \\ \mathrm{H} & 2.655106 & 0.027110 & 0.000000 \\ \mathrm{C} & -1.943395 & 0.175050 & 0.000000 \\ \mathrm{C} & -1.939606 & 1.536306 & 0.000000 \\ \mathrm{C} & -0.721233 & 2.261074 & 0.000000 \\ \mathrm{C} & 0.467665 & 1.592475 & 0.000000 \\ \mathrm{H} & -2.871628 & -0.378198 & 0.000000 \\ \mathrm{H} & -2.870754 & 2.083873 & 0.000000 \\ \mathrm{H} & -0.739275 & 3.342679 & 0.000000 \\ \mathrm{O} & 1.676431 & 2.220855 & 0.000000 \\ \mathrm{H} & 1.569873 & 3.173520 & 0.000000\end{array}$

1-naphthol, trans $\mathrm{S}_{1}$

$\begin{array}{rrrr}\mathrm{C} & -0.951482 & -0.397287 & 0.000000 \\ \mathrm{C} & -1.304179 & -1.749013 & 0.000000 \\ \mathrm{C} & -0.311121 & -2.773351 & 0.000000 \\ \mathrm{C} & 1.010835 & -2.424761 & 0.000000 \\ \mathrm{C} & 1.408137 & -1.052726 & 0.000000 \\ \mathrm{C} & 0.446852 & -0.040985 & 0.000000 \\ \mathrm{H} & -2.350801 & -2.016951 & 0.000000 \\ \mathrm{H} & -0.611591 & -3.809612 & 0.000000 \\ \mathrm{H} & 1.778240 & -3.184941 & 0.000000 \\ \mathrm{H} & 2.454886 & -0.793221 & 0.000000 \\ \mathrm{C} & -1.897085 & 0.639121 & 0.000000 \\ \mathrm{C} & -1.516309 & 2.015521 & 0.000000 \\ \mathrm{C} & -0.204454 & 2.372269 & 0.000000 \\ \mathrm{C} & 0.783451 & 1.324376 & 0.000000 \\ \mathrm{H} & -2.948512 & 0.389282 & 0.000000 \\ \mathrm{H} & -2.287396 & 2.771564 & 0.000000 \\ \mathrm{H} & 0.108563 & 3.406921 & 0.000000 \\ \mathrm{O} & 2.087965 & 1.636576 & 0.000000 \\ \mathrm{H} & 2.228323 & 2.586291 & 0.000000\end{array}$

2-naphthol, cis $\mathrm{S}_{0}$
$\begin{array}{lll}\text { C } & 0.597143 & 2.010257\end{array}$
0.000000
$\begin{array}{llll}\text { C } & 0.614914 & 0.591980 & 0.000000\end{array}$
$\begin{array}{llll}\text { C } & -0.604574 & -0.115649 & 0.000000\end{array}$
$\begin{array}{llll}\text { C } & -1.821526 & 0.615380 & 0.000000\end{array}$
$\begin{array}{llll}\text { C } & -1.811837 & 1.980030 & 0.000000\end{array}$
$\begin{array}{llll}\text { C } & -0.585738 & 2.690893 & 0.000000\end{array}$ 


$\begin{array}{lrrr}\mathrm{C} & 1.832483 & -0.143794 & 0.000000 \\ \mathrm{C} & 1.829486 & -1.503249 & 0.000000 \\ \mathrm{C} & 0.596843 & -2.206914 & 0.000000 \\ \mathrm{C} & -0.589746 & -1.536461 & 0.000000 \\ \mathrm{H} & -1.530513 & -2.073258 & 0.000000 \\ \mathrm{H} & 1.539517 & 2.541958 & 0.000000 \\ \mathrm{H} & -0.595416 & 3.771094 & 0.000000 \\ \mathrm{H} & -2.743262 & 2.527068 & 0.000000 \\ \mathrm{H} & -2.757982 & 0.073594 & 0.000000 \\ \mathrm{H} & 2.769910 & 0.396345 & 0.000000 \\ \mathrm{H} & 2.742125 & -2.079748 & 0.000000 \\ \mathrm{O} & 0.693018 & -3.566235 & 0.000000 \\ \mathrm{H} & -0.174856 & -3.973281 & 0.000000\end{array}$

2-naphthol, cis $\mathrm{S}_{1}$

$\begin{array}{lrrl}\mathrm{C} & 0.606443 & 1.985131 & 0.000000 \\ \mathrm{C} & 0.652771 & 0.584512 & 0.000000 \\ \mathrm{C} & -0.638830 & -0.118931 & 0.000000 \\ \mathrm{C} & -1.840265 & 0.608633 & 0.000000 \\ \mathrm{C} & -1.846268 & 2.011369 & 0.000000 \\ \mathrm{C} & -0.618753 & 2.675653 & 0.000000 \\ \mathrm{C} & 1.854219 & -0.148011 & 0.000000 \\ \mathrm{C} & 1.880712 & -1.547347 & 0.000000 \\ \mathrm{C} & 0.650508 & -2.204377 & 0.000000 \\ \mathrm{C} & -0.593291 & -1.515179 & 0.000000 \\ \mathrm{H} & -1.512661 & -2.088115 & 0.000000 \\ \mathrm{H} & 1.531024 & 2.545711 & 0.000000 \\ \mathrm{H} & -0.598723 & 3.757042 & 0.000000 \\ \mathrm{H} & -2.772274 & 2.561888 & 0.000000 \\ \mathrm{H} & -2.773815 & 0.061895 & 0.000000 \\ \mathrm{H} & 2.785517 & 0.401879 & 0.000000 \\ \mathrm{H} & 2.791745 & -2.120356 & 0.000000 \\ \mathrm{O} & 0.664588 & -3.542672 & 0.000000 \\ \mathrm{H} & -0.222637 & -3.908715 & 0.000000\end{array}$

2-naphthol, trans $\mathrm{S}_{0}$

$\begin{array}{rrrc}\text { C } & 0.509827 & 2.012916 & 0.000000 \\ \text { C } & 0.533768 & 0.592678 & 0.000000 \\ \text { C } & -0.683182 & -0.122464 & 0.000000 \\ \text { C } & -1.904782 & 0.605238 & 0.000000 \\ \text { C } & -1.900152 & 1.968416 & 0.000000 \\ \text { C } & -0.675514 & 2.686227 & 0.000000 \\ \text { C } & 1.750736 & -0.135642 & 0.000000 \\ \text { C } & 1.751568 & -1.498544 & 0.000000\end{array}$




$\begin{array}{lrrr}\mathrm{C} & 0.522917 & -2.207810 & 0.000000 \\ \mathrm{C} & -0.664585 & -1.538909 & 0.000000 \\ \mathrm{H} & -1.588117 & -2.100383 & 0.000000 \\ \mathrm{H} & 1.449567 & 2.549293 & 0.000000 \\ \mathrm{H} & -0.691136 & 3.766399 & 0.000000 \\ \mathrm{H} & -2.833490 & 2.512235 & 0.000000 \\ \mathrm{H} & -2.837729 & 0.057806 & 0.000000 \\ \mathrm{H} & 2.687409 & 0.405763 & 0.000000 \\ \mathrm{H} & 2.685011 & -2.045866 & 0.000000 \\ \mathrm{O} & 0.500920 & -3.571690 & 0.000000 \\ \mathrm{H} & 1.386974 & -3.935664 & 0.000000\end{array}$

2-naphthol, trans $\mathrm{S}_{1}$

$\begin{array}{rrrc}\mathrm{C} & 0.517162 & 1.987632 & 0.000000 \\ \mathrm{C} & 0.566444 & 0.587973 & 0.000000 \\ \mathrm{C} & -0.719368 & -0.117968 & 0.000000 \\ \mathrm{C} & -1.921599 & 0.604164 & 0.000000 \\ \mathrm{C} & -1.933632 & 2.009854 & 0.000000 \\ \mathrm{C} & -0.711942 & 2.676039 & 0.000000 \\ \mathrm{C} & 1.770558 & -0.139327 & 0.000000 \\ \mathrm{C} & 1.803586 & -1.541589 & 0.000000 \\ \mathrm{C} & 0.570878 & -2.199004 & 0.000000 \\ \mathrm{C} & -0.670898 & -1.514958 & 0.000000 \\ \mathrm{H} & -1.569951 & -2.113933 & 0.000000 \\ \mathrm{H} & 1.439614 & 2.551804 & 0.000000 \\ \mathrm{H} & -0.693472 & 3.757488 & 0.000000 \\ \mathrm{H} & -2.862380 & 2.555724 & 0.000000 \\ \mathrm{H} & -2.852456 & 0.053138 & 0.000000 \\ \mathrm{H} & 2.700001 & 0.413731 & 0.000000 \\ \mathrm{H} & 2.732085 & -2.090313 & 0.000000 \\ \mathrm{O} & 0.483765 & -3.535062 & 0.000000 \\ \mathrm{H} & 1.351603 & -3.945382 & 0.000000\end{array}$

5-methoxysalicylic acid $\mathrm{S}_{0}$
$\begin{array}{llll}\text { C } & 2.275916 & -0.168122 & 0.000000\end{array}$
$\begin{array}{llll}\text { C } & 1.565547 & -1.340714 & 0.000000\end{array}$
$\begin{array}{llll}\text { C } & 0.161085 & -1.336962 & 0.000000\end{array}$
$\begin{array}{llll}\text { C } & -0.510430 & -0.137087 & 0.000000\end{array}$
$\begin{array}{llll}\text { C } & 0.217479 & 1.066406 & 0.000000\end{array}$
C $\quad 1.609630 \quad 1.065280 \quad 0.000000$
$\begin{array}{llll}\mathrm{O} & -0.435323 & -2.558067 & 0.000000\end{array}$
C $\quad-1.848943 \quad-2.592370 \quad 0.000000$
$\begin{array}{llll}\text { C } & -0.483603 & 2.361705 & 0.000000\end{array}$
$\begin{array}{llll}\mathrm{O} & -1.813402 & 2.284583 & 0.000000\end{array}$ 


$\begin{array}{lrrr}\mathrm{O} & 2.356770 & 2.179522 & 0.000000 \\ \mathrm{O} & 0.074507 & 3.434659 & 0.000000 \\ \mathrm{H} & -2.119590 & -3.642750 & 0.000000 \\ \mathrm{H} & -2.242631 & -2.107708 & -0.894885 \\ \mathrm{H} & -2.242631 & -2.107708 & 0.894885 \\ \mathrm{H} & -1.587329 & -0.081843 & 0.000000 \\ \mathrm{H} & 3.355611 & -0.160765 & 0.000000 \\ \mathrm{H} & 2.067620 & -2.297241 & 0.000000 \\ \mathrm{H} & 1.773732 & 2.960027 & 0.000000 \\ \mathrm{H} & -2.173996 & 3.179156 & 0.000000\end{array}$

5-methoxysalicylic acid $\mathrm{S}_{1}$

$\begin{array}{lrrc}\mathrm{C} & 2.184466 & -0.136226 & 0.000000 \\ \mathrm{C} & 1.728019 & -1.447974 & 0.000000 \\ \mathrm{C} & 0.329504 & -1.683285 & 0.000000 \\ \mathrm{C} & -0.617851 & -0.635354 & 0.000000 \\ \mathrm{C} & -0.179908 & 0.686687 & 0.000000 \\ \mathrm{C} & 1.278150 & 0.930915 & 0.000000 \\ \mathrm{O} & -0.025088 & -2.964286 & 0.000000 \\ \mathrm{C} & -1.402067 & -3.321580 & 0.000000 \\ \mathrm{C} & -1.024200 & 1.821500 & 0.000000 \\ \mathrm{O} & -2.347980 & 1.601670 & 0.000000 \\ \mathrm{O} & 1.675715 & 2.160609 & 0.000000 \\ \mathrm{O} & -0.597690 & 3.003858 & 0.000000 \\ \mathrm{H} & -1.416883 & -4.405607 & 0.000000 \\ \mathrm{H} & -1.888020 & -2.938112 & -0.896288 \\ \mathrm{H} & -1.888020 & -2.938112 & 0.896288 \\ \mathrm{H} & -1.677890 & -0.832551 & 0.000000 \\ \mathrm{H} & 3.237389 & 0.102788 & 0.000000 \\ \mathrm{H} & 2.391192 & -2.296441 & 0.000000 \\ \mathrm{H} & 0.803477 & 2.764345 & 0.000000 \\ \mathrm{H} & -2.790264 & 2.457933 & 0.000000\end{array}$

m-dimethoxybenzene, trans $\mathrm{S}_{0}$
$\begin{array}{llll}\text { C } & 0.000000 & -0.705259 & -0.183780\end{array}$
$\begin{array}{llll}\text { C } & 0.000000 & 0.705259 & -0.183780\end{array}$
$\begin{array}{llll}\text { C } & 0.000000 & 1.386952 & 1.019089\end{array}$
$\begin{array}{llll}\text { C } & 0.000000 & 0.688579 & 2.230013\end{array}$
$\begin{array}{llll}\text { C } & 0.000000 & -0.688579 & 2.230013\end{array}$
$\begin{array}{llll}\text { C } & 0.000000 & -1.386952 & 1.019089\end{array}$
$\begin{array}{llll}\mathrm{O} & 0.000000 & 1.297150 & -1.399232\end{array}$
$\begin{array}{llll}\mathrm{H} & 0.000000 & 2.465612 & 1.031517\end{array}$
$\begin{array}{llll}\mathrm{H} & 0.000000 & 1.238064 & 3.159249\end{array}$
H $\quad 0.000000 \quad-1.238064 \quad 3.159249$ 


$\begin{array}{lrrr}\mathrm{H} & 0.000000 & -2.465612 & 1.031517 \\ \mathrm{O} & 0.000000 & -1.297150 & -1.399232 \\ \mathrm{C} & 0.000000 & -2.709376 & -1.441333 \\ \mathrm{H} & 0.000000 & -2.970873 & -2.494316 \\ \mathrm{H} & 0.894662 & -3.108602 & -0.960593 \\ \mathrm{H} & -0.894662 & -3.108602 & -0.960593 \\ \mathrm{C} & 0.000000 & 2.709376 & -1.441333 \\ \mathrm{H} & 0.000000 & 2.970873 & -2.494316 \\ \mathrm{H} & -0.894662 & 3.108602 & -0.960593 \\ \mathrm{H} & 0.894662 & 3.108602 & -0.960593\end{array}$

$m$-dimethoxybenzene, trans $\mathrm{S}_{1}$

$\begin{array}{rrrc}\mathrm{C} & -0.262004 & -1.068355 & -1.433878 \\ \mathrm{C} & -0.288000 & 0.170774 & -0.709791 \\ \mathrm{C} & -0.288000 & 0.170774 & 0.709791 \\ \mathrm{C} & -0.262004 & -1.068355 & 1.433878 \\ \mathrm{C} & 0.307157 & -2.119290 & 0.708265 \\ \mathrm{C} & 0.307157 & -2.119290 & -0.708265 \\ \mathrm{O} & -0.127385 & 1.361990 & -1.289470 \\ \mathrm{C} & 0.002253 & 1.417983 & -2.703219 \\ \mathrm{O} & -0.127385 & 1.361990 & 1.289470 \\ \mathrm{C} & 0.002253 & 1.417983 & 2.703219 \\ \mathrm{H} & -0.463115 & -1.133451 & 2.488220 \\ \mathrm{H} & 0.722329 & -2.976926 & 1.222872 \\ \mathrm{H} & 0.722329 & -2.976926 & -1.222872 \\ \mathrm{H} & -0.463115 & -1.133451 & -2.488220 \\ \mathrm{H} & 0.124936 & 2.469463 & -2.938060 \\ \mathrm{H} & 0.875026 & 0.850558 & -3.023201 \\ \mathrm{H} & -0.898107 & 1.029693 & -3.178366 \\ \mathrm{H} & 0.124936 & 2.469463 & 2.938060 \\ \mathrm{H} & -0.898107 & 1.029693 & 3.178366 \\ \mathrm{H} & 0.875026 & 0.850558 & 3.023201\end{array}$

3P-propionic acid, gauche $\mathrm{S}_{0}$

$\begin{array}{lrrr}\mathrm{C} & -0.997823 & 0.897258 & -1.157947 \\ \mathrm{C} & -0.364573 & 0.686669 & 0.064999 \\ \mathrm{C} & -1.009995 & -0.075788 & 1.034632 \\ \mathrm{C} & -2.263794 & -0.620239 & 0.782036 \\ \mathrm{C} & -2.886714 & -0.406880 & -0.440202 \\ \mathrm{C} & -2.249973 & 0.355204 & -1.412538 \\ \mathrm{C} & 1.018369 & 1.256476 & 0.310277 \\ \mathrm{C} & 2.108271 & 0.326633 & -0.246166 \\ \mathrm{C} & 2.237446 & -0.926500 & 0.591691 \\ \mathrm{O} & 1.973225 & -1.010491 & 1.754610\end{array}$




$\begin{array}{lrrr}\mathrm{O} & 2.728589 & -1.965653 & -0.096003 \\ \mathrm{H} & 1.898057 & 0.034211 & -1.271828 \\ \mathrm{H} & 3.085282 & 0.812424 & -0.216717 \\ \mathrm{H} & 1.182855 & 1.394309 & 1.376168 \\ \mathrm{H} & 1.102536 & 2.222489 & -0.184534 \\ \mathrm{H} & -0.521319 & -0.249195 & 1.983345 \\ \mathrm{H} & -2.754661 & -1.209572 & 1.543425 \\ \mathrm{H} & -3.861977 & -0.828374 & -0.634765 \\ \mathrm{H} & -2.729511 & 0.530977 & -2.364911 \\ \mathrm{H} & -0.507065 & 1.495285 & -1.915767 \\ \mathrm{H} & 2.812785 & -2.719262 & 0.500203\end{array}$

3P-propionic acid, gauche $\mathrm{S}_{1}$

$\begin{array}{rrrr}\mathrm{C} & -0.955350 & 0.980372 & -1.139363 \\ \mathrm{C} & -0.301491 & 0.809316 & 0.111672 \\ \mathrm{C} & -0.903080 & 0.019239 & 1.128055 \\ \mathrm{C} & -2.038058 & -0.775318 & 0.831533 \\ \mathrm{C} & -2.664141 & -0.625646 & -0.429906 \\ \mathrm{C} & -2.076497 & 0.168348 & -1.443823 \\ \mathrm{C} & 1.073072 & 1.375981 & 0.319311 \\ \mathrm{C} & 2.154645 & 0.412547 & -0.238133 \\ \mathrm{C} & 2.066970 & -0.892927 & 0.519663 \\ \mathrm{O} & 2.257490 & -1.004064 & 1.696018 \\ \mathrm{O} & 1.736147 & -1.936255 & -0.247653 \\ \mathrm{H} & 1.997946 & 0.230406 & -1.296180 \\ \mathrm{H} & 3.143326 & 0.830813 & -0.061015 \\ \mathrm{H} & 1.260055 & 1.521656 & 1.381871 \\ \mathrm{H} & 1.160968 & 2.330199 & -0.199514 \\ \mathrm{H} & -0.462643 & 0.001134 & 2.114448 \\ \mathrm{H} & -2.446813 & -1.444262 & 1.571600 \\ \mathrm{H} & -3.607414 & -1.116737 & -0.620667 \\ \mathrm{H} & -2.494139 & 0.168273 & -2.438700 \\ \mathrm{H} & -0.553113 & 1.664921 & -1.870979 \\ \mathrm{H} & 1.652130 & -2.718014 & 0.311772\end{array}$

3P-propionic acid, anti $\mathrm{S}_{0}$

$\begin{array}{lrrr}\mathrm{C} & 1.427400 & -0.447595 & 0.000000 \\ \mathrm{C} & 0.547345 & 0.808213 & 0.000000 \\ \mathrm{C} & 2.898660 & -0.104058 & 0.000000 \\ \mathrm{H} & 1.230775 & -1.063312 & 0.877581 \\ \mathrm{H} & 1.230775 & -1.063312 & -0.877581 \\ \mathrm{C} & -0.913989 & 0.410653 & 0.000000 \\ \mathrm{H} & 0.775664 & 1.407406 & 0.878482 \\ \mathrm{H} & 0.775664 & 1.407406 & -0.878482\end{array}$




$\begin{array}{lrrr}\mathrm{C} & -1.590532 & 0.202752 & 1.198504 \\ \mathrm{C} & -2.917401 & -0.208325 & 1.201178 \\ \mathrm{C} & -3.583554 & -0.415566 & 0.000000 \\ \mathrm{C} & -2.917401 & -0.208325 & -1.201178 \\ \mathrm{C} & -1.590532 & 0.202752 & -1.198504 \\ \mathrm{H} & -1.075803 & 0.369230 & 2.135918 \\ \mathrm{H} & -3.431508 & -0.362440 & 2.138838 \\ \mathrm{H} & -4.616314 & -0.732600 & 0.000000 \\ \mathrm{H} & -3.431508 & -0.362440 & -2.138838 \\ \mathrm{H} & -1.075803 & 0.369230 & -2.135918 \\ \mathrm{O} & 3.678562 & -1.197145 & 0.000000 \\ \mathrm{O} & 3.360218 & 0.997600 & 0.000000 \\ \mathrm{H} & 4.602121 & -0.918801 & 0.000000\end{array}$

3P-propionic acid, anti $\mathrm{S}_{1}$

$\begin{array}{lrrr}\mathrm{C} & 1.394962 & -0.454671 & 0.000000 \\ \mathrm{C} & 0.554561 & 0.835810 & 0.000000 \\ \mathrm{C} & 2.874231 & -0.145470 & 0.000000 \\ \mathrm{H} & 1.179531 & -1.060469 & 0.879161 \\ \mathrm{H} & 1.179531 & -1.060469 & -0.879161 \\ \mathrm{C} & -0.909456 & 0.503850 & 0.000000 \\ \mathrm{H} & 0.804164 & 1.422432 & 0.882410 \\ \mathrm{H} & 0.804164 & 1.422432 & -0.882410 \\ \mathrm{C} & -1.592290 & 0.310756 & 1.230790 \\ \mathrm{C} & -2.883435 & -0.273204 & 1.232141 \\ \mathrm{C} & -3.551304 & -0.472724 & 0.000000 \\ \mathrm{C} & -2.883435 & -0.273204 & -1.232141 \\ \mathrm{C} & -1.592290 & 0.310756 & -1.230790 \\ \mathrm{H} & -1.107326 & 0.575993 & 2.158810 \\ \mathrm{H} & -3.369894 & -0.524063 & 2.161465 \\ \mathrm{H} & -4.589765 & -0.770749 & 0.000000 \\ \mathrm{H} & -3.369894 & -0.524063 & -2.161465 \\ \mathrm{H} & -1.107326 & 0.575993 & -2.158810 \\ \mathrm{O} & 3.629561 & -1.255057 & 0.000000 \\ \mathrm{O} & 3.358851 & 0.946869 & 0.000000 \\ \mathrm{H} & 4.559700 & -0.999425 & 0.000000\end{array}$

fluorine $\mathrm{S}_{0}$
$\begin{array}{llll}\text { C } & 0.000000 & 0.000000 & -1.801700\end{array}$
$\begin{array}{llll}\text { C } & 0.000000 & 1.182082 & -0.848048\end{array}$
$\begin{array}{llll}\text { C } & 0.000000 & 0.738954 & 0.477236\end{array}$
$\begin{array}{llll}\text { C } & 0.000000 & -0.738954 & 0.477236\end{array}$
$\begin{array}{llll}\text { C } & 0.000000 & 1.642540 & 1.529072\end{array}$
$\begin{array}{llll}\text { C } & 0.000000 & 3.001949 & 1.239173\end{array}$ 


$\begin{array}{lrrr}\mathrm{C} & 0.000000 & 3.445774 & -0.080527 \\ \mathrm{C} & 0.000000 & 2.535731 & -1.134216 \\ \mathrm{H} & 0.000000 & 2.884672 & -2.157931 \\ \mathrm{H} & 0.000000 & 4.505948 & -0.287928 \\ \mathrm{H} & 0.000000 & 3.721175 & 2.045062 \\ \mathrm{H} & 0.000000 & 1.299832 & 2.554260 \\ \mathrm{C} & 0.000000 & -1.642540 & 1.529072 \\ \mathrm{C} & 0.000000 & -3.001949 & 1.239173 \\ \mathrm{C} & 0.000000 & -3.445774 & -0.080527 \\ \mathrm{C} & 0.000000 & -2.535731 & -1.134216 \\ \mathrm{C} & 0.000000 & -1.182082 & -0.848048 \\ \mathrm{H} & 0.000000 & -1.299832 & 2.554260 \\ \mathrm{H} & 0.000000 & -3.721175 & 2.045062 \\ \mathrm{H} & 0.000000 & -4.505948 & -0.287928 \\ \mathrm{H} & 0.000000 & -2.884672 & -2.157931 \\ \mathrm{H} & -0.887121 & 0.000000 & -2.435292 \\ \mathrm{H} & 0.887121 & 0.000000 & -2.435292\end{array}$

fluorine $\mathrm{S}_{1}$

$\begin{array}{lrrr}\mathrm{C} & 0.000000 & 0.000000 & -1.886431 \\ \mathrm{C} & 0.000000 & 1.180690 & -0.924825 \\ \mathrm{C} & 0.000000 & 0.692471 & 0.445134 \\ \mathrm{C} & 0.000000 & -0.692471 & 0.445134 \\ \mathrm{C} & 0.000000 & 1.629897 & 1.537542 \\ \mathrm{C} & 0.000000 & 2.966263 & 1.234023 \\ \mathrm{C} & 0.000000 & 3.425237 & -0.101844 \\ \mathrm{C} & 0.000000 & 2.519546 & -1.186524 \\ \mathrm{H} & 0.000000 & 2.889933 & -2.202076 \\ \mathrm{H} & 0.000000 & 4.487462 & -0.294838 \\ \mathrm{H} & 0.000000 & 3.696005 & 2.031199 \\ \mathrm{H} & 0.000000 & 1.280675 & 2.558733 \\ \mathrm{C} & 0.000000 & -1.629897 & 1.537542 \\ \mathrm{C} & 0.000000 & -2.966263 & 1.234023 \\ \mathrm{C} & 0.000000 & -3.425237 & -0.101844 \\ \mathrm{C} & 0.000000 & -2.519546 & -1.186524 \\ \mathrm{C} & 0.000000 & -1.180690 & -0.924825 \\ \mathrm{H} & 0.000000 & -1.280675 & 2.558733 \\ \mathrm{H} & 0.000000 & -3.696005 & 2.031199 \\ \mathrm{H} & 0.000000 & -4.487462 & -0.294838 \\ \mathrm{H} & 0.000000 & -2.889933 & -2.202076 \\ \mathrm{H} & -0.886515 & 0.000000 & -2.521147 \\ \mathrm{H} & 0.886515 & 0.000000 & -2.521147 \\ & & & \end{array}$

phenanthrene $S_{0}$ 


$\begin{array}{rrrr}\mathrm{C} & 0.731077 & 0.000000 & -0.345993 \\ \mathrm{C} & -0.731077 & 0.000000 & -0.345993 \\ \mathrm{C} & -1.413983 & 0.000000 & 0.881101 \\ \mathrm{C} & -0.671880 & 0.000000 & 2.119102 \\ \mathrm{C} & 0.671880 & 0.000000 & 2.119102 \\ \mathrm{C} & 1.413983 & 0.000000 & 0.881101 \\ \mathrm{C} & -1.490633 & 0.000000 & -1.533828 \\ \mathrm{C} & -2.822943 & 0.000000 & 0.900767 \\ \mathrm{H} & -1.226254 & 0.000000 & 3.047567 \\ \mathrm{H} & 1.226254 & 0.000000 & 3.047567 \\ \mathrm{C} & 1.490633 & 0.000000 & -1.533828 \\ \mathrm{C} & 2.863662 & 0.000000 & -1.497324 \\ \mathrm{C} & 3.540887 & 0.000000 & -0.267649 \\ \mathrm{C} & 2.822943 & 0.000000 & 0.900767 \\ \mathrm{H} & 0.998730 & 0.000000 & -2.493302 \\ \mathrm{H} & 3.424740 & 0.000000 & -2.420567 \\ \mathrm{H} & 4.620967 & 0.000000 & -0.245239 \\ \mathrm{H} & 3.330614 & 0.000000 & 1.855455 \\ \mathrm{C} & -3.540887 & 0.000000 & -0.267649 \\ \mathrm{C} & -2.863662 & 0.000000 & -1.497324 \\ \mathrm{H} & -0.998730 & 0.000000 & -2.493302 \\ \mathrm{H} & -3.424740 & 0.000000 & -2.420567 \\ \mathrm{H} & -4.620967 & 0.000000 & -0.245239 \\ \mathrm{H} & -3.330614 & 0.000000 & 1.855455\end{array}$

phenanthrene $S_{1}$

$\begin{array}{rrrr}\mathrm{C} & 0.707510 & 0.000000 & -0.391527 \\ \mathrm{C} & -0.707510 & 0.000000 & -0.391527 \\ \mathrm{C} & -1.431089 & 0.000000 & 0.884951 \\ \mathrm{C} & -0.694849 & 0.000000 & 2.080288 \\ \mathrm{C} & 0.694849 & 0.000000 & 2.080288 \\ \mathrm{C} & 1.431089 & 0.000000 & 0.884951 \\ \mathrm{C} & -1.487991 & 0.000000 & -1.572882 \\ \mathrm{C} & -2.837750 & 0.000000 & 0.887238 \\ \mathrm{H} & -1.229243 & 0.000000 & 3.020269 \\ \mathrm{H} & 1.229243 & 0.000000 & 3.020269 \\ \mathrm{C} & 1.487991 & 0.000000 & -1.572882 \\ \mathrm{C} & 2.872738 & 0.000000 & -1.525954 \\ \mathrm{C} & 3.560063 & 0.000000 & -0.295804 \\ \mathrm{C} & 2.837750 & 0.000000 & 0.887238 \\ \mathrm{H} & 1.007679 & 0.000000 & -2.538039 \\ \mathrm{H} & 3.432230 & 0.000000 & -2.450067 \\ \mathrm{H} & 4.639135 & 0.000000 & -0.277728 \\ \mathrm{H} & 3.352257 & 0.000000 & 1.838294 \\ \mathrm{C} & -3.560063 & 0.000000 & -0.295804\end{array}$




$\begin{array}{llll}\mathrm{C} & -2.872738 & 0.000000 & -1.525954 \\ \mathrm{H} & -1.007679 & 0.000000 & -2.538039 \\ \mathrm{H} & -3.432230 & 0.000000 & -2.450067 \\ \mathrm{H} & -4.639135 & 0.000000 & -0.277728 \\ \mathrm{H} & -3.352257 & 0.000000 & 1.838294\end{array}$

2,4,6,8-decatetraene $\mathrm{S}_{0}$

$\begin{array}{lrrc}\mathrm{C} & -2.587806 & -5.012726 & 0.000000 \\ \mathrm{C} & -2.462107 & -3.510306 & 0.000000 \\ \mathrm{C} & -1.306449 & -2.852543 & 0.000000 \\ \mathrm{C} & -1.208545 & -1.390019 & 0.000000 \\ \mathrm{C} & -0.048531 & -0.729380 & 0.000000 \\ \mathrm{C} & 0.048531 & 0.729380 & 0.000000 \\ \mathrm{C} & 1.208545 & 1.390019 & 0.000000 \\ \mathrm{C} & 1.306449 & 2.852543 & 0.000000 \\ \mathrm{C} & 2.462107 & 3.510306 & 0.000000 \\ \mathrm{C} & 2.587806 & 5.012726 & 0.000000 \\ \mathrm{H} & -0.372313 & -3.403653 & 0.000000 \\ \mathrm{H} & -2.141011 & -0.836391 & 0.000000 \\ \mathrm{H} & 0.883801 & -1.283077 & 0.000000 \\ \mathrm{H} & -0.883801 & 1.283077 & 0.000000 \\ \mathrm{H} & 2.141011 & 0.836391 & 0.000000 \\ \mathrm{H} & 0.372313 & 3.403653 & 0.000000 \\ \mathrm{H} & 3.387371 & 2.943934 & 0.000000 \\ \mathrm{H} & -3.387371 & -2.943934 & 0.000000 \\ \mathrm{H} & 1.604559 & 5.478821 & 0.000000 \\ \mathrm{H} & 3.136249 & 5.345955 & 0.881652 \\ \mathrm{H} & 3.136249 & 5.345955 & -0.881652 \\ \mathrm{H} & -1.604559 & -5.478821 & 0.000000 \\ \mathrm{H} & -3.136249 & -5.345955 & 0.881652 \\ \mathrm{H} & -3.136249 & -5.345955 & -0.881652\end{array}$

2,4,6,8-decatetraene $S_{1}$

$\begin{array}{cccc}\text { C } & -2.570626 & -5.018272 & 0.000000 \\ \text { C } & -2.511594 & -3.519349 & 0.000000 \\ \text { C } & -1.350112 & -2.804847 & 0.000000 \\ \text { C } & -1.271988 & -1.395450 & 0.000000 \\ \text { C } & -0.050311 & -0.692018 & 0.000000 \\ \text { C } & 0.050311 & 0.692018 & 0.000000 \\ \text { C } & 1.271988 & 1.395450 & 0.000000 \\ \text { C } & 1.350112 & 2.804847 & 0.000000 \\ \text { C } & 2.511594 & 3.519349 & 0.000000 \\ \text { C } & 2.570626 & 5.018272 & 0.000000 \\ \text { H } & -0.412129 & -3.351398 & 0.000000\end{array}$




$\begin{array}{rrrr}\mathrm{H} & -2.193689 & -0.826009 & 0.000000 \\ \mathrm{H} & 0.864595 & -1.273647 & 0.000000 \\ \mathrm{H} & -0.864595 & 1.273647 & 0.000000 \\ \mathrm{H} & 2.193689 & 0.826009 & 0.000000 \\ \mathrm{H} & 0.412129 & 3.351398 & 0.000000 \\ \mathrm{H} & 3.454033 & 2.983297 & 0.000000 \\ \mathrm{H} & -3.454033 & -2.983297 & 0.000000 \\ \mathrm{H} & 1.568073 & 5.442552 & 0.000000 \\ \mathrm{H} & 3.106382 & 5.384106 & 0.880246 \\ \mathrm{H} & 3.106382 & 5.384106 & -0.880246 \\ \mathrm{H} & -1.568073 & -5.442552 & 0.000000 \\ \mathrm{H} & -3.106382 & -5.384106 & 0.880246 \\ \mathrm{H} & -3.106382 & -5.384106 & -0.880246\end{array}$

tetracene $\mathrm{S}_{0}$

$\begin{array}{lrrr}\mathrm{C} & 3.696673 & 1.408690 & 0.000000 \\ \mathrm{C} & 2.428322 & 0.718624 & 0.000000 \\ \mathrm{C} & 2.428322 & -0.718624 & 0.000000 \\ \mathrm{C} & 3.696673 & -1.408690 & 0.000000 \\ \mathrm{C} & 4.856710 & -0.721340 & 0.000000 \\ \mathrm{C} & 4.856710 & 0.721340 & 0.000000 \\ \mathrm{C} & 1.230646 & 1.399958 & 0.000000 \\ \mathrm{C} & 0.000000 & 0.715341 & 0.000000 \\ \mathrm{C} & 0.000000 & -0.715341 & 0.000000 \\ \mathrm{C} & 1.230646 & -1.399958 & 0.000000 \\ \mathrm{C} & -1.230646 & 1.399958 & 0.000000 \\ \mathrm{C} & -2.428322 & 0.718624 & 0.000000 \\ \mathrm{C} & -2.428322 & -0.718624 & 0.000000 \\ \mathrm{C} & -1.230646 & -1.399958 & 0.000000 \\ \mathrm{C} & -3.696673 & 1.408690 & 0.000000 \\ \mathrm{C} & -4.856710 & 0.721340 & 0.000000 \\ \mathrm{C} & -4.856710 & -0.721340 & 0.000000 \\ \mathrm{C} & -3.696673 & -1.408690 & 0.000000 \\ \mathrm{H} & -3.691184 & -2.490138 & 0.000000 \\ \mathrm{H} & -5.801240 & -1.245180 & 0.000000 \\ \mathrm{H} & -5.801240 & 1.245180 & 0.000000 \\ \mathrm{H} & -3.691184 & 2.490138 & 0.000000 \\ \mathrm{H} & -1.229837 & -2.482490 & 0.000000 \\ \mathrm{H} & -1.229837 & 2.482490 & 0.000000 \\ \mathrm{H} & 1.229837 & -2.482490 & 0.000000 \\ \mathrm{H} & 1.229837 & 2.482490 & 0.000000 \\ \mathrm{H} & 3.691184 & -2.490138 & 0.000000 \\ \mathrm{H} & 5.801240 & -1.245180 & 0.000000 \\ \mathrm{H} & 5.801240 & 1.245180 & 0.000000 \\ \mathrm{H} & 3.691184 & 2.490138 & 0.000000\end{array}$


tetracene $\mathrm{S}_{1}$

$\begin{array}{lrrr}\mathrm{C} & 3.690492 & 1.393072 & 0.000000 \\ \mathrm{C} & 2.464734 & 0.713807 & 0.000000 \\ \mathrm{C} & 2.464734 & -0.713807 & 0.000000 \\ \mathrm{C} & 3.690492 & -1.393072 & 0.000000 \\ \mathrm{C} & 4.890824 & -0.697692 & 0.000000 \\ \mathrm{C} & 4.890824 & 0.697692 & 0.000000 \\ \mathrm{C} & 1.222238 & 1.398478 & 0.000000 \\ \mathrm{C} & 0.000000 & 0.727748 & 0.000000 \\ \mathrm{C} & 0.000000 & -0.727748 & 0.000000 \\ \mathrm{C} & 1.222238 & -1.398478 & 0.000000 \\ \mathrm{C} & -1.222238 & 1.398478 & 0.000000 \\ \mathrm{C} & -2.464734 & 0.713807 & 0.000000 \\ \mathrm{C} & -2.464734 & -0.713807 & 0.000000 \\ \mathrm{C} & -1.222238 & -1.398478 & 0.000000 \\ \mathrm{C} & -3.690492 & 1.393072 & 0.000000 \\ \mathrm{C} & -4.890824 & 0.697692 & 0.000000 \\ \mathrm{C} & -4.890824 & -0.697692 & 0.000000 \\ \mathrm{C} & -3.690492 & -1.393072 & 0.000000 \\ \mathrm{H} & -3.690633 & -2.474552 & 0.000000 \\ \mathrm{H} & -5.825522 & -1.238317 & 0.000000 \\ \mathrm{H} & -5.825522 & 1.238317 & 0.000000 \\ \mathrm{H} & -3.690633 & 2.474552 & 0.000000 \\ \mathrm{H} & -1.227258 & -2.480857 & 0.000000 \\ \mathrm{H} & -1.227258 & 2.480857 & 0.000000 \\ \mathrm{H} & 1.227258 & -2.480857 & 0.000000 \\ \mathrm{H} & 1.227258 & 2.480857 & 0.000000 \\ \mathrm{H} & 3.690633 & -2.474552 & 0.000000 \\ \mathrm{H} & 5.825522 & -1.238317 & 0.000000 \\ \mathrm{H} & 5.825522 & 1.238317 & 0.000000 \\ \mathrm{H} & 3.690633 & 2.474552 & 0.000000\end{array}$

perylene $\mathrm{S}_{0}$

$\begin{array}{lrrr}\mathrm{C} & 0.213259 & 1.457242 & -2.403586 \\ \mathrm{C} & 0.064538 & 0.739762 & -1.243101 \\ \mathrm{C} & 0.011320 & 1.440309 & -0.000353 \\ \mathrm{C} & 0.036177 & 2.853112 & -0.000162 \\ \mathrm{C} & 0.159206 & 3.553891 & -1.226624 \\ \mathrm{C} & 0.262406 & 2.867309 & -2.398984 \\ \mathrm{C} & -0.068890 & 3.557396 & 1.225779 \\ \mathrm{C} & -0.183977 & 2.873930 & 2.398924 \\ \mathrm{C} & -0.168419 & 1.463201 & 2.403987 \\ \mathrm{C} & -0.039278 & 0.741651 & 1.243466\end{array}$




$\begin{array}{lrrc}\mathrm{C} & -0.064538 & -0.739762 & -1.243101 \\ \mathrm{C} & -0.011320 & -1.440309 & -0.000353 \\ \mathrm{C} & 0.039278 & -0.741651 & 1.243466 \\ \mathrm{C} & -0.036177 & -2.853112 & -0.000162 \\ \mathrm{C} & 0.068890 & -3.557396 & 1.225779 \\ \mathrm{C} & 0.183977 & -2.873930 & 2.398924 \\ \mathrm{C} & 0.168419 & -1.463201 & 2.403987 \\ \mathrm{C} & -0.213259 & -1.457242 & -2.403586 \\ \mathrm{C} & -0.262406 & -2.867309 & -2.398984 \\ \mathrm{C} & -0.159206 & -3.553891 & -1.226624 \\ \mathrm{H} & -0.061556 & 4.638491 & 1.209538 \\ \mathrm{H} & -0.281777 & 3.403542 & 3.335280 \\ \mathrm{H} & -0.261466 & 0.960926 & 3.353979 \\ \mathrm{H} & 0.061556 & -4.638491 & 1.209538 \\ \mathrm{H} & 0.281777 & -3.403542 & 3.335280 \\ \mathrm{H} & 0.261466 & -0.960926 & 3.353979 \\ \mathrm{H} & -0.179956 & -4.634816 & -1.210737 \\ \mathrm{H} & -0.376175 & -3.394060 & -3.335149 \\ \mathrm{H} & -0.301818 & -0.951569 & -3.352255 \\ \mathrm{H} & 0.179956 & 4.634816 & -1.210737 \\ \mathrm{H} & 0.376175 & 3.394060 & -3.335149 \\ \mathrm{H} & 0.301818 & 0.951569 & -3.352255\end{array}$

perylene $S_{1}$

$\begin{array}{rrrr}\text { C } & -0.006561 & 1.462266 & -2.444351 \\ \mathrm{C} & -0.006760 & 0.717610 & -1.234732 \\ \mathrm{C} & 0.015806 & 1.413868 & 0.000001 \\ \mathrm{C} & 0.006650 & 2.842635 & -0.000001 \\ \mathrm{C} & 0.009580 & 3.534744 & -1.225428 \\ \mathrm{C} & -0.001105 & 2.843493 & -2.428620 \\ \mathrm{C} & 0.009775 & 3.534749 & 1.225419 \\ \mathrm{C} & -0.000693 & 2.843500 & 2.428618 \\ \mathrm{C} & -0.006189 & 1.462276 & 2.444354 \\ \mathrm{C} & -0.006669 & 0.717611 & 1.234738 \\ \mathrm{C} & 0.006760 & -0.717610 & -1.234732 \\ \mathrm{C} & -0.015806 & -1.413868 & 0.000001 \\ \mathrm{C} & 0.006669 & -0.717611 & 1.234738 \\ \mathrm{C} & -0.006650 & -2.842635 & -0.000001 \\ \mathrm{C} & -0.009775 & -3.534749 & 1.225419 \\ \mathrm{C} & 0.000693 & -2.843500 & 2.428618 \\ \mathrm{C} & 0.006189 & -1.462276 & 2.444354 \\ \mathrm{C} & 0.006561 & -1.462266 & -2.444351 \\ \mathrm{C} & 0.001105 & -2.843493 & -2.428620 \\ \mathrm{C} & -0.009580 & -3.534744 & -1.225428 \\ \mathrm{H} & 0.013113 & 4.615767 & 1.216165\end{array}$




$\begin{array}{rrrr}\mathrm{H} & -0.004160 & 3.388256 & 3.361405 \\ \mathrm{H} & -0.030497 & 0.957213 & 3.394822 \\ \mathrm{H} & -0.013113 & -4.615767 & 1.216165 \\ \mathrm{H} & 0.004160 & -3.388256 & 3.361405 \\ \mathrm{H} & 0.030497 & -0.957213 & 3.394822 \\ \mathrm{H} & -0.012907 & -4.615762 & -1.216177 \\ \mathrm{H} & 0.004791 & -3.388244 & -3.361410 \\ \mathrm{H} & 0.031154 & -0.957194 & -3.394808 \\ \mathrm{H} & 0.012907 & 4.615762 & -1.216177 \\ \mathrm{H} & -0.004791 & 3.388244 & -3.361410 \\ \mathrm{H} & -0.031154 & 0.957194 & -3.394808\end{array}$

\section{CAM-B3LYP/TZVP geometries - Group I}

pyrazine $\mathrm{S}_{0}$

$\begin{array}{rrrr}\mathrm{C} & 1.126638 & -0.693086 & 0.000000 \\ \mathrm{C} & 1.126638 & 0.693086 & 0.000000 \\ \mathrm{~N} & 0.000000 & 1.393917 & 0.000000 \\ \mathrm{C} & -1.126638 & 0.693086 & 0.000000 \\ \mathrm{C} & -1.126638 & -0.693086 & 0.000000 \\ \mathrm{~N} & 0.000000 & -1.393917 & 0.000000 \\ \mathrm{H} & -2.056557 & -1.249635 & 0.000000 \\ \mathrm{H} & 2.056557 & -1.249635 & 0.000000 \\ \mathrm{H} & 2.056557 & 1.249635 & 0.000000 \\ \mathrm{H} & -2.056557 & 1.249635 & 0.000000\end{array}$

pyrazine $S_{1}$

$\begin{array}{rrrr}\mathrm{C} & -1.200798 & 0.664784 & 0.000000 \\ \mathrm{C} & -1.116245 & -0.735393 & 0.000000 \\ \mathrm{~N} & 0.024738 & -1.348730 & 0.000000 \\ \mathrm{C} & 1.200798 & -0.664784 & 0.000000 \\ \mathrm{C} & 1.116245 & 0.735393 & 0.000000 \\ \mathrm{~N} & -0.024738 & 1.348730 & 0.000000 \\ \mathrm{H} & 2.043089 & 1.301406 & 0.000000 \\ \mathrm{H} & -2.152341 & 1.170187 & 0.000000 \\ \mathrm{H} & -2.043089 & -1.301406 & 0.000000 \\ \mathrm{H} & 2.152341 & -1.170187 & 0.000000\end{array}$

2,6-difluoropyridine $\mathrm{S}_{0}$
$\begin{array}{llll}\text { C } & 1.111700 & 0.000000 & -0.872572\end{array}$
$\begin{array}{llll}\mathrm{N} & 0.000000 & 0.000000 & -1.560292\end{array}$
$\begin{array}{llll}\text { C } & -1.111700 & 0.000000 & -0.872572\end{array}$ 


$\begin{array}{cccr}\mathrm{C} & -1.198699 & 0.000000 & 0.505117 \\ \mathrm{C} & 0.000000 & 0.000000 & 1.197729 \\ \mathrm{C} & 1.198699 & 0.000000 & 0.505117 \\ \mathrm{~F} & -2.237911 & 0.000000 & -1.586722 \\ \mathrm{H} & -2.159967 & 0.000000 & 0.995903 \\ \mathrm{H} & 0.000000 & 0.000000 & 2.279529 \\ \mathrm{H} & 2.159967 & 0.000000 & 0.995903 \\ \mathrm{~F} & 2.237911 & 0.000000 & -1.586722\end{array}$

2,6-difluoropyridine $\mathrm{S}_{1}$

$\begin{array}{crrr}\mathrm{C} & -1.098440 & 0.000000 & -0.334304 \\ \mathrm{~N} & 0.000000 & 0.000000 & -1.095748 \\ \mathrm{C} & 1.098440 & 0.000000 & -0.334304 \\ \mathrm{C} & 1.189266 & 0.000000 & 1.070349 \\ \mathrm{C} & 0.000000 & 0.000000 & 1.841500 \\ \mathrm{C} & -1.189266 & 0.000000 & 1.070349 \\ \mathrm{~F} & 2.236219 & 0.000000 & -1.008796 \\ \mathrm{H} & 2.173883 & 0.000000 & 1.515958 \\ \mathrm{H} & 0.000000 & 0.000000 & 2.917130 \\ \mathrm{H} & -2.173883 & 0.000000 & 1.515958 \\ \mathrm{~F} & -2.236219 & 0.000000 & -1.008796\end{array}$

2-fluoropyridine $\mathrm{S}_{0}$

$\begin{array}{crrr}\mathrm{C} & -1.310995 & 0.501789 & 0.000000 \\ \mathrm{~N} & -1.360589 & -0.797986 & 0.000000 \\ \mathrm{C} & -0.195811 & -1.448482 & 0.000000 \\ \mathrm{C} & 1.026468 & -0.806791 & 0.000000 \\ \mathrm{C} & 1.040100 & 0.582038 & 0.000000 \\ \mathrm{C} & -0.157202 & 1.267851 & 0.000000 \\ \mathrm{H} & -0.257899 & -2.530087 & 0.000000 \\ \mathrm{H} & 1.943741 & -1.378362 & 0.000000 \\ \mathrm{H} & 1.976444 & 1.124599 & 0.000000 \\ \mathrm{H} & -0.215350 & 2.346400 & 0.000000 \\ \mathrm{~F} & -2.488918 & 1.139020 & 0.000000\end{array}$

2-fluoropyridine $\mathrm{S}_{1}$
$\begin{array}{llll}\text { C } & -1.317414 & 0.490002 & 0.000000\end{array}$
$\begin{array}{llll}\mathrm{N} & -1.430927 & -0.839560 & 0.000000\end{array}$
$\begin{array}{llll}\text { C } & -0.224103 & -1.461784 & 0.000000\end{array}$
$\begin{array}{llll}\text { C } & 1.027188 & -0.790600 & 0.000000\end{array}$
$\begin{array}{llll}\text { C } & 1.103480 & 0.616504 & 0.000000\end{array}$
$\begin{array}{llll}\mathrm{C} & -0.151773 & 1.279234 & 0.000000\end{array}$
$\begin{array}{llll}\mathrm{H} & -0.248252 & -2.543375 & 0.000000\end{array}$ 


$\begin{array}{lrrl}\mathrm{H} & 1.924979 & -1.394126 & 0.000000 \\ \mathrm{H} & 2.039234 & 1.149238 & 0.000000 \\ \mathrm{H} & -0.252822 & 2.354472 & 0.000000 \\ \mathrm{~F} & -2.469600 & 1.139983 & 0.000000\end{array}$

2-hydroxypyridine $\mathrm{S}_{0}$

$\begin{array}{crrc}\mathrm{C} & -0.975419 & 0.749724 & 0.000000 \\ \mathrm{C} & -0.967066 & -0.643193 & 0.000000 \\ \mathrm{~N} & 0.130848 & -1.370917 & 0.000000 \\ \mathrm{C} & 1.302196 & -0.733083 & 0.000000 \\ \mathrm{C} & 1.414472 & 0.640806 & 0.000000 \\ \mathrm{C} & 0.242471 & 1.390767 & 0.000000 \\ \mathrm{O} & -2.146016 & -1.296833 & 0.000000 \\ \mathrm{H} & 2.183298 & -1.364118 & 0.000000 \\ \mathrm{H} & 2.386783 & 1.112285 & 0.000000 \\ \mathrm{H} & 0.283943 & 2.472494 & 0.000000 \\ \mathrm{H} & -1.914486 & 1.283609 & 0.000000 \\ \mathrm{H} & -1.941025 & -2.241539 & 0.000000\end{array}$

2-hydroxypyridine $\mathrm{S}_{1}$

$\begin{array}{rrrl}\mathrm{C} & -0.994649 & 0.765596 & 0.000000 \\ \mathrm{C} & -0.966536 & -0.636072 & 0.000000 \\ \mathrm{~N} & 0.119780 & -1.447653 & 0.000000 \\ \mathrm{C} & 1.291327 & -0.772203 & 0.000000 \\ \mathrm{C} & 1.388416 & 0.641945 & 0.000000 \\ \mathrm{C} & 0.248794 & 1.458865 & 0.000000 \\ \mathrm{O} & -2.123267 & -1.287401 & 0.000000 \\ \mathrm{H} & 2.191987 & -1.371478 & 0.000000 \\ \mathrm{H} & 2.378554 & 1.078908 & 0.000000 \\ \mathrm{H} & 0.307784 & 2.534343 & 0.000000 \\ \mathrm{H} & -1.950822 & 1.267662 & 0.000000 \\ \mathrm{H} & -1.891367 & -2.232514 & 0.000000\end{array}$

2-pyridone $\mathrm{S}_{0}$

$\begin{array}{lrrc}\mathrm{C} & -1.243081 & 0.709583 & 0.000000 \\ \mathrm{~N} & -1.133918 & -0.684603 & 0.000000 \\ \mathrm{C} & 0.034263 & -1.379744 & 0.000000 \\ \mathrm{C} & 1.218260 & -0.732192 & 0.000000 \\ \mathrm{C} & 1.200380 & 0.691020 & 0.000000 \\ \mathrm{C} & 0.038857 & 1.382517 & 0.000000 \\ \mathrm{H} & -2.018084 & -1.172703 & 0.000000 \\ \mathrm{H} & -0.053947 & -2.457214 & 0.000000 \\ \mathrm{H} & 2.144931 & -1.284642 & 0.000000\end{array}$




$\begin{array}{rrrr}\mathrm{H} & 2.141153 & 1.228025 & 0.000000 \\ \mathrm{H} & 0.011164 & 2.462536 & 0.000000 \\ \mathrm{O} & -2.339978 & 1.237429 & 0.000000\end{array}$

2-pyridone $\mathrm{S}_{1}$

$\begin{array}{rrrr}\mathrm{C} & -1.384599 & 0.106118 & 0.000000 \\ \mathrm{~N} & -0.728719 & -1.137655 & 0.000000 \\ \mathrm{C} & 0.622217 & -1.250713 & 0.000000 \\ \mathrm{C} & 1.408882 & -0.075470 & 0.000000 \\ \mathrm{C} & 0.828280 & 1.169318 & 0.000000 \\ \mathrm{C} & -0.607467 & 1.263564 & 0.000000 \\ \mathrm{H} & -1.340875 & -1.941085 & 0.000000 \\ \mathrm{H} & 1.042674 & -2.243798 & 0.000000 \\ \mathrm{H} & 2.483619 & -0.192264 & 0.000000 \\ \mathrm{H} & 1.433584 & 2.062871 & 0.000000 \\ \mathrm{H} & -1.125532 & 2.209565 & 0.000000 \\ \mathrm{O} & -2.632064 & 0.029538 & 0.000000\end{array}$

2-methylpyrimidine $\mathrm{S}_{0}$

$\begin{array}{crrc}\mathrm{C} & 0.000149 & 1.958213 & 0.000000 \\ \mathrm{C} & -1.170494 & 1.221631 & 0.000000 \\ \mathrm{~N} & -1.174808 & -0.103013 & 0.000000 \\ \mathrm{C} & 0.010659 & -0.715555 & 0.000000 \\ \mathrm{~N} & 1.189218 & -0.095813 & 0.000000 \\ \mathrm{C} & 1.174075 & 1.232225 & 0.000000 \\ \mathrm{H} & -2.140604 & 1.707485 & 0.000000 \\ \mathrm{H} & 2.140930 & 1.724141 & 0.000000 \\ \mathrm{H} & -0.004492 & 3.038679 & 0.000000 \\ \mathrm{C} & 0.004735 & -2.210752 & 0.000000 \\ \mathrm{H} & 1.022039 & -2.592045 & 0.000000 \\ \mathrm{H} & -0.525709 & -2.582597 & 0.877206 \\ \mathrm{H} & -0.525709 & -2.582597 & -0.877206\end{array}$

2-methylpyrimidine $\mathrm{S}_{1}$

$\begin{array}{rrrc}\mathrm{C} & -0.014082 & 2.006782 & 0.000000 \\ \mathrm{C} & -1.164577 & 1.276476 & 0.000000 \\ \mathrm{~N} & -1.144871 & -0.093712 & 0.000000 \\ \mathrm{C} & 0.010975 & -0.782542 & 0.000000 \\ \mathrm{~N} & 1.081798 & -0.065577 & 0.000000 \\ \mathrm{C} & 1.202046 & 1.288173 & 0.000000 \\ \mathrm{H} & -2.142634 & 1.738165 & 0.000000 \\ \mathrm{H} & 2.187282 & 1.722059 & 0.000000 \\ \mathrm{H} & -0.025632 & 3.088036 & 0.000000\end{array}$




$$
\begin{array}{lrrr}
\mathrm{C} & 0.012849 & -2.266032 & 0.000000 \\
\mathrm{H} & 1.030629 & -2.648559 & 0.000000 \\
\mathrm{H} & -0.516891 & -2.631640 & 0.880246 \\
\mathrm{H} & -0.516891 & -2.631640 & -0.880246
\end{array}
$$

5-methylpyrimidine $\mathrm{S}_{0}$

$\begin{array}{lrrr}\mathrm{C} & 0.871515 & 0.848194 & 0.000000 \\ \mathrm{C} & -0.358437 & 0.209931 & 0.000000 \\ \mathrm{C} & -0.298403 & -1.177335 & 0.000000 \\ \mathrm{~N} & 0.843012 & -1.851747 & 0.000000 \\ \mathrm{C} & 1.955566 & -1.128097 & 0.000000 \\ \mathrm{~N} & 2.027672 & 0.194169 & 0.000000 \\ \mathrm{H} & 2.893288 & -1.671550 & 0.000000 \\ \mathrm{H} & -1.208900 & -1.769857 & 0.000000 \\ \mathrm{H} & 0.930122 & 1.932581 & 0.000000 \\ \mathrm{C} & -1.657719 & 0.956735 & 0.000000 \\ \mathrm{H} & -1.488895 & 2.032976 & 0.000000 \\ \mathrm{H} & -2.254410 & 0.711990 & -0.879967 \\ \mathrm{H} & -2.254410 & 0.711990 & 0.879967\end{array}$

5-methylpyrimidine $\mathrm{S}_{1}$

$\begin{array}{rrrr}\mathrm{C} & 0.841549 & 0.854907 & 0.000000 \\ \mathrm{C} & -0.375000 & 0.242265 & 0.000000 \\ \mathrm{C} & -0.346163 & -1.188052 & 0.000000 \\ \mathrm{~N} & 0.878507 & -1.750817 & 0.000000 \\ \mathrm{C} & 2.030309 & -1.177049 & 0.000000 \\ \mathrm{~N} & 2.025904 & 0.165507 & 0.000000 \\ \mathrm{H} & 2.954871 & -1.734694 & 0.000000 \\ \mathrm{H} & -1.217100 & -1.822350 & 0.000000 \\ \mathrm{H} & 0.928063 & 1.934185 & 0.000000 \\ \mathrm{C} & -1.673971 & 0.977883 & 0.000000 \\ \mathrm{H} & -1.513704 & 2.055182 & 0.000000 \\ \mathrm{H} & -2.266637 & 0.721523 & -0.880670 \\ \mathrm{H} & -2.266637 & 0.721523 & 0.880670\end{array}$

7-azaindole $\mathrm{S}_{0}$
$\begin{array}{llll}\mathrm{N} & -0.632597 & 1.839006 & 0.000000\end{array}$
$\begin{array}{llll}\mathrm{C} & -0.672026 & 0.470824 & 0.000000\end{array}$
$\begin{array}{llll}\text { C } & 0.661916 & 0.006420 & 0.000000\end{array}$
$\begin{array}{llll}\text { C } & 1.503764 & 1.164723 & 0.000000\end{array}$
$\begin{array}{llll}\text { C } & 0.681332 & 2.246926 & 0.000000\end{array}$
$\begin{array}{llll}\text { C } & 0.840004 & -1.373183 & 0.000000\end{array}$
$\begin{array}{llll}\text { C } & -0.290722 & -2.165804 & 0.000000\end{array}$ 


$\begin{array}{rrrr}\mathrm{C} & -1.558909 & -1.576474 & 0.000000 \\ \mathrm{~N} & -1.769072 & -0.268149 & 0.000000 \\ \mathrm{H} & -2.445134 & -2.200768 & 0.000000 \\ \mathrm{H} & 1.829444 & -1.813411 & 0.000000 \\ \mathrm{H} & -0.210263 & -3.244224 & 0.000000 \\ \mathrm{H} & -1.446213 & 2.428989 & 0.000000 \\ \mathrm{H} & 2.580595 & 1.189421 & 0.000000 \\ \mathrm{H} & 0.927900 & 3.295703 & 0.000000\end{array}$

7-azaindole $\mathrm{S}_{1}$

$\begin{array}{lrrr}\mathrm{N} & -0.606518 & 1.870308 & 0.000000 \\ \mathrm{C} & -0.660848 & 0.451139 & 0.000000 \\ \mathrm{C} & 0.656903 & -0.003559 & 0.000000 \\ \mathrm{C} & 1.485706 & 1.139277 & 0.000000 \\ \mathrm{C} & 0.648560 & 2.299935 & 0.000000 \\ \mathrm{C} & 0.884110 & -1.404387 & 0.000000 \\ \mathrm{C} & -0.302975 & -2.177185 & 0.000000 \\ \mathrm{C} & -1.545096 & -1.595597 & 0.000000 \\ \mathrm{~N} & -1.789401 & -0.223242 & 0.000000 \\ \mathrm{H} & -2.434776 & -2.211649 & 0.000000 \\ \mathrm{H} & 1.870336 & -1.840792 & 0.000000 \\ \mathrm{H} & -0.243384 & -3.257579 & 0.000000 \\ \mathrm{H} & -1.439407 & 2.441367 & 0.000000 \\ \mathrm{H} & 2.563526 & 1.168979 & 0.000000 \\ \mathrm{H} & 0.913272 & 3.343006 & 0.000000\end{array}$

7-hydroxyquinoline, cis $\mathrm{S}_{0}$

$\begin{array}{lrrl}\mathrm{N} & 0.458493 & 1.964297 & 0.000000 \\ \mathrm{C} & 0.431489 & 0.606591 & 0.000000 \\ \mathrm{C} & -0.782474 & -0.123022 & 0.000000 \\ \mathrm{C} & -1.985892 & 0.607493 & 0.000000 \\ \mathrm{C} & -1.941347 & 1.970511 & 0.000000 \\ \mathrm{C} & -0.681895 & 2.601298 & 0.000000 \\ \mathrm{C} & 1.658933 & -0.090535 & 0.000000 \\ \mathrm{C} & 1.671652 & -1.455721 & 0.000000 \\ \mathrm{C} & 0.464640 & -2.190860 & 0.000000 \\ \mathrm{C} & -0.727487 & -1.537438 & 0.000000 \\ \mathrm{H} & -0.625945 & 3.685536 & 0.000000 \\ \mathrm{H} & -1.654225 & -2.098454 & 0.000000 \\ \mathrm{H} & 0.521088 & -3.270887 & 0.000000 \\ \mathrm{O} & 2.813801 & -2.193483 & 0.000000 \\ \mathrm{H} & 2.572317 & 0.491901 & 0.000000 \\ \mathrm{H} & -2.931392 & 0.077776 & 0.000000 \\ \mathrm{H} & -2.843039 & 2.567458 & 0.000000\end{array}$




\section{H $\quad 3.581283 \quad-1.612451 \quad 0.000000$}

7-hydroxyquinoline, cis $\mathrm{S}_{1}$

$\begin{array}{rrrr}\mathrm{N} & 0.485682 & 1.967441 & 0.000000 \\ \mathrm{C} & 0.434426 & 0.629561 & 0.000000 \\ \mathrm{C} & -0.789075 & -0.139878 & 0.000000 \\ \mathrm{C} & -1.990217 & 0.592843 & 0.000000 \\ \mathrm{C} & -1.915898 & 1.992017 & 0.000000 \\ \mathrm{C} & -0.686639 & 2.635838 & 0.000000 \\ \mathrm{C} & 1.632799 & -0.087088 & 0.000000 \\ \mathrm{C} & 1.649932 & -1.512095 & 0.000000 \\ \mathrm{C} & 0.481586 & -2.258462 & 0.000000 \\ \mathrm{C} & -0.720212 & -1.552601 & 0.000000 \\ \mathrm{H} & -0.623761 & 3.715622 & 0.000000 \\ \mathrm{H} & -1.653139 & -2.103518 & 0.000000 \\ \mathrm{H} & 0.524144 & -3.336392 & 0.000000 \\ \mathrm{O} & 2.822098 & -2.153817 & 0.000000 \\ \mathrm{H} & 2.563358 & 0.469450 & 0.000000 \\ \mathrm{H} & -2.945301 & 0.085769 & 0.000000 \\ \mathrm{H} & -2.824590 & 2.580508 & 0.000000 \\ \mathrm{H} & 3.554804 & -1.525190 & 0.000000\end{array}$

7-hydroxyquinoline, trans $\mathrm{S}_{0}$

$\begin{array}{rrrr}\mathrm{N} & 0.508595 & 2.037070 & 0.000000 \\ \mathrm{C} & 0.479524 & 0.677018 & 0.000000 \\ \mathrm{C} & -0.739720 & -0.046939 & 0.000000 \\ \mathrm{C} & -1.942326 & 0.688323 & 0.000000 \\ \mathrm{C} & -1.892499 & 2.049519 & 0.000000 \\ \mathrm{C} & -0.628479 & 2.676162 & 0.000000 \\ \mathrm{C} & 1.703301 & -0.019970 & 0.000000 \\ \mathrm{C} & 1.712853 & -1.385380 & 0.000000 \\ \mathrm{C} & 0.502179 & -2.114025 & 0.000000 \\ \mathrm{C} & -0.691669 & -1.457844 & 0.000000 \\ \mathrm{H} & -0.569863 & 3.760408 & 0.000000 \\ \mathrm{H} & -1.619250 & -2.017379 & 0.000000 \\ \mathrm{H} & 0.530913 & -3.198147 & 0.000000 \\ \mathrm{O} & 2.914761 & -2.024262 & 0.000000 \\ \mathrm{H} & 2.627199 & 0.540425 & 0.000000 \\ \mathrm{H} & -2.889600 & 0.161721 & 0.000000 \\ \mathrm{H} & -2.791499 & 2.650565 & 0.000000 \\ \mathrm{H} & 2.785562 & -2.977246 & 0.000000\end{array}$

7-hydroxyquinoline, trans $\mathrm{S}_{1}$ 


$\begin{array}{rrrr}\mathrm{N} & 0.528782 & 2.042898 & 0.000000 \\ \mathrm{C} & 0.476496 & 0.706896 & 0.000000 \\ \mathrm{C} & -0.748336 & -0.057311 & 0.000000 \\ \mathrm{C} & -1.948817 & 0.676486 & 0.000000 \\ \mathrm{C} & -1.872661 & 2.076280 & 0.000000 \\ \mathrm{C} & -0.643802 & 2.714842 & 0.000000 \\ \mathrm{C} & 1.676785 & -0.012078 & 0.000000 \\ \mathrm{C} & 1.686984 & -1.433712 & 0.000000 \\ \mathrm{C} & 0.515530 & -2.179517 & 0.000000 \\ \mathrm{C} & -0.685750 & -1.468864 & 0.000000 \\ \mathrm{H} & -0.577352 & 3.794540 & 0.000000 \\ \mathrm{H} & -1.620338 & -2.017161 & 0.000000 \\ \mathrm{H} & 0.536428 & -3.260720 & 0.000000 \\ \mathrm{O} & 2.903964 & -1.987389 & 0.000000 \\ \mathrm{H} & 2.618575 & 0.518487 & 0.000000 \\ \mathrm{H} & -2.904415 & 0.170212 & 0.000000 \\ \mathrm{H} & -2.780097 & 2.666637 & 0.000000 \\ \mathrm{H} & 2.838007 & -2.950506 & 0.000000\end{array}$

2-hydroxyquinoline, enol $\mathrm{S}_{0}$

$\begin{array}{rrrr}\mathrm{C} & 0.429143 & 1.898097 & 0.000000 \\ \mathrm{C} & 0.482386 & 0.488197 & 0.000000 \\ \mathrm{C} & -0.720152 & -0.255952 & 0.000000 \\ \mathrm{C} & -1.949176 & 0.437409 & 0.000000 \\ \mathrm{C} & -1.971621 & 1.802873 & 0.000000 \\ \mathrm{C} & -0.773482 & 2.544117 & 0.000000 \\ \mathrm{C} & 1.692263 & -0.244167 & 0.000000 \\ \mathrm{C} & 1.660355 & -1.600606 & 0.000000 \\ \mathrm{C} & 0.392495 & -2.243849 & 0.000000 \\ \mathrm{~N} & -0.735863 & -1.615302 & 0.000000 \\ \mathrm{O} & 0.316386 & -3.595966 & 0.000000 \\ \mathrm{H} & 1.356619 & 2.458494 & 0.000000 \\ \mathrm{H} & -0.811641 & 3.625552 & 0.000000 \\ \mathrm{H} & -2.919726 & 2.325467 & 0.000000 \\ \mathrm{H} & -2.858171 & -0.148607 & 0.000000 \\ \mathrm{H} & 2.637574 & 0.285258 & 0.000000 \\ \mathrm{H} & 2.572898 & -2.184131 & 0.000000 \\ \mathrm{H} & 1.199712 & -3.976884 & 0.000000\end{array}$

2-hydroxyquinoline, enol $\mathrm{S}_{1}$
$\begin{array}{llll}\text { C } & 0.435605 & 1.876917 & 0.000000\end{array}$
$\begin{array}{llll}\text { C } & 0.526310 & 0.488600 & 0.000000\end{array}$
$\begin{array}{llll}\text { C } & -0.724817 & -0.259788 & 0.000000\end{array}$
$\begin{array}{llll}\text { C } & -1.955818 & 0.438790 & 0.000000\end{array}$ 


$\begin{array}{lrrr}\mathrm{C} & -2.007409 & 1.834403 & 0.000000 \\ \mathrm{C} & -0.813681 & 2.539039 & 0.000000 \\ \mathrm{C} & 1.729299 & -0.234739 & 0.000000 \\ \mathrm{C} & 1.691423 & -1.635562 & 0.000000 \\ \mathrm{C} & 0.431835 & -2.237399 & 0.000000 \\ \mathrm{~N} & -0.746555 & -1.598965 & 0.000000 \\ \mathrm{O} & 0.295120 & -3.568761 & 0.000000 \\ \mathrm{H} & 1.343194 & 2.467761 & 0.000000 \\ \mathrm{H} & -0.824001 & 3.621664 & 0.000000 \\ \mathrm{H} & -2.955562 & 2.351690 & 0.000000 \\ \mathrm{H} & -2.856458 & -0.160332 & 0.000000 \\ \mathrm{H} & 2.672900 & 0.293793 & 0.000000 \\ \mathrm{H} & 2.593890 & -2.230748 & 0.000000 \\ \mathrm{H} & 1.164716 & -3.986343 & 0.000000\end{array}$

2-hydroxyquinoline, keto $\mathrm{S}_{0}$

$\begin{array}{rrrc}\mathrm{N} & 0.558396 & 1.702496 & 0.000000 \\ \mathrm{C} & 0.599782 & 0.325580 & 0.000000 \\ \mathrm{C} & -0.610436 & -0.381849 & 0.000000 \\ \mathrm{C} & -1.832616 & 0.378040 & 0.000000 \\ \mathrm{C} & -1.830552 & 1.718607 & 0.000000 \\ \mathrm{C} & -0.586253 & 2.482013 & 0.000000 \\ \mathrm{C} & 1.813554 & -0.363395 & 0.000000 \\ \mathrm{C} & 1.817077 & -1.740039 & 0.000000 \\ \mathrm{C} & 0.620399 & -2.457260 & 0.000000 \\ \mathrm{C} & -0.576705 & -1.779259 & 0.000000 \\ \mathrm{H} & -1.513687 & -2.322908 & 0.000000 \\ \mathrm{H} & 0.636053 & -3.538584 & 0.000000 \\ \mathrm{H} & 2.761368 & -2.268851 & 0.000000 \\ \mathrm{H} & 2.744886 & 0.189704 & 0.000000 \\ \mathrm{H} & -2.770637 & -0.165295 & 0.000000 \\ \mathrm{H} & -2.740712 & 2.300766 & 0.000000 \\ \mathrm{O} & -0.513116 & 3.695883 & 0.000000 \\ \mathrm{H} & 1.423220 & 2.224372 & 0.000000\end{array}$

2-hydroxyquinoline, keto $\mathrm{S}_{1}$

$\begin{array}{rrrc}\mathrm{N} & 0.561891 & 1.673208 & 0.000000 \\ \mathrm{C} & 0.611327 & 0.326586 & 0.000000 \\ \mathrm{C} & -0.664707 & -0.380517 & 0.000000 \\ \mathrm{C} & -1.851858 & 0.363784 & 0.000000 \\ \mathrm{C} & -1.850780 & 1.761603 & 0.000000 \\ \mathrm{C} & -0.628350 & 2.467717 & 0.000000 \\ \mathrm{C} & 1.832505 & -0.360353 & 0.000000 \\ \mathrm{C} & 1.851321 & -1.744184 & 0.000000\end{array}$




$\begin{array}{lrrr}\mathrm{C} & 0.629776 & -2.442330 & 0.000000 \\ \mathrm{C} & -0.589819 & -1.785574 & 0.000000 \\ \mathrm{H} & -1.509481 & -2.355350 & 0.000000 \\ \mathrm{H} & 0.642519 & -3.524790 & 0.000000 \\ \mathrm{H} & 2.788489 & -2.281049 & 0.000000 \\ \mathrm{H} & 2.754335 & 0.208635 & 0.000000 \\ \mathrm{H} & -2.791566 & -0.172300 & 0.000000 \\ \mathrm{H} & -2.758959 & 2.342567 & 0.000000 \\ \mathrm{O} & -0.438912 & 3.679936 & 0.000000 \\ \mathrm{H} & 1.412268 & 2.222418 & 0.000000\end{array}$

dibenzofuran $\mathrm{S}_{0}$

$\begin{array}{lrrr}\mathrm{C} & 1.715739 & 0.000000 & -1.352856 \\ \mathrm{C} & 0.724613 & 0.000000 & -0.379243 \\ \mathrm{C} & 1.094766 & 0.000000 & 0.966047 \\ \mathrm{C} & 2.406849 & 0.000000 & 1.389215 \\ \mathrm{C} & 3.379107 & 0.000000 & 0.402622 \\ \mathrm{C} & 3.039417 & 0.000000 & -0.950764 \\ \mathrm{C} & -0.724613 & 0.000000 & -0.379243 \\ \mathrm{C} & -1.094766 & 0.000000 & 0.966047 \\ \mathrm{O} & 0.000000 & 0.000000 & 1.789497 \\ \mathrm{C} & -1.715739 & 0.000000 & -1.352856 \\ \mathrm{C} & -3.039417 & 0.000000 & -0.950764 \\ \mathrm{C} & -3.379107 & 0.000000 & 0.402622 \\ \mathrm{C} & -2.406849 & 0.000000 & 1.389215 \\ \mathrm{H} & -2.655757 & 0.000000 & 2.441222 \\ \mathrm{H} & -4.422836 & 0.000000 & 0.688430 \\ \mathrm{H} & -3.825107 & 0.000000 & -1.694645 \\ \mathrm{H} & -1.459965 & 0.000000 & -2.404609 \\ \mathrm{H} & 1.459965 & 0.000000 & -2.404609 \\ \mathrm{H} & 3.825107 & 0.000000 & -1.694645 \\ \mathrm{H} & 4.422836 & 0.000000 & 0.688430 \\ \mathrm{H} & 2.655757 & 0.000000 & 2.441222\end{array}$

dibenzofuran $\mathrm{S}_{1}$

$\begin{array}{lrrr}\mathrm{C} & 0.000000 & 1.724796 & -1.527066 \\ \mathrm{C} & 0.000000 & 0.710328 & -0.565997 \\ \mathrm{C} & 0.000000 & 1.102348 & 0.803136 \\ \mathrm{C} & 0.000000 & 2.404037 & 1.252324 \\ \mathrm{C} & 0.000000 & 3.409429 & 0.264317 \\ \mathrm{C} & 0.000000 & 3.062612 & -1.085893 \\ \mathrm{C} & 0.000000 & -0.710328 & -0.565997 \\ \mathrm{C} & 0.000000 & -1.102348 & 0.803136 \\ \mathrm{O} & 0.000000 & 0.000000 & 1.600711\end{array}$




$\begin{array}{lrrr}\mathrm{C} & 0.000000 & -1.724796 & -1.527066 \\ \mathrm{C} & 0.000000 & -3.062612 & -1.085893 \\ \mathrm{C} & 0.000000 & -3.409429 & 0.264317 \\ \mathrm{C} & 0.000000 & -2.404037 & 1.252324 \\ \mathrm{H} & 0.000000 & -2.631930 & 2.308544 \\ \mathrm{H} & 0.000000 & -4.449428 & 0.555870 \\ \mathrm{H} & 0.000000 & -3.850921 & -1.827623 \\ \mathrm{H} & 0.000000 & -1.499749 & -2.584257 \\ \mathrm{H} & 0.000000 & 1.499749 & -2.584257 \\ \mathrm{H} & 0.000000 & 3.850921 & -1.827623 \\ \mathrm{H} & 0.000000 & 4.449428 & 0.555870 \\ \mathrm{H} & 0.000000 & 2.631930 & 2.308544\end{array}$

pyrrolo[3,2-h]quinoline $\mathrm{S}_{0}$

$\begin{array}{lrrr}\mathrm{C} & 0.135014 & -0.682836 & 0.000000 \\ \mathrm{~N} & 0.064113 & -2.030548 & 0.000000 \\ \mathrm{C} & 1.191460 & -2.701010 & 0.000000 \\ \mathrm{C} & 2.455897 & -2.093768 & 0.000000 \\ \mathrm{C} & 2.532180 & -0.726880 & 0.000000 \\ \mathrm{C} & 1.354218 & 0.037613 & 0.000000 \\ \mathrm{H} & 1.109760 & -3.783011 & 0.000000 \\ \mathrm{H} & 3.346807 & -2.706853 & 0.000000 \\ \mathrm{H} & 3.492866 & -0.225349 & 0.000000 \\ \mathrm{C} & -1.050752 & 0.084265 & 0.000000 \\ \mathrm{C} & -1.055012 & 1.478388 & 0.000000 \\ \mathrm{C} & 0.182587 & 2.170287 & 0.000000 \\ \mathrm{C} & 1.345257 & 1.466063 & 0.000000 \\ \mathrm{H} & 0.198576 & 3.252875 & 0.000000 \\ \mathrm{H} & 2.296948 & 1.983055 & 0.000000 \\ \mathrm{C} & -2.423190 & 1.883231 & 0.000000 \\ \mathrm{C} & -3.174622 & 0.743992 & 0.000000 \\ \mathrm{~N} & -2.343148 & -0.345743 & 0.000000 \\ \mathrm{H} & -2.610516 & -1.315212 & 0.000000 \\ \mathrm{H} & -2.803814 & 2.891062 & 0.000000 \\ \mathrm{H} & -4.244619 & 0.620378 & 0.000000\end{array}$

pyrrolo[3,2-h]quinoline $\mathrm{S}_{1}$

$\begin{array}{lrrr}\mathrm{C} & 0.149985 & -0.697502 & 0.000000 \\ \mathrm{~N} & 0.020136 & -2.020323 & 0.000000 \\ \mathrm{C} & 1.201461 & -2.725524 & 0.000000 \\ \mathrm{C} & 2.433170 & -2.130836 & 0.000000 \\ \mathrm{C} & 2.557059 & -0.724374 & 0.000000 \\ \mathrm{C} & 1.362324 & 0.029270 & 0.000000 \\ \mathrm{H} & 1.102332 & -3.802959 & 0.000000\end{array}$




$\begin{array}{rrrr}\mathrm{H} & 3.319831 & -2.751684 & 0.000000 \\ \mathrm{H} & 3.523191 & -0.240911 & 0.000000 \\ \mathrm{C} & -1.025428 & 0.101004 & 0.000000 \\ \mathrm{C} & -1.059031 & 1.545086 & 0.000000 \\ \mathrm{C} & 0.138689 & 2.220427 & 0.000000 \\ \mathrm{C} & 1.319961 & 1.449328 & 0.000000 \\ \mathrm{H} & 0.189459 & 3.299867 & 0.000000 \\ \mathrm{H} & 2.269080 & 1.972495 & 0.000000 \\ \mathrm{C} & -2.453604 & 1.902255 & 0.000000 \\ \mathrm{C} & -3.161213 & 0.749769 & 0.000000 \\ \mathrm{~N} & -2.285648 & -0.339460 & 0.000000 \\ \mathrm{H} & -2.511291 & -1.323314 & 0.000000 \\ \mathrm{H} & -2.864182 & 2.897528 & 0.000000 \\ \mathrm{H} & -4.226292 & 0.589857 & 0.000000\end{array}$

carbazole $\mathrm{S}_{0}$

$\begin{array}{lrrr}\mathrm{C} & 1.127104 & 0.000000 & -0.894816 \\ \mathrm{~N} & 0.000000 & 0.000000 & -1.692349 \\ \mathrm{C} & -1.127104 & 0.000000 & -0.894816 \\ \mathrm{C} & -0.723412 & 0.000000 & 0.453714 \\ \mathrm{C} & 0.723412 & 0.000000 & 0.453714 \\ \mathrm{C} & -1.688604 & 0.000000 & 1.454391 \\ \mathrm{C} & -3.024463 & 0.000000 & 1.101394 \\ \mathrm{C} & -3.406196 & 0.000000 & -0.242165 \\ \mathrm{C} & -2.467272 & 0.000000 & -1.256281 \\ \mathrm{C} & 2.467272 & 0.000000 & -1.256281 \\ \mathrm{C} & 3.406196 & 0.000000 & -0.242165 \\ \mathrm{C} & 3.024463 & 0.000000 & 1.101394 \\ \mathrm{C} & 1.688604 & 0.000000 & 1.454391 \\ \mathrm{H} & -2.770316 & 0.000000 & -2.295702 \\ \mathrm{H} & -4.458599 & 0.000000 & -0.495409 \\ \mathrm{H} & -3.784948 & 0.000000 & 1.870996 \\ \mathrm{H} & -1.398452 & 0.000000 & 2.497649 \\ \mathrm{H} & 1.398452 & 0.000000 & 2.497649 \\ \mathrm{H} & 3.784948 & 0.000000 & 1.870996 \\ \mathrm{H} & 4.458599 & 0.000000 & -0.495409 \\ \mathrm{H} & 2.770316 & 0.000000 & -2.295702 \\ \mathrm{H} & 0.000000 & 0.000000 & -2.696154\end{array}$

carbazole $\mathrm{S}_{1}$
$\begin{array}{llll}\text { C } & 1.139156 & 0.000000 & -0.836014\end{array}$
$\begin{array}{llll}\mathrm{N} & 0.000000 & 0.000000 & -1.607657\end{array}$
$\begin{array}{llll}\text { C } & -1.139156 & 0.000000 & -0.836014\end{array}$
$\begin{array}{llll}\text { C } & -0.713749 & 0.000000 & 0.528843\end{array}$ 


$\begin{array}{crrr}\mathrm{C} & 0.713749 & 0.000000 & 0.528843 \\ \mathrm{C} & -1.700122 & 0.000000 & 1.516629 \\ \mathrm{C} & -3.049788 & 0.000000 & 1.116601 \\ \mathrm{C} & -3.438853 & 0.000000 & -0.217253 \\ \mathrm{C} & -2.459446 & 0.000000 & -1.234615 \\ \mathrm{C} & 2.459446 & 0.000000 & -1.234615 \\ \mathrm{C} & 3.438853 & 0.000000 & -0.217253 \\ \mathrm{C} & 3.049788 & 0.000000 & 1.116601 \\ \mathrm{C} & 1.700122 & 0.000000 & 1.516629 \\ \mathrm{H} & -2.735254 & 0.000000 & -2.280992 \\ \mathrm{H} & -4.487201 & 0.000000 & -0.477487 \\ \mathrm{H} & -3.814522 & 0.000000 & 1.883299 \\ \mathrm{H} & -1.443590 & 0.000000 & 2.566641 \\ \mathrm{H} & 1.443590 & 0.000000 & 2.566641 \\ \mathrm{H} & 3.814522 & 0.000000 & 1.883299 \\ \mathrm{H} & 4.487201 & 0.000000 & -0.477487 \\ \mathrm{H} & 2.735254 & 0.000000 & -2.280992 \\ \mathrm{H} & 0.000000 & 0.000000 & -2.616273\end{array}$

tryptamine, A-ph $\mathrm{S}_{0}$

$\begin{array}{rrrr}\mathrm{C} & -2.127166 & 0.137939 & 0.887979 \\ \mathrm{~N} & -1.251262 & 0.129636 & 1.952323 \\ \mathrm{C} & 0.031976 & 0.045046 & 1.472796 \\ \mathrm{C} & -0.049982 & -0.005671 & 0.067059 \\ \mathrm{C} & -1.444496 & 0.055496 & -0.284920 \\ \mathrm{C} & 1.249336 & 0.014004 & 2.146159 \\ \mathrm{C} & 2.396375 & -0.067106 & 1.386596 \\ \mathrm{C} & 2.339055 & -0.113869 & -0.013678 \\ \mathrm{C} & 1.131371 & -0.083780 & -0.675833 \\ \mathrm{C} & -2.017520 & 0.006219 & -1.663713 \\ \mathrm{C} & -1.985835 & -1.389839 & -2.281624 \\ \mathrm{~N} & -2.484456 & -1.351334 & -3.651820 \\ \mathrm{H} & 1.296935 & 0.053530 & 3.227401 \\ \mathrm{H} & 3.359863 & -0.092966 & 1.879167 \\ \mathrm{H} & 3.260395 & -0.173297 & -0.578447 \\ \mathrm{H} & 1.099110 & -0.116398 & -1.757892 \\ \mathrm{H} & -1.510808 & 0.199143 & 2.919223 \\ \mathrm{H} & -3.190066 & 0.208496 & 1.055640 \\ \mathrm{H} & -1.474193 & 0.682918 & -2.327380 \\ \mathrm{H} & -3.052329 & 0.360410 & -1.641272 \\ \mathrm{H} & -2.540473 & -2.076168 & -1.627483 \\ \mathrm{H} & -0.954774 & -1.746352 & -2.308394 \\ \mathrm{H} & -3.466453 & -1.105914 & -3.676117 \\ \mathrm{H} & -2.382015 & -2.247763 & -4.108400\end{array}$


tryptamine, A-ph $\mathrm{S}_{1}$

$\begin{array}{rrrr}\mathrm{C} & -2.175983 & 0.157086 & 0.908481 \\ \mathrm{~N} & -1.290780 & 0.127032 & 1.914400 \\ \mathrm{C} & 0.025906 & 0.052035 & 1.439577 \\ \mathrm{C} & -0.053830 & 0.045692 & 0.041016 \\ \mathrm{C} & -1.427218 & 0.108274 & -0.306877 \\ \mathrm{C} & 1.207514 & -0.031697 & 2.154771 \\ \mathrm{C} & 2.404842 & -0.112721 & 1.350328 \\ \mathrm{C} & 2.357637 & -0.116866 & -0.017544 \\ \mathrm{C} & 1.130513 & -0.038964 & -0.732595 \\ \mathrm{C} & -2.006247 & 0.045586 & -1.666071 \\ \mathrm{C} & -1.965545 & -1.377332 & -2.243413 \\ \mathrm{~N} & -2.501728 & -1.383019 & -3.596327 \\ \mathrm{H} & 1.245773 & -0.039297 & 3.233939 \\ \mathrm{H} & 3.361318 & -0.172066 & 1.852958 \\ \mathrm{H} & 3.283468 & -0.177411 & -0.575411 \\ \mathrm{H} & 1.108036 & -0.008415 & -1.812071 \\ \mathrm{H} & -1.532511 & 0.169680 & 2.892120 \\ \mathrm{H} & -3.236448 & 0.248672 & 1.067753 \\ \mathrm{H} & -1.445865 & 0.692020 & -2.344351 \\ \mathrm{H} & -3.041107 & 0.398739 & -1.653732 \\ \mathrm{H} & -2.488285 & -2.054818 & -1.555267 \\ \mathrm{H} & -0.924782 & -1.701481 & -2.282326 \\ \mathrm{H} & -3.505883 & -1.257242 & -3.600283 \\ \mathrm{H} & -2.296225 & -2.251107 & -4.071745\end{array}$

tryptamine, A-py $\mathrm{S}_{0}$

$\begin{array}{rrrc}\mathrm{C} & -2.119976 & 0.112485 & 0.865240 \\ \mathrm{~N} & -1.254226 & 0.119316 & 1.937452 \\ \mathrm{C} & 0.034188 & 0.046167 & 1.470674 \\ \mathrm{C} & -0.033930 & -0.013703 & 0.064254 \\ \mathrm{C} & -1.425728 & 0.029988 & -0.300938 \\ \mathrm{C} & 1.245041 & 0.032238 & 2.156165 \\ \mathrm{C} & 2.400663 & -0.040477 & 1.408971 \\ \mathrm{C} & 2.357722 & -0.096350 & 0.008578 \\ \mathrm{C} & 1.156219 & -0.083871 & -0.665236 \\ \mathrm{C} & -1.996495 & -0.025519 & -1.680707 \\ \mathrm{C} & -1.946290 & -1.418038 & -2.304355 \\ \mathrm{~N} & -2.519516 & -1.391672 & -3.645946 \\ \mathrm{H} & 1.281110 & 0.078135 & 3.237593 \\ \mathrm{H} & 3.359324 & -0.053082 & 1.911320 \\ \mathrm{H} & 3.285198 & -0.150031 & -0.546670 \\ \mathrm{H} & 1.136242 & -0.126662 & -1.747254 \\ \mathrm{H} & -1.523879 & 0.189972 & 2.901539\end{array}$




$\begin{array}{lrrr}\mathrm{H} & -3.185182 & 0.172918 & 1.021418 \\ \mathrm{H} & -1.454431 & 0.670151 & -2.330387 \\ \mathrm{H} & -3.035460 & 0.308134 & -1.673360 \\ \mathrm{H} & -2.543589 & -2.097059 & -1.693034 \\ \mathrm{H} & -0.914117 & -1.791013 & -2.274275 \\ \mathrm{H} & -1.955697 & -0.828801 & -4.270916 \\ \mathrm{H} & -2.577279 & -2.320837 & -4.041017\end{array}$

tryptamine, A-py $\mathrm{S}_{1}$

$\begin{array}{lrrr}\mathrm{C} & -2.169197 & 0.117784 & 0.884078 \\ \mathrm{~N} & -1.295521 & 0.101664 & 1.899236 \\ \mathrm{C} & 0.027873 & 0.047055 & 1.438466 \\ \mathrm{C} & -0.036524 & 0.036877 & 0.039785 \\ \mathrm{C} & -1.406721 & 0.079696 & -0.322890 \\ \mathrm{C} & 1.203562 & -0.013517 & 2.167010 \\ \mathrm{C} & 2.409446 & -0.076637 & 1.374983 \\ \mathrm{C} & 2.377775 & -0.084776 & 0.006469 \\ \mathrm{C} & 1.157195 & -0.028904 & -0.721877 \\ \mathrm{C} & -1.976563 & 0.014818 & -1.684883 \\ \mathrm{C} & -1.911188 & -1.405443 & -2.270064 \\ \mathrm{~N} & -2.512867 & -1.423499 & -3.594317 \\ \mathrm{H} & 1.230836 & -0.015670 & 3.246455 \\ \mathrm{H} & 3.361580 & -0.118907 & 1.887622 \\ \mathrm{H} & 3.310869 & -0.131924 & -0.540495 \\ \mathrm{H} & 1.145739 & -0.005915 & -1.801685 \\ \mathrm{H} & -1.548821 & 0.139999 & 2.874180 \\ \mathrm{H} & -3.233252 & 0.187645 & 1.030070 \\ \mathrm{H} & -1.416186 & 0.683824 & -2.344759 \\ \mathrm{H} & -3.017686 & 0.340819 & -1.690899 \\ \mathrm{H} & -2.483113 & -2.076850 & -1.626888 \\ \mathrm{H} & -0.868058 & -1.743271 & -2.244569 \\ \mathrm{H} & -1.937033 & -0.933650 & -4.267449 \\ \mathrm{H} & -2.642236 & -2.368839 & -3.928478\end{array}$

tryptamine, A-up $\mathrm{S}_{0}$

$\begin{array}{rrrr}\mathrm{C} & -2.119182 & 0.127812 & 0.877004 \\ \mathrm{~N} & -1.251303 & 0.106990 & 1.947162 \\ \mathrm{C} & 0.035811 & 0.036365 & 1.476224 \\ \mathrm{C} & -0.035117 & 0.007042 & 0.069180 \\ \mathrm{C} & -1.427273 & 0.068485 & -0.292219 \\ \mathrm{C} & 1.247838 & 0.000246 & 2.158819 \\ \mathrm{C} & 2.401329 & -0.064284 & 1.407707 \\ \mathrm{C} & 2.355435 & -0.090713 & 0.006540 \\ \mathrm{C} & 1.152905 & -0.055714 & -0.664469\end{array}$




$\begin{array}{crcc}\mathrm{C} & -1.994553 & 0.032151 & -1.673879 \\ \mathrm{C} & -1.956635 & -1.362056 & -2.315728 \\ \mathrm{~N} & -2.465541 & -1.439128 & -3.676486 \\ \mathrm{H} & 1.286355 & 0.022731 & 3.240878 \\ \mathrm{H} & 3.360873 & -0.094148 & 1.907600 \\ \mathrm{H} & 3.281401 & -0.139779 & -0.551561 \\ \mathrm{H} & 1.130938 & -0.076618 & -1.747085 \\ \mathrm{H} & -1.518687 & 0.155219 & 2.913279 \\ \mathrm{H} & -3.183643 & 0.189971 & 1.037806 \\ \mathrm{H} & -1.447950 & 0.728397 & -2.318350 \\ \mathrm{H} & -3.030813 & 0.381272 & -1.654571 \\ \mathrm{H} & -2.530093 & -2.054066 & -1.695645 \\ \mathrm{H} & -0.931486 & -1.736776 & -2.314887 \\ \mathrm{H} & -1.928534 & -0.856293 & -4.305934 \\ \mathrm{H} & -3.430836 & -1.139197 & -3.728145\end{array}$

tryptamine, A-up $\mathrm{S}_{1}$

$\begin{array}{lrrr}\mathrm{C} & -2.166086 & 0.121045 & 0.890316 \\ \mathrm{~N} & -1.289862 & 0.078877 & 1.903754 \\ \mathrm{C} & 0.032515 & 0.034711 & 1.441710 \\ \mathrm{C} & -0.032477 & 0.059532 & 0.042952 \\ \mathrm{C} & -1.403595 & 0.118992 & -0.317650 \\ \mathrm{C} & 1.208689 & -0.043725 & 2.167501 \\ \mathrm{C} & 2.414015 & -0.090965 & 1.374405 \\ \mathrm{C} & 2.381135 & -0.067269 & 0.006390 \\ \mathrm{C} & 1.159844 & 0.008723 & -0.719676 \\ \mathrm{C} & -1.965439 & 0.078246 & -1.680162 \\ \mathrm{C} & -1.958145 & -1.361313 & -2.263727 \\ \mathrm{~N} & -2.469552 & -1.479958 & -3.611088 \\ \mathrm{H} & 1.236275 & -0.070510 & 3.246660 \\ \mathrm{H} & 3.366214 & -0.148480 & 1.885338 \\ \mathrm{H} & 3.313370 & -0.105370 & -0.542728 \\ \mathrm{H} & 1.148476 & 0.049226 & -1.799049 \\ \mathrm{H} & -1.541930 & 0.093711 & 2.879715 \\ \mathrm{H} & -3.229298 & 0.191839 & 1.041603 \\ \mathrm{H} & -1.372412 & 0.713243 & -2.343265 \\ \mathrm{H} & -2.991514 & 0.455049 & -1.686450 \\ \mathrm{H} & -2.544634 & -2.009960 & -1.611155 \\ \mathrm{H} & -0.934186 & -1.736567 & -2.240924 \\ \mathrm{H} & -1.923933 & -0.948745 & -4.276403 \\ \mathrm{H} & -3.436250 & -1.192412 & -3.684847\end{array}$

tryptamine, $\mathrm{Ph}$-out $\mathrm{S}_{0}$

$\begin{array}{llll}\text { C } & 0.006284 & -0.000803 & 0.083307\end{array}$ 


$\begin{array}{lrrr}\mathrm{C} & 0.046411 & 0.065233 & 1.490896 \\ \mathrm{C} & 1.243668 & 0.079899 & 2.200474 \\ \mathrm{C} & 2.414299 & 0.031937 & 1.475506 \\ \mathrm{C} & 2.398851 & -0.031299 & 0.074459 \\ \mathrm{C} & 1.212111 & -0.047710 & -0.624845 \\ \mathrm{~N} & -1.251363 & 0.111085 & 1.934179 \\ \mathrm{H} & 1.257385 & 0.129452 & 3.282285 \\ \mathrm{H} & 3.363355 & 0.042770 & 1.995978 \\ \mathrm{H} & 3.337862 & -0.067562 & -0.462602 \\ \mathrm{H} & 1.207060 & -0.112952 & -1.705095 \\ \mathrm{C} & -1.380831 & 0.012387 & -0.307655 \\ \mathrm{C} & -2.097058 & 0.085861 & 0.847111 \\ \mathrm{H} & -1.538953 & 0.188795 & 2.892583 \\ \mathrm{H} & -3.165558 & 0.133194 & 0.985176 \\ \mathrm{C} & -1.938206 & -0.044062 & -1.696444 \\ \mathrm{H} & -1.303240 & 0.532718 & -2.372781 \\ \mathrm{C} & -2.083072 & -1.456305 & -2.261302 \\ \mathrm{H} & -2.923056 & 0.429169 & -1.704597 \\ \mathrm{H} & -2.545262 & -1.387228 & -3.248735 \\ \mathrm{~N} & -0.785489 & -2.100347 & -2.421802 \\ \mathrm{H} & -2.774041 & -2.020649 & -1.618857 \\ \mathrm{H} & -0.339991 & -2.248807 & -1.524749 \\ \mathrm{H} & -0.876192 & -3.000668 & -2.873279\end{array}$

tryptamine, $\mathrm{Ph}$-out $\mathrm{S}_{1}$

$\begin{array}{lrrr}\mathrm{C} & 0.000726 & 0.022294 & 0.065705 \\ \mathrm{C} & 0.036031 & 0.081590 & 1.466585 \\ \mathrm{C} & 1.195086 & 0.058066 & 2.221574 \\ \mathrm{C} & 2.421315 & -0.009204 & 1.461104 \\ \mathrm{C} & 2.419272 & -0.067823 & 0.094005 \\ \mathrm{C} & 1.215236 & -0.063971 & -0.663530 \\ \mathrm{~N} & -1.294627 & 0.140112 & 1.898965 \\ \mathrm{H} & 1.196899 & 0.101869 & 3.300613 \\ \mathrm{H} & 3.361652 & -0.015026 & 1.996558 \\ \mathrm{H} & 3.364169 & -0.117978 & -0.431985 \\ \mathrm{H} & 1.226684 & -0.086697 & -1.742890 \\ \mathrm{C} & -1.362706 & 0.047600 & -0.325950 \\ \mathrm{C} & -2.148484 & 0.116608 & 0.867081 \\ \mathrm{H} & -1.566719 & 0.208812 & 2.867197 \\ \mathrm{H} & -3.215397 & 0.185332 & 0.991318 \\ \mathrm{C} & -1.934841 & -0.042539 & -1.689502 \\ \mathrm{H} & -1.301449 & 0.494349 & -2.397976 \\ \mathrm{C} & -2.077226 & -1.490716 & -2.189837 \\ \mathrm{H} & -2.923889 & 0.422097 & -1.696401 \\ \mathrm{H} & -2.677788 & -1.468510 & -3.101576\end{array}$




$$
\begin{array}{llll}
\mathrm{N} & -0.781994 & -2.057546 & -2.516446 \\
\mathrm{H} & -2.644683 & -2.062902 & -1.442078 \\
\mathrm{H} & -0.156225 & -2.072452 & -1.719027 \\
\mathrm{H} & -0.866050 & -2.999245 & -2.874299
\end{array}
$$

tryptamine, $\mathrm{Ph}$-up $\mathrm{S}_{0}$

$\begin{array}{lrrc}\mathrm{C} & -2.072974 & 0.022076 & 0.815215 \\ \mathrm{~N} & -1.240309 & 0.016932 & 1.912272 \\ \mathrm{C} & 0.062724 & 0.034519 & 1.484301 \\ \mathrm{C} & 0.038984 & 0.044721 & 0.074761 \\ \mathrm{C} & -1.341763 & 0.034322 & -0.332888 \\ \mathrm{C} & 1.251412 & 0.047334 & 2.208087 \\ \mathrm{C} & 2.430961 & 0.075091 & 1.496998 \\ \mathrm{C} & 2.432938 & 0.091286 & 0.094516 \\ \mathrm{C} & 1.253978 & 0.076404 & -0.617176 \\ \mathrm{C} & -1.874583 & 0.010333 & -1.731197 \\ \mathrm{C} & -2.118527 & -1.403089 & -2.283133 \\ \mathrm{~N} & -0.957187 & -2.270843 & -2.380916 \\ \mathrm{H} & 1.251865 & 0.037895 & 3.291013 \\ \mathrm{H} & 3.373445 & 0.086217 & 2.028984 \\ \mathrm{H} & 3.378227 & 0.117388 & -0.431871 \\ \mathrm{H} & 1.269338 & 0.091511 & -1.699743 \\ \mathrm{H} & -1.541238 & 0.025026 & 2.869794 \\ \mathrm{H} & -3.143751 & 0.023061 & 0.943229 \\ \mathrm{H} & -1.182550 & 0.536466 & -2.395187 \\ \mathrm{H} & -2.817527 & 0.562833 & -1.773353 \\ \mathrm{H} & -2.563933 & -1.321954 & -3.277683 \\ \mathrm{H} & -2.858059 & -1.903461 & -1.653535 \\ \mathrm{H} & -0.511483 & -2.390066 & -1.480027 \\ \mathrm{H} & -0.266338 & -1.894372 & -3.017403\end{array}$

tryptamine, $\mathrm{Ph}$-up $\mathrm{S}_{1}$

$\begin{array}{lrrr}\mathrm{C} & -2.151453 & 0.127600 & 0.860527 \\ \mathrm{~N} & -1.299993 & 0.118961 & 1.894852 \\ \mathrm{C} & 0.032092 & 0.077191 & 1.463315 \\ \mathrm{C} & -0.000570 & 0.072152 & 0.062512 \\ \mathrm{C} & -1.363822 & 0.100662 & -0.330442 \\ \mathrm{C} & 1.190704 & 0.016739 & 2.219228 \\ \mathrm{C} & 2.415539 & -0.028657 & 1.458612 \\ \mathrm{C} & 2.417714 & -0.030587 & 0.090057 \\ \mathrm{C} & 1.214830 & 0.013470 & -0.668947 \\ \mathrm{C} & -1.917377 & 0.004665 & -1.691594 \\ \mathrm{C} & -2.019741 & -1.472018 & -2.191342 \\ \mathrm{~N} & -0.775866 & -2.149664 & -2.445028\end{array}$




$\begin{array}{rrrr}\mathrm{H} & 1.190649 & 0.011576 & 3.299023 \\ \mathrm{H} & 3.355247 & -0.062841 & 1.994244 \\ \mathrm{H} & 3.364475 & -0.061887 & -0.434095 \\ \mathrm{H} & 1.233936 & 0.082815 & -1.746316 \\ \mathrm{H} & -1.574154 & 0.159983 & 2.863924 \\ \mathrm{H} & -3.218865 & 0.189840 & 0.985004 \\ \mathrm{H} & -1.294159 & 0.559042 & -2.396517 \\ \mathrm{H} & -2.921527 & 0.436685 & -1.718152 \\ \mathrm{H} & -2.605083 & -1.464153 & -3.112870 \\ \mathrm{H} & -2.591233 & -2.044651 & -1.459078 \\ \mathrm{H} & -0.163123 & -2.170273 & -1.638519 \\ \mathrm{H} & -0.264561 & -1.737039 & -3.213309\end{array}$

tryptamine, Py-out $\mathrm{S}_{0}$

$\begin{array}{lrrr}\mathrm{C} & 0.519294 & 0.453394 & 0.223581 \\ \mathrm{C} & 0.594960 & 0.528072 & 1.629079 \\ \mathrm{C} & 1.807683 & 0.489349 & 2.310137 \\ \mathrm{C} & 2.957747 & 0.375704 & 1.559311 \\ \mathrm{C} & 2.907449 & 0.304146 & 0.159995 \\ \mathrm{C} & 1.704065 & 0.342137 & -0.509820 \\ \mathrm{~N} & -0.689226 & 0.640653 & 2.100500 \\ \mathrm{H} & 1.849549 & 0.548118 & 3.390729 \\ \mathrm{H} & 3.917799 & 0.343008 & 2.058085 \\ \mathrm{H} & 3.830831 & 0.218916 & -0.398105 \\ \mathrm{H} & 1.678409 & 0.288264 & -1.591065 \\ \mathrm{C} & -0.873045 & 0.531603 & -0.136288 \\ \mathrm{C} & -1.560135 & 0.643323 & 1.032312 \\ \mathrm{H} & -0.952460 & 0.729393 & 3.064929 \\ \mathrm{H} & -2.623282 & 0.728323 & 1.189975 \\ \mathrm{C} & -1.450377 & 0.470433 & -1.514366 \\ \mathrm{H} & -0.892091 & 1.136874 & -2.177607 \\ \mathrm{C} & -1.445305 & -0.934917 & -2.114609 \\ \mathrm{H} & -2.481389 & 0.828187 & -1.492954 \\ \mathrm{H} & -0.407293 & -1.283702 & -2.204036 \\ \mathrm{~N} & -2.288262 & -1.827603 & -1.329387 \\ \mathrm{H} & -1.855234 & -0.883511 & -3.125492 \\ \mathrm{H} & -2.324166 & -2.752612 & -1.736600 \\ \mathrm{H} & -1.925502 & -1.917551 & -0.388287\end{array}$

tryptamine, Py-out $\mathrm{S}_{1}$
$\begin{array}{llll}\text { C } & 0.523012 & 0.481543 & 0.206252\end{array}$
$\begin{array}{llll}\text { C } & 0.599395 & 0.532166 & 1.603340\end{array}$
$\begin{array}{llll}\text { C } & 1.780104 & 0.473300 & 2.324959\end{array}$
$\begin{array}{llll}\text { C } & 2.978108 & 0.367374 & 1.525475\end{array}$ 


$\begin{array}{lrrr}\mathrm{C} & 2.934860 & 0.319934 & 0.158351 \\ \mathrm{C} & 1.708721 & 0.372555 & -0.562284 \\ \mathrm{~N} & -0.718555 & 0.628363 & 2.073132 \\ \mathrm{H} & 1.816687 & 0.512095 & 3.403417 \\ \mathrm{H} & 3.933804 & 0.325164 & 2.031488 \\ \mathrm{H} & 3.862402 & 0.241820 & -0.394444 \\ \mathrm{H} & 1.688790 & 0.357937 & -1.641978 \\ \mathrm{C} & -0.850339 & 0.549832 & -0.146678 \\ \mathrm{C} & -1.600951 & 0.634699 & 1.065685 \\ \mathrm{H} & -0.962856 & 0.688636 & 3.049348 \\ \mathrm{H} & -2.664082 & 0.706034 & 1.216251 \\ \mathrm{C} & -1.429545 & 0.484524 & -1.506189 \\ \mathrm{H} & -0.839579 & 1.115913 & -2.175763 \\ \mathrm{C} & -1.431967 & -0.943341 & -2.074812 \\ \mathrm{H} & -2.456248 & 0.854367 & -1.499736 \\ \mathrm{H} & -0.394460 & -1.294619 & -2.132074 \\ \mathrm{~N} & -2.302551 & -1.809256 & -1.292388 \\ \mathrm{H} & -1.822948 & -0.900623 & -3.092339 \\ \mathrm{H} & -2.449765 & -2.695468 & -1.756541 \\ \mathrm{H} & -1.902048 & -2.002960 & -0.382484\end{array}$

tryptamine, Py-up $\mathrm{S}_{0}$

$\begin{array}{lrrr}\mathrm{C} & -2.116581 & 0.250027 & 0.894500 \\ \mathrm{~N} & -1.242144 & 0.230250 & 1.959779 \\ \mathrm{C} & 0.038046 & 0.097347 & 1.482852 \\ \mathrm{C} & -0.044017 & 0.027829 & 0.077872 \\ \mathrm{C} & -1.436160 & 0.129321 & -0.277183 \\ \mathrm{C} & 1.252578 & 0.036842 & 2.159224 \\ \mathrm{C} & 2.397362 & -0.093918 & 1.403478 \\ \mathrm{C} & 2.340581 & -0.161350 & 0.004138 \\ \mathrm{C} & 1.135614 & -0.101884 & -0.660776 \\ \mathrm{C} & -2.012058 & 0.076228 & -1.654301 \\ \mathrm{C} & -2.003153 & -1.331574 & -2.272589 \\ \mathrm{~N} & -2.788120 & -2.335784 & -1.575259 \\ \mathrm{H} & 1.299568 & 0.091553 & 3.239781 \\ \mathrm{H} & 3.358667 & -0.144308 & 1.898286 \\ \mathrm{H} & 3.260143 & -0.261478 & -0.557656 \\ \mathrm{H} & 1.105249 & -0.153387 & -1.742010 \\ \mathrm{H} & -1.499644 & 0.322341 & 2.925418 \\ \mathrm{H} & -3.176406 & 0.361143 & 1.059227 \\ \mathrm{H} & -1.457069 & 0.751062 & -2.313713 \\ \mathrm{H} & -3.042093 & 0.443599 & -1.632534 \\ \mathrm{H} & -0.975121 & -1.694334 & -2.333376 \\ \mathrm{H} & -2.368721 & -1.268334 & -3.299925 \\ \mathrm{H} & -3.767579 & -2.080896 & -1.546555\end{array}$




$$
\text { H } \quad-2.470421 \quad-2.440675 \quad-0.619788
$$

tryptamine, Py-up $\mathrm{S}_{1}$

$\begin{array}{rrrr}\mathrm{C} & -2.162122 & 0.253744 & 0.932010 \\ \mathrm{~N} & -1.271406 & 0.234403 & 1.934691 \\ \mathrm{C} & 0.039853 & 0.110826 & 1.457586 \\ \mathrm{C} & -0.046592 & 0.061249 & 0.059969 \\ \mathrm{C} & -1.420851 & 0.152804 & -0.284882 \\ \mathrm{C} & 1.222056 & 0.023785 & 2.171138 \\ \mathrm{C} & 2.414355 & -0.112280 & 1.366960 \\ \mathrm{C} & 2.361162 & -0.159305 & 0.000701 \\ \mathrm{C} & 1.132477 & -0.076081 & -0.712574 \\ \mathrm{C} & -2.003268 & 0.087338 & -1.639307 \\ \mathrm{C} & -1.983978 & -1.343905 & -2.237443 \\ \mathrm{~N} & -2.798782 & -2.332802 & -1.566706 \\ \mathrm{H} & 1.264366 & 0.057323 & 3.249690 \\ \mathrm{H} & 3.370861 & -0.179532 & 1.868353 \\ \mathrm{H} & 3.282446 & -0.261554 & -0.558529 \\ \mathrm{H} & 1.108222 & -0.090367 & -1.792285 \\ \mathrm{H} & -1.506946 & 0.309839 & 2.912053 \\ \mathrm{H} & -3.218646 & 0.380002 & 1.093239 \\ \mathrm{H} & -1.426512 & 0.732543 & -2.308896 \\ \mathrm{H} & -3.032961 & 0.454721 & -1.630847 \\ \mathrm{H} & -0.952789 & -1.699551 & -2.250371 \\ \mathrm{H} & -2.310905 & -1.276047 & -3.276544 \\ \mathrm{H} & -3.777755 & -2.079288 & -1.552309 \\ \mathrm{H} & -2.493765 & -2.498237 & -0.616782\end{array}$

porphycene $\mathrm{S}_{0}$

$\begin{array}{rrrr}\mathrm{C} & -3.425118 & 1.914094 & 0.000000 \\ \mathrm{C} & -2.416675 & 0.907691 & 0.000000 \\ \mathrm{~N} & -1.221180 & 1.542741 & 0.000000 \\ \mathrm{C} & -1.387482 & 2.890295 & 0.000000 \\ \mathrm{C} & -2.796146 & 3.126731 & 0.000000 \\ \mathrm{C} & -0.362915 & 3.840292 & 0.000000 \\ \mathrm{C} & 1.012696 & 3.690492 & 0.000000 \\ \mathrm{C} & 1.828253 & 2.550165 & 0.000000 \\ \mathrm{H} & -0.263033 & 1.127622 & 0.000000 \\ \mathrm{~N} & 1.414174 & 1.263874 & 0.000000 \\ \mathrm{C} & 3.277141 & 2.589097 & 0.000000 \\ \mathrm{C} & 3.703790 & 1.304686 & 0.000000 \\ \mathrm{C} & 2.511847 & 0.490895 & 0.000000 \\ \mathrm{C} & 2.416675 & -0.907691 & 0.000000 \\ \mathrm{C} & 3.425118 & -1.914094 & 0.000000\end{array}$




$\begin{array}{cccc}\mathrm{C} & 2.796146 & -3.126731 & 0.000000 \\ \mathrm{C} & 1.387482 & -2.890295 & 0.000000 \\ \mathrm{~N} & 1.221180 & -1.542741 & 0.000000 \\ \mathrm{C} & 0.362915 & -3.840292 & 0.000000 \\ \mathrm{C} & -1.012696 & -3.690492 & 0.000000 \\ \mathrm{C} & -1.828253 & -2.550165 & 0.000000 \\ \mathrm{C} & -3.277141 & -2.589097 & 0.000000 \\ \mathrm{C} & -3.703790 & -1.304686 & 0.000000 \\ \mathrm{C} & -2.511847 & -0.490895 & 0.000000 \\ \mathrm{~N} & -1.414174 & -1.263874 & 0.000000 \\ \mathrm{H} & 0.263033 & -1.127622 & 0.000000 \\ \mathrm{H} & 3.255876 & -4.102042 & 0.000000 \\ \mathrm{H} & 4.486886 & -1.729918 & 0.000000 \\ \mathrm{H} & 0.719633 & -4.863482 & 0.000000 \\ \mathrm{H} & -1.568292 & -4.621445 & 0.000000 \\ \mathrm{H} & -3.878519 & -3.485064 & 0.000000 \\ \mathrm{H} & -4.720547 & -0.945506 & 0.000000 \\ \mathrm{H} & -4.486886 & 1.729918 & 0.000000 \\ \mathrm{H} & -3.255876 & 4.102042 & 0.000000 \\ \mathrm{H} & -0.719633 & 4.863482 & 0.000000 \\ \mathrm{H} & 1.568292 & 4.621445 & 0.000000 \\ \mathrm{H} & 3.878519 & 3.485064 & 0.000000 \\ \mathrm{H} & 4.720547 & 0.945506 & 0.000000\end{array}$

porphycene $\mathrm{S}_{1}$

$\begin{array}{rrrr}\mathrm{C} & -3.419140 & 1.952876 & 0.000000 \\ \mathrm{C} & -2.429520 & 0.933620 & 0.000000 \\ \mathrm{~N} & -1.212514 & 1.559943 & 0.000000 \\ \mathrm{C} & -1.366396 & 2.901674 & 0.000000 \\ \mathrm{C} & -2.769771 & 3.159345 & 0.000000 \\ \mathrm{C} & -0.318564 & 3.853811 & 0.000000 \\ \mathrm{C} & 1.041962 & 3.688460 & 0.000000 \\ \mathrm{C} & 1.868750 & 2.528200 & 0.000000 \\ \mathrm{H} & -0.263333 & 1.131274 & 0.000000 \\ \mathrm{~N} & 1.439230 & 1.254773 & 0.000000 \\ \mathrm{C} & 3.309418 & 2.558183 & 0.000000 \\ \mathrm{C} & 3.732268 & 1.264329 & 0.000000 \\ \mathrm{C} & 2.547690 & 0.461757 & 0.000000 \\ \mathrm{C} & 2.429520 & -0.933620 & 0.000000 \\ \mathrm{C} & 3.419140 & -1.952876 & 0.000000 \\ \mathrm{C} & 2.769771 & -3.159345 & 0.000000 \\ \mathrm{C} & 1.366396 & -2.901674 & 0.000000 \\ \mathrm{~N} & 1.212514 & -1.559943 & 0.000000 \\ \mathrm{C} & 0.318564 & -3.853811 & 0.000000 \\ \mathrm{C} & -1.041962 & -3.688460 & 0.000000\end{array}$




$\begin{array}{lrrr}\mathrm{C} & -1.868750 & -2.528200 & 0.000000 \\ \mathrm{C} & -3.309418 & -2.558183 & 0.000000 \\ \mathrm{C} & -3.732268 & -1.264329 & 0.000000 \\ \mathrm{C} & -2.547690 & -0.461757 & 0.000000 \\ \mathrm{~N} & -1.439230 & -1.254773 & 0.000000 \\ \mathrm{H} & 0.263333 & -1.131274 & 0.000000 \\ \mathrm{H} & 3.213998 & -4.141802 & 0.000000 \\ \mathrm{H} & 4.483406 & -1.784436 & 0.000000 \\ \mathrm{H} & 0.667768 & -4.879172 & 0.000000 \\ \mathrm{H} & -1.609734 & -4.612530 & 0.000000 \\ \mathrm{H} & -3.917775 & -3.449502 & 0.000000 \\ \mathrm{H} & -4.747746 & -0.902395 & 0.000000 \\ \mathrm{H} & -4.483406 & 1.784436 & 0.000000 \\ \mathrm{H} & -3.213998 & 4.141802 & 0.000000 \\ \mathrm{H} & -0.667768 & 4.879172 & 0.000000 \\ \mathrm{H} & 1.609734 & 4.612530 & 0.000000 \\ \mathrm{H} & 3.917775 & 3.449502 & 0.000000 \\ \mathrm{H} & 4.747746 & 0.902395 & 0.000000\end{array}$

porphine $\mathrm{S}_{0}$

$\begin{array}{rrrr}\mathrm{N} & 0.000000 & -2.019344 & 0.000000 \\ \mathrm{~N} & 2.098453 & 0.000000 & 0.000000 \\ \mathrm{C} & 1.079234 & -2.835028 & 0.000000 \\ \mathrm{C} & 0.672351 & -4.230773 & 0.000000 \\ \mathrm{C} & 2.406382 & -2.424451 & 0.000000 \\ \mathrm{C} & 2.872650 & -1.122013 & 0.000000 \\ \mathrm{C} & 4.230767 & -0.680300 & 0.000000 \\ \mathrm{H} & 1.342627 & -5.076502 & 0.000000 \\ \mathrm{H} & 3.161591 & -3.199760 & 0.000000 \\ \mathrm{H} & 5.084732 & -1.338377 & 0.000000 \\ \mathrm{H} & 1.086278 & 0.000000 & 0.000000 \\ \mathrm{~N} & 0.000000 & 2.019344 & 0.000000 \\ \mathrm{~N} & -2.098453 & 0.000000 & 0.000000 \\ \mathrm{C} & 1.079234 & 2.835028 & 0.000000 \\ \mathrm{C} & -1.079234 & 2.835028 & 0.000000 \\ \mathrm{C} & -1.079234 & -2.835028 & 0.000000 \\ \mathrm{C} & 0.672351 & 4.230773 & 0.000000 \\ \mathrm{C} & -0.672351 & 4.230773 & 0.000000 \\ \mathrm{C} & -0.672351 & -4.230773 & 0.000000 \\ \mathrm{C} & 2.406382 & 2.424451 & 0.000000 \\ \mathrm{C} & -2.406382 & 2.424451 & 0.000000 \\ \mathrm{C} & -2.406382 & -2.424451 & 0.000000 \\ \mathrm{C} & 2.872650 & 1.122013 & 0.000000 \\ \mathrm{C} & -2.872650 & 1.122013 & 0.000000 \\ \mathrm{C} & -2.872650 & -1.122013 & 0.000000\end{array}$




$\begin{array}{rrrr}\mathrm{C} & 4.230767 & 0.680300 & 0.000000 \\ \mathrm{C} & -4.230767 & 0.680300 & 0.000000 \\ \mathrm{C} & -4.230767 & -0.680300 & 0.000000 \\ \mathrm{H} & 1.342627 & 5.076502 & 0.000000 \\ \mathrm{H} & -1.342627 & 5.076502 & 0.000000 \\ \mathrm{H} & -1.342627 & -5.076502 & 0.000000 \\ \mathrm{H} & 3.161591 & 3.199760 & 0.000000 \\ \mathrm{H} & -3.161591 & 3.199760 & 0.000000 \\ \mathrm{H} & -3.161591 & -3.199760 & 0.000000 \\ \mathrm{H} & 5.084732 & 1.338377 & 0.000000 \\ \mathrm{H} & -5.084732 & 1.338377 & 0.000000 \\ \mathrm{H} & -5.084732 & -1.338377 & 0.000000 \\ \mathrm{H} & -1.086278 & 0.000000 & 0.000000\end{array}$

porphine $\mathrm{S}_{1}$

$\begin{array}{rrrr}\mathrm{N} & 0.000000 & -2.038321 & 0.000000 \\ \mathrm{~N} & 2.096582 & 0.000000 & 0.000000 \\ \mathrm{C} & 1.082390 & -2.857640 & 0.000000 \\ \mathrm{C} & 0.673776 & -4.249774 & 0.000000 \\ \mathrm{C} & 2.411675 & -2.433131 & 0.000000 \\ \mathrm{C} & 2.874776 & -1.124934 & 0.000000 \\ \mathrm{C} & 4.232112 & -0.681987 & 0.000000 \\ \mathrm{H} & 1.342303 & -5.096634 & 0.000000 \\ \mathrm{H} & 3.171371 & -3.203976 & 0.000000 \\ \mathrm{H} & 5.086822 & -1.338950 & 0.000000 \\ \mathrm{H} & 1.086034 & 0.000000 & 0.000000 \\ \mathrm{~N} & 0.000000 & 2.038321 & 0.000000 \\ \mathrm{~N} & -2.096582 & 0.000000 & 0.000000 \\ \mathrm{C} & 1.082390 & 2.857640 & 0.000000 \\ \mathrm{C} & -1.082390 & 2.857640 & 0.000000 \\ \mathrm{C} & -1.082390 & -2.857640 & 0.000000 \\ \mathrm{C} & 0.673776 & 4.249774 & 0.000000 \\ \mathrm{C} & -0.673776 & 4.249774 & 0.000000 \\ \mathrm{C} & -0.673776 & -4.249774 & 0.000000 \\ \mathrm{C} & 2.411675 & 2.433131 & 0.000000 \\ \mathrm{C} & -2.411675 & 2.433131 & 0.000000 \\ \mathrm{C} & -2.411675 & -2.433131 & 0.000000 \\ \mathrm{C} & 2.874776 & 1.124934 & 0.000000 \\ \mathrm{C} & -2.874776 & 1.124934 & 0.000000 \\ \mathrm{C} & -2.874776 & -1.124934 & 0.000000 \\ \mathrm{C} & 4.232112 & 0.681987 & 0.000000 \\ \mathrm{C} & -4.232112 & 0.681987 & 0.000000 \\ \mathrm{C} & -4.232112 & -0.681987 & 0.000000 \\ \mathrm{H} & 1.342303 & 5.096634 & 0.000000 \\ \mathrm{H} & -1.342303 & 5.096634 & 0.000000\end{array}$




$\begin{array}{crcc}\mathrm{H} & -1.342303 & -5.096634 & 0.000000 \\ \mathrm{H} & 3.171371 & 3.203976 & 0.000000 \\ \mathrm{H} & -3.171371 & 3.203976 & 0.000000 \\ \mathrm{H} & -3.171371 & -3.203976 & 0.000000 \\ \mathrm{H} & 5.086822 & 1.338950 & 0.000000 \\ \mathrm{H} & -5.086822 & 1.338950 & 0.000000 \\ \mathrm{H} & -5.086822 & -1.338950 & 0.000000 \\ \mathrm{H} & -1.086034 & 0.000000 & 0.000000\end{array}$

chlorin $\mathrm{S}_{0}$

$\begin{array}{rrrr}\text { C } & 0.000000 & -4.224022 & 0.919736 \\ \mathrm{C} & 0.000000 & -2.872242 & 1.371718 \\ \mathrm{~N} & 0.000000 & -2.088595 & 0.240423 \\ \mathrm{C} & 0.000000 & -2.853798 & -0.876366 \\ \mathrm{C} & 0.000000 & -4.215322 & -0.441359 \\ \mathrm{C} & 0.000000 & -2.410082 & 2.665576 \\ \mathrm{C} & 0.000000 & -1.078578 & 3.089395 \\ \mathrm{~N} & 0.000000 & 0.000000 & 2.272230 \\ \mathrm{C} & 0.000000 & 1.078578 & 3.089395 \\ \mathrm{C} & 0.000000 & 0.676100 & 4.475087 \\ \mathrm{C} & 0.000000 & -0.676100 & 4.475087 \\ \mathrm{C} & 0.000000 & 2.410082 & 2.665576 \\ \mathrm{C} & 0.000000 & 2.872242 & 1.371718 \\ \mathrm{~N} & 0.000000 & 2.088595 & 0.240423 \\ \mathrm{C} & 0.000000 & 2.853798 & -0.876366 \\ \mathrm{C} & 0.000000 & 4.215322 & -0.441359 \\ \mathrm{C} & 0.000000 & 4.224022 & 0.919736 \\ \mathrm{C} & 0.000000 & 2.400686 & -2.195162 \\ \mathrm{C} & 0.000000 & 1.097881 & -2.641672 \\ \mathrm{C} & 0.000000 & 0.763950 & -4.122143 \\ \mathrm{C} & 0.000000 & -0.763950 & -4.122143 \\ \mathrm{C} & 0.000000 & -1.097881 & -2.641672 \\ \mathrm{~N} & 0.000000 & 0.000000 & -1.868151 \\ \mathrm{C} & 0.000000 & -2.400686 & -2.195162 \\ \mathrm{H} & -0.877728 & 1.186590 & -4.612119 \\ \mathrm{H} & 0.000000 & 3.176407 & -2.950166 \\ \mathrm{H} & 0.000000 & 5.064362 & -1.105512 \\ \mathrm{H} & 0.000000 & 1.344712 & 5.322220 \\ \mathrm{H} & 0.000000 & -1.344712 & 5.322220 \\ \mathrm{H} & -0.877728 & -1.186590 & -4.612119 \\ \mathrm{H} & 0.000000 & 3.167771 & 3.439245 \\ \mathrm{H} & 0.000000 & -3.167771 & 3.439245 \\ \mathrm{H} & 0.000000 & -3.176407 & -2.950166 \\ \mathrm{H} & 0.000000 & 5.083292 & 1.571215 \\ \mathrm{H} & 0.000000 & -5.083292 & 1.571215\end{array}$




$\begin{array}{rrrr}\mathrm{H} & 0.000000 & -5.064362 & -1.105512 \\ \mathrm{H} & 0.877728 & -1.186590 & -4.612119 \\ \mathrm{H} & 0.877728 & 1.186590 & -4.612119 \\ \mathrm{H} & 0.000000 & -1.078484 & 0.259958 \\ \mathrm{H} & 0.000000 & 1.078484 & 0.259958\end{array}$

chlorin $\mathrm{S}_{1}$

$\begin{array}{rrrr}\mathrm{C} & 0.000000 & -4.231223 & 0.920279 \\ \mathrm{C} & 0.000000 & -2.878436 & 1.368042 \\ \mathrm{~N} & 0.000000 & -2.095421 & 0.241319 \\ \mathrm{C} & 0.000000 & -2.868065 & -0.883550 \\ \mathrm{C} & 0.000000 & -4.224746 & -0.446445 \\ \mathrm{C} & 0.000000 & -2.412720 & 2.675777 \\ \mathrm{C} & 0.000000 & -1.082452 & 3.088975 \\ \mathrm{~N} & 0.000000 & 0.000000 & 2.270397 \\ \mathrm{C} & 0.000000 & 1.082452 & 3.088975 \\ \mathrm{C} & 0.000000 & 0.672116 & 4.485783 \\ \mathrm{C} & 0.000000 & -0.672116 & 4.485783 \\ \mathrm{C} & 0.000000 & 2.412720 & 2.675777 \\ \mathrm{C} & 0.000000 & 2.878436 & 1.368042 \\ \mathrm{~N} & 0.000000 & 2.095421 & 0.241319 \\ \mathrm{C} & 0.000000 & 2.868065 & -0.883550 \\ \mathrm{C} & 0.000000 & 4.224746 & -0.446445 \\ \mathrm{C} & 0.000000 & 4.231223 & 0.920279 \\ \mathrm{C} & 0.000000 & 2.409967 & -2.202678 \\ \mathrm{C} & 0.000000 & 1.099257 & -2.645850 \\ \mathrm{C} & 0.000000 & 0.763209 & -4.121688 \\ \mathrm{C} & 0.000000 & -0.763209 & -4.121688 \\ \mathrm{C} & 0.000000 & -1.099257 & -2.645850 \\ \mathrm{~N} & 0.000000 & 0.000000 & -1.864889 \\ \mathrm{C} & 0.000000 & -2.409967 & -2.202678 \\ \mathrm{H} & -0.877316 & 1.186532 & -4.613369 \\ \mathrm{H} & 0.000000 & 3.181430 & -2.961770 \\ \mathrm{H} & 0.000000 & 5.076126 & -1.107753 \\ \mathrm{H} & 0.000000 & 1.342288 & 5.331470 \\ \mathrm{H} & 0.000000 & -1.342288 & 5.331470 \\ \mathrm{H} & -0.877316 & -1.186532 & -4.613369 \\ \mathrm{H} & 0.000000 & 3.168635 & 3.449984 \\ \mathrm{H} & 0.000000 & -3.168635 & 3.449984 \\ \mathrm{H} & 0.000000 & -3.181430 & -2.961770 \\ \mathrm{H} & 0.000000 & 5.088813 & 1.573617 \\ \mathrm{H} & 0.000000 & -5.088813 & 1.573617 \\ \mathrm{H} & 0.000000 & -5.076126 & -1.107753 \\ \mathrm{H} & 0.877316 & -1.186532 & -4.613369 \\ \mathrm{H} & 0.877316 & 1.186532 & -4.613369\end{array}$




$$
\begin{array}{rrrr}
H & 0.000000 & -1.085675 & 0.258483 \\
H & 0.000000 & 1.085675 & 0.258483
\end{array}
$$

Zn-tetraphenylporphine $\mathrm{S}_{0}$

$\begin{array}{lrrr}\mathrm{C} & -3.781829 & 4.191866 & 1.161199 \\ \mathrm{C} & -3.485735 & 3.485722 & 0.000021 \\ \mathrm{C} & -4.191893 & 3.781803 & -1.161152 \\ \mathrm{C} & -5.171242 & 4.762205 & -1.162367 \\ \mathrm{C} & -5.459463 & 5.459414 & 0.000028 \\ \mathrm{C} & -4.762247 & 5.171199 & 1.162419 \\ \mathrm{C} & -2.428862 & 2.428860 & 0.000018 \\ \mathrm{C} & -1.098186 & 2.849287 & -0.005399 \\ \mathrm{~N} & 0.000000 & 2.038877 & 0.019262 \\ \mathrm{C} & 1.098186 & 2.849287 & -0.005399 \\ \mathrm{C} & 0.674913 & 4.224611 & -0.056007 \\ \mathrm{C} & -0.674913 & 4.224611 & -0.056007 \\ \mathrm{C} & 2.428862 & 2.428860 & 0.000018 \\ \mathrm{C} & 3.485735 & 3.485722 & 0.000021 \\ \mathrm{C} & 4.191893 & 3.781803 & -1.161152 \\ \mathrm{C} & 5.171242 & 4.762205 & -1.162367 \\ \mathrm{C} & 5.459463 & 5.459414 & 0.000028 \\ \mathrm{C} & 4.762247 & 5.171199 & 1.162419 \\ \mathrm{C} & 3.781829 & 4.191866 & 1.161199 \\ \mathrm{C} & -2.849286 & 1.098184 & 0.005405 \\ \mathrm{~N} & -2.038877 & 0.000000 & -0.019341 \\ \mathrm{C} & -2.849286 & -1.098184 & 0.005405 \\ \mathrm{C} & -4.224612 & -0.674913 & 0.055969 \\ \mathrm{C} & -4.224612 & 0.674913 & 0.055969 \\ \mathrm{C} & -2.428862 & -2.428860 & 0.000018 \\ \mathrm{C} & -3.485735 & -3.485722 & 0.000021 \\ \mathrm{C} & -4.191893 & -3.781803 & -1.161152 \\ \mathrm{C} & -5.171242 & -4.762205 & -1.162367 \\ \mathrm{C} & -5.459463 & -5.459414 & 0.000028 \\ \mathrm{C} & -4.762247 & -5.171199 & 1.162419 \\ \mathrm{C} & -3.781829 & -4.191866 & 1.161199 \\ \mathrm{C} & 2.849286 & 1.098184 & 0.005405 \\ \mathrm{~N} & 2.038877 & 0.000000 & -0.019341 \\ \mathrm{C} & 2.849286 & -1.098184 & 0.005405 \\ \mathrm{C} & 4.224612 & -0.674913 & 0.055969 \\ \mathrm{C} & 4.224612 & 0.674913 & 0.055969 \\ \mathrm{C} & 2.428862 & -2.428860 & 0.000018 \\ \mathrm{C} & 3.485735 & -3.485722 & 0.000021 \\ \mathrm{C} & 4.191893 & -3.781803 & -1.161152 \\ & 5.459463 & -5.459414 & 0.000028\end{array}$




$\begin{array}{rrrr}\mathrm{C} & 4.762247 & -5.171199 & 1.162419 \\ \mathrm{C} & 3.781829 & -4.191866 & 1.161199 \\ \mathrm{C} & 1.098186 & -2.849287 & -0.005399 \\ \mathrm{~N} & 0.000000 & -2.038877 & 0.019262 \\ \mathrm{C} & -1.098186 & -2.849287 & -0.005399 \\ \mathrm{C} & -0.674913 & -4.224611 & -0.056007 \\ \mathrm{C} & 0.674913 & -4.224611 & -0.056007 \\ \mathrm{H} & 1.333648 & -5.075397 & -0.097286 \\ \mathrm{H} & 5.075398 & -1.333649 & 0.097240 \\ \mathrm{H} & 1.333648 & 5.075397 & -0.097286 \\ \mathrm{H} & -1.333648 & 5.075397 & -0.097286 \\ \mathrm{H} & -1.333648 & -5.075397 & -0.097286 \\ \mathrm{H} & 5.075398 & 1.333649 & 0.097240 \\ \mathrm{H} & -5.075398 & 1.333649 & 0.097240 \\ \mathrm{H} & -5.075398 & -1.333649 & 0.097240 \\ \mathrm{H} & 3.966672 & 3.239101 & -2.070609 \\ \mathrm{H} & 5.709145 & 4.983043 & -2.075622 \\ \mathrm{H} & 6.224902 & 6.224840 & 0.000030 \\ \mathrm{H} & 4.983093 & 5.709093 & 2.075678 \\ \mathrm{H} & 3.239123 & 3.966650 & 2.070654 \\ \mathrm{H} & 3.966672 & -3.239101 & -2.070609 \\ \mathrm{H} & 5.709145 & -4.983043 & -2.075622 \\ \mathrm{H} & 6.224902 & -6.224840 & 0.000030 \\ \mathrm{H} & 4.983093 & -5.709093 & 2.075678 \\ \mathrm{H} & 3.239123 & -3.966650 & 2.070654 \\ \mathrm{H} & -3.966672 & -3.239101 & -2.070609 \\ \mathrm{H} & -5.709145 & -4.983043 & -2.075622 \\ \mathrm{H} & -6.224902 & -6.224840 & 0.000030 \\ \mathrm{H} & -4.983093 & -5.709093 & 2.075678 \\ \mathrm{H} & -3.239123 & -3.966650 & 2.070654 \\ \mathrm{H} & -3.966672 & 3.239101 & -2.070609 \\ \mathrm{H} & -5.709145 & 4.983043 & -2.075622 \\ \mathrm{H} & -6.224902 & 6.224840 & 0.000030 \\ \mathrm{H} & -4.983093 & 5.709093 & 2.075678 \\ \mathrm{H} & -3.239123 & 3.966650 & 2.070654 \\ \mathrm{Zn} & 0.000000 & 0.000000 & -0.000080 \\ & & & \end{array}$

Zn-tetraphenylporphine $\mathrm{S}_{1}$

$\begin{array}{lrrr}\text { C } & -3.660136 & 4.324011 & 1.102030 \\ \mathrm{C} & -3.488384 & 3.490809 & -0.000070 \\ \mathrm{C} & -4.320261 & 3.664632 & -1.102996 \\ \mathrm{C} & -5.295231 & 4.648601 & -1.106131 \\ \mathrm{C} & -5.458659 & 5.470793 & -0.002423 \\ \mathrm{C} & -4.639010 & 5.304331 & 1.102514 \\ \mathrm{C} & -2.438491 & 2.434906 & 0.000547\end{array}$




$\begin{array}{rrrr}\mathrm{C} & -1.101094 & 2.849967 & -0.011951 \\ \mathrm{~N} & 0.000000 & 2.036096 & 0.051257 \\ \mathrm{C} & 1.101094 & 2.849967 & -0.011951 \\ \mathrm{C} & 0.676238 & 4.218954 & -0.127356 \\ \mathrm{C} & -0.676238 & 4.218954 & -0.127356 \\ \mathrm{C} & 2.438491 & 2.434906 & 0.000547 \\ \mathrm{C} & 3.488384 & 3.490809 & -0.000070 \\ \mathrm{C} & 4.320261 & 3.664632 & -1.102996 \\ \mathrm{C} & 5.295231 & 4.648601 & -1.106131 \\ \mathrm{C} & 5.458659 & 5.470793 & -0.002423 \\ \mathrm{C} & 4.639010 & 5.304331 & 1.102514 \\ \mathrm{C} & 3.660136 & 4.324011 & 1.102030 \\ \mathrm{C} & -2.868834 & 1.098492 & 0.014618 \\ \mathrm{~N} & -2.057658 & 0.000000 & -0.049373 \\ \mathrm{C} & -2.868834 & -1.098492 & 0.014618 \\ \mathrm{C} & -4.237785 & -0.676872 & 0.129700 \\ \mathrm{C} & -4.237785 & 0.676872 & 0.129700 \\ \mathrm{C} & -2.438491 & -2.434906 & 0.000547 \\ \mathrm{C} & -3.488384 & -3.490809 & -0.000070 \\ \mathrm{C} & -4.320261 & -3.664632 & -1.102996 \\ \mathrm{C} & -5.295231 & -4.648601 & -1.106131 \\ \mathrm{C} & -5.458659 & -5.470793 & -0.002423 \\ \mathrm{C} & -4.639010 & -5.304331 & 1.102514 \\ \mathrm{C} & -3.660136 & -4.324011 & 1.102030 \\ \mathrm{C} & 2.868834 & 1.098492 & 0.014618 \\ \mathrm{~N} & 2.057658 & 0.000000 & -0.049373 \\ \mathrm{C} & 2.868834 & -1.098492 & 0.014618 \\ \mathrm{C} & 4.237785 & -0.676872 & 0.129700 \\ \mathrm{C} & 4.237785 & 0.676872 & 0.129700 \\ \mathrm{C} & 2.438491 & -2.434906 & 0.000547 \\ \mathrm{C} & 3.488384 & -3.490809 & -0.000070 \\ \mathrm{C} & 4.320261 & -3.664632 & -1.102996 \\ \mathrm{C} & 5.295231 & -4.648601 & -1.106131 \\ \mathrm{C} & 5.458659 & -5.470793 & -0.002423 \\ \mathrm{C} & 4.639010 & -5.304331 & 1.102514 \\ \mathrm{C} & 3.660136 & -4.324011 & 1.102030 \\ \mathrm{C} & 1.101094 & -2.849967 & -0.011951 \\ \mathrm{~N} & 0.000000 & -2.036096 & 0.051257 \\ \mathrm{C} & -1.101094 & -2.849967 & -0.011951 \\ \mathrm{C} & -0.676238 & -4.218954 & -0.127356 \\ \mathrm{C} & 0.676238 & -4.218954 & -0.127356 \\ \mathrm{H} & 1.332297 & -5.067337 & -0.219545 \\ \mathrm{H} & 5.085239 & -1.334140 & 0.220808 \\ \mathrm{H} & 1.332297 & 5.067337 & -0.219545 \\ \mathrm{H} & -1.332297 & 5.067337 & -0.219545 \\ \mathrm{H} & -1.332297 & -5.067337 & -0.219545\end{array}$




$\begin{array}{crrr}\mathrm{H} & 5.085239 & 1.334140 & 0.220808 \\ \mathrm{H} & -5.085239 & 1.334140 & 0.220808 \\ \mathrm{H} & -5.085239 & -1.334140 & 0.220808 \\ \mathrm{H} & 4.192725 & 3.025630 & -1.967566 \\ \mathrm{H} & 5.927750 & 4.774807 & -1.975676 \\ \mathrm{H} & 6.222005 & 6.238268 & -0.003450 \\ \mathrm{H} & 4.762953 & 5.938681 & 1.971051 \\ \mathrm{H} & 3.022871 & 4.194048 & 1.967475 \\ \mathrm{H} & 4.192725 & -3.025630 & -1.967566 \\ \mathrm{H} & 5.927750 & -4.774807 & -1.975676 \\ \mathrm{H} & 6.222005 & -6.238268 & -0.003450 \\ \mathrm{H} & 4.762953 & -5.938681 & 1.971051 \\ \mathrm{H} & 3.022871 & -4.194048 & 1.967475 \\ \mathrm{H} & -4.192725 & -3.025630 & -1.967566 \\ \mathrm{H} & -5.927750 & -4.774807 & -1.975676 \\ \mathrm{H} & -6.222005 & -6.238268 & -0.003450 \\ \mathrm{H} & -4.762953 & -5.938681 & 1.971051 \\ \mathrm{H} & -3.022871 & -4.194048 & 1.967475 \\ \mathrm{H} & -4.192725 & 3.025630 & -1.967566 \\ \mathrm{H} & -5.927750 & 4.774807 & -1.975676 \\ \mathrm{H} & -6.222005 & 6.238268 & -0.003450 \\ \mathrm{H} & -4.762953 & 5.938681 & 1.971051 \\ \mathrm{H} & -3.022871 & 4.194048 & 1.967475 \\ \mathrm{Zn} & 0.000000 & 0.000000 & 0.001256\end{array}$

tetraphenylporphine $\mathrm{S}_{0}$

$\begin{array}{lrrc}\mathrm{C} & -0.679158 & 4.221493 & 0.063330 \\ \mathrm{C} & -1.123724 & 2.865922 & 0.006731 \\ \mathrm{~N} & 0.000001 & 2.088925 & -0.022180 \\ \mathrm{C} & 1.123724 & 2.865924 & 0.006761 \\ \mathrm{C} & 0.679155 & 4.221494 & 0.063351 \\ \mathrm{C} & 2.442638 & 2.421401 & -0.006017 \\ \mathrm{C} & 2.842506 & 1.081499 & -0.022606 \\ \mathrm{~N} & 2.025494 & 0.000001 & 0.007579 \\ \mathrm{C} & 2.842506 & -1.081497 & -0.022634 \\ \mathrm{C} & 4.235793 & -0.671254 & -0.089221 \\ \mathrm{C} & 4.235793 & 0.671257 & -0.089202 \\ \mathrm{C} & 2.442638 & -2.421399 & -0.006067 \\ \mathrm{C} & 1.123724 & -2.865922 & 0.006731 \\ \mathrm{~N} & -0.000001 & -2.088925 & -0.022180 \\ \mathrm{C} & -1.123724 & -2.865924 & 0.006761 \\ \mathrm{C} & -0.679155 & -4.221494 & 0.063351 \\ \mathrm{C} & 0.679158 & -4.221493 & 0.063330 \\ \mathrm{C} & -2.442638 & -2.421401 & -0.006017 \\ \mathrm{C} & -2.842506 & -1.081499 & -0.022606\end{array}$




$\begin{array}{rrrr}\mathrm{N} & -2.025494 & -0.000001 & 0.007579 \\ \mathrm{C} & -2.842506 & 1.081497 & -0.022634 \\ \mathrm{C} & -4.235793 & 0.671254 & -0.089221 \\ \mathrm{C} & -4.235793 & -0.671257 & -0.089202 \\ \mathrm{C} & -2.442638 & 2.421399 & -0.006067 \\ \mathrm{H} & -5.083260 & -1.333740 & -0.141064 \\ \mathrm{C} & -3.503002 & -3.474741 & 0.001163 \\ \mathrm{H} & -1.332960 & -5.075367 & 0.106923 \\ \mathrm{H} & -0.000002 & -1.077376 & -0.033019 \\ \mathrm{H} & 5.083260 & -1.333735 & -0.141103 \\ \mathrm{H} & 5.083260 & 1.333740 & -0.141064 \\ \mathrm{H} & -5.083260 & 1.333735 & -0.141103 \\ \mathrm{C} & 3.503001 & -3.474741 & 0.001068 \\ \mathrm{C} & 3.503002 & 3.474741 & 0.001163 \\ \mathrm{C} & -3.503001 & 3.474741 & 0.001068 \\ \mathrm{H} & 1.332966 & -5.075365 & 0.106882 \\ \mathrm{H} & 1.332960 & 5.075367 & 0.106923 \\ \mathrm{H} & -1.332966 & 5.075365 & 0.106882 \\ \mathrm{H} & 0.000002 & 1.077376 & -0.033019 \\ \mathrm{C} & 3.791463 & -4.198853 & -1.150977 \\ \mathrm{C} & 4.775072 & -5.175038 & -1.144340 \\ \mathrm{C} & 5.482351 & -5.442248 & 0.016949 \\ \mathrm{C} & 5.201179 & -4.727379 & 1.170377 \\ \mathrm{C} & 4.218739 & -3.750226 & 1.161532 \\ \mathrm{H} & 3.242348 & -3.988436 & -2.060165 \\ \mathrm{H} & 4.991010 & -5.726219 & -2.050812 \\ \mathrm{H} & 6.250593 & -6.204832 & 0.022965 \\ \mathrm{H} & 5.747434 & -4.931498 & 2.082539 \\ \mathrm{H} & 3.999590 & -3.192723 & 2.063434 \\ \mathrm{C} & -3.791486 & -4.198885 & -1.150857 \\ \mathrm{C} & -4.775098 & -5.175067 & -1.144175 \\ \mathrm{C} & -5.482358 & -5.442242 & 0.017133 \\ \mathrm{C} & -5.201164 & -4.727341 & 1.170536 \\ \mathrm{C} & -4.218722 & -3.750191 & 1.161647 \\ \mathrm{H} & -3.242387 & -3.988495 & -2.060061 \\ \mathrm{H} & -4.991053 & -5.726274 & -2.050628 \\ \mathrm{H} & -6.250602 & -6.204824 & 0.023184 \\ \mathrm{H} & -5.747404 & -4.931433 & 2.082713 \\ \mathrm{H} & -3.999556 & -3.192663 & 2.063528 \\ \mathrm{C} & -3.791463 & 4.198853 & -1.150977 \\ \mathrm{C} & -4.775072 & 5.175038 & -1.144340 \\ \mathrm{C} & -5.482351 & 5.442248 & 0.016949 \\ \mathrm{C} & -5.201179 & 4.727379 & 1.170377 \\ \mathrm{C} & -4.218739 & 3.750226 & 1.161532 \\ \mathrm{H} & -3.242348 & 3.988436 & -2.060165 \\ \mathrm{H} & -4.991010 & 5.726219 & -2.050812\end{array}$




$\begin{array}{lrrr}\mathrm{H} & -6.250593 & 6.204832 & 0.022965 \\ \mathrm{H} & -5.747434 & 4.931498 & 2.082539 \\ \mathrm{H} & -3.999590 & 3.192723 & 2.063434 \\ \mathrm{C} & 3.791486 & 4.198885 & -1.150857 \\ \mathrm{C} & 4.775098 & 5.175067 & -1.144175 \\ \mathrm{C} & 5.482358 & 5.442242 & 0.017133 \\ \mathrm{C} & 5.201164 & 4.727341 & 1.170536 \\ \mathrm{C} & 4.218722 & 3.750191 & 1.161647 \\ \mathrm{H} & 3.242387 & 3.988495 & -2.060061 \\ \mathrm{H} & 4.991053 & 5.726274 & -2.050628 \\ \mathrm{H} & 6.250602 & 6.204824 & 0.023184 \\ \mathrm{H} & 5.747404 & 4.931433 & 2.082713 \\ \mathrm{H} & 3.999556 & 3.192663 & 2.063528\end{array}$

tetraphenylporphine $\mathrm{S}_{1}$

$\begin{array}{lrrr}\mathrm{C} & 4.211672 & 0.744179 & 0.127178 \\ \mathrm{C} & 2.853628 & 1.170941 & 0.016072 \\ \mathrm{~N} & 2.091756 & 0.032144 & -0.045475 \\ \mathrm{C} & 2.887556 & -1.083381 & 0.011999 \\ \mathrm{C} & 4.232155 & -0.616373 & 0.124435 \\ \mathrm{C} & 2.466323 & -2.413868 & -0.017505 \\ \mathrm{C} & 1.127698 & -2.848806 & -0.055584 \\ \mathrm{~N} & 0.030977 & -2.046743 & 0.005809 \\ \mathrm{C} & -1.040912 & -2.881916 & -0.051669 \\ \mathrm{C} & -0.609508 & -4.258300 & -0.176654 \\ \mathrm{C} & 0.738025 & -4.237719 & -0.179320 \\ \mathrm{C} & -2.392168 & -2.488040 & -0.010232 \\ \mathrm{C} & -2.853628 & -1.170941 & 0.016072 \\ \mathrm{~N} & -2.091756 & -0.032144 & -0.045475 \\ \mathrm{C} & -2.887556 & 1.083381 & 0.011999 \\ \mathrm{C} & -4.232155 & 0.616373 & 0.124435 \\ \mathrm{C} & -4.211672 & -0.744179 & 0.127178 \\ \mathrm{C} & -2.466323 & 2.413868 & -0.017505 \\ \mathrm{C} & -1.127698 & 2.848806 & -0.055584 \\ \mathrm{~N} & -0.030977 & 2.046743 & 0.005809 \\ \mathrm{C} & 1.040912 & 2.881916 & -0.051669 \\ \mathrm{C} & 0.609508 & 4.258300 & -0.176654 \\ \mathrm{C} & -0.738025 & 4.237719 & -0.179320 \\ \mathrm{C} & 2.392168 & 2.488040 & -0.010232 \\ \mathrm{H} & -1.410364 & 5.073285 & -0.273086 \\ \mathrm{C} & -3.534126 & 3.452797 & -0.005160 \\ \mathrm{H} & -5.094488 & 1.254564 & 0.207512 \\ \mathrm{H} & -1.082026 & -0.017198 & -0.069035 \\ \mathrm{H} & -1.256477 & -5.113936 & -0.267921 \\ \mathrm{H} & 1.410364 & -5.073285 & -0.273086\end{array}$




$\begin{array}{cccc}\mathrm{H} & 1.256477 & 5.113936 & -0.267921 \\ \mathrm{C} & -3.427199 & -3.559443 & 0.009541 \\ \mathrm{C} & 3.534126 & -3.452797 & -0.005160 \\ \mathrm{C} & 3.427199 & 3.559443 & 0.009541 \\ \mathrm{H} & -5.054536 & -1.407534 & 0.212772 \\ \mathrm{H} & 5.094488 & -1.254564 & 0.207512 \\ \mathrm{H} & 5.054536 & 1.407534 & 0.212772 \\ \mathrm{H} & 1.082026 & 0.017198 & -0.069035 \\ \mathrm{C} & -4.266741 & -3.760097 & -1.082356 \\ \mathrm{C} & -5.226721 & -4.759097 & -1.062021 \\ \mathrm{C} & -5.365065 & -5.569061 & 0.053649 \\ \mathrm{C} & -4.535499 & -5.376426 & 1.147272 \\ \mathrm{C} & -3.572132 & -4.381589 & 1.123637 \\ \mathrm{H} & -4.157029 & -3.131723 & -1.957142 \\ \mathrm{H} & -5.866391 & -4.906462 & -1.922944 \\ \mathrm{H} & -6.116088 & -6.348413 & 0.070753 \\ \mathrm{H} & -4.639328 & -6.002337 & 2.024491 \\ \mathrm{H} & -2.925319 & -4.232310 & 1.978799 \\ \mathrm{C} & -4.374005 & 3.624058 & -1.101773 \\ \mathrm{C} & -5.365065 & 4.592331 & -1.089092 \\ \mathrm{C} & -5.534597 & 5.400377 & 0.023672 \\ \mathrm{C} & -4.704989 & 5.236786 & 1.121975 \\ \mathrm{C} & -3.710606 & 4.272748 & 1.105986 \\ \mathrm{H} & -4.240257 & 2.996927 & -1.974099 \\ \mathrm{H} & -6.004716 & 4.717126 & -1.953588 \\ \mathrm{H} & -6.309972 & 6.155613 & 0.034949 \\ \mathrm{H} & -4.833071 & 5.861299 & 1.996981 \\ \mathrm{H} & -3.063980 & 4.146001 & 1.964924 \\ \mathrm{C} & 4.266741 & 3.760097 & -1.082356 \\ \mathrm{C} & 5.226721 & 4.759097 & -1.062021 \\ \mathrm{C} & 5.365065 & 5.569061 & 0.053649 \\ \mathrm{C} & 4.535499 & 5.376426 & 1.147272 \\ \mathrm{C} & 3.572132 & 4.381589 & 1.123637 \\ \mathrm{H} & 4.157029 & 3.131723 & -1.957142 \\ \mathrm{H} & 5.866391 & 4.906462 & -1.922944 \\ \mathrm{H} & 6.116088 & 6.348413 & 0.070753 \\ \mathrm{H} & 4.639328 & 6.002337 & 2.024491 \\ \mathrm{C} & 2.925319 & 4.232310 & 1.978799 \\ \mathrm{C} & 5.365065 & -4.592331 & -1.089092 \\ \mathrm{H} & 4.704989 & -5.236786 & 1.121975 \\ \mathrm{H} & -3.272748 & 1.105986 \\ \mathrm{H} & -2.996927 & -1.974099 \\ \mathrm{H} & -6.155613 & 0.034949\end{array}$




$\begin{array}{llll}\mathrm{H} & 4.833071 & -5.861299 & 1.996981 \\ \mathrm{H} & 3.063980 & -4.146001 & 1.964924\end{array}$

CAM-B3LYP/TZVP geometries - Group II

tetrafluorobenzene $\mathrm{S}_{0}$

$\begin{array}{lrrr}\text { C } & -1.186683 & 0.691323 & 0.000000 \\ \text { C } & -1.186683 & -0.691323 & 0.000000 \\ \text { C } & 0.000000 & -1.393072 & 0.000000 \\ \text { C } & 1.186683 & -0.691323 & 0.000000 \\ \text { C } & 1.186683 & 0.691323 & 0.000000 \\ \text { C } & 0.000000 & 1.393072 & 0.000000 \\ \text { F } & -2.350141 & -1.347609 & 0.000000 \\ \text { H } & 0.000000 & -2.473768 & 0.000000 \\ \text { F } & 2.350141 & -1.347609 & 0.000000 \\ \text { F } & 2.350141 & 1.347609 & 0.000000 \\ \text { H } & 0.000000 & 2.473768 & 0.000000 \\ \text { F } & -2.350141 & 1.347609 & 0.000000\end{array}$

tetrafluorobenzene $\mathrm{S}_{1}$

$\begin{array}{lrrr}\text { C } & 1.161874 & -0.705860 & 0.107230 \\ \text { C } & 1.161874 & 0.705860 & 0.107230 \\ \text { C } & 0.000000 & 1.461433 & 0.305642 \\ \text { C } & -1.161874 & 0.705860 & 0.107230 \\ \text { C } & -1.161874 & -0.705860 & 0.107230 \\ \text { C } & 0.000000 & -1.461433 & 0.305642 \\ \text { F } & 2.305639 & 1.315120 & -0.201809 \\ \text { H } & 0.000000 & 2.505500 & 0.581639 \\ \text { F } & -2.305639 & 1.315120 & -0.201809 \\ \text { F } & -2.305639 & -1.315120 & -0.201809 \\ \text { H } & 0.000000 & -2.505500 & 0.581639 \\ \text { F } & 2.305639 & -1.315120 & -0.201809\end{array}$

benzonitrile $\mathrm{S}_{0}$

$\begin{array}{rrrr}\mathrm{C} & 0.000000 & 0.000000 & -1.052066 \\ \mathrm{C} & -1.207438 & 0.000000 & -0.357767 \\ \mathrm{C} & -1.201751 & 0.000000 & 1.024940 \\ \mathrm{C} & 0.000000 & 0.000000 & 1.716328 \\ \mathrm{C} & 1.201751 & 0.000000 & 1.024940 \\ \mathrm{C} & 1.207438 & 0.000000 & -0.357767 \\ \mathrm{H} & -2.139765 & 0.000000 & -0.905875 \\ \mathrm{H} & -2.139223 & 0.000000 & 1.565028\end{array}$




$\begin{array}{lrrr}\mathrm{H} & 0.000000 & 0.000000 & 2.798559 \\ \mathrm{H} & 2.139223 & 0.000000 & 1.565028 \\ \mathrm{H} & 2.139765 & 0.000000 & -0.905875 \\ \mathrm{C} & 0.000000 & 0.000000 & -2.484211 \\ \mathrm{~N} & 0.000000 & 0.000000 & -3.631253\end{array}$

benzonitrile $S_{1}$

$\begin{array}{lrrr}\mathrm{C} & 0.000000 & 0.000000 & -1.102010 \\ \mathrm{C} & -1.222482 & 0.000000 & -0.372116 \\ \mathrm{C} & -1.212237 & 0.000000 & 1.043259 \\ \mathrm{C} & 0.000000 & 0.000000 & 1.760446 \\ \mathrm{C} & 1.212237 & 0.000000 & 1.043259 \\ \mathrm{C} & 1.222482 & 0.000000 & -0.372116 \\ \mathrm{H} & -2.160935 & 0.000000 & -0.907206 \\ \mathrm{H} & -2.155430 & 0.000000 & 1.571136 \\ \mathrm{H} & 0.000000 & 0.000000 & 2.839835 \\ \mathrm{H} & 2.155430 & 0.000000 & 1.571136 \\ \mathrm{H} & 2.160935 & 0.000000 & -0.907206 \\ \mathrm{C} & 0.000000 & 0.000000 & -2.507017 \\ \mathrm{~N} & 0.000000 & 0.000000 & -3.661400\end{array}$

$o$-fluorophenol, cis $\mathrm{S}_{0}$

$\begin{array}{lrrc}\mathrm{C} & 0.325657 & 1.448449 & 0.000000 \\ \mathrm{C} & -0.869174 & 0.771496 & 0.000000 \\ \mathrm{C} & -0.945298 & -0.612362 & 0.000000 \\ \mathrm{C} & 0.237143 & -1.333229 & 0.000000 \\ \mathrm{C} & 1.452925 & -0.669010 & 0.000000 \\ \mathrm{C} & 1.503981 & 0.716555 & 0.000000 \\ \mathrm{~F} & -2.045263 & 1.450873 & 0.000000 \\ \mathrm{O} & -2.136514 & -1.261125 & 0.000000 \\ \mathrm{H} & 0.182453 & -2.413452 & 0.000000 \\ \mathrm{H} & 2.370002 & -1.242943 & 0.000000 \\ \mathrm{H} & 2.455436 & 1.230132 & 0.000000 \\ \mathrm{H} & 0.321513 & 2.529968 & 0.000000 \\ \mathrm{H} & -2.852852 & -0.615372 & 0.000000\end{array}$

$o$-fluorophenol, cis $\mathrm{S}_{1}$

$\begin{array}{lrrr}\mathrm{C} & 1.236481 & -0.232152 & -0.738760 \\ \mathrm{C} & 0.008256 & -0.082187 & -0.089730 \\ \mathrm{C} & -0.005818 & -0.031112 & 1.347279 \\ \mathrm{C} & 1.174634 & 0.029406 & 2.090682 \\ \mathrm{C} & 2.388866 & 0.103481 & 1.411219 \\ \mathrm{C} & 2.403585 & -0.012111 & 0.013324\end{array}$




$\begin{array}{crcc}\mathrm{F} & -0.978965 & 0.738551 & -0.685362 \\ \mathrm{O} & -1.172339 & -0.097660 & 1.984180 \\ \mathrm{H} & 1.124950 & -0.115848 & 3.162694 \\ \mathrm{H} & 3.316569 & 0.203679 & 1.955552 \\ \mathrm{H} & 3.346873 & 0.090754 & -0.511835 \\ \mathrm{H} & 1.286452 & -0.366883 & -1.810260 \\ \mathrm{H} & -1.874165 & -0.227918 & 1.320956\end{array}$

o-fluorophenol, trans $\mathrm{S}_{0}$

$\begin{array}{cccc}\mathrm{C} & 0.289912 & 1.257003 & 0.000000 \\ \mathrm{C} & -0.942206 & 0.614352 & 0.000000 \\ \mathrm{C} & -0.958505 & -0.771617 & 0.000000 \\ \mathrm{C} & 0.227356 & -1.491188 & 0.000000 \\ \mathrm{C} & 1.443690 & -0.832382 & 0.000000 \\ \mathrm{C} & 1.471877 & 0.555294 & 0.000000 \\ \mathrm{O} & -2.058713 & 1.386554 & 0.000000 \\ \mathrm{H} & -1.911198 & -1.289514 & 0.000000 \\ \mathrm{H} & 0.192912 & -2.572410 & 0.000000 \\ \mathrm{H} & 2.370210 & -1.389768 & 0.000000 \\ \mathrm{H} & 2.402614 & 1.106400 & 0.000000 \\ \mathrm{~F} & 0.313786 & 2.599728 & 0.000000 \\ \mathrm{H} & -2.841745 & 0.827548 & 0.000000\end{array}$

$o$-fluorophenol, trans $\mathrm{S}_{1}$

$\begin{array}{lrrc}\mathrm{C} & 0.817781 & 1.031378 & 0.102194 \\ \mathrm{C} & -0.585987 & 0.970015 & 0.084215 \\ \mathrm{C} & -1.270300 & -0.287388 & 0.099697 \\ \mathrm{C} & -0.469443 & -1.422165 & -0.074348 \\ \mathrm{C} & 0.938629 & -1.334720 & -0.065852 \\ \mathrm{C} & 1.616324 & -0.126383 & 0.164828 \\ \mathrm{O} & -1.250980 & 2.113356 & -0.101746 \\ \mathrm{H} & -2.296888 & -0.352565 & 0.440903 \\ \mathrm{H} & -0.928150 & -2.396054 & -0.185546 \\ \mathrm{H} & 1.511945 & -2.241286 & -0.218410 \\ \mathrm{H} & 2.685429 & -0.061767 & 0.289269 \\ \mathrm{~F} & 1.394509 & 2.208957 & -0.172578 \\ \mathrm{H} & -2.162897 & 1.898622 & -0.362625\end{array}$

m-fluorophenol, cis $\mathrm{S}_{0}$
$\begin{array}{llll}\mathrm{C} & 0.308844 & 1.403467 & 0.000000\end{array}$
$\begin{array}{llll}\text { C } & -0.924405 & 0.785082 & 0.000000\end{array}$
$\begin{array}{llll}\text { C } & -0.962838 & -0.602140 & 0.000000\end{array}$
$\begin{array}{llll}\text { C } & 0.214703 & -1.338456 & 0.000000\end{array}$ 


$\begin{array}{cccc}\mathrm{C} & 1.429125 & -0.678568 & 0.000000 \\ \mathrm{C} & 1.496432 & 0.707980 & 0.000000 \\ \mathrm{O} & -2.134176 & -1.293843 & 0.000000 \\ \mathrm{~F} & 0.339697 & 2.750416 & 0.000000 \\ \mathrm{H} & 0.157419 & -2.417776 & 0.000000 \\ \mathrm{H} & 2.345546 & -1.253959 & 0.000000 \\ \mathrm{H} & 2.436526 & 1.240258 & 0.000000 \\ \mathrm{H} & -1.825613 & 1.385161 & 0.000000 \\ \mathrm{H} & -2.881260 & -0.687622 & 0.000000\end{array}$

$m$-fluorophenol, cis $\mathrm{S}_{1}$

$\begin{array}{cccc}\mathrm{C} & 1.225767 & -0.039253 & -0.684813 \\ \mathrm{C} & -0.044543 & 0.024216 & -0.099103 \\ \mathrm{C} & -0.021003 & 0.030141 & 1.323643 \\ \mathrm{C} & 1.164470 & 0.019534 & 2.083588 \\ \mathrm{C} & 2.424304 & 0.100658 & 1.440104 \\ \mathrm{C} & 2.429754 & -0.032525 & 0.034013 \\ \mathrm{O} & -1.172818 & -0.015334 & 2.009285 \\ \mathrm{~F} & 1.300013 & -0.118905 & -2.023244 \\ \mathrm{H} & 1.075616 & -0.097726 & 3.154793 \\ \mathrm{H} & 3.343116 & 0.131291 & 2.001472 \\ \mathrm{H} & 3.353417 & -0.120815 & -0.522486 \\ \mathrm{H} & -0.918170 & 0.310569 & -0.668792 \\ \mathrm{H} & -1.904563 & -0.191851 & 1.401490\end{array}$

$m$-fluorophenol, trans $\mathrm{S}_{0}$

$\begin{array}{cccc}\mathrm{C} & 0.268354 & 1.299035 & 0.000000 \\ \mathrm{C} & -0.944971 & 0.625945 & 0.000000 \\ \mathrm{C} & -0.980164 & -0.762444 & 0.000000 \\ \mathrm{C} & 0.207577 & -1.475988 & 0.000000 \\ \mathrm{C} & 1.430847 & -0.827883 & 0.000000 \\ \mathrm{C} & 1.423860 & 0.552399 & 0.000000 \\ \mathrm{O} & -2.072182 & 1.387400 & 0.000000 \\ \mathrm{H} & -1.930792 & -1.282565 & 0.000000 \\ \mathrm{H} & 0.177303 & -2.557604 & 0.000000 \\ \mathrm{H} & 2.368128 & -1.365070 & 0.000000 \\ \mathrm{~F} & 2.602102 & 1.204227 & 0.000000 \\ \mathrm{H} & 0.301876 & 2.378849 & 0.000000 \\ \mathrm{H} & -2.851939 & 0.823709 & 0.000000\end{array}$

$m$-fluorophenol, trans $\mathrm{S}_{1}$
$\begin{array}{llll}\text { C } & 1.079930 & -0.016077 & 0.000000\end{array}$
$\begin{array}{llll}\text { C } & 0.327142 & 1.179962 & 0.000000\end{array}$ 


$\begin{array}{rrrc}\mathrm{C} & -1.088421 & 1.208884 & 0.000000 \\ \mathrm{C} & -1.819495 & -0.006210 & 0.000000 \\ \mathrm{C} & -1.083634 & -1.215802 & 0.000000 \\ \mathrm{C} & 0.317644 & -1.194042 & 0.000000 \\ \mathrm{O} & 1.034791 & 2.319871 & 0.000000 \\ \mathrm{H} & -1.597459 & 2.164740 & 0.000000 \\ \mathrm{H} & -2.896919 & -0.012931 & 0.000000 \\ \mathrm{H} & -1.581485 & -2.175620 & 0.000000 \\ \mathrm{~F} & 0.966927 & -2.369159 & 0.000000 \\ \mathrm{H} & 2.156831 & -0.011598 & 0.000000 \\ \mathrm{H} & 0.443002 & 3.082130 & 0.000000\end{array}$

$p$-fluorophenol $\mathrm{S}_{0}$

$\begin{array}{lrrr}\mathrm{C} & 0.271326 & 1.311308 & 0.000000 \\ \mathrm{C} & -0.945792 & 0.644374 & 0.000000 \\ \mathrm{C} & -0.976933 & -0.742146 & 0.000000 \\ \mathrm{C} & 0.206609 & -1.465258 & 0.000000 \\ \mathrm{C} & 1.401973 & -0.784040 & 0.000000 \\ \mathrm{C} & 1.453829 & 0.594404 & 0.000000 \\ \mathrm{O} & -2.077680 & 1.407246 & 0.000000 \\ \mathrm{H} & -1.925895 & -1.266010 & 0.000000 \\ \mathrm{H} & 0.203595 & -2.546481 & 0.000000 \\ \mathrm{~F} & 2.557162 & -1.484183 & 0.000000 \\ \mathrm{H} & 2.412666 & 1.094295 & 0.000000 \\ \mathrm{H} & 0.276365 & 2.392549 & 0.000000 \\ \mathrm{H} & -2.857246 & 0.843932 & 0.000000\end{array}$

$p$-fluorophenol $\mathrm{S}_{1}$

$\begin{array}{rrrr}\mathrm{C} & 1.262725 & 0.678029 & 0.000000 \\ \mathrm{C} & 0.027237 & 1.357636 & 0.000000 \\ \mathrm{C} & -1.232970 & 0.707949 & 0.000000 \\ \mathrm{C} & -1.247438 & -0.702885 & 0.000000 \\ \mathrm{C} & -0.006482 & -1.359880 & 0.000000 \\ \mathrm{C} & 1.248108 & -0.734186 & 0.000000 \\ \mathrm{O} & 0.091625 & 2.694728 & 0.000000 \\ \mathrm{H} & -2.147003 & 1.285952 & 0.000000 \\ \mathrm{H} & -2.157966 & -1.280929 & 0.000000 \\ \mathrm{~F} & -0.026673 & -2.692359 & 0.000000 \\ \mathrm{H} & 2.146406 & -1.330571 & 0.000000 \\ \mathrm{H} & 2.176747 & 1.250999 & 0.000000 \\ \mathrm{H} & -0.797621 & 3.071110 & 0.000000\end{array}$

phenylacetylene $\mathrm{S}_{0}$ 


$\begin{array}{rrrr}\mathrm{C} & 1.203167 & 0.000000 & -0.013243 \\ \mathrm{C} & 0.000000 & 0.000000 & -0.719475 \\ \mathrm{C} & -1.203167 & 0.000000 & -0.013243 \\ \mathrm{C} & -1.199541 & 0.000000 & 1.369818 \\ \mathrm{C} & 0.000000 & 0.000000 & 2.064871 \\ \mathrm{C} & 1.199541 & 0.000000 & 1.369818 \\ \mathrm{C} & 0.000000 & 0.000000 & -2.148902 \\ \mathrm{C} & 0.000000 & 0.000000 & -3.346027 \\ \mathrm{H} & -2.136964 & 0.000000 & -0.559262 \\ \mathrm{H} & -2.138522 & 0.000000 & 1.908147 \\ \mathrm{H} & 0.000000 & 0.000000 & 3.147186 \\ \mathrm{H} & 2.138522 & 0.000000 & 1.908147 \\ \mathrm{H} & 2.136964 & 0.000000 & -0.559262 \\ \mathrm{H} & 0.000000 & 0.000000 & -4.408593\end{array}$

phenylacetylene $S_{1}$

$\begin{array}{rrrr}\mathrm{C} & 1.228227 & 0.000000 & -0.029104 \\ \mathrm{C} & 0.000000 & 0.000000 & -0.752371 \\ \mathrm{C} & -1.228227 & 0.000000 & -0.029104 \\ \mathrm{C} & -1.219473 & 0.000000 & 1.387051 \\ \mathrm{C} & 0.000000 & 0.000000 & 2.090545 \\ \mathrm{C} & 1.219473 & 0.000000 & 1.387051 \\ \mathrm{C} & 0.000000 & 0.000000 & -2.151889 \\ \mathrm{C} & 0.000000 & 0.000000 & -3.357382 \\ \mathrm{H} & -2.160799 & 0.000000 & -0.573398 \\ \mathrm{H} & -2.156416 & 0.000000 & 1.925257 \\ \mathrm{H} & 0.000000 & 0.000000 & 3.171344 \\ \mathrm{H} & 2.156416 & 0.000000 & 1.925257 \\ \mathrm{H} & 2.160799 & 0.000000 & -0.573398 \\ \mathrm{H} & 0.000000 & 0.000000 & -4.419858\end{array}$

aniline $\mathrm{S}_{0}$

$\begin{array}{rrrr}\mathrm{C} & 0.916126 & 0.026431 & 0.000000 \\ \mathrm{C} & 0.204032 & 0.034474 & 1.198934 \\ \mathrm{C} & -1.179193 & 0.042897 & 1.193596 \\ \mathrm{C} & -1.884273 & 0.047695 & 0.000000 \\ \mathrm{C} & -1.179193 & 0.042897 & -1.193596 \\ \mathrm{C} & 0.204032 & 0.034474 & -1.198934 \\ \mathrm{H} & 0.742897 & 0.036277 & 2.139356 \\ \mathrm{H} & -2.965853 & 0.054105 & 0.000000 \\ \mathrm{H} & -1.711666 & 0.047051 & 2.136536 \\ \mathrm{H} & -1.711666 & 0.047051 & -2.136536 \\ \mathrm{H} & 0.742897 & 0.036277 & -2.139356 \\ \mathrm{~N} & 2.306144 & 0.066950 & 0.000000\end{array}$




$$
\begin{array}{lrrr}
\mathrm{H} & 2.757863 & -0.258285 & -0.838507 \\
\mathrm{H} & 2.757863 & -0.258285 & 0.838507
\end{array}
$$

aniline $\mathrm{S}_{1}$

$\begin{array}{lrrr}\mathrm{C} & 0.894691 & -0.034154 & 0.000000 \\ \mathrm{C} & 0.223350 & -0.008481 & 1.243689 \\ \mathrm{C} & -1.192413 & 0.045594 & 1.234105 \\ \mathrm{C} & -1.863008 & 0.071162 & 0.000000 \\ \mathrm{C} & -1.192413 & 0.045594 & -1.234105 \\ \mathrm{C} & 0.223350 & -0.008481 & -1.243689 \\ \mathrm{H} & 0.789843 & -0.030162 & 2.164494 \\ \mathrm{H} & -2.945622 & 0.112424 & 0.000000 \\ \mathrm{H} & -1.750587 & 0.066903 & 2.157758 \\ \mathrm{H} & -1.750587 & 0.066903 & -2.157758 \\ \mathrm{H} & 0.789843 & -0.030162 & -2.164494 \\ \mathrm{~N} & 2.247736 & -0.085962 & 0.000000 \\ \mathrm{H} & 2.762903 & -0.105599 & -0.863656 \\ \mathrm{H} & 2.762903 & -0.105599 & 0.863656\end{array}$

catechol $\mathrm{S}_{0}$

$\begin{array}{rrrr}\mathrm{C} & 0.469742 & 1.273766 & 0.000000 \\ \mathrm{C} & -0.743385 & 0.616338 & 0.000000 \\ \mathrm{C} & -0.793566 & -0.776151 & 0.000000 \\ \mathrm{C} & 0.383164 & -1.499226 & 0.000000 \\ \mathrm{C} & 1.604138 & -0.840033 & 0.000000 \\ \mathrm{C} & 1.650669 & 0.542907 & 0.000000 \\ \mathrm{O} & -1.966575 & 1.241307 & 0.000000 \\ \mathrm{O} & -1.989362 & -1.420296 & 0.000000 \\ \mathrm{H} & 0.324672 & -2.579391 & 0.000000 \\ \mathrm{H} & 2.520756 & -1.414535 & 0.000000 \\ \mathrm{H} & 2.600523 & 1.060078 & 0.000000 \\ \mathrm{H} & 0.494094 & 2.357942 & 0.000000 \\ \mathrm{H} & -1.861668 & 2.196739 & 0.000000 \\ \mathrm{H} & -2.693200 & -0.759446 & 0.000000\end{array}$

catechol $\mathrm{S}_{1}$

$\begin{array}{lrrr}\text { C } & 0.473156 & 1.342957 & 0.000000 \\ \text { C } & -0.755741 & 0.623035 & 0.000000 \\ \text { C } & -0.800023 & -0.782568 & 0.000000 \\ \text { C } & 0.387459 & -1.568612 & 0.000000 \\ \text { C } & 1.595670 & -0.856688 & 0.000000 \\ \text { C } & 1.637107 & 0.555859 & 0.000000 \\ \text { O } & -1.964324 & 1.243592 & 0.000000\end{array}$




$\begin{array}{rrrr}\mathrm{O} & -1.982436 & -1.417538 & 0.000000 \\ \mathrm{H} & 0.324017 & -2.643920 & 0.000000 \\ \mathrm{H} & 2.532991 & -1.396210 & 0.000000 \\ \mathrm{H} & 2.603729 & 1.041775 & 0.000000 \\ \mathrm{H} & 0.496029 & 2.422275 & 0.000000 \\ \mathrm{H} & -1.851169 & 2.199748 & 0.000000 \\ \mathrm{H} & -2.696464 & -0.763704 & 0.000000\end{array}$

resorcinol, isomer $1 \mathrm{~S}_{0}$

$\begin{array}{rrrr}\mathrm{C} & 0.075040 & 1.142820 & 0.000000 \\ \mathrm{C} & -1.133930 & 0.463028 & 0.000000 \\ \mathrm{C} & -1.165581 & -0.923019 & 0.000000 \\ \mathrm{C} & 0.032970 & -1.621802 & 0.000000 \\ \mathrm{C} & 1.247574 & -0.964970 & 0.000000 \\ \mathrm{C} & 1.258959 & 0.424447 & 0.000000 \\ \mathrm{O} & -2.265631 & 1.222432 & 0.000000 \\ \mathrm{H} & -2.112239 & -1.450053 & 0.000000 \\ \mathrm{H} & 0.014476 & -2.704058 & 0.000000 \\ \mathrm{H} & 2.185545 & -1.501559 & 0.000000 \\ \mathrm{O} & 2.474016 & 1.039297 & 0.000000 \\ \mathrm{H} & 0.069183 & 2.226010 & 0.000000 \\ \mathrm{H} & -3.040860 & 0.652732 & 0.000000 \\ \mathrm{H} & 2.360478 & 1.994686 & 0.000000\end{array}$

resorcinol, isomer $1 \mathrm{~S}_{1}$
$\begin{array}{llll}\text { C } & -0.001616 & 1.057442 & 0.052315\end{array}$
$\begin{array}{llll}\text { C } & -1.189976 & 0.304495 & -0.000880\end{array}$
$\begin{array}{llll}\text { C } & -1.200368 & -1.106964 & -0.034018\end{array}$
$\begin{array}{llll}\text { C } & -0.000087 & -1.846211 & 0.093131\end{array}$
$\begin{array}{llll}\text { C } & 1.205940 & -1.107828 & 0.005193\end{array}$
$\begin{array}{llll}C & 1.190976 & 0.299580 & 0.028607\end{array}$
$\begin{array}{llll}\mathrm{O} & -2.343328 & 1.006256 & -0.037351\end{array}$
$\mathrm{H} \quad-2.147427 \quad-1.617529-0.171640$
$\begin{array}{llll}\mathrm{H} & -0.001409 & -2.923333 & 0.091992\end{array}$
$\begin{array}{llll}\mathrm{H} & 2.163028 & -1.599239 & -0.106829\end{array}$
$\begin{array}{llll}\mathrm{O} & 2.392403 & 0.914873 & -0.014556\end{array}$
$\begin{array}{llll}\mathrm{H} & -0.022942 & 2.110012 & 0.295595\end{array}$
$\mathrm{H} \quad-3.097882 \quad 0.407091 \quad-0.003706$
H $2.263681 \quad 1.857476 \quad-0.178202$

resorcinol, isomer $2 \mathrm{~S}_{0}$
$\begin{array}{llll}\text { C } & 1.194078 & 0.000000 & -0.543984\end{array}$
$\begin{array}{llll}\text { C } & 0.000000 & 0.000000 & -1.243606\end{array}$ 


$\begin{array}{rrrr}\mathrm{C} & -1.194078 & 0.000000 & -0.543984 \\ \mathrm{C} & -1.205154 & 0.000000 & 0.846152 \\ \mathrm{C} & 0.000000 & 0.000000 & 1.526314 \\ \mathrm{C} & 1.205154 & 0.000000 & 0.846152 \\ \mathrm{O} & -2.340820 & 0.000000 & -1.279033 \\ \mathrm{O} & 2.340820 & 0.000000 & -1.279033 \\ \mathrm{H} & -2.143875 & 0.000000 & 1.387192 \\ \mathrm{H} & 0.000000 & 0.000000 & 2.608675 \\ \mathrm{H} & 2.143875 & 0.000000 & 1.387192 \\ \mathrm{H} & 0.000000 & 0.000000 & -2.324124 \\ \mathrm{H} & -3.104600 & 0.000000 & -0.694197 \\ \mathrm{H} & 3.104600 & 0.000000 & -0.694197\end{array}$

resorcinol, isomer $2 \mathrm{~S}_{1}$

$\begin{array}{rrrr}\mathrm{C} & 1.193235 & 0.000000 & -0.328062 \\ \mathrm{C} & 0.000000 & 0.000000 & -1.075730 \\ \mathrm{C} & -1.193235 & 0.000000 & -0.328062 \\ \mathrm{C} & -1.209683 & 0.000000 & 1.084361 \\ \mathrm{C} & 0.000000 & 0.000000 & 1.822068 \\ \mathrm{C} & 1.209683 & 0.000000 & 1.084361 \\ \mathrm{O} & -2.345722 & 0.000000 & -1.034603 \\ \mathrm{O} & 2.345722 & 0.000000 & -1.034603 \\ \mathrm{H} & -2.163289 & 0.000000 & 1.599425 \\ \mathrm{H} & 0.000000 & 0.000000 & 2.899433 \\ \mathrm{H} & 2.163289 & 0.000000 & 1.599425 \\ \mathrm{H} & 0.000000 & 0.000000 & -2.152619 \\ \mathrm{H} & -3.101891 & 0.000000 & -0.437241 \\ \mathrm{H} & 3.101891 & 0.000000 & -0.437241\end{array}$

resorcinol, isomer $3 \mathrm{~S}_{0}$

$\begin{array}{rrrr}\mathrm{C} & 1.199404 & 0.000000 & -0.285915 \\ \mathrm{C} & 0.000000 & 0.000000 & -0.985474 \\ \mathrm{C} & -1.199404 & 0.000000 & -0.285915 \\ \mathrm{C} & -1.207924 & 0.000000 & 1.099811 \\ \mathrm{C} & 0.000000 & 0.000000 & 1.775560 \\ \mathrm{C} & 1.207924 & 0.000000 & 1.099811 \\ \mathrm{O} & -2.404405 & 0.000000 & -0.922515 \\ \mathrm{O} & 2.404405 & 0.000000 & -0.922515 \\ \mathrm{H} & -2.153779 & 0.000000 & 1.622423 \\ \mathrm{H} & 0.000000 & 0.000000 & 2.858013 \\ \mathrm{H} & 2.153779 & 0.000000 & 1.622423 \\ \mathrm{H} & 0.000000 & 0.000000 & -2.071269 \\ \mathrm{H} & 2.278122 & 0.000000 & -1.875979 \\ \mathrm{H} & -2.278122 & 0.000000 & -1.875979\end{array}$


resorcinol, isomer $3 \mathrm{~S}_{1}$

$\begin{array}{lrrc}\mathrm{C} & -0.019486 & -0.302054 & 1.185559 \\ \mathrm{C} & -0.099985 & -1.070347 & 0.000000 \\ \mathrm{C} & -0.019486 & -0.302054 & -1.185559 \\ \mathrm{C} & 0.026138 & 1.105174 & -1.197281 \\ \mathrm{C} & -0.081257 & 1.844475 & 0.000000 \\ \mathrm{C} & 0.026138 & 1.105174 & 1.197281 \\ \mathrm{O} & 0.053602 & -0.914514 & -2.385967 \\ \mathrm{O} & 0.053602 & -0.914514 & 2.385967 \\ \mathrm{H} & 0.174133 & 1.591892 & -2.152597 \\ \mathrm{H} & -0.085686 & 2.921725 & 0.000000 \\ \mathrm{H} & 0.174133 & 1.591892 & 2.152597 \\ \mathrm{H} & -0.578892 & -2.046753 & 0.000000 \\ \mathrm{H} & 0.233155 & -1.854372 & 2.248621 \\ \mathrm{H} & 0.233155 & -1.854372 & -2.248621\end{array}$

hydroquinone, cis $\mathrm{S}_{0}$

$\begin{array}{rrrr}\mathrm{C} & -0.694479 & 0.000000 & 1.087016 \\ \mathrm{C} & 0.694479 & 0.000000 & 1.087016 \\ \mathrm{C} & 1.389775 & 0.000000 & -0.108784 \\ \mathrm{C} & 0.690038 & 0.000000 & -1.307801 \\ \mathrm{C} & -0.690038 & 0.000000 & -1.307801 \\ \mathrm{C} & -1.389775 & 0.000000 & -0.108784 \\ \mathrm{O} & 2.756833 & 0.000000 & -0.175184 \\ \mathrm{O} & -2.756833 & 0.000000 & -0.175184 \\ \mathrm{H} & 1.244299 & 0.000000 & -2.236574 \\ \mathrm{H} & -1.244299 & 0.000000 & -2.236574 \\ \mathrm{H} & -1.229478 & 0.000000 & 2.030069 \\ \mathrm{H} & 1.229478 & 0.000000 & 2.030069 \\ \mathrm{H} & -3.128678 & 0.000000 & 0.711548 \\ \mathrm{H} & 3.128678 & 0.000000 & 0.711548\end{array}$

hydroquinone, cis $\mathrm{S}_{1}$

$\begin{array}{lrrr}\text { C } & 0.704564 & 0.000000 & -1.128276 \\ \mathrm{C} & -0.704564 & 0.000000 & -1.128276 \\ \mathrm{C} & -1.369667 & 0.000000 & 0.120154 \\ \mathrm{C} & -0.705057 & 0.000000 & 1.358680 \\ \mathrm{C} & 0.705057 & 0.000000 & 1.358680 \\ \mathrm{C} & 1.369667 & 0.000000 & 0.120154 \\ \mathrm{O} & -2.711859 & 0.000000 & 0.164058 \\ \mathrm{O} & 2.711859 & 0.000000 & 0.164058 \\ \mathrm{H} & -1.286432 & 0.000000 & 2.267263\end{array}$




$\begin{array}{rrrr}\mathrm{H} & 1.286432 & 0.000000 & 2.267263 \\ \mathrm{H} & 1.271744 & 0.000000 & -2.049389 \\ \mathrm{H} & -1.271744 & 0.000000 & -2.049389 \\ \mathrm{H} & 3.070453 & 0.000000 & -0.731720 \\ \mathrm{H} & -3.070453 & 0.000000 & -0.731720\end{array}$

hydroquinone, trans $\mathrm{S}_{0}$

$\begin{array}{rrrr}\mathrm{C} & 0.020605 & 1.384747 & 0.000000 \\ \mathrm{C} & -1.191399 & 0.715628 & 0.000000 \\ \mathrm{C} & -1.206971 & -0.671228 & 0.000000 \\ \mathrm{C} & -0.020605 & -1.384747 & 0.000000 \\ \mathrm{C} & 1.191399 & -0.715628 & 0.000000 \\ \mathrm{C} & 1.206971 & 0.671228 & 0.000000 \\ \mathrm{O} & -2.333135 & 1.470542 & 0.000000 \\ \mathrm{H} & -2.151452 & -1.204045 & 0.000000 \\ \mathrm{H} & -0.024114 & -2.466229 & 0.000000 \\ \mathrm{O} & 2.333135 & -1.470542 & 0.000000 \\ \mathrm{H} & 2.151452 & 1.204045 & 0.000000 \\ \mathrm{H} & 0.024114 & 2.466229 & 0.000000 \\ \mathrm{H} & -3.105019 & 0.897163 & 0.000000 \\ \mathrm{H} & 3.105019 & -0.897163 & 0.000000\end{array}$

hydroquinone, trans $\mathrm{S}_{1}$

$\begin{array}{rrrr}\mathrm{C} & 1.258672 & 0.674511 & 0.000000 \\ \mathrm{C} & 0.035123 & 1.369117 & 0.000000 \\ \mathrm{C} & -1.227719 & 0.734873 & 0.000000 \\ \mathrm{C} & -1.258672 & -0.674511 & 0.000000 \\ \mathrm{C} & -0.035123 & -1.369117 & 0.000000 \\ \mathrm{C} & 1.227719 & -0.734873 & 0.000000 \\ \mathrm{O} & 0.117417 & 2.709501 & 0.000000 \\ \mathrm{H} & -2.135915 & 1.322417 & 0.000000 \\ \mathrm{H} & -2.179979 & -1.236075 & 0.000000 \\ \mathrm{O} & -0.117417 & -2.709501 & 0.000000 \\ \mathrm{H} & 2.135915 & -1.322417 & 0.000000 \\ \mathrm{H} & 2.179979 & 1.236075 & 0.000000 \\ \mathrm{H} & -0.768647 & 3.092264 & 0.000000 \\ \mathrm{H} & 0.768647 & -3.092264 & 0.000000\end{array}$

salicylic acid $\mathrm{S}_{0}$
$\begin{array}{llll}\text { C } & 1.097780 & 1.593760 & 0.000000\end{array}$
$\begin{array}{llll}\text { C } & 1.128712 & 0.199448 & 0.000000\end{array}$
$\begin{array}{llll}\text { C } & -0.083564 & -0.513452 & 0.000000\end{array}$
$\begin{array}{llll}\text { C } & -1.295025 & 0.187039 & 0.000000\end{array}$ 


$\begin{array}{rrrr}\mathrm{C} & -1.314298 & 1.560960 & 0.000000 \\ \mathrm{C} & -0.106342 & 2.258862 & 0.000000 \\ \mathrm{O} & 2.324727 & -0.398227 & 0.000000 \\ \mathrm{C} & -0.043942 & -1.973699 & 0.000000 \\ \mathrm{O} & -1.242627 & -2.570814 & 0.000000 \\ \mathrm{O} & 0.981116 & -2.633574 & 0.000000 \\ \mathrm{H} & -2.217359 & -0.376509 & 0.000000 \\ \mathrm{H} & 2.039808 & 2.124648 & 0.000000 \\ \mathrm{H} & -2.254499 & 2.094663 & 0.000000 \\ \mathrm{H} & -0.112346 & 3.341638 & 0.000000 \\ \mathrm{H} & 2.183394 & -1.368552 & 0.000000 \\ \mathrm{H} & -1.085534 & -3.526191 & 0.000000\end{array}$

salicylic acid $\mathrm{S}_{1}$

$\begin{array}{rrrl}\mathrm{C} & 0.998895 & 1.907910 & 0.000000 \\ \mathrm{C} & 0.963048 & 0.510481 & 0.000000 \\ \mathrm{C} & -0.295912 & -0.224507 & 0.000000 \\ \mathrm{C} & -1.454356 & 0.536532 & 0.000000 \\ \mathrm{C} & -1.397816 & 1.957010 & 0.000000 \\ \mathrm{C} & -0.195398 & 2.649683 & 0.000000 \\ \mathrm{O} & 2.075174 & -0.175535 & 0.000000 \\ \mathrm{C} & -0.235228 & -1.648195 & 0.000000 \\ \mathrm{O} & -1.417518 & -2.298520 & 0.000000 \\ \mathrm{O} & 0.842191 & -2.303335 & 0.000000 \\ \mathrm{H} & -2.415087 & 0.042100 & 0.000000 \\ \mathrm{H} & 1.969660 & 2.384447 & 0.000000 \\ \mathrm{H} & -2.331811 & 2.503754 & 0.000000 \\ \mathrm{H} & -0.174288 & 3.728857 & 0.000000 \\ \mathrm{H} & 1.794048 & -1.189994 & 0.000000 \\ \mathrm{H} & -1.215023 & -3.243742 & 0.000000\end{array}$

$o$-cresol, cis $\mathrm{S}_{0}$

$\begin{array}{rrrr}\mathrm{C} & 1.958136 & 0.510775 & 0.000000 \\ \mathrm{C} & 0.728232 & 1.146657 & 0.000000 \\ \mathrm{C} & -0.462755 & 0.431383 & 0.000000 \\ \mathrm{C} & -0.386917 & -0.961464 & 0.000000 \\ \mathrm{C} & 0.837367 & -1.610945 & 0.000000 \\ \mathrm{C} & 2.007484 & -0.874364 & 0.000000 \\ \mathrm{C} & -1.796966 & 1.123001 & 0.000000 \\ \mathrm{O} & -1.504723 & -1.747103 & 0.000000 \\ \mathrm{H} & 0.850150 & -2.692629 & 0.000000 \\ \mathrm{H} & 2.961170 & -1.386270 & 0.000000 \\ \mathrm{H} & 2.870241 & 1.092435 & 0.000000 \\ \mathrm{H} & 0.684300 & 2.229438 & 0.000000\end{array}$




$\begin{array}{rrrr}\mathrm{H} & -2.296493 & -1.201650 & 0.000000 \\ \mathrm{H} & -2.389536 & 0.868425 & 0.883926 \\ \mathrm{H} & -1.670155 & 2.203897 & 0.000000 \\ \mathrm{H} & -2.389536 & 0.868425 & -0.883926\end{array}$

$o$-cresol, cis $\mathrm{S}_{1}$

$\begin{array}{lrrr}\mathrm{C} & 1.859914 & -0.063995 & -0.778855 \\ \mathrm{C} & 0.626147 & -0.021409 & -1.456760 \\ \mathrm{C} & -0.586310 & -0.015500 & -0.714103 \\ \mathrm{C} & -0.467590 & 0.059042 & 0.703229 \\ \mathrm{C} & 0.769595 & 0.052681 & 1.390802 \\ \mathrm{C} & 1.948777 & -0.035445 & 0.626843 \\ \mathrm{C} & -1.895303 & -0.035114 & -1.422930 \\ \mathrm{O} & -1.555332 & 0.121898 & 1.484584 \\ \mathrm{H} & 0.767677 & 0.132121 & 2.466770 \\ \mathrm{H} & 2.915333 & -0.063116 & 1.107667 \\ \mathrm{H} & 2.772603 & -0.117448 & -1.358717 \\ \mathrm{H} & 0.595609 & -0.051539 & -2.536155 \\ \mathrm{H} & -2.353513 & 0.159545 & 0.942045 \\ \mathrm{H} & -2.738568 & -0.350191 & -0.801350 \\ \mathrm{H} & -2.152757 & 0.944564 & -1.847780 \\ \mathrm{H} & -1.856862 & -0.735586 & -2.262879\end{array}$

$o$-cresol, trans $\mathrm{S}_{0}$

$\begin{array}{lrrr}\mathrm{C} & 2.414497 & 0.000025 & 0.030414 \\ \mathrm{C} & 1.244693 & -0.000020 & -0.713872 \\ \mathrm{C} & 0.035249 & 0.000002 & -0.019103 \\ \mathrm{C} & 0.003746 & 0.000058 & 1.365828 \\ \mathrm{C} & 1.188594 & 0.000117 & 2.085232 \\ \mathrm{C} & 2.399802 & 0.000077 & 1.418169 \\ \mathrm{C} & 1.248163 & -0.000109 & -2.213143 \\ \mathrm{O} & -1.106437 & -0.000040 & -0.771706 \\ \mathrm{H} & -0.949855 & 0.000079 & 1.883048 \\ \mathrm{H} & 1.157826 & 0.000152 & 3.167081 \\ \mathrm{H} & 3.329683 & 0.000126 & 1.971053 \\ \mathrm{H} & 3.362535 & 0.000010 & -0.494414 \\ \mathrm{H} & -1.877165 & -0.000047 & -0.196205 \\ \mathrm{H} & 2.267667 & -0.000064 & -2.596187 \\ \mathrm{H} & 0.729925 & -0.875437 & -2.608041 \\ \mathrm{H} & 0.729785 & 0.875069 & -2.608184\end{array}$

$o$-cresol, trans $\mathrm{S}_{1}$

$\begin{array}{llll}\text { C } & 2.426456 & 0.221340 & -0.007304\end{array}$ 


$\begin{array}{rrrr}\mathrm{C} & 1.218014 & 0.083617 & -0.750224 \\ \mathrm{C} & 0.015001 & -0.029249 & -0.015154 \\ \mathrm{C} & -0.043753 & -0.092309 & 1.407221 \\ \mathrm{C} & 1.176311 & -0.044379 & 2.102135 \\ \mathrm{C} & 2.386342 & 0.096417 & 1.390682 \\ \mathrm{C} & 1.250504 & -0.008929 & -2.232239 \\ \mathrm{O} & -1.125349 & -0.126239 & -0.716947 \\ \mathrm{H} & -0.996779 & -0.009352 & 1.913152 \\ \mathrm{H} & 1.194028 & -0.092923 & 3.181543 \\ \mathrm{H} & 3.313541 & 0.121732 & 1.949346 \\ \mathrm{H} & 3.366256 & 0.340756 & -0.525542 \\ \mathrm{H} & -1.846425 & -0.367482 & -0.119307 \\ \mathrm{H} & 1.855763 & -0.866260 & -2.552930 \\ \mathrm{H} & 0.258119 & -0.105413 & -2.664356 \\ \mathrm{H} & 1.730661 & 0.878671 & -2.660097\end{array}$

$m$-cresol, cis $\mathrm{S}_{0}$

$\begin{array}{rrrr}\mathrm{C} & 1.158281 & -0.002895 & 2.079475 \\ \mathrm{C} & 2.365371 & 0.013171 & 1.409132 \\ \mathrm{C} & 2.410589 & 0.021785 & 0.021521 \\ \mathrm{C} & 1.235926 & 0.012824 & -0.717009 \\ \mathrm{C} & 0.019952 & -0.001444 & -0.040149 \\ \mathrm{C} & -0.019436 & -0.009887 & 1.344795 \\ \mathrm{C} & 1.261250 & 0.002214 & -2.220184 \\ \mathrm{O} & -1.192404 & -0.023001 & 2.040816 \\ \mathrm{H} & 1.106198 & -0.007807 & 3.159574 \\ \mathrm{H} & 3.287805 & 0.021140 & 1.975952 \\ \mathrm{H} & 3.365418 & 0.036504 & -0.488554 \\ \mathrm{H} & -0.908477 & -0.004969 & -0.603525 \\ \mathrm{H} & -1.936040 & -0.026722 & 1.430601 \\ \mathrm{H} & 2.262011 & 0.206806 & -2.598281 \\ \mathrm{H} & 0.951100 & -0.969703 & -2.610029 \\ \mathrm{H} & 0.584754 & 0.751355 & -2.634184\end{array}$

$m$-cresol, cis $\mathrm{S}_{1}$

$\begin{array}{lrrr}\mathrm{C} & 1.164303 & 0.009301 & 2.118137 \\ \mathrm{C} & 2.405285 & 0.063389 & 1.444389 \\ \mathrm{C} & 2.405313 & 0.031711 & 0.034035 \\ \mathrm{C} & 1.220631 & 0.002706 & -0.741684 \\ \mathrm{C} & -0.024773 & -0.016972 & -0.068726 \\ \mathrm{C} & -0.015208 & -0.007285 & 1.348906 \\ \mathrm{C} & 1.265520 & -0.010437 & -2.229292 \\ \mathrm{O} & -1.171679 & -0.035063 & 2.029415 \\ \mathrm{H} & 1.084868 & -0.043853 & 3.193828\end{array}$




$\begin{array}{rrrr}\mathrm{H} & 3.334478 & 0.085541 & 1.992027 \\ \mathrm{H} & 3.354236 & 0.039211 & -0.488635 \\ \mathrm{H} & -0.958852 & 0.076452 & -0.608725 \\ \mathrm{H} & -1.909973 & -0.131620 & 1.414454 \\ \mathrm{H} & 2.286252 & 0.061127 & -2.603050 \\ \mathrm{H} & 0.820988 & -0.927622 & -2.634171 \\ \mathrm{H} & 0.690891 & 0.822785 & -2.650970\end{array}$

$m$-cresol, trans $\mathrm{S}_{0}$

$\begin{array}{rrrr}\mathrm{C} & -1.187329 & -1.095452 & 0.000000 \\ \mathrm{C} & 0.199101 & -1.206717 & 0.000000 \\ \mathrm{C} & 0.966239 & -0.049359 & 0.000000 \\ \mathrm{C} & 0.362083 & 1.197037 & 0.000000 \\ \mathrm{C} & -1.022007 & 1.305269 & 0.000000 \\ \mathrm{C} & -1.788010 & 0.152634 & 0.000000 \\ \mathrm{C} & 0.869519 & -2.552541 & 0.000000 \\ \mathrm{O} & 1.180034 & 2.288993 & 0.000000 \\ \mathrm{H} & -1.494480 & 2.281252 & 0.000000 \\ \mathrm{H} & -2.867776 & 0.231447 & 0.000000 \\ \mathrm{H} & -1.798140 & -1.989253 & 0.000000 \\ \mathrm{H} & 2.048053 & -0.099295 & 0.000000 \\ \mathrm{H} & 0.652438 & 3.093287 & 0.000000 \\ \mathrm{H} & 0.136924 & -3.358521 & 0.000000 \\ \mathrm{H} & 1.504219 & -2.675128 & -0.879417 \\ \mathrm{H} & 1.504219 & -2.675128 & 0.879417\end{array}$

m-cresol, trans $\mathrm{S}_{1}$

$\begin{array}{rrrr}\mathrm{C} & -1.198992 & -1.055988 & 0.000000 \\ \mathrm{C} & 0.204237 & -1.230976 & 0.000000 \\ \mathrm{C} & 1.021763 & -0.072493 & 0.000000 \\ \mathrm{C} & 0.396294 & 1.191041 & 0.000000 \\ \mathrm{C} & -1.008234 & 1.367234 & 0.000000 \\ \mathrm{C} & -1.821016 & 0.213064 & 0.000000 \\ \mathrm{C} & 0.825075 & -2.584279 & 0.000000 \\ \mathrm{O} & 1.212828 & 2.257128 & 0.000000 \\ \mathrm{H} & -1.430987 & 2.363681 & 0.000000 \\ \mathrm{H} & -2.897234 & 0.292102 & 0.000000 \\ \mathrm{H} & -1.825540 & -1.939727 & 0.000000 \\ \mathrm{H} & 2.100297 & -0.125001 & 0.000000 \\ \mathrm{H} & 0.694568 & 3.071367 & 0.000000 \\ \mathrm{H} & 0.072185 & -3.372416 & 0.000000 \\ \mathrm{H} & 1.465619 & -2.729860 & -0.877324 \\ \mathrm{H} & 1.465619 & -2.729860 & 0.877324\end{array}$


$p$-cresol $\mathrm{S}_{0}$

$\begin{array}{lrrr}\mathrm{C} & 0.710131 & 0.020948 & -1.184474 \\ \mathrm{C} & -0.671032 & 0.021673 & -1.192956 \\ \mathrm{C} & -1.366619 & 0.000222 & 0.007625 \\ \mathrm{C} & -0.668648 & -0.023426 & 1.203270 \\ \mathrm{C} & 0.718908 & -0.023747 & 1.192856 \\ \mathrm{C} & 1.434657 & -0.000081 & 0.005327 \\ \mathrm{O} & -2.730646 & 0.000040 & -0.052928 \\ \mathrm{C} & 2.938360 & 0.020428 & -0.005266 \\ \mathrm{H} & -1.204082 & -0.044010 & 2.146359 \\ \mathrm{H} & 1.250700 & -0.044495 & 2.136729 \\ \mathrm{H} & 1.240780 & 0.035617 & -2.129569 \\ \mathrm{H} & -1.227414 & 0.036259 & -2.120595 \\ \mathrm{H} & -3.097784 & -0.017252 & 0.835892 \\ \mathrm{H} & 3.341309 & -0.703043 & -0.715613 \\ \mathrm{H} & 3.320039 & 1.003685 & -0.289638 \\ \mathrm{H} & 3.342901 & -0.217441 & 0.978281\end{array}$

$p$-cresol $\mathrm{S}_{1}$

$\begin{array}{lrrr}\mathrm{C} & 0.730193 & -0.064961 & -1.233023 \\ \mathrm{C} & -0.680660 & -0.026247 & -1.240486 \\ \mathrm{C} & -1.349209 & -0.003169 & -0.001500 \\ \mathrm{C} & -0.678526 & 0.007825 & 1.245349 \\ \mathrm{C} & 0.730968 & -0.025956 & 1.233753 \\ \mathrm{C} & 1.427285 & -0.035723 & -0.001011 \\ \mathrm{O} & -2.689070 & 0.021327 & -0.046936 \\ \mathrm{C} & 2.911624 & 0.034202 & -0.000979 \\ \mathrm{H} & -1.243236 & 0.018417 & 2.168470 \\ \mathrm{H} & 1.289980 & -0.023203 & 2.158430 \\ \mathrm{H} & 1.285752 & -0.086030 & -2.159255 \\ \mathrm{H} & -1.261612 & -0.011539 & -2.150032 \\ \mathrm{H} & -3.048063 & 0.054447 & 0.848590 \\ \mathrm{H} & 3.332520 & -0.457401 & -0.879317 \\ \mathrm{H} & 3.241105 & 1.080060 & -0.025649 \\ \mathrm{H} & 3.332497 & -0.416669 & 0.898887\end{array}$

$o$-methoxyphenol $\mathrm{S}_{0}$
$\begin{array}{llll}\text { C } & 2.025133 & -0.090351 & 0.000000\end{array}$
$\begin{array}{llll}\text { C } & 0.874244 & 0.692766 & 0.000000\end{array}$
$\begin{array}{llll}\text { C } & -0.365119 & 0.083411 & 0.000000\end{array}$
$\begin{array}{llll}\text { C } & -0.464328 & -1.312309 & 0.000000\end{array}$
$\begin{array}{llll}\text { C } & 0.680132 & -2.080865 & 0.000000\end{array}$
$\begin{array}{llll}\text { C } & 1.927255 & -1.468088 & 0.000000\end{array}$ 


$\begin{array}{rrrr}\mathrm{O} & -1.574471 & 0.723219 & 0.000000 \\ \mathrm{O} & -1.685552 & -1.904388 & 0.000000 \\ \mathrm{H} & 0.579425 & -3.157957 & 0.000000 \\ \mathrm{H} & 2.821334 & -2.077287 & 0.000000 \\ \mathrm{H} & 2.994465 & 0.389727 & 0.000000 \\ \mathrm{H} & 0.957300 & 1.770203 & 0.000000 \\ \mathrm{C} & -1.587110 & 2.136222 & 0.000000 \\ \mathrm{H} & -2.353589 & -1.206579 & 0.000000 \\ \mathrm{H} & -2.632693 & 2.431741 & 0.000000 \\ \mathrm{H} & -1.098212 & 2.535268 & 0.892149 \\ \mathrm{H} & -1.098212 & 2.535268 & -0.892149\end{array}$

$o$-methoxyphenol $\mathrm{S}_{1}$

$\begin{array}{lrrc}\mathrm{C} & 2.483882 & 0.073422 & 0.066521 \\ \mathrm{C} & 1.383723 & -0.226578 & -0.750391 \\ \mathrm{C} & 0.117036 & -0.217904 & -0.095517 \\ \mathrm{C} & 0.023633 & -0.142124 & 1.311910 \\ \mathrm{C} & 1.179894 & -0.082892 & 2.136331 \\ \mathrm{C} & 2.387447 & 0.143919 & 1.471867 \\ \mathrm{O} & -1.071068 & -0.018773 & -0.726187 \\ \mathrm{O} & -1.174145 & 0.046759 & 1.884264 \\ \mathrm{H} & 1.093968 & -0.189976 & 3.206346 \\ \mathrm{H} & 3.282332 & 0.367554 & 2.039430 \\ \mathrm{H} & 3.451011 & 0.248185 & -0.391322 \\ \mathrm{H} & 1.504168 & -0.520889 & -1.782126 \\ \mathrm{C} & -1.094517 & 0.096486 & -2.144047 \\ \mathrm{H} & -1.851849 & 0.048676 & 1.187757 \\ \mathrm{H} & -2.128285 & 0.297846 & -2.409436 \\ \mathrm{H} & -0.455769 & 0.913563 & -2.479138 \\ \mathrm{H} & -0.772900 & -0.837284 & -2.608183\end{array}$

$m$-methoxyphenol, isomer $1 \mathrm{~S}_{0}$

$\begin{array}{rrrr}\mathrm{C} & 0.926760 & 1.441862 & 0.000000 \\ \mathrm{C} & -0.258604 & 0.713753 & 0.000000 \\ \mathrm{C} & -0.227099 & -0.675230 & 0.000000 \\ \mathrm{C} & 1.002036 & -1.328438 & 0.000000 \\ \mathrm{C} & 2.187295 & -0.616258 & 0.000000 \\ \mathrm{C} & 2.132308 & 0.769267 & 0.000000 \\ \mathrm{H} & -1.139065 & -1.256923 & 0.000000 \\ \mathrm{O} & 1.084342 & -2.689646 & 0.000000 \\ \mathrm{H} & 3.126852 & -1.150215 & 0.000000 \\ \mathrm{H} & 3.054899 & 1.335575 & 0.000000 \\ \mathrm{H} & 0.875590 & 2.521421 & 0.000000 \\ \mathrm{O} & -1.405071 & 1.441372 & 0.000000\end{array}$




$\begin{array}{rrrr}\mathrm{H} & 0.203413 & -3.075339 & 0.000000 \\ \mathrm{C} & -2.641290 & 0.760439 & 0.000000 \\ \mathrm{H} & -3.408666 & 1.529736 & 0.000000 \\ \mathrm{H} & -2.756851 & 0.139312 & -0.892528 \\ \mathrm{H} & -2.756851 & 0.139312 & 0.892528\end{array}$

m-methoxyphenol, isomer $1 \mathrm{~S}_{1}$

$\begin{array}{lrrc}\mathrm{C} & 1.166747 & -0.008986 & -0.619083 \\ \mathrm{C} & -0.028855 & -0.034679 & 0.126326 \\ \mathrm{C} & -0.046066 & -0.081657 & 1.542699 \\ \mathrm{C} & 1.236074 & -0.010859 & 2.141124 \\ \mathrm{C} & 2.438732 & 0.013528 & 1.412696 \\ \mathrm{C} & 2.430621 & -0.113524 & 0.007499 \\ \mathrm{H} & -0.890037 & -0.467380 & 2.100169 \\ \mathrm{O} & 1.344642 & 0.091978 & 3.483633 \\ \mathrm{H} & 3.356511 & 0.164485 & 1.966008 \\ \mathrm{H} & 3.343339 & -0.128811 & -0.564406 \\ \mathrm{H} & 1.076527 & 0.105786 & -1.691357 \\ \mathrm{O} & -1.152017 & 0.030215 & -0.615752 \\ \mathrm{H} & 0.476434 & 0.303360 & 3.852855 \\ \mathrm{C} & -2.414139 & 0.032791 & 0.030398 \\ \mathrm{H} & -3.146204 & 0.241779 & -0.744519 \\ \mathrm{H} & -2.626972 & -0.942212 & 0.473995 \\ \mathrm{H} & -2.457027 & 0.804186 & 0.800453\end{array}$

$m$-methoxyphenol, isomer $2 \mathrm{~S}_{0}$

$\begin{array}{lrrc}\mathrm{C} & 0.345716 & 2.154374 & 0.000000 \\ \mathrm{C} & -0.833655 & 1.442986 & 0.000000 \\ \mathrm{C} & -0.789989 & 0.048735 & 0.000000 \\ \mathrm{C} & 0.426447 & -0.615033 & 0.000000 \\ \mathrm{C} & 1.603245 & 0.125469 & 0.000000 \\ \mathrm{C} & 1.576581 & 1.508702 & 0.000000 \\ \mathrm{O} & -1.991613 & -0.580618 & 0.000000 \\ \mathrm{C} & -2.014374 & -1.993376 & 0.000000 \\ \mathrm{O} & 2.766899 & -0.585911 & 0.000000 \\ \mathrm{H} & 0.501159 & -1.691557 & 0.000000 \\ \mathrm{H} & 2.499202 & 2.076901 & 0.000000 \\ \mathrm{H} & 0.314768 & 3.236424 & 0.000000 \\ \mathrm{H} & -1.796025 & 1.934799 & 0.000000 \\ \mathrm{H} & -3.063409 & -2.276620 & 0.000000 \\ \mathrm{H} & -1.530933 & -2.400845 & -0.891660 \\ \mathrm{H} & -1.530933 & -2.400845 & 0.891660 \\ \mathrm{H} & 3.516902 & 0.016424 & 0.000000\end{array}$


$m$-methoxyphenol, isomer $2 \mathrm{~S}_{1}$

$\begin{array}{lrrr}\mathrm{C} & 1.240065 & -0.081338 & -0.704666 \\ \mathrm{C} & 0.057655 & -0.013241 & 0.077199 \\ \mathrm{C} & 0.111257 & -0.033897 & 1.485376 \\ \mathrm{C} & 1.335190 & -0.049364 & 2.197536 \\ \mathrm{C} & 2.497182 & -0.000720 & 1.401753 \\ \mathrm{C} & 2.466549 & 0.028512 & -0.007821 \\ \mathrm{O} & -1.090278 & -0.012290 & 2.094867 \\ \mathrm{C} & -1.151384 & 0.024824 & 3.510328 \\ \mathrm{O} & 3.672151 & 0.040657 & 2.070036 \\ \mathrm{H} & 1.416483 & -0.163442 & 3.264172 \\ \mathrm{H} & 3.400274 & 0.135096 & -0.548914 \\ \mathrm{H} & 1.202386 & -0.064945 & -1.781093 \\ \mathrm{H} & -0.918423 & 0.056955 & -0.382685 \\ \mathrm{H} & -2.206918 & 0.078112 & 3.761271 \\ \mathrm{H} & -0.719438 & -0.878479 & 3.945370 \\ \mathrm{H} & -0.634992 & 0.902900 & 3.902227 \\ \mathrm{H} & 4.407370 & 0.030661 & 1.446943\end{array}$

p-methoxyphenol, cis $\mathrm{S}_{0}$

$\begin{array}{lrrr}\mathrm{C} & -0.314502 & 0.727474 & 0.000000 \\ \mathrm{C} & -1.542998 & 0.070422 & 0.000000 \\ \mathrm{C} & -1.603213 & -1.304986 & 0.000000 \\ \mathrm{C} & -0.431990 & -2.054523 & 0.000000 \\ \mathrm{C} & 0.788055 & -1.408390 & 0.000000 \\ \mathrm{C} & 0.852599 & -0.017438 & 0.000000 \\ \mathrm{O} & -0.556347 & -3.417174 & 0.000000 \\ \mathrm{O} & -0.365742 & 2.090249 & 0.000000 \\ \mathrm{C} & 0.851898 & 2.800953 & 0.000000 \\ \mathrm{H} & 1.821375 & 0.459807 & 0.000000 \\ \mathrm{H} & -2.446408 & 0.665419 & 0.000000 \\ \mathrm{H} & -2.554525 & -1.819790 & 0.000000 \\ \mathrm{H} & 1.708194 & -1.982310 & 0.000000 \\ \mathrm{H} & 0.587473 & 3.855091 & 0.000000 \\ \mathrm{H} & 1.446046 & 2.580510 & 0.891758 \\ \mathrm{H} & 1.446046 & 2.580510 & -0.891758 \\ \mathrm{H} & 0.314038 & -3.825802 & 0.000000\end{array}$

p-methoxyphenol, cis $\mathrm{S}_{1}$
$\begin{array}{llll}\text { C } & 1.326247 & -0.003279 & -0.614298\end{array}$
$\begin{array}{llll}\text { C } & 0.053660 & -0.006999 & 0.003085\end{array}$
$\begin{array}{llll}\text { C } & -0.015347 & 0.000171 & 1.411404\end{array}$
$\begin{array}{llll}\text { C } & 1.187018 & -0.001108 & 2.133462\end{array}$ 


$\begin{array}{rrrr}\mathrm{C} & 2.464213 & 0.001625 & 1.522817 \\ \mathrm{C} & 2.537179 & -0.003951 & 0.114710 \\ \mathrm{O} & 1.086863 & -0.000193 & 3.473175 \\ \mathrm{O} & 1.289539 & -0.003888 & -1.951492 \\ \mathrm{C} & 2.492547 & 0.003665 & -2.706507 \\ \mathrm{H} & 3.492575 & -0.006320 & -0.383369 \\ \mathrm{H} & -0.825485 & -0.004430 & -0.621780 \\ \mathrm{H} & -0.951871 & 0.008271 & 1.947006 \\ \mathrm{H} & 3.358449 & -0.016326 & 2.131669 \\ \mathrm{H} & 2.186413 & 0.002403 & -3.748293 \\ \mathrm{H} & 3.076947 & 0.899918 & -2.495293 \\ \mathrm{H} & 3.087268 & -0.886112 & -2.496590 \\ \mathrm{H} & 1.967317 & 0.016536 & 3.867295\end{array}$

p-methoxyphenol, trans $\mathrm{S}_{0}$

$\begin{array}{crcc}\mathrm{C} & -0.655589 & 1.299015 & 0.000000 \\ \mathrm{C} & -1.884127 & 0.668631 & 0.000000 \\ \mathrm{C} & -1.958538 & -0.718713 & 0.000000 \\ \mathrm{C} & -0.793604 & -1.461408 & 0.000000 \\ \mathrm{C} & 0.443087 & -0.831084 & 0.000000 \\ \mathrm{C} & 0.517870 & 0.555059 & 0.000000 \\ \mathrm{O} & -3.148124 & -1.395468 & 0.000000 \\ \mathrm{H} & 1.337016 & -1.437268 & 0.000000 \\ \mathrm{O} & 1.680305 & 1.268758 & 0.000000 \\ \mathrm{H} & -0.585483 & 2.378393 & 0.000000 \\ \mathrm{H} & -2.791709 & 1.262373 & 0.000000 \\ \mathrm{H} & -3.879806 & -0.771762 & 0.000000 \\ \mathrm{H} & -0.860121 & -2.541017 & 0.000000 \\ \mathrm{C} & 2.897730 & 0.556842 & 0.000000 \\ \mathrm{H} & 3.685759 & 1.305281 & 0.000000 \\ \mathrm{H} & 2.997663 & -0.068827 & 0.891660 \\ \mathrm{H} & 2.997663 & -0.068827 & -0.891660\end{array}$

p-methoxyphenol, trans $\mathrm{S}_{1}$

$\begin{array}{lrrc}\mathrm{C} & 0.121884 & -0.053727 & -1.388400 \\ \mathrm{C} & -1.276846 & -0.017639 & -1.215589 \\ \mathrm{C} & -1.778540 & 0.027565 & 0.101167 \\ \mathrm{C} & -0.957767 & 0.057985 & 1.244663 \\ \mathrm{C} & 0.442479 & 0.033762 & 1.075221 \\ \mathrm{C} & 0.950507 & -0.018135 & -0.241016 \\ \mathrm{O} & -3.102922 & 0.050375 & 0.325503 \\ \mathrm{H} & 1.096606 & 0.059235 & 1.930755 \\ \mathrm{O} & 2.260010 & -0.043332 & -0.513247 \\ \mathrm{H} & 0.587111 & -0.093626 & -2.360860\end{array}$




$\begin{array}{lrrr}\mathrm{H} & -1.953590 & -0.047425 & -2.059015 \\ \mathrm{H} & -3.576789 & 0.033731 & -0.514801 \\ \mathrm{H} & -1.419409 & 0.098078 & 2.219131 \\ \mathrm{C} & 3.213944 & -0.015400 & 0.539045 \\ \mathrm{H} & 4.186092 & -0.043815 & 0.056188 \\ \mathrm{H} & 3.118870 & 0.900494 & 1.123302 \\ \mathrm{H} & 3.099150 & -0.883817 & 1.188655\end{array}$

1-naphthol, cis $\mathrm{S}_{0}$

$\begin{array}{lrrr}\mathrm{C} & 0.825817 & 1.513809 & 0.012161 \\ \mathrm{C} & 0.524045 & 0.123155 & -0.002309 \\ \mathrm{C} & -0.841040 & -0.266313 & -0.005030 \\ \mathrm{C} & -1.852049 & 0.720826 & -0.015333 \\ \mathrm{C} & -1.517745 & 2.042902 & -0.020849 \\ \mathrm{C} & -0.172332 & 2.445878 & -0.004073 \\ \mathrm{C} & 1.518222 & -0.883582 & -0.019790 \\ \mathrm{C} & 1.182981 & -2.205926 & -0.018801 \\ \mathrm{C} & -0.170567 & -2.593293 & -0.006221 \\ \mathrm{C} & -1.151483 & -1.646873 & -0.002690 \\ \mathrm{O} & 2.110317 & 1.967521 & 0.025287 \\ \mathrm{H} & -2.194352 & -1.939881 & -0.000243 \\ \mathrm{H} & -0.427126 & -3.644735 & -0.004765 \\ \mathrm{H} & 1.958089 & -2.960901 & -0.034296 \\ \mathrm{H} & 2.570120 & -0.623204 & -0.050246 \\ \mathrm{H} & -2.888974 & 0.410313 & -0.020405 \\ \mathrm{H} & -2.291544 & 2.799648 & -0.031477 \\ \mathrm{H} & 0.094999 & 3.493751 & 0.004367 \\ \mathrm{H} & 2.722631 & 1.246916 & 0.194710\end{array}$

1-naphthol, cis $\mathrm{S}_{1}$

$\begin{array}{lrrr}\text { C } & 0.866857 & 1.462420 & 0.015677 \\ \text { C } & 0.536204 & 0.090161 & -0.053035 \\ \text { C } & -0.850381 & -0.246657 & 0.013282 \\ \text { C } & -1.791067 & 0.797582 & -0.013948 \\ \text { C } & -1.421148 & 2.164323 & -0.026919 \\ \text { C } & -0.103319 & 2.503748 & -0.033632 \\ \text { C } & 1.498180 & -0.928875 & -0.121149 \\ \text { C } & 1.090849 & -2.291432 & -0.051283 \\ \text { C } & -0.233445 & -2.614346 & 0.038308 \\ \text { C } & -1.223445 & -1.599637 & 0.054496 \\ \text { O } & 2.137203 & 1.861711 & 0.134025 \\ \text { H } & -2.271302 & -1.859924 & 0.116826 \\ \text { H } & -0.537068 & -3.651629 & 0.090713 \\ \text { H } & 1.843114 & -3.068579 & -0.077384\end{array}$




$\begin{array}{rrrr}\mathrm{H} & 2.544698 & -0.710522 & -0.296205 \\ \mathrm{H} & -2.843233 & 0.541203 & -0.018529 \\ \mathrm{H} & -2.188063 & 2.925760 & -0.055116 \\ \mathrm{H} & 0.242052 & 3.527693 & -0.027122 \\ \mathrm{H} & 2.703315 & 1.096990 & 0.310974\end{array}$

1-naphthol, trans $\mathrm{S}_{0}$

$\begin{array}{lrrl}\mathrm{C} & -0.712753 & -0.538719 & 0.000000 \\ \mathrm{C} & -0.670250 & -1.952335 & 0.000000 \\ \mathrm{C} & 0.519841 & -2.619299 & 0.000000 \\ \mathrm{C} & 1.734530 & -1.906443 & 0.000000 \\ \mathrm{C} & 1.732185 & -0.541578 & 0.000000 \\ \mathrm{C} & 0.512663 & 0.171079 & 0.000000 \\ \mathrm{H} & -1.605412 & -2.499110 & 0.000000 \\ \mathrm{H} & 0.534700 & -3.701804 & 0.000000 \\ \mathrm{H} & 2.672567 & -2.446426 & 0.000000 \\ \mathrm{H} & 2.659004 & 0.014388 & 0.000000 \\ \mathrm{C} & -1.935421 & 0.175664 & 0.000000 \\ \mathrm{C} & -1.932970 & 1.537565 & 0.000000 \\ \mathrm{C} & -0.721842 & 2.256931 & 0.000000 \\ \mathrm{C} & 0.470713 & 1.591043 & 0.000000 \\ \mathrm{H} & -2.866910 & -0.375912 & 0.000000 \\ \mathrm{H} & -2.867076 & 2.084365 & 0.000000 \\ \mathrm{H} & -0.738040 & 3.341135 & 0.000000 \\ \mathrm{O} & 1.674970 & 2.228541 & 0.000000 \\ \mathrm{H} & 1.539511 & 3.180904 & 0.000000\end{array}$

1-naphthol, trans $\mathrm{S}_{1}$

$\begin{array}{lrrr}\mathrm{C} & -0.941877 & -0.392248 & 0.000000 \\ \mathrm{C} & -1.303061 & -1.745262 & 0.000000 \\ \mathrm{C} & -0.312576 & -2.763182 & 0.000000 \\ \mathrm{C} & 1.011187 & -2.423905 & 0.000000 \\ \mathrm{C} & 1.413322 & -1.060588 & 0.000000 \\ \mathrm{C} & 0.450162 & -0.044876 & 0.000000 \\ \mathrm{H} & -2.352320 & -2.010083 & 0.000000 \\ \mathrm{H} & -0.614079 & -3.801914 & 0.000000 \\ \mathrm{H} & 1.772408 & -3.193303 & 0.000000 \\ \mathrm{H} & 2.462002 & -0.803688 & 0.000000 \\ \mathrm{C} & -1.889808 & 0.644091 & 0.000000 \\ \mathrm{C} & -1.519399 & 2.012600 & 0.000000 \\ \mathrm{C} & -0.203086 & 2.359926 & 0.000000 \\ \mathrm{C} & 0.779902 & 1.324231 & 0.000000 \\ \mathrm{H} & -2.941534 & 0.386646 & 0.000000 \\ \mathrm{H} & -2.288901 & 2.772469 & 0.000000\end{array}$




$\begin{array}{llll}\mathrm{H} & 0.112739 & 3.396359 & 0.000000 \\ \mathrm{O} & 2.087939 & 1.642357 & 0.000000 \\ \mathrm{H} & 2.201301 & 2.599445 & 0.000000\end{array}$

2-naphthol, cis $\mathrm{S}_{0}$

$\begin{array}{rrrr}\mathrm{C} & 0.594244 & 2.004333 & 0.000000 \\ \mathrm{C} & 0.617687 & 0.592309 & 0.000000 \\ \mathrm{C} & -0.608081 & -0.119094 & 0.000000 \\ \mathrm{C} & -1.816781 & 0.614688 & 0.000000 \\ \mathrm{C} & -1.806826 & 1.979556 & 0.000000 \\ \mathrm{C} & -0.588371 & 2.685648 & 0.000000 \\ \mathrm{C} & 1.826552 & -0.144668 & 0.000000 \\ \mathrm{C} & 1.825741 & -1.504830 & 0.000000 \\ \mathrm{C} & 0.601849 & -2.206518 & 0.000000 \\ \mathrm{C} & -0.585602 & -1.533254 & 0.000000 \\ \mathrm{H} & -1.525006 & -2.076144 & 0.000000 \\ \mathrm{H} & 1.535721 & 2.540714 & 0.000000 \\ \mathrm{H} & -0.594577 & 3.767819 & 0.000000 \\ \mathrm{H} & -2.741190 & 2.526316 & 0.000000 \\ \mathrm{H} & -2.756475 & 0.075405 & 0.000000 \\ \mathrm{H} & 2.766411 & 0.394055 & 0.000000 \\ \mathrm{H} & 2.745609 & -2.073674 & 0.000000 \\ \mathrm{O} & 0.694438 & -3.566383 & 0.000000 \\ \mathrm{H} & -0.185352 & -3.956266 & 0.000000\end{array}$

2-naphthol, cis $\mathrm{S}_{1}$

$\begin{array}{rrrr}\mathrm{C} & 0.599708 & 1.984720 & 0.000000 \\ \mathrm{C} & 0.644140 & 0.585418 & 0.000000 \\ \mathrm{C} & -0.625850 & -0.117665 & 0.000000 \\ \mathrm{C} & -1.826833 & 0.606229 & 0.000000 \\ \mathrm{C} & -1.836265 & 2.013455 & 0.000000 \\ \mathrm{C} & -0.626091 & 2.680267 & 0.000000 \\ \mathrm{C} & 1.844628 & -0.149834 & 0.000000 \\ \mathrm{C} & 1.874854 & -1.551181 & 0.000000 \\ \mathrm{C} & 0.656914 & -2.211285 & 0.000000 \\ \mathrm{C} & -0.581128 & -1.515810 & 0.000000 \\ \mathrm{H} & -1.503973 & -2.086903 & 0.000000 \\ \mathrm{H} & 1.526709 & 2.543941 & 0.000000 \\ \mathrm{H} & -0.606628 & 3.763077 & 0.000000 \\ \mathrm{H} & -2.769867 & 2.556717 & 0.000000 \\ \mathrm{H} & -2.762354 & 0.059909 & 0.000000 \\ \mathrm{H} & 2.778167 & 0.398997 & 0.000000 \\ \mathrm{H} & 2.795698 & -2.113056 & 0.000000 \\ \mathrm{O} & 0.660621 & -3.554346 & 0.000000\end{array}$


$\begin{array}{llll}\mathrm{H} & -0.242442 & -3.892639 & 0.000000\end{array}$

2-naphthol, trans $\mathrm{S}_{0}$

$\begin{array}{rrrr}\text { C } & 0.507207 & 2.006428 & 0.000000 \\ \mathrm{C} & 0.536291 & 0.592943 & 0.000000 \\ \mathrm{C} & -0.687118 & -0.125515 & 0.000000 \\ \mathrm{C} & -1.899781 & 0.605351 & 0.000000 \\ \mathrm{C} & -1.894555 & 1.968972 & 0.000000 \\ \mathrm{C} & -0.677685 & 2.681260 & 0.000000 \\ \mathrm{C} & 1.745285 & -0.137230 & 0.000000 \\ \mathrm{C} & 1.745306 & -1.500264 & 0.000000 \\ \mathrm{C} & 0.524740 & -2.207408 & 0.000000 \\ \mathrm{C} & -0.663617 & -1.536156 & 0.000000 \\ \mathrm{H} & -1.589005 & -2.097200 & 0.000000 \\ \mathrm{H} & 1.446297 & 2.547069 & 0.000000 \\ \mathrm{H} & -0.689256 & 3.763414 & 0.000000 \\ \mathrm{H} & -2.830803 & 2.512531 & 0.000000 \\ \mathrm{H} & -2.836411 & 0.061246 & 0.000000 \\ \mathrm{H} & 2.684513 & 0.402487 & 0.000000 \\ \mathrm{H} & 2.681545 & -2.047890 & 0.000000 \\ \mathrm{O} & 0.500049 & -3.571640 & 0.000000 \\ \mathrm{H} & 1.397008 & -3.918398 & 0.000000\end{array}$

2-naphthol, trans $\mathrm{S}_{1}$

$\begin{array}{rrrr}\mathrm{C} & 0.511268 & 1.989097 & 0.000000 \\ \mathrm{C} & 0.558321 & 0.590120 & 0.000000 \\ \mathrm{C} & -0.707049 & -0.117636 & 0.000000 \\ \mathrm{C} & -1.908808 & 0.602430 & 0.000000 \\ \mathrm{C} & -1.922353 & 2.011311 & 0.000000 \\ \mathrm{C} & -0.717057 & 2.682088 & 0.000000 \\ \mathrm{C} & 1.761381 & -0.140051 & 0.000000 \\ \mathrm{C} & 1.793429 & -1.543487 & 0.000000 \\ \mathrm{C} & 0.573060 & -2.205666 & 0.000000 \\ \mathrm{C} & -0.663154 & -1.516622 & 0.000000 \\ \mathrm{H} & -1.570366 & -2.106089 & 0.000000 \\ \mathrm{H} & 1.436923 & 2.550735 & 0.000000 \\ \mathrm{H} & -0.700847 & 3.764946 & 0.000000 \\ \mathrm{H} & -2.858409 & 2.550587 & 0.000000 \\ \mathrm{H} & -2.842256 & 0.053016 & 0.000000 \\ \mathrm{H} & 2.693762 & 0.410799 & 0.000000 \\ \mathrm{H} & 2.727056 & -2.089399 & 0.000000 \\ \mathrm{O} & 0.477497 & -3.545821 & 0.000000 \\ \mathrm{H} & 1.357605 & -3.940351 & 0.000000\end{array}$


5-methoxysalicylic acid $\mathrm{S}_{0}$

$\begin{array}{lrrc}\mathrm{C} & 2.271540 & -0.165280 & 0.000000 \\ \mathrm{C} & 1.561506 & -1.335905 & 0.000000 \\ \mathrm{C} & 0.160623 & -1.329833 & 0.000000 \\ \mathrm{C} & -0.506443 & -0.129256 & 0.000000 \\ \mathrm{C} & 0.212994 & 1.079716 & 0.000000 \\ \mathrm{C} & 1.611308 & 1.067292 & 0.000000 \\ \mathrm{O} & -0.433487 & -2.554681 & 0.000000 \\ \mathrm{C} & -1.843735 & -2.605192 & 0.000000 \\ \mathrm{C} & -0.478430 & 2.365512 & 0.000000 \\ \mathrm{O} & -1.817090 & 2.287719 & 0.000000 \\ \mathrm{O} & 2.363344 & 2.177246 & 0.000000 \\ \mathrm{O} & 0.081051 & 3.448468 & 0.000000 \\ \mathrm{H} & -2.108944 & -3.658908 & 0.000000 \\ \mathrm{H} & -2.261136 & -2.128638 & -0.891691 \\ \mathrm{H} & -2.261136 & -2.128638 & 0.891691 \\ \mathrm{H} & -1.583765 & -0.079573 & 0.000000 \\ \mathrm{H} & 3.352847 & -0.165756 & 0.000000 \\ \mathrm{H} & 2.068713 & -2.291768 & 0.000000 \\ \mathrm{H} & 1.764229 & 2.952123 & 0.000000 \\ \mathrm{H} & -2.153967 & 3.195354 & 0.000000\end{array}$

5-methoxysalicylic acid $\mathrm{S}_{1}$

$\begin{array}{rrrc}\mathrm{C} & 2.171237 & -0.134137 & 0.000000 \\ \mathrm{C} & 1.725428 & -1.452908 & 0.000000 \\ \mathrm{C} & 0.338802 & -1.679005 & 0.000000 \\ \mathrm{C} & -0.598140 & -0.616736 & 0.000000 \\ \mathrm{C} & -0.163555 & 0.704034 & 0.000000 \\ \mathrm{C} & 1.269569 & 0.929075 & 0.000000 \\ \mathrm{O} & -0.032804 & -2.960491 & 0.000000 \\ \mathrm{C} & -1.409883 & -3.320591 & 0.000000 \\ \mathrm{C} & -1.034489 & 1.834032 & 0.000000 \\ \mathrm{O} & -2.361149 & 1.554206 & 0.000000 \\ \mathrm{O} & 1.714831 & 2.167636 & 0.000000 \\ \mathrm{O} & -0.647862 & 3.025337 & 0.000000 \\ \mathrm{H} & -1.426676 & -4.405896 & 0.000000 \\ \mathrm{H} & -1.909553 & -2.945068 & -0.893410 \\ \mathrm{H} & -1.909553 & -2.945068 & 0.893410 \\ \mathrm{H} & -1.658309 & -0.814457 & 0.000000 \\ \mathrm{H} & 3.225999 & 0.103759 & 0.000000 \\ \mathrm{H} & 2.400305 & -2.294438 & 0.000000 \\ \mathrm{H} & 0.895794 & 2.775492 & 0.000000 \\ \mathrm{H} & -2.817938 & 2.406000 & 0.000000\end{array}$


m-dimethoxybenzene, trans $\mathrm{S}_{0}$

$\begin{array}{rrrr}\mathrm{C} & 0.000000 & -0.130248 & 0.704691 \\ \mathrm{C} & 0.000000 & -0.130248 & -0.704691 \\ \mathrm{C} & 0.000000 & 1.072673 & -1.384407 \\ \mathrm{C} & 0.000000 & 2.279784 & -0.687682 \\ \mathrm{C} & 0.000000 & 2.279784 & 0.687682 \\ \mathrm{C} & 0.000000 & 1.072673 & 1.384407 \\ \mathrm{O} & 0.000000 & -1.351252 & -1.292315 \\ \mathrm{H} & 0.000000 & 1.084409 & -2.464339 \\ \mathrm{H} & 0.000000 & 3.211405 & -1.237717 \\ \mathrm{H} & 0.000000 & 3.211405 & 1.237717 \\ \mathrm{H} & 0.000000 & 1.084409 & 2.464339 \\ \mathrm{O} & 0.000000 & -1.351252 & 1.292315 \\ \mathrm{C} & 0.000000 & -1.411814 & 2.701415 \\ \mathrm{H} & 0.000000 & -2.468304 & 2.955848 \\ \mathrm{H} & 0.891574 & -0.939933 & 3.124002 \\ \mathrm{H} & -0.891573 & -0.939933 & 3.124002 \\ \mathrm{C} & 0.000000 & -1.411814 & -2.701415 \\ \mathrm{H} & 0.000000 & -2.468304 & -2.955848 \\ \mathrm{H} & -0.891573 & -0.939933 & -3.124002 \\ \mathrm{H} & 0.891574 & -0.939933 & -3.124002\end{array}$

$m$-dimethoxybenzene, trans $\mathrm{S}_{1}$

$\begin{array}{rrrr}\text { C } & -0.105744 & -1.065382 & -1.442139 \\ \mathrm{C} & -0.236637 & 0.155905 & -0.706583 \\ \mathrm{C} & -0.236637 & 0.155905 & 0.706583 \\ \mathrm{C} & -0.105744 & -1.065382 & 1.442139 \\ \mathrm{C} & 0.271177 & -2.191474 & 0.703569 \\ \mathrm{C} & 0.271177 & -2.191474 & -0.703569 \\ \mathrm{O} & -0.195077 & 1.367415 & -1.287685 \\ \mathrm{C} & -0.017584 & 1.459201 & -2.693605 \\ \mathrm{O} & -0.195077 & 1.367415 & 1.287685 \\ \mathrm{C} & -0.017584 & 1.459201 & 2.693605 \\ \mathrm{H} & -0.337620 & -1.129070 & 2.494005 \\ \mathrm{H} & 0.541425 & -3.104908 & 1.220805 \\ \mathrm{H} & 0.541425 & -3.104908 & -1.220805 \\ \mathrm{H} & -0.337620 & -1.129070 & -2.494005 \\ \mathrm{H} & 0.031663 & 2.521898 & -2.913155 \\ \mathrm{H} & 0.905274 & 0.970640 & -3.007980 \\ \mathrm{H} & -0.863787 & 1.018214 & -3.223481 \\ \mathrm{H} & 0.031663 & 2.521898 & 2.913155 \\ \mathrm{H} & -0.863787 & 1.018214 & 3.223481 \\ \mathrm{H} & 0.905274 & 0.970640 & 3.007980\end{array}$


3P-propionic acid, gauche $\mathrm{S}_{0}$

$\begin{array}{rrrr}\mathrm{C} & -1.031237 & 0.900857 & -1.163368 \\ \mathrm{C} & -0.387874 & 0.662816 & 0.047287 \\ \mathrm{C} & -1.048459 & -0.089094 & 1.012899 \\ \mathrm{C} & -2.317795 & -0.591587 & 0.772144 \\ \mathrm{C} & -2.947077 & -0.349887 & -0.438167 \\ \mathrm{C} & -2.299136 & 0.399473 & -1.407538 \\ \mathrm{C} & 1.001175 & 1.198460 & 0.289916 \\ \mathrm{C} & 2.104942 & 0.278745 & -0.231319 \\ \mathrm{C} & 2.309852 & -0.947603 & 0.615281 \\ \mathrm{O} & 1.997096 & -1.068042 & 1.768414 \\ \mathrm{O} & 2.941060 & -1.925872 & -0.062189 \\ \mathrm{H} & 1.917001 & -0.031957 & -1.259125 \\ \mathrm{H} & 3.064494 & 0.804459 & -0.237530 \\ \mathrm{H} & 1.155314 & 1.363572 & 1.356130 \\ \mathrm{H} & 1.103821 & 2.165597 & -0.204777 \\ \mathrm{H} & -0.556343 & -0.286726 & 1.956567 \\ \mathrm{H} & -2.817845 & -1.173309 & 1.536272 \\ \mathrm{H} & -3.939470 & -0.740093 & -0.624439 \\ \mathrm{H} & -2.784936 & 0.599498 & -2.354285 \\ \mathrm{H} & -0.535635 & 1.494908 & -1.923327 \\ \mathrm{H} & 3.071061 & -2.664234 & 0.551163\end{array}$

3P-propionic acid, gauche $\mathrm{S}_{1}$

$\begin{array}{rrrr}\mathrm{C} & -1.064518 & 0.994597 & -1.171777 \\ \mathrm{C} & -0.360253 & 0.695808 & 0.027104 \\ \mathrm{C} & -0.996935 & -0.088640 & 1.027644 \\ \mathrm{C} & -2.306508 & -0.585182 & 0.824392 \\ \mathrm{C} & -2.989797 & -0.292679 & -0.377891 \\ \mathrm{C} & -2.370341 & 0.491328 & -1.378705 \\ \mathrm{C} & 1.041661 & 1.174468 & 0.206128 \\ \mathrm{C} & 2.098199 & 0.180954 & -0.298957 \\ \mathrm{C} & 2.328465 & -0.972440 & 0.638669 \\ \mathrm{O} & 2.096421 & -0.981074 & 1.817085 \\ \mathrm{O} & 2.887192 & -2.024117 & 0.009989 \\ \mathrm{H} & 1.842992 & -0.206638 & -1.284854 \\ \mathrm{H} & 3.064497 & 0.684224 & -0.402085 \\ \mathrm{H} & 1.237706 & 1.375863 & 1.260773 \\ \mathrm{H} & 1.172601 & 2.110185 & -0.341847 \\ \mathrm{H} & -0.462910 & -0.309338 & 1.940795 \\ \mathrm{H} & -2.780165 & -1.189111 & 1.584657 \\ \mathrm{H} & -3.990353 & -0.670785 & -0.535105 \\ \mathrm{H} & -2.896090 & 0.708499 & -2.297446 \\ \mathrm{H} & -0.595871 & 1.613321 & -1.925146\end{array}$


$\begin{array}{llll}\text { H } & 3.044018 & -2.709255 & 0.676579\end{array}$

3P-propionic acid, anti $\mathrm{S}_{0}$

$\begin{array}{rrrr}\mathrm{C} & 1.453981 & -0.447375 & 0.000000 \\ \mathrm{C} & 0.541157 & 0.774888 & 0.000000 \\ \mathrm{C} & 2.912935 & -0.089913 & 0.000000 \\ \mathrm{H} & 1.264058 & -1.080334 & 0.869883 \\ \mathrm{H} & 1.264058 & -1.080334 & -0.869883 \\ \mathrm{C} & -0.914488 & 0.387330 & 0.000000 \\ \mathrm{H} & 0.766447 & 1.389373 & 0.872198 \\ \mathrm{H} & 0.766447 & 1.389373 & -0.872198 \\ \mathrm{C} & -1.598828 & 0.189804 & 1.194256 \\ \mathrm{C} & -2.928831 & -0.198901 & 1.197186 \\ \mathrm{C} & -3.598243 & -0.396049 & 0.000000 \\ \mathrm{C} & -2.928831 & -0.198901 & -1.197186 \\ \mathrm{C} & -1.598828 & 0.189804 & -1.194256 \\ \mathrm{H} & -1.085111 & 0.350415 & 2.135347 \\ \mathrm{H} & -3.445512 & -0.343375 & 2.137618 \\ \mathrm{H} & -4.638156 & -0.696227 & 0.000000 \\ \mathrm{H} & -3.445512 & -0.343375 & -2.137618 \\ \mathrm{H} & -1.085111 & 0.350415 & -2.135347 \\ \mathrm{O} & 3.695742 & -1.188598 & 0.000000 \\ \mathrm{O} & 3.369502 & 1.020105 & 0.000000 \\ \mathrm{H} & 4.615964 & -0.886803 & 0.000000\end{array}$

3P-propionic acid, anti $\mathrm{S}_{1}$

$\begin{array}{rrrr}\mathrm{C} & 1.461858 & -0.434996 & 0.000000 \\ \mathrm{C} & 0.557058 & 0.802192 & 0.000000 \\ \mathrm{C} & 2.922388 & -0.083999 & 0.000000 \\ \mathrm{H} & 1.265122 & -1.063378 & 0.871124 \\ \mathrm{H} & 1.265122 & -1.063378 & -0.871124 \\ \mathrm{C} & -0.887568 & 0.436574 & 0.000000 \\ \mathrm{H} & 0.792508 & 1.408755 & 0.876489 \\ \mathrm{H} & 0.792508 & 1.408755 & -0.876489 \\ \mathrm{C} & -1.575885 & 0.230082 & 1.226310 \\ \mathrm{C} & -2.919184 & -0.213877 & 1.227736 \\ \mathrm{C} & -3.586022 & -0.431083 & 0.000000 \\ \mathrm{C} & -2.919184 & -0.213877 & -1.227736 \\ \mathrm{C} & -1.575885 & 0.230082 & -1.226310 \\ \mathrm{H} & -1.063924 & 0.417465 & 2.160534 \\ \mathrm{H} & -3.435198 & -0.383785 & 2.161536 \\ \mathrm{H} & -4.614749 & -0.763779 & 0.000000 \\ \mathrm{H} & -3.435198 & -0.383785 & -2.161536 \\ \mathrm{H} & -1.063924 & 0.417465 & -2.160534\end{array}$




$\begin{array}{rrrr}\mathrm{O} & 3.698460 & -1.187054 & 0.000000 \\ \mathrm{O} & 3.383607 & 1.024017 & 0.000000 \\ \mathrm{H} & 4.620715 & -0.891237 & 0.000000\end{array}$

fluorine $\mathrm{S}_{0}$

$\begin{array}{rrrr}\mathrm{C} & 0.000000 & 0.000000 & -1.787580 \\ \mathrm{C} & 0.000000 & 1.178043 & -0.845990 \\ \mathrm{C} & 0.000000 & 0.733455 & 0.480116 \\ \mathrm{C} & 0.000000 & -0.733455 & 0.480116 \\ \mathrm{C} & 0.000000 & 1.642463 & 1.526259 \\ \mathrm{C} & 0.000000 & 2.998209 & 1.235301 \\ \mathrm{C} & 0.000000 & 3.439266 & -0.081898 \\ \mathrm{C} & 0.000000 & 2.529108 & -1.131295 \\ \mathrm{H} & 0.000000 & 2.879716 & -2.156537 \\ \mathrm{H} & 0.000000 & 4.501283 & -0.291474 \\ \mathrm{H} & 0.000000 & 3.720603 & 2.041570 \\ \mathrm{H} & 0.000000 & 1.305275 & 2.555417 \\ \mathrm{C} & 0.000000 & -1.642463 & 1.526259 \\ \mathrm{C} & 0.000000 & -2.998209 & 1.235301 \\ \mathrm{C} & 0.000000 & -3.439266 & -0.081898 \\ \mathrm{C} & 0.000000 & -2.529108 & -1.131295 \\ \mathrm{C} & 0.000000 & -1.178043 & -0.845990 \\ \mathrm{H} & 0.000000 & -1.305275 & 2.555417 \\ \mathrm{H} & 0.000000 & -3.720603 & 2.041570 \\ \mathrm{H} & 0.000000 & -4.501283 & -0.291474 \\ \mathrm{H} & 0.000000 & -2.879716 & -2.156537 \\ \mathrm{H} & -0.878852 & 0.000000 & -2.437670 \\ \mathrm{H} & 0.878852 & 0.000000 & -2.437670\end{array}$

fluorine $\mathrm{S}_{1}$

$\begin{array}{rrrr}\mathrm{C} & 0.000000 & 0.000000 & -1.865103 \\ \mathrm{C} & 0.000000 & 1.177176 & -0.917084 \\ \mathrm{C} & 0.000000 & 0.693799 & 0.450512 \\ \mathrm{C} & 0.000000 & -0.693799 & 0.450512 \\ \mathrm{C} & 0.000000 & 1.636378 & 1.531691 \\ \mathrm{C} & 0.000000 & 2.970821 & 1.227402 \\ \mathrm{C} & 0.000000 & 3.425738 & -0.106303 \\ \mathrm{C} & 0.000000 & 2.515629 & -1.180924 \\ \mathrm{H} & 0.000000 & 2.883794 & -2.199754 \\ \mathrm{H} & 0.000000 & 4.489407 & -0.304757 \\ \mathrm{H} & 0.000000 & 3.702949 & 2.025402 \\ \mathrm{H} & 0.000000 & 1.293306 & 2.557615 \\ \mathrm{C} & 0.000000 & -1.636378 & 1.531691 \\ \mathrm{C} & 0.000000 & -2.970821 & 1.227402\end{array}$




$\begin{array}{rrrr}\mathrm{C} & 0.000000 & -3.425738 & -0.106303 \\ \mathrm{C} & 0.000000 & -2.515629 & -1.180924 \\ \mathrm{C} & 0.000000 & -1.177176 & -0.917084 \\ \mathrm{H} & 0.000000 & -1.293306 & 2.557615 \\ \mathrm{H} & 0.000000 & -3.702949 & 2.025402 \\ \mathrm{H} & 0.000000 & -4.489407 & -0.304757 \\ \mathrm{H} & 0.000000 & -2.883794 & -2.199754 \\ \mathrm{H} & -0.877899 & 0.000000 & -2.519086 \\ \mathrm{H} & 0.877899 & 0.000000 & -2.519086\end{array}$

phenanthrene $\mathrm{S}_{0}$

$\begin{array}{lrrr}\mathrm{C} & 0.726726 & 0.000000 & -0.347651 \\ \mathrm{C} & -0.726726 & 0.000000 & -0.347651 \\ \mathrm{C} & -1.413368 & 0.000000 & 0.884912 \\ \mathrm{C} & -0.672691 & 0.000000 & 2.110532 \\ \mathrm{C} & 0.672691 & 0.000000 & 2.110532 \\ \mathrm{C} & 1.413368 & 0.000000 & 0.884912 \\ \mathrm{C} & -1.490475 & 0.000000 & -1.528679 \\ \mathrm{C} & -2.819359 & 0.000000 & 0.898237 \\ \mathrm{H} & -1.223862 & 0.000000 & 3.043133 \\ \mathrm{H} & 1.223862 & 0.000000 & 3.043133 \\ \mathrm{C} & 1.490475 & 0.000000 & -1.528679 \\ \mathrm{C} & 2.861570 & 0.000000 & -1.493127 \\ \mathrm{C} & 3.536901 & 0.000000 & -0.268036 \\ \mathrm{C} & 2.819359 & 0.000000 & 0.898237 \\ \mathrm{H} & 0.997549 & 0.000000 & -2.490163 \\ \mathrm{H} & 3.422963 & 0.000000 & -2.418624 \\ \mathrm{H} & 4.618963 & 0.000000 & -0.244586 \\ \mathrm{H} & 3.329371 & 0.000000 & 1.854143 \\ \mathrm{C} & -3.536901 & 0.000000 & -0.268036 \\ \mathrm{C} & -2.861570 & 0.000000 & -1.493127 \\ \mathrm{H} & -0.997549 & 0.000000 & -2.490163 \\ \mathrm{H} & -3.422963 & 0.000000 & -2.418624 \\ \mathrm{H} & -4.618963 & 0.000000 & -0.244586 \\ \mathrm{H} & -3.329371 & 0.000000 & 1.854143\end{array}$

phenanthrene $S_{1}$

$\begin{array}{lrrr}\mathrm{C} & 0.709519 & 0.000000 & -0.387242 \\ \mathrm{C} & -0.709519 & 0.000000 & -0.387242 \\ \mathrm{C} & -1.429730 & 0.000000 & 0.882422 \\ \mathrm{C} & -0.692451 & 0.000000 & 2.076318 \\ \mathrm{C} & 0.692451 & 0.000000 & 2.076318 \\ \mathrm{C} & 1.429730 & 0.000000 & 0.882422 \\ \mathrm{C} & -1.489233 & 0.000000 & -1.566172\end{array}$




$\begin{array}{lrrr}\mathrm{C} & -2.831483 & 0.000000 & 0.882826 \\ \mathrm{H} & -1.227732 & 0.000000 & 3.017858 \\ \mathrm{H} & 1.227732 & 0.000000 & 3.017858 \\ \mathrm{C} & 1.489233 & 0.000000 & -1.566172 \\ \mathrm{C} & 2.872888 & 0.000000 & -1.523173 \\ \mathrm{C} & 3.556527 & 0.000000 & -0.299424 \\ \mathrm{C} & 2.831483 & 0.000000 & 0.882826 \\ \mathrm{H} & 1.005009 & 0.000000 & -2.531474 \\ \mathrm{H} & 3.431670 & 0.000000 & -2.449948 \\ \mathrm{H} & 4.637455 & 0.000000 & -0.278352 \\ \mathrm{H} & 3.347192 & 0.000000 & 1.835401 \\ \mathrm{C} & -3.556527 & 0.000000 & -0.299424 \\ \mathrm{C} & -2.872888 & 0.000000 & -1.523173 \\ \mathrm{H} & -1.005009 & 0.000000 & -2.531474 \\ \mathrm{H} & -3.431670 & 0.000000 & -2.449948 \\ \mathrm{H} & -4.637455 & 0.000000 & -0.278352 \\ \mathrm{H} & -3.347192 & 0.000000 & 1.835401\end{array}$

2,4,6,8-decatetraene $\mathrm{S}_{0}$

$\begin{array}{crcc}\mathrm{C} & -2.584003 & -4.998839 & 0.000000 \\ \mathrm{C} & -2.459872 & -3.512210 & 0.000000 \\ \mathrm{C} & -1.310598 & -2.839535 & 0.000000 \\ \mathrm{C} & -1.207364 & -1.395444 & 0.000000 \\ \mathrm{C} & -0.051613 & -0.719831 & 0.000000 \\ \mathrm{C} & 0.051613 & 0.719831 & 0.000000 \\ \mathrm{C} & 1.207364 & 1.395444 & 0.000000 \\ \mathrm{C} & 1.310598 & 2.839535 & 0.000000 \\ \mathrm{C} & 2.459872 & 3.512210 & 0.000000 \\ \mathrm{C} & 2.584003 & 4.998839 & 0.000000 \\ \mathrm{H} & -0.374619 & -3.393339 & 0.000000 \\ \mathrm{H} & -2.141615 & -0.839436 & 0.000000 \\ \mathrm{H} & 0.882237 & -1.276547 & 0.000000 \\ \mathrm{H} & -0.882237 & 1.276547 & 0.000000 \\ \mathrm{H} & 2.141615 & 0.839436 & 0.000000 \\ \mathrm{H} & 0.374619 & 3.393339 & 0.000000 \\ \mathrm{H} & 3.390332 & 2.949512 & 0.000000 \\ \mathrm{H} & -3.390332 & -2.949512 & 0.000000 \\ \mathrm{H} & 1.606111 & 5.481032 & 0.000000 \\ \mathrm{H} & 3.135261 & 5.348146 & 0.876907 \\ \mathrm{H} & 3.135261 & 5.348146 & -0.876907 \\ \mathrm{H} & -1.606111 & -5.481032 & 0.000000 \\ \mathrm{H} & -3.135261 & -5.348146 & 0.876907 \\ \mathrm{H} & -3.135261 & -5.348146 & -0.876907\end{array}$

2,4,6,8-decatetraene $\mathrm{S}_{1}$ 


$\begin{array}{crcc}\mathrm{C} & -2.571448 & -5.008247 & 0.000000 \\ \mathrm{C} & -2.509330 & -3.526186 & 0.000000 \\ \mathrm{C} & -1.352108 & -2.802873 & 0.000000 \\ \mathrm{C} & -1.264488 & -1.400912 & 0.000000 \\ \mathrm{C} & -0.053807 & -0.691272 & 0.000000 \\ \mathrm{C} & 0.053807 & 0.691272 & 0.000000 \\ \mathrm{C} & 1.264488 & 1.400912 & 0.000000 \\ \mathrm{C} & 1.352108 & 2.802873 & 0.000000 \\ \mathrm{C} & 2.509330 & 3.526186 & 0.000000 \\ \mathrm{C} & 2.571448 & 5.008247 & 0.000000 \\ \mathrm{H} & -0.412680 & -3.352765 & 0.000000 \\ \mathrm{H} & -2.189008 & -0.829778 & 0.000000 \\ \mathrm{H} & 0.865591 & -1.271607 & 0.000000 \\ \mathrm{H} & -0.865591 & 1.271607 & 0.000000 \\ \mathrm{H} & 2.189008 & 0.829778 & 0.000000 \\ \mathrm{H} & 0.412680 & 3.352765 & 0.000000 \\ \mathrm{H} & 3.454885 & 2.990065 & 0.000000 \\ \mathrm{H} & -3.454885 & -2.990065 & 0.000000 \\ \mathrm{H} & 1.574710 & 5.451261 & 0.000000 \\ \mathrm{H} & 3.111568 & 5.390810 & 0.874652 \\ \mathrm{H} & 3.111568 & 5.390810 & -0.874652 \\ \mathrm{H} & -1.574710 & -5.451261 & 0.000000 \\ \mathrm{H} & -3.111568 & -5.390810 & 0.874652 \\ \mathrm{H} & -3.111568 & -5.390810 & -0.874652\end{array}$

tetracene $\mathrm{S}_{0}$

$\begin{array}{rrrr}\mathrm{C} & 3.690563 & 1.400776 & 0.000000 \\ \mathrm{C} & 2.431230 & 0.719368 & 0.000000 \\ \mathrm{C} & 2.431230 & -0.719368 & 0.000000 \\ \mathrm{C} & 3.690563 & -1.400776 & 0.000000 \\ \mathrm{C} & 4.854769 & -0.713899 & 0.000000 \\ \mathrm{C} & 4.854769 & 0.713899 & 0.000000 \\ \mathrm{C} & 1.228704 & 1.395845 & 0.000000 \\ \mathrm{C} & 0.000000 & 0.718241 & 0.000000 \\ \mathrm{C} & 0.000000 & -0.718241 & 0.000000 \\ \mathrm{C} & 1.228704 & -1.395845 & 0.000000 \\ \mathrm{C} & -1.228704 & 1.395845 & 0.000000 \\ \mathrm{C} & -2.431230 & 0.719368 & 0.000000 \\ \mathrm{C} & -2.431230 & -0.719368 & 0.000000 \\ \mathrm{C} & -1.228704 & -1.395845 & 0.000000 \\ \mathrm{C} & -3.690563 & 1.400776 & 0.000000 \\ \mathrm{C} & -4.854769 & 0.713899 & 0.000000 \\ \mathrm{C} & -4.854769 & -0.713899 & 0.000000 \\ \mathrm{C} & -3.690563 & -1.400776 & 0.000000\end{array}$




$\begin{array}{rrrr}\mathrm{H} & -3.688873 & -2.484125 & 0.000000 \\ \mathrm{H} & -5.799320 & -1.242510 & 0.000000 \\ \mathrm{H} & -5.799320 & 1.242510 & 0.000000 \\ \mathrm{H} & -3.688873 & 2.484125 & 0.000000 \\ \mathrm{H} & -1.228845 & -2.480006 & 0.000000 \\ \mathrm{H} & -1.228845 & 2.480006 & 0.000000 \\ \mathrm{H} & 1.228845 & -2.480006 & 0.000000 \\ \mathrm{H} & 1.228845 & 2.480006 & 0.000000 \\ \mathrm{H} & 3.688873 & -2.484125 & 0.000000 \\ \mathrm{H} & 5.799320 & -1.242510 & 0.000000 \\ \mathrm{H} & 5.799320 & 1.242510 & 0.000000 \\ \mathrm{H} & 3.688873 & 2.484125 & 0.000000\end{array}$

tetracene $\mathrm{S}_{1}$

$\begin{array}{rrrr}\text { C } & 3.689198 & 1.390320 & 0.000000 \\ \mathrm{C} & 2.462092 & 0.715666 & 0.000000 \\ \mathrm{C} & 2.462092 & -0.715666 & 0.000000 \\ \mathrm{C} & 3.689198 & -1.390320 & 0.000000 \\ \mathrm{C} & 4.887624 & -0.695571 & 0.000000 \\ \mathrm{C} & 4.887624 & 0.695571 & 0.000000 \\ \mathrm{C} & 1.223430 & 1.392767 & 0.000000 \\ \mathrm{C} & 0.000000 & 0.724114 & 0.000000 \\ \mathrm{C} & 0.000000 & -0.724114 & 0.000000 \\ \mathrm{C} & 1.223430 & -1.392767 & 0.000000 \\ \mathrm{C} & -1.223430 & 1.392767 & 0.000000 \\ \mathrm{C} & -2.462092 & 0.715666 & 0.000000 \\ \mathrm{C} & -2.462092 & -0.715666 & 0.000000 \\ \mathrm{C} & -1.223430 & -1.392767 & 0.000000 \\ \mathrm{C} & -3.689198 & 1.390320 & 0.000000 \\ \mathrm{C} & -4.887624 & 0.695571 & 0.000000 \\ \mathrm{C} & -4.887624 & -0.695571 & 0.000000 \\ \mathrm{C} & -3.689198 & -1.390320 & 0.000000 \\ \mathrm{H} & -3.691320 & -2.473665 & 0.000000 \\ \mathrm{H} & -5.824337 & -1.237316 & 0.000000 \\ \mathrm{H} & -5.824337 & 1.237316 & 0.000000 \\ \mathrm{H} & -3.691320 & 2.473665 & 0.000000 \\ \mathrm{H} & -1.226337 & -2.477029 & 0.000000 \\ \mathrm{H} & -1.226337 & 2.477029 & 0.000000 \\ \mathrm{H} & 1.226337 & -2.477029 & 0.000000 \\ \mathrm{H} & 1.226337 & 2.477029 & 0.000000 \\ \mathrm{H} & 3.691320 & -2.473665 & 0.000000 \\ \mathrm{H} & 5.824337 & -1.237316 & 0.000000 \\ \mathrm{H} & 5.824337 & 1.237316 & 0.000000 \\ \mathrm{H} & 3.691320 & 2.473665 & 0.000000\end{array}$


perylene $\mathrm{S}_{0}$

$\begin{array}{lrrc}\mathrm{C} & 0.020469 & 1.468858 & -2.409118 \\ \mathrm{C} & 0.010705 & 0.737778 & -1.243158 \\ \mathrm{C} & 0.014976 & 1.434379 & -0.000006 \\ \mathrm{C} & 0.034825 & 2.854787 & 0.000001 \\ \mathrm{C} & 0.043954 & 3.552723 & -1.226699 \\ \mathrm{C} & 0.037065 & 2.869712 & -2.405256 \\ \mathrm{C} & 0.044042 & 3.552710 & 1.226709 \\ \mathrm{C} & 0.037244 & 2.869688 & 2.405261 \\ \mathrm{C} & 0.020627 & 1.468834 & 2.409110 \\ \mathrm{C} & 0.010750 & 0.737773 & 1.243142 \\ \mathrm{C} & -0.010705 & -0.737778 & -1.243158 \\ \mathrm{C} & -0.014976 & -1.434379 & -0.000006 \\ \mathrm{C} & -0.010750 & -0.737773 & 1.243142 \\ \mathrm{C} & -0.034825 & -2.854787 & 0.000001 \\ \mathrm{C} & -0.044042 & -3.552710 & 1.226709 \\ \mathrm{C} & -0.037244 & -2.869688 & 2.405261 \\ \mathrm{C} & -0.020627 & -1.468834 & 2.409110 \\ \mathrm{C} & -0.020469 & -1.468858 & -2.409118 \\ \mathrm{C} & -0.037065 & -2.869712 & -2.405256 \\ \mathrm{C} & -0.043954 & -3.552723 & -1.226699 \\ \mathrm{H} & 0.057471 & 4.635465 & 1.211851 \\ \mathrm{H} & 0.044807 & 3.402370 & 3.347483 \\ \mathrm{H} & 0.016543 & 0.968243 & 3.365893 \\ \mathrm{H} & -0.057471 & -4.635465 & 1.211851 \\ \mathrm{H} & -0.044807 & -3.402370 & 3.347483 \\ \mathrm{H} & -0.016543 & -0.968243 & 3.365893 \\ \mathrm{H} & -0.057381 & -4.635478 & -1.211830 \\ \mathrm{H} & -0.044546 & -3.402405 & -3.347473 \\ \mathrm{H} & -0.016298 & -0.968285 & -3.365909 \\ \mathrm{H} & 0.057381 & 4.635478 & -1.211830 \\ \mathrm{H} & 0.044546 & 3.402405 & -3.347473 \\ \mathrm{H} & 0.016298 & 0.968285 & -3.365909 \\ & & & \end{array}$

perylene $\mathrm{S}_{1}$
$\begin{array}{llll}\text { C } & -0.005708 & 1.463360 & -2.439428\end{array}$
$\begin{array}{llll}\text { C } & -0.005949 & 0.716897 & -1.234963\end{array}$
$\begin{array}{llll}\text { C } & 0.010831 & 1.414748 & 0.000000\end{array}$
$\begin{array}{llll}\text { C } & 0.007285 & 2.843320 & -0.000001\end{array}$
$\begin{array}{llll}\text { C } & 0.008726 & 3.531924 & -1.225232\end{array}$
$\begin{array}{llll}\text { C } & -0.000845 & 2.840990 & -2.424417\end{array}$
$\begin{array}{llll}\text { C } & 0.008896 & 3.531925 & 1.225227\end{array}$
$\begin{array}{llll}\text { C } & -0.000494 & 2.840990 & 2.424416\end{array}$
$\begin{array}{llll}\text { C } & -0.005408 & 1.463362 & 2.439428\end{array}$ 


$\begin{array}{rrrr}\mathrm{C} & -0.005872 & 0.716897 & 1.234964 \\ \mathrm{C} & 0.005949 & -0.716897 & -1.234963 \\ \mathrm{C} & -0.010831 & -1.414748 & 0.000000 \\ \mathrm{C} & 0.005872 & -0.716897 & 1.234964 \\ \mathrm{C} & -0.007285 & -2.843320 & -0.000001 \\ \mathrm{C} & -0.008896 & -3.531925 & 1.225227 \\ \mathrm{C} & 0.000494 & -2.840990 & 2.424416 \\ \mathrm{C} & 0.005408 & -1.463362 & 2.439428 \\ \mathrm{C} & 0.005708 & -1.463360 & -2.439428 \\ \mathrm{C} & 0.000845 & -2.840990 & -2.424417 \\ \mathrm{C} & -0.008726 & -3.531924 & -1.225232 \\ \mathrm{H} & 0.012501 & 4.614770 & 1.217489 \\ \mathrm{H} & -0.003736 & 3.386210 & 3.359586 \\ \mathrm{H} & -0.025869 & 0.954872 & 3.390632 \\ \mathrm{H} & -0.012501 & -4.614770 & 1.217489 \\ \mathrm{H} & 0.003736 & -3.386210 & 3.359586 \\ \mathrm{H} & 0.025869 & -0.954872 & 3.390632 \\ \mathrm{H} & -0.012318 & -4.614769 & -1.217494 \\ \mathrm{H} & 0.004272 & -3.386208 & -3.359588 \\ \mathrm{H} & 0.026384 & -0.954866 & -3.390625 \\ \mathrm{H} & 0.012318 & 4.614769 & -1.217494 \\ \mathrm{H} & -0.004272 & 3.386208 & -3.359588 \\ \mathrm{H} & -0.026384 & 0.954866 & -3.390625\end{array}$

$\omega$ B97X-D/TZVP geometries - Group I

pyrazine $\mathrm{S}_{0}$

$\begin{array}{rrrr}\mathrm{C} & 1.126592 & -0.694160 & 0.000000 \\ \mathrm{C} & 1.126592 & 0.694160 & 0.000000 \\ \mathrm{~N} & 0.000000 & 1.398578 & 0.000000 \\ \mathrm{C} & -1.126592 & 0.694160 & 0.000000 \\ \mathrm{C} & -1.126592 & -0.694160 & 0.000000 \\ \mathrm{~N} & 0.000000 & -1.398578 & 0.000000 \\ \mathrm{H} & -2.057654 & -1.250015 & 0.000000 \\ \mathrm{H} & 2.057654 & -1.250015 & 0.000000 \\ \mathrm{H} & 2.057654 & 1.250015 & 0.000000 \\ \mathrm{H} & -2.057654 & 1.250015 & 0.000000\end{array}$

pyrazine $\mathrm{S}_{1}$
$\begin{array}{llll}\text { C } & -1.201919 & 0.666965 & 0.000000\end{array}$
$\begin{array}{llll}\text { C } & -1.115993 & -0.736831 & 0.000000\end{array}$
$\begin{array}{llll}\mathrm{N} & 0.024036 & -1.351302 & 0.000000\end{array}$
$\begin{array}{llll}\text { C } & 1.201919 & -0.666965 & 0.000000\end{array}$ 


$\begin{array}{crrr}\mathrm{C} & 1.115993 & 0.736831 & 0.000000 \\ \mathrm{~N} & -0.024036 & 1.351302 & 0.000000 \\ \mathrm{H} & 2.043272 & 1.303340 & 0.000000 \\ \mathrm{H} & -2.154094 & 1.172365 & 0.000000 \\ \mathrm{H} & -2.043272 & -1.303340 & 0.000000 \\ \mathrm{H} & 2.154094 & -1.172365 & 0.000000\end{array}$

2,6-difluoropyridine $\mathrm{S}_{0}$

$\begin{array}{lrrr}\mathrm{C} & 1.113535 & 0.000000 & -0.874793 \\ \mathrm{~N} & 0.000000 & 0.000000 & -1.564382 \\ \mathrm{C} & -1.113535 & 0.000000 & -0.874793 \\ \mathrm{C} & -1.200200 & 0.000000 & 0.506069 \\ \mathrm{C} & 0.000000 & 0.000000 & 1.199018 \\ \mathrm{C} & 1.200200 & 0.000000 & 0.506069 \\ \mathrm{~F} & -2.235049 & 0.000000 & -1.588266 \\ \mathrm{H} & -2.160909 & 0.000000 & 0.999139 \\ \mathrm{H} & 0.000000 & 0.000000 & 2.281486 \\ \mathrm{H} & 2.160909 & 0.000000 & 0.999139 \\ \mathrm{~F} & 2.235049 & 0.000000 & -1.588266\end{array}$

2,6-difluoropyridine $\mathrm{S}_{1}$

$\begin{array}{cccr}\mathrm{C} & -1.100137 & 0.000000 & -0.336695 \\ \mathrm{~N} & 0.000000 & 0.000000 & -1.101220 \\ \mathrm{C} & 1.100137 & 0.000000 & -0.336695 \\ \mathrm{C} & 1.190973 & 0.000000 & 1.071140 \\ \mathrm{C} & 0.000000 & 0.000000 & 1.843319 \\ \mathrm{C} & -1.190973 & 0.000000 & 1.071140 \\ \mathrm{~F} & 2.234620 & 0.000000 & -1.010234 \\ \mathrm{H} & 2.175086 & 0.000000 & 1.519528 \\ \mathrm{H} & 0.000000 & 0.000000 & 2.919716 \\ \mathrm{H} & -2.175086 & 0.000000 & 1.519528 \\ \mathrm{~F} & -2.234620 & 0.000000 & -1.010234\end{array}$

2-fluoropyridine $\mathrm{S}_{0}$

$\begin{array}{lrrr}\mathrm{C} & -1.314175 & 0.502701 & 0.000000 \\ \mathrm{~N} & -1.364244 & -0.800234 & 0.000000 \\ \mathrm{C} & -0.197164 & -1.448794 & 0.000000 \\ \mathrm{C} & 1.027725 & -0.807150 & 0.000000 \\ \mathrm{C} & 1.041564 & 0.583054 & 0.000000 \\ \mathrm{C} & -0.157185 & 1.269639 & 0.000000 \\ \mathrm{H} & -0.258206 & -2.531153 & 0.000000 \\ \mathrm{H} & 1.944981 & -1.379809 & 0.000000 \\ \mathrm{H} & 1.978048 & 1.126681 & 0.000000\end{array}$




$$
\begin{array}{llll}
\mathrm{H} & -0.212562 & 2.348985 & 0.000000 \\
\mathrm{~F} & -2.488790 & 1.136072 & 0.000000
\end{array}
$$

2-fluoropyridine $S_{1}$

$$
\begin{array}{lrrr}
\mathrm{C} & -1.320113 & 0.490466 & 0.000000 \\
\mathrm{~N} & -1.435972 & -0.842879 & 0.000000 \\
\mathrm{C} & -0.225455 & -1.462101 & 0.000000 \\
\mathrm{C} & 1.028241 & -0.790934 & 0.000000 \\
\mathrm{C} & 1.105939 & 0.618223 & 0.000000 \\
\mathrm{C} & -0.151377 & 1.281085 & 0.000000 \\
\mathrm{H} & -0.248651 & -2.544628 & 0.000000 \\
\mathrm{H} & 1.926349 & -1.395311 & 0.000000 \\
\mathrm{H} & 2.042512 & 1.150884 & 0.000000 \\
\mathrm{H} & -0.251829 & 2.357157 & 0.000000 \\
\mathrm{~F} & -2.469652 & 1.138029 & 0.000000
\end{array}
$$

2-hydroxypyridine $\mathrm{S}_{0}$

$$
\begin{array}{cccc}
\mathrm{C} & -0.977282 & 0.750290 & 0.000000 \\
\mathrm{C} & -0.968870 & -0.645270 & 0.000000 \\
\mathrm{~N} & 0.130665 & -1.375633 & 0.000000 \\
\mathrm{C} & 1.301788 & -0.734813 & 0.000000 \\
\mathrm{C} & 1.415124 & 0.641237 & 0.000000 \\
\mathrm{C} & 0.242162 & 1.392300 & 0.000000 \\
\mathrm{O} & -2.145356 & -1.297677 & 0.000000 \\
\mathrm{H} & 2.183951 & -1.365466 & 0.000000 \\
\mathrm{H} & 2.388323 & 1.112262 & 0.000000 \\
\mathrm{H} & 0.282720 & 2.474694 & 0.000000 \\
\mathrm{H} & -1.915815 & 1.286413 & 0.000000 \\
\mathrm{H} & -1.937410 & -2.238338 & 0.000000
\end{array}
$$

2-hydroxypyridine $\mathrm{S}_{1}$
$\begin{array}{llll}\text { C } & -0.996238 & 0.765707 & 0.000000\end{array}$
$\begin{array}{llll}\text { C } & -0.967553 & -0.638541 & 0.000000\end{array}$
$\begin{array}{llll}\mathrm{N} & 0.119988 & -1.452990 & 0.000000\end{array}$
$\begin{array}{llll}\text { C } & 1.291613 & -0.773581 & 0.000000\end{array}$
$\begin{array}{llll}\text { C } & 1.389703 & 0.642923 & 0.000000\end{array}$
$\begin{array}{llll}\mathrm{C} & 0.248720 & 1.460931 & 0.000000\end{array}$
$\begin{array}{llll}\mathrm{O} & -2.123820 & -1.288373 & 0.000000\end{array}$
$\begin{array}{llll}\mathrm{H} & 2.193384 & -1.372582 & 0.000000\end{array}$
$\begin{array}{llll}\mathrm{H} & 2.380976 & 1.079063 & 0.000000\end{array}$
$\begin{array}{llll}\mathrm{H} & 0.306040 & 2.537232 & 0.000000\end{array}$
$\begin{array}{llll}\mathrm{H} & -1.952243 & 1.269676 & 0.000000\end{array}$
$\mathrm{H} \quad-1.890571 \quad-2.229465 \quad 0.000000$ 
2-pyridone $\mathrm{S}_{0}$

$\begin{array}{cccc}\mathrm{C} & -1.245908 & 0.711039 & 0.000000 \\ \mathrm{~N} & -1.134849 & -0.685198 & 0.000000 \\ \mathrm{C} & 0.033211 & -1.380423 & 0.000000 \\ \mathrm{C} & 1.219543 & -0.732271 & 0.000000 \\ \mathrm{C} & 1.201960 & 0.691440 & 0.000000 \\ \mathrm{C} & 0.038325 & 1.384003 & 0.000000 \\ \mathrm{H} & -2.016501 & -1.175246 & 0.000000 \\ \mathrm{H} & -0.055131 & -2.458415 & 0.000000 \\ \mathrm{H} & 2.146013 & -1.286157 & 0.000000 \\ \mathrm{H} & 2.143128 & 1.228927 & 0.000000 \\ \mathrm{H} & 0.013277 & 2.464767 & 0.000000 \\ \mathrm{O} & -2.343068 & 1.237544 & 0.000000\end{array}$

2-pyridone $\mathrm{S}_{1}$

$\begin{array}{lrrr}\mathrm{C} & -1.385877 & 0.105025 & 0.000000 \\ \mathrm{~N} & -0.729806 & -1.137644 & 0.000000 \\ \mathrm{C} & 0.622464 & -1.251891 & 0.000000 \\ \mathrm{C} & 1.410373 & -0.075580 & 0.000000 \\ \mathrm{C} & 0.829292 & 1.171006 & 0.000000 \\ \mathrm{C} & -0.608728 & 1.264887 & 0.000000 \\ \mathrm{H} & -1.338648 & -1.941928 & 0.000000 \\ \mathrm{H} & 1.041103 & -2.246412 & 0.000000 \\ \mathrm{H} & 2.485697 & -0.193346 & 0.000000 \\ \mathrm{H} & 1.434898 & 2.065137 & 0.000000 \\ \mathrm{H} & -1.126779 & 2.211721 & 0.000000 \\ \mathrm{O} & -2.633988 & 0.029016 & 0.000000\end{array}$

2-methylpyrimidine $\mathrm{S}_{0}$

$\begin{array}{lrrr}\mathrm{C} & 0.000010 & 1.959836 & 0.000000 \\ \mathrm{C} & -1.171845 & 1.221707 & 0.000000 \\ \mathrm{~N} & -1.177940 & -0.104703 & 0.000000 \\ \mathrm{C} & 0.011026 & -0.714707 & 0.000000 \\ \mathrm{~N} & 1.192930 & -0.097074 & 0.000000 \\ \mathrm{C} & 1.175538 & 1.232599 & 0.000000 \\ \mathrm{H} & -2.142372 & 1.707974 & 0.000000 \\ \mathrm{H} & 2.142537 & 1.725460 & 0.000000 \\ \mathrm{H} & -0.004752 & 3.040846 & 0.000000 \\ \mathrm{C} & 0.004636 & -2.211945 & 0.000000 \\ \mathrm{H} & 1.022376 & -2.594804 & 0.000000 \\ \mathrm{H} & -0.526077 & -2.582594 & 0.878412 \\ \mathrm{H} & -0.526077 & -2.582594 & -0.878412\end{array}$


2-methylpyrimidine $\mathrm{S}_{1}$

$\begin{array}{rrrr}\mathrm{C} & -0.015112 & 2.008449 & 0.000000 \\ \mathrm{C} & -1.164519 & 1.275446 & 0.000000 \\ \mathrm{~N} & -1.151615 & -0.095134 & 0.000000 \\ \mathrm{C} & 0.010047 & -0.782129 & 0.000000 \\ \mathrm{~N} & 1.081654 & -0.064703 & 0.000000 \\ \mathrm{C} & 1.205467 & 1.288168 & 0.000000 \\ \mathrm{H} & -2.142648 & 1.739126 & 0.000000 \\ \mathrm{H} & 2.190982 & 1.722638 & 0.000000 \\ \mathrm{H} & -0.026396 & 3.090205 & 0.000000 \\ \mathrm{C} & 0.013373 & -2.267314 & 0.000000 \\ \mathrm{H} & 1.031795 & -2.650422 & 0.000000 \\ \mathrm{H} & -0.516514 & -2.632170 & 0.881352 \\ \mathrm{H} & -0.516514 & -2.632170 & -0.881352\end{array}$

5-methylpyrimidine $\mathrm{S}_{0}$

$\begin{array}{rrrr}\mathrm{C} & 0.873486 & 0.849529 & 0.000000 \\ \mathrm{C} & -0.358465 & 0.210927 & 0.000000 \\ \mathrm{C} & -0.298845 & -1.178206 & 0.000000 \\ \mathrm{~N} & 0.842862 & -1.855702 & 0.000000 \\ \mathrm{C} & 1.955122 & -1.128249 & 0.000000 \\ \mathrm{~N} & 2.031736 & 0.195783 & 0.000000 \\ \mathrm{H} & 2.893351 & -1.672443 & 0.000000 \\ \mathrm{H} & -1.210831 & -1.769625 & 0.000000 \\ \mathrm{H} & 0.932052 & 1.934682 & 0.000000 \\ \mathrm{C} & -1.659313 & 0.957450 & 0.000000 \\ \mathrm{H} & -1.492107 & 2.034861 & 0.000000 \\ \mathrm{H} & -2.254524 & 0.710486 & -0.881136 \\ \mathrm{H} & -2.254524 & 0.710486 & 0.881136\end{array}$

5-methylpyrimidine $\mathrm{S}_{1}$

$\begin{array}{rrrr}\mathrm{C} & 0.844197 & 0.855364 & 0.000000 \\ \mathrm{C} & -0.374431 & 0.243401 & 0.000000 \\ \mathrm{C} & -0.347427 & -1.189463 & 0.000000 \\ \mathrm{~N} & 0.878286 & -1.751904 & 0.000000 \\ \mathrm{C} & 2.030627 & -1.176904 & 0.000000 \\ \mathrm{~N} & 2.030804 & 0.168482 & 0.000000 \\ \mathrm{H} & 2.954334 & -1.736816 & 0.000000 \\ \mathrm{H} & -1.219791 & -1.822797 & 0.000000 \\ \mathrm{H} & 0.929792 & 1.935586 & 0.000000 \\ \mathrm{C} & -1.675746 & 0.978158 & 0.000000 \\ \mathrm{H} & -1.517087 & 2.056642 & 0.000000\end{array}$



H $\quad-2.266783$
$0.720130 \quad-0.882019$
H $\quad-2.266783$
0.720130
0.882019

7-azaindole $\mathrm{S}_{0}$

$\begin{array}{rrrr}\mathrm{N} & -0.632523 & 1.838772 & 0.000000 \\ \mathrm{C} & -0.671656 & 0.470508 & 0.000000 \\ \mathrm{C} & 0.664109 & 0.006216 & 0.000000 \\ \mathrm{C} & 1.505727 & 1.165037 & 0.000000 \\ \mathrm{C} & 0.680323 & 2.247332 & 0.000000 \\ \mathrm{C} & 0.841887 & -1.374650 & 0.000000 \\ \mathrm{C} & -0.291322 & -2.167012 & 0.000000 \\ \mathrm{C} & -1.559927 & -1.576027 & 0.000000 \\ \mathrm{~N} & -1.772548 & -0.265991 & 0.000000 \\ \mathrm{H} & -2.446993 & -2.200374 & 0.000000 \\ \mathrm{H} & 1.831241 & -1.816180 & 0.000000 \\ \mathrm{H} & -0.210960 & -3.245925 & 0.000000 \\ \mathrm{H} & -1.444343 & 2.428629 & 0.000000 \\ \mathrm{H} & 2.582848 & 1.192402 & 0.000000 \\ \mathrm{H} & 0.924155 & 3.297263 & 0.000000\end{array}$

7-azaindole $\mathrm{S}_{1}$

$\begin{array}{lrrr}\mathrm{N} & -0.606525 & 1.868952 & 0.000000 \\ \mathrm{C} & -0.659819 & 0.450117 & 0.000000 \\ \mathrm{C} & 0.658981 & -0.005005 & 0.000000 \\ \mathrm{C} & 1.487366 & 1.140017 & 0.000000 \\ \mathrm{C} & 0.648086 & 2.300750 & 0.000000 \\ \mathrm{C} & 0.887321 & -1.406213 & 0.000000 \\ \mathrm{C} & -0.303837 & -2.177947 & 0.000000 \\ \mathrm{C} & -1.546151 & -1.593446 & 0.000000 \\ \mathrm{~N} & -1.793772 & -0.220463 & 0.000000 \\ \mathrm{H} & -2.436620 & -2.210100 & 0.000000 \\ \mathrm{H} & 1.873628 & -1.843907 & 0.000000 \\ \mathrm{H} & -0.245109 & -3.259010 & 0.000000 \\ \mathrm{H} & -1.438135 & 2.439128 & 0.000000 \\ \mathrm{H} & 2.565785 & 1.171808 & 0.000000 \\ \mathrm{H} & 0.908810 & 3.345339 & 0.000000\end{array}$

7-hydroxyquinoline, cis $\mathrm{S}_{0}$
$\mathrm{N} \quad 0.462083$
1.966702
0.000000
$\begin{array}{llll}\text { C } & 0.432078 & 0.607499 & 0.000000\end{array}$
$\begin{array}{llll}\text { C } & -0.782681 & -0.123445 & 0.000000\end{array}$
$\begin{array}{llll}\text { C } & -1.987771 & 0.607015 & 0.000000\end{array}$
$\begin{array}{llll}\text { C } & -1.942580 & 1.971820 & 0.000000\end{array}$ 


$\begin{array}{rrrr}\mathrm{C} & -0.681311 & 2.602251 & 0.000000 \\ \mathrm{C} & 1.660860 & -0.090127 & 0.000000 \\ \mathrm{C} & 1.675189 & -1.458078 & 0.000000 \\ \mathrm{C} & 0.465756 & -2.193586 & 0.000000 \\ \mathrm{C} & -0.727689 & -1.539064 & 0.000000 \\ \mathrm{H} & -0.625715 & 3.687220 & 0.000000 \\ \mathrm{H} & -1.655021 & -2.100528 & 0.000000 \\ \mathrm{H} & 0.519950 & -3.274295 & 0.000000 \\ \mathrm{O} & 2.815156 & -2.193761 & 0.000000 \\ \mathrm{H} & 2.574521 & 0.493513 & 0.000000 \\ \mathrm{H} & -2.934306 & 0.077738 & 0.000000 \\ \mathrm{H} & -2.844787 & 2.568937 & 0.000000 \\ \mathrm{H} & 3.576269 & -1.609801 & 0.000000\end{array}$

7-hydroxyquinoline, cis $\mathrm{S}_{1}$

$\begin{array}{lrrl}\mathrm{N} & 0.489293 & 1.970500 & 0.000000 \\ \mathrm{C} & 0.434861 & 0.630861 & 0.000000 \\ \mathrm{C} & -0.789810 & -0.140222 & 0.000000 \\ \mathrm{C} & -1.992433 & 0.592158 & 0.000000 \\ \mathrm{C} & -1.917040 & 1.993079 & 0.000000 \\ \mathrm{C} & -0.685525 & 2.636655 & 0.000000 \\ \mathrm{C} & 1.635036 & -0.085523 & 0.000000 \\ \mathrm{C} & 1.653426 & -1.512826 & 0.000000 \\ \mathrm{C} & 0.482370 & -2.261077 & 0.000000 \\ \mathrm{C} & -0.720674 & -1.555256 & 0.000000 \\ \mathrm{H} & -0.623355 & 3.717381 & 0.000000 \\ \mathrm{H} & -1.654376 & -2.106305 & 0.000000 \\ \mathrm{H} & 0.524863 & -3.339574 & 0.000000 \\ \mathrm{O} & 2.822547 & -2.154979 & 0.000000 \\ \mathrm{H} & 2.565805 & 0.472666 & 0.000000 \\ \mathrm{H} & -2.948686 & 0.085729 & 0.000000 \\ \mathrm{H} & -2.826179 & 2.582041 & 0.000000 \\ \mathrm{H} & 3.549876 & -1.525299 & 0.000000\end{array}$

7-hydroxyquinoline, trans $\mathrm{S}_{0}$

$\begin{array}{rrrr}\mathrm{N} & 0.512068 & 2.039031 & 0.000000 \\ \mathrm{C} & 0.479618 & 0.677459 & 0.000000 \\ \mathrm{C} & -0.740548 & -0.047663 & 0.000000 \\ \mathrm{C} & -1.944848 & 0.687850 & 0.000000 \\ \mathrm{C} & -1.893596 & 2.050826 & 0.000000 \\ \mathrm{C} & -0.628027 & 2.676827 & 0.000000 \\ \mathrm{C} & 1.705019 & -0.019571 & 0.000000 \\ \mathrm{C} & 1.716128 & -1.387577 & 0.000000 \\ \mathrm{C} & 0.502991 & -2.116510 & 0.000000\end{array}$




$\begin{array}{rrrr}\mathrm{C} & -0.692393 & -1.459957 & 0.000000 \\ \mathrm{H} & -0.568484 & 3.761713 & 0.000000 \\ \mathrm{H} & -1.620559 & -2.019879 & 0.000000 \\ \mathrm{H} & 0.529709 & -3.201472 & 0.000000 \\ \mathrm{O} & 2.916141 & -2.023651 & 0.000000 \\ \mathrm{H} & 2.629319 & 0.541553 & 0.000000 \\ \mathrm{H} & -2.893093 & 0.161673 & 0.000000 \\ \mathrm{H} & -2.793005 & 2.652276 & 0.000000 \\ \mathrm{H} & 2.783538 & -2.972906 & 0.000000\end{array}$

7-hydroxyquinoline, trans $\mathrm{S}_{1}$

$\begin{array}{rrrr}\mathrm{N} & 0.532460 & 2.045610 & 0.000000 \\ \mathrm{C} & 0.476743 & 0.707951 & 0.000000 \\ \mathrm{C} & -0.749280 & -0.057700 & 0.000000 \\ \mathrm{C} & -1.951229 & 0.675746 & 0.000001 \\ \mathrm{C} & -1.873671 & 2.077192 & 0.000000 \\ \mathrm{C} & -0.642711 & 2.715411 & -0.000001 \\ \mathrm{C} & 1.678777 & -0.010926 & 0.000000 \\ \mathrm{C} & 1.690105 & -1.434785 & 0.000000 \\ \mathrm{C} & 0.515973 & -2.182401 & 0.000000 \\ \mathrm{C} & -0.686412 & -1.471611 & 0.000000 \\ \mathrm{H} & -0.575893 & 3.795937 & -0.000001 \\ \mathrm{H} & -1.621700 & -2.020146 & 0.000000 \\ \mathrm{H} & 0.536190 & -3.264352 & -0.000001 \\ \mathrm{O} & 2.904949 & -1.987352 & 0.000000 \\ \mathrm{H} & 2.620986 & 0.520645 & 0.000001 \\ \mathrm{H} & -2.907587 & 0.169388 & 0.000001 \\ \mathrm{H} & -2.781560 & 2.668066 & 0.000000 \\ \mathrm{H} & 2.833838 & -2.946651 & 0.000000\end{array}$

2-hydroxyquinoline, enol $\mathrm{S}_{0}$

$\begin{array}{rrrr}\mathrm{C} & 0.430279 & 1.899594 & 0.000000 \\ \mathrm{C} & 0.483564 & 0.488133 & 0.000000 \\ \mathrm{C} & -0.719646 & -0.257813 & 0.000000 \\ \mathrm{C} & -1.950364 & 0.436077 & 0.000000 \\ \mathrm{C} & -1.973122 & 1.803286 & 0.000000 \\ \mathrm{C} & -0.774080 & 2.545782 & 0.000000 \\ \mathrm{C} & 1.694822 & -0.244154 & 0.000000 \\ \mathrm{C} & 1.662434 & -1.602407 & 0.000000 \\ \mathrm{C} & 0.392946 & -2.246793 & 0.000000 \\ \mathrm{~N} & -0.738865 & -1.618172 & 0.000000 \\ \mathrm{O} & 0.314642 & -3.596006 & 0.000000 \\ \mathrm{H} & 1.358567 & 2.460079 & 0.000000 \\ \mathrm{H} & -0.813104 & 3.627818 & 0.000000\end{array}$




$\begin{array}{cccc}\mathrm{H} & -2.921903 & 2.325808 & 0.000000 \\ \mathrm{H} & -2.860802 & -0.149060 & 0.000000 \\ \mathrm{H} & 2.641284 & 0.284578 & 0.000000 \\ \mathrm{H} & 2.577007 & -2.184134 & 0.000000 \\ \mathrm{H} & 1.196342 & -3.972615 & 0.000000\end{array}$

2-hydroxyquinoline, enol $\mathrm{S}_{1}$

$\begin{array}{lrrr}\mathrm{C} & 0.437265 & 1.879017 & 0.000000 \\ \mathrm{C} & 0.527176 & 0.488831 & 0.000000 \\ \mathrm{C} & -0.725495 & -0.261810 & 0.000000 \\ \mathrm{C} & -1.957950 & 0.437161 & 0.000000 \\ \mathrm{C} & -2.009010 & 1.833977 & 0.000000 \\ \mathrm{C} & -0.812858 & 2.540148 & 0.000000 \\ \mathrm{C} & 1.731979 & -0.234920 & 0.000000 \\ \mathrm{C} & 1.693498 & -1.636541 & 0.000000 \\ \mathrm{C} & 0.430939 & -2.239969 & 0.000000 \\ \mathrm{~N} & -0.750373 & -1.602812 & 0.000000 \\ \mathrm{O} & 0.293907 & -3.568967 & 0.000000 \\ \mathrm{H} & 1.345976 & 2.469533 & 0.000000 \\ \mathrm{H} & -0.824524 & 3.623555 & 0.000000 \\ \mathrm{H} & -2.957417 & 2.351972 & 0.000000 \\ \mathrm{H} & -2.860259 & -0.160906 & 0.000000 \\ \mathrm{H} & 2.676694 & 0.293125 & 0.000000 \\ \mathrm{H} & 2.597932 & -2.230235 & 0.000000 \\ \mathrm{H} & 1.162510 & -3.981140 & 0.000000\end{array}$

2-hydroxyquinoline, keto $\mathrm{S}_{0}$

$\begin{array}{lrrr}\mathrm{N} & 0.558774 & 1.703464 & 0.000000 \\ \mathrm{C} & 0.599789 & 0.326721 & 0.000000 \\ \mathrm{C} & -0.611362 & -0.381931 & 0.000000 \\ \mathrm{C} & -1.834765 & 0.377909 & 0.000000 \\ \mathrm{C} & -1.832319 & 1.720371 & 0.000000 \\ \mathrm{C} & -0.586135 & 2.485549 & 0.000000 \\ \mathrm{C} & 1.815492 & -0.362681 & 0.000000 \\ \mathrm{C} & 1.818945 & -1.740744 & 0.000000 \\ \mathrm{C} & 0.620963 & -2.459164 & 0.000000 \\ \mathrm{C} & -0.577545 & -1.781007 & 0.000000 \\ \mathrm{H} & -1.515437 & -2.324555 & 0.000000 \\ \mathrm{H} & 0.637522 & -3.541073 & 0.000000 \\ \mathrm{H} & 2.763719 & -2.269764 & 0.000000 \\ \mathrm{H} & 2.748013 & 0.189598 & 0.000000 \\ \mathrm{H} & -2.773887 & -0.164978 & 0.000000 \\ \mathrm{H} & -2.744131 & 2.301142 & 0.000000 \\ \mathrm{O} & -0.511635 & 3.698923 & 0.000000\end{array}$




\section{$\begin{array}{llll}\mathrm{H} & 1.424018 & 2.222240 & 0.000000\end{array}$}

2-hydroxyquinoline, keto $\mathrm{S}_{1}$

$\begin{array}{rrrr}\mathrm{N} & 0.561762 & 1.674688 & 0.000000 \\ \mathrm{C} & 0.611422 & 0.327088 & 0.000000 \\ \mathrm{C} & -0.664831 & -0.380667 & 0.000000 \\ \mathrm{C} & -1.854114 & 0.364178 & 0.000000 \\ \mathrm{C} & -1.852717 & 1.763415 & 0.000000 \\ \mathrm{C} & -0.627738 & 2.470617 & 0.000000 \\ \mathrm{C} & 1.834539 & -0.359567 & 0.000000 \\ \mathrm{C} & 1.853091 & -1.745202 & 0.000000 \\ \mathrm{C} & 0.630468 & -2.444381 & 0.000000 \\ \mathrm{C} & -0.590668 & -1.787326 & 0.000000 \\ \mathrm{H} & -1.511442 & -2.356664 & 0.000000 \\ \mathrm{H} & 0.644037 & -3.527546 & 0.000000 \\ \mathrm{H} & 2.790930 & -2.282060 & 0.000000 \\ \mathrm{H} & 2.757640 & 0.208551 & 0.000000 \\ \mathrm{H} & -2.794645 & -0.171901 & 0.000000 \\ \mathrm{H} & -2.762017 & 2.343835 & 0.000000 \\ \mathrm{O} & -0.438526 & 3.682378 & 0.000000 \\ \mathrm{H} & 1.412809 & 2.220575 & 0.000000\end{array}$

dibenzofuran $\mathrm{S}_{0}$

$\begin{array}{rrrr}\mathrm{C} & 1.715971 & 0.000000 & -1.355127 \\ \mathrm{C} & 0.724691 & 0.000000 & -0.380542 \\ \mathrm{C} & 1.092263 & 0.000000 & 0.966753 \\ \mathrm{C} & 2.406232 & 0.000000 & 1.391364 \\ \mathrm{C} & 3.379421 & 0.000000 & 0.403634 \\ \mathrm{C} & 3.040967 & 0.000000 & -0.951636 \\ \mathrm{C} & -0.724691 & 0.000000 & -0.380542 \\ \mathrm{C} & -1.092263 & 0.000000 & 0.966753 \\ \mathrm{O} & 0.000000 & 0.000000 & 1.788936 \\ \mathrm{C} & -1.715971 & 0.000000 & -1.355127 \\ \mathrm{C} & -3.040967 & 0.000000 & -0.951636 \\ \mathrm{C} & -3.379421 & 0.000000 & 0.403634 \\ \mathrm{C} & -2.406232 & 0.000000 & 1.391364 \\ \mathrm{H} & -2.655325 & 0.000000 & 2.443819 \\ \mathrm{H} & -4.423646 & 0.000000 & 0.690091 \\ \mathrm{H} & -3.827324 & 0.000000 & -1.695508 \\ \mathrm{H} & -1.459073 & 0.000000 & -2.407149 \\ \mathrm{H} & 1.459073 & 0.000000 & -2.407149 \\ \mathrm{H} & 3.827324 & 0.000000 & -1.695508 \\ \mathrm{H} & 4.423646 & 0.000000 & 0.690091 \\ \mathrm{H} & 2.655325 & 0.000000 & 2.443819\end{array}$




\begin{tabular}{|c|c|c|c|}
\hline $\mathrm{C}$ & 0.000000 & 1.724658 & -1.529927 \\
\hline $\mathrm{C}$ & 0.000000 & 0.710466 & -0.567015 \\
\hline $\mathrm{C}$ & 0.000000 & 1.099937 & 0.804579 \\
\hline $\mathrm{C}$ & 0.000000 & 2.403705 & 1.254868 \\
\hline $\mathrm{C}$ & 0.000000 & 3.409314 & 0.265446 \\
\hline $\mathrm{C}$ & 0.000000 & 3.063611 & -1.087429 \\
\hline $\mathrm{C}$ & 0.000000 & -0.710466 & -0.567015 \\
\hline $\mathrm{C}$ & 0.000000 & -1.099937 & 0.804579 \\
\hline $\mathrm{O}$ & 0.000000 & 0.000000 & 1.601742 \\
\hline $\mathrm{C}$ & 0.000000 & -1.724658 & -1.529927 \\
\hline $\mathrm{C}$ & 0.000000 & -3.063611 & -1.087429 \\
\hline $\mathrm{C}$ & 0.000000 & -3.409314 & 0.265446 \\
\hline $\mathrm{C}$ & 0.000000 & -2.403705 & 1.254868 \\
\hline $\mathrm{H}$ & 0.000000 & -2.632291 & 2.311505 \\
\hline $\mathrm{H}$ & 0.000000 & -4.449990 & 0.557191 \\
\hline $\mathrm{H}$ & 0.000000 & -3.852849 & -1.828994 \\
\hline $\mathrm{H}$ & 0.000000 & -1.498031 & -2.587384 \\
\hline $\mathrm{H}$ & 0.000000 & 1.498031 & -2.587384 \\
\hline $\mathrm{H}$ & 0.000000 & 3.852849 & -1.828994 \\
\hline $\mathrm{H}$ & 0.000000 & 4.449990 & 0.557191 \\
\hline $\mathrm{H}$ & 0.000000 & 2.632291 & 2.311505 \\
\hline
\end{tabular}

pyrrolo[3,2-h]quinoline $\mathrm{S}_{0}$

$\begin{array}{lrrr}\mathrm{C} & 0.135542 & -0.683711 & 0.000000 \\ \mathrm{~N} & 0.060662 & -2.032509 & 0.000000 \\ \mathrm{C} & 1.190478 & -2.702245 & 0.000000 \\ \mathrm{C} & 2.456698 & -2.095733 & 0.000000 \\ \mathrm{C} & 2.534774 & -0.727240 & 0.000000 \\ \mathrm{C} & 1.355370 & 0.038130 & 0.000000 \\ \mathrm{H} & 1.107378 & -3.784785 & 0.000000 \\ \mathrm{H} & 3.347870 & -2.709471 & 0.000000 \\ \mathrm{H} & 3.496494 & -0.226185 & 0.000000 \\ \mathrm{C} & -1.051132 & 0.084963 & 0.000000 \\ \mathrm{C} & -1.055783 & 1.480251 & 0.000000 \\ \mathrm{C} & 0.182571 & 2.173044 & 0.000000 \\ \mathrm{C} & 1.346719 & 1.468149 & 0.000000 \\ \mathrm{H} & 0.198437 & 3.256216 & 0.000000 \\ \mathrm{H} & 2.299121 & 1.985345 & 0.000000 \\ \mathrm{C} & -2.424958 & 1.884705 & 0.000000 \\ \mathrm{C} & -3.175342 & 0.742661 & 0.000000 \\ \mathrm{~N} & -2.342854 & -0.345772 & 0.000000 \\ \mathrm{H} & -2.609106 & -1.314081 & 0.000000\end{array}$




$$
\begin{array}{llll}
\mathrm{H} & -2.807416 & 2.892297 & 0.000000 \\
\mathrm{H} & -4.245515 & 0.615971 & 0.000000
\end{array}
$$

pyrrolo[3,2- $h]$ quinoline $\mathrm{S}_{1}$

$\begin{array}{lrrr}\mathrm{C} & 0.150529 & -0.698387 & 0.000000 \\ \mathrm{~N} & 0.016748 & -2.022064 & 0.000000 \\ \mathrm{C} & 1.200401 & -2.726185 & 0.000000 \\ \mathrm{C} & 2.434116 & -2.132611 & 0.000000 \\ \mathrm{C} & 2.560128 & -0.724461 & 0.000000 \\ \mathrm{C} & 1.364760 & 0.029635 & 0.000000 \\ \mathrm{H} & 1.100041 & -3.804296 & 0.000000 \\ \mathrm{H} & 3.320646 & -2.754763 & 0.000000 \\ \mathrm{H} & 3.527049 & -0.240693 & 0.000000 \\ \mathrm{C} & -1.025019 & 0.102131 & 0.000000 \\ \mathrm{C} & -1.059672 & 1.546717 & 0.000000 \\ \mathrm{C} & 0.138378 & 2.222952 & 0.000000 \\ \mathrm{C} & 1.322356 & 1.450181 & 0.000000 \\ \mathrm{H} & 0.189713 & 3.302900 & 0.000000 \\ \mathrm{H} & 2.271918 & 1.974321 & 0.000000 \\ \mathrm{C} & -2.455961 & 1.903752 & 0.000000 \\ \mathrm{C} & -3.161345 & 0.747839 & 0.000000 \\ \mathrm{~N} & -2.286293 & -0.339122 & 0.000000 \\ \mathrm{H} & -2.512650 & -1.321295 & 0.000000 \\ \mathrm{H} & -2.869245 & 2.898246 & 0.000000 \\ \mathrm{H} & -4.226606 & 0.585204 & 0.000000\end{array}$

carbazole $\mathrm{S}_{0}$

$\begin{array}{lrrr}\mathrm{C} & 1.126464 & 0.000000 & -0.895839 \\ \mathrm{~N} & 0.000000 & 0.000000 & -1.693132 \\ \mathrm{C} & -1.126464 & 0.000000 & -0.895839 \\ \mathrm{C} & -0.723491 & 0.000000 & 0.454005 \\ \mathrm{C} & 0.723491 & 0.000000 & 0.454005 \\ \mathrm{C} & -1.688453 & 0.000000 & 1.456250 \\ \mathrm{C} & -3.025878 & 0.000000 & 1.102669 \\ \mathrm{C} & -3.407554 & 0.000000 & -0.242696 \\ \mathrm{C} & -2.468178 & 0.000000 & -1.258403 \\ \mathrm{C} & 2.468178 & 0.000000 & -1.258403 \\ \mathrm{C} & 3.407554 & 0.000000 & -0.242696 \\ \mathrm{C} & 3.025878 & 0.000000 & 1.102669 \\ \mathrm{C} & 1.688453 & 0.000000 & 1.456250 \\ \mathrm{H} & -2.770815 & 0.000000 & -2.298438 \\ \mathrm{H} & -4.460661 & 0.000000 & -0.495732 \\ \mathrm{H} & -3.786958 & 0.000000 & 1.872359 \\ \mathrm{H} & -1.396505 & 0.000000 & 2.499617\end{array}$




$\begin{array}{lrrr}\mathrm{H} & 1.396505 & 0.000000 & 2.499617 \\ \mathrm{H} & 3.786958 & 0.000000 & 1.872359 \\ \mathrm{H} & 4.460661 & 0.000000 & -0.495732 \\ \mathrm{H} & 2.770815 & 0.000000 & -2.298438 \\ \mathrm{H} & 0.000000 & 0.000000 & -2.695411\end{array}$

carbazole $\mathrm{S}_{1}$

$\begin{array}{lrrr}\mathrm{C} & 1.139196 & 0.000000 & -0.837246 \\ \mathrm{~N} & 0.000000 & 0.000000 & -1.609079 \\ \mathrm{C} & -1.139196 & 0.000000 & -0.837246 \\ \mathrm{C} & -0.713809 & 0.000000 & 0.528859 \\ \mathrm{C} & 0.713809 & 0.000000 & 0.528859 \\ \mathrm{C} & -1.699899 & 0.000000 & 1.518733 \\ \mathrm{C} & -3.050842 & 0.000000 & 1.118256 \\ \mathrm{C} & -3.440246 & 0.000000 & -0.217911 \\ \mathrm{C} & -2.460676 & 0.000000 & -1.236839 \\ \mathrm{C} & 2.460676 & 0.000000 & -1.236839 \\ \mathrm{C} & 3.440246 & 0.000000 & -0.217911 \\ \mathrm{C} & 3.050842 & 0.000000 & 1.118256 \\ \mathrm{C} & 1.699899 & 0.000000 & 1.518733 \\ \mathrm{H} & -2.736001 & 0.000000 & -2.283937 \\ \mathrm{H} & -4.489382 & 0.000000 & -0.477756 \\ \mathrm{H} & -3.816146 & 0.000000 & 1.885179 \\ \mathrm{H} & -1.441519 & 0.000000 & 2.568949 \\ \mathrm{H} & 1.441519 & 0.000000 & 2.568949 \\ \mathrm{H} & 3.816146 & 0.000000 & 1.885179 \\ \mathrm{H} & 4.489382 & 0.000000 & -0.477756 \\ \mathrm{H} & 2.736001 & 0.000000 & -2.283937 \\ \mathrm{H} & 0.000000 & 0.000000 & -2.616120\end{array}$

tryptamine, A-ph $\mathrm{S}_{0}$

$\begin{array}{rrrr}\mathrm{C} & -2.136691 & 0.149661 & 0.896260 \\ \mathrm{~N} & -1.256006 & 0.129814 & 1.956382 \\ \mathrm{C} & 0.024747 & 0.042809 & 1.471355 \\ \mathrm{C} & -0.063601 & 0.003413 & 0.064965 \\ \mathrm{C} & -1.458196 & 0.074317 & -0.281239 \\ \mathrm{C} & 1.247355 & -0.001075 & 2.138316 \\ \mathrm{C} & 2.390062 & -0.083318 & 1.369306 \\ \mathrm{C} & 2.324891 & -0.118843 & -0.032768 \\ \mathrm{C} & 1.112010 & -0.076396 & -0.688009 \\ \mathrm{C} & -2.025696 & 0.021300 & -1.662068 \\ \mathrm{C} & -1.972558 & -1.382388 & -2.266093 \\ \mathrm{~N} & -2.439471 & -1.362933 & -3.647784 \\ \mathrm{H} & 1.301486 & 0.028550 & 3.220052\end{array}$




$\begin{array}{lrrr}\mathrm{H} & 3.357048 & -0.119448 & 1.855658 \\ \mathrm{H} & 3.243063 & -0.178036 & -0.603712 \\ \mathrm{H} & 1.069261 & -0.099918 & -1.770816 \\ \mathrm{H} & -1.510798 & 0.192826 & 2.923498 \\ \mathrm{H} & -3.199065 & 0.221802 & 1.070035 \\ \mathrm{H} & -1.478116 & 0.697359 & -2.324303 \\ \mathrm{H} & -3.063882 & 0.367830 & -1.648637 \\ \mathrm{H} & -2.537549 & -2.063653 & -1.613499 \\ \mathrm{H} & -0.936888 & -1.730096 & -2.262492 \\ \mathrm{H} & -3.420904 & -1.118912 & -3.689675 \\ \mathrm{H} & -2.337914 & -2.272285 & -4.077362\end{array}$

tryptamine, A-ph $\mathrm{S}_{1}$

$\begin{array}{rrrr}\mathrm{C} & -2.187524 & 0.174583 & 0.915465 \\ \mathrm{~N} & -1.296583 & 0.128982 & 1.916608 \\ \mathrm{C} & 0.017460 & 0.053434 & 1.435168 \\ \mathrm{C} & -0.068276 & 0.066366 & 0.036792 \\ \mathrm{C} & -1.443215 & 0.137133 & -0.303741 \\ \mathrm{C} & 1.202946 & -0.055596 & 2.144129 \\ \mathrm{C} & 2.394819 & -0.137225 & 1.328342 \\ \mathrm{C} & 2.340486 & -0.123519 & -0.040873 \\ \mathrm{C} & 1.107969 & -0.023563 & -0.748959 \\ \mathrm{C} & -2.017010 & 0.064494 & -1.665029 \\ \mathrm{C} & -1.947160 & -1.367948 & -2.221193 \\ \mathrm{~N} & -2.449806 & -1.401528 & -3.586456 \\ \mathrm{H} & 1.246588 & -0.084171 & 3.223341 \\ \mathrm{H} & 3.354841 & -0.211490 & 1.823773 \\ \mathrm{H} & 3.262937 & -0.184388 & -0.605440 \\ \mathrm{H} & 1.075552 & 0.024611 & -1.828507 \\ \mathrm{H} & -1.532556 & 0.160895 & 2.894592 \\ \mathrm{H} & -3.246976 & 0.270593 & 1.082665 \\ \mathrm{H} & -1.454928 & 0.710013 & -2.344031 \\ \mathrm{H} & -3.056835 & 0.404440 & -1.662890 \\ \mathrm{H} & -2.478642 & -2.039195 & -1.531593 \\ \mathrm{H} & -0.899710 & -1.677360 & -2.227184 \\ \mathrm{H} & -3.454042 & -1.281896 & -3.609677 \\ \mathrm{H} & -2.237766 & -2.285286 & -4.027973\end{array}$

tryptamine, A-py $\mathrm{S}_{0}$
C $\quad-2.130746$
$\begin{array}{ll}0.127534 & 0.872237\end{array}$
$\begin{array}{llll}\mathrm{N} & -1.260246 & 0.121451 & 1.940453\end{array}$
$\begin{array}{llll}\text { C } & 0.025719 & 0.044174 & 1.468330\end{array}$
$\begin{array}{llll}\text { C } & -0.048690 & -0.004058 & 0.061052\end{array}$
$\begin{array}{llll}\text { C } & -1.440739 & 0.050837 & -0.298604\end{array}$ 


$\begin{array}{lrrr}\mathrm{C} & 1.241977 & 0.015156 & 2.147727 \\ \mathrm{C} & 2.393465 & -0.060242 & 1.391330 \\ \mathrm{C} & 2.342855 & -0.104093 & -0.011093 \\ \mathrm{C} & 1.136023 & -0.077256 & -0.678296 \\ \mathrm{C} & -2.006326 & -0.009335 & -1.680269 \\ \mathrm{C} & -1.929469 & -1.409210 & -2.289663 \\ \mathrm{~N} & -2.489867 & -1.409272 & -3.636865 \\ \mathrm{H} & 1.284568 & 0.050722 & 3.229806 \\ \mathrm{H} & 3.355611 & -0.084758 & 1.887863 \\ \mathrm{H} & 3.267282 & -0.158514 & -0.572382 \\ \mathrm{H} & 1.105617 & -0.110072 & -1.761328 \\ \mathrm{H} & -1.524999 & 0.186331 & 2.904815 \\ \mathrm{H} & -3.195484 & 0.191026 & 1.034230 \\ \mathrm{H} & -1.463456 & 0.688187 & -2.328551 \\ \mathrm{H} & -3.049763 & 0.312752 & -1.680846 \\ \mathrm{H} & -2.521514 & -2.088931 & -1.672409 \\ \mathrm{H} & -0.889607 & -1.762826 & -2.242060 \\ \mathrm{H} & -1.924409 & -0.842549 & -4.256428 \\ \mathrm{H} & -2.507890 & -2.344662 & -4.019938\end{array}$

tryptamine, A-py $\mathrm{S}_{1}$

$\begin{array}{rrrr}\mathrm{C} & -2.181706 & 0.136905 & 0.889905 \\ \mathrm{~N} & -1.302310 & 0.106307 & 1.900617 \\ \mathrm{C} & 0.018550 & 0.048987 & 1.433577 \\ \mathrm{C} & -0.051838 & 0.056020 & 0.034945 \\ \mathrm{C} & -1.423589 & 0.107900 & -0.320745 \\ \mathrm{C} & 1.198302 & -0.036806 & 2.156452 \\ \mathrm{C} & 2.399048 & -0.102688 & 1.353480 \\ \mathrm{C} & 2.360383 & -0.094881 & -0.016540 \\ \mathrm{C} & 1.134211 & -0.017201 & -0.738481 \\ \mathrm{C} & -1.988006 & 0.032065 & -1.684771 \\ \mathrm{C} & -1.890522 & -1.396971 & -2.249189 \\ \mathrm{~N} & -2.478482 & -1.446474 & -3.578943 \\ \mathrm{H} & 1.230957 & -0.058377 & 3.236141 \\ \mathrm{H} & 3.354847 & -0.159839 & 1.859360 \\ \mathrm{H} & 3.290378 & -0.143670 & -0.569797 \\ \mathrm{H} & 1.112960 & 0.023293 & -1.818625 \\ \mathrm{H} & -1.549894 & 0.135981 & 2.875775 \\ \mathrm{H} & -3.244929 & 0.212280 & 1.043318 \\ \mathrm{H} & -1.427786 & 0.701662 & -2.345307 \\ \mathrm{H} & -3.034327 & 0.342791 & -1.700900 \\ \mathrm{H} & -2.456480 & -2.067524 & -1.598286 \\ \mathrm{H} & -0.838927 & -1.711277 & -2.204667 \\ \mathrm{H} & -1.904022 & -0.945766 & -4.244749 \\ \mathrm{H} & -2.556907 & -2.400337 & -3.903471\end{array}$


tryptamine, A-up $\mathrm{S}_{0}$

$\begin{array}{lrrr}\mathrm{C} & -2.130242 & 0.140397 & 0.887508 \\ \mathrm{~N} & -1.256281 & 0.107867 & 1.952331 \\ \mathrm{C} & 0.027934 & 0.035141 & 1.474468 \\ \mathrm{C} & -0.050984 & 0.017654 & 0.066991 \\ \mathrm{C} & -1.443770 & 0.088471 & -0.287017 \\ \mathrm{C} & 1.246264 & -0.014590 & 2.148932 \\ \mathrm{C} & 2.394570 & -0.080424 & 1.386902 \\ \mathrm{C} & 2.339180 & -0.094971 & -0.015913 \\ \mathrm{C} & 1.130469 & -0.046906 & -0.678290 \\ \mathrm{C} & -2.005569 & 0.045753 & -1.670523 \\ \mathrm{C} & -1.943315 & -1.357685 & -2.295719 \\ \mathrm{~N} & -2.416039 & -1.457685 & -3.668084 \\ \mathrm{H} & 1.292809 & -0.002509 & 3.231331 \\ \mathrm{H} & 3.358259 & -0.121013 & 1.879334 \\ \mathrm{H} & 3.261276 & -0.143931 & -0.581438 \\ \mathrm{H} & 1.096666 & -0.058015 & -1.761638 \\ \mathrm{H} & -1.517698 & 0.149976 & 2.918873 \\ \mathrm{H} & -3.194091 & 0.203769 & 1.055532 \\ \mathrm{H} & -1.455415 & 0.740321 & -2.315204 \\ \mathrm{H} & -3.045504 & 0.386327 & -1.661982 \\ \mathrm{H} & -2.527147 & -2.044669 & -1.678193 \\ \mathrm{H} & -0.913750 & -1.721845 & -2.261255 \\ \mathrm{H} & -1.867712 & -0.869047 & -4.281924 \\ \mathrm{H} & -3.378671 & -1.154479 & -3.741785\end{array}$

tryptamine, A-up $\mathrm{S}_{1}$

$\begin{array}{lrrr}\mathrm{C} & -2.180883 & 0.145780 & 0.901530 \\ \mathrm{~N} & -1.297020 & 0.089569 & 1.908394 \\ \mathrm{C} & 0.021904 & 0.040781 & 1.437432 \\ \mathrm{C} & -0.051817 & 0.082754 & 0.039285 \\ \mathrm{C} & -1.425262 & 0.150977 & -0.311360 \\ \mathrm{C} & 1.203095 & -0.067273 & 2.154543 \\ \mathrm{C} & 2.401422 & -0.121524 & 1.347918 \\ \mathrm{C} & 2.359028 & -0.081384 & -0.021015 \\ \mathrm{C} & 1.131076 & 0.021415 & -0.737734 \\ \mathrm{C} & -1.983727 & 0.093940 & -1.674963 \\ \mathrm{C} & -1.925674 & -1.354278 & -2.239924 \\ \mathrm{~N} & -2.405091 & -1.505480 & -3.595966 \\ \mathrm{H} & 1.237937 & -0.114878 & 3.233363 \\ \mathrm{H} & 3.358030 & -0.197848 & 1.849643 \\ \mathrm{H} & 3.286795 & -0.124571 & -0.578451 \\ \mathrm{H} & 1.108361 & 0.081972 & -1.816945\end{array}$




$\begin{array}{llll}\mathrm{H} & -1.541312 & 0.096882 & 2.884858 \\ \mathrm{H} & -3.242646 & 0.225805 & 1.062367 \\ \mathrm{H} & -1.400475 & 0.738218 & -2.339133 \\ \mathrm{H} & -3.020347 & 0.441899 & -1.690096 \\ \mathrm{H} & -2.507917 & -2.008393 & -1.587534 \\ \mathrm{H} & -0.890159 & -1.697500 & -2.188194 \\ \mathrm{H} & -1.860175 & -0.957754 & -4.247916 \\ \mathrm{H} & -3.373922 & -1.231188 & -3.686882\end{array}$

tryptamine, Ph-out $\mathrm{S}_{0}$

$\begin{array}{lrrr}\mathrm{C} & 0.001685 & 0.007399 & 0.078860 \\ \mathrm{C} & 0.041777 & 0.061712 & 1.487609 \\ \mathrm{C} & 1.241394 & 0.069060 & 2.196924 \\ \mathrm{C} & 2.411406 & 0.023194 & 1.467684 \\ \mathrm{C} & 2.394727 & -0.030428 & 0.064566 \\ \mathrm{C} & 1.205749 & -0.038049 & -0.634013 \\ \mathrm{~N} & -1.255762 & 0.102794 & 1.931077 \\ \mathrm{H} & 1.256506 & 0.110673 & 3.279460 \\ \mathrm{H} & 3.362160 & 0.027579 & 1.986475 \\ \mathrm{H} & 3.333125 & -0.067060 & -0.474609 \\ \mathrm{H} & 1.195439 & -0.097244 & -1.715542 \\ \mathrm{C} & -1.384495 & 0.023417 & -0.312094 \\ \mathrm{C} & -2.101842 & 0.084845 & 0.844465 \\ \mathrm{H} & -1.542588 & 0.171048 & 2.888915 \\ \mathrm{H} & -3.171009 & 0.127811 & 0.983139 \\ \mathrm{C} & -1.935346 & -0.034896 & -1.703502 \\ \mathrm{H} & -1.292018 & 0.535630 & -2.378581 \\ \mathrm{C} & -2.075156 & -1.454963 & -2.254870 \\ \mathrm{H} & -2.920012 & 0.439565 & -1.720029 \\ \mathrm{H} & -2.536171 & -1.397735 & -3.244450 \\ \mathrm{~N} & -0.774373 & -2.095673 & -2.403576 \\ \mathrm{H} & -2.766467 & -2.012719 & -1.604812 \\ \mathrm{H} & -0.334414 & -2.221804 & -1.500129 \\ \mathrm{H} & -0.869346 & -3.010048 & -2.823756\end{array}$

tryptamine, $\mathrm{Ph}$-out $\mathrm{S}_{1}$

$\begin{array}{rrrr}\mathrm{C} & -0.001035 & 0.038091 & 0.058060 \\ \mathrm{C} & 0.030862 & 0.074441 & 1.460091 \\ \mathrm{C} & 1.189910 & 0.039022 & 2.217908 \\ \mathrm{C} & 2.417034 & -0.018142 & 1.454153 \\ \mathrm{C} & 2.417173 & -0.055450 & 0.084646 \\ \mathrm{C} & 1.212251 & -0.036755 & -0.675457 \\ \mathrm{~N} & -1.300632 & 0.120616 & 1.890815 \\ \mathrm{H} & 1.190390 & 0.064643 & 3.298066\end{array}$




$\begin{array}{lrrr}\mathrm{H} & 3.358008 & -0.033902 & 1.989849 \\ \mathrm{H} & 3.362812 & -0.100172 & -0.441757 \\ \mathrm{H} & 1.219838 & -0.049605 & -1.756012 \\ \mathrm{C} & -1.364073 & 0.062288 & -0.335129 \\ \mathrm{C} & -2.153618 & 0.108802 & 0.857357 \\ \mathrm{H} & -1.573449 & 0.171696 & 2.858365 \\ \mathrm{H} & -3.221686 & 0.169542 & 0.981276 \\ \mathrm{C} & -1.927257 & -0.028885 & -1.702979 \\ \mathrm{H} & -1.281670 & 0.497420 & -2.409349 \\ \mathrm{C} & -2.070322 & -1.485736 & -2.183179 \\ \mathrm{H} & -2.914372 & 0.440521 & -1.721550 \\ \mathrm{H} & -2.658362 & -1.476197 & -3.104102 \\ \mathrm{~N} & -0.772322 & -2.062944 & -2.480412 \\ \mathrm{H} & -2.651741 & -2.040434 & -1.431215 \\ \mathrm{H} & -0.162538 & -2.057300 & -1.670353 \\ \mathrm{H} & -0.860211 & -3.017440 & -2.799882\end{array}$

tryptamine, $\mathrm{Ph}$-up $\mathrm{S}_{0}$

$\begin{array}{lrrc}\mathrm{C} & -2.091455 & 0.054002 & 0.824724 \\ \mathrm{~N} & -1.251591 & 0.042680 & 1.915948 \\ \mathrm{C} & 0.048440 & 0.039019 & 1.479731 \\ \mathrm{C} & 0.016071 & 0.044304 & 0.069653 \\ \mathrm{C} & -1.366732 & 0.052863 & -0.329189 \\ \mathrm{C} & 1.243939 & 0.033736 & 2.195910 \\ \mathrm{C} & 2.418965 & 0.035722 & 1.473515 \\ \mathrm{C} & 2.411510 & 0.045425 & 0.069391 \\ \mathrm{C} & 1.225742 & 0.050131 & -0.634180 \\ \mathrm{C} & -1.902812 & 0.013323 & -1.725376 \\ \mathrm{C} & -2.076067 & -1.414720 & -2.273648 \\ \mathrm{~N} & -0.871150 & -2.223454 & -2.359275 \\ \mathrm{H} & 1.252498 & 0.029230 & 3.279219 \\ \mathrm{H} & 3.366316 & 0.031293 & 1.998190 \\ \mathrm{H} & 3.353316 & 0.049676 & -0.464936 \\ \mathrm{H} & 1.229225 & 0.058265 & -1.717751 \\ \mathrm{H} & -1.545223 & 0.058193 & 2.874127 \\ \mathrm{H} & -3.161941 & 0.067901 & 0.958733 \\ \mathrm{H} & -1.234875 & 0.571581 & -2.389717 \\ \mathrm{H} & -2.871218 & 0.521277 & -1.762245 \\ \mathrm{H} & -2.519560 & -1.358960 & -3.271561 \\ \mathrm{H} & -2.792551 & -1.947895 & -1.642842 \\ \mathrm{H} & -0.435184 & -2.311094 & -1.449695 \\ \mathrm{H} & -0.192014 & -1.792867 & -2.973666\end{array}$

tryptamine, $\mathrm{Ph}$-up $\mathrm{S}_{1}$ 


$\begin{array}{lrrr}\mathrm{C} & -2.162406 & 0.144579 & 0.858379 \\ \mathrm{~N} & -1.307159 & 0.124121 & 1.890110 \\ \mathrm{C} & 0.023315 & 0.079730 & 1.454281 \\ \mathrm{C} & -0.012823 & 0.090157 & 0.053293 \\ \mathrm{C} & -1.377388 & 0.124398 & -0.334909 \\ \mathrm{C} & 1.184665 & -0.003373 & 2.206896 \\ \mathrm{C} & 2.406048 & -0.058181 & 1.437569 \\ \mathrm{C} & 2.404133 & -0.045787 & 0.067499 \\ \mathrm{C} & 1.197246 & 0.024414 & -0.687814 \\ \mathrm{C} & -1.927387 & 0.011131 & -1.696277 \\ \mathrm{C} & -1.993700 & -1.479074 & -2.171360 \\ \mathrm{~N} & -0.733543 & -2.134562 & -2.402430 \\ \mathrm{H} & 1.188337 & -0.023252 & 3.287062 \\ \mathrm{H} & 3.348387 & -0.111049 & 1.968589 \\ \mathrm{H} & 3.348636 & -0.085740 & -0.461381 \\ \mathrm{H} & 1.208442 & 0.108693 & -1.765224 \\ \mathrm{H} & -1.577108 & 0.157821 & 2.859077 \\ \mathrm{H} & -3.229767 & 0.209927 & 0.986937 \\ \mathrm{H} & -1.308241 & 0.566132 & -2.405366 \\ \mathrm{H} & -2.940066 & 0.422665 & -1.731611 \\ \mathrm{H} & -2.572877 & -1.499826 & -3.097436 \\ \mathrm{H} & -2.558611 & -2.048339 & -1.430167 \\ \mathrm{H} & -0.135066 & -2.125423 & -1.584429 \\ \mathrm{H} & -0.219409 & -1.699555 & -3.156198\end{array}$

tryptamine, Py-out $\mathrm{S}_{0}$

$\begin{array}{lrrr}\mathrm{C} & 0.507277 & 0.456685 & 0.219205 \\ \mathrm{C} & 0.584157 & 0.520991 & 1.625928 \\ \mathrm{C} & 1.799394 & 0.472366 & 2.305266 \\ \mathrm{C} & 2.948017 & 0.358096 & 1.549150 \\ \mathrm{C} & 2.895166 & 0.294562 & 0.147731 \\ \mathrm{C} & 1.689299 & 0.342556 & -0.519922 \\ \mathrm{~N} & -0.698998 & 0.636546 & 2.098783 \\ \mathrm{H} & 1.845009 & 0.524122 & 3.386521 \\ \mathrm{H} & 3.909421 & 0.320409 & 2.046239 \\ \mathrm{H} & 3.818269 & 0.209183 & -0.411995 \\ \mathrm{H} & 1.656089 & 0.295696 & -1.602158 \\ \mathrm{C} & -0.883582 & 0.547422 & -0.139479 \\ \mathrm{C} & -1.570541 & 0.652215 & 1.031548 \\ \mathrm{H} & -0.959981 & 0.722996 & 3.062545 \\ \mathrm{H} & -2.634174 & 0.737176 & 1.189495 \\ \mathrm{C} & -1.453065 & 0.482254 & -1.520451 \\ \mathrm{H} & -0.890257 & 1.147009 & -2.182387 \\ \mathrm{C} & -1.429816 & -0.931195 & -2.106053 \\ \mathrm{H} & -2.487380 & 0.833364 & -1.507499\end{array}$




$\begin{array}{llll}\mathrm{H} & -0.384589 & -1.265064 & -2.185857 \\ \mathrm{~N} & -2.263031 & -1.822623 & -1.309042 \\ \mathrm{H} & -1.837442 & -0.896856 & -3.119328 \\ \mathrm{H} & -2.260750 & -2.757932 & -1.692456 \\ \mathrm{H} & -1.898472 & -1.879980 & -0.365765\end{array}$

tryptamine, Py-out $\mathrm{S}_{1}$

$\begin{array}{lrrr}\mathrm{C} & 0.494969 & 0.487034 & 0.200014 \\ \mathrm{C} & 0.578249 & 0.529592 & 1.597166 \\ \mathrm{C} & 1.761974 & 0.439518 & 2.313791 \\ \mathrm{C} & 2.953552 & 0.314875 & 1.503497 \\ \mathrm{C} & 2.902553 & 0.274635 & 0.134778 \\ \mathrm{C} & 1.671538 & 0.354775 & -0.579951 \\ \mathrm{~N} & -0.735196 & 0.652721 & 2.073271 \\ \mathrm{H} & 1.805903 & 0.467137 & 3.392908 \\ \mathrm{H} & 3.912009 & 0.255780 & 2.004109 \\ \mathrm{H} & 3.826866 & 0.184233 & -0.422807 \\ \mathrm{H} & 1.640385 & 0.351327 & -1.660418 \\ \mathrm{C} & -0.878459 & 0.585866 & -0.145754 \\ \mathrm{C} & -1.622386 & 0.683521 & 1.069813 \\ \mathrm{H} & -0.972669 & 0.714283 & 3.049595 \\ \mathrm{H} & -2.684243 & 0.768135 & 1.225972 \\ \mathrm{C} & -1.456568 & 0.507801 & -1.505573 \\ \mathrm{H} & -0.885763 & 1.159440 & -2.173696 \\ \mathrm{C} & -1.394181 & -0.924331 & -2.064533 \\ \mathrm{H} & -2.497003 & 0.838619 & -1.499526 \\ \mathrm{H} & -0.338592 & -1.221716 & -2.124845 \\ \mathrm{~N} & -2.212182 & -1.821690 & -1.260531 \\ \mathrm{H} & -1.796673 & -0.910986 & -3.079110 \\ \mathrm{H} & -2.282733 & -2.729860 & -1.698671 \\ \mathrm{H} & -1.791359 & -1.960718 & -0.349512\end{array}$

tryptamine, Py-up $\mathrm{S}_{0}$

$\begin{array}{rrrr}\mathrm{C} & -2.129404 & 0.260062 & 0.886313 \\ \mathrm{~N} & -1.257130 & 0.225641 & 1.952640 \\ \mathrm{C} & 0.023069 & 0.089946 & 1.477122 \\ \mathrm{C} & -0.056657 & 0.033310 & 0.070543 \\ \mathrm{C} & -1.446214 & 0.148489 & -0.286366 \\ \mathrm{C} & 1.238120 & 0.017001 & 2.154740 \\ \mathrm{C} & 2.383239 & -0.114622 & 1.396433 \\ \mathrm{C} & 2.327445 & -0.171730 & -0.005188 \\ \mathrm{C} & 1.121957 & -0.099678 & -0.670958 \\ \mathrm{C} & -2.013636 & 0.090486 & -1.666424 \\ \mathrm{C} & -1.988141 & -1.327980 & -2.265998\end{array}$




$\begin{array}{lrrr}\mathrm{N} & -2.738876 & -2.334695 & -1.534393 \\ \mathrm{H} & 1.286019 & 0.062683 & 3.236130 \\ \mathrm{H} & 3.344429 & -0.172140 & 1.891977 \\ \mathrm{H} & 3.247967 & -0.272438 & -0.566497 \\ \mathrm{H} & 1.087041 & -0.142374 & -1.753286 \\ \mathrm{H} & -1.514722 & 0.313427 & 2.917174 \\ \mathrm{H} & -3.190179 & 0.370865 & 1.048893 \\ \mathrm{H} & -1.455396 & 0.762778 & -2.326416 \\ \mathrm{H} & -3.047353 & 0.450151 & -1.652534 \\ \mathrm{H} & -0.952269 & -1.669224 & -2.336713 \\ \mathrm{H} & -2.373480 & -1.287758 & -3.287862 \\ \mathrm{H} & -3.716213 & -2.076930 & -1.474513 \\ \mathrm{H} & -2.391097 & -2.405650 & -0.585930\end{array}$

tryptamine, Py-up $\mathrm{S}_{1}$

$\begin{array}{rrrr}\mathrm{C} & -2.181705 & 0.282688 & 0.919422 \\ \mathrm{~N} & -1.292917 & 0.240523 & 1.923696 \\ \mathrm{C} & 0.017757 & 0.105449 & 1.447916 \\ \mathrm{C} & -0.065787 & 0.074084 & 0.049144 \\ \mathrm{C} & -1.438004 & 0.185801 & -0.296748 \\ \mathrm{C} & 1.198009 & -0.011215 & 2.163459 \\ \mathrm{C} & 2.389796 & -0.152763 & 1.355427 \\ \mathrm{C} & 2.338467 & -0.182692 & -0.013067 \\ \mathrm{C} & 1.110056 & -0.073480 & -0.727829 \\ \mathrm{C} & -2.013561 & 0.109096 & -1.654110 \\ \mathrm{C} & -1.957879 & -1.332783 & -2.228863 \\ \mathrm{~N} & -2.709689 & -2.337451 & -1.507915 \\ \mathrm{H} & 1.239953 & 0.003106 & 3.243035 \\ \mathrm{H} & 3.345623 & -0.235574 & 1.857335 \\ \mathrm{H} & 3.260565 & -0.288238 & -0.571591 \\ \mathrm{H} & 1.082010 & -0.070218 & -1.808483 \\ \mathrm{H} & -1.528403 & 0.308442 & 2.900121 \\ \mathrm{H} & -3.238267 & 0.415046 & 1.079370 \\ \mathrm{H} & -1.442969 & 0.758739 & -2.325567 \\ \mathrm{H} & -3.051160 & 0.455411 & -1.651618 \\ \mathrm{H} & -0.912923 & -1.647571 & -2.265531 \\ \mathrm{H} & -2.320624 & -1.297987 & -3.258322 \\ \mathrm{H} & -3.689138 & -2.094991 & -1.439616 \\ \mathrm{H} & -2.350689 & -2.463793 & -0.570755\end{array}$

porphycene $\mathrm{S}_{0}$
C $\quad-3.426107$
$1.908650 \quad 0.000000$
$\begin{array}{llll}\mathrm{C} & -2.414021 & 0.904517 & 0.000000\end{array}$
$\begin{array}{llll}\mathrm{N} & -1.219694 & 1.540251 & 0.000000\end{array}$ 


$\begin{array}{rrrr}\mathrm{C} & -1.388187 & 2.887551 & 0.000000 \\ \mathrm{C} & -2.798748 & 3.122976 & 0.000000 \\ \mathrm{C} & -0.365749 & 3.841322 & 0.000000 \\ \mathrm{C} & 1.011357 & 3.693551 & 0.000000 \\ \mathrm{C} & 1.827033 & 2.551962 & 0.000000 \\ \mathrm{H} & -0.262248 & 1.126277 & 0.000000 \\ \mathrm{~N} & 1.410506 & 1.265932 & 0.000000 \\ \mathrm{C} & 3.277546 & 2.591727 & 0.000000 \\ \mathrm{C} & 3.703838 & 1.305721 & 0.000000 \\ \mathrm{C} & 2.509259 & 0.494150 & 0.000000 \\ \mathrm{C} & 2.414021 & -0.904517 & 0.000000 \\ \mathrm{C} & 3.426107 & -1.908650 & 0.000000 \\ \mathrm{C} & 2.798748 & -3.122976 & 0.000000 \\ \mathrm{C} & 1.388187 & -2.887551 & 0.000000 \\ \mathrm{~N} & 1.219694 & -1.540251 & 0.000000 \\ \mathrm{C} & 0.365749 & -3.841322 & 0.000000 \\ \mathrm{C} & -1.011357 & -3.693551 & 0.000000 \\ \mathrm{C} & -1.827033 & -2.551962 & 0.000000 \\ \mathrm{C} & -3.277546 & -2.591727 & 0.000000 \\ \mathrm{C} & -3.703838 & -1.305721 & 0.000000 \\ \mathrm{C} & -2.509259 & -0.494150 & 0.000000 \\ \mathrm{~N} & -1.410506 & -1.265932 & 0.000000 \\ \mathrm{H} & 0.262248 & -1.126277 & 0.000000 \\ \mathrm{H} & 3.259870 & -4.098221 & 0.000000 \\ \mathrm{H} & 4.487759 & -1.720331 & 0.000000 \\ \mathrm{H} & 0.726422 & -4.864024 & 0.000000 \\ \mathrm{H} & -1.566746 & -4.625688 & 0.000000 \\ \mathrm{H} & -3.879094 & -3.488401 & 0.000000 \\ \mathrm{H} & -4.719979 & -0.942648 & 0.000000 \\ \mathrm{H} & -4.487759 & 1.720331 & 0.000000 \\ \mathrm{H} & -3.259870 & 4.098221 & 0.000000 \\ \mathrm{H} & -0.726422 & 4.864024 & 0.000000 \\ \mathrm{H} & 1.566746 & 4.625688 & 0.000000 \\ \mathrm{H} & 3.879094 & 3.488401 & 0.000000 \\ \mathrm{H} & 4.719979 & 0.942648 & 0.000000\end{array}$

porphycene $\mathrm{S}_{1}$

$\begin{array}{rrrr}\mathrm{C} & -3.449300 & 1.897580 & 0.000000 \\ \mathrm{C} & -2.440755 & 0.894518 & 0.000000 \\ \mathrm{~N} & -1.234786 & 1.538552 & 0.000000 \\ \mathrm{C} & -1.409367 & 2.878775 & 0.000000 \\ \mathrm{C} & -2.819092 & 3.114784 & 0.000000 \\ \mathrm{C} & -0.378948 & 3.849272 & 0.000000 \\ \mathrm{C} & 0.986943 & 3.705727 & 0.000000 \\ \mathrm{C} & 1.831329 & 2.558286 & 0.000000\end{array}$




$\begin{array}{lrrr}\mathrm{H} & -0.281510 & 1.123822 & 0.000000 \\ \mathrm{~N} & 1.418460 & 1.277172 & 0.000000 \\ \mathrm{C} & 3.272308 & 2.610748 & 0.000000 \\ \mathrm{C} & 3.714631 & 1.321686 & 0.000000 \\ \mathrm{C} & 2.539125 & 0.503498 & 0.000000 \\ \mathrm{C} & 2.440755 & -0.894518 & 0.000000 \\ \mathrm{C} & 3.449300 & -1.897580 & 0.000000 \\ \mathrm{C} & 2.819092 & -3.114784 & 0.000000 \\ \mathrm{C} & 1.409367 & -2.878775 & 0.000000 \\ \mathrm{~N} & 1.234786 & -1.538552 & 0.000000 \\ \mathrm{C} & 0.378948 & -3.849272 & 0.000000 \\ \mathrm{C} & -0.986943 & -3.705727 & 0.000000 \\ \mathrm{C} & -1.831329 & -2.558286 & 0.000000 \\ \mathrm{C} & -3.272308 & -2.610748 & 0.000000 \\ \mathrm{C} & -3.714631 & -1.321686 & 0.000000 \\ \mathrm{C} & -2.539125 & -0.503498 & 0.000000 \\ \mathrm{~N} & -1.418460 & -1.277172 & 0.000000 \\ \mathrm{H} & 0.281510 & -1.123822 & 0.000000 \\ \mathrm{H} & 3.278487 & -4.090822 & 0.000000 \\ \mathrm{H} & 4.510719 & -1.708828 & 0.000000 \\ \mathrm{H} & 0.746900 & -4.868862 & 0.000000 \\ \mathrm{H} & -1.540133 & -4.639691 & 0.000000 \\ \mathrm{H} & -3.866773 & -3.512173 & 0.000000 \\ \mathrm{H} & -4.734732 & -0.970943 & 0.000000 \\ \mathrm{H} & -4.510719 & 1.708828 & 0.000000 \\ \mathrm{H} & -3.278487 & 4.090822 & 0.000000 \\ \mathrm{H} & -0.746900 & 4.868862 & 0.000000 \\ \mathrm{H} & 1.540133 & 4.639691 & 0.000000 \\ \mathrm{H} & 3.866773 & 3.512173 & 0.000000 \\ \mathrm{H} & 4.734732 & 0.970943 & 0.000000\end{array}$

porphine $\mathrm{S}_{0}$

$\begin{array}{lrrr}\mathrm{N} & 0.000000 & -2.015135 & 0.000000 \\ \mathrm{~N} & 2.098550 & 0.000000 & 0.000000 \\ \mathrm{C} & 1.078102 & -2.833716 & 0.000000 \\ \mathrm{C} & 0.673100 & -4.231206 & 0.000000 \\ \mathrm{C} & 2.406649 & -2.424730 & 0.000000 \\ \mathrm{C} & 2.872955 & -1.121263 & 0.000000 \\ \mathrm{C} & 4.232738 & -0.680867 & 0.000000 \\ \mathrm{H} & 1.345451 & -5.076108 & 0.000000 \\ \mathrm{H} & 3.162566 & -3.200337 & 0.000000 \\ \mathrm{H} & 5.085796 & -1.341008 & 0.000000 \\ \mathrm{H} & 1.086460 & 0.000000 & 0.000000 \\ \mathrm{~N} & 0.000000 & 2.015135 & 0.000000 \\ \mathrm{~N} & -2.098550 & 0.000000 & 0.000000\end{array}$




$\begin{array}{lrrr}\mathrm{C} & 1.078102 & 2.833716 & 0.000000 \\ \mathrm{C} & -1.078102 & 2.833716 & 0.000000 \\ \mathrm{C} & -1.078102 & -2.833716 & 0.000000 \\ \mathrm{C} & 0.673100 & 4.231206 & 0.000000 \\ \mathrm{C} & -0.673100 & 4.231206 & 0.000000 \\ \mathrm{C} & -0.673100 & -4.231206 & 0.000000 \\ \mathrm{C} & 2.406649 & 2.424730 & 0.000000 \\ \mathrm{C} & -2.406649 & 2.424730 & 0.000000 \\ \mathrm{C} & -2.406649 & -2.424730 & 0.000000 \\ \mathrm{C} & 2.872955 & 1.121263 & 0.000000 \\ \mathrm{C} & -2.872955 & 1.121263 & 0.000000 \\ \mathrm{C} & -2.872955 & -1.121263 & 0.000000 \\ \mathrm{C} & 4.232738 & 0.680867 & 0.000000 \\ \mathrm{C} & -4.232738 & 0.680867 & 0.000000 \\ \mathrm{C} & -4.232738 & -0.680867 & 0.000000 \\ \mathrm{H} & 1.345451 & 5.076108 & 0.000000 \\ \mathrm{H} & -1.345451 & 5.076108 & 0.000000 \\ \mathrm{H} & -1.345451 & -5.076108 & 0.000000 \\ \mathrm{H} & 3.162566 & 3.200337 & 0.000000 \\ \mathrm{H} & -3.162566 & 3.200337 & 0.000000 \\ \mathrm{H} & -3.162566 & -3.200337 & 0.000000 \\ \mathrm{H} & 5.085796 & 1.341008 & 0.000000 \\ \mathrm{H} & -5.085796 & 1.341008 & 0.000000 \\ \mathrm{H} & -5.085796 & -1.341008 & 0.000000 \\ \mathrm{H} & -1.086460 & 0.000000 & 0.000000\end{array}$

porphine $\mathrm{S}_{1}$

$\begin{array}{rrrr}\mathrm{N} & 0.000000 & -2.035219 & 0.000000 \\ \mathrm{~N} & 2.096875 & 0.000000 & 0.000000 \\ \mathrm{C} & 1.081451 & -2.857396 & 0.000000 \\ \mathrm{C} & 0.674602 & -4.251194 & 0.000000 \\ \mathrm{C} & 2.412312 & -2.434047 & 0.000000 \\ \mathrm{C} & 2.875258 & -1.124396 & 0.000000 \\ \mathrm{C} & 4.234376 & -0.682555 & 0.000000 \\ \mathrm{H} & 1.345192 & -5.097220 & 0.000000 \\ \mathrm{H} & 3.172749 & -3.205137 & 0.000000 \\ \mathrm{H} & 5.088254 & -1.341509 & 0.000000 \\ \mathrm{H} & 1.086533 & 0.000000 & 0.000000 \\ \mathrm{~N} & 0.000000 & 2.035219 & 0.000000 \\ \mathrm{~N} & -2.096875 & 0.000000 & 0.000000 \\ \mathrm{C} & 1.081451 & 2.857396 & 0.000000 \\ \mathrm{C} & -1.081451 & 2.857396 & 0.000000 \\ \mathrm{C} & -1.081451 & -2.857396 & 0.000000 \\ \mathrm{C} & 0.674602 & 4.251194 & 0.000000 \\ \mathrm{C} & -0.674602 & 4.251194 & 0.000000\end{array}$




$\begin{array}{rrrr}\mathrm{C} & -0.674602 & -4.251194 & 0.000000 \\ \mathrm{C} & 2.412312 & 2.434047 & 0.000000 \\ \mathrm{C} & -2.412312 & 2.434047 & 0.000000 \\ \mathrm{C} & -2.412312 & -2.434047 & 0.000000 \\ \mathrm{C} & 2.875258 & 1.124396 & 0.000000 \\ \mathrm{C} & -2.875258 & 1.124396 & 0.000000 \\ \mathrm{C} & -2.875258 & -1.124396 & 0.000000 \\ \mathrm{C} & 4.234376 & 0.682555 & 0.000000 \\ \mathrm{C} & -4.234376 & 0.682555 & 0.000000 \\ \mathrm{C} & -4.234376 & -0.682555 & 0.000000 \\ \mathrm{H} & 1.345192 & 5.097220 & 0.000000 \\ \mathrm{H} & -1.345192 & 5.097220 & 0.000000 \\ \mathrm{H} & -1.345192 & -5.097220 & 0.000000 \\ \mathrm{H} & 3.172749 & 3.205137 & 0.000000 \\ \mathrm{H} & -3.172749 & 3.205137 & 0.000000 \\ \mathrm{H} & -3.172749 & -3.205137 & 0.000000 \\ \mathrm{H} & 5.088254 & 1.341509 & 0.000000 \\ \mathrm{H} & -5.088254 & 1.341509 & 0.000000 \\ \mathrm{H} & -5.088254 & -1.341509 & 0.000000 \\ \mathrm{H} & -1.086533 & 0.000000 & 0.000000\end{array}$

chlorin $\mathrm{S}_{0}$

$\begin{array}{rrrr}\mathrm{C} & 0.000000 & -4.225967 & 0.920130 \\ \mathrm{C} & 0.000000 & -2.872187 & 1.370753 \\ \mathrm{~N} & 0.000000 & -2.088534 & 0.239440 \\ \mathrm{C} & 0.000000 & -2.854042 & -0.875869 \\ \mathrm{C} & 0.000000 & -4.217498 & -0.441817 \\ \mathrm{C} & 0.000000 & -2.410575 & 2.664947 \\ \mathrm{C} & 0.000000 & -1.077250 & 3.088950 \\ \mathrm{~N} & 0.000000 & 0.000000 & 2.268892 \\ \mathrm{C} & 0.000000 & 1.077250 & 3.088950 \\ \mathrm{C} & 0.000000 & 0.677214 & 4.475402 \\ \mathrm{C} & 0.000000 & -0.677214 & 4.475402 \\ \mathrm{C} & 0.000000 & 2.410575 & 2.664947 \\ \mathrm{C} & 0.000000 & 2.872187 & 1.370753 \\ \mathrm{~N} & 0.000000 & 2.088534 & 0.239440 \\ \mathrm{C} & 0.000000 & 2.854042 & -0.875869 \\ \mathrm{C} & 0.000000 & 4.217498 & -0.441817 \\ \mathrm{C} & 0.000000 & 4.225967 & 0.920130 \\ \mathrm{C} & 0.000000 & 2.401203 & -2.196148 \\ \mathrm{C} & 0.000000 & 1.096846 & -2.639855 \\ \mathrm{C} & 0.000000 & 0.764066 & -4.121717 \\ \mathrm{C} & 0.000000 & -0.764066 & -4.121717 \\ \mathrm{C} & 0.000000 & -1.096846 & -2.639855 \\ \mathrm{~N} & 0.000000 & 0.000000 & -1.862602\end{array}$




$\begin{array}{lrrr}\mathrm{C} & 0.000000 & -2.401203 & -2.196148 \\ \mathrm{H} & -0.879124 & 1.187848 & -4.609335 \\ \mathrm{H} & 0.000000 & 3.176398 & -2.952507 \\ \mathrm{H} & 0.000000 & 5.065571 & -1.108085 \\ \mathrm{H} & 0.000000 & 1.347846 & 5.321773 \\ \mathrm{H} & 0.000000 & -1.347846 & 5.321773 \\ \mathrm{H} & -0.879124 & -1.187848 & -4.609335 \\ \mathrm{H} & 0.000000 & 3.169436 & 3.438555 \\ \mathrm{H} & 0.000000 & -3.169436 & 3.438555 \\ \mathrm{H} & 0.000000 & -3.176398 & -2.952507 \\ \mathrm{H} & 0.000000 & 5.084300 & 1.573779 \\ \mathrm{H} & 0.000000 & -5.084300 & 1.573779 \\ \mathrm{H} & 0.000000 & -5.065571 & -1.108085 \\ \mathrm{H} & 0.879124 & -1.187848 & -4.609335 \\ \mathrm{H} & 0.879124 & 1.187848 & -4.609335 \\ \mathrm{H} & 0.000000 & -1.078405 & 0.257788 \\ \mathrm{H} & 0.000000 & 1.078405 & 0.257788\end{array}$

chlorin $\mathrm{S}_{1}$

$\begin{array}{lrrr}\mathrm{C} & 0.000000 & -4.233148 & 0.920686 \\ \mathrm{C} & 0.000000 & -2.878519 & 1.367108 \\ \mathrm{~N} & 0.000000 & -2.095396 & 0.241172 \\ \mathrm{C} & 0.000000 & -2.868421 & -0.883310 \\ \mathrm{C} & 0.000000 & -4.226799 & -0.447168 \\ \mathrm{C} & 0.000000 & -2.413086 & 2.676614 \\ \mathrm{C} & 0.000000 & -1.081469 & 3.088492 \\ \mathrm{~N} & 0.000000 & 0.000000 & 2.267117 \\ \mathrm{C} & 0.000000 & 1.081469 & 3.088492 \\ \mathrm{C} & 0.000000 & 0.672831 & 4.487312 \\ \mathrm{C} & 0.000000 & -0.672831 & 4.487312 \\ \mathrm{C} & 0.000000 & 2.413086 & 2.676614 \\ \mathrm{C} & 0.000000 & 2.878519 & 1.367108 \\ \mathrm{~N} & 0.000000 & 2.095396 & 0.241172 \\ \mathrm{C} & 0.000000 & 2.868421 & -0.883310 \\ \mathrm{C} & 0.000000 & 4.226799 & -0.447168 \\ \mathrm{C} & 0.000000 & 4.233148 & 0.920686 \\ \mathrm{C} & 0.000000 & 2.410569 & -2.203467 \\ \mathrm{C} & 0.000000 & 1.098156 & -2.645554 \\ \mathrm{C} & 0.000000 & 0.763491 & -4.122884 \\ \mathrm{C} & 0.000000 & -0.763491 & -4.122884 \\ \mathrm{C} & 0.000000 & -1.098156 & -2.645554 \\ \mathrm{~N} & 0.000000 & 0.000000 & -1.861418 \\ \mathrm{C} & 0.000000 & -2.410569 & -2.203467 \\ \mathrm{H} & -0.878689 & 1.187729 & -4.612451 \\ \mathrm{H} & 0.000000 & 3.181945 & -2.963565\end{array}$




$\begin{array}{lrrr}\mathrm{H} & 0.000000 & 5.077342 & -1.110461 \\ \mathrm{H} & 0.000000 & 1.345124 & 5.332115 \\ \mathrm{H} & 0.000000 & -1.345124 & 5.332115 \\ \mathrm{H} & -0.878689 & -1.187729 & -4.612451 \\ \mathrm{H} & 0.000000 & 3.169935 & 3.450849 \\ \mathrm{H} & 0.000000 & -3.169935 & 3.450849 \\ \mathrm{H} & 0.000000 & -3.181945 & -2.963565 \\ \mathrm{H} & 0.000000 & 5.089821 & 1.576125 \\ \mathrm{H} & 0.000000 & -5.089821 & 1.576125 \\ \mathrm{H} & 0.000000 & -5.077342 & -1.110461 \\ \mathrm{H} & 0.878689 & -1.187729 & -4.612451 \\ \mathrm{H} & 0.878689 & 1.187729 & -4.612451 \\ \mathrm{H} & 0.000000 & -1.085734 & 0.257999 \\ \mathrm{H} & 0.000000 & 1.085734 & 0.257999\end{array}$

Zn-tetraphenylporphine $\mathrm{S}_{0}$

$\begin{array}{lrrr}\text { C } & -3.794409 & 4.173228 & 1.168451 \\ \mathrm{C} & -3.483601 & 3.483572 & -0.000012 \\ \mathrm{C} & -4.172756 & 3.793817 & -1.168925 \\ \mathrm{C} & -5.153363 & 4.774894 & -1.170219 \\ \mathrm{C} & -5.457417 & 5.457241 & -0.000901 \\ \mathrm{C} & -4.775567 & 5.153764 & 1.168854 \\ \mathrm{C} & -2.427570 & 2.427577 & 0.000380 \\ \mathrm{C} & -1.096971 & 2.850581 & 0.004692 \\ \mathrm{~N} & 0.000000 & 2.039337 & 0.023637 \\ \mathrm{C} & 1.096971 & 2.850581 & 0.004692 \\ \mathrm{C} & 0.675802 & 4.228310 & -0.032225 \\ \mathrm{C} & -0.675802 & 4.228310 & -0.032225 \\ \mathrm{C} & 2.427570 & 2.427577 & 0.000380 \\ \mathrm{C} & 3.483601 & 3.483572 & -0.000012 \\ \mathrm{C} & 4.172756 & 3.793817 & -1.168925 \\ \mathrm{C} & 5.153363 & 4.774894 & -1.170219 \\ \mathrm{C} & 5.457417 & 5.457241 & -0.000901 \\ \mathrm{C} & 4.775567 & 5.153764 & 1.168854 \\ \mathrm{C} & 3.794409 & 4.173228 & 1.168451 \\ \mathrm{C} & -2.850560 & 1.096972 & -0.003798 \\ \mathrm{~N} & -2.039312 & 0.000000 & -0.022547 \\ \mathrm{C} & -2.850560 & -1.096972 & -0.003798 \\ \mathrm{C} & -4.228296 & -0.675801 & 0.032828 \\ \mathrm{C} & -4.228296 & 0.675801 & 0.032828 \\ \mathrm{C} & -2.427570 & -2.427577 & 0.000380 \\ \mathrm{C} & -3.483601 & -3.483572 & -0.000012 \\ \mathrm{C} & -4.172756 & -3.793817 & -1.168925 \\ \mathrm{C} & -5.153363 & -4.774894 & -1.170219 \\ \mathrm{C} & -5.457417 & -5.457241 & -0.000901\end{array}$




$\begin{array}{rrrr}\mathrm{C} & -4.775567 & -5.153764 & 1.168854 \\ \mathrm{C} & -3.794409 & -4.173228 & 1.168451 \\ \mathrm{C} & 2.850560 & 1.096972 & -0.003798 \\ \mathrm{~N} & 2.039312 & 0.000000 & -0.022547 \\ \mathrm{C} & 2.850560 & -1.096972 & -0.003798 \\ \mathrm{C} & 4.228296 & -0.675801 & 0.032828 \\ \mathrm{C} & 4.228296 & 0.675801 & 0.032828 \\ \mathrm{C} & 2.427570 & -2.427577 & 0.000380 \\ \mathrm{C} & 3.483601 & -3.483572 & -0.000012 \\ \mathrm{C} & 4.172756 & -3.793817 & -1.168925 \\ \mathrm{C} & 5.153363 & -4.774894 & -1.170219 \\ \mathrm{C} & 5.457417 & -5.457241 & -0.000901 \\ \mathrm{C} & 4.775567 & -5.153764 & 1.168854 \\ \mathrm{C} & 3.794409 & -4.173228 & 1.168451 \\ \mathrm{C} & 1.096971 & -2.850581 & 0.004692 \\ \mathrm{~N} & 0.000000 & -2.039337 & 0.023637 \\ \mathrm{C} & -1.096971 & -2.850581 & 0.004692 \\ \mathrm{C} & -0.675802 & -4.228310 & -0.032225 \\ \mathrm{C} & 0.675802 & -4.228310 & -0.032225 \\ \mathrm{H} & 1.338339 & -5.078020 & -0.064247 \\ \mathrm{H} & 5.078013 & -1.338339 & 0.064665 \\ \mathrm{H} & 1.338339 & 5.078020 & -0.064247 \\ \mathrm{H} & -1.338339 & 5.078020 & -0.064247 \\ \mathrm{H} & -1.338339 & -5.078020 & -0.064247 \\ \mathrm{H} & 5.078013 & 1.338339 & 0.064665 \\ \mathrm{H} & -5.078013 & 1.338339 & 0.064665 \\ \mathrm{H} & -5.078013 & -1.338339 & 0.064665 \\ \mathrm{H} & 3.933503 & 3.260681 & -2.081343 \\ \mathrm{H} & 5.679073 & 5.007759 & -2.088201 \\ \mathrm{H} & 6.223220 & 6.222982 & -0.001237 \\ \mathrm{H} & 5.008892 & 5.679864 & 2.086497 \\ \mathrm{H} & 3.261666 & 3.934433 & 2.081218 \\ \mathrm{H} & 3.933503 & -3.260681 & -2.081343 \\ \mathrm{H} & 5.679073 & -5.007759 & -2.088201 \\ \mathrm{H} & 6.223220 & -6.222982 & -0.001237 \\ \mathrm{H} & 5.008892 & -5.679864 & 2.086497 \\ \mathrm{H} & 3.261666 & -3.934433 & 2.081218 \\ \mathrm{H} & -3.933503 & -3.260681 & -2.081343 \\ \mathrm{H} & -5.679073 & -5.007759 & -2.088201 \\ \mathrm{H} & -6.223220 & -6.222982 & -0.001237 \\ \mathrm{H} & -5.008892 & -5.679864 & 2.086497 \\ \mathrm{H} & -3.261666 & -3.934433 & 2.081218 \\ \mathrm{H} & -3.933503 & 3.260681 & -2.081343 \\ \mathrm{H} & -5.679073 & 5.007759 & -2.088201 \\ \mathrm{H} & -6.223220 & 6.222982 & -0.001237 \\ \mathrm{H} & -5.008892 & 5.679864 & 2.086497\end{array}$




$\begin{array}{rrrr}\mathrm{H} & -3.261666 & 3.934433 & 2.081218 \\ \mathrm{Zn} & 0.000000 & 0.000000 & 0.000545\end{array}$

Zn-tetraphenylporphine $\mathrm{S}_{1}$

\begin{tabular}{rrrr}
$\mathrm{C}$ & -3.638403 & 4.433470 & 1.002576 \\
$\mathrm{C}$ & -3.491458 & 3.481672 & -0.004867 \\
$\mathrm{C}$ & -4.355402 & 3.525226 & -1.098682 \\
$\mathrm{C}$ & -5.338988 & 4.497419 & -1.185656 \\
$\mathrm{C}$ & -5.476977 & 5.440430 & -0.176786 \\
$\mathrm{C}$ & -4.624730 & 5.404899 & 0.917651 \\
$\mathrm{C}$ & -2.437175 & 2.435956 & 0.069830 \\
$\mathrm{C}$ & -1.100244 & 2.853263 & 0.037415 \\
$\mathrm{~N}$ & 0.000000 & 2.043099 & 0.146063 \\
$\mathrm{C}$ & 1.100244 & 2.853263 & 0.037415 \\
$\mathrm{C}$ & 0.676759 & 4.215399 & -0.156451 \\
$\mathrm{C}$ & -0.676759 & 4.215399 & -0.156451 \\
$\mathrm{C}$ & 2.437175 & 2.435956 & 0.069830 \\
$\mathrm{C}$ & 3.491458 & 3.481672 & -0.004867 \\
$\mathrm{C}$ & 4.355402 & 3.525226 & -1.098682 \\
$\mathrm{C}$ & 5.338988 & 4.497419 & -1.185656 \\
$\mathrm{C}$ & 5.476977 & 5.440430 & -0.176786 \\
$\mathrm{C}$ & 4.624730 & 5.404899 & 0.917651 \\
$\mathrm{C}$ & 3.638403 & 4.433470 & 1.002576 \\
$\mathrm{C}$ & -2.866272 & 1.097442 & 0.135001 \\
$\mathrm{~N}$ & -2.055160 & 0.000000 & 0.058115 \\
$\mathrm{C}$ & -2.866272 & -1.097442 & 0.135001 \\
$\mathrm{C}$ & -4.231103 & -0.678306 & 0.290902 \\
$\mathrm{C}$ & -4.231103 & 0.678306 & 0.290902 \\
$\mathrm{C}$ & -2.437175 & -2.435956 & 0.069830 \\
$\mathrm{C}$ & -3.491458 & -3.481672 & -0.004867 \\
$\mathrm{C}$ & -4.355402 & -3.525226 & -1.098682 \\
$\mathrm{C}$ & -5.338988 & -4.497419 & -1.185656 \\
$\mathrm{C}$ & -5.476977 & -5.440430 & -0.176786 \\
$\mathrm{C}$ & -4.624730 & -5.404899 & 0.917651 \\
$\mathrm{C}$ & -3.638403 & -4.433470 & 1.002576 \\
$\mathrm{C}$ & 2.866272 & 1.097442 & 0.135001 \\
$\mathrm{~N}$ & 2.055160 & 0.000000 & 0.058115 \\
$\mathrm{C}$ & 2.866272 & -1.097442 & 0.135001 \\
$\mathrm{C}$ & 4.231103 & -0.678306 & 0.290902 \\
$\mathrm{C}$ & 4.231103 & 0.678306 & 0.290902 \\
$\mathrm{C}$ & 2.437175 & -2.435956 & 0.069830 \\
$\mathrm{C}$ & 3.491458 & -3.481672 & -0.004867 \\
$\mathrm{C}$ & 4.355402 & -3.525226 & -1.098682 \\
$\mathrm{C}$ & 5.338988 & -4.497419 & -1.185656 \\
$\mathrm{C}$ & 5.476977 & -5.440430 & -0.176786 \\
& & & \\
\hline
\end{tabular}




$\begin{array}{rrrr}\mathrm{C} & 4.624730 & -5.404899 & 0.917651 \\ \mathrm{C} & 3.638403 & -4.433470 & 1.002576 \\ \mathrm{C} & 1.100244 & -2.853263 & 0.037415 \\ \mathrm{~N} & 0.000000 & -2.043099 & 0.146063 \\ \mathrm{C} & -1.100244 & -2.853263 & 0.037415 \\ \mathrm{C} & -0.676759 & -4.215399 & -0.156451 \\ \mathrm{C} & 0.676759 & -4.215399 & -0.156451 \\ \mathrm{H} & 1.334190 & -5.056604 & -0.301239 \\ \mathrm{H} & 5.075211 & -1.337733 & 0.406084 \\ \mathrm{H} & 1.334190 & 5.056604 & -0.301239 \\ \mathrm{H} & -1.334190 & 5.056604 & -0.301239 \\ \mathrm{H} & -1.334190 & -5.056604 & -0.301239 \\ \mathrm{H} & 5.075211 & 1.337733 & 0.406084 \\ \mathrm{H} & -5.075211 & 1.337733 & 0.406084 \\ \mathrm{H} & -5.075211 & -1.337733 & 0.406084 \\ \mathrm{H} & 4.245689 & 2.790051 & -1.886769 \\ \mathrm{H} & 5.997823 & 4.520647 & -2.045143 \\ \mathrm{H} & 6.245927 & 6.200186 & -0.242721 \\ \mathrm{H} & 4.728899 & 6.135145 & 1.710843 \\ \mathrm{H} & 2.974441 & 4.403884 & 1.858174 \\ \mathrm{H} & 4.245689 & -2.790051 & -1.886769 \\ \mathrm{H} & 5.997823 & -4.520647 & -2.045143 \\ \mathrm{H} & 6.245927 & -6.200186 & -0.242721 \\ \mathrm{H} & 4.728899 & -6.135145 & 1.710843 \\ \mathrm{H} & 2.974441 & -4.403884 & 1.858174 \\ \mathrm{H} & -4.245689 & -2.790051 & -1.886769 \\ \mathrm{H} & -5.997823 & -4.520647 & -2.045143 \\ \mathrm{H} & -6.245927 & -6.200186 & -0.242721 \\ \mathrm{H} & -4.728899 & -6.135145 & 1.710843 \\ \mathrm{H} & -2.974441 & -4.403884 & 1.858174 \\ \mathrm{H} & -4.245689 & 2.790051 & -1.886769 \\ \mathrm{H} & -5.997823 & 4.520647 & -2.045143 \\ \mathrm{H} & -6.245927 & 6.200186 & -0.242721 \\ \mathrm{H} & -4.728899 & 6.135145 & 1.710843 \\ \mathrm{H} & -2.974441 & 4.403884 & 1.858174 \\ \mathrm{Zn} & 0.000000 & 0.000000 & 0.106741\end{array}$

tetraphenylporphine $\mathrm{S}_{0}$

$\begin{array}{lrrr}\mathrm{C} & 0.679811 & -4.224469 & 0.017654 \\ \mathrm{C} & 1.121948 & -2.866372 & -0.028042 \\ \mathrm{~N} & 0.000004 & -2.089072 & -0.055013 \\ \mathrm{C} & -1.121948 & -2.866364 & -0.028174 \\ \mathrm{C} & -0.679826 & -4.224464 & 0.017563 \\ \mathrm{C} & -2.440126 & -2.419417 & -0.026284 \\ \mathrm{C} & -2.839789 & -1.079428 & -0.034475\end{array}$




$\begin{array}{rrrr}\mathrm{N} & -2.019312 & 0.000004 & -0.018840 \\ \mathrm{C} & -2.839789 & 1.079438 & -0.034346 \\ \mathrm{C} & -4.236146 & 0.672102 & -0.073308 \\ \mathrm{C} & -4.236146 & -0.672088 & -0.073396 \\ \mathrm{C} & -2.440127 & 2.419427 & -0.026054 \\ \mathrm{C} & -1.121948 & 2.866372 & -0.028042 \\ \mathrm{~N} & -0.000004 & 2.089072 & -0.055013 \\ \mathrm{C} & 1.121948 & 2.866364 & -0.028174 \\ \mathrm{C} & 0.679826 & 4.224464 & 0.017563 \\ \mathrm{C} & -0.679811 & 4.224469 & 0.017654 \\ \mathrm{C} & 2.440126 & 2.419417 & -0.026284 \\ \mathrm{C} & 2.839789 & 1.079428 & -0.034475 \\ \mathrm{~N} & 2.019312 & -0.000004 & -0.018840 \\ \mathrm{C} & 2.839789 & -1.079438 & -0.034346 \\ \mathrm{C} & 4.236146 & -0.672102 & -0.073308 \\ \mathrm{C} & 4.236146 & 0.672088 & -0.073396 \\ \mathrm{C} & 2.440127 & -2.419427 & -0.026054 \\ \mathrm{H} & 5.082692 & 1.338903 & -0.106413 \\ \mathrm{C} & 3.500526 & 3.471079 & -0.000648 \\ \mathrm{H} & 1.337956 & 5.076770 & 0.053769 \\ \mathrm{H} & -0.000010 & 1.077508 & -0.060184 \\ \mathrm{H} & -5.082692 & 1.338921 & -0.106239 \\ \mathrm{H} & -5.082692 & -1.338903 & -0.106413 \\ \mathrm{H} & 5.082692 & -1.338921 & -0.106239 \\ \mathrm{C} & -3.500530 & 3.471079 & -0.000185 \\ \mathrm{C} & -3.500526 & -3.471079 & -0.000648 \\ \mathrm{C} & 3.500530 & -3.471079 & -0.000185 \\ \mathrm{H} & -1.337931 & 5.076779 & 0.053945 \\ \mathrm{H} & -1.337956 & -5.076770 & 0.053769 \\ \mathrm{H} & 1.337931 & -5.076779 & 0.053945 \\ \mathrm{H} & 0.000010 & -1.077508 & -0.060184 \\ \mathrm{C} & -3.827509 & 4.178651 & -1.153343 \\ \mathrm{C} & -4.813283 & 5.154456 & -1.125572 \\ \mathrm{C} & -5.482345 & 5.435201 & 0.057077 \\ \mathrm{C} & -5.161164 & 4.735296 & 1.211471 \\ \mathrm{C} & -4.176166 & 3.759326 & 1.182286 \\ \mathrm{H} & -3.306236 & 3.956159 & -2.076807 \\ \mathrm{H} & -5.060803 & 5.693909 & -2.031677 \\ \mathrm{H} & -6.251902 & 6.196863 & 0.078886 \\ \mathrm{H} & -5.677320 & 4.949990 & 2.139210 \\ \mathrm{H} & -3.923961 & 3.211297 & 2.082266 \\ \mathrm{C} & 3.827387 & 4.178484 & -1.153942 \\ \mathrm{C} & 4.813148 & 5.154307 & -1.126411 \\ \mathrm{C} & 5.482317 & 5.435239 & 0.056132 \\ \mathrm{C} & 5.161257 & 4.735501 & 1.210661 \\ \mathrm{C} & 4.176271 & 3.759511 & 1.181715\end{array}$




$\begin{array}{lrrr}\mathrm{H} & 3.306028 & 3.955847 & -2.077323 \\ \mathrm{H} & 5.060571 & 5.693630 & -2.032620 \\ \mathrm{H} & 6.251864 & 6.196916 & 0.077756 \\ \mathrm{H} & 5.677497 & 4.950341 & 2.138318 \\ \mathrm{H} & 3.924161 & 3.211612 & 2.081802 \\ \mathrm{C} & 3.827509 & -4.178651 & -1.153343 \\ \mathrm{C} & 4.813283 & -5.154456 & -1.125572 \\ \mathrm{C} & 5.482345 & -5.435201 & 0.057077 \\ \mathrm{C} & 5.161164 & -4.735296 & 1.211471 \\ \mathrm{C} & 4.176166 & -3.759326 & 1.182286 \\ \mathrm{H} & 3.306236 & -3.956159 & -2.076807 \\ \mathrm{H} & 5.060803 & -5.693909 & -2.031677 \\ \mathrm{H} & 6.251902 & -6.196863 & 0.078886 \\ \mathrm{H} & 5.677320 & -4.949990 & 2.139210 \\ \mathrm{H} & 3.923961 & -3.211297 & 2.082266 \\ \mathrm{C} & -3.827387 & -4.178484 & -1.153942 \\ \mathrm{C} & -4.813148 & -5.154307 & -1.126411 \\ \mathrm{C} & -5.482317 & -5.435239 & 0.056132 \\ \mathrm{C} & -5.161257 & -4.735501 & 1.210661 \\ \mathrm{C} & -4.176271 & -3.759511 & 1.181715 \\ \mathrm{H} & -3.306028 & -3.955847 & -2.077323 \\ \mathrm{H} & -5.060571 & -5.693630 & -2.032620 \\ \mathrm{H} & -6.251864 & -6.196916 & 0.077756 \\ \mathrm{H} & -5.677497 & -4.950341 & 2.138318 \\ \mathrm{H} & -3.924161 & -3.211612 & 2.081802\end{array}$

tetraphenylporphine $\mathrm{S}_{1}$

$\begin{array}{rrrr}\mathrm{C} & -0.680902 & 4.215562 & 0.167017 \\ \mathrm{C} & -1.126339 & 2.871822 & -0.028628 \\ \mathrm{~N} & 0.000000 & 2.098296 & -0.137062 \\ \mathrm{C} & 1.126339 & 2.871822 & -0.028628 \\ \mathrm{C} & 0.680902 & 4.215562 & 0.167017 \\ \mathrm{C} & 2.449764 & 2.428349 & -0.083809 \\ \mathrm{C} & 2.858950 & 1.082609 & -0.180449 \\ \mathrm{~N} & 2.038913 & 0.000000 & -0.102335 \\ \mathrm{C} & 2.858950 & -1.082609 & -0.180449 \\ \mathrm{C} & 4.237114 & -0.674889 & -0.357624 \\ \mathrm{C} & 4.237114 & 0.674889 & -0.357624 \\ \mathrm{C} & 2.449764 & -2.428349 & -0.083808 \\ \mathrm{C} & 1.126339 & -2.871822 & -0.028628 \\ \mathrm{~N} & 0.000000 & -2.098296 & -0.137062 \\ \mathrm{C} & -1.126339 & -2.871822 & -0.028628 \\ \mathrm{C} & -0.680902 & -4.215562 & 0.167017 \\ \mathrm{C} & 0.680902 & -4.215562 & 0.167017 \\ \mathrm{C} & -2.449764 & -2.428349 & -0.083809\end{array}$




\begin{tabular}{|c|c|c|c|}
\hline $\mathrm{C}$ & -2.858950 & & \\
\hline $\mathrm{N}$ & -2.038913 & & \\
\hline & -2.858950 & 082609 & \\
\hline C & -4.237114 & & \\
\hline & -4.237114 & -0.674889 & \\
\hline & -2.449764 & & \\
\hline $\mathrm{H}$ & -5.077753 & -1.337557 & \\
\hline & -3.509 & -3.468768 & \\
\hline $\mathrm{H}$ & -1.333 & -5.060634 & \\
\hline & 0.000 & & \\
\hline & 5.07 & & \\
\hline & 5.07 & & \\
\hline & & & \\
\hline & 3.50 & -3.4 & \\
\hline & & & \\
\hline & -3.5 & & \\
\hline 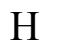 & & & \\
\hline [ & & & \\
\hline & -1.3 & & \\
\hline [ & & & \\
\hline & 3.6 & & \\
\hline C & & & \\
\hline & & & \\
\hline & & & \\
\hline & & & \\
\hline $\mathrm{H}$ & & & \\
\hline $\mathrm{H}$ & & & \\
\hline & 6.27 & -6.1 & \\
\hline $\mathrm{H}$ & & & \\
\hline $\mathrm{H}$ & 4.26 & -2.7 & \\
\hline $\mathrm{C}$ & -3.6 & -4. & \\
\hline & -4.64 & -5.3 & \\
\hline 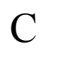 & -5.50 & -5.4 & \\
\hline$C$ & -5.36 & -4.4 & \\
\hline $\mathrm{C}$ & & & \\
\hline $\mathrm{H}$ & -2.99 & -4. & \\
\hline $\mathrm{H}$ & -4.75 & -6.1 & \\
\hline $\mathrm{H}$ & -6.276 & -6.1 & \\
\hline & -6.026 & & \\
\hline $\mathrm{H}$ & -4.266 & -2.750146 & \\
\hline & -3.657 & 4.430781 & \\
\hline $\mathrm{C}$ & & & \\
\hline & -5.503409 & 5.416219 & \\
\hline $\mathrm{C}$ & -5.364148 & 4.462289 & \\
\hline & -4.376224 & 3.495211 & 1.091618 \\
\hline & & 4.412474 & -1.852977 \\
\hline
\end{tabular}




$\begin{array}{lrrr}\mathrm{H} & -4.754444 & 6.135031 & -1.690307 \\ \mathrm{H} & -6.276049 & 6.171630 & 0.260418 \\ \mathrm{H} & -6.026082 & 4.472523 & 2.043848 \\ \mathrm{H} & -4.266295 & 2.750145 & 1.870243 \\ \mathrm{C} & 3.657650 & 4.430781 & -0.997645 \\ \mathrm{C} & 4.648530 & 5.396996 & -0.904622 \\ \mathrm{C} & 5.503408 & 5.416220 & 0.188017 \\ \mathrm{C} & 5.364147 & 4.462290 & 1.186533 \\ \mathrm{C} & 4.376223 & 3.495212 & 1.091617 \\ \mathrm{H} & 2.992835 & 4.412473 & -1.852979 \\ \mathrm{H} & 4.754444 & 6.135031 & -1.690309 \\ \mathrm{H} & 6.276048 & 6.171631 & 0.260417 \\ \mathrm{H} & 6.026081 & 4.472524 & 2.043847 \\ \mathrm{H} & 4.266295 & 2.750146 & 1.870242\end{array}$

\section{$\omega \mathrm{B97X}-\mathrm{D} / \mathrm{TZVP}$ geometries - Group II}

tetrafluorobenzene $\mathrm{S}_{0}$

$\begin{array}{lrrr}\text { C } & -1.190075 & 0.692879 & 0.000000 \\ \text { C } & -1.190075 & -0.692879 & 0.000000 \\ \text { C } & 0.000000 & -1.393186 & 0.000000 \\ \text { C } & 1.190075 & -0.692879 & 0.000000 \\ \text { C } & 1.190075 & 0.692879 & 0.000000 \\ \text { C } & 0.000000 & 1.393186 & 0.000000 \\ \text { F } & -2.348528 & -1.350973 & 0.000000 \\ \text { H } & 0.000000 & -2.474377 & 0.000000 \\ \text { F } & 2.348528 & -1.350973 & 0.000000 \\ \text { F } & 2.348528 & 1.350973 & 0.000000 \\ \text { H } & 0.000000 & 2.474377 & 0.000000 \\ \text { F } & -2.348528 & 1.350973 & 0.000000\end{array}$

tetrafluorobenzene $S_{1}$

$\begin{array}{lrrr}\text { C } & 1.165416 & -0.707629 & 0.106010 \\ \text { C } & 1.165416 & 0.707629 & 0.106010 \\ \text { C } & 0.000000 & 1.460638 & 0.314596 \\ \text { C } & -1.165416 & 0.707629 & 0.106010 \\ \text { C } & -1.165416 & -0.707629 & 0.106010 \\ \text { C } & 0.000000 & -1.460638 & 0.314596 \\ \text { F } & 2.302175 & 1.320477 & -0.207028 \\ \text { H } & 0.000000 & 2.506338 & 0.585564 \\ \text { F } & -2.302175 & 1.320477 & -0.207028 \\ \text { F } & -2.302175 & -1.320477 & -0.207028 \\ \text { H } & 0.000000 & -2.506338 & 0.585564\end{array}$




\begin{tabular}{crrr}
$\mathrm{F}$ & 2.302175 & -1.320477 & -0.207028 \\
\multicolumn{4}{c}{ benzonitrile $\mathrm{S}_{0}$} \\
& & \\
$\mathrm{C}$ & 0.000000 & 0.000000 & -1.052359 \\
$\mathrm{C}$ & -1.209394 & 0.000000 & -0.358347 \\
$\mathrm{C}$ & -1.203298 & 0.000000 & 1.026169 \\
$\mathrm{C}$ & 0.000000 & 0.000000 & 1.718045 \\
$\mathrm{C}$ & 1.203298 & 0.000000 & 1.026169 \\
$\mathrm{C}$ & 1.209394 & 0.000000 & -0.358347 \\
$\mathrm{H}$ & -2.142509 & 0.000000 & -0.906441 \\
$\mathrm{H}$ & -2.141450 & 0.000000 & 1.566169 \\
$\mathrm{H}$ & 0.000000 & 0.000000 & 2.800928 \\
$\mathrm{H}$ & 2.141450 & 0.000000 & 1.566169 \\
$\mathrm{H}$ & 2.142509 & 0.000000 & -0.906441 \\
$\mathrm{C}$ & 0.000000 & 0.000000 & -2.485997 \\
$\mathrm{~N}$ & 0.000000 & 0.000000 & -3.635708
\end{tabular}

benzonitrile $\mathrm{S}_{1}$

$\begin{array}{rrrr}\mathrm{C} & 0.000000 & 0.000000 & -1.103118 \\ \mathrm{C} & -1.224594 & 0.000000 & -0.372923 \\ \mathrm{C} & -1.213948 & 0.000000 & 1.044661 \\ \mathrm{C} & 0.000000 & 0.000000 & 1.763218 \\ \mathrm{C} & 1.213948 & 0.000000 & 1.044661 \\ \mathrm{C} & 1.224594 & 0.000000 & -0.372923 \\ \mathrm{H} & -2.164038 & 0.000000 & -0.907904 \\ \mathrm{H} & -2.158061 & 0.000000 & 1.572303 \\ \mathrm{H} & 0.000000 & 0.000000 & 2.843306 \\ \mathrm{H} & 2.158061 & 0.000000 & 1.572303 \\ \mathrm{H} & 2.164038 & 0.000000 & -0.907904 \\ \mathrm{C} & 0.000000 & 0.000000 & -2.509341 \\ \mathrm{~N} & 0.000000 & 0.000000 & -3.666337\end{array}$

$o$-fluorophenol, cis $\mathrm{S}_{0}$

$\begin{array}{rrrc}\text { C } & 0.325631 & 1.450017 & 0.000000 \\ \text { C } & -0.873382 & 0.775161 & 0.000000 \\ \text { C } & -0.949819 & -0.612738 & 0.000000 \\ \text { C } & 0.235499 & -1.333541 & 0.000000 \\ \text { C } & 1.453131 & -0.669889 & 0.000000 \\ \text { C } & 1.505071 & 0.717104 & 0.000000 \\ \text { F } & -2.043338 & 1.456780 & 0.000000 \\ \text { O } & -2.135864 & -1.265100 & 0.000000 \\ \text { H } & 0.181564 & -2.414490 & 0.000000 \\ \text { H } & 2.370532 & -1.244470 & 0.000000\end{array}$




$\begin{array}{cccc}\mathrm{H} & 2.457038 & 1.230964 & 0.000000 \\ \mathrm{H} & 0.323745 & 2.532215 & 0.000000 \\ \mathrm{H} & -2.849797 & -0.622033 & 0.000000\end{array}$

$o$-fluorophenol, cis $\mathrm{S}_{1}$

$\begin{array}{lrrr}\mathrm{C} & 1.233191 & -0.253729 & -0.739098 \\ \mathrm{C} & 0.003837 & -0.064179 & -0.094437 \\ \mathrm{C} & -0.011901 & -0.031599 & 1.347849 \\ \mathrm{C} & 1.169996 & 0.037327 & 2.090550 \\ \mathrm{C} & 2.383969 & 0.113820 & 1.408852 \\ \mathrm{C} & 2.400419 & -0.017838 & 0.011254 \\ \mathrm{~F} & -0.944145 & 0.808574 & -0.681529 \\ \mathrm{O} & -1.174969 & -0.126644 & 1.988732 \\ \mathrm{H} & 1.124184 & -0.111265 & 3.163104 \\ \mathrm{H} & 3.312636 & 0.218883 & 1.952022 \\ \mathrm{H} & 3.343758 & 0.088293 & -0.514971 \\ \mathrm{H} & 1.285252 & -0.399859 & -1.809659 \\ \mathrm{H} & -1.870848 & -0.261783 & 1.327272\end{array}$

$o$-fluorophenol, trans $\mathrm{S}_{0}$

$\begin{array}{lrrc}\mathrm{C} & 0.289639 & 1.261312 & 0.000000 \\ \mathrm{C} & -0.945170 & 0.617245 & 0.000000 \\ \mathrm{C} & -0.960331 & -0.771243 & 0.000000 \\ \mathrm{C} & 0.226498 & -1.491828 & 0.000000 \\ \mathrm{C} & 1.443936 & -0.832606 & 0.000000 \\ \mathrm{C} & 1.472727 & 0.556576 & 0.000000 \\ \mathrm{O} & -2.061160 & 1.385949 & 0.000000 \\ \mathrm{H} & -1.913036 & -1.290533 & 0.000000 \\ \mathrm{H} & 0.191189 & -2.573655 & 0.000000 \\ \mathrm{H} & 2.371353 & -1.389672 & 0.000000 \\ \mathrm{H} & 2.405447 & 1.105408 & 0.000000 \\ \mathrm{~F} & 0.315689 & 2.600486 & 0.000000 \\ \mathrm{H} & -2.836790 & 0.822561 & 0.000000\end{array}$

$o$-fluorophenol, trans $\mathrm{S}_{1}$
$\begin{array}{llll}\text { C } & 0.819142 & 1.036153 & 0.097799\end{array}$
$\begin{array}{llll}\text { C } & -0.587655 & 0.972496 & 0.075299\end{array}$
$\begin{array}{llll}\text { C } & -1.271577 & -0.286637 & 0.092386\end{array}$
$\begin{array}{llll}\text { C } & -0.468878 & -1.425560 & -0.072756\end{array}$
$\begin{array}{llll}\text { C } & 0.941234 & -1.337804 & -0.060523\end{array}$
$\begin{array}{llll}\text { C } & 1.618781 & -0.126951 & 0.168682\end{array}$
$\begin{array}{llll}\mathrm{O} & -1.254034 & 2.116280 & -0.102752\end{array}$

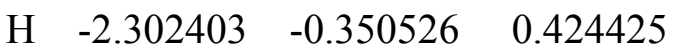




$\begin{array}{cccc}\mathrm{H} & -0.928885 & -2.399220 & -0.185774 \\ \mathrm{H} & 1.514603 & -2.245405 & -0.211196 \\ \mathrm{H} & 2.688735 & -0.060731 & 0.289059 \\ \mathrm{~F} & 1.398291 & 2.208052 & -0.180274 \\ \mathrm{H} & -2.167385 & 1.899852 & -0.334376\end{array}$

$m$-fluorophenol, cis $\mathrm{S}_{0}$

$\begin{array}{cccc}\mathrm{C} & 0.308305 & 1.407719 & 0.000000 \\ \mathrm{C} & -0.926308 & 0.786040 & 0.000000 \\ \mathrm{C} & -0.966425 & -0.603834 & 0.000000 \\ \mathrm{C} & 0.213936 & -1.340421 & 0.000000 \\ \mathrm{C} & 1.429086 & -0.679114 & 0.000000 \\ \mathrm{C} & 1.497368 & 0.708923 & 0.000000 \\ \mathrm{O} & -2.134772 & -1.293948 & 0.000000 \\ \mathrm{~F} & 0.338561 & 2.750210 & 0.000000 \\ \mathrm{H} & 0.158548 & -2.420521 & 0.000000 \\ \mathrm{H} & 2.346533 & -1.254091 & 0.000000 \\ \mathrm{H} & 2.439526 & 1.238762 & 0.000000 \\ \mathrm{H} & -1.828853 & 1.385571 & 0.000000 \\ \mathrm{H} & -2.875506 & -0.685297 & 0.000000\end{array}$

$m$-fluorophenol, cis $\mathrm{S}_{1}$

$\begin{array}{lccc}\mathrm{C} & 1.227323 & -0.034197 & -0.690424 \\ \mathrm{C} & -0.045016 & 0.021400 & -0.099570 \\ \mathrm{C} & -0.023833 & 0.024410 & 1.325157 \\ \mathrm{C} & 1.163991 & 0.015135 & 2.086492 \\ \mathrm{C} & 2.425517 & 0.102010 & 1.441109 \\ \mathrm{C} & 2.432611 & -0.027908 & 0.032016 \\ \mathrm{O} & -1.174209 & -0.019844 & 2.011117 \\ \mathrm{~F} & 1.299085 & -0.112236 & -2.025107 \\ \mathrm{H} & 1.076903 & -0.102568 & 3.158385 \\ \mathrm{H} & 3.345500 & 0.128923 & 2.002121 \\ \mathrm{H} & 3.359178 & -0.116980 & -0.520772 \\ \mathrm{H} & -0.926567 & 0.282252 & -0.670564 \\ \mathrm{H} & -1.905124 & -0.160396 & 1.399990\end{array}$

$m$-fluorophenol, trans $\mathrm{S}_{0}$
$\begin{array}{llll}\text { C } & 0.267698 & 1.300851 & 0.000000\end{array}$
$\begin{array}{llll}\mathrm{C} & -0.948347 & 0.627963 & 0.000000\end{array}$
$\begin{array}{llll}\text { C } & -0.982184 & -0.763397 & 0.000000\end{array}$
$\begin{array}{llll}\text { C } & 0.207263 & -1.476692 & 0.000000\end{array}$
$\begin{array}{llll}\text { C } & 1.432133 & -0.828530 & 0.000000\end{array}$
$\begin{array}{llll}\text { C } & 1.426850 & 0.554799 & 0.000000\end{array}$ 


$\begin{array}{cccc}\mathrm{O} & -2.072177 & 1.388489 & 0.000000 \\ \mathrm{H} & -1.932663 & -1.285313 & 0.000000 \\ \mathrm{H} & 0.177108 & -2.559013 & 0.000000 \\ \mathrm{H} & 2.368606 & -1.368276 & 0.000000 \\ \mathrm{~F} & 2.600832 & 1.205081 & 0.000000 \\ \mathrm{H} & 0.301039 & 2.381407 & 0.000000 \\ \mathrm{H} & -2.846159 & 0.822640 & 0.000000\end{array}$

$m$-fluorophenol, trans $\mathrm{S}_{1}$

$\begin{array}{cccc}\mathrm{C} & 1.082637 & -0.015607 & 0.000000 \\ \mathrm{C} & 0.328680 & 1.182642 & 0.000000 \\ \mathrm{C} & -1.089600 & 1.211052 & 0.000000 \\ \mathrm{C} & -1.820895 & -0.006146 & 0.000000 \\ \mathrm{C} & -1.084380 & -1.217630 & 0.000000 \\ \mathrm{C} & 0.319789 & -1.196630 & 0.000000 \\ \mathrm{O} & 1.036412 & 2.321194 & 0.000000 \\ \mathrm{H} & -1.601410 & 2.166778 & 0.000000 \\ \mathrm{H} & -2.899131 & -0.013045 & 0.000000 \\ \mathrm{H} & -1.584983 & -2.176685 & 0.000000 \\ \mathrm{~F} & 0.967775 & -2.368742 & 0.000000 \\ \mathrm{H} & 2.160743 & -0.010935 & 0.000000 \\ \mathrm{H} & 0.443214 & 3.077902 & 0.000000\end{array}$

$p$-fluorophenol $\mathrm{S}_{0}$

$\begin{array}{lccc}\mathrm{C} & 0.270870 & 1.312827 & 0.000000 \\ \mathrm{C} & -0.948793 & 0.646172 & 0.000000 \\ \mathrm{C} & -0.978580 & -0.742686 & 0.000000 \\ \mathrm{C} & 0.206181 & -1.466566 & 0.000000 \\ \mathrm{C} & 1.404319 & -0.785552 & 0.000000 \\ \mathrm{C} & 1.454716 & 0.595214 & 0.000000 \\ \mathrm{O} & -2.078281 & 1.407654 & 0.000000 \\ \mathrm{H} & -1.926860 & -1.269192 & 0.000000 \\ \mathrm{H} & 0.200069 & -2.548332 & 0.000000 \\ \mathrm{~F} & 2.556315 & -1.483821 & 0.000000 \\ \mathrm{H} & 2.412684 & 1.097972 & 0.000000 \\ \mathrm{H} & 0.279071 & 2.394628 & 0.000000 \\ \mathrm{H} & -2.851729 & 0.841670 & 0.000000\end{array}$

p-fluorophenol $\mathrm{S}_{1}$
$\begin{array}{llll}\text { C } & 1.263780 & 0.679252 & 0.000000\end{array}$
$\begin{array}{llll}\text { C } & 0.026867 & 1.360728 & 0.000000\end{array}$
$\begin{array}{llll}\text { C } & -1.235073 & 0.709296 & 0.000000\end{array}$
$\begin{array}{llll}\text { C } & -1.249069 & -0.703769 & 0.000000\end{array}$ 


$\begin{array}{crrr}\mathrm{C} & -0.006473 & -1.363196 & 0.000000 \\ \mathrm{C} & 1.249982 & -0.735176 & 0.000000 \\ \mathrm{O} & 0.092302 & 2.696686 & 0.000000 \\ \mathrm{H} & -2.152607 & 1.284464 & 0.000000 \\ \mathrm{H} & -2.162086 & -1.279171 & 0.000000 \\ \mathrm{~F} & -0.026798 & -2.692661 & 0.000000 \\ \mathrm{H} & 2.150996 & -1.328615 & 0.000000 \\ \mathrm{H} & 2.180344 & 1.249862 & 0.000000 \\ \mathrm{H} & -0.795468 & 3.067893 & 0.000000\end{array}$

phenylacetylene $\mathrm{S}_{0}$

$\begin{array}{lrrr}\mathrm{C} & 1.205124 & 0.000000 & -0.013496 \\ \mathrm{C} & 0.000000 & 0.000000 & -0.719264 \\ \mathrm{C} & -1.205124 & 0.000000 & -0.013496 \\ \mathrm{C} & -1.201144 & 0.000000 & 1.371385 \\ \mathrm{C} & 0.000000 & 0.000000 & 2.066841 \\ \mathrm{C} & 1.201144 & 0.000000 & 1.371385 \\ \mathrm{C} & 0.000000 & 0.000000 & -2.150087 \\ \mathrm{C} & 0.000000 & 0.000000 & -3.349816 \\ \mathrm{H} & -2.139644 & 0.000000 & -0.559741 \\ \mathrm{H} & -2.140764 & 0.000000 & 1.909715 \\ \mathrm{H} & 0.000000 & 0.000000 & 3.149824 \\ \mathrm{H} & 2.140764 & 0.000000 & 1.909715 \\ \mathrm{H} & 2.139644 & 0.000000 & -0.559741 \\ \mathrm{H} & 0.000000 & 0.000000 & -4.413246\end{array}$

phenylacetylene $S_{1}$

$\begin{array}{rrrr}\mathrm{C} & 1.230217 & 0.000000 & -0.029426 \\ \mathrm{C} & 0.000000 & 0.000000 & -0.753141 \\ \mathrm{C} & -1.230217 & 0.000000 & -0.029426 \\ \mathrm{C} & -1.221175 & 0.000000 & 1.388879 \\ \mathrm{C} & 0.000000 & 0.000000 & 2.094101 \\ \mathrm{C} & 1.221175 & 0.000000 & 1.388879 \\ \mathrm{C} & 0.000000 & 0.000000 & -2.154127 \\ \mathrm{C} & 0.000000 & 0.000000 & -3.361975 \\ \mathrm{H} & -2.163946 & 0.000000 & -0.573573 \\ \mathrm{H} & -2.159253 & 0.000000 & 1.926565 \\ \mathrm{H} & 0.000000 & 0.000000 & 3.175591 \\ \mathrm{H} & 2.159253 & 0.000000 & 1.926565 \\ \mathrm{H} & 2.163946 & 0.000000 & -0.573573 \\ \mathrm{H} & 0.000000 & 0.000000 & -4.425339\end{array}$

aniline $\mathrm{S}_{0}$ 


$\begin{array}{rrrr}\mathrm{C} & 0.918650 & 0.028889 & 0.000000 \\ \mathrm{C} & 0.205445 & 0.036481 & 1.200563 \\ \mathrm{C} & -1.179513 & 0.042784 & 1.194974 \\ \mathrm{C} & -1.885209 & 0.046565 & 0.000000 \\ \mathrm{C} & -1.179513 & 0.042784 & -1.194974 \\ \mathrm{C} & 0.205445 & 0.036481 & -1.200563 \\ \mathrm{H} & 0.743289 & 0.038524 & 2.142278 \\ \mathrm{H} & -2.967453 & 0.051522 & 0.000000 \\ \mathrm{H} & -1.711895 & 0.046141 & 2.138655 \\ \mathrm{H} & -1.711895 & 0.046141 & -2.138655 \\ \mathrm{H} & 0.743289 & 0.038524 & -2.142278 \\ \mathrm{~N} & 2.309788 & 0.074438 & 0.000000 \\ \mathrm{H} & 2.754790 & -0.264633 & -0.836091 \\ \mathrm{H} & 2.754790 & -0.264633 & 0.836091\end{array}$

aniline $\mathrm{S}_{1}$

$\begin{array}{rrrr}\mathrm{C} & 0.896410 & -0.034209 & 0.000000 \\ \mathrm{C} & 0.224365 & -0.008501 & 1.245726 \\ \mathrm{C} & -1.193706 & 0.045652 & 1.235879 \\ \mathrm{C} & -1.865164 & 0.071237 & 0.000000 \\ \mathrm{C} & -1.193706 & 0.045652 & -1.235879 \\ \mathrm{C} & 0.224365 & -0.008501 & -1.245726 \\ \mathrm{H} & 0.789306 & -0.030128 & 2.168566 \\ \mathrm{H} & -2.948618 & 0.112502 & 0.000000 \\ \mathrm{H} & -1.751324 & 0.066931 & 2.160773 \\ \mathrm{H} & -1.751324 & 0.066931 & -2.160773 \\ \mathrm{H} & 0.789306 & -0.030128 & -2.168566 \\ \mathrm{~N} & 2.250438 & -0.086079 & 0.000000 \\ \mathrm{H} & 2.764821 & -0.105691 & -0.862830 \\ \mathrm{H} & 2.764821 & -0.105691 & 0.862830\end{array}$

catechol $\mathrm{S}_{0}$

$\begin{array}{rrrr}\mathrm{C} & 0.468888 & 1.275724 & 0.000000 \\ \mathrm{C} & -0.747269 & 0.618916 & 0.000000 \\ \mathrm{C} & -0.797917 & -0.777169 & 0.000000 \\ \mathrm{C} & 0.381619 & -1.500170 & 0.000000 \\ \mathrm{C} & 1.604090 & -0.840566 & 0.000000 \\ \mathrm{C} & 1.651206 & 0.543893 & 0.000000 \\ \mathrm{O} & -1.966803 & 1.243269 & 0.000000 \\ \mathrm{O} & -1.989815 & -1.422401 & 0.000000 \\ \mathrm{H} & 0.324440 & -2.581087 & 0.000000 \\ \mathrm{H} & 2.521361 & -1.415228 & 0.000000 \\ \mathrm{H} & 2.601556 & 1.061396 & 0.000000 \\ \mathrm{H} & 0.494922 & 2.360616 & 0.000000\end{array}$




$$
\begin{array}{lrrr}
H & -1.856890 & 2.194592 & 0.000000 \\
H & -2.689389 & -0.761785 & 0.000000
\end{array}
$$

catechol $\mathrm{S}_{1}$

$\begin{array}{lrrr}\mathrm{C} & 0.472756 & 1.345294 & 0.000000 \\ \mathrm{C} & -0.759700 & 0.625751 & 0.000000 \\ \mathrm{C} & -0.804322 & -0.783195 & 0.000000 \\ \mathrm{C} & 0.386040 & -1.569796 & 0.000000 \\ \mathrm{C} & 1.596291 & -0.857396 & 0.000000 \\ \mathrm{C} & 1.638341 & 0.556944 & 0.000000 \\ \mathrm{O} & -1.965487 & 1.246446 & 0.000000 \\ \mathrm{O} & -1.983725 & -1.420438 & 0.000000 \\ \mathrm{H} & 0.323604 & -2.645996 & 0.000000 \\ \mathrm{H} & 2.534144 & -1.397444 & 0.000000 \\ \mathrm{H} & 2.605272 & 1.043746 & 0.000000 \\ \mathrm{H} & 0.497894 & 2.425512 & 0.000000 \\ \mathrm{H} & -1.846830 & 2.198425 & 0.000000 \\ \mathrm{H} & -2.694278 & -0.767853 & 0.000000\end{array}$

resorcinol, isomer $1 \mathrm{~S}_{0}$

$\begin{array}{lrrr}\mathrm{C} & 0.074948 & 1.145263 & 0.000000 \\ \mathrm{C} & -1.136365 & 0.465368 & 0.000000 \\ \mathrm{C} & -1.167203 & -0.923257 & 0.000000 \\ \mathrm{C} & 0.032782 & -1.622076 & 0.000000 \\ \mathrm{C} & 1.248906 & -0.965275 & 0.000000 \\ \mathrm{C} & 1.261476 & 0.426455 & 0.000000 \\ \mathrm{O} & -2.265811 & 1.223301 & 0.000000 \\ \mathrm{H} & -2.113605 & -1.452109 & 0.000000 \\ \mathrm{H} & 0.014020 & -2.704999 & 0.000000 \\ \mathrm{H} & 2.186473 & -1.503742 & 0.000000 \\ \mathrm{O} & 2.474200 & 1.039819 & 0.000000 \\ \mathrm{H} & 0.069081 & 2.229215 & 0.000000 \\ \mathrm{H} & -3.034742 & 0.650821 & 0.000000 \\ \mathrm{H} & 2.355840 & 1.991205 & 0.000000\end{array}$

resorcinol, isomer $1 \mathrm{~S}_{1}$

$\begin{array}{lrrc}\text { C } & -0.002107 & 1.058724 & 0.041210 \\ \text { C } & -1.194444 & 0.305432 & 0.001851 \\ \text { C } & -1.204143 & -1.109046 & -0.029612 \\ \text { C } & 0.000128 & -1.848313 & 0.084408 \\ \text { C } & 1.208723 & -1.108937 & 0.000537 \\ \text { C } & 1.194158 & 0.301182 & 0.022183 \\ \text { O } & -2.345051 & 1.008046 & -0.027049\end{array}$




$\begin{array}{rrrr}\mathrm{H} & -2.152621 & -1.622220 & -0.152454 \\ \mathrm{H} & -0.000705 & -2.926251 & 0.077732 \\ \mathrm{H} & 2.166217 & -1.602825 & -0.101841 \\ \mathrm{O} & 2.394703 & 0.914655 & -0.014953 \\ \mathrm{H} & -0.023165 & 2.119116 & 0.251661 \\ \mathrm{H} & -3.094265 & 0.407386 & 0.001936 \\ \mathrm{H} & 2.263566 & 1.859172 & -0.135959\end{array}$

resorcinol, isomer $2 \mathrm{~S}_{0}$

$\begin{array}{rrrr}\mathrm{C} & 1.196539 & 0.000000 & -0.545932 \\ \mathrm{C} & 0.000000 & 0.000000 & -1.245687 \\ \mathrm{C} & -1.196539 & 0.000000 & -0.545932 \\ \mathrm{C} & -1.206662 & 0.000000 & 0.846715 \\ \mathrm{C} & 0.000000 & 0.000000 & 1.526795 \\ \mathrm{C} & 1.206662 & 0.000000 & 0.846715 \\ \mathrm{O} & -2.340785 & 0.000000 & -1.279919 \\ \mathrm{O} & 2.340785 & 0.000000 & -1.279919 \\ \mathrm{H} & -2.145105 & 0.000000 & 1.389607 \\ \mathrm{H} & 0.000000 & 0.000000 & 2.609812 \\ \mathrm{H} & 2.145105 & 0.000000 & 1.389607 \\ \mathrm{H} & 0.000000 & 0.000000 & -2.326845 \\ \mathrm{H} & -3.098467 & 0.000000 & -0.692748 \\ \mathrm{H} & 3.098467 & 0.000000 & -0.692748\end{array}$

resorcinol, isomer $2 \mathrm{~S}_{1}$

$\begin{array}{lrrr}\mathrm{C} & 1.196142 & 0.000000 & -0.330092 \\ \mathrm{C} & 0.000000 & 0.000000 & -1.078252 \\ \mathrm{C} & -1.196142 & 0.000000 & -0.330092 \\ \mathrm{C} & -1.211557 & 0.000000 & 1.085453 \\ \mathrm{C} & 0.000000 & 0.000000 & 1.823752 \\ \mathrm{C} & 1.211557 & 0.000000 & 1.085453 \\ \mathrm{O} & -2.346148 & 0.000000 & -1.036232 \\ \mathrm{O} & 2.346148 & 0.000000 & -1.036232 \\ \mathrm{H} & -2.164904 & 0.000000 & 1.602996 \\ \mathrm{H} & 0.000000 & 0.000000 & 2.901957 \\ \mathrm{H} & 2.164904 & 0.000000 & 1.602996 \\ \mathrm{H} & 0.000000 & 0.000000 & -2.156188 \\ \mathrm{H} & -3.096611 & 0.000000 & -0.437303 \\ \mathrm{H} & 3.096611 & 0.000000 & -0.437303\end{array}$

resorcinol, isomer $3 \mathrm{~S}_{0}$

$\begin{array}{llll}\mathrm{C} & 1.201972 & 0.000000 & -0.288231 \\ \mathrm{C} & 0.000000 & 0.000000 & -0.988087\end{array}$ 


$\begin{array}{cccc}\mathrm{C} & -1.201972 & 0.000000 & -0.288231 \\ \mathrm{C} & -1.209412 & 0.000000 & 1.099885 \\ \mathrm{C} & 0.000000 & 0.000000 & 1.775607 \\ \mathrm{C} & 1.209412 & 0.000000 & 1.099885 \\ \mathrm{O} & -2.404757 & 0.000000 & -0.923097 \\ \mathrm{O} & 2.404757 & 0.000000 & -0.923097 \\ \mathrm{H} & -2.154819 & 0.000000 & 1.624458 \\ \mathrm{H} & 0.000000 & 0.000000 & 2.858754 \\ \mathrm{H} & 2.154819 & 0.000000 & 1.624458 \\ \mathrm{H} & 0.000000 & 0.000000 & -2.074774 \\ \mathrm{H} & 2.273951 & 0.000000 & -1.872525 \\ \mathrm{H} & -2.273951 & 0.000000 & -1.872525\end{array}$

resorcinol, isomer $3 \mathrm{~S}_{1}$

$\begin{array}{lrcc}\mathrm{C} & -0.017378 & -0.303289 & 1.189076 \\ \mathrm{C} & -0.088870 & -1.073187 & 0.000000 \\ \mathrm{C} & -0.017378 & -0.303289 & -1.189076 \\ \mathrm{C} & 0.025146 & 1.106322 & -1.199998 \\ \mathrm{C} & -0.082445 & 1.846383 & 0.000000 \\ \mathrm{C} & 0.025146 & 1.106322 & 1.199998 \\ \mathrm{O} & 0.051338 & -0.914410 & -2.388472 \\ \mathrm{O} & 0.051338 & -0.914410 & 2.388472 \\ \mathrm{H} & 0.172466 & 1.595653 & -2.154842 \\ \mathrm{H} & -0.083125 & 2.924368 & 0.000000 \\ \mathrm{H} & 0.172466 & 1.595653 & 2.154842 \\ \mathrm{H} & -0.564217 & -2.053091 & 0.000000 \\ \mathrm{H} & 0.207836 & -1.853795 & 2.248000 \\ \mathrm{H} & 0.207836 & -1.853795 & -2.248000\end{array}$

hydroquinone, cis $\mathrm{S}_{0}$
$\begin{array}{llll}\text { C } & -0.695194 & 0.000000 & 1.088471\end{array}$
$\begin{array}{llll}\text { C } & 0.695194 & 0.000000 & 1.088471\end{array}$
$\begin{array}{llll}\text { C } & 1.392736 & 0.000000 & -0.108739\end{array}$
$\begin{array}{llll}\text { C } & 0.690831 & 0.000000 & -1.308894\end{array}$
$\begin{array}{llll}\text { C } & -0.690831 & 0.000000 & -1.308894\end{array}$
$\begin{array}{llll}\text { C } & -1.392736 & 0.000000 & -0.108739\end{array}$
$\begin{array}{llll}\text { O } & 2.756967 & 0.000000 & -0.175089\end{array}$
$\begin{array}{llll}\mathrm{O} & -2.756967 & 0.000000 & -0.175089\end{array}$
$\mathrm{H} \quad 1.242827 \quad 0.000000 \quad-2.239692$
$\begin{array}{llll}\mathrm{H} & -1.242827 & 0.000000 & -2.239692\end{array}$
$\begin{array}{llll}\mathrm{H} & -1.228323 & 0.000000 & 2.033358\end{array}$
$\begin{array}{llll}\mathrm{H} & 1.228323 & 0.000000 & 2.033358\end{array}$
$\begin{array}{llll}\mathrm{H} & -3.122025 & 0.000000 & 0.710874\end{array}$
$\begin{array}{llll}\mathrm{H} & 3.122025 & 0.000000 & 0.710874\end{array}$ 
hydroquinone, cis $\mathrm{S}_{1}$

$\begin{array}{rrrr}\mathrm{C} & 0.705606 & 0.000000 & -1.130607 \\ \mathrm{C} & -0.705606 & 0.000000 & -1.130607 \\ \mathrm{C} & -1.373081 & 0.000000 & 0.119583 \\ \mathrm{C} & -0.706026 & 0.000000 & 1.359923 \\ \mathrm{C} & 0.706026 & 0.000000 & 1.359923 \\ \mathrm{C} & 1.373081 & 0.000000 & 0.119583 \\ \mathrm{O} & -2.713241 & 0.000000 & 0.164787 \\ \mathrm{O} & 2.713241 & 0.000000 & 0.164787 \\ \mathrm{H} & -1.285005 & 0.000000 & 2.270945 \\ \mathrm{H} & 1.285005 & 0.000000 & 2.270945 \\ \mathrm{H} & 1.269867 & 0.000000 & -2.054655 \\ \mathrm{H} & -1.269867 & 0.000000 & -2.054655 \\ \mathrm{H} & 3.066865 & 0.000000 & -0.729206 \\ \mathrm{H} & -3.066865 & 0.000000 & -0.729206\end{array}$

hydroquinone, trans $\mathrm{S}_{0}$

$\begin{array}{rrrr}\mathrm{C} & 0.020587 & 1.386237 & 0.000000 \\ \mathrm{C} & -1.193914 & 0.717202 & 0.000000 \\ \mathrm{C} & -1.208247 & -0.671944 & 0.000000 \\ \mathrm{C} & -0.020587 & -1.386237 & 0.000000 \\ \mathrm{C} & 1.193914 & -0.717202 & 0.000000 \\ \mathrm{C} & 1.208247 & 0.671944 & 0.000000 \\ \mathrm{O} & -2.333138 & 1.470792 & 0.000000 \\ \mathrm{H} & -2.152114 & -1.207242 & 0.000000 \\ \mathrm{H} & -0.027160 & -2.468274 & 0.000000 \\ \mathrm{O} & 2.333138 & -1.470792 & 0.000000 \\ \mathrm{H} & 2.152114 & 1.207242 & 0.000000 \\ \mathrm{H} & 0.027160 & 2.468274 & 0.000000 \\ \mathrm{H} & -3.098905 & 0.894760 & 0.000000 \\ \mathrm{H} & 3.098905 & -0.894760 & 0.000000\end{array}$

hydroquinone, trans $\mathrm{S}_{1}$

$\begin{array}{rrrr}\mathrm{C} & 1.260421 & 0.675717 & 0.000000 \\ \mathrm{C} & 0.035022 & 1.372550 & 0.000000 \\ \mathrm{C} & -1.229472 & 0.735648 & 0.000000 \\ \mathrm{C} & -1.260421 & -0.675717 & 0.000000 \\ \mathrm{C} & -0.035022 & -1.372550 & 0.000000 \\ \mathrm{C} & 1.229472 & -0.735648 & 0.000000 \\ \mathrm{O} & 0.117990 & 2.710888 & 0.000000 \\ \mathrm{H} & -2.140771 & 1.320444 & 0.000000 \\ \mathrm{H} & -2.184141 & -1.234845 & 0.000000\end{array}$




$\begin{array}{rrrr}\mathrm{O} & -0.117990 & -2.710888 & 0.000000 \\ \mathrm{H} & 2.140771 & -1.320444 & 0.000000 \\ \mathrm{H} & 2.184141 & 1.234845 & 0.000000 \\ \mathrm{H} & -0.766900 & 3.088239 & 0.000000 \\ \mathrm{H} & 0.766900 & -3.088239 & 0.000000\end{array}$

salicylic acid $\mathrm{S}_{0}$

$\begin{array}{lrrl}\mathrm{C} & 1.099223 & 1.594702 & 0.000000 \\ \mathrm{C} & 1.131595 & 0.197905 & 0.000000 \\ \mathrm{C} & -0.082828 & -0.516042 & 0.000000 \\ \mathrm{C} & -1.295258 & 0.185944 & 0.000000 \\ \mathrm{C} & -1.315032 & 1.561661 & 0.000000 \\ \mathrm{C} & -0.106424 & 2.260372 & 0.000000 \\ \mathrm{O} & 2.327938 & -0.394916 & 0.000000 \\ \mathrm{C} & -0.045247 & -1.980176 & 0.000000 \\ \mathrm{O} & -1.244839 & -2.572580 & 0.000000 \\ \mathrm{O} & 0.978707 & -2.641653 & 0.000000 \\ \mathrm{H} & -2.219633 & -0.375764 & 0.000000 \\ \mathrm{H} & 2.040492 & 2.128122 & 0.000000 \\ \mathrm{H} & -2.256493 & 2.094385 & 0.000000 \\ \mathrm{H} & -0.111247 & 3.343784 & 0.000000 \\ \mathrm{H} & 2.187671 & -1.361419 & 0.000000 \\ \mathrm{H} & -1.088624 & -3.524326 & 0.000000\end{array}$

salicylic acid $\mathrm{S}_{1}$

$\begin{array}{lrrl}\mathrm{C} & 1.000549 & 1.907801 & 0.000000 \\ \mathrm{C} & 0.964098 & 0.508366 & 0.000000 \\ \mathrm{C} & -0.294418 & -0.229145 & 0.000000 \\ \mathrm{C} & -1.455182 & 0.533850 & 0.000000 \\ \mathrm{C} & -1.398996 & 1.954973 & 0.000000 \\ \mathrm{C} & -0.194973 & 2.650517 & 0.000000 \\ \mathrm{O} & 2.082283 & -0.170219 & 0.000000 \\ \mathrm{C} & -0.236377 & -1.656984 & 0.000000 \\ \mathrm{O} & -1.424590 & -2.295962 & 0.000000 \\ \mathrm{O} & 0.834368 & -2.315843 & 0.000000 \\ \mathrm{H} & -2.416701 & 0.039427 & 0.000000 \\ \mathrm{H} & 1.971064 & 2.386267 & 0.000000 \\ \mathrm{H} & -2.334107 & 2.501246 & 0.000000 \\ \mathrm{H} & -0.173595 & 3.730231 & 0.000000 \\ \mathrm{H} & 1.813725 & -1.168995 & 0.000000 \\ \mathrm{H} & -1.226566 & -3.238583 & 0.000000\end{array}$

$o$-cresol, cis $\mathrm{S}_{0}$ 


$\begin{array}{rrrr}\mathrm{C} & 1.958858 & 0.511414 & 0.000000 \\ \mathrm{C} & 0.727259 & 1.147098 & 0.000000 \\ \mathrm{C} & -0.465069 & 0.430939 & 0.000000 \\ \mathrm{C} & -0.390196 & -0.964936 & 0.000000 \\ \mathrm{C} & 0.837403 & -1.613456 & 0.000000 \\ \mathrm{C} & 2.008525 & -0.875420 & 0.000000 \\ \mathrm{C} & -1.799553 & 1.125999 & 0.000000 \\ \mathrm{O} & -1.503874 & -1.750337 & 0.000000 \\ \mathrm{H} & 0.853118 & -2.695809 & 0.000000 \\ \mathrm{H} & 2.962766 & -1.387549 & 0.000000 \\ \mathrm{H} & 2.871601 & 1.093213 & 0.000000 \\ \mathrm{H} & 0.682797 & 2.230703 & 0.000000 \\ \mathrm{H} & -2.291265 & -1.203656 & 0.000000 \\ \mathrm{H} & -2.391387 & 0.872195 & 0.885698 \\ \mathrm{H} & -1.669597 & 2.207417 & 0.000000 \\ \mathrm{H} & -2.391387 & 0.872195 & -0.885698\end{array}$

$o$-cresol, cis $\mathrm{S}_{1}$

$\begin{array}{rrrc}\mathrm{C} & 1.865283 & -0.089529 & -0.778563 \\ \mathrm{C} & 0.628549 & -0.102988 & -1.456899 \\ \mathrm{C} & -0.586533 & -0.065984 & -0.716224 \\ \mathrm{C} & -0.468980 & 0.056153 & 0.700510 \\ \mathrm{C} & 0.769674 & 0.085107 & 1.389143 \\ \mathrm{C} & 1.954734 & -0.003994 & 0.627069 \\ \mathrm{C} & -1.901430 & -0.010960 & -1.418334 \\ \mathrm{O} & -1.559442 & 0.145174 & 1.473546 \\ \mathrm{H} & 0.767326 & 0.165075 & 2.465948 \\ \mathrm{H} & 2.921423 & 0.005459 & 1.109465 \\ \mathrm{H} & 2.779219 & -0.141239 & -1.357886 \\ \mathrm{H} & 0.599426 & -0.178593 & -2.535089 \\ \mathrm{H} & -2.350385 & 0.120836 & 0.925220 \\ \mathrm{H} & -2.726883 & -0.460388 & -0.854260 \\ \mathrm{H} & -2.198096 & 1.017640 & -1.670896 \\ \mathrm{H} & -1.844464 & -0.561260 & -2.360341\end{array}$

$o$-cresol, trans $\mathrm{S}_{0}$

$\begin{array}{lrrc}\text { C } & 2.414760 & 0.000016 & 0.029819 \\ \text { C } & 1.243521 & -0.000035 & -0.715508 \\ \text { C } & 0.031163 & -0.000007 & -0.020679 \\ \text { C } & 0.001347 & 0.000066 & 1.367138 \\ \text { C } & 1.187870 & 0.000111 & 2.087053 \\ \text { C } & 2.400416 & 0.000089 & 1.419118 \\ \text { C } & 1.248783 & -0.000112 & -2.216587 \\ \text { O } & -1.107942 & -0.000064 & -0.770780\end{array}$




$\begin{array}{rrrc}\mathrm{H} & -0.951407 & 0.000089 & 1.887433 \\ \mathrm{H} & 1.156189 & 0.000168 & 3.169466 \\ \mathrm{H} & 3.331159 & 0.000133 & 1.971801 \\ \mathrm{H} & 3.363318 & -0.000006 & -0.495751 \\ \mathrm{H} & -1.872661 & -0.000050 & -0.192906 \\ \mathrm{H} & 2.269856 & -0.000059 & -2.598043 \\ \mathrm{H} & 0.731230 & -0.877120 & -2.610757 \\ \mathrm{H} & 0.731104 & 0.876780 & -2.610847\end{array}$

$o$-cresol, trans $\mathrm{S}_{1}$

$\begin{array}{lrrr}\mathrm{C} & 2.426102 & 0.224799 & -0.008524 \\ \mathrm{C} & 1.215756 & 0.083545 & -0.752722 \\ \mathrm{C} & 0.012322 & -0.044316 & -0.015397 \\ \mathrm{C} & -0.042739 & -0.109463 & 1.409017 \\ \mathrm{C} & 1.177798 & -0.035161 & 2.106960 \\ \mathrm{C} & 2.387550 & 0.114734 & 1.393075 \\ \mathrm{C} & 1.251599 & -0.007140 & -2.236777 \\ \mathrm{O} & -1.126123 & -0.143948 & -0.717595 \\ \mathrm{H} & -0.997581 & -0.071355 & 1.918786 \\ \mathrm{H} & 1.194886 & -0.083570 & 3.186973 \\ \mathrm{H} & 3.315693 & 0.157107 & 1.950452 \\ \mathrm{H} & 3.365277 & 0.350709 & -0.528274 \\ \mathrm{H} & -1.851584 & -0.339247 & -0.116099 \\ \mathrm{H} & 1.786235 & -0.912191 & -2.555527 \\ \mathrm{H} & 0.257577 & -0.024908 & -2.678255 \\ \mathrm{H} & 1.805923 & 0.840407 & -2.656112\end{array}$

$m$-cresol, cis $\mathrm{S}_{0}$

$\begin{array}{cccc}\mathrm{C} & 1.157217 & -0.002186 & 2.081693 \\ \mathrm{C} & 2.366096 & 0.016352 & 1.410975 \\ \mathrm{C} & 2.412714 & 0.024848 & 0.022064 \\ \mathrm{C} & 1.236874 & 0.013736 & -0.717628 \\ \mathrm{C} & 0.018878 & -0.003094 & -0.041090 \\ \mathrm{C} & -0.022950 & -0.011696 & 1.346251 \\ \mathrm{C} & 1.260261 & 0.002830 & -2.222415 \\ \mathrm{O} & -1.193526 & -0.027678 & 2.040422 \\ \mathrm{H} & 1.107676 & -0.007600 & 3.162457 \\ \mathrm{H} & 3.288120 & 0.025833 & 1.979663 \\ \mathrm{H} & 3.368463 & 0.040762 & -0.487932 \\ \mathrm{H} & -0.908119 & -0.008938 & -0.608443 \\ \mathrm{H} & -1.930974 & -0.033623 & 1.427785 \\ \mathrm{H} & 2.265432 & 0.187565 & -2.601484 \\ \mathrm{H} & 0.929075 & -0.964120 & -2.608875 \\ \mathrm{H} & 0.597063 & 0.766379 & -2.633493\end{array}$




\begin{tabular}{lrrr}
\multicolumn{5}{l}{$m$-cresol, cis $\mathrm{S}_{1}$} & & \\
$\mathrm{C}$ & 1.163401 & -0.000284 & 2.121890 \\
$\mathrm{C}$ & 2.406454 & 0.046905 & 1.446383 \\
$\mathrm{C}$ & 2.407585 & 0.033247 & 0.033659 \\
$\mathrm{C}$ & 1.221605 & 0.015054 & -0.743503 \\
$\mathrm{C}$ & -0.025840 & -0.006178 & -0.068934 \\
$\mathrm{C}$ & -0.019021 & -0.007831 & 1.351090 \\
$\mathrm{C}$ & 1.264648 & -0.009254 & -2.232926 \\
$\mathrm{O}$ & -1.173591 & -0.034301 & 2.031412 \\
$\mathrm{H}$ & 1.087132 & -0.048745 & 3.198636 \\
$\mathrm{H}$ & 3.335788 & 0.062939 & 1.995550 \\
$\mathrm{H}$ & 3.357889 & 0.043374 & -0.488497 \\
$\mathrm{H}$ & -0.960092 & 0.065878 & -0.613882 \\
$\mathrm{H}$ & -1.909511 & -0.086523 & 1.413992 \\
$\mathrm{H}$ & 2.280107 & 0.118510 & -2.608985 \\
$\mathrm{H}$ & 0.875994 & -0.957578 & -2.625987 \\
$\mathrm{H}$ & 0.639730 & 0.784156 & -2.659961
\end{tabular}

$m$-cresol, trans $\mathrm{S}_{0}$

$\begin{array}{rrrr}\mathrm{C} & -1.190482 & -1.095944 & 0.000000 \\ \mathrm{C} & 0.197535 & -1.207783 & 0.000000 \\ \mathrm{C} & 0.966938 & -0.050110 & 0.000000 \\ \mathrm{C} & 0.364285 & 1.199433 & 0.000000 \\ \mathrm{C} & -1.022514 & 1.307597 & 0.000000 \\ \mathrm{C} & -1.790170 & 0.154060 & 0.000000 \\ \mathrm{C} & 0.871933 & -2.553668 & 0.000000 \\ \mathrm{O} & 1.181725 & 2.288015 & 0.000000 \\ \mathrm{H} & -1.496463 & 2.283599 & 0.000000 \\ \mathrm{H} & -2.870476 & 0.234636 & 0.000000 \\ \mathrm{H} & -1.802250 & -1.990074 & 0.000000 \\ \mathrm{H} & 2.049335 & -0.104097 & 0.000000 \\ \mathrm{H} & 0.651584 & 3.086672 & 0.000000 \\ \mathrm{H} & 0.140201 & -3.361634 & 0.000000 \\ \mathrm{H} & 1.506954 & -2.671089 & -0.880593 \\ \mathrm{H} & 1.506954 & -2.671089 & 0.880593\end{array}$

$m$-cresol, trans $\mathrm{S}_{1}$

$\begin{array}{rrrr}\mathrm{C} & -1.201144 & -1.056549 & 0.000000 \\ \mathrm{C} & 0.203842 & -1.232460 & 0.000000 \\ \mathrm{C} & 1.023174 & -0.072508 & 0.000000 \\ \mathrm{C} & 0.398279 & 1.194269 & 0.000000 \\ \mathrm{C} & -1.009531 & 1.370609 & 0.000000\end{array}$




$\begin{array}{rrrr}\mathrm{C} & -1.823343 & 0.214542 & 0.000000 \\ \mathrm{C} & 0.827063 & -2.586648 & 0.000000 \\ \mathrm{O} & 1.214570 & 2.258051 & 0.000000 \\ \mathrm{H} & -1.434818 & 2.366899 & 0.000000 \\ \mathrm{H} & -2.900328 & 0.294130 & 0.000000 \\ \mathrm{H} & -1.828742 & -1.940768 & 0.000000 \\ \mathrm{H} & 2.102576 & -0.127759 & 0.000000 \\ \mathrm{H} & 0.694904 & 3.067283 & 0.000000 \\ \mathrm{H} & 0.074045 & -3.375934 & 0.000000 \\ \mathrm{H} & 1.467967 & -2.729071 & -0.878247 \\ \mathrm{H} & 1.467967 & -2.729071 & 0.878247\end{array}$

$p$-cresol $\mathrm{S}_{0}$

$\begin{array}{lrrr}\mathrm{C} & 0.710104 & -0.004750 & -1.185951 \\ \mathrm{C} & -0.673386 & -0.002220 & -1.195109 \\ \mathrm{C} & -1.370759 & -0.000291 & 0.006643 \\ \mathrm{C} & -0.670070 & -0.003025 & 1.204837 \\ \mathrm{C} & 0.718340 & -0.005499 & 1.194504 \\ \mathrm{C} & 1.435075 & -0.004665 & 0.004642 \\ \mathrm{O} & -2.731893 & -0.000728 & -0.052961 \\ \mathrm{C} & 2.940206 & 0.022760 & -0.004509 \\ \mathrm{H} & -1.203922 & -0.006582 & 2.149853 \\ \mathrm{H} & 1.249894 & -0.010642 & 2.139669 \\ \mathrm{H} & 1.240653 & -0.009489 & -2.131933 \\ \mathrm{H} & -1.227135 & -0.005239 & -2.125094 \\ \mathrm{H} & -3.092061 & -0.002023 & 0.835411 \\ \mathrm{H} & 3.341769 & -0.587927 & -0.814972 \\ \mathrm{H} & 3.315363 & 1.040218 & -0.143403 \\ \mathrm{H} & 3.349382 & -0.354521 & 0.933674\end{array}$

$p$-cresol $\mathrm{S}_{1}$

$\begin{array}{lrrr}\mathrm{C} & 0.729958 & -0.065125 & -1.234435 \\ \mathrm{C} & -0.683139 & -0.026510 & -1.242281 \\ \mathrm{C} & -1.353847 & -0.001100 & -0.001203 \\ \mathrm{C} & -0.680506 & 0.008956 & 1.247450 \\ \mathrm{C} & 0.731232 & -0.030652 & 1.235687 \\ \mathrm{C} & 1.428311 & -0.039455 & -0.000934 \\ \mathrm{O} & -2.691885 & 0.025344 & -0.047724 \\ \mathrm{C} & 2.914629 & 0.033796 & -0.001013 \\ \mathrm{H} & -1.242031 & 0.032416 & 2.173327 \\ \mathrm{H} & 1.289333 & -0.027484 & 2.161978 \\ \mathrm{H} & 1.284478 & -0.085182 & -2.162403 \\ \mathrm{H} & -1.261700 & -0.017765 & -2.154284 \\ \mathrm{H} & -3.045856 & 0.048176 & 0.846577\end{array}$




$$
\begin{array}{rrrr}
\mathrm{H} & 3.336921 & -0.449739 & -0.884042 \\
\mathrm{H} & 3.238172 & 1.082307 & -0.017523 \\
\mathrm{H} & 3.337480 & -0.422602 & 0.896113
\end{array}
$$

$o$-methoxyphenol $\mathrm{S}_{0}$

$\begin{array}{rrrr}\mathrm{C} & 2.025384 & -0.088782 & 0.000000 \\ \mathrm{C} & 0.873213 & 0.695179 & 0.000000 \\ \mathrm{C} & -0.368392 & 0.085891 & 0.000000 \\ \mathrm{C} & -0.468603 & -1.312644 & 0.000000 \\ \mathrm{C} & 0.678637 & -2.081440 & 0.000000 \\ \mathrm{C} & 1.926917 & -1.467997 & 0.000000 \\ \mathrm{O} & -1.575668 & 0.723707 & 0.000000 \\ \mathrm{O} & -1.686395 & -1.905720 & 0.000000 \\ \mathrm{H} & 0.579118 & -3.159256 & 0.000000 \\ \mathrm{H} & 2.821447 & -2.077646 & 0.000000 \\ \mathrm{H} & 2.994906 & 0.392073 & 0.000000 \\ \mathrm{H} & 0.958686 & 1.773404 & 0.000000 \\ \mathrm{C} & -1.585449 & 2.133580 & 0.000000 \\ \mathrm{H} & -2.350962 & -1.208893 & 0.000000 \\ \mathrm{H} & -2.631419 & 2.431249 & 0.000000 \\ \mathrm{H} & -1.095710 & 2.533648 & 0.893038 \\ \mathrm{H} & -1.095710 & 2.533648 & -0.893038\end{array}$

$o$-methoxyphenol $\mathrm{S}_{1}$

$\begin{array}{lrrc}\mathrm{C} & 2.483298 & 0.060915 & 0.061300 \\ \mathrm{C} & 1.380039 & -0.247316 & -0.753112 \\ \mathrm{C} & 0.111860 & -0.231150 & -0.095840 \\ \mathrm{C} & 0.018333 & -0.136815 & 1.312761 \\ \mathrm{C} & 1.177984 & -0.070492 & 2.137419 \\ \mathrm{C} & 2.386994 & 0.149825 & 1.467563 \\ \mathrm{O} & -1.077084 & -0.065164 & -0.728880 \\ \mathrm{O} & -1.177700 & 0.053222 & 1.885558 \\ \mathrm{H} & 1.092907 & -0.163544 & 3.209334 \\ \mathrm{H} & 3.283067 & 0.379523 & 2.031918 \\ \mathrm{H} & 3.451387 & 0.223898 & -0.399722 \\ \mathrm{H} & 1.499362 & -0.551319 & -1.782815 \\ \mathrm{C} & -1.089012 & 0.106791 & -2.138500 \\ \mathrm{H} & -1.851142 & 0.044682 & 1.190437 \\ \mathrm{H} & -2.128755 & 0.273314 & -2.408385 \\ \mathrm{H} & -0.482358 & 0.965334 & -2.432438 \\ \mathrm{H} & -0.720620 & -0.791713 & -2.638517\end{array}$

m-methoxyphenol, isomer $1 \mathrm{~S}_{0}$ 


$\begin{array}{crrc}\mathrm{C} & 0.925547 & 1.443215 & 0.000000 \\ \mathrm{C} & -0.262003 & 0.714839 & 0.000000 \\ \mathrm{C} & -0.229601 & -0.676474 & 0.000000 \\ \mathrm{C} & 1.000897 & -1.331906 & 0.000000 \\ \mathrm{C} & 2.187703 & -0.617497 & 0.000000 \\ \mathrm{C} & 2.131951 & 0.769371 & 0.000000 \\ \mathrm{H} & -1.143017 & -1.258056 & 0.000000 \\ \mathrm{O} & 1.082672 & -2.690177 & 0.000000 \\ \mathrm{H} & 3.129126 & -1.149268 & 0.000000 \\ \mathrm{H} & 3.055458 & 1.335394 & 0.000000 \\ \mathrm{H} & 0.876287 & 2.523500 & 0.000000 \\ \mathrm{O} & -1.405516 & 1.442774 & 0.000000 \\ \mathrm{H} & 0.202438 & -3.069448 & 0.000000 \\ \mathrm{C} & -2.637776 & 0.759975 & 0.000000 \\ \mathrm{H} & -3.407998 & 1.527554 & 0.000000 \\ \mathrm{H} & -2.753084 & 0.138102 & -0.893779 \\ \mathrm{H} & -2.753084 & 0.138102 & 0.893779\end{array}$

$m$-methoxyphenol, isomer $1 \mathrm{~S}_{1}$

$\begin{array}{lrrr}\mathrm{C} & 1.166960 & -0.012607 & -0.623077 \\ \mathrm{C} & -0.031588 & -0.041738 & 0.121802 \\ \mathrm{C} & -0.048594 & -0.095588 & 1.540577 \\ \mathrm{C} & 1.233127 & -0.007775 & 2.143046 \\ \mathrm{C} & 2.437705 & 0.017548 & 1.413688 \\ \mathrm{C} & 2.431185 & -0.124694 & 0.006494 \\ \mathrm{H} & -0.894046 & -0.480631 & 2.098609 \\ \mathrm{O} & 1.339566 & 0.104770 & 3.483230 \\ \mathrm{H} & 3.356082 & 0.184383 & 1.962985 \\ \mathrm{H} & 3.346045 & -0.136333 & -0.563417 \\ \mathrm{H} & 1.080191 & 0.119408 & -1.694481 \\ \mathrm{O} & -1.152967 & 0.029705 & -0.619352 \\ \mathrm{H} & 0.465459 & 0.274140 & 3.848884 \\ \mathrm{C} & -2.408179 & 0.039906 & 0.033775 \\ \mathrm{H} & -3.148197 & 0.225135 & -0.740820 \\ \mathrm{H} & -2.612331 & -0.925869 & 0.504086 \\ \mathrm{H} & -2.452110 & 0.830242 & 0.786712\end{array}$

$m$-methoxyphenol, isomer $2 \mathrm{~S}_{0}$

$\begin{array}{rrrr}\mathrm{C} & 0.345516 & 2.154283 & 0.000000 \\ \mathrm{C} & -0.835435 & 1.442532 & 0.000000 \\ \mathrm{C} & -0.791913 & 0.046177 & 0.000000 \\ \mathrm{C} & 0.426787 & -0.617891 & 0.000000 \\ \mathrm{C} & 1.606017 & 0.122925 & 0.000000 \\ \mathrm{C} & 1.577695 & 1.508886 & 0.000000\end{array}$




$\begin{array}{lrrr}\mathrm{O} & -1.992340 & -0.580302 & 0.000000 \\ \mathrm{C} & -2.012785 & -1.990374 & 0.000000 \\ \mathrm{O} & 2.767045 & -0.587050 & 0.000000 \\ \mathrm{H} & 0.501551 & -1.695551 & 0.000000 \\ \mathrm{H} & 2.500070 & 2.078860 & 0.000000 \\ \mathrm{H} & 0.314133 & 3.236982 & 0.000000 \\ \mathrm{H} & -1.797209 & 1.936686 & 0.000000 \\ \mathrm{H} & -3.062350 & -2.275356 & 0.000000 \\ \mathrm{H} & -1.528997 & -2.399029 & -0.892621 \\ \mathrm{H} & -1.528997 & -2.399029 & 0.892621 \\ \mathrm{H} & 3.511199 & 0.017259 & 0.000000\end{array}$

$m$-methoxyphenol, isomer $2 \mathrm{~S}_{1}$

$\begin{array}{cccc}\mathrm{C} & 1.239477 & -0.080793 & -0.705434 \\ \mathrm{C} & 0.055342 & -0.016781 & 0.077264 \\ \mathrm{C} & 0.108829 & -0.039134 & 1.488048 \\ \mathrm{C} & 1.335425 & -0.053973 & 2.200619 \\ \mathrm{C} & 2.500331 & -0.001915 & 1.404098 \\ \mathrm{C} & 2.468056 & 0.030786 & -0.008425 \\ \mathrm{O} & -1.092000 & -0.017342 & 2.094941 \\ \mathrm{C} & -1.149723 & 0.027194 & 3.507532 \\ \mathrm{O} & 3.672490 & 0.039489 & 2.072611 \\ \mathrm{H} & 1.417939 & -0.175305 & 3.267567 \\ \mathrm{H} & 3.401222 & 0.141423 & -0.551358 \\ \mathrm{H} & 1.200903 & -0.062696 & -1.782625 \\ \mathrm{H} & -0.920064 & 0.056437 & -0.385263 \\ \mathrm{H} & -2.205534 & 0.085964 & 3.760000 \\ \mathrm{H} & -0.720319 & -0.876189 & 3.948488 \\ \mathrm{H} & -0.629616 & 0.906580 & 3.895602 \\ \mathrm{H} & 4.402374 & 0.036257 & 1.448236\end{array}$

p-methoxyphenol, cis $\mathrm{S}_{0}$

$\begin{array}{lrrr}\mathrm{C} & -0.314687 & 0.731128 & 0.000000 \\ \mathrm{C} & -1.544019 & 0.071872 & 0.000000 \\ \mathrm{C} & -1.604355 & -1.305267 & 0.000000 \\ \mathrm{C} & -0.431891 & -2.056913 & 0.000000 \\ \mathrm{C} & 0.789472 & -1.407889 & 0.000000 \\ \mathrm{C} & 0.854032 & -0.015751 & 0.000000 \\ \mathrm{O} & -0.555071 & -3.416663 & 0.000000 \\ \mathrm{O} & -0.367737 & 2.091435 & 0.000000 \\ \mathrm{C} & 0.850117 & 2.796759 & 0.000000 \\ \mathrm{H} & 1.824581 & 0.460084 & 0.000000 \\ \mathrm{H} & -2.449873 & 0.664308 & 0.000000 \\ \mathrm{H} & -2.557470 & -1.817882 & 0.000000\end{array}$




$\begin{array}{rrrr}\mathrm{H} & 1.712015 & -1.979338 & 0.000000 \\ \mathrm{H} & 0.590779 & 3.853123 & 0.000000 \\ \mathrm{H} & 1.444476 & 2.574536 & 0.893028 \\ \mathrm{H} & 1.444476 & 2.574536 & -0.893028 \\ \mathrm{H} & 0.315154 & -3.818059 & 0.000000\end{array}$

$p$-methoxyphenol, cis $\mathrm{S}_{1}$

$\begin{array}{lrrr}\mathrm{C} & 1.326446 & 0.000234 & -0.618166 \\ \mathrm{C} & 0.052732 & 0.000320 & 0.001694 \\ \mathrm{C} & -0.016674 & 0.000252 & 1.411899 \\ \mathrm{C} & 1.187869 & 0.000204 & 2.136277 \\ \mathrm{C} & 2.466297 & -0.001441 & 1.522576 \\ \mathrm{C} & 2.539591 & 0.001123 & 0.112537 \\ \mathrm{O} & 1.086955 & 0.000722 & 3.473952 \\ \mathrm{O} & 1.287017 & 0.000298 & -1.953875 \\ \mathrm{C} & 2.490515 & -0.000451 & -2.703169 \\ \mathrm{H} & 3.497354 & 0.002397 & -0.383266 \\ \mathrm{H} & -0.829065 & -0.001260 & -0.620893 \\ \mathrm{H} & -0.955485 & 0.001103 & 1.944974 \\ \mathrm{H} & 3.363958 & -0.000838 & 2.128346 \\ \mathrm{H} & 2.189279 & -0.000945 & -3.747344 \\ \mathrm{H} & 3.080344 & 0.893820 & -2.491117 \\ \mathrm{H} & 3.079929 & -0.894738 & -2.490078 \\ \mathrm{H} & 1.966468 & -0.000817 & 3.862653\end{array}$

$p$-methoxyphenol, trans $\mathrm{S}_{0}$

$\begin{array}{cccc}\mathrm{C} & -0.654783 & 1.300329 & 0.000000 \\ \mathrm{C} & -1.884643 & 0.669470 & 0.000000 \\ \mathrm{C} & -1.960956 & -0.720172 & 0.000000 \\ \mathrm{C} & -0.793019 & -1.462854 & 0.000000 \\ \mathrm{C} & 0.444726 & -0.832089 & 0.000000 \\ \mathrm{C} & 0.521051 & 0.556235 & 0.000000 \\ \mathrm{O} & -3.147656 & -1.395785 & 0.000000 \\ \mathrm{H} & 1.338449 & -1.440523 & 0.000000 \\ \mathrm{O} & 1.680257 & 1.270309 & 0.000000 \\ \mathrm{H} & -0.586584 & 2.380479 & 0.000000 \\ \mathrm{H} & -2.791779 & 1.265293 & 0.000000 \\ \mathrm{H} & -3.873406 & -0.770198 & 0.000000 \\ \mathrm{H} & -0.857542 & -2.543220 & 0.000000 \\ \mathrm{C} & 2.894036 & 0.557057 & 0.000000 \\ \mathrm{H} & 3.684132 & 1.304487 & 0.000000 \\ \mathrm{H} & 2.993854 & -0.069418 & 0.892869 \\ \mathrm{H} & 2.993854 & -0.069418 & -0.892869\end{array}$


p-methoxyphenol, trans $\mathrm{S}_{1}$

$\begin{array}{rrrc}\mathrm{C} & 0.123420 & -0.064102 & -1.389958 \\ \mathrm{C} & -1.276449 & -0.007929 & -1.217404 \\ \mathrm{C} & -1.781121 & 0.028870 & 0.101798 \\ \mathrm{C} & -0.958096 & 0.046090 & 1.247252 \\ \mathrm{C} & 0.443887 & 0.025140 & 1.077284 \\ \mathrm{C} & 0.953933 & -0.030270 & -0.241024 \\ \mathrm{O} & -3.103397 & 0.055967 & 0.326404 \\ \mathrm{H} & 1.096820 & 0.049766 & 1.935188 \\ \mathrm{O} & 2.261489 & -0.061894 & -0.514806 \\ \mathrm{H} & 0.586608 & -0.106489 & -2.364129 \\ \mathrm{H} & -1.951487 & -0.012379 & -2.063884 \\ \mathrm{H} & -3.571584 & 0.038298 & -0.513319 \\ \mathrm{H} & -1.417946 & 0.070014 & 2.223901 \\ \mathrm{C} & 3.210561 & -0.006556 & 0.537152 \\ \mathrm{H} & 4.184805 & -0.060379 & 0.058822 \\ \mathrm{H} & 3.121364 & 0.931392 & 1.089168 \\ \mathrm{H} & 3.087983 & -0.851229 & 1.218254\end{array}$

1-naphthol, cis $\mathrm{S}_{0}$

$\begin{array}{lrrc}\mathrm{C} & 0.829178 & 1.516602 & 0.013851 \\ \mathrm{C} & 0.525938 & 0.123523 & -0.001733 \\ \mathrm{C} & -0.840479 & -0.265946 & -0.005573 \\ \mathrm{C} & -1.853127 & 0.721254 & -0.016026 \\ \mathrm{C} & -1.518536 & 2.045171 & -0.019641 \\ \mathrm{C} & -0.172278 & 2.448949 & -0.002123 \\ \mathrm{C} & 1.520478 & -0.885235 & -0.017108 \\ \mathrm{C} & 1.184270 & -2.209187 & -0.017024 \\ \mathrm{C} & -0.170814 & -2.596226 & -0.006826 \\ \mathrm{C} & -1.152227 & -1.648062 & -0.004411 \\ \mathrm{O} & 2.109643 & 1.971592 & 0.028328 \\ \mathrm{H} & -2.196122 & -1.940227 & -0.003115 \\ \mathrm{H} & -0.427962 & -3.648154 & -0.005805 \\ \mathrm{H} & 1.959891 & -2.964355 & -0.030867 \\ \mathrm{H} & 2.574426 & -0.628517 & -0.044493 \\ \mathrm{H} & -2.890886 & 0.411057 & -0.022042 \\ \mathrm{H} & -2.293090 & 2.801851 & -0.030029 \\ \mathrm{H} & 0.092589 & 3.498068 & 0.007064 \\ \mathrm{H} & 2.719118 & 1.247853 & 0.177572\end{array}$

1-naphthol, cis $\mathrm{S}_{1}$

$\begin{array}{lrrr}\mathrm{C} & 0.870041 & 1.464260 & 0.019719 \\ \mathrm{C} & 0.538240 & 0.090413 & -0.055840\end{array}$ 


$\begin{array}{rrrr}\mathrm{C} & -0.849955 & -0.247089 & 0.013440 \\ \mathrm{C} & -1.792253 & 0.797572 & -0.017008 \\ \mathrm{C} & -1.421815 & 2.165783 & -0.029367 \\ \mathrm{C} & -0.102684 & 2.506124 & -0.032097 \\ \mathrm{C} & 1.500458 & -0.930459 & -0.127214 \\ \mathrm{C} & 1.091459 & -2.294346 & -0.054484 \\ \mathrm{C} & -0.233851 & -2.617293 & 0.040012 \\ \mathrm{C} & -1.224478 & -1.600639 & 0.056792 \\ \mathrm{O} & 2.137212 & 1.865368 & 0.144136 \\ \mathrm{H} & -2.273261 & -1.859056 & 0.125163 \\ \mathrm{H} & -0.537811 & -3.654888 & 0.097500 \\ \mathrm{H} & 1.844582 & -3.071539 & -0.080167 \\ \mathrm{H} & 2.547908 & -0.715909 & -0.307584 \\ \mathrm{H} & -2.845344 & 0.541626 & -0.025034 \\ \mathrm{H} & -2.188900 & 2.927742 & -0.058954 \\ \mathrm{H} & 0.240460 & 3.531558 & -0.023781 \\ \mathrm{H} & 2.699992 & 1.100761 & 0.314749\end{array}$

1-naphthol, trans $\mathrm{S}_{0}$

$\begin{array}{crcc}\mathrm{C} & -0.712349 & -0.538113 & 0.000000 \\ \mathrm{C} & -0.670858 & -1.953294 & 0.000000 \\ \mathrm{C} & 0.520177 & -2.621842 & 0.000000 \\ \mathrm{C} & 1.736386 & -1.908793 & 0.000000 \\ \mathrm{C} & 1.734833 & -0.542314 & 0.000000 \\ \mathrm{C} & 0.514394 & 0.172015 & 0.000000 \\ \mathrm{H} & -1.606744 & -2.500363 & 0.000000 \\ \mathrm{H} & 0.534875 & -3.704945 & 0.000000 \\ \mathrm{H} & 2.674375 & -2.449969 & 0.000000 \\ \mathrm{H} & 2.663671 & 0.012012 & 0.000000 \\ \mathrm{C} & -1.936662 & 0.176064 & 0.000000 \\ \mathrm{C} & -1.934078 & 1.539508 & 0.000000 \\ \mathrm{C} & -0.722266 & 2.259945 & 0.000000 \\ \mathrm{C} & 0.473517 & 1.594312 & 0.000000 \\ \mathrm{H} & -2.868491 & -0.376394 & 0.000000 \\ \mathrm{H} & -2.868210 & 2.087377 & 0.000000 \\ \mathrm{H} & -0.741416 & 3.344774 & 0.000000 \\ \mathrm{O} & 1.674569 & 2.230856 & 0.000000 \\ \mathrm{H} & 1.534288 & 3.179153 & 0.000000\end{array}$

1-naphthol, trans $\mathrm{S}_{1}$
$\begin{array}{llll}\text { C } & -0.941854 & -0.392516 & 0.000000\end{array}$
$\begin{array}{llll}\text { C } & -1.304364 & -1.746245 & 0.000000\end{array}$
$\begin{array}{llll}\text { C } & -0.313070 & -2.765667 & 0.000000\end{array}$
$\begin{array}{llll}\text { C } & 1.012175 & -2.425642 & 0.000000\end{array}$ 


$\begin{array}{rrrr}\mathrm{C} & 1.415857 & -1.061346 & 0.000000 \\ \mathrm{C} & 0.451730 & -0.044009 & 0.000000 \\ \mathrm{H} & -2.354470 & -2.011301 & 0.000000 \\ \mathrm{H} & -0.614491 & -3.805077 & 0.000000 \\ \mathrm{H} & 1.773243 & -3.196178 & 0.000000 \\ \mathrm{H} & 2.465863 & -0.806019 & 0.000000 \\ \mathrm{C} & -1.891147 & 0.644606 & 0.000000 \\ \mathrm{C} & -1.519924 & 2.013958 & 0.000000 \\ \mathrm{C} & -0.202466 & 2.363316 & 0.000000 \\ \mathrm{C} & 0.783314 & 1.326628 & 0.000000 \\ \mathrm{H} & -2.943708 & 0.386951 & 0.000000 \\ \mathrm{H} & -2.289210 & 2.775018 & 0.000000 \\ \mathrm{H} & 0.109629 & 3.401623 & 0.000000 \\ \mathrm{O} & 2.089155 & 1.643392 & 0.000000 \\ \mathrm{H} & 2.198059 & 2.597580 & 0.000000\end{array}$

2-naphthol, cis $\mathrm{S}_{0}$

$\begin{array}{rrrr}\mathrm{C} & 0.595314 & 2.005969 & 0.000000 \\ \mathrm{C} & 0.618104 & 0.591945 & 0.000000 \\ \mathrm{C} & -0.608142 & -0.119569 & 0.000000 \\ \mathrm{C} & -1.818998 & 0.614280 & 0.000000 \\ \mathrm{C} & -1.809115 & 1.980795 & 0.000000 \\ \mathrm{C} & -0.588772 & 2.687823 & 0.000000 \\ \mathrm{C} & 1.828660 & -0.145663 & 0.000000 \\ \mathrm{C} & 1.827919 & -1.507312 & 0.000000 \\ \mathrm{C} & 0.602019 & -2.211611 & 0.000000 \\ \mathrm{C} & -0.586726 & -1.535408 & 0.000000 \\ \mathrm{H} & -1.528444 & -2.076063 & 0.000000 \\ \mathrm{H} & 1.537751 & 2.542295 & 0.000000 \\ \mathrm{H} & -0.596096 & 3.770623 & 0.000000 \\ \mathrm{H} & -2.743660 & 2.528355 & 0.000000 \\ \mathrm{H} & -2.759259 & 0.074377 & 0.000000 \\ \mathrm{H} & 2.769370 & 0.393156 & 0.000000 \\ \mathrm{H} & 2.749383 & -2.074773 & 0.000000 \\ \mathrm{O} & 0.694854 & -3.567850 & 0.000000 \\ \mathrm{H} & -0.184170 & -3.951359 & 0.000000\end{array}$

2-naphthol, cis $\mathrm{S}_{1}$

$\begin{array}{rrrr}\mathrm{C} & 0.601059 & 1.986360 & 0.000000 \\ \mathrm{C} & 0.644524 & 0.585488 & 0.000000 \\ \mathrm{C} & -0.627040 & -0.118223 & 0.000000 \\ \mathrm{C} & -1.829442 & 0.605946 & 0.000000 \\ \mathrm{C} & -1.838315 & 2.014181 & 0.000000 \\ \mathrm{C} & -0.625689 & 2.681974 & 0.000000\end{array}$




$\begin{array}{lrrr}\mathrm{C} & 1.846623 & -0.150834 & 0.000000 \\ \mathrm{C} & 1.876937 & -1.552969 & 0.000000 \\ \mathrm{C} & 0.656183 & -2.215349 & 0.000000 \\ \mathrm{C} & -0.583199 & -1.518025 & 0.000000 \\ \mathrm{H} & -1.508355 & -2.087286 & 0.000000 \\ \mathrm{H} & 1.528810 & 2.545923 & 0.000000 \\ \mathrm{H} & -0.607210 & 3.765539 & 0.000000 \\ \mathrm{H} & -2.771865 & 2.558683 & 0.000000 \\ \mathrm{H} & -2.765840 & 0.059514 & 0.000000 \\ \mathrm{H} & 2.781127 & 0.397956 & 0.000000 \\ \mathrm{H} & 2.799038 & -2.113883 & 0.000000 \\ \mathrm{O} & 0.662025 & -3.555688 & 0.000000 \\ \mathrm{H} & -0.239360 & -3.889298 & 0.000000\end{array}$

2-naphthol, trans $\mathrm{S}_{0}$

$\begin{array}{lrrl}\mathrm{C} & 0.508357 & 2.008204 & 0.000000 \\ \mathrm{C} & 0.536952 & 0.592684 & 0.000000 \\ \mathrm{C} & -0.686860 & -0.126040 & 0.000000 \\ \mathrm{C} & -1.901735 & 0.604818 & 0.000000 \\ \mathrm{C} & -1.896733 & 1.970086 & 0.000000 \\ \mathrm{C} & -0.678060 & 2.683435 & 0.000000 \\ \mathrm{C} & 1.747697 & -0.137877 & 0.000000 \\ \mathrm{C} & 1.747794 & -1.502401 & 0.000000 \\ \mathrm{C} & 0.525104 & -2.212387 & 0.000000 \\ \mathrm{C} & -0.664393 & -1.538394 & 0.000000 \\ \mathrm{H} & -1.591967 & -2.097355 & 0.000000 \\ \mathrm{H} & 1.448396 & 2.548819 & 0.000000 \\ \mathrm{H} & -0.690859 & 3.766218 & 0.000000 \\ \mathrm{H} & -2.833216 & 2.514356 & 0.000000 \\ \mathrm{H} & -2.838862 & 0.059981 & 0.000000 \\ \mathrm{H} & 2.687826 & 0.401869 & 0.000000 \\ \mathrm{H} & 2.685516 & -2.048869 & 0.000000 \\ \mathrm{O} & 0.499750 & -3.572968 & 0.000000 \\ \mathrm{H} & 1.395304 & -3.914178 & 0.000000\end{array}$

2-naphthol, trans $\mathrm{S}_{1}$

$\begin{array}{rrrc}\text { C } & 0.512669 & 1.990663 & 0.000000 \\ \text { C } & 0.558929 & 0.590202 & 0.000000 \\ \text { C } & -0.707895 & -0.118301 & 0.000000 \\ \text { C } & -1.911096 & 0.601924 & 0.000000 \\ \text { C } & -1.924306 & 2.011888 & 0.000000 \\ \text { C } & -0.716761 & 2.683687 & 0.000000 \\ \text { C } & 1.763711 & -0.140806 & 0.000000 \\ \text { C } & 1.796017 & -1.545102 & 0.000000\end{array}$




$\begin{array}{lrrr}\mathrm{C} & 0.572773 & -2.209607 & 0.000000 \\ \mathrm{C} & -0.664738 & -1.518922 & 0.000000 \\ \mathrm{H} & -1.573629 & -2.107377 & 0.000000 \\ \mathrm{H} & 1.439027 & 2.552746 & 0.000000 \\ \mathrm{H} & -0.701520 & 3.767297 & 0.000000 \\ \mathrm{H} & -2.860393 & 2.552265 & 0.000000 \\ \mathrm{H} & -2.845348 & 0.052257 & 0.000000 \\ \mathrm{H} & 2.697059 & 0.410027 & 0.000000 \\ \mathrm{H} & 2.730848 & -2.090270 & 0.000000 \\ \mathrm{O} & 0.477716 & -3.547101 & 0.000000 \\ \mathrm{H} & 1.356935 & -3.935463 & 0.000000\end{array}$

5-methoxysalicylic acid $\mathrm{S}_{0}$

$\begin{array}{lrrc}\mathrm{C} & 2.272840 & -0.166110 & 0.000000 \\ \mathrm{C} & 1.562782 & -1.338568 & 0.000000 \\ \mathrm{C} & 0.160269 & -1.333433 & 0.000000 \\ \mathrm{C} & -0.506557 & -0.129831 & 0.000000 \\ \mathrm{C} & 0.212277 & 1.080477 & 0.000000 \\ \mathrm{C} & 1.613050 & 1.068956 & 0.000000 \\ \mathrm{O} & -0.433283 & -2.555658 & 0.000000 \\ \mathrm{C} & -1.841277 & -2.599721 & 0.000000 \\ \mathrm{C} & -0.484343 & 2.367686 & 0.000000 \\ \mathrm{O} & -1.821285 & 2.283563 & 0.000000 \\ \mathrm{O} & 2.367255 & 2.175247 & 0.000000 \\ \mathrm{O} & 0.072386 & 3.452114 & 0.000000 \\ \mathrm{H} & -2.111069 & -3.653185 & 0.000000 \\ \mathrm{H} & -2.258398 & -2.121260 & -0.892577 \\ \mathrm{H} & -2.258398 & -2.121260 & 0.892577 \\ \mathrm{H} & -1.585188 & -0.080314 & 0.000000 \\ \mathrm{H} & 3.354792 & -0.168612 & 0.000000 \\ \mathrm{H} & 2.072664 & -2.293732 & 0.000000 \\ \mathrm{H} & 1.769274 & 2.946116 & 0.000000 \\ \mathrm{H} & -2.157774 & 3.187522 & 0.000000\end{array}$

5-methoxysalicylic acid $\mathrm{S}_{1}$

$\begin{array}{lccc}\text { C } & 2.172361 & -0.134474 & 0.000000 \\ \text { C } & 1.726380 & -1.455296 & 0.000000 \\ \text { C } & 0.337331 & -1.680945 & 0.000000 \\ \text { C } & -0.599956 & -0.616658 & 0.000000 \\ \text { C } & -0.164946 & 0.706215 & 0.000000 \\ \text { C } & 1.269800 & 0.930612 & 0.000000 \\ \text { O } & -0.032424 & -2.961178 & 0.000000 \\ \text { C } & -1.408202 & -3.315742 & 0.000000 \\ \text { C } & -1.040802 & 1.836633 & 0.000000\end{array}$




$\begin{array}{rrrr}\mathrm{O} & -2.364135 & 1.548269 & 0.000000 \\ \mathrm{O} & 1.721161 & 2.166719 & 0.000000 \\ \mathrm{O} & -0.659245 & 3.027734 & 0.000000 \\ \mathrm{H} & -1.428307 & -4.401882 & 0.000000 \\ \mathrm{H} & -1.908274 & -2.939135 & -0.894330 \\ \mathrm{H} & -1.908274 & -2.939135 & 0.894330 \\ \mathrm{H} & -1.661387 & -0.814295 & 0.000000 \\ \mathrm{H} & 3.228252 & 0.101403 & 0.000000 \\ \mathrm{H} & 2.403662 & -2.295623 & 0.000000 \\ \mathrm{H} & 0.910953 & 2.772005 & 0.000000 \\ \mathrm{H} & -2.821896 & 2.395550 & 0.000000\end{array}$

$m$-dimethoxybenzene, trans $\mathrm{S}_{0}$

$\begin{array}{rrrr}\mathrm{C} & 0.000000 & -0.705948 & -0.173781 \\ \mathrm{C} & 0.000000 & 0.705948 & -0.173781 \\ \mathrm{C} & 0.000000 & 1.385864 & 1.031483 \\ \mathrm{C} & 0.000000 & 0.688501 & 2.239424 \\ \mathrm{C} & 0.000000 & -0.688501 & 2.239424 \\ \mathrm{C} & 0.000000 & -1.385864 & 1.031483 \\ \mathrm{O} & 0.000000 & 1.290965 & -1.393415 \\ \mathrm{H} & 0.000000 & 2.466955 & 1.045149 \\ \mathrm{H} & 0.000000 & 1.238499 & 3.171725 \\ \mathrm{H} & 0.000000 & -1.238499 & 3.171725 \\ \mathrm{H} & 0.000000 & -2.466955 & 1.045149 \\ \mathrm{O} & 0.000000 & -1.290965 & -1.393415 \\ \mathrm{C} & 0.000000 & -2.697537 & -1.451916 \\ \mathrm{H} & 0.000000 & -2.953195 & -2.509065 \\ \mathrm{H} & 0.892643 & -3.121230 & -0.979792 \\ \mathrm{H} & -0.892642 & -3.121230 & -0.979792 \\ \mathrm{C} & 0.000000 & 2.697537 & -1.451916 \\ \mathrm{H} & 0.000000 & 2.953195 & -2.509065 \\ \mathrm{H} & -0.892642 & 3.121230 & -0.979792 \\ \mathrm{H} & 0.892643 & 3.121230 & -0.979792\end{array}$

m-dimethoxybenzene, trans $\mathrm{S}_{1}$
$\begin{array}{llll}\text { C } & -0.112151 & -1.064444 & -1.443656\end{array}$
$\begin{array}{llll}\text { C } & -0.240357 & 0.159830 & -0.707865\end{array}$
$\begin{array}{llll}\text { C } & -0.240357 & 0.159830 & 0.707865\end{array}$
$\begin{array}{llll}\text { C } & -0.112151 & -1.064444 & 1.443656\end{array}$
$\begin{array}{llll}\text { C } & 0.274496 & -2.189662 & 0.704489\end{array}$
$\begin{array}{llll}\text { C } & 0.274496 & -2.189662 & -0.704489\end{array}$
$\begin{array}{lllll}\mathrm{O} & -0.194224 & 1.370588 & -1.286950\end{array}$
C $\quad-0.018547 \quad 1.456777 \quad-2.690454$
$\begin{array}{llll}\mathrm{O} & -0.194224 & 1.370588 & 1.286950\end{array}$ 


$\begin{array}{lrrr}\mathrm{C} & -0.018547 & 1.456777 & 2.690454 \\ \mathrm{H} & -0.337937 & -1.128803 & 2.497503 \\ \mathrm{H} & 0.551139 & -3.101592 & 1.222007 \\ \mathrm{H} & 0.551139 & -3.101592 & -1.222007 \\ \mathrm{H} & -0.337937 & -1.128803 & -2.497503 \\ \mathrm{H} & 0.041866 & 2.519216 & -2.912676 \\ \mathrm{H} & 0.899871 & 0.957603 & -3.006360 \\ \mathrm{H} & -0.871065 & 1.022926 & -3.219156 \\ \mathrm{H} & 0.041866 & 2.519216 & 2.912676 \\ \mathrm{H} & -0.871065 & 1.022926 & 3.219156 \\ \mathrm{H} & 0.899871 & 0.957603 & 3.006360\end{array}$

3P-propionic acid, gauche $\mathrm{S}_{0}$

$\begin{array}{lrrr}\mathrm{C} & -1.001615 & 0.877957 & -1.163588 \\ \mathrm{C} & -0.377923 & 0.680475 & 0.066174 \\ \mathrm{C} & -1.043854 & -0.057870 & 1.040202 \\ \mathrm{C} & -2.302163 & -0.587105 & 0.789234 \\ \mathrm{C} & -2.912586 & -0.385617 & -0.439757 \\ \mathrm{C} & -2.258413 & 0.350445 & -1.417739 \\ \mathrm{C} & 1.006041 & 1.228595 & 0.313552 \\ \mathrm{C} & 2.106132 & 0.311059 & -0.224207 \\ \mathrm{C} & 2.276999 & -0.945147 & 0.591312 \\ \mathrm{O} & 2.020850 & -1.062780 & 1.759679 \\ \mathrm{O} & 2.802616 & -1.948915 & -0.133507 \\ \mathrm{H} & 1.923263 & 0.038761 & -1.264294 \\ \mathrm{H} & 3.072961 & 0.823819 & -0.197072 \\ \mathrm{H} & 1.163328 & 1.383542 & 1.381665 \\ \mathrm{H} & 1.104447 & 2.200128 & -0.174990 \\ \mathrm{H} & -0.565015 & -0.224960 & 1.997492 \\ \mathrm{H} & -2.806179 & -1.159580 & 1.558469 \\ \mathrm{H} & -3.895003 & -0.797793 & -0.634711 \\ \mathrm{H} & -2.728956 & 0.517087 & -2.379147 \\ \mathrm{H} & -0.498858 & 1.458431 & -1.930363 \\ \mathrm{H} & 2.913937 & -2.700554 & 0.461606\end{array}$

3P-propionic acid, gauche $\mathrm{S}_{1}$
$\begin{array}{llll}\text { C } & -1.000003 & 0.957072 & -1.180010\end{array}$
$\begin{array}{llll}\text { C } & -0.364258 & 0.713874 & 0.070607\end{array}$
$\begin{array}{llll}\text { C } & -1.034641 & -0.070238 & 1.051571\end{array}$
$\begin{array}{llll}\text { C } & -2.312153 & -0.621068 & 0.780644\end{array}$
$\begin{array}{llll}\text { C } & -2.926939 & -0.384767 & -0.472542\end{array}$
$\begin{array}{llll}\text { C } & -2.271992 & 0.397038 & -1.455716\end{array}$
$\begin{array}{llll}\text { C } & 1.017497 & 1.225113 & 0.312489\end{array}$
$\begin{array}{llll}\text { C } & 2.102783 & 0.277472 & -0.224262\end{array}$ 


$\begin{array}{lrrr}\mathrm{C} & 2.282937 & -0.951624 & 0.629502 \\ \mathrm{O} & 2.071922 & -1.021714 & 1.810788 \\ \mathrm{O} & 2.765968 & -1.988382 & -0.077503 \\ \mathrm{H} & 1.891335 & -0.022724 & -1.250911 \\ \mathrm{H} & 3.071481 & 0.787591 & -0.233822 \\ \mathrm{H} & 1.185572 & 1.377628 & 1.380240 \\ \mathrm{H} & 1.139215 & 2.189115 & -0.189079 \\ \mathrm{H} & -0.546774 & -0.256171 & 1.998879 \\ \mathrm{H} & -2.811205 & -1.223556 & 1.526557 \\ \mathrm{H} & -3.901661 & -0.804308 & -0.682796 \\ \mathrm{H} & -2.744097 & 0.568435 & -2.413264 \\ \mathrm{H} & -0.504501 & 1.571282 & -1.921136 \\ \mathrm{H} & 2.889523 & -2.720076 & 0.539763\end{array}$

3P-propionic acid, anti $\mathrm{S}_{0}$

$\begin{array}{lrrr}\mathrm{C} & 1.444628 & -0.438974 & 0.000000 \\ \mathrm{C} & 0.542706 & 0.793252 & 0.000000 \\ \mathrm{C} & 2.910438 & -0.097015 & 0.000000 \\ \mathrm{H} & 1.244903 & -1.068411 & 0.871260 \\ \mathrm{H} & 1.244903 & -1.068411 & -0.871260 \\ \mathrm{C} & -0.913249 & 0.403354 & 0.000000 \\ \mathrm{H} & 0.770485 & 1.405498 & 0.874095 \\ \mathrm{H} & 0.770485 & 1.405498 & -0.874095 \\ \mathrm{C} & -1.594853 & 0.197345 & 1.196039 \\ \mathrm{C} & -2.922397 & -0.205464 & 1.198904 \\ \mathrm{C} & -3.590258 & -0.409381 & 0.000000 \\ \mathrm{C} & -2.922397 & -0.205464 & -1.198904 \\ \mathrm{C} & -1.594853 & 0.197345 & -1.196039 \\ \mathrm{H} & -1.080788 & 0.360447 & 2.137365 \\ \mathrm{H} & -3.437253 & -0.356623 & 2.139946 \\ \mathrm{H} & -4.627482 & -0.721048 & 0.000000 \\ \mathrm{H} & -3.437253 & -0.356623 & -2.139946 \\ \mathrm{H} & -1.080788 & 0.360447 & -2.137365 \\ \mathrm{O} & 3.679184 & -1.203607 & 0.000000 \\ \mathrm{O} & 3.378260 & 1.009327 & 0.000000 \\ \mathrm{H} & 4.598420 & -0.910168 & 0.000000\end{array}$

3P-propionic acid, anti $\mathrm{S}_{1}$

$\begin{array}{rrrr}\mathrm{C} & 1.449721 & -0.424782 & 0.000000 \\ \mathrm{C} & 0.559268 & 0.824870 & 0.000000 \\ \mathrm{C} & 2.917957 & -0.093357 & 0.000000 \\ \mathrm{H} & 1.241345 & -1.048916 & 0.872563 \\ \mathrm{H} & 1.241345 & -1.048916 & -0.872563 \\ \mathrm{C} & -0.886617 & 0.459936 & 0.000000\end{array}$




$\begin{array}{lrrr}\mathrm{H} & 0.798754 & 1.428458 & 0.878371 \\ \mathrm{H} & 0.798754 & 1.428458 & -0.878371 \\ \mathrm{C} & -1.571538 & 0.241367 & 1.228302 \\ \mathrm{C} & -2.910289 & -0.223234 & 1.229706 \\ \mathrm{C} & -3.575506 & -0.448635 & 0.000000 \\ \mathrm{C} & -2.910289 & -0.223234 & -1.229706 \\ \mathrm{C} & -1.571538 & 0.241367 & -1.228302 \\ \mathrm{H} & -1.060187 & 0.431762 & 2.163319 \\ \mathrm{H} & -3.423001 & -0.402029 & 2.164486 \\ \mathrm{H} & -4.601049 & -0.793454 & 0.000000 \\ \mathrm{H} & -3.423001 & -0.402029 & -2.164486 \\ \mathrm{H} & -1.060187 & 0.431762 & -2.163319 \\ \mathrm{O} & 3.676595 & -1.206444 & 0.000000 \\ \mathrm{O} & 3.393570 & 1.009587 & 0.000000 \\ \mathrm{H} & 4.598519 & -0.921375 & 0.000000\end{array}$

fluorine $\mathrm{S}_{0}$

$\begin{array}{lrrr}\mathrm{C} & 0.000000 & 0.000000 & -1.788917 \\ \mathrm{C} & 0.000000 & 1.178221 & -0.846844 \\ \mathrm{C} & 0.000000 & 0.733667 & 0.480369 \\ \mathrm{C} & 0.000000 & -0.733667 & 0.480369 \\ \mathrm{C} & 0.000000 & 1.642494 & 1.528146 \\ \mathrm{C} & 0.000000 & 2.999838 & 1.236696 \\ \mathrm{C} & 0.000000 & 3.441299 & -0.082271 \\ \mathrm{C} & 0.000000 & 2.530596 & -1.133132 \\ \mathrm{H} & 0.000000 & 2.880854 & -2.159075 \\ \mathrm{H} & 0.000000 & 4.504035 & -0.291399 \\ \mathrm{H} & 0.000000 & 3.723201 & 2.042810 \\ \mathrm{H} & 0.000000 & 1.303236 & 2.557366 \\ \mathrm{C} & 0.000000 & -1.642494 & 1.528146 \\ \mathrm{C} & 0.000000 & -2.999838 & 1.236696 \\ \mathrm{C} & 0.000000 & -3.441299 & -0.082271 \\ \mathrm{C} & 0.000000 & -2.530596 & -1.133132 \\ \mathrm{C} & 0.000000 & -1.178221 & -0.846844 \\ \mathrm{H} & 0.000000 & -1.303236 & 2.557366 \\ \mathrm{H} & 0.000000 & -3.723201 & 2.042810 \\ \mathrm{H} & 0.000000 & -4.504035 & -0.291399 \\ \mathrm{H} & 0.000000 & -2.880854 & -2.159075 \\ \mathrm{H} & -0.880062 & 0.000000 & -2.438198 \\ \mathrm{H} & 0.880062 & 0.000000 & -2.438198\end{array}$

fluorine $\mathrm{S}_{1}$

$\begin{array}{llll}\text { C } & 0.000000 & 0.000000 & -1.867246 \\ \text { C } & 0.000000 & 1.176935 & -0.918246\end{array}$ 


$\begin{array}{rrrr}\text { C } & 0.000000 & 0.693672 & 0.451614 \\ \mathrm{C} & 0.000000 & -0.693672 & 0.451614 \\ \mathrm{C} & 0.000000 & 1.636972 & 1.534128 \\ \mathrm{C} & 0.000000 & 2.972540 & 1.229081 \\ \mathrm{C} & 0.000000 & 3.427328 & -0.107100 \\ \mathrm{C} & 0.000000 & 2.517256 & -1.182477 \\ \mathrm{H} & 0.000000 & 2.885357 & -2.201926 \\ \mathrm{H} & 0.000000 & 4.491769 & -0.305302 \\ \mathrm{H} & 0.000000 & 3.706021 & 2.026550 \\ \mathrm{H} & 0.000000 & 1.291996 & 2.560269 \\ \mathrm{C} & 0.000000 & -1.636972 & 1.534128 \\ \mathrm{C} & 0.000000 & -2.972540 & 1.229081 \\ \mathrm{C} & 0.000000 & -3.427328 & -0.107100 \\ \mathrm{C} & 0.000000 & -2.517256 & -1.182477 \\ \mathrm{C} & 0.000000 & -1.176935 & -0.918246 \\ \mathrm{H} & 0.000000 & -1.291996 & 2.560269 \\ \mathrm{H} & 0.000000 & -3.706021 & 2.026550 \\ \mathrm{H} & 0.000000 & -4.491769 & -0.305302 \\ \mathrm{H} & 0.000000 & -2.885357 & -2.201926 \\ \mathrm{H} & -0.879159 & 0.000000 & -2.520807 \\ \mathrm{H} & 0.879159 & 0.000000 & -2.520807\end{array}$

phenanthrene $\mathrm{S}_{0}$

$\begin{array}{lrrr}\mathrm{C} & 0.727605 & 0.000000 & -0.348543 \\ \mathrm{C} & -0.727605 & 0.000000 & -0.348543 \\ \mathrm{C} & -1.414337 & 0.000000 & 0.885060 \\ \mathrm{C} & -0.673364 & 0.000000 & 2.112335 \\ \mathrm{C} & 0.673364 & 0.000000 & 2.112335 \\ \mathrm{C} & 1.414337 & 0.000000 & 0.885060 \\ \mathrm{C} & -1.492235 & 0.000000 & -1.530841 \\ \mathrm{C} & -2.821886 & 0.000000 & 0.899714 \\ \mathrm{H} & -1.224114 & 0.000000 & 3.046024 \\ \mathrm{H} & 1.224114 & 0.000000 & 3.046024 \\ \mathrm{C} & 1.492235 & 0.000000 & -1.530841 \\ \mathrm{C} & 2.864988 & 0.000000 & -1.494209 \\ \mathrm{C} & 3.540450 & 0.000000 & -0.267866 \\ \mathrm{C} & 2.821886 & 0.000000 & 0.899714 \\ \mathrm{H} & 1.000295 & 0.000000 & -2.493885 \\ \mathrm{H} & 3.426500 & 0.000000 & -2.420336 \\ \mathrm{H} & 4.623125 & 0.000000 & -0.243706 \\ \mathrm{H} & 3.331975 & 0.000000 & 1.856345 \\ \mathrm{C} & -3.540450 & 0.000000 & -0.267866 \\ \mathrm{C} & -2.864988 & 0.000000 & -1.494209 \\ \mathrm{H} & -1.000295 & 0.000000 & -2.493885 \\ \mathrm{H} & -3.426500 & 0.000000 & -2.420336\end{array}$




$$
\begin{array}{llll}
\mathrm{H} & -4.623125 & 0.000000 & -0.243706 \\
\mathrm{H} & -3.331975 & 0.000000 & 1.856345
\end{array}
$$

phenanthrene $\mathrm{S}_{1}$

$\begin{array}{lrrr}\mathrm{C} & 0.710306 & 0.000000 & -0.388439 \\ \mathrm{C} & -0.710306 & 0.000000 & -0.388439 \\ \mathrm{C} & -1.430443 & 0.000000 & 0.882816 \\ \mathrm{C} & -0.692884 & 0.000000 & 2.079108 \\ \mathrm{C} & 0.692884 & 0.000000 & 2.079108 \\ \mathrm{C} & 1.430443 & 0.000000 & 0.882816 \\ \mathrm{C} & -1.491145 & 0.000000 & -1.568723 \\ \mathrm{C} & -2.834017 & 0.000000 & 0.884005 \\ \mathrm{H} & -1.228274 & 0.000000 & 3.021438 \\ \mathrm{H} & 1.228274 & 0.000000 & 3.021438 \\ \mathrm{C} & 1.491145 & 0.000000 & -1.568723 \\ \mathrm{C} & 2.876107 & 0.000000 & -1.524931 \\ \mathrm{C} & 3.559650 & 0.000000 & -0.298870 \\ \mathrm{C} & 2.834017 & 0.000000 & 0.884005 \\ \mathrm{H} & 1.007800 & 0.000000 & -2.535589 \\ \mathrm{H} & 3.435321 & 0.000000 & -2.452159 \\ \mathrm{H} & 4.641289 & 0.000000 & -0.276940 \\ \mathrm{H} & 3.349906 & 0.000000 & 1.837323 \\ \mathrm{C} & -3.559650 & 0.000000 & -0.298870 \\ \mathrm{C} & -2.876107 & 0.000000 & -1.524931 \\ \mathrm{H} & -1.007800 & 0.000000 & -2.535589 \\ \mathrm{H} & -3.435321 & 0.000000 & -2.452159 \\ \mathrm{H} & -4.641289 & 0.000000 & -0.276940 \\ \mathrm{H} & -3.349906 & 0.000000 & 1.837323\end{array}$

2,4,6,8-decatetraene $\mathrm{S}_{0}$

$\begin{array}{lrrc}\mathrm{C} & -2.586709 & -5.003423 & 0.000000 \\ \mathrm{C} & -2.461332 & -3.515122 & 0.000000 \\ \mathrm{C} & -1.309287 & -2.843463 & 0.000000 \\ \mathrm{C} & -1.207860 & -1.396783 & 0.000000 \\ \mathrm{C} & -0.050266 & -0.721317 & 0.000000 \\ \mathrm{C} & 0.050266 & 0.721317 & 0.000000 \\ \mathrm{C} & 1.207860 & 1.396783 & 0.000000 \\ \mathrm{C} & 1.309287 & 2.843463 & 0.000000 \\ \mathrm{C} & 2.461332 & 3.515122 & 0.000000 \\ \mathrm{C} & 2.586709 & 5.003423 & 0.000000 \\ \mathrm{H} & -0.372781 & -3.397905 & 0.000000 \\ \mathrm{H} & -2.142904 & -0.840442 & 0.000000 \\ \mathrm{H} & 0.884631 & -1.277875 & 0.000000 \\ \mathrm{H} & -0.884631 & 1.277875 & 0.000000\end{array}$




$\begin{array}{crcc}\mathrm{H} & 2.142904 & 0.840442 & 0.000000 \\ \mathrm{H} & 0.372781 & 3.397905 & 0.000000 \\ \mathrm{H} & 3.391958 & 2.951394 & 0.000000 \\ \mathrm{H} & -3.391958 & -2.951394 & 0.000000 \\ \mathrm{H} & 1.608657 & 5.487268 & 0.000000 \\ \mathrm{H} & 3.138404 & 5.350363 & 0.878211 \\ \mathrm{H} & 3.138404 & 5.350363 & -0.878211 \\ \mathrm{H} & -1.608657 & -5.487268 & 0.000000 \\ \mathrm{H} & -3.138404 & -5.350363 & 0.878211 \\ \mathrm{H} & -3.138404 & -5.350363 & -0.878211\end{array}$

2,4,6,8-decatetraene $\mathrm{S}_{1}$

$\begin{array}{rrrr}\mathrm{C} & -2.573564 & -5.012489 & 0.000000 \\ \mathrm{C} & -2.510593 & -3.528600 & 0.000000 \\ \mathrm{C} & -1.351412 & -2.805981 & 0.000000 \\ \mathrm{C} & -1.265896 & -1.401692 & 0.000000 \\ \mathrm{C} & -0.052793 & -0.692103 & 0.000000 \\ \mathrm{C} & 0.052793 & 0.692103 & 0.000000 \\ \mathrm{C} & 1.265896 & 1.401692 & 0.000000 \\ \mathrm{C} & 1.351412 & 2.805981 & 0.000000 \\ \mathrm{C} & 2.510593 & 3.528600 & 0.000000 \\ \mathrm{C} & 2.573564 & 5.012489 & 0.000000 \\ \mathrm{H} & -0.411037 & -3.356117 & 0.000000 \\ \mathrm{H} & -2.191544 & -0.830581 & 0.000000 \\ \mathrm{H} & 0.867800 & -1.272427 & 0.000000 \\ \mathrm{H} & -0.867800 & 1.272427 & 0.000000 \\ \mathrm{H} & 2.191544 & 0.830581 & 0.000000 \\ \mathrm{H} & 0.411037 & 3.356117 & 0.000000 \\ \mathrm{H} & 3.456437 & 2.991480 & 0.000000 \\ \mathrm{H} & -3.456437 & -2.991480 & 0.000000 \\ \mathrm{H} & 1.576476 & 5.457103 & 0.000000 \\ \mathrm{H} & 3.114139 & 5.392648 & 0.875916 \\ \mathrm{H} & 3.114139 & 5.392648 & -0.875916 \\ \mathrm{H} & -1.576476 & -5.457103 & 0.000000 \\ \mathrm{H} & -3.114139 & -5.392648 & 0.875916 \\ \mathrm{H} & -3.114139 & -5.392648 & -0.875916\end{array}$

tetracene $\mathrm{S}_{0}$
$\begin{array}{llll}\text { C } & 3.693077 & 1.402433 & 0.000000\end{array}$
$\begin{array}{llll}\text { C } & 2.432938 & 0.719694 & 0.000000\end{array}$
$\begin{array}{llll}\text { C } & 2.432938 & -0.719694 & 0.000000\end{array}$
$\begin{array}{llll}\text { C } & 3.693077 & -1.402433 & 0.000000\end{array}$
$\begin{array}{llll}\text { C } & 4.858787 & -0.714479 & 0.000000\end{array}$
$\begin{array}{llll}\text { C } & 4.858787 & 0.714479 & 0.000000\end{array}$ 


$\begin{array}{rrrr}\mathrm{C} & 1.229444 & 1.397567 & 0.000000 \\ \mathrm{C} & 0.000000 & 0.718485 & 0.000000 \\ \mathrm{C} & 0.000000 & -0.718485 & 0.000000 \\ \mathrm{C} & 1.229444 & -1.397567 & 0.000000 \\ \mathrm{C} & -1.229444 & 1.397567 & 0.000000 \\ \mathrm{C} & -2.432938 & 0.719694 & 0.000000 \\ \mathrm{C} & -2.432938 & -0.719694 & 0.000000 \\ \mathrm{C} & -1.229444 & -1.397567 & 0.000000 \\ \mathrm{C} & -3.693077 & 1.402433 & 0.000000 \\ \mathrm{C} & -4.858787 & 0.714479 & 0.000000 \\ \mathrm{C} & -4.858787 & -0.714479 & 0.000000 \\ \mathrm{C} & -3.693077 & -1.402433 & 0.000000 \\ \mathrm{H} & -3.691569 & -2.486555 & 0.000000 \\ \mathrm{H} & -5.803998 & -1.243103 & 0.000000 \\ \mathrm{H} & -5.803998 & 1.243103 & 0.000000 \\ \mathrm{H} & -3.691569 & 2.486555 & 0.000000 \\ \mathrm{H} & -1.229490 & -2.482607 & 0.000000 \\ \mathrm{H} & -1.229490 & 2.482607 & 0.000000 \\ \mathrm{H} & 1.229490 & -2.482607 & 0.000000 \\ \mathrm{H} & 1.229490 & 2.482607 & 0.000000 \\ \mathrm{H} & 3.691569 & -2.486555 & 0.000000 \\ \mathrm{H} & 5.803998 & -1.243103 & 0.000000 \\ \mathrm{H} & 5.803998 & 1.243103 & 0.000000 \\ \mathrm{H} & 3.691569 & 2.486555 & 0.000000\end{array}$

tetracene $\mathrm{S}_{1}$

$\begin{array}{rrrr}\mathrm{C} & 3.691965 & 1.391948 & 0.000000 \\ \mathrm{C} & 2.464241 & 0.715861 & 0.000000 \\ \mathrm{C} & 2.464241 & -0.715861 & 0.000000 \\ \mathrm{C} & 3.691965 & -1.391948 & 0.000000 \\ \mathrm{C} & 4.891172 & -0.696456 & 0.000000 \\ \mathrm{C} & 4.891172 & 0.696456 & 0.000000 \\ \mathrm{C} & 1.223946 & 1.394742 & 0.000000 \\ \mathrm{C} & 0.000000 & 0.725030 & 0.000000 \\ \mathrm{C} & 0.000000 & -0.725030 & 0.000000 \\ \mathrm{C} & 1.223946 & -1.394742 & 0.000000 \\ \mathrm{C} & -1.223946 & 1.394742 & 0.000000 \\ \mathrm{C} & -2.464241 & 0.715861 & 0.000000 \\ \mathrm{C} & -2.464241 & -0.715861 & 0.000000 \\ \mathrm{C} & -1.223946 & -1.394742 & 0.000000 \\ \mathrm{C} & -3.691965 & 1.391948 & 0.000000 \\ \mathrm{C} & -4.891172 & 0.696456 & 0.000000 \\ \mathrm{C} & -4.891172 & -0.696456 & 0.000000 \\ \mathrm{C} & -3.691965 & -1.391948 & 0.000000 \\ \mathrm{H} & -3.694051 & -2.476109 & 0.000000\end{array}$




$\begin{array}{rrrr}\mathrm{H} & -5.828756 & -1.237944 & 0.000000 \\ \mathrm{H} & -5.828756 & 1.237944 & 0.000000 \\ \mathrm{H} & -3.694051 & 2.476109 & 0.000000 \\ \mathrm{H} & -1.227118 & -2.479952 & 0.000000 \\ \mathrm{H} & -1.227118 & 2.479952 & 0.000000 \\ \mathrm{H} & 1.227118 & -2.479952 & 0.000000 \\ \mathrm{H} & 1.227118 & 2.479952 & 0.000000 \\ \mathrm{H} & 3.694051 & -2.476109 & 0.000000 \\ \mathrm{H} & 5.828756 & -1.237944 & 0.000000 \\ \mathrm{H} & 5.828756 & 1.237944 & 0.000000 \\ \mathrm{H} & 3.694051 & 2.476109 & 0.000000\end{array}$

perylene $\mathrm{S}_{0}$

$\begin{array}{lrrc}\mathrm{C} & 0.020457 & 1.470072 & -2.411257 \\ \mathrm{C} & 0.010909 & 0.738598 & -1.244129 \\ \mathrm{C} & 0.014165 & 1.435531 & -0.000001 \\ \mathrm{C} & 0.034466 & 2.856861 & 0.000001 \\ \mathrm{C} & 0.043357 & 3.555984 & -1.227677 \\ \mathrm{C} & 0.036684 & 2.872513 & -2.407520 \\ \mathrm{C} & 0.044072 & 3.555970 & 1.227683 \\ \mathrm{C} & 0.038085 & 2.872485 & 2.407522 \\ \mathrm{C} & 0.021645 & 1.470047 & 2.411253 \\ \mathrm{C} & 0.011249 & 0.738591 & 1.244122 \\ \mathrm{C} & -0.010909 & -0.738598 & -1.244129 \\ \mathrm{C} & -0.014165 & -1.435531 & -0.000001 \\ \mathrm{C} & -0.011249 & -0.738591 & 1.244122 \\ \mathrm{C} & -0.034466 & -2.856861 & 0.000001 \\ \mathrm{C} & -0.044072 & -3.555970 & 1.227683 \\ \mathrm{C} & -0.038085 & -2.872485 & 2.407522 \\ \mathrm{C} & -0.021645 & -1.470047 & 2.411253 \\ \mathrm{C} & -0.020457 & -1.470072 & -2.411257 \\ \mathrm{C} & -0.036684 & -2.872513 & -2.407520 \\ \mathrm{C} & -0.043357 & -3.555984 & -1.227677 \\ \mathrm{H} & 0.057468 & 4.639517 & 1.212885 \\ \mathrm{H} & 0.046179 & 3.405013 & 3.350428 \\ \mathrm{H} & 0.018459 & 0.970035 & 3.369459 \\ \mathrm{H} & -0.057468 & -4.639517 & 1.212885 \\ \mathrm{H} & -0.046179 & -3.405013 & 3.350428 \\ \mathrm{H} & -0.018459 & -0.970035 & 3.369459 \\ \mathrm{H} & -0.056710 & -4.639531 & -1.212874 \\ \mathrm{H} & -0.044105 & -3.405052 & -3.350424 \\ \mathrm{H} & -0.016699 & -0.970076 & -3.369469 \\ \mathrm{H} & 0.056710 & 4.639531 & -1.212874 \\ \mathrm{H} & 0.044105 & 3.405052 & -3.350424 \\ \mathrm{H} & 0.016699 & 0.970076 & -3.369469\end{array}$


perylene $S_{1}$

$\begin{array}{rrrr}\mathrm{C} & -0.005324 & 1.464949 & -2.441539 \\ \mathrm{C} & -0.006132 & 0.717493 & -1.235824 \\ \mathrm{C} & 0.011180 & 1.415581 & -0.000002 \\ \mathrm{C} & 0.006750 & 2.845701 & 0.000000 \\ \mathrm{C} & 0.008250 & 3.535384 & -1.226021 \\ \mathrm{C} & -0.000608 & 2.843944 & -2.426471 \\ \mathrm{C} & 0.008418 & 3.535383 & 1.226019 \\ \mathrm{C} & -0.000268 & 2.843940 & 2.426471 \\ \mathrm{C} & -0.005034 & 1.464946 & 2.441537 \\ \mathrm{C} & -0.006057 & 0.717492 & 1.235822 \\ \mathrm{C} & 0.006132 & -0.717493 & -1.235824 \\ \mathrm{C} & -0.011180 & -1.415581 & -0.000002 \\ \mathrm{C} & 0.006057 & -0.717492 & 1.235822 \\ \mathrm{C} & -0.006750 & -2.845701 & 0.000000 \\ \mathrm{C} & -0.008418 & -3.535383 & 1.226019 \\ \mathrm{C} & 0.000268 & -2.843940 & 2.426471 \\ \mathrm{C} & 0.005034 & -1.464946 & 2.441537 \\ \mathrm{C} & 0.005324 & -1.464949 & -2.441539 \\ \mathrm{C} & 0.000608 & -2.843944 & -2.426471 \\ \mathrm{C} & -0.008250 & -3.535384 & -1.226021 \\ \mathrm{H} & 0.011676 & 4.619057 & 1.218371 \\ \mathrm{H} & -0.003034 & 3.388968 & 3.362398 \\ \mathrm{H} & -0.023248 & 0.957508 & 3.394608 \\ \mathrm{H} & -0.011676 & -4.619057 & 1.218371 \\ \mathrm{H} & 0.003034 & -3.388968 & 3.362398 \\ \mathrm{H} & 0.023248 & -0.957508 & 3.394608 \\ \mathrm{H} & -0.011493 & -4.619058 & -1.218371 \\ \mathrm{H} & 0.003556 & -3.388972 & -3.362398 \\ \mathrm{H} & 0.023741 & -0.957512 & -3.394606 \\ \mathrm{H} & 0.011493 & 4.619058 & -1.218371 \\ \mathrm{H} & -0.003556 & 3.388972 & -3.362398 \\ \mathrm{H} & -0.023741 & 0.957512 & -3.394606\end{array}$

\section{CIS/TZVP geometries - Group I}

pyrazine $\mathrm{S}_{0}$
$\begin{array}{llll}\text { C } & 1.119913 & -0.691798 & 0.000000\end{array}$
$\begin{array}{llll}\text { C } & 1.119913 & 0.691798 & 0.000000\end{array}$
$\begin{array}{llll}\mathrm{N} & 0.000000 & 1.382381 & 0.000000\end{array}$
$\begin{array}{llll}\text { C } & -1.119913 & 0.691798 & 0.000000\end{array}$
$\begin{array}{llll}\text { C } & -1.119913 & -0.691798 & 0.000000\end{array}$ 


$\begin{array}{rrrr}\mathrm{N} & 0.000000 & -1.382381 & 0.000000 \\ \mathrm{H} & -2.041739 & -1.243106 & 0.000000 \\ \mathrm{H} & 2.041739 & -1.243106 & 0.000000 \\ \mathrm{H} & 2.041739 & 1.243106 & 0.000000 \\ \mathrm{H} & -2.041739 & 1.243106 & 0.000000\end{array}$

pyrazine $S_{1}$
$\begin{array}{llll}\text { C } & -1.175508 & 0.652729 & 0.000000\end{array}$
$\begin{array}{llll}\text { C } & -1.128640 & -0.730686 & 0.000000\end{array}$
$\begin{array}{llll}\mathrm{N} & 0.045453 & -1.348772 & 0.000000\end{array}$
$\begin{array}{llll}\text { C } & 1.175508 & -0.652729 & 0.000000\end{array}$
$\begin{array}{llll}\text { C } & 1.128640 & 0.730686 & 0.000000\end{array}$
$\begin{array}{llll}\mathrm{N} & -0.045453 & 1.348772 & 0.000000\end{array}$
$\begin{array}{llll}\mathrm{H} & 2.043899 & 1.289942 & 0.000000\end{array}$
$\begin{array}{llll}\mathrm{H} & -2.125909 & 1.149788 & 0.000000\end{array}$
$\begin{array}{llll}\mathrm{H} & -2.043899 & -1.289942 & 0.000000\end{array}$
$\begin{array}{llll}\mathrm{H} & 2.125909 & -1.149788 & 0.000000\end{array}$

2,6-difluoropyridine $\mathrm{S}_{0}$

$\begin{array}{crrr}\mathrm{C} & 1.106153 & 0.000000 & -0.870702 \\ \mathrm{~N} & 0.000000 & 0.000000 & -1.552051 \\ \mathrm{C} & -1.106153 & 0.000000 & -0.870702 \\ \mathrm{C} & -1.196102 & 0.000000 & 0.503126 \\ \mathrm{C} & 0.000000 & 0.000000 & 1.192910 \\ \mathrm{C} & 1.196102 & 0.000000 & 0.503126 \\ \mathrm{~F} & -2.208601 & 0.000000 & -1.575047 \\ \mathrm{H} & -2.150344 & 0.000000 & 0.989051 \\ \mathrm{H} & 0.000000 & 0.000000 & 2.266705 \\ \mathrm{H} & 2.150344 & 0.000000 & 0.989051 \\ \mathrm{~F} & 2.208601 & 0.000000 & -1.575047\end{array}$

2,6-difluoropyridine $\mathrm{S}_{1}$

$\begin{array}{crrr}\mathrm{C} & -1.094635 & 0.000000 & -0.335202 \\ \mathrm{~N} & 0.000000 & 0.000000 & -1.081589 \\ \mathrm{C} & 1.094635 & 0.000000 & -0.335202 \\ \mathrm{C} & 1.185118 & 0.000000 & 1.070084 \\ \mathrm{C} & 0.000000 & 0.000000 & 1.833574 \\ \mathrm{C} & -1.185118 & 0.000000 & 1.070084 \\ \mathrm{~F} & 2.209290 & 0.000000 & -0.997560 \\ \mathrm{H} & 2.162145 & 0.000000 & 1.510722 \\ \mathrm{H} & 0.000000 & 0.000000 & 2.901220 \\ \mathrm{H} & -2.162145 & 0.000000 & 1.510722 \\ \mathrm{~F} & -2.209290 & 0.000000 & -0.997560\end{array}$


2-fluoropyridine $\mathrm{S}_{0}$

$\begin{array}{cccc}\mathrm{C} & -1.309124 & 0.498341 & 0.000000 \\ \mathrm{~N} & -1.353954 & -0.791334 & 0.000000 \\ \mathrm{C} & -0.196510 & -1.438978 & 0.000000 \\ \mathrm{C} & 1.023665 & -0.806413 & 0.000000 \\ \mathrm{C} & 1.035391 & 0.581675 & 0.000000 \\ \mathrm{C} & -0.156476 & 1.264635 & 0.000000 \\ \mathrm{H} & -0.260198 & -2.511213 & 0.000000 \\ \mathrm{H} & 1.933180 & -1.374460 & 0.000000 \\ \mathrm{H} & 1.964772 & 1.120155 & 0.000000 \\ \mathrm{H} & -0.215835 & 2.334603 & 0.000000 \\ \mathrm{~F} & -2.464921 & 1.122979 & 0.000000\end{array}$

2-fluoropyridine $\mathrm{S}_{1}$

$\begin{array}{crcc}\mathrm{C} & -1.325639 & 0.481428 & 0.000000 \\ \mathrm{~N} & -1.422900 & -0.823849 & 0.000000 \\ \mathrm{C} & -0.223731 & -1.450704 & 0.000000 \\ \mathrm{C} & 1.031191 & -0.785145 & 0.000000 \\ \mathrm{C} & 1.098819 & 0.608280 & 0.000000 \\ \mathrm{C} & -0.150842 & 1.271306 & 0.000000 \\ \mathrm{H} & -0.251064 & -2.522886 & 0.000000 \\ \mathrm{H} & 1.919696 & -1.386593 & 0.000000 \\ \mathrm{H} & 2.025727 & 1.141107 & 0.000000 \\ \mathrm{H} & -0.250963 & 2.338016 & 0.000000 \\ \mathrm{~F} & -2.450305 & 1.129032 & 0.000000\end{array}$

2-hydroxypyridine $\mathrm{S}_{0}$

$\begin{array}{cccc}\mathrm{C} & -0.971433 & 0.745298 & 0.000000 \\ \mathrm{C} & -0.957184 & -0.648367 & 0.000000 \\ \mathrm{~N} & 0.132768 & -1.363847 & 0.000000 \\ \mathrm{C} & 1.297731 & -0.727519 & 0.000000 \\ \mathrm{C} & 1.414289 & 0.638744 & 0.000000 \\ \mathrm{C} & 0.239091 & 1.385216 & 0.000000 \\ \mathrm{O} & -2.125226 & -1.292532 & 0.000000 \\ \mathrm{H} & 2.171361 & -1.353384 & 0.000000 \\ \mathrm{H} & 2.378509 & 1.107778 & 0.000000 \\ \mathrm{H} & 0.278336 & 2.459081 & 0.000000 \\ \mathrm{H} & -1.905024 & 1.271527 & 0.000000 \\ \mathrm{H} & -1.953217 & -2.221994 & 0.000000\end{array}$

2-hydroxypyridine $\mathrm{S}_{1}$ 


$\begin{array}{crcc}\mathrm{C} & -0.981827 & 0.757327 & 0.000000 \\ \mathrm{C} & -0.956167 & -0.657276 & 0.000000 \\ \mathrm{~N} & 0.127867 & -1.440522 & 0.000000 \\ \mathrm{C} & 1.287950 & -0.765365 & 0.000000 \\ \mathrm{C} & 1.390606 & 0.649685 & 0.000000 \\ \mathrm{C} & 0.248966 & 1.452672 & 0.000000 \\ \mathrm{O} & -2.105502 & -1.279928 & 0.000000 \\ \mathrm{H} & 2.184318 & -1.355455 & 0.000000 \\ \mathrm{H} & 2.372794 & 1.082273 & 0.000000 \\ \mathrm{H} & 0.298822 & 2.520901 & 0.000000 \\ \mathrm{H} & -1.933929 & 1.248847 & 0.000000 \\ \mathrm{H} & -1.933898 & -2.213157 & 0.000000\end{array}$

2-pyridone $\mathrm{S}_{0}$

$\begin{array}{crrc}\mathrm{C} & -1.241952 & 0.697150 & 0.000000 \\ \mathrm{~N} & -1.136492 & -0.679731 & 0.000000 \\ \mathrm{C} & 0.035177 & -1.372205 & 0.000000 \\ \mathrm{C} & 1.210510 & -0.738471 & 0.000000 \\ \mathrm{C} & 1.193286 & 0.697565 & 0.000000 \\ \mathrm{C} & 0.043613 & 1.383143 & 0.000000 \\ \mathrm{H} & -2.002652 & -1.169462 & 0.000000 \\ \mathrm{H} & -0.055119 & -2.440944 & 0.000000 \\ \mathrm{H} & 2.130781 & -1.285554 & 0.000000 \\ \mathrm{H} & 2.128556 & 1.227357 & 0.000000 \\ \mathrm{H} & 0.011074 & 2.454429 & 0.000000 \\ \mathrm{O} & -2.316783 & 1.226734 & 0.000000\end{array}$

2-pyridone $\mathrm{S}_{1}$

$\begin{array}{rrrr}\mathrm{C} & -1.410538 & 0.098867 & 0.000000 \\ \mathrm{~N} & -0.731474 & -1.155873 & 0.000000 \\ \mathrm{C} & 0.607078 & -1.241950 & 0.000000 \\ \mathrm{C} & 1.403725 & -0.074996 & 0.000000 \\ \mathrm{C} & 0.823594 & 1.174654 & 0.000000 \\ \mathrm{C} & -0.594628 & 1.265035 & 0.000000 \\ \mathrm{H} & -1.313625 & -1.962335 & 0.000000 \\ \mathrm{H} & 1.040917 & -2.221883 & 0.000000 \\ \mathrm{H} & 2.468346 & -0.201082 & 0.000000 \\ \mathrm{H} & 1.423676 & 2.061748 & 0.000000 \\ \mathrm{H} & -1.107460 & 2.205529 & 0.000000 \\ \mathrm{O} & -2.609610 & 0.052276 & 0.000000\end{array}$

2-methylpyrimidine $\mathrm{S}_{0}$

$\begin{array}{llll}\text { C } & 0.000341 & 1.955396 & 0.000000\end{array}$ 


$\begin{array}{cccc}\mathrm{C} & -1.164690 & 1.214686 & 0.000000 \\ \mathrm{~N} & -1.164939 & -0.099229 & 0.000000 \\ \mathrm{C} & 0.011723 & -0.705935 & 0.000000 \\ \mathrm{~N} & 1.179861 & -0.091881 & 0.000000 \\ \mathrm{C} & 1.168043 & 1.226665 & 0.000000 \\ \mathrm{H} & -2.127556 & 1.694539 & 0.000000 \\ \mathrm{H} & 2.127510 & 1.712849 & 0.000000 \\ \mathrm{H} & -0.004715 & 3.027129 & 0.000000 \\ \mathrm{C} & 0.004248 & -2.205887 & 0.000000 \\ \mathrm{H} & 1.014683 & -2.585839 & 0.000000 \\ \mathrm{H} & -0.522259 & -2.571247 & 0.873902 \\ \mathrm{H} & -0.522259 & -2.571247 & -0.873902\end{array}$

2-methylpyrimidine $\mathrm{S}_{1}$

$\begin{array}{lrrr}\mathrm{C} & -0.000859 & 1.998572 & 0.000000 \\ \mathrm{C} & -1.182733 & 1.283941 & 0.000000 \\ \mathrm{~N} & -1.090912 & -0.072114 & 0.000000 \\ \mathrm{C} & 0.013938 & -0.777868 & 0.000000 \\ \mathrm{~N} & 1.107408 & -0.075282 & 0.000000 \\ \mathrm{C} & 1.178976 & 1.284852 & 0.000000 \\ \mathrm{H} & -2.157172 & 1.724546 & 0.000000 \\ \mathrm{H} & 2.151017 & 1.730882 & 0.000000 \\ \mathrm{H} & -0.003540 & 3.072205 & 0.000000 \\ \mathrm{C} & 0.003281 & -2.267701 & 0.000000 \\ \mathrm{H} & 1.017097 & -2.639613 & 0.000000 \\ \mathrm{H} & -0.518251 & -2.631214 & 0.877045 \\ \mathrm{H} & -0.518251 & -2.631214 & -0.877045\end{array}$

5-methylpyrimidine $\mathrm{S}_{0}$

$\begin{array}{rrrr}\mathrm{C} & 0.872829 & 0.841051 & 0.000000 \\ \mathrm{C} & -0.354774 & 0.209043 & 0.000000 \\ \mathrm{C} & -0.290663 & -1.174606 & 0.000000 \\ \mathrm{~N} & 0.843292 & -1.840779 & 0.000000 \\ \mathrm{C} & 1.947262 & -1.123002 & 0.000000 \\ \mathrm{~N} & 2.018878 & 0.187467 & 0.000000 \\ \mathrm{H} & 2.876173 & -1.661892 & 0.000000 \\ \mathrm{H} & -1.191396 & -1.764891 & 0.000000 \\ \mathrm{H} & 0.936849 & 1.915460 & 0.000000 \\ \mathrm{C} & -1.661555 & 0.956996 & 0.000000 \\ \mathrm{H} & -1.491834 & 2.026348 & 0.000000 \\ \mathrm{H} & -2.252531 & 0.714393 & -0.876214 \\ \mathrm{H} & -2.252531 & 0.714393 & 0.876214\end{array}$

5-methylpyrimidine $\mathrm{S}_{1}$ 


$\begin{array}{cccc}\mathrm{C} & 0.838161 & 0.869041 & 0.000000 \\ \mathrm{C} & -0.372646 & 0.227326 & 0.000000 \\ \mathrm{C} & -0.337977 & -1.175467 & 0.000000 \\ \mathrm{~N} & 0.873369 & -1.766804 & 0.000000 \\ \mathrm{C} & 2.030494 & -1.167487 & 0.000000 \\ \mathrm{~N} & 1.991898 & 0.133052 & 0.000000 \\ \mathrm{H} & 2.951141 & -1.712024 & 0.000000 \\ \mathrm{H} & -1.209396 & -1.796099 & 0.000000 \\ \mathrm{H} & 0.940793 & 1.934420 & 0.000000 \\ \mathrm{C} & -1.675996 & 0.972888 & 0.000000 \\ \mathrm{H} & -1.508575 & 2.041946 & 0.000000 \\ \mathrm{H} & -2.260637 & 0.719608 & -0.877124 \\ \mathrm{H} & -2.260637 & 0.719608 & 0.877124\end{array}$

7-azaindole $\mathrm{S}_{0}$

$\begin{array}{lrrr}\mathrm{N} & -0.631860 & 1.828575 & 0.000000 \\ \mathrm{C} & -0.668599 & 0.467496 & 0.000000 \\ \mathrm{C} & 0.654924 & 0.003603 & 0.000000 \\ \mathrm{C} & 1.500305 & 1.170502 & 0.000000 \\ \mathrm{C} & 0.679874 & 2.236844 & 0.000000 \\ \mathrm{C} & 0.835614 & -1.370110 & 0.000000 \\ \mathrm{C} & -0.291553 & -2.163463 & 0.000000 \\ \mathrm{C} & -1.552479 & -1.567733 & 0.000000 \\ \mathrm{~N} & -1.757231 & -0.269597 & 0.000000 \\ \mathrm{H} & -2.432837 & -2.184826 & 0.000000 \\ \mathrm{H} & 1.817918 & -1.806457 & 0.000000 \\ \mathrm{H} & -0.213950 & -3.233638 & 0.000000 \\ \mathrm{H} & -1.432420 & 2.412693 & 0.000000 \\ \mathrm{H} & 2.569225 & 1.197723 & 0.000000 \\ \mathrm{H} & 0.923089 & 3.278389 & 0.000000\end{array}$

7-azaindole $\mathrm{S}_{1}$

$\begin{array}{rrrr}\mathrm{N} & -0.626758 & 1.862105 & 0.000000 \\ \mathrm{C} & -0.682955 & 0.472899 & 0.000000 \\ \mathrm{C} & 0.671037 & 0.009565 & 0.000000 \\ \mathrm{C} & 1.482620 & 1.130654 & 0.000000 \\ \mathrm{C} & 0.645619 & 2.274435 & 0.000000 \\ \mathrm{C} & 0.876014 & -1.408330 & 0.000000 \\ \mathrm{C} & -0.287884 & -2.173277 & 0.000000 \\ \mathrm{C} & -1.538514 & -1.587141 & 0.000000 \\ \mathrm{~N} & -1.769776 & -0.217416 & 0.000000 \\ \mathrm{H} & -2.423589 & -2.192650 & 0.000000 \\ \mathrm{H} & 1.855243 & -1.843365 & 0.000000\end{array}$




$\begin{array}{rrrr}\mathrm{H} & -0.229727 & -3.245316 & 0.000000 \\ \mathrm{H} & -1.434720 & 2.440264 & 0.000000 \\ \mathrm{H} & 2.551580 & 1.168095 & 0.000000 \\ \mathrm{H} & 0.911821 & 3.309497 & 0.000000\end{array}$

7-hydroxyquinoline, cis $\mathrm{S}_{0}$

$\begin{array}{rrrr}\mathrm{N} & 0.448850 & 1.957454 & 0.000000 \\ \mathrm{C} & 0.424250 & 0.605537 & 0.000000 \\ \mathrm{C} & -0.777931 & -0.119356 & 0.000000 \\ \mathrm{C} & -1.984427 & 0.609940 & 0.000000 \\ \mathrm{C} & -1.941319 & 1.964871 & 0.000000 \\ \mathrm{C} & -0.676064 & 2.591236 & 0.000000 \\ \mathrm{C} & 1.657509 & -0.089854 & 0.000000 \\ \mathrm{C} & 1.672064 & -1.445060 & 0.000000 \\ \mathrm{C} & 0.463064 & -2.185875 & 0.000000 \\ \mathrm{C} & -0.721188 & -1.537287 & 0.000000 \\ \mathrm{H} & -0.620488 & 3.666412 & 0.000000 \\ \mathrm{H} & -1.639742 & -2.095745 & 0.000000 \\ \mathrm{H} & 0.519586 & -3.257296 & 0.000000 \\ \mathrm{O} & 2.803899 & -2.176314 & 0.000000 \\ \mathrm{H} & 2.564260 & 0.486687 & 0.000000 \\ \mathrm{H} & -2.923051 & 0.085126 & 0.000000 \\ \mathrm{H} & -2.834048 & 2.560003 & 0.000000 \\ \mathrm{H} & 3.564777 & -1.620468 & 0.000000\end{array}$

7-hydroxyquinoline, cis $\mathrm{S}_{1}$

$\begin{array}{rrrl}\mathrm{N} & 0.470301 & 1.964486 & 0.000000 \\ \mathrm{C} & 0.436670 & 0.624204 & 0.000000 \\ \mathrm{C} & -0.797632 & -0.140284 & 0.000000 \\ \mathrm{C} & -1.986618 & 0.590565 & 0.000000 \\ \mathrm{C} & -1.916261 & 1.990922 & 0.000000 \\ \mathrm{C} & -0.680099 & 2.624593 & 0.000000 \\ \mathrm{C} & 1.628014 & -0.078832 & 0.000000 \\ \mathrm{C} & 1.652920 & -1.509716 & 0.000000 \\ \mathrm{C} & 0.488411 & -2.239210 & 0.000000 \\ \mathrm{C} & -0.724648 & -1.548870 & 0.000000 \\ \mathrm{H} & -0.618657 & 3.696139 & 0.000000 \\ \mathrm{H} & -1.642834 & -2.107123 & 0.000000 \\ \mathrm{H} & 0.526542 & -3.309891 & 0.000000 \\ \mathrm{O} & 2.819341 & -2.148828 & 0.000000 \\ \mathrm{H} & 2.551201 & 0.471595 & 0.000000 \\ \mathrm{H} & -2.936741 & 0.091029 & 0.000000 \\ \mathrm{H} & -2.815945 & 2.576407 & 0.000000 \\ \mathrm{H} & 3.546032 & -1.547177 & 0.000000\end{array}$


7-hydroxyquinoline, trans $\mathrm{S}_{0}$

$\begin{array}{crrr}\mathrm{N} & 0.500186 & 2.028408 & 0.000000 \\ \mathrm{C} & 0.472565 & 0.673141 & 0.000000 \\ \mathrm{C} & -0.735908 & -0.045026 & 0.000000 \\ \mathrm{C} & -1.941678 & 0.691117 & 0.000000 \\ \mathrm{C} & -1.891738 & 2.043350 & 0.000000 \\ \mathrm{C} & -0.620211 & 2.664855 & 0.000000 \\ \mathrm{C} & 1.700277 & -0.022838 & 0.000000 \\ \mathrm{C} & 1.710500 & -1.378882 & 0.000000 \\ \mathrm{C} & 0.498150 & -2.111328 & 0.000000 \\ \mathrm{C} & -0.687645 & -1.457882 & 0.000000 \\ \mathrm{H} & -0.561429 & 3.740018 & 0.000000 \\ \mathrm{H} & -1.607484 & -2.014186 & 0.000000 \\ \mathrm{H} & 0.526707 & -3.186750 & 0.000000 \\ \mathrm{O} & 2.899113 & -2.018022 & 0.000000 \\ \mathrm{H} & 2.617171 & 0.532400 & 0.000000 \\ \mathrm{H} & -2.882506 & 0.170190 & 0.000000 \\ \mathrm{H} & -2.780959 & 2.643755 & 0.000000 \\ \mathrm{H} & 2.784870 & -2.952301 & 0.000000\end{array}$

7-hydroxyquinoline, trans $\mathrm{S}_{1}$

$\begin{array}{lrrr}\mathrm{N} & 0.514428 & 2.033436 & 0.000004 \\ \mathrm{C} & 0.476196 & 0.697918 & 0.000055 \\ \mathrm{C} & -0.759535 & -0.058951 & 0.000119 \\ \mathrm{C} & -1.945953 & 0.675837 & 0.000076 \\ \mathrm{C} & -1.870844 & 2.077102 & -0.000006 \\ \mathrm{C} & -0.635712 & 2.702245 & -0.000055 \\ \mathrm{C} & 1.668681 & -0.010994 & 0.000067 \\ \mathrm{C} & 1.686379 & -1.440274 & 0.000015 \\ \mathrm{C} & 0.520208 & -2.165076 & -0.000049 \\ \mathrm{C} & -0.693976 & -1.465110 & 0.000000 \\ \mathrm{H} & -0.565969 & 3.773147 & -0.000103 \\ \mathrm{H} & -1.614071 & -2.020299 & -0.000036 \\ \mathrm{H} & 0.533448 & -3.238742 & -0.000116 \\ \mathrm{O} & 2.898388 & -1.988881 & -0.000012 \\ \mathrm{H} & 2.603000 & 0.514352 & 0.000077 \\ \mathrm{H} & -2.897960 & 0.179712 & 0.000075 \\ \mathrm{H} & -2.768481 & 2.665718 & -0.000042 \\ \mathrm{H} & 2.851774 & -2.931157 & -0.000070\end{array}$

2-hydroxyquinoline, enol $\mathrm{S}_{0}$

$\begin{array}{llll}\text { C } & 0.425688 & 1.898267 & 0.000000\end{array}$ 


$\begin{array}{rrrr}\mathrm{C} & 0.478705 & 0.485722 & 0.000000 \\ \mathrm{C} & -0.710099 & -0.255204 & 0.000000 \\ \mathrm{C} & -1.943661 & 0.435259 & 0.000000 \\ \mathrm{C} & -1.967830 & 1.792334 & 0.000000 \\ \mathrm{C} & -0.769220 & 2.539827 & 0.000000 \\ \mathrm{C} & 1.692033 & -0.247997 & 0.000000 \\ \mathrm{C} & 1.660753 & -1.595149 & 0.000000 \\ \mathrm{C} & 0.387180 & -2.237292 & 0.000000 \\ \mathrm{~N} & -0.725674 & -1.611628 & 0.000000 \\ \mathrm{O} & 0.320230 & -3.574717 & 0.000000 \\ \mathrm{H} & 1.345371 & 2.455346 & 0.000000 \\ \mathrm{H} & -0.811112 & 3.612820 & 0.000000 \\ \mathrm{H} & -2.909427 & 2.309886 & 0.000000 \\ \mathrm{H} & -2.846098 & -0.144971 & 0.000000 \\ \mathrm{H} & 2.630787 & 0.275866 & 0.000000 \\ \mathrm{H} & 2.563145 & -2.178231 & 0.000000 \\ \mathrm{H} & 1.179228 & -3.960139 & 0.000000\end{array}$

2-hydroxyquinoline, enol $\mathrm{S}_{1}$

$\begin{array}{rrrr}\mathrm{C} & 0.437383 & 1.880614 & 0.000000 \\ \mathrm{C} & 0.532426 & 0.492735 & 0.000000 \\ \mathrm{C} & -0.714876 & -0.268715 & 0.000000 \\ \mathrm{C} & -1.950696 & 0.432492 & 0.000000 \\ \mathrm{C} & -1.999654 & 1.827617 & 0.000000 \\ \mathrm{C} & -0.816175 & 2.539123 & 0.000000 \\ \mathrm{C} & 1.722290 & -0.234367 & 0.000000 \\ \mathrm{C} & 1.683288 & -1.632124 & 0.000000 \\ \mathrm{C} & 0.441086 & -2.238072 & 0.000000 \\ \mathrm{~N} & -0.729121 & -1.584053 & 0.000000 \\ \mathrm{O} & 0.297554 & -3.556769 & 0.000000 \\ \mathrm{H} & 1.333812 & 2.472574 & 0.000000 \\ \mathrm{H} & -0.830694 & 3.612798 & 0.000000 \\ \mathrm{H} & -2.943502 & 2.336111 & 0.000000 \\ \mathrm{H} & -2.847109 & -0.156950 & 0.000000 \\ \mathrm{H} & 2.664453 & 0.280869 & 0.000000 \\ \mathrm{H} & 2.583064 & -2.216622 & 0.000000 \\ \mathrm{H} & 1.136464 & -3.987240 & 0.000000\end{array}$

2-hydroxyquinoline, keto $\mathrm{S}_{0}$
$\begin{array}{lll}\mathrm{N} & 0.557934 & 1.701681\end{array}$
0.000000
$\begin{array}{llll}\text { C } & 0.596423 & 0.324358 & 0.000000\end{array}$
$\begin{array}{llll}\text { C } & -0.603739 & -0.380800 & 0.000000\end{array}$
$\begin{array}{llll}\text { C } & -1.834210 & 0.384230 & 0.000000\end{array}$
$\begin{array}{llll}\text { C } & -1.832040 & 1.713520 & 0.000000\end{array}$ 


$\begin{array}{rrrr}\mathrm{C} & -0.576265 & 2.472927 & 0.000000 \\ \mathrm{C} & 1.808695 & -0.361181 & 0.000000 \\ \mathrm{C} & 1.812486 & -1.734032 & 0.000000 \\ \mathrm{C} & 0.620069 & -2.452757 & 0.000000 \\ \mathrm{C} & -0.572477 & -1.774567 & 0.000000 \\ \mathrm{H} & -1.501336 & -2.315671 & 0.000000 \\ \mathrm{H} & 0.635981 & -3.525783 & 0.000000 \\ \mathrm{H} & 2.750555 & -2.257420 & 0.000000 \\ \mathrm{H} & 2.733291 & 0.186567 & 0.000000 \\ \mathrm{H} & -2.765034 & -0.154132 & 0.000000 \\ \mathrm{H} & -2.732678 & 2.294488 & 0.000000 \\ \mathrm{O} & -0.510461 & 3.667595 & 0.000000 \\ \mathrm{H} & 1.412827 & 2.210997 & 0.000000\end{array}$

2-hydroxyquinoline, keto $\mathrm{S}_{1}$

$\begin{array}{rrrc}\mathrm{N} & 0.573337 & 1.684186 & 0.000000 \\ \mathrm{C} & 0.618970 & 0.343240 & 0.000000 \\ \mathrm{C} & -0.663857 & -0.382218 & 0.000000 \\ \mathrm{C} & -1.842858 & 0.357893 & 0.000000 \\ \mathrm{C} & -1.839924 & 1.747512 & 0.000000 \\ \mathrm{C} & -0.609775 & 2.471645 & 0.000000 \\ \mathrm{C} & 1.828084 & -0.352758 & 0.000000 \\ \mathrm{C} & 1.837967 & -1.729227 & 0.000000 \\ \mathrm{C} & 0.613152 & -2.441544 & 0.000000 \\ \mathrm{C} & -0.592550 & -1.794648 & 0.000000 \\ \mathrm{H} & -1.507769 & -2.355536 & 0.000000 \\ \mathrm{H} & 0.637013 & -3.515060 & 0.000000 \\ \mathrm{H} & 2.767660 & -2.263762 & 0.000000 \\ \mathrm{H} & 2.748330 & 0.202412 & 0.000000 \\ \mathrm{H} & -2.778794 & -0.168305 & 0.000000 \\ \mathrm{H} & -2.743304 & 2.321940 & 0.000000 \\ \mathrm{O} & -0.462726 & 3.660389 & 0.000000 \\ \mathrm{H} & 1.417043 & 2.213851 & 0.000000\end{array}$

dibenzofuran $\mathrm{S}_{0}$

$\begin{array}{lrrr}\mathrm{C} & 1.718334 & 0.000000 & -1.350759 \\ \mathrm{C} & 0.728115 & 0.000000 & -0.381192 \\ \mathrm{C} & 1.087410 & 0.000000 & 0.958201 \\ \mathrm{C} & 2.395811 & 0.000000 & 1.385032 \\ \mathrm{C} & 3.369716 & 0.000000 & 0.405652 \\ \mathrm{C} & 3.037489 & 0.000000 & -0.946699 \\ \mathrm{C} & -0.728115 & 0.000000 & -0.381192 \\ \mathrm{C} & -1.087410 & 0.000000 & 0.958201 \\ \mathrm{O} & 0.000000 & 0.000000 & 1.767801\end{array}$




$\begin{array}{rrrr}\mathrm{C} & -1.718334 & 0.000000 & -1.350759 \\ \mathrm{C} & -3.037489 & 0.000000 & -0.946699 \\ \mathrm{C} & -3.369716 & 0.000000 & 0.405652 \\ \mathrm{C} & -2.395811 & 0.000000 & 1.385032 \\ \mathrm{H} & -2.639875 & 0.000000 & 2.429762 \\ \mathrm{H} & -4.404459 & 0.000000 & 0.693725 \\ \mathrm{H} & -3.820003 & 0.000000 & -1.682073 \\ \mathrm{H} & -1.468269 & 0.000000 & -2.395383 \\ \mathrm{H} & 1.468269 & 0.000000 & -2.395383 \\ \mathrm{H} & 3.820003 & 0.000000 & -1.682073 \\ \mathrm{H} & 4.404459 & 0.000000 & 0.693725 \\ \mathrm{H} & 2.639875 & 0.000000 & 2.429762\end{array}$

dibenzofuran $\mathrm{S}_{1}$

$\begin{array}{lrrr}\mathrm{C} & 0.000000 & 1.722768 & -1.525625 \\ \mathrm{C} & 0.000000 & 0.712647 & -0.565651 \\ \mathrm{C} & 0.000000 & 1.088521 & 0.801328 \\ \mathrm{C} & 0.000000 & 2.393449 & 1.249251 \\ \mathrm{C} & 0.000000 & 3.392963 & 0.266794 \\ \mathrm{C} & 0.000000 & 3.056541 & -1.086778 \\ \mathrm{C} & 0.000000 & -0.712647 & -0.565651 \\ \mathrm{C} & 0.000000 & -1.088521 & 0.801328 \\ \mathrm{O} & 0.000000 & 0.000000 & 1.584247 \\ \mathrm{C} & 0.000000 & -1.722768 & -1.525625 \\ \mathrm{C} & 0.000000 & -3.056541 & -1.086778 \\ \mathrm{C} & 0.000000 & -3.392963 & 0.266794 \\ \mathrm{C} & 0.000000 & -2.393449 & 1.249251 \\ \mathrm{H} & 0.000000 & -2.620960 & 2.296993 \\ \mathrm{H} & 0.000000 & -4.424784 & 0.558812 \\ \mathrm{H} & 0.000000 & -3.842027 & -1.818726 \\ \mathrm{H} & 0.000000 & -1.499035 & -2.574811 \\ \mathrm{H} & 0.000000 & 1.499035 & -2.574811 \\ \mathrm{H} & 0.000000 & 3.842027 & -1.818726 \\ \mathrm{H} & 0.000000 & 4.424784 & 0.558812 \\ \mathrm{H} & 0.000000 & 2.620960 & 2.296993\end{array}$

pyrrolo[3,2-h]quinoline $\mathrm{S}_{0}$

$\begin{array}{lrrr}\mathrm{C} & 0.140550 & -0.680697 & 0.000000 \\ \mathrm{~N} & 0.072872 & -2.023730 & 0.000000 \\ \mathrm{C} & 1.183915 & -2.690122 & 0.000000 \\ \mathrm{C} & 2.454471 & -2.087718 & 0.000000 \\ \mathrm{C} & 2.529441 & -0.729249 & 0.000000 \\ \mathrm{C} & 1.348012 & 0.033963 & 0.000000 \\ \mathrm{H} & 1.103388 & -3.763242 & 0.000000\end{array}$




$\begin{array}{lrrl}\mathrm{H} & 3.336225 & -2.699234 & 0.000000 \\ \mathrm{H} & 3.482648 & -0.231783 & 0.000000 \\ \mathrm{C} & -1.050396 & 0.086367 & 0.000000 \\ \mathrm{C} & -1.050804 & 1.465630 & 0.000000 \\ \mathrm{C} & 0.187734 & 2.165436 & 0.000000 \\ \mathrm{C} & 1.342504 & 1.467603 & 0.000000 \\ \mathrm{H} & 0.199369 & 3.239769 & 0.000000 \\ \mathrm{H} & 2.287340 & 1.979665 & 0.000000 \\ \mathrm{C} & -2.423916 & 1.874093 & 0.000000 \\ \mathrm{C} & -3.164226 & 0.741294 & 0.000000 \\ \mathrm{~N} & -2.338764 & -0.344906 & 0.000000 \\ \mathrm{H} & -2.610639 & -1.298160 & 0.000000 \\ \mathrm{H} & -2.802877 & 2.874236 & 0.000000 \\ \mathrm{H} & -4.226836 & 0.620786 & 0.000000\end{array}$

pyrrolo[3,2-h]quinoline $\mathrm{S}_{1}$

$\begin{array}{lrrr}\mathrm{C} & 0.141493 & -0.701806 & 0.000000 \\ \mathrm{~N} & 0.047222 & -2.010593 & 0.000000 \\ \mathrm{C} & 1.206849 & -2.717387 & 0.000000 \\ \mathrm{C} & 2.438365 & -2.128044 & 0.000000 \\ \mathrm{C} & 2.545718 & -0.721129 & 0.000000 \\ \mathrm{C} & 1.373697 & 0.035500 & 0.000000 \\ \mathrm{H} & 1.102657 & -3.785349 & 0.000000 \\ \mathrm{H} & 3.322482 & -2.736627 & 0.000000 \\ \mathrm{H} & 3.507440 & -0.244309 & 0.000000 \\ \mathrm{C} & -1.032543 & 0.088490 & 0.000000 \\ \mathrm{C} & -1.054076 & 1.527488 & 0.000000 \\ \mathrm{C} & 0.140071 & 2.204396 & 0.000000 \\ \mathrm{C} & 1.332174 & 1.448153 & 0.000000 \\ \mathrm{H} & 0.184919 & 3.276043 & 0.000000 \\ \mathrm{H} & 2.268036 & 1.976361 & 0.000000 \\ \mathrm{C} & -2.446691 & 1.893162 & 0.000000 \\ \mathrm{C} & -3.154980 & 0.748762 & 0.000000 \\ \mathrm{~N} & -2.302364 & -0.340130 & 0.000000 \\ \mathrm{H} & -2.556471 & -1.300265 & 0.000000 \\ \mathrm{H} & -2.849698 & 2.883067 & 0.000000 \\ \mathrm{H} & -4.214310 & 0.604214 & 0.000000\end{array}$

carbazole $\mathrm{S}_{0}$
$\begin{array}{llll}\text { C } & 1.123087 & 0.000000 & -0.888285\end{array}$
$\begin{array}{llll}\mathrm{N} & 0.000000 & 0.000000 & -1.684939\end{array}$
$\begin{array}{llll}\mathrm{C} & -1.123087 & 0.000000 & -0.888285\end{array}$
$\begin{array}{llll}\text { C } & -0.726771 & 0.000000 & 0.452157\end{array}$
$\begin{array}{llll}\text { C } & 0.726771 & 0.000000 & 0.452157\end{array}$ 


$\begin{array}{lrrr}\mathrm{C} & -1.690284 & 0.000000 & 1.450594 \\ \mathrm{C} & -3.022065 & 0.000000 & 1.098381 \\ \mathrm{C} & -3.398744 & 0.000000 & -0.244270 \\ \mathrm{C} & -2.460519 & 0.000000 & -1.252872 \\ \mathrm{C} & 2.460519 & 0.000000 & -1.252872 \\ \mathrm{C} & 3.398744 & 0.000000 & -0.244270 \\ \mathrm{C} & 3.022065 & 0.000000 & 1.098381 \\ \mathrm{C} & 1.690284 & 0.000000 & 1.450594 \\ \mathrm{H} & -2.758587 & 0.000000 & -2.285235 \\ \mathrm{H} & -4.442747 & 0.000000 & -0.498316 \\ \mathrm{H} & -3.778253 & 0.000000 & 1.860774 \\ \mathrm{H} & -1.404373 & 0.000000 & 2.486477 \\ \mathrm{H} & 1.404373 & 0.000000 & 2.486477 \\ \mathrm{H} & 3.778253 & 0.000000 & 1.860774 \\ \mathrm{H} & 4.442747 & 0.000000 & -0.498316 \\ \mathrm{H} & 2.758587 & 0.000000 & -2.285235 \\ \mathrm{H} & 0.000000 & 0.000000 & -2.674830\end{array}$

carbazole $\mathrm{S}_{1}$

$\begin{array}{rrrr}\mathrm{C} & 1.126687 & 0.000000 & -0.893671 \\ \mathrm{~N} & 0.000000 & 0.000000 & -1.682595 \\ \mathrm{C} & -1.126687 & 0.000000 & -0.893671 \\ \mathrm{C} & -0.692299 & 0.000000 & 0.482288 \\ \mathrm{C} & 0.692299 & 0.000000 & 0.482288 \\ \mathrm{C} & -1.672236 & 0.000000 & 1.521290 \\ \mathrm{C} & -2.997196 & 0.000000 & 1.159453 \\ \mathrm{C} & -3.389362 & 0.000000 & -0.185096 \\ \mathrm{C} & -2.447623 & 0.000000 & -1.235818 \\ \mathrm{C} & 2.447623 & 0.000000 & -1.235818 \\ \mathrm{C} & 3.389362 & 0.000000 & -0.185096 \\ \mathrm{C} & 2.997196 & 0.000000 & 1.159453 \\ \mathrm{C} & 1.672236 & 0.000000 & 1.521290 \\ \mathrm{H} & -2.768922 & 0.000000 & -2.260174 \\ \mathrm{H} & -4.435875 & 0.000000 & -0.425957 \\ \mathrm{H} & -3.757298 & 0.000000 & 1.918088 \\ \mathrm{H} & -1.371639 & 0.000000 & 2.551181 \\ \mathrm{H} & 1.371639 & 0.000000 & 2.551181 \\ \mathrm{H} & 3.757298 & 0.000000 & 1.918088 \\ \mathrm{H} & 4.435875 & 0.000000 & -0.425957 \\ \mathrm{H} & 2.768922 & 0.000000 & -2.260174 \\ \mathrm{H} & 0.000000 & 0.000000 & -2.673198\end{array}$

tryptamine, A-ph $\mathrm{S}_{0}$
C -2.112375
0.130567
0.880029 


$\begin{array}{lrcc}\mathrm{N} & -1.243455 & 0.126344 & 1.945278 \\ \mathrm{C} & 0.035250 & 0.044752 & 1.468184 \\ \mathrm{C} & -0.037905 & -0.011212 & 0.073385 \\ \mathrm{C} & -1.439573 & 0.044297 & -0.282959 \\ \mathrm{C} & 1.249389 & 0.020734 & 2.147315 \\ \mathrm{C} & 2.395376 & -0.057776 & 1.396872 \\ \mathrm{C} & 2.346134 & -0.110030 & -0.002566 \\ \mathrm{C} & 1.144592 & -0.087517 & -0.665019 \\ \mathrm{C} & -2.020046 & 0.000579 & -1.665915 \\ \mathrm{C} & -1.994447 & -1.393566 & -2.292389 \\ \mathrm{~N} & -2.504266 & -1.348706 & -3.654683 \\ \mathrm{H} & 1.290792 & 0.063848 & 3.220471 \\ \mathrm{H} & 3.349692 & -0.077730 & 1.890183 \\ \mathrm{H} & 3.263265 & -0.167392 & -0.558757 \\ \mathrm{H} & 1.118042 & -0.124522 & -1.738678 \\ \mathrm{H} & -1.502655 & 0.204822 & 2.897122 \\ \mathrm{H} & -3.167933 & 0.200202 & 1.044532 \\ \mathrm{H} & -1.482662 & 0.679741 & -2.319151 \\ \mathrm{H} & -3.047401 & 0.353172 & -1.632052 \\ \mathrm{H} & -2.546940 & -2.078378 & -1.649099 \\ \mathrm{H} & -0.973214 & -1.753710 & -2.329969 \\ \mathrm{H} & -3.472399 & -1.100133 & -3.670605 \\ \mathrm{H} & -2.414673 & -2.236007 & -4.104159\end{array}$

tryptamine, A-ph $\mathrm{S}_{1}$

$\begin{array}{lrrr}\mathrm{C} & -2.136292 & 0.113816 & 0.883141 \\ \mathrm{~N} & -1.279456 & 0.098157 & 1.919516 \\ \mathrm{C} & 0.027855 & 0.042913 & 1.466354 \\ \mathrm{C} & -0.040246 & 0.026632 & 0.043889 \\ \mathrm{C} & -1.386123 & 0.068560 & -0.316408 \\ \mathrm{C} & 1.201812 & -0.005973 & 2.164102 \\ \mathrm{C} & 2.416314 & -0.066308 & 1.382552 \\ \mathrm{C} & 2.383283 & -0.079606 & 0.005520 \\ \mathrm{C} & 1.177619 & -0.033787 & -0.705898 \\ \mathrm{C} & -1.971140 & 0.023240 & -1.691210 \\ \mathrm{C} & -2.007229 & -1.394952 & -2.267598 \\ \mathrm{~N} & -2.560154 & -1.379988 & -3.611970 \\ \mathrm{H} & 1.239082 & -0.000864 & 3.236424 \\ \mathrm{H} & 3.357897 & -0.100340 & 1.896893 \\ \mathrm{H} & 3.308660 & -0.122865 & -0.537984 \\ \mathrm{H} & 1.167058 & -0.027440 & -1.778556 \\ \mathrm{H} & -1.543796 & 0.140435 & 2.874824 \\ \mathrm{H} & -3.194395 & 0.178565 & 1.020749 \\ \mathrm{H} & -1.395856 & 0.651530 & -2.361076 \\ \mathrm{H} & -2.980349 & 0.426365 & -1.667455\end{array}$




$$
\begin{array}{llll}
\mathrm{H} & -2.555767 & -2.042184 & -1.583041 \\
\mathrm{H} & -0.994562 & -1.776857 & -2.321637 \\
\mathrm{H} & -3.531301 & -1.143644 & -3.604135 \\
\mathrm{H} & -2.470344 & -2.273027 & -4.049665
\end{array}
$$

tryptamine, A-py $\mathrm{S}_{0}$

$\begin{array}{lrrc}\mathrm{C} & -2.105244 & 0.105561 & 0.857924 \\ \mathrm{~N} & -1.246277 & 0.114955 & 1.930794 \\ \mathrm{C} & 0.037411 & 0.045045 & 1.465916 \\ \mathrm{C} & -0.022248 & -0.017636 & 0.070506 \\ \mathrm{C} & -1.421230 & 0.021756 & -0.298771 \\ \mathrm{C} & 1.245109 & 0.036787 & 2.156652 \\ \mathrm{C} & 2.399365 & -0.032483 & 1.418075 \\ \mathrm{C} & 2.364125 & -0.091410 & 0.018598 \\ \mathrm{C} & 1.168619 & -0.084907 & -0.655060 \\ \mathrm{C} & -1.998567 & -0.028524 & -1.683109 \\ \mathrm{C} & -1.959752 & -1.421088 & -2.311421 \\ \mathrm{~N} & -2.531092 & -1.388790 & -3.649944 \\ \mathrm{H} & 1.275430 & 0.084570 & 3.229974 \\ \mathrm{H} & 3.348924 & -0.040242 & 1.920772 \\ \mathrm{H} & 3.287097 & -0.142053 & -0.528567 \\ \mathrm{H} & 1.153950 & -0.129143 & -1.728719 \\ \mathrm{H} & -1.515322 & 0.191768 & 2.880071 \\ \mathrm{H} & -3.163012 & 0.164700 & 1.011555 \\ \mathrm{H} & -1.459723 & 0.664635 & -2.325438 \\ \mathrm{H} & -3.028357 & 0.308603 & -1.664261 \\ \mathrm{H} & -2.556324 & -2.094357 & -1.706688 \\ \mathrm{H} & -0.938922 & -1.800966 & -2.292539 \\ \mathrm{H} & -1.971363 & -0.837779 & -4.268351 \\ \mathrm{H} & -2.592684 & -2.306612 & -4.038860\end{array}$

tryptamine, A-py $\mathrm{S}_{1}$

$\begin{array}{lrrr}\mathrm{C} & -2.128327 & 0.083400 & 0.862836 \\ \mathrm{~N} & -1.280994 & 0.077969 & 1.906083 \\ \mathrm{C} & 0.031046 & 0.039471 & 1.465050 \\ \mathrm{C} & -0.023727 & 0.021998 & 0.042332 \\ \mathrm{C} & -1.366809 & 0.047778 & -0.330037 \\ \mathrm{C} & 1.199040 & 0.007114 & 2.174364 \\ \mathrm{C} & 2.421271 & -0.037451 & 1.403899 \\ \mathrm{C} & 2.401687 & -0.052063 & 0.027006 \\ \mathrm{C} & 1.201725 & -0.022851 & -0.695902 \\ \mathrm{C} & -1.946471 & -0.001052 & -1.706770 \\ \mathrm{C} & -1.973281 & -1.418973 & -2.284738 \\ \mathrm{~N} & -2.575784 & -1.411725 & -3.607969\end{array}$




$\begin{array}{rrrr}\mathrm{H} & 1.226181 & 0.013710 & 3.246924 \\ \mathrm{H} & 3.358348 & -0.058840 & 1.927120 \\ \mathrm{H} & 3.332670 & -0.083966 & -0.507685 \\ \mathrm{H} & 1.202116 & -0.020396 & -1.768633 \\ \mathrm{H} & -1.554628 & 0.115430 & 2.859052 \\ \mathrm{H} & -3.188585 & 0.132858 & 0.989699 \\ \mathrm{H} & -1.365020 & 0.639484 & -2.363981 \\ \mathrm{H} & -2.958209 & 0.388433 & -1.699269 \\ \mathrm{H} & -2.577900 & -2.048846 & -1.641900 \\ \mathrm{H} & -0.963031 & -1.825975 & -2.271947 \\ \mathrm{H} & -2.001902 & -0.922184 & -4.263982 \\ \mathrm{H} & -2.699506 & -2.340943 & -3.952452\end{array}$

tryptamine, A-up $\mathrm{S}_{0}$

$\begin{array}{lrrr}\mathrm{C} & -2.104477 & 0.121018 & 0.868686 \\ \mathrm{~N} & -1.243724 & 0.106309 & 1.939744 \\ \mathrm{C} & 0.038851 & 0.036822 & 1.471429 \\ \mathrm{C} & -0.023303 & -0.000483 & 0.075434 \\ \mathrm{C} & -1.422539 & 0.054796 & -0.290591 \\ \mathrm{C} & 1.247611 & 0.008422 & 2.159902 \\ \mathrm{C} & 2.399936 & -0.055511 & 1.418053 \\ \mathrm{C} & 2.362077 & -0.089747 & 0.017716 \\ \mathrm{C} & 1.165766 & -0.063103 & -0.653695 \\ \mathrm{C} & -1.997157 & 0.023693 & -1.676451 \\ \mathrm{C} & -1.964853 & -1.367089 & -2.326116 \\ \mathrm{~N} & -2.485301 & -1.426498 & -3.680598 \\ \mathrm{H} & 1.280015 & 0.036666 & 3.233829 \\ \mathrm{H} & 3.350278 & -0.078939 & 1.918735 \\ \mathrm{H} & 3.283744 & -0.137962 & -0.531759 \\ \mathrm{H} & 1.149266 & -0.089499 & -1.727900 \\ \mathrm{H} & -1.510809 & 0.164346 & 2.890923 \\ \mathrm{H} & -3.161587 & 0.182739 & 1.026143 \\ \mathrm{H} & -1.456947 & 0.722294 & -2.311319 \\ \mathrm{H} & -3.025964 & 0.371643 & -1.644852 \\ \mathrm{H} & -2.533791 & -2.057024 & -1.713232 \\ \mathrm{H} & -0.947889 & -1.741078 & -2.340254 \\ \mathrm{H} & -1.959624 & -0.845445 & -4.300751 \\ \mathrm{H} & -3.438341 & -1.128461 & -3.719835\end{array}$

tryptamine, A-up $\mathrm{S}_{1}$
$\begin{array}{llll}\text { C } & -2.125635 & 0.092663 & 0.871536\end{array}$
$\begin{array}{llll}\mathrm{N} & -1.276478 & 0.065080 & 1.913226\end{array}$
$\begin{array}{llll}\text { C } & 0.034777 & 0.029268 & 1.469906\end{array}$
$\begin{array}{llll}\text { C } & -0.021736 & 0.035712 & 0.046925\end{array}$ 


$\begin{array}{lrrr}\mathrm{C} & -1.365216 & 0.076333 & -0.323111 \\ \mathrm{C} & 1.203657 & -0.015802 & 2.176559 \\ \mathrm{C} & 2.424813 & -0.052062 & 1.404258 \\ \mathrm{C} & 2.403239 & -0.044685 & 0.027452 \\ \mathrm{C} & 1.202206 & -0.000380 & -0.692995 \\ \mathrm{C} & -1.941264 & 0.047637 & -1.701128 \\ \mathrm{C} & -2.004048 & -1.373901 & -2.290150 \\ \mathrm{~N} & -2.549481 & -1.459120 & -3.630423 \\ \mathrm{H} & 1.232285 & -0.025607 & 3.249082 \\ \mathrm{H} & 3.362350 & -0.085556 & 1.925936 \\ \mathrm{H} & 3.333129 & -0.072099 & -0.509288 \\ \mathrm{H} & 1.201492 & 0.014208 & -1.765669 \\ \mathrm{H} & -1.548626 & 0.084607 & 2.867187 \\ \mathrm{H} & -3.185431 & 0.142056 & 1.002154 \\ \mathrm{H} & -1.343595 & 0.674993 & -2.355910 \\ \mathrm{H} & -2.942293 & 0.470799 & -1.685549 \\ \mathrm{H} & -2.600649 & -2.002238 & -1.638464 \\ \mathrm{H} & -1.006860 & -1.798017 & -2.299245 \\ \mathrm{H} & -1.999919 & -0.943605 & -4.286384 \\ \mathrm{H} & -3.485496 & -1.112362 & -3.672683\end{array}$

tryptamine, $\mathrm{Ph}$-out $\mathrm{S}_{0}$

$\begin{array}{lrrr}\mathrm{C} & 0.026935 & 0.000649 & 0.095941 \\ \mathrm{C} & 0.055720 & 0.067663 & 1.492276 \\ \mathrm{C} & 1.248330 & 0.096098 & 2.209252 \\ \mathrm{C} & 2.418572 & 0.060568 & 1.494723 \\ \mathrm{C} & 2.413972 & -0.003977 & 0.094496 \\ \mathrm{C} & 1.234660 & -0.034709 & -0.606083 \\ \mathrm{~N} & -1.238434 & 0.100274 & 1.931027 \\ \mathrm{H} & 1.254141 & 0.146671 & 3.282877 \\ \mathrm{H} & 3.357430 & 0.082392 & 2.016882 \\ \mathrm{H} & 3.349464 & -0.030361 & -0.432740 \\ \mathrm{H} & 1.238662 & -0.096206 & -1.677954 \\ \mathrm{C} & -1.365652 & -0.000371 & -0.302310 \\ \mathrm{C} & -2.074663 & 0.068835 & 0.841515 \\ \mathrm{H} & -1.528236 & 0.180461 & 2.873938 \\ \mathrm{H} & -3.136276 & 0.107095 & 0.975000 \\ \mathrm{C} & -1.924324 & -0.050659 & -1.698328 \\ \mathrm{H} & -1.284511 & 0.515551 & -2.366488 \\ \mathrm{C} & -2.104248 & -1.457456 & -2.271349 \\ \mathrm{H} & -2.894116 & 0.437353 & -1.702529 \\ \mathrm{H} & -2.567170 & -1.367263 & -3.248725 \\ \mathrm{~N} & -0.833706 & -2.142180 & -2.445820 \\ \mathrm{H} & -2.799610 & -2.008021 & -1.636311 \\ \mathrm{H} & -0.402290 & -2.333549 & -1.565090\end{array}$




$$
\begin{array}{llll}
\mathrm{H} & -0.959680 & -3.014747 & -2.914989
\end{array}
$$

tryptamine, $\mathrm{Ph}$-out $\mathrm{S}_{1}$

$\begin{array}{rrrr}\mathrm{C} & 0.034878 & 0.015460 & 0.070572 \\ \mathrm{C} & 0.055523 & 0.056330 & 1.496017 \\ \mathrm{C} & 1.206175 & 0.094099 & 2.231389 \\ \mathrm{C} & 2.448281 & 0.095276 & 1.493371 \\ \mathrm{C} & 2.461170 & 0.050805 & 0.116944 \\ \mathrm{C} & 1.280031 & 0.003720 & -0.634291 \\ \mathrm{~N} & -1.265984 & 0.051020 & 1.906939 \\ \mathrm{H} & 1.205671 & 0.129871 & 3.303911 \\ \mathrm{H} & 3.371871 & 0.127511 & 2.039382 \\ \mathrm{H} & 3.404481 & 0.047577 & -0.396644 \\ \mathrm{H} & 1.305119 & -0.050565 & -1.704836 \\ \mathrm{C} & -1.300019 & -0.002284 & -0.333072 \\ \mathrm{C} & -2.090618 & 0.017573 & 0.844941 \\ \mathrm{H} & -1.561776 & 0.083971 & 2.853429 \\ \mathrm{H} & -3.154350 & 0.029849 & 0.949742 \\ \mathrm{C} & -1.862222 & -0.038582 & -1.720096 \\ \mathrm{H} & -1.177153 & 0.448750 & -2.403283 \\ \mathrm{C} & -2.149617 & -1.450916 & -2.241475 \\ \mathrm{H} & -2.791265 & 0.525059 & -1.732507 \\ \mathrm{H} & -2.667351 & -1.359515 & -3.190235 \\ \mathrm{~N} & -0.921750 & -2.189051 & -2.472299 \\ \mathrm{H} & -2.830576 & -1.950492 & -1.550672 \\ \mathrm{H} & -0.406718 & -2.322086 & -1.626278 \\ \mathrm{H} & -1.108813 & -3.089260 & -2.861736\end{array}$

tryptamine, Ph-up $\mathrm{S}_{0}$

$\begin{array}{lrrc}\mathrm{C} & -2.053068 & 0.011825 & 0.813050 \\ \mathrm{~N} & -1.228051 & 0.015327 & 1.911007 \\ \mathrm{C} & 0.070165 & 0.039241 & 1.485406 \\ \mathrm{C} & 0.055080 & 0.042291 & 0.087042 \\ \mathrm{C} & -1.331968 & 0.019705 & -0.325650 \\ \mathrm{C} & 1.255109 & 0.062812 & 2.214886 \\ \mathrm{C} & 2.432922 & 0.094535 & 1.512899 \\ \mathrm{C} & 2.443012 & 0.104302 & 0.111311 \\ \mathrm{C} & 1.270686 & 0.078686 & -0.600505 \\ \mathrm{C} & -1.870381 & 0.000761 & -1.730029 \\ \mathrm{C} & -2.137522 & -1.403345 & -2.295505 \\ \mathrm{~N} & -0.993516 & -2.285823 & -2.416783 \\ \mathrm{H} & 1.249617 & 0.058369 & 3.289637 \\ \mathrm{H} & 3.365792 & 0.113934 & 2.045483 \\ \mathrm{H} & 3.383390 & 0.133522 & -0.406930\end{array}$




$\begin{array}{rrrr}\mathrm{H} & 1.292156 & 0.089460 & -1.674580 \\ \mathrm{H} & -1.528910 & 0.030461 & 2.853834 \\ \mathrm{H} & -3.116410 & 0.008418 & 0.937905 \\ \mathrm{H} & -1.181640 & 0.525233 & -2.386725 \\ \mathrm{H} & -2.802544 & 0.557835 & -1.761978 \\ \mathrm{H} & -2.583962 & -1.298352 & -3.279408 \\ \mathrm{H} & -2.874757 & -1.898019 & -1.672311 \\ \mathrm{H} & -0.557038 & -2.441633 & -1.532007 \\ \mathrm{H} & -0.304510 & -1.909917 & -3.034990\end{array}$

tryptamine, $\mathrm{Ph}$-up $\mathrm{S}_{1}$

$\begin{array}{lrrc}\mathrm{C} & -2.072536 & -0.027096 & 0.821624 \\ \mathrm{~N} & -1.255496 & -0.018843 & 1.888536 \\ \mathrm{C} & 0.068254 & 0.036137 & 1.488124 \\ \mathrm{C} & 0.056772 & 0.064292 & 0.063267 \\ \mathrm{C} & -1.273847 & 0.025511 & -0.350676 \\ \mathrm{C} & 1.213651 & 0.055668 & 2.233212 \\ \mathrm{C} & 2.458852 & 0.117643 & 1.504208 \\ \mathrm{C} & 2.482006 & 0.147242 & 0.127998 \\ \mathrm{C} & 1.305570 & 0.119349 & -0.632486 \\ \mathrm{C} & -1.817730 & 0.012675 & -1.742561 \\ \mathrm{C} & -2.167180 & -1.396065 & -2.263251 \\ \mathrm{~N} & -1.062187 & -2.319474 & -2.404276 \\ \mathrm{H} & 1.206426 & 0.032759 & 3.305921 \\ \mathrm{H} & 3.378536 & 0.139004 & 2.057296 \\ \mathrm{H} & 3.428532 & 0.192888 & -0.377530 \\ \mathrm{H} & 1.341487 & 0.150242 & -1.703953 \\ \mathrm{H} & -1.557645 & -0.038646 & 2.833528 \\ \mathrm{H} & -3.136752 & -0.048773 & 0.919947 \\ \mathrm{H} & -1.100365 & 0.473636 & -2.413447 \\ \mathrm{H} & -2.718798 & 0.621110 & -1.777214 \\ \mathrm{H} & -2.644297 & -1.289286 & -3.231633 \\ \mathrm{H} & -2.901849 & -1.845725 & -1.604005 \\ \mathrm{H} & -0.590706 & -2.467524 & -1.536110 \\ \mathrm{H} & -0.387039 & -1.987116 & -3.061429\end{array}$

tryptamine, Py-out $\mathrm{S}_{0}$

$\begin{array}{lrrr}\mathrm{C} & 0.530159 & 0.447303 & 0.239489 \\ \mathrm{C} & 0.604614 & 0.539816 & 1.632815 \\ \mathrm{C} & 1.817648 & 0.511437 & 2.313272 \\ \mathrm{C} & 2.962660 & 0.391330 & 1.566563 \\ \mathrm{C} & 2.912859 & 0.302085 & 0.169347 \\ \mathrm{C} & 1.711948 & 0.329177 & -0.494552 \\ \mathrm{~N} & -0.672343 & 0.657813 & 2.107157\end{array}$




$\begin{array}{lrrr}\mathrm{H} & 1.859253 & 0.583164 & 3.384877 \\ \mathrm{H} & 3.916122 & 0.367014 & 2.061265 \\ \mathrm{H} & 3.828881 & 0.212427 & -0.384423 \\ \mathrm{H} & 1.686429 & 0.262541 & -1.566674 \\ \mathrm{C} & -0.871071 & 0.520118 & -0.118309 \\ \mathrm{C} & -1.541544 & 0.649261 & 1.042438 \\ \mathrm{H} & -0.930094 & 0.764457 & 3.056739 \\ \mathrm{H} & -2.596092 & 0.738232 & 1.202418 \\ \mathrm{C} & -1.460785 & 0.458438 & -1.498260 \\ \mathrm{H} & -0.912788 & 1.129435 & -2.154063 \\ \mathrm{C} & -1.457013 & -0.938888 & -2.120731 \\ \mathrm{H} & -2.484747 & 0.812569 & -1.464735 \\ \mathrm{H} & -0.430323 & -1.295106 & -2.205321 \\ \mathrm{~N} & -2.310126 & -1.848713 & -1.373415 \\ \mathrm{H} & -1.851460 & -0.863606 & -3.128540 \\ \mathrm{H} & -2.356640 & -2.742080 & -1.817553 \\ \mathrm{H} & -1.955526 & -1.988223 & -0.449783\end{array}$

tryptamine, Py-out $\mathrm{S}_{1}$

$\begin{array}{lrrr}\mathrm{C} & 0.531075 & 0.461432 & 0.217959 \\ \mathrm{C} & 0.608923 & 0.532285 & 1.638165 \\ \mathrm{C} & 1.787664 & 0.508267 & 2.330470 \\ \mathrm{C} & 2.996690 & 0.412575 & 1.543760 \\ \mathrm{C} & 2.954841 & 0.347121 & 0.168720 \\ \mathrm{C} & 1.744007 & 0.367874 & -0.536416 \\ \mathrm{~N} & -0.694351 & 0.618877 & 2.097226 \\ \mathrm{H} & 1.832224 & 0.562703 & 3.401088 \\ \mathrm{H} & 3.941443 & 0.394448 & 2.053104 \\ \mathrm{H} & 3.876444 & 0.279056 & -0.378663 \\ \mathrm{H} & 1.727604 & 0.324526 & -1.608036 \\ \mathrm{C} & -0.817842 & 0.507778 & -0.135123 \\ \mathrm{C} & -1.558598 & 0.601981 & 1.067470 \\ \mathrm{H} & -0.952537 & 0.685507 & 3.052949 \\ \mathrm{H} & -2.616550 & 0.661235 & 1.207388 \\ \mathrm{C} & -1.414525 & 0.456920 & -1.506782 \\ \mathrm{H} & -0.824837 & 1.083023 & -2.169189 \\ \mathrm{C} & -1.477662 & -0.953212 & -2.101710 \\ \mathrm{H} & -2.418809 & 0.865201 & -1.479718 \\ \mathrm{H} & -0.466847 & -1.353631 & -2.171975 \\ \mathrm{~N} & -2.377413 & -1.807538 & -1.343637 \\ \mathrm{H} & -1.864506 & -0.875014 & -3.111673 \\ \mathrm{H} & -2.505839 & -2.685481 & -1.802322 \\ \mathrm{H} & -2.010610 & -1.995944 & -0.433065\end{array}$

tryptamine, Py-up $\mathrm{S}_{0}$ 


$\begin{array}{lrrr}\mathrm{C} & -2.100904 & 0.258932 & 0.902488 \\ \mathrm{~N} & -1.228159 & 0.248627 & 1.964334 \\ \mathrm{C} & 0.044387 & 0.106483 & 1.484836 \\ \mathrm{C} & -0.036683 & 0.017749 & 0.091999 \\ \mathrm{C} & -1.437490 & 0.117881 & -0.261092 \\ \mathrm{C} & 1.259120 & 0.053866 & 2.161153 \\ \mathrm{C} & 2.398607 & -0.086571 & 1.410030 \\ \mathrm{C} & 2.342240 & -0.172469 & 0.012661 \\ \mathrm{C} & 1.140007 & -0.121654 & -0.646810 \\ \mathrm{C} & -2.028312 & 0.067610 & -1.639615 \\ \mathrm{C} & -2.012519 & -1.326532 & -2.287700 \\ \mathrm{~N} & -2.794981 & -2.348198 & -1.619362 \\ \mathrm{H} & 1.305813 & 0.122491 & 3.232719 \\ \mathrm{H} & 3.353216 & -0.130298 & 1.901119 \\ \mathrm{H} & 3.254332 & -0.279259 & -0.544418 \\ \mathrm{H} & 1.109830 & -0.186578 & -1.718878 \\ \mathrm{H} & -1.479738 & 0.360762 & 2.914933 \\ \mathrm{H} & -3.152050 & 0.375393 & 1.068577 \\ \mathrm{H} & -1.490733 & 0.754612 & -2.288880 \\ \mathrm{H} & -3.054314 & 0.423351 & -1.599280 \\ \mathrm{H} & -0.991775 & -1.683936 & -2.358430 \\ \mathrm{H} & -2.378663 & -1.236073 & -3.305117 \\ \mathrm{H} & -3.760205 & -2.092748 & -1.571234 \\ \mathrm{H} & -2.472505 & -2.493819 & -0.685141\end{array}$

tryptamine, Py-up $\mathrm{S}_{1}$

$\begin{array}{rrrr}\mathrm{C} & -2.127658 & 0.238335 & 0.927590 \\ \mathrm{~N} & -1.258450 & 0.234501 & 1.954189 \\ \mathrm{C} & 0.039169 & 0.107255 & 1.489042 \\ \mathrm{C} & -0.047725 & 0.032135 & 0.069100 \\ \mathrm{C} & -1.396290 & 0.114556 & -0.278112 \\ \mathrm{C} & 1.219521 & 0.050739 & 2.175206 \\ \mathrm{C} & 2.422071 & -0.084521 & 1.384523 \\ \mathrm{C} & 2.371763 & -0.152926 & 0.009868 \\ \mathrm{C} & 1.159170 & -0.097547 & -0.689405 \\ \mathrm{C} & -1.998272 & 0.067389 & -1.646203 \\ \mathrm{C} & -2.020432 & -1.339758 & -2.272379 \\ \mathrm{~N} & -2.827556 & -2.331506 & -1.591433 \\ \mathrm{H} & 1.270287 & 0.106436 & 3.245513 \\ \mathrm{H} & 3.367822 & -0.130519 & 1.890050 \\ \mathrm{H} & 3.288092 & -0.250652 & -0.541693 \\ \mathrm{H} & 1.137565 & -0.141972 & -1.760868 \\ \mathrm{H} & -1.509149 & 0.318607 & 2.910461 \\ \mathrm{H} & -3.180740 & 0.347756 & 1.074572\end{array}$




$\begin{array}{llll}\mathrm{H} & -1.435374 & 0.724662 & -2.303398 \\ \mathrm{H} & -3.012607 & 0.455941 & -1.606887 \\ \mathrm{H} & -1.006637 & -1.717433 & -2.333420 \\ \mathrm{H} & -2.383459 & -1.251094 & -3.290616 \\ \mathrm{H} & -3.785009 & -2.051535 & -1.533117 \\ \mathrm{H} & -2.497579 & -2.499218 & -0.663670\end{array}$

porphycene $\mathrm{S}_{0}$

$\begin{array}{lrrr}\mathrm{C} & 2.693304 & -2.865313 & -0.004806 \\ \mathrm{C} & 2.066720 & -1.629182 & -0.001041 \\ \mathrm{~N} & 0.735020 & -1.849623 & -0.000954 \\ \mathrm{C} & 0.468725 & -3.166100 & -0.004886 \\ \mathrm{C} & 1.689611 & -3.833095 & -0.007483 \\ \mathrm{C} & -0.850660 & -3.746147 & -0.008613 \\ \mathrm{C} & -2.080066 & -3.200688 & -0.010267 \\ \mathrm{C} & -2.530238 & -1.821402 & -0.006917 \\ \mathrm{H} & 0.006481 & -1.157697 & 0.002886 \\ \mathrm{~N} & -1.770839 & -0.765058 & 0.003704 \\ \mathrm{C} & -3.932037 & -1.440934 & -0.013463 \\ \mathrm{C} & -3.961890 & -0.093966 & -0.007730 \\ \mathrm{C} & -2.588363 & 0.330941 & 0.006036 \\ \mathrm{C} & -2.051217 & 1.589614 & -0.009494 \\ \mathrm{C} & -2.690312 & 2.898568 & -0.000935 \\ \mathrm{C} & -1.727168 & 3.823691 & 0.004475 \\ \mathrm{C} & -0.432299 & 3.147555 & 0.003653 \\ \mathrm{~N} & -0.723888 & 1.817740 & -0.005326 \\ \mathrm{C} & 0.788788 & 3.731538 & 0.007389 \\ \mathrm{C} & 2.103376 & 3.155622 & 0.006157 \\ \mathrm{C} & 2.540853 & 1.873341 & 0.003680 \\ \mathrm{C} & 3.946504 & 1.472367 & 0.004038 \\ \mathrm{C} & 3.971758 & 0.137013 & 0.002890 \\ \mathrm{C} & 2.571787 & -0.286085 & 0.001999 \\ \mathrm{~N} & 1.770565 & 0.730503 & 0.002463 \\ \mathrm{H} & -0.000017 & 1.118851 & -0.005061 \\ \mathrm{H} & -1.847793 & 4.887317 & 0.010480 \\ \mathrm{H} & -3.747691 & 3.058649 & -0.002861 \\ \mathrm{H} & 0.772501 & 4.805497 & 0.013174 \\ \mathrm{H} & 2.889043 & 3.890346 & 0.007971 \\ \mathrm{H} & 4.780213 & 2.145059 & 0.005111 \\ \mathrm{H} & 4.824204 & -0.509624 & 0.002785 \\ \mathrm{H} & 3.749346 & -3.036711 & -0.005812 \\ \mathrm{H} & 1.819884 & -4.895208 & -0.011193 \\ \mathrm{H} & -0.825030 & -4.821457 & -0.012383 \\ \mathrm{H} & -2.890473 & -3.907884 & -0.015584 \\ \mathrm{H} & -4.764119 & -2.115035 & -0.021813\end{array}$


$\begin{array}{llll}\mathrm{H} & -4.824705 & 0.540230 & -0.009669\end{array}$

porphycene $\mathrm{S}_{1}$

$\begin{array}{rrrr}\mathrm{C} & 2.695503 & -2.885387 & -0.004003 \\ \mathrm{C} & 2.070031 & -1.614288 & -0.002370 \\ \mathrm{~N} & 0.728080 & -1.841668 & -0.005012 \\ \mathrm{C} & 0.458937 & -3.152375 & -0.007953 \\ \mathrm{C} & 1.707165 & -3.830127 & -0.007375 \\ \mathrm{C} & -0.834740 & -3.745531 & -0.010952 \\ \mathrm{C} & -2.079611 & -3.190545 & -0.011496 \\ \mathrm{C} & -2.532819 & -1.833181 & -0.009408 \\ \mathrm{H} & -0.001852 & -1.146407 & -0.004891 \\ \mathrm{~N} & -1.771229 & -0.751648 & -0.006571 \\ \mathrm{C} & -3.930135 & -1.456721 & -0.009935 \\ \mathrm{C} & -3.965426 & -0.106939 & -0.007396 \\ \mathrm{C} & -2.593123 & 0.319332 & -0.005642 \\ \mathrm{C} & -2.072512 & 1.615045 & -0.001294 \\ \mathrm{C} & -2.698026 & 2.886310 & 0.000311 \\ \mathrm{C} & -1.709698 & 3.830895 & 0.003400 \\ \mathrm{C} & -0.461311 & 3.153250 & 0.003384 \\ \mathrm{~N} & -0.730853 & 1.842226 & 0.000579 \\ \mathrm{C} & 0.831792 & 3.745990 & 0.006268 \\ \mathrm{C} & 2.077156 & 3.190879 & 0.006777 \\ \mathrm{C} & 2.529918 & 1.834024 & 0.004961 \\ \mathrm{C} & 3.927634 & 1.457413 & 0.006474 \\ \mathrm{C} & 3.963000 & 0.107925 & 0.003872 \\ \mathrm{C} & 2.590361 & -0.318563 & 0.000682 \\ \mathrm{~N} & 1.768504 & 0.752195 & 0.001447 \\ \mathrm{H} & -0.001260 & 1.146742 & 0.000360 \\ \mathrm{H} & -1.829033 & 4.894304 & 0.005546 \\ \mathrm{H} & -3.753994 & 3.055890 & -0.000487 \\ \mathrm{H} & 0.803407 & 4.820207 & 0.008098 \\ \mathrm{H} & 2.882546 & 3.903741 & 0.009307 \\ \mathrm{H} & 4.757010 & 2.135346 & 0.009143 \\ \mathrm{H} & 4.824306 & -0.527315 & 0.004106 \\ \mathrm{H} & 3.751508 & -3.054801 & -0.002778 \\ \mathrm{H} & 1.826278 & -4.893548 & -0.009281 \\ \mathrm{H} & -0.806070 & -4.819730 & -0.012850 \\ \mathrm{H} & -2.885021 & -3.903415 & -0.013772 \\ \mathrm{H} & -4.759696 & -2.134436 & -0.011889 \\ \mathrm{H} & -4.826851 & 0.528146 & -0.006759\end{array}$

porphine $\mathrm{S}_{0}$

$\begin{array}{llll}\mathrm{N} & -0.000001 & 2.042314 & 0.023541\end{array}$ 


$\begin{array}{rrrr}\mathrm{N} & 0.000000 & 0.000000 & 2.089283 \\ \mathrm{C} & 0.000001 & 2.828326 & 1.056648 \\ \mathrm{C} & 0.000005 & 4.241536 & 0.664795 \\ \mathrm{C} & -0.000002 & 2.405611 & 2.427989 \\ \mathrm{C} & -0.000002 & 1.128736 & 2.854355 \\ \mathrm{C} & -0.000005 & 0.664705 & 4.241744 \\ \mathrm{H} & 0.000007 & 5.072830 & 1.339905 \\ \mathrm{H} & -0.000004 & 3.174081 & 3.176654 \\ \mathrm{H} & -0.000007 & 1.320750 & 5.087397 \\ \mathrm{H} & 0.000002 & 0.000000 & 1.097020 \\ \mathrm{~N} & -0.000001 & -2.042314 & 0.023541 \\ \mathrm{~N} & 0.000004 & 0.000000 & -2.099306 \\ \mathrm{C} & 0.000001 & -2.828326 & 1.056648 \\ \mathrm{C} & 0.000002 & -2.859196 & -1.094559 \\ \mathrm{C} & 0.000002 & 2.859196 & -1.094559 \\ \mathrm{C} & 0.000005 & -4.241536 & 0.664795 \\ \mathrm{C} & 0.000006 & -4.254021 & -0.667305 \\ \mathrm{C} & 0.000006 & 4.254021 & -0.667305 \\ \mathrm{C} & -0.000002 & -2.405611 & 2.427989 \\ \mathrm{C} & 0.000002 & -2.449038 & -2.377176 \\ \mathrm{C} & 0.000002 & 2.449038 & -2.377176 \\ \mathrm{C} & -0.000002 & -1.128736 & 2.854355 \\ \mathrm{C} & -0.000001 & -1.110260 & -2.871546 \\ \mathrm{C} & -0.000001 & 1.110260 & -2.871546 \\ \mathrm{C} & -0.000005 & -0.664705 & 4.241744 \\ \mathrm{C} & -0.000010 & -0.695568 & -4.195676 \\ \mathrm{C} & -0.000010 & 0.695568 & -4.195676 \\ \mathrm{H} & 0.000007 & -5.072830 & 1.339905 \\ \mathrm{H} & 0.000009 & -5.100349 & -1.324015 \\ \mathrm{H} & 0.000009 & 5.100349 & -1.324015 \\ \mathrm{H} & -0.000004 & -3.174081 & 3.176654 \\ \mathrm{H} & 0.000005 & -3.210066 & -3.135666 \\ \mathrm{H} & 0.000005 & 3.210066 & -3.135666 \\ \mathrm{H} & -0.000007 & -1.320750 & 5.087397 \\ \mathrm{H} & -0.000015 & -1.343120 & -5.047561 \\ \mathrm{H} & -0.000015 & 1.343120 & -5.047561 \\ \mathrm{H} & 0.000011 & 0.000000 & -1.106047\end{array}$

porphine $\mathrm{S}_{1}$

$\begin{array}{lccc}\mathrm{N} & 0.000054 & 2.041297 & -0.002699 \\ \mathrm{~N} & -0.000046 & -0.000004 & 2.091690 \\ \mathrm{C} & 0.000012 & 2.844784 & 1.072619 \\ \mathrm{C} & 0.000047 & 4.246404 & 0.664600 \\ \mathrm{C} & 0.000036 & 2.429051 & 2.399730 \\ \mathrm{C} & 0.000017 & 1.119249 & 2.860882\end{array}$




$\begin{array}{rrrr}\mathrm{C} & -0.000003 & 0.679450 & 4.216848 \\ \mathrm{H} & 0.000314 & 5.084797 & 1.330910 \\ \mathrm{H} & 0.000136 & 3.193311 & 3.153288 \\ \mathrm{H} & -0.000061 & 1.331464 & 5.065287 \\ \mathrm{H} & -0.000167 & -0.000159 & 1.098371 \\ \mathrm{~N} & -0.000006 & -2.041301 & -0.002670 \\ \mathrm{~N} & 0.000164 & -0.000033 & -2.097258 \\ \mathrm{C} & -0.000044 & -2.844705 & 1.072636 \\ \mathrm{C} & -0.000014 & -2.844688 & -1.078097 \\ \mathrm{C} & 0.000015 & 2.844708 & -1.078054 \\ \mathrm{C} & -0.000052 & -4.246274 & 0.664672 \\ \mathrm{C} & 0.000010 & -4.246251 & -0.670201 \\ \mathrm{C} & -0.000131 & 4.246376 & -0.670199 \\ \mathrm{C} & -0.000064 & -2.428952 & 2.399838 \\ \mathrm{C} & -0.000012 & -2.428970 & -2.405285 \\ \mathrm{C} & -0.000003 & 2.429004 & -2.405207 \\ \mathrm{C} & 0.000013 & -1.119300 & 2.861081 \\ \mathrm{C} & 0.000011 & -1.119310 & -2.866621 \\ \mathrm{C} & 0.000018 & 1.119293 & -2.866460 \\ \mathrm{C} & 0.000018 & -0.679327 & 4.216947 \\ \mathrm{C} & -0.000061 & -0.679361 & -4.222506 \\ \mathrm{C} & 0.000007 & 0.679456 & -4.222402 \\ \mathrm{H} & -0.000094 & -5.084998 & 1.330569 \\ \mathrm{H} & 0.000074 & -5.084993 & -1.336104 \\ \mathrm{H} & -0.000442 & 5.084761 & -1.336543 \\ \mathrm{H} & -0.000262 & -3.193464 & 3.153146 \\ \mathrm{H} & 0.000053 & -3.193672 & -3.158408 \\ \mathrm{H} & 0.000034 & 3.193486 & -3.158554 \\ \mathrm{H} & 0.000021 & -1.331181 & 5.065513 \\ \mathrm{H} & -0.000143 & -1.331556 & -5.070807 \\ \mathrm{H} & 0.000142 & 1.331783 & -5.070605 \\ \mathrm{H} & 0.000407 & -0.000175 & -1.103946\end{array}$

chlorin $\mathrm{S}_{0}$

$\begin{array}{rrrr}\mathrm{C} & 0.000000 & 4.236937 & 0.732878 \\ \mathrm{C} & 0.000000 & 2.855212 & 1.209525 \\ \mathrm{~N} & 0.000000 & 2.078559 & 0.073814 \\ \mathrm{C} & 0.000000 & 2.833890 & -1.050294 \\ \mathrm{C} & 0.000000 & 4.227526 & -0.595589 \\ \mathrm{C} & 0.000000 & 2.436490 & 2.480368 \\ \mathrm{C} & 0.000000 & 1.059708 & 2.917346 \\ \mathrm{~N} & 0.000000 & 0.030837 & 2.125441 \\ \mathrm{C} & 0.000000 & -1.088571 & 2.938465 \\ \mathrm{C} & 0.000000 & -0.670963 & 4.325197 \\ \mathrm{C} & 0.000000 & 0.668192 & 4.319290\end{array}$




$\begin{array}{rrrr}\mathrm{C} & 0.000000 & -2.373527 & 2.512413 \\ \mathrm{C} & 0.000000 & -2.865591 & 1.185520 \\ \mathrm{~N} & 0.000000 & -2.092758 & 0.057725 \\ \mathrm{C} & 0.000000 & -2.865535 & -1.040934 \\ \mathrm{C} & 0.000000 & -4.189610 & -0.623408 \\ \mathrm{C} & 0.000000 & -4.185360 & 0.769102 \\ \mathrm{C} & 0.000000 & -2.384596 & -2.401244 \\ \mathrm{C} & 0.000000 & -1.126842 & -2.845181 \\ \mathrm{C} & 0.000000 & -0.755094 & -4.320351 \\ \mathrm{C} & 0.000000 & 0.774552 & -4.281953 \\ \mathrm{C} & 0.000000 & 1.063396 & -2.794841 \\ \mathrm{~N} & 0.000000 & 0.016071 & -2.052804 \\ \mathrm{C} & 0.000000 & 2.419049 & -2.336801 \\ \mathrm{H} & 0.873747 & -1.153303 & -4.820031 \\ \mathrm{H} & 0.000000 & -3.163832 & -3.141468 \\ \mathrm{H} & 0.000000 & -5.042093 & -1.269569 \\ \mathrm{H} & 0.000000 & -1.329091 & 5.170707 \\ \mathrm{H} & 0.000000 & 1.337886 & 5.155004 \\ \mathrm{H} & 0.873121 & 1.213998 & -4.750431 \\ \mathrm{H} & 0.000000 & -3.134138 & 3.272242 \\ \mathrm{H} & 0.000000 & 3.189702 & 3.245081 \\ \mathrm{H} & 0.000000 & 3.182919 & -3.090369 \\ \mathrm{H} & 0.000000 & -5.037027 & 1.417859 \\ \mathrm{H} & 0.000000 & 5.088918 & 1.381023 \\ \mathrm{H} & 0.000000 & 5.067246 & -1.258948 \\ \mathrm{H} & -0.873121 & 1.213998 & -4.750431 \\ \mathrm{H} & -0.873748 & -1.153303 & -4.820031 \\ \mathrm{H} & 0.000000 & 1.086976 & 0.089271 \\ \mathrm{H} & 0.000000 & -1.101228 & 0.056114 \\ & & & \end{array}$

chlorin $\mathrm{S}_{1}$

$\begin{array}{lrrr}\mathrm{C} & 0.000212 & 4.202302 & 0.742931 \\ \mathrm{C} & 0.000276 & 2.851816 & 1.190864 \\ \mathrm{~N} & 0.000064 & 2.075799 & 0.061848 \\ \mathrm{C} & -0.000046 & 2.838515 & -1.051235 \\ \mathrm{C} & 0.000162 & 4.197542 & -0.616607 \\ \mathrm{C} & 0.000364 & 2.395111 & 2.494751 \\ \mathrm{C} & 0.000275 & 1.065828 & 2.917674 \\ \mathrm{~N} & 0.000113 & -0.009624 & 2.114977 \\ \mathrm{C} & 0.000033 & -1.082887 & 2.920492 \\ \mathrm{C} & -0.000116 & -0.675656 & 4.317657 \\ \mathrm{C} & 0.000222 & 0.662266 & 4.315875 \\ \mathrm{C} & -0.000174 & -2.413282 & 2.501095 \\ \mathrm{C} & -0.000226 & -2.873386 & 1.198409 \\ \mathrm{~N} & -0.000097 & -2.100559 & 0.067230\end{array}$




$\begin{array}{rrrr}\text { C } & -0.000096 & -2.866346 & -1.043704 \\ \mathrm{C} & -0.000276 & -4.224213 & -0.605282 \\ \mathrm{C} & -0.000419 & -4.225136 & 0.754236 \\ \mathrm{C} & 0.000149 & -2.417480 & -2.368251 \\ \mathrm{C} & 0.000318 & -1.110328 & -2.809767 \\ \mathrm{C} & 0.001029 & -0.782260 & -4.289818 \\ \mathrm{C} & -0.000876 & 0.745801 & -4.291813 \\ \mathrm{C} & -0.000265 & 1.077729 & -2.812612 \\ \mathrm{~N} & 0.000026 & -0.015317 & -2.041908 \\ \mathrm{C} & -0.000237 & 2.386014 & -2.374558 \\ \mathrm{H} & 0.875490 & -1.202348 & -4.772155 \\ \mathrm{H} & 0.000234 & -3.186286 & -3.117252 \\ \mathrm{H} & -0.000257 & -5.070787 & -1.259446 \\ \mathrm{H} & -0.000540 & -1.340144 & 5.157590 \\ \mathrm{H} & 0.000426 & 1.328955 & 5.154067 \\ \mathrm{H} & 0.871525 & 1.167011 & -4.776858 \\ \mathrm{H} & -0.000266 & -3.166509 & 3.266059 \\ \mathrm{H} & 0.000434 & 3.150280 & 3.257806 \\ \mathrm{H} & -0.000486 & 3.152824 & -3.125611 \\ \mathrm{H} & -0.000673 & -5.075337 & 1.404531 \\ \mathrm{H} & 0.000081 & 5.054311 & 1.390852 \\ \mathrm{H} & 0.000334 & 5.042256 & -1.273177 \\ \mathrm{H} & -0.875311 & 1.164614 & -4.775308 \\ \mathrm{H} & -0.871331 & -1.204753 & -4.773820 \\ \mathrm{H} & -0.000064 & 1.083332 & 0.070471 \\ \mathrm{H} & -0.000014 & -1.108070 & 0.072957\end{array}$

Zn-tetraphenylporphine $\mathrm{S}_{0}$

$\begin{array}{rrrr}\text { C } & -3.993804 & -3.868162 & 1.093851 \\ \mathrm{C} & -3.477445 & -3.381828 & -0.098931 \\ \mathrm{C} & -3.963839 & -3.901804 & -1.289958 \\ \mathrm{C} & -4.943191 & -4.879008 & -1.290596 \\ \mathrm{C} & -5.451288 & -5.353436 & -0.095830 \\ \mathrm{C} & -4.973127 & -4.845217 & 1.097526 \\ \mathrm{C} & -2.411301 & -2.320942 & -0.100723 \\ \mathrm{C} & -2.797102 & -1.003893 & -0.129261 \\ \mathrm{~N} & -1.990555 & 0.089228 & -0.136279 \\ \mathrm{C} & -2.780867 & 1.196890 & -0.165847 \\ \mathrm{C} & -4.182837 & 0.769931 & -0.178622 \\ \mathrm{C} & -4.192847 & -0.556877 & -0.156701 \\ \mathrm{C} & -2.374554 & 2.505568 & -0.181984 \\ \mathrm{C} & -3.423191 & 3.583272 & -0.214533 \\ \mathrm{C} & -3.898714 & 4.074911 & -1.421834 \\ \mathrm{C} & -4.861390 & 5.067950 & -1.453930 \\ \mathrm{C} & -5.363165 & 5.586834 & -0.275031\end{array}$




$\begin{array}{rrrr}\mathrm{C} & -4.895766 & 5.106765 & 0.934081 \\ \mathrm{C} & -3.932763 & 4.113939 & 0.961987 \\ \mathrm{C} & -1.057555 & -2.757507 & -0.070863 \\ \mathrm{~N} & 0.017070 & -1.979853 & -0.067042 \\ \mathrm{C} & 1.131041 & -2.796360 & -0.033684 \\ \mathrm{C} & 0.680728 & -4.169799 & -0.015831 \\ \mathrm{C} & -0.657480 & -4.151320 & -0.038981 \\ \mathrm{C} & 2.434357 & -2.387521 & -0.020725 \\ \mathrm{C} & 3.498235 & -3.449760 & 0.015871 \\ \mathrm{C} & 4.014414 & -3.970769 & -1.160350 \\ \mathrm{C} & 4.992794 & -4.947862 & -1.127594 \\ \mathrm{C} & 5.468745 & -5.413938 & 0.083619 \\ \mathrm{C} & 4.960853 & -4.897512 & 1.260972 \\ \mathrm{C} & 3.982639 & -3.920327 & 1.226379 \\ \mathrm{C} & -1.012097 & 2.923624 & -0.169151 \\ \mathrm{~N} & 0.049591 & 2.129411 & -0.140643 \\ \mathrm{C} & 1.175818 & 2.928870 & -0.138126 \\ \mathrm{C} & 0.748513 & 4.307238 & -0.167754 \\ \mathrm{C} & -0.591325 & 4.309332 & -0.186688 \\ \mathrm{C} & 2.474029 & 2.498305 & -0.112224 \\ \mathrm{C} & 3.553916 & 3.544944 & -0.116403 \\ \mathrm{C} & 4.075394 & 4.013940 & -1.311931 \\ \mathrm{C} & 5.068574 & 4.976528 & -1.316765 \\ \mathrm{C} & 5.553745 & 5.480014 & -0.124345 \\ \mathrm{C} & 5.040275 & 5.015545 & 1.072096 \\ \mathrm{C} & 4.047157 & 4.052923 & 1.075215 \\ \mathrm{C} & 2.906616 & 1.142126 & -0.081192 \\ \mathrm{~N} & 2.095554 & 0.058347 & -0.071209 \\ \mathrm{C} & 2.888683 & -1.035513 & -0.039793 \\ \mathrm{C} & 4.246663 & -0.639116 & -0.029572 \\ \mathrm{C} & 4.257626 & 0.723930 & -0.055742 \\ \mathrm{H} & 5.113763 & 1.363045 & -0.057287 \\ \mathrm{H} & 1.398646 & 5.155535 & -0.173137 \\ \mathrm{H} & -5.022989 & 1.429676 & -0.201394 \\ \mathrm{H} & -5.042679 & -1.204457 & -0.157695 \\ \mathrm{H} & 5.092153 & -1.291760 & -0.006006 \\ \mathrm{H} & -1.243145 & 5.155048 & -0.210186 \\ \mathrm{H} & -1.323048 & -4.986601 & -0.035191 \\ \mathrm{H} & 1.317692 & -5.027640 & 0.010635 \\ \mathrm{H} & -3.513041 & 3.676421 & -2.342261 \\ \mathrm{H} & -5.218009 & 5.434640 & -2.398992 \\ \mathrm{H} & -6.111318 & 6.357592 & -0.298300 \\ \mathrm{H} & -5.279522 & 5.503524 & 1.856090 \\ \mathrm{H} & -3.573241 & 3.746425 & 1.905596 \\ \mathrm{H} & 3.702948 & 3.624815 & -2.241528 \\ \mathrm{H} & 5.461733 & 5.331429 & -2.251642\end{array}$




$\begin{array}{crrc}\mathrm{H} & 6.325588 & 6.227315 & -0.127422 \\ \mathrm{H} & 5.411373 & 5.400929 & 2.003917 \\ \mathrm{H} & 3.652606 & 3.694268 & 2.007941 \\ \mathrm{H} & 3.649451 & -3.610622 & -2.104489 \\ \mathrm{H} & 5.381633 & -5.343311 & -2.047898 \\ \mathrm{H} & 6.228992 & -6.172592 & 0.109762 \\ \mathrm{H} & 5.324539 & -5.253802 & 2.207188 \\ \mathrm{H} & 3.592972 & -3.520791 & 2.144437 \\ \mathrm{H} & -3.573422 & -3.537853 & -2.222618 \\ \mathrm{H} & -5.307482 & -5.268314 & -2.223621 \\ \mathrm{H} & -6.212639 & -6.111516 & -0.094654 \\ \mathrm{H} & -5.361103 & -5.207931 & 2.031721 \\ \mathrm{H} & -3.626666 & -3.477759 & 2.025286 \\ \mathrm{Zn} & 0.048469 & 0.074087 & -0.103258\end{array}$

Zn-tetraphenylporphine $\mathrm{S}_{1}$

$\begin{array}{lrrr}\mathrm{C} & -3.994186 & -3.991565 & 1.193058 \\ \mathrm{C} & -3.492014 & -3.493210 & -0.000028 \\ \mathrm{C} & -3.994192 & -3.991684 & -1.193060 \\ \mathrm{C} & -4.974370 & -4.967858 & -1.194496 \\ \mathrm{C} & -5.467148 & -5.458762 & 0.000075 \\ \mathrm{C} & -4.974359 & -4.967744 & 1.194595 \\ \mathrm{C} & -2.427179 & -2.433447 & -0.000084 \\ \mathrm{C} & -2.851542 & -1.093644 & -0.000091 \\ \mathrm{~N} & -2.049390 & 0.000806 & -0.000092 \\ \mathrm{C} & -2.850369 & 1.096168 & -0.000051 \\ \mathrm{C} & -4.226596 & 0.675547 & -0.000041 \\ \mathrm{C} & -4.227307 & -0.671554 & -0.000049 \\ \mathrm{C} & -2.424578 & 2.435445 & -0.000025 \\ \mathrm{C} & -3.488242 & 3.496382 & 0.000007 \\ \mathrm{C} & -3.989883 & 3.995359 & -1.193039 \\ \mathrm{C} & -4.969016 & 4.972582 & -1.194496 \\ \mathrm{C} & -5.461247 & 5.464061 & 0.000064 \\ \mathrm{C} & -4.969017 & 4.972511 & 1.194597 \\ \mathrm{C} & -3.989882 & 3.995292 & 1.193084 \\ \mathrm{C} & -1.097832 & -2.853120 & -0.000094 \\ \mathrm{~N} & -0.001152 & -2.053295 & -0.000121 \\ \mathrm{C} & 1.094712 & -2.854332 & -0.000105 \\ \mathrm{C} & 0.667173 & -4.239148 & -0.000051 \\ \mathrm{C} & -0.671824 & -4.238410 & -0.000061 \\ \mathrm{C} & 2.424457 & -2.436108 & -0.000103 \\ \mathrm{C} & 3.488160 & -3.497005 & -0.000034 \\ \mathrm{C} & 3.989803 & -3.996024 & -1.193062 \\ \mathrm{C} & 4.969041 & -4.973141 & -1.194484 \\ \mathrm{C} & 5.461362 & -5.464487 & 0.000093\end{array}$




$\begin{array}{rrrr}\mathrm{C} & 4.969079 & -4.972949 & 1.194608 \\ \mathrm{C} & 3.989837 & -3.995835 & 1.193059 \\ \mathrm{C} & -1.094718 & 2.853732 & -0.000028 \\ \mathrm{~N} & 0.001076 & 2.052754 & -0.000066 \\ \mathrm{C} & 1.097774 & 2.852563 & -0.000025 \\ \mathrm{C} & 0.671796 & 4.237799 & 0.000021 \\ \mathrm{C} & -0.667261 & 4.238511 & 0.000022 \\ \mathrm{C} & 2.427139 & 2.432841 & -0.000027 \\ \mathrm{C} & 3.491972 & 3.492603 & 0.000005 \\ \mathrm{C} & 3.994205 & 3.990979 & -1.193042 \\ \mathrm{C} & 4.974568 & 4.966968 & -1.194498 \\ \mathrm{C} & 5.467414 & 5.457829 & 0.000063 \\ \mathrm{C} & 4.974535 & 4.966930 & 1.194595 \\ \mathrm{C} & 3.994176 & 3.990939 & 1.193083 \\ \mathrm{C} & 2.851516 & 1.093074 & -0.000048 \\ \mathrm{~N} & 2.049352 & -0.001416 & -0.000100 \\ \mathrm{C} & 2.850313 & -1.096721 & -0.000129 \\ \mathrm{C} & 4.226495 & -0.676153 & -0.000044 \\ \mathrm{C} & 4.227251 & 0.670982 & -0.000081 \\ \mathrm{H} & 5.075064 & 1.321011 & -0.000201 \\ \mathrm{H} & 1.323906 & 5.083967 & 0.000058 \\ \mathrm{H} & -5.073696 & 1.326503 & -0.000043 \\ \mathrm{H} & -5.075128 & -1.321574 & -0.000006 \\ \mathrm{H} & 5.073618 & -1.327087 & 0.000113 \\ \mathrm{H} & -1.318468 & 5.085377 & 0.000057 \\ \mathrm{H} & -1.324271 & -5.084317 & -0.000051 \\ \mathrm{H} & 1.318687 & -5.085773 & 0.000008 \\ \mathrm{H} & -3.611692 & 3.616790 & -2.124796 \\ \mathrm{H} & -5.345724 & 5.348274 & -2.128097 \\ \mathrm{H} & -6.221626 & 6.223061 & 0.000087 \\ \mathrm{H} & -5.345732 & 5.348143 & 2.128219 \\ \mathrm{H} & -3.611683 & 3.616676 & 2.124818 \\ \mathrm{H} & 3.615470 & 3.612957 & -2.124800 \\ \mathrm{H} & 5.351765 & 5.342171 & -2.128099 \\ \mathrm{H} & 6.228795 & 6.215824 & 0.000086 \\ \mathrm{H} & 5.351703 & 5.342108 & 2.128218 \\ \mathrm{H} & 3.615421 & 3.612881 & 2.124817 \\ \mathrm{H} & 3.611508 & -3.617595 & -2.124833 \\ \mathrm{H} & 5.345813 & -5.348803 & -2.128073 \\ \mathrm{H} & 6.221871 & -6.223357 & 0.000143 \\ \mathrm{H} & 5.345885 & -5.348456 & 2.128245 \\ \mathrm{H} & 3.611567 & -3.617262 & 2.124781 \\ \mathrm{H} & -3.615612 & -3.613532 & -2.124828 \\ \mathrm{H} & -5.351496 & -5.343152 & -2.128090 \\ \mathrm{H} & -6.228389 & -6.216897 & 0.000116 \\ \mathrm{H} & -5.351471 & -5.342954 & 2.128228\end{array}$




$$
\begin{array}{cccc}
\mathrm{H} & -3.615609 & -3.613314 & 2.124786 \\
\mathrm{Zn} & -0.000017 & -0.000581 & -0.000114
\end{array}
$$

tetraphenylporphine $\mathrm{S}_{0}$

$\begin{array}{lrrr}\mathrm{C} & 0.238368 & 4.740425 & -0.121451 \\ \mathrm{C} & -0.309614 & 3.399101 & -0.336874 \\ \mathrm{~N} & 0.739952 & 2.541011 & -0.146940 \\ \mathrm{C} & 1.891504 & 3.212075 & 0.165502 \\ \mathrm{C} & 1.527196 & 4.630932 & 0.172670 \\ \mathrm{C} & 3.115810 & 2.702290 & 0.417097 \\ \mathrm{C} & 3.427307 & 1.287230 & 0.397428 \\ \mathrm{~N} & 2.595364 & 0.323091 & 0.142676 \\ \mathrm{C} & 3.320041 & -0.861851 & 0.233845 \\ \mathrm{C} & 4.703976 & -0.542905 & 0.573697 \\ \mathrm{C} & 4.777042 & 0.780313 & 0.675926 \\ \mathrm{C} & 2.847094 & -2.113322 & 0.043308 \\ \mathrm{C} & 1.493629 & -2.472155 & -0.294369 \\ \mathrm{~N} & 0.464840 & -1.608019 & -0.483873 \\ \mathrm{C} & -0.668949 & -2.288393 & -0.788322 \\ \mathrm{C} & -0.351783 & -3.637465 & -0.793562 \\ \mathrm{C} & 0.997976 & -3.752120 & -0.485604 \\ \mathrm{C} & -1.958817 & -1.705029 & -1.054140 \\ \mathrm{C} & -2.260497 & -0.387848 & -1.039776 \\ \mathrm{~N} & -1.387821 & 0.661555 & -0.766433 \\ \mathrm{C} & -2.081888 & 1.755218 & -0.860224 \\ \mathrm{C} & -3.481921 & 1.481577 & -1.208590 \\ \mathrm{C} & -3.584878 & 0.160905 & -1.317894 \\ \mathrm{C} & -1.587025 & 3.101787 & -0.656102 \\ \mathrm{H} & -4.454926 & -0.410262 & -1.560943 \\ \mathrm{C} & -3.049202 & -2.689607 & -1.373697 \\ \mathrm{H} & -1.030724 & -4.435809 & -0.997927 \\ \mathrm{H} & 0.531672 & -0.620360 & -0.408687 \\ \mathrm{H} & 5.489886 & -1.254533 & 0.708702 \\ \mathrm{H} & 5.632365 & 1.375624 & 0.911044 \\ \mathrm{H} & -4.247597 & 2.214367 & -1.342800 \\ \mathrm{C} & 3.794799 & -3.271073 & 0.191264 \\ \mathrm{C} & 4.233275 & 3.655620 & 0.737036 \\ \mathrm{C} & -2.564796 & 4.232920 & -0.812232 \\ \mathrm{H} & 1.561134 & -4.655903 & -0.407409 \\ \mathrm{H} & 2.213792 & 5.421453 & 0.384353 \\ \mathrm{H} & -0.335786 & 5.638048 & -0.197436 \\ \mathrm{H} & 0.674005 & 1.554799 & -0.225617 \\ \mathrm{C} & 5.5466075 & -4.817056 & -0.769166 \\ & & -5.414614 & 0.464316\end{array}$




$\begin{array}{lrrr}\mathrm{C} & 4.849167 & -4.942774 & 1.560645 \\ \mathrm{C} & 3.976889 & -3.878158 & 1.423758 \\ \mathrm{H} & 4.355947 & -3.291644 & -1.865272 \\ \mathrm{H} & 5.904235 & -5.177208 & -1.626610 \\ \mathrm{H} & 6.224864 & -6.240949 & 0.570268 \\ \mathrm{H} & 4.982894 & -5.401632 & 2.522982 \\ \mathrm{H} & 3.436265 & -3.515311 & 2.278387 \\ \mathrm{C} & -3.271578 & -3.093441 & -2.680847 \\ \mathrm{C} & -4.274482 & -3.999027 & -2.975433 \\ \mathrm{C} & -5.063809 & -4.514484 & -1.964418 \\ \mathrm{C} & -4.844394 & -4.120260 & -0.657942 \\ \mathrm{C} & -3.841094 & -3.214511 & -0.364570 \\ \mathrm{H} & -2.660106 & -2.695966 & -3.469833 \\ \mathrm{H} & -4.438318 & -4.299928 & -3.993846 \\ \mathrm{H} & -5.843185 & -5.217767 & -2.192885 \\ \mathrm{H} & -5.452768 & -4.516405 & 0.134200 \\ \mathrm{H} & -3.673026 & -2.912095 & 0.652608 \\ \mathrm{C} & -2.757309 & 4.839336 & -2.045406 \\ \mathrm{C} & -3.660345 & 5.877077 & -2.192089 \\ \mathrm{C} & -4.389085 & 6.321997 & -1.104910 \\ \mathrm{C} & -4.207891 & 5.724002 & 0.128192 \\ \mathrm{C} & -3.303176 & 4.687204 & 0.271234 \\ \mathrm{H} & -2.196207 & 4.496273 & -2.895269 \\ \mathrm{H} & -3.793011 & 6.336714 & -3.154326 \\ \mathrm{H} & -5.091896 & 7.126772 & -1.217690 \\ \mathrm{H} & -4.769736 & 6.062847 & 0.979237 \\ \mathrm{H} & -3.167500 & 4.226356 & 1.232484 \\ \mathrm{C} & 5.031570 & 4.177071 & -0.271089 \\ \mathrm{C} & 6.063673 & 5.050491 & 0.021826 \\ \mathrm{C} & 6.314899 & 5.413802 & 1.331743 \\ \mathrm{C} & 5.528130 & 4.898735 & 2.344986 \\ \mathrm{C} & 4.496742 & 4.025788 & 2.048169 \\ \mathrm{H} & 4.842931 & 3.897079 & -1.291292 \\ \mathrm{H} & 6.669659 & 5.445700 & -0.772746 \\ \mathrm{H} & 7.116328 & 6.091715 & 1.560812 \\ \mathrm{H} & 5.715743 & 5.175092 & 3.366352 \\ \mathrm{H} & 3.889810 & 3.627993 & 2.840739\end{array}$

tetraphenylporphine $\mathrm{S}_{1}$

$\begin{array}{lrrr}\mathrm{C} & -0.677738 & 4.212363 & 0.000219 \\ \mathrm{C} & -1.122135 & 2.857072 & 0.000110 \\ \mathrm{~N} & 0.000099 & 2.084468 & 0.000049 \\ \mathrm{C} & 1.122126 & 2.856993 & 0.000146 \\ \mathrm{C} & 0.677867 & 4.212357 & 0.000249 \\ \mathrm{C} & 2.446583 & 2.416638 & 0.000106\end{array}$




$\begin{array}{rrrr}\mathrm{C} & 2.853458 & 1.077393 & 0.000044 \\ \mathrm{~N} & 2.048154 & -0.000776 & 0.000081 \\ \mathrm{C} & 2.853450 & -1.078998 & 0.000020 \\ \mathrm{C} & 4.254145 & -0.667174 & -0.000081 \\ \mathrm{C} & 4.254145 & 0.665544 & -0.000112 \\ \mathrm{C} & 2.446563 & -2.418153 & 0.000086 \\ \mathrm{C} & 1.122102 & -2.858537 & 0.000179 \\ \mathrm{~N} & 0.000072 & -2.086054 & 0.000081 \\ \mathrm{C} & -1.122177 & -2.858627 & 0.000206 \\ \mathrm{C} & -0.677795 & -4.213863 & 0.000431 \\ \mathrm{C} & 0.677856 & -4.213855 & 0.000407 \\ \mathrm{C} & -2.446446 & -2.418227 & 0.000134 \\ \mathrm{C} & -2.853518 & -1.078927 & 0.000044 \\ \mathrm{~N} & -2.048130 & -0.000774 & 0.000101 \\ \mathrm{C} & -2.853459 & 1.077376 & 0.000002 \\ \mathrm{C} & -4.253989 & 0.665664 & -0.000185 \\ \mathrm{C} & -4.254034 & -0.667170 & -0.000121 \\ \mathrm{C} & -2.446358 & 2.416701 & 0.000038 \\ \mathrm{H} & -5.097063 & -1.323498 & -0.000184 \\ \mathrm{C} & -3.507496 & -3.481551 & 0.000090 \\ \mathrm{H} & -1.322466 & -5.065111 & 0.000584 \\ \mathrm{H} & 0.000067 & -1.093308 & -0.000064 \\ \mathrm{H} & 5.097035 & -1.323682 & -0.000106 \\ \mathrm{H} & 5.097089 & 1.321980 & -0.000275 \\ \mathrm{H} & -5.097034 & 1.321968 & -0.000377 \\ \mathrm{C} & 3.507487 & -3.481574 & -0.000055 \\ \mathrm{C} & 3.507551 & 3.480028 & 0.000085 \\ \mathrm{C} & -3.507414 & 3.480022 & -0.000047 \\ \mathrm{H} & 1.322590 & -5.065046 & 0.000535 \\ \mathrm{H} & 1.322891 & 5.063325 & 0.000338 \\ \mathrm{H} & -1.322699 & 5.063388 & 0.000266 \\ \mathrm{H} & 0.000096 & 1.091723 & -0.000042 \\ \mathrm{C} & 4.006015 & -3.982115 & -1.193557 \\ \mathrm{C} & 4.984059 & -4.960377 & -1.194966 \\ \mathrm{C} & 5.475995 & -5.451982 & -0.000305 \\ \mathrm{C} & 4.984489 & -4.960249 & 1.194482 \\ \mathrm{C} & 4.006446 & -3.981986 & 1.193321 \\ \mathrm{H} & 3.627887 & -3.602582 & -2.124925 \\ \mathrm{H} & 5.360508 & -5.336408 & -2.128509 \\ \mathrm{H} & 6.235886 & -6.211471 & -0.000400 \\ \mathrm{H} & 5.361264 & -5.336190 & 2.127929 \\ \mathrm{H} & 3.628645 & -3.602362 & 2.124784 \\ \mathrm{C} & -4.006077 & -3.982104 & -1.193378 \\ \mathrm{C} & -4.984231 & -4.960257 & -1.194696 \\ \mathrm{C} & -5.476161 & -5.451758 & 0.000009 \\ \mathrm{C} & -4.984621 & -4.959964 & 1.194754\end{array}$




$\begin{array}{lrrr}\mathrm{C} & -4.006462 & -3.981816 & 1.193517 \\ \mathrm{H} & -3.627911 & -3.602680 & -2.124774 \\ \mathrm{H} & -5.360748 & -5.336304 & -2.128204 \\ \mathrm{H} & -6.236056 & -6.211243 & -0.000022 \\ \mathrm{H} & -5.361450 & -5.335775 & 2.128231 \\ \mathrm{H} & -3.628571 & -3.602194 & 2.124944 \\ \mathrm{C} & -4.006107 & 3.980421 & -1.193534 \\ \mathrm{C} & -4.984590 & 4.958246 & -1.194892 \\ \mathrm{C} & -5.476694 & 5.449622 & -0.000208 \\ \mathrm{C} & -4.985008 & 4.958022 & 1.194557 \\ \mathrm{C} & -4.006507 & 3.980214 & 1.193358 \\ \mathrm{H} & -3.627805 & 3.601099 & -2.124915 \\ \mathrm{H} & -5.361109 & 5.334252 & -2.128417 \\ \mathrm{H} & -6.236765 & 6.208931 & -0.000270 \\ \mathrm{H} & -5.361884 & 5.333823 & 2.128019 \\ \mathrm{H} & -3.628483 & 3.600765 & 2.124801 \\ \mathrm{C} & 4.006252 & 3.980539 & -1.193360 \\ \mathrm{C} & 4.984540 & 4.958560 & -1.194662 \\ \mathrm{C} & 5.476483 & 5.450026 & 0.000053 \\ \mathrm{C} & 4.984840 & 4.958299 & 1.194783 \\ \mathrm{C} & 4.006567 & 3.980264 & 1.193514 \\ \mathrm{H} & 3.628099 & 3.601136 & -2.124769 \\ \mathrm{H} & 5.361097 & 5.334585 & -2.128164 \\ \mathrm{H} & 6.236399 & 6.209490 & 0.000040 \\ \mathrm{H} & 5.361609 & 5.334143 & 2.128273 \\ \mathrm{H} & 3.628685 & 3.600619 & 2.124935\end{array}$

\section{CIS/TZVP geometries - Group II}

tetrafluorobenzene $\mathrm{S}_{0}$

$\begin{array}{lrrr}\text { C } & -1.184138 & 0.687535 & 0.000000 \\ \mathrm{C} & -1.184138 & -0.687535 & 0.000000 \\ \mathrm{C} & 0.000000 & -1.385263 & 0.000000 \\ \mathrm{C} & 1.184138 & -0.687535 & 0.000000 \\ \mathrm{C} & 1.184138 & 0.687535 & 0.000000 \\ \mathrm{C} & 0.000000 & 1.385263 & 0.000000 \\ \mathrm{~F} & -2.327895 & -1.338482 & 0.000000 \\ \mathrm{H} & 0.000000 & -2.457630 & 0.000000 \\ \mathrm{~F} & 2.327895 & -1.338482 & 0.000000 \\ \mathrm{~F} & 2.327895 & 1.338482 & 0.000000 \\ \mathrm{H} & 0.000000 & 2.457630 & 0.000000 \\ \mathrm{~F} & -2.327895 & 1.338482 & 0.000000\end{array}$

tetrafluorobenzene $\mathrm{S}_{1}$ 


$\begin{array}{lccc}\text { C } & 1.163878 & -0.704358 & 0.096236 \\ \text { C } & 1.163878 & 0.704358 & 0.096236 \\ \text { C } & 0.000000 & 1.451411 & 0.288401 \\ \text { C } & -1.163878 & 0.704358 & 0.096236 \\ \text { C } & -1.163878 & -0.704358 & 0.096236 \\ \text { C } & 0.000000 & -1.451411 & 0.288401 \\ \text { F } & 2.296001 & 1.310623 & -0.150438 \\ \text { H } & 0.000000 & 2.496649 & 0.518126 \\ \text { F } & -2.296001 & 1.310623 & -0.150438 \\ \text { F } & -2.296001 & -1.310623 & -0.150438 \\ \text { H } & 0.000000 & -2.496649 & 0.518126 \\ \text { F } & 2.296001 & -1.310623 & -0.150438\end{array}$

benzonitrile $\mathrm{S}_{0}$

$\begin{array}{rrrr}\mathrm{C} & 0.000000 & 0.000000 & -1.045841 \\ \mathrm{C} & -1.203930 & 0.000000 & -0.356787 \\ \mathrm{C} & -1.199689 & 0.000000 & 1.023288 \\ \mathrm{C} & 0.000000 & 0.000000 & 1.712111 \\ \mathrm{C} & 1.199689 & 0.000000 & 1.023288 \\ \mathrm{C} & 1.203930 & 0.000000 & -0.356787 \\ \mathrm{H} & -2.129423 & 0.000000 & -0.900005 \\ \mathrm{H} & -2.129730 & 0.000000 & 1.559299 \\ \mathrm{H} & 0.000000 & 0.000000 & 2.786056 \\ \mathrm{H} & 2.129730 & 0.000000 & 1.559299 \\ \mathrm{H} & 2.129423 & 0.000000 & -0.900005 \\ \mathrm{C} & 0.000000 & 0.000000 & -2.487747 \\ \mathrm{~N} & 0.000000 & 0.000000 & -3.616159\end{array}$

benzonitrile $\mathrm{S}_{1}$

$\begin{array}{crrr}\mathrm{C} & 0.000000 & 0.000000 & -1.089630 \\ \mathrm{C} & -1.219457 & 0.000000 & -0.371095 \\ \mathrm{C} & -1.211706 & 0.000000 & 1.039983 \\ \mathrm{C} & 0.000000 & 0.000000 & 1.753539 \\ \mathrm{C} & 1.211706 & 0.000000 & 1.039983 \\ \mathrm{C} & 1.219457 & 0.000000 & -0.371095 \\ \mathrm{H} & -2.150596 & 0.000000 & -0.901941 \\ \mathrm{H} & -2.146503 & 0.000000 & 1.565245 \\ \mathrm{H} & 0.000000 & 0.000000 & 2.824748 \\ \mathrm{H} & 2.146503 & 0.000000 & 1.565245 \\ \mathrm{H} & 2.150596 & 0.000000 & -0.901941 \\ \mathrm{C} & 0.000000 & 0.000000 & -2.510024 \\ \mathrm{~N} & 0.000000 & 0.000000 & -3.643018\end{array}$


$o$-fluorophenol, cis $\mathrm{S}_{0}$

$\begin{array}{lrrr}\mathrm{C} & 0.325180 & 1.444103 & 0.000000 \\ \mathrm{C} & -0.870657 & 0.773818 & 0.000000 \\ \mathrm{C} & -0.946457 & -0.603976 & 0.000000 \\ \mathrm{C} & 0.231893 & -1.324813 & 0.000000 \\ \mathrm{C} & 1.446673 & -0.666489 & 0.000000 \\ \mathrm{C} & 1.500275 & 0.715269 & 0.000000 \\ \mathrm{~F} & -2.020305 & 1.454497 & 0.000000 \\ \mathrm{O} & -2.124930 & -1.254443 & 0.000000 \\ \mathrm{H} & 0.177981 & -2.396702 & 0.000000 \\ \mathrm{H} & 2.355210 & -1.238854 & 0.000000 \\ \mathrm{H} & 2.445116 & 1.224109 & 0.000000 \\ \mathrm{H} & 0.323962 & 2.517486 & 0.000000 \\ \mathrm{H} & -2.843929 & -0.644025 & 0.000000\end{array}$

$o$-fluorophenol, cis $\mathrm{S}_{1}$

$\begin{array}{lrcc}\mathrm{C} & 1.232849 & -0.209328 & -0.749934 \\ \mathrm{C} & 0.007874 & 0.041548 & -0.093731 \\ \mathrm{C} & -0.029581 & -0.018457 & 1.362581 \\ \mathrm{C} & 1.146866 & 0.117642 & 2.079403 \\ \mathrm{C} & 2.345430 & 0.129461 & 1.394763 \\ \mathrm{C} & 2.379746 & -0.075140 & -0.010011 \\ \mathrm{~F} & -0.813199 & 0.979989 & -0.641996 \\ \mathrm{O} & -1.163527 & -0.292113 & 1.986872 \\ \mathrm{H} & 1.117515 & 0.069892 & 3.153031 \\ \mathrm{H} & 3.268801 & 0.219667 & 1.933762 \\ \mathrm{H} & 3.335130 & -0.113945 & -0.502429 \\ \mathrm{H} & 1.267751 & -0.362366 & -1.812282 \\ \mathrm{H} & -1.840274 & -0.486851 & 1.349911\end{array}$

$o$-fluorophenol, trans $\mathrm{S}_{0}$

$\begin{array}{lrrr}\mathrm{C} & 0.286179 & 1.255124 & 0.000000 \\ \mathrm{C} & -0.940035 & 0.612370 & 0.000000 \\ \mathrm{C} & -0.956084 & -0.768483 & 0.000000 \\ \mathrm{C} & 0.227837 & -1.486829 & 0.000000 \\ \mathrm{C} & 1.439322 & -0.829822 & 0.000000 \\ \mathrm{C} & 1.464103 & 0.555418 & 0.000000 \\ \mathrm{O} & -2.051273 & 1.373105 & 0.000000 \\ \mathrm{H} & -1.900238 & -1.284372 & 0.000000 \\ \mathrm{H} & 0.193289 & -2.559997 & 0.000000 \\ \mathrm{H} & 2.359873 & -1.381413 & 0.000000 \\ \mathrm{H} & 2.388546 & 1.101146 & 0.000000 \\ \mathrm{~F} & 0.313218 & 2.577822 & 0.000000\end{array}$




$$
\begin{aligned}
& \mathrm{H} \quad-2.824746 \quad 0.835932 \quad 0.000000 \\
& o \text {-fluorophenol, trans } \mathrm{S}_{1}
\end{aligned}
$$

$\begin{array}{cccc}\mathrm{C} & 0.820928 & 1.032082 & 0.083764 \\ \mathrm{C} & -0.579335 & 0.973500 & 0.068808 \\ \mathrm{C} & -1.260560 & -0.297462 & 0.114992 \\ \mathrm{C} & -0.473477 & -1.429403 & -0.054454 \\ \mathrm{C} & 0.930447 & -1.339056 & -0.072774 \\ \mathrm{C} & 1.606158 & -0.113538 & 0.127140 \\ \mathrm{O} & -1.251338 & 2.094500 & -0.113164 \\ \mathrm{H} & -2.291343 & -0.345571 & 0.411485 \\ \mathrm{H} & -0.929898 & -2.397768 & -0.136018 \\ \mathrm{H} & 1.504609 & -2.236789 & -0.207124 \\ \mathrm{H} & 2.670395 & -0.048540 & 0.217912 \\ \mathrm{~F} & 1.391368 & 2.209689 & -0.049520 \\ \mathrm{H} & -2.137985 & 1.898356 & -0.391048\end{array}$

$m$-fluorophenol, cis $\mathrm{S}_{0}$

$\begin{array}{cccc}\mathrm{C} & 0.308876 & 1.402711 & 0.000000 \\ \mathrm{C} & -0.921832 & 0.784396 & 0.000000 \\ \mathrm{C} & -0.962043 & -0.598087 & 0.000000 \\ \mathrm{C} & 0.212078 & -1.334709 & 0.000000 \\ \mathrm{C} & 1.421878 & -0.675592 & 0.000000 \\ \mathrm{C} & 1.491797 & 0.708482 & 0.000000 \\ \mathrm{O} & -2.124270 & -1.278725 & 0.000000 \\ \mathrm{~F} & 0.338038 & 2.726550 & 0.000000 \\ \mathrm{H} & 0.157500 & -2.405823 & 0.000000 \\ \mathrm{H} & 2.331233 & -1.247179 & 0.000000 \\ \mathrm{H} & 2.426578 & 1.233596 & 0.000000 \\ \mathrm{H} & -1.816511 & 1.379223 & 0.000000 \\ \mathrm{H} & -2.863322 & -0.694842 & 0.000000\end{array}$

$m$-fluorophenol, cis $\mathrm{S}_{1}$

$\begin{array}{rrrr}\text { C } & 1.229195 & -0.017390 & -0.689376 \\ \text { C } & -0.027911 & 0.028632 & -0.097249 \\ \text { C } & -0.023573 & 0.020605 & 1.325334 \\ \text { C } & 1.163944 & -0.000168 & 2.081553 \\ \text { C } & 2.415114 & 0.072902 & 1.437725 \\ \text { C } & 2.432577 & -0.028787 & 0.029556 \\ \text { O } & -1.165839 & 0.000388 & 1.997114 \\ \text { F } & 1.297193 & -0.055165 & -2.006427 \\ \text { H } & 1.077799 & -0.067882 & 3.148122 \\ \text { H } & 3.326112 & 0.093145 & 1.997295\end{array}$




$$
\begin{array}{rrrr}
\mathrm{H} & 3.352393 & -0.095143 & -0.518487 \\
\mathrm{H} & -0.920463 & 0.170344 & -0.672290 \\
\mathrm{H} & -1.901181 & -0.121481 & 1.417080
\end{array}
$$

$m$-fluorophenol, trans $\mathrm{S}_{0}$

$\begin{array}{cccc}\mathrm{C} & 0.266447 & 1.294683 & 0.000000 \\ \mathrm{C} & -0.943865 & 0.622491 & 0.000000 \\ \mathrm{C} & -0.978756 & -0.761539 & 0.000000 \\ \mathrm{C} & 0.208644 & -1.470495 & 0.000000 \\ \mathrm{C} & 1.428177 & -0.824808 & 0.000000 \\ \mathrm{C} & 1.420392 & 0.552891 & 0.000000 \\ \mathrm{O} & -2.061309 & 1.374466 & 0.000000 \\ \mathrm{H} & -1.920678 & -1.280058 & 0.000000 \\ \mathrm{H} & 0.179270 & -2.544149 & 0.000000 \\ \mathrm{H} & 2.357833 & -1.358921 & 0.000000 \\ \mathrm{~F} & 2.577825 & 1.194661 & 0.000000 \\ \mathrm{H} & 0.299091 & 2.366180 & 0.000000 \\ \mathrm{H} & -2.833072 & 0.834606 & 0.000000\end{array}$

$m$-fluorophenol, trans $\mathrm{S}_{1}$

$\begin{array}{lccc}\mathrm{C} & 1.068035 & -0.016680 & 0.000000 \\ \mathrm{C} & 0.322588 & 1.186064 & 0.000000 \\ \mathrm{C} & -1.091036 & 1.205139 & 0.000000 \\ \mathrm{C} & -1.810835 & -0.005637 & 0.000000 \\ \mathrm{C} & -1.080795 & -1.217337 & 0.000000 \\ \mathrm{C} & 0.319472 & -1.190648 & 0.000000 \\ \mathrm{O} & 1.025558 & 2.309694 & 0.000000 \\ \mathrm{H} & -1.601529 & 2.150321 & 0.000000 \\ \mathrm{H} & -2.880456 & -0.013064 & 0.000000 \\ \mathrm{H} & -1.576751 & -2.168183 & 0.000000 \\ \mathrm{~F} & 0.961768 & -2.343653 & 0.000000 \\ \mathrm{H} & 2.136969 & -0.011188 & 0.000000 \\ \mathrm{H} & 0.465863 & 3.069318 & 0.000000\end{array}$

$p$-fluorophenol $\mathrm{S}_{0}$

$\begin{array}{rrrr}\mathrm{C} & 0.268541 & 1.305214 & 0.000000 \\ \mathrm{C} & -0.945827 & 0.640376 & 0.000000 \\ \mathrm{C} & -0.973711 & -0.740460 & 0.000000 \\ \mathrm{C} & 0.209283 & -1.460937 & 0.000000 \\ \mathrm{C} & 1.401166 & -0.784719 & 0.000000 \\ \mathrm{C} & 1.447884 & 0.590943 & 0.000000 \\ \mathrm{O} & -2.069663 & 1.394879 & 0.000000 \\ \mathrm{H} & -1.913493 & -1.263655 & 0.000000\end{array}$




$\begin{array}{lcrc}\mathrm{H} & 0.203102 & -2.534109 & 0.000000 \\ \mathrm{~F} & 2.539792 & -1.471715 & 0.000000 \\ \mathrm{H} & 2.397596 & 1.090898 & 0.000000 \\ \mathrm{H} & 0.276378 & 2.378161 & 0.000000 \\ \mathrm{H} & -2.841068 & 0.855113 & 0.000000\end{array}$

p-fluorophenol $\mathrm{S}_{1}$

$\begin{array}{lrrr}\mathrm{C} & 1.253911 & 0.674251 & 0.000000 \\ \mathrm{C} & 0.022604 & 1.363750 & 0.000000 \\ \mathrm{C} & -1.231598 & 0.701503 & 0.000000 \\ \mathrm{C} & -1.241574 & -0.700958 & 0.000000 \\ \mathrm{C} & -0.005779 & -1.365169 & 0.000000 \\ \mathrm{C} & 1.240989 & -0.729674 & 0.000000 \\ \mathrm{O} & 0.086377 & 2.683763 & 0.000000 \\ \mathrm{H} & -2.141540 & 1.269950 & 0.000000 \\ \mathrm{H} & -2.147048 & -1.272262 & 0.000000 \\ \mathrm{~F} & -0.024440 & -2.675399 & 0.000000 \\ \mathrm{H} & 2.136479 & -1.315970 & 0.000000 \\ \mathrm{H} & 2.162611 & 1.240397 & 0.000000 \\ \mathrm{H} & -0.774296 & 3.071413 & 0.000000\end{array}$

phenylacetylene $\mathrm{S}_{0}$

$\begin{array}{rrrr}\mathrm{C} & 1.199604 & 0.000000 & -0.013134 \\ \mathrm{C} & 0.000000 & 0.000000 & -0.714140 \\ \mathrm{C} & -1.199604 & 0.000000 & -0.013134 \\ \mathrm{C} & -1.197000 & 0.000000 & 1.367308 \\ \mathrm{C} & 0.000000 & 0.000000 & 2.060171 \\ \mathrm{C} & 1.197000 & 0.000000 & 1.367308 \\ \mathrm{C} & 0.000000 & 0.000000 & -2.154070 \\ \mathrm{C} & 0.000000 & 0.000000 & -3.337778 \\ \mathrm{H} & -2.126836 & 0.000000 & -0.553597 \\ \mathrm{H} & -2.128742 & 0.000000 & 1.901356 \\ \mathrm{H} & 0.000000 & 0.000000 & 3.134206 \\ \mathrm{H} & 2.128742 & 0.000000 & 1.901356 \\ \mathrm{H} & 2.126836 & 0.000000 & -0.553597 \\ \mathrm{H} & 0.000000 & 0.000000 & -4.392274\end{array}$

phenylacetylene $S_{1}$
$\begin{array}{llll}\text { C } & 1.240007 & 0.000000 & -0.004572\end{array}$
$\begin{array}{llll}\text { C } & 0.000000 & 0.000000 & -0.749582\end{array}$
$\begin{array}{llll}\mathrm{C} & -1.240007 & 0.000000 & -0.004572\end{array}$
$\begin{array}{llll}\text { C } & -1.218229 & 0.000000 & 1.358853\end{array}$
$\begin{array}{llll}\text { C } & 0.000000 & 0.000000 & 2.061997\end{array}$ 


$\begin{array}{lrrr}\mathrm{C} & 1.218229 & 0.000000 & 1.358853 \\ \mathrm{C} & 0.000000 & 0.000000 & -2.118564 \\ \mathrm{C} & 0.000000 & 0.000000 & -3.350419 \\ \mathrm{H} & -2.165002 & 0.000000 & -0.547583 \\ \mathrm{H} & -2.142240 & 0.000000 & 1.906256 \\ \mathrm{H} & 0.000000 & 0.000000 & 3.135295 \\ \mathrm{H} & 2.142240 & 0.000000 & 1.906256 \\ \mathrm{H} & 2.165002 & 0.000000 & -0.547583 \\ \mathrm{H} & 0.000000 & 0.000000 & -4.404634\end{array}$

aniline $\mathrm{S}_{0}$

$\begin{array}{crrr}\mathrm{C} & 0.914102 & 0.035741 & 0.000000 \\ \mathrm{C} & 0.205928 & 0.040823 & 1.195653 \\ \mathrm{C} & -1.174574 & 0.042175 & 1.189750 \\ \mathrm{C} & -1.879108 & 0.043237 & 0.000000 \\ \mathrm{C} & -1.174574 & 0.042175 & -1.189750 \\ \mathrm{C} & 0.205928 & 0.040823 & -1.195653 \\ \mathrm{H} & 0.738958 & 0.046205 & 2.129681 \\ \mathrm{H} & -2.952648 & 0.044134 & 0.000000 \\ \mathrm{H} & -1.702722 & 0.044109 & 2.125929 \\ \mathrm{H} & -1.702722 & 0.044109 & -2.125929 \\ \mathrm{H} & 0.738958 & 0.046205 & -2.129681 \\ \mathrm{~N} & 2.308240 & 0.083285 & 0.000000 \\ \mathrm{H} & 2.737123 & -0.276505 & -0.822090 \\ \mathrm{H} & 2.737123 & -0.276505 & 0.822090\end{array}$

aniline $S_{1}$

$\begin{array}{rrrr}\mathrm{C} & 0.907674 & -0.034655 & 0.000000 \\ \mathrm{C} & 0.218746 & -0.008286 & 1.242442 \\ \mathrm{C} & -1.185796 & 0.045329 & 1.230458 \\ \mathrm{C} & -1.864187 & 0.071200 & 0.000000 \\ \mathrm{C} & -1.185796 & 0.045329 & -1.230458 \\ \mathrm{C} & 0.218746 & -0.008286 & -1.242442 \\ \mathrm{H} & 0.777265 & -0.029618 & 2.158010 \\ \mathrm{H} & -2.937506 & 0.112083 & 0.000000 \\ \mathrm{H} & -1.738728 & 0.066448 & 2.147942 \\ \mathrm{H} & -1.738728 & 0.066448 & -2.147942 \\ \mathrm{H} & 0.777265 & -0.029618 & -2.158010 \\ \mathrm{~N} & 2.244756 & -0.085869 & 0.000000 \\ \mathrm{H} & 2.753139 & -0.105262 & -0.850465 \\ \mathrm{H} & 2.753139 & -0.105262 & 0.850465\end{array}$

catechol $\mathrm{S}_{0}$ 


$\begin{array}{lrrr}\mathrm{C} & 0.467510 & 1.270066 & 0.000000 \\ \mathrm{C} & -0.744621 & 0.619153 & 0.000000 \\ \mathrm{C} & -0.795681 & -0.768410 & 0.000000 \\ \mathrm{C} & 0.376866 & -1.489606 & 0.000000 \\ \mathrm{C} & 1.598242 & -0.836258 & 0.000000 \\ \mathrm{C} & 1.646443 & 0.541422 & 0.000000 \\ \mathrm{O} & -1.948807 & 1.255143 & 0.000000 \\ \mathrm{O} & -1.977527 & -1.417535 & 0.000000 \\ \mathrm{H} & 0.319349 & -2.561585 & 0.000000 \\ \mathrm{H} & 2.506161 & -1.409516 & 0.000000 \\ \mathrm{H} & 2.589208 & 1.054946 & 0.000000 \\ \mathrm{H} & 0.494297 & 2.345936 & 0.000000 \\ \mathrm{H} & -1.845198 & 2.190711 & 0.000000 \\ \mathrm{H} & -2.686243 & -0.794467 & 0.000000\end{array}$

catechol $\mathrm{S}_{1}$

$\begin{array}{rrrr}\mathrm{C} & 0.469694 & 1.333616 & 0.000000 \\ \mathrm{C} & -0.755959 & 0.631002 & 0.000000 \\ \mathrm{C} & -0.804486 & -0.776122 & 0.000000 \\ \mathrm{C} & 0.383474 & -1.553739 & 0.000000 \\ \mathrm{C} & 1.593490 & -0.856463 & 0.000000 \\ \mathrm{C} & 1.638262 & 0.554716 & 0.000000 \\ \mathrm{O} & -1.947913 & 1.252820 & 0.000000 \\ \mathrm{O} & -1.970974 & -1.409238 & 0.000000 \\ \mathrm{H} & 0.318142 & -2.621104 & 0.000000 \\ \mathrm{H} & 2.519690 & -1.398053 & 0.000000 \\ \mathrm{H} & 2.595423 & 1.039844 & 0.000000 \\ \mathrm{H} & 0.494518 & 2.404981 & 0.000000 \\ \mathrm{H} & -1.845913 & 2.189925 & 0.000000 \\ \mathrm{H} & -2.687449 & -0.792185 & 0.000000\end{array}$

resorcinol, isomer $1 \mathrm{~S}_{0}$

$\begin{array}{rrrr}\mathrm{C} & 0.072561 & 1.140292 & 0.000000 \\ \mathrm{C} & -1.133734 & 0.459619 & 0.000000 \\ \mathrm{C} & -1.164461 & -0.921551 & 0.000000 \\ \mathrm{C} & 0.035206 & -1.615144 & 0.000000 \\ \mathrm{C} & 1.245694 & -0.961240 & 0.000000 \\ \mathrm{C} & 1.253816 & 0.426430 & 0.000000 \\ \mathrm{O} & -2.255072 & 1.209193 & 0.000000 \\ \mathrm{H} & -2.101948 & -1.447832 & 0.000000 \\ \mathrm{H} & 0.018083 & -2.689528 & 0.000000 \\ \mathrm{H} & 2.175947 & -1.494624 & 0.000000 \\ \mathrm{O} & 2.454103 & 1.039360 & 0.000000 \\ \mathrm{H} & 0.066654 & 2.214950 & 0.000000\end{array}$




$$
\begin{array}{rrrr}
\mathrm{H} & -3.022983 & 0.663973 & 0.000000 \\
\mathrm{H} & 2.356134 & 1.976094 & 0.000000
\end{array}
$$

resorcinol, isomer $1 \mathrm{~S}_{1}$

$\begin{array}{lrrc}\mathrm{C} & -0.004473 & 1.039645 & 0.119709 \\ \mathrm{C} & -1.191854 & 0.300811 & 0.017841 \\ \mathrm{C} & -1.198644 & -1.112275 & -0.033723 \\ \mathrm{C} & 0.000507 & -1.836874 & 0.114115 \\ \mathrm{C} & 1.202607 & -1.105645 & -0.022701 \\ \mathrm{C} & 1.188412 & 0.303105 & 0.021506 \\ \mathrm{O} & -2.329001 & 0.995699 & -0.029681 \\ \mathrm{H} & -2.136032 & -1.621482 & -0.173032 \\ \mathrm{H} & 0.002543 & -2.906059 & 0.132206 \\ \mathrm{H} & 2.147673 & -1.594126 & -0.162845 \\ \mathrm{O} & 2.372089 & 0.915617 & -0.038927 \\ \mathrm{H} & -0.025779 & 2.103028 & 0.246005 \\ \mathrm{H} & -3.080075 & 0.425852 & -0.046472 \\ \mathrm{H} & 2.263020 & 1.848827 & -0.124352\end{array}$

resorcinol, isomer $2 \mathrm{~S}_{0}$

$\begin{array}{rrrr}\mathrm{C} & 1.191286 & 0.000000 & -0.541124 \\ \mathrm{C} & 0.000000 & 0.000000 & -1.238879 \\ \mathrm{C} & -1.191286 & 0.000000 & -0.541124 \\ \mathrm{C} & -1.203479 & 0.000000 & 0.845905 \\ \mathrm{C} & 0.000000 & 0.000000 & 1.521843 \\ \mathrm{C} & 1.203479 & 0.000000 & 0.845905 \\ \mathrm{O} & -2.327452 & 0.000000 & -1.266717 \\ \mathrm{O} & 2.327452 & 0.000000 & -1.266717 \\ \mathrm{H} & -2.134133 & 0.000000 & 1.384177 \\ \mathrm{H} & 0.000000 & 0.000000 & 2.596331 \\ \mathrm{H} & 2.134133 & 0.000000 & 1.384177 \\ \mathrm{H} & 0.000000 & 0.000000 & -2.310912 \\ \mathrm{H} & -3.084478 & 0.000000 & -0.706673 \\ \mathrm{H} & 3.084478 & 0.000000 & -0.706673\end{array}$

resorcinol, isomer $2 \mathrm{~S}_{1}$
$\begin{array}{llll}\text { C } & 1.194456 & 0.000000 & -0.326711\end{array}$
$\begin{array}{llll}\text { C } & 0.000000 & 0.000000 & -1.062097\end{array}$
$\begin{array}{llll}\text { C } & -1.194456 & 0.000000 & -0.326711\end{array}$
$\begin{array}{llll}\text { C } & -1.208921 & 0.000000 & 1.087762\end{array}$
$\begin{array}{llll}\text { C } & 0.000000 & 0.000000 & 1.814857\end{array}$
$\begin{array}{llll}\text { C } & 1.208921 & 0.000000 & 1.087762\end{array}$
$\begin{array}{llll}\mathrm{O} & -2.331630 & 0.000000 & -1.026423\end{array}$ 


$\begin{array}{rrrr}\mathrm{O} & 2.331630 & 0.000000 & -1.026423 \\ \mathrm{H} & -2.152973 & 0.000000 & 1.601482 \\ \mathrm{H} & 0.000000 & 0.000000 & 2.884567 \\ \mathrm{H} & 2.152973 & 0.000000 & 1.601482 \\ \mathrm{H} & 0.000000 & 0.000000 & -2.131084 \\ \mathrm{H} & -3.084102 & 0.000000 & -0.458774 \\ \mathrm{H} & 3.084102 & 0.000000 & -0.458774\end{array}$

resorcinol, isomer $3 \mathrm{~S}_{0}$

$\begin{array}{crcc}\mathrm{C} & 1.196800 & 0.000000 & -0.287090 \\ \mathrm{C} & 0.000000 & 0.000000 & -0.984729 \\ \mathrm{C} & -1.196800 & 0.000000 & -0.287090 \\ \mathrm{C} & -1.206556 & 0.000000 & 1.094951 \\ \mathrm{C} & 0.000000 & 0.000000 & 1.766625 \\ \mathrm{C} & 1.206556 & 0.000000 & 1.094951 \\ \mathrm{O} & -2.386886 & 0.000000 & -0.921889 \\ \mathrm{O} & 2.386886 & 0.000000 & -0.921889 \\ \mathrm{H} & -2.143470 & 0.000000 & 1.616551 \\ \mathrm{H} & 0.000000 & 0.000000 & 2.841170 \\ \mathrm{H} & 2.143470 & 0.000000 & 1.616551 \\ \mathrm{H} & 0.000000 & 0.000000 & -2.061987 \\ \mathrm{H} & 2.275681 & 0.000000 & -1.856823 \\ \mathrm{H} & -2.275681 & 0.000000 & -1.856823\end{array}$

resorcinol, isomer $3 \mathrm{~S}_{1}$

$\begin{array}{lrcc}\mathrm{C} & -0.032943 & -0.303105 & 1.189630 \\ \mathrm{C} & -0.158965 & -1.047721 & 0.000000 \\ \mathrm{C} & -0.032943 & -0.303105 & -1.189630 \\ \mathrm{C} & 0.114075 & 1.098345 & -1.197979 \\ \mathrm{C} & 0.043291 & 1.835151 & 0.000000 \\ \mathrm{C} & 0.114075 & 1.098345 & 1.197979 \\ \mathrm{O} & -0.032943 & -0.911755 & -2.375722 \\ \mathrm{O} & -0.032943 & -0.911755 & 2.375722 \\ \mathrm{H} & 0.283384 & 1.574999 & -2.144727 \\ \mathrm{H} & 0.100286 & 2.902975 & 0.000000 \\ \mathrm{H} & 0.283384 & 1.574999 & 2.144727 \\ \mathrm{H} & -0.563801 & -2.043488 & 0.000000 \\ \mathrm{H} & 0.072143 & -1.844434 & 2.269995 \\ \mathrm{H} & 0.072143 & -1.844434 & -2.269995\end{array}$

hydroquinone, cis $\mathrm{S}_{0}$
$\begin{array}{llll}\text { C } & -0.694287 & 0.000000 & 1.084628\end{array}$
$\begin{array}{llll}\text { C } & 0.694287 & 0.000000 & 1.084628\end{array}$ 


$\begin{array}{rrrr}\mathrm{C} & 1.387977 & 0.000000 & -0.103938 \\ \mathrm{C} & 0.687607 & 0.000000 & -1.300157 \\ \mathrm{C} & -0.687607 & 0.000000 & -1.300157 \\ \mathrm{C} & -1.387977 & 0.000000 & -0.103938 \\ \mathrm{O} & 2.743743 & 0.000000 & -0.170091 \\ \mathrm{O} & -2.743743 & 0.000000 & -0.170091 \\ \mathrm{H} & 1.234548 & 0.000000 & -2.223739 \\ \mathrm{H} & -1.234548 & 0.000000 & -2.223739 \\ \mathrm{H} & -1.222271 & 0.000000 & 2.022185 \\ \mathrm{H} & 1.222271 & 0.000000 & 2.022185 \\ \mathrm{H} & -3.123003 & 0.000000 & 0.691402 \\ \mathrm{H} & 3.123003 & 0.000000 & 0.691402\end{array}$

hydroquinone, cis $\mathrm{S}_{1}$

$\begin{array}{rrrr}\mathrm{C} & 0.700238 & 0.000000 & -1.128155 \\ \mathrm{C} & -0.700238 & 0.000000 & -1.128155 \\ \mathrm{C} & -1.376455 & 0.000000 & 0.116264 \\ \mathrm{C} & -0.700927 & 0.000000 & 1.348985 \\ \mathrm{C} & 0.700927 & 0.000000 & 1.348985 \\ \mathrm{C} & 1.376455 & 0.000000 & 0.116264 \\ \mathrm{O} & -2.701442 & 0.000000 & 0.160713 \\ \mathrm{O} & 2.701442 & 0.000000 & 0.160713 \\ \mathrm{H} & -1.273723 & 0.000000 & 2.253690 \\ \mathrm{H} & 1.273723 & 0.000000 & 2.253690 \\ \mathrm{H} & 1.260068 & 0.000000 & -2.044018 \\ \mathrm{H} & -1.260068 & 0.000000 & -2.044018 \\ \mathrm{H} & 3.072490 & 0.000000 & -0.706708 \\ \mathrm{H} & -3.072490 & 0.000000 & -0.706708\end{array}$

hydroquinone, trans $\mathrm{S}_{0}$

$\begin{array}{lrrr}\mathrm{C} & 0.016827 & 1.379574 & 0.000000 \\ \mathrm{C} & -1.191033 & 0.712685 & 0.000000 \\ \mathrm{C} & -1.202153 & -0.669565 & 0.000000 \\ \mathrm{C} & -0.016827 & -1.379574 & 0.000000 \\ \mathrm{C} & 1.191033 & -0.712685 & 0.000000 \\ \mathrm{C} & 1.202153 & 0.669565 & 0.000000 \\ \mathrm{O} & -2.325987 & 1.457698 & 0.000000 \\ \mathrm{H} & -2.137135 & -1.202344 & 0.000000 \\ \mathrm{H} & -0.022892 & -2.452866 & 0.000000 \\ \mathrm{O} & 2.325987 & -1.457698 & 0.000000 \\ \mathrm{H} & 2.137135 & 1.202344 & 0.000000 \\ \mathrm{H} & 0.022892 & 2.452866 & 0.000000 \\ \mathrm{H} & -3.089595 & 0.907335 & 0.000000 \\ \mathrm{H} & 3.089595 & -0.907335 & 0.000000\end{array}$


hydroquinone, trans $\mathrm{S}_{1}$

$\begin{array}{rrrr}\mathrm{C} & 1.251077 & 0.672915 & 0.000000 \\ \mathrm{C} & 0.030374 & 1.375869 & 0.000000 \\ \mathrm{C} & -1.225420 & 0.728121 & 0.000000 \\ \mathrm{C} & -1.251077 & -0.672915 & 0.000000 \\ \mathrm{C} & -0.030374 & -1.375869 & 0.000000 \\ \mathrm{C} & 1.225420 & -0.728121 & 0.000000 \\ \mathrm{O} & 0.110451 & 2.699301 & 0.000000 \\ \mathrm{H} & -2.130424 & 1.304964 & 0.000000 \\ \mathrm{H} & -2.166249 & -1.229198 & 0.000000 \\ \mathrm{O} & -0.110451 & -2.699301 & 0.000000 \\ \mathrm{H} & 2.130424 & -1.304964 & 0.000000 \\ \mathrm{H} & 2.166249 & 1.229198 & 0.000000 \\ \mathrm{H} & -0.747260 & 3.092448 & 0.000000 \\ \mathrm{H} & 0.747260 & -3.092448 & 0.000000\end{array}$

salicylic acid $\mathrm{S}_{0}$

$\begin{array}{lrrr}\mathrm{C} & 1.093627 & 1.588646 & 0.000000 \\ \mathrm{C} & 1.127369 & 0.195888 & 0.000000 \\ \mathrm{C} & -0.075113 & -0.517079 & 0.000000 \\ \mathrm{C} & -1.286005 & 0.182690 & 0.000000 \\ \mathrm{C} & -1.313018 & 1.551093 & 0.000000 \\ \mathrm{C} & -0.107059 & 2.248848 & 0.000000 \\ \mathrm{O} & 2.325075 & -0.378241 & 0.000000 \\ \mathrm{C} & -0.049343 & -1.987996 & 0.000000 \\ \mathrm{O} & -1.241992 & -2.558694 & 0.000000 \\ \mathrm{O} & 0.948983 & -2.647176 & 0.000000 \\ \mathrm{H} & -2.201736 & -0.374296 & 0.000000 \\ \mathrm{H} & 2.026205 & 2.118696 & 0.000000 \\ \mathrm{H} & -2.247714 & 2.077601 & 0.000000 \\ \mathrm{H} & -0.114209 & 3.323476 & 0.000000 \\ \mathrm{H} & 2.238825 & -1.325075 & 0.000000 \\ \mathrm{H} & -1.123895 & -3.498384 & 0.000000\end{array}$

salicylic acid $\mathrm{S}_{1}$

$\begin{array}{rrrr}\text { C } & 1.001698 & 1.905713 & 0.000000 \\ \text { C } & 0.977776 & 0.507266 & 0.000000 \\ \text { C } & -0.292801 & -0.244894 & 0.000000 \\ \text { C } & -1.455409 & 0.521609 & 0.000000 \\ \text { C } & -1.400843 & 1.928497 & 0.000000 \\ \text { C } & -0.188620 & 2.634168 & 0.000000 \\ \text { O } & 2.098957 & -0.136861 & 0.000000\end{array}$




$\begin{array}{rrrr}\mathrm{C} & -0.251298 & -1.669106 & 0.000000 \\ \mathrm{O} & -1.437990 & -2.272396 & 0.000000 \\ \mathrm{O} & 0.774064 & -2.333830 & 0.000000 \\ \mathrm{H} & -2.409297 & 0.034384 & 0.000000 \\ \mathrm{H} & 1.962117 & 2.383487 & 0.000000 \\ \mathrm{H} & -2.326280 & 2.472604 & 0.000000 \\ \mathrm{H} & -0.178486 & 3.704999 & 0.000000 \\ \mathrm{H} & 1.925485 & -1.091951 & 0.000000 \\ \mathrm{H} & -1.288493 & -3.206744 & 0.000000\end{array}$

$o$-cresol, cis $\mathrm{S}_{0}$

$\begin{array}{lrrr}\mathrm{C} & 1.953582 & 0.508840 & 0.000000 \\ \mathrm{C} & 0.725628 & 1.140762 & 0.000000 \\ \mathrm{C} & -0.463501 & 0.427608 & 0.000000 \\ \mathrm{C} & -0.387461 & -0.960492 & 0.000000 \\ \mathrm{C} & 0.834770 & -1.606857 & 0.000000 \\ \mathrm{C} & 2.002252 & -0.872739 & 0.000000 \\ \mathrm{C} & -1.798694 & 1.132588 & 0.000000 \\ \mathrm{O} & -1.491859 & -1.745608 & 0.000000 \\ \mathrm{H} & 0.850118 & -2.680200 & 0.000000 \\ \mathrm{H} & 2.948544 & -1.381477 & 0.000000 \\ \mathrm{H} & 2.858135 & 1.087377 & 0.000000 \\ \mathrm{H} & 0.685051 & 2.215270 & 0.000000 \\ \mathrm{H} & -2.280713 & -1.233656 & 0.000000 \\ \mathrm{H} & -2.386964 & 0.881678 & 0.879051 \\ \mathrm{H} & -1.661925 & 2.205235 & 0.000000 \\ \mathrm{H} & -2.386964 & 0.881678 & -0.879051\end{array}$

$o$-cresol, cis $\mathrm{S}_{1}$

$\begin{array}{rrrc}\mathrm{C} & 1.863404 & -0.090020 & -0.778872 \\ \mathrm{C} & 0.626277 & -0.076173 & -1.449966 \\ \mathrm{C} & -0.579992 & -0.051570 & -0.715366 \\ \mathrm{C} & -0.473756 & 0.067851 & 0.698409 \\ \mathrm{C} & 0.766211 & 0.081460 & 1.378052 \\ \mathrm{C} & 1.943936 & -0.021257 & 0.624079 \\ \mathrm{C} & -1.898817 & -0.030045 & -1.428030 \\ \mathrm{O} & -1.550023 & 0.157789 & 1.470448 \\ \mathrm{H} & 0.765751 & 0.168304 & 2.445230 \\ \mathrm{H} & 2.900498 & -0.030878 & 1.107413 \\ \mathrm{H} & 2.768356 & -0.149311 & -1.353443 \\ \mathrm{H} & 0.598008 & -0.134095 & -2.520051 \\ \mathrm{H} & -2.344663 & 0.177471 & 0.964253 \\ \mathrm{H} & -2.698760 & -0.503537 & -0.865439 \\ \mathrm{H} & -2.218153 & 0.984752 & -1.670219\end{array}$




$$
\begin{aligned}
& \mathrm{H} \quad-1.818857 \quad-0.570231-2.364087 \\
& \text { o-cresol, trans } \mathrm{S}_{0}
\end{aligned}
$$

$\begin{array}{lrrc}\mathrm{C} & 2.406282 & 0.000015 & 0.030402 \\ \mathrm{C} & 1.239505 & -0.000039 & -0.709937 \\ \mathrm{C} & 0.033141 & -0.000011 & -0.015299 \\ \mathrm{C} & 0.005166 & 0.000067 & 1.364752 \\ \mathrm{C} & 1.189434 & 0.000108 & 2.081673 \\ \mathrm{C} & 2.395564 & 0.000094 & 1.416657 \\ \mathrm{C} & 1.248036 & -0.000116 & -2.216621 \\ \mathrm{O} & -1.103075 & -0.000063 & -0.754260 \\ \mathrm{H} & -0.939284 & 0.000092 & 1.881211 \\ \mathrm{H} & 1.159203 & 0.000173 & 3.155566 \\ \mathrm{H} & 3.319946 & 0.000134 & 1.963038 \\ \mathrm{H} & 3.348096 & -0.000007 & -0.488304 \\ \mathrm{H} & -1.863845 & -0.000048 & -0.199741 \\ \mathrm{H} & 2.264170 & -0.000067 & -2.589972 \\ \mathrm{H} & 0.738240 & -0.872407 & -2.609558 \\ \mathrm{H} & 0.738128 & 0.872074 & -2.609636\end{array}$

o-cresol, trans $\mathrm{S}_{1}$

$\begin{array}{lrrr}\mathrm{C} & 2.425238 & 0.147782 & -0.006540 \\ \mathrm{C} & 1.221256 & 0.025504 & -0.743477 \\ \mathrm{C} & 0.010122 & -0.068031 & -0.010879 \\ \mathrm{C} & -0.034312 & -0.095589 & 1.405201 \\ \mathrm{C} & 1.176092 & 0.006760 & 2.102932 \\ \mathrm{C} & 2.390506 & 0.111938 & 1.393837 \\ \mathrm{C} & 1.251286 & 0.007149 & -2.237016 \\ \mathrm{O} & -1.118154 & -0.153692 & -0.706555 \\ \mathrm{H} & -0.979589 & -0.133749 & 1.911996 \\ \mathrm{H} & 1.185991 & -0.000489 & 3.175098 \\ \mathrm{H} & 3.308392 & 0.178059 & 1.946821 \\ \mathrm{H} & 3.359666 & 0.231676 & -0.524919 \\ \mathrm{H} & -1.857958 & -0.271529 & -0.133098 \\ \mathrm{H} & 1.873376 & -0.813829 & -2.591035 \\ \mathrm{H} & 0.267043 & -0.096612 & -2.666238 \\ \mathrm{H} & 1.699733 & 0.924652 & -2.616148\end{array}$

$m$-cresol, cis $\mathrm{S}_{0}$

$\begin{array}{lrrr}\text { C } & 1.155694 & 0.009572 & 2.075526 \\ \text { C } & 2.358090 & 0.040568 & 1.408482 \\ \text { C } & 2.408380 & 0.038989 & 0.021972 \\ \text { C } & 1.238914 & 0.005971 & -0.713063\end{array}$




$\begin{array}{crcc}\mathrm{C} & 0.022551 & -0.025385 & -0.039381 \\ \mathrm{C} & -0.018903 & -0.023618 & 1.339707 \\ \mathrm{C} & 1.260787 & 0.003870 & -2.221798 \\ \mathrm{O} & -1.182584 & -0.053460 & 2.026759 \\ \mathrm{H} & 1.104922 & 0.010392 & 3.147311 \\ \mathrm{H} & 3.272694 & 0.066344 & 1.972239 \\ \mathrm{H} & 3.358432 & 0.063470 & -0.478606 \\ \mathrm{H} & -0.897313 & -0.051320 & -0.599850 \\ \mathrm{H} & -1.919412 & -0.075991 & 1.440930 \\ \mathrm{H} & 2.275697 & 0.026257 & -2.596614 \\ \mathrm{H} & 0.776973 & -0.883740 & -2.615404 \\ \mathrm{H} & 0.737379 & 0.867452 & -2.618260\end{array}$

$m$-cresol, cis $\mathrm{S}_{1}$

$\begin{array}{cccc}\mathrm{C} & 1.162473 & 0.007249 & 2.108918 \\ \mathrm{C} & 2.399564 & 0.043749 & 1.440449 \\ \mathrm{C} & 2.408844 & 0.032910 & 0.033459 \\ \mathrm{C} & 1.220582 & 0.006939 & -0.736315 \\ \mathrm{C} & -0.018232 & -0.035591 & -0.068394 \\ \mathrm{C} & -0.022724 & -0.020406 & 1.345147 \\ \mathrm{C} & 1.264947 & 0.006342 & -2.231823 \\ \mathrm{O} & -1.165176 & -0.041866 & 2.020648 \\ \mathrm{H} & 1.084672 & 0.005909 & 3.177618 \\ \mathrm{H} & 3.319193 & 0.065591 & 1.988652 \\ \mathrm{H} & 3.350958 & 0.052027 & -0.481779 \\ \mathrm{H} & -0.945387 & -0.046980 & -0.610559 \\ \mathrm{H} & -1.905298 & -0.073580 & 1.436400 \\ \mathrm{H} & 2.281699 & 0.046853 & -2.600445 \\ \mathrm{H} & 0.793400 & -0.888635 & -2.634677 \\ \mathrm{H} & 0.722767 & 0.858859 & -2.637360\end{array}$

$m$-cresol, trans $\mathrm{S}_{0}$

$\begin{array}{rrrr}\mathrm{C} & -1.187231 & -1.093515 & 0.000000 \\ \mathrm{C} & 0.196136 & -1.201986 & 0.000000 \\ \mathrm{C} & 0.961655 & -0.047565 & 0.000000 \\ \mathrm{C} & 0.358963 & 1.195564 & 0.000000 \\ \mathrm{C} & -1.021092 & 1.303628 & 0.000000 \\ \mathrm{C} & -1.783689 & 0.152585 & 0.000000 \\ \mathrm{C} & 0.871632 & -2.551457 & 0.000000 \\ \mathrm{O} & 1.167379 & 2.279404 & 0.000000 \\ \mathrm{H} & -1.491825 & 2.271080 & 0.000000 \\ \mathrm{H} & -2.855460 & 0.231208 & 0.000000 \\ \mathrm{H} & -1.796309 & -1.978245 & 0.000000 \\ \mathrm{H} & 2.034933 & -0.097726 & 0.000000\end{array}$




$$
\begin{array}{rrrr}
\mathrm{H} & 0.663606 & 3.074871 & 0.000000 \\
\mathrm{H} & 0.144098 & -3.352716 & 0.000000 \\
\mathrm{H} & 1.501145 & -2.668304 & -0.875498 \\
\mathrm{H} & 1.501145 & -2.668304 & 0.875498
\end{array}
$$

$m$-cresol, trans $\mathrm{S}_{1}$

$\begin{array}{rrrr}\mathrm{C} & -1.201533 & -1.058420 & 0.000000 \\ \mathrm{C} & 0.201834 & -1.222914 & 0.000000 \\ \mathrm{C} & 1.012793 & -0.071485 & 0.000000 \\ \mathrm{C} & 0.397002 & 1.196333 & 0.000000 \\ \mathrm{C} & -1.008891 & 1.360680 & 0.000000 \\ \mathrm{C} & -1.816928 & 0.211920 & 0.000000 \\ \mathrm{C} & 0.828207 & -2.582602 & 0.000000 \\ \mathrm{O} & 1.204199 & 2.249642 & 0.000000 \\ \mathrm{H} & -1.432656 & 2.347532 & 0.000000 \\ \mathrm{H} & -2.885040 & 0.292679 & 0.000000 \\ \mathrm{H} & -1.823868 & -1.933691 & 0.000000 \\ \mathrm{H} & 2.083098 & -0.125820 & 0.000000 \\ \mathrm{H} & 0.714884 & 3.056216 & 0.000000 \\ \mathrm{H} & 0.079687 & -3.364566 & 0.000000 \\ \mathrm{H} & 1.461846 & -2.720244 & -0.874210 \\ \mathrm{H} & 1.461846 & -2.720244 & 0.874210\end{array}$

$p$-cresol $\mathrm{S}_{0}$

$\begin{array}{lrcc}\mathrm{C} & 0.705667 & 0.058977 & -1.177256 \\ \mathrm{C} & -0.670230 & 0.054414 & -1.185551 \\ \mathrm{C} & -1.365325 & -0.000781 & 0.012240 \\ \mathrm{C} & -0.669488 & -0.050562 & 1.200402 \\ \mathrm{C} & 0.717915 & -0.045092 & 1.189588 \\ \mathrm{C} & 1.431460 & 0.009372 & 0.009436 \\ \mathrm{O} & -2.717960 & -0.003015 & -0.047445 \\ \mathrm{C} & 2.941057 & 0.015508 & -0.007295 \\ \mathrm{H} & -1.197737 & -0.093685 & 2.137152 \\ \mathrm{H} & 1.241093 & -0.084498 & 2.128053 \\ \mathrm{H} & 1.229670 & 0.102148 & -2.115895 \\ \mathrm{H} & -1.220022 & 0.092980 & -2.106708 \\ \mathrm{H} & -3.092423 & -0.041786 & 0.815515 \\ \mathrm{H} & 3.331426 & -0.835424 & -0.555880 \\ \mathrm{H} & 3.325150 & 0.914008 & -0.479084 \\ \mathrm{H} & 3.341307 & -0.027187 & 0.998027\end{array}$

$p$-cresol $\mathrm{S}_{1}$

$\begin{array}{llll}\text { C } & 0.722126 & -0.070978 & -1.228435\end{array}$ 


$\begin{array}{crrr}\mathrm{C} & -0.680210 & -0.052273 & -1.232496 \\ \mathrm{C} & -1.355860 & -0.003644 & 0.006336 \\ \mathrm{C} & -0.672866 & 0.040529 & 1.240756 \\ \mathrm{C} & 0.729087 & -0.015972 & 1.227400 \\ \mathrm{C} & 1.427821 & -0.032042 & -0.007957 \\ \mathrm{O} & -2.679777 & 0.013095 & -0.041239 \\ \mathrm{C} & 2.919183 & 0.036281 & -0.004364 \\ \mathrm{H} & -1.226062 & 0.087150 & 2.159935 \\ \mathrm{H} & 1.282255 & -0.008346 & 2.146007 \\ \mathrm{H} & 1.265342 & -0.098209 & -2.152376 \\ \mathrm{H} & -1.255913 & -0.073743 & -2.135395 \\ \mathrm{H} & -3.052659 & 0.049146 & 0.824846 \\ \mathrm{H} & 3.334868 & -0.335316 & -0.932428 \\ \mathrm{H} & 3.246114 & 1.067337 & 0.123819 \\ \mathrm{H} & 3.328102 & -0.537633 & 0.820882\end{array}$

$o$-methoxyphenol $\mathrm{S}_{0}$

$\begin{array}{rrrr}\mathrm{C} & 2.024329 & -0.094988 & 0.000000 \\ \mathrm{C} & 0.876286 & 0.688136 & 0.000000 \\ \mathrm{C} & -0.363146 & 0.089479 & 0.000000 \\ \mathrm{C} & -0.463813 & -1.301900 & 0.000000 \\ \mathrm{C} & 0.674159 & -2.070476 & 0.000000 \\ \mathrm{C} & 1.923091 & -1.466269 & 0.000000 \\ \mathrm{O} & -1.555241 & 0.737082 & 0.000000 \\ \mathrm{O} & -1.672254 & -1.898715 & 0.000000 \\ \mathrm{H} & 0.572158 & -3.139133 & 0.000000 \\ \mathrm{H} & 2.807280 & -2.075608 & 0.000000 \\ \mathrm{H} & 2.986797 & 0.380851 & 0.000000 \\ \mathrm{H} & 0.966004 & 1.756395 & 0.000000 \\ \mathrm{C} & -1.586018 & 2.136320 & 0.000000 \\ \mathrm{H} & -2.347891 & -1.239641 & 0.000000 \\ \mathrm{H} & -2.627380 & 2.419180 & 0.000000 \\ \mathrm{H} & -1.107181 & 2.539644 & 0.885625 \\ \mathrm{H} & -1.107181 & 2.539644 & -0.885625\end{array}$

$o$-methoxyphenol $\mathrm{S}_{1}$
$\begin{array}{llll}\text { C } & 2.506247 & -0.000104 & 0.074940\end{array}$
$\begin{array}{llll}\text { C } & 1.391585 & -0.245299 & -0.742452\end{array}$
$\begin{array}{llll}\text { C } & 0.126445 & -0.175283 & -0.111179\end{array}$
$\begin{array}{llll}\text { C } & 0.022083 & -0.063521 & 1.293084\end{array}$
$\begin{array}{llll}\text { C } & 1.172128 & -0.023698 & 2.124873\end{array}$
$\begin{array}{llll}\text { C } & 2.399151 & 0.117331 & 1.476938\end{array}$
O $\quad-1.050863 \quad-0.173430 \quad-0.753322$
$\begin{array}{llll}\mathrm{O} & -1.169131 & 0.049162 & 1.863273\end{array}$ 


$\begin{array}{rrrr}\mathrm{H} & 1.058749 & -0.013942 & 3.188380 \\ \mathrm{H} & 3.292397 & 0.286121 & 2.047933 \\ \mathrm{H} & 3.479140 & 0.075871 & -0.373800 \\ \mathrm{H} & 1.495670 & -0.439488 & -1.788058 \\ \mathrm{C} & -1.103712 & 0.099761 & -2.134909 \\ \mathrm{H} & -1.848722 & 0.006661 & 1.205981 \\ \mathrm{H} & -2.150152 & 0.112804 & -2.396250 \\ \mathrm{H} & -0.658174 & 1.061757 & -2.355536 \\ \mathrm{H} & -0.604280 & -0.674713 & -2.701814\end{array}$

$m$-methoxyphenol, isomer $1 \mathrm{~S}_{0}$

$\begin{array}{lrrr}\mathrm{C} & 0.917996 & 1.432600 & 0.000000 \\ \mathrm{C} & -0.262252 & 0.701223 & 0.000000 \\ \mathrm{C} & -0.222765 & -0.683424 & 0.000000 \\ \mathrm{C} & 1.006652 & -1.330571 & 0.000000 \\ \mathrm{C} & 2.185569 & -0.617352 & 0.000000 \\ \mathrm{C} & 2.121483 & 0.765120 & 0.000000 \\ \mathrm{H} & -1.123415 & -1.266340 & 0.000000 \\ \mathrm{O} & 1.087514 & -2.678367 & 0.000000 \\ \mathrm{H} & 3.121906 & -1.139975 & 0.000000 \\ \mathrm{H} & 3.034685 & 1.331514 & 0.000000 \\ \mathrm{H} & 0.866904 & 2.503725 & 0.000000 \\ \mathrm{O} & -1.400171 & 1.416381 & 0.000000 \\ \mathrm{H} & 0.231870 & -3.070995 & 0.000000 \\ \mathrm{C} & -2.640549 & 0.770802 & 0.000000 \\ \mathrm{H} & -3.386675 & 1.550230 & 0.000000 \\ \mathrm{H} & -2.769375 & 0.157714 & -0.885925 \\ \mathrm{H} & -2.769375 & 0.157714 & 0.885925\end{array}$

m-methoxyphenol, isomer $1 \mathrm{~S}_{1}$

$\begin{array}{lrcc}\mathrm{C} & 1.163582 & 0.010557 & -0.614379 \\ \mathrm{C} & -0.034907 & -0.058432 & 0.130581 \\ \mathrm{C} & -0.024256 & -0.185150 & 1.532414 \\ \mathrm{C} & 1.237960 & -0.018865 & 2.140729 \\ \mathrm{C} & 2.437870 & 0.039228 & 1.407440 \\ \mathrm{C} & 2.421461 & -0.138380 & 0.012253 \\ \mathrm{H} & -0.896947 & -0.397966 & 2.113299 \\ \mathrm{O} & 1.340437 & 0.085443 & 3.466679 \\ \mathrm{H} & 3.346081 & 0.224556 & 1.949149 \\ \mathrm{H} & 3.325801 & -0.150794 & -0.558308 \\ \mathrm{H} & 1.076489 & 0.174699 & -1.671681 \\ \mathrm{O} & -1.148358 & -0.005165 & -0.592674 \\ \mathrm{H} & 0.489710 & 0.197026 & 3.859806 \\ \mathrm{C} & -2.407791 & 0.045613 & 0.027633\end{array}$




$$
\begin{array}{rrrr}
\mathrm{H} & -3.128409 & 0.134057 & -0.769871 \\
\mathrm{H} & -2.605272 & -0.861008 & 0.586679 \\
\mathrm{H} & -2.485141 & 0.904580 & 0.682990
\end{array}
$$

$m$-methoxyphenol, isomer $2 \mathrm{~S}_{0}$

$\begin{array}{lrrc}\mathrm{C} & 0.341951 & 2.144885 & 0.000000 \\ \mathrm{C} & -0.829668 & 1.432392 & 0.000000 \\ \mathrm{C} & -0.778998 & 0.039099 & 0.000000 \\ \mathrm{C} & 0.437163 & -0.613611 & 0.000000 \\ \mathrm{C} & 1.609156 & 0.132467 & 0.000000 \\ \mathrm{C} & 1.576877 & 1.509349 & 0.000000 \\ \mathrm{O} & -1.965699 & -0.588882 & 0.000000 \\ \mathrm{C} & -2.020197 & -1.987484 & 0.000000 \\ \mathrm{O} & 2.764566 & -0.566109 & 0.000000 \\ \mathrm{H} & 0.517865 & -1.680898 & 0.000000 \\ \mathrm{H} & 2.488041 & 2.080103 & 0.000000 \\ \mathrm{H} & 0.305750 & 3.218826 & 0.000000 \\ \mathrm{H} & -1.785647 & 1.918256 & 0.000000 \\ \mathrm{H} & -3.067264 & -2.248228 & 0.000000 \\ \mathrm{H} & -1.550188 & -2.402085 & -0.885265 \\ \mathrm{H} & -1.550188 & -2.402085 & 0.885265 \\ \mathrm{H} & 3.506470 & 0.014015 & 0.000000\end{array}$

$m$-methoxyphenol, isomer $2 \mathrm{~S}_{1}$

$\begin{array}{lrrr}\mathrm{C} & 1.241059 & -0.129770 & -0.692806 \\ \mathrm{C} & 0.064815 & 0.004758 & 0.084846 \\ \mathrm{C} & 0.117122 & -0.054271 & 1.495691 \\ \mathrm{C} & 1.340160 & -0.161000 & 2.182547 \\ \mathrm{C} & 2.496844 & -0.016978 & 1.398028 \\ \mathrm{C} & 2.459827 & 0.040633 & -0.012379 \\ \mathrm{O} & -1.063856 & -0.009893 & 2.103233 \\ \mathrm{C} & -1.147163 & 0.041819 & 3.505004 \\ \mathrm{O} & 3.654411 & 0.055758 & 2.057833 \\ \mathrm{H} & 1.426152 & -0.249620 & 3.242628 \\ \mathrm{H} & 3.378359 & 0.196432 & -0.550782 \\ \mathrm{H} & 1.198258 & -0.141059 & -1.761320 \\ \mathrm{H} & -0.896384 & 0.147823 & -0.370217 \\ \mathrm{H} & -2.198782 & 0.096786 & 3.737940 \\ \mathrm{H} & -0.726791 & -0.849351 & 3.954432 \\ \mathrm{H} & -0.643900 & 0.919123 & 3.893022 \\ \mathrm{H} & 4.385000 & 0.108810 & 1.464198\end{array}$

$p$-methoxyphenol, cis $\mathrm{S}_{0}$ 


$\begin{array}{rrrr}\mathrm{C} & -0.298711 & 0.723305 & 0.000000 \\ \mathrm{C} & -1.527514 & 0.068988 & 0.000000 \\ \mathrm{C} & -1.593806 & -1.300349 & 0.000000 \\ \mathrm{C} & -0.428303 & -2.056247 & 0.000000 \\ \mathrm{C} & 0.785905 & -1.416852 & 0.000000 \\ \mathrm{C} & 0.856106 & -0.025657 & 0.000000 \\ \mathrm{O} & -0.558227 & -3.407131 & 0.000000 \\ \mathrm{O} & -0.347579 & 2.074966 & 0.000000 \\ \mathrm{C} & 0.841437 & 2.806298 & 0.000000 \\ \mathrm{H} & 1.821829 & 0.438720 & 0.000000 \\ \mathrm{H} & -2.423770 & 0.659684 & 0.000000 \\ \mathrm{H} & -2.541872 & -1.803711 & 0.000000 \\ \mathrm{H} & 1.698903 & -1.986567 & 0.000000 \\ \mathrm{H} & 0.559287 & 3.848109 & 0.000000 \\ \mathrm{H} & 1.435709 & 2.601162 & 0.885190 \\ \mathrm{H} & 1.435709 & 2.601162 & -0.885190 \\ \mathrm{H} & 0.284896 & -3.825860 & 0.000000\end{array}$

p-methoxyphenol, cis $\mathrm{S}_{1}$

$\begin{array}{rrrr}\mathrm{C} & 1.339739 & -0.018753 & -0.618621 \\ \mathrm{C} & 0.070333 & -0.074564 & 0.009632 \\ \mathrm{C} & -0.002847 & 0.041004 & 1.403861 \\ \mathrm{C} & 1.184375 & 0.001539 & 2.140907 \\ \mathrm{C} & 2.460895 & -0.086867 & 1.520597 \\ \mathrm{C} & 2.539540 & 0.028111 & 0.127327 \\ \mathrm{O} & 1.087181 & 0.038309 & 3.464040 \\ \mathrm{O} & 1.305553 & -0.010335 & -1.937826 \\ \mathrm{C} & 2.483141 & 0.020315 & -2.707350 \\ \mathrm{H} & 3.491142 & 0.071585 & -0.358362 \\ \mathrm{H} & -0.802971 & -0.121466 & -0.607952 \\ \mathrm{H} & -0.936471 & 0.121089 & 1.922898 \\ \mathrm{H} & 3.345255 & -0.170379 & 2.123575 \\ \mathrm{H} & 2.162497 & 0.020306 & -3.736668 \\ \mathrm{H} & 3.053543 & 0.918083 & -2.506336 \\ \mathrm{H} & 3.092546 & -0.854524 & -2.518708 \\ \mathrm{H} & 1.940079 & 0.076533 & 3.865987\end{array}$

p-methoxyphenol, trans $\mathrm{S}_{0}$
$\begin{array}{llll}\text { C } & -0.647981 & 1.287485 & 0.000000\end{array}$
$\begin{array}{llll}\text { C } & -1.876955 & 0.665909 & 0.000000\end{array}$
$\begin{array}{llll}\text { C } & -1.961395 & -0.716734 & 0.000000\end{array}$
$\begin{array}{llll}\text { C } & -0.803908 & -1.460215 & 0.000000\end{array}$
$\begin{array}{llll}\text { C } & 0.434333 & -0.837245 & 0.000000\end{array}$
$\begin{array}{llll}\text { C } & 0.520366 & 0.542507 & 0.000000\end{array}$ 


$\begin{array}{cccc}\mathrm{O} & -3.146795 & -1.378584 & 0.000000 \\ \mathrm{H} & 1.315237 & -1.447485 & 0.000000 \\ \mathrm{O} & 1.676511 & 1.245533 & 0.000000 \\ \mathrm{H} & -0.578076 & 2.358525 & 0.000000 \\ \mathrm{H} & -2.772591 & 1.262654 & 0.000000 \\ \mathrm{H} & -3.868868 & -0.774815 & 0.000000 \\ \mathrm{H} & -0.870223 & -2.531554 & 0.000000 \\ \mathrm{C} & 2.896467 & 0.566097 & 0.000000 \\ \mathrm{H} & 3.666472 & 1.322448 & 0.000000 \\ \mathrm{H} & 3.008698 & -0.052274 & 0.885174 \\ \mathrm{H} & 3.008698 & -0.052274 & -0.885174\end{array}$

p-methoxyphenol, trans $\mathrm{S}_{1}$

$\begin{array}{lrrr}\mathrm{C} & 0.120651 & 0.012804 & -1.376018 \\ \mathrm{C} & -1.267810 & -0.081315 & -1.209453 \\ \mathrm{C} & -1.786885 & 0.028199 & 0.092178 \\ \mathrm{C} & -0.957699 & 0.114116 & 1.232693 \\ \mathrm{C} & 0.430558 & -0.000204 & 1.078395 \\ \mathrm{C} & 0.958162 & -0.008949 & -0.230204 \\ \mathrm{O} & -3.095298 & 0.049384 & 0.313635 \\ \mathrm{H} & 1.069359 & -0.023196 & 1.935307 \\ \mathrm{O} & 2.250766 & -0.034396 & -0.495034 \\ \mathrm{H} & 0.582249 & 0.021538 & -2.342210 \\ \mathrm{H} & -1.928651 & -0.160857 & -2.051722 \\ \mathrm{H} & -3.577215 & 0.013642 & -0.496757 \\ \mathrm{H} & -1.416479 & 0.207691 & 2.196061 \\ \mathrm{C} & 3.212893 & -0.024850 & 0.532049 \\ \mathrm{H} & 4.171022 & -0.050638 & 0.038397 \\ \mathrm{H} & 3.135829 & 0.876738 & 1.126474 \\ \mathrm{H} & 3.109339 & -0.895399 & 1.166910\end{array}$

1-naphthol, cis $\mathrm{S}_{0}$
$\begin{array}{llll}\text { C } & 0.828491 & 1.506092 & -0.013623\end{array}$
$\begin{array}{llll}\text { C } & 0.520593 & 0.114978 & -0.021618\end{array}$
$\begin{array}{llll}\text { C } & -0.833569 & -0.268582 & -0.000295\end{array}$
$\begin{array}{llll}\text { C } & -1.845770 & 0.722764 & -0.008407\end{array}$
$\begin{array}{llll}\text { C } & -1.510248 & 2.034122 & -0.031533\end{array}$
$\begin{array}{llll}\text { C } & -0.157839 & 2.434169 & -0.027277\end{array}$
$\begin{array}{llll}\text { C } & 1.517021 & -0.894694 & -0.057641\end{array}$
$\begin{array}{llll}\text { C } & 1.179636 & -2.207279 & -0.043631\end{array}$
$\begin{array}{llll}\text { C } & -0.178090 & -2.592671 & 0.000146\end{array}$
$\begin{array}{llll}\text { C } & -1.151130 & -1.650689 & 0.016278\end{array}$
$\begin{array}{llll}\mathrm{O} & 2.114053 & 1.938882 & -0.030015\end{array}$
H $\quad-2.185926 \quad-1.939820 \quad 0.039012$ 


$\begin{array}{rrrr}\mathrm{H} & -0.433402 & -3.635968 & 0.012047 \\ \mathrm{H} & 1.944882 & -2.960188 & -0.076174 \\ \mathrm{H} & 2.555261 & -0.627767 & -0.121043 \\ \mathrm{H} & -2.875813 & 0.418325 & 0.000690 \\ \mathrm{H} & -2.275049 & 2.788389 & -0.041798 \\ \mathrm{H} & 0.106127 & 3.474250 & -0.025744 \\ \mathrm{H} & 2.680783 & 1.345696 & 0.430623\end{array}$

1-naphthol, cis $\mathrm{S}_{1}$

$\begin{array}{lrrr}\mathrm{C} & 0.880234 & 1.459042 & 0.009613 \\ \mathrm{C} & 0.545924 & 0.095420 & -0.041875 \\ \mathrm{C} & -0.856283 & -0.244991 & 0.020859 \\ \mathrm{C} & -1.797918 & 0.786952 & -0.006504 \\ \mathrm{C} & -1.417604 & 2.149965 & -0.019077 \\ \mathrm{C} & -0.100731 & 2.485725 & -0.032532 \\ \mathrm{C} & 1.485595 & -0.938433 & -0.094906 \\ \mathrm{C} & 1.080130 & -2.296234 & -0.050777 \\ \mathrm{C} & -0.244470 & -2.617973 & 0.024669 \\ \mathrm{C} & -1.219813 & -1.594874 & 0.045796 \\ \mathrm{O} & 2.145355 & 1.881578 & 0.071019 \\ \mathrm{H} & -2.260837 & -1.854681 & 0.093693 \\ \mathrm{H} & -0.555795 & -3.644665 & 0.057223 \\ \mathrm{H} & 1.828954 & -3.064771 & -0.080883 \\ \mathrm{H} & 2.533847 & -0.732366 & -0.208758 \\ \mathrm{H} & -2.842623 & 0.537684 & 0.001732 \\ \mathrm{H} & -2.174793 & 2.909640 & -0.042306 \\ \mathrm{H} & 0.234308 & 3.504187 & -0.031126 \\ \mathrm{H} & 2.736519 & 1.178786 & 0.284121\end{array}$

1-naphthol, trans $\mathrm{S}_{0}$

$\begin{array}{lrrc}\mathrm{C} & -0.706760 & -0.534195 & 0.000000 \\ \mathrm{C} & -0.666913 & -1.950873 & 0.000000 \\ \mathrm{C} & 0.515852 & -2.613177 & 0.000000 \\ \mathrm{C} & 1.734093 & -1.899086 & 0.000000 \\ \mathrm{C} & 1.731050 & -0.542901 & 0.000000 \\ \mathrm{C} & 0.508001 & 0.171096 & 0.000000 \\ \mathrm{H} & -1.594289 & -2.494039 & 0.000000 \\ \mathrm{H} & 0.531613 & -3.687553 & 0.000000 \\ \mathrm{H} & 2.664219 & -2.436281 & 0.000000 \\ \mathrm{H} & 2.651756 & 0.005805 & 0.000000 \\ \mathrm{C} & -1.934763 & 0.178808 & 0.000000 \\ \mathrm{C} & -1.931683 & 1.530726 & 0.000000 \\ \mathrm{C} & -0.717277 & 2.254205 & 0.000000 \\ \mathrm{C} & 0.466876 & 1.594177 & 0.000000\end{array}$




$\begin{array}{rrrr}\mathrm{H} & -2.858888 & -0.368686 & 0.000000 \\ \mathrm{H} & -2.858148 & 2.074579 & 0.000000 \\ \mathrm{H} & -0.737544 & 3.329886 & 0.000000 \\ \mathrm{O} & 1.660350 & 2.226588 & 0.000000 \\ \mathrm{H} & 1.542465 & 3.160912 & 0.000000\end{array}$

1-naphthol, trans $\mathrm{S}_{1}$

$\begin{array}{lrrl}\mathrm{C} & -0.948639 & -0.386186 & 0.000000 \\ \mathrm{C} & -1.297413 & -1.740220 & 0.000000 \\ \mathrm{C} & -0.313119 & -2.755328 & 0.000000 \\ \mathrm{C} & 1.011271 & -2.415982 & 0.000000 \\ \mathrm{C} & 1.405507 & -1.055324 & 0.000000 \\ \mathrm{C} & 0.452552 & -0.035467 & 0.000000 \\ \mathrm{H} & -2.337181 & -2.010739 & 0.000000 \\ \mathrm{H} & -0.615081 & -3.785185 & 0.000000 \\ \mathrm{H} & 1.769017 & -3.176599 & 0.000000 \\ \mathrm{H} & 2.447367 & -0.804653 & 0.000000 \\ \mathrm{C} & -1.898634 & 0.640295 & 0.000000 \\ \mathrm{C} & -1.520670 & 2.001942 & 0.000000 \\ \mathrm{C} & -0.201262 & 2.345602 & 0.000000 \\ \mathrm{C} & 0.786334 & 1.326326 & 0.000000 \\ \mathrm{H} & -2.942693 & 0.388221 & 0.000000 \\ \mathrm{H} & -2.278897 & 2.761232 & 0.000000 \\ \mathrm{H} & 0.104490 & 3.376276 & 0.000000 \\ \mathrm{O} & 2.088804 & 1.645320 & 0.000000 \\ \mathrm{H} & 2.212566 & 2.579543 & 0.000000\end{array}$

2-naphthol, cis $\mathrm{S}_{0}$

$\begin{array}{lrrc}\mathrm{C} & 0.591233 & 2.004377 & 0.000000 \\ \mathrm{C} & 0.611060 & 0.589171 & 0.000000 \\ \mathrm{C} & -0.603313 & -0.115912 & 0.000000 \\ \mathrm{C} & -1.815700 & 0.617632 & 0.000000 \\ \mathrm{C} & -1.804818 & 1.974062 & 0.000000 \\ \mathrm{C} & -0.583146 & 2.682696 & 0.000000 \\ \mathrm{C} & 1.823392 & -0.150263 & 0.000000 \\ \mathrm{C} & 1.821859 & -1.501116 & 0.000000 \\ \mathrm{C} & 0.592995 & -2.204478 & 0.000000 \\ \mathrm{C} & -0.584666 & -1.535193 & 0.000000 \\ \mathrm{H} & -1.517847 & -2.071584 & 0.000000 \\ \mathrm{H} & 1.525610 & 2.536368 & 0.000000 \\ \mathrm{H} & -0.590783 & 3.756672 & 0.000000 \\ \mathrm{H} & -2.731395 & 2.518146 & 0.000000 \\ \mathrm{H} & -2.748489 & 0.083347 & 0.000000 \\ \mathrm{H} & 2.756862 & 0.382790 & 0.000000\end{array}$




$\begin{array}{rrrr}\mathrm{H} & 2.734518 & -2.065588 & 0.000000 \\ \mathrm{O} & 0.688112 & -3.551406 & 0.000000 \\ \mathrm{H} & -0.165495 & -3.949713 & 0.000000\end{array}$

2-naphthol, cis $\mathrm{S}_{1}$

$\begin{array}{lrrl}\mathrm{C} & 0.598786 & 1.987485 & 0.000000 \\ \mathrm{C} & 0.643804 & 0.591484 & 0.000000 \\ \mathrm{C} & -0.619154 & -0.122601 & 0.000000 \\ \mathrm{C} & -1.818235 & 0.605736 & 0.000000 \\ \mathrm{C} & -1.825282 & 2.013154 & 0.000000 \\ \mathrm{C} & -0.627984 & 2.686870 & 0.000000 \\ \mathrm{C} & 1.834053 & -0.145910 & 0.000000 \\ \mathrm{C} & 1.851192 & -1.551215 & 0.000000 \\ \mathrm{C} & 0.655896 & -2.220008 & 0.000000 \\ \mathrm{C} & -0.578977 & -1.513546 & 0.000000 \\ \mathrm{H} & -1.497951 & -2.074006 & 0.000000 \\ \mathrm{H} & 1.517508 & 2.544434 & 0.000000 \\ \mathrm{H} & -0.610812 & 3.760682 & 0.000000 \\ \mathrm{H} & -2.756378 & 2.545415 & 0.000000 \\ \mathrm{H} & -2.750653 & 0.071084 & 0.000000 \\ \mathrm{H} & 2.768600 & 0.384303 & 0.000000 \\ \mathrm{H} & 2.772065 & -2.099162 & 0.000000 \\ \mathrm{O} & 0.659968 & -3.558935 & 0.000000 \\ \mathrm{H} & -0.216434 & -3.905256 & 0.000000\end{array}$

2-naphthol, trans $\mathrm{S}_{0}$

$\begin{array}{rrrr}\mathrm{C} & 0.504333 & 2.007410 & 0.000000 \\ \mathrm{C} & 0.531795 & 0.589629 & 0.000000 \\ \mathrm{C} & -0.679879 & -0.123966 & 0.000000 \\ \mathrm{C} & -1.897752 & 0.606258 & 0.000000 \\ \mathrm{C} & -1.892738 & 1.960708 & 0.000000 \\ \mathrm{C} & -0.672417 & 2.677477 & 0.000000 \\ \mathrm{C} & 1.743480 & -0.140839 & 0.000000 \\ \mathrm{C} & 1.744720 & -1.495550 & 0.000000 \\ \mathrm{C} & 0.521154 & -2.204992 & 0.000000 \\ \mathrm{C} & -0.658612 & -1.538242 & 0.000000 \\ \mathrm{H} & -1.578142 & -2.092517 & 0.000000 \\ \mathrm{H} & 1.435871 & 2.544454 & 0.000000 \\ \mathrm{H} & -0.686965 & 3.751410 & 0.000000 \\ \mathrm{H} & -2.821546 & 2.500992 & 0.000000 \\ \mathrm{H} & -2.826903 & 0.066180 & 0.000000 \\ \mathrm{H} & 2.676044 & 0.393776 & 0.000000 \\ \mathrm{H} & 2.673996 & -2.038112 & 0.000000 \\ \mathrm{O} & 0.504890 & -3.557187 & 0.000000\end{array}$


$\begin{array}{llll}\mathrm{H} & 1.378682 & -3.906887 & 0.000000\end{array}$

2-naphthol, trans $\mathrm{S}_{1}$

$\begin{array}{rrrr}\mathrm{C} & 0.510035 & 1.992893 & 0.000000 \\ \mathrm{C} & 0.561055 & 0.597044 & 0.000000 \\ \mathrm{C} & -0.698192 & -0.124197 & 0.000000 \\ \mathrm{C} & -1.899384 & 0.598865 & 0.000000 \\ \mathrm{C} & -1.912600 & 2.006577 & 0.000000 \\ \mathrm{C} & -0.719545 & 2.686464 & 0.000000 \\ \mathrm{C} & 1.754282 & -0.132776 & 0.000000 \\ \mathrm{C} & 1.776587 & -1.541263 & 0.000000 \\ \mathrm{C} & 0.581140 & -2.213032 & 0.000000 \\ \mathrm{C} & -0.655177 & -1.514442 & 0.000000 \\ \mathrm{H} & -1.557504 & -2.095856 & 0.000000 \\ \mathrm{H} & 1.426258 & 2.554166 & 0.000000 \\ \mathrm{H} & -0.707732 & 3.760372 & 0.000000 \\ \mathrm{H} & -2.846482 & 2.534084 & 0.000000 \\ \mathrm{H} & -2.828844 & 0.059447 & 0.000000 \\ \mathrm{H} & 2.686846 & 0.400974 & 0.000000 \\ \mathrm{H} & 2.710635 & -2.071555 & 0.000000 \\ \mathrm{O} & 0.483039 & -3.547630 & 0.000000 \\ \mathrm{H} & 1.335581 & -3.950127 & 0.000000\end{array}$

5-methoxysalicylic acid $\mathrm{S}_{0}$

$\begin{array}{cccc}\mathrm{C} & 2.259664 & -0.164748 & 0.000000 \\ \mathrm{C} & 1.548458 & -1.326558 & 0.000000 \\ \mathrm{C} & 0.148156 & -1.317063 & 0.000000 \\ \mathrm{C} & -0.503156 & -0.118564 & 0.000000 \\ \mathrm{C} & 0.220348 & 1.090337 & 0.000000 \\ \mathrm{C} & 1.606265 & 1.071773 & 0.000000 \\ \mathrm{O} & -0.444646 & -2.531836 & 0.000000 \\ \mathrm{C} & -1.839651 & -2.611681 & 0.000000 \\ \mathrm{C} & -0.487594 & 2.381298 & 0.000000 \\ \mathrm{O} & -1.807438 & 2.286516 & 0.000000 \\ \mathrm{O} & 2.376652 & 2.158917 & 0.000000 \\ \mathrm{O} & 0.051173 & 3.448783 & 0.000000 \\ \mathrm{H} & -2.084726 & -3.662574 & 0.000000 \\ \mathrm{H} & -2.263604 & -2.148203 & -0.885320 \\ \mathrm{H} & -2.263604 & -2.148203 & 0.885320 \\ \mathrm{H} & -1.570886 & -0.065143 & 0.000000 \\ \mathrm{H} & 3.332419 & -0.172294 & 0.000000 \\ \mathrm{H} & 2.052112 & -2.274751 & 0.000000 \\ \mathrm{H} & 1.839618 & 2.942327 & 0.000000 \\ \mathrm{H} & -2.169541 & 3.161668 & 0.000000\end{array}$


5-methoxysalicylic acid $\mathrm{S}_{1}$

$\begin{array}{lrrc}\mathrm{C} & 2.165277 & -0.131319 & 0.000000 \\ \mathrm{C} & 1.708264 & -1.441446 & 0.000000 \\ \mathrm{C} & 0.313963 & -1.667898 & 0.000000 \\ \mathrm{C} & -0.612338 & -0.604042 & 0.000000 \\ \mathrm{C} & -0.175213 & 0.718484 & 0.000000 \\ \mathrm{C} & 1.276347 & 0.938930 & 0.000000 \\ \mathrm{O} & -0.046739 & -2.935724 & 0.000000 \\ \mathrm{C} & -1.401140 & -3.325123 & 0.000000 \\ \mathrm{C} & -1.052562 & 1.843584 & 0.000000 \\ \mathrm{O} & -2.353880 & 1.547729 & 0.000000 \\ \mathrm{O} & 1.746846 & 2.151748 & 0.000000 \\ \mathrm{O} & -0.692993 & 3.009664 & 0.000000 \\ \mathrm{H} & -1.395443 & -4.402825 & 0.000000 \\ \mathrm{H} & -1.907274 & -2.965604 & -0.886753 \\ \mathrm{H} & -1.907274 & -2.965604 & 0.886753 \\ \mathrm{H} & -1.662613 & -0.800821 & 0.000000 \\ \mathrm{H} & 3.213869 & 0.094569 & 0.000000 \\ \mathrm{H} & 2.372190 & -2.280982 & 0.000000 \\ \mathrm{H} & 1.016360 & 2.784363 & 0.000000 \\ \mathrm{H} & -2.833594 & 2.363095 & 0.000000\end{array}$

$m$-dimethoxybenzene, trans $\mathrm{S}_{0}$

$\begin{array}{lrrr}\mathrm{C} & 0.000000 & -0.703933 & -0.158156 \\ \mathrm{C} & 0.000000 & 0.703933 & -0.158156 \\ \mathrm{C} & 0.000000 & 1.378328 & 1.039280 \\ \mathrm{C} & 0.000000 & 0.683603 & 2.247728 \\ \mathrm{C} & 0.000000 & -0.683603 & 2.247728 \\ \mathrm{C} & 0.000000 & -1.378328 & 1.039280 \\ \mathrm{O} & 0.000000 & 1.289453 & -1.368165 \\ \mathrm{H} & 0.000000 & 2.449697 & 1.054671 \\ \mathrm{H} & 0.000000 & 1.232010 & 3.170954 \\ \mathrm{H} & 0.000000 & -1.232010 & 3.170954 \\ \mathrm{H} & 0.000000 & -2.449697 & 1.054671 \\ \mathrm{O} & 0.000000 & -1.289453 & -1.368165 \\ \mathrm{C} & 0.000000 & -2.682344 & -1.461782 \\ \mathrm{H} & 0.000000 & -2.913927 & -2.515844 \\ \mathrm{H} & 0.885146 & -3.113274 & -1.004333 \\ \mathrm{H} & -0.885146 & -3.113274 & -1.004333 \\ \mathrm{C} & 0.000000 & 2.682344 & -1.461782 \\ \mathrm{H} & 0.000000 & 2.913927 & -2.515844 \\ \mathrm{H} & -0.885146 & 3.113274 & -1.004333 \\ \mathrm{H} & 0.885146 & 3.113274 & -1.004333\end{array}$


$m$-dimethoxybenzene, trans $\mathrm{S}_{1}$

$\begin{array}{rrrr}\text { C } & -0.115071 & -1.078424 & -1.434195 \\ \mathrm{C} & -0.175187 & 0.148375 & -0.708193 \\ \mathrm{C} & -0.175187 & 0.148375 & 0.708193 \\ \mathrm{C} & -0.115071 & -1.078424 & 1.434195 \\ \mathrm{C} & 0.226494 & -2.217676 & 0.704365 \\ \mathrm{C} & 0.226494 & -2.217676 & -0.704365 \\ \mathrm{O} & -0.197561 & 1.340984 & -1.286013 \\ \mathrm{C} & -0.024229 & 1.470661 & -2.674599 \\ \mathrm{O} & -0.197561 & 1.340984 & 1.286013 \\ \mathrm{C} & -0.024229 & 1.470661 & 2.674599 \\ \mathrm{H} & -0.229252 & -1.108238 & 2.495750 \\ \mathrm{H} & 0.455128 & -3.132457 & 1.219562 \\ \mathrm{H} & 0.455128 & -3.132457 & -1.219562 \\ \mathrm{H} & -0.229252 & -1.108238 & -2.495750 \\ \mathrm{H} & -0.051110 & 2.529948 & -2.875643 \\ \mathrm{H} & 0.929286 & 1.065172 & -2.990190 \\ \mathrm{H} & -0.825407 & 0.984095 & -3.216971 \\ \mathrm{H} & -0.051110 & 2.529948 & 2.875643 \\ \mathrm{H} & -0.825407 & 0.984095 & 3.216971 \\ \mathrm{H} & 0.929286 & 1.065172 & 2.990190\end{array}$

3P-propionic acid, gauche $\mathrm{S}_{0}$

$\begin{array}{rrrr}\mathrm{C} & -1.018021 & 0.861276 & -1.164967 \\ \mathrm{C} & -0.397370 & 0.650286 & 0.059378 \\ \mathrm{C} & -1.082372 & -0.061790 & 1.032334 \\ \mathrm{C} & -2.351993 & -0.554066 & 0.785735 \\ \mathrm{C} & -2.957293 & -0.341375 & -0.437660 \\ \mathrm{C} & -2.284689 & 0.369590 & -1.414916 \\ \mathrm{C} & 0.995530 & 1.187637 & 0.312704 \\ \mathrm{C} & 2.111784 & 0.286715 & -0.223537 \\ \mathrm{C} & 2.335657 & -0.959367 & 0.594764 \\ \mathrm{O} & 2.049601 & -1.099008 & 1.734114 \\ \mathrm{O} & 2.952531 & -1.908855 & -0.100940 \\ \mathrm{H} & 1.933497 & -0.000289 & -1.251375 \\ \mathrm{H} & 3.058869 & 0.820262 & -0.208106 \\ \mathrm{H} & 1.144615 & 1.341492 & 1.372836 \\ \mathrm{H} & 1.092583 & 2.154761 & -0.167277 \\ \mathrm{H} & -0.617631 & -0.238057 & 1.984171 \\ \mathrm{H} & -2.867193 & -1.102766 & 1.552693 \\ \mathrm{H} & -3.943778 & -0.722057 & -0.627755 \\ \mathrm{H} & -2.748186 & 0.546521 & -2.368162 \\ \mathrm{H} & -0.509399 & 1.421714 & -1.929951\end{array}$




\section{$\begin{array}{llll}\mathrm{H} & 3.103269 & -2.652645 & 0.465928\end{array}$}

3P-propionic acid, gauche $\mathrm{S}_{1}$

$\begin{array}{lrrr}\mathrm{C} & -1.041781 & 0.950698 & -1.176418 \\ \mathrm{C} & -0.385401 & 0.671311 & 0.048043 \\ \mathrm{C} & -1.061136 & -0.094313 & 1.029660 \\ \mathrm{C} & -2.354269 & -0.598284 & 0.782605 \\ \mathrm{C} & -2.987271 & -0.331379 & -0.447897 \\ \mathrm{C} & -2.330344 & 0.438786 & -1.428954 \\ \mathrm{C} & 1.009381 & 1.175856 & 0.283645 \\ \mathrm{C} & 2.109485 & 0.239788 & -0.240338 \\ \mathrm{C} & 2.358963 & -0.959419 & 0.638422 \\ \mathrm{O} & 2.105320 & -1.037767 & 1.791306 \\ \mathrm{O} & 2.961170 & -1.942513 & -0.021796 \\ \mathrm{H} & 1.897963 & -0.099777 & -1.245152 \\ \mathrm{H} & 3.055818 & 0.773493 & -0.282132 \\ \mathrm{H} & 1.168639 & 1.344492 & 1.340788 \\ \mathrm{H} & 1.123473 & 2.129853 & -0.220417 \\ \mathrm{H} & -0.571801 & -0.302547 & 1.960370 \\ \mathrm{H} & -2.852017 & -1.185523 & 1.528726 \\ \mathrm{H} & -3.970197 & -0.715475 & -0.640060 \\ \mathrm{H} & -2.814745 & 0.640949 & -2.364097 \\ \mathrm{H} & -0.553269 & 1.555808 & -1.916082 \\ \mathrm{H} & 3.132032 & -2.654047 & 0.579779\end{array}$

3P-propionic acid, anti $\mathrm{S}_{0}$

$\begin{array}{rrrr}\mathrm{C} & 1.461421 & -0.448419 & 0.000000 \\ \mathrm{C} & 0.542169 & 0.772932 & 0.000000 \\ \mathrm{C} & 2.923152 & -0.086839 & 0.000000 \\ \mathrm{H} & 1.279830 & -1.075736 & 0.866125 \\ \mathrm{H} & 1.279830 & -1.075736 & -0.866125 \\ \mathrm{C} & -0.918444 & 0.380759 & 0.000000 \\ \mathrm{H} & 0.760276 & 1.383126 & 0.867163 \\ \mathrm{H} & 0.760276 & 1.383126 & -0.867163 \\ \mathrm{C} & -1.602569 & 0.186550 & 1.191153 \\ \mathrm{C} & -2.931039 & -0.196760 & 1.193658 \\ \mathrm{C} & -3.600406 & -0.391097 & 0.000000 \\ \mathrm{C} & -2.931039 & -0.196760 & -1.193658 \\ \mathrm{C} & -1.602569 & 0.186550 & -1.191153 \\ \mathrm{H} & -1.095425 & 0.342484 & 2.126930 \\ \mathrm{H} & -3.443576 & -0.338634 & 2.127416 \\ \mathrm{H} & -4.633462 & -0.685614 & 0.000000 \\ \mathrm{H} & -3.443576 & -0.338634 & -2.127416 \\ \mathrm{H} & -1.095425 & 0.342484 & -2.126930\end{array}$



$\begin{array}{llll}\text { O } & 3.698834 & -1.167953 & 0.000000\end{array}$
$\begin{array}{llll}\mathrm{O} & 3.369472 & 1.008237 & 0.000000\end{array}$
H $\quad 4.605110 \quad-0.892744 \quad 0.000000$

3P-propionic acid, anti $\mathrm{S}_{1}$

$\begin{array}{rrrr}\mathrm{C} & 1.474277 & -0.437697 & 0.000000 \\ \mathrm{C} & 0.560049 & 0.794021 & 0.000000 \\ \mathrm{C} & 2.936653 & -0.078407 & 0.000000 \\ \mathrm{H} & 1.289412 & -1.061835 & 0.867040 \\ \mathrm{H} & 1.289412 & -1.061835 & -0.867040 \\ \mathrm{C} & -0.892946 & 0.420996 & 0.000000 \\ \mathrm{H} & 0.783558 & 1.399387 & 0.870100 \\ \mathrm{H} & 0.783558 & 1.399387 & -0.870100 \\ \mathrm{C} & -1.582872 & 0.222275 & 1.220681 \\ \mathrm{C} & -2.924421 & -0.209393 & 1.222649 \\ \mathrm{C} & -3.593139 & -0.421650 & 0.000000 \\ \mathrm{C} & -2.924421 & -0.209393 & -1.222649 \\ \mathrm{C} & -1.582872 & 0.222275 & -1.220681 \\ \mathrm{H} & -1.076911 & 0.401859 & 2.149943 \\ \mathrm{H} & -3.436968 & -0.371199 & 2.150315 \\ \mathrm{H} & -4.616140 & -0.744428 & 0.000000 \\ \mathrm{H} & -3.436968 & -0.371199 & -2.150315 \\ \mathrm{H} & -1.076911 & 0.401859 & -2.149943 \\ \mathrm{O} & 3.709384 & -1.161190 & 0.000000 \\ \mathrm{O} & 3.384355 & 1.015948 & 0.000000 \\ \mathrm{H} & 4.616536 & -0.888623 & 0.000000\end{array}$

fluorine $\mathrm{S}_{0}$
$\begin{array}{llll}\text { C } & 0.000000 & 0.000000 & -1.789671\end{array}$
$\begin{array}{llll}\text { C } & 0.000000 & 1.178032 & -0.841661\end{array}$
$\begin{array}{llll}\text { C } & 0.000000 & 0.736387 & 0.478887\end{array}$
$\begin{array}{llll}\text { C } & 0.000000 & -0.736387 & 0.478887\end{array}$
$\begin{array}{llll}\text { C } & 0.000000 & 1.642459 & 1.522693\end{array}$
$\begin{array}{llll}\mathrm{C} & 0.000000 & 2.995467 & 1.232637\end{array}$
$\begin{array}{llll}\text { C } & 0.000000 & 3.434957 & -0.081028\end{array}$
$\begin{array}{llll}\text { C } & 0.000000 & 2.525709 & -1.127216\end{array}$
$\mathrm{H} \quad 0.000000 \quad 2.873810 \quad-2.144530$
$\mathrm{H} \quad 0.000000 \quad 4.488733 \quad-0.289808$
$\mathrm{H} \quad 0.000000 \quad 3.712104 \quad 2.032966$
$\begin{array}{llll}\mathrm{H} & 0.000000 & 1.310078 & 2.544376\end{array}$
$\begin{array}{llll}\text { C } & 0.000000 & -1.642459 & 1.522693\end{array}$
$\begin{array}{llll}\text { C } & 0.000000 & -2.995467 & 1.232637\end{array}$
$\begin{array}{llll}\text { C } & 0.000000 & -3.434957 & -0.081028\end{array}$
$\begin{array}{llll}\text { C } & 0.000000 & -2.525709 & -1.127216\end{array}$ 


$\begin{array}{rrrr}\mathrm{C} & 0.000000 & -1.178032 & -0.841661 \\ \mathrm{H} & 0.000000 & -1.310078 & 2.544376 \\ \mathrm{H} & 0.000000 & -3.712104 & 2.032966 \\ \mathrm{H} & 0.000000 & -4.488733 & -0.289808 \\ \mathrm{H} & 0.000000 & -2.873810 & -2.144530 \\ \mathrm{H} & -0.874918 & 0.000000 & -2.432470 \\ \mathrm{H} & 0.874918 & 0.000000 & -2.432470\end{array}$

fluorine $\mathrm{S}_{1}$

$\begin{array}{lrrr}\mathrm{C} & 0.000000 & 0.000000 & -1.870905 \\ \mathrm{C} & 0.000000 & 1.175814 & -0.915451 \\ \mathrm{C} & 0.000000 & 0.694047 & 0.447338 \\ \mathrm{C} & 0.000000 & -0.694047 & 0.447338 \\ \mathrm{C} & 0.000000 & 1.634503 & 1.525762 \\ \mathrm{C} & 0.000000 & 2.966107 & 1.227864 \\ \mathrm{C} & 0.000000 & 3.421047 & -0.104723 \\ \mathrm{C} & 0.000000 & 2.512768 & -1.174724 \\ \mathrm{H} & 0.000000 & 2.880863 & -2.184897 \\ \mathrm{H} & 0.000000 & 4.476613 & -0.302311 \\ \mathrm{H} & 0.000000 & 3.690130 & 2.021693 \\ \mathrm{H} & 0.000000 & 1.294884 & 2.544131 \\ \mathrm{C} & 0.000000 & -1.634503 & 1.525762 \\ \mathrm{C} & 0.000000 & -2.966107 & 1.227864 \\ \mathrm{C} & 0.000000 & -3.421047 & -0.104723 \\ \mathrm{C} & 0.000000 & -2.512768 & -1.174724 \\ \mathrm{C} & 0.000000 & -1.175814 & -0.915451 \\ \mathrm{H} & 0.000000 & -1.294884 & 2.544131 \\ \mathrm{H} & 0.000000 & -3.690130 & 2.021693 \\ \mathrm{H} & 0.000000 & -4.476613 & -0.302311 \\ \mathrm{H} & 0.000000 & -2.880863 & -2.184897 \\ \mathrm{H} & -0.874325 & 0.000000 & -2.517068 \\ \mathrm{H} & 0.874325 & 0.000000 & -2.517068\end{array}$

phenanthrene $\mathrm{S}_{0}$

$\begin{array}{lrrc}\mathrm{C} & 0.729575 & 0.000000 & -0.346734 \\ \mathrm{C} & -0.729575 & 0.000000 & -0.346734 \\ \mathrm{C} & -1.410497 & 0.000000 & 0.877087 \\ \mathrm{C} & -0.667571 & 0.000000 & 2.108929 \\ \mathrm{C} & 0.667571 & 0.000000 & 2.108929 \\ \mathrm{C} & 1.410497 & 0.000000 & 0.877087 \\ \mathrm{C} & -1.495287 & 0.000000 & -1.528349 \\ \mathrm{C} & -2.816605 & 0.000000 & 0.895209 \\ \mathrm{H} & -1.214077 & 0.000000 & 3.034265 \\ \mathrm{H} & 1.214077 & 0.000000 & 3.034265\end{array}$




$\begin{array}{lrrr}\mathrm{C} & 1.495287 & 0.000000 & -1.528349 \\ \mathrm{C} & 2.859365 & 0.000000 & -1.490136 \\ \mathrm{C} & 3.533281 & 0.000000 & -0.263524 \\ \mathrm{C} & 2.816605 & 0.000000 & 0.895209 \\ \mathrm{H} & 1.011373 & 0.000000 & -2.484005 \\ \mathrm{H} & 3.418411 & 0.000000 & -2.407475 \\ \mathrm{H} & 4.607118 & 0.000000 & -0.239052 \\ \mathrm{H} & 3.322002 & 0.000000 & 1.843877 \\ \mathrm{C} & -3.533281 & 0.000000 & -0.263524 \\ \mathrm{C} & -2.859365 & 0.000000 & -1.490136 \\ \mathrm{H} & -1.011373 & 0.000000 & -2.484005 \\ \mathrm{H} & -3.418411 & 0.000000 & -2.407475 \\ \mathrm{H} & -4.607118 & 0.000000 & -0.239052 \\ \mathrm{H} & -3.322002 & 0.000000 & 1.843877\end{array}$

phenanthrene $\mathrm{S}_{1}$

$\begin{array}{lrrr}\mathrm{C} & 0.727112 & 0.000000 & -0.390335 \\ \mathrm{C} & -0.727112 & 0.000000 & -0.390335 \\ \mathrm{C} & -1.425656 & 0.000000 & 0.877054 \\ \mathrm{C} & -0.699281 & 0.000000 & 2.065882 \\ \mathrm{C} & 0.699281 & 0.000000 & 2.065882 \\ \mathrm{C} & 1.425656 & 0.000000 & 0.877054 \\ \mathrm{C} & -1.492614 & 0.000000 & -1.551215 \\ \mathrm{C} & -2.842978 & 0.000000 & 0.874656 \\ \mathrm{H} & -1.224763 & 0.000000 & 3.002564 \\ \mathrm{H} & 1.224763 & 0.000000 & 3.002564 \\ \mathrm{C} & 1.492614 & 0.000000 & -1.551215 \\ \mathrm{C} & 2.881748 & 0.000000 & -1.518431 \\ \mathrm{C} & 3.556006 & 0.000000 & -0.296327 \\ \mathrm{C} & 2.842978 & 0.000000 & 0.874656 \\ \mathrm{H} & 1.015524 & 0.000000 & -2.510392 \\ \mathrm{H} & 3.433179 & 0.000000 & -2.439454 \\ \mathrm{H} & 4.629441 & 0.000000 & -0.273508 \\ \mathrm{H} & 3.356462 & 0.000000 & 1.818545 \\ \mathrm{C} & -3.556006 & 0.000000 & -0.296327 \\ \mathrm{C} & -2.881748 & 0.000000 & -1.518431 \\ \mathrm{H} & -1.015524 & 0.000000 & -2.510392 \\ \mathrm{H} & -3.433179 & 0.000000 & -2.439454 \\ \mathrm{H} & -4.629441 & 0.000000 & -0.273508 \\ \mathrm{H} & -3.356462 & 0.000000 & 1.818545\end{array}$

2,4,6,8-decatetraene $\mathrm{S}_{0}$
$\begin{array}{llll}\text { C } & -2.585127 & -5.001881 & 0.000000\end{array}$
$\begin{array}{llll}\text { C } & -2.451256 & -3.508803 & 0.000000\end{array}$ 


$\begin{array}{crcc}\mathrm{C} & -1.306844 & -2.847380 & 0.000000 \\ \mathrm{C} & -1.201272 & -1.390661 & 0.000000 \\ \mathrm{C} & -0.052409 & -0.726658 & 0.000000 \\ \mathrm{C} & 0.052409 & 0.726658 & 0.000000 \\ \mathrm{C} & 1.201272 & 1.390661 & 0.000000 \\ \mathrm{C} & 1.306844 & 2.847380 & 0.000000 \\ \mathrm{C} & 2.451256 & 3.508803 & 0.000000 \\ \mathrm{C} & 2.585127 & 5.001881 & 0.000000 \\ \mathrm{H} & -0.377916 & -3.395494 & 0.000000 \\ \mathrm{H} & -2.128690 & -0.840485 & 0.000000 \\ \mathrm{H} & 0.874980 & -1.276820 & 0.000000 \\ \mathrm{H} & -0.874980 & 1.276820 & 0.000000 \\ \mathrm{H} & 2.128690 & 0.840485 & 0.000000 \\ \mathrm{H} & 0.377916 & 3.395494 & 0.000000 \\ \mathrm{H} & 3.374662 & 2.950132 & 0.000000 \\ \mathrm{H} & -3.374662 & -2.950132 & 0.000000 \\ \mathrm{H} & 1.616092 & 5.486508 & 0.000000 \\ \mathrm{H} & 3.134544 & 5.340033 & 0.873820 \\ \mathrm{H} & 3.134544 & 5.340033 & -0.873820 \\ \mathrm{H} & -1.616092 & -5.486508 & 0.000000 \\ \mathrm{H} & -3.134544 & -5.340033 & 0.873820 \\ \mathrm{H} & -3.134544 & -5.340033 & -0.873820\end{array}$

2,4,6,8-decatetraene $\mathrm{S}_{1}$

$\begin{array}{crcc}\mathrm{C} & -2.568345 & -5.009460 & 0.000000 \\ \mathrm{C} & -2.503345 & -3.517927 & 0.000000 \\ \mathrm{C} & -1.353064 & -2.800485 & 0.000000 \\ \mathrm{C} & -1.265471 & -1.393190 & 0.000000 \\ \mathrm{C} & -0.055924 & -0.691385 & 0.000000 \\ \mathrm{C} & 0.055924 & 0.691385 & 0.000000 \\ \mathrm{C} & 1.265471 & 1.393190 & 0.000000 \\ \mathrm{C} & 1.353064 & 2.800485 & 0.000000 \\ \mathrm{C} & 2.503345 & 3.517927 & 0.000000 \\ \mathrm{C} & 2.568345 & 5.009460 & 0.000000 \\ \mathrm{H} & -0.419752 & -3.343306 & 0.000000 \\ \mathrm{H} & -2.181927 & -0.825749 & 0.000000 \\ \mathrm{H} & 0.853646 & -1.270543 & 0.000000 \\ \mathrm{H} & -0.853646 & 1.270543 & 0.000000 \\ \mathrm{H} & 2.181927 & 0.825749 & 0.000000 \\ \mathrm{H} & 0.419752 & 3.343306 & 0.000000 \\ \mathrm{H} & 3.444470 & 2.990216 & 0.000000 \\ \mathrm{H} & -3.444470 & -2.990216 & 0.000000 \\ \mathrm{H} & 1.577593 & 5.449537 & 0.000000 \\ \mathrm{H} & 3.103418 & 5.381666 & 0.872708 \\ \mathrm{H} & 3.103418 & 5.381666 & -0.872708\end{array}$




$$
\begin{array}{lrrr}
H & -1.577593 & -5.449537 & 0.000000 \\
H & -3.103418 & -5.381666 & 0.872708 \\
H & -3.103418 & -5.381666 & -0.872708
\end{array}
$$

tetracene $\mathrm{S}_{0}$

$$
\begin{array}{lrrr}
\mathrm{C} & 3.691873 & 1.402323 & 0.000000 \\
\mathrm{C} & 2.422048 & 0.717717 & 0.000000 \\
\mathrm{C} & 2.422048 & -0.717717 & 0.000000 \\
\mathrm{C} & 3.691873 & -1.402323 & 0.000000 \\
\mathrm{C} & 4.843459 & -0.720115 & 0.000000 \\
\mathrm{C} & 4.843459 & 0.720115 & 0.000000 \\
\mathrm{C} & 1.229971 & 1.392474 & 0.000000 \\
\mathrm{C} & 0.000000 & 0.712265 & 0.000000 \\
\mathrm{C} & 0.000000 & -0.712265 & 0.000000 \\
\mathrm{C} & 1.229971 & -1.392474 & 0.000000 \\
\mathrm{C} & -1.229971 & 1.392474 & 0.000000 \\
\mathrm{C} & -2.422048 & 0.717717 & 0.000000 \\
\mathrm{C} & -2.422048 & -0.717717 & 0.000000 \\
\mathrm{C} & -1.229971 & -1.392474 & 0.000000 \\
\mathrm{C} & -3.691873 & 1.402323 & 0.000000 \\
\mathrm{C} & -4.843459 & 0.720115 & 0.000000 \\
\mathrm{C} & -4.843459 & -0.720115 & 0.000000 \\
\mathrm{C} & -3.691873 & -1.402323 & 0.000000 \\
\mathrm{H} & -3.691500 & -2.477182 & 0.000000 \\
\mathrm{H} & -5.783038 & -1.240832 & 0.000000 \\
\mathrm{H} & -5.783038 & 1.240832 & 0.000000 \\
\mathrm{H} & -3.691500 & 2.477182 & 0.000000 \\
\mathrm{H} & -1.229010 & -2.467879 & 0.000000 \\
\mathrm{H} & -1.229010 & 2.467879 & 0.000000 \\
\mathrm{H} & 1.229010 & -2.467879 & 0.000000 \\
\mathrm{H} & 1.229010 & 2.467879 & 0.000000 \\
\mathrm{H} & 3.691500 & -2.477182 & 0.000000 \\
\mathrm{H} & 5.783038 & -1.240832 & 0.000000 \\
\mathrm{H} & 5.783038 & 1.240832 & 0.000000 \\
\mathrm{H} & 3.691500 & 2.477182 & 0.000000
\end{array}
$$

tetracene $\mathrm{S}_{1}$
$\begin{array}{llll}\mathrm{C} & 3.685273 & 1.388742 & 0.000000\end{array}$
$\begin{array}{llll}\text { C } & 2.456465 & 0.713753 & 0.000000\end{array}$
$\begin{array}{llll}\text { C } & 2.456465 & -0.713753 & 0.000000\end{array}$
$\begin{array}{llll}\text { C } & 3.685273 & -1.388742 & 0.000000\end{array}$
$\begin{array}{llll}\text { C } & 4.875019 & -0.697487 & 0.000000\end{array}$
$\begin{array}{llll}\text { C } & 4.875019 & 0.697487 & 0.000000\end{array}$
$\begin{array}{llll}\text { C } & 1.220803 & 1.392516 & 0.000000\end{array}$ 


$\begin{array}{rrrr}\mathrm{C} & 0.000000 & 0.725795 & 0.000000 \\ \mathrm{C} & 0.000000 & -0.725795 & 0.000000 \\ \mathrm{C} & 1.220803 & -1.392516 & 0.000000 \\ \mathrm{C} & -1.220803 & 1.392516 & 0.000000 \\ \mathrm{C} & -2.456465 & 0.713753 & 0.000000 \\ \mathrm{C} & -2.456465 & -0.713753 & 0.000000 \\ \mathrm{C} & -1.220803 & -1.392516 & 0.000000 \\ \mathrm{C} & -3.685273 & 1.388742 & 0.000000 \\ \mathrm{C} & -4.875019 & 0.697487 & 0.000000 \\ \mathrm{C} & -4.875019 & -0.697487 & 0.000000 \\ \mathrm{C} & -3.685273 & -1.388742 & 0.000000 \\ \mathrm{H} & -3.690248 & -2.463627 & 0.000000 \\ \mathrm{H} & -5.805447 & -1.233683 & 0.000000 \\ \mathrm{H} & -5.805447 & 1.233683 & 0.000000 \\ \mathrm{H} & -3.690248 & 2.463627 & 0.000000 \\ \mathrm{H} & -1.225399 & -2.467908 & 0.000000 \\ \mathrm{H} & -1.225399 & 2.467908 & 0.000000 \\ \mathrm{H} & 1.225399 & -2.467908 & 0.000000 \\ \mathrm{H} & 1.225399 & 2.467908 & 0.000000 \\ \mathrm{H} & 3.690248 & -2.463627 & 0.000000 \\ \mathrm{H} & 5.805447 & -1.233683 & 0.000000 \\ \mathrm{H} & 5.805447 & 1.233683 & 0.000000 \\ \mathrm{H} & 3.690248 & 2.463627 & 0.000000\end{array}$

perylene $\mathrm{S}_{0}$

$\begin{array}{lrrr}\mathrm{C} & 0.051437 & 1.467800 & -2.402395 \\ \mathrm{C} & 0.019534 & 0.741925 & -1.245358 \\ \mathrm{C} & 0.013711 & 1.439045 & -0.000029 \\ \mathrm{C} & 0.034623 & 2.849370 & -0.000030 \\ \mathrm{C} & 0.062211 & 3.551049 & -1.227122 \\ \mathrm{C} & 0.073404 & 2.872795 & -2.397424 \\ \mathrm{C} & 0.024990 & 3.551583 & 1.227010 \\ \mathrm{C} & 0.001543 & 2.873801 & 2.397416 \\ \mathrm{C} & -0.009507 & 1.468692 & 2.402458 \\ \mathrm{C} & 0.002714 & 0.742193 & 1.245413 \\ \mathrm{C} & -0.019534 & -0.741925 & -1.245358 \\ \mathrm{C} & -0.013711 & -1.439045 & -0.000029 \\ \mathrm{C} & -0.002714 & -0.742193 & 1.245413 \\ \mathrm{C} & -0.034623 & -2.849370 & -0.000030 \\ \mathrm{C} & -0.024990 & -3.551583 & 1.227010 \\ \mathrm{C} & -0.001543 & -2.873801 & 2.397416 \\ \mathrm{C} & 0.009507 & -1.468692 & 2.402458 \\ \mathrm{C} & -0.051437 & -1.467800 & -2.402395 \\ \mathrm{C} & -0.073404 & -2.872795 & -2.397424 \\ \mathrm{C} & -0.062211 & -3.551049 & -1.227122\end{array}$




$\begin{array}{rrrr}\mathrm{H} & 0.036989 & 4.625822 & 1.212378 \\ \mathrm{H} & -0.007667 & 3.400842 & 3.333391 \\ \mathrm{H} & -0.026538 & 0.979283 & 3.354326 \\ \mathrm{H} & -0.036989 & -4.625822 & 1.212378 \\ \mathrm{H} & 0.007667 & -3.400842 & 3.333391 \\ \mathrm{H} & 0.026538 & -0.979283 & 3.354326 \\ \mathrm{H} & -0.076894 & -4.625256 & -1.212537 \\ \mathrm{H} & -0.097795 & -3.399394 & -3.333375 \\ \mathrm{H} & -0.062001 & -0.977921 & -3.354120 \\ \mathrm{H} & 0.076894 & 4.625256 & -1.212537 \\ \mathrm{H} & 0.097795 & 3.399394 & -3.333375 \\ \mathrm{H} & 0.062001 & 0.977921 & -3.354120\end{array}$

perylene $S_{1}$

$\begin{array}{lrrc}\mathrm{C} & -0.005126 & 1.464236 & -2.433756 \\ \mathrm{C} & -0.005965 & 0.718628 & -1.232950 \\ \mathrm{C} & 0.012929 & 1.413482 & 0.000000 \\ \mathrm{C} & 0.006558 & 2.841563 & 0.000000 \\ \mathrm{C} & 0.008812 & 3.529821 & -1.223424 \\ \mathrm{C} & -0.000748 & 2.840933 & -2.417294 \\ \mathrm{C} & 0.008868 & 3.529821 & 1.223419 \\ \mathrm{C} & -0.000589 & 2.840932 & 2.417292 \\ \mathrm{C} & -0.004943 & 1.464237 & 2.433757 \\ \mathrm{C} & -0.005922 & 0.718627 & 1.232951 \\ \mathrm{C} & 0.005965 & -0.718628 & -1.232950 \\ \mathrm{C} & -0.012929 & -1.413482 & 0.000000 \\ \mathrm{C} & 0.005922 & -0.718627 & 1.232951 \\ \mathrm{C} & -0.006558 & -2.841563 & 0.000000 \\ \mathrm{C} & -0.008868 & -3.529821 & 1.223419 \\ \mathrm{C} & 0.000589 & -2.840932 & 2.417292 \\ \mathrm{C} & 0.004943 & -1.464237 & 2.433757 \\ \mathrm{C} & 0.005126 & -1.464236 & -2.433756 \\ \mathrm{C} & 0.000748 & -2.840933 & -2.417294 \\ \mathrm{C} & -0.008812 & -3.529821 & -1.223424 \\ \mathrm{H} & 0.011658 & 4.604214 & 1.218676 \\ \mathrm{H} & -0.003610 & 3.381279 & 3.345700 \\ \mathrm{H} & -0.025155 & 0.966102 & 3.379705 \\ \mathrm{H} & -0.011658 & -4.604214 & 1.218676 \\ \mathrm{H} & 0.003610 & -3.381279 & 3.345700 \\ \mathrm{H} & 0.025155 & -0.966102 & 3.379705 \\ \mathrm{H} & -0.011614 & -4.604213 & -1.218680 \\ \mathrm{H} & 0.003848 & -3.381279 & -3.345703 \\ \mathrm{H} & 0.025477 & -0.966099 & -3.379699 \\ \mathrm{H} & 0.011614 & 4.604213 & -1.218680 \\ \mathrm{H} & -0.003848 & 3.381279 & -3.345703\end{array}$


$\begin{array}{llll}\mathrm{H} & -0.025477 & 0.966099 & -3.379699\end{array}$

\section{CC2/TZVP geometries - Group I}

pyrazine $\mathrm{S}_{0}$

$\begin{array}{lrrr}\mathrm{C} & 1.132312 & -0.696476 & -0.000010 \\ \mathrm{C} & 1.132312 & 0.696476 & 0.000009 \\ \mathrm{~N} & 0.000000 & 1.417550 & 0.000034 \\ \mathrm{C} & -1.132312 & 0.696476 & 0.000008 \\ \mathrm{C} & -1.132312 & -0.696476 & -0.000005 \\ \mathrm{~N} & 0.000000 & -1.417550 & -0.000031 \\ \mathrm{H} & -2.063169 & -1.251290 & 0.000030 \\ \mathrm{H} & 2.063169 & -1.251290 & 0.000014 \\ \mathrm{H} & 2.063169 & 1.251290 & -0.000015 \\ \mathrm{H} & -2.063169 & 1.251290 & -0.000033\end{array}$

pyrazine $S_{1}$

$\begin{array}{lrrc}\mathrm{C} & -1.221966 & 0.696174 & 0.095215 \\ \mathrm{C} & -1.116217 & -0.752525 & 0.001529 \\ \mathrm{~N} & 0.024588 & -1.353955 & -0.002941 \\ \mathrm{C} & 1.222069 & -0.695840 & -0.111868 \\ \mathrm{C} & 1.116279 & 0.752896 & -0.017983 \\ \mathrm{~N} & -0.024476 & 1.354371 & -0.013466 \\ \mathrm{H} & 2.037554 & 1.325435 & 0.035557 \\ \mathrm{H} & -2.139277 & 1.188962 & -0.192179 \\ \mathrm{H} & -2.037373 & -1.325270 & -0.051791 \\ \mathrm{H} & 2.139454 & -1.188466 & 0.175601\end{array}$

2,6-difluoropyridine $\mathrm{S}_{0}$

$\begin{array}{rrrr}\text { C } & 1.116685 & -0.007903 & -0.874860 \\ \text { N } & 0.000002 & -0.008794 & -1.579366 \\ \mathrm{C} & -1.116690 & -0.007917 & -0.874847 \\ \mathrm{C} & -1.205210 & 0.020919 & 0.509832 \\ \mathrm{C} & 0.000059 & -0.014327 & 1.205917 \\ \mathrm{C} & 1.205275 & 0.021004 & 0.509830 \\ \mathrm{~F} & -2.251262 & -0.015093 & -1.592732 \\ \mathrm{H} & -2.166392 & 0.004172 & 1.000970 \\ \mathrm{H} & -0.000151 & 0.009323 & 2.287500 \\ \mathrm{H} & 2.166446 & 0.004281 & 1.000953 \\ \mathrm{~F} & 2.251238 & -0.015100 & -1.592776\end{array}$

2,6-difluoropyridine $\mathrm{S}_{1}$ 


$\begin{array}{lrcc}\mathrm{C} & -1.088394 & 0.067505 & -0.335738 \\ \mathrm{~N} & -0.000012 & 0.299896 & -1.096388 \\ \mathrm{C} & 1.088393 & 0.067217 & -0.335890 \\ \mathrm{C} & 1.167565 & -0.033359 & 1.071112 \\ \mathrm{C} & 0.000203 & 0.310270 & 1.850896 \\ \mathrm{C} & -1.167366 & -0.033020 & 1.071275 \\ \mathrm{~F} & 2.223806 & -0.093369 & -1.017477 \\ \mathrm{H} & 2.088249 & -0.387428 & 1.517333 \\ \mathrm{H} & 0.000234 & 0.198078 & 2.923963 \\ \mathrm{H} & -2.088055 & -0.386815 & 1.517705 \\ \mathrm{~F} & -2.223919 & -0.092764 & -1.017172\end{array}$

2-fluoropyridine $\mathrm{S}_{0}$

$\begin{array}{lrrr}\mathrm{C} & -1.315242 & 0.503842 & -0.002550 \\ \mathrm{~N} & -1.378092 & -0.809457 & -0.005316 \\ \mathrm{C} & -0.194903 & -1.455969 & -0.011208 \\ \mathrm{C} & 1.033990 & -0.809937 & 0.018311 \\ \mathrm{C} & 1.047974 & 0.585735 & -0.020778 \\ \mathrm{C} & -0.156749 & 1.275184 & 0.018141 \\ \mathrm{H} & -0.256586 & -2.537307 & -0.002495 \\ \mathrm{H} & 1.950431 & -1.382943 & -0.005071 \\ \mathrm{H} & 1.983461 & 1.129554 & -0.002644 \\ \mathrm{H} & -0.214695 & 2.353990 & 0.003091 \\ \mathrm{~F} & -2.499601 & 1.147300 & -0.000851\end{array}$

2-fluoropyridine $\mathrm{S}_{1}$

$\begin{array}{lrrr}\mathrm{C} & -1.328210 & 0.566339 & 0.056675 \\ \mathrm{~N} & -1.263248 & -0.744195 & 0.169861 \\ \mathrm{C} & -0.195189 & -1.550472 & -0.228877 \\ \mathrm{C} & 1.026232 & -0.803185 & -0.021075 \\ \mathrm{C} & 1.039699 & 0.574547 & 0.034738 \\ \mathrm{C} & -0.170318 & 1.345349 & 0.045327 \\ \mathrm{H} & -0.265056 & -2.597210 & 0.033767 \\ \mathrm{H} & 1.939439 & -1.379722 & 0.072588 \\ \mathrm{H} & 1.991896 & 1.087476 & 0.085546 \\ \mathrm{H} & -0.226927 & 2.390701 & -0.221054 \\ \mathrm{~F} & -2.547534 & 1.109682 & 0.014692\end{array}$

2-hydroxypyridine $\mathrm{S}_{0}$
$\begin{array}{llll}\text { C } & -0.982106 & 0.753709 & 0.021568\end{array}$
$\begin{array}{llll}\text { C } & -0.971751 & -0.645112 & -0.003482\end{array}$
$\begin{array}{llll}\mathrm{N} & 0.130422 & -1.391271 & -0.004135\end{array}$ 


$\begin{array}{crrc}\mathrm{C} & 1.309236 & -0.737579 & -0.011748 \\ \mathrm{C} & 1.419825 & 0.644552 & 0.015711 \\ \mathrm{C} & 0.242948 & 1.399259 & -0.022718 \\ \mathrm{O} & -2.163094 & -1.297231 & -0.000328 \\ \mathrm{H} & 2.190151 & -1.368457 & -0.002627 \\ \mathrm{H} & 2.393116 & 1.114143 & -0.012308 \\ \mathrm{H} & 0.283761 & 2.480930 & -0.006174 \\ \mathrm{H} & -1.921527 & 1.288070 & 0.006512 \\ \mathrm{H} & -1.930980 & -2.241014 & -0.002406\end{array}$

2-hydroxypyridine $\mathrm{S}_{1}$

$\begin{array}{rrrc}\mathrm{C} & -0.991824 & 0.769006 & -0.002048 \\ \mathrm{C} & -0.974892 & -0.632270 & -0.087292 \\ \mathrm{~N} & 0.120764 & -1.455874 & -0.257855 \\ \mathrm{C} & 1.276550 & -0.774094 & -0.008828 \\ \mathrm{C} & 1.379796 & 0.644219 & 0.054547 \\ \mathrm{C} & 0.253583 & 1.472110 & -0.212144 \\ \mathrm{O} & -2.130508 & -1.298795 & 0.080236 \\ \mathrm{H} & 2.174341 & -1.376006 & 0.070484 \\ \mathrm{H} & 2.351876 & 1.075552 & 0.263445 \\ \mathrm{H} & 0.310303 & 2.546897 & -0.135154 \\ \mathrm{H} & -1.909272 & 1.268033 & 0.281770 \\ \mathrm{H} & -1.860668 & -2.239331 & 0.021958\end{array}$

2-pyridone $\mathrm{S}_{0}$

$\begin{array}{cccc}\mathrm{C} & -1.248997 & 0.720313 & -0.007055 \\ \mathrm{~N} & -1.133194 & -0.687822 & 0.035222 \\ \mathrm{C} & 0.035472 & -1.390355 & -0.011605 \\ \mathrm{C} & 1.230131 & -0.731593 & 0.001251 \\ \mathrm{C} & 1.209981 & 0.688579 & -0.022468 \\ \mathrm{C} & 0.033481 & 1.385575 & 0.039132 \\ \mathrm{H} & -2.020431 & -1.177107 & -0.002802 \\ \mathrm{H} & -0.056199 & -2.467886 & -0.006811 \\ \mathrm{H} & 2.153364 & -1.287962 & -0.051143 \\ \mathrm{H} & 2.147770 & 1.230931 & -0.011825 \\ \mathrm{H} & 0.014142 & 2.466617 & 0.023221 \\ \mathrm{O} & -2.365518 & 1.250720 & -0.052479\end{array}$

2-pyridone $\mathrm{S}_{1}$
C -1.314200
$\begin{array}{ll}0.118032 & 0.000001\end{array}$
$\begin{array}{llll}\mathrm{N} & -0.749905 & -1.124015 & -0.000001\end{array}$
C $\quad 0.647505 \quad-1.254548 \quad 0.000005$
$\begin{array}{llll}\text { C } & 1.416959 & -0.087429 & 0.000000\end{array}$ 


$\begin{array}{rrrr}\mathrm{C} & 0.836955 & 1.160651 & -0.000007 \\ \mathrm{C} & -0.627768 & 1.267426 & 0.000009 \\ \mathrm{H} & -1.342656 & -1.941777 & -0.000010 \\ \mathrm{H} & 1.030665 & -2.262269 & 0.000016 \\ \mathrm{H} & 2.494042 & -0.197860 & 0.000002 \\ \mathrm{H} & 1.433461 & 2.062545 & -0.000029 \\ \mathrm{H} & -1.138047 & 2.219314 & 0.000035 \\ \mathrm{O} & -2.739501 & 0.013261 & -0.000021\end{array}$

2-methylpyrimidine $\mathrm{S}_{0}$

$\begin{array}{rrrc}\mathrm{C} & 0.000075 & 1.965544 & 0.000008 \\ \mathrm{C} & -1.178402 & 1.227299 & -0.000006 \\ \mathrm{~N} & -1.194000 & -0.112814 & -0.000020 \\ \mathrm{C} & 0.010164 & -0.720009 & -0.000019 \\ \mathrm{~N} & 1.207895 & -0.105487 & -0.000017 \\ \mathrm{C} & 1.182223 & 1.237436 & -0.000005 \\ \mathrm{H} & -2.147131 & 1.715125 & -0.000003 \\ \mathrm{H} & 2.147849 & 1.731041 & -0.000006 \\ \mathrm{H} & -0.004421 & 3.046736 & 0.000034 \\ \mathrm{C} & 0.002807 & -2.217025 & 0.000004 \\ \mathrm{H} & 1.022014 & -2.594181 & 0.000010 \\ \mathrm{H} & -0.524542 & -2.586819 & 0.879243 \\ \mathrm{H} & -0.524541 & -2.586845 & -0.879224\end{array}$

2-methylpyrimidine $\mathrm{S}_{1}$

$\begin{array}{rrrr}\mathrm{C} & -0.013075 & 2.013633 & 0.042691 \\ \mathrm{C} & -1.164813 & 1.275896 & -0.013216 \\ \mathrm{~N} & -1.175471 & -0.106484 & -0.104208 \\ \mathrm{C} & 0.009189 & -0.787737 & -0.024946 \\ \mathrm{~N} & 1.094488 & -0.071623 & 0.019272 \\ \mathrm{C} & 1.222304 & 1.307619 & -0.081878 \\ \mathrm{H} & -2.142924 & 1.738512 & 0.032564 \\ \mathrm{H} & 2.198046 & 1.724258 & 0.113759 \\ \mathrm{H} & -0.032675 & 3.094219 & 0.103190 \\ \mathrm{C} & 0.010782 & -2.271853 & 0.015272 \\ \mathrm{H} & 1.029905 & -2.649598 & 0.058460 \\ \mathrm{H} & -0.547447 & -2.612724 & 0.887158 \\ \mathrm{H} & -0.487919 & -2.654053 & -0.875431\end{array}$

5-methylpyrimidine $\mathrm{S}_{0}$
C $\quad 0.873400$
$\begin{array}{ll}0.856727 & -0.000052\end{array}$
$\begin{array}{llll}\text { C } & -0.362588 & 0.212922 & -0.000036\end{array}$
$\begin{array}{llll}\text { C } & -0.305203 & -1.181890 & -0.000024\end{array}$ 


$\begin{array}{lrrr}\mathrm{N} & 0.843530 & -1.873866 & 0.000047 \\ \mathrm{C} & 1.961205 & -1.131710 & 0.000033 \\ \mathrm{~N} & 2.047600 & 0.204749 & -0.000035 \\ \mathrm{H} & 2.898275 & -1.675121 & 0.000096 \\ \mathrm{H} & -1.217695 & -1.771427 & -0.000135 \\ \mathrm{H} & 0.929045 & 1.941415 & -0.000059 \\ \mathrm{C} & -1.663765 & 0.959118 & 0.000029 \\ \mathrm{H} & -1.491982 & 2.035254 & 0.000035 \\ \mathrm{H} & -2.255949 & 0.711917 & -0.881659 \\ \mathrm{H} & -2.255875 & 0.711895 & 0.881760\end{array}$

5-methylpyrimidine $\mathrm{S}_{1}$

$\begin{array}{lrrr}\mathrm{C} & 0.843957 & 0.855065 & -0.042151 \\ \mathrm{C} & -0.381205 & 0.240939 & -0.000252 \\ \mathrm{C} & -0.366549 & -1.195932 & -0.094792 \\ \mathrm{~N} & 0.880869 & -1.769674 & 0.055378 \\ \mathrm{C} & 2.038319 & -1.177702 & 0.036737 \\ \mathrm{~N} & 2.053249 & 0.180802 & -0.063505 \\ \mathrm{H} & 2.961839 & -1.732028 & 0.109494 \\ \mathrm{H} & -1.226427 & -1.822869 & 0.091291 \\ \mathrm{H} & 0.927893 & 1.936051 & -0.030512 \\ \mathrm{C} & -1.679913 & 0.980960 & 0.024932 \\ \mathrm{H} & -1.512608 & 2.057927 & 0.020188 \\ \mathrm{H} & -2.282980 & 0.721734 & -0.847442 \\ \mathrm{H} & -2.255711 & 0.726950 & 0.917160\end{array}$

7-azaindole $\mathrm{S}_{0}$

$\begin{array}{rrrc}\mathrm{N} & -0.633428 & 1.844450 & -0.027670 \\ \mathrm{C} & -0.672231 & 0.471758 & 0.000315 \\ \mathrm{C} & 0.671684 & 0.008041 & 0.072066 \\ \mathrm{C} & 1.512841 & 1.161908 & 0.043283 \\ \mathrm{C} & 0.681570 & 2.257594 & 0.029873 \\ \mathrm{C} & 0.849985 & -1.378569 & 0.002932 \\ \mathrm{C} & -0.290569 & -2.172746 & 0.019239 \\ \mathrm{C} & -1.565530 & -1.581934 & -0.002075 \\ \mathrm{~N} & -1.789631 & -0.258675 & -0.007221 \\ \mathrm{H} & -2.451646 & -2.206672 & 0.002838 \\ \mathrm{H} & 1.838502 & -1.822788 & 0.014148 \\ \mathrm{H} & -0.212525 & -3.251810 & -0.012919 \\ \mathrm{H} & -1.452167 & 2.431210 & 0.031550 \\ \mathrm{H} & 2.589458 & 1.190436 & 0.084744 \\ \mathrm{H} & 0.923706 & 3.307799 & 0.012064\end{array}$

7-azaindole $\mathrm{S}_{1}$ 


$\begin{array}{lrrc}\mathrm{N} & -0.604342 & 1.878510 & 0.006554 \\ \mathrm{C} & -0.658562 & 0.440528 & 0.093263 \\ \mathrm{C} & 0.664695 & -0.005829 & 0.164623 \\ \mathrm{C} & 1.485353 & 1.143227 & -0.006317 \\ \mathrm{C} & 0.651201 & 2.313655 & -0.041672 \\ \mathrm{C} & 0.900452 & -1.415664 & 0.023219 \\ \mathrm{C} & -0.294972 & -2.178317 & -0.028143 \\ \mathrm{C} & -1.552001 & -1.595754 & -0.028866 \\ \mathrm{~N} & -1.817124 & -0.214022 & 0.004268 \\ \mathrm{H} & -2.438550 & -2.218403 & -0.046871 \\ \mathrm{H} & 1.876955 & -1.859475 & 0.160369 \\ \mathrm{H} & -0.242297 & -3.259656 & -0.086476 \\ \mathrm{H} & -1.445654 & 2.447954 & 0.027168 \\ \mathrm{H} & 2.561413 & 1.164074 & -0.106726 \\ \mathrm{H} & 0.913563 & 3.358995 & -0.041397\end{array}$

7-hydroxyquinoline, cis $\mathrm{S}_{0}$

$\begin{array}{rrrr}\mathrm{N} & 0.478954 & 1.977243 & -0.005393 \\ \mathrm{C} & 0.435679 & 0.608774 & -0.015798 \\ \mathrm{C} & -0.787677 & -0.126213 & 0.046304 \\ \mathrm{C} & -1.994269 & 0.603444 & -0.010172 \\ \mathrm{C} & -1.946887 & 1.979728 & -0.000400 \\ \mathrm{C} & -0.686149 & 2.612810 & -0.001503 \\ \mathrm{C} & 1.664310 & -0.087965 & 0.035419 \\ \mathrm{C} & 1.677086 & -1.465562 & -0.010129 \\ \mathrm{C} & 0.469709 & -2.202270 & 0.018132 \\ \mathrm{C} & -0.734362 & -1.541912 & -0.005491 \\ \mathrm{H} & -0.630733 & 3.697094 & 0.000162 \\ \mathrm{H} & -1.661583 & -2.104490 & 0.006617 \\ \mathrm{H} & 0.520898 & -3.283302 & -0.010301 \\ \mathrm{O} & 2.827314 & -2.210121 & -0.011445 \\ \mathrm{H} & 2.580309 & 0.493511 & 0.026423 \\ \mathrm{H} & -2.940802 & 0.073152 & -0.005746 \\ \mathrm{H} & -2.850151 & 2.575911 & -0.021860 \\ \mathrm{H} & 3.578355 & -1.599823 & -0.034820\end{array}$

7-hydroxyquinoline, cis $\mathrm{S}_{1}$

$\begin{array}{lrrr}\mathrm{N} & 0.508421 & 1.984892 & 0.122155 \\ \mathrm{C} & 0.452050 & 0.635444 & 0.090359 \\ \mathrm{C} & -0.792009 & -0.152511 & 0.001136 \\ \mathrm{C} & -2.002126 & 0.590132 & -0.034439 \\ \mathrm{C} & -1.925829 & 1.991351 & -0.047087 \\ \mathrm{C} & -0.683099 & 2.649226 & 0.031982\end{array}$




$\begin{array}{rrrr}\mathrm{C} & 1.645464 & -0.081135 & -0.049706 \\ \mathrm{C} & 1.655849 & -1.501603 & -0.013840 \\ \mathrm{C} & 0.475555 & -2.269384 & 0.000822 \\ \mathrm{C} & -0.735590 & -1.562560 & 0.060039 \\ \mathrm{H} & -0.629445 & 3.729957 & 0.070508 \\ \mathrm{H} & -1.671810 & -2.110917 & 0.083554 \\ \mathrm{H} & 0.529153 & -3.346725 & 0.057995 \\ \mathrm{O} & 2.829330 & -2.170305 & -0.032811 \\ \mathrm{H} & 2.578079 & 0.473596 & -0.105569 \\ \mathrm{H} & -2.952832 & 0.078930 & -0.131134 \\ \mathrm{H} & -2.834098 & 2.582832 & -0.063584 \\ \mathrm{H} & 3.552938 & -1.521209 & -0.040383\end{array}$

7-hydroxyquinoline, trans $\mathrm{S}_{0}$

$\begin{array}{rrrc}\mathrm{N} & 0.528693 & 2.049916 & 0.006179 \\ \mathrm{C} & 0.483208 & 0.679429 & 0.018282 \\ \mathrm{C} & -0.745498 & -0.049706 & -0.045458 \\ \mathrm{C} & -1.950998 & 0.684222 & 0.009598 \\ \mathrm{C} & -1.898317 & 2.059085 & -0.000097 \\ \mathrm{C} & -0.633416 & 2.687776 & 0.000762 \\ \mathrm{C} & 1.708784 & -0.016778 & -0.029001 \\ \mathrm{C} & 1.718507 & -1.394511 & 0.014513 \\ \mathrm{C} & 0.507294 & -2.124780 & -0.020298 \\ \mathrm{C} & -0.698802 & -1.462474 & 0.005620 \\ \mathrm{H} & -0.575237 & 3.772051 & -0.001960 \\ \mathrm{H} & -1.626537 & -2.024181 & -0.010476 \\ \mathrm{H} & 0.533364 & -3.209732 & 0.007502 \\ \mathrm{O} & 2.934771 & -2.028297 & 0.018112 \\ \mathrm{H} & 2.633987 & 0.544563 & -0.014646 \\ \mathrm{H} & -2.899209 & 0.156830 & 0.004322 \\ \mathrm{H} & -2.799140 & 2.659050 & 0.020362 \\ \mathrm{H} & 2.778524 & -2.982444 & 0.016682\end{array}$

7-hydroxyquinoline, trans $\mathrm{S}_{1}$

$\begin{array}{lrrr}\mathrm{N} & 0.552286 & 2.061080 & -0.103761 \\ \mathrm{C} & 0.494068 & 0.715171 & -0.083634 \\ \mathrm{C} & -0.747967 & -0.068759 & 0.006441 \\ \mathrm{C} & -1.958116 & 0.672108 & 0.025127 \\ \mathrm{C} & -1.881812 & 2.074321 & 0.039401 \\ \mathrm{C} & -0.641490 & 2.728698 & -0.022917 \\ \mathrm{C} & 1.691274 & -0.004538 & 0.040472 \\ \mathrm{C} & 1.695023 & -1.422026 & 0.012412 \\ \mathrm{C} & 0.510328 & -2.193045 & 0.012815 \\ \mathrm{C} & -0.697285 & -1.480656 & -0.052548\end{array}$




$\begin{array}{rrrr}\mathrm{H} & -0.583681 & 3.809547 & -0.050677 \\ \mathrm{H} & -1.636291 & -2.024510 & -0.080138 \\ \mathrm{H} & 0.545716 & -3.272621 & -0.063352 \\ \mathrm{O} & 2.918634 & -1.990813 & 0.033558 \\ \mathrm{H} & 2.634257 & 0.526757 & 0.086773 \\ \mathrm{H} & -2.910075 & 0.161172 & 0.112358 \\ \mathrm{H} & -2.790879 & 2.664926 & 0.052297 \\ \mathrm{H} & 2.805989 & -2.956789 & 0.035373\end{array}$

2-hydroxyquinoline, enol $\mathrm{S}_{0}$

$\begin{array}{cccc}\mathrm{C} & 0.435920 & 1.902880 & -0.008012 \\ \mathrm{C} & 0.488062 & 0.490456 & 0.042669 \\ \mathrm{C} & -0.724323 & -0.258442 & -0.025892 \\ \mathrm{C} & -1.955898 & 0.434253 & 0.016119 \\ \mathrm{C} & -1.979370 & 1.812787 & -0.013757 \\ \mathrm{C} & -0.778418 & 2.554728 & 0.011997 \\ \mathrm{C} & 1.700007 & -0.240076 & -0.010578 \\ \mathrm{C} & 1.665927 & -1.610784 & 0.008764 \\ \mathrm{C} & 0.397134 & -2.252443 & -0.005002 \\ \mathrm{~N} & -0.755064 & -1.626855 & -0.010198 \\ \mathrm{O} & 0.311858 & -3.615276 & -0.001913 \\ \mathrm{H} & 1.364552 & 2.463746 & 0.005182 \\ \mathrm{H} & -0.813339 & 3.636854 & -0.014973 \\ \mathrm{H} & -2.929022 & 2.333760 & 0.003534 \\ \mathrm{H} & -2.867164 & -0.150752 & 0.003893 \\ \mathrm{H} & 2.647155 & 0.288350 & 0.001275 \\ \mathrm{H} & 2.580687 & -2.192663 & -0.014867 \\ \mathrm{H} & 1.211293 & -3.970524 & 0.011759\end{array}$

2-hydroxyquinoline, enol $\mathrm{S}_{1}$

$\begin{array}{rrrr}\mathrm{C} & 0.444754 & 1.885534 & 0.059552 \\ \mathrm{C} & 0.529263 & 0.490921 & -0.019263 \\ \mathrm{C} & -0.743674 & -0.263215 & 0.035368 \\ \mathrm{C} & -1.970178 & 0.433298 & -0.046106 \\ \mathrm{C} & -2.015635 & 1.836054 & -0.000434 \\ \mathrm{C} & -0.804491 & 2.550129 & 0.013096 \\ \mathrm{C} & 1.739646 & -0.231138 & -0.014120 \\ \mathrm{C} & 1.696388 & -1.641224 & -0.113643 \\ \mathrm{C} & 0.418892 & -2.245540 & 0.011204 \\ \mathrm{~N} & -0.773552 & -1.622820 & 0.039256 \\ \mathrm{O} & 0.299548 & -3.588960 & 0.095275 \\ \mathrm{H} & 1.358758 & 2.470271 & 0.061775 \\ \mathrm{H} & -0.817578 & 3.633663 & 0.038352 \\ \mathrm{H} & -2.960702 & 2.359459 & -0.048019\end{array}$




$\begin{array}{lrrc}\mathrm{H} & -2.875619 & -0.161631 & -0.058100 \\ \mathrm{H} & 2.682986 & 0.302163 & -0.032013 \\ \mathrm{H} & 2.596198 & -2.243015 & -0.075036 \\ \mathrm{H} & 1.194994 & -3.963949 & 0.052855\end{array}$

2-hydroxyquinoline, keto $\mathrm{S}_{0}$

$\begin{array}{crrr}\mathrm{N} & 0.559103 & 1.705863 & 0.051021 \\ \mathrm{C} & 0.600113 & 0.326298 & -0.003773 \\ \mathrm{C} & -0.618020 & -0.385197 & 0.055942 \\ \mathrm{C} & -1.837254 & 0.371432 & 0.014986 \\ \mathrm{C} & -1.831206 & 1.729669 & 0.025944 \\ \mathrm{C} & -0.592506 & 2.495760 & -0.017353 \\ \mathrm{C} & 1.821096 & -0.362996 & 0.027862 \\ \mathrm{C} & 1.827211 & -1.748349 & -0.021586 \\ \mathrm{C} & 0.623893 & -2.469610 & 0.000231 \\ \mathrm{C} & -0.582803 & -1.789625 & -0.009052 \\ \mathrm{H} & -1.520892 & -2.333599 & 0.000757 \\ \mathrm{H} & 0.639377 & -3.551076 & -0.038897 \\ \mathrm{H} & 2.773211 & -2.275512 & -0.015145 \\ \mathrm{H} & 2.752478 & 0.192422 & 0.019244 \\ \mathrm{H} & -2.779010 & -0.167614 & 0.027594 \\ \mathrm{H} & -2.746823 & 2.304879 & -0.004553 \\ \mathrm{O} & -0.513764 & 3.728029 & -0.084819 \\ \mathrm{H} & 1.427394 & 2.225992 & -0.024727\end{array}$

2-hydroxyquinoline, keto $\mathrm{S}_{1}$

$\begin{array}{rrrr}\mathrm{N} & 0.551573 & 1.713787 & -0.083991 \\ \mathrm{C} & 0.589791 & 0.314377 & 0.009527 \\ \mathrm{C} & -0.661439 & -0.386310 & -0.043069 \\ \mathrm{C} & -1.854349 & 0.331961 & 0.009255 \\ \mathrm{C} & -1.837641 & 1.786539 & -0.013238 \\ \mathrm{C} & -0.637419 & 2.377512 & -0.001731 \\ \mathrm{C} & 1.811086 & -0.355651 & -0.027324 \\ \mathrm{C} & 1.843645 & -1.745092 & 0.019920 \\ \mathrm{C} & 0.633410 & -2.464352 & -0.003649 \\ \mathrm{C} & -0.580888 & -1.809580 & 0.017450 \\ \mathrm{H} & -1.507857 & -2.372741 & 0.008002 \\ \mathrm{H} & 0.655219 & -3.547307 & 0.028100 \\ \mathrm{H} & 2.792897 & -2.264875 & 0.001007 \\ \mathrm{H} & 2.735090 & 0.215162 & -0.027849 \\ \mathrm{H} & -2.804832 & -0.187637 & -0.018923 \\ \mathrm{H} & -2.745355 & 2.370975 & 0.029464 \\ \mathrm{O} & -0.390372 & 3.792518 & 0.014386 \\ \mathrm{H} & 1.407441 & 2.230724 & 0.082663\end{array}$


dibenzofuran $\mathrm{S}_{0}$

\begin{tabular}{|c|c|c|c|}
\hline $\mathrm{C}$ & 1.718043 & -0.024648 & \\
\hline $\mathrm{C}$ & 0.721693 & 0.023729 & \\
\hline & 1.096909 & -0.015936 & \\
\hline$C$ & 2.415858 & 0.027937 & \\
\hline & 3.391199 & -0.011217 & 0.4 \\
\hline $\mathrm{C}$ & 3.048707 & & -0.9 \\
\hline $\mathrm{C}$ & -0.721882 & -0.023801 & -0.383134 \\
\hline$C$ & -1.097 & 987 & \\
\hline 0 & -0.000086 & 0.000102 & 1.809526 \\
\hline$C$ & -1.7 & & \\
\hline $\mathrm{C}$ & -3.048897 & -0.008251 & -0.957607 \\
\hline $\mathrm{C}$ & -3.39 & 223 & \\
\hline 0 & -2.416030 & 7955 & 1.3 \\
\hline $\mathrm{H}$ & -2.667901 & -0.022244 & 2.4 \\
\hline $\mathrm{H}$ & -4.436061 & -0.013071 & 0.689327 \\
\hline $\mathrm{H}$ & -3.835704 & 0.022209 & -1.700924 \\
\hline $\mathrm{H}$ & -1.460049 & 0.022348 & -2.416237 \\
\hline$-\sigma_{-1}$ & 1.459858 & -0.022315 & -2.416247 \\
\hline $\mathrm{H}$ & 3.835511 & -0.022600 & -1.700933 \\
\hline 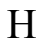 & 4.435890 & 0.013320 & 0.689268 \\
\hline & 2.667689 & 0.022032 & 2.452047 \\
\hline
\end{tabular}

dibenzofuran $\mathrm{S}_{1}$

$\begin{array}{lrrr}\mathrm{C} & 1.729871 & -0.039130 & -1.384414 \\ \mathrm{C} & 0.708804 & 0.019534 & -0.417123 \\ \mathrm{C} & 1.112701 & -0.030178 & 0.960803 \\ \mathrm{C} & 2.416453 & 0.026759 & 1.416851 \\ \mathrm{C} & 3.428889 & -0.016299 & 0.418547 \\ \mathrm{C} & 3.077187 & 0.002703 & -0.939634 \\ \mathrm{C} & -0.708818 & -0.020191 & -0.417100 \\ \mathrm{C} & -1.112705 & 0.030118 & 0.960789 \\ \mathrm{O} & 0.000018 & 0.000068 & 1.779282 \\ \mathrm{C} & -1.729867 & 0.038449 & -1.384413 \\ \mathrm{C} & -3.077196 & -0.002555 & -0.939628 \\ \mathrm{C} & -3.428894 & 0.016658 & 0.418547 \\ \mathrm{C} & -2.416452 & -0.026410 & 1.416855 \\ \mathrm{H} & -2.645944 & -0.029657 & 2.474263 \\ \mathrm{H} & -4.470111 & -0.025096 & 0.707648 \\ \mathrm{H} & -3.865392 & 0.015351 & -1.683315 \\ \mathrm{H} & -1.501495 & 0.037635 & -2.443095 \\ \mathrm{H} & 1.501501 & -0.039000 & -2.443094 \\ \mathrm{H} & 3.865397 & -0.014413 & -1.683326\end{array}$




$$
\begin{array}{llll}
\mathrm{H} & 4.470113 & 0.025388 & 0.707632 \\
\mathrm{H} & 2.645941 & 0.030266 & 2.474259
\end{array}
$$

pyrrolo[3,2- $h]$ quinoline $\mathrm{S}_{0}$

$\begin{array}{lrrr}\mathrm{C} & 0.131210 & -0.683975 & 0.021407 \\ \mathrm{~N} & 0.043007 & -2.043064 & 0.016264 \\ \mathrm{C} & 1.193811 & -2.712924 & 0.011867 \\ \mathrm{C} & 2.462022 & -2.103807 & -0.000977 \\ \mathrm{C} & 2.543456 & -0.724555 & 0.008691 \\ \mathrm{C} & 1.360885 & 0.041597 & -0.030531 \\ \mathrm{H} & 1.112486 & -3.795250 & 0.012985 \\ \mathrm{H} & 3.353301 & -2.718225 & 0.013883 \\ \mathrm{H} & 3.505880 & -0.223170 & -0.002156 \\ \mathrm{C} & -1.050335 & 0.084428 & -0.045043 \\ \mathrm{C} & -1.058400 & 1.492509 & 0.018439 \\ \mathrm{C} & 0.179754 & 2.185273 & -0.015664 \\ \mathrm{C} & 1.354018 & 1.470487 & 0.000452 \\ \mathrm{H} & 0.199724 & 3.269103 & 0.008564 \\ \mathrm{H} & 2.307227 & 1.988009 & -0.022208 \\ \mathrm{C} & -2.427724 & 1.894257 & 0.014446 \\ \mathrm{C} & -3.185496 & 0.741919 & 0.006450 \\ \mathrm{~N} & -2.346368 & -0.350145 & -0.021679 \\ \mathrm{H} & -2.609014 & -1.325280 & -0.035366 \\ \mathrm{H} & -2.813930 & 2.900546 & 0.039352 \\ \mathrm{H} & -4.255501 & 0.612271 & 0.000820\end{array}$

pyrrolo[3,2- $h]$ quinoline $\mathrm{S}_{1}$

$\begin{array}{rrrr}\mathrm{C} & 0.120810 & -0.687139 & 0.056770 \\ \mathrm{~N} & -0.016071 & -2.042845 & 0.067359 \\ \mathrm{C} & 1.168716 & -2.733270 & 0.023155 \\ \mathrm{C} & 2.428846 & -2.115388 & -0.023292 \\ \mathrm{C} & 2.561146 & -0.713162 & 0.016754 \\ \mathrm{C} & 1.368382 & 0.066649 & -0.006102 \\ \mathrm{H} & 1.086146 & -3.813833 & 0.014909 \\ \mathrm{H} & 3.313957 & -2.741472 & -0.040931 \\ \mathrm{H} & 3.532626 & -0.235282 & -0.030612 \\ \mathrm{C} & -1.018342 & 0.095400 & -0.046268 \\ \mathrm{C} & -1.053095 & 1.536047 & 0.006885 \\ \mathrm{C} & 0.174006 & 2.246321 & -0.019361 \\ \mathrm{C} & 1.347563 & 1.488258 & 0.016892 \\ \mathrm{H} & 0.199115 & 3.327717 & 0.016354 \\ \mathrm{H} & 2.306119 & 1.998250 & 0.003163 \\ \mathrm{C} & -2.408797 & 1.890515 & -0.003297 \\ \mathrm{C} & -3.166350 & 0.697653 & -0.010061\end{array}$




$$
\begin{array}{lrrc}
\mathrm{N} & -2.327276 & -0.364451 & -0.037860 \\
\mathrm{H} & -2.549547 & -1.352946 & -0.021092 \\
\mathrm{H} & -2.830059 & 2.883790 & 0.024161 \\
\mathrm{H} & -4.237883 & 0.569187 & -0.007525
\end{array}
$$

carbazole $\mathrm{S}_{0}$

$\begin{array}{lrrr}\mathrm{C} & 1.129525 & 0.016007 & -0.900599 \\ \mathrm{~N} & 0.000017 & -0.000036 & -1.699881 \\ \mathrm{C} & -1.129486 & -0.016188 & -0.900590 \\ \mathrm{C} & -0.720604 & 0.021537 & 0.457585 \\ \mathrm{C} & 0.720646 & -0.021463 & 0.457586 \\ \mathrm{C} & -1.690343 & -0.031159 & 1.465330 \\ \mathrm{C} & -3.033499 & 0.002941 & 1.108236 \\ \mathrm{C} & -3.418791 & -0.008701 & -0.244278 \\ \mathrm{C} & -2.475948 & 0.034849 & -1.266056 \\ \mathrm{C} & 2.475986 & -0.035086 & -1.266063 \\ \mathrm{C} & 3.418834 & 0.008432 & -0.244289 \\ \mathrm{C} & 3.033543 & -0.002742 & 1.108229 \\ \mathrm{C} & 1.690387 & 0.031471 & 1.465314 \\ \mathrm{H} & -2.780872 & 0.030329 & -2.306207 \\ \mathrm{H} & -4.471932 & 0.017504 & -0.496668 \\ \mathrm{H} & -3.795254 & -0.031753 & 1.877155 \\ \mathrm{H} & -1.397803 & -0.032583 & 2.509260 \\ \mathrm{H} & 1.397853 & 0.033086 & 2.509246 \\ \mathrm{H} & 3.795297 & 0.032309 & 1.877133 \\ \mathrm{H} & 4.471964 & -0.018286 & -0.496670 \\ \mathrm{H} & 2.780911 & -0.030442 & -2.306213 \\ \mathrm{H} & 0.000031 & -0.001168 & -2.708311\end{array}$

carbazole $\mathrm{S}_{1}$

$\begin{array}{lrrr}\mathrm{C} & 1.149322 & -0.033060 & -0.883628 \\ \mathrm{~N} & -0.000015 & -0.000079 & -1.659642 \\ \mathrm{C} & -1.149333 & 0.033046 & -0.883614 \\ \mathrm{C} & -0.712461 & -0.019598 & 0.489690 \\ \mathrm{C} & 0.712489 & 0.019636 & 0.489674 \\ \mathrm{C} & -1.703266 & 0.036732 & 1.485445 \\ \mathrm{C} & -3.063017 & -0.003389 & 1.083400 \\ \mathrm{C} & -3.457134 & 0.016308 & -0.258328 \\ \mathrm{C} & -2.471840 & -0.025599 & -1.286885 \\ \mathrm{C} & 2.471835 & 0.025388 & -1.286897 \\ \mathrm{C} & 3.457115 & -0.016461 & -0.258335 \\ \mathrm{C} & 3.063014 & 0.003427 & 1.083397 \\ \mathrm{C} & 1.703271 & -0.036619 & 1.485445 \\ \mathrm{H} & -2.749337 & -0.020023 & -2.334688\end{array}$




$\begin{array}{rrrr}\mathrm{H} & -4.506760 & -0.024475 & -0.516679 \\ \mathrm{H} & -3.827823 & 0.013041 & 1.851786 \\ \mathrm{H} & -1.442265 & 0.029534 & 2.536754 \\ \mathrm{H} & 1.442224 & -0.029228 & 2.536743 \\ \mathrm{H} & 3.827859 & -0.012911 & 1.851744 \\ \mathrm{H} & 4.506753 & 0.024329 & -0.516648 \\ \mathrm{H} & 2.749365 & 0.019439 & -2.334688 \\ \mathrm{H} & 0.000004 & 0.000565 & -2.675006\end{array}$

tryptamine, A-ph $\mathrm{S}_{0}$

$\begin{array}{rrrr}\mathrm{C} & -2.153233 & 0.159997 & 0.898065 \\ \mathrm{~N} & -1.269424 & 0.088138 & 1.961600 \\ \mathrm{C} & 0.018036 & 0.040116 & 1.469308 \\ \mathrm{C} & -0.077636 & -0.026918 & 0.053543 \\ \mathrm{C} & -1.465396 & 0.094782 & -0.291804 \\ \mathrm{C} & 1.238699 & -0.057727 & 2.140673 \\ \mathrm{C} & 2.390600 & -0.091009 & 1.364492 \\ \mathrm{C} & 2.322802 & -0.130965 & -0.042503 \\ \mathrm{C} & 1.105013 & -0.049490 & -0.703877 \\ \mathrm{C} & -2.032992 & 0.033456 & -1.669585 \\ \mathrm{C} & -1.943732 & -1.371639 & -2.259071 \\ \mathrm{~N} & -2.425069 & -1.370124 & -3.643133 \\ \mathrm{H} & 1.294853 & -0.033562 & 3.223083 \\ \mathrm{H} & 3.355848 & -0.156841 & 1.851611 \\ \mathrm{H} & 3.242444 & -0.152639 & -0.614452 \\ \mathrm{H} & 1.064770 & -0.068765 & -1.787367 \\ \mathrm{H} & -1.509317 & 0.293308 & 2.919268 \\ \mathrm{H} & -3.213963 & 0.253070 & 1.075620 \\ \mathrm{H} & -1.500406 & 0.719099 & -2.334494 \\ \mathrm{H} & -3.081430 & 0.348489 & -1.652921 \\ \mathrm{H} & -2.487698 & -2.056920 & -1.595426 \\ \mathrm{H} & -0.899396 & -1.688415 & -2.263693 \\ \mathrm{H} & -3.418138 & -1.158240 & -3.653453 \\ \mathrm{H} & -2.322641 & -2.294819 & -4.048112\end{array}$

tryptamine, A-ph $\mathrm{S}_{1}$

$\begin{array}{rrrr}\mathrm{C} & -2.220468 & 0.196745 & 0.898833 \\ \mathrm{~N} & -1.324159 & 0.128626 & 1.892948 \\ \mathrm{C} & 0.013421 & 0.106450 & 1.404003 \\ \mathrm{C} & -0.075426 & 0.228265 & 0.008377 \\ \mathrm{C} & -1.467114 & 0.243293 & -0.323945 \\ \mathrm{C} & 1.172221 & -0.226160 & 2.110132 \\ \mathrm{C} & 2.368861 & -0.215556 & 1.293873 \\ \mathrm{C} & 2.311688 & -0.126980 & -0.083405\end{array}$




$\begin{array}{lrrr}\mathrm{C} & 1.083947 & 0.024305 & -0.798841 \\ \mathrm{C} & -2.026003 & 0.114270 & -1.683314 \\ \mathrm{C} & -1.893484 & -1.345583 & -2.153360 \\ \mathrm{~N} & -2.409151 & -1.472584 & -3.514283 \\ \mathrm{H} & 1.207990 & -0.274708 & 3.190789 \\ \mathrm{H} & 3.329261 & -0.351200 & 1.778057 \\ \mathrm{H} & 3.238994 & -0.163360 & -0.644822 \\ \mathrm{H} & 1.070782 & 0.221833 & -1.863768 \\ \mathrm{H} & -1.554241 & 0.142191 & 2.881481 \\ \mathrm{H} & -3.273440 & 0.354469 & 1.075782 \\ \mathrm{H} & -1.466844 & 0.740938 & -2.380883 \\ \mathrm{H} & -3.078880 & 0.409859 & -1.703446 \\ \mathrm{H} & -2.391304 & -1.992906 & -1.418792 \\ \mathrm{H} & -0.831889 & -1.598305 & -2.151431 \\ \mathrm{H} & -3.423767 & -1.458095 & -3.507420 \\ \mathrm{H} & -2.128421 & -2.363424 & -3.909232\end{array}$

tryptamine, A-py $\mathrm{S}_{0}$

$\begin{array}{lrrr}\mathrm{C} & -2.145768 & 0.137033 & 0.873374 \\ \mathrm{~N} & -1.271835 & 0.083093 & 1.945508 \\ \mathrm{C} & 0.020567 & 0.043113 & 1.466837 \\ \mathrm{C} & -0.060330 & -0.036357 & 0.050274 \\ \mathrm{C} & -1.445855 & 0.067870 & -0.309323 \\ \mathrm{C} & 1.234948 & -0.037473 & 2.151774 \\ \mathrm{C} & 2.395589 & -0.064902 & 1.388671 \\ \mathrm{C} & 2.343158 & -0.116051 & -0.018582 \\ \mathrm{C} & 1.131482 & -0.052700 & -0.692927 \\ \mathrm{C} & -2.012443 & -0.001821 & -1.687393 \\ \mathrm{C} & -1.907182 & -1.404300 & -2.279985 \\ \mathrm{~N} & -2.500247 & -1.428761 & -3.620300 \\ \mathrm{H} & 1.278962 & -0.005175 & 3.234532 \\ \mathrm{H} & 3.356098 & -0.117550 & 1.886582 \\ \mathrm{H} & 3.268960 & -0.133144 & -0.580712 \\ \mathrm{H} & 1.104332 & -0.084472 & -1.776555 \\ \mathrm{H} & -1.524105 & 0.285846 & 2.900498 \\ \mathrm{H} & -3.209254 & 0.220109 & 1.038504 \\ \mathrm{H} & -1.482331 & 0.700404 & -2.341201 \\ \mathrm{H} & -3.064304 & 0.292798 & -1.683509 \\ \mathrm{H} & -2.471833 & -2.089934 & -1.645402 \\ \mathrm{H} & -0.857597 & -1.723837 & -2.250551 \\ \mathrm{H} & -1.950269 & -0.842457 & -4.240827 \\ \mathrm{H} & -2.460830 & -2.368946 & -4.000170\end{array}$

tryptamine, A-py $\mathrm{S}_{1}$ 


$\begin{array}{rrrr}\mathrm{C} & -2.213633 & 0.153099 & 0.873657 \\ \mathrm{~N} & -1.329203 & 0.094557 & 1.876563 \\ \mathrm{C} & 0.014827 & 0.101502 & 1.400496 \\ \mathrm{C} & -0.060965 & 0.227903 & 0.005473 \\ \mathrm{C} & -1.448235 & 0.216404 & -0.341148 \\ \mathrm{C} & 1.173405 & -0.213931 & 2.118072 \\ \mathrm{C} & 2.375252 & -0.180851 & 1.312242 \\ \mathrm{C} & 2.332632 & -0.083781 & -0.065440 \\ \mathrm{C} & 1.109422 & 0.048723 & -0.793159 \\ \mathrm{C} & -1.996547 & 0.078269 & -1.702876 \\ \mathrm{C} & -1.853681 & -1.383857 & -2.165804 \\ \mathrm{~N} & -2.422010 & -1.526432 & -3.504670 \\ \mathrm{H} & 1.201260 & -0.259940 & 3.199026 \\ \mathrm{H} & 3.333238 & -0.302156 & 1.805229 \\ \mathrm{H} & 3.267005 & -0.102791 & -0.615878 \\ \mathrm{H} & 1.104422 & 0.263942 & -1.854914 \\ \mathrm{H} & -1.570171 & 0.100586 & 2.862408 \\ \mathrm{H} & -3.273149 & 0.278249 & 1.036786 \\ \mathrm{H} & -1.431081 & 0.715669 & -2.388435 \\ \mathrm{H} & -3.051456 & 0.355938 & -1.744466 \\ \mathrm{H} & -2.422357 & -2.019120 & -1.482914 \\ \mathrm{H} & -0.795730 & -1.659224 & -2.081357 \\ \mathrm{H} & -1.814788 & -1.072489 & -4.180161 \\ \mathrm{H} & -2.458543 & -2.507886 & -3.759624\end{array}$

tryptamine, A-up $\mathrm{S}_{0}$

$\begin{array}{lrrr}\mathrm{C} & -2.144987 & 0.153065 & 0.884295 \\ \mathrm{~N} & -1.270022 & 0.070931 & 1.953588 \\ \mathrm{C} & 0.021689 & 0.034848 & 1.472623 \\ \mathrm{C} & -0.060937 & -0.010702 & 0.054865 \\ \mathrm{C} & -1.446174 & 0.111303 & -0.300771 \\ \mathrm{C} & 1.236288 & -0.069297 & 2.153988 \\ \mathrm{C} & 2.395627 & -0.087879 & 1.388694 \\ \mathrm{C} & 2.341314 & -0.108049 & -0.019243 \\ \mathrm{C} & 1.129504 & -0.020481 & -0.690555 \\ \mathrm{C} & -2.007825 & 0.055666 & -1.681336 \\ \mathrm{C} & -1.908015 & -1.350028 & -2.290966 \\ \mathrm{~N} & -2.414370 & -1.488285 & -3.655497 \\ \mathrm{H} & 1.281864 & -0.062149 & 3.237112 \\ \mathrm{H} & 3.356279 & -0.158765 & 1.884027 \\ \mathrm{H} & 3.266292 & -0.121316 & -0.582716 \\ \mathrm{H} & 1.101097 & -0.029878 & -1.774574 \\ \mathrm{H} & -1.520531 & 0.249058 & 2.913958 \\ \mathrm{H} & -3.207760 & 0.237115 & 1.053857 \\ \mathrm{H} & -1.477097 & 0.760322 & -2.331449\end{array}$




$\begin{array}{llll}\mathrm{H} & -3.058940 & 0.361277 & -1.668717 \\ \mathrm{H} & -2.456368 & -2.044572 & -1.651238 \\ \mathrm{H} & -0.865183 & -1.672140 & -2.280597 \\ \mathrm{H} & -1.904431 & -0.866367 & -4.275176 \\ \mathrm{H} & -3.386071 & -1.195766 & -3.690926\end{array}$

tryptamine, A-up $\mathrm{S}_{1}$

$\begin{array}{lrrr}\mathrm{C} & -2.214751 & 0.168285 & 0.883544 \\ \mathrm{~N} & -1.324386 & 0.079913 & 1.882034 \\ \mathrm{C} & 0.016835 & 0.092087 & 1.405242 \\ \mathrm{C} & -0.061922 & 0.254683 & 0.013445 \\ \mathrm{C} & -1.451285 & 0.270521 & -0.330193 \\ \mathrm{C} & 1.175745 & -0.243089 & 2.110932 \\ \mathrm{C} & 2.376796 & -0.198111 & 1.304309 \\ \mathrm{C} & 2.329909 & -0.068191 & -0.069953 \\ \mathrm{C} & 1.105414 & 0.091643 & -0.790529 \\ \mathrm{C} & -1.989329 & 0.143196 & -1.692249 \\ \mathrm{C} & -1.889783 & -1.346817 & -2.140028 \\ \mathrm{~N} & -2.330129 & -1.616013 & -3.496459 \\ \mathrm{H} & 1.204597 & -0.322421 & 3.189948 \\ \mathrm{H} & 3.334835 & -0.340699 & 1.791245 \\ \mathrm{H} & 3.261951 & -0.079634 & -0.624547 \\ \mathrm{H} & 1.100435 & 0.327493 & -1.847932 \\ \mathrm{H} & -1.562888 & 0.058027 & 2.868404 \\ \mathrm{H} & -3.270869 & 0.305622 & 1.058815 \\ \mathrm{H} & -1.393931 & 0.744061 & -2.383874 \\ \mathrm{H} & -3.033718 & 0.462609 & -1.744451 \\ \mathrm{H} & -2.484845 & -1.951228 & -1.452625 \\ \mathrm{H} & -0.847518 & -1.652702 & -2.035571 \\ \mathrm{H} & -1.758643 & -1.110122 & -4.164506 \\ \mathrm{H} & -3.291294 & -1.321189 & -3.631778\end{array}$

tryptamine, $\mathrm{Ph}$-out $\mathrm{S}_{0}$

$\begin{array}{rrrr}\mathrm{C} & -0.015055 & -0.036602 & 0.060616 \\ \mathrm{C} & 0.035322 & 0.051111 & 1.478580 \\ \mathrm{C} & 1.236786 & 0.004010 & 2.189159 \\ \mathrm{C} & 2.412478 & 0.009736 & 1.449364 \\ \mathrm{C} & 2.388934 & -0.043967 & 0.041060 \\ \mathrm{C} & 1.191690 & -0.022270 & -0.660956 \\ \mathrm{~N} & -1.266537 & 0.060681 & 1.932946 \\ \mathrm{H} & 1.257194 & 0.039080 & 3.272587 \\ \mathrm{H} & 3.364060 & -0.013682 & 1.966528 \\ \mathrm{H} & 3.326288 & -0.036556 & -0.501939 \\ \mathrm{H} & 1.179275 & -0.073744 & -1.742827\end{array}$




$\begin{array}{lrrr}\mathrm{C} & -1.396948 & 0.033513 & -0.325935 \\ \mathrm{C} & -2.120365 & 0.092360 & 0.844747 \\ \mathrm{H} & -1.541227 & 0.263082 & 2.881908 \\ \mathrm{H} & -3.187411 & 0.167972 & 0.990428 \\ \mathrm{C} & -1.952041 & -0.025578 & -1.711818 \\ \mathrm{H} & -1.323195 & 0.556600 & -2.391250 \\ \mathrm{C} & -2.055105 & -1.452928 & -2.245992 \\ \mathrm{H} & -2.950357 & 0.421849 & -1.719988 \\ \mathrm{H} & -2.481857 & -1.426280 & -3.251468 \\ \mathrm{~N} & -0.725337 & -2.058559 & -2.338510 \\ \mathrm{H} & -2.750277 & -2.010678 & -1.602629 \\ \mathrm{H} & -0.349112 & -2.200246 & -1.405347 \\ \mathrm{H} & -0.792228 & -2.974793 & -2.770053\end{array}$

tryptamine, $\mathrm{Ph}$-out $\mathrm{S}_{1}$

$\begin{array}{rrrr}\mathrm{C} & -0.014032 & 0.159474 & 0.014538 \\ \mathrm{C} & 0.022384 & 0.119830 & 1.416656 \\ \mathrm{C} & 1.161465 & -0.151952 & 2.185910 \\ \mathrm{C} & 2.386350 & -0.127208 & 1.418556 \\ \mathrm{C} & 2.389709 & -0.086428 & 0.037474 \\ \mathrm{C} & 1.186620 & -0.040735 & -0.737073 \\ \mathrm{~N} & -1.332827 & 0.139592 & 1.859250 \\ \mathrm{H} & 1.156743 & -0.128058 & 3.268120 \\ \mathrm{H} & 3.330266 & -0.184151 & 1.949165 \\ \mathrm{H} & 3.341894 & -0.105644 & -0.481251 \\ \mathrm{H} & 1.212276 & 0.133392 & -1.806028 \\ \mathrm{C} & -1.391940 & 0.158381 & -0.368533 \\ \mathrm{C} & -2.186457 & 0.159323 & 0.832685 \\ \mathrm{H} & -1.598512 & 0.195413 & 2.837015 \\ \mathrm{H} & -3.250627 & 0.285320 & 0.960641 \\ \mathrm{C} & -1.954394 & -0.024570 & -1.723341 \\ \mathrm{H} & -1.318948 & 0.464205 & -2.464932 \\ \mathrm{C} & -2.031074 & -1.522587 & -2.082359 \\ \mathrm{H} & -2.959106 & 0.403632 & -1.768517 \\ \mathrm{H} & -2.691758 & -1.627951 & -2.945665 \\ \mathrm{~N} & -0.709668 & -2.014212 & -2.447577 \\ \mathrm{H} & -2.499696 & -2.050594 & -1.238535 \\ \mathrm{H} & -0.033085 & -1.808053 & -1.711329 \\ \mathrm{H} & -0.730592 & -3.022295 & -2.565659\end{array}$

tryptamine, $\mathrm{Ph}$-up $\mathrm{S}_{0}$
$\begin{array}{llll}\text { C } & -2.105790 & 0.049529 & 0.813440\end{array}$
$\begin{array}{llll}\mathrm{N} & -1.262551 & -0.013270 & 1.907953\end{array}$
$\begin{array}{llll}\text { C } & 0.043482 & 0.024794 & 1.470590\end{array}$ 


$\begin{array}{rrrr}\text { C } & 0.008781 & 0.011919 & 0.049167 \\ \mathrm{C} & -1.368538 & 0.069484 & -0.350457 \\ \mathrm{C} & 1.238570 & -0.037373 & 2.190869 \\ \mathrm{C} & 2.422036 & 0.027679 & 1.466784 \\ \mathrm{C} & 2.415095 & 0.052556 & 0.057490 \\ \mathrm{C} & 1.223695 & 0.094767 & -0.652765 \\ \mathrm{C} & -1.902146 & 0.021984 & -1.743939 \\ \mathrm{C} & -2.073004 & -1.417587 & -2.257698 \\ \mathrm{~N} & -0.859156 & -2.226403 & -2.302565 \\ \mathrm{H} & 1.247139 & -0.059535 & 3.274737 \\ \mathrm{H} & 3.368186 & -0.007110 & 1.992839 \\ \mathrm{H} & 3.357503 & 0.110890 & -0.473381 \\ \mathrm{H} & 1.231971 & 0.122908 & -1.736450 \\ \mathrm{H} & -1.553123 & 0.115126 & 2.865074 \\ \mathrm{H} & -3.175952 & 0.083835 & 0.951252 \\ \mathrm{H} & -1.227069 & 0.563272 & -2.415397 \\ \mathrm{H} & -2.871038 & 0.530195 & -1.787397 \\ \mathrm{H} & -2.501789 & -1.392195 & -3.262627 \\ \mathrm{H} & -2.791099 & -1.929740 & -1.612679 \\ \mathrm{H} & -0.432308 & -2.254484 & -1.381131 \\ \mathrm{H} & -0.179248 & -1.791612 & -2.918647\end{array}$

tryptamine, $\mathrm{Ph}$-up $\mathrm{S}_{1}$

$\begin{array}{lrrr}\mathrm{C} & -2.188868 & 0.173502 & 0.833036 \\ \mathrm{~N} & -1.334748 & 0.126101 & 1.860751 \\ \mathrm{C} & 0.019169 & 0.115961 & 1.419327 \\ \mathrm{C} & -0.016505 & 0.199430 & 0.019401 \\ \mathrm{C} & -1.393926 & 0.206727 & -0.365044 \\ \mathrm{C} & 1.160116 & -0.173206 & 2.179782 \\ \mathrm{C} & 2.383861 & -0.129626 & 1.413228 \\ \mathrm{C} & 2.388024 & -0.048948 & 0.034112 \\ \mathrm{C} & 1.184524 & 0.021704 & -0.739490 \\ \mathrm{C} & -1.934424 & 0.020821 & -1.716958 \\ \mathrm{C} & -1.957079 & -1.508319 & -2.077983 \\ \mathrm{~N} & -0.674375 & -2.126538 & -2.306753 \\ \mathrm{H} & 1.155902 & -0.181320 & 3.262187 \\ \mathrm{H} & 3.327908 & -0.206491 & 1.941183 \\ \mathrm{H} & 3.340934 & -0.049821 & -0.483843 \\ \mathrm{H} & 1.215202 & 0.267372 & -1.794984 \\ \mathrm{H} & -1.601491 & 0.156385 & 2.839073 \\ \mathrm{H} & -3.253228 & 0.294203 & 0.964689 \\ \mathrm{H} & -1.314580 & 0.533525 & -2.455640 \\ \mathrm{H} & -2.959081 & 0.399626 & -1.778510 \\ \mathrm{H} & -2.566657 & -1.616695 & -2.977403 \\ \mathrm{H} & -2.466395 & -2.035088 & -1.268376\end{array}$




$$
\begin{array}{llll}
\mathrm{H} & -0.032432 & -1.962742 & -1.530737 \\
\mathrm{H} & -0.228190 & -1.726953 & -3.125958
\end{array}
$$

tryptamine, Py-out $\mathrm{S}_{0}$

$\begin{array}{rrrr}\mathrm{C} & 0.475627 & 0.435031 & 0.195963 \\ \mathrm{C} & 0.559148 & 0.516085 & 1.612907 \\ \mathrm{C} & 1.768763 & 0.390655 & 2.299321 \\ \mathrm{C} & 2.927839 & 0.312117 & 1.537226 \\ \mathrm{C} & 2.874139 & 0.255481 & 0.130386 \\ \mathrm{C} & 1.666736 & 0.365848 & -0.546011 \\ \mathrm{~N} & -0.731189 & 0.605446 & 2.091245 \\ \mathrm{H} & 1.813386 & 0.428196 & 3.381898 \\ \mathrm{H} & 3.885202 & 0.224258 & 2.036243 \\ \mathrm{H} & 3.798938 & 0.198083 & -0.430697 \\ \mathrm{H} & 1.639841 & 0.332194 & -1.629311 \\ \mathrm{C} & -0.905161 & 0.593663 & -0.163830 \\ \mathrm{C} & -1.602924 & 0.685221 & 1.018885 \\ \mathrm{H} & -0.976359 & 0.817149 & 3.046253 \\ \mathrm{H} & -2.663995 & 0.797673 & 1.180793 \\ \mathrm{C} & -1.478622 & 0.510172 & -1.538839 \\ \mathrm{H} & -0.942526 & 1.186132 & -2.212505 \\ \mathrm{C} & -1.399123 & -0.913105 & -2.089635 \\ \mathrm{H} & -2.527518 & 0.816167 & -1.520410 \\ \mathrm{H} & -0.342461 & -1.192526 & -2.196102 \\ \mathrm{~N} & -2.155190 & -1.815814 & -1.219816 \\ \mathrm{H} & -1.852556 & -0.940916 & -3.082740 \\ \mathrm{H} & -2.123867 & -2.759220 & -1.593350 \\ \mathrm{H} & -1.708107 & -1.847990 & -0.307856\end{array}$

tryptamine, Py-out $\mathrm{S}_{1}$

$\begin{array}{lrrr}\mathrm{C} & 0.418701 & 0.627579 & 0.133455 \\ \mathrm{C} & 0.489639 & 0.577264 & 1.530944 \\ \mathrm{C} & 1.612002 & 0.158281 & 2.266738 \\ \mathrm{C} & 2.805995 & 0.055297 & 1.460353 \\ \mathrm{C} & 2.777542 & 0.121579 & 0.079871 \\ \mathrm{C} & 1.566779 & 0.320438 & -0.659851 \\ \mathrm{~N} & -0.848350 & 0.729488 & 2.013114 \\ \mathrm{H} & 1.646460 & 0.188384 & 3.348371 \\ \mathrm{H} & 3.752070 & -0.121936 & 1.960184 \\ \mathrm{H} & 3.708341 & 0.003243 & -0.464234 \\ \mathrm{H} & 1.588024 & 0.528042 & -1.723036 \\ \mathrm{C} & -0.965203 & 0.778355 & -0.208658 \\ \mathrm{C} & -1.718369 & 0.849753 & 1.013041 \\ \mathrm{H} & -1.085194 & 0.768159 & 2.998881\end{array}$




$\begin{array}{lrrr}\mathrm{H} & -2.773646 & 1.012449 & 1.165066 \\ \mathrm{C} & -1.560560 & 0.586448 & -1.546844 \\ \mathrm{H} & -1.103153 & 1.271105 & -2.267578 \\ \mathrm{C} & -1.288326 & -0.863778 & -1.994404 \\ \mathrm{H} & -2.637231 & 0.764044 & -1.512224 \\ \mathrm{H} & -0.239559 & -0.941320 & -2.294453 \\ \mathrm{~N} & -1.638545 & -1.784314 & -0.908171 \\ \mathrm{H} & -1.917746 & -1.082717 & -2.858040 \\ \mathrm{H} & -1.729367 & -2.724958 & -1.280167 \\ \mathrm{H} & -0.860311 & -1.820895 & -0.252369\end{array}$

tryptamine, Py-up $\mathrm{S}_{0}$

$\begin{array}{lrrr}\mathrm{C} & -2.158818 & 0.286381 & 0.881282 \\ \mathrm{~N} & -1.285278 & 0.196574 & 1.951457 \\ \mathrm{C} & 0.002088 & 0.088421 & 1.468600 \\ \mathrm{C} & -0.086538 & 0.006629 & 0.052527 \\ \mathrm{C} & -1.466726 & 0.178460 & -0.303760 \\ \mathrm{C} & 1.211957 & -0.052680 & 2.151546 \\ \mathrm{C} & 2.367182 & -0.147676 & 1.385785 \\ \mathrm{C} & 2.308244 & -0.206558 & -0.020774 \\ \mathrm{C} & 1.100610 & -0.081282 & -0.693726 \\ \mathrm{C} & -2.035239 & 0.109682 & -1.679885 \\ \mathrm{C} & -1.950835 & -1.313220 & -2.258695 \\ \mathrm{~N} & -2.632486 & -2.341982 & -1.480373 \\ \mathrm{H} & 1.260371 & -0.015267 & 3.233905 \\ \mathrm{H} & 3.324806 & -0.248372 & 1.881799 \\ \mathrm{H} & 3.230230 & -0.279327 & -0.584524 \\ \mathrm{H} & 1.070013 & -0.119009 & -1.776760 \\ \mathrm{H} & -1.523267 & 0.418427 & 2.905971 \\ \mathrm{H} & -3.215870 & 0.427531 & 1.048990 \\ \mathrm{H} & -1.503709 & 0.800675 & -2.343958 \\ \mathrm{H} & -3.084626 & 0.422317 & -1.661190 \\ \mathrm{H} & -0.901809 & -1.603979 & -2.341378 \\ \mathrm{H} & -2.368141 & -1.317214 & -3.268213 \\ \mathrm{H} & -3.617687 & -2.108915 & -1.393249 \\ \mathrm{H} & -2.255949 & -2.349995 & -0.536489\end{array}$

tryptamine, Py-up $S_{1}$

$\begin{array}{rrrr}\mathrm{C} & -2.271255 & 0.416939 & 0.872478 \\ \mathrm{~N} & -1.389256 & 0.314942 & 1.873585 \\ \mathrm{C} & -0.057858 & 0.149488 & 1.392646 \\ \mathrm{C} & -0.134158 & 0.193461 & -0.008424 \\ \mathrm{C} & -1.517397 & 0.336432 & -0.349038 \\ \mathrm{C} & 1.065382 & -0.249340 & 2.125202\end{array}$




$\begin{array}{lrrr}\mathrm{C} & 2.264005 & -0.361063 & 1.320891 \\ \mathrm{C} & 2.226707 & -0.317063 & -0.058621 \\ \mathrm{C} & 1.016171 & -0.117768 & -0.794903 \\ \mathrm{C} & -2.101715 & 0.178412 & -1.693059 \\ \mathrm{C} & -1.855740 & -1.271208 & -2.200011 \\ \mathrm{~N} & -2.231265 & -2.332829 & -1.282209 \\ \mathrm{H} & 1.092944 & -0.239481 & 3.207188 \\ \mathrm{H} & 3.209354 & -0.538518 & 1.821043 \\ \mathrm{H} & 3.153880 & -0.440659 & -0.607499 \\ \mathrm{H} & 1.033721 & 0.056323 & -1.863827 \\ \mathrm{H} & -1.618808 & 0.389105 & 2.859581 \\ \mathrm{H} & -3.311372 & 0.655826 & 1.032616 \\ \mathrm{H} & -1.627673 & 0.872937 & -2.394637 \\ \mathrm{H} & -3.176104 & 0.382121 & -1.670358 \\ \mathrm{H} & -0.792606 & -1.383549 & -2.409042 \\ \mathrm{H} & -2.403798 & -1.397525 & -3.135487 \\ \mathrm{H} & -3.177755 & -2.207653 & -0.937855 \\ \mathrm{H} & -1.606883 & -2.339698 & -0.481344\end{array}$

porphycene $\mathrm{S}_{0}$

$\begin{array}{lrrr}\mathrm{C} & -3.443303 & 1.914343 & -0.001322 \\ \mathrm{C} & -2.412824 & 0.916434 & -0.001417 \\ \mathrm{~N} & -1.214195 & 1.564133 & -0.001513 \\ \mathrm{C} & -1.399770 & 2.918984 & -0.001582 \\ \mathrm{C} & -2.818154 & 3.149631 & -0.001468 \\ \mathrm{C} & -0.372394 & 3.885827 & -0.001672 \\ \mathrm{C} & 1.017453 & 3.732163 & -0.001602 \\ \mathrm{C} & 1.831359 & 2.574455 & -0.001448 \\ \mathrm{H} & -0.223446 & 1.162588 & -0.001703 \\ \mathrm{~N} & 1.397588 & 1.278350 & -0.001610 \\ \mathrm{C} & 3.283961 & 2.608116 & -0.001164 \\ \mathrm{C} & 3.709484 & 1.299806 & -0.001079 \\ \mathrm{C} & 2.505620 & 0.495081 & -0.001506 \\ \mathrm{C} & 2.412861 & -0.916435 & -0.001143 \\ \mathrm{C} & 3.443339 & -1.914348 & -0.001262 \\ \mathrm{C} & 2.818189 & -3.149633 & -0.001432 \\ \mathrm{C} & 1.399807 & -2.918986 & -0.001528 \\ \mathrm{~N} & 1.214229 & -1.564134 & -0.001331 \\ \mathrm{C} & 0.372430 & -3.885828 & -0.001518 \\ \mathrm{C} & -1.017417 & -3.732162 & -0.001448 \\ \mathrm{C} & -1.831322 & -2.574454 & -0.001301 \\ \mathrm{C} & -3.283927 & -2.608119 & -0.001282 \\ \mathrm{C} & -3.709448 & -1.299807 & -0.001302 \\ \mathrm{C} & -2.505585 & -0.495083 & -0.001212 \\ \mathrm{~N} & -1.397553 & -1.278350 & -0.001311\end{array}$




$\begin{array}{lrrr}\mathrm{H} & 0.223483 & -1.162585 & -0.001269 \\ \mathrm{H} & 3.290714 & -4.130971 & -0.001389 \\ \mathrm{H} & 4.513395 & -1.714241 & -0.001057 \\ \mathrm{H} & 0.735559 & -4.918223 & -0.001564 \\ \mathrm{H} & -1.583281 & -4.669628 & -0.001707 \\ \mathrm{H} & -3.898122 & -3.508744 & -0.001275 \\ \mathrm{H} & -4.734265 & -0.930071 & -0.001418 \\ \mathrm{H} & -4.513359 & 1.714236 & -0.001198 \\ \mathrm{H} & -3.290683 & 4.130967 & -0.001516 \\ \mathrm{H} & -0.735524 & 4.918222 & -0.001979 \\ \mathrm{H} & 1.583316 & 4.669630 & -0.001340 \\ \mathrm{H} & 3.898157 & 3.508741 & -0.000989 \\ \mathrm{H} & 4.734301 & 0.930070 & -0.000758\end{array}$

porphycene $\mathrm{S}_{1}$

$\begin{array}{lrrr}\mathrm{C} & -3.465924 & 1.905468 & 0.000137 \\ \mathrm{C} & -2.441130 & 0.907580 & 0.000149 \\ \mathrm{~N} & -1.235320 & 1.554219 & -0.000123 \\ \mathrm{C} & -1.419358 & 2.910930 & -0.000047 \\ \mathrm{C} & -2.834942 & 3.143522 & 0.000064 \\ \mathrm{C} & -0.389590 & 3.883386 & 0.000093 \\ \mathrm{C} & 1.002879 & 3.742032 & 0.000034 \\ \mathrm{C} & 1.847362 & 2.596972 & 0.000127 \\ \mathrm{H} & -0.258778 & 1.148585 & -0.000221 \\ \mathrm{~N} & 1.415076 & 1.287634 & -0.000267 \\ \mathrm{C} & 3.287030 & 2.636969 & 0.000173 \\ \mathrm{C} & 3.726745 & 1.323238 & -0.000189 \\ \mathrm{C} & 2.544340 & 0.509564 & -0.000484 \\ \mathrm{C} & 2.441130 & -0.907581 & 0.000133 \\ \mathrm{C} & 3.465925 & -1.905468 & 0.000277 \\ \mathrm{C} & 2.834940 & -3.143523 & 0.000098 \\ \mathrm{C} & 1.419359 & -2.910930 & -0.000164 \\ \mathrm{~N} & 1.235319 & -1.554218 & -0.000292 \\ \mathrm{C} & 0.389590 & -3.883387 & -0.000180 \\ \mathrm{C} & -1.002878 & -3.742032 & 0.000016 \\ \mathrm{C} & -1.847358 & -2.596970 & 0.000002 \\ \mathrm{C} & -3.287029 & -2.636968 & 0.000007 \\ \mathrm{C} & -3.726743 & -1.323239 & -0.000119 \\ \mathrm{C} & -2.544338 & -0.509564 & -0.000183 \\ \mathrm{~N} & -1.415076 & -1.287633 & 0.000029 \\ \mathrm{H} & 0.258775 & -1.148588 & -0.000178 \\ \mathrm{H} & 3.305004 & -4.126042 & 0.000216 \\ \mathrm{H} & 4.536219 & -1.707192 & 0.000679 \\ \mathrm{H} & 0.757052 & -4.913829 & -0.000176 \\ \mathrm{H} & -1.555207 & -4.688018 & 0.000026\end{array}$




$\begin{array}{rrrr}\mathrm{H} & -3.897453 & -3.540294 & 0.000105 \\ \mathrm{H} & -4.756182 & -0.966321 & -0.000235 \\ \mathrm{H} & -4.536220 & 1.707199 & 0.000199 \\ \mathrm{H} & -3.305012 & 4.126038 & 0.000095 \\ \mathrm{H} & -0.757048 & 4.913828 & 0.000121 \\ \mathrm{H} & 1.555201 & 4.688021 & 0.000163 \\ \mathrm{H} & 3.897454 & 3.540294 & 0.000420 \\ \mathrm{H} & 4.756183 & 0.966320 & -0.000503\end{array}$

porphine $\mathrm{S}_{0}$

$\begin{array}{rrrr}\mathrm{N} & -0.000673 & -2.016906 & -0.000407 \\ \mathrm{~N} & 2.124854 & 0.000830 & -0.000362 \\ \mathrm{C} & 1.084459 & -2.848978 & -0.000804 \\ \mathrm{C} & 0.683636 & -4.249976 & -0.000963 \\ \mathrm{C} & 2.427230 & -2.441751 & -0.000827 \\ \mathrm{C} & 2.903545 & -1.127012 & -0.000544 \\ \mathrm{C} & 4.270451 & -0.690061 & -0.000400 \\ \mathrm{H} & 1.361468 & -5.103595 & -0.001337 \\ \mathrm{H} & 3.187716 & -3.227824 & -0.000999 \\ \mathrm{H} & 5.130837 & -1.357422 & -0.000421 \\ \mathrm{H} & 1.100525 & 0.002140 & -0.000394 \\ \mathrm{~N} & 0.000611 & 2.016997 & -0.000165 \\ \mathrm{~N} & -2.124930 & -0.000749 & 0.000231 \\ \mathrm{C} & 1.085271 & 2.849731 & -0.000177 \\ \mathrm{C} & -1.084527 & 2.849064 & -0.000091 \\ \mathrm{C} & -1.085339 & -2.849640 & -0.000193 \\ \mathrm{C} & 0.683647 & 4.250476 & -0.000178 \\ \mathrm{C} & -0.683707 & 4.250061 & -0.000151 \\ \mathrm{C} & -0.683713 & -4.250382 & -0.000549 \\ \mathrm{C} & 2.428191 & 2.442964 & -0.000073 \\ \mathrm{C} & -2.427286 & 2.441823 & 0.000137 \\ \mathrm{C} & -2.428270 & -2.442879 & 0.000103 \\ \mathrm{C} & 2.904360 & 1.128167 & -0.000170 \\ \mathrm{C} & -2.903628 & 1.127095 & 0.000496 \\ \mathrm{C} & -2.904439 & -1.128083 & 0.000597 \\ \mathrm{C} & 4.270969 & 0.690377 & -0.000138 \\ \mathrm{C} & -4.270533 & 0.690149 & 0.001060 \\ \mathrm{C} & -4.271047 & -0.690291 & 0.001166 \\ \mathrm{H} & 1.360978 & 5.104494 & -0.000260 \\ \mathrm{H} & -1.361558 & 5.103664 & -0.000129 \\ \mathrm{H} & -1.361045 & -5.104399 & -0.000570 \\ \mathrm{H} & 3.188535 & 3.229165 & 0.000085 \\ \mathrm{H} & -3.187770 & 3.227897 & 0.000173 \\ \mathrm{H} & -3.188594 & -3.229104 & 0.000571 \\ \mathrm{H} & 5.131852 & 1.357075 & -0.000115\end{array}$




$$
\begin{array}{rrrr}
\mathrm{H} & -5.130935 & 1.357481 & 0.001422 \\
\mathrm{H} & -5.131904 & -1.357022 & 0.001462 \\
\mathrm{H} & -1.100601 & -0.002017 & -0.000074
\end{array}
$$

porphine $\mathrm{S}_{1}$

$\begin{array}{lrrr}\mathrm{N} & 0.000000 & -2.042770 & -0.000007 \\ \mathrm{~N} & 2.120309 & 0.000001 & -0.000005 \\ \mathrm{C} & 1.090183 & -2.882272 & 0.000020 \\ \mathrm{C} & 0.686908 & -4.274771 & 0.000010 \\ \mathrm{C} & 2.435181 & -2.451582 & 0.000038 \\ \mathrm{C} & 2.903544 & -1.130610 & 0.000022 \\ \mathrm{C} & 4.269331 & -0.691926 & 0.000017 \\ \mathrm{H} & 1.362144 & -5.130325 & -0.000004 \\ \mathrm{H} & 3.203591 & -3.229939 & 0.000072 \\ \mathrm{H} & 5.130677 & -1.357872 & 0.000021 \\ \mathrm{H} & 1.098450 & 0.000002 & -0.000028 \\ \mathrm{~N} & 0.000001 & 2.042768 & -0.000014 \\ \mathrm{~N} & -2.120309 & 0.000000 & 0.000008 \\ \mathrm{C} & 1.090183 & 2.882272 & -0.000022 \\ \mathrm{C} & -1.090182 & 2.882272 & 0.000017 \\ \mathrm{C} & -1.090183 & -2.882273 & -0.000019 \\ \mathrm{C} & 0.686908 & 4.274772 & -0.000013 \\ \mathrm{C} & -0.686907 & 4.274772 & 0.000028 \\ \mathrm{C} & -0.686908 & -4.274773 & -0.000001 \\ \mathrm{C} & 2.435181 & 2.451583 & -0.000030 \\ \mathrm{C} & -2.435180 & 2.451583 & 0.000028 \\ \mathrm{C} & -2.435182 & -2.451583 & -0.000036 \\ \mathrm{C} & 2.903544 & 1.130611 & -0.000007 \\ \mathrm{C} & -2.903544 & 1.130611 & 0.000007 \\ \mathrm{C} & -2.903545 & -1.130611 & -0.000019 \\ \mathrm{C} & 4.269331 & 0.691925 & -0.000002 \\ \mathrm{C} & -4.269331 & 0.691925 & 0.000002 \\ \mathrm{C} & -4.269332 & -0.691926 & -0.000016 \\ \mathrm{H} & 1.362143 & 5.130326 & -0.000040 \\ \mathrm{H} & -1.362142 & 5.130327 & 0.000058 \\ \mathrm{H} & -1.362143 & -5.130327 & 0.000014 \\ \mathrm{H} & 3.203590 & 3.229941 & -0.000090 \\ \mathrm{H} & -3.203589 & 3.229942 & 0.000070 \\ \mathrm{H} & -3.203592 & -3.229942 & -0.000084 \\ \mathrm{H} & 5.130676 & 1.357871 & -0.000021 \\ \mathrm{H} & -5.130676 & 1.357872 & 0.000015 \\ \mathrm{H} & -5.130678 & -1.357872 & -0.000025 \\ \mathrm{H} & -1.098451 & 0.000000 & 0.000035\end{array}$

chlorin $\mathrm{S}_{0}$ 


$\begin{array}{lrrr}\mathrm{C} & -0.077908 & -4.264318 & 0.921875 \\ \mathrm{C} & -0.070915 & -2.903863 & 1.367143 \\ \mathrm{~N} & -0.031485 & -2.115759 & 0.248919 \\ \mathrm{C} & -0.003524 & -2.885448 & -0.888732 \\ \mathrm{C} & -0.034765 & -4.250847 & -0.460600 \\ \mathrm{C} & -0.080493 & -2.426964 & 2.686036 \\ \mathrm{C} & -0.041414 & -1.086594 & 3.084538 \\ \mathrm{~N} & 0.000290 & 0.000014 & 2.252061 \\ \mathrm{C} & 0.041872 & 1.086477 & 3.084745 \\ \mathrm{C} & 0.027803 & 0.681392 & 4.487916 \\ \mathrm{C} & -0.027655 & -0.681755 & 4.487788 \\ \mathrm{C} & 0.081036 & 2.426901 & 2.686424 \\ \mathrm{C} & 0.071115 & 2.903883 & 1.367562 \\ \mathrm{~N} & 0.031075 & 2.115732 & 0.249383 \\ \mathrm{C} & 0.003395 & 2.885369 & -0.888300 \\ \mathrm{C} & 0.034944 & 4.250784 & -0.460216 \\ \mathrm{C} & 0.078270 & 4.264318 & 0.922252 \\ \mathrm{C} & -0.052808 & 2.418470 & -2.208109 \\ \mathrm{C} & -0.062330 & 1.098775 & -2.658397 \\ \mathrm{C} & -0.204216 & 0.736019 & -4.128196 \\ \mathrm{C} & 0.203883 & -0.735658 & -4.128318 \\ \mathrm{C} & 0.061916 & -1.098693 & -2.658604 \\ \mathrm{~N} & -0.000313 & -0.000036 & -1.866708 \\ \mathrm{C} & 0.052550 & -2.418457 & -2.208516 \\ \mathrm{H} & -1.256686 & 0.858973 & -4.433110 \\ \mathrm{H} & -0.094007 & 3.198046 & -2.974376 \\ \mathrm{H} & 0.024865 & 5.106624 & -1.133606 \\ \mathrm{H} & 0.054598 & 1.359682 & 5.340706 \\ \mathrm{H} & -0.054755 & -1.360199 & 5.340446 \\ \mathrm{H} & -0.410556 & -1.370445 & -4.781807 \\ \mathrm{H} & 0.111525 & 3.185902 & 3.473198 \\ \mathrm{H} & -0.110595 & -3.186056 & 3.472738 \\ \mathrm{H} & 0.093922 & -3.197938 & -2.974868 \\ \mathrm{H} & 0.109420 & 5.130297 & 1.581439 \\ \mathrm{H} & -0.108893 & -5.130263 & 1.581116 \\ \mathrm{H} & -0.024617 & -5.106723 & -1.133942 \\ \mathrm{H} & 1.256370 & -0.858561 & -4.433192 \\ \mathrm{H} & 0.410259 & 1.370929 & -4.781531 \\ \mathrm{H} & -0.011888 & -1.094045 & 0.281994 \\ \mathrm{H} & 0.010717 & 1.094035 & 0.282836\end{array}$

chlorin $\mathrm{S}_{1}$

$\begin{array}{llll}\text { C } & -0.092030 & -4.271878 & 0.921706 \\ \text { C } & -0.079824 & -2.909381 & 1.364025\end{array}$ 


$\begin{array}{rrrr}\mathrm{N} & -0.028069 & -2.122512 & 0.241597 \\ \mathrm{C} & 0.001720 & -2.901576 & -0.895768 \\ \mathrm{C} & -0.039383 & -4.263350 & -0.465664 \\ \mathrm{C} & -0.096280 & -2.434293 & 2.688453 \\ \mathrm{C} & -0.048644 & -1.088044 & 3.094971 \\ \mathrm{~N} & 0.000070 & -0.000022 & 2.259476 \\ \mathrm{C} & 0.048980 & 1.087874 & 3.095139 \\ \mathrm{C} & 0.032973 & 0.681507 & 4.498843 \\ \mathrm{C} & -0.032256 & -0.681902 & 4.498741 \\ \mathrm{C} & 0.096521 & 2.434175 & 2.688781 \\ \mathrm{C} & 0.079766 & 2.909365 & 1.364389 \\ \mathrm{~N} & 0.027924 & 2.122497 & 0.241958 \\ \mathrm{C} & -0.001914 & 2.901562 & -0.895396 \\ \mathrm{C} & 0.039082 & 4.263336 & -0.465275 \\ \mathrm{C} & 0.091790 & 4.271866 & 0.922094 \\ \mathrm{C} & -0.066030 & 2.429066 & -2.219545 \\ \mathrm{C} & -0.079013 & 1.099708 & -2.659703 \\ \mathrm{C} & -0.232653 & 0.727267 & -4.121192 \\ \mathrm{C} & 0.232473 & -0.726922 & -4.121304 \\ \mathrm{C} & 0.078857 & -1.099569 & -2.659869 \\ \mathrm{~N} & -0.000032 & 0.000014 & -1.857001 \\ \mathrm{C} & 0.065743 & -2.428986 & -2.219887 \\ \mathrm{H} & -1.295803 & 0.800549 & -4.407289 \\ \mathrm{H} & -0.114098 & 3.202471 & -2.990929 \\ \mathrm{H} & 0.029663 & 5.121694 & -1.135250 \\ \mathrm{H} & 0.064612 & 1.359585 & 5.351417 \\ \mathrm{H} & -0.063625 & -1.360123 & 5.351211 \\ \mathrm{H} & -0.340423 & -1.385802 & -4.788687 \\ \mathrm{H} & 0.135893 & 3.194057 & 3.473757 \\ \mathrm{H} & -0.135388 & -3.194262 & 3.473360 \\ \mathrm{H} & 0.113920 & -3.202308 & -2.991345 \\ \mathrm{H} & 0.130994 & 5.135727 & 1.583579 \\ \mathrm{H} & -0.131304 & -5.135735 & 1.583193 \\ \mathrm{H} & -0.030086 & -5.121710 & -1.135637 \\ \mathrm{H} & 1.295621 & -0.800169 & -4.407424 \\ \mathrm{H} & 0.340234 & 1.386243 & -4.788489 \\ \mathrm{H} & -0.003863 & -1.101864 & 0.264203 \\ \mathrm{H} & 0.003885 & 1.101845 & 0.264747\end{array}$

Zn-tetraphenylporphine $\mathrm{S}_{0}$
$\begin{array}{llll}\text { C } & -3.649443 & 4.475233 & 1.081245\end{array}$
C $\quad-3.548912 \quad 3.549288-0.000140$
$\begin{array}{llll}\text { C } & -4.475322 & 3.650398 & -1.081060\end{array}$
$\begin{array}{llll}\text { C } & -5.478980 & 4.655125 & -1.081409\end{array}$
$\begin{array}{llll}\text { C } & -5.572391 & 5.572742 & 0.000858\end{array}$ 


\begin{tabular}{|c|c|c|c|}
\hline & -4.654296 & 5.478764 & \\
\hline 0 & -2.486693 & 2.486821 & -0.000602 \\
\hline & -1.127779 & 2.908162 & -0.046502 \\
\hline I) & 0.000134 & 2.062098 & \\
\hline & 1.128134 & 2.908065 & -0.0 \\
\hline 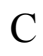 & 0.696481 & & -0.177995 \\
\hline & -0.695990 & 4.303621 & -0.177721 \\
\hline 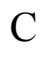 & 2.487009 & 2.486578 & -0.0 \\
\hline $\mathrm{C}$ & 3.549339 & 3.54 & \\
\hline 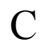 & 4.475444 & 3.650 & -1.0 \\
\hline C & 5.47 & & \\
\hline$C$ & 5.5718 & $5.5^{\prime}$ & \\
\hline C & 4.65 & & \\
\hline$C$ & 3.65 & 4.4 & \\
\hline $\mathrm{C}$ & -2.90 & & \\
\hline $\mathrm{N}$ & -2.062179 & -0.0 & -0.0 \\
\hline C & -2.90 & & \\
\hline $\mathrm{C}$ & -4.3( & -0.6 & \\
\hline $\mathrm{C}$ & -4.31 & & 0.1 \\
\hline $\mathrm{C}$ & -2.4 & & \\
\hline C & -3.5 & -3.5 & 00087 \\
\hline $\mathrm{C}$ & $-4.4^{\prime}$ & -3.6 & \\
\hline C & $-5.4^{\prime}$ & -4.6 & $-1 .($ \\
\hline $\mathrm{C}$ & -5.5 & -5.5 & -0 . \\
\hline $\mathrm{C}$ & -4.6 & -5.4 & \\
\hline $\mathrm{C}$ & -3.6 & & \\
\hline $\mathrm{C}$ & 2.90 & & \\
\hline $\mathrm{N}$ & 2.06 & -0.0 & -0 . \\
\hline $\mathrm{C}$ & 2.9 & & \\
\hline $\mathrm{C}$ & 4.30 & -0.6 & 0 . \\
\hline $\mathrm{C}$ & 4.303792 & 0.6 & 76660 \\
\hline $\mathrm{C}$ & 2.486756 & -2.4 & 0.000134 \\
\hline $\mathrm{C}$ & 3.549045 & -3.5 & 00020 \\
\hline $\mathrm{C}$ & 4.474928 & -3.6 & -1 . \\
\hline $\mathrm{C}$ & 5.47 & -4.6 & -1 \\
\hline $\mathrm{C}$ & $5.5^{\circ}$ & -5.5 & 00082 \\
\hline $\mathrm{C}$ & 4.65 & -5.4 & \\
\hline $\mathrm{C}$ & 3.650358 & -4.475344 & 1.081373 \\
\hline $\mathrm{C}$ & 1.127837 & -2.90 & -0.0 \\
\hline $\mathrm{N}$ & -0.000079 & -2.062384 & 0.015180 \\
\hline $\mathrm{C}$ & -1.128079 & -2.908370 & -0.045337 \\
\hline $\mathrm{C}$ & -0.696428 & -4.303878 & -0.176475 \\
\hline $\mathrm{C}$ & 0.696048 & -4.303971 & -0.176243 \\
\hline $\mathrm{H}$ & 1.360249 & -5.171261 & -0.287680 \\
\hline $\mathrm{H}$ & 5.170855 & -1.360825 & 0.289203 \\
\hline & 1.360740 & 5.170561 & -0.291023 \\
\hline
\end{tabular}




$\begin{array}{crrc}\mathrm{H} & -1.360190 & 5.170889 & -0.289336 \\ \mathrm{H} & -1.360694 & -5.170968 & -0.289065 \\ \mathrm{H} & 5.170972 & 1.360121 & 0.288942 \\ \mathrm{H} & -5.170918 & 1.360669 & 0.287659 \\ \mathrm{H} & -5.170794 & -1.360275 & 0.290462 \\ \mathrm{H} & 4.395036 & 2.945370 & -1.926017 \\ \mathrm{H} & 6.181530 & 4.726904 & -1.927993 \\ \mathrm{H} & 6.350969 & 6.352802 & 0.000840 \\ \mathrm{H} & 4.723613 & 6.182637 & 1.928384 \\ \mathrm{H} & 2.945366 & 4.392872 & 1.926845 \\ \mathrm{H} & 4.393511 & -2.945793 & -1.926623 \\ \mathrm{H} & 6.182518 & -4.724809 & -1.927985 \\ \mathrm{H} & 6.352275 & -6.351761 & -0.000074 \\ \mathrm{H} & 4.726066 & -6.181829 & 1.928458 \\ \mathrm{H} & 2.945263 & -4.394610 & 1.926285 \\ \mathrm{H} & -4.393876 & -2.945432 & -1.926486 \\ \mathrm{H} & -6.181369 & -4.725964 & -1.928947 \\ \mathrm{H} & -6.352118 & -6.351882 & -0.000245 \\ \mathrm{H} & -4.725856 & -6.181952 & 1.928242 \\ \mathrm{H} & -2.946479 & -4.393311 & 1.927088 \\ \mathrm{H} & -4.394285 & 2.945899 & -1.926442 \\ \mathrm{H} & -6.183207 & 4.724998 & -1.926728 \\ \mathrm{H} & -6.351437 & 6.352257 & 0.001058 \\ \mathrm{H} & -4.724241 & 6.182089 & 1.928738 \\ \mathrm{H} & -2.943777 & 4.394533 & 1.925685 \\ \mathrm{Zn} & 0.000029 & -0.000145 & -0.000013\end{array}$

Zn-tetraphenylporphine $\mathrm{S}_{1}$

$\begin{array}{lrrr}\mathrm{C} & -3.559097 & 4.578152 & 0.997228 \\ \mathrm{C} & -3.549605 & 3.555317 & -0.000076 \\ \mathrm{C} & -4.568772 & 3.563345 & -1.001786 \\ \mathrm{C} & -5.565765 & 4.573484 & -1.010941 \\ \mathrm{C} & -5.567191 & 5.587097 & -0.012853 \\ \mathrm{C} & -4.560710 & 5.584633 & 0.991522 \\ \mathrm{C} & -2.496084 & 2.491798 & 0.004914 \\ \mathrm{C} & -1.131607 & 2.898022 & -0.077217 \\ \mathrm{~N} & 0.000043 & 2.049956 & 0.040706 \\ \mathrm{C} & 1.131692 & 2.898033 & -0.077197 \\ \mathrm{C} & 0.697645 & 4.276564 & -0.308252 \\ \mathrm{C} & -0.697563 & 4.276552 & -0.308291 \\ \mathrm{C} & 2.496157 & 2.491801 & 0.004967 \\ \mathrm{C} & 3.549689 & 3.555309 & 0.000007 \\ \mathrm{C} & 4.568830 & 3.563386 & -1.001730 \\ \mathrm{C} & 5.565661 & 4.573683 & -1.011024 \\ \mathrm{C} & 5.566959 & 5.587401 & -0.013041\end{array}$




$\begin{array}{rrrr}\mathrm{C} & 4.560611 & 5.584783 & 0.991467 \\ \mathrm{C} & 3.559156 & 4.578146 & 0.997309 \\ \mathrm{C} & -2.933463 & 1.129736 & 0.098775 \\ \mathrm{~N} & -2.087254 & -0.000013 & -0.025652 \\ \mathrm{C} & -2.933468 & -1.129764 & 0.098685 \\ \mathrm{C} & -4.310098 & -0.699690 & 0.331221 \\ \mathrm{C} & -4.310104 & 0.699650 & 0.331226 \\ \mathrm{C} & -2.496092 & -2.491826 & 0.004779 \\ \mathrm{C} & -3.549616 & -3.555342 & -0.000159 \\ \mathrm{C} & -4.568869 & -3.563327 & -1.001782 \\ \mathrm{C} & -5.565911 & -4.573418 & -1.010846 \\ \mathrm{C} & -5.567291 & -5.587035 & -0.012761 \\ \mathrm{C} & -4.560778 & -5.584559 & 0.991583 \\ \mathrm{C} & -3.559051 & -4.578191 & 0.997131 \\ \mathrm{C} & 2.933518 & 1.129728 & 0.098843 \\ \mathrm{~N} & 2.087315 & -0.000024 & -0.025544 \\ \mathrm{C} & 2.933513 & -1.129785 & 0.098830 \\ \mathrm{C} & 4.310140 & -0.699705 & 0.331306 \\ \mathrm{C} & 4.310140 & 0.699637 & 0.331343 \\ \mathrm{C} & 2.496146 & -2.491850 & 0.004902 \\ \mathrm{C} & 3.549673 & -3.555363 & -0.000040 \\ \mathrm{C} & 4.568900 & -3.563378 & -1.001689 \\ \mathrm{C} & 5.565837 & -4.573571 & -1.010853 \\ \mathrm{C} & 5.567116 & -5.587284 & -0.012866 \\ \mathrm{C} & 4.560652 & -5.584757 & 0.991527 \\ \mathrm{C} & 3.559087 & -4.578228 & 0.997235 \\ \mathrm{C} & 1.131679 & -2.898074 & -0.077293 \\ \mathrm{~N} & 0.000034 & -2.049996 & 0.040615 \\ \mathrm{C} & -1.131620 & -2.898050 & -0.077388 \\ \mathrm{C} & -0.697577 & -4.276582 & -0.308445 \\ \mathrm{C} & 0.697632 & -4.276588 & -0.308448 \\ \mathrm{H} & 1.359535 & -5.132532 & -0.495025 \\ \mathrm{H} & 5.165099 & -1.363677 & 0.515290 \\ \mathrm{H} & 1.359552 & 5.132537 & -0.494679 \\ \mathrm{H} & -1.359470 & 5.132501 & -0.494828 \\ \mathrm{H} & -1.359487 & -5.132552 & -0.494873 \\ \mathrm{H} & 5.165083 & 1.363600 & 0.515428 \\ \mathrm{H} & -5.165068 & 1.363619 & 0.515197 \\ \mathrm{H} & -5.165031 & -1.363665 & 0.515316 \\ \mathrm{H} & 4.560918 & 2.785041 & -1.783705 \\ \mathrm{H} & 6.337709 & 4.573089 & -1.798034 \\ \mathrm{H} & 6.342932 & 6.369996 & -0.018706 \\ \mathrm{H} & 4.559517 & 6.361651 & 1.773716 \\ \mathrm{H} & 2.787273 & 4.567487 & 1.785437 \\ \mathrm{H} & 4.560958 & -2.785084 & -1.783715 \\ \mathrm{H} & 6.337999 & -4.572886 & -1.797752 \\ & & & \end{array}$




$\begin{array}{crrc}\mathrm{H} & 6.343158 & -6.369811 & -0.018442 \\ \mathrm{H} & 4.559561 & -6.361601 & 1.773799 \\ \mathrm{H} & 2.787049 & -4.567700 & 1.785212 \\ \mathrm{H} & -4.560867 & -2.785092 & -1.783866 \\ \mathrm{H} & -6.338067 & -4.572737 & -1.797750 \\ \mathrm{H} & -6.343450 & -6.369447 & -0.018224 \\ \mathrm{H} & -4.559798 & -6.361293 & 1.773965 \\ \mathrm{H} & -2.786858 & -4.567812 & 1.784959 \\ \mathrm{H} & -4.560736 & 2.785109 & -1.783869 \\ \mathrm{H} & -6.337829 & 4.572860 & -1.797936 \\ \mathrm{H} & -6.343399 & 6.369460 & -0.018299 \\ \mathrm{H} & -4.559618 & 6.361514 & 1.773759 \\ \mathrm{H} & -2.787083 & 4.567634 & 1.785230 \\ \mathrm{Zn} & 0.000174 & -0.000020 & 0.005686\end{array}$

tetraphenylporphine $\mathrm{S}_{0}$

$\begin{array}{lccc}\mathrm{C} & -2.958596 & 4.476938 & 1.417891 \\ \mathrm{C} & -3.433628 & 3.339426 & 0.729757 \\ \mathrm{C} & -4.803847 & 3.259484 & 0.402912 \\ \mathrm{C} & -5.680581 & 4.282053 & 0.779368 \\ \mathrm{C} & -5.203710 & 5.395879 & 1.483466 \\ \mathrm{C} & -3.841326 & 5.489384 & 1.802719 \\ \mathrm{C} & -2.494273 & 2.281443 & 0.342264 \\ \mathrm{C} & -1.198909 & 2.696375 & -0.165891 \\ \mathrm{~N} & -0.060422 & 2.075585 & 0.156618 \\ \mathrm{C} & 0.935040 & 2.830165 & -0.448173 \\ \mathrm{C} & 0.376107 & 3.964953 & -1.175917 \\ \mathrm{C} & -0.982103 & 3.877282 & -1.001714 \\ \mathrm{C} & 2.281207 & 2.494074 & -0.342558 \\ \mathrm{C} & 3.339314 & 3.433382 & -0.729821 \\ \mathrm{C} & 4.477119 & 2.958189 & -1.417362 \\ \mathrm{C} & 5.489599 & 3.840885 & -1.802179 \\ \mathrm{C} & 5.396245 & 5.203239 & -1.482739 \\ \mathrm{C} & 4.282102 & 5.680269 & -0.779251 \\ \mathrm{C} & 3.259108 & 4.803709 & -0.403518 \\ \mathrm{C} & -2.830266 & 0.935224 & 0.447848 \\ \mathrm{~N} & -2.075566 & -0.060192 & -0.156875 \\ \mathrm{C} & -2.696161 & -1.198739 & 0.165745 \\ \mathrm{C} & -3.877380 & -0.982003 & 1.001134 \\ \mathrm{C} & -3.964475 & 0.376111 & 1.176372 \\ \mathrm{C} & -2.281213 & -2.494076 & -0.342552 \\ \mathrm{C} & -3.339316 & -3.433385 & -0.729824 \\ \mathrm{C} & -4.477116 & -2.958192 & -1.417373 \\ \mathrm{C} & -5.489592 & -3.840888 & -1.802198 \\ \mathrm{C} & -5.396239 & -5.203243 & -1.482761\end{array}$




$\begin{array}{rrrc}\text { C } & -4.282102 & -5.680272 & -0.779263 \\ \mathrm{C} & -3.259113 & -4.803712 & -0.403520 \\ \mathrm{C} & 2.696147 & 1.198744 & 0.165761 \\ \mathrm{~N} & 2.075562 & 0.060192 & -0.156864 \\ \mathrm{C} & 2.830251 & -0.935216 & 0.447884 \\ \mathrm{C} & 3.964443 & -0.376094 & 1.176427 \\ \mathrm{C} & 3.877339 & 0.982019 & 1.001190 \\ \mathrm{C} & 2.494262 & -2.281437 & 0.342306 \\ \mathrm{C} & 3.433634 & -3.339416 & 0.729769 \\ \mathrm{C} & 4.803845 & -3.259462 & 0.402894 \\ \mathrm{C} & 5.680592 & -4.282036 & 0.779310 \\ \mathrm{C} & 5.203742 & -5.395874 & 1.483402 \\ \mathrm{C} & 3.841368 & -5.489387 & 1.802691 \\ \mathrm{C} & 2.958624 & -4.476939 & 1.417901 \\ \mathrm{C} & 1.198901 & -2.696377 & -0.165849 \\ \mathrm{~N} & 0.060413 & -2.075572 & 0.156627 \\ \mathrm{C} & -0.935046 & -2.830171 & -0.448143 \\ \mathrm{C} & -0.376109 & -3.964982 & -1.175850 \\ \mathrm{C} & 0.982100 & -3.877313 & -1.001633 \\ \mathrm{H} & 1.756446 & -4.518823 & -1.422778 \\ \mathrm{H} & 4.665270 & -0.927636 & 1.801201 \\ \mathrm{H} & 0.927942 & 4.667160 & -1.798878 \\ \mathrm{H} & -1.756449 & 4.518769 & -1.422898 \\ \mathrm{H} & -0.927939 & -4.667204 & -1.798798 \\ \mathrm{H} & 4.519853 & 1.756366 & 1.420803 \\ \mathrm{H} & -4.665302 & 0.927657 & 1.801141 \\ \mathrm{H} & -4.519926 & -1.756343 & 1.420711 \\ \mathrm{H} & 4.540356 & 1.897567 & -1.671617 \\ \mathrm{H} & 6.356422 & 3.466371 & -2.350797 \\ \mathrm{H} & 6.193872 & 5.889573 & -1.774686 \\ \mathrm{H} & 4.216506 & 6.736856 & -0.510754 \\ \mathrm{H} & 2.405059 & 5.164021 & 0.173462 \\ \mathrm{H} & 5.163786 & -2.406204 & -0.175477 \\ \mathrm{H} & 6.737264 & -4.216182 & 0.511217 \\ \mathrm{H} & 5.890270 & -6.193050 & 1.776124 \\ \mathrm{H} & 3.466849 & -6.356405 & 2.350998 \\ \mathrm{H} & 1.898119 & -4.539944 & 1.672701 \\ \mathrm{H} & -4.540352 & -1.897570 & -1.671628 \\ \mathrm{H} & -6.356416 & -3.466373 & -2.350816 \\ \mathrm{H} & -6.193861 & -5.889578 & -1.774718 \\ \mathrm{H} & -4.216508 & -6.736859 & -0.510764 \\ \mathrm{H} & -2.405071 & -5.164023 & 0.173472 \\ \mathrm{H} & -5.163805 & 2.406240 & -0.175470 \\ \mathrm{H} & -6.737261 & 4.216206 & 0.511305 \\ \mathrm{H} & -5.890229 & 6.193048 & 1.776225 \\ \mathrm{H} & -3.466790 & 6.356397 & 2.351021\end{array}$




$$
\begin{array}{llll}
\mathrm{H} & -1.898083 & 4.539938 & 1.672662
\end{array}
$$

tetraphenylporphine $\mathrm{S}_{1}$

$\begin{array}{crcc}\mathrm{C} & -4.358744 & -0.086474 & 0.309902 \\ \mathrm{C} & -3.073394 & -0.729534 & 0.070998 \\ \mathrm{~N} & -2.124662 & 0.303280 & -0.060583 \\ \mathrm{C} & -2.746162 & 1.560461 & 0.070933 \\ \mathrm{C} & -4.160157 & 1.303094 & 0.310018 \\ \mathrm{C} & -2.114204 & 2.836207 & -0.024936 \\ \mathrm{C} & -0.699634 & 3.056521 & -0.151345 \\ \mathrm{~N} & 0.293360 & 2.053310 & -0.013922 \\ \mathrm{C} & 1.527436 & 2.738573 & -0.151133 \\ \mathrm{C} & 1.300475 & 4.167913 & -0.412506 \\ \mathrm{C} & -0.081726 & 4.365235 & -0.412698 \\ \mathrm{C} & 2.823808 & 2.131157 & -0.024537 \\ \mathrm{C} & 3.073349 & 0.729508 & 0.071236 \\ \mathrm{~N} & 2.124633 & -0.303305 & -0.060514 \\ \mathrm{C} & 2.746113 & -1.560487 & 0.071124 \\ \mathrm{C} & 4.160079 & -1.303122 & 0.310425 \\ \mathrm{C} & 4.358664 & 0.086441 & 0.310350 \\ \mathrm{C} & 2.114174 & -2.836229 & -0.024829 \\ \mathrm{C} & 0.699604 & -3.056546 & -0.151317 \\ \mathrm{~N} & -0.293398 & -2.053348 & -0.013930 \\ \mathrm{C} & -1.527471 & -2.738608 & -0.151258 \\ \mathrm{C} & -1.300492 & -4.167939 & -0.412675 \\ \mathrm{C} & 0.081709 & -4.365251 & -0.412761 \\ \mathrm{C} & -2.823842 & -2.131192 & -0.024763 \\ \mathrm{H} & 0.617373 & -5.302614 & -0.616584 \\ \mathrm{C} & 3.005826 & -4.040475 & 0.004036 \\ \mathrm{H} & 4.916277 & -2.076199 & 0.495911 \\ \mathrm{H} & 1.104964 & -0.157398 & -0.125363 \\ \mathrm{H} & 2.077058 & 4.917989 & -0.616063 \\ \mathrm{H} & -0.617373 & 5.302604 & -0.616544 \\ \mathrm{H} & -2.077056 & -4.918006 & -0.616337 \\ \mathrm{C} & 4.016871 & 3.037661 & 0.005086 \\ \mathrm{C} & -3.005830 & 4.040469 & 0.004040 \\ \mathrm{C} & -4.016900 & -3.037698 & 0.005010 \\ \mathrm{H} & 5.301211 & 0.616755 & 0.495449 \\ \mathrm{H} & -4.916383 & 2.076170 & 0.495397 \\ \mathrm{H} & -5.301323 & -0.616792 & 0.494831 \\ \mathrm{H} & -1.104977 & 0.157370 & -0.125214 \\ \mathrm{C} & 5.053122 & 2.915657 & -0.970314 \\ \mathrm{C} & 6.178197 & 3.781956 & -0.941493 \\ \mathrm{C} & 6.286978 & 4.779817 & 0.065497 \\ \mathrm{C} & 5.260037 & 4.908267 & 1.041572 \\ & & & \end{array}$




$\begin{array}{lrrr}\mathrm{C} & 4.132334 & 4.046989 & 1.009648 \\ \mathrm{H} & 4.960782 & 2.152252 & -1.761514 \\ \mathrm{H} & 6.964523 & 3.682934 & -1.707832 \\ \mathrm{H} & 7.160616 & 5.451240 & 0.088496 \\ \mathrm{H} & 5.340245 & 5.675377 & 1.829252 \\ \mathrm{H} & 3.341707 & 4.138222 & 1.773706 \\ \mathrm{C} & 4.034570 & -4.212972 & -0.971685 \\ \mathrm{C} & 4.874010 & -5.358206 & -0.941887 \\ \mathrm{C} & 4.699753 & -6.346756 & 0.065088 \\ \mathrm{C} & 3.678150 & -6.183037 & 1.041483 \\ \mathrm{C} & 2.834805 & -5.041884 & 1.008591 \\ \mathrm{H} & 4.157415 & -3.455451 & -1.764394 \\ \mathrm{H} & 5.658116 & -5.481681 & -1.706949 \\ \mathrm{H} & 5.350279 & -7.236083 & 0.087321 \\ \mathrm{H} & 3.542607 & -6.940734 & 1.830693 \\ \mathrm{H} & 2.048690 & -4.909755 & 1.771328 \\ \mathrm{C} & -5.053220 & -2.915745 & -0.970319 \\ \mathrm{C} & -6.178287 & -3.782050 & -0.941375 \\ \mathrm{C} & -6.287023 & -4.779813 & 0.065717 \\ \mathrm{C} & -5.259991 & -4.908240 & 1.041699 \\ \mathrm{C} & -4.132296 & -4.046955 & 1.009651 \\ \mathrm{H} & -4.960955 & -2.152359 & -1.761547 \\ \mathrm{H} & -6.964623 & -3.683131 & -1.707715 \\ \mathrm{H} & -7.160728 & -5.451141 & 0.088900 \\ \mathrm{H} & -5.340099 & -5.675364 & 1.829374 \\ \mathrm{H} & -3.341635 & -4.138117 & 1.773682 \\ \mathrm{C} & -4.034639 & 4.213029 & -0.971600 \\ \mathrm{C} & -4.873937 & 5.358366 & -0.941799 \\ \mathrm{C} & -4.699593 & 6.346865 & 0.065210 \\ \mathrm{C} & -3.677889 & 6.183110 & 1.041495 \\ \mathrm{C} & -2.834692 & 5.041849 & 1.008607 \\ \mathrm{H} & -4.157703 & 3.455429 & -1.764199 \\ \mathrm{H} & -5.657945 & 5.482001 & -1.706935 \\ \mathrm{H} & -5.350194 & 7.236132 & 0.087615 \\ \mathrm{H} & -3.542090 & 6.940914 & 1.830559 \\ \mathrm{H} & -2.048656 & 4.909572 & 1.771400 \\ & & & \end{array}$

CC2/TZVP geometries - Group II

tetrafluorobenzene $\mathrm{S}_{0}$
$\begin{array}{llll}\text { C } & -1.194054 & 0.695303 & 0.022817\end{array}$
$\begin{array}{llll}\text { C } & -1.194055 & -0.695303 & -0.022808\end{array}$
$\begin{array}{llll}\text { C } & 0.000000 & -1.399322 & 0.021462\end{array}$
$\begin{array}{llll}\text { C } & 1.194055 & -0.695303 & -0.022812\end{array}$ 


$\begin{array}{lrrc}\mathrm{C} & 1.194054 & 0.695303 & 0.022820 \\ \mathrm{C} & 0.000000 & 1.399321 & -0.021484 \\ \mathrm{~F} & -2.362659 & -1.359184 & -0.025382 \\ \mathrm{H} & 0.000000 & -2.479980 & -0.001106 \\ \mathrm{~F} & 2.362659 & -1.359184 & -0.025398 \\ \mathrm{~F} & 2.362658 & 1.359184 & 0.025449 \\ \mathrm{H} & 0.000000 & 2.479980 & 0.001009 \\ \mathrm{~F} & -2.362658 & 1.359184 & 0.025434\end{array}$

tetrafluorobenzene $\mathrm{S}_{1}$

$\begin{array}{lrrr}\text { C } & 1.166960 & -0.711234 & 0.115373 \\ \text { C } & 1.166964 & 0.711234 & 0.115387 \\ \text { C } & -0.000001 & 1.469124 & 0.373482 \\ \text { C } & -1.166962 & 0.711232 & 0.115379 \\ \text { C } & -1.166961 & -0.711236 & 0.115381 \\ \text { C } & 0.000001 & -1.469121 & 0.373493 \\ \text { F } & 2.306126 & 1.327556 & -0.233182 \\ \text { H } & -0.000002 & 2.530999 & 0.560300 \\ \text { F } & -2.306119 & 1.327551 & -0.233212 \\ \text { F } & -2.306108 & -1.327560 & -0.233235 \\ \text { H } & 0.000002 & -2.530989 & 0.560350 \\ \text { F } & 2.306101 & -1.327555 & -0.233265\end{array}$

benzonitrile $\mathrm{S}_{0}$

$\begin{array}{lrrr}\mathrm{C} & -0.000010 & 0.025790 & -1.056730 \\ \mathrm{C} & -1.214659 & -0.010425 & -0.359015 \\ \mathrm{C} & -1.208794 & 0.016785 & 1.032265 \\ \mathrm{C} & 0.000001 & -0.020520 & 1.727824 \\ \mathrm{C} & 1.208787 & 0.016910 & 1.032253 \\ \mathrm{C} & 1.214639 & -0.010625 & -0.359021 \\ \mathrm{H} & -2.147214 & 0.003873 & -0.908478 \\ \mathrm{H} & -2.146928 & -0.004919 & 1.572103 \\ \mathrm{H} & 0.000006 & -0.009214 & 2.810409 \\ \mathrm{H} & 2.146936 & -0.004016 & 1.572095 \\ \mathrm{H} & 2.147195 & 0.002979 & -0.908499 \\ \mathrm{C} & 0.000005 & 0.012533 & -2.487537 \\ \mathrm{~N} & 0.000036 & 0.008606 & -3.667663\end{array}$

benzonitrile $\mathrm{S}_{1}$
$\begin{array}{llll}\text { C } & -0.000029 & -0.036809 & -1.108243\end{array}$
$\begin{array}{llll}\text { C } & -1.236835 & 0.045478 & -0.374399\end{array}$
$\begin{array}{llll}\text { C } & -1.226562 & -0.019502 & 1.052690\end{array}$
$\begin{array}{llll}\text { C } & -0.000163 & 0.009101 & 1.773846\end{array}$ 


$\begin{array}{rrrr}\mathrm{C} & 1.226300 & -0.019619 & 1.052803 \\ \mathrm{C} & 1.236711 & 0.045379 & -0.374285 \\ \mathrm{H} & -2.172328 & 0.091909 & -0.915700 \\ \mathrm{H} & -2.169219 & -0.029871 & 1.583773 \\ \mathrm{H} & -0.000217 & -0.032688 & 2.853597 \\ \mathrm{H} & 2.168906 & -0.030117 & 1.583973 \\ \mathrm{H} & 2.172261 & 0.091773 & -0.915491 \\ \mathrm{C} & 0.000023 & -0.051767 & -2.512719 \\ \mathrm{~N} & 0.000050 & -0.073472 & -3.699667\end{array}$

$o$-fluorophenol, cis $\mathrm{S}_{0}$

$\begin{array}{lrrc}\mathrm{C} & 0.326477 & 1.456627 & -0.021519 \\ \mathrm{C} & -0.874486 & 0.775240 & 0.033162 \\ \mathrm{C} & -0.951262 & -0.616225 & -0.015326 \\ \mathrm{C} & 0.237272 & -1.340415 & 0.030029 \\ \mathrm{C} & 1.459919 & -0.673735 & -0.015721 \\ \mathrm{C} & 1.510642 & 0.719859 & 0.013834 \\ \mathrm{~F} & -2.056405 & 1.459990 & 0.030688 \\ \mathrm{O} & -2.146838 & -1.276476 & -0.008118 \\ \mathrm{H} & 0.182577 & -2.421179 & 0.013940 \\ \mathrm{H} & 2.376533 & -1.248459 & 0.004271 \\ \mathrm{H} & 2.461310 & 1.234633 & -0.018833 \\ \mathrm{H} & 0.322177 & 2.538366 & -0.007999 \\ \mathrm{H} & -2.847907 & -0.608244 & -0.038407\end{array}$

$o$-fluorophenol, cis $\mathrm{S}_{1}$

$\begin{array}{lrrc}\mathrm{C} & 0.302974 & 1.489119 & 0.102234 \\ \mathrm{C} & -0.938492 & 0.812242 & 0.192136 \\ \mathrm{C} & -0.945101 & -0.621953 & 0.031399 \\ \mathrm{C} & 0.244019 & -1.384825 & 0.040711 \\ \mathrm{C} & 1.480284 & -0.699325 & -0.101628 \\ \mathrm{C} & 1.495152 & 0.707352 & -0.006532 \\ \mathrm{~F} & -2.068415 & 1.442358 & -0.337210 \\ \mathrm{O} & -2.127061 & -1.262108 & -0.057082 \\ \mathrm{H} & 0.181765 & -2.455589 & 0.185471 \\ \mathrm{H} & 2.408885 & -1.249076 & -0.151811 \\ \mathrm{H} & 2.439626 & 1.231203 & -0.096475 \\ \mathrm{H} & 0.347993 & 2.565400 & 0.193737 \\ \mathrm{H} & -2.821621 & -0.574818 & 0.005050\end{array}$

$o$-fluorophenol, trans $\mathrm{S}_{0}$
$\begin{array}{llll}\text { C } & 0.290603 & 1.263513 & -0.027360\end{array}$
$\begin{array}{llll}\text { C } & -0.948119 & 0.618122 & 0.019812\end{array}$ 


$\begin{array}{lccc}\mathrm{C} & -0.964891 & -0.774925 & -0.029574 \\ \mathrm{C} & 0.226259 & -1.499904 & 0.017661 \\ \mathrm{C} & 1.449488 & -0.836866 & -0.014132 \\ \mathrm{C} & 1.479614 & 0.558352 & 0.019614 \\ \mathrm{O} & -2.068790 & 1.401062 & 0.020688 \\ \mathrm{H} & -1.919436 & -1.290406 & -0.010639 \\ \mathrm{H} & 0.190378 & -2.581139 & -0.004394 \\ \mathrm{H} & 2.376085 & -1.393962 & 0.016100 \\ \mathrm{H} & 2.410892 & 1.108933 & 0.004459 \\ \mathrm{~F} & 0.312871 & 2.613839 & -0.031886 \\ \mathrm{H} & -2.836768 & 0.813317 & 0.026569\end{array}$

$o$-fluorophenol, trans $\mathrm{S}_{1}$

$\begin{array}{cccc}\mathrm{C} & 0.294895 & 1.287805 & 0.095768 \\ \mathrm{C} & -0.955703 & 0.625840 & 0.108497 \\ \mathrm{C} & -1.039322 & -0.813402 & 0.094419 \\ \mathrm{C} & 0.189944 & -1.496213 & -0.063220 \\ \mathrm{C} & 1.432035 & -0.802405 & -0.090294 \\ \mathrm{C} & 1.530360 & 0.580257 & 0.199641 \\ \mathrm{O} & -2.056134 & 1.392181 & -0.076599 \\ \mathrm{H} & -1.928494 & -1.313168 & 0.464227 \\ \mathrm{H} & 0.194090 & -2.572188 & -0.188346 \\ \mathrm{H} & 2.336746 & -1.373430 & -0.264048 \\ \mathrm{H} & 2.468291 & 1.110446 & 0.252191 \\ \mathrm{~F} & 0.309563 & 2.598346 & -0.221036 \\ \mathrm{H} & -2.776280 & 0.775930 & -0.311199\end{array}$

$m$-fluorophenol, cis $\mathrm{S}_{0}$

$\begin{array}{lccc}\text { C } & 0.308648 & 1.409675 & -0.023069 \\ \text { C } & -0.931100 & 0.787517 & 0.018043 \\ \text { C } & -0.968301 & -0.606849 & -0.019208 \\ \text { C } & 0.213980 & -1.347056 & 0.032204 \\ \text { C } & 1.435081 & -0.683214 & -0.009772 \\ \text { C } & 1.503172 & 0.710917 & 0.017374 \\ \text { O } & -2.146108 & -1.307839 & -0.023519 \\ \text { F } & 0.337613 & 2.765365 & -0.038051 \\ \text { H } & 0.158983 & -2.426885 & 0.017132 \\ \text { H } & 2.351843 & -1.257969 & 0.016256 \\ \text { H } & 2.443747 & 1.242233 & -0.008836 \\ \text { H } & -1.833571 & 1.386286 & -0.005438 \\ \text { H } & -2.876203 & -0.674725 & -0.058210\end{array}$

$m$-fluorophenol, cis $\mathrm{S}_{1}$ 


$\begin{array}{lrrr}\mathrm{C} & 1.224275 & -0.033628 & -0.691728 \\ \mathrm{C} & -0.062016 & 0.008299 & -0.107548 \\ \mathrm{C} & -0.029965 & 0.041815 & 1.325962 \\ \mathrm{C} & 1.163384 & 0.015364 & 2.089714 \\ \mathrm{C} & 2.436873 & 0.166402 & 1.443089 \\ \mathrm{C} & 2.439547 & -0.042887 & 0.032538 \\ \mathrm{O} & -1.189248 & -0.020221 & 2.030759 \\ \mathrm{~F} & 1.304777 & -0.121802 & -2.039260 \\ \mathrm{H} & 1.078521 & -0.132201 & 3.158525 \\ \mathrm{H} & 3.355345 & 0.146798 & 2.008183 \\ \mathrm{H} & 3.360929 & -0.165429 & -0.522219 \\ \mathrm{H} & -0.923965 & 0.341062 & -0.673169 \\ \mathrm{H} & -1.903100 & -0.203573 & 1.395101\end{array}$

$m$-fluorophenol, trans $\mathrm{S}_{0}$

$\begin{array}{cccc}\mathrm{C} & 0.267796 & 1.306157 & -0.019020 \\ \mathrm{C} & -0.950866 & 0.628838 & 0.018027 \\ \mathrm{C} & -0.987391 & -0.765850 & -0.034547 \\ \mathrm{C} & 0.206542 & -1.483723 & 0.012412 \\ \mathrm{C} & 1.437077 & -0.832517 & -0.017528 \\ \mathrm{C} & 1.429642 & 0.555214 & 0.019376 \\ \mathrm{O} & -2.082116 & 1.402924 & 0.024855 \\ \mathrm{H} & -1.938543 & -1.285652 & -0.016940 \\ \mathrm{H} & 0.176052 & -2.565336 & -0.012938 \\ \mathrm{H} & 2.373178 & -1.371644 & 0.008878 \\ \mathrm{~F} & 2.614701 & 1.212798 & 0.031407 \\ \mathrm{H} & 0.301813 & 2.386270 & 0.005159 \\ \mathrm{H} & -2.847888 & 0.812529 & 0.038721\end{array}$

$m$-fluorophenol, trans $\mathrm{S}_{1}$

$\begin{array}{cccc}\mathrm{C} & 1.097301 & -0.014641 & -0.008205 \\ \mathrm{C} & 0.335759 & 1.187092 & 0.017814 \\ \mathrm{C} & -1.090132 & 1.213243 & -0.041429 \\ \mathrm{C} & -1.832169 & -0.004974 & 0.115956 \\ \mathrm{C} & -1.091051 & -1.223342 & -0.015078 \\ \mathrm{C} & 0.321163 & -1.198751 & 0.003039 \\ \mathrm{O} & 1.050622 & 2.341959 & -0.014853 \\ \mathrm{H} & -1.597591 & 2.161829 & -0.176782 \\ \mathrm{H} & -2.910409 & -0.009152 & 0.079275 \\ \mathrm{H} & -1.589800 & -2.180804 & -0.086270 \\ \mathrm{~F} & 0.971378 & -2.383820 & -0.046971 \\ \mathrm{H} & 2.166164 & -0.016559 & 0.136900 \\ \mathrm{H} & 0.426460 & 3.082103 & 0.062668\end{array}$


$p$-fluorophenol $\mathrm{S}_{0}$

$\begin{array}{lrrr}\mathrm{C} & 0.270997 & 1.319382 & 0.020482 \\ \mathrm{C} & -0.951683 & 0.648459 & -0.025603 \\ \mathrm{C} & -0.984004 & -0.745117 & 0.028366 \\ \mathrm{C} & 0.204996 & -1.473821 & -0.011809 \\ \mathrm{C} & 1.406945 & -0.787278 & 0.022726 \\ \mathrm{C} & 1.460613 & 0.599064 & -0.014032 \\ \mathrm{O} & -2.087825 & 1.421607 & -0.031483 \\ \mathrm{H} & -1.933395 & -1.269065 & 0.009655 \\ \mathrm{H} & 0.199555 & -2.555071 & 0.011900 \\ \mathrm{~F} & 2.568325 & -1.491260 & 0.024185 \\ \mathrm{H} & 2.418764 & 1.100486 & 0.004759 \\ \mathrm{H} & 0.279349 & 2.400940 & -0.002426 \\ \mathrm{H} & -2.852656 & 0.831663 & -0.069371\end{array}$

p-fluorophenol $\mathrm{S}_{1}$

$\begin{array}{lrrc}\text { C } & 1.272486 & 0.684500 & -0.018224 \\ \mathrm{C} & 0.028721 & 1.366021 & 0.033943 \\ \mathrm{C} & -1.243384 & 0.715265 & -0.047389 \\ \mathrm{C} & -1.258412 & -0.706988 & 0.020053 \\ \mathrm{C} & -0.005503 & -1.369073 & -0.031544 \\ \mathrm{C} & 1.259879 & -0.742160 & 0.026463 \\ \mathrm{O} & 0.099179 & 2.719698 & 0.056930 \\ \mathrm{H} & -2.156247 & 1.293654 & 0.018664 \\ \mathrm{H} & -2.170250 & -1.284455 & -0.004108 \\ \mathrm{~F} & -0.029298 & -2.713960 & -0.053220 \\ \mathrm{H} & 2.159332 & -1.336647 & -0.020517 \\ \mathrm{H} & 2.188702 & 1.255354 & 0.010283 \\ \mathrm{H} & -0.809098 & 3.065682 & 0.043400\end{array}$

phenylacetylene $S_{0}$

$\begin{array}{lrrr}\mathrm{C} & 1.210406 & 0.018728 & -0.013252 \\ \mathrm{C} & 0.000015 & -0.016217 & -0.723134 \\ \mathrm{C} & -1.210386 & 0.018729 & -0.013267 \\ \mathrm{C} & -1.207061 & -0.018298 & 1.378197 \\ \mathrm{C} & -0.000003 & 0.014483 & 2.077112 \\ \mathrm{C} & 1.207064 & -0.018299 & 1.378212 \\ \mathrm{C} & 0.000017 & 0.001361 & -2.150600 \\ \mathrm{C} & -0.000034 & -0.014615 & -3.371679 \\ \mathrm{H} & -2.144618 & 0.007450 & -0.560781 \\ \mathrm{H} & -2.146478 & 0.000746 & 1.916861 \\ \mathrm{H} & -0.000010 & -0.005522 & 3.159771 \\ \mathrm{H} & 2.146474 & 0.000747 & 1.916889\end{array}$




$$
\begin{array}{cccc}
\mathrm{H} & 2.144647 & 0.007448 & -0.560753 \\
\mathrm{H} & -0.000033 & -0.002858 & -4.433597
\end{array}
$$

phenylacetylene $S_{1}$

$\begin{array}{lrrc}\mathrm{C} & 1.239804 & -0.057202 & -0.028718 \\ \mathrm{C} & -0.000065 & 0.008134 & -0.764307 \\ \mathrm{C} & -1.239973 & -0.057345 & -0.028796 \\ \mathrm{C} & -1.230322 & 0.024109 & 1.398690 \\ \mathrm{C} & -0.000151 & 0.001046 & 2.111689 \\ \mathrm{C} & 1.230059 & 0.024282 & 1.398764 \\ \mathrm{C} & -0.000021 & 0.023336 & -2.162320 \\ \mathrm{C} & 0.000001 & 0.044640 & -3.390638 \\ \mathrm{H} & -2.174053 & -0.086342 & -0.574023 \\ \mathrm{H} & -2.169204 & 0.032721 & 1.936624 \\ \mathrm{H} & -0.000191 & 0.042889 & 3.192424 \\ \mathrm{H} & 2.168905 & 0.033105 & 1.936759 \\ \mathrm{H} & 2.173915 & -0.086162 & -0.573895 \\ \mathrm{H} & 0.000033 & 0.059497 & -4.452932\end{array}$

aniline $\mathrm{S}_{0}$

$\begin{array}{rrrr}\mathrm{C} & 0.922330 & 0.022617 & 0.000000 \\ \mathrm{C} & 0.208862 & 0.065117 & 1.204279 \\ \mathrm{C} & -1.182526 & 0.026753 & 1.201552 \\ \mathrm{C} & -1.889537 & 0.055861 & 0.000000 \\ \mathrm{C} & -1.182525 & 0.026744 & -1.201552 \\ \mathrm{C} & 0.208862 & 0.065126 & -1.204278 \\ \mathrm{H} & 0.749070 & 0.056510 & 2.144737 \\ \mathrm{H} & -2.971272 & 0.029320 & 0.000000 \\ \mathrm{H} & -1.714716 & 0.045194 & 2.144929 \\ \mathrm{H} & -1.714716 & 0.045150 & -2.144929 \\ \mathrm{H} & 0.749071 & 0.056551 & -2.144736 \\ \mathrm{~N} & 2.323920 & 0.098014 & 0.000001 \\ \mathrm{H} & 2.746592 & -0.296470 & -0.829522 \\ \mathrm{H} & 2.746592 & -0.296478 & 0.829520\end{array}$

aniline $\mathrm{S}_{1}$
$\begin{array}{llll}\text { C } & 0.905082 & -0.039080 & -0.000017\end{array}$
$\begin{array}{llll}\text { C } & 0.225917 & -0.053362 & 1.252461\end{array}$
$\begin{array}{llll}\text { C } & -1.197092 & 0.086255 & 1.245296\end{array}$
$\begin{array}{llll}\text { C } & -1.876271 & 0.076713 & 0.000004\end{array}$
$\begin{array}{llll}\text { C } & -1.197109 & 0.086244 & -1.245298\end{array}$
$\begin{array}{llll}\text { C } & 0.225902 & -0.053351 & -1.252479\end{array}$
H $\quad 0.794491 \quad-0.076235 \quad 2.173740$ 


$\begin{array}{rrrr}\mathrm{H} & -2.959387 & 0.118248 & 0.000013 \\ \mathrm{H} & -1.756541 & 0.078281 & 2.170241 \\ \mathrm{H} & -1.756566 & 0.078274 & -2.170238 \\ \mathrm{H} & 0.794467 & -0.076159 & -2.173766 \\ \mathrm{~N} & 2.264733 & -0.196898 & -0.000002 \\ \mathrm{H} & 2.766229 & -0.014494 & -0.859000 \\ \mathrm{H} & 2.766136 & -0.014455 & 0.859044\end{array}$

catechol $\mathrm{S}_{0}$

$\begin{array}{cccc}\mathrm{C} & 0.470329 & 1.282780 & 0.005144 \\ \mathrm{C} & -0.748522 & 0.620160 & -0.055463 \\ \mathrm{C} & -0.799587 & -0.778209 & 0.011580 \\ \mathrm{C} & 0.383199 & -1.506985 & -0.022058 \\ \mathrm{C} & 1.609756 & -0.844023 & 0.022321 \\ \mathrm{C} & 1.657491 & 0.547701 & -0.013749 \\ \mathrm{O} & -1.981236 & 1.245517 & -0.086658 \\ \mathrm{O} & -2.000450 & -1.431782 & 0.005062 \\ \mathrm{H} & 0.325002 & -2.587596 & 0.002942 \\ \mathrm{H} & 2.526478 & -1.418925 & 0.009909 \\ \mathrm{H} & 2.606743 & 1.065735 & 0.021265 \\ \mathrm{H} & 0.493081 & 2.367053 & -0.028964 \\ \mathrm{H} & -1.852255 & 2.188324 & 0.080298 \\ \mathrm{H} & -2.688591 & -0.749173 & 0.048671\end{array}$

catechol $\mathrm{S}_{1}$

$\begin{array}{lrrr}\mathrm{C} & 0.460556 & 1.364438 & -0.122967 \\ \mathrm{C} & -0.774501 & 0.630263 & -0.208979 \\ \mathrm{C} & -0.805872 & -0.788320 & -0.136873 \\ \mathrm{C} & 0.392814 & -1.567824 & -0.170028 \\ \mathrm{C} & 1.569421 & -0.850525 & 0.113800 \\ \mathrm{C} & 1.615538 & 0.569773 & 0.070586 \\ \mathrm{O} & -1.981314 & 1.244583 & 0.009756 \\ \mathrm{O} & -1.979784 & -1.415884 & 0.104727 \\ \mathrm{H} & 0.348987 & -2.642560 & -0.259941 \\ \mathrm{H} & 2.495838 & -1.386320 & 0.282781 \\ \mathrm{H} & 2.571432 & 1.057463 & 0.225612 \\ \mathrm{H} & 0.527000 & 2.385230 & -0.481929 \\ \mathrm{H} & -1.777840 & 2.116573 & 0.407413 \\ \mathrm{H} & -2.662275 & -0.716889 & 0.166043\end{array}$

resorcinol, isomer $1 \mathrm{~S}_{0}$
C $\quad 0.075430$
$\begin{array}{ll}1.148970 & -0.039355\end{array}$
C -1.139441
$0.466397 \quad 0.011987$ 


$\begin{array}{crrr}\mathrm{C} & -1.172002 & -0.926346 & -0.026347 \\ \mathrm{C} & 0.032671 & -1.629925 & 0.014669 \\ \mathrm{C} & 1.253980 & -0.968819 & -0.024041 \\ \mathrm{C} & 1.264923 & 0.427135 & 0.010454 \\ \mathrm{O} & -2.274976 & 1.239077 & 0.019863 \\ \mathrm{H} & -2.119339 & -1.452384 & 0.004594 \\ \mathrm{H} & 0.013507 & -2.712383 & -0.001478 \\ \mathrm{H} & 2.190872 & -1.507832 & 0.002664 \\ \mathrm{O} & 2.491871 & 1.042073 & 0.011476 \\ \mathrm{H} & 0.071811 & 2.232997 & -0.025902 \\ \mathrm{H} & -3.036782 & 0.643602 & 0.026030 \\ \mathrm{H} & 2.347585 & 1.997750 & 0.043667\end{array}$

resorcinol, isomer $1 \mathrm{~S}_{1}$

$\begin{array}{lrrc}\mathrm{C} & 0.000719 & 1.062144 & 0.208484 \\ \mathrm{C} & -1.181428 & 0.315357 & 0.033336 \\ \mathrm{C} & -1.183734 & -1.104907 & -0.034234 \\ \mathrm{C} & -0.005628 & -1.848836 & 0.330327 \\ \mathrm{C} & 1.192121 & -1.109919 & -0.000012 \\ \mathrm{C} & 1.186865 & 0.302553 & 0.042198 \\ \mathrm{O} & -2.338662 & 1.020313 & -0.110210 \\ \mathrm{H} & -2.076294 & -1.612944 & -0.390618 \\ \mathrm{H} & -0.007863 & -2.927004 & 0.263024 \\ \mathrm{H} & 2.096971 & -1.609420 & -0.326880 \\ \mathrm{O} & 2.394527 & 0.912739 & -0.123122 \\ \mathrm{H} & -0.013626 & 2.108479 & 0.480091 \\ \mathrm{H} & -3.074543 & 0.388038 & -0.101302 \\ \mathrm{H} & 2.221567 & 1.859529 & -0.251435\end{array}$

resorcinol, isomer $2 \mathrm{~S}_{0}$
$\begin{array}{llll}\text { C } & 1.199695 & 0.012450 & -0.546893\end{array}$
$\begin{array}{llll}\text { C } & -0.000001 & -0.034398 & -1.249910\end{array}$
$\begin{array}{llll}\text { C } & -1.199698 & 0.012476 & -0.546897\end{array}$
$\begin{array}{llll}\text { C } & -1.211472 & -0.027520 & 0.849654\end{array}$
$\begin{array}{llll}\text { C } & 0.000004 & 0.013835 & 1.534709\end{array}$
$\begin{array}{llll}\text { C } & 1.211465 & -0.027869 & 0.849649\end{array}$
$\begin{array}{llll}\mathrm{O} & -2.350874 & 0.017801 & -1.294862\end{array}$
$\begin{array}{llll}\mathrm{O} & 2.350870 & 0.018305 & -1.294858\end{array}$
$\begin{array}{llll}\mathrm{H} & -2.150318 & 0.001059 & 1.390829\end{array}$
H $\quad 0.000004 \quad-0.005296 \quad 2.617275$
$\begin{array}{llll}\mathrm{H} & 2.150329 & -0.000024 & 1.390830\end{array}$
$\begin{array}{llll}\mathrm{H} & 0.000001 & -0.015283 & -2.331208\end{array}$
$\mathrm{H} \quad-3.100525 \quad 0.030478 \quad-0.684403$
$\begin{array}{llll}\mathrm{H} & 3.100518 & 0.030994 & -0.684395\end{array}$ 
resorcinol, isomer $2 \mathrm{~S}_{1}$

$\begin{array}{rrrr}\mathrm{C} & 1.178491 & -0.040905 & -0.339129 \\ \mathrm{C} & -0.000078 & -0.224576 & -1.086385 \\ \mathrm{C} & -1.178641 & -0.041110 & -0.339072 \\ \mathrm{C} & -1.176775 & 0.008963 & 1.080547 \\ \mathrm{C} & 0.000007 & -0.404094 & 1.816302 \\ \mathrm{C} & 1.176685 & 0.009162 & 1.080490 \\ \mathrm{O} & -2.334157 & 0.130889 & -1.038564 \\ \mathrm{O} & 2.333944 & 0.131288 & -1.038676 \\ \mathrm{H} & -2.045077 & 0.409853 & 1.598019 \\ \mathrm{H} & 0.000029 & -0.335998 & 2.895338 \\ \mathrm{H} & 2.044945 & 0.410189 & 1.597926 \\ \mathrm{H} & -0.000090 & -0.355401 & -2.156871 \\ \mathrm{H} & -3.068138 & 0.131650 & -0.404253 \\ \mathrm{H} & 3.067951 & 0.132216 & -0.404395\end{array}$

resorcinol, isomer $3 \mathrm{~S}_{0}$

$\begin{array}{rrrl}\mathrm{C} & 1.205202 & -0.007510 & -0.288981 \\ \mathrm{C} & -0.000049 & 0.052436 & -0.990823 \\ \mathrm{C} & -1.205296 & -0.007353 & -0.288961 \\ \mathrm{C} & -1.214489 & 0.022594 & 1.103628 \\ \mathrm{C} & -0.000031 & -0.015612 & 1.783592 \\ \mathrm{C} & 1.214422 & 0.022428 & 1.103608 \\ \mathrm{O} & -2.421473 & -0.004083 & -0.927192 \\ \mathrm{O} & 2.421369 & -0.004383 & -0.927231 \\ \mathrm{H} & -2.159798 & -0.007413 & 1.627611 \\ \mathrm{H} & -0.000023 & -0.004421 & 2.866309 \\ \mathrm{H} & 2.159736 & -0.007702 & 1.627576 \\ \mathrm{H} & -0.000058 & 0.050234 & -2.077520 \\ \mathrm{H} & 2.263039 & -0.056339 & -1.879511 \\ \mathrm{H} & -2.263168 & -0.056080 & -1.879473\end{array}$

resorcinol, isomer $3 \mathrm{~S}_{1}$

$\begin{array}{crcc}\mathrm{C} & -0.025281 & -0.305189 & 1.188623 \\ \mathrm{C} & -0.126022 & -1.087051 & 0.000000 \\ \mathrm{C} & -0.025282 & -0.305190 & -1.188622 \\ \mathrm{C} & 0.029588 & 1.110182 & -1.200239 \\ \mathrm{C} & -0.174391 & 1.857384 & 0.000000 \\ \mathrm{C} & 0.029591 & 1.110182 & 1.200240 \\ \mathrm{O} & 0.080079 & -0.915946 & -2.403758 \\ \mathrm{O} & 0.080091 & -0.915943 & 2.403759 \\ \mathrm{H} & 0.231907 & 1.597605 & -2.146462\end{array}$




$\begin{array}{rrrc}\mathrm{H} & -0.122737 & 2.934961 & 0.000000 \\ \mathrm{H} & 0.231911 & 1.597606 & 2.146462 \\ \mathrm{H} & -0.662995 & -2.034395 & -0.000002 \\ \mathrm{H} & 0.262967 & -1.854733 & 2.226560 \\ \mathrm{H} & 0.262986 & -1.854730 & -2.226560\end{array}$

hydroquinone, cis $\mathrm{S}_{0}$

$\begin{array}{lrrr}\mathrm{C} & -0.697930 & 0.025648 & 1.094290 \\ \mathrm{C} & 0.697931 & -0.025667 & 1.094289 \\ \mathrm{C} & 1.396065 & 0.020265 & -0.109318 \\ \mathrm{C} & 0.694083 & -0.018529 & -1.315155 \\ \mathrm{C} & -0.694083 & 0.018525 & -1.315155 \\ \mathrm{C} & -1.396065 & -0.020268 & -0.109318 \\ \mathrm{O} & 2.771401 & 0.009918 & -0.181177 \\ \mathrm{O} & -2.771400 & -0.009893 & -0.181177 \\ \mathrm{H} & 1.246101 & -0.000221 & -2.245784 \\ \mathrm{H} & -1.246101 & 0.000207 & -2.245784 \\ \mathrm{H} & -1.233454 & 0.008025 & 2.037625 \\ \mathrm{H} & 1.233455 & -0.008043 & 2.037624 \\ \mathrm{H} & -3.116952 & -0.064001 & 0.719809 \\ \mathrm{H} & 3.116951 & 0.064036 & 0.719809\end{array}$

hydroquinone, cis $\mathrm{S}_{1}$

$\begin{array}{rrrc}\mathrm{C} & 0.707425 & -0.061906 & -1.136999 \\ \mathrm{C} & -0.707610 & 0.062018 & -1.136956 \\ \mathrm{C} & -1.378069 & -0.016046 & 0.122875 \\ \mathrm{C} & -0.712666 & 0.014026 & 1.370218 \\ \mathrm{C} & 0.712654 & -0.014073 & 1.370176 \\ \mathrm{C} & 1.377972 & 0.016090 & 0.122787 \\ \mathrm{O} & -2.735563 & -0.017089 & 0.168521 \\ \mathrm{O} & 2.735469 & 0.017135 & 0.168337 \\ \mathrm{H} & -1.291558 & -0.040389 & 2.280386 \\ \mathrm{H} & 1.291599 & 0.040365 & 2.280309 \\ \mathrm{H} & 1.277410 & -0.053933 & -2.058016 \\ \mathrm{H} & -1.277654 & 0.053933 & -2.057936 \\ \mathrm{H} & 3.061796 & 0.023877 & -0.746338 \\ \mathrm{H} & -3.061954 & -0.023595 & -0.746132\end{array}$

hydroquinone, trans $\mathrm{S}_{0}$
$\begin{array}{llll}\text { C } & 0.021575 & 1.393440 & 0.014949\end{array}$
$\begin{array}{llll}\text { C } & -1.196308 & 0.719522 & -0.025689\end{array}$
$\begin{array}{llll}\text { C } & -1.213846 & -0.674875 & 0.023364\end{array}$
$\begin{array}{llll}\text { C } & -0.021562 & -1.393444 & -0.015002\end{array}$ 


$\begin{array}{rrrr}\mathrm{C} & 1.196319 & -0.719538 & 0.025717 \\ \mathrm{C} & 1.213855 & 0.674860 & -0.023380 \\ \mathrm{O} & -2.342034 & 1.483919 & -0.023517 \\ \mathrm{H} & -2.159116 & -1.207273 & 0.007812 \\ \mathrm{H} & -0.028608 & -2.475270 & 0.009726 \\ \mathrm{O} & 2.342043 & -1.483929 & 0.023904 \\ \mathrm{H} & 2.159106 & 1.207289 & -0.007699 \\ \mathrm{H} & 0.028560 & 2.475264 & -0.009777 \\ \mathrm{H} & -3.099116 & 0.884570 & -0.063949 \\ \mathrm{H} & 3.099132 & -0.884534 & 0.063542\end{array}$

hydroquinone, trans $\mathrm{S}_{1}$

$\begin{array}{rrrr}\mathrm{C} & 1.267059 & 0.678625 & 0.001008 \\ \mathrm{C} & 0.034279 & 1.376834 & 0.037810 \\ \mathrm{C} & -1.239932 & 0.743316 & -0.054752 \\ \mathrm{C} & -1.266908 & -0.678528 & -0.001153 \\ \mathrm{C} & -0.034129 & -1.376739 & -0.037923 \\ \mathrm{C} & 1.240085 & -0.743221 & 0.054599 \\ \mathrm{O} & 0.125709 & 2.731480 & 0.062467 \\ \mathrm{H} & -2.145340 & 1.327952 & 0.052777 \\ \mathrm{H} & -2.189664 & -1.240562 & -0.001734 \\ \mathrm{O} & -0.125559 & -2.731387 & -0.062470 \\ \mathrm{H} & 2.145492 & -1.327859 & -0.052936 \\ \mathrm{H} & 2.189815 & 1.240659 & 0.001584 \\ \mathrm{H} & -0.775276 & 3.087186 & -0.012512 \\ \mathrm{H} & 0.775422 & -3.087083 & 0.012607\end{array}$

salicylic acid $\mathrm{S}_{0}$

$\begin{array}{rrrr}\mathrm{C} & 1.103972 & 1.598226 & 0.015859 \\ \mathrm{C} & 1.131325 & 0.198111 & -0.007634 \\ \mathrm{C} & -0.085564 & -0.518278 & 0.067541 \\ \mathrm{C} & -1.302179 & 0.184809 & 0.008195 \\ \mathrm{C} & -1.319639 & 1.568964 & 0.017878 \\ \mathrm{C} & -0.107212 & 2.270415 & -0.022229 \\ \mathrm{O} & 2.339945 & -0.403573 & -0.011982 \\ \mathrm{C} & -0.042220 & -1.978721 & 0.017293 \\ \mathrm{O} & -1.257552 & -2.575346 & -0.007099 \\ \mathrm{O} & 0.999789 & -2.647419 & 0.000618 \\ \mathrm{H} & -2.226376 & -0.376559 & 0.030884 \\ \mathrm{H} & 2.046519 & 2.129422 & -0.012323 \\ \mathrm{H} & -2.261095 & 2.100866 & -0.015125 \\ \mathrm{H} & -0.110354 & 3.353590 & -0.022040 \\ \mathrm{H} & 2.161151 & -1.373934 & -0.013168 \\ \mathrm{H} & -1.070384 & -3.530558 & -0.033660\end{array}$


salicylic acid $\mathrm{S}_{1}$

$\begin{array}{rrrr}\mathrm{C} & 0.998588 & 1.934591 & 0.043317 \\ \mathrm{C} & 0.982717 & 0.551326 & 0.147177 \\ \mathrm{C} & -0.267035 & -0.145494 & 0.064688 \\ \mathrm{C} & -1.432519 & 0.547063 & -0.011876 \\ \mathrm{C} & -1.409798 & 1.991682 & -0.096172 \\ \mathrm{C} & -0.225602 & 2.666257 & -0.014143 \\ \mathrm{O} & 2.112436 & -0.166972 & 0.231590 \\ \mathrm{C} & -0.182514 & -1.631533 & 0.182477 \\ \mathrm{O} & -1.350834 & -2.310645 & 0.266262 \\ \mathrm{O} & 0.817667 & -2.238680 & -0.467132 \\ \mathrm{H} & -2.379487 & 0.021420 & -0.043479 \\ \mathrm{H} & 1.953705 & 2.444509 & 0.083782 \\ \mathrm{H} & -2.352322 & 2.524809 & -0.109277 \\ \mathrm{H} & -0.199795 & 3.747515 & -0.059195 \\ \mathrm{H} & 1.584109 & -1.562092 & -0.274719 \\ \mathrm{H} & -1.138737 & -3.236810 & 0.056702\end{array}$

$o$-cresol, cis $\mathrm{S}_{0}$

$\begin{array}{rrrr}\mathrm{C} & 1.965914 & 0.514882 & -0.005295 \\ \mathrm{C} & 0.729339 & 1.154708 & 0.029687 \\ \mathrm{C} & -0.467740 & 0.435910 & -0.029869 \\ \mathrm{C} & -0.391334 & -0.963734 & 0.015080 \\ \mathrm{C} & 0.837657 & -1.619316 & -0.033779 \\ \mathrm{C} & 2.014624 & -0.879009 & 0.007904 \\ \mathrm{C} & -1.803682 & 1.125590 & -0.028619 \\ \mathrm{O} & -1.516435 & -1.756197 & -0.006205 \\ \mathrm{H} & 0.851680 & -2.701760 & -0.025567 \\ \mathrm{H} & 2.967835 & -1.392115 & -0.016472 \\ \mathrm{H} & 2.878399 & 1.096002 & 0.027272 \\ \mathrm{H} & 0.684778 & 2.238327 & 0.019293 \\ \mathrm{H} & -2.288640 & -1.190391 & 0.128009 \\ \mathrm{H} & -2.388597 & 0.874831 & 0.861645 \\ \mathrm{H} & -1.676626 & 2.206969 & -0.037928 \\ \mathrm{H} & -2.395378 & 0.855603 & -0.907810\end{array}$

$o$-cresol, cis $\mathrm{S}_{1}$
$\begin{array}{llll}\text { C } & 1.881299 & -0.096475 & -0.780467\end{array}$
$\begin{array}{llll}\text { C } & 0.632778 & -0.066505 & -1.467391\end{array}$
$\begin{array}{llll}\text { C } & -0.592989 & -0.100484 & -0.722253\end{array}$
$\begin{array}{llll}\text { C } & -0.470960 & 0.073440 & 0.700175\end{array}$
$\begin{array}{llll}\text { C } & 0.768770 & 0.053645 & 1.400387\end{array}$ 


$\begin{array}{rrrc}\mathrm{C} & 1.968664 & 0.017614 & 0.632335 \\ \mathrm{C} & -1.909867 & -0.026204 & -1.420838 \\ \mathrm{O} & -1.578423 & 0.167068 & 1.483584 \\ \mathrm{H} & 0.764037 & 0.154038 & 2.476232 \\ \mathrm{H} & 2.932535 & -0.006557 & 1.120650 \\ \mathrm{H} & 2.794265 & -0.152601 & -1.361131 \\ \mathrm{H} & 0.606060 & -0.148196 & -2.546180 \\ \mathrm{H} & -2.356181 & 0.154479 & 0.901106 \\ \mathrm{H} & -2.724190 & -0.494592 & -0.857576 \\ \mathrm{H} & -2.210974 & 1.007005 & -1.642354 \\ \mathrm{H} & -1.855402 & -0.555164 & -2.373869\end{array}$

o-cresol, trans $\mathrm{S}_{0}$

$\begin{array}{lrrr}\mathrm{C} & 2.422255 & 0.025429 & 0.028315 \\ \mathrm{C} & 1.246048 & -0.022810 & -0.720026 \\ \mathrm{C} & 0.031003 & 0.016422 & -0.022156 \\ \mathrm{C} & -0.003501 & -0.034131 & 1.370317 \\ \mathrm{C} & 1.187366 & 0.013069 & 2.094071 \\ \mathrm{C} & 2.406968 & -0.006658 & 1.423520 \\ \mathrm{C} & 1.246715 & -0.010587 & -2.220625 \\ \mathrm{O} & -1.113672 & 0.015595 & -0.787335 \\ \mathrm{H} & -0.958291 & -0.022708 & 1.886845 \\ \mathrm{H} & 1.155287 & -0.010483 & 3.176123 \\ \mathrm{H} & 3.336127 & 0.023149 & 1.977778 \\ \mathrm{H} & 3.370675 & 0.013857 & -0.497589 \\ \mathrm{H} & -1.871439 & 0.022124 & -0.186239 \\ \mathrm{H} & 2.266920 & -0.029237 & -2.602688 \\ \mathrm{H} & 0.709485 & -0.873511 & -2.617009 \\ \mathrm{H} & 0.746757 & 0.880479 & -2.603331\end{array}$

o-cresol, trans $\mathrm{S}_{1}$

$\begin{array}{lrcc}\mathrm{C} & 2.430624 & 0.285876 & -0.006912 \\ \mathrm{C} & 1.218001 & 0.081171 & -0.756480 \\ \mathrm{C} & 0.003830 & 0.006533 & -0.017479 \\ \mathrm{C} & -0.057267 & -0.106211 & 1.414608 \\ \mathrm{C} & 1.176525 & -0.055176 & 2.113621 \\ \mathrm{C} & 2.398353 & 0.075358 & 1.396444 \\ \mathrm{C} & 1.257113 & -0.031206 & -2.241454 \\ \mathrm{O} & -1.145766 & -0.086857 & -0.737911 \\ \mathrm{H} & -1.004909 & 0.028526 & 1.923820 \\ \mathrm{H} & 1.196112 & -0.139102 & 3.192188 \\ \mathrm{H} & 3.327956 & 0.070656 & 1.953492 \\ \mathrm{H} & 3.372588 & 0.380864 & -0.530096 \\ \mathrm{H} & -1.848896 & -0.339336 & -0.113506\end{array}$




$\begin{array}{lrrr}\mathrm{H} & 1.929703 & -0.840509 & -2.544742 \\ \mathrm{H} & 0.272388 & -0.220723 & -2.663015 \\ \mathrm{H} & 1.652328 & 0.890135 & -2.682598\end{array}$

$m$-cresol, cis $\mathrm{S}_{0}$

$\begin{array}{rrrr}\mathrm{C} & 1.156771 & -0.043478 & 2.087580 \\ \mathrm{C} & 2.372588 & -0.007574 & 1.413444 \\ \mathrm{C} & 2.415126 & -0.019666 & 0.018714 \\ \mathrm{C} & 1.233435 & 0.032687 & -0.723730 \\ \mathrm{C} & 0.012803 & -0.006625 & -0.042648 \\ \mathrm{C} & -0.025897 & 0.016865 & 1.351091 \\ \mathrm{C} & 1.261216 & 0.003489 & -2.228231 \\ \mathrm{O} & -1.204927 & 0.024997 & 2.056910 \\ \mathrm{H} & 1.107329 & -0.035031 & 3.168426 \\ \mathrm{H} & 3.295125 & -0.037273 & 1.980347 \\ \mathrm{H} & 3.370081 & 0.007452 & -0.492525 \\ \mathrm{H} & -0.916797 & 0.027980 & -0.604725 \\ \mathrm{H} & -1.931495 & 0.077027 & 1.420868 \\ \mathrm{H} & 2.180752 & 0.447731 & -2.609086 \\ \mathrm{H} & 1.207869 & -1.022522 & -2.598297 \\ \mathrm{H} & 0.418316 & 0.553310 & -2.648190\end{array}$

$m$-cresol, cis $\mathrm{S}_{1}$

$\begin{array}{crcc}\mathrm{C} & 1.163392 & -0.001265 & 2.133670 \\ \mathrm{C} & 2.417368 & 0.067233 & 1.451860 \\ \mathrm{C} & 2.419079 & 0.009628 & 0.031046 \\ \mathrm{C} & 1.221292 & 0.031496 & -0.753016 \\ \mathrm{C} & -0.029634 & -0.091271 & -0.070883 \\ \mathrm{C} & -0.022704 & -0.002920 & 1.358009 \\ \mathrm{C} & 1.262644 & 0.019616 & -2.244084 \\ \mathrm{O} & -1.191897 & -0.013380 & 2.050650 \\ \mathrm{H} & 1.084740 & 0.009615 & 3.211595 \\ \mathrm{H} & 3.347105 & 0.045853 & 2.001604 \\ \mathrm{H} & 3.369113 & 0.010858 & -0.492039 \\ \mathrm{H} & -0.968017 & -0.051644 & -0.613736 \\ \mathrm{H} & -1.915435 & -0.060387 & 1.403795 \\ \mathrm{H} & 2.283088 & 0.117160 & -2.615076 \\ \mathrm{H} & 0.842478 & -0.911251 & -2.641828 \\ \mathrm{H} & 0.669663 & 0.840028 & -2.661630\end{array}$

$m$-cresol, trans $\mathrm{S}_{0}$
$\begin{array}{llll}\text { C } & -1.146116 & -1.135567 & -0.016245\end{array}$
$\begin{array}{llll}\text { C } & -1.197632 & 0.261383 & 0.023644\end{array}$ 


$\begin{array}{rrrr}\mathrm{C} & -0.001995 & 0.981274 & -0.016476 \\ \mathrm{C} & 1.223432 & 0.320394 & 0.023272 \\ \mathrm{C} & 1.271949 & -1.073835 & -0.024708 \\ \mathrm{C} & 0.080975 & -1.795185 & 0.012201 \\ \mathrm{C} & -2.512956 & 0.993316 & 0.013398 \\ \mathrm{O} & 2.354371 & 1.101574 & 0.032135 \\ \mathrm{H} & 2.227495 & -1.587410 & -0.006372 \\ \mathrm{H} & 0.114578 & -2.877562 & -0.011499 \\ \mathrm{H} & -2.065566 & -1.708061 & 0.007671 \\ \mathrm{H} & -0.008627 & 2.065066 & 0.003542 \\ \mathrm{H} & 3.121687 & 0.513226 & 0.037837 \\ \mathrm{H} & -3.349535 & 0.295162 & 0.019643 \\ \mathrm{H} & -2.605568 & 1.640931 & 0.886865 \\ \mathrm{H} & -2.600819 & 1.620506 & -0.875070\end{array}$

$m$-cresol, trans $\mathrm{S}_{1}$

$\begin{array}{rrrr}\mathrm{C} & -1.206683 & -1.065166 & 0.027831 \\ \mathrm{C} & 0.207611 & -1.242890 & -0.057409 \\ \mathrm{C} & 1.031767 & -0.070948 & 0.007228 \\ \mathrm{C} & 0.401723 & 1.202468 & -0.030378 \\ \mathrm{C} & -1.012945 & 1.380756 & 0.041904 \\ \mathrm{C} & -1.833148 & 0.215724 & -0.006463 \\ \mathrm{C} & 0.831338 & -2.597514 & -0.014544 \\ \mathrm{O} & 1.233083 & 2.277302 & -0.045279 \\ \mathrm{H} & -1.436515 & 2.377700 & 0.012012 \\ \mathrm{H} & -2.909875 & 0.296357 & 0.039122 \\ \mathrm{H} & -1.835604 & -1.948140 & 0.058266 \\ \mathrm{H} & 2.111655 & -0.126922 & -0.017915 \\ \mathrm{H} & 0.683095 & 3.078038 & -0.031734 \\ \mathrm{H} & 0.084480 & -3.382815 & -0.135414 \\ \mathrm{H} & 1.577203 & -2.712347 & -0.806943 \\ \mathrm{H} & 1.349458 & -2.766299 & 0.936802\end{array}$

$p$-cresol $\mathrm{S}_{0}$

$\begin{array}{lccc}\text { C } & 0.713270 & 0.002969 & -1.192326 \\ \text { C } & -0.676918 & -0.032340 & -1.201559 \\ \text { C } & -1.373517 & 0.012004 & 0.005781 \\ \text { C } & -0.672641 & -0.031390 & 1.209924 \\ \text { C } & 0.721585 & 0.009523 & 1.198896 \\ \text { C } & 1.441299 & -0.026180 & 0.003279 \\ \text { O } & -2.746927 & 0.008798 & -0.059135 \\ \text { C } & 2.945183 & 0.027285 & -0.003972 \\ \text { H } & -1.209366 & -0.019110 & 2.153296 \\ \text { H } & 1.252703 & -0.012029 & 2.144161\end{array}$




$\begin{array}{rrrc}\mathrm{H} & 1.243266 & -0.019696 & -2.138349 \\ \mathrm{H} & -1.231718 & -0.021257 & -2.131026 \\ \mathrm{H} & -3.086883 & 0.040498 & 0.845431 \\ \mathrm{H} & 3.353626 & -0.559652 & -0.827413 \\ \mathrm{H} & 3.303437 & 1.052703 & -0.117968 \\ \mathrm{H} & 3.355158 & -0.366750 & 0.926280\end{array}$

$p$-cresol $\mathrm{S}_{1}$

$\begin{array}{lrrr}\mathrm{C} & 0.734126 & -0.101607 & -1.244035 \\ \mathrm{C} & -0.690532 & -0.015610 & -1.251585 \\ \mathrm{C} & -1.361670 & -0.017089 & -0.002498 \\ \mathrm{C} & -0.685599 & 0.051497 & 1.254374 \\ \mathrm{C} & 0.734388 & -0.060035 & 1.243257 \\ \mathrm{C} & 1.436692 & -0.033054 & -0.003856 \\ \mathrm{O} & -2.718053 & 0.018526 & -0.053623 \\ \mathrm{C} & 2.926098 & 0.046801 & -0.000990 \\ \mathrm{H} & -1.252187 & 0.046169 & 2.178050 \\ \mathrm{H} & 1.294540 & -0.039490 & 2.169028 \\ \mathrm{H} & 1.289288 & -0.093978 & -2.172603 \\ \mathrm{H} & -1.271662 & -0.020640 & -2.162663 \\ \mathrm{H} & -3.046789 & 0.053562 & 0.860093 \\ \mathrm{H} & 3.345646 & -0.422155 & -0.892202 \\ \mathrm{H} & 3.255388 & 1.091358 & 0.010993 \\ \mathrm{H} & 3.341873 & -0.438877 & 0.883549\end{array}$

$o$-methoxyphenol $\mathrm{S}_{0}$

$\begin{array}{lrrr}\mathrm{C} & 2.024701 & -0.080278 & -0.016249 \\ \mathrm{C} & 0.866228 & 0.704072 & 0.018397 \\ \mathrm{C} & -0.378314 & 0.084600 & -0.035305 \\ \mathrm{C} & -0.471865 & -1.317573 & 0.024666 \\ \mathrm{C} & 0.680678 & -2.088220 & -0.020003 \\ \mathrm{C} & 1.931215 & -1.467575 & 0.016218 \\ \mathrm{O} & -1.600515 & 0.716326 & -0.043060 \\ \mathrm{O} & -1.696908 & -1.923682 & 0.021616 \\ \mathrm{H} & 0.584262 & -3.166313 & -0.000967 \\ \mathrm{H} & 2.827865 & -2.073217 & -0.006059 \\ \mathrm{H} & 2.992084 & 0.404191 & 0.011684 \\ \mathrm{H} & 0.947825 & 1.781878 & -0.005561 \\ \mathrm{C} & -1.577812 & 2.140381 & -0.008426 \\ \mathrm{H} & -2.353129 & -1.208360 & 0.046258 \\ \mathrm{H} & -2.617841 & 2.452201 & -0.007687 \\ \mathrm{H} & -1.084013 & 2.498149 & 0.897167 \\ \mathrm{H} & -1.073139 & 2.543127 & -0.888601\end{array}$


$o$-methoxyphenol $\mathrm{S}_{1}$

$\begin{array}{rrrr}\mathrm{C} & 2.467351 & 0.064215 & 0.040079 \\ \mathrm{C} & 1.368095 & -0.298288 & -0.772034 \\ \mathrm{C} & 0.096112 & -0.320988 & -0.091524 \\ \mathrm{C} & 0.014257 & -0.192795 & 1.321773 \\ \mathrm{C} & 1.190243 & -0.146576 & 2.143715 \\ \mathrm{C} & 2.367027 & 0.198649 & 1.451311 \\ \mathrm{O} & -1.108650 & -0.120956 & -0.717322 \\ \mathrm{O} & -1.180897 & 0.087201 & 1.899650 \\ \mathrm{H} & 1.106348 & -0.159135 & 3.219785 \\ \mathrm{H} & 3.252618 & 0.492445 & 2.003306 \\ \mathrm{H} & 3.425386 & 0.265532 & -0.428490 \\ \mathrm{H} & 1.505046 & -0.696535 & -1.768957 \\ \mathrm{C} & -1.069365 & 0.137492 & -2.133823 \\ \mathrm{H} & -1.840667 & 0.095214 & 1.177283 \\ \mathrm{H} & -2.102022 & 0.310010 & -2.420256 \\ \mathrm{H} & -0.457069 & 1.013313 & -2.343051 \\ \mathrm{H} & -0.675255 & -0.728809 & -2.663365\end{array}$

$m$-methoxyphenol, isomer $1 \mathrm{~S}_{0}$

$\begin{array}{rrrc}\mathrm{C} & 0.929806 & 1.452917 & -0.023685 \\ \mathrm{C} & -0.264104 & 0.725422 & -0.010247 \\ \mathrm{C} & -0.236526 & -0.670829 & -0.072149 \\ \mathrm{C} & 0.995096 & -1.330410 & 0.002475 \\ \mathrm{C} & 2.190174 & -0.620240 & -0.016015 \\ \mathrm{C} & 2.140094 & 0.772833 & 0.024059 \\ \mathrm{H} & -1.148217 & -1.254325 & -0.078146 \\ \mathrm{O} & 1.076610 & -2.702881 & -0.005806 \\ \mathrm{H} & 3.127845 & -1.157225 & 0.023769 \\ \mathrm{H} & 3.064944 & 1.336173 & 0.024184 \\ \mathrm{H} & 0.882862 & 2.532924 & 0.009431 \\ \mathrm{O} & -1.412635 & 1.468514 & -0.016259 \\ \mathrm{H} & 0.180051 & -3.057090 & 0.067193 \\ \mathrm{C} & -2.639242 & 0.748413 & 0.020047 \\ \mathrm{H} & -3.420516 & 1.502480 & 0.013984 \\ \mathrm{H} & -2.747942 & 0.107920 & -0.858389 \\ \mathrm{H} & -2.718185 & 0.145568 & 0.927907\end{array}$

m-methoxyphenol, isomer $1 \mathrm{~S}_{1}$
$\begin{array}{llll}\text { C } & 0.928429 & 1.434202 & 0.006914\end{array}$
$\begin{array}{llll}\text { C } & -0.275520 & 0.688908 & 0.028377\end{array}$
$\begin{array}{llll}\text { C } & -0.314487 & -0.744911 & 0.027937\end{array}$
$\begin{array}{llll}\text { C } & 0.983366 & -1.339298 & 0.008279\end{array}$ 


$\begin{array}{crrc}\mathrm{C} & 2.197099 & -0.609945 & -0.014318 \\ \mathrm{C} & 2.199776 & 0.800965 & 0.182158 \\ \mathrm{H} & -1.136655 & -1.290252 & 0.483434 \\ \mathrm{O} & 1.104697 & -2.695222 & -0.104013 \\ \mathrm{H} & 3.112804 & -1.165761 & -0.175746 \\ \mathrm{H} & 3.115723 & 1.369972 & 0.147766 \\ \mathrm{H} & 0.844283 & 2.506123 & -0.125901 \\ \mathrm{O} & -1.399338 & 1.453622 & -0.067252 \\ \mathrm{H} & 0.223361 & -3.032733 & -0.339520 \\ \mathrm{C} & -2.658226 & 0.768885 & -0.020432 \\ \mathrm{H} & -3.401550 & 1.513153 & -0.288522 \\ \mathrm{H} & -2.670752 & -0.053726 & -0.735214 \\ \mathrm{H} & -2.853008 & 0.396021 & 0.986052\end{array}$

$m$-methoxyphenol, isomer $2 \mathrm{~S}_{0}$

$\begin{array}{lrrr}\mathrm{C} & 0.346150 & 2.162963 & -0.022800 \\ \mathrm{C} & -0.842167 & 1.450969 & 0.027551 \\ \mathrm{C} & -0.802992 & 0.050069 & 0.008549 \\ \mathrm{C} & 0.417739 & -0.622007 & 0.058036 \\ \mathrm{C} & 1.601175 & 0.118224 & -0.009201 \\ \mathrm{C} & 1.580884 & 1.508960 & 0.017226 \\ \mathrm{O} & -2.019459 & -0.572909 & 0.014667 \\ \mathrm{C} & -2.004016 & -1.996764 & -0.018648 \\ \mathrm{O} & 2.767441 & -0.609966 & -0.021135 \\ \mathrm{H} & 0.491932 & -1.699392 & 0.053748 \\ \mathrm{H} & 2.506358 & 2.071826 & -0.027155 \\ \mathrm{H} & 0.319488 & 3.245500 & -0.017418 \\ \mathrm{H} & -1.802534 & 1.947550 & -0.000372 \\ \mathrm{H} & -3.046826 & -2.299204 & -0.010876 \\ \mathrm{H} & -1.519803 & -2.365293 & -0.925614 \\ \mathrm{H} & -1.499319 & -2.405733 & 0.859280 \\ \mathrm{H} & 3.505039 & 0.015298 & -0.010498\end{array}$

$m$-methoxyphenol, isomer $2 \mathrm{~S}_{1}$

$\begin{array}{lrrr}\mathrm{C} & 1.254741 & -0.347933 & -0.713657 \\ \mathrm{C} & 0.071258 & -0.041443 & 0.065704 \\ \mathrm{C} & 0.105540 & -0.118570 & 1.476557 \\ \mathrm{C} & 1.319118 & -0.321221 & 2.191607 \\ \mathrm{C} & 2.465545 & -0.042362 & 1.423886 \\ \mathrm{C} & 2.433348 & 0.056125 & 0.006340 \\ \mathrm{O} & -1.103043 & 0.033179 & 2.077548 \\ \mathrm{C} & -1.111674 & 0.090778 & 3.509999 \\ \mathrm{O} & 3.632231 & 0.140394 & 2.106581 \\ \mathrm{H} & 1.395169 & -0.485638 & 3.253464\end{array}$




$\begin{array}{rrrr}\mathrm{H} & 3.299450 & 0.458386 & -0.513884 \\ \mathrm{H} & 1.225129 & -0.242029 & -1.789009 \\ \mathrm{H} & -0.847283 & 0.293628 & -0.402975 \\ \mathrm{H} & -2.152298 & 0.231287 & 3.784507 \\ \mathrm{H} & -0.740387 & -0.841133 & 3.936353 \\ \mathrm{H} & -0.510355 & 0.929152 & 3.862644 \\ \mathrm{H} & 4.348641 & 0.207402 & 1.456225\end{array}$

p-methoxyphenol, cis $\mathrm{S}_{0}$

$\begin{array}{lrrr}\mathrm{C} & -0.321112 & 0.738440 & -0.010933 \\ \mathrm{C} & -1.554235 & 0.074749 & 0.016107 \\ \mathrm{C} & -1.611073 & -1.309127 & -0.023772 \\ \mathrm{C} & -0.431378 & -2.057028 & 0.022512 \\ \mathrm{C} & 0.794713 & -1.403923 & -0.014899 \\ \mathrm{C} & 0.857210 & -0.006145 & 0.038868 \\ \mathrm{O} & -0.555998 & -3.428738 & 0.008140 \\ \mathrm{O} & -0.386370 & 2.108503 & -0.004224 \\ \mathrm{C} & 0.858921 & 2.793980 & -0.023589 \\ \mathrm{H} & 1.827047 & 0.469960 & 0.025297 \\ \mathrm{H} & -2.460992 & 0.665521 & -0.008026 \\ \mathrm{H} & -2.562876 & -1.824260 & -0.013891 \\ \mathrm{H} & 1.716734 & -1.975923 & 0.005936 \\ \mathrm{H} & 0.611990 & 3.851506 & -0.018535 \\ \mathrm{H} & 1.455650 & 2.555330 & 0.860380 \\ \mathrm{H} & 1.429498 & 2.555397 & -0.924677 \\ \mathrm{H} & 0.330640 & -3.807871 & 0.074768\end{array}$

$p$-methoxyphenol, cis $\mathrm{S}_{1}$

$\begin{array}{lrrc}\mathrm{C} & -0.323419 & 0.719129 & 0.014513 \\ \mathrm{C} & -1.599691 & 0.096717 & 0.030380 \\ \mathrm{C} & -1.664241 & -1.327664 & -0.023676 \\ \mathrm{C} & -0.448886 & -2.044776 & 0.008319 \\ \mathrm{C} & 0.835011 & -1.423263 & -0.049641 \\ \mathrm{C} & 0.905014 & -0.010205 & 0.084157 \\ \mathrm{O} & -0.546356 & -3.400493 & -0.007327 \\ \mathrm{O} & -0.369628 & 2.072427 & 0.026775 \\ \mathrm{C} & 0.869624 & 2.796992 & -0.023463 \\ \mathrm{H} & 1.861623 & 0.487413 & 0.107173 \\ \mathrm{H} & -2.481378 & 0.717183 & -0.039024 \\ \mathrm{H} & -2.600460 & -1.865807 & 0.002119 \\ \mathrm{H} & 1.731456 & -2.031428 & -0.019897 \\ \mathrm{H} & 0.586227 & 3.843652 & 0.015798 \\ \mathrm{H} & 1.491814 & 2.546829 & 0.834994 \\ \mathrm{H} & 1.397714 & 2.583190 & -0.952071\end{array}$




$$
\begin{array}{llll}
\mathrm{H} & 0.355577 & -3.759878 & -0.009128 \\
&
\end{array}
$$

$\begin{array}{rrrr}\text { C } & -0.657412 & 1.310628 & 0.020688 \\ \mathrm{C} & -1.890500 & 0.672571 & -0.029652 \\ \mathrm{C} & -1.959809 & -0.722148 & 0.017253 \\ \mathrm{C} & -0.786334 & -1.466287 & -0.013940 \\ \mathrm{C} & 0.455263 & -0.829618 & 0.036042 \\ \mathrm{C} & 0.524600 & 0.565712 & -0.008122 \\ \mathrm{O} & -3.152284 & -1.411891 & 0.004179 \\ \mathrm{H} & 1.348870 & -1.437001 & 0.025278 \\ \mathrm{O} & 1.686223 & 1.295629 & 0.000244 \\ \mathrm{H} & -0.592986 & 2.390901 & -0.003161 \\ \mathrm{H} & -2.800487 & 1.263780 & -0.022324 \\ \mathrm{H} & -3.869445 & -0.764734 & 0.032825 \\ \mathrm{H} & -0.846681 & -2.546794 & 0.007954 \\ \mathrm{C} & 2.896001 & 0.548493 & -0.014168 \\ \mathrm{H} & 3.694774 & 1.284256 & -0.008366 \\ \mathrm{H} & 2.979580 & -0.086639 & 0.871146 \\ \mathrm{H} & 2.972039 & -0.067078 & -0.913891\end{array}$

p-methoxyphenol, trans $\mathrm{S}_{1}$

$\begin{array}{rrrr}\text { C } & -0.664374 & 1.347815 & 0.020070 \\ \text { C } & -1.934372 & 0.705965 & -0.054437 \\ \text { C } & -1.950806 & -0.715242 & 0.018602 \\ \text { C } & -0.782774 & -1.515355 & -0.010253 \\ \text { C } & 0.487752 & -0.879202 & 0.074639 \\ \text { C } & 0.510579 & 0.545276 & -0.003891 \\ \mathrm{O} & -3.129516 & -1.391707 & 0.019202 \\ \text { H } & 1.391592 & -1.466098 & 0.034820 \\ \mathrm{O} & 1.657920 & 1.264280 & -0.010306 \\ \mathrm{H} & -0.556977 & 2.422754 & -0.007641 \\ \mathrm{H} & -2.856838 & 1.267175 & 0.033139 \\ \mathrm{H} & -3.842165 & -0.734801 & -0.043778 \\ \mathrm{H} & -0.884498 & -2.591257 & -0.006142 \\ \mathrm{C} & 2.904905 & 0.550626 & -0.015790 \\ \mathrm{H} & 3.670145 & 1.318666 & 0.027408 \\ \mathrm{H} & 2.974142 & -0.098393 & 0.856172 \\ \mathrm{H} & 3.005276 & -0.030521 & -0.931815\end{array}$

1-naphthol, cis $\mathrm{S}_{0}$
C $\quad 0.832471$
$\begin{array}{ll}1.514057 & 0.020374\end{array}$
$\begin{array}{llll}\text { C } & 0.530042 & 0.123724 & -0.044874\end{array}$ 


$\begin{array}{lrrr}\mathrm{C} & -0.845962 & -0.268885 & 0.027338 \\ \mathrm{C} & -1.857944 & 0.719539 & -0.027105 \\ \mathrm{C} & -1.520341 & 2.055127 & -0.025232 \\ \mathrm{C} & -0.171222 & 2.457310 & -0.036356 \\ \mathrm{C} & 1.527917 & -0.882740 & -0.043504 \\ \mathrm{C} & 1.186771 & -2.218316 & -0.055142 \\ \mathrm{C} & -0.167280 & -2.606598 & 0.020460 \\ \mathrm{C} & -1.159178 & -1.651080 & 0.006273 \\ \mathrm{O} & 2.129384 & 1.969079 & 0.027869 \\ \mathrm{H} & -2.202985 & -1.944791 & 0.038523 \\ \mathrm{H} & -0.426499 & -3.658222 & 0.012046 \\ \mathrm{H} & 1.964543 & -2.972034 & -0.068241 \\ \mathrm{H} & 2.576874 & -0.617169 & -0.124834 \\ \mathrm{H} & -2.896411 & 0.409722 & -0.005325 \\ \mathrm{H} & -2.295150 & 2.811299 & -0.062388 \\ \mathrm{H} & 0.098655 & 3.505407 & -0.008121 \\ \mathrm{H} & 2.696324 & 1.254578 & 0.348232\end{array}$

1-naphthol, cis $\mathrm{S}_{1}$

$\begin{array}{lrrr}\mathrm{C} & 0.857354 & 1.459218 & 0.030466 \\ \mathrm{C} & 0.531592 & 0.087472 & -0.130319 \\ \mathrm{C} & -0.853595 & -0.245732 & 0.005918 \\ \mathrm{C} & -1.792384 & 0.814882 & -0.044926 \\ \mathrm{C} & -1.427730 & 2.185885 & -0.032562 \\ \mathrm{C} & -0.097674 & 2.523032 & -0.028411 \\ \mathrm{C} & 1.526567 & -0.915445 & -0.187587 \\ \mathrm{C} & 1.116232 & -2.285509 & -0.069641 \\ \mathrm{C} & -0.213527 & -2.604282 & 0.093376 \\ \mathrm{C} & -1.230855 & -1.609161 & 0.040720 \\ \mathrm{O} & 2.125363 & 1.821133 & 0.252697 \\ \mathrm{H} & -2.274444 & -1.872168 & 0.169690 \\ \mathrm{H} & -0.503452 & -3.644871 & 0.190473 \\ \mathrm{H} & 1.868908 & -3.065310 & -0.055130 \\ \mathrm{H} & 2.535038 & -0.681492 & -0.518546 \\ \mathrm{H} & -2.846551 & 0.555056 & -0.061167 \\ \mathrm{H} & -2.193325 & 2.948952 & -0.084089 \\ \mathrm{H} & 0.258572 & 3.544684 & 0.004328 \\ \mathrm{H} & 2.613913 & 0.983645 & 0.424689\end{array}$

1-naphthol, trans $\mathrm{S}_{0}$
$\begin{array}{llll}\text { C } & -0.717001 & -0.541062 & 0.026701\end{array}$
$\begin{array}{llll}\text { C } & -0.676689 & -1.957218 & -0.007484\end{array}$
$\begin{array}{llll}\text { C } & 0.524530 & -2.631748 & 0.016845\end{array}$
$\begin{array}{llll}\text { C } & 1.741272 & -1.917371 & -0.018909\end{array}$ 


$\begin{array}{crcc}\mathrm{C} & 1.740910 & -0.538935 & 0.002368 \\ \mathrm{C} & 0.518809 & 0.174260 & -0.033992 \\ \mathrm{H} & -1.613536 & -2.503899 & 0.008608 \\ \mathrm{H} & 0.536529 & -3.714917 & -0.000744 \\ \mathrm{H} & 2.681083 & -2.455756 & 0.001902 \\ \mathrm{H} & 2.670781 & 0.014195 & -0.012662 \\ \mathrm{C} & -1.941548 & 0.172683 & -0.012254 \\ \mathrm{C} & -1.940344 & 1.548488 & 0.009823 \\ \mathrm{C} & -0.726812 & 2.267886 & -0.024812 \\ \mathrm{C} & 0.476450 & 1.594715 & 0.015375 \\ \mathrm{H} & -2.874151 & -0.379356 & 0.007779 \\ \mathrm{H} & -2.875741 & 2.094349 & -0.014023 \\ \mathrm{H} & -0.739549 & 3.352963 & 0.004282 \\ \mathrm{O} & 1.691507 & 2.234057 & 0.028264 \\ \mathrm{H} & 1.523511 & 3.186657 & 0.002934\end{array}$

1-naphthol, trans $\mathrm{S}_{1}$

$\begin{array}{lrrr}\mathrm{C} & -0.734021 & -0.565861 & 0.034501 \\ \mathrm{C} & -0.690677 & -1.970513 & 0.006734 \\ \mathrm{C} & 0.528810 & -2.669930 & 0.035885 \\ \mathrm{C} & 1.750442 & -1.939216 & -0.026973 \\ \mathrm{C} & 1.755765 & -0.537321 & -0.011047 \\ \mathrm{C} & 0.547942 & 0.190200 & -0.039848 \\ \mathrm{H} & -1.628850 & -2.516220 & 0.025043 \\ \mathrm{H} & 0.539393 & -3.751607 & 0.015796 \\ \mathrm{H} & 2.694122 & -2.470928 & -0.016602 \\ \mathrm{H} & 2.695325 & 0.000262 & -0.039388 \\ \mathrm{C} & -1.936643 & 0.164871 & -0.025566 \\ \mathrm{C} & -1.945635 & 1.573522 & -0.002426 \\ \mathrm{C} & -0.735003 & 2.305944 & -0.026718 \\ \mathrm{C} & 0.488126 & 1.599002 & 0.028610 \\ \mathrm{H} & -2.878252 & -0.373851 & -0.018206 \\ \mathrm{H} & -2.889864 & 2.102864 & -0.028185 \\ \mathrm{H} & -0.740069 & 3.388065 & 0.038616 \\ \mathrm{O} & 1.682804 & 2.261401 & 0.062068 \\ \mathrm{H} & 1.496297 & 3.209305 & -0.012295\end{array}$

2-naphthol, cis $\mathrm{S}_{0}$
$\begin{array}{llll}\text { C } & 0.601006 & 2.009624 & -0.013179\end{array}$
$\begin{array}{llll}\text { C } & 0.623307 & 0.595128 & 0.034783\end{array}$

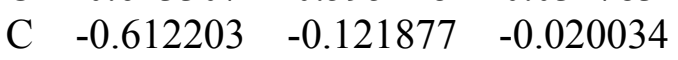
$\begin{array}{llll}\text { C } & -1.824494 & 0.611000 & 0.014936\end{array}$
$\begin{array}{llll}\text { C } & -1.815331 & 1.989081 & -0.016329\end{array}$
$\begin{array}{llll}\text { C } & -0.593818 & 2.696460 & 0.008046\end{array}$ 


$\begin{array}{lrrr}\mathrm{C} & 1.835157 & -0.141148 & -0.005104 \\ \mathrm{C} & 1.833461 & -1.514835 & 0.021541 \\ \mathrm{C} & 0.607160 & -2.216839 & -0.009588 \\ \mathrm{C} & -0.591362 & -1.537604 & 0.028672 \\ \mathrm{H} & -1.532585 & -2.080862 & 0.020186 \\ \mathrm{H} & 1.543753 & 2.546616 & -0.005061 \\ \mathrm{H} & -0.598441 & 3.779179 & -0.021273 \\ \mathrm{H} & -2.751352 & 2.534427 & 0.001139 \\ \mathrm{H} & -2.764691 & 0.069589 & 0.004560 \\ \mathrm{H} & 2.776458 & 0.397758 & 0.006283 \\ \mathrm{H} & 2.755524 & -2.081851 & -0.001940 \\ \mathrm{O} & 0.702744 & -3.586098 & -0.007995 \\ \mathrm{H} & -0.194303 & -3.947741 & -0.039644\end{array}$

2-naphthol, cis $\mathrm{S}_{1}$

$\begin{array}{lrrr}\mathrm{C} & 0.610578 & 1.996912 & 0.012878 \\ \mathrm{C} & 0.654584 & 0.587455 & -0.029321 \\ \mathrm{C} & -0.642389 & -0.124581 & 0.030921 \\ \mathrm{C} & -1.845740 & 0.603479 & -0.000782 \\ \mathrm{C} & -1.850045 & 2.012955 & 0.033834 \\ \mathrm{C} & -0.614514 & 2.690632 & -0.012794 \\ \mathrm{C} & 1.860958 & -0.141962 & 0.011854 \\ \mathrm{C} & 1.888644 & -1.550035 & -0.032145 \\ \mathrm{C} & 0.641114 & -2.215407 & 0.003165 \\ \mathrm{C} & -0.595159 & -1.528262 & -0.042694 \\ \mathrm{H} & -1.520779 & -2.099474 & -0.051871 \\ \mathrm{H} & 1.541740 & 2.553071 & -0.010665 \\ \mathrm{H} & -0.598882 & 3.774606 & -0.004226 \\ \mathrm{H} & -2.780471 & 2.563558 & 0.004920 \\ \mathrm{H} & -2.782268 & 0.054837 & 0.011296 \\ \mathrm{H} & 2.794053 & 0.411669 & 0.006631 \\ \mathrm{H} & 2.804192 & -2.120725 & 0.023128 \\ \mathrm{O} & 0.674185 & -3.571178 & 0.005880 \\ \mathrm{H} & -0.239809 & -3.897542 & 0.039990\end{array}$

2-naphthol, trans $\mathrm{S}_{0}$

$\begin{array}{rrrc}\text { C } & 0.514175 & 2.011873 & -0.012637 \\ \text { C } & 0.541739 & 0.596333 & 0.034265 \\ \text { C } & -0.691304 & -0.127881 & -0.021640 \\ \text { C } & -1.907231 & 0.601902 & 0.014524 \\ \text { C } & -1.902738 & 1.979071 & -0.015361 \\ \text { C } & -0.683181 & 2.692424 & 0.009899 \\ \text { C } & 1.753909 & -0.133569 & -0.006667 \\ \text { C } & 1.752431 & -1.510073 & 0.022683\end{array}$




$\begin{array}{lrrr}\mathrm{C} & 0.529358 & -2.217726 & -0.015178 \\ \mathrm{C} & -0.669975 & -1.540531 & 0.020961 \\ \mathrm{H} & -1.598259 & -2.100046 & 0.007478 \\ \mathrm{H} & 1.454623 & 2.552968 & -0.003520 \\ \mathrm{H} & -0.692630 & 3.775181 & -0.018313 \\ \mathrm{H} & -2.840716 & 2.521068 & 0.002886 \\ \mathrm{H} & -2.844605 & 0.056072 & 0.003265 \\ \mathrm{H} & 2.694895 & 0.405800 & 0.008667 \\ \mathrm{H} & 2.689929 & -2.057290 & -0.002060 \\ \mathrm{O} & 0.494305 & -3.591065 & -0.017917 \\ \mathrm{H} & 1.405285 & -3.914507 & -0.011335\end{array}$

2-naphthol, trans $\mathrm{S}_{1}$

$\begin{array}{lrrr}\mathrm{C} & 0.521579 & 1.996127 & 0.013275 \\ \mathrm{C} & 0.566382 & 0.588313 & -0.029091 \\ \mathrm{C} & -0.725581 & -0.123639 & 0.035726 \\ \mathrm{C} & -1.928234 & 0.600326 & -0.001651 \\ \mathrm{C} & -1.936550 & 2.011488 & 0.028335 \\ \mathrm{C} & -0.705289 & 2.688628 & -0.015403 \\ \mathrm{C} & 1.777913 & -0.136328 & 0.010402 \\ \mathrm{C} & 1.809766 & -1.544316 & -0.045586 \\ \mathrm{C} & 0.555668 & -2.210453 & 0.008233 \\ \mathrm{C} & -0.679333 & -1.530137 & -0.023776 \\ \mathrm{H} & -1.590504 & -2.118050 & -0.022098 \\ \mathrm{H} & 1.451759 & 2.554286 & -0.009427 \\ \mathrm{H} & -0.689689 & 3.772769 & -0.009160 \\ \mathrm{H} & -2.868240 & 2.559814 & -0.003830 \\ \mathrm{H} & -2.863048 & 0.049041 & 0.012686 \\ \mathrm{H} & 2.708599 & 0.421643 & 0.007490 \\ \mathrm{H} & 2.734753 & -2.101371 & 0.040379 \\ \mathrm{O} & 0.477438 & -3.563567 & 0.019039 \\ \mathrm{H} & 1.382620 & -3.914576 & -0.015543\end{array}$

5-methoxysalicylic acid $\mathrm{S}_{0}$

$\begin{array}{lrrc}\mathrm{C} & 2.282492 & -0.166885 & -0.023695 \\ \mathrm{C} & 1.571678 & -1.349125 & 0.024437 \\ \mathrm{C} & 0.165372 & -1.345722 & -0.001997 \\ \mathrm{C} & -0.512790 & -0.136877 & 0.025782 \\ \mathrm{C} & 0.206397 & 1.076049 & -0.055626 \\ \mathrm{C} & 1.613915 & 1.066630 & 0.002526 \\ \mathrm{O} & -0.424657 & -2.579793 & 0.023314 \\ \mathrm{C} & -1.847978 & -2.589205 & -0.017007 \\ \mathrm{C} & -0.485273 & 2.360885 & -0.007692 \\ \mathrm{O} & -1.839146 & 2.271660 & -0.006841\end{array}$




$\begin{array}{lrrr}\mathrm{O} & 2.369413 & 2.188871 & -0.010503 \\ \mathrm{O} & 0.081259 & 3.461747 & 0.021972 \\ \mathrm{H} & -2.134179 & -3.636328 & 0.004188 \\ \mathrm{H} & -2.219268 & -2.122727 & -0.932645 \\ \mathrm{H} & -2.269168 & -2.077552 & 0.851691 \\ \mathrm{H} & -1.590849 & -0.087985 & 0.015339 \\ \mathrm{H} & 3.364708 & -0.166792 & -0.008190 \\ \mathrm{H} & 2.084836 & -2.302742 & 0.018332 \\ \mathrm{H} & 1.734137 & 2.942519 & 0.009751 \\ \mathrm{H} & -2.150877 & 3.193370 & 0.031168\end{array}$

5-methoxysalicylic acid $\mathrm{S}_{1}$

$\begin{array}{lrrr}\mathrm{C} & 2.187631 & -0.128980 & 0.027036 \\ \mathrm{C} & 1.744198 & -1.468066 & -0.010913 \\ \mathrm{C} & 0.357863 & -1.699836 & -0.010516 \\ \mathrm{C} & -0.593271 & -0.629631 & -0.071658 \\ \mathrm{C} & -0.166608 & 0.689066 & 0.061304 \\ \mathrm{C} & 1.275589 & 0.924428 & -0.016495 \\ \mathrm{O} & -0.017786 & -2.997015 & -0.021697 \\ \mathrm{C} & -1.422620 & -3.307417 & 0.011576 \\ \mathrm{C} & -1.032894 & 1.819337 & 0.039083 \\ \mathrm{O} & -2.377376 & 1.554908 & 0.030408 \\ \mathrm{O} & 1.695296 & 2.187933 & -0.055144 \\ \mathrm{O} & -0.615984 & 3.032465 & 0.033125 \\ \mathrm{H} & -1.468847 & -4.391139 & 0.004379 \\ \mathrm{H} & -1.922946 & -2.907775 & -0.869796 \\ \mathrm{H} & -1.878131 & -2.916032 & 0.920544 \\ \mathrm{H} & -1.653945 & -0.828169 & -0.122126 \\ \mathrm{H} & 3.243340 & 0.111176 & 0.012432 \\ \mathrm{H} & 2.426162 & -2.303559 & 0.053525 \\ \mathrm{H} & 0.788304 & 2.756918 & -0.023397 \\ \mathrm{H} & -2.795923 & 2.432169 & 0.008329\end{array}$

$m$-dimethoxybenzene, trans $\mathrm{S}_{0}$

$\begin{array}{lrrc}\mathrm{C} & 0.019326 & -0.707497 & -0.186559 \\ \mathrm{C} & -0.020036 & 0.707822 & -0.186514 \\ \mathrm{C} & 0.039539 & 1.393667 & 1.023335 \\ \mathrm{C} & -0.004763 & 0.692761 & 2.235662 \\ \mathrm{C} & 0.005574 & -0.692530 & 2.235614 \\ \mathrm{C} & -0.039523 & -1.393388 & 1.023302 \\ \mathrm{O} & -0.016384 & 1.287036 & -1.421220 \\ \mathrm{H} & 0.033566 & 2.474189 & 1.037364 \\ \mathrm{H} & 0.028143 & 1.242611 & 3.167497 \\ \mathrm{H} & -0.026756 & -1.242409 & 3.167458\end{array}$




$\begin{array}{lrrr}\mathrm{H} & -0.033551 & -2.473912 & 1.037276 \\ \mathrm{O} & 0.014884 & -1.286661 & -1.421275 \\ \mathrm{C} & 0.017920 & -2.709550 & -1.443775 \\ \mathrm{H} & 0.018126 & -2.982230 & -2.494889 \\ \mathrm{H} & 0.910732 & -3.111911 & -0.959257 \\ \mathrm{H} & -0.874543 & -3.114070 & -0.960411 \\ \mathrm{C} & -0.018190 & 2.709928 & -1.443654 \\ \mathrm{H} & -0.018556 & 2.982670 & -2.494750 \\ \mathrm{H} & -0.910459 & 3.113082 & -0.958791 \\ \mathrm{H} & 0.874814 & 3.113592 & -0.960574\end{array}$

$m$-dimethoxybenzene, trans $\mathrm{S}_{1}$

$\begin{array}{lrrr}\mathrm{C} & -0.223104 & -1.078765 & -1.451238 \\ \mathrm{C} & -0.292630 & 0.173334 & -0.712389 \\ \mathrm{C} & -0.339888 & 0.166958 & 0.703597 \\ \mathrm{C} & -0.168738 & -1.060484 & 1.447456 \\ \mathrm{C} & 0.287677 & -2.161056 & 0.704633 \\ \mathrm{C} & 0.332064 & -2.142912 & -0.714057 \\ \mathrm{O} & -0.005374 & 1.388862 & -1.265630 \\ \mathrm{C} & 0.035790 & 1.441301 & -2.698036 \\ \mathrm{O} & -0.314923 & 1.393509 & 1.286864 \\ \mathrm{C} & -0.030670 & 1.433942 & 2.696271 \\ \mathrm{H} & -0.485603 & -1.163893 & 2.476520 \\ \mathrm{H} & 0.604175 & -3.061977 & 1.220592 \\ \mathrm{H} & 0.718757 & -3.017015 & -1.229684 \\ \mathrm{H} & -0.372337 & -1.135397 & -2.518251 \\ \mathrm{H} & 0.187619 & 2.489133 & -2.938708 \\ \mathrm{H} & 0.860203 & 0.842972 & -3.085745 \\ \mathrm{H} & -0.907999 & 1.095128 & -3.121253 \\ \mathrm{H} & 0.004925 & 2.490034 & 2.944763 \\ \mathrm{H} & -0.824401 & 0.946782 & 3.262153 \\ \mathrm{H} & 0.924710 & 0.955905 & 2.908954\end{array}$

3P-propionic acid, gauche $\mathrm{S}_{0}$

$\begin{array}{lrrr}\mathrm{C} & -0.906288 & 0.830637 & -1.167266 \\ \mathrm{C} & -0.294276 & 0.714904 & 0.085774 \\ \mathrm{C} & -0.939629 & -0.022023 & 1.084427 \\ \mathrm{C} & -2.139529 & -0.680868 & 0.816270 \\ \mathrm{C} & -2.747363 & -0.547073 & -0.431171 \\ \mathrm{C} & -2.103714 & 0.172241 & -1.438665 \\ \mathrm{C} & 1.047757 & 1.344597 & 0.340041 \\ \mathrm{C} & 2.175173 & 0.443833 & -0.186575 \\ \mathrm{C} & 2.121135 & -0.874902 & 0.534991 \\ \mathrm{O} & 2.197085 & -1.008483 & 1.745818\end{array}$




$\begin{array}{lrrr}\mathrm{O} & 1.947264 & -1.923144 & -0.315289 \\ \mathrm{H} & 2.071474 & 0.282057 & -1.258679 \\ \mathrm{H} & 3.146873 & 0.898622 & 0.016495 \\ \mathrm{H} & 1.193612 & 1.496714 & 1.410840 \\ \mathrm{H} & 1.111352 & 2.315917 & -0.155001 \\ \mathrm{H} & -0.473300 & -0.116930 & 2.058787 \\ \mathrm{H} & -2.631929 & -1.241133 & 1.601908 \\ \mathrm{H} & -3.680227 & -1.056182 & -0.639089 \\ \mathrm{H} & -2.569888 & 0.278953 & -2.410655 \\ \mathrm{H} & -0.416482 & 1.403484 & -1.948323 \\ \mathrm{H} & 1.890910 & -2.711240 & 0.255373\end{array}$

3P-propionic acid, gauche $\mathrm{S}_{1}$

$\begin{array}{rrrr}\mathrm{C} & -0.827279 & 0.840778 & -1.215723 \\ \mathrm{C} & -0.265847 & 0.762556 & 0.102832 \\ \mathrm{C} & -0.919917 & -0.045207 & 1.095831 \\ \mathrm{C} & -2.159817 & -0.691555 & 0.797996 \\ \mathrm{C} & -2.690898 & -0.630448 & -0.526102 \\ \mathrm{C} & -2.068145 & 0.193590 & -1.511925 \\ \mathrm{C} & 1.066597 & 1.367160 & 0.381580 \\ \mathrm{C} & 2.201424 & 0.448943 & -0.127260 \\ \mathrm{C} & 2.074290 & -0.876353 & 0.569854 \\ \mathrm{O} & 2.243058 & -1.053311 & 1.765632 \\ \mathrm{O} & 1.693259 & -1.869126 & -0.280497 \\ \mathrm{H} & 2.128335 & 0.314642 & -1.205436 \\ \mathrm{H} & 3.170950 & 0.880858 & 0.126233 \\ \mathrm{H} & 1.199369 & 1.514504 & 1.455783 \\ \mathrm{H} & 1.159083 & 2.336607 & -0.116312 \\ \mathrm{H} & -0.491501 & -0.112915 & 2.088347 \\ \mathrm{H} & -2.653988 & -1.288317 & 1.552853 \\ \mathrm{H} & -3.614454 & -1.142875 & -0.760467 \\ \mathrm{H} & -2.492647 & 0.264656 & -2.504382 \\ \mathrm{H} & -0.334829 & 1.445263 & -1.968131 \\ \mathrm{H} & 1.582965 & -2.659459 & 0.279294\end{array}$

3P-propionic acid, anti $\mathrm{S}_{0}$

$\begin{array}{rrrr}\text { C } & 1.437268 & -0.440757 & -0.000041 \\ \mathrm{C} & 0.547009 & 0.797733 & -0.000009 \\ \mathrm{C} & 2.903407 & -0.099426 & 0.000014 \\ \mathrm{H} & 1.230634 & -1.062598 & 0.874405 \\ \mathrm{H} & 1.230683 & -1.062514 & -0.874559 \\ \mathrm{C} & -0.904329 & 0.405838 & 0.000000 \\ \mathrm{H} & 0.774403 & 1.405464 & 0.877432 \\ \mathrm{H} & 0.774385 & 1.405496 & -0.877432\end{array}$




$\begin{array}{lrrr}\mathrm{C} & -1.593819 & 0.219419 & 1.202504 \\ \mathrm{C} & -2.914676 & -0.226306 & 1.205306 \\ \mathrm{C} & -3.593178 & -0.404723 & 0.000011 \\ \mathrm{C} & -2.914649 & -0.226418 & -1.205286 \\ \mathrm{C} & -1.593852 & 0.219487 & -1.202496 \\ \mathrm{H} & -1.075039 & 0.375941 & 2.142806 \\ \mathrm{H} & -3.435809 & -0.362484 & 2.145162 \\ \mathrm{H} & -4.621477 & -0.744005 & 0.000015 \\ \mathrm{H} & -3.435679 & -0.362963 & -2.145146 \\ \mathrm{H} & -1.075183 & 0.376337 & -2.142804 \\ \mathrm{O} & 3.670184 & -1.228858 & 0.000019 \\ \mathrm{O} & 3.380635 & 1.021704 & 0.000049 \\ \mathrm{H} & 4.591920 & -0.915049 & 0.000049\end{array}$

3P-propionic acid, anti $\mathrm{S}_{1}$

$\begin{array}{lrrr}\mathrm{C} & 1.439050 & -0.422540 & 0.000239 \\ \mathrm{C} & 0.569694 & 0.837939 & 0.000186 \\ \mathrm{C} & 2.910513 & -0.104463 & -0.000213 \\ \mathrm{H} & 1.220157 & -1.037710 & 0.876190 \\ \mathrm{H} & 1.219701 & -1.038101 & -0.875323 \\ \mathrm{C} & -0.876785 & 0.482880 & 0.000084 \\ \mathrm{H} & 0.813220 & 1.436636 & 0.880984 \\ \mathrm{H} & 0.813344 & 1.436651 & -0.880568 \\ \mathrm{C} & -1.574884 & 0.283647 & 1.238564 \\ \mathrm{C} & -2.897479 & -0.260317 & 1.239121 \\ \mathrm{C} & -3.578138 & -0.460685 & -0.000109 \\ \mathrm{C} & -2.897309 & -0.260297 & -1.239242 \\ \mathrm{C} & -1.574711 & 0.283658 & -1.238495 \\ \mathrm{H} & -1.066863 & 0.488011 & 2.173432 \\ \mathrm{H} & -3.412487 & -0.437102 & 2.173704 \\ \mathrm{H} & -4.594506 & -0.832019 & -0.000182 \\ \mathrm{H} & -3.412191 & -0.437060 & -2.173899 \\ \mathrm{H} & -1.066556 & 0.488022 & -2.173290 \\ \mathrm{O} & 3.658191 & -1.246495 & -0.000125 \\ \mathrm{O} & 3.405437 & 1.008881 & -0.000616 \\ \mathrm{H} & 4.585230 & -0.948377 & -0.000442\end{array}$

fluorine $\mathrm{S}_{0}$
$\begin{array}{llll}\text { C } & 0.000144 & -0.000004 & -1.796462\end{array}$
$\begin{array}{llll}\text { C } & -0.022764 & 1.178508 & -0.853528\end{array}$
$\begin{array}{llll}\text { C } & 0.018471 & 0.731286 & 0.481328\end{array}$
$\begin{array}{llll}\text { C } & -0.017956 & -0.731281 & 0.481334\end{array}$
$\begin{array}{llll}\text { C } & -0.020053 & 1.643949 & 1.536366\end{array}$
C $\quad 0.013302 \quad 3.007220 \quad 1.242973$ 


$\begin{array}{lrrr}\mathrm{C} & -0.012488 & 3.452072 & -0.083605 \\ \mathrm{C} & 0.017614 & 2.538451 & -1.140788 \\ \mathrm{H} & 0.005715 & 2.890330 & -2.166707 \\ \mathrm{H} & 0.010580 & 4.515096 & -0.291436 \\ \mathrm{H} & -0.008213 & 3.730731 & 2.049124 \\ \mathrm{H} & -0.008691 & 1.304751 & 2.566257 \\ \mathrm{C} & 0.020392 & -1.643958 & 1.536367 \\ \mathrm{C} & -0.013591 & -3.007214 & 1.242974 \\ \mathrm{C} & 0.011967 & -3.452076 & -0.083605 \\ \mathrm{C} & -0.017607 & -2.538441 & -1.140790 \\ \mathrm{C} & 0.023138 & -1.178510 & -0.853523 \\ \mathrm{H} & 0.009501 & -1.304757 & 2.566261 \\ \mathrm{H} & 0.007595 & -3.730735 & 2.049125 \\ \mathrm{H} & -0.011827 & -4.515084 & -0.291436 \\ \mathrm{H} & -0.005460 & -2.890321 & -2.166705 \\ \mathrm{H} & -0.883317 & -0.019887 & -2.441710 \\ \mathrm{H} & 0.883546 & 0.019875 & -2.441791\end{array}$

fluorine $\mathrm{S}_{1}$

$\begin{array}{lrrc}\mathrm{C} & -0.000040 & -0.000005 & -1.828953 \\ \mathrm{C} & -0.024477 & 1.166821 & -0.879148 \\ \mathrm{C} & 0.029077 & 0.707165 & 0.475425 \\ \mathrm{C} & -0.029142 & -0.707213 & 0.475374 \\ \mathrm{C} & -0.012449 & 1.613518 & 1.567608 \\ \mathrm{C} & 0.020713 & 2.986995 & 1.264376 \\ \mathrm{C} & -0.015106 & 3.438194 & -0.072980 \\ \mathrm{C} & 0.018278 & 2.540883 & -1.164176 \\ \mathrm{H} & -0.005704 & 2.906470 & -2.183393 \\ \mathrm{H} & -0.004072 & 4.505159 & -0.263688 \\ \mathrm{H} & 0.002426 & 3.717710 & 2.063970 \\ \mathrm{H} & 0.015824 & 1.256166 & 2.589086 \\ \mathrm{C} & 0.012403 & -1.613586 & 1.567472 \\ \mathrm{C} & -0.020699 & -2.986966 & 1.264332 \\ \mathrm{C} & 0.015168 & -3.438167 & -0.072968 \\ \mathrm{C} & -0.018262 & -2.540828 & -1.164075 \\ \mathrm{C} & 0.024299 & -1.166874 & -0.879124 \\ \mathrm{H} & -0.015777 & -1.256211 & 2.588954 \\ \mathrm{H} & -0.002505 & -3.717649 & 2.063960 \\ \mathrm{H} & 0.004235 & -4.505126 & -0.263719 \\ \mathrm{H} & 0.005784 & -2.906416 & -2.183299 \\ \mathrm{H} & -0.882643 & -0.020516 & -2.482573 \\ \mathrm{H} & 0.882666 & 0.020477 & -2.482443\end{array}$

phenanthrene $\mathrm{S}_{0}$ 


$\begin{array}{rrrr}\text { C } & 0.725016 & -0.020753 & -0.350497 \\ \mathrm{C} & -0.725013 & 0.020339 & -0.350505 \\ \mathrm{C} & -1.418401 & -0.023214 & 0.892901 \\ \mathrm{C} & -0.681207 & 0.011950 & 2.117636 \\ \mathrm{C} & 0.681200 & -0.012164 & 2.117642 \\ \mathrm{C} & 1.418403 & 0.022880 & 0.892908 \\ \mathrm{C} & -1.491028 & -0.022899 & -1.537101 \\ \mathrm{C} & -2.830421 & 0.017433 & 0.906310 \\ \mathrm{H} & -1.231757 & -0.003197 & 3.052653 \\ \mathrm{H} & 1.231741 & 0.003080 & 3.052664 \\ \mathrm{C} & 1.491018 & 0.022616 & -1.537095 \\ \mathrm{C} & 2.873368 & -0.010422 & -1.500676 \\ \mathrm{C} & 3.552324 & 0.011179 & -0.270231 \\ \mathrm{C} & 2.830433 & -0.017377 & 0.906311 \\ \mathrm{H} & 0.997581 & 0.021305 & -2.500151 \\ \mathrm{H} & 3.433689 & 0.015855 & -2.427611 \\ \mathrm{H} & 4.635029 & -0.012088 & -0.246743 \\ \mathrm{H} & 3.341131 & -0.006896 & 1.863585 \\ \mathrm{C} & -3.552324 & -0.010812 & -0.270233 \\ \mathrm{C} & -2.873365 & 0.010654 & -1.500679 \\ \mathrm{H} & -0.997578 & -0.021781 & -2.500150 \\ \mathrm{H} & -3.433697 & -0.015432 & -2.427614 \\ \mathrm{H} & -4.635017 & 0.012963 & -0.246724 \\ \mathrm{H} & -3.341124 & 0.006780 & 1.863579\end{array}$

phenanthrene $S_{1}$

$\begin{array}{rrrr}\mathrm{C} & 0.711336 & -0.013540 & -0.388781 \\ \mathrm{C} & -0.711347 & 0.013685 & -0.388781 \\ \mathrm{C} & -1.436624 & -0.022723 & 0.891556 \\ \mathrm{C} & -0.700431 & 0.019324 & 2.088669 \\ \mathrm{C} & 0.700446 & -0.019530 & 2.088668 \\ \mathrm{C} & 1.436635 & 0.022618 & 0.891555 \\ \mathrm{C} & -1.491215 & -0.058091 & -1.572937 \\ \mathrm{C} & -2.843381 & 0.035882 & 0.889914 \\ \mathrm{H} & -1.237507 & 0.010239 & 3.032157 \\ \mathrm{H} & 1.237524 & -0.010415 & 3.032155 \\ \mathrm{C} & 1.491211 & 0.058408 & -1.572923 \\ \mathrm{C} & 2.888898 & 0.011088 & -1.534368 \\ \mathrm{C} & 3.574882 & 0.004340 & -0.305840 \\ \mathrm{C} & 2.843384 & -0.036123 & 0.889888 \\ \mathrm{H} & 1.002281 & 0.086585 & -2.538340 \\ \mathrm{H} & 3.445326 & 0.051253 & -2.463197 \\ \mathrm{H} & 4.656710 & -0.033027 & -0.283890 \\ \mathrm{H} & 3.360650 & -0.044511 & 1.844061 \\ \mathrm{C} & -3.574878 & -0.004372 & -0.305821\end{array}$




$\begin{array}{lrrr}\mathrm{C} & -2.888907 & -0.010946 & -1.534355 \\ \mathrm{H} & -1.002291 & -0.085909 & -2.538366 \\ \mathrm{H} & -3.445349 & -0.051160 & -2.463174 \\ \mathrm{H} & -4.656705 & 0.033023 & -0.283863 \\ \mathrm{H} & -3.360647 & 0.043904 & 1.844090\end{array}$

2,4,6,8-decatetraene $\mathrm{S}_{0}$

$\begin{array}{lrrr}\mathrm{C} & -2.588135 & -5.006750 & -0.008319 \\ \mathrm{C} & -2.475492 & -3.517110 & -0.005991 \\ \mathrm{C} & -1.311313 & -2.839386 & 0.028122 \\ \mathrm{C} & -1.215988 & -1.399751 & 0.031986 \\ \mathrm{C} & -0.046572 & -0.717349 & -0.043219 \\ \mathrm{C} & 0.046614 & 0.717272 & -0.042290 \\ \mathrm{C} & 1.216240 & 1.399564 & 0.030617 \\ \mathrm{C} & 1.311561 & 2.839203 & 0.028564 \\ \mathrm{C} & 2.475636 & 3.516976 & -0.008036 \\ \mathrm{C} & 2.588280 & 5.006617 & -0.008464 \\ \mathrm{H} & -0.376700 & -3.396888 & 0.038949 \\ \mathrm{H} & -2.148467 & -0.839151 & 0.061315 \\ \mathrm{H} & 0.886333 & -1.277422 & -0.070649 \\ \mathrm{H} & -0.886363 & 1.277383 & -0.066257 \\ \mathrm{H} & 2.148792 & 0.838921 & 0.056590 \\ \mathrm{H} & 0.376987 & 3.396690 & 0.043027 \\ \mathrm{H} & 3.404285 & 2.950978 & -0.026518 \\ \mathrm{H} & -3.404194 & -2.951091 & -0.020789 \\ \mathrm{H} & 1.603546 & 5.474136 & 0.011162 \\ \mathrm{H} & 3.149210 & 5.357809 & 0.860634 \\ \mathrm{H} & 3.117431 & 5.361014 & -0.895887 \\ \mathrm{H} & -1.603345 & -5.474292 & 0.007651 \\ \mathrm{H} & -3.146453 & -5.359250 & 0.861931 \\ \mathrm{H} & -3.119946 & -5.359818 & -0.894680\end{array}$

2,4,6,8-decatetraene $S_{1}$

$\begin{array}{lrrr}\text { C } & -2.572020 & -5.015465 & 0.016090 \\ \text { C } & -2.528879 & -3.530134 & -0.006184 \\ \text { C } & -1.360502 & -2.795016 & -0.012443 \\ \text { C } & -1.279565 & -1.396130 & -0.069460 \\ \text { C } & -0.056911 & -0.687150 & -0.079545 \\ \text { C } & 0.057220 & 0.687437 & 0.078955 \\ \text { C } & 1.279834 & 1.396485 & 0.068716 \\ \text { C } & 1.360625 & 2.795406 & 0.012327 \\ \text { C } & 2.528926 & 3.530643 & 0.005762 \\ \text { C } & 2.571903 & 5.015986 & -0.015942 \\ \text { H } & -0.419167 & -3.344526 & 0.002516\end{array}$




$\begin{array}{lrrc}\mathrm{H} & -2.203813 & -0.819801 & -0.059353 \\ \mathrm{H} & 0.859516 & -1.270727 & -0.161764 \\ \mathrm{H} & -0.859214 & 1.270963 & 0.161501 \\ \mathrm{H} & 2.204112 & 0.820225 & 0.057609 \\ \mathrm{H} & 0.419232 & 3.344837 & -0.001847 \\ \mathrm{H} & 3.476872 & 2.996052 & 0.023250 \\ \mathrm{H} & -3.476763 & -2.995452 & -0.024310 \\ \mathrm{H} & 1.566329 & 5.438422 & -0.037900 \\ \mathrm{H} & 3.088577 & 5.417086 & 0.863704 \\ \mathrm{H} & 3.118008 & 5.391151 & -0.889014 \\ \mathrm{H} & -1.566503 & -5.437990 & 0.038987 \\ \mathrm{H} & -3.118827 & -5.390220 & 0.888896 \\ \mathrm{H} & -3.088055 & -5.416882 & -0.863789\end{array}$

tetracene $\mathrm{S}_{0}$

$\begin{array}{lrrr}\mathrm{C} & 3.697460 & 1.406324 & -0.010274 \\ \mathrm{C} & 2.445456 & 0.722233 & 0.032128 \\ \mathrm{C} & 2.445460 & -0.722235 & -0.032143 \\ \mathrm{C} & 3.697468 & -1.406320 & 0.010254 \\ \mathrm{C} & 4.878973 & -0.711782 & -0.011127 \\ \mathrm{C} & 4.878969 & 0.711792 & 0.011119 \\ \mathrm{C} & 1.230844 & 1.406856 & -0.012165 \\ \mathrm{C} & 0.000469 & 0.724335 & -0.031617 \\ \mathrm{C} & 0.000470 & -0.724351 & 0.031506 \\ \mathrm{C} & 1.230851 & -1.406864 & 0.012139 \\ \mathrm{C} & -1.230160 & 1.405441 & 0.005839 \\ \mathrm{C} & -2.445787 & 0.722275 & -0.024558 \\ \mathrm{C} & -2.445790 & -0.722283 & 0.024522 \\ \mathrm{C} & -1.230164 & -1.405454 & -0.005854 \\ \mathrm{C} & -3.697621 & 1.406465 & 0.022612 \\ \mathrm{C} & -4.878977 & 0.711912 & -0.007678 \\ \mathrm{C} & -4.878984 & -0.711900 & 0.007645 \\ \mathrm{C} & -3.697632 & -1.406464 & -0.022562 \\ \mathrm{H} & -3.695570 & -2.491550 & -0.018955 \\ \mathrm{H} & -5.821429 & -1.245705 & -0.019758 \\ \mathrm{H} & -5.821421 & 1.245727 & 0.019591 \\ \mathrm{H} & -3.695549 & 2.491551 & 0.019198 \\ \mathrm{H} & -1.232261 & -2.492539 & 0.007411 \\ \mathrm{H} & -1.232253 & 2.492528 & -0.007291 \\ \mathrm{H} & 1.231283 & -2.494059 & 0.011842 \\ \mathrm{H} & 1.231267 & 2.494050 & -0.011792 \\ \mathrm{H} & 3.696011 & -2.491377 & 0.000133 \\ \mathrm{H} & 5.821314 & -1.245914 & 0.014029 \\ \mathrm{H} & 5.821307 & 1.245929 & -0.014019 \\ \mathrm{H} & 3.695996 & 2.491381 & -0.000172\end{array}$


tetracene $\mathrm{S}_{1}$

$\begin{array}{lrrr}\mathrm{C} & 3.700743 & 1.398915 & -0.018765 \\ \mathrm{C} & 2.472418 & 0.721041 & 0.025104 \\ \mathrm{C} & 2.472418 & -0.721043 & -0.025195 \\ \mathrm{C} & 3.700740 & -1.398921 & 0.018693 \\ \mathrm{C} & 4.911153 & -0.697736 & -0.013082 \\ \mathrm{C} & 4.911150 & 0.697733 & 0.013262 \\ \mathrm{C} & 1.228462 & 1.398181 & -0.012284 \\ \mathrm{C} & -0.000674 & 0.726742 & 0.035428 \\ \mathrm{C} & -0.000676 & -0.726735 & -0.035573 \\ \mathrm{C} & 1.228456 & -1.398177 & 0.012165 \\ \mathrm{C} & -1.229291 & 1.400327 & 0.014837 \\ \mathrm{C} & -2.471889 & 0.720945 & -0.035040 \\ \mathrm{C} & -2.471886 & -0.720937 & 0.035040 \\ \mathrm{C} & -1.229291 & -1.400321 & -0.014855 \\ \mathrm{C} & -3.700515 & 1.398486 & 0.007700 \\ \mathrm{C} & -4.911091 & 0.697673 & -0.014370 \\ \mathrm{C} & -4.911086 & -0.697679 & 0.014512 \\ \mathrm{C} & -3.700508 & -1.398482 & -0.007775 \\ \mathrm{H} & -3.701754 & -2.484109 & 0.007726 \\ \mathrm{H} & -5.847421 & -1.242687 & -0.005949 \\ \mathrm{H} & -5.847423 & 1.242676 & 0.006356 \\ \mathrm{H} & -3.701769 & 2.484108 & -0.008138 \\ \mathrm{H} & -1.230000 & -2.488628 & -0.021784 \\ \mathrm{H} & -1.230004 & 2.488633 & 0.021926 \\ \mathrm{H} & 1.230823 & -2.486452 & 0.004762 \\ \mathrm{H} & 1.230832 & 2.486455 & -0.004833 \\ \mathrm{H} & 3.701376 & -2.484613 & 0.009215 \\ \mathrm{H} & 5.847662 & -1.242290 & 0.010515 \\ \mathrm{H} & 5.847667 & 1.242287 & -0.010049 \\ \mathrm{H} & 3.701378 & 2.484609 & -0.009552\end{array}$

perylene $S_{0}$

$\begin{array}{lrrr}\mathrm{C} & -0.246293 & 1.460286 & -2.409583 \\ \mathrm{C} & -0.047902 & 0.731711 & -1.239451 \\ \mathrm{C} & -0.006235 & 1.433285 & 0.002568 \\ \mathrm{C} & 0.057814 & 2.865107 & -0.002361 \\ \mathrm{C} & -0.115221 & 3.559864 & -1.223052 \\ \mathrm{C} & -0.243599 & 2.864518 & -2.406883 \\ \mathrm{C} & 0.175778 & 3.557180 & 1.226262 \\ \mathrm{C} & 0.347454 & 2.857714 & 2.402365 \\ \mathrm{C} & 0.265595 & 1.455820 & 2.412865 \\ \mathrm{C} & 0.095061 & 0.727514 & 1.238448\end{array}$




$\begin{array}{lrrr}\mathrm{C} & 0.048113 & -0.731704 & -1.239440 \\ \mathrm{C} & 0.006272 & -1.433277 & 0.002573 \\ \mathrm{C} & -0.095142 & -0.727505 & 1.238441 \\ \mathrm{C} & -0.057808 & -2.865097 & -0.002361 \\ \mathrm{C} & -0.175766 & -3.557175 & 1.226261 \\ \mathrm{C} & -0.347442 & -2.857714 & 2.402366 \\ \mathrm{C} & -0.265637 & -1.455816 & 2.412860 \\ \mathrm{C} & 0.246406 & -1.460294 & -2.409579 \\ \mathrm{C} & 0.243542 & -2.864527 & -2.406893 \\ \mathrm{C} & 0.115126 & -3.559862 & -1.223060 \\ \mathrm{H} & 0.209940 & 4.641505 & 1.215909 \\ \mathrm{H} & 0.469950 & 3.389517 & 3.338662 \\ \mathrm{H} & 0.383709 & 0.937095 & 3.356110 \\ \mathrm{H} & -0.209818 & -4.641504 & 1.215918 \\ \mathrm{H} & -0.469831 & -3.389519 & 3.338677 \\ \mathrm{H} & -0.383831 & -0.937094 & 3.356096 \\ \mathrm{H} & 0.091204 & -4.644489 & -1.217335 \\ \mathrm{H} & 0.391648 & -3.399329 & -3.337783 \\ \mathrm{H} & 0.350062 & -0.945610 & -3.356753 \\ \mathrm{H} & -0.091499 & 4.644495 & -1.217314 \\ \mathrm{H} & -0.391897 & 3.399307 & -3.337749 \\ \mathrm{H} & -0.349753 & 0.945599 & -3.356777\end{array}$

perylene $S_{1}$

$\begin{array}{lrrr}\text { C } & 0.020386 & 1.465986 & -2.455490 \\ \mathrm{C} & -0.018081 & 0.715380 & -1.240064 \\ \mathrm{C} & 0.020600 & 1.418238 & -0.000003 \\ \mathrm{C} & -0.022910 & 2.856830 & 0.000002 \\ \mathrm{C} & 0.018043 & 3.547303 & -1.229671 \\ \mathrm{C} & -0.014018 & 2.850374 & -2.439297 \\ \mathrm{C} & 0.018394 & 3.547300 & 1.229665 \\ \mathrm{C} & -0.013239 & 2.850370 & 2.439302 \\ \mathrm{C} & 0.021109 & 1.465981 & 2.455481 \\ \mathrm{C} & -0.017882 & 0.715385 & 1.240065 \\ \mathrm{C} & 0.018076 & -0.715380 & -1.240063 \\ \mathrm{C} & -0.020599 & -1.418238 & -0.000003 \\ \mathrm{C} & 0.017887 & -0.715386 & 1.240066 \\ \mathrm{C} & 0.022909 & -2.856831 & 0.000002 \\ \mathrm{C} & -0.018392 & -3.547300 & 1.229665 \\ \mathrm{C} & 0.013239 & -2.850370 & 2.439302 \\ \mathrm{C} & -0.021108 & -1.465981 & 2.455481 \\ \mathrm{C} & -0.020386 & -1.465986 & -2.455490 \\ \mathrm{C} & 0.014017 & -2.850374 & -2.439297 \\ \mathrm{C} & -0.018046 & -3.547303 & -1.229671 \\ \mathrm{H} & 0.001171 & 4.632554 & 1.221336\end{array}$




$\begin{array}{rrrl}\mathrm{H} & 0.008460 & 3.397393 & 3.375060 \\ \mathrm{H} & -0.001842 & 0.953742 & 3.407547 \\ \mathrm{H} & -0.001160 & -4.632554 & 1.221337 \\ \mathrm{H} & -0.008463 & -3.397393 & 3.375060 \\ \mathrm{H} & 0.001838 & -0.953742 & 3.407548 \\ \mathrm{H} & -0.000811 & -4.632558 & -1.221326 \\ \mathrm{H} & -0.007285 & -3.397391 & -3.375068 \\ \mathrm{H} & 0.003001 & -0.953743 & -3.407545 \\ \mathrm{H} & 0.000800 & 4.632558 & -1.221326 \\ \mathrm{H} & 0.007284 & 3.397391 & -3.375068 \\ \mathrm{H} & -0.002992 & 0.953743 & -3.407545\end{array}$

